
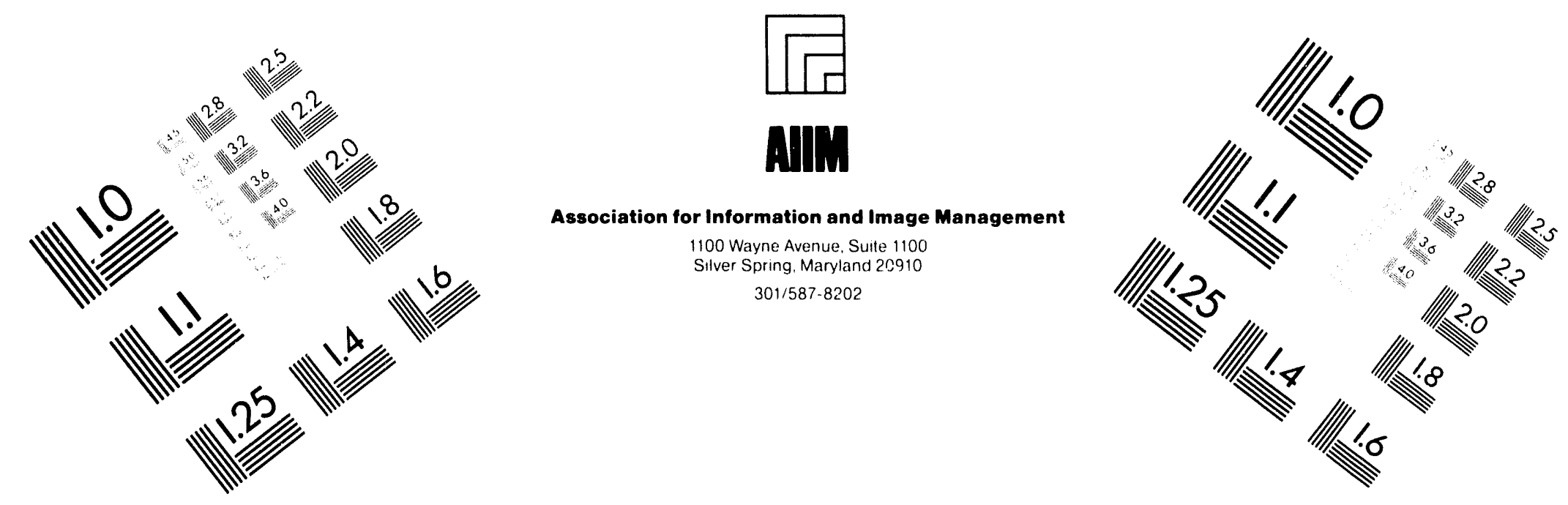

\title{
Centimeter
}

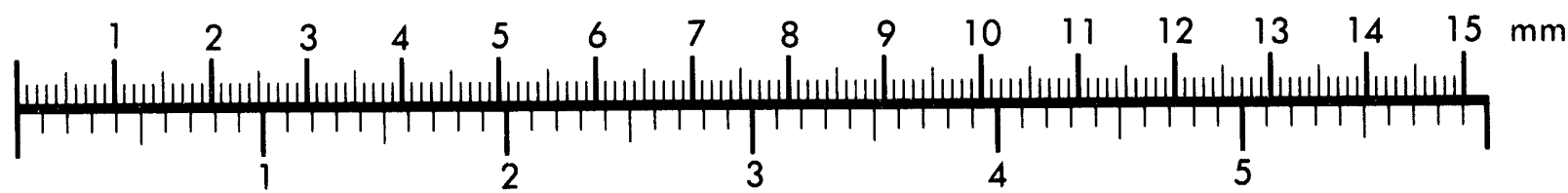

Inches
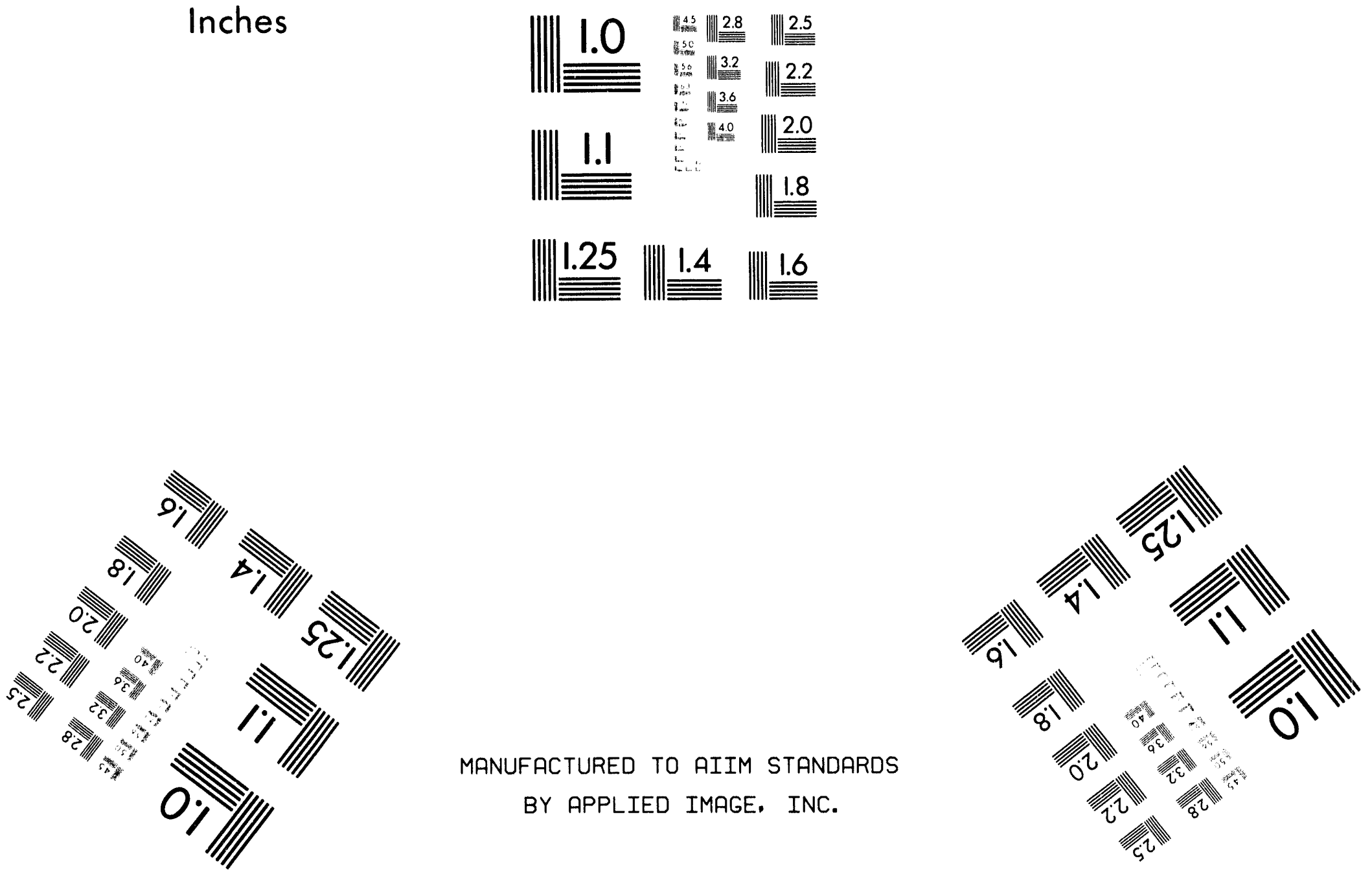

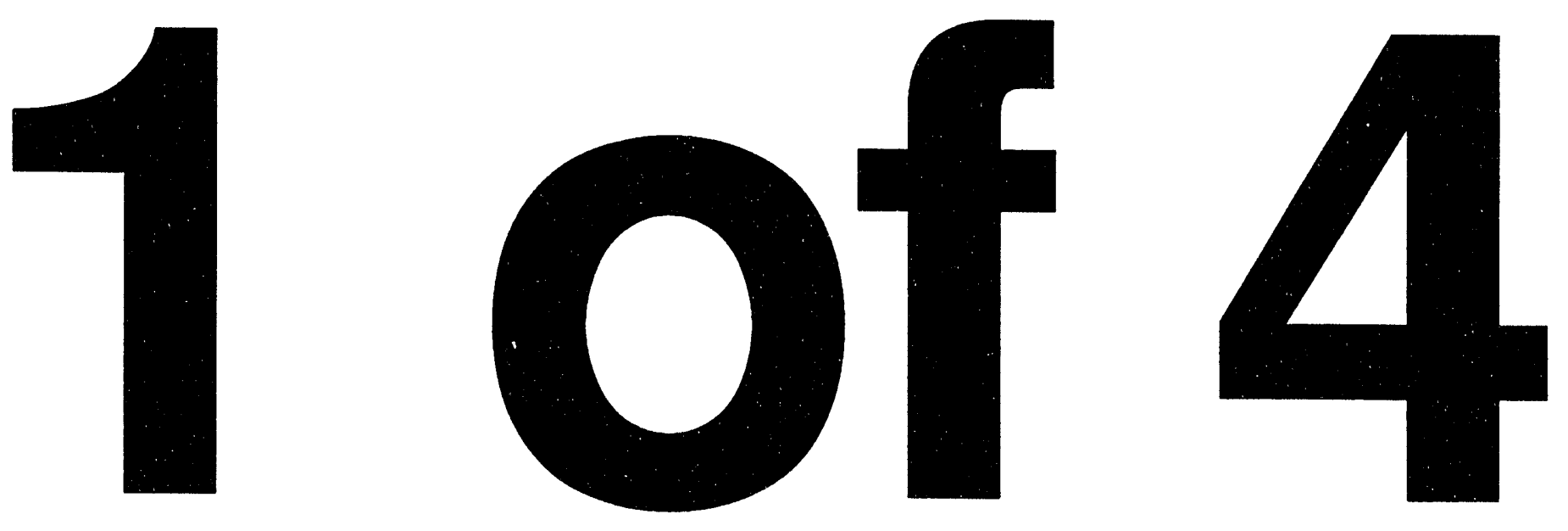


\title{
NRTSC \\ NUCLEAR REACTOR TECHNOLOGY \\ AND SCIENTIFIC COMPUTATIONS
}

\author{
WSR C-TR-92-534 \\ Task No.: 92-104-1
}

KEYWORDS:

ONSET OF FLOW INSTABILTY

ONSET OF SIGNIFICANT VOID

DEMAND CURVES

LOSS OF COOLANT ACCDENT

ANNULUS

RETENTION:

PERMANENT

\section{NUCLEATE BOILING PRESSURE DROP IN AN ANNULUS, BOOK 4 (U)}

James A. Block, Christopher Crowley, Francis X. Dolan, Richard G. Sam, and Brant $H$. Stoedefalke

ISSUED: NOVEMBER, 1992

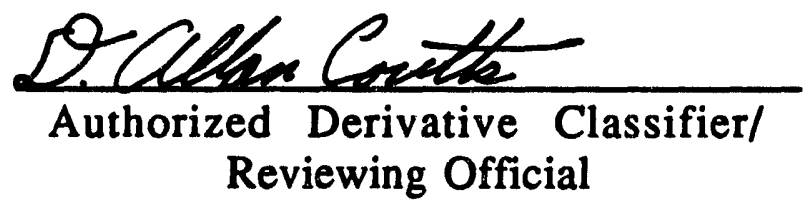

SRL SAVANNAH RIVER TECHNOLOGY CENTER, AIKEN, SC 29808 Westinghouse Savannah River Corporation Prepared for the U.S. Department of Energy under Contract DE-AC'J9-89SR18035 
Final Report

\title{
NUCLEATE BOILNG PRESSURE DROP
} IN AN ANNULUS

Volume 3

DATA PLOTS AND SUMMMARY FILES

\author{
Prepared by \\ James A. Block \\ Christopher J. Crowley \\ Francis X. Dolan \\ Richard G. Sam \\ Brant H.Stoedefalke \\ Prepared for \\ U.S. Department of Energy \\ and \\ Savannah River Laboratory \\ Subcontract AX-721102
}

CREARE INC.

TN-499

HANOVER, NEW HAMPSHIRE 03755

NOVEMBER, 1990 
TABLE OF CONTENTS

Volume 2

TABLE OF CONTENTS $\ldots \ldots \ldots \ldots \ldots \ldots \ldots \ldots \ldots \ldots \ldots \ldots \ldots \ldots \ldots \ldots \ldots$

LIST OF FIGURES $\ldots \ldots \ldots \ldots \ldots \ldots \ldots \ldots \ldots \ldots \ldots \ldots \ldots \ldots \ldots \ldots \ldots \ldots \ldots$

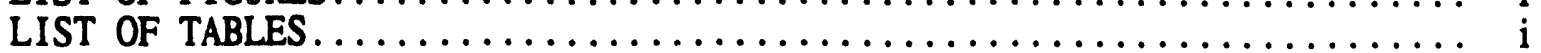

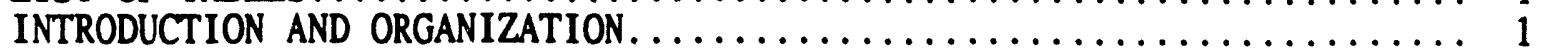

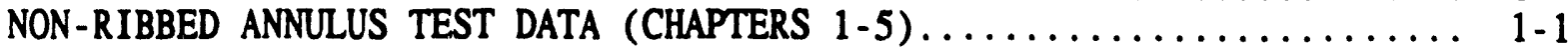

RIBBED ANNULUS TEST DATA (CHAPTEKS $6-11$ )................. $6-1$

Volume 3

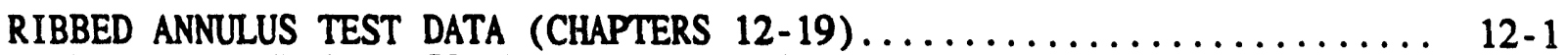

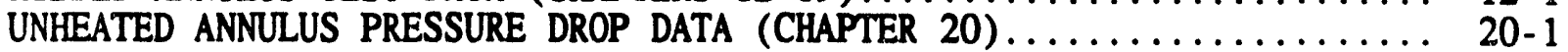

\section{LIST OF FIGURES}

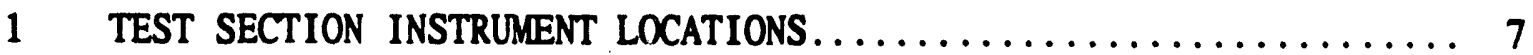

\section{LIST OF TABLES}

1 ANNULUS TEST DATA SUMMARIES ....................... 3

2 DEFINITIONS AND NOMENCLATURE FOR TEST DATA SUMMARY FILES $\ldots \ldots \ldots, 4$

3 INSTRUMENT IDENTIFICATION NUMBERS FOR ANNULUS MEASUREMENTS . . . . 6

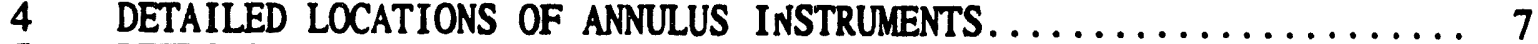

5 DEFECTIVE INSTRUMENTS FOR HEATED ANNULUS TEST SERIES .......... 9 
(5reare

TN-499

\section{Chapter 12}

NUCLEATE BOILING PRESSURE DROP IN AN ANNULUS

Test Geometry: Ribbed (4)

Test Series: 15

Test Date: August 15,1990 


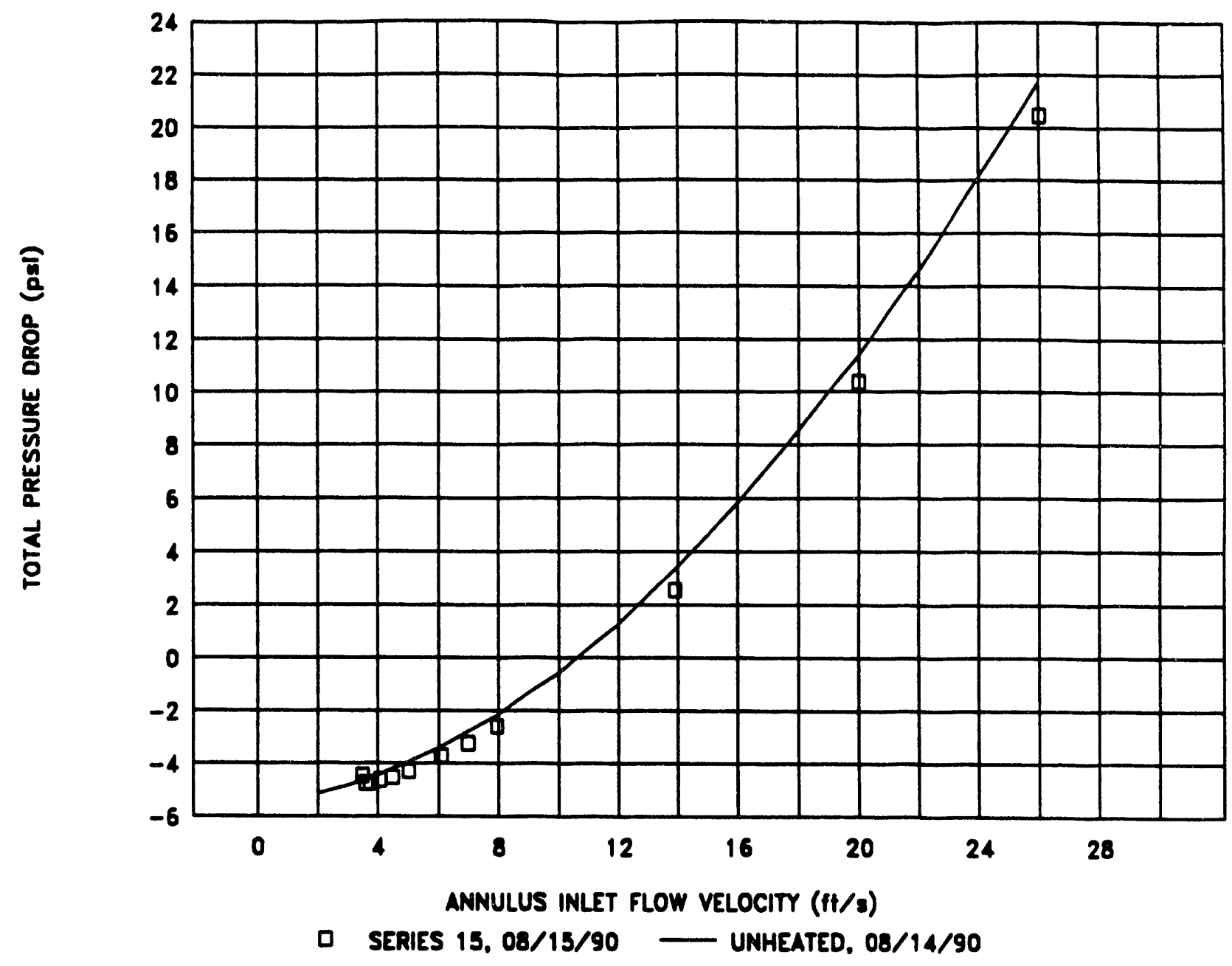

\begin{tabular}{|c|c|c|}
\hline $\begin{array}{c}\text { TEST } \\
\text { NUMBER }\end{array}$ & $\begin{array}{c}\text { INLET } \\
\text { VELOCITY(a) }\end{array}$ & $\begin{array}{c}\text { PRESSURE } \\
\text { DROP(b) }\end{array}$ \\
\hline & $(f / s)$ & (psi) \\
\hline Aug15_01 & 26.03 & 20.46 \\
\hline Aug15_02 & 19.98 & 10.35 \\
\hline Aug15_03 & 13.90 & 2.56 \\
\hline Augi5_04 & 7.94 & -2.60 \\
\hline Aug15_05 & 6.99 & -3.25 \\
\hline Aug15_06 & 6.10 & -3.72 \\
\hline Aug15_07 & 5.00 & -4.29 \\
\hline Aug15_08 & 4.47 & -4.52 \\
\hline Aug15_09 & 4.06 & -4.64 \\
\hline Aug15_12 & 3.73 & -4.73 \\
\hline $4018 \%$ & $80 \%$ & 18 \\
\hline Aug15_11 & 3.49 & -4.46 \\
\hline
\end{tabular}

\begin{tabular}{|l|l|}
\hline \multicolumn{2}{|c|}{ NOMINAL TEST CONDITIONS } \\
\hline Annulus geometry & ribbed (4) \\
Inlet temperature & $86^{\circ} \mathrm{F}$ \\
Inlet pressure & $40 \mathrm{psia}$ \\
Wall heat flux & $100 \mathrm{kBtu} / \mathrm{hr}-\mathrm{ft} 2$ \\
Flux distribution & tilt \\
He saturation pressure & $5 \mathrm{psig}$ \\
\hline
\end{tabular}
NOTES:
(a) Iniet velocity calculated from PFM000001
(b) Pressure drop calculated from PAN000136
(c) Shading notes test at minimum pressure drop 

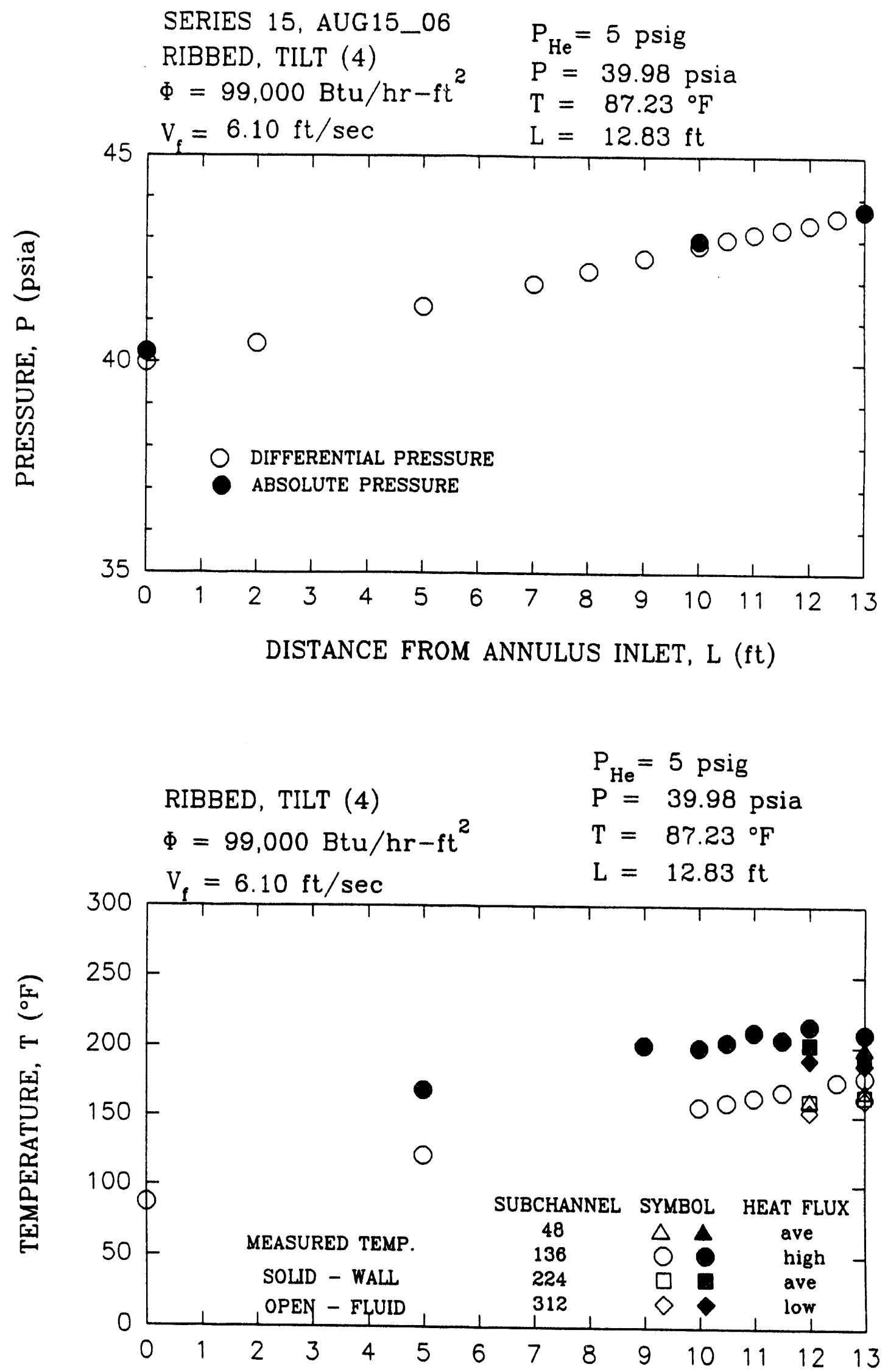

DISTANCE FROM ANNULUS INLET, L (ft) 

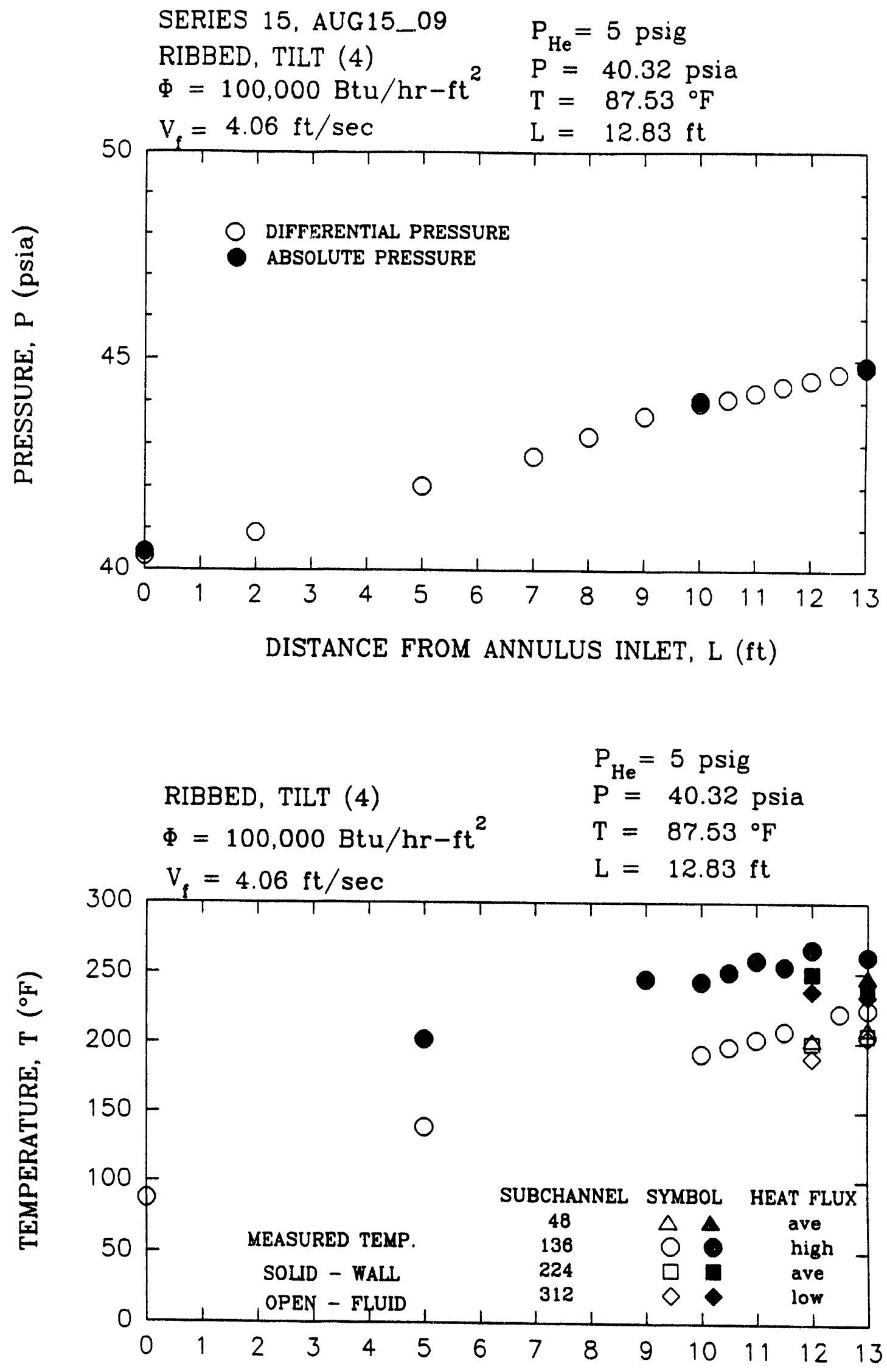

DISTANCE FROM ANNULUS INLET, L (ft) 

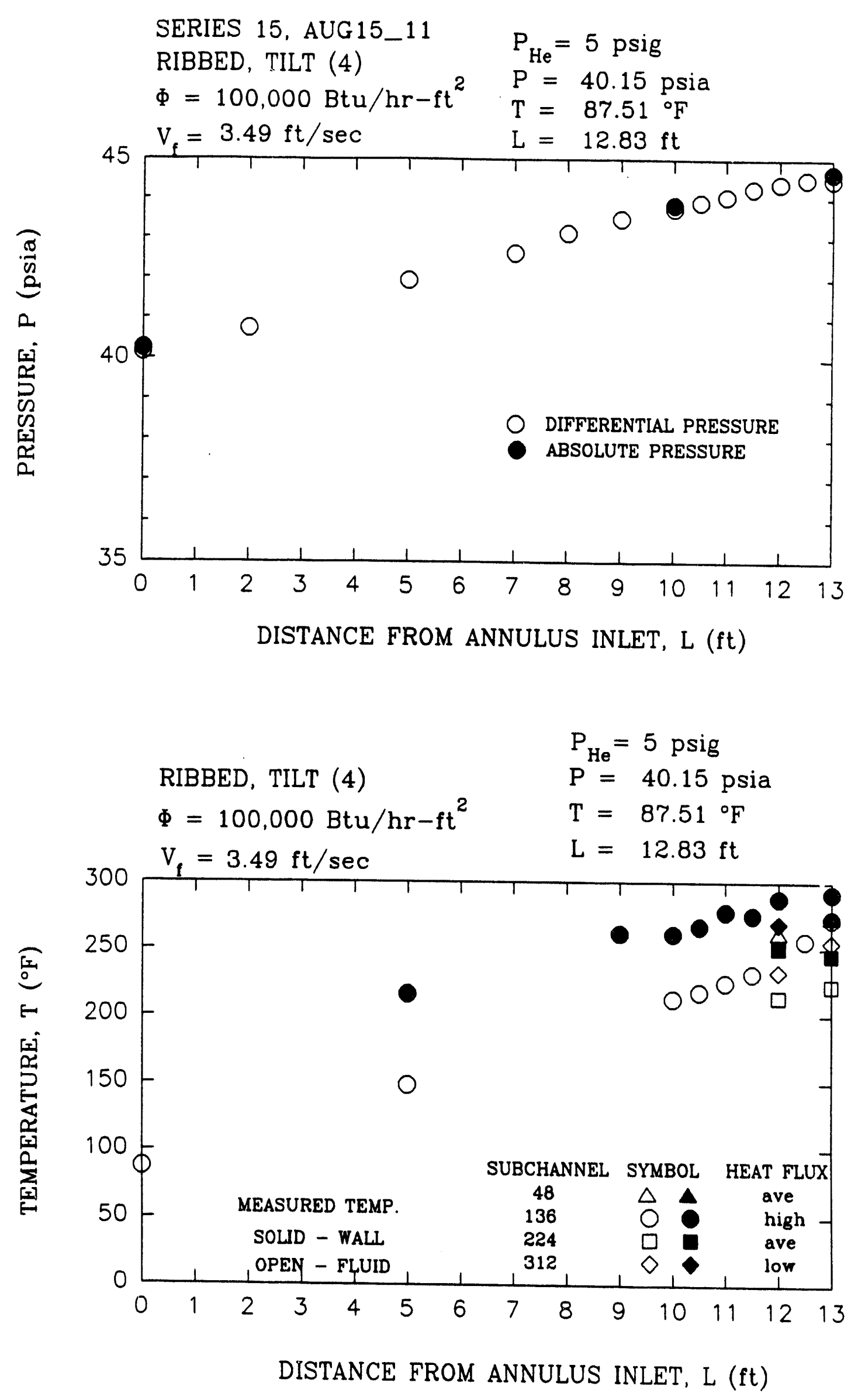


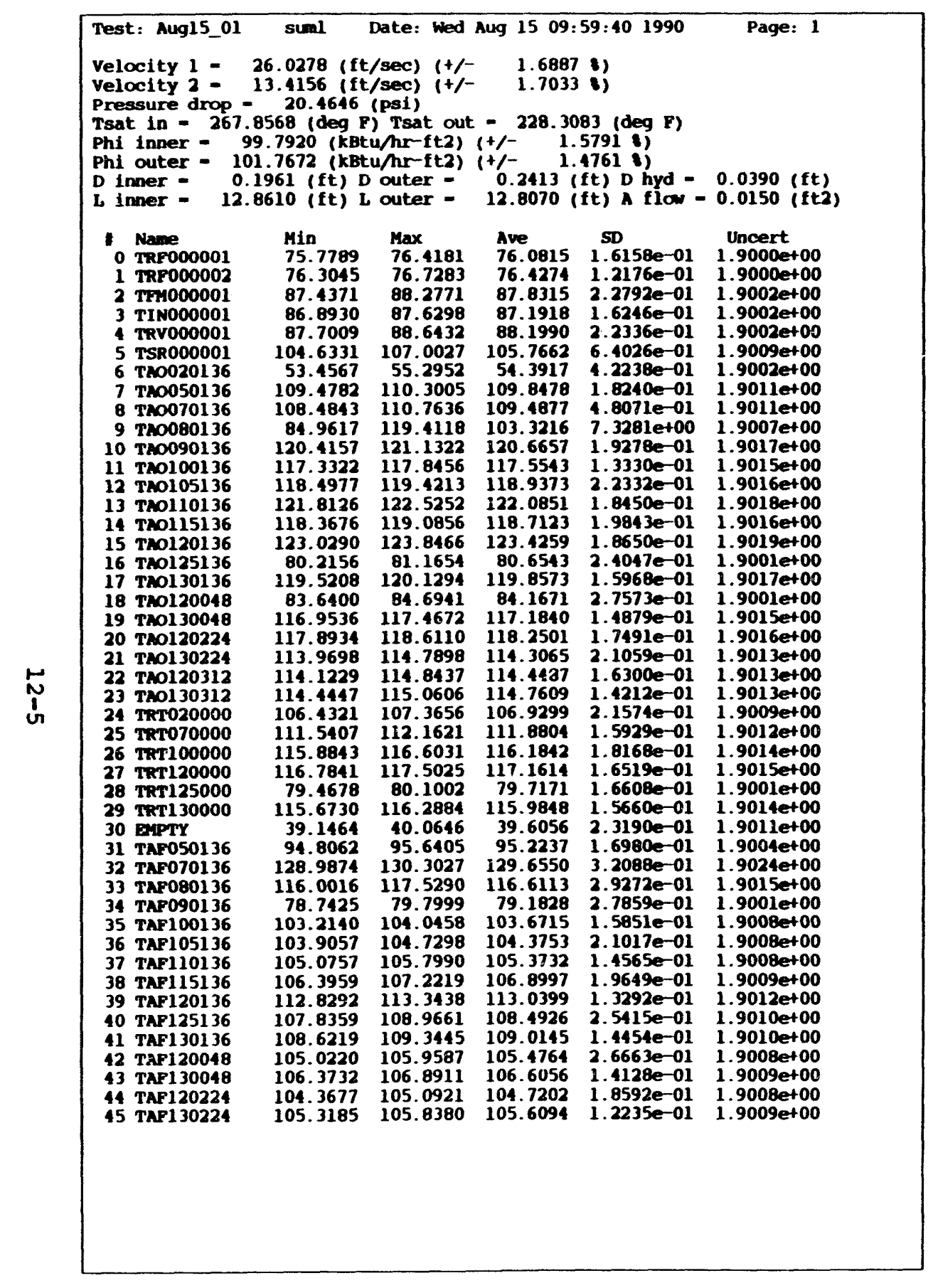

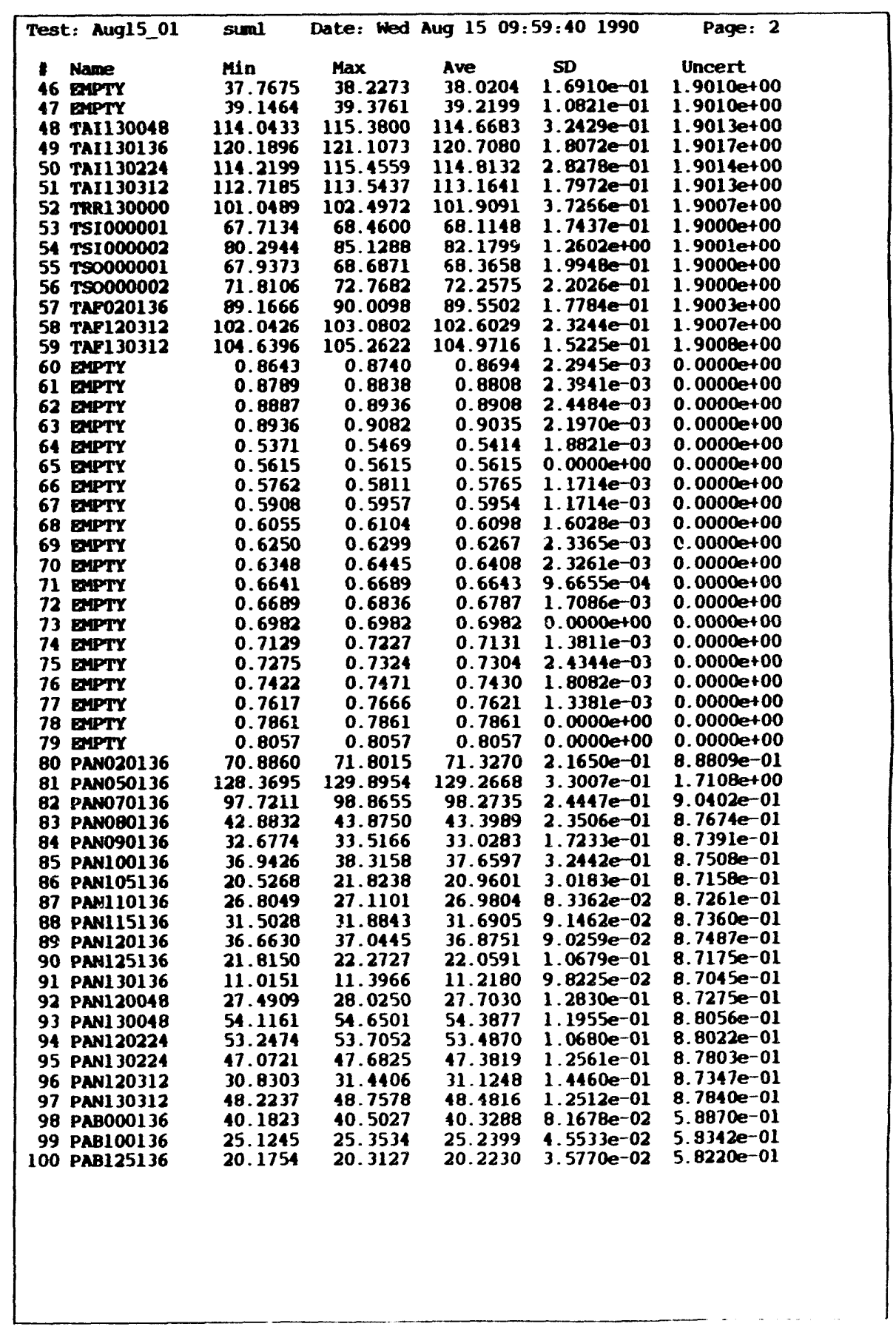




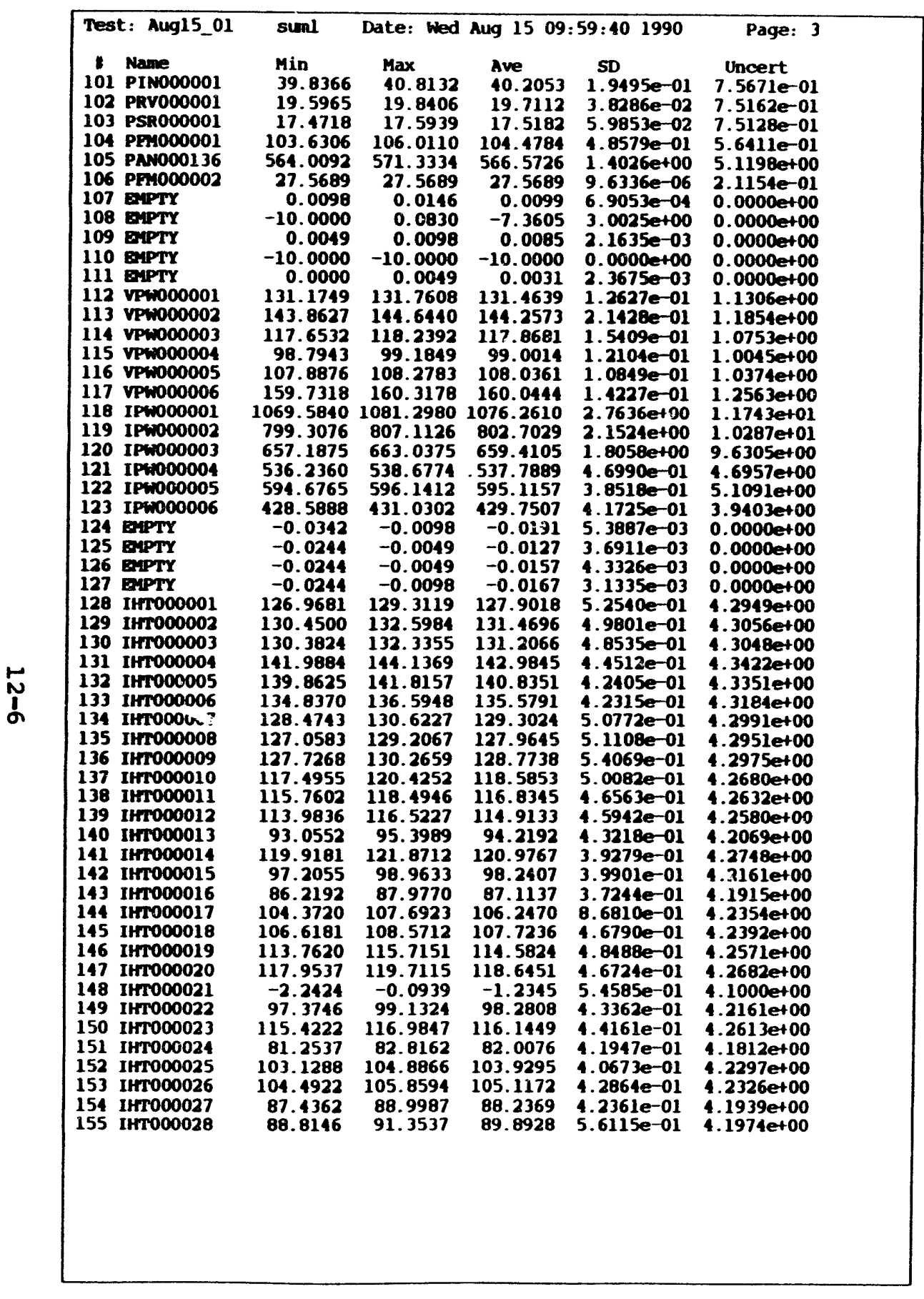

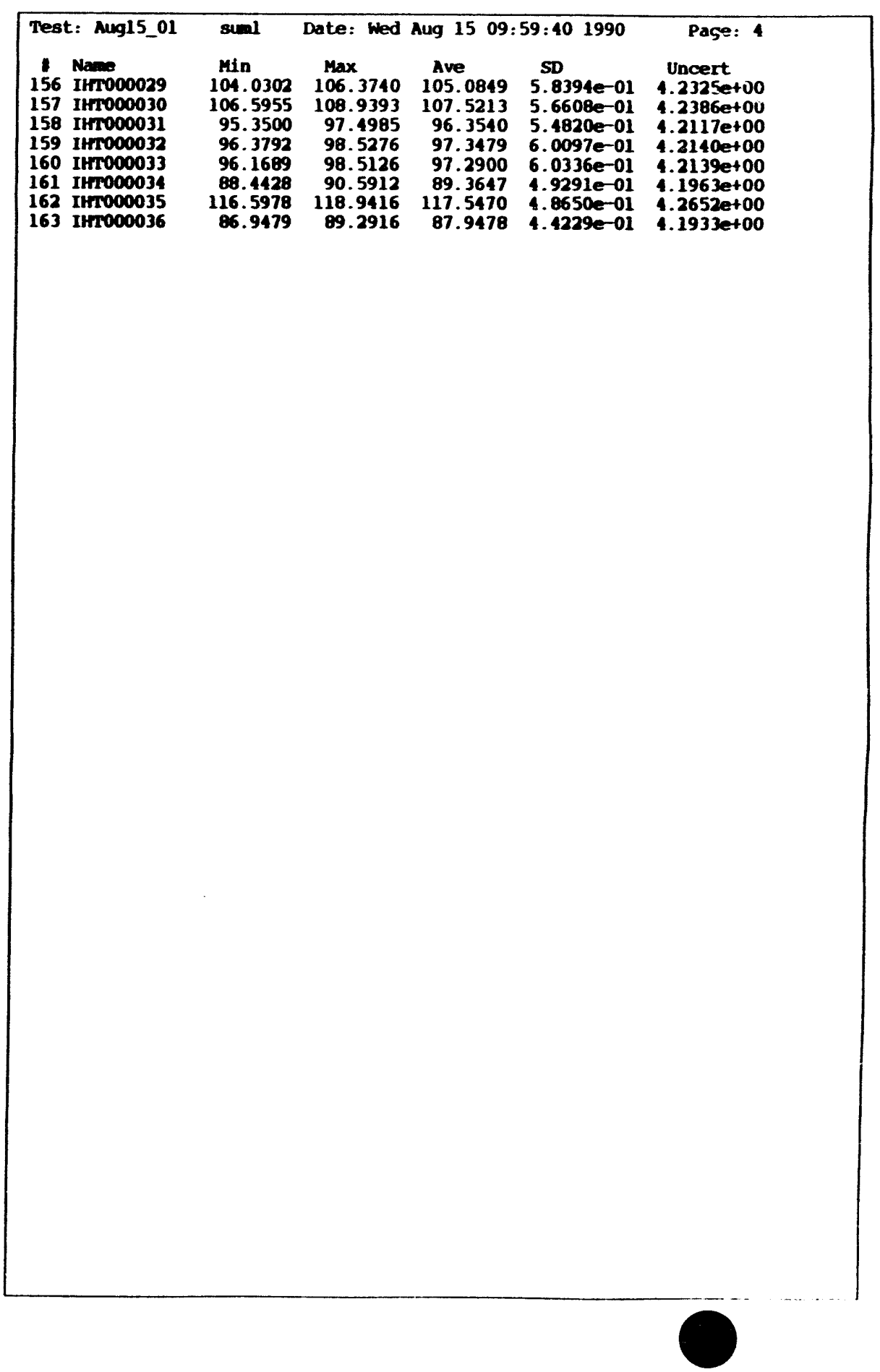




\begin{tabular}{|c|c|c|c|c|c|c|}
\hline \multicolumn{2}{|r|}{ st: Aug15_0 } & \multicolumn{4}{|c|}{510} & \multirow[b]{2}{*}{$\begin{array}{l}\text { Page: } 1 \\
\\
0.0390 \text { (ft) } \\
0.0150 \text { (ft ) }\end{array}$} \\
\hline & $\begin{array}{l}\text { ty } 1= \\
\text { ty } 2= \\
\text { re drop } \\
\text { n= } 26 \\
\text { ner }= \\
\text { ter }= \\
x= \\
y=1\end{array}$ & 48 & i) & $\begin{array}{l}1.7 \\
1.7\end{array}$ & eq & \\
\hline & me & $\begin{array}{r}\operatorname{Min} \\
75.7789 \\
76.3045 \\
85.3379 \\
84.6824 \\
85.7136 \\
104.3228 \\
53.8900 \\
113.6768 \\
112.4186 \\
79.1997\end{array}$ & $\begin{array}{r}\operatorname{Max} \\
76.6311 \\
76.7283 \\
86.3893 \\
85.7361 \\
86.5544 \\
106.6949 \\
55.5119 \\
114.9073 \\
114.3814 \\
135.4904\end{array}$ & $\begin{array}{r}\text { Ave } \\
76.2307 \\
76.5715 \\
85.8575 \\
85.3585 \\
86.1524 \\
105.5015 \\
54.8264 \\
114.2294 \\
113.3301\end{array}$ & $\begin{array}{c}\text { SD } \\
2.0479 e-01 \\
1.2705 e-01 \\
2.0969 e-01 \\
1.8942 e-01 \\
2.1067 e-01 \\
5.4985 e-01 \\
3.8742 e-01 \\
2.3792 e-01 \\
4.6512 e-01 \\
1.0261 e+01 \\
1.9530 e-01 \\
1.8822 e-01 \\
2.8153 e-01 \\
1.9835 e-01 \\
1.9342 e-01 \\
2.0478 e-01 \\
1.7804 e+00 \\
2.1152 e-01 \\
3.3439 e-01 \\
1.8369 e-01 \\
1.5542 e-01 \\
2.1282 e-01\end{array}$ & $\begin{array}{l}\text { Uncert } \\
1.9000 \text { et } 00\end{array}$ \\
\hline
\end{tabular}

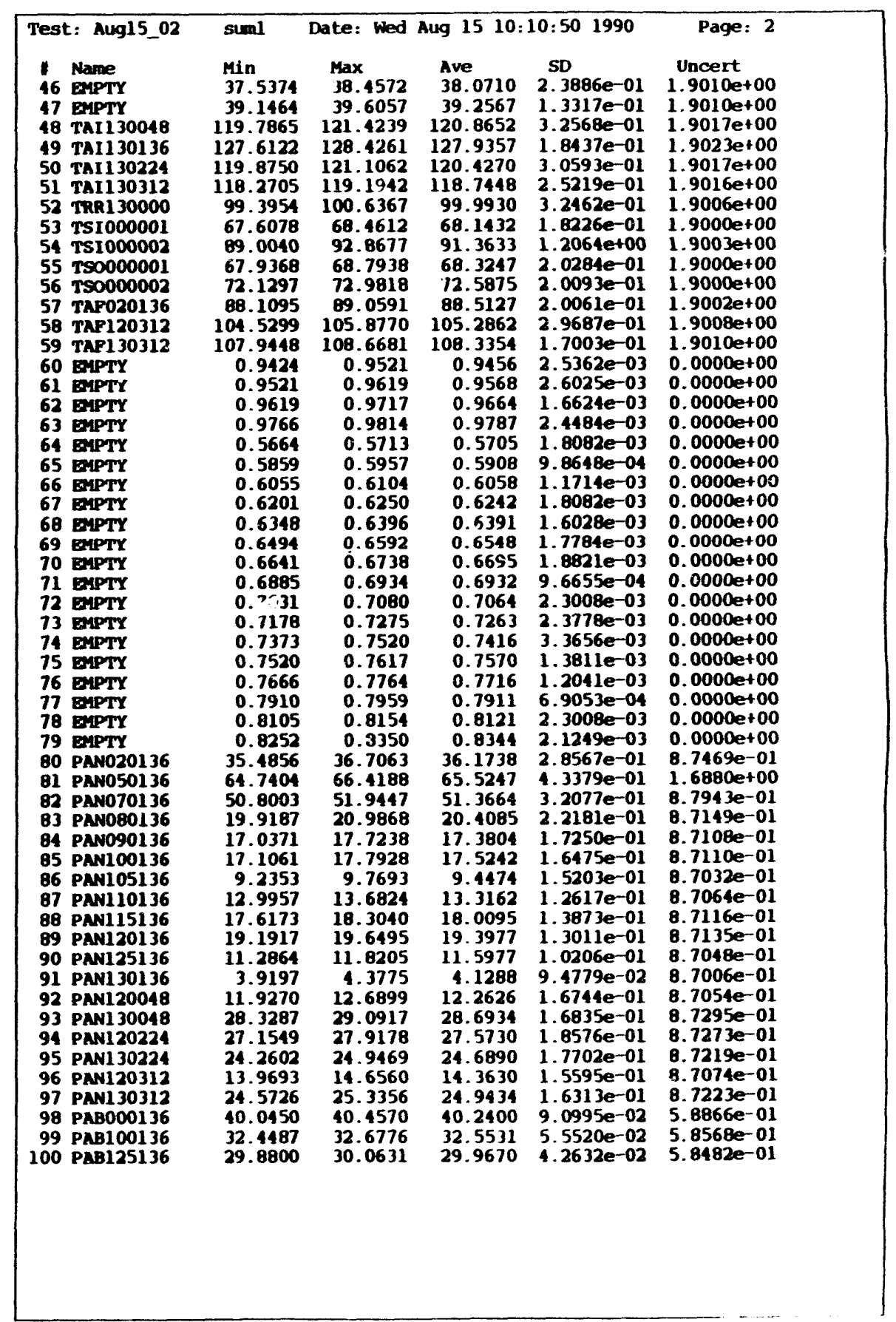




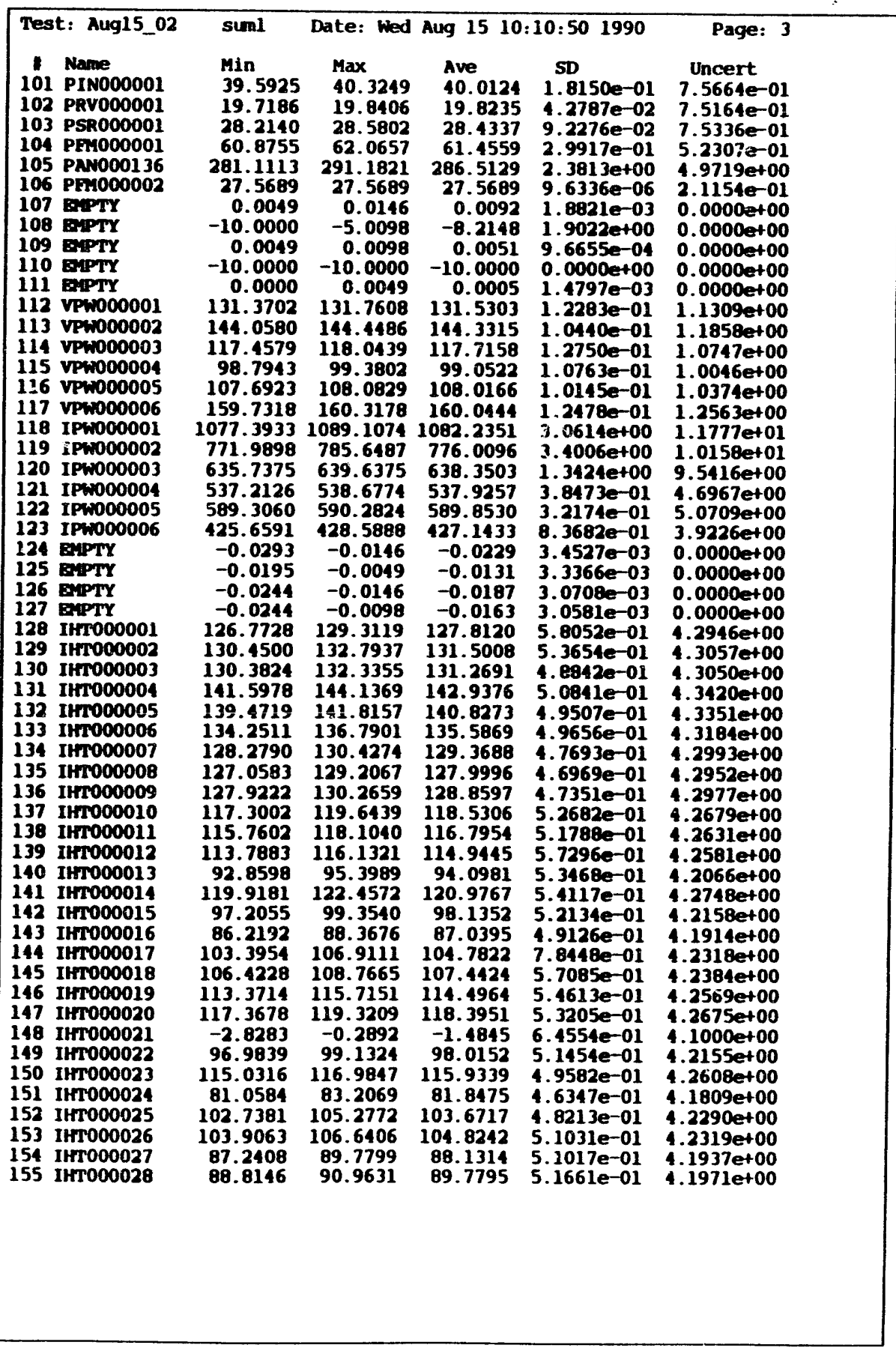

\begin{tabular}{|c|c|c|c|c|c|}
\hline Test: Au & suml & Date: Hed & Aug 15 10: & 90 & Page: \\
\hline 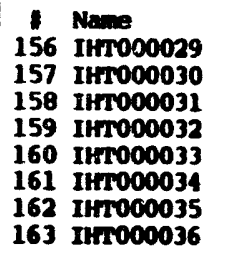 & $\begin{array}{r}\operatorname{Min} \\
103.8349 \\
106.2049 \\
95.1547 \\
96.1839 \\
95.9736 \\
88.6381 \\
116.5978 \\
87.1432\end{array}$ & $\begin{array}{r}\operatorname{Max} \\
106.1786 \\
108.5487 \\
97.4985 \\
98.3323 \\
98.3173 \\
90.5912 \\
118.5509 \\
89.0963\end{array}$ & $\begin{array}{r}\text { Ave } \\
104.8505 \\
107.2245 \\
96.1235 \\
97.1565 \\
97.1298 \\
89.3725 \\
117.4884 \\
87.9986\end{array}$ & $\begin{array}{l}\text { SD } \\
4.8004 \mathrm{e}-01 \\
4.7726 \mathrm{e}-01 \\
4.6682 \mathrm{e}-01 \\
4.9676 \mathrm{e}-01 \\
4.9417 \mathrm{e}-01 \\
4.0349 \mathrm{e}-01 \\
4.0464 \mathrm{e}-01 \\
4.0219 \mathrm{e}-01\end{array}$ & 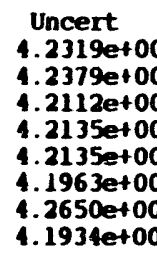 \\
\hline
\end{tabular}


Test: Aug15 03 suml Date: Hed Aug 15 10:26:35 1990 Page: 1

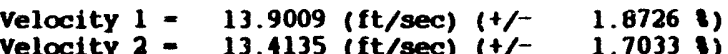

Pressure drop - 2.5648 (psi)

Tsat in -267.3005 (deg F) Tsat out -263.2042 (deg F)

Phi inner - 98.4054 (kBtu/hr-ft2) $(+/-\quad 1.5925$ \&)

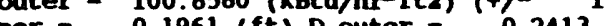

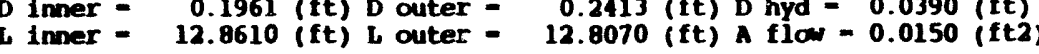

0

0 TRP000001

1 TRF000002

3 TIN000001

4 Trv000001

5 TSR000001

6 Th0020136

7 Th0050136

8 Th0070136

9 Th0080136

11 Th0090136

12 T20105136

13 Tho110136

14 TM0115136

16 Th0125136

17 Tho1 30136

$18 \mathrm{THO120048}$

19
20
ThO120224

21 ThO130224

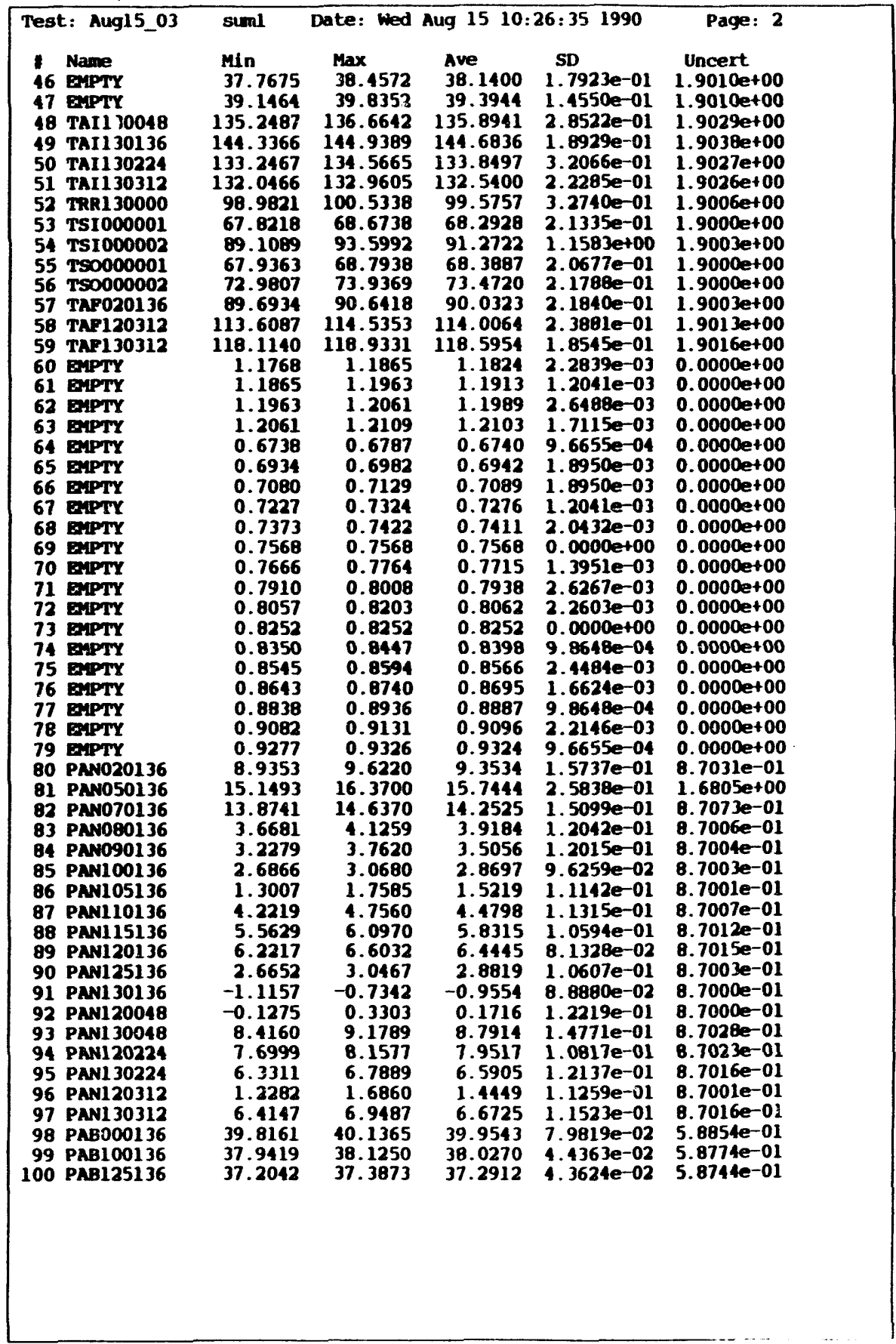




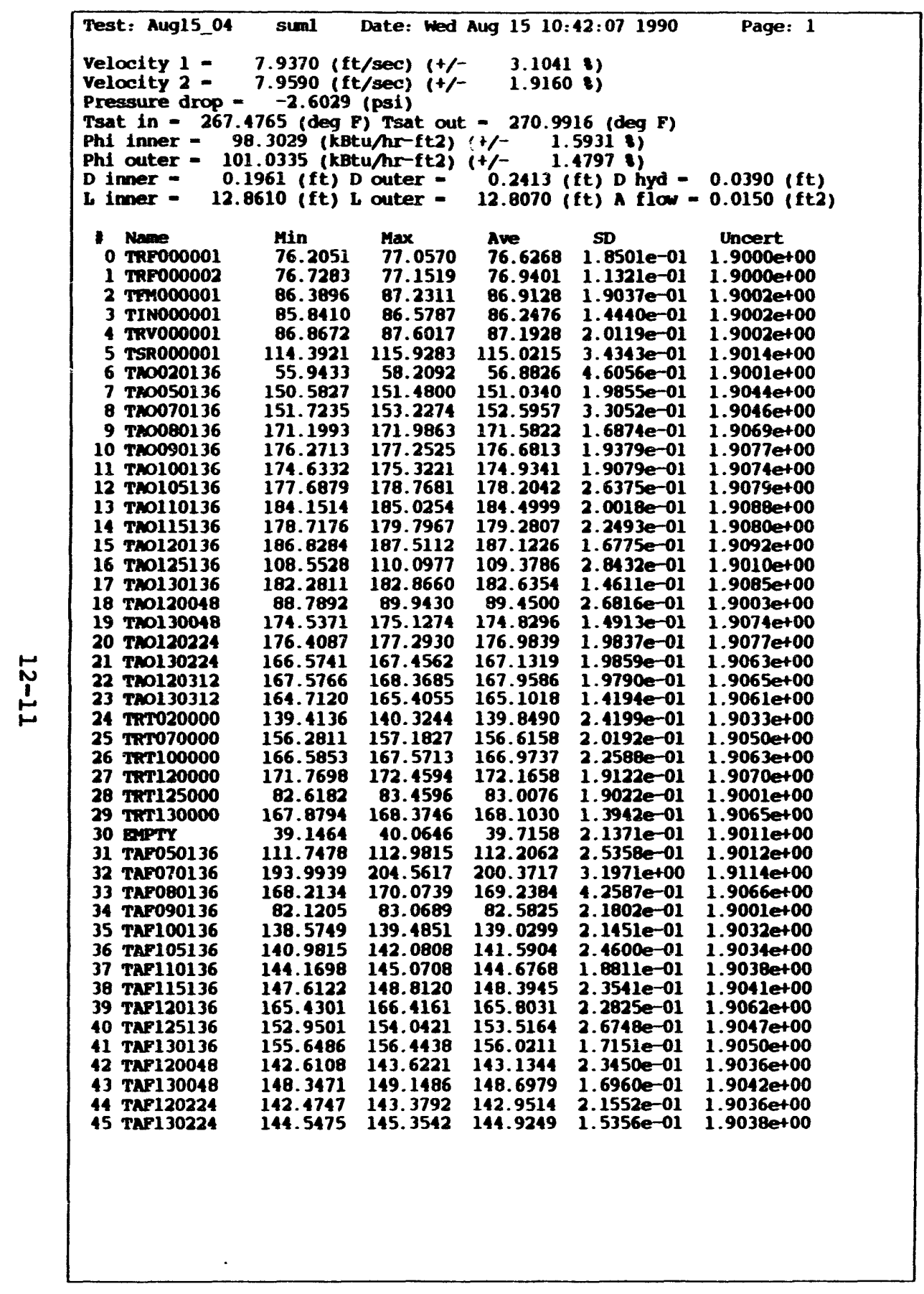

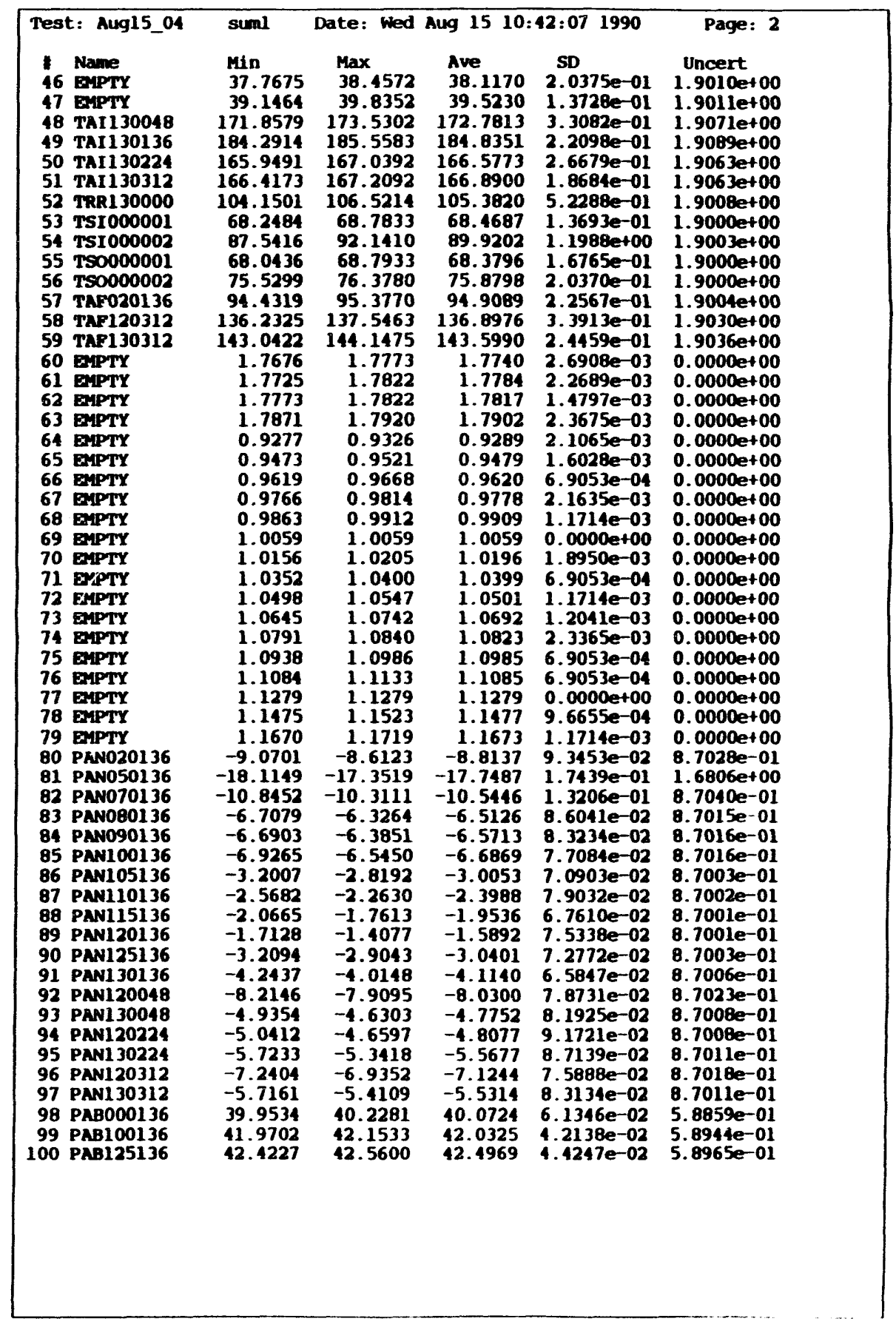



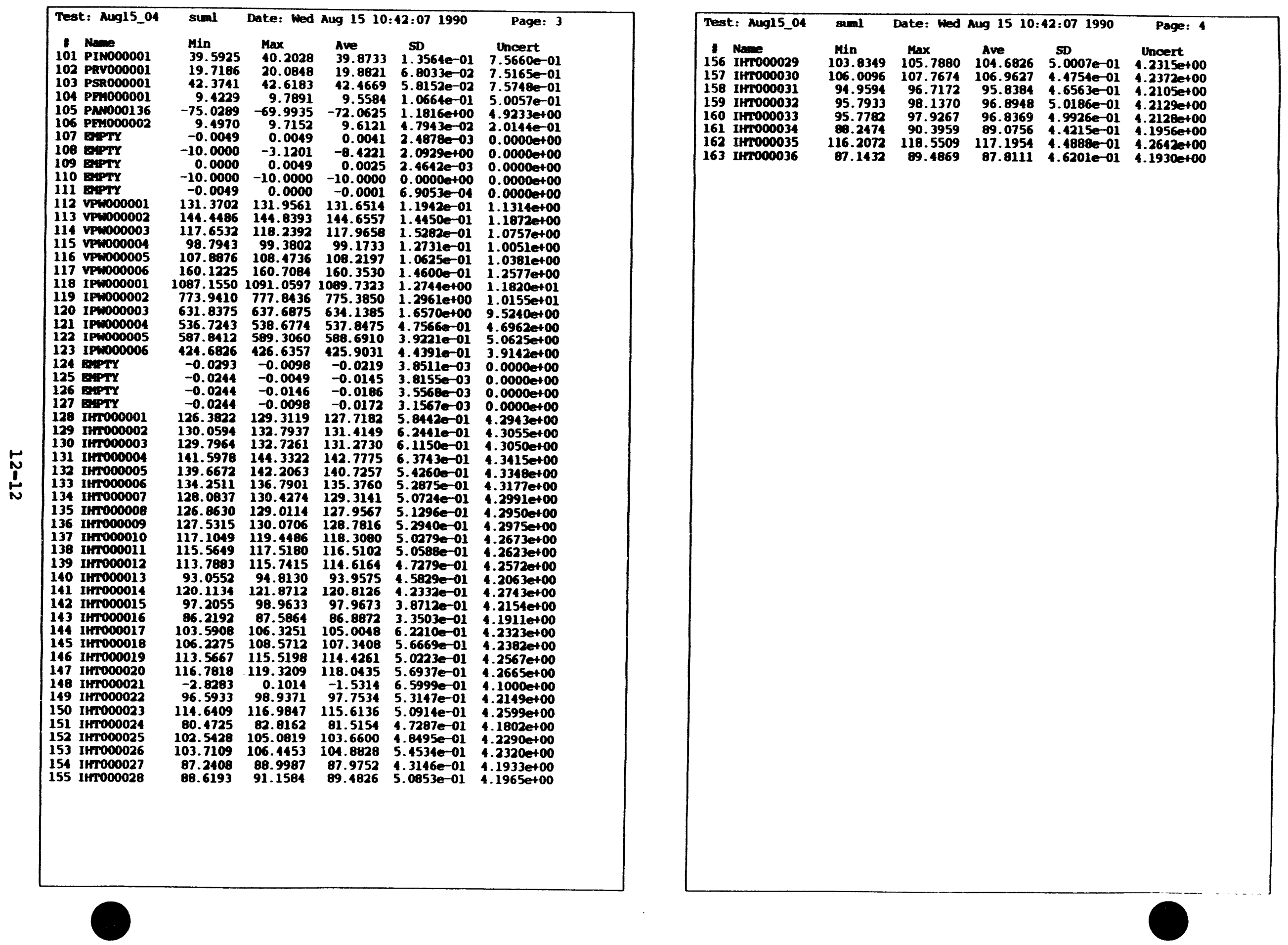


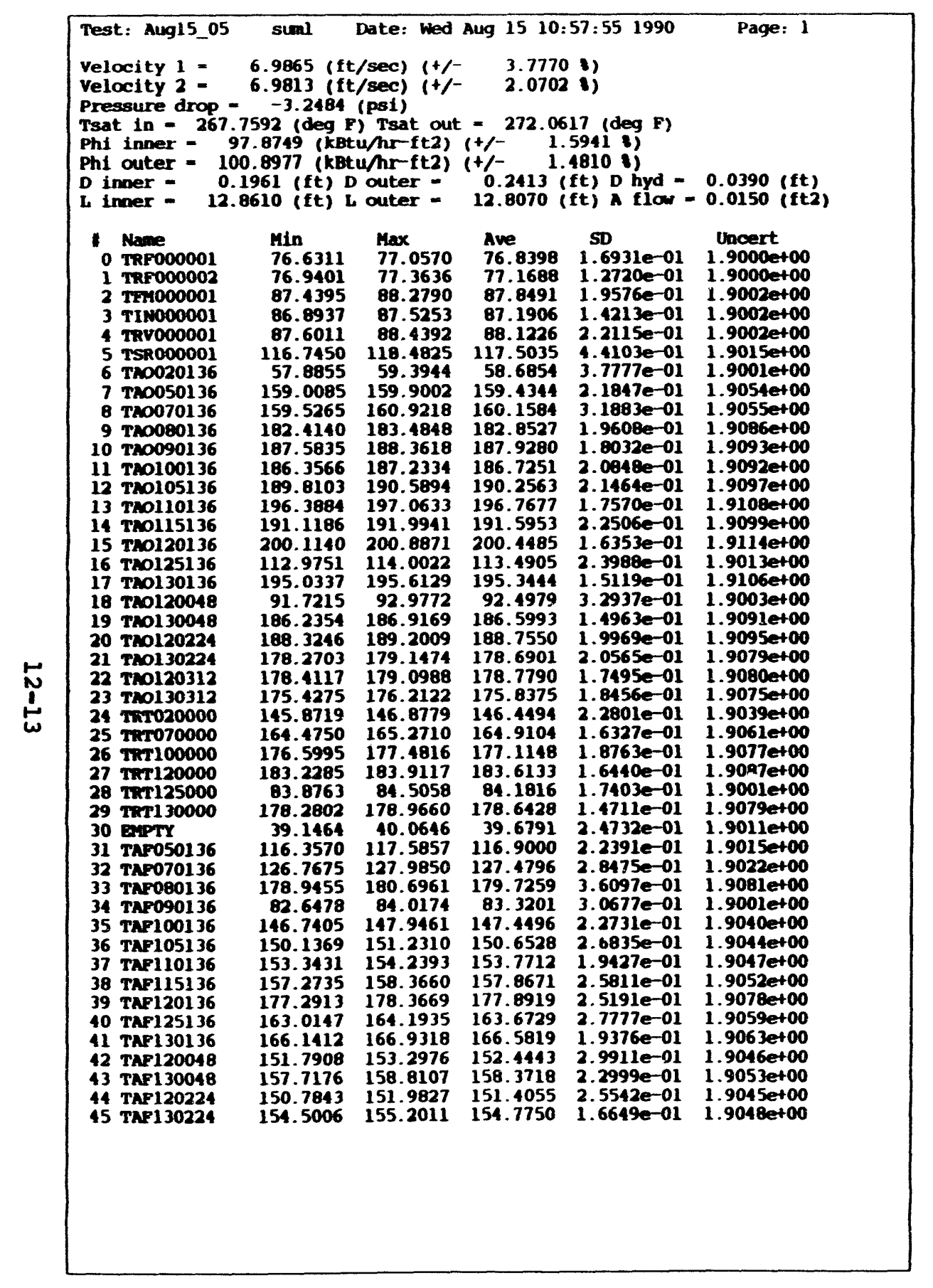

\begin{tabular}{|c|c|c|c|c|c|}
\hline 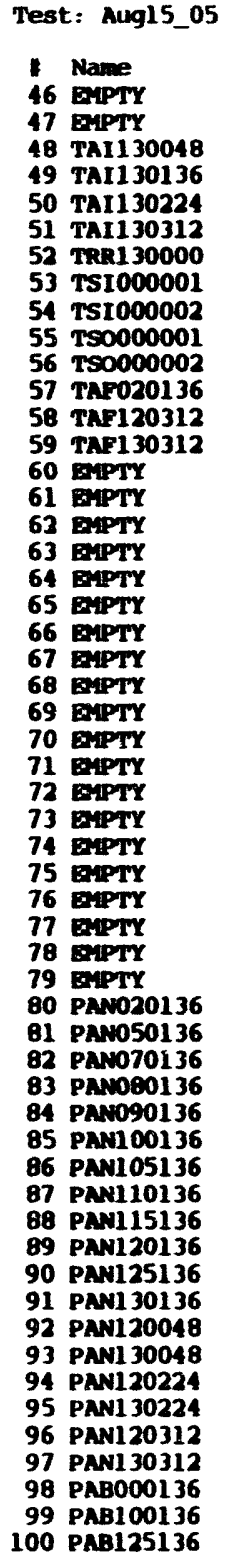 & $\begin{array}{l}\text { suml } \\
\text { Min } \\
37.5374 \\
39.3761 \\
184.2063 \\
197.4587 \\
176.8095 \\
177.0443 \\
103.7395 \\
66.0369 \\
87.8588 \\
67.8286 \\
76.1665 \\
96.9517 \\
144.3911 \\
151.9477 \\
1.9775 \\
1.9824 \\
1.9873 \\
1.9922 \\
1.0205 \\
1.0400 \\
1.0498 \\
1.0693 \\
1.0791 \\
1.0938 \\
1.1035 \\
1.1230 \\
1.1377 \\
1.1523 \\
1.1670 \\
1.1816 \\
1.1914 \\
1.2109 \\
1.2305 \\
1.2500 \\
-11.2063 \\
-22.0821 \\
-13.6681 \\
-7.8523 \\
-7.9110 \\
-8.1472 \\
-3.7347 \\
-3.3312 \\
-3.0583 \\
-2.6284 \\
-3.8198 \\
-4.6252 \\
-9.2065 \\
-6.5376 \\
-6.4906 \\
-7.1729 \\
-8.3085 \\
-7.0894 \\
40.1365 \\
42.5653 \\
13.2009\end{array}$ & 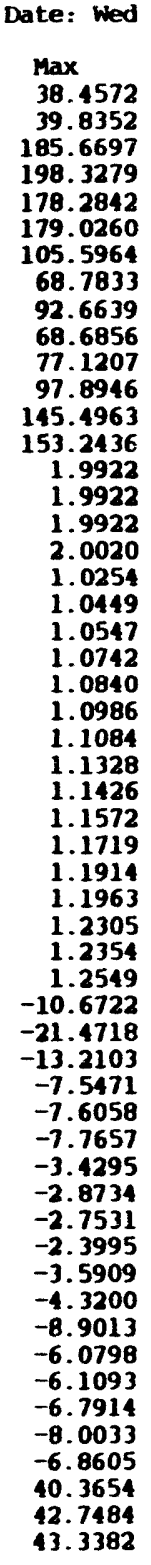 & 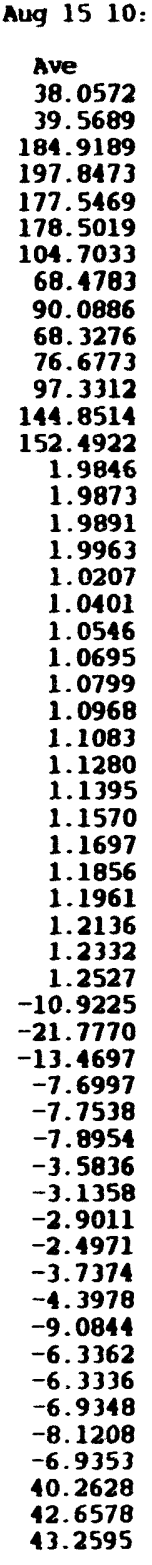 & 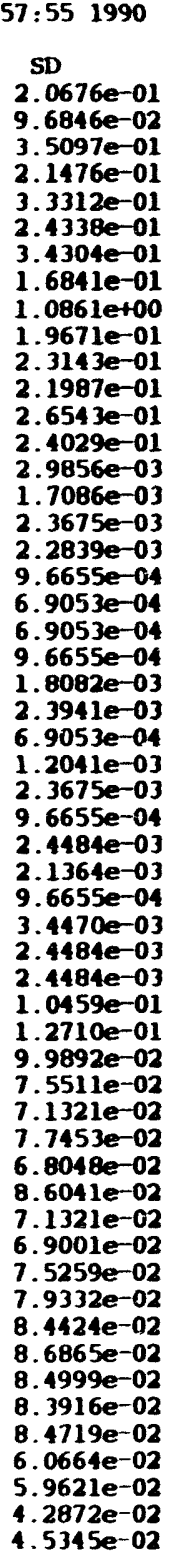 & 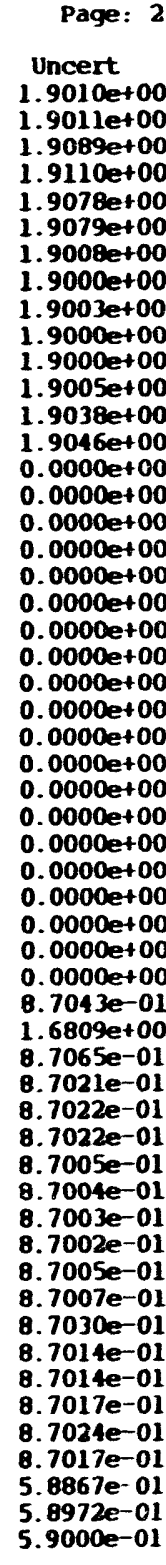 \\
\hline
\end{tabular}




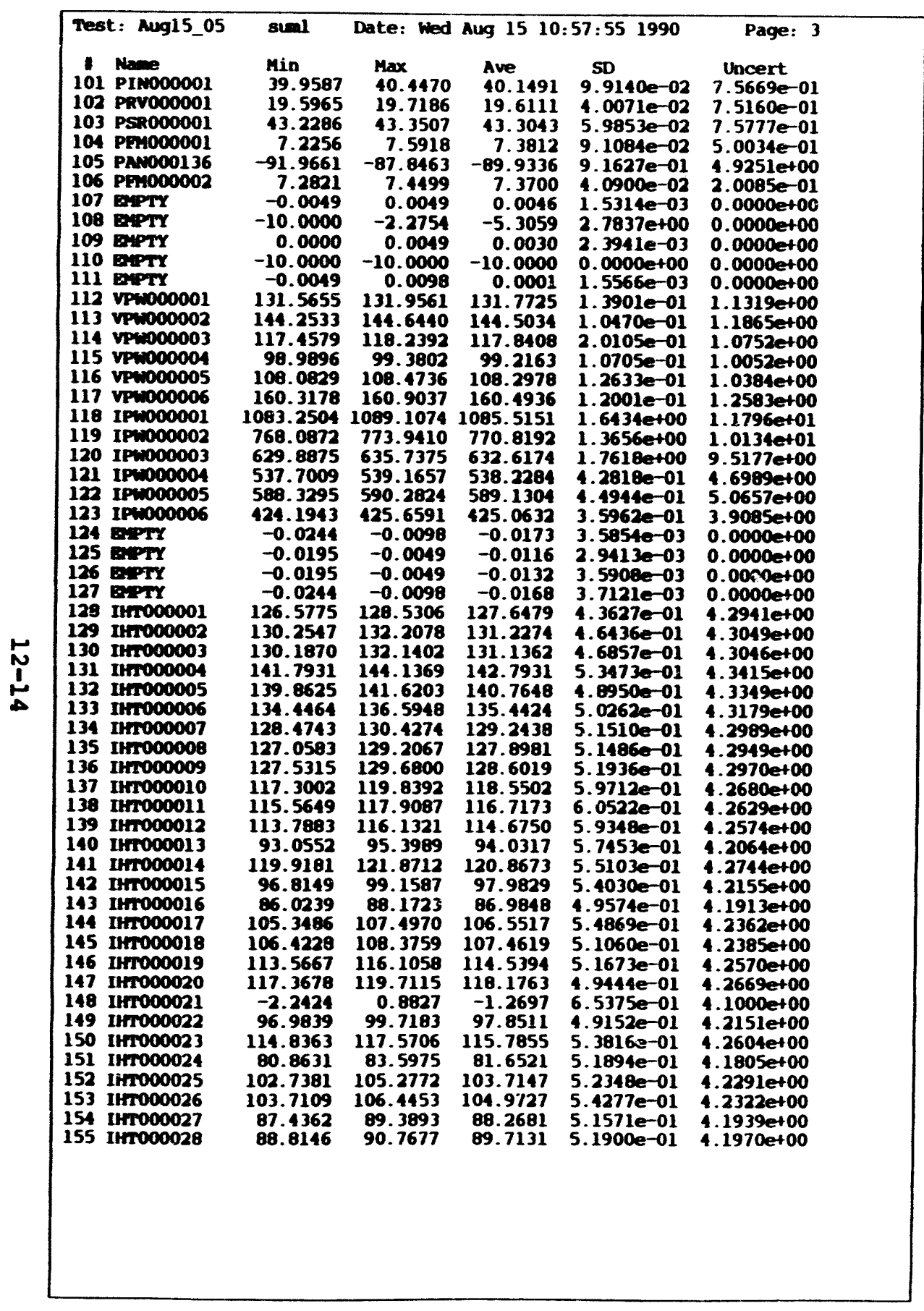

\begin{tabular}{|c|c|c|c|c|c|c|}
\hline \multicolumn{2}{|c|}{ Test: Aug15_0 } & suml & Date: Wed & Aug 151 & 990 & Page \\
\hline $\begin{array}{l}1 \\
156 \\
157 \\
158 \\
159 \\
160 \\
161 \\
162 \\
163\end{array}$ & 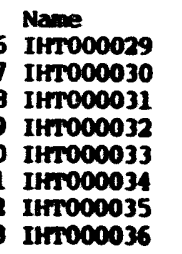 & $\begin{array}{r}\text { Min } \\
103.8349 \\
106.2049 \\
95.1547 \\
96.1839 \\
95.9736 \\
88.2474 \\
115.4025 \\
87.1432\end{array}$ & $\begin{array}{r}\operatorname{Max} \\
106.1786 \\
108.5487 \\
97.1079 \\
98.1370 \\
98.1220 \\
90.0053 \\
118.3556 \\
88.7057\end{array}$ & $\begin{array}{r}\text { Ave } \\
104.8193 \\
107.1190 \\
96.1040 \\
97.1644 \\
97.1181 \\
89.1928 \\
117.3087 \\
87.8970\end{array}$ & $\begin{array}{c}\text { SD } \\
5.5798 \mathrm{e}-01 \\
5.8586 \mathrm{e}-01 \\
5.1450 \mathrm{e}-01 \\
5.3596 \mathrm{e}-01 \\
5.3235 \mathrm{e}-01 \\
4.2565 \mathrm{e}-01 \\
4.5636 \mathrm{e}-01 \\
4.3586 \mathrm{e}-01\end{array}$ & $\begin{array}{c}\text { Uncert } \\
4.2319 \mathrm{e}+00 \\
4.2376 \mathrm{e}+00 \\
4.2111 \mathrm{e}+00 \\
4.2136 \mathrm{e}+00 \\
4.2135 \mathrm{e}+00 \\
4.1959 \mathrm{e}+00 \\
4.2645 \mathrm{e}+00 \\
4.1932 \mathrm{e}+00\end{array}$ \\
\hline
\end{tabular}




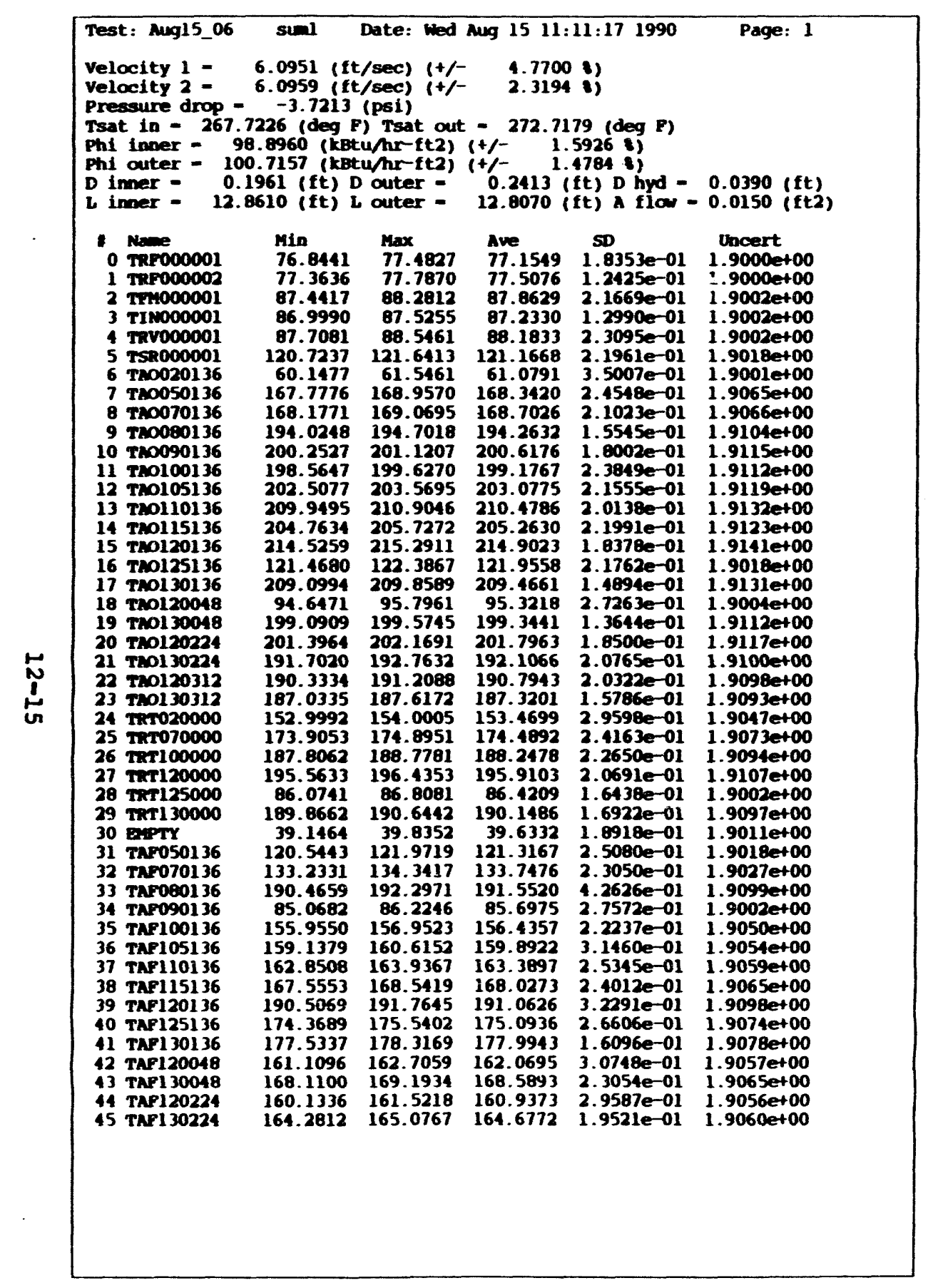

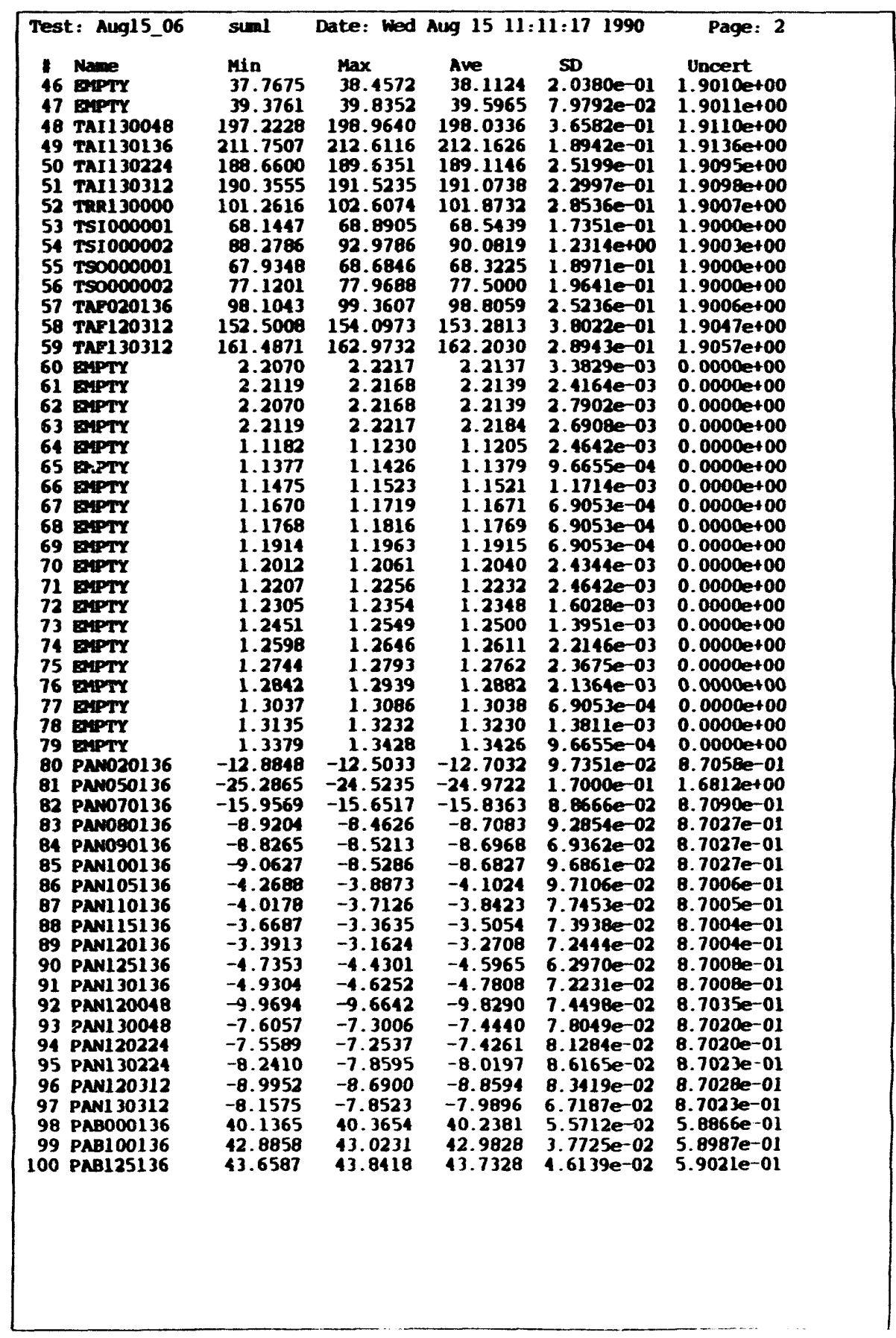



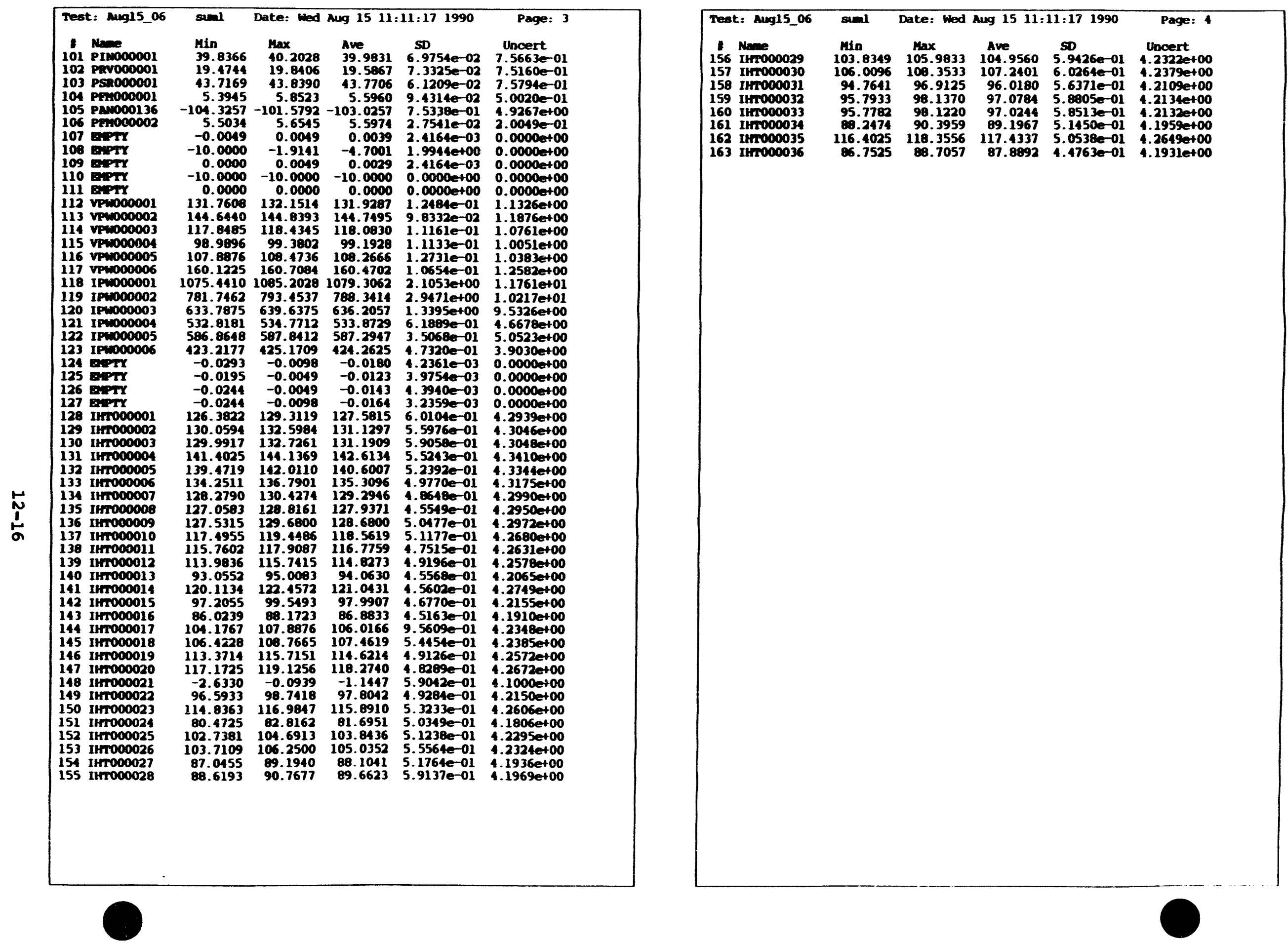


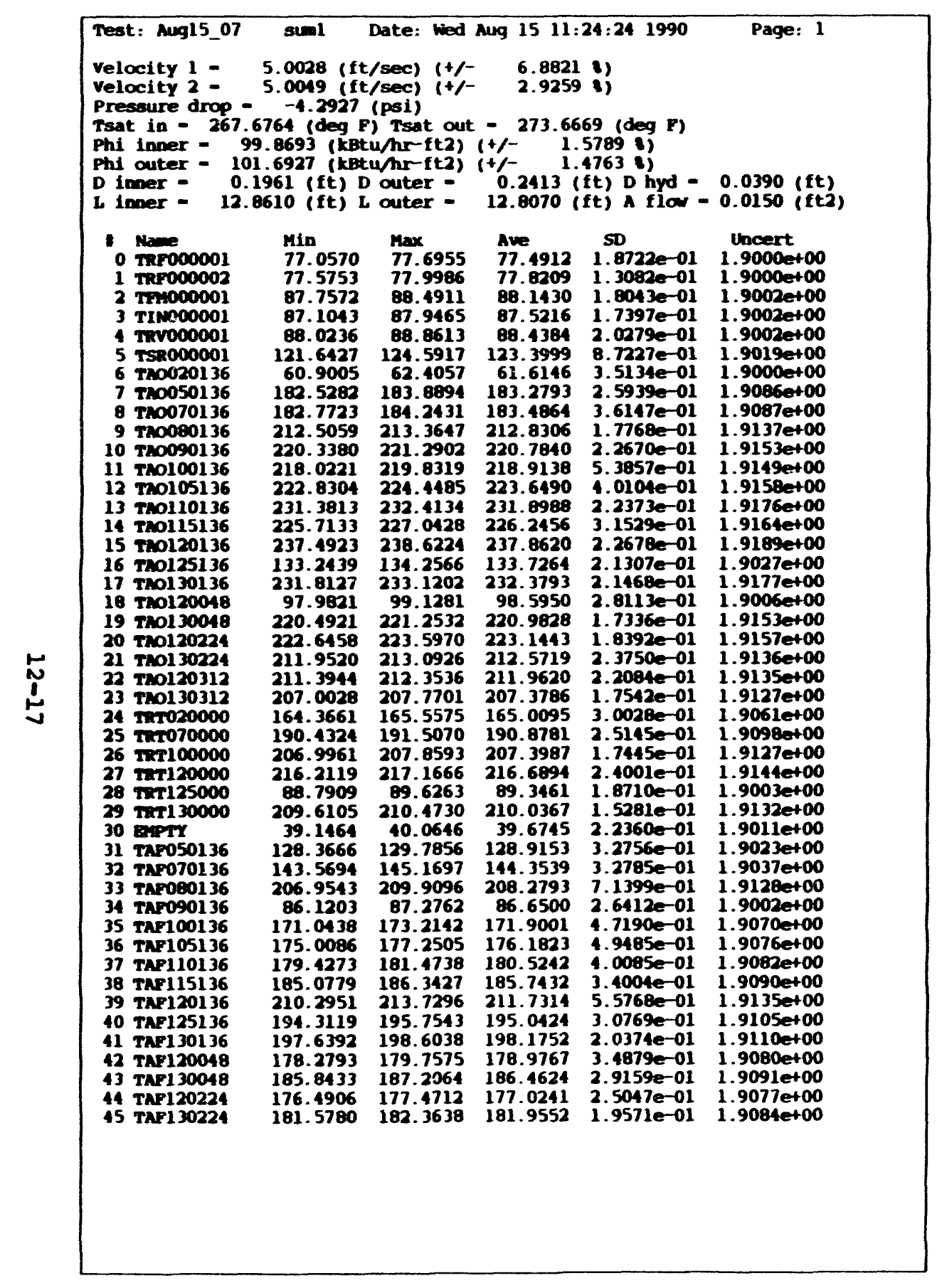

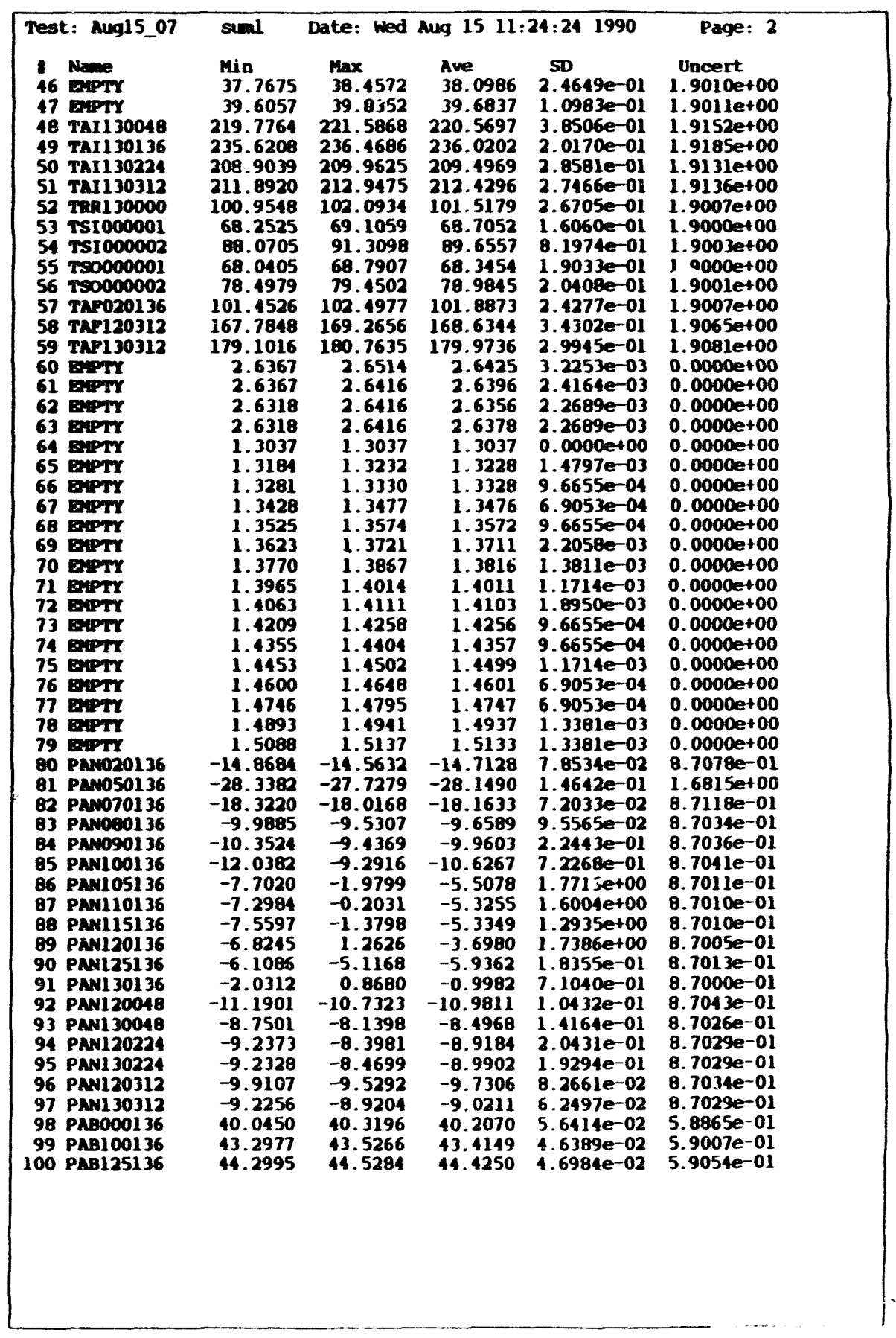



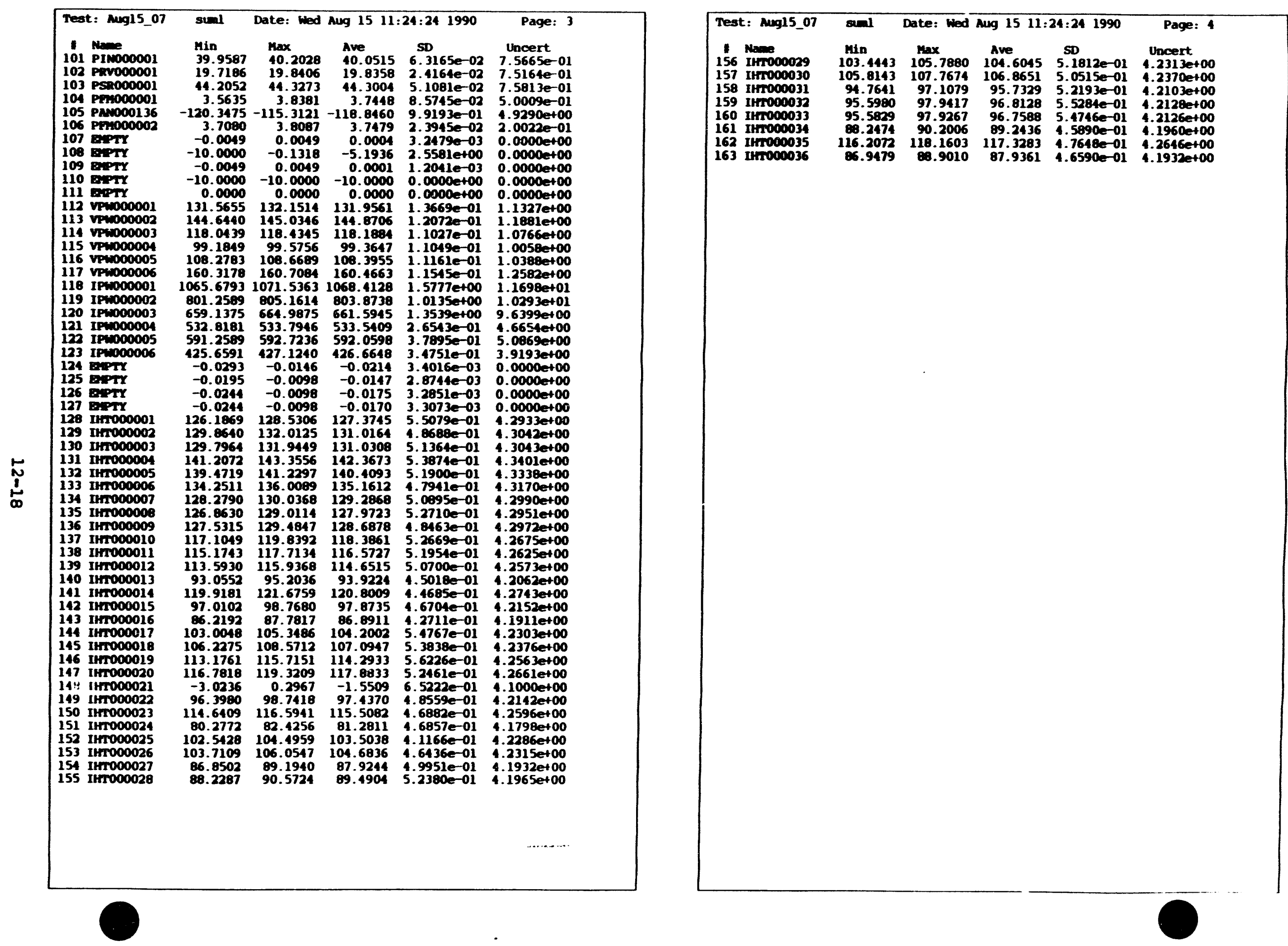


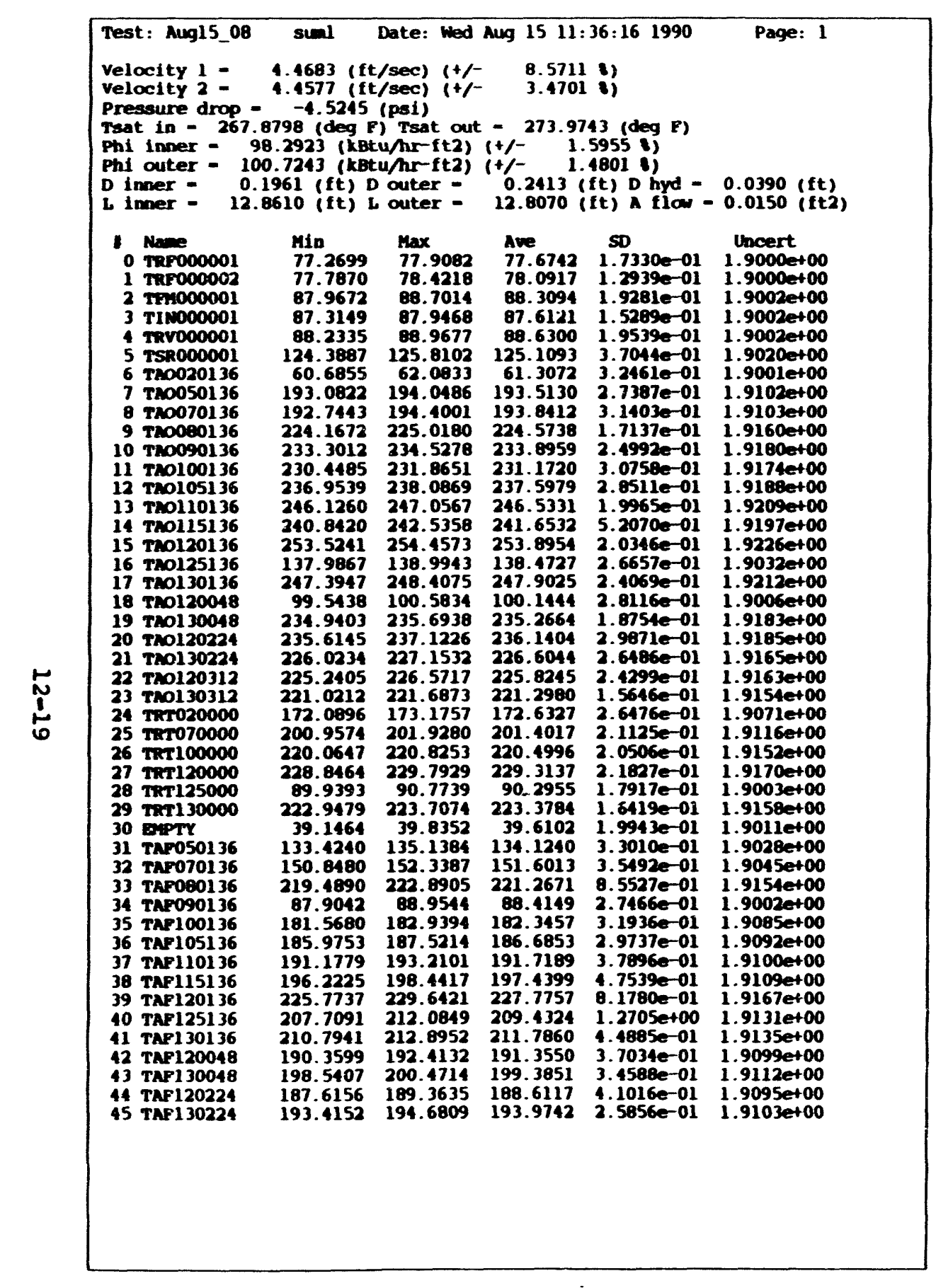

\begin{tabular}{|c|c|c|c|c|c|}
\hline 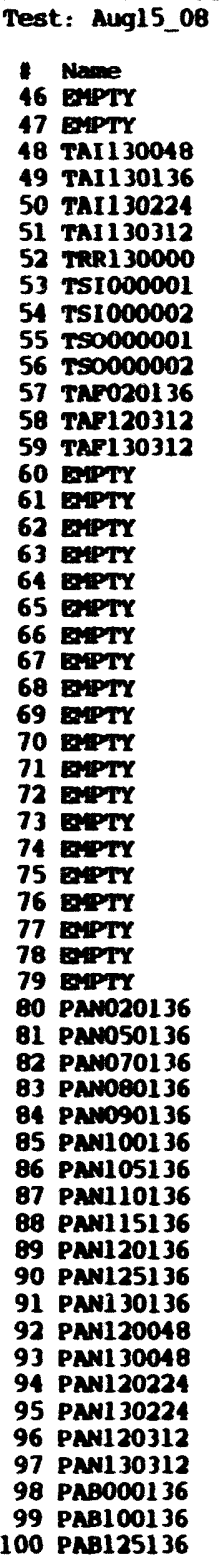 & \begin{aligned} \multicolumn{1}{c}{ suml } \\
Min \\
37.7675 \\
39.6057 \\
235.0399 \\
250.7794 \\
222.6942 \\
225.7319 \\
103.8490 \\
68.4664 \\
87.9661 \\
67.6261 \\
79.5554 \\
102.7057 \\
178.6982 \\
191.5619 \\
2.9395 \\
2.9395 \\
2.9346 \\
2.9346 \\
1.4404 \\
1.4551 \\
1.4648 \\
1.4795 \\
1.4844 \\
1.4990 \\
1.5088 \\
1.5283 \\
1.5381 \\
1.5527 \\
1.5625 \\
1.5771 \\
1.5820 \\
1.5967 \\
1.6162 \\
1.6357 \\
-15.6313 \\
-30.0167 \\
-19.4664 \\
-12.5062 \\
-13.0990 \\
-12.2670 \\
-7.7020 \\
-4.9333 \\
-5.8812 \\
-6.8245 \\
-6.1086 \\
-7.9058 \\
-12.1056 \\
-10.3523 \\
-12.3654 \\
-10.9113 \\
-10.5210 \\
-9.5308 \\
10.2739 \\
13.7555 \\
44.3911\end{aligned} & $\begin{aligned} \text { Date: } \text { Wed } \\
\text { Max } \\
38.4572 \\
40.0646 \\
236.9249 \\
252.4542 \\
224.0289 \\
227.1570 \\
105.0880 \\
69.1071 \\
90.7894 \\
68.8974 \\
80.6126 \\
104.2713 \\
180.2653 \\
193.5014 \\
2.9639 \\
2.9541 \\
2.9443 \\
2.9443 \\
1.4453 \\
1.4600 \\
1.4746 \\
1.4844 \\
1.4941 \\
1.5088 \\
1.5186 \\
1.5381 \\
1.5430 \\
1.5576 \\
1.5723 \\
1.5820 \\
1.5918 \\
1.6064 \\
1.6211 \\
1.6406 \\
-15.3262 \\
-29.4064 \\
-19.0849 \\
-10.1411 \\
-7.3769 \\
-10.7412 \\
-4.8028 \\
-0.8135 \\
2.6637 \\
4.9247 \\
-0.6917 \\
-3.9385 \\
-9.5879 \\
-3.1807 \\
-6.9485 \\
-6.9440 \\
-9.8344 \\
-9.2256 \\
40.4112 \\
13.9844 \\
44.8488\end{aligned}$ & $\begin{aligned} \text { Aug } 1511: \\
\text { Ave } \\
38.0802 \\
39.7801 \\
235.9879 \\
251.7077 \\
223.3865 \\
226.5326 \\
104.3793 \\
68.8190 \\
89.0694 \\
68.3683 \\
80.1033 \\
103.7515 \\
179.3747 \\
192.6194 \\
2.9530 \\
2.9471 \\
2.9410 \\
2.9409 \\
1.4409 \\
1.4578 \\
1.4688 \\
1.4821 \\
1.4899 \\
1.5038 \\
1.5132 \\
1.5313 \\
1.5395 \\
1.5545 \\
1.5647 \\
1.5773 \\
1.5868 \\
1.6016 \\
1.6189 \\
1.6366 \\
-15.4223 \\
-29.7665 \\
-19.2558 \\
-11.7250 \\
-10.5218 \\
-11.8718 \\
-6.0342 \\
-2.9985 \\
-3.1392 \\
-3.4066 \\
-4.4469 \\
-4.9654 \\
-11.7959 \\
-7.1770 \\
-10.1895 \\
-7.8412 \\
-10.1731 \\
-9.3995 \\
40.3443 \\
13.8590 \\
44.6511\end{aligned}$ & $\begin{array}{l}36: 161990 \\
\text { SD } \\
2.2642 e-01 \\
1.0934 e-01 \\
3.8865 e-01 \\
3.1277 e-01 \\
3.0859 e-01 \\
2.8606 e-01 \\
2.7669 e-01 \\
1.8602 e-01 \\
6.0169 e-01 \\
2.2329 e-01 \\
2.0900 e-01 \\
3.2133 e-01 \\
1.1223 e-01 \\
3.9262 e-01 \\
4.7607 e-03 \\
1.0864 e-03 \\
3.0309 e-03 \\
2.6562 e-03 \\
1.4797 e-03 \\
2.4484 e-03 \\
2.1364 e-03 \\
2.4583 e-03 \\
1.9754 e-03 \\
1.2041 e-03 \\
2.0337 e-03 \\
2.6100 e-03 \\
2.2146 e-03 \\
2.3675 e-03 \\
2.6488 e-03 \\
9.6655 e-04 \\
1.2041 e-03 \\
9.8648 e-04 \\
2.4484 e-03 \\
1.8950 e-03 \\
7.3616 e-02 \\
1.3703 e-01 \\
8.3803 e-02 \\
6.2924 e-01 \\
1.6995 e+00 \\
4.8122 e-01 \\
6.7293 e-01 \\
9.6957 e-01 \\
1.6847 e+00 \\
2.2692 e+00 \\
1.1185 e+00 \\
7.3925 e-01 \\
6.0946 e-01 \\
1.1366 e+00 \\
1.1255 e+00 \\
8.4136 e-01 \\
2.4428 e-01 \\
6.3627 e-02 \\
1.4555 e-02 \\
1.7886 e-02 \\
7.6509 e-02\end{array}$ & $\begin{array}{c}\text { Page: } 2 \\
\text { Uncert } \\
1.9010 e+00 \\
1.9011 e+00 \\
1.9185 e+00 \\
1.9221 e+00 \\
1.9158 e+00 \\
1.9164 e+00 \\
1.9008 e+00 \\
1.9000 e+00 \\
1.9002 e+00 \\
1.9000 e+00 \\
1.9001 e+00 \\
1.9006 e+00 \\
1.9080 e+00 \\
1.9101 e+00 \\
0.0000 e+00 \\
0.0000 e+00 \\
0.0000 e+00 \\
0.0000 e+00 \\
0.0000 e+00 \\
0.0000 e+00 \\
0.0000 e+00 \\
0.0000 e+00 \\
0.0000 e+00 \\
0.0000 e+00 \\
0.0000 e+00 \\
0.0000 e+00 \\
0.0000 e+00 \\
0.0000 e+00 \\
0.0000 e+00 \\
0.0000 e+00 \\
0.0000 e+00 \\
0.0000 e+00 \\
0.0000 e+00 \\
0.0000 e+00 \\
8.7085 e-01 \\
1.6816 e+00 \\
8.7133 e-01 \\
8.7049 e-01 \\
8.7040 e-01 \\
8.7051 e-01 \\
8.7013 e-01 \\
8.7003 e-01 \\
8.7004 e-01 \\
8.7004 e-01 \\
8.7007 e-01 \\
8.7009 e-01 \\
8.7050 e-01 \\
8.7019 e-01 \\
8.7037 e-01 \\
8.7022 e-01 \\
8.7037 e-01 \\
8.7032 e-01 \\
5.8870 e-01 \\
5.9027 e-01 \\
5.9064 e-01\end{array}$ \\
\hline
\end{tabular}




\begin{tabular}{|c|c|c|c|c|c|}
\hline 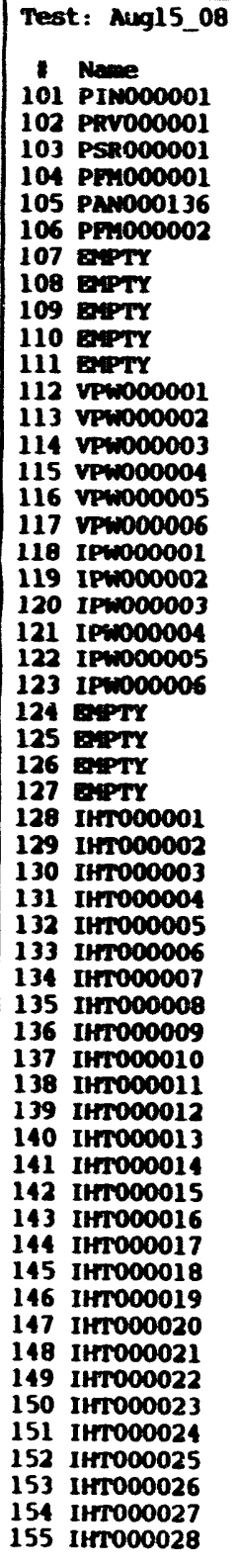 & 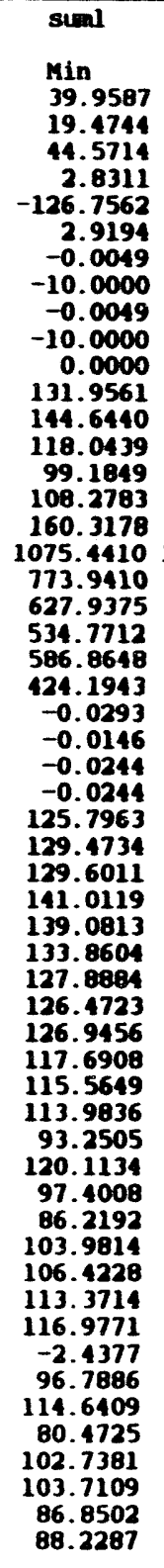 & $\begin{aligned} & \text { Date: } \text { med } \\
& \text { Max } \\
& 40.2028 \\
& 19.5965 \\
& 44.6935 \\
& 3.1057 \\
&-123.5518 \\
& 3.0200 \\
& 0.0049 \\
&-0.8594 \\
& 0.0098 \\
&-10.0000 \\
& 0.0000 \\
& 132.3468 \\
& 115.0346 \\
& 118.4345 \\
& 99.5756 \\
& 108.6689 \\
& 160.7084 \\
& 1085.2028 \\
& 779.7949 \\
& 633.7875 \\
& 536.7243 \\
& 588.3295 \\
& 125.6591 \\
&-0.0098 \\
&-0.0998 \\
&-0.0049 \\
&-0.0998 \\
& 128.9213 \\
& 132.2078 \\
& 132.5308 \\
& 144.3322 \\
& 142.0110 \\
& 136.7901 \\
& 130.8181 \\
& 129.4020 \\
& 130.2659 \\
& 119.6439 \\
& 117.7134 \\
& 115.9368 \\
& 95.2036 \\
& 122.0666 \\
& 98.9633 \\
& 87.7917 \\
& 107.3017 \\
& 108.7665 \\
& 115.7151 \\
& 119.1256 \\
&-0.0939 \\
& 98.5464 \\
& 116.7894 \\
& 82.4256 \\
& 104.8866 \\
& 106.2500 \\
& 99.1940 \\
& 90.7677\end{aligned}$ & $\begin{aligned} \text { Aug } 1511 \text { : } \\
\text { Ave } \\
40.0735 \\
19.5819 \\
44.6569 \\
2.9739 \\
-125.2639 \\
2.9596 \\
0.0037 \\
-4.2841 \\
0.0030 \\
-10.0000 \\
0.0000 \\
132.0850 \\
114.8510 \\
118.2041 \\
99.3764 \\
108.5634 \\
160.5483 \\
1080.5951 \\
776.3997 \\
629.8875 \\
535.3866 \\
587.7145 \\
124.8972 \\
-0.0185 \\
-0.0144 \\
-0.0138 \\
-0.0151 \\
127.4760 \\
131.0672 \\
131.1441 \\
112.654 \\
140.6828 \\
135.4144 \\
129.418 \\
128.0543 \\
128.7191 \\
118.5384 \\
116.7407 \\
114.7726 \\
94.1450 \\
120.9220 \\
98.0649 \\
86.8716 \\
105.7900 \\
107.2158 \\
114.4378 \\
118.0044 \\
-1.2619 \\
97.7144 \\
115.6371 \\
01.4217 \\
103.7069 \\
104.9570 \\
88.1509 \\
89.5295\end{aligned}$ & 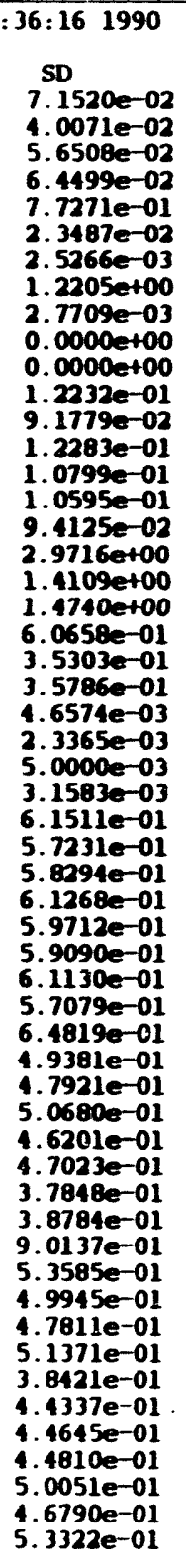 & 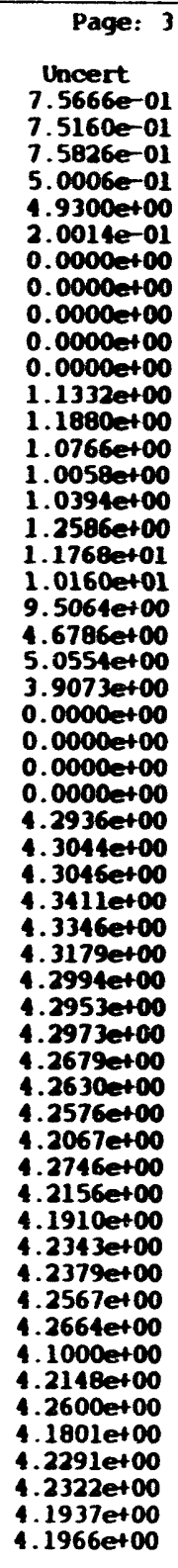 \\
\hline
\end{tabular}

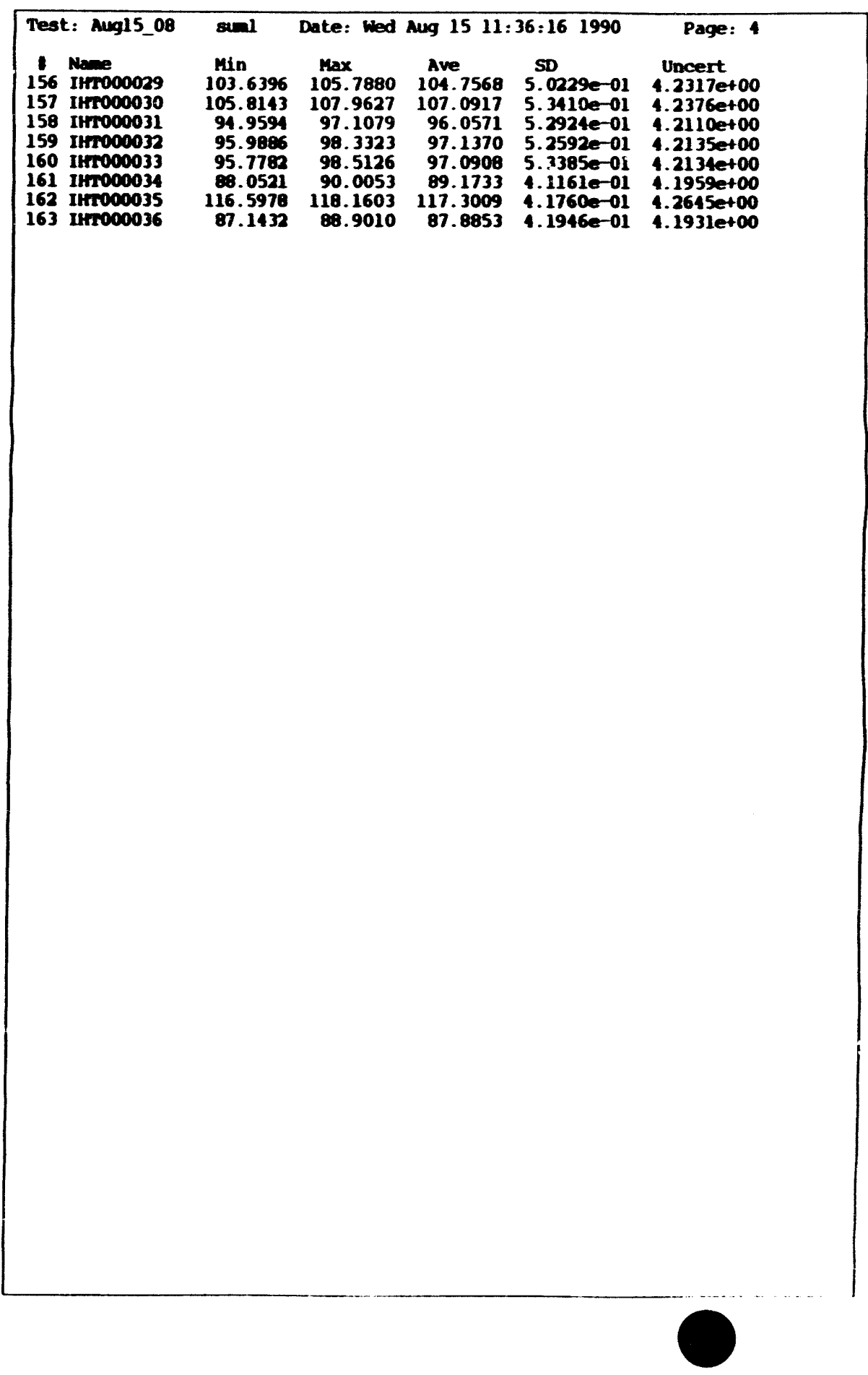




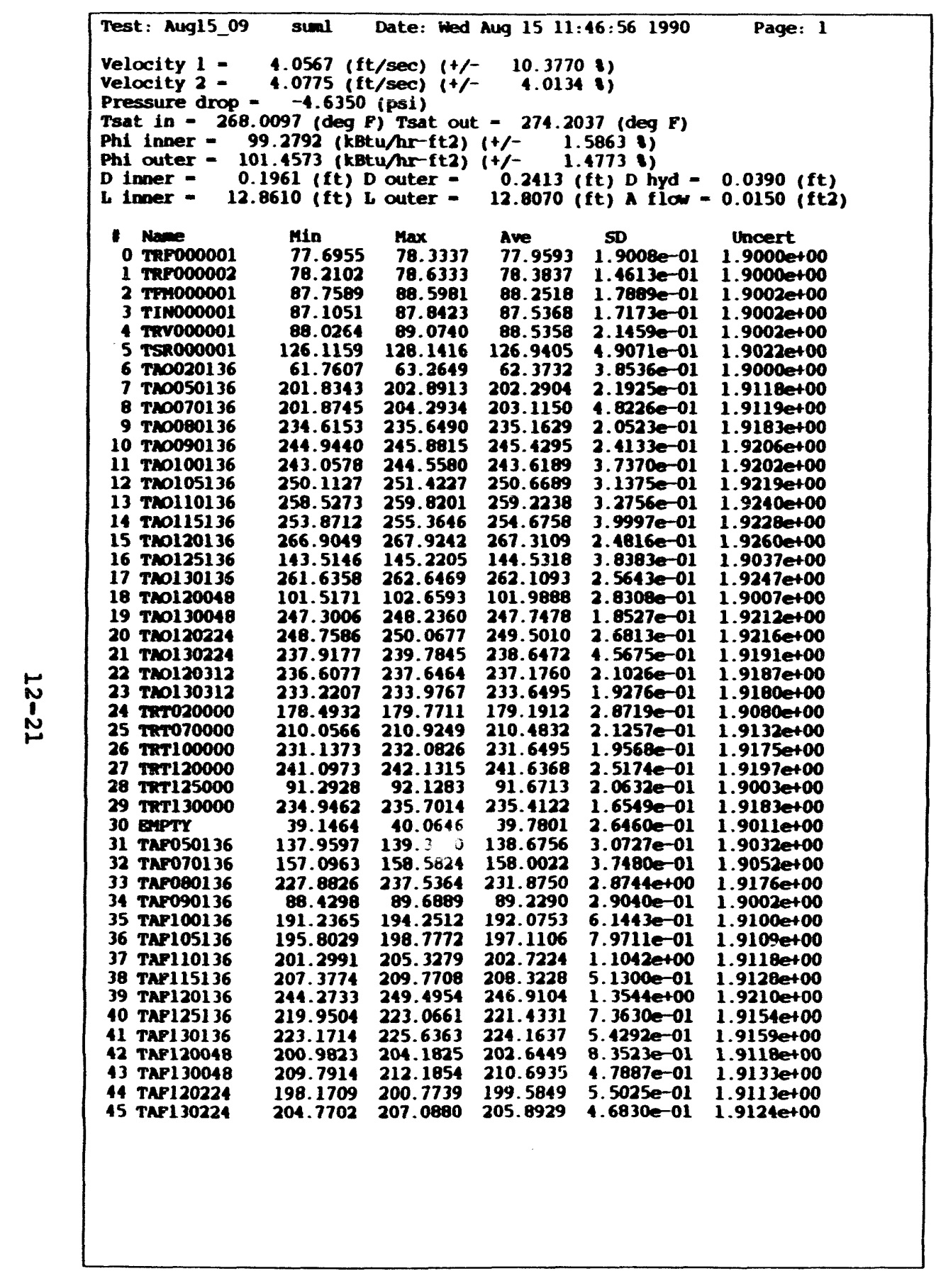

\begin{tabular}{|c|c|c|c|c|c|c|}
\hline 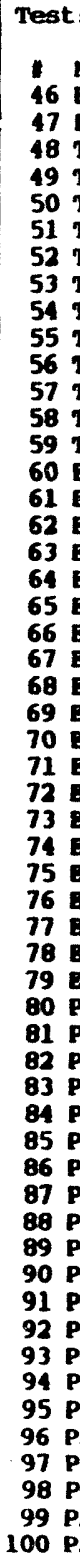 & 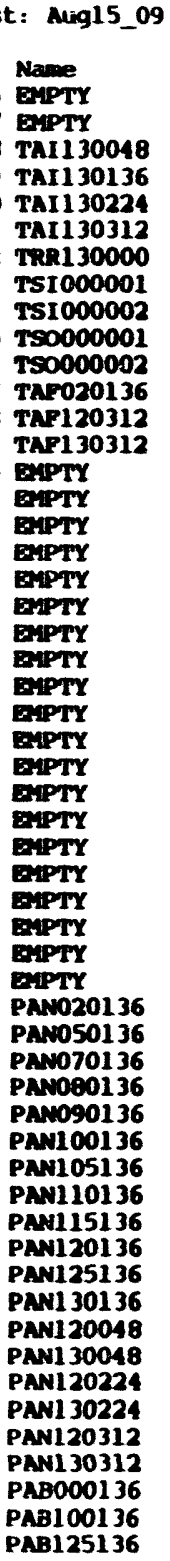 & \begin{tabular}{r}
\multicolumn{1}{c}{ suml } \\
Min \\
37.7675 \\
39.1464 \\
248.1926 \\
265.5216 \\
235.2282 \\
238.6972 \\
107.5588 \\
68.4687 \\
88.1795 \\
67.9329 \\
80.7187 \\
104.1662 \\
188.2644 \\
202.8618 \\
3.2129 \\
3.2080 \\
3.2031 \\
3.1982 \\
1.5527 \\
1.5674 \\
1.5771 \\
1.5918 \\
1.6016 \\
1.6113 \\
1.6211 \\
1.6357 \\
1.6455 \\
1.6602 \\
1.6699 \\
1.6846 \\
1.6943 \\
1.7041 \\
1.7139 \\
1.7432 \\
-15.9365 \\
-31.3900 \\
-19.9242 \\
-13.9558 \\
-13.1753 \\
-10.0545 \\
-6.0998 \\
-7.2222 \\
-5.5760 \\
-6.3668 \\
-5.9560 \\
-5.7696 \\
-11.4953 \\
-10.8864 \\
-8.4744 \\
-13.1238 \\
-11.5129 \\
-8.92004 \\
10.3196 \\
13.8928 \\
44.7573
\end{tabular} & $\begin{array}{r}\text { Date: } \text { wed } \\
\text { Max } \\
38.4572 \\
40.0646 \\
250.3438 \\
266.9984 \\
236.1737 \\
239.7337 \\
109.3094 \\
69.3308 \\
91.4180 \\
68.7898 \\
81.6699 \\
106.0418 \\
190.2098 \\
204.5929 \\
3.2324 \\
3.2227 \\
3.2080 \\
3.2080 \\
1.5576 \\
1.5723 \\
1.5820 \\
1.5967 \\
1.6064 \\
1.6162 \\
1.6260 \\
1.6455 \\
1.6504 \\
1.6650 \\
1.6748 \\
1.6895 \\
1.6992 \\
1.7139 \\
1.7285 \\
1.7480 \\
-15.7076 \\
-30.6271 \\
-19.3138 \\
-10.9803 \\
-12.1835 \\
-6.3161 \\
6.1835 \\
-1.8816 \\
-2.7531 \\
3.4751 \\
-2.4465 \\
-4.0911 \\
-7.9858 \\
-7.1480 \\
-1.7605 \\
-2.5190 \\
-10.6736 \\
-8.1575 \\
10.5485 \\
44.1675 \\
14.9404\end{array}$ & $\begin{aligned} \text { Aug } 1511 \text { : } \\
\text { Ave } \\
38.1124 \\
39.8398 \\
249.2534 \\
266.1360 \\
235.7145 \\
239.2983 \\
108.3476 \\
68.9311 \\
89.1797 \\
68.3505 \\
81.1433 \\
105.0937 \\
189.0761 \\
203.6556 \\
3.2209 \\
3.2135 \\
3.2047 \\
3.2032 \\
1.5568 \\
1.5718 \\
1.5817 \\
1.5952 \\
1.6018 \\
1.6159 \\
1.6254 \\
1.6408 \\
1.6497 \\
1.6644 \\
1.6745 \\
1.6848 \\
1.6944 \\
1.7089 \\
1.7243 \\
1.7433 \\
-15.8022 \\
-30.8834 \\
-19.6800 \\
-13.2051 \\
-12.8793 \\
-8.3287 \\
-2.6529 \\
-4.4252 \\
-4.2653 \\
-4.3389 \\
-4.2943 \\
-5.0387 \\
-9.3926 \\
-9.1911 \\
-7.1148 \\
-9.6021 \\
-11.0307 \\
-8.5466 \\
40.4322 \\
44.0311 \\
44.8205\end{aligned}$ & $\begin{array}{c}: 46: 561990 \\
50 \\
2.1910 e-01 \\
1.8250 e-01 \\
4.4585 e-01 \\
3.0013 e-01 \\
3.2137 e-01 \\
2.3921 e-01 \\
4.6767 e-01 \\
1.8346 e-01 \\
7.5046 e-01 \\
1.6014 e-01 \\
2.3911 e-01 \\
4.0778 e-01 \\
1.6098 e-01 \\
1.0372 e-01 \\
4.8085 e-03 \\
3.9013 e-03 \\
2.3008 e-03 \\
2.0903 e-03 \\
1.6082 e-03 \\
1.4797 e-03 \\
1.1714 e-03 \\
2.2603 e-03 \\
9.6655 e-04 \\
1.1714 e-03 \\
1.6028 e-03 \\
1.3811 e-03 \\
1.7115 e-03 \\
1.7115 e-03 \\
1.1714 e-03 \\
9.6655 e-04 \\
6.9053 e-04 \\
1.5566 e-03 \\
2.4184 e-03 \\
6.9053 e-04 \\
7.1570 e-02 \\
1.7285 e-01 \\
1.2710 e-01 \\
9.4871 e-01 \\
2.8154 e-01 \\
9.9223 e-01 \\
2.2141 e+00 \\
1.4255 e+00 \\
7.0224 e-01 \\
1.7016 e+00 \\
9.2499 e-01 \\
3.9058 e-01 \\
7.6700 e-01 \\
9.7549 e-01 \\
1.0071 e+00 \\
1.5179 e+00 \\
1.9155 e-01 \\
1.9814 e-01 \\
5.0832 e-02 \\
6.5714 e-02 \\
4.9709 e-02\end{array}$ & $\begin{array}{c}\text { Page: } 2 \\
\text { Uncert } \\
1.9010 e+00 \\
1.9011 e+00 \\
1.9215 e+00 \\
1.9257 e+00 \\
1.9184 e+00 \\
1.9192 e+00 \\
1.9010 e+00 \\
1.9000 e+00 \\
1.9002 e+00 \\
1.9000 e+00 \\
1.9001 e+00 \\
1.9008 e+00 \\
1.9095 e+00 \\
1.9120 e+00 \\
0.0000 e+00 \\
0.0000 e+00 \\
0.0000 e+00 \\
0.0000 e+00 \\
0.0000 e+00 \\
0.0000 e+00 \\
0.0000 e+00 \\
0.0000 e+00 \\
0.0000 e+00 \\
0.0000 e+00 \\
0.0000 e+00 \\
0.0000 e+00 \\
0.0000 e+00 \\
0.0000 e+00 \\
0.0000 e+00 \\
0.0000 e+00 \\
0.0000 e+00 \\
0.0000 e+00 \\
0.0000 e+00 \\
0.0000 e+00 \\
8.7090 e-01 \\
1.6818 e+00 \\
8.7139 e-01 \\
8.7063 e-01 \\
8.7060 e-01 \\
8.7025 e-01 \\
8.7003 e-01 \\
8.7007 e-01 \\
8.7007 e-01 \\
8.7007 e-01 \\
8.7007 e-01 \\
8.7009 e-01 \\
8.7032 e-01 \\
8.7030 e-01 \\
8.7018 e-01 \\
8.7033 e-01 \\
8.7044 e-01 \\
8.7026 e-01 \\
5.8874 e-01 \\
5.9035 e-01 \\
5.9072 e-01 \\
\end{array}$ \\
\hline
\end{tabular}



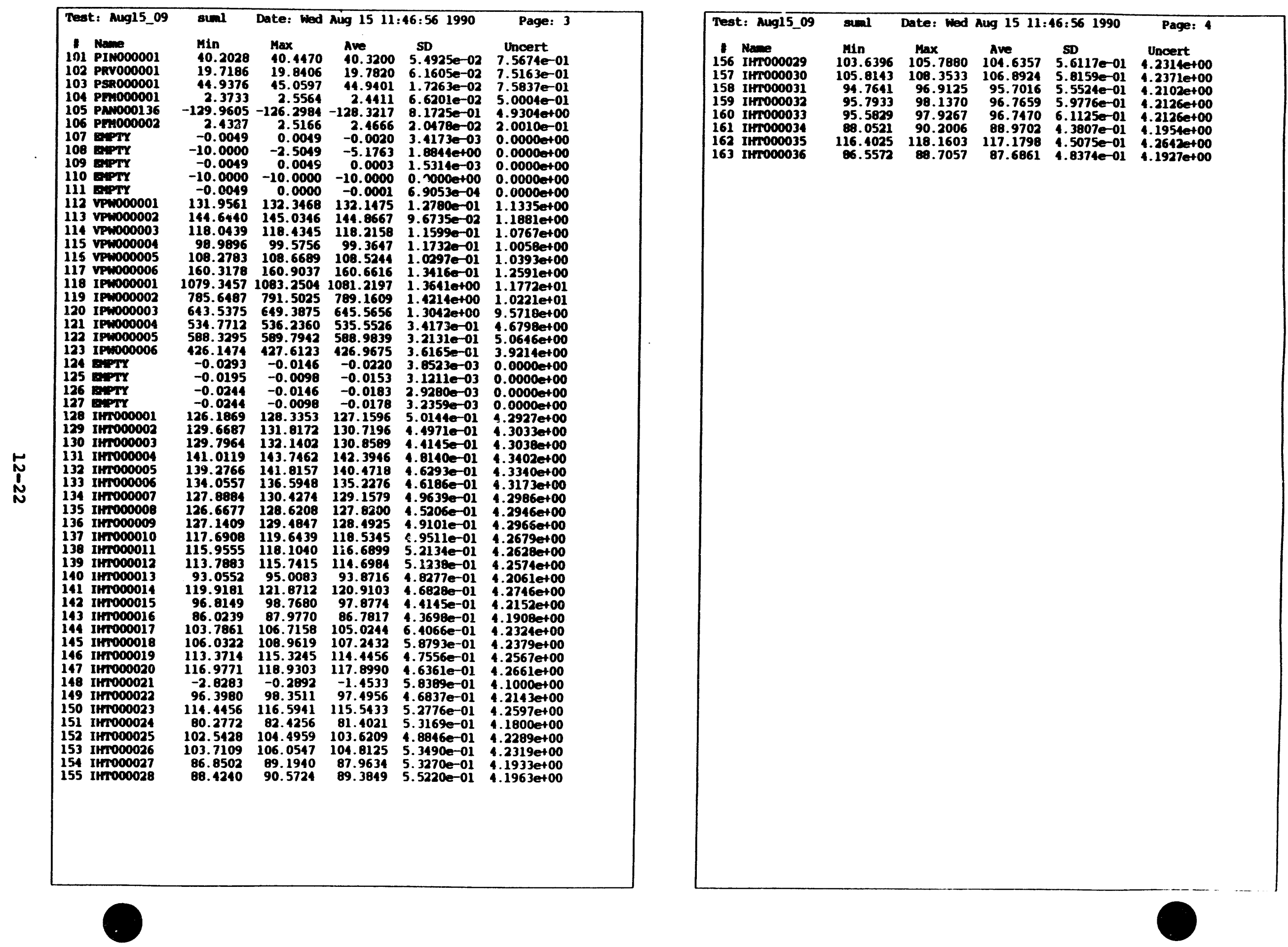


\begin{tabular}{|c|c|c|c|c|c|c|c|}
\hline \multicolumn{2}{|r|}{ st: Aug15_10 } & & te & & \multicolumn{2}{|c|}{ Aug $15 \quad 11: 59: 501$} & ge \\
\hline \multicolumn{2}{|c|}{$\begin{array}{l}\text { Velocity 1 = } \\
\text { Velocity } 2= \\
\text { Pressure drop } \\
\text { Tsat in = 268 } \\
\text { Phi inner = } \\
\text { Phi outer = } \\
\text { D inner = } \\
\text { L inner = } 12\end{array}$} & \multicolumn{2}{|c|}{$\begin{array}{l}3.5905 \text { (ft/sec) } \\
3.5419 \text { (ft/sec) } \\
-4.744 \text { (psi) } \\
124 \text { (deg F) Tsat } \\
.4487 \text { (kBtu/hr-f } \\
.4557 \text { (kBtu/hr f } \\
961 \text { (ft) D outer } \\
9610 \text { (ft) L outer }\end{array}$} & & \multicolumn{2}{|c|}{ 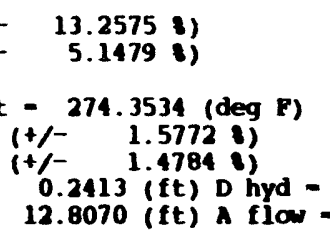 } & 0 \\
\hline 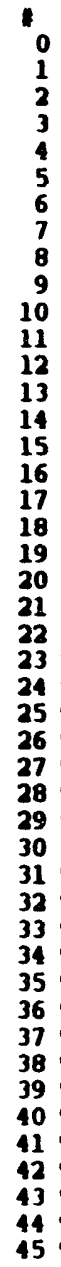 & $\begin{array}{l}\text { me } \\
F 000001 \\
F 000002 \\
\text { roo0001 } \\
\text { No00001 }\end{array}$ & $\begin{array}{l}\text { Min } \\
77.9082 \\
78.4218 \\
87.7605 \\
87.2109 \\
88.2383 \\
126.3216 \\
61.9758 \\
213.8697 \\
217.9572 \\
252.3438\end{array}$ & $\begin{array}{l}\operatorname{Max} \\
78.5 \\
78.8 \\
88.5 \\
87.8 \\
88.9 \\
129.0 \\
63.4 \\
215.8 \\
220.15 \\
253.27\end{array}$ & & $\begin{array}{l}\text { Ave } \\
78.3039 \\
78.6967 \\
88.2468 \\
87.5225 \\
88.5360 \\
127.6417 \\
62.6634 \\
214.6390 \\
218.9879 \\
252.8422\end{array}$ & $\begin{array}{c}\text { SD } \\
1.8235 e-01 \\
1.4332 e-01 \\
2.1066 e-01 \\
1.3937 e-01 \\
1.7471 e-01 \\
6.5518 e-01 \\
3.0222 e-01 \\
3.7257 e-01 \\
5.0151 e-01 \\
2.0560 e-01 \\
2.8614 e-01 \\
5.0500 e-01 \\
3.2359 e-01 \\
3.1493 e-01 \\
2.7771 e-01 \\
2.0993 e-01 \\
3.7195 e-01 \\
1.9783 e-01 \\
3.5677 e-01 \\
1.9299 e-01 \\
2.4457 e-01 \\
4.0378 e-01 \\
2.6453 e-01 \\
1.8761 e-01 \\
3.6779 e-01 \\
2.5704 e-01 \\
2.7222 e-01 \\
2.9961 e-01 \\
1.5463 e-01 \\
1.5302 e-01 \\
1.9432 e-01 \\
5.4616 e-01 \\
2.0176\end{array}$ & 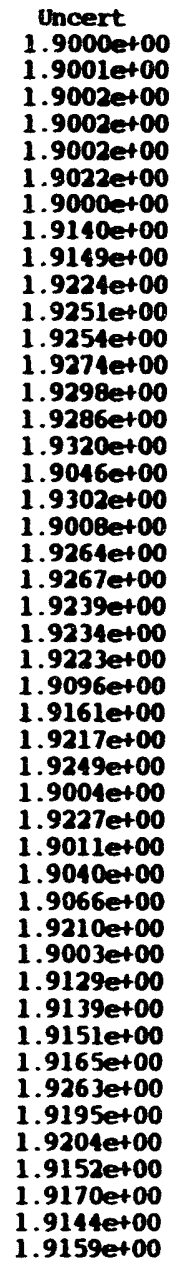 \\
\hline
\end{tabular}

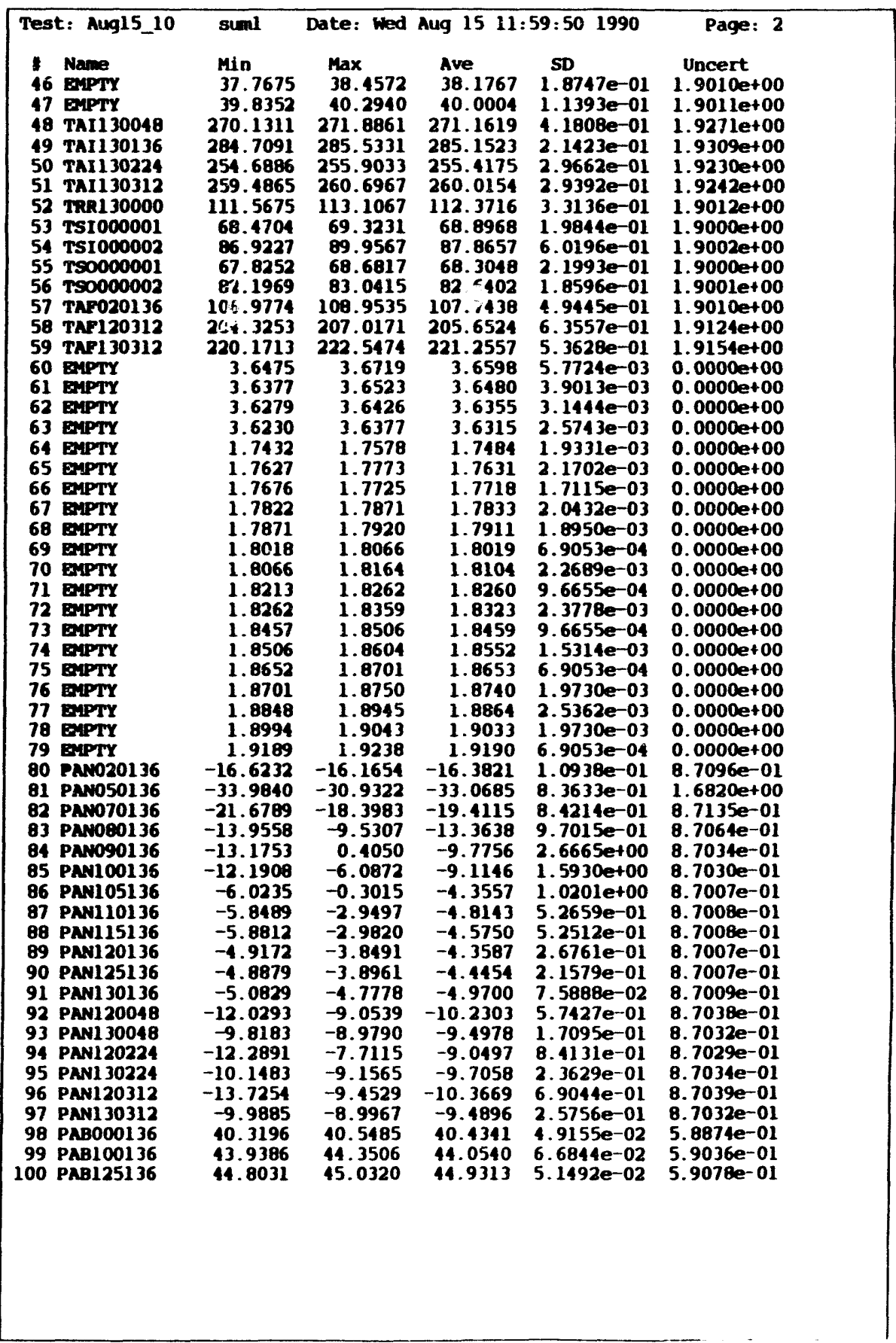



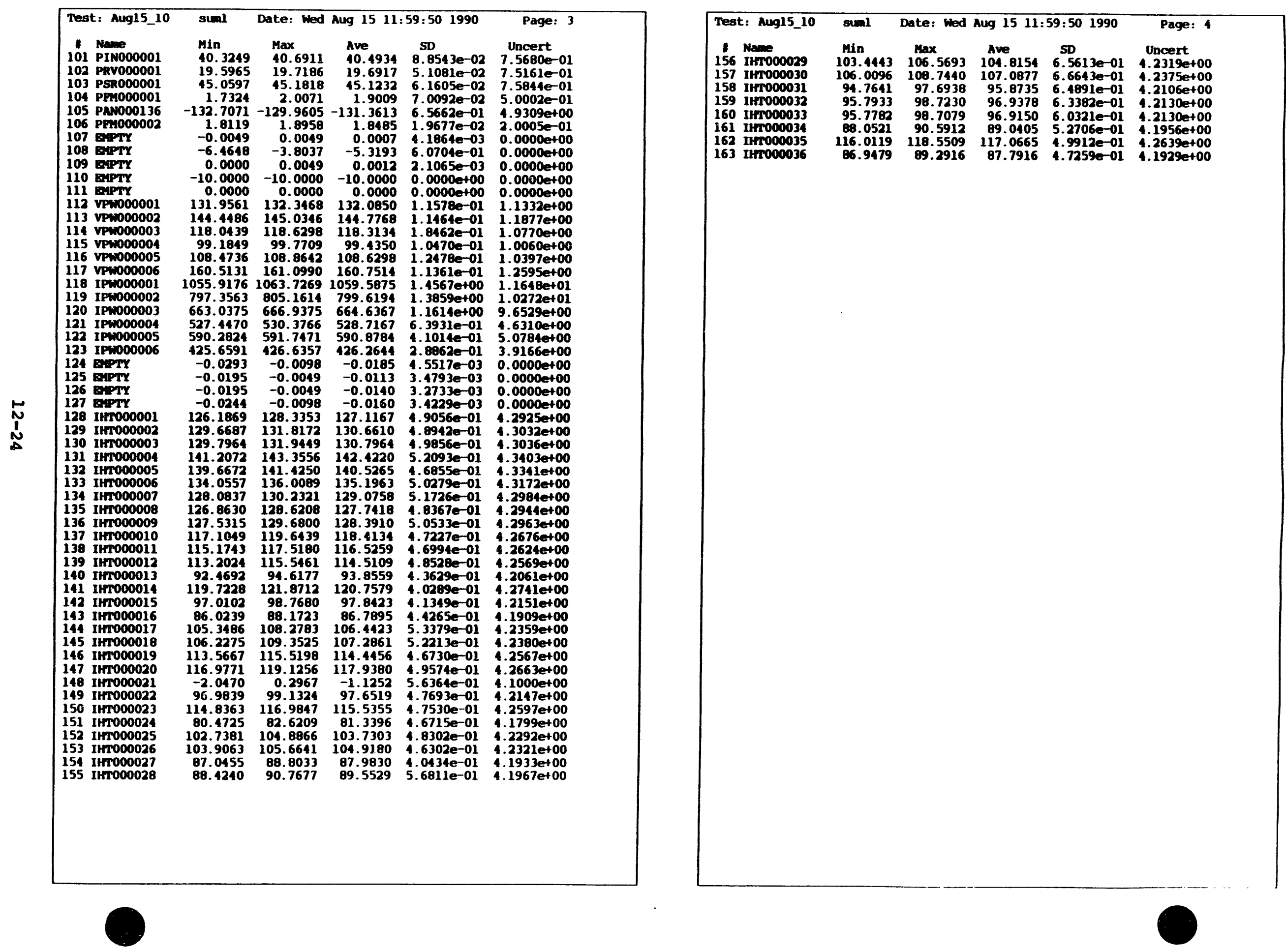


\begin{tabular}{|c|c|c|c|c|c|c|c|}
\hline \multicolumn{2}{|c|}{ st: Aug15_11 } & & \multicolumn{2}{|c|}{ Aug $15 \quad 12: 10: 311990$} & age: 1 \\
\hline ese & $\begin{array}{l}y 1= \\
y 2= \\
\text { e drop } \\
=26 \\
\text { er }= \\
=1 \\
=1 \\
=1\end{array}$ & $\begin{array}{l}4878 \\
4671 \\
-4.457 \\
9003 \\
603\end{array}$ & & & $\begin{array}{r}14.0 \\
\quad 5.3 \\
-\quad 273 \\
+/- \\
+/- \\
0.211 \\
12.807\end{array}$ & $\operatorname{deg}$ F) & $\begin{array}{l}.0390 \text { (ft) } \\
.0150 \text { (ftt2 }\end{array}$ \\
\hline $\begin{array}{ll} & 1 \\
0 & 1 \\
1 & T \\
2 & T \\
3 & 1 \\
4 & T\end{array}$ & 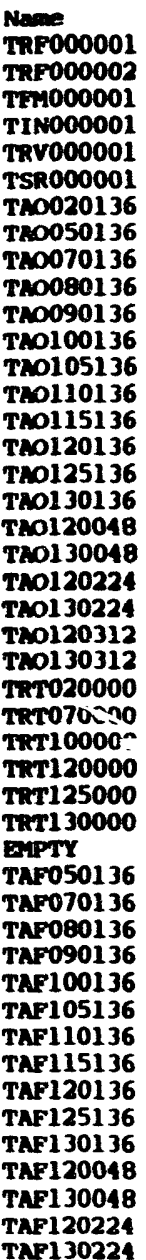 & $\begin{array}{r}\text { Min } \\
78.1210 \\
78.8448 \\
87.7608 \\
87.1056 \\
88.1357 \\
128.8554 \\
64.0152 \\
213.9675 \\
216.2315 \\
250.1171 \\
260.4025 \\
259.3820 \\
263.9109 \\
274.2297 \\
271.7955 \\
284.6807 \\
154.8019 \\
287.0795 \\
106.4901 \\
294.2249 \\
247.8257 \\
242.2121 \\
262.2662 \\
263.6442 \\
191.2149 \\
230.0960 \\
262.2429 \\
271.6081 \\
95.3487 \\
288.5680 \\
39.3761 \\
146.7789 \\
169.6110 \\
240.9057 \\
91.2581 \\
209.1341 \\
214.6955 \\
222.8545 \\
229.7293 \\
266.6286 \\
250.2769 \\
265.0103 \\
245.8566\end{array}$ & $\begin{array}{r}\operatorname{Max} \\
78.9 \\
79.2 \\
88.6 \\
88.1 \\
89.0 \\
132.7 \\
65.6 \\
216.9 \\
218.7 \\
251.7 \\
261.8 \\
262.6 \\
270.3 \\
281.1 \\
279.3 \\
290.5 \\
157.7 \\
294.2 \\
107.6 \\
338.4 \\
253.2 \\
248.3 \\
277.0 \\
284.9 \\
192.7 \\
235.0 \\
267.0 \\
290.4 \\
96.3 \\
298.1 \\
40.0\end{array}$ & $\begin{array}{l}6 \\
6 \\
0 \\
3 \\
8 \\
5 \\
4 \\
3 \\
9 \\
6 \\
9 \\
9 \\
0 \\
9 \\
3 \\
8 \\
9 \\
3 \\
1 \\
1\end{array}$ & $\begin{array}{r}\text { Ave } \\
78.6186 \\
79.0224 \\
88.2292 \\
87.5081 \\
88.6322 \\
130.4295 \\
64.6912 \\
215.6772 \\
217.5182 \\
250.8453 \\
261.2690 \\
261.0610 \\
266.7615 \\
277.6674 \\
275.2627 \\
287.7831 \\
156.3939 \\
291.6056 \\
107.0964 \\
317.3937 \\
250.7763 \\
245.2076\end{array}$ & $\begin{array}{c}\text { SD } \\
1.9970 e-01 \\
1.3749 e-01 \\
2.0574 e-01 \\
1.9097 e-01 \\
1.9616 e-01 \\
1.1412 e+00 \\
3.6716 e-01 \\
6.2231 e-01 \\
5.8880 e-01 \\
4.5837 e-01 \\
3.8760 e-01 \\
8.4415 e-01 \\
1.7713 e+00 \\
2.1944 e+00 \\
2.1837 e+00 \\
1.8522 e+00 \\
9.0879 e-01 \\
1.9840 e+00 \\
2.8712 e-01 \\
1.6353 e+01 \\
1.7723 e+00 \\
1.8486 e+00 \\
3.7675 e+00 \\
7.2131 e+00 \\
3.6184 e-01 \\
1.2788 e+00 \\
1.3615 e+00 \\
6.2635 e+00 \\
1.8818 e-01 \\
3.0055 e+00 \\
2.2185 e-01 \\
7.5047 e-01 \\
1.1518 e+00 \\
1.5672 e+00 \\
3.1786 e-01 \\
2.5277 e+00 \\
1.9099 e+00 \\
9.1709 e-01 \\
8.0632 e-01 \\
1.5348 e+00 \\
3.9758 e+00 \\
1.8073 e+00 \\
1.1029 e+01 \\
1.8806 e-01 \\
1.0812 e+00 \\
2.2448 e+00\end{array}$ & $\begin{array}{l}\text { Uncert } \\
1.9001 e+00 \\
1.9001 e+00 \\
1.9002 e+00 \\
1.9002 e+00 \\
1.9002 e+00 \\
1.9025 e+00 \\
1.9000 e+00 \\
1.9142 e+00 \\
1.9146 e+00 \\
1.9219 e+00 \\
1.9245 e+00 \\
1.9244 e+00 \\
1.9259 e+00 \\
1.9288 e+00 \\
1.9282 e+00 \\
1.9317 e+00 \\
1.9050 e+00 \\
1.9328 e+00 \\
1.9009 e+00 \\
1.9408 e+00 \\
1.9219 e+00 \\
1.9206 e+00 \\
1.9264 e+00 \\
1.9276 e+00 \\
1.9100 e+00 \\
1.9176 e+00 \\
1.9254 e+00 \\
1.9291 e+00 \\
1.9004 e+00 \\
1.9334 e+00 \\
1.9011 e+00 \\
1.9041 e+00 \\
1.9069 e+00 \\
1.9205 e+00 \\
1.9003 e+00 \\
1.9136 e+00 \\
1.9146 e+00 \\
1.9160 e+00 \\
1.9175 e+00 \\
1.9266 e+00 \\
1.9232 e+00 \\
1.9275 e+00 \\
1.9247 e+00 \\
1.9279 e+00 \\
1.9139 e+00 \\
1.9155 e+00\end{array}$ \\
\hline
\end{tabular}

\begin{tabular}{|c|c|c|c|c|c|}
\hline 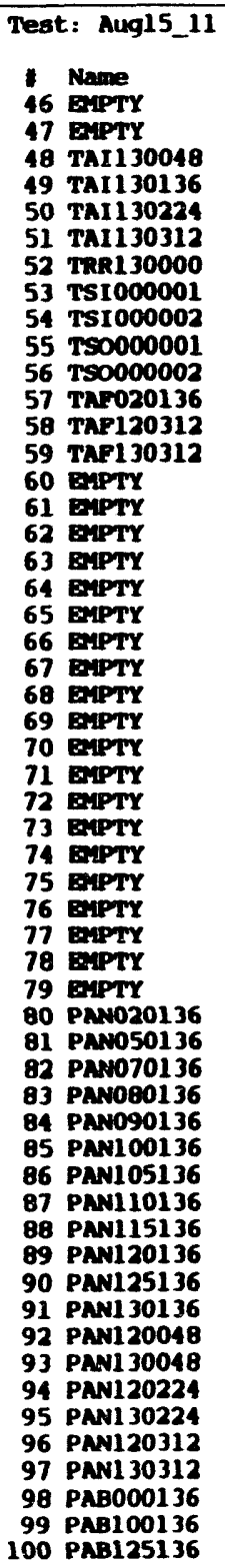 & \begin{aligned} \multicolumn{1}{c}{ suml } \\
Min \\
37.7675 \\
39.6057 \\
279.0680 \\
289.0895 \\
260.5658 \\
246.5950 \\
112.9013 \\
68.7908 \\
84.0930 \\
68.0386 \\
84.2000 \\
107.3937 \\
223.4476 \\
240.4848 \\
4.2041 \\
1.2188 \\
4.2188 \\
1.2383 \\
2.0215 \\
2.0361 \\
2.0508 \\
2.0703 \\
2.0850 \\
2.1045 \\
2.1240 \\
2.1484 \\
2.1680 \\
2.1973 \\
2.2119 \\
2.2363 \\
2.2510 \\
2.2754 \\
2.2949 \\
2.3193 \\
-17.1572 \\
-34.4418 \\
-20.6871 \\
-14.0321 \\
-12.3360 \\
-10.2071 \\
-7.7020 \\
-5.4674 \\
-7.1782 \\
-4.6883 \\
-3.7435 \\
-0.1238 \\
-11.9530 \\
-0.8919 \\
-12.4417 \\
-6.4099 \\
-13.8017 \\
-3.0458 \\
40.0908 \\
43.7097 \\
14.5284\end{aligned} & $\begin{aligned} \text { Date: Wed } \\
\text { Max } \\
38.4572 \\
40.5234 \\
281.5490 \\
294.5481 \\
285.0720 \\
249.6871 \\
116.7902 \\
69.6433 \\
86.2976 \\
68.7884 \\
85.2536 \\
109.1605 \\
267.0311 \\
275.5027 \\
5.0342 \\
4.9805 \\
4.9219 \\
4.8877 \\
2.3047 \\
2.3096 \\
2.3145 \\
2.3193 \\
2.3242 \\
2.3291 \\
2.3291 \\
2.3389 \\
2.3389 \\
2.3486 \\
2.3486 \\
2.3535 \\
2.3584 \\
2.3633 \\
2.3730 \\
2.3828 \\
-16.0891 \\
-30.7796 \\
-16.9487 \\
-13.7269 \\
-7.6058 \\
-2.5777 \\
-1.2933 \\
-0.2794 \\
-4.1264 \\
-2.0943 \\
-0.3103 \\
0.7917 \\
-0.5090 \\
1.7784 \\
-4.2019 \\
-5.1129 \\
-2.0524 \\
1.3793 \\
40.5027 \\
44.0759 \\
44.8488\end{aligned}$ & $\begin{aligned} \text { Aug } 1512: \\
\text { Ave } \\
38.1354 \\
10.0187 \\
280.2021 \\
292.6074 \\
271.3645 \\
248.3929 \\
115.0863 \\
69.2025 \\
85.3300 \\
68.4433 \\
84.7376 \\
108.3477 \\
232.4007 \\
254.9168 \\
4.5565 \\
1.5728 \\
4.5845 \\
4.6025 \\
2.1874 \\
2.2076 \\
2.2213 \\
2.2367 \\
2.2476 \\
2.2612 \\
2.2709 \\
2.2881 \\
2.2958 \\
2.3083 \\
2.3162 \\
2.3255 \\
2.3309 \\
2.3407 \\
2.3529 \\
2.3657 \\
-16.4797 \\
-33.0074 \\
-18.8591 \\
-13.8230 \\
-10.0366 \\
-7.3690 \\
-4.4565 \\
-4.0560 \\
-5.7866 \\
-3.4050 \\
-2.7257 \\
0.2012 \\
-9.0691 \\
0.3639 \\
-6.2344 \\
-5.8728 \\
-7.8233 \\
-1.2300 \\
40.2491 \\
13.8379 \\
14.6493\end{aligned}$ & $\begin{array}{c}10.31 \text { 1990 } \\
\text { SD } \\
2.0772 e-01 \\
2.6221 e-01 \\
5.5442 e-01 \\
1.5377 e+00 \\
8.6051 e+00 \\
7.8694 e-01 \\
1.1094 e+00 \\
1.9132 e-01 \\
1.6469 e-01 \\
1.8131 e-01 \\
2.1220 e-01 \\
4.5668 e-01 \\
1.1109 e+01 \\
1.1807 e+01 \\
2.9804 e-01 \\
2.8472 e-01 \\
2.6498 e-01 \\
2.4073 e-01 \\
1.0253 e-01 \\
9.7360 e-02 \\
9.1162 e-02 \\
8.4284 e-02 \\
7.7902 e-02 \\
7.0609 e-02 \\
6.3333 e-02 \\
5.6583 e-02 \\
4.9743 e-02 \\
1.2770 e-02 \\
3.7105 e-02 \\
3.1332 e-02 \\
2.6903 e-02 \\
2.2900 e-02 \\
2.0280 e-02 \\
1.7626 e-02 \\
2.0135 e-01 \\
1.0999 e+00 \\
1.1805 e+00 \\
6.8604 e-02 \\
1.0752 e+00 \\
1.4269 e+00 \\
1.2786 e+00 \\
1.0730 e+00 \\
6.8976 e-01 \\
5.3676 e-01 \\
7.6132 e-01 \\
2.0321 e-01 \\
3.5923 e+00 \\
5.3422 e-01 \\
1.5403 e+00 \\
3.2514 e-01 \\
2.6257 e+00 \\
1.3947 e+00 \\
9.5311 e-02 \\
8.8706 e-02 \\
7.3266 e-02\end{array}$ & $\begin{array}{c}\text { Page: } 2 \\
\text { Uncert } \\
1.9010 e+00 \\
1.9011 e+00 \\
1.9295 e+00 \\
1.9331 e+00 \\
1.9271 e+00 \\
1.9213 e+00 \\
1.9014 e+00 \\
1.9000 e+00 \\
1.9002 e+00 \\
1.9000 e+00 \\
1.9001 e+00 \\
1.9010 e+00 \\
1.9177 e+00 \\
1.9229 e+00 \\
0.0000 e+00 \\
0.0000 e+00 \\
0.0000 e+00 \\
0.0000 e+00 \\
0.0000 e+00 \\
0.0000 e+00 \\
0.0000 e+00 \\
0.0000 e+00 \\
0.0000 e+00 \\
0.0000 e+00 \\
0.0000 e+00 \\
0.0000 e+00 \\
0.0000 e+00 \\
0.0000 e+00 \\
0.0000 e+00 \\
0.0000 e+00 \\
0.0000 e+00 \\
0.0000 e+00 \\
0.000 e+00 \\
0.0000 e+00 \\
8.7097 e-01 \\
1.6820 e+00 \\
8.7128 e-01 \\
8.7069 e-01 \\
8.7036 e-01 \\
8.7020 e-01 \\
8.7007 e-01 \\
8.7006 e-01 \\
8.7012 e-01 \\
8.7004 e-01 \\
8.7003 e-01 \\
8.7000 e-01 \\
8.7030 e-01 \\
8.7000 e-01 \\
8.7014 e-01 \\
8.7012 e-01 \\
8.7022 e-01 \\
8.7001 e-01 \\
5.8866 e-01 \\
5.9026 e-01 \\
5.9064 e-01 \\
\end{array}$ \\
\hline
\end{tabular}



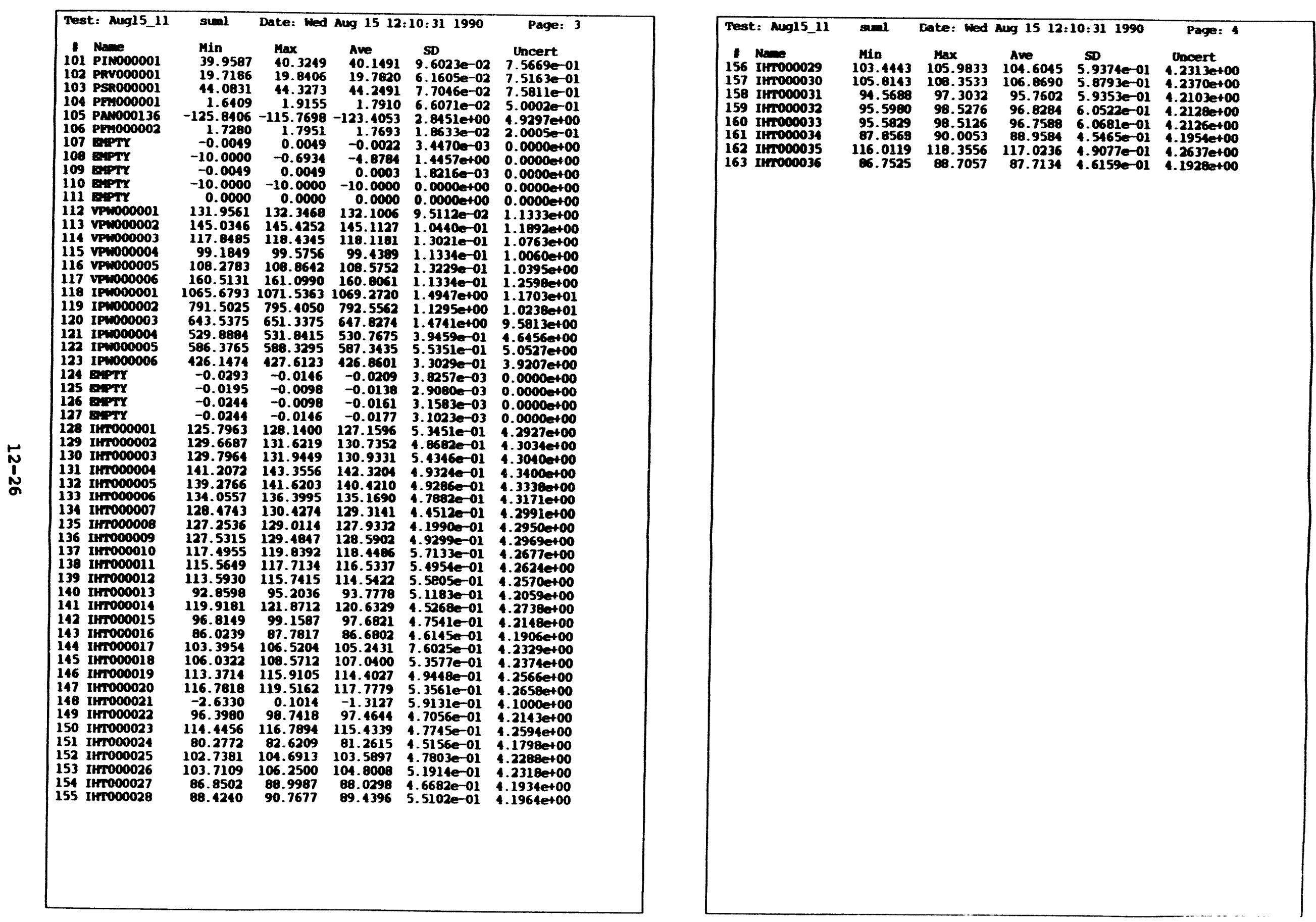


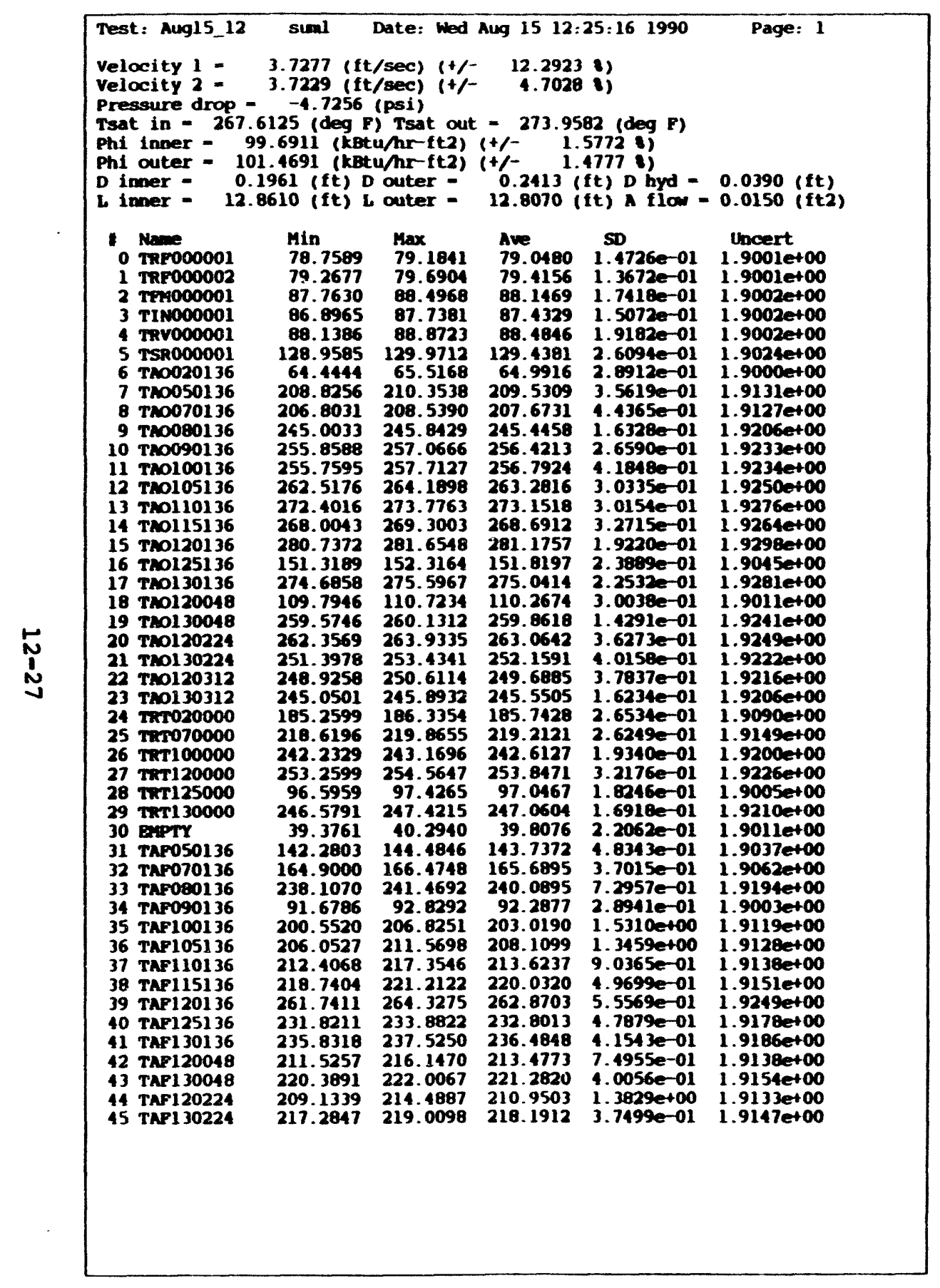

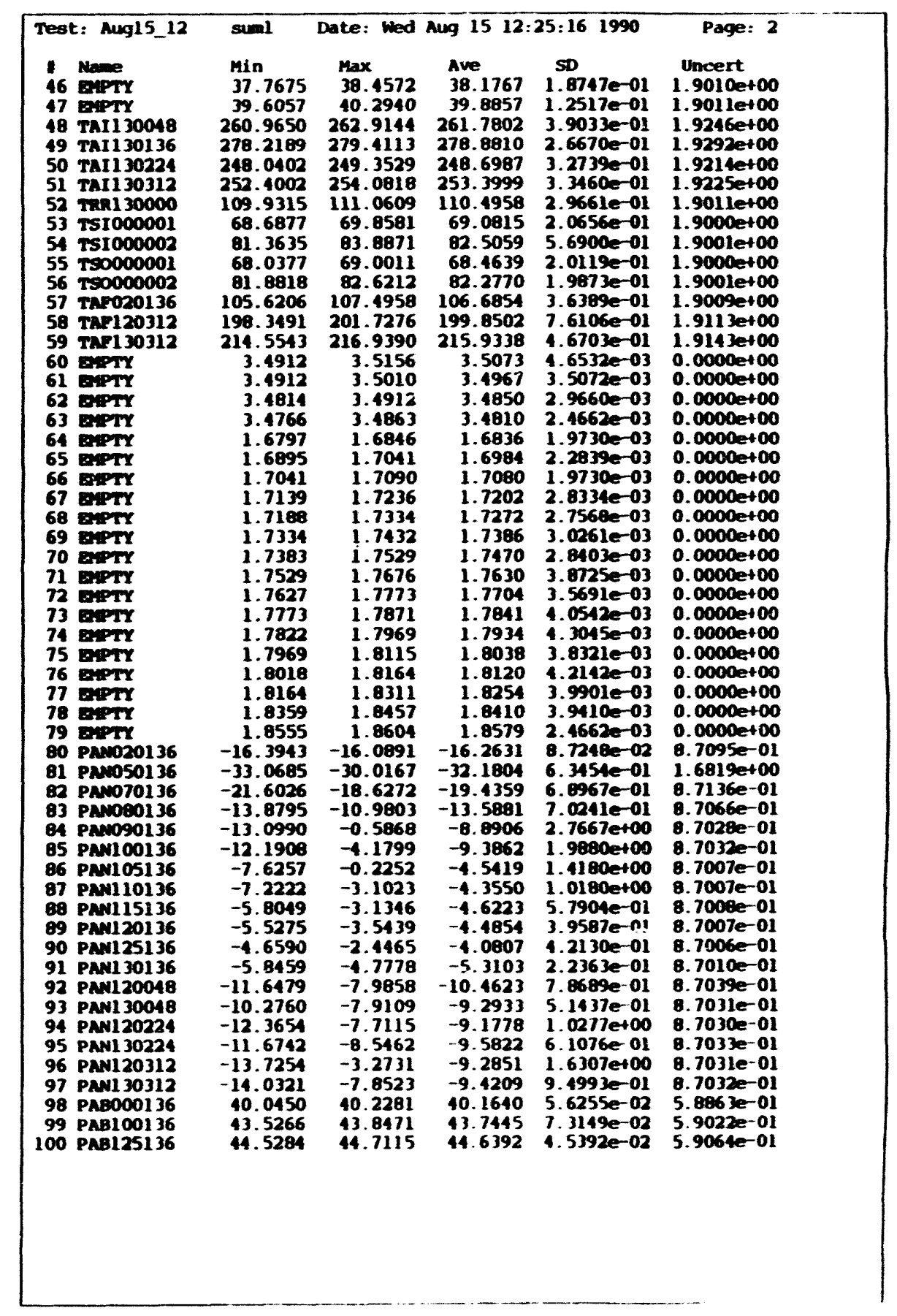


DEFECTIVE INSTRUIENTS FOR THE RIBBED GEOMETRY TEST SERIES

\begin{tabular}{|c|c|c|}
\hline Series & Tests & Defective Instruments \\
\hline 15 & Aug15_01 to Aug15_12 & TAO120048 TAF070136 TRT125000 \\
& & TAO020136 TAF080136 TRR130000 \\
& & TAO070136 TAF090136 \\
& & TAO125136 TAF120136 \\
& & IHTO00021 IHTO00024 IHTO00033 \\
\hline
\end{tabular}


Chapter 13

NUCLEATE BOILING PRESSURE DROP IN AN ANNULUS

Test Geometry: Ribbed (4)

Test Series: 16

Test Date: August 15, 1990 

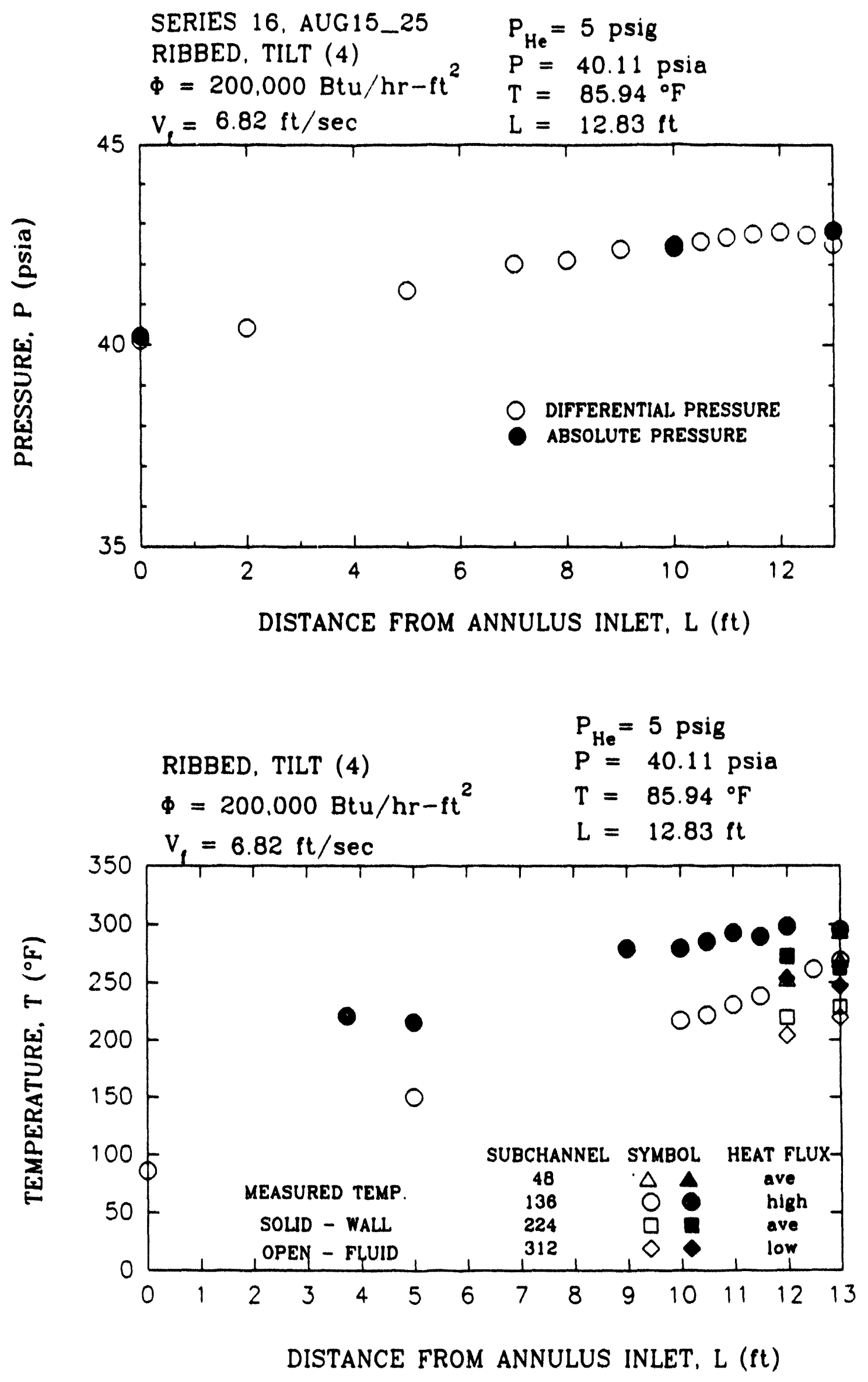


\begin{tabular}{|c|c|c|c|c|c|c|}
\hline \multicolumn{7}{|r|}{ Page: 1} \\
\hline \multicolumn{4}{|c|}{ 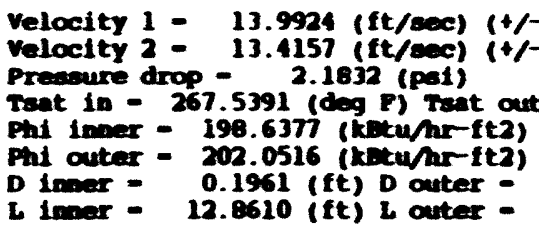 } & $\begin{aligned} & 1.8678 \\
& 1.7033 \\
&- 264.23 \\
&(+/- 1 . \\
& 1+/-2413 ! \\
& 0.2413 \\
& 12.8070 !\end{aligned}$ & ; & $\begin{array}{l}0.0390 \text { (ft) } \\
0.0150 \text { (fte) }\end{array}$ \\
\hline $\begin{array}{l}8 \\
9 \\
10 \\
11 \\
12 \\
13 \\
14 \\
15 \\
16 \\
17 \\
18 \\
19 \\
20 \\
21 \\
22 \\
23 \\
24 \\
25 \\
26 \\
27 \\
28 \\
29 \\
30 \\
31 \\
32 \\
33 \\
34 \\
35 \\
36 \\
37 \\
38 \\
39 \\
40 \\
41 \\
12 \\
43 \\
44\end{array}$ & 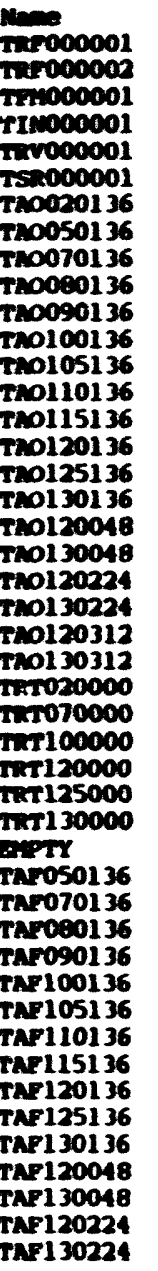 & $\begin{array}{r}\text { Min } \\
81.7317 \\
82.2238 \\
87.1600 \\
86.9000 \\
88.0539 \\
128.6758 \\
19.9924 \\
164.9653 \\
160.6148 \\
130.3011 \\
193.1341 \\
186.6695 \\
189.9134 \\
199.0304 \\
191.1183 \\
205.8116 \\
135.8921 \\
198.0750 \\
104.1219 \\
186.8496 \\
191.5462 \\
181.2385 \\
179.3987 \\
179.1694 \\
150.8922 \\
168.3349 \\
181.4116 \\
184.0270 \\
94.9643 \\
181.5270 \\
39.6057 \\
117.2075 \\
130.9522 \\
177.7345 \\
93.4651 \\
148.2484 \\
151.5795 \\
155.1649 \\
159.3809 \\
179.8638 \\
165.3253 \\
168.0399 \\
150.2616 \\
156.5446 \\
150.9055 \\
155.0906\end{array}$ & 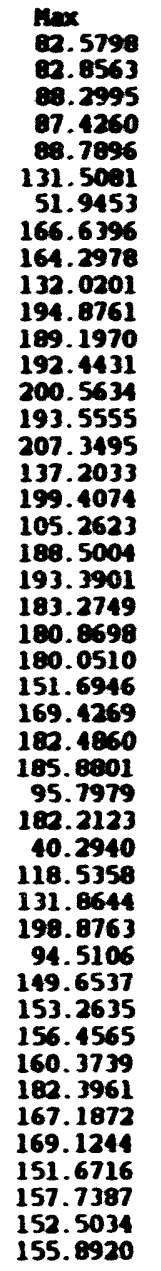 & $\begin{array}{r}10 \\
62.0922 \\
82.5063 \\
87.8509 \\
67.1357 \\
89.4491 \\
130.5043 \\
51.0513 \\
165.7924 \\
161.6150 \\
131.0181 \\
193.9159 \\
187.5567 \\
190.6690 \\
199.5456 \\
192.2153 \\
205.4344 \\
136.4693 \\
198.5902 \\
104.6400 \\
187.4014 \\
192.0550 \\
181.9949 \\
180.0346 \\
179.5227 \\
151.3416 \\
168.9027 \\
182.0056 \\
184.9092 \\
95.5140 \\
181.9226 \\
39.8352 \\
117.9200 \\
131.4310 \\
185.3090 \\
94.0466 \\
149.0877 \\
152.4972 \\
155.7689 \\
159.9133 \\
180.7991 \\
166.0640 \\
168.6346 \\
150.9745 \\
157.1694 \\
151.8601 \\
155.5835\end{array}$ & 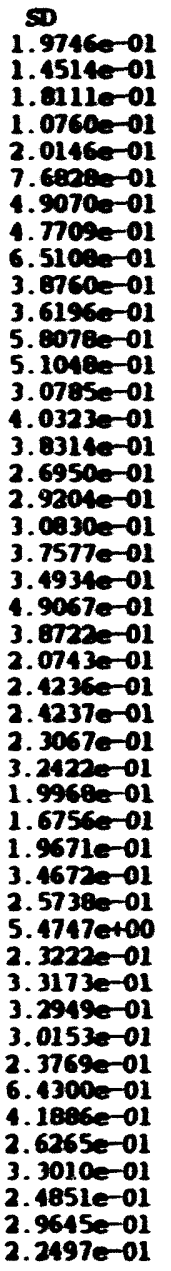 & 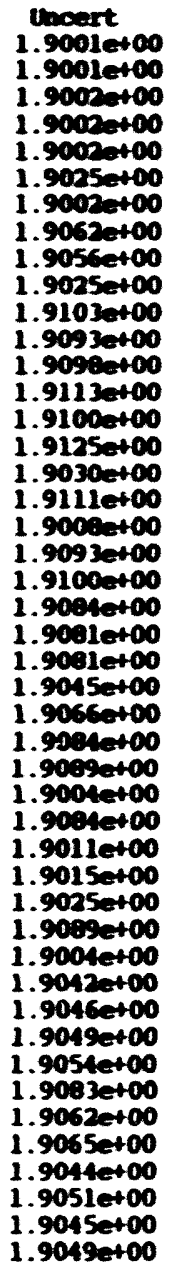 \\
\hline
\end{tabular}

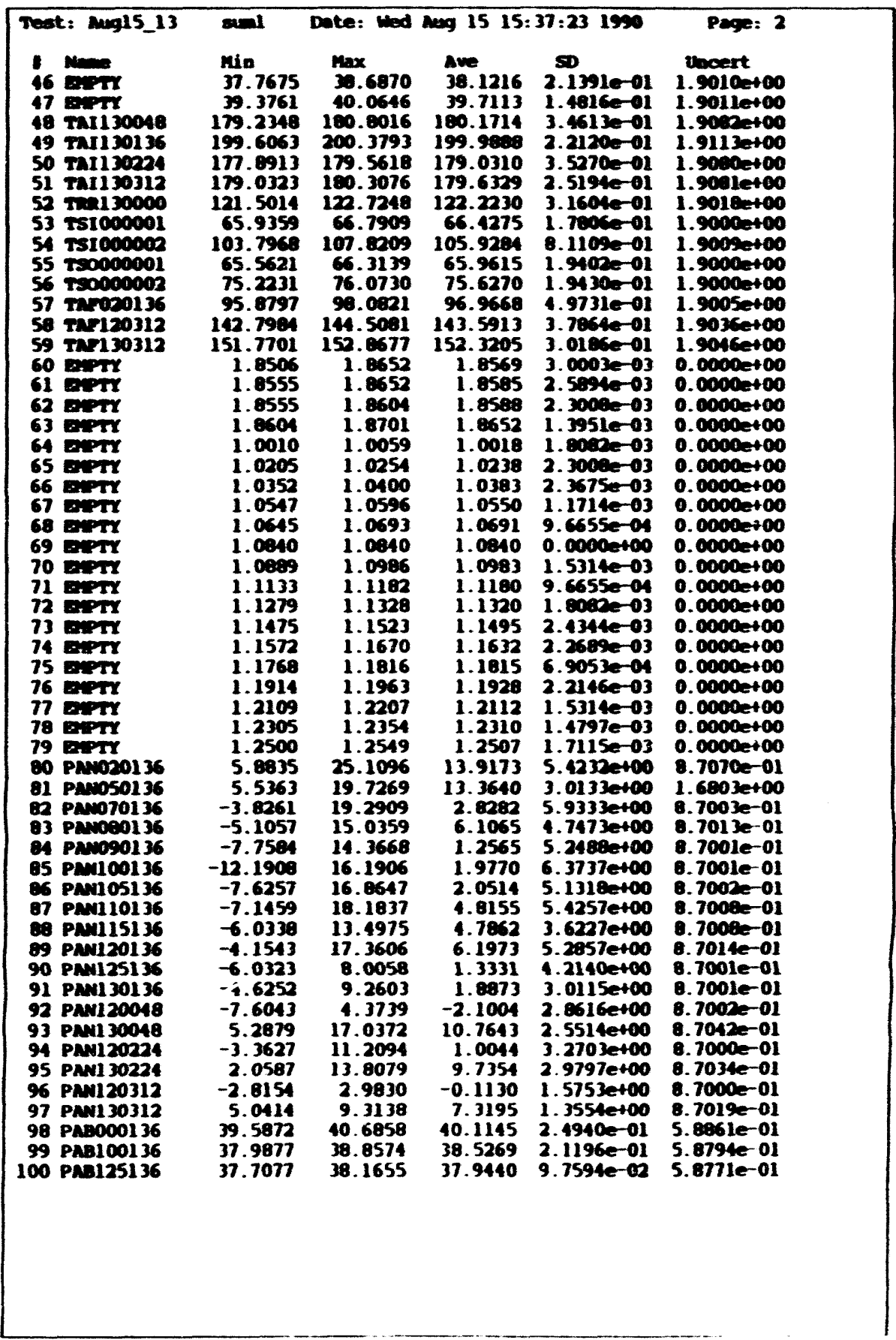




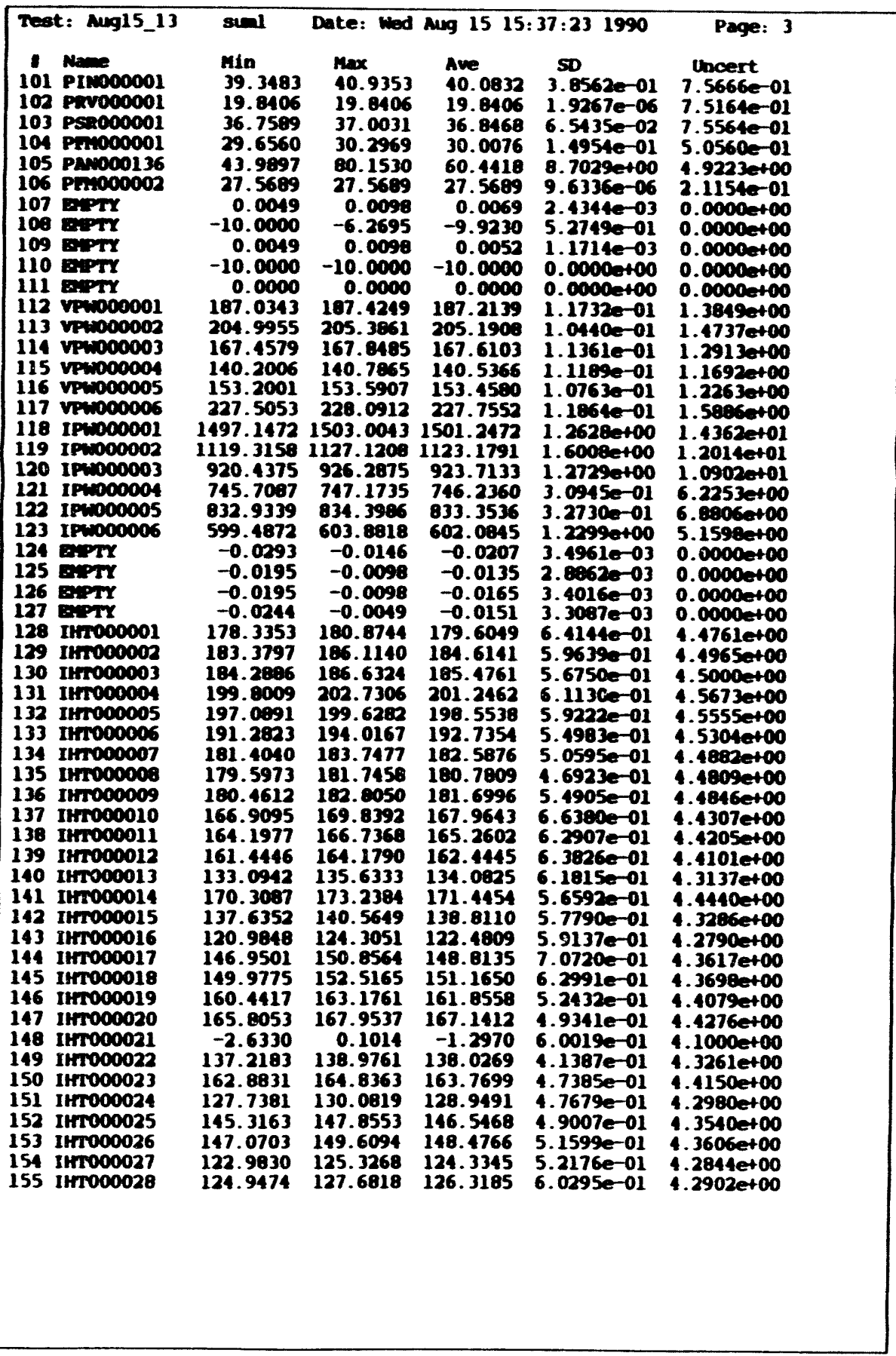

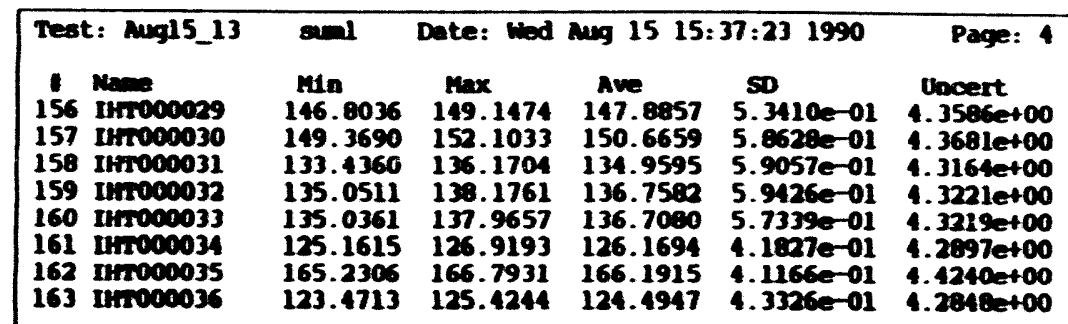




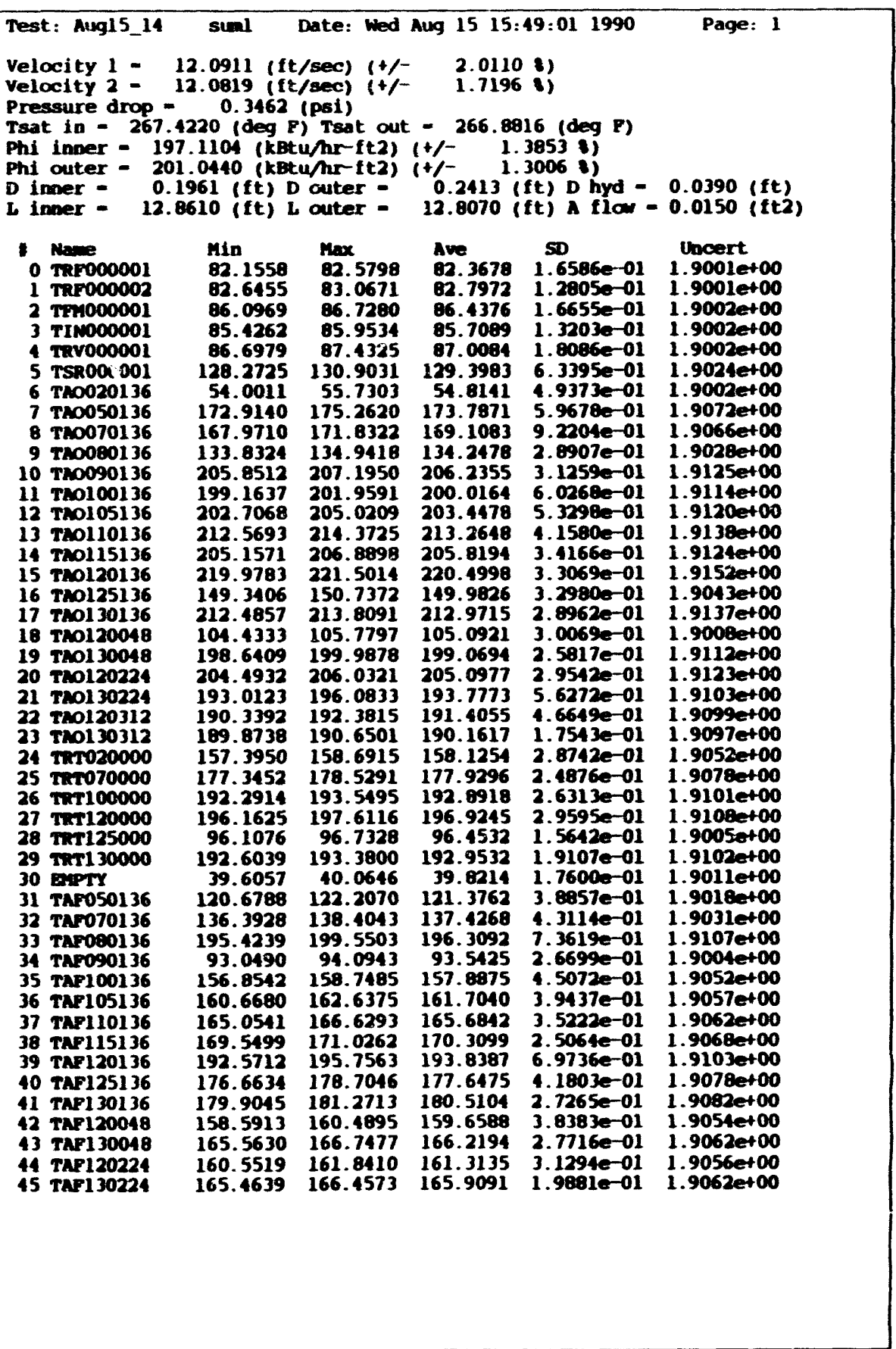

\begin{tabular}{|c|c|c|c|c|c|c|}
\hline $\begin{array}{l}\text { Test } \\
1 \\
16 \\
17 \\
48 \\
19 \\
50 \\
51 \\
52 \\
53 \\
54 \\
55 \\
56 \\
57 \\
58 \\
59 \\
60 \\
61 \\
62 \\
63 \\
64 \\
65 \\
66 \\
67 \\
68 \\
69 \\
70 \\
71 \\
72 \\
73 \\
74 \\
75 \\
76 \\
77 \\
78 \\
79 \\
80 \\
81 \\
82 \\
83 \\
84 \\
85 \\
86 \\
87 \\
88 \\
89 \\
90 \\
91 \\
92 \\
93 \\
94 \\
95 \\
96 \\
97 \\
98 \\
99 \\
100\end{array}$ & 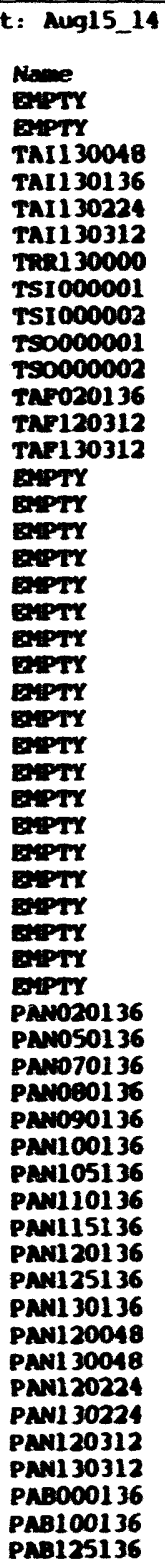 & \begin{tabular}{r}
\multicolumn{1}{c}{ suml } \\
Min \\
37.7675 \\
39.3761 \\
192.0055 \\
214.8312 \\
189.9284 \\
191.9186 \\
127.0890 \\
66.2575 \\
106.0694 \\
65.6691 \\
75.9665 \\
96.6127 \\
150.4191 \\
160.5182 \\
2.0605 \\
2.0557 \\
2.0605 \\
2.0605 \\
1.0899 \\
1.1094 \\
1.1230 \\
1.1426 \\
1.1523 \\
1.1670 \\
1.1768 \\
1.2012 \\
1.2109 \\
1.2305 \\
1.2451 \\
1.2598 \\
1.2744 \\
1.2891 \\
1.3086 \\
1.3281 \\
-2.2799 \\
-7.5663 \\
-13.2103 \\
-13.8795 \\
-13.0990 \\
-12.1145 \\
-7.7020 \\
-7.0696 \\
-7.5597 \\
-6.6720 \\
-6.0323 \\
-3.0230 \\
-12.0293 \\
-2.7992 \\
-9.8477 \\
-5.8759 \\
-9.4529 \\
0.9215 \\
39.5872 \\
39.4525 \\
39.3099
\end{tabular} & $\begin{array}{r}\text { Date: } \text { Hed } \\
\text { Max } \\
38.4572 \\
39.8352 \\
193.4615 \\
215.8807 \\
191.4867 \\
193.0846 \\
128.6094 \\
66.8977 \\
108.4416 \\
66.5276 \\
76.9213 \\
98.7100 \\
152.1169 \\
162.4995 \\
2.0752 \\
2.0703 \\
2.0703 \\
2.0703 \\
1.0938 \\
1.1133 \\
1.1279 \\
1.1426 \\
1.1572 \\
1.1719 \\
1.1816 \\
1.2061 \\
1.2158 \\
1.2354 \\
1.2451 \\
1.2646 \\
1.2793 \\
1.3086 \\
1.3135 \\
1.3379 \\
14.4285 \\
12.2501 \\
8.4572 \\
14.1204 \\
17.1134 \\
12.3759 \\
12.2870 \\
12.8432 \\
9.8354 \\
12.4778 \\
3.8859 \\
7.6581 \\
4.5264 \\
16.2743 \\
13.2694 \\
12.5872 \\
0.8468 \\
6.8724 \\
40.5027 \\
40.0934 \\
39.9966\end{array}$ & $\begin{array}{r}\text { Aug } 1515: \\
\text { Ave } \\
38.1767 \\
39.7204 \\
192.6210 \\
215.3922 \\
190.7114 \\
192.5174 \\
127.8024 \\
66.5824 \\
107.3570 \\
66.0596 \\
76.4545 \\
97.1775 \\
151.3977 \\
161.1689 \\
2.0657 \\
2.0646 \\
2.0636 \\
2.0681 \\
1.0892 \\
1.1102 \\
1.1239 \\
1.1426 \\
1.1527 \\
1.1683 \\
1.1815 \\
1.2014 \\
1.2137 \\
1.2307 \\
1.2451 \\
1.2605 \\
1.2745 \\
1.2896 \\
1.3094 \\
1.3306 \\
6.2345 \\
3.0033 \\
-3.4691 \\
-0.8592 \\
-1.4138 \\
-0.3133 \\
0.8368 \\
2.5984 \\
0.0453 \\
3.6171 \\
-1.9765 \\
2.9081 \\
-6.5575 \\
6.9969 \\
-1.0510 \\
5.6537 \\
-4.2848 \\
3.8527 \\
40.0358 \\
39.8169 \\
39.6743\end{array}$ & $\begin{array}{l}49.01 \text { 1990 } \\
5 . \\
2.1432 e-01 \\
1.3320 e-01 \\
1.2263 e-01 \\
2.4862 e-01 \\
3.3276 e-01 \\
2.5925 e-01 \\
3.6522 e-01 \\
1.5557 e-01 \\
6.0329 e-01 \\
1.9238 e-01 \\
2.2273 e-01 \\
4.5560 e-01 \\
4.1457 e-01 \\
3.1209 e-01 \\
1.1161 e-03 \\
3.6057 e-03 \\
2.5894 e-03 \\
2.8266 e-03 \\
1.1714 e-03 \\
2.3675 e-03 \\
1.8950 e-03 \\
0.0000 e+00 \\
1.3381 e-03 \\
2.1635 e-03 \\
6.9053 e-04 \\
9.6655 e-04 \\
2.4484 e-03 \\
9.6655 e-04 \\
0.0000 e+00 \\
1.8002 e-03 \\
6.9053 e-04 \\
2.8334 e-03 \\
1.8062 e-03 \\
2.6562 e-03 \\
1.6861 e+00 \\
4.2906 e+00 \\
6.7466 e+00 \\
6.0673 e+00 \\
6.7487 e+00 \\
6.6332 e+00 \\
5.8312 e+00 \\
5.1247 e+00 \\
4.2034 e+00 \\
3.8061 e+00 \\
2.9621 e+00 \\
2.2435 e+00 \\
4.4181 e+00 \\
1.2685 e+00 \\
5.3827 e+00 \\
3.7000 e+00 \\
2.2299 e+00 \\
1.8121 e+00 \\
2.4953 e-01 \\
1.7522 e-01 \\
1.2578 e-01\end{array}$ & 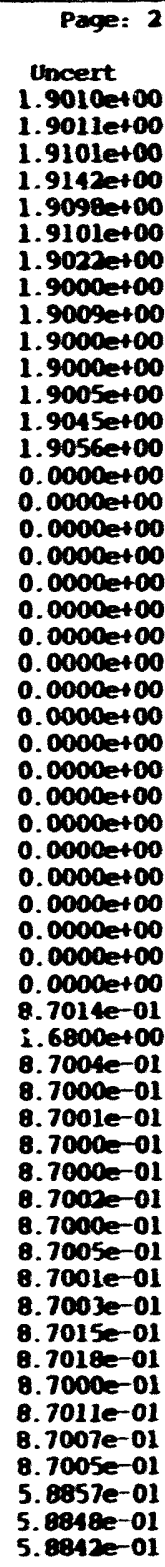 \\
\hline
\end{tabular}



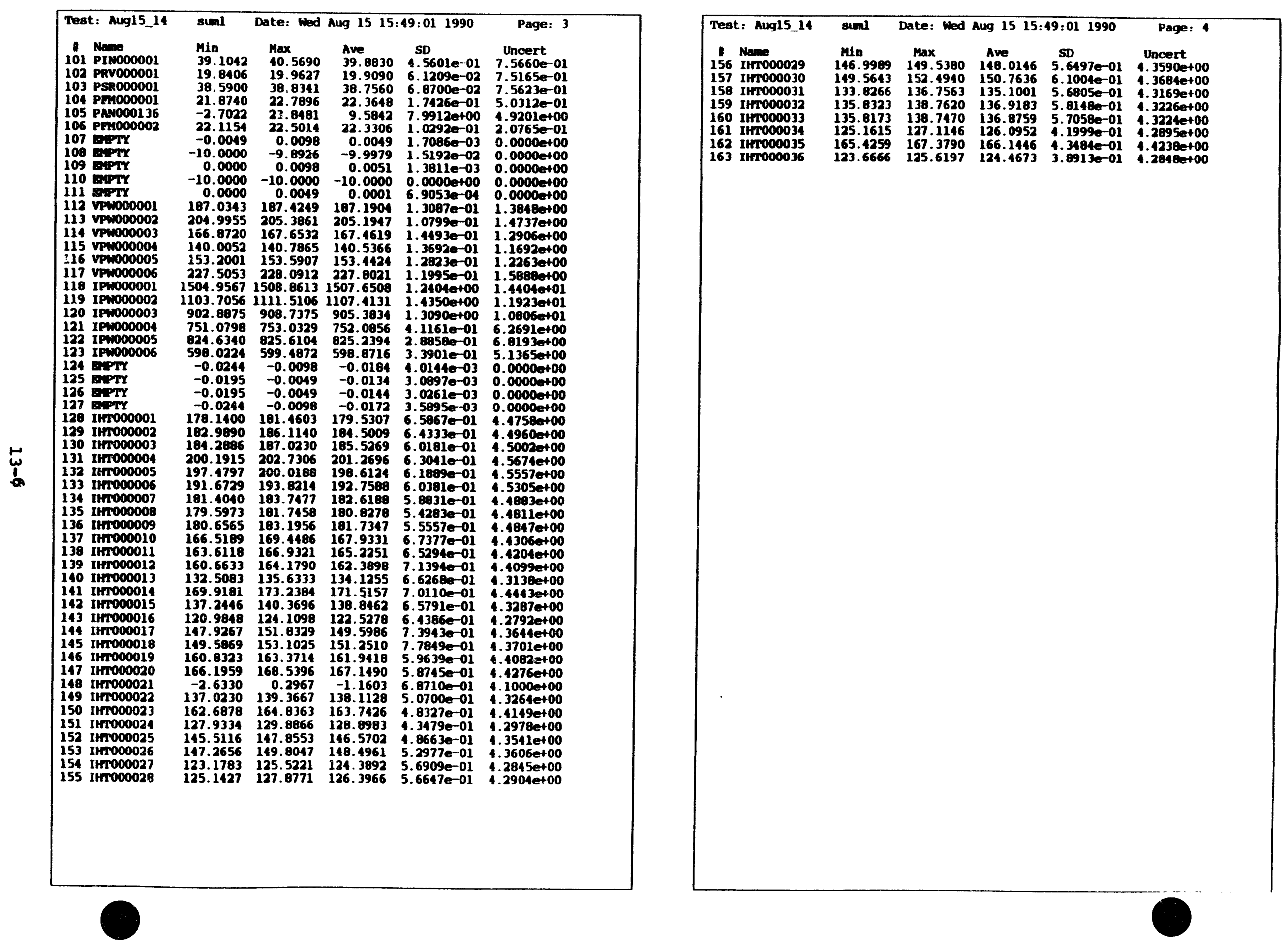
Test: Aug15 15 Sunl Date: Wed Aug 15 16:00:27 1990

Page: 1

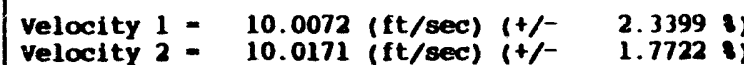

velocity 2 - 10.0171 (ft/sec) $(+/-1.7722$ i)

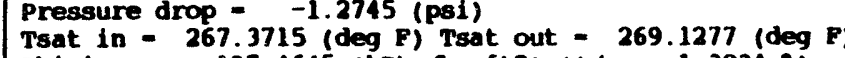

Tsat in - 267.3715 (deg F) Tsat out $=269.1277$ (deg

D inner - 0.1961 (ft) D outer - 0.2413 (ft) D hyd $=0.0390$ (ft)

$L$ inner = 12.8610 (ft) 2 outer - 12.8070 (ft) A flow $=0.0150$ (ft2)

1 Name

1 TRF000002

2 TFM00001

3 TIN000001

5 TSR000001

6 TMO020136

T T20070136

9 TM0080136

10 T20090136

11 TMO100136

13 T20110136

14 TMO115136

15 TMO120136

$16 \mathrm{Th} 0125136$

18 THO120048

19 Thol 30048

$\stackrel{w}{0}$

\begin{tabular}{|c|c|c|c|c|}
\hline $\begin{array}{l}\text { Min } \\
82.3678 \\
82.8563 \\
86.4139 \\
85.8483 \\
86.9091 \\
33.9321 \\
57.2414 \\
86.1583 \\
82.1752 \\
38.8565 \\
25.2953 \\
18.8037 \\
22.5504 \\
33.8685 \\
26.0065 \\
141.5500 \\
60.6744 \\
35.9884 \\
03.8126 \\
13.5307 \\
24.3709 \\
12.1044 \\
0.97 .9424 \\
07.4134 \\
69.1186 \\
93.0511 \\
10.4668 \\
15.4675 \\
92.1682 \\
10.5924 \\
39.3761 \\
28.2991 \\
47.2036 \\
12.7155 \\
90.2279 \\
72.2300 \\
76.8177 \\
81.4093 \\
86.5609 \\
14.9041\end{array}$ & $\begin{array}{r}\text { Max } \\
83.0036 \\
83.4885 \\
87.1493 \\
86.3753 \\
87.7486 \\
136.0479 \\
59.8273 \\
188.6770 \\
186.6836 \\
140.4636 \\
226.6225 \\
220.6122 \\
224.1682 \\
234.9001 \\
228.2849 \\
242.8666 \\
161.7654 \\
236.8296 \\
104.9533 \\
219.7667 \\
225.7944 \\
214.2875 \\
209.4805 \\
209.1366 \\
170.8024 \\
194.5148 \\
211.8064 \\
217.1854 \\
92.7935 \\
211.4544 \\
40.0646 \\
130.8302 \\
149.2961 \\
214.3307 \\
91.3800 \\
174.5955 \\
178.4691 \\
183.6474 \\
188.6982 \\
220.1281 \\
197.2445 \\
200.7464 \\
176.2847 \\
184.1098 \\
178.1767 \\
183.7275\end{array}$ & $\begin{array}{r}\text { Ave } \\
82.6900 \\
83.1008 \\
86.7957 \\
86.1305 \\
87.3452 \\
134.9270 \\
58.5696 \\
187.2134 \\
183.7487 \\
139.4957 \\
225.7542 \\
219.7408 \\
223.2740 \\
234.4541 \\
227.1305 \\
242.1708 \\
161.1309 \\
236.3887 \\
104.5135 \\
219.0549 \\
224.9141 \\
213.1339 \\
208.6006 \\
208.3469 \\
170.0910 \\
193.6450 \\
211.0793 \\
216.1967 \\
92.4475 \\
211.0175 \\
39.7296 \\
129.3368 \\
148.0337 \\
213.5737 \\
90.7598 \\
173.1564 \\
177.4655 \\
182.2439 \\
187.5138 \\
217.1593 \\
196.4846 \\
199.9420 \\
175.4747 \\
183.1063 \\
177.3549 \\
107\end{array}$ & $\begin{array}{c}\text { SD } \\
1.9262 e-01 \\
1.6134 e-01 \\
1.8458 e-01 \\
1.2846 e-01 \\
1.9095 e-01 \\
1.2659 e-01 \\
1.3985 e-01 \\
5.1710 e-01 \\
1.0183 e+00 \\
3.5373 e-01 \\
2.6974 e-01 \\
4.1070 e-01 \\
3.4296 e-01 \\
2.1624 e-01 \\
4.6721 e-01 \\
3.2499 e-01 \\
2.7368 e-01 \\
1.8623 e-01 \\
2.6813 e-01 \\
2.6556 e-01 \\
2.5231 e-01 \\
4.7211 e-01 \\
3.6194 e-01 \\
3.7403 e-01 \\
2.9492 e-01 \\
3.0262 e-01 \\
3.0008 e-01 \\
3.3931 e-01 \\
1.5405 e-01 \\
1.6553 e-01 \\
2.2815 e-01 \\
4.6080 e-01 \\
4.5929 e-01 \\
1.4543 e-01 \\
2.9820 e-01 \\
5.3372 e-01\end{array}$ & $\begin{array}{l}\text { Uncert } \\
1.9001 e+00 \\
1.9001 e+00 \\
1.9002 e+00 \\
1.9002 e+00 \\
1.9002 e+00 \\
1.9028 e+00 \\
1.9001 e+00 \\
1.9092 e+00 \\
1.9087 e+00 \\
1.9033 e+00 \\
1.9163 e+00 \\
1.9150 e+00 \\
1.9158 e+00 \\
1.9181 e+00 \\
1.9166 e+00 \\
1.9199 e+00 \\
1.9056 e+00 \\
1.9186 e+00 \\
1.9008 e+00 \\
1.9149 e+00 \\
1.9161 e+00 \\
1.9138 e+00 \\
1.9129 e+00 \\
1.9129 e+00 \\
1.9067 e+00 \\
1.9103 e+00 \\
1.9134 e+00 \\
1.9143 e+00 \\
1.9003 e+00 \\
1.9133 e+00 \\
1.9011 e+00 \\
1.9024 e+00 \\
1.9041 e+00 \\
1.9138 e+00 \\
1.9003 e+00 \\
1.9072 e+00 \\
1.9078 e+00 \\
1.9085 e+00 \\
1.9093 e+00 \\
1.9145 e+00\end{array}$ \\
\hline
\end{tabular}

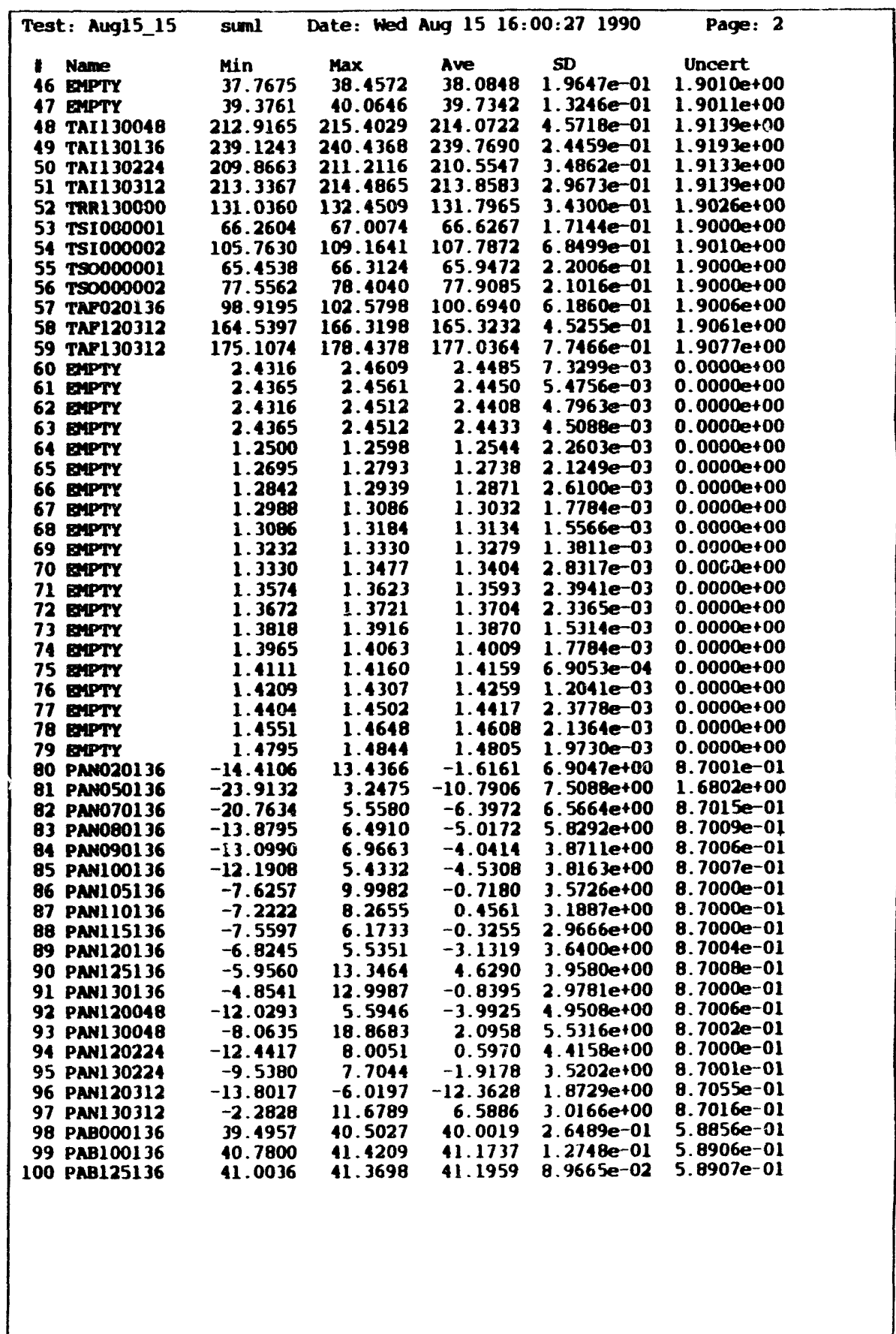




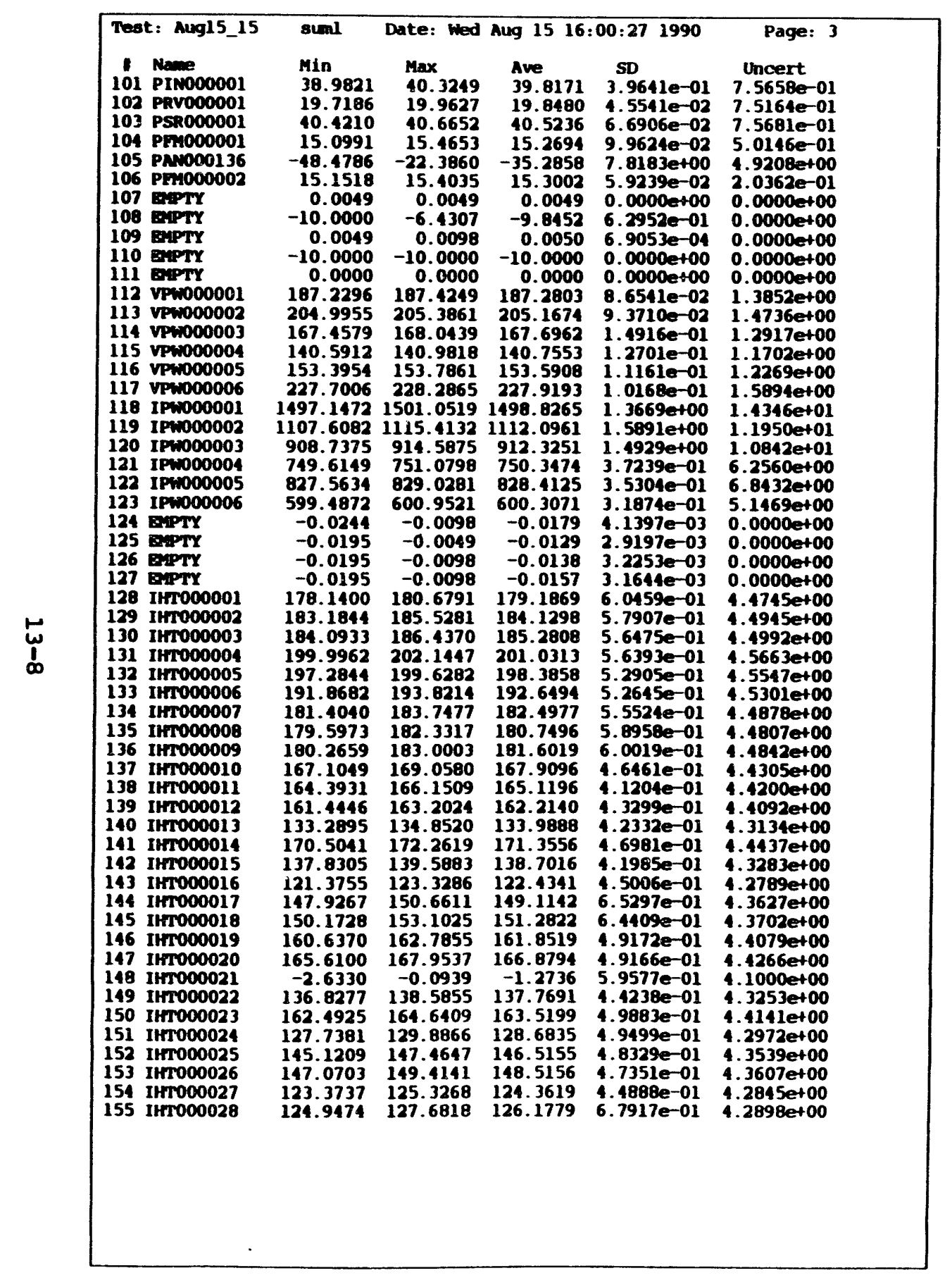

\begin{tabular}{|c|c|c|c|c|c|}
\hline Test: Aug15_15 & sumal & Date: Wed & Aug 15 16: & $00: 271990$ & Page: \\
\hline 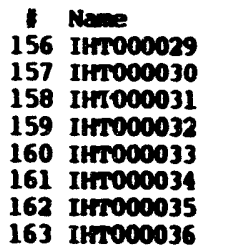 & $\begin{array}{l}\text { Min } \\
146.6083 \\
149.5643 \\
133.8266 \\
135.6370 \\
135.6220 \\
124.9662 \\
164.8400 \\
123.2760\end{array}$ & $\begin{array}{l}\operatorname{Max} \\
149.1474 \\
151.9080 \\
136.1704 \\
138.1761 \\
138.1611 \\
127.5053 \\
167.5744 \\
125.8150\end{array}$ & $\begin{array}{l}\text { Ave } \\
147.8076 \\
150.5800 \\
134.8891 \\
136.7504 \\
136.7158 \\
126.1108 \\
166.0587 \\
124.4595\end{array}$ & $\begin{array}{l}\text { SD } \\
6.3136 e-01 \\
5.9451 e-01 \\
6.0381 e-01 \\
6.4144 e-01 \\
6.4356 e-01 \\
5.1450 e-01 \\
5.2432 e-01 \\
4.9820 e-01\end{array}$ & $\begin{array}{l}\text { Uncert } \\
4.3583 e+00 \\
4.3678 e+00 \\
4.3162 e+00 \\
4.3220 e+00 \\
1.3219 e+00 \\
1.2896 e+00 \\
4.4235 e+00 \\
4.2847 e+00\end{array}$ \\
\hline
\end{tabular}

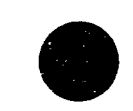




\begin{tabular}{|c|c|c|c|c|c|c|}
\hline \multicolumn{2}{|r|}{ st: Aug15_16 } & ml & te: & & age: \\
\hline \multicolumn{3}{|c|}{ 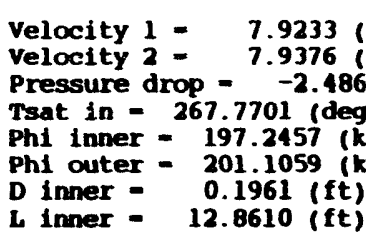 } & c) 1 & $1-$ & $\operatorname{leg}$ & $\begin{array}{l}0390 \text { (ft } \\
0150 \text { ift }\end{array}$ \\
\hline . & we & $\begin{array}{r}\text { Min } \\
83.0036 \\
83.2778 \\
86.1149 \\
85.7433 \\
87.1212 \\
137.6578 \\
54.1099 \\
205.6096 \\
203.6085 \\
149.1477 \\
252.6219 \\
248.7856 \\
253.5789 \\
267.6825 \\
260.2149 \\
277.7132 \\
180.0465 \\
271.2038 \\
110.3232 \\
249.7563 \\
255.3990 \\
241.8864 \\
235.9538 \\
234.6593 \\
186.72 \\
215.0490 \\
237.4703 \\
243.3672 \\
98.8059 \\
237.6039 \\
39.3761 \\
139.5986 \\
163.1609 \\
245.3255 \\
95.7641 \\
195.2240 \\
200.6463 \\
206.7015 \\
213.2357 \\
258.6257 \\
225.0962\end{array}$ & $\begin{array}{l}\operatorname{Max} \\
83.4272 \\
83.6991 \\
87.0453 \\
86.3752 \\
87.7515 \\
139.8689 \\
55.5153\end{array}$ & $\begin{array}{r}\text { Ave } \\
83.1942 \\
83.5728 \\
86.7242 \\
86.0237 \\
87.4009 \\
138.8180 \\
54.7822 \\
206.3573 \\
204.9713 \\
149.8153 \\
253.6097 \\
249.7587 \\
254.6861 \\
268.5618\end{array}$ & $\begin{array}{c}\text { SD } \\
1.6155 e-01 \\
1.3460 e-01 \\
1.607 e-01 \\
1.3224 e-01 \\
1.5926 e-01 \\
5.7181 e-01 \\
3.7469 e-01 \\
1.1289 e-01 \\
7.0518 e-01 \\
2.7662 e-01 \\
1.0332 e-01 \\
5.5208 e-01 \\
5.1992 e-01 \\
2.6406 e-01 \\
3.8828 e-01 \\
2.4868 e-01 \\
2.9298 e-01 \\
2.7689 e-01 \\
3.1407 e-01 \\
3.0768 e-01 \\
3.3938 e-01 \\
6.1825 e-01 \\
3.3439 e-01 \\
2.6047 e-01 \\
3.5778 e-01 \\
4.1529 e-01 \\
3.1069 e-01 \\
3.9475 e-01 \\
1.5992 e-01 \\
1.6922 e-01 \\
2.2121 e-01 \\
6.8344 e-01\end{array}$ & 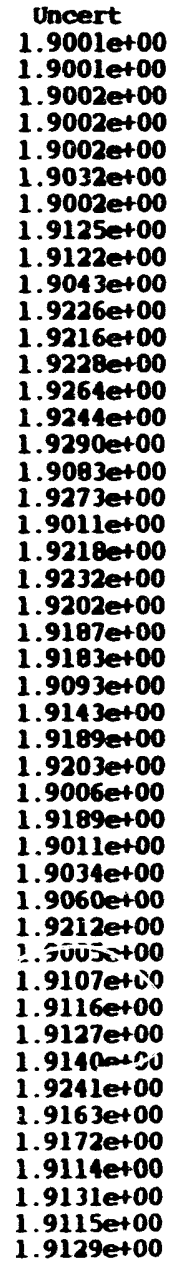 \\
\hline
\end{tabular}

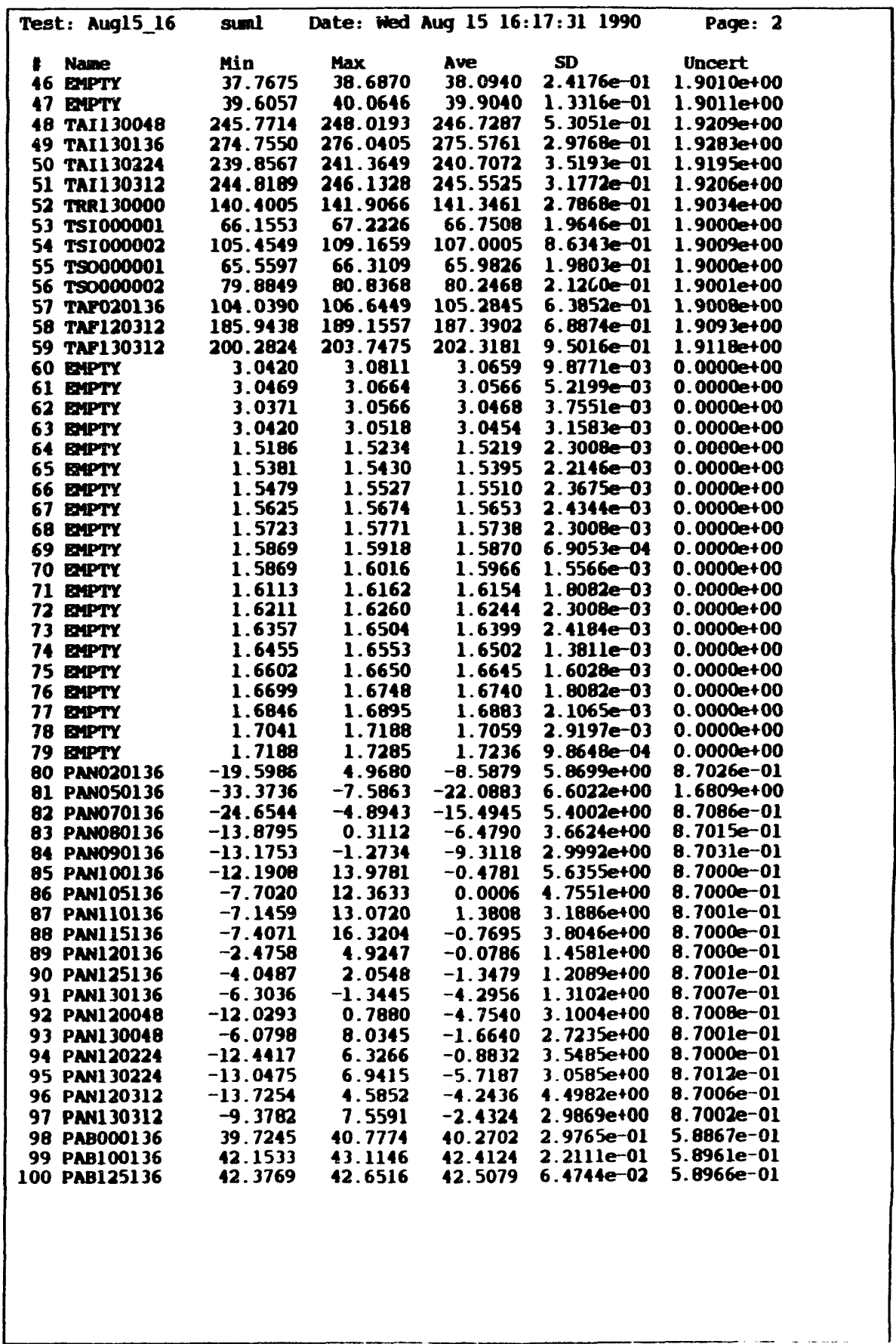



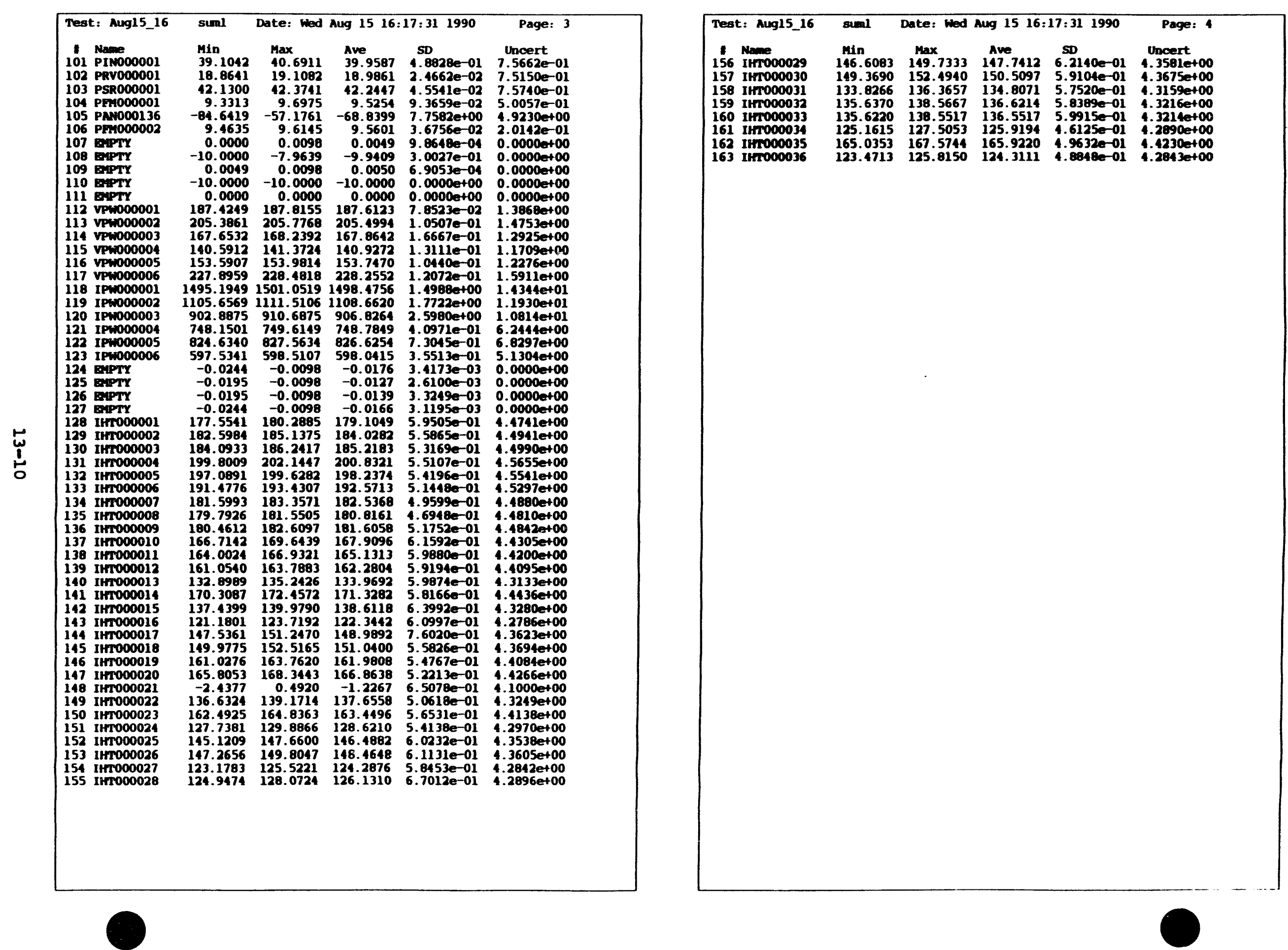


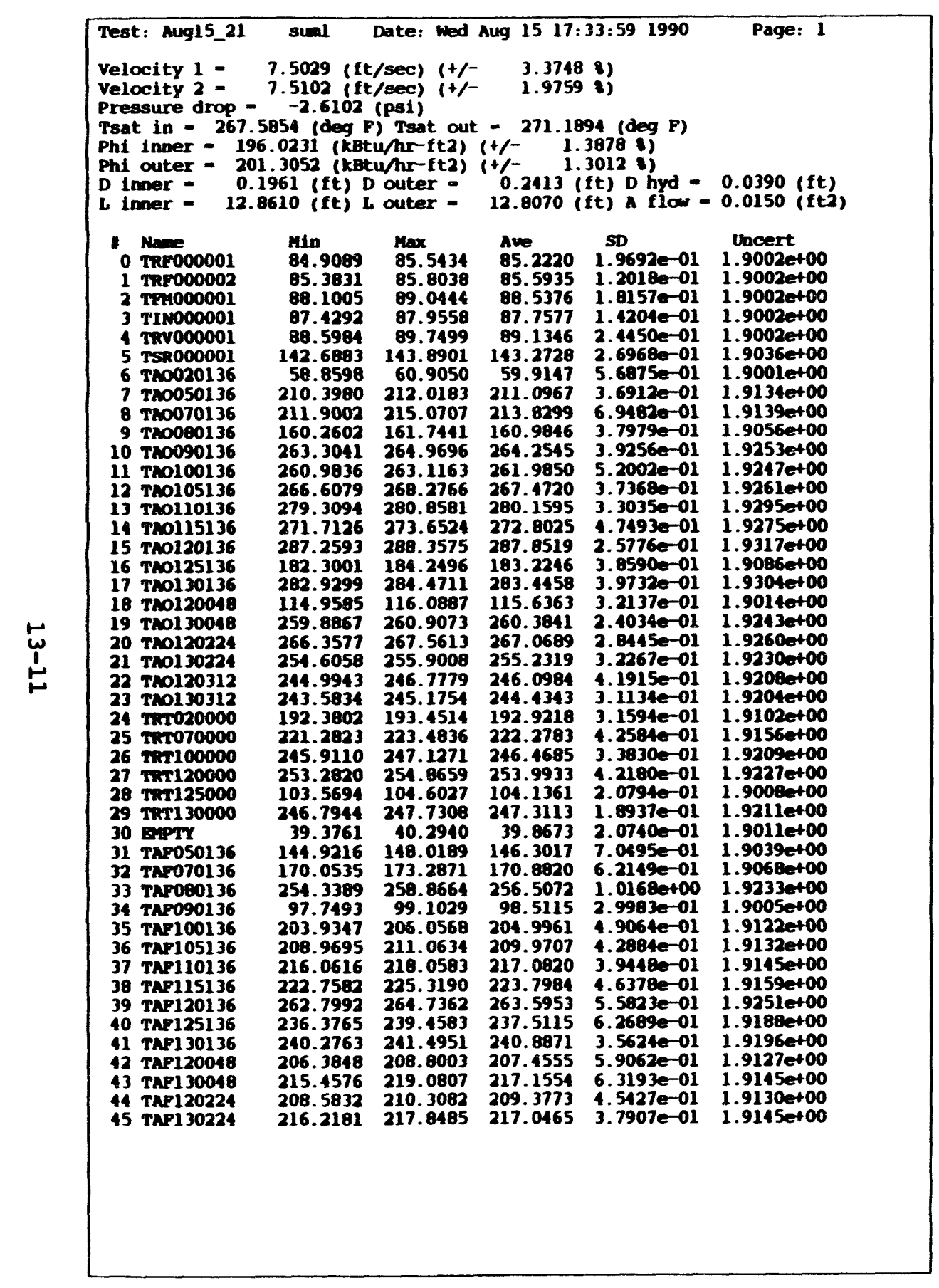

\begin{tabular}{|c|c|c|c|c|c|}
\hline 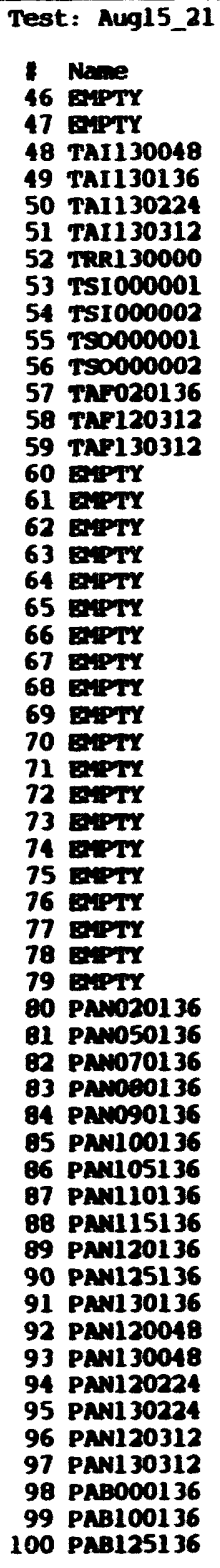 & 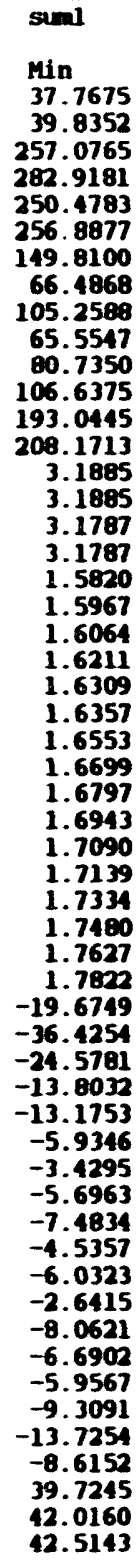 & 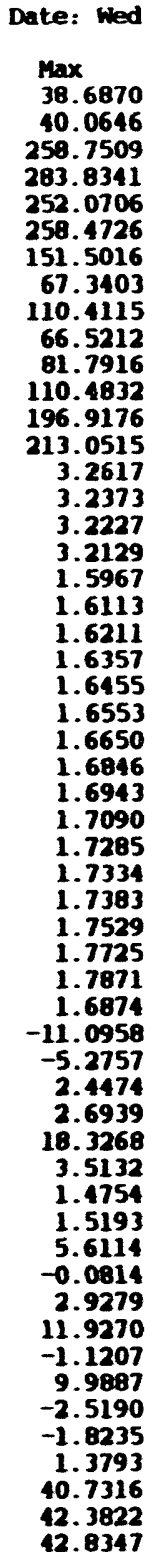 & 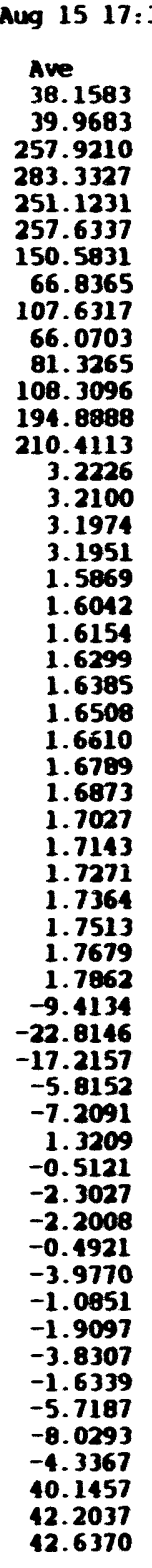 & 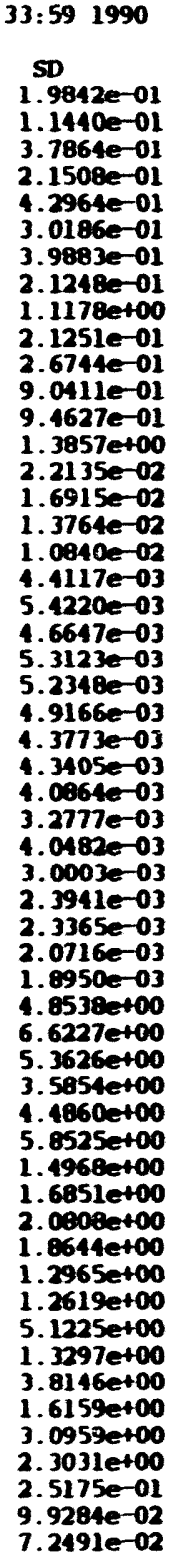 & 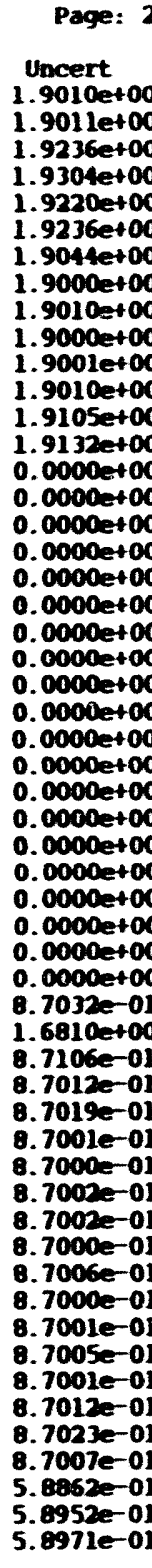 \\
\hline
\end{tabular}



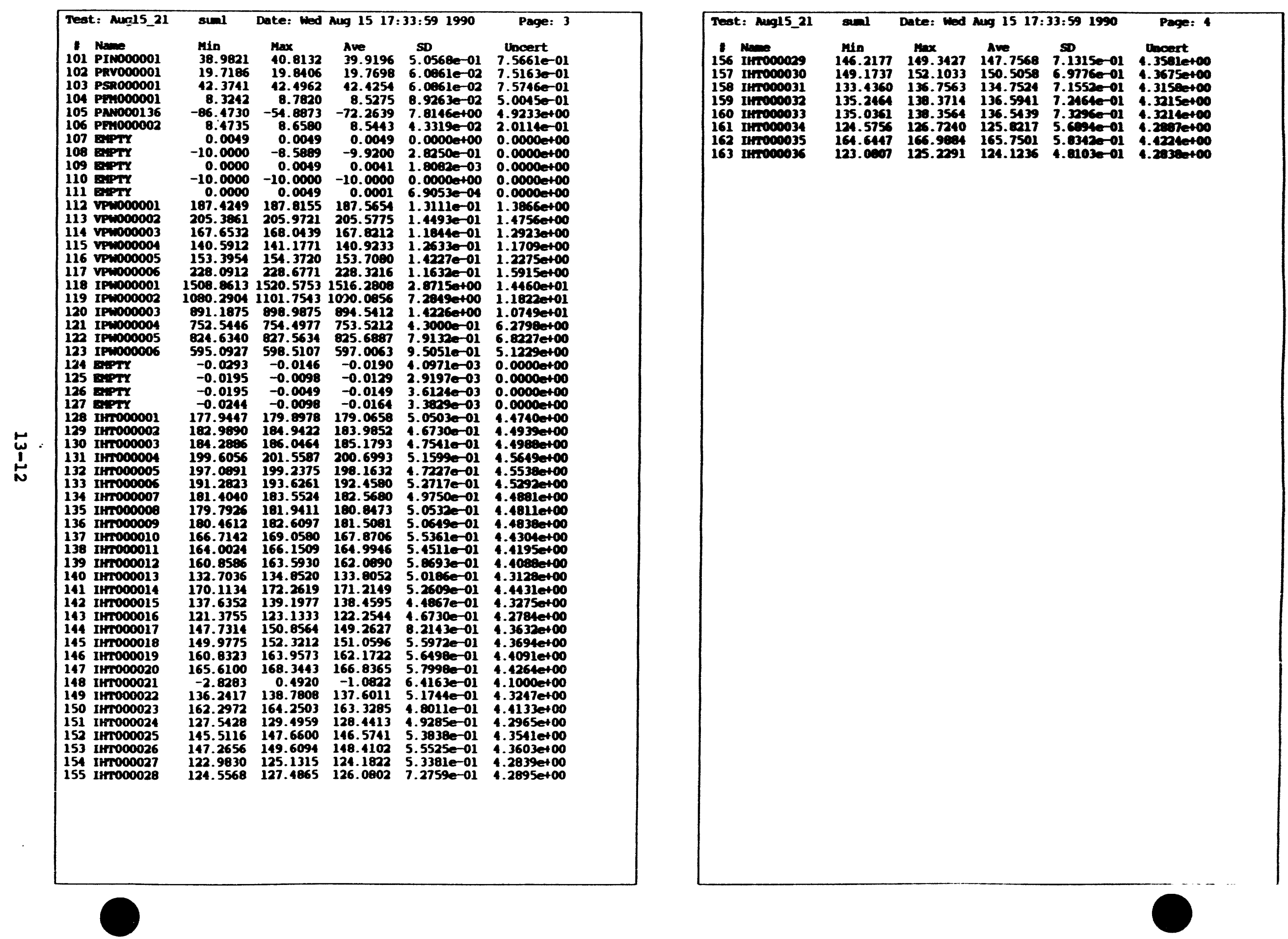
Test: Mug15_22 siml Date: Wod Aug 15 17:45:00 1990 Page: 1

Velocity 1 - 7.3075 (ft/sec) $(t /-\quad 3.5153$ i)

velocity $2=7.3006$ (ft/sec)

Tsat in - 267.5064 (deg F) Tsat out - 271.1713 (deg F)

Phi inner = 196.0148 (kBtu/hr ft2) $(+/-21.3604$ है)

D Iner - 0.1961 (ft) D outer = 0.2413 (ft) D hyd - 0.0390 (ft) L iner - 12.8610 (ft) $\mathrm{L}$ outer - 12.8070 (ft) A flow - 0.0150 (ftz)

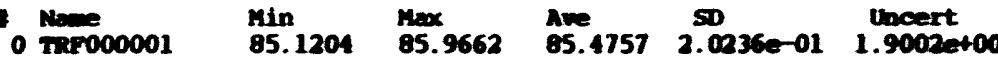

$1 \mathrm{mP000002} \quad 85.5935$ 86.0140 85.8711 1.2341e-01 $1.90020+00$

$\begin{array}{llllll}87.6828 & 08.4186 & 88.0481 & 1.92190-01 & 1.9002 e+00\end{array}$

$\begin{array}{llllllll}3 & \text { TrN000001 } & 87.1138 & 87.7463 & 87.3374 & 1.37310-01 & 1.90020000\end{array}$

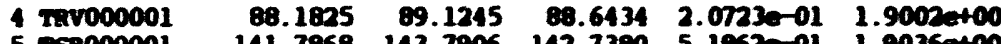

$\begin{array}{lrrrrrr}5 & \mathrm{TSR} 000001 & 141.7868 & 143.7906 & 142.7380 & 5.18620-01 & 1.90360+00 \\ 6 & \mathrm{Tm} 0020136 & 60.9045 & 63.4825 & 62.0352 & 5.25440-01 & 1.90000+00\end{array}$

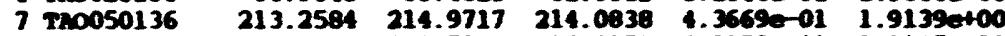

$\begin{array}{llllllll}8 & \text { T20070136 } 214.5903 & 218.5214 & 216.8159 & 6.91700-01 & 1.9145 e+00\end{array}$

9 T20080136 $160.2637 \quad 161.7459 \quad 161.3023 \quad 2.79440-01 \quad 1.90560+\infty 0$

$\begin{array}{llllllll}10 & \mathrm{~T} .0090136 & 267.0005 & 269.0313 & 268.3527 & 4.76750-01 & 1.9263 \mathrm{et00}\end{array}$

$\begin{array}{lllllll}11 & \mathbf{T} 010136 & 265.7076 & 268.0170 & 266.7166 & 5.26810-01 & 1.9259 e+00\end{array}$

$\begin{array}{lllllll}12 & 720105136 & 271.6996 & 273.0851 & 272.4424 & 3.31800-01 & 1.92740+00\end{array}$

13 T20110136 283.8599 $285.0420284 .57092 .68270-01 \quad 1.93060+00$

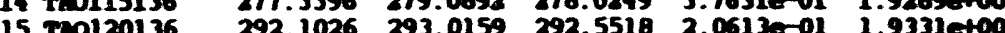

1670125136 183.7630 185.0293 194.5346 2.7105001 1.900.000

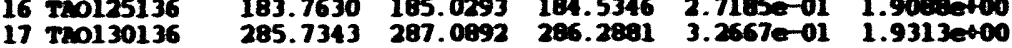

$\begin{array}{llllllllll}18 & \text { T20120048 } & 112.4921 & 113.5223 & 113.0074 & 2.94740-01 & 1.90120400\end{array}$

$\begin{array}{lllllllll}19 & \mathbf{T 2 0 1 3 0 0 4 8} 263.4021 & 264.6051 & 263.9060 & 2.72100-01 & 1.92520+00\end{array}$

$\begin{array}{llllllll}20 & \mathrm{~T} 10120224 & 270.0864 & 272.1786 & 271.6181 & 3.07410-01 & 1.92720+00\end{array}$

22 Tal20112 $249.4955 \quad 251.2735 \quad 250.2296 \quad 4.1789-01 \quad 1.9218=+\infty$

23 Tro130312 247.1378 248.3531 247.7135 $2.6185=01$ 1.92120400

24 TrT020000 $194.3258 \quad 196.0752 \quad 195.1659$ 3.3761e-01 1.9105 etol

25 TrT070000 $224.5358 \quad 226.4466 \quad 225.5102 \quad 4.16150-01 \quad 1.91620400$

26 Imr100000 248.8064 250. 2590 249.7692 $3.13410-01$ 1.9216et00

28 rar120000 97171598.1055 97.6942 $1.95730-01$ 1.9005.400

$28 \operatorname{mr} 30000 \quad 248.7569249 .8780249 .2802 \quad 2.24910-01$ 1.9215

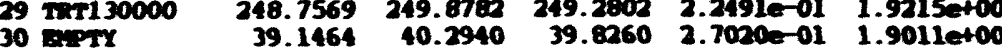

$\begin{array}{llllllll}31 & \text { TAF } 50136 & 145.1431 & 149.1196 & 147.5058 & 7.51400-01 & 1.90400+00\end{array}$

$\begin{array}{llllllll}32 & \text { TMF070136 } & 171.6247 & 174.3651 & 172.6345 & 5.78960-01 & 1.9071 e+00\end{array}$

$\begin{array}{llllllll}33 & \text { TAP060136 } & 257.5769 & 260.6967 & 259.3969 & 7.5706 e-01 & 1.9240 e+00\end{array}$

$\begin{array}{lllllll}34 \text { Tar090136 } & 96.1887 & 97.4393 & 96.7145 & 3.2761 e-01 & 1.9005 e+00\end{array}$

$\begin{array}{llllllll}35 & \text { TNF100136 } 206.8275 & 209.2336 & 206.2096 & 4.9827 e-01 & 1.9128 e+00\end{array}$

$\begin{array}{lllllll}36 & \text { TAF105136 } & 212.2032 & 214.7654 & 213.2673 & 5.3322 e-01 & 1.9138 e+00 \\ 37 & \text { TAF110136 } & 220.0537 & 221.8558 & 220.7287 & 4.44740-01 & 1.9152 e+00\end{array}$

37 TAF110136

38 TAP115136

40 TAF125136

11 TAF130136

42 TAP120048

13 TAF130048

45 TNF130224 $226.3589228 .4417 \quad 227.2910$ 4.7267e-01 $1.91660+00$

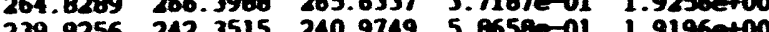

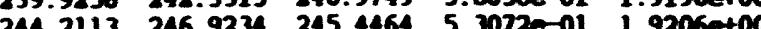

244.2113246 .9234245 .4464 5.30720-01 $1.9206 \mathrm{e}+00$

$\begin{array}{lllll}209.7652 & 212.7548 & 211.3979 & 7.2705 e-01 & 1.9134 \text { e+00 } \\ 219.1755 & 221.9330 & 220.5763 & 6.1342 e-01 & 1.9152 e+00\end{array}$ $\begin{array}{lllll}211.9344 & 214.4193 & 212.9020 & 5.0224 e-01 & 1.9137 e+00 \\ 219.8591 & 222.9194 & 221.0836 & 6.7100 e-01 & 1.9153 e+00\end{array}$

\begin{tabular}{|c|c|c|c|c|c|}
\hline 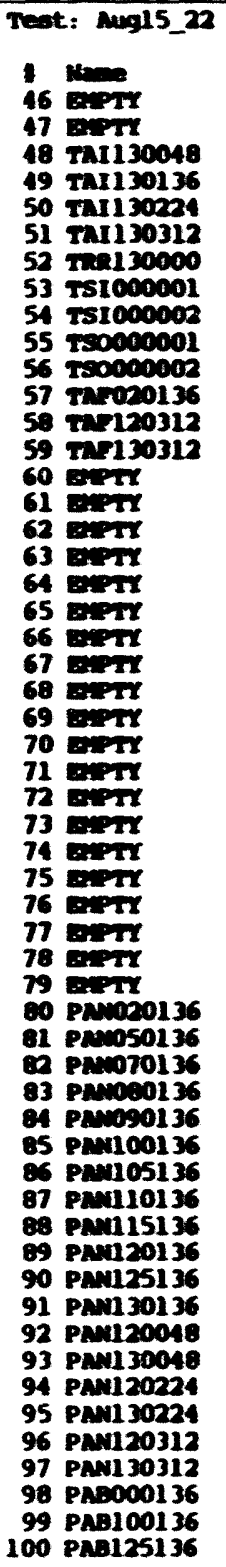 & $\begin{aligned} &=1 \\
& \text { Min } \\
& 37.7675 \\
& 39.8352 \\
& 259.9600 \\
& 286.2911 \\
& 253.6614 \\
& 260.5203 \\
& 151.7034 \\
& 66.5936 \\
& 105.0603 \\
& 65.6620 \\
& 81.1578 \\
& 107.0520 \\
& 195.6600 \\
& 211.1391 \\
& 3.2617 \\
& 3.2568 \\
& 3.2520 \\
& 3.2471 \\
& 1.6064 \\
& 1.6260 \\
& 1.6357 \\
& 1.6504 \\
& 1.6553 \\
& 1.6699 \\
& 1.6797 \\
& 1.6992 \\
& 1.7011 \\
& 1.7236 \\
& 1.7334 \\
& 1.7480 \\
& 1.7529 \\
& 1.7676 \\
& 1.7871 \\
& 1.8018 \\
&-19.6749 \\
&-34.6995 \\
&-24.6544 \\
&-12.7351 \\
&-13.1753 \\
&-6.1635 \\
&-7.5494 \\
&-7.2202 \\
&-5.1162 \\
&-2.9335 \\
&-2.8260 \\
&-2.7178 \\
&-9.9694 \\
&-7.9872 \\
&-9.4662 \\
&-8.4699 \\
&-13.3439 \\
&-8.9204 \\
& 39.5672 \\
& 12.0160 \\
& 42.4227\end{aligned}$ & 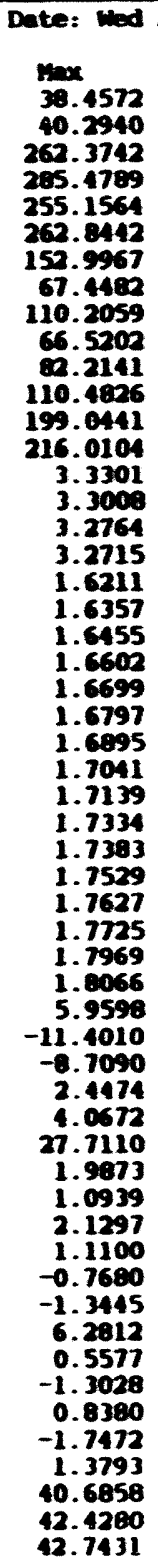 & 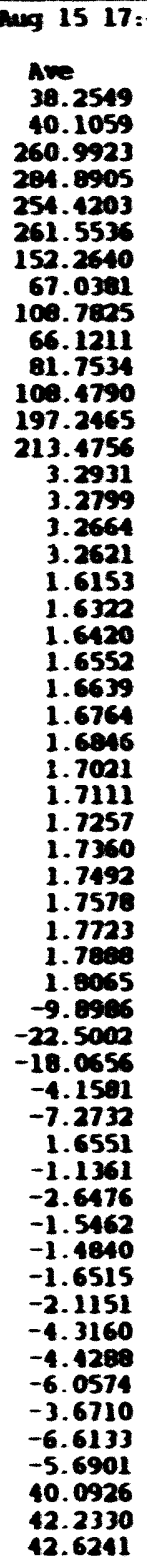 & 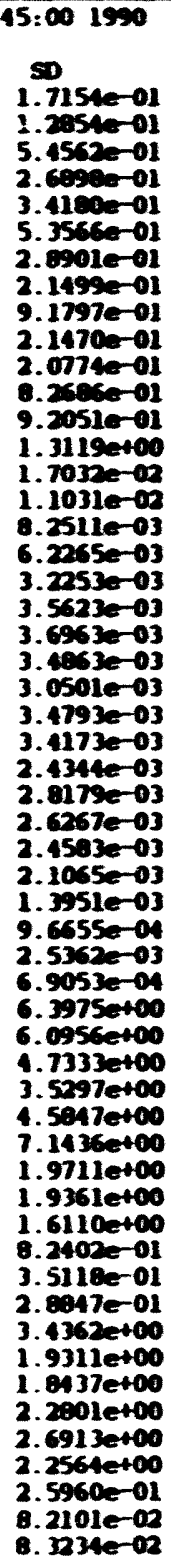 & 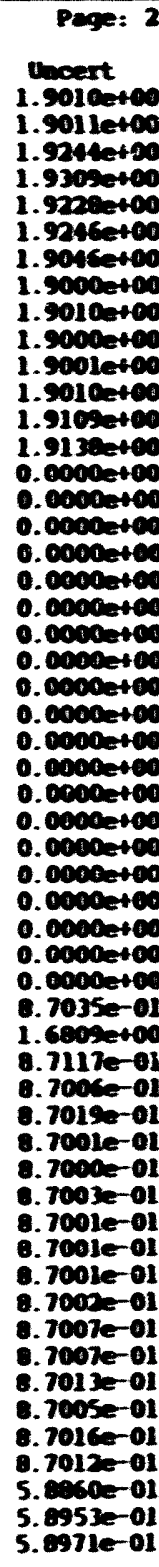 \\
\hline
\end{tabular}




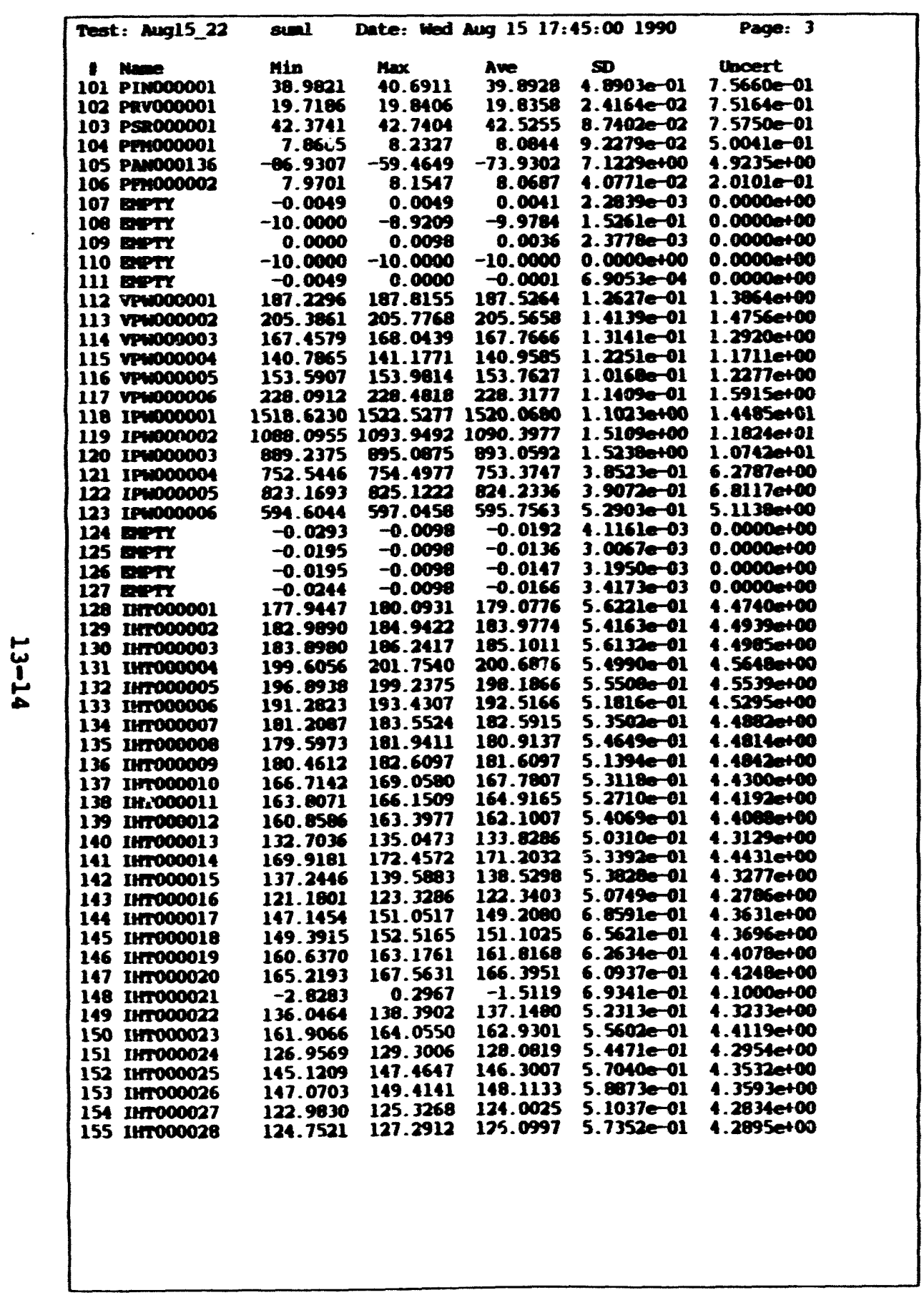

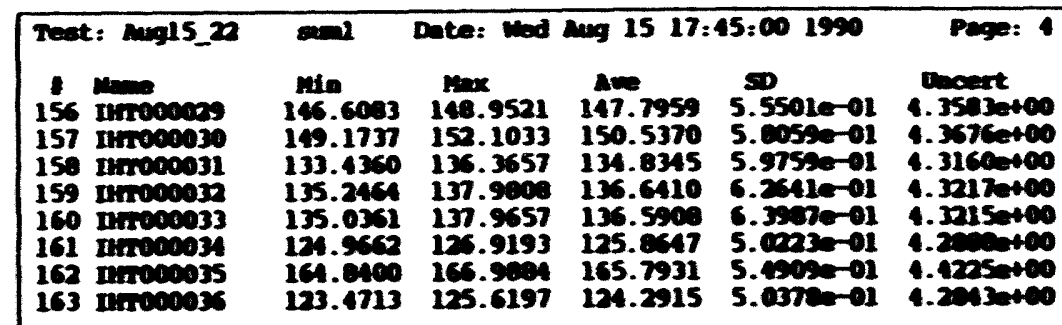




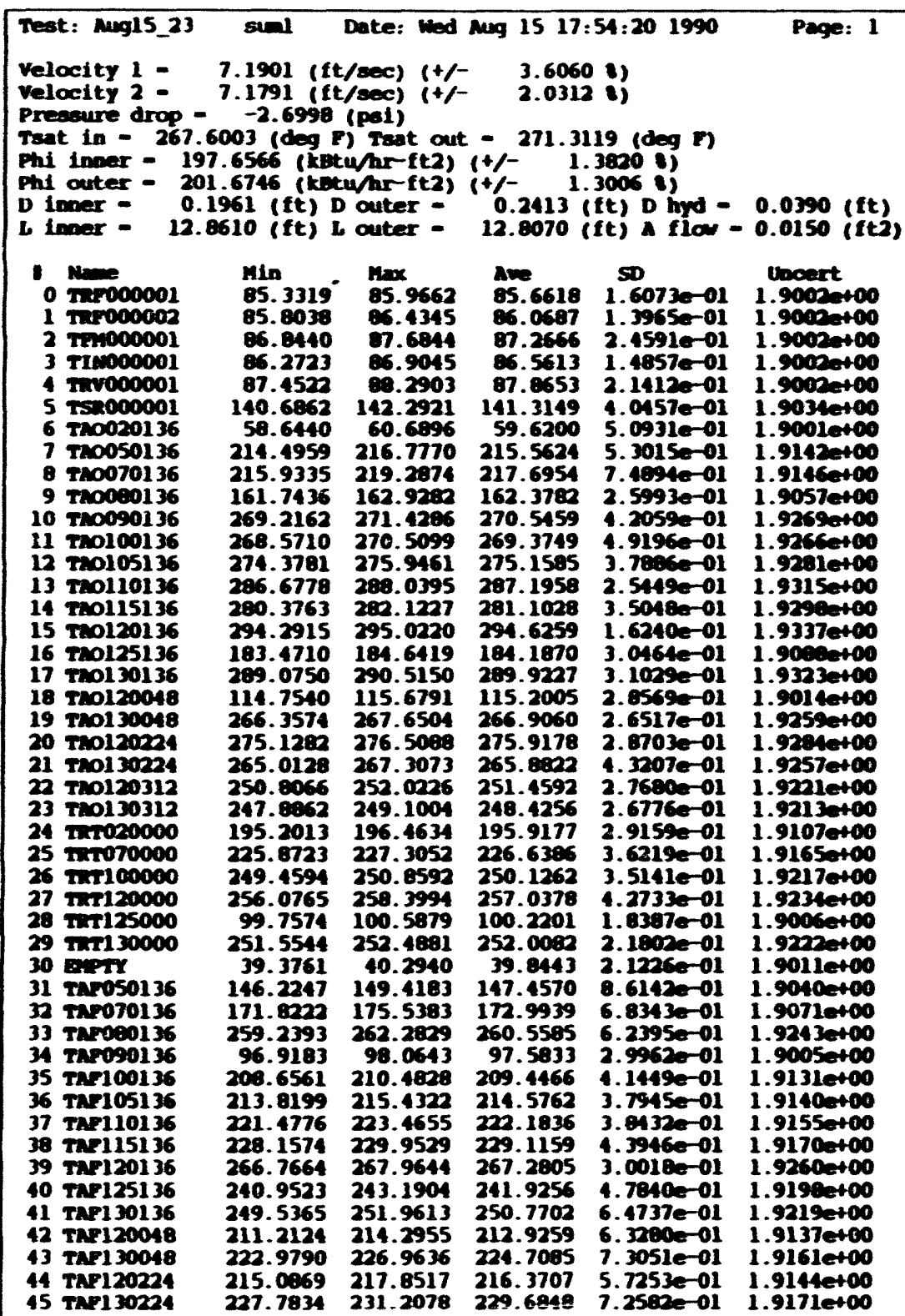

\begin{tabular}{|c|c|c|c|c|c|}
\hline 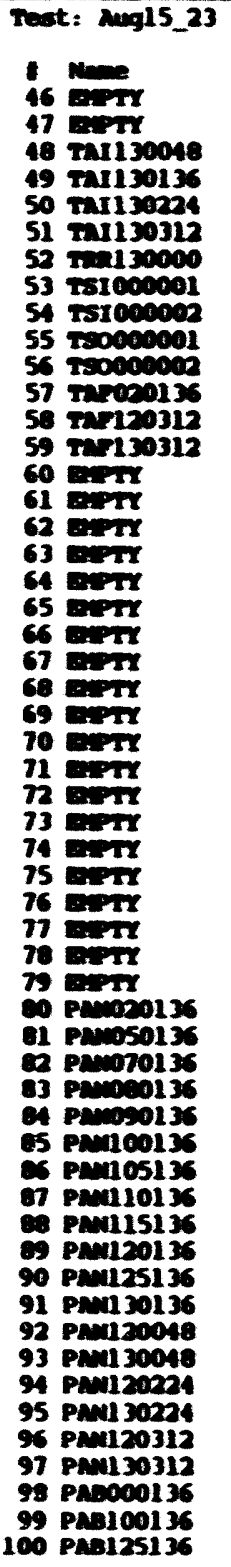 & 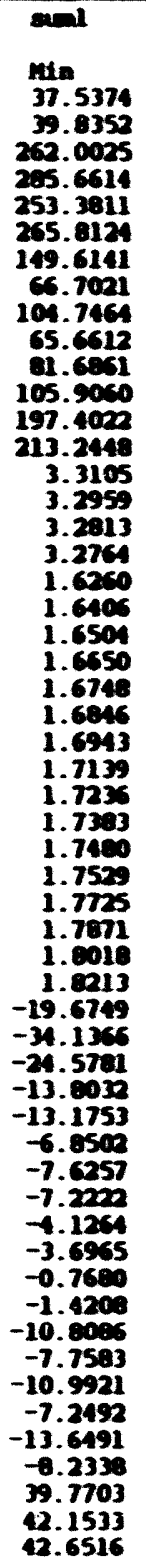 & 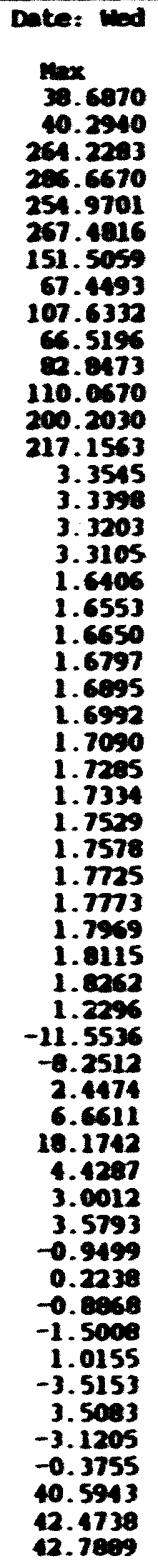 & 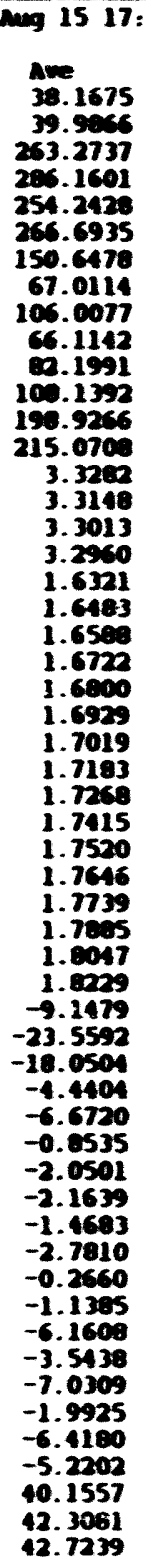 & 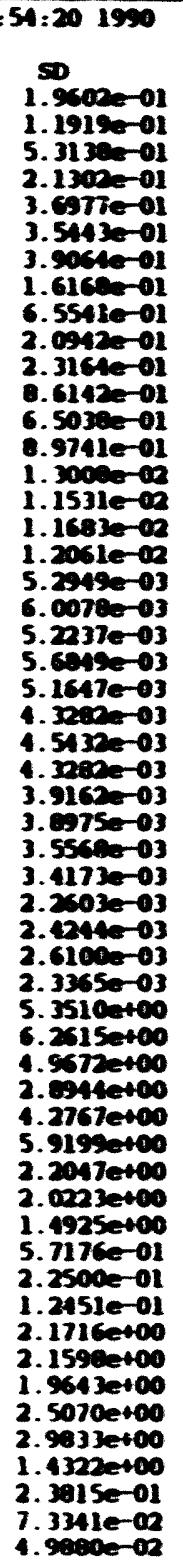 & 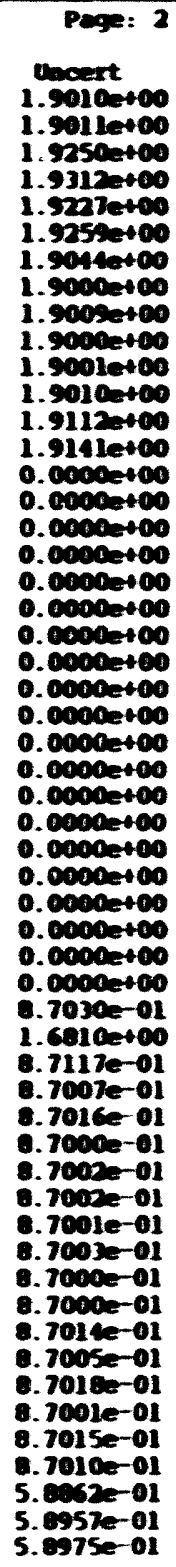 \\
\hline
\end{tabular}




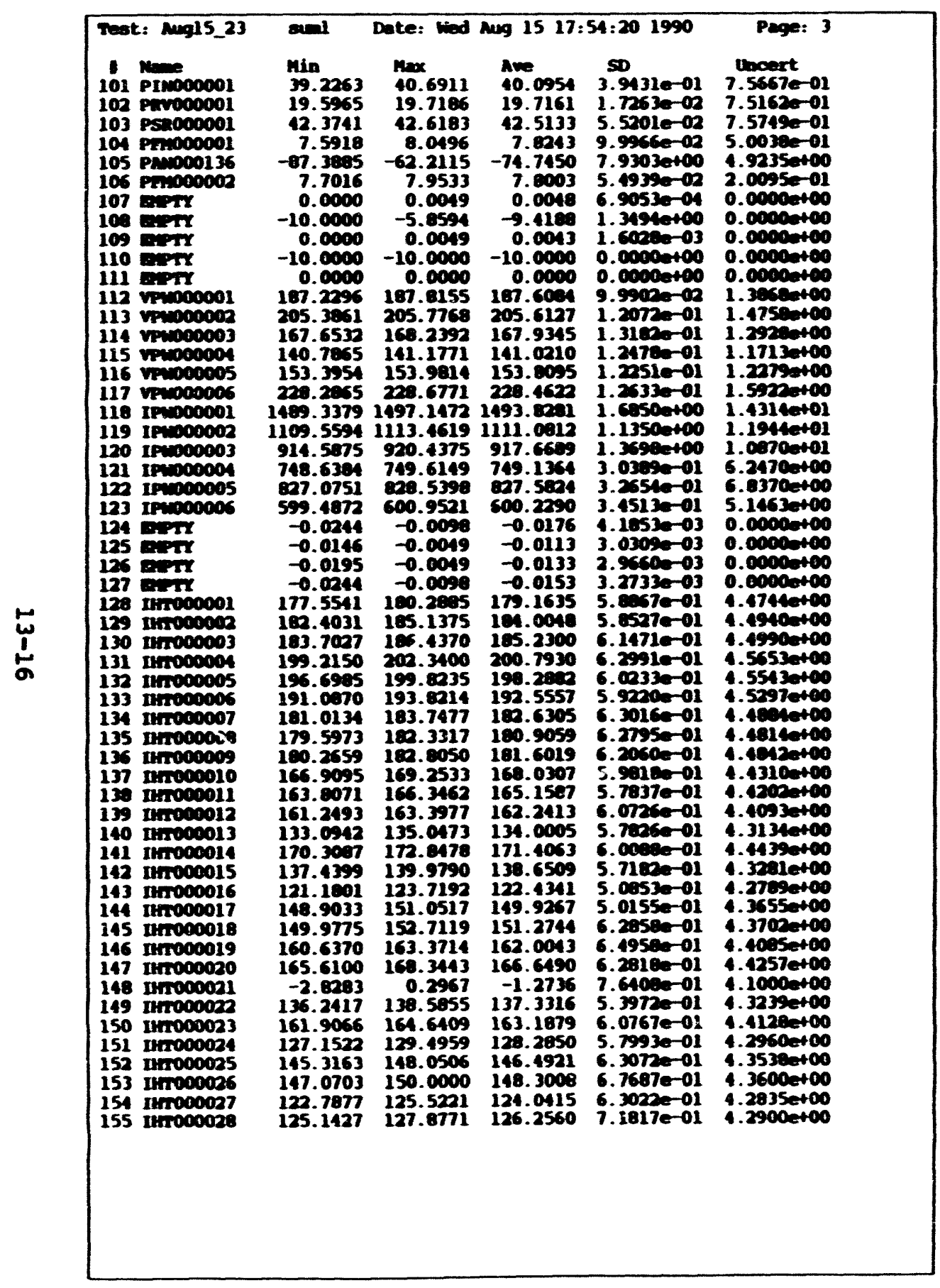

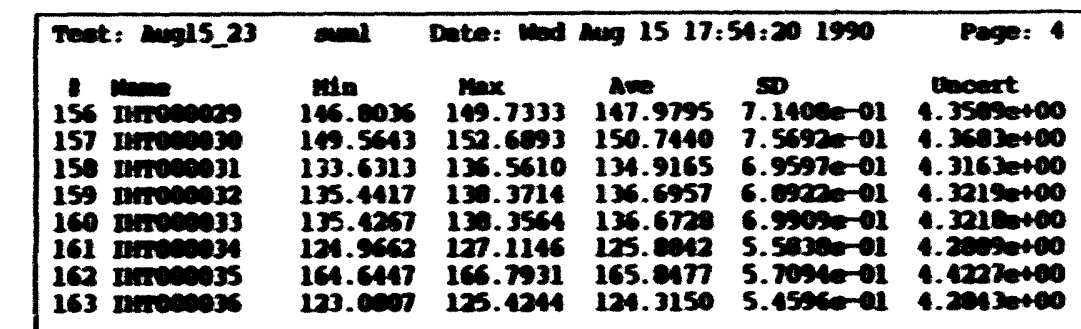




\begin{tabular}{|c|c|c|c|c|c|}
\hline \multirow[b]{2}{*}{$\begin{array}{l}\text { Test: Mugl5_24 } \\
\text { Velocity } 1=- \\
\text { Velocity } 2= \\
\text { Pressure drop - } \\
\text { Trat in - } 267.1 \\
\text { Phi inner = } 197 \\
\text { Phi outer - } 201 \\
\text { D inver = } 0.1 \\
\text { L inser - } 12.8\end{array}$} & \multirow[b]{2}{*}{$\begin{array}{l}\text { sen } \\
7.0480 \text { (f } \\
7.0367 \text { (f } \\
-2.7401 \\
601 \text { (deg } \\
.1527 \text { (k: } \\
2262 \text { (k) } \\
961 \text { (ft) } \\
1610 \text { (ft) }\end{array}$} & \multirow[b]{2}{*}{ 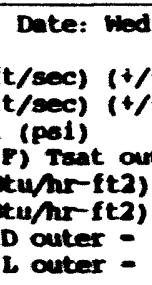 } & \multirow{2}{*}{\multicolumn{2}{|c|}{ 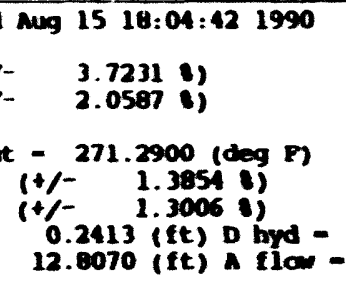 }} & \multirow[b]{2}{*}{$\begin{array}{l}\text { Page: } 1 \\
0.0390 \text { (ft) } \\
0.0150 \text { (ftz) }\end{array}$} \\
\hline & & & & & \\
\hline 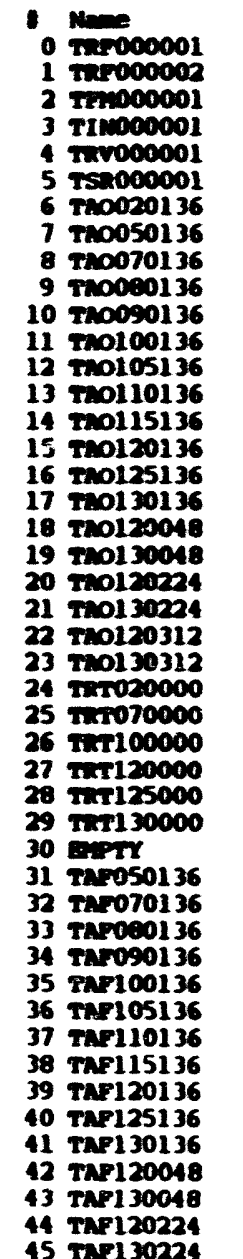 & $\begin{array}{l}\text { Min } \\
85.5434 \\
86.0140 \\
86.3213 \\
85.6409 \\
86.8252 \\
142.5930 \\
58.4205 \\
216.8709 \\
217.9467 \\
163.0256 \\
272.3496 \\
272.0774 \\
278.8022 \\
290.1241 \\
284.4171 \\
296.3874 \\
185.0297 \\
291.5952 \\
111.1554 \\
271.3326 \\
279.3578 \\
270.4200 \\
251.9207 \\
249.0969 \\
196.6577 \\
228.0671 \\
251.0455 \\
250.1213 \\
96.8635 \\
254.3497 \\
39.3761 \\
146.6238 \\
172.9975 \\
260.6259 \\
94.0008 \\
210.7710 \\
216.3793 \\
224.5071 \\
231.6522 \\
268.4256 \\
246.7252 \\
253.3579 \\
215.2565 \\
230.1605 \\
219.4691 \\
234.9095\end{array}$ & 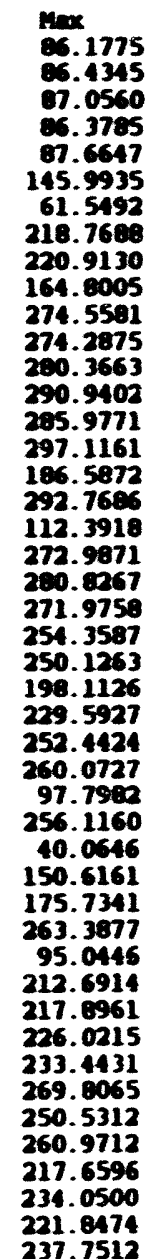 & $\begin{array}{l}110 \\
85.0609 \\
86.2621 \\
86.6736 \\
85.6994 \\
87.2540 \\
144.4735 \\
59.6953 \\
217.8253 \\
219.5012 \\
164.0675 \\
273.6150 \\
273.4621 \\
279.5827 \\
290.5451 \\
285.1294 \\
296.7122 \\
185.7391 \\
292.2309 \\
111.7493 \\
272.1597 \\
279.9720 \\
271.1466 \\
253.6409 \\
249.6296 \\
197.4454 \\
228.9298 \\
251.9473 \\
259.0622 \\
97.3789 \\
255.1373 \\
39.8305 \\
148.1907 \\
173.9792 \\
262.2275 \\
94.5318 \\
211.9002 \\
217.2926 \\
225.2263 \\
232.4626 \\
269.0068 \\
248.4870 \\
255.8791 \\
216.3951 \\
232.3638 \\
220.4096 \\
236.2740\end{array}$ & 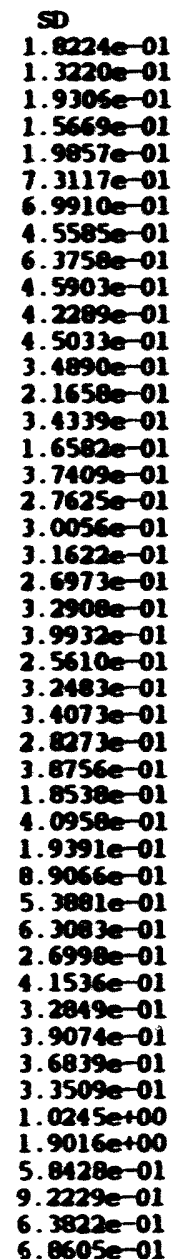 & 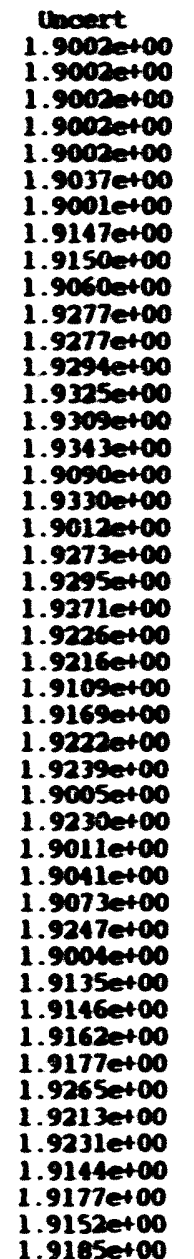 \\
\hline
\end{tabular}

\begin{tabular}{|c|c|c|c|c|c|}
\hline 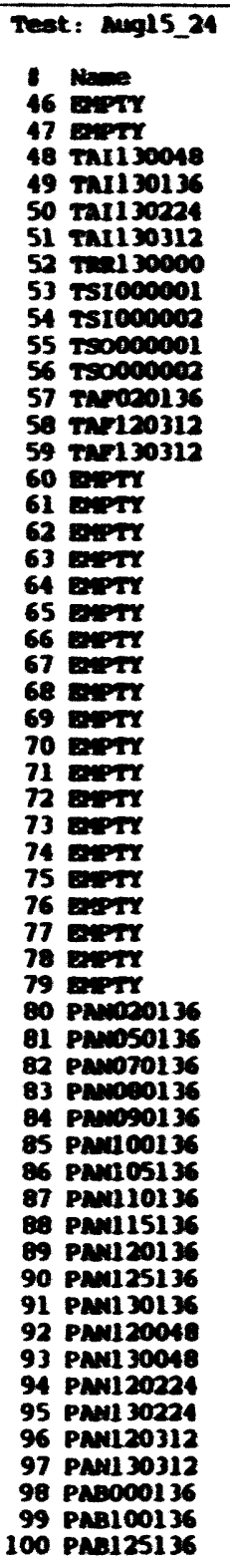 & 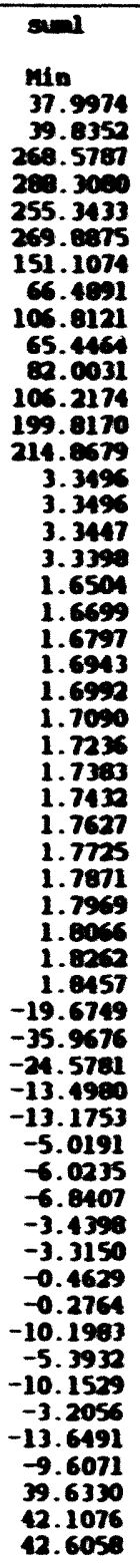 & 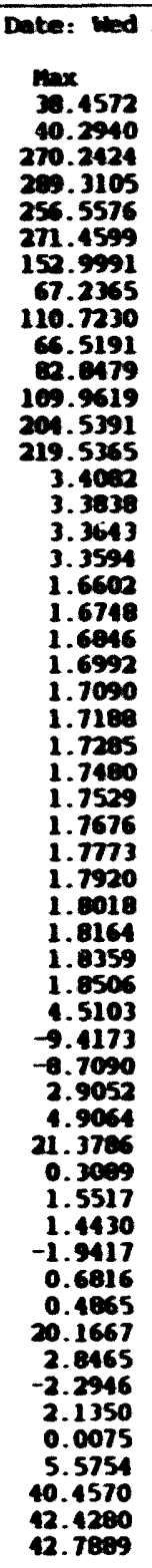 & 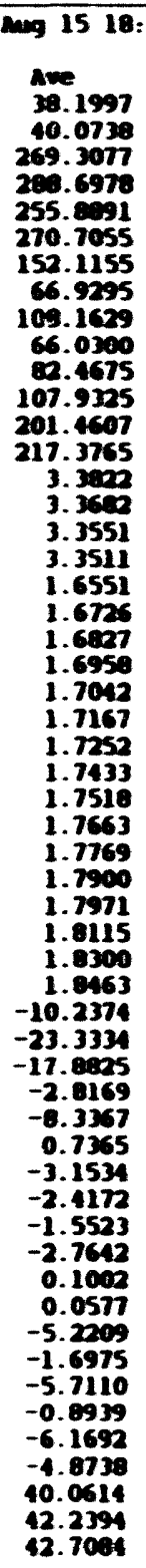 & 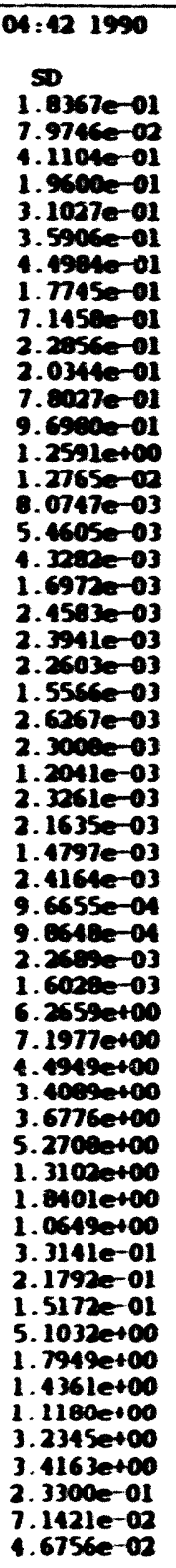 & 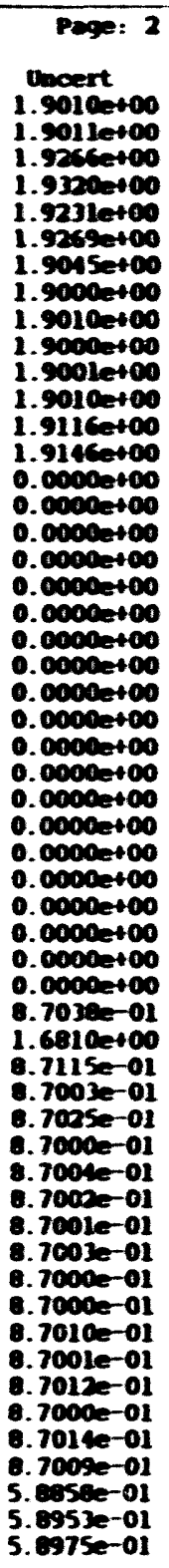 \\
\hline
\end{tabular}




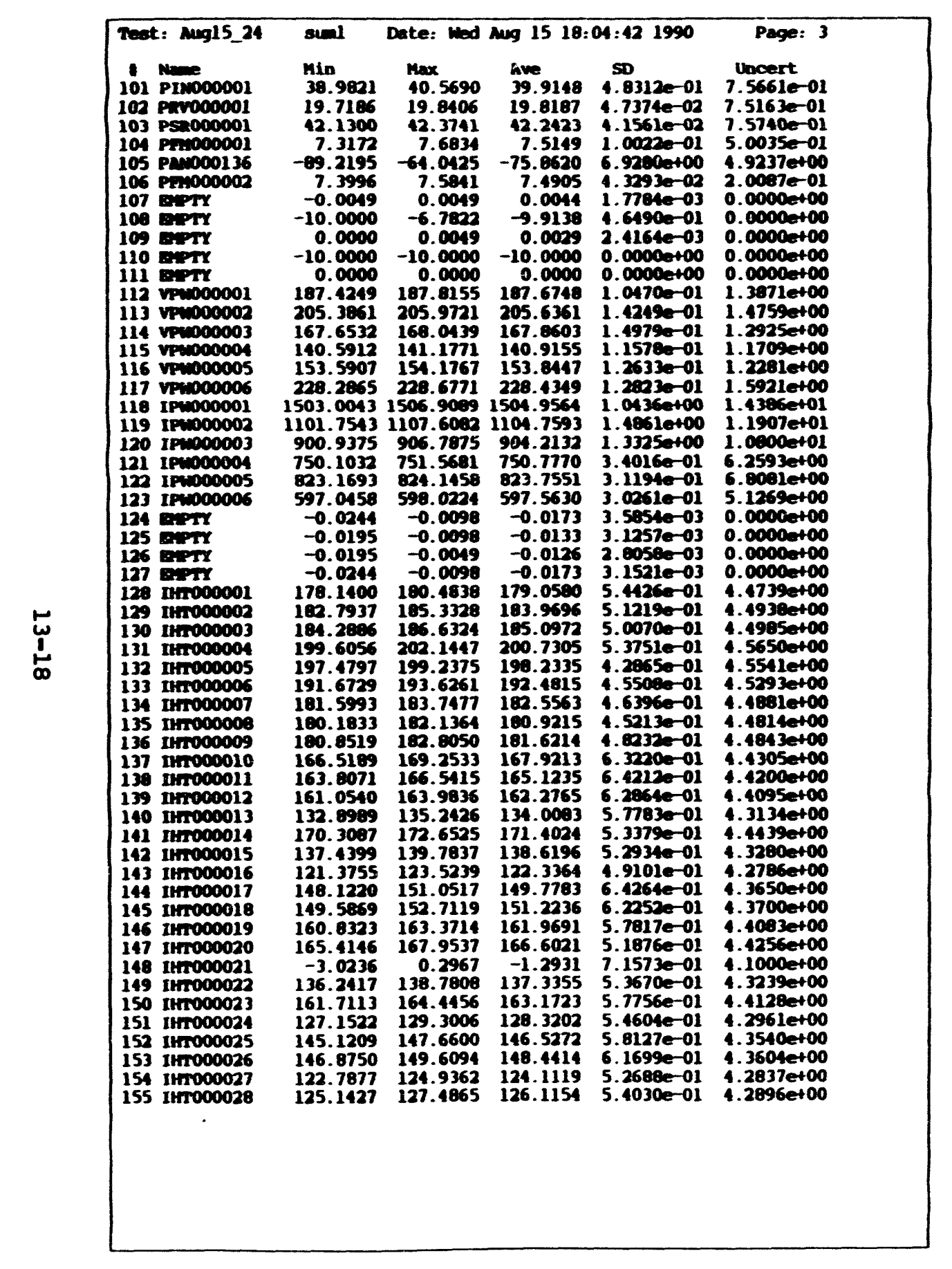

\begin{tabular}{|c|c|c|c|c|c|}
\hline Teot: Mar & an & Date: Wad & $\operatorname{Ang} 1518: 0$ & $04: 421990$ & Page: 4 \\
\hline 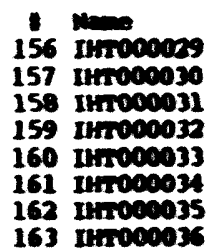 & $\begin{array}{l}\text { Min } \\
146.6003 \\
149.5643 \\
134.0219 \\
135.6370 \\
135.4267 \\
124.9662 \\
164.6447 \\
123.0607\end{array}$ & $\begin{array}{l}\max \\
149.1474 \\
151.7127 \\
136.5610 \\
139.3714 \\
139.3564 \\
127.1146 \\
167.1837 \\
125.4244\end{array}$ & $\begin{array}{l}\text { Ave } \\
147.8701 \\
150.6893 \\
134.9751 \\
136.7777 \\
136.7470 \\
125.8530 \\
165.8430 \\
134.2090\end{array}$ & $\begin{array}{l}50 \\
5.52720-01 \\
5.20750-01 \\
5.99690-01 \\
5.91020-01 \\
6.18360-01 \\
5.00720-01 \\
5.50900-01 \\
5.09370-01\end{array}$ & $\begin{array}{l}\text { Docert } \\
4.35050+00 \\
1.36010+00 \\
4.3165=+00 \\
1.32210+00 \\
1.32200+00 \\
4.2006=+00 \\
4.12270+00 \\
4.20410+00\end{array}$ \\
\hline
\end{tabular}




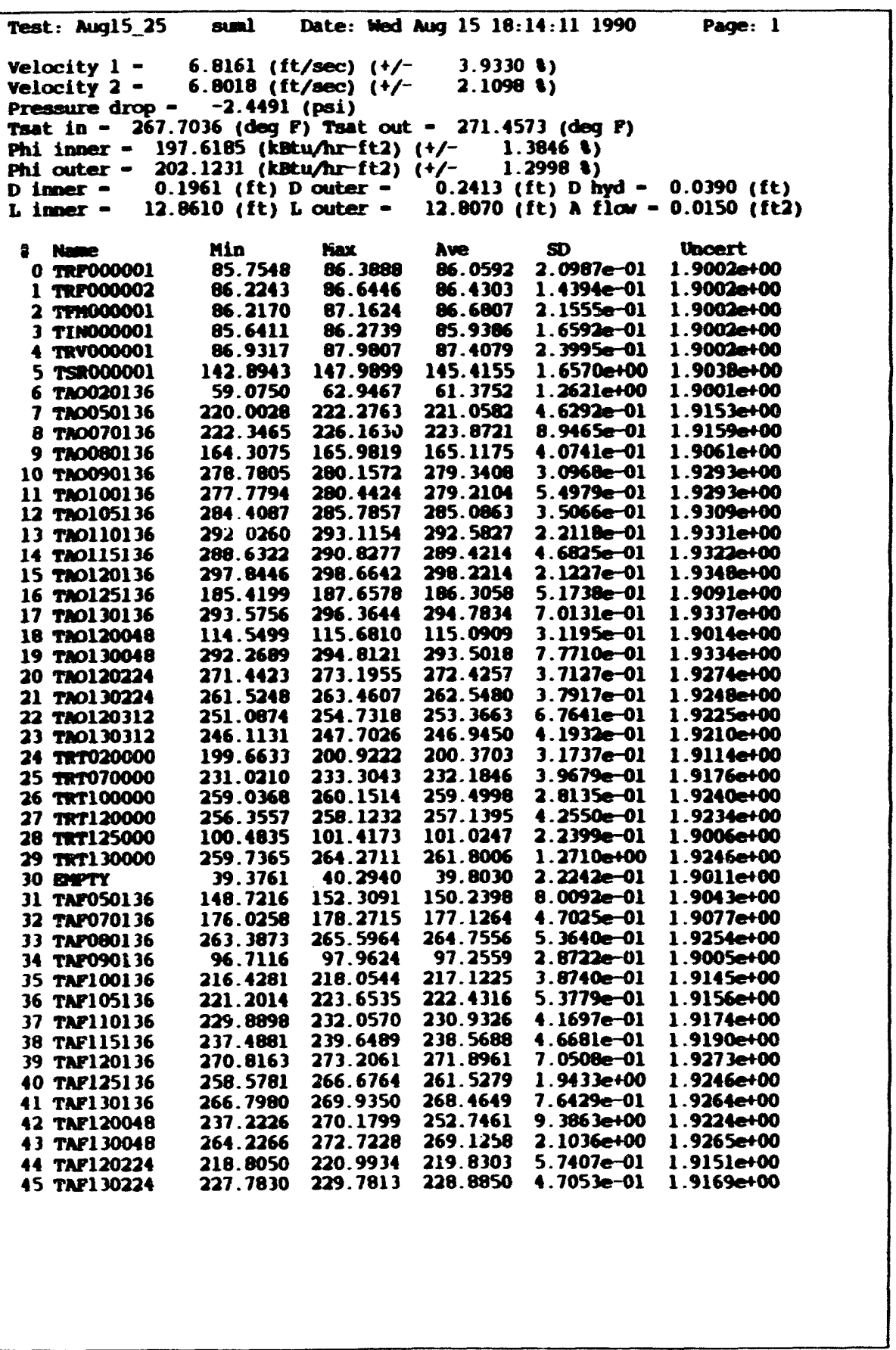

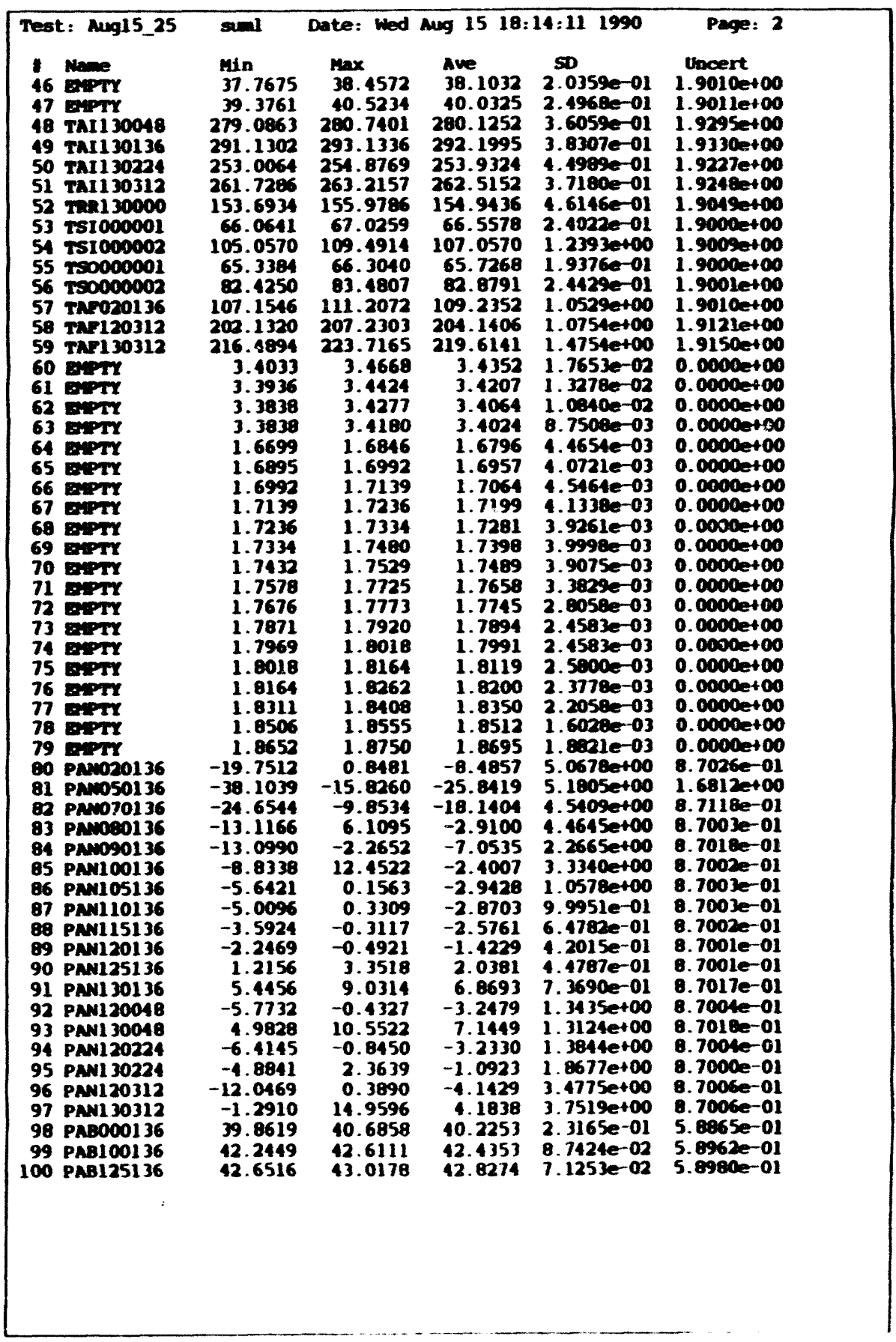




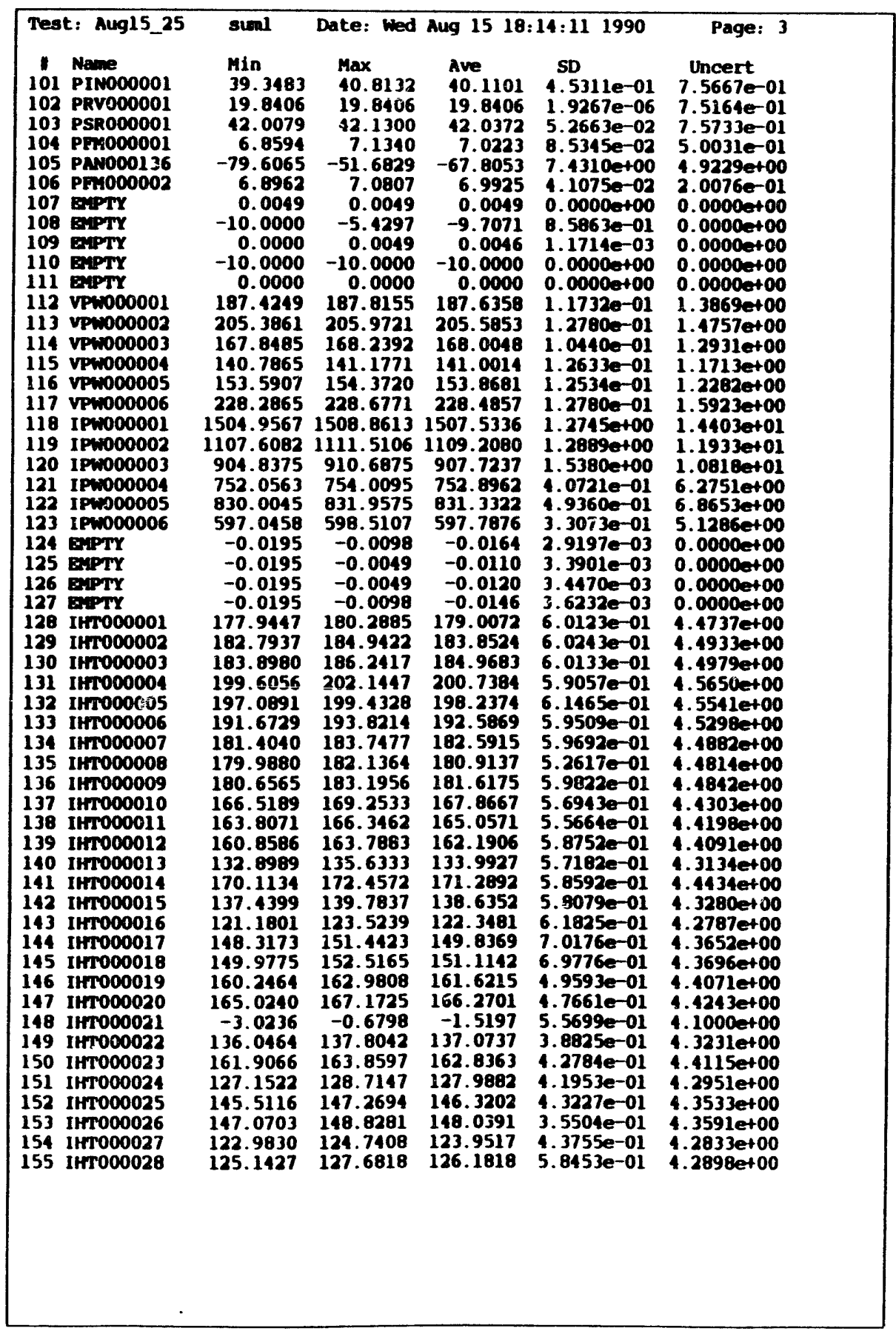
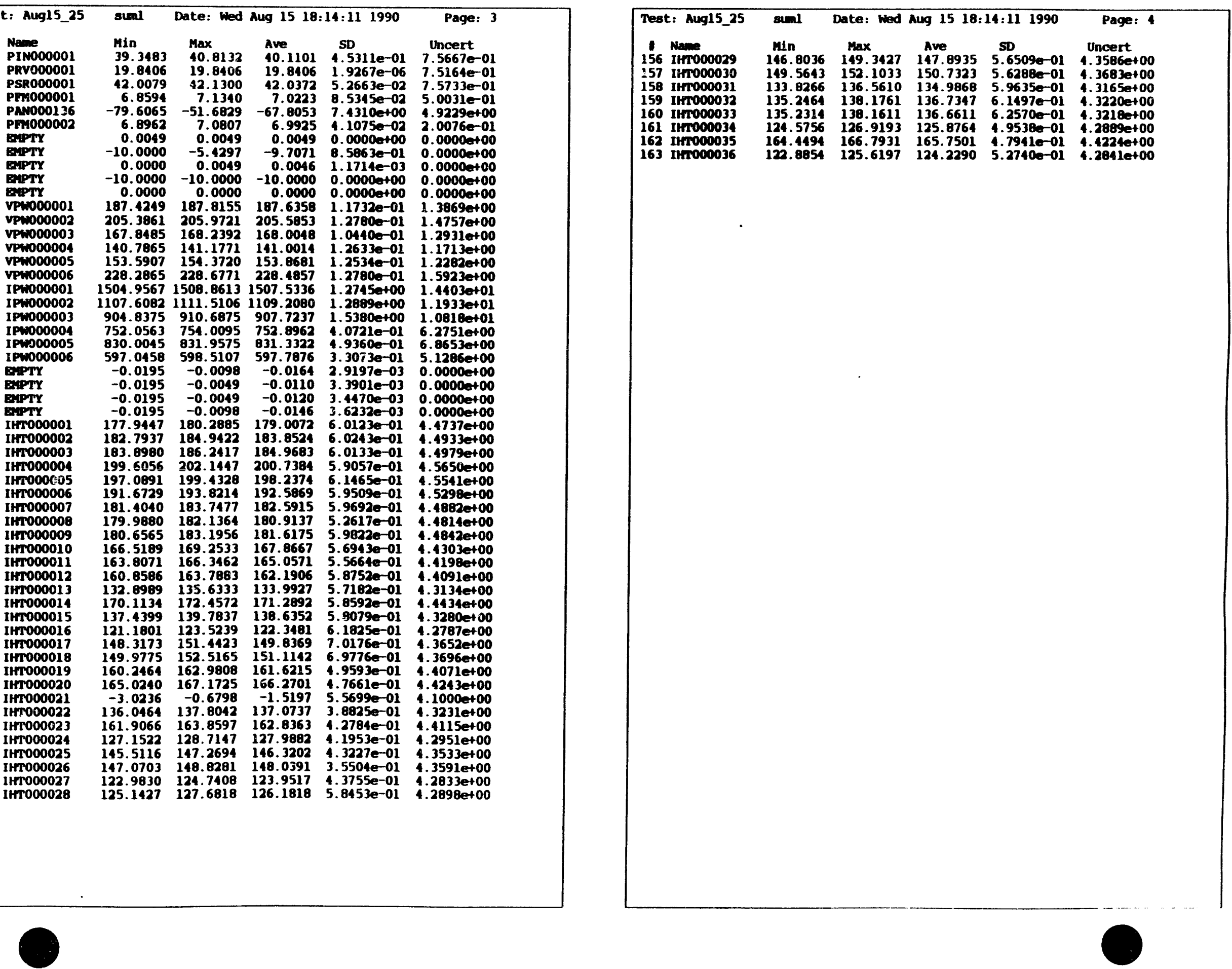


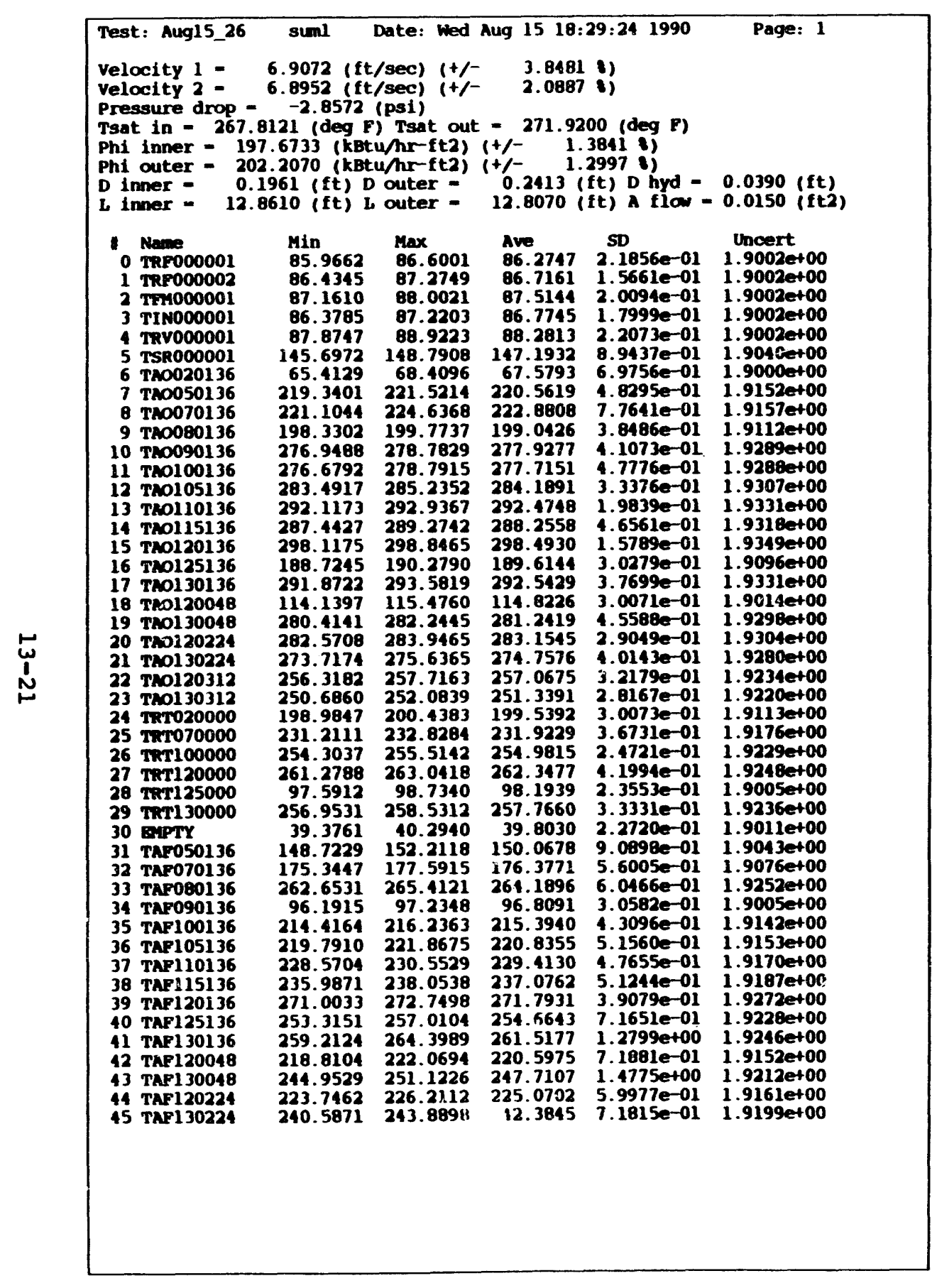

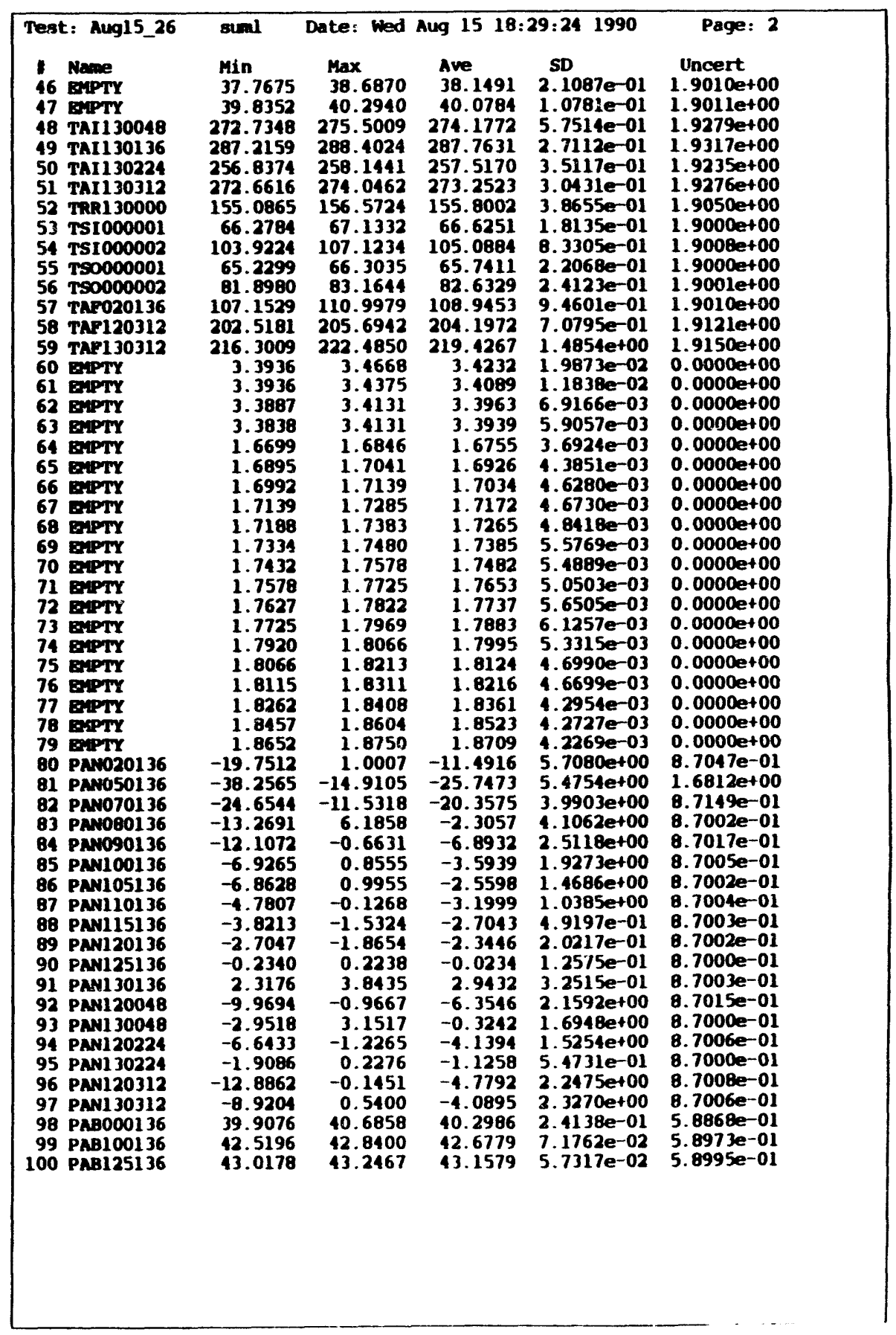




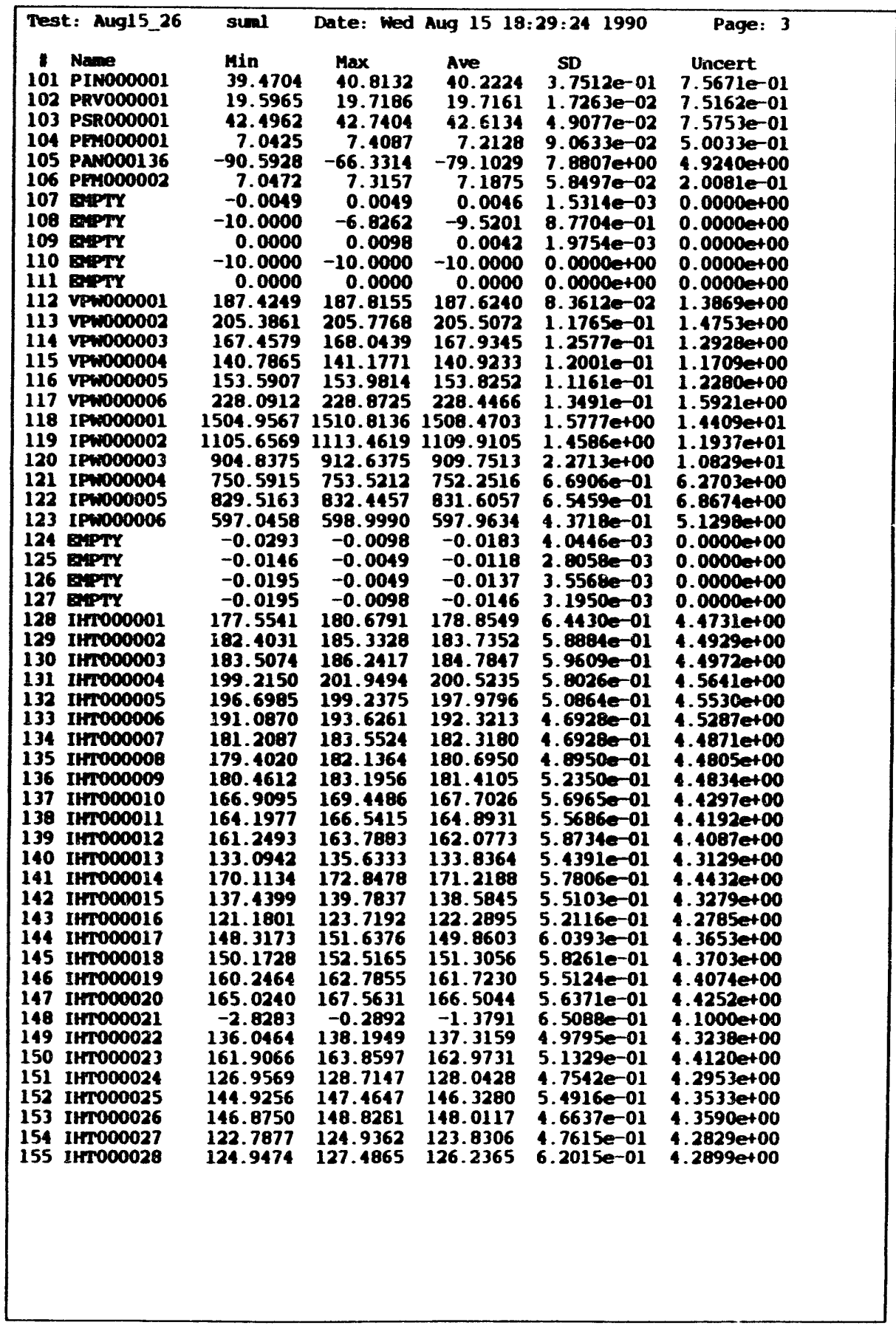

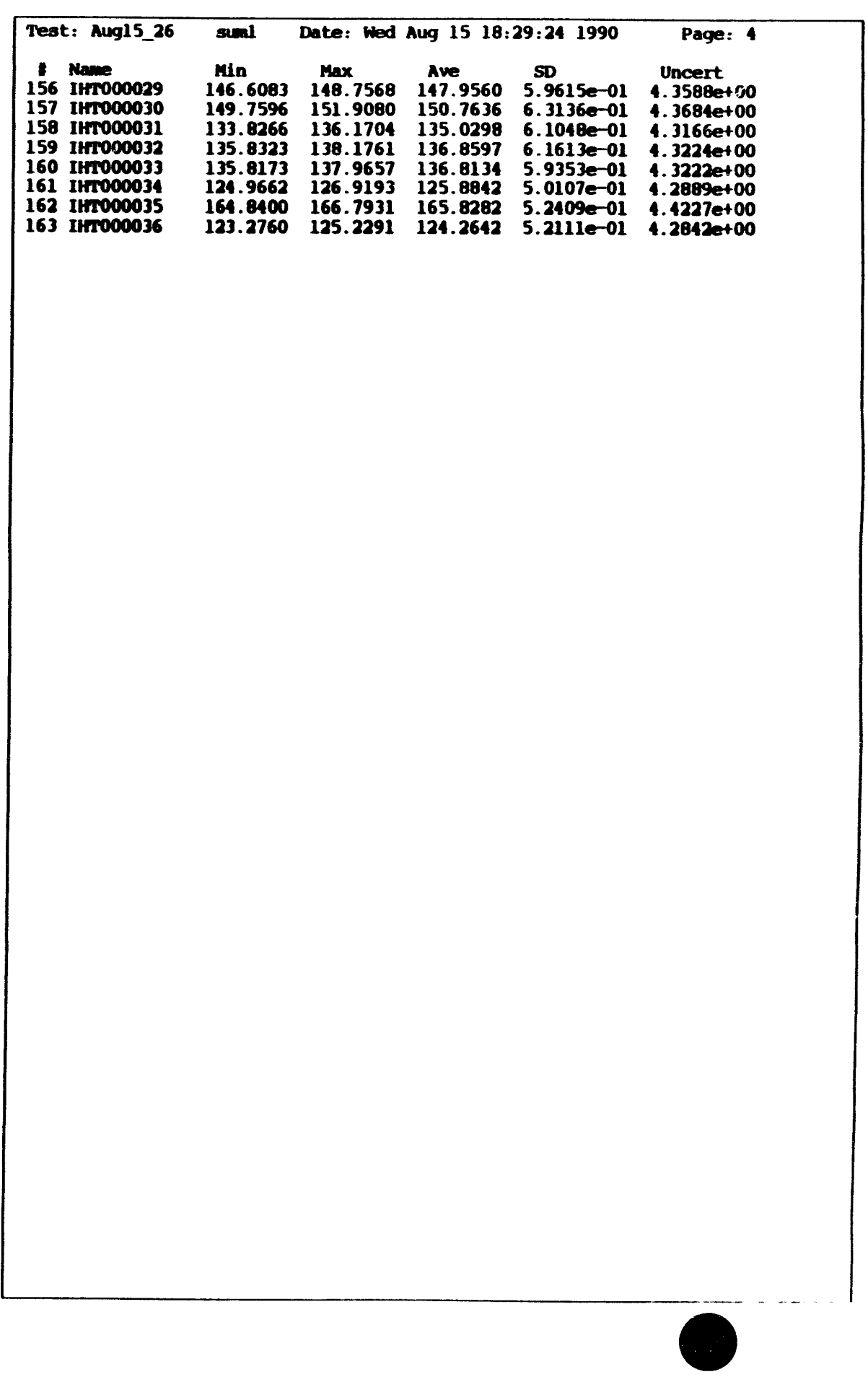


DEFECTIVE INSTRUMENTS FOR THE RIBBED GEOMETRY TEST SERIES

\begin{tabular}{|c|c|cc|}
\hline Series & Tests & \multicolumn{1}{c|}{ Defective Instruments } \\
\hline 16 & Aug15_13 to Aug15_26 & TAO120048 TAF070136 & TRT125000 \\
& & TAOS20136 TAF080136 & TRR130000 \\
& & TAO070136 TAF090136 & \\
& & TAO080136 TAF120136 & \\
& & TAO125136 & \\
& & IHTO00021 IHTO00024 IHTO00033 \\
\hline
\end{tabular}




\section{Chapter 14}

NUCLEATE BOILING PRESSURE DROP IN AN ANNULUS

$$
\begin{array}{ll}
\text { Test Geometry: } & \text { Ribbed (4) } \\
\text { Test Series: } & 5 \\
\text { Test Date: } & \text { August } 16,1990
\end{array}
$$




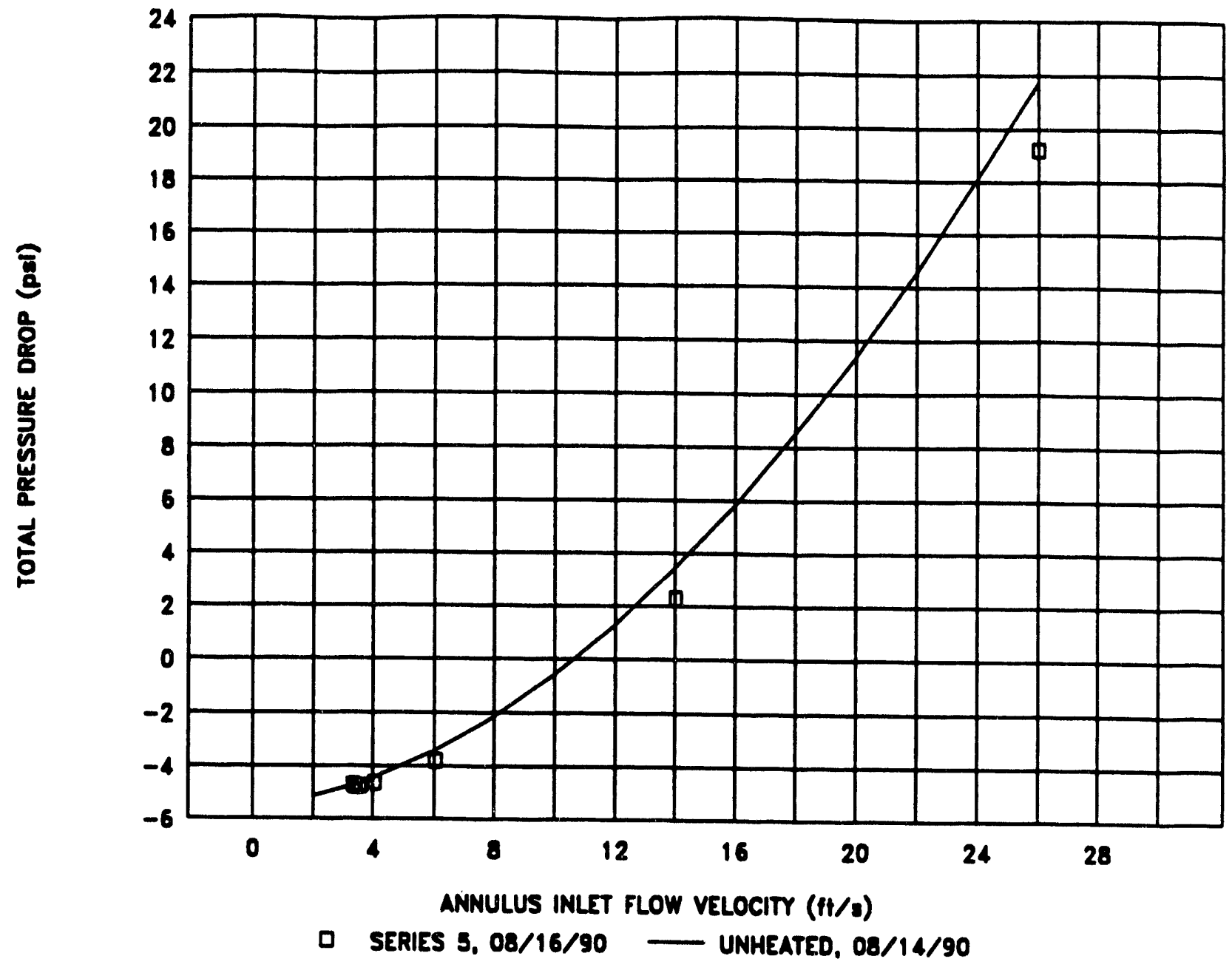

\begin{tabular}{|c|c|c|}
\hline $\begin{array}{c}\text { TEST } \\
\text { NUMBER }\end{array}$ & $\begin{array}{c}\text { INLET } \\
\text { VELOCITY(a) }\end{array}$ & $\begin{array}{c}\text { PAESSURE } \\
\text { DROP(b) }\end{array}$ \\
\hline & $(\mathrm{ft} / \mathrm{s})$ & (psi) \\
\hline Aug16_01 & 26.00 & 19.20 \\
\hline Aug16_02 & 14.02 & 2.29 \\
\hline Aug16_03 & 6.05 & -3.79 \\
\hline Aug16_04 & 4.03 & -4.65 \\
\hline Aug16_07 & 3.56 & -4.76 \\
\hline $40 \% \div 6 \%$ & $6 \%$ & 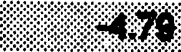 \\
\hline Aug16_05 & 3.34 & -4.70 \\
\hline
\end{tabular}

\begin{tabular}{|l|l|}
\hline \multicolumn{2}{|c|}{ NOMINAL TEST CONDITIONS } \\
\hline Annulus geometry & ribbed (4) \\
Inlet temperature & $86^{\circ} \mathrm{F}$ \\
Inlet pressure & $40 \mathrm{psia}$ \\
Wall heat flux & $100 \mathrm{kBtu} / \mathrm{hr}-\mathrm{tt} 2$ \\
Flux distribution & Uniform \\
He saturation pressure & $5 \mathrm{psig}$ \\
\hline NOTES: \\
(a) Inlot velocity calculated from PFM000001 \\
(b) Preseure drop calculated from PANO00136 \\
(c) Shading notes teat at minimum pressure drop
\end{tabular}

DEMAND CURVE AND DATA FOR SERIES 5 - RIBBED ANNULUS 

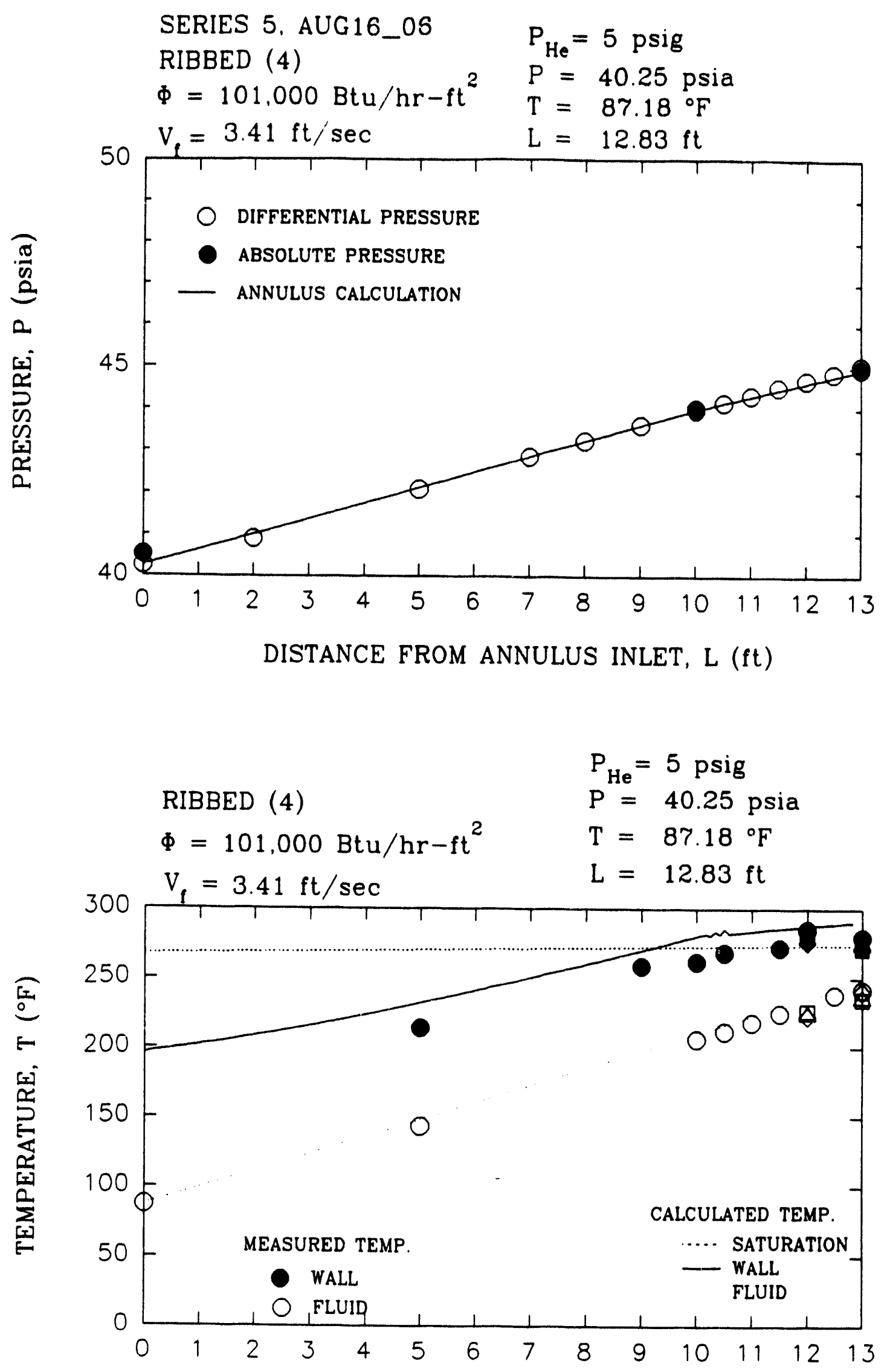

DISTANCE FROM ANNULUS INLET, L (ft) 


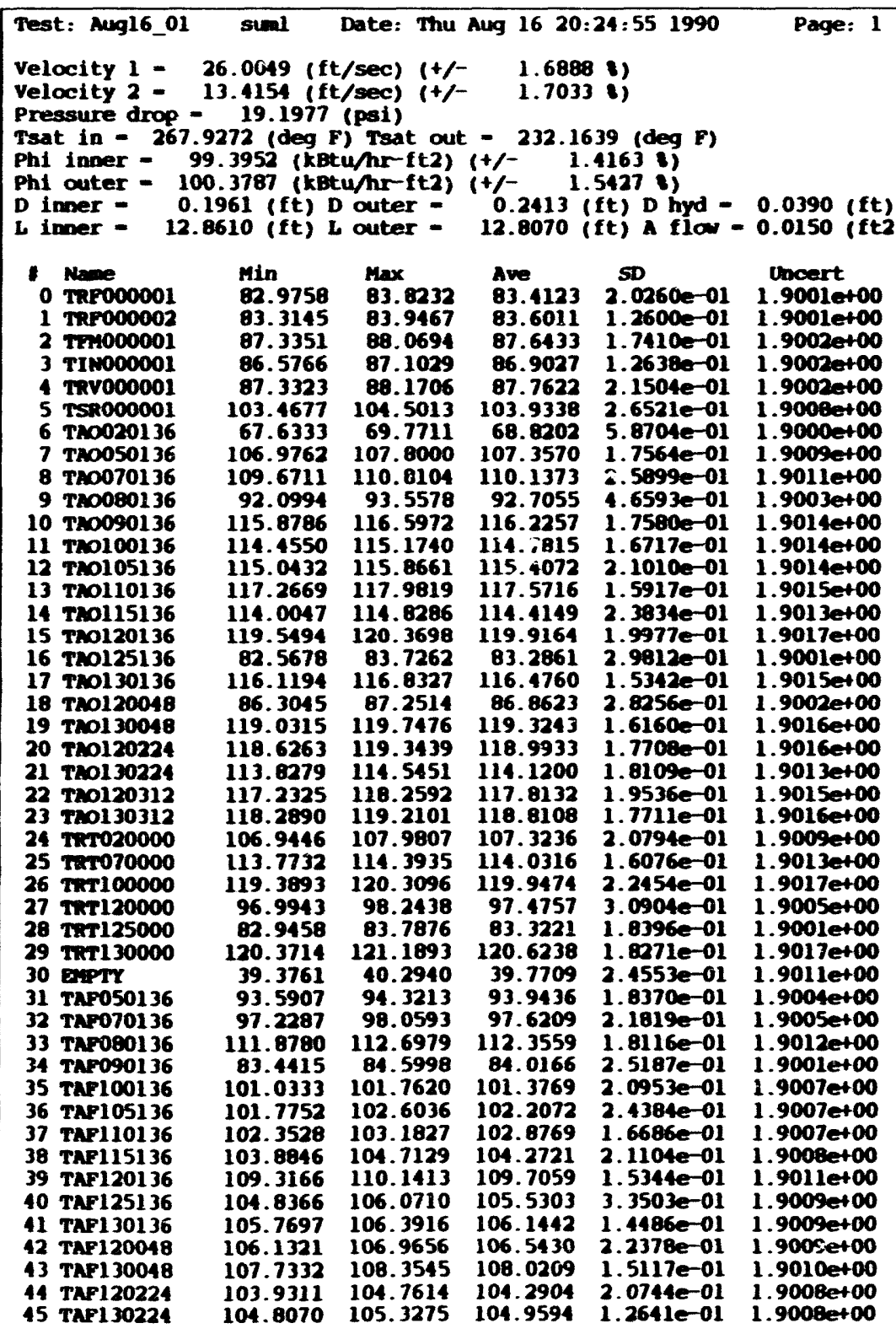

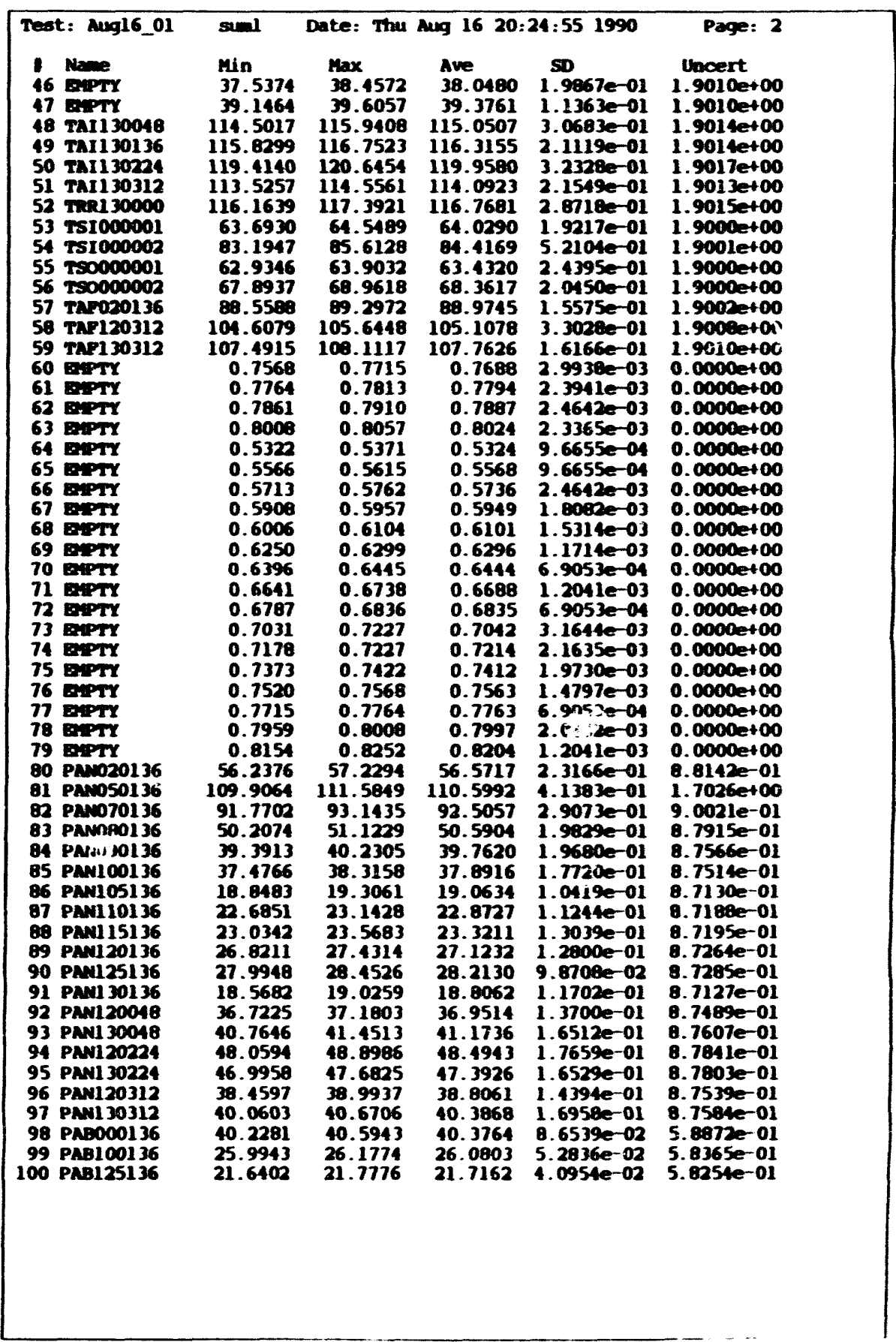




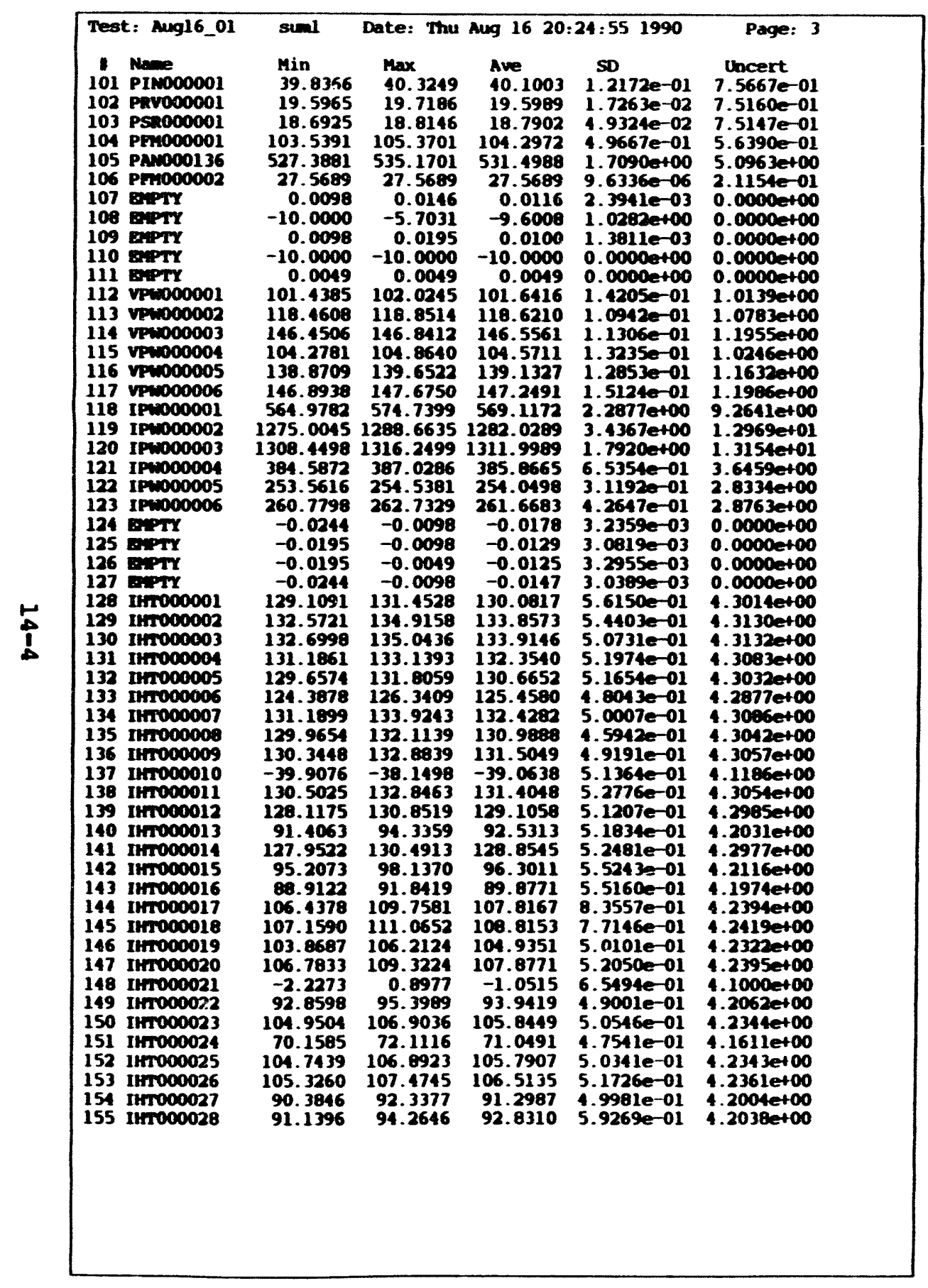

\begin{tabular}{|c|c|c|c|c|c|}
\hline Test: Mug16_01 & an & Date: Thu & $\operatorname{mang} 1620$ & $24: 551$ & Page: \\
\hline 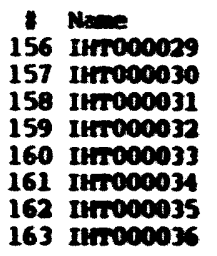 & $\begin{array}{l}\text { Min } \\
104.8152 \\
107.0125 \\
105.6415 \\
107.1326 \\
106.9599 \\
93.9378 \\
124.1136 \\
92.4091\end{array}$ & $\begin{array}{l}\operatorname{Max} \\
107.5496 \\
109.9422 \\
108.5712 \\
109.8670 \\
109.8096 \\
95.8909 \\
126.4573 \\
94.5575\end{array}$ & $\begin{array}{c}\text { Ave } \\
106.3270 \\
108.6336 \\
107.1572 \\
108.4608 \\
108.4013 \\
95.0472 \\
125.3285 \\
93.5966\end{array}$ & $\begin{array}{l}50 \\
5.6171 e-01 \\
5.96150-01 \\
6.2335=-01 \\
5.7045 e-01 \\
5.9174 e-01 \\
4.8396 e-01 \\
4.9014 e-01 \\
4.51350-01\end{array}$ & $\begin{array}{l}t \\
e+00 \\
e+00 \\
e+00 \\
e+00 \\
e+00 \\
e+00 \\
0\end{array}$ \\
\hline
\end{tabular}


Test: $\operatorname{Aug} 16$

Velocity $1=14.0221$ (ft/sec) $(t /-\quad 1.8662$ : $)$

Pressure drop - 2.2906 (Psi)

Trat in - 267.6289 (deg F) Tsat out $=264.0803$ (deg F)

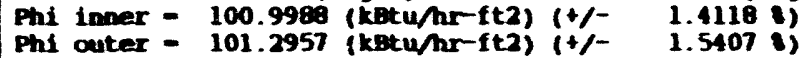

D inner - 0.1961 (ft) D outer - 0.2413 (ft) D hyd - 0.0390 (ft)

L inner - 12.8610 (ft) L outer - 12.8070 (ft) A fiow - 0.0150 (ft2)

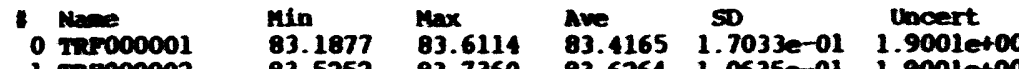

$\begin{array}{lllllll}1 \text { TIFro00002 } & 83.5252 & 83.7360 & 83.6264 & 1.0635 \mathrm{e}-01 & 1.9001 \mathrm{e}+00\end{array}$

$\begin{array}{lllllll}2 & \mathrm{~T} 7 \mathrm{H} 000001 & 87.4391 & 8.1744 & 87.8509 & 1.74550-01 & 1.9002 \mathrm{e}+00\end{array}$

$\begin{array}{lllllll}3 & \text { Tim000001 } & 86.8917 & 87.3134 & 87.1069 & 1.18420-01 & 1.9002 e+00\end{array}$

\begin{tabular}{lllllll}
\hline Imv000001 & 87.5414 & 88.4845 & 83.0152 & $2.2907 \mathrm{e}-01$ & $1.9002 \mathrm{et} 00$
\end{tabular}

$\begin{array}{lllllll}5 \text { Tsioco001 } & 107.2816 & 110.5710 & 109.1280 & 8.6679 e-01 & 1.90100+00\end{array}$

$\begin{array}{lllllll}6 & \mathrm{TnO020136} & 69.0231 & 70.1981 & 69.5658 & 3.2500 \mathrm{e}-01 & 1.9000 \mathrm{et} 00\end{array}$

7 T20050136 122.2913 123.1049 122.7057 1.8950e-01 1.90190+00

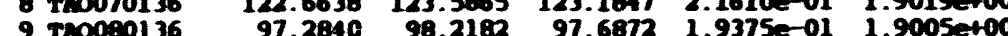

$\begin{array}{llllllll}10 & 130090136 & 136.7039 & 137.4105 & 137.0512 & 2.0100 e-01 & 1.9030+00\end{array}$

11 T20100136 135.011 135.7197 135.3677 1.6643e-01 1.9029et00

$\begin{array}{llllllll}12 & \text { rnol05136 } & 136.1614 & 137.0727 & 136.6313 & 2.00240-01 & 1.9030 e+00\end{array}$

$\begin{array}{llllllll}13 & \mathrm{Tm} 0110136 & 139.9280 & 140.5308 & 140.2534 & 1.5779 e-01 & 1.9033 e+00\end{array}$

$\begin{array}{llllllll}14 & \text { T20115136 } & 135.8262 & 136.6360 & 136.1827 & 2.004 \theta e-01 & 1.9029 e+00\end{array}$

15 Tmo120136

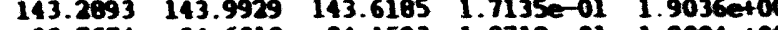

$93.767494 .601894 .1593 \quad 1.9718=01 \quad 1.9004$ et 00

$138.2755 \quad 138.8759 \quad 138.5989 \quad 1.3522 \mathrm{e}-01 \quad 1.9032 \mathrm{et} 00$

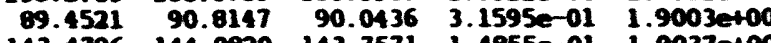
$\begin{array}{lllll}143.4796 & 144.0020 & 143.7571 & 1.4855 e-01 & 1.9037 e+00\end{array}$ 142.1557142 .9609 142.5907 1.5409e-01 1.9035 et00

141.3258142 .1325 141.6970 1.6597e-01 1.9035000

141.87541425789 142.2574 1.62580-01 1.9035e00

$120.6546 \quad 121.4752 \quad 121.0528 \quad 1.9506=-01 \quad 1.9018=+00$

$133.0089 \quad 134.5223 \quad 134.1557 \quad 1.56720-01 \quad 1.9028 e+00$

$142.4500 \quad 143.1538 \quad 142.7919 \quad 1.6861$ e-01 $1.9036 e+00$

$110.7593 \quad 111.5834111 .1587 \quad 1.7257 e-01 \quad 1.9011$ et00

$85.038285 .772985 .34931 .70720-01$ 1.9002et00

$144.4232145 .0256 \quad 144.7286 \quad 1.3612 e-01 \quad 1.9038 e+00$

$39.1464 \quad 40.2940 \quad 39.75712 .6071$ e-01 1.9011 e+00 99.4037 $100.3379 \quad 99.9061 \quad 1.8791 \mathrm{e}-01 \quad 1.9006 \mathrm{el} 00$ $105.6934 \quad 106.7236106 .1793 \quad 2.04060-01$ 1.9009e+00 130.8468 131.5527 131.2298 1.7335e-01 $1.9025 e+00$ $113.3523 \quad 114.1770 \quad 113.7319 \quad 1.67210-01 \quad 1.90130000$ $114.5767115 .5975 \quad 115.2007 \quad 2.3735 e-01 \quad 1.9014 e+00$ $116.3338 \quad 116.8472 \quad 116.5722 \quad 1.3346 e-01 \quad 1.9015 e+00$ $118.1714 \quad 118.9899118 .6253 \quad 1.6271 \mathrm{e}-01$ 1.9016et00 $128.2634 \quad 128.7717 \quad 128.5439 \quad 1.2895 e-01 \quad 1.9023 e+00$ 120.6543121 .6703 121.1212 2.4611e-01 1.9013e+00 121.9711 122.6862 $122.3349 \quad 1.2255 e-01 \quad 1.9018$ eto $122.3717 \quad 123.3984 \quad 122.9920 \quad 2.1857 e-01$ 1.9019et00 $125.3626 \quad 126.0765 \quad 125.7380 \quad 1.4785 e-01 \quad 1.9021 e+00$ $\begin{array}{lllll}118.4384 & 119.2575 & 118.8028 & 2.0067 e-01 & 1.9016 e+00 \\ 120.3280 & 120.8416 & 120.5828 & 1.2500 e-01 & 1.9017 \mathrm{et00}\end{array}$

\begin{tabular}{|c|c|c|c|c|c|}
\hline 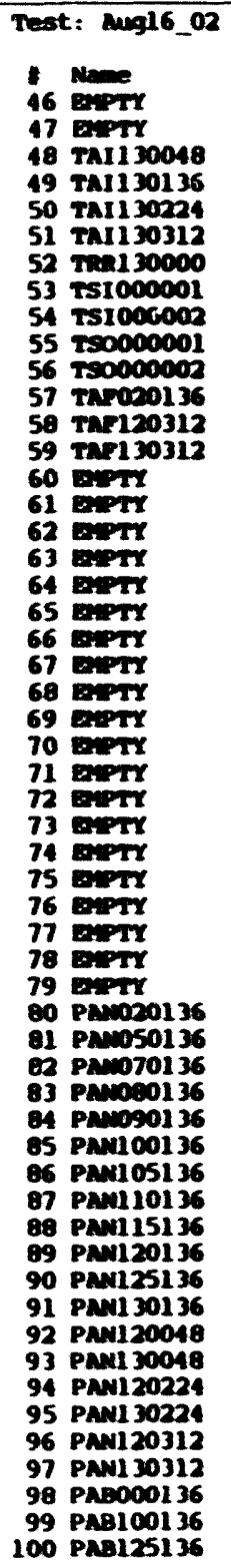 & 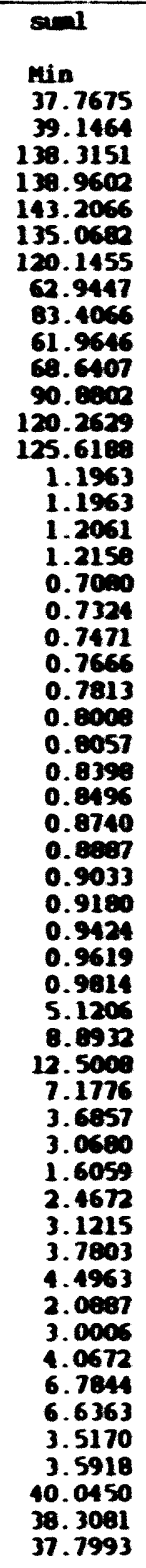 & 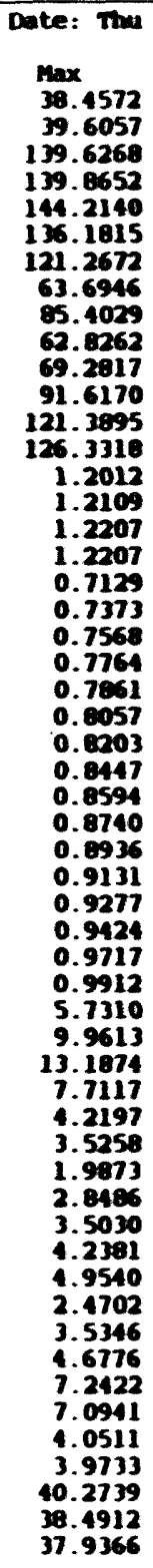 & 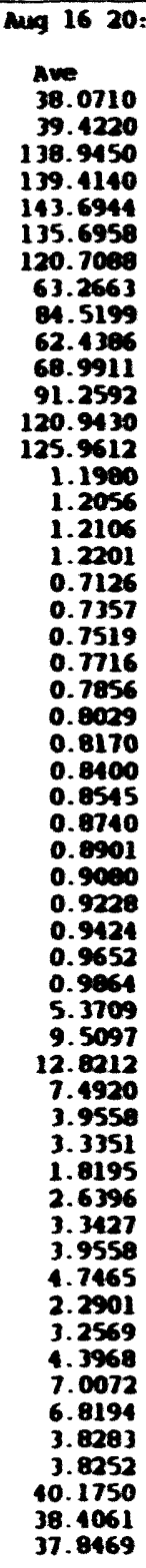 & 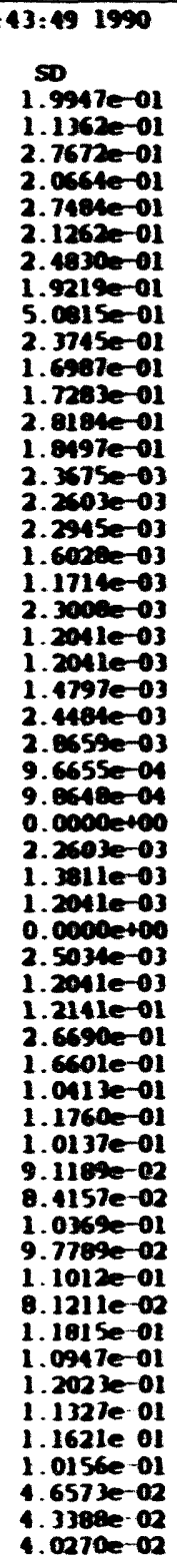 & 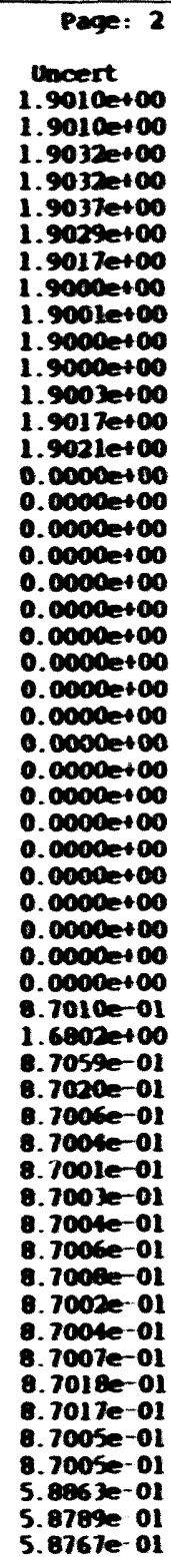 \\
\hline
\end{tabular}




\begin{tabular}{|c|c|c|c|c|c|}
\hline 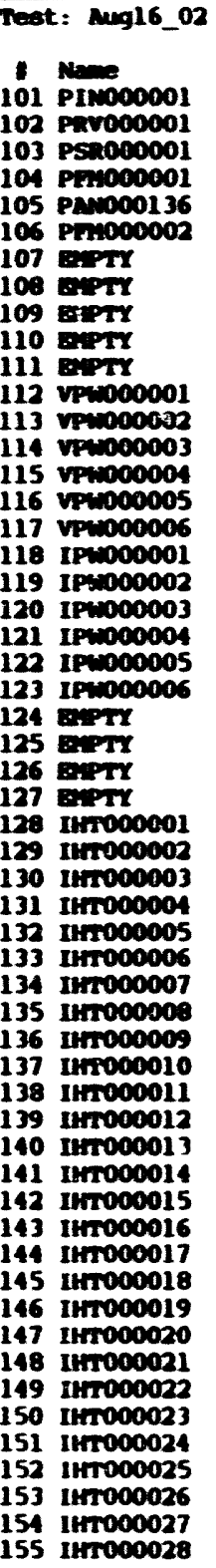 & $\begin{array}{r}\text { sal } \\
\text { Min } \\
39.9587 \\
19.8406 \\
37.0031 \\
29.74776 \\
60.4692 \\
27.5689 \\
0.0049 \\
-10.0000 \\
0.0049 \\
-10.0000 \\
0.0000 \\
101.6339 \\
118.2655 \\
146.6459 \\
104.2781 \\
139.2616 \\
147.2844 \\
547.4072 \\
1314.0299 \\
1329.8999 \\
383.1223 \\
254.5301 \\
263.2212 \\
-0.0342 \\
-0.0146 \\
-0.0195 \\
-0.0244 \\
128.7184 \\
132.5721 \\
132.8951 \\
131.1861 \\
129.4621 \\
124.5831 \\
131.1899 \\
129.7701 \\
130.5401 \\
-40.1029 \\
130.3072 \\
127.9222 \\
91.6016 \\
127.7569 \\
95.2073 \\
89.9122 \\
107.0238 \\
107.9402 \\
104.0640 \\
106.9787 \\
-2.2273 \\
93.0551 \\
104.9504 \\
70.3538 \\
105.1345 \\
105.9120 \\
90.7752 \\
92.1161 \\
\end{array}$ & $\begin{array}{r}\text { Date: Thu } \\
\text { Max } \\
40.2028 \\
19.9627 \\
37.3693 \\
30.7547 \\
65.9623 \\
27.5689 \\
0.0098 \\
-10.0000 \\
0.0096 \\
-10.0000 \\
0.0049 \\
102.2190 \\
119.0467 \\
147.0365 \\
105.0593 \\
139.6522 \\
147.8703 \\
555.2165 \\
1319.8937 \\
1333.7999 \\
384.5872 \\
255.5145 \\
264.6860 \\
-0.0098 \\
-0.0049 \\
-0.0049 \\
-0.0090 \\
131.2575 \\
135.3065 \\
135.4312 \\
133.5299 \\
132.3918 \\
126.9268 \\
133.9243 \\
132.1139 \\
132.4932 \\
-37.9515 \\
132.1557 \\
130.2659 \\
93.7500 \\
130.1007 \\
97.3558 \\
90.6701 \\
109.3675 \\
109.6980 \\
105.8218 \\
108.7365 \\
-0.0769 \\
94.8130 \\
106.5129 \\
71.7210 \\
106.5017 \\
107.4745 \\
92.3377 \\
94.0693\end{array}$ & $\begin{array}{r}\text { Aug } 1620: \\
\text { Ave } \\
10.1174 \\
19.9505 \\
37.1129 \\
30.1357 \\
63.4172 \\
27.5689 \\
0.0077 \\
-10.0000 \\
0.0050 \\
-10.0000 \\
0.0006 \\
101.9307 \\
118.7382 \\
146.7905 \\
104.7938 \\
139.4020 \\
147.4757 \\
550.6480 \\
1316.4894 \\
1332.4745 \\
383.0450 \\
255.0752 \\
264.0511 \\
-0.0177 \\
-0.0118 \\
-0.0126 \\
-0.0156 \\
129.9449 \\
133.8300 \\
134.0357 \\
132.3423 \\
130.7277 \\
125.5009 \\
132.4860 \\
131.0201 \\
131.4776 \\
-39.0092 \\
131.4790 \\
129.1566 \\
92.6953 \\
128.9639 \\
96.3753 \\
69.9709 \\
108.1410 \\
108.8426 \\
104.9155 \\
107.7755 \\
-1.1802 \\
93.9067 \\
105.6691 \\
70.9280 \\
105.7985 \\
106.5331 \\
91.1197 \\
92.9169\end{array}$ & 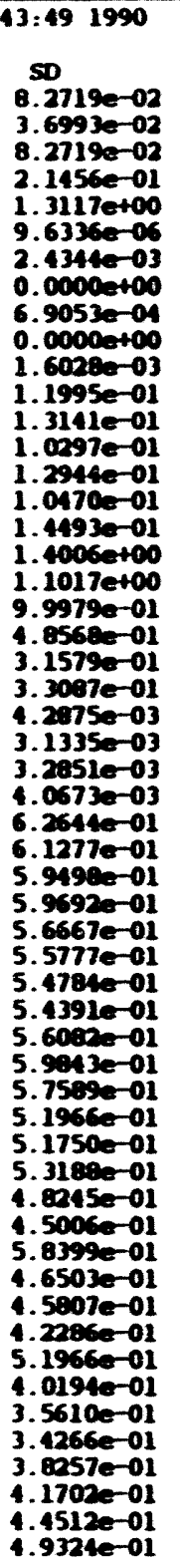 & 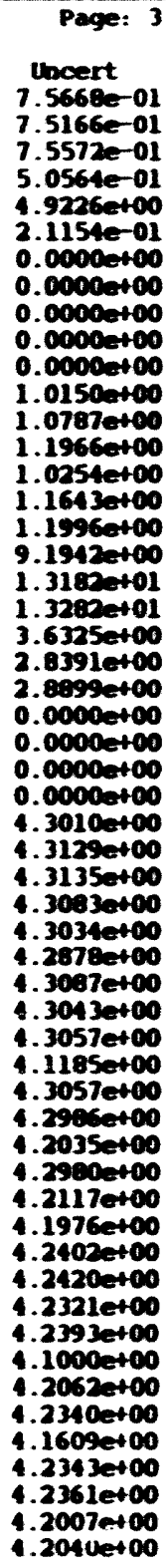 \\
\hline
\end{tabular}

\begin{tabular}{|c|c|c|c|c|c|}
\hline sot: & & 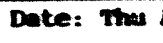 & 8 & & \\
\hline 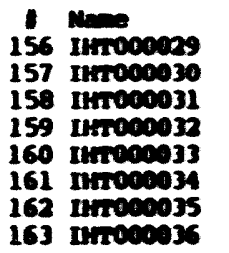 & $\begin{array}{l}\text { Min } \\
105.1012 \\
107.5904 \\
106.0320 \\
107.5233 \\
107.5450 \\
94.3204 \\
124.5042 \\
92.9950\end{array}$ & $\begin{array}{c}\operatorname{nax} \\
107.5496 \\
110.1375 \\
100.7665 \\
109.8670 \\
109.6943 \\
96.0662 \\
126.2620 \\
94.5575\end{array}$ & $\begin{array}{l}\text { Ave } \\
106.3934 \\
108.6610 \\
107.1377 \\
100.1725 \\
109.4209 \\
95.1253 \\
125.3265 \\
93.7130\end{array}$ & 5 & \\
\hline
\end{tabular}




\begin{tabular}{|c|c|c|c|c|c|}
\hline $\begin{array}{l}\text { Test: Mug16_03 } \\
\text { Velocity } 1= \\
\text { Velocity } 2= \\
\text { Pressure drop } \\
\text { Tsat in = } 268 . \\
\text { Phi inner = } 10 \\
\text { Phi outer = } 10 \\
\text { D Inner = } 0 . \\
\text { L inner - } 12 .\end{array}$ & $\begin{array}{l}\text { sum } \\
6.0508 \text { (ft } \\
6.0463 \text { ift } \\
-3.7904 \\
110 \text { (deg F } \\
.9309 \text { (kBt } \\
.1407 \text { (kBt } \\
961 \text { (ft) D } \\
1610 \text { (ft) }\end{array}$ & $\begin{array}{l}\text { Date: Thu } \\
\text { /sec) }(+/- \\
\text { /sec) } 1+/- \\
\text { (psi) } \\
\text { Tsat out }\end{array}$ & $\begin{array}{r}\operatorname{Meg} 1620: \\
4.8312 \\
2.3378 \\
=273.06 \\
1+/-\quad 1 \\
1+/-21 \\
0.2413 \\
12.8070\end{array}$ & $\begin{array}{l}57: 371990 \\
\text { ()) } \\
\text { 8) } \\
83 \text { (dog F) } \\
1121 \text { E) } \\
5413 \text { : ) } \\
\text { (t) D hyd - } \\
\text { ft) A flow - }\end{array}$ & $\begin{array}{l}0.0390 \text { (ft) } \\
0.0150 \text { (ft2) }\end{array}$ \\
\hline 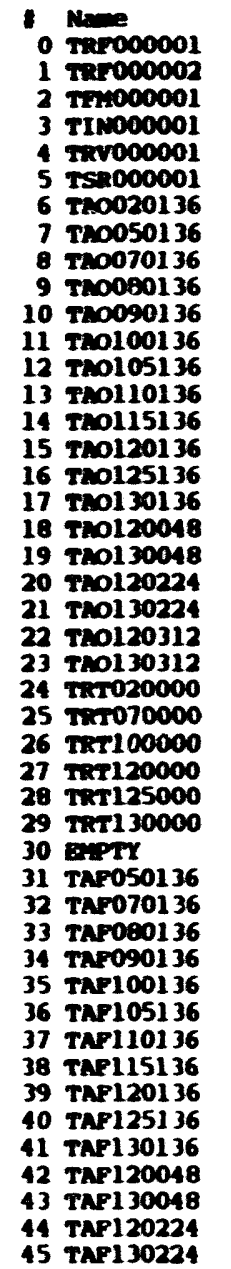 & $\begin{array}{l}\text { Min } \\
82.9758 \\
83.5252 \\
85.4462 \\
84.7852 \\
85.5545 \\
112.5161 \\
71.1572 \\
157.7375 \\
162.5800 \\
113.4272 \\
187.5410 \\
187.9815 \\
191.0051 \\
190.7922 \\
192.0822 \\
203.3894 \\
125.2538 \\
195.6059 \\
89.8706 \\
204.9493 \\
201.8569 \\
189.5952 \\
202.2125 \\
201.1751 \\
151.6622 \\
182.0822 \\
198.5398 \\
144.2882 \\
83.8690 \\
204.6777 \\
39.3761 \\
114.0247 \\
127.1166 \\
178.3287 \\
84.7034 \\
146.3105 \\
149.7575 \\
152.8316 \\
156.6431 \\
178.6742 \\
162.7707 \\
166.0631 \\
166.4412 \\
173.3508 \\
157.9397 \\
163.0295\end{array}$ & $\begin{array}{l}\text { Max } \\
83.8232 \\
83.9467 \\
86.2883 \\
85.4181 \\
86.6027 \\
113.9538 \\
73.7153 \\
158.9270 \\
163.9735 \\
115.0653 \\
189.4172 \\
189.2460 \\
192.3667 \\
193.6772 \\
193.1512 \\
204.3539 \\
126.4741 \\
196.1695 \\
91.2333 \\
205.6240 \\
202.6293 \\
190.7548 \\
203.2745 \\
201.9470 \\
152.9640 \\
183.1638 \\
199.5055 \\
145.3933 \\
84.8330 \\
205.6391 \\
10.2940 \\
115.1528 \\
128.3343 \\
179.4018 \\
85.6505 \\
147.2150 \\
150.8519 \\
153.8276 \\
157.9355 \\
180.0376 \\
164.4432 \\
166.8533 \\
167.8321 \\
174.2369 \\
159.1321 \\
163.8261\end{array}$ & $\begin{array}{l}\text { Ave } \\
8.3 .1758 \\
85.1191 \\
85.9300 \\
85.1773 \\
86.1331 \\
113.3133 \\
72.3858 \\
158.1740 \\
163.2809 \\
114.2132 \\
187.8839 \\
186.5613 \\
191.5225 \\
192.5129 \\
192.5915 \\
203.9025 \\
125.8985 \\
196.0109 \\
90.6049 \\
205.2619 \\
202.1698 \\
190.1510 \\
202.6876 \\
201.1570 \\
152.1673 \\
182.6759 \\
199.0070 \\
144.7986 \\
84.4550 \\
205.0965 \\
39.8168 \\
114.7838 \\
127.7695 \\
178.8204 \\
85.2215 \\
146.8208 \\
150.1610 \\
153.2058 \\
157.1064 \\
179.3914 \\
163.6482 \\
166.4538 \\
167.2200 \\
173.7919 \\
158.6187 \\
163.4075\end{array}$ & 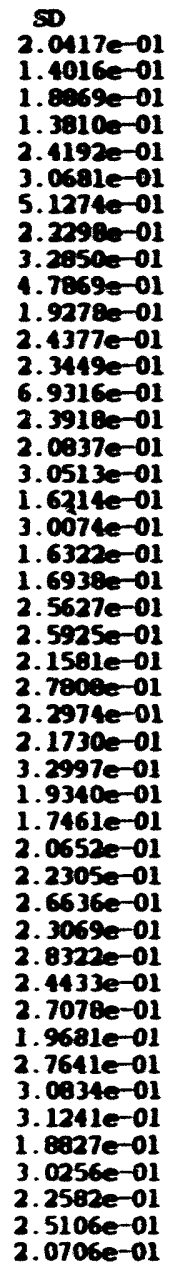 & 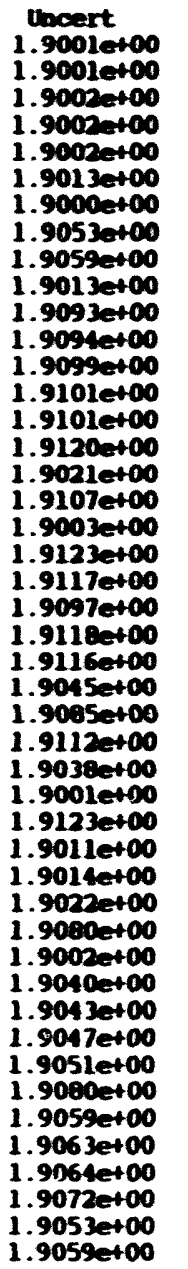 \\
\hline
\end{tabular}

\begin{tabular}{|c|c|c|c|c|c|}
\hline 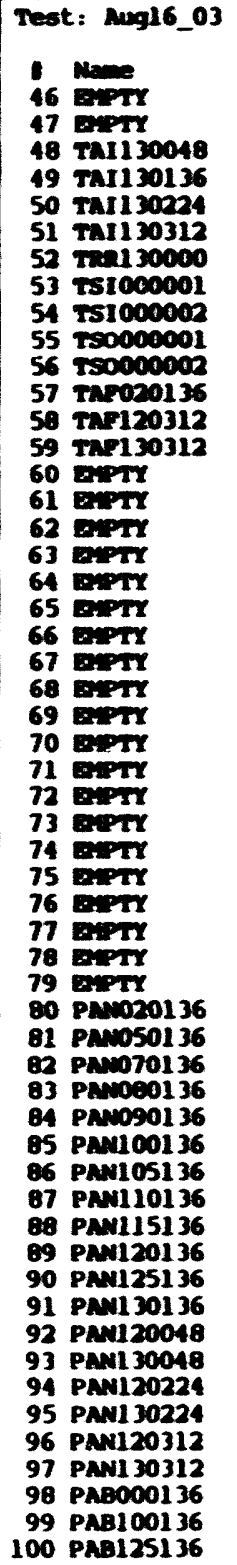 & $\begin{array}{l}\text { san } \\
\text { Min } \\
37.7675 \\
39.6057 \\
200.9904 \\
199.4441 \\
203.4391 \\
192.5624 \\
127.6660 \\
61.6749 \\
76.3421 \\
60.2380 \\
70.9855 \\
94.7745 \\
162.9681 \\
174.5414 \\
2.3779 \\
2.3730 \\
2.3633 \\
2.3730 \\
1.2150 \\
1.2354 \\
1.2500 \\
1.2646 \\
1.2744 \\
1.2991 \\
1.2989 \\
1.3232 \\
1.3281 \\
1.3477 \\
1.3623 \\
1.3770 \\
1.3867 \\
1.4014 \\
1.4258 \\
1.4453 \\
-14.0292 \\
-27.2701 \\
-16.2621 \\
-8.1575 \\
-8.8265 \\
-8.6812 \\
-4.4977 \\
-4.3230 \\
-4.2027 \\
-3.8491 \\
-3.6672 \\
-4.3200 \\
-9.0539 \\
-8.5213 \\
-7.5589 \\
-8.0121 \\
-8.3848 \\
-8.6915 \\
40.1112 \\
43.1604 \\
43.9333\end{array}$ & $\begin{aligned} \text { Date: Thu } \\
\text { Max } \\
38.4572 \\
39.8352 \\
203.1187 \\
200.4009 \\
204.7907 \\
193.9219 \\
129.1661 \\
62.6271 \\
60.5631 \\
61.3162 \\
71.8386 \\
95.7190 \\
164.6524 \\
175.7197 \\
2.3877 \\
2.3828 \\
2.3779 \\
2.3779 \\
1.2256 \\
1.2402 \\
1.2549 \\
1.2695 \\
1.2691 \\
1.2939 \\
1.3037 \\
1.3232 \\
1.3379 \\
1.3525 \\
1.3672 \\
1.3818 \\
1.3916 \\
1.4111 \\
1.4307 \\
1.4502 \\
-13.6477 \\
-26.3546 \\
-15.8043 \\
-7.7760 \\
-8.5213 \\
-8.4523 \\
-4.2688 \\
-4.0178 \\
-3.6975 \\
-3.5439 \\
-3.4383 \\
-1.0911 \\
-8.7487 \\
-8.2161 \\
-7.3300 \\
-7.6306 \\
-8.0033 \\
-8.3101 \\
40.5943 \\
43.3435 \\
44.0706\end{aligned}$ & $\begin{array}{r}\text { Aug } 1620: \\
\text { Ave } \\
38.1583 \\
39.7250 \\
201.8270 \\
199.8798 \\
204.1769 \\
193.2077 \\
128.3318 \\
62.2533 \\
78.1249 \\
60.8637 \\
71.4206 \\
95.2193 \\
163.6338 \\
175.0670 \\
2.3850 \\
2.3795 \\
2.3740 \\
2.3762 \\
1.2180 \\
1.2380 \\
1.2501 \\
1.2656 \\
1.2773 \\
1.2929 \\
1.3036 \\
1.3232 \\
1.3331 \\
1.3519 \\
1.3625 \\
1.3797 \\
1.3909 \\
1.4063 \\
1.4259 \\
1.1455 \\
-13.9117 \\
-26.7208 \\
-16.0499 \\
-7.9667 \\
-8.6861 \\
-8.5637 \\
-4.3817 \\
-4.1307 \\
-4.0761 \\
-3.6797 \\
-3.5161 \\
-4.2254 \\
-8.9303 \\
-8.3641 \\
-7.4780 \\
-7.8061 \\
-8.2368 \\
-8.5145 \\
40.5009 \\
43.2401 \\
43.9874\end{array}$ & $\begin{array}{l}57: 371990 \\
50 \\
2.0379 e-01 \\
1.1583 e-01 \\
1.2743 e-01 \\
2.0553 e-01 \\
3.3926 e-01 \\
2.6015 e-01 \\
3.5583 e-01 \\
1.8794 e-01 \\
9.4644 e-01 \\
2.3970 e-01 \\
2.0056 e-01 \\
2.5317 e-01 \\
3.5146 e-01 \\
2.7558 e-01 \\
2.8179 e-03 \\
2.5034 e-03 \\
3.1195 e-03 \\
2.3675 e-03 \\
2.6396 e-03 \\
2.4583 e-03 \\
6.9053 e-04 \\
1.9730 e-03 \\
2.9594 e-03 \\
2.0432 e-03 \\
6.9053 e-04 \\
0.0000 e+00 \\
1.2041 e-03 \\
1.7115 e-03 \\
9.6655 e-04 \\
2.4484 e-03 \\
1.7115 e-03 \\
1.2041 e-03 \\
6.9053 e-04 \\
9.6655 e-04 \\
8.8026 e-02 \\
1.8238 e-01 \\
1.0931 e-01 \\
9.0206 e-02 \\
7.7621 e-02 \\
6.5775 e-02 \\
5.1676 e-02 \\
6.5829 e-02 \\
7.6465 e-02 \\
7.7514 e-02 \\
6.2592 e-02 \\
7.1570 e-02 \\
6.6992 e-02 \\
8.3590 e-02 \\
6.6135 e-02 \\
7.4972 e-02 \\
9.6763 e-02 \\
8.9133 e-02 \\
1.8020 e-02 \\
5.2974 e-02 \\
4.6875 e-02\end{array}$ & 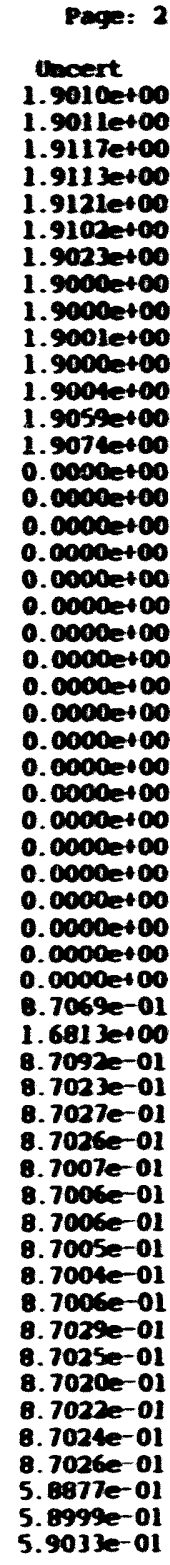 \\
\hline
\end{tabular}




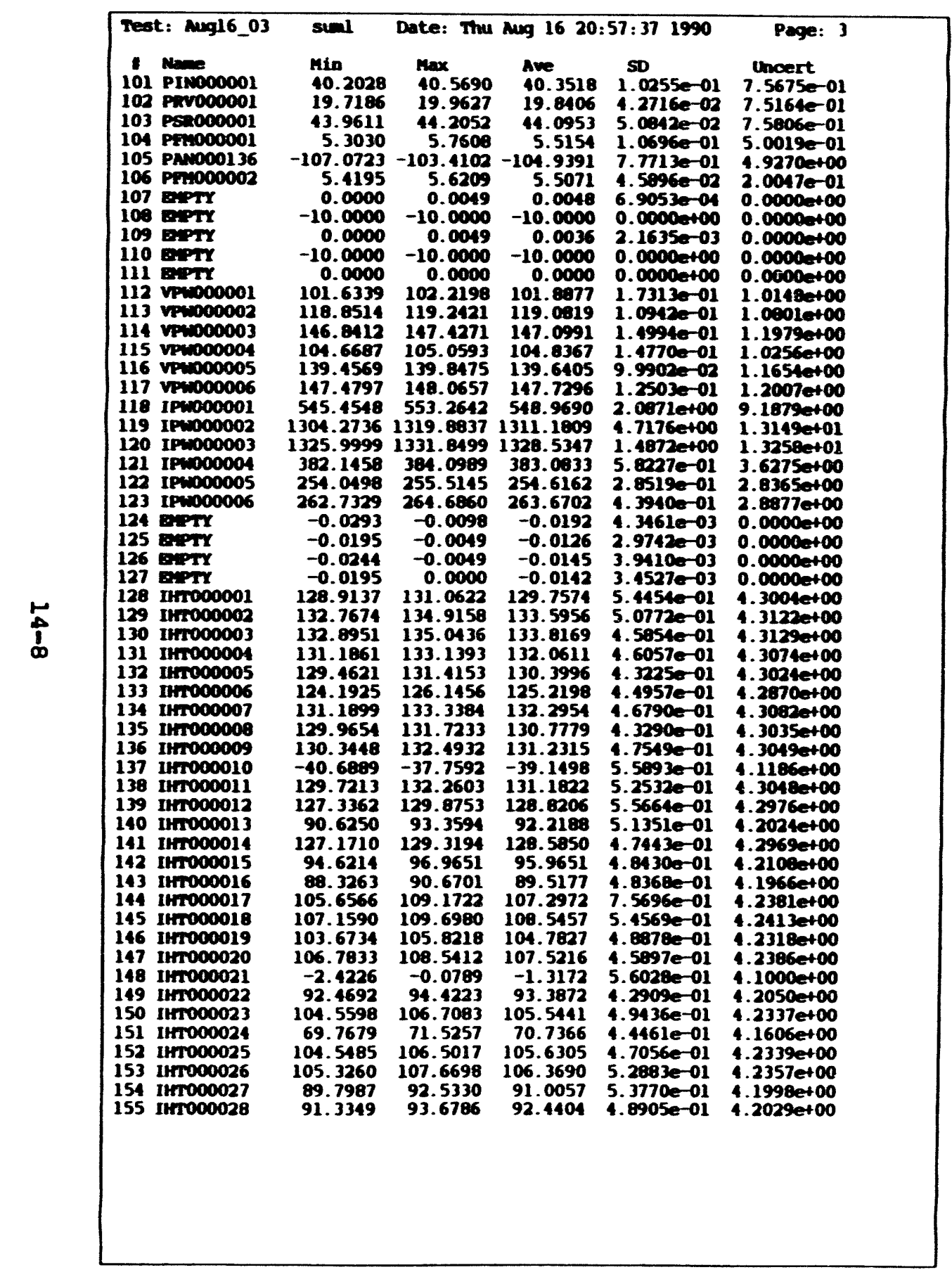

\begin{tabular}{|c|c|c|c|c|c|}
\hline at: & & 2 & 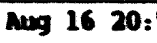 & 990 & e: 4 \\
\hline 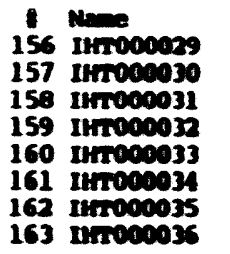 & $\begin{array}{l}\min \\
105.2059 \\
107.1031 \\
105.8368 \\
107.1326 \\
107.1552 \\
93.7425 \\
124.1136 \\
92.4091\end{array}$ & $\begin{array}{l}10 x \\
107.3543 \\
109.5515 \\
107.7900 \\
109.0858 \\
109.1083 \\
95.5003 \\
125.8714 \\
94.1669\end{array}$ & $\begin{array}{l}\text { Ave } \\
106.2020 \\
108.4578 \\
106.9150 \\
100.1678 \\
108.1357 \\
94.7034 \\
125.0629 \\
93.3363\end{array}$ & $\begin{array}{l}50 \\
1.7062 \\
4.4613 \\
4.2543 \\
4.1432 \\
4.2021 \\
4.0786 \\
3.9064 \\
3.8371\end{array}$ & 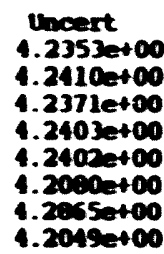 \\
\hline
\end{tabular}




\begin{tabular}{|c|c|c|c|c|c|c|}
\hline & Aug16_04 & suml & tee: Th & $\operatorname{lng} 16$ & $: 141990$ & Page: 1 \\
\hline & $\begin{array}{l}x \text { city } 1= \\
\text { xity } 2= \\
\text { ssure drop }- \\
\text { in }=268 . \\
\text { inner }=10 \\
\text { outer }=10 \\
\text { mer }=0 \\
\text { mer }=12 .\end{array}$ & $\begin{array}{r}4.0279 \\
4.0111 \\
-4.646 \\
124 \text { (deg } \\
.7989 \text { lk }\end{array}$ & $\begin{array}{l}\text { c) } \\
\text { c) } \\
\text { i) }\end{array}$ & $\begin{array}{r}10.5232 \\
4.1262 \\
=\quad 274.11 \\
1+/--1 \\
1+/-1 \\
0.2413 \\
12.8070\end{array}$ & $\begin{array}{l}\text { ) } \\
(\operatorname{deg} F) \\
25 \text { :) } \\
31 \text { i) } \\
\text { D hyd - } \\
\text { A flou = }\end{array}$ & $\begin{array}{l}0.0390 \text { (ft) } \\
0.0150 \text { (ftz) }\end{array}$ \\
\hline & 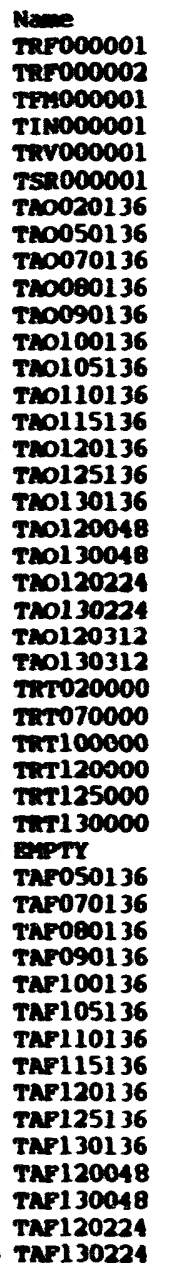 & $\begin{array}{r}\text { Hin } \\
83.1877 \\
83.5252 \\
86.4961 \\
85.9438 \\
86.4970 \\
120.4851 \\
73.0759 \\
191.9991 \\
200.2083 \\
219.8658 \\
232.1243 \\
232.2951 \\
238.1066 \\
-143.8467 \\
240.6069 \\
254.9194 \\
151.3200 \\
246.0701 \\
94.3672 \\
258.1590 \\
251.6117 \\
237.9866 \\
254.2709 \\
252.4283 \\
178.7374 \\
222.5586 \\
249.5895 \\
173.1723 \\
86.3976 \\
256.4720 \\
39.3761 \\
130.7075 \\
149.6879 \\
211.5889 \\
86.5947 \\
180.1443 \\
184.6250 \\
189.2438 \\
194.8335 \\
226.0311 \\
205.0696 \\
209.1772 \\
210.2126 \\
220.2279 \\
197.0072 \\
204.8336\end{array}$ & $\begin{array}{r}\operatorname{Max} \\
83.8232 \\
83.9467 \\
87.1273 \\
86.3664 \\
87.3358 \\
124.1456 \\
74.8951 \\
193.7363 \\
202.2432 \\
220.6245 \\
233.2579 \\
233.8051 \\
239.8970 \\
-46.4865 \\
242.8634 \\
255.9454 \\
153.5119 \\
247.0937 \\
95.6190 \\
259.0864 \\
252.7314 \\
239.8530 \\
255.4842 \\
253.4540 \\
180.2102 \\
223.8989 \\
250.1301 \\
174.9400 \\
87.3397 \\
257.1225 \\
10.2940 \\
132.2231 \\
151.5781 \\
230.7612 \\
87.7493 \\
182.0067 \\
186.2721 \\
191.4696 \\
197.8253 \\
230.5544 \\
208.1190 \\
211.9481 \\
213.0082 \\
224.0296 \\
200.6721 \\
205.8973\end{array}$ & $\begin{array}{r}\text { Ave } \\
83.5394 \\
83.7613 \\
86.8430 \\
86.1068 \\
86.9559 \\
122.8029 \\
74.0063 \\
192.8349 \\
201.2574 \\
220.2591 \\
232.6931 \\
232.9893 \\
238.9985 \\
293.3255 \\
241.9030 \\
255.3727 \\
152.3227 \\
246.6419 \\
95.0458 \\
258.6325 \\
252.1469 \\
238.9700 \\
254.8737 \\
252.8595 \\
179.4543 \\
223.1165 \\
250.0839 \\
173.7874 \\
86.9066 \\
256.7789 \\
39.8719 \\
131.4417 \\
150.6890 \\
218.2602 \\
87.1596 \\
180.9622 \\
185.4062 \\
190.1984 \\
196.3806 \\
227.9595 \\
206.4149 \\
210.9189 \\
211.2294 \\
221.4739 \\
199.0663 \\
205.3423\end{array}$ & $\begin{array}{l}\text { SD } \\
1.7957 e-01 \\
1.2512 e-01 \\
1.7268 e-01 \\
1.1902 e-01 \\
2.1879 e-01 \\
9.7701 e-01 \\
4.5236 e-01 \\
3.2366 e-01 \\
4.5311 e-01 \\
1.7759 e-01 \\
2.5611 e-01 \\
3.1524 e-01 \\
4.4626 e-01 \\
2.3167 e+01 \\
6.3015 e-01 \\
2.3060 e-01 \\
4.6705 e-01 \\
2.5281 e-01 \\
2.9828 e-01 \\
2.2956 e-01 \\
2.8386 e-01 \\
4.1913 e-01 \\
3.4308 e-01 \\
2.5302 e-01 \\
3.4710 e-01 \\
3.1154 e-01 \\
1.9614 e-01 \\
4.6734 e-01 \\
2.1778 e-01 \\
1.7463 e-01 \\
2.2863 e-01 \\
3.6341 e-01 \\
3.6640 e-01 \\
6.6847 e+00 \\
2.9299 e-01 \\
4.4666 e-01 \\
1.4764 e-01 \\
5.2414 e-01 \\
7.5894 e-01 \\
1.2301 e+00 \\
6.1740 e-01 \\
6.6703 e-01 \\
6.1623 e-01 \\
8.8170 e-01 \\
9.9489 e-01 \\
2.9417 e-01\end{array}$ & 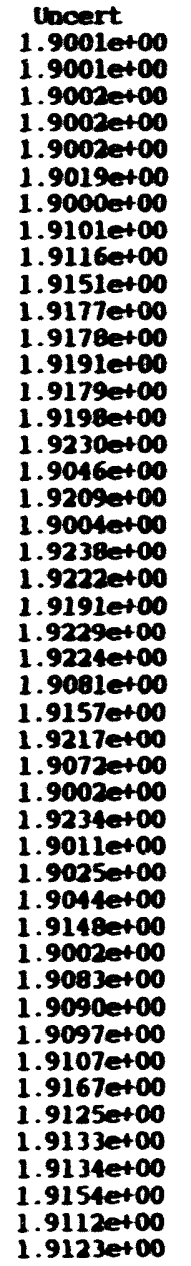 \\
\hline
\end{tabular}

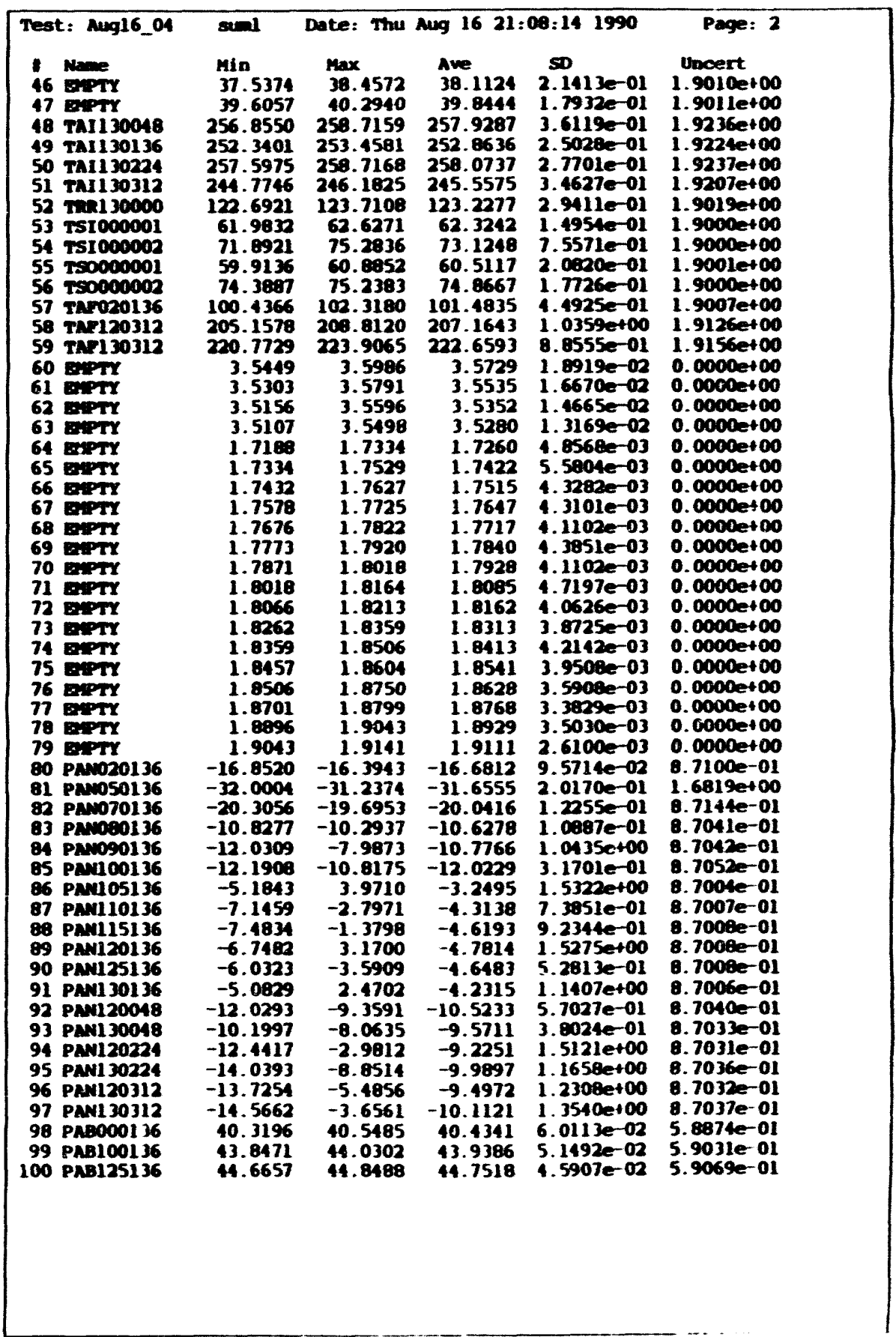



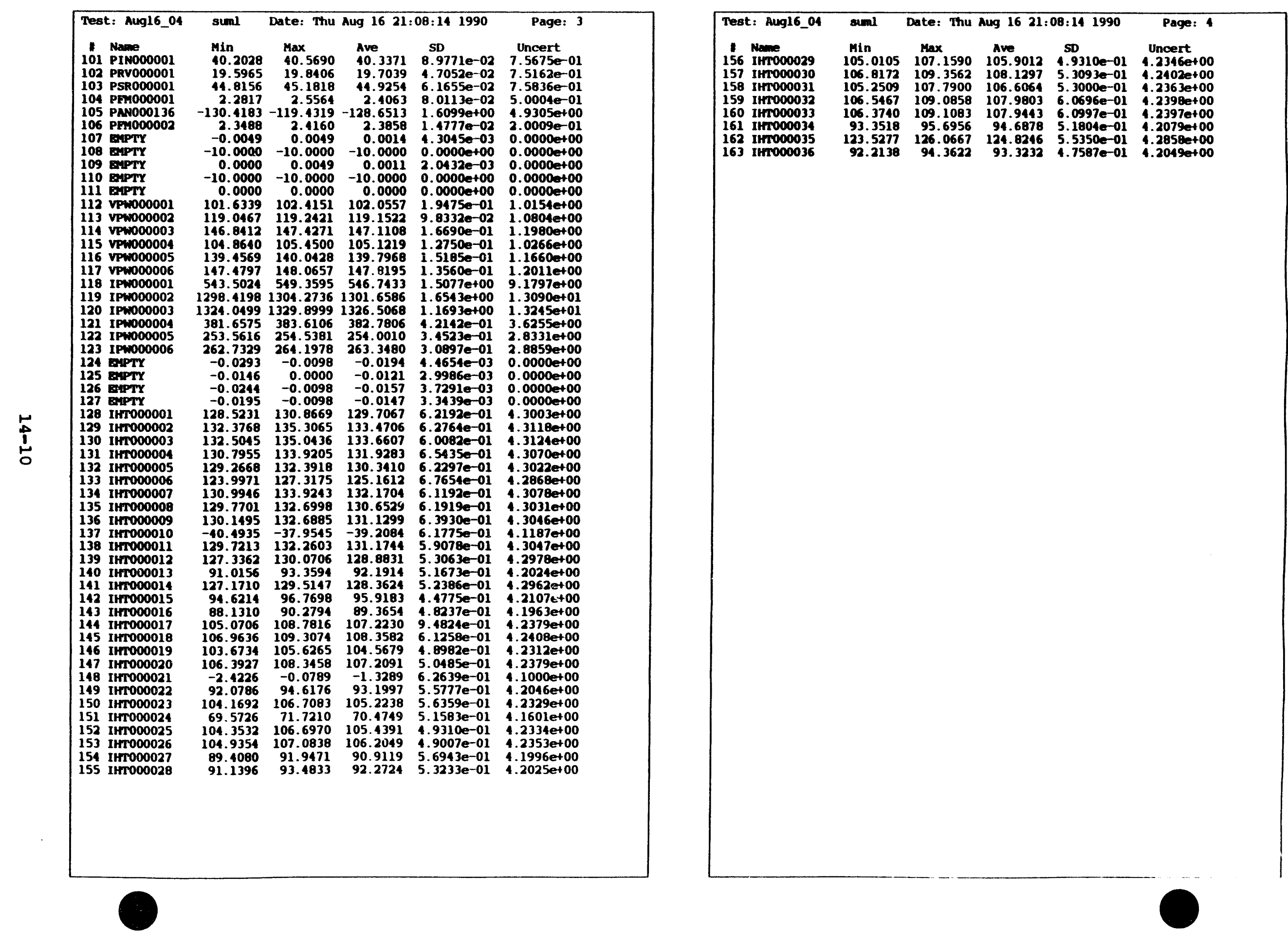
Test: Aug16_05 suml Date: Thu Aug 16 21:19:54 1990 Page: 1

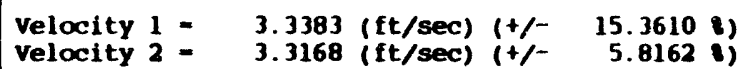

Velocity $2=-3.3168$ (ft/sec)
Pressure drop $-\quad-4.7038$ (psi)

Tsat in $=268.2000(\mathrm{deg}$ F) Tsat out $=274.3966(\mathrm{deg}$ F)

Phi inner $=100.4335$ (kBtu/Mr-ft2) (t/- 1.4136 : $)$
Phi outer $=100.6932$ (kBtu/hr-ft2) $(+/-1.5428$ )

$D$ inner - 0.1961 (ft) D outer $=0.2413$ (f $t$ ) D hyd - 0.0390 (ft)

L inner $=12.8610$ (ft) L outer - 12.8070 (ft) a flow - 0.0150 (ft2)

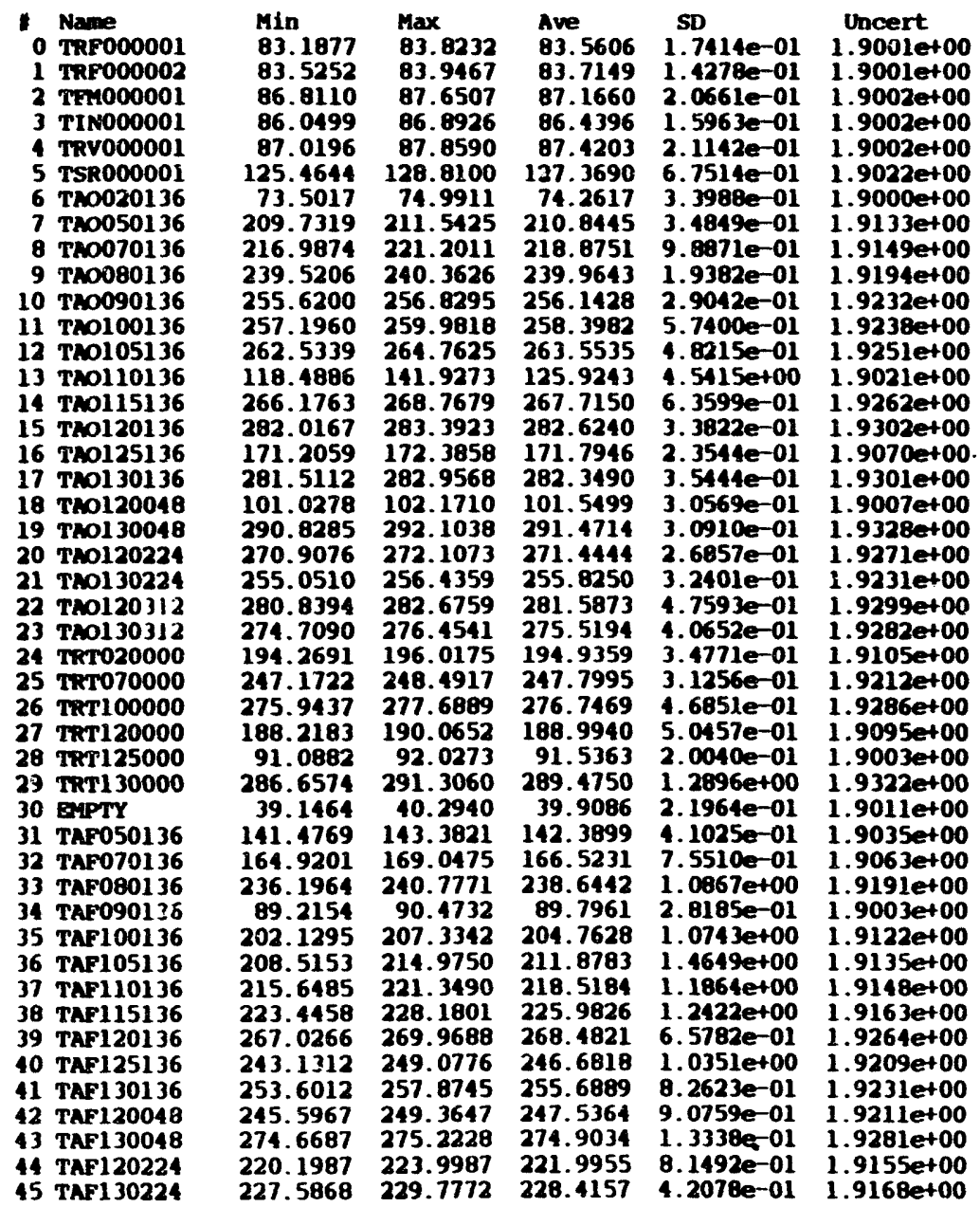

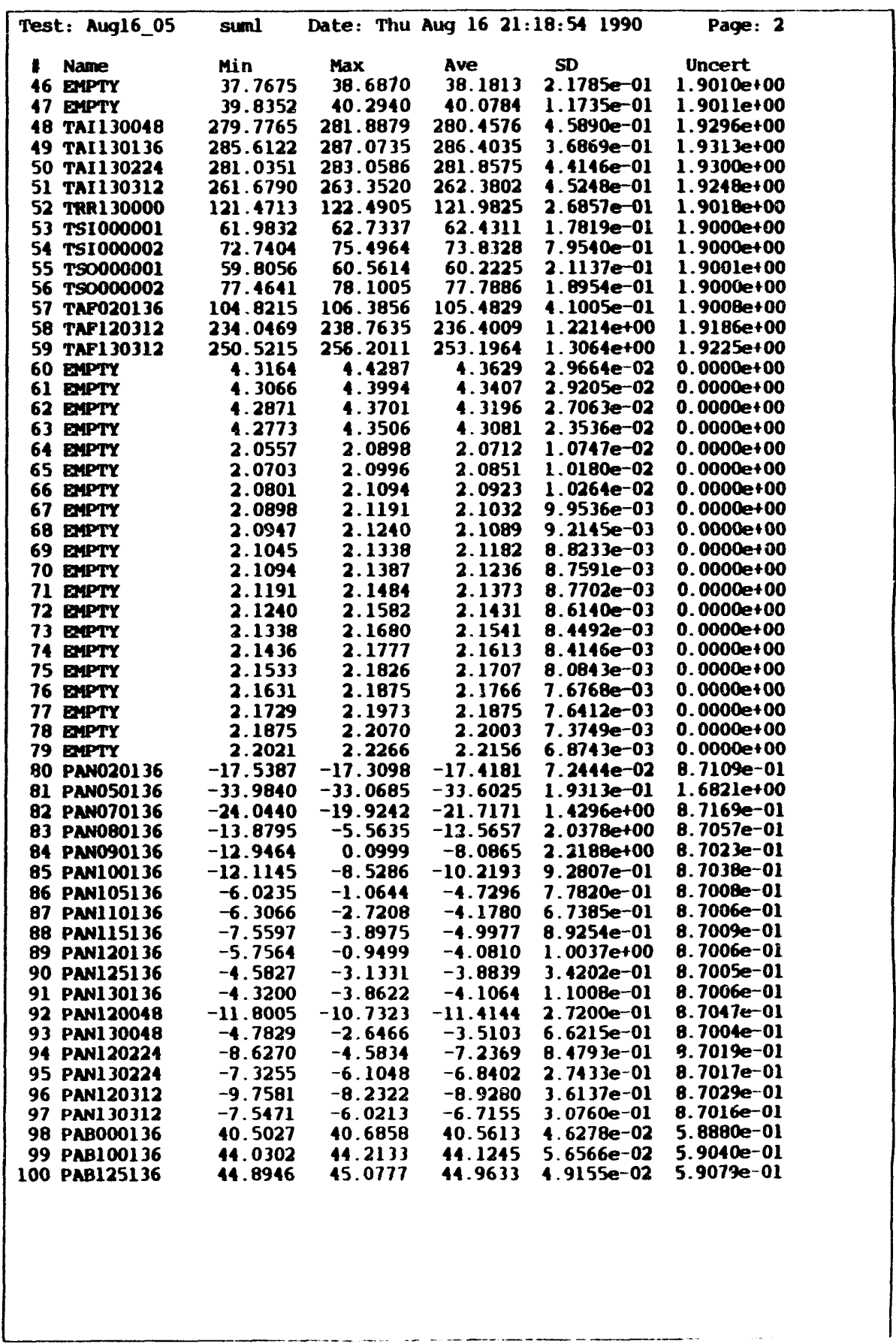




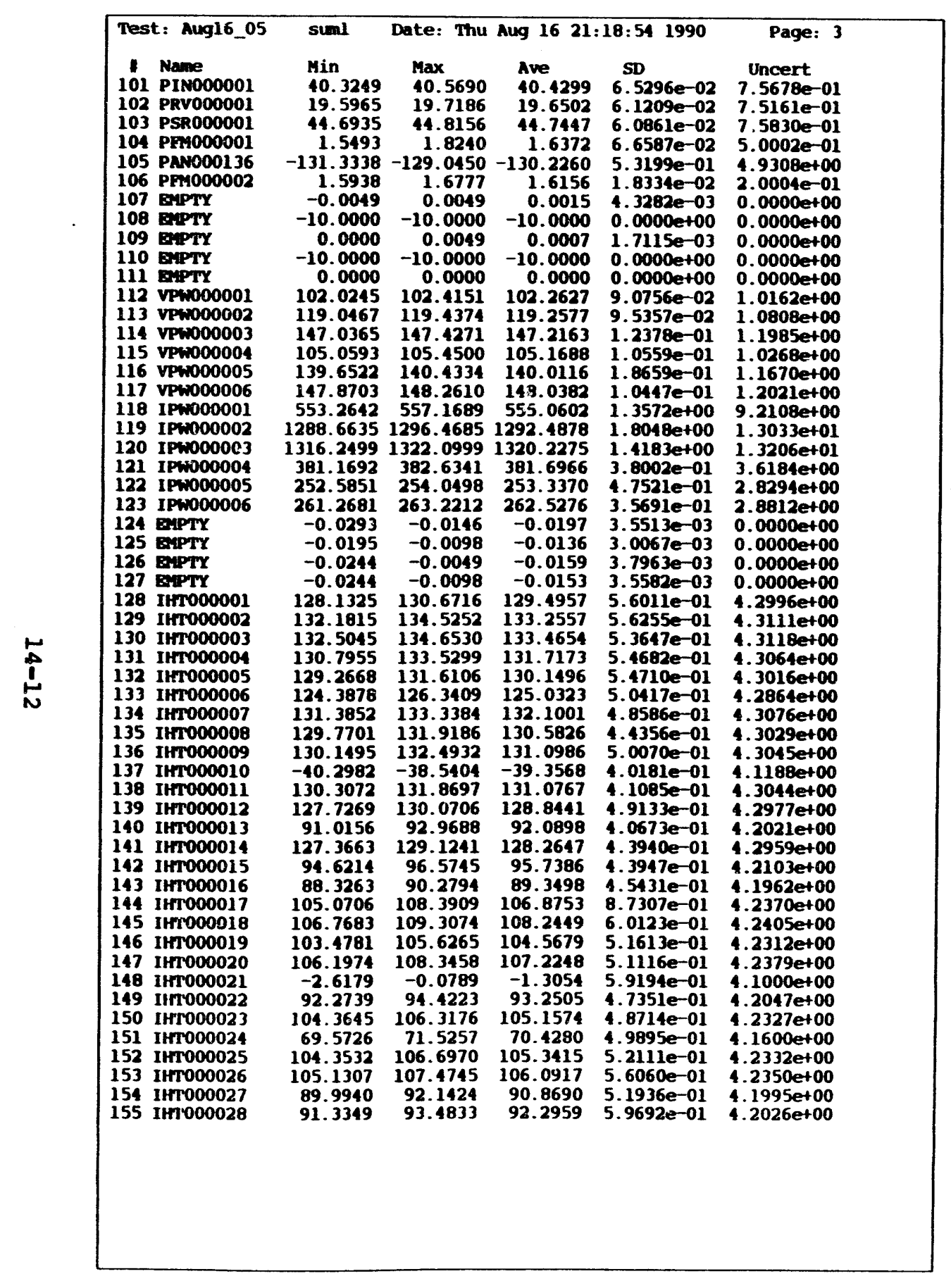

\begin{tabular}{|c|c|c|c|c|c|}
\hline Test: Aug16_05 & simal & Date: Thu & Aug 16 21: & $18: 541990$ & Page: 4 \\
\hline 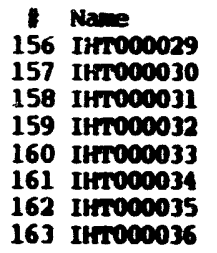 & $\begin{array}{l}\text { Min } \\
104.8152 \\
107.0125 \\
105.4462 \\
106.7420 \\
106.7646 \\
93.7425 \\
123.9183 \\
92.2138\end{array}$ & $\begin{array}{l}\text { Max } \\
107.1590 \\
109.1609 \\
107.5947 \\
109.0858 \\
109.1083 \\
95.6956 \\
125.6761 \\
93.9716\end{array}$ & $\begin{array}{c}\text { Ave } \\
105.8387 \\
108.0516 \\
106.4853 \\
107.8045 \\
107.7724 \\
94.4495 \\
124.6175 \\
93.1044\end{array}$ & $\begin{array}{l}\text { SD } \\
5.9524 e-01 \\
5.5164 e-01 \\
5.5586 e-01 \\
5.7610 e-01 \\
5.6409 e-01 \\
4.0025 e-01 \\
4.2332 e-01 \\
3.9094 e-01\end{array}$ & $\begin{array}{c}\text { Uncert } \\
4.2344 \text { e+00 } \\
1.2400 \mathrm{e}+00 \\
4.2360 \mathrm{e}+00 \\
4.2394 \mathrm{e}+00 \\
4.2393 \mathrm{e}+00 \\
4.2074 \mathrm{e}+00 \\
4.2852 \mathrm{e}+00 \\
4.2044 \mathrm{e}+00\end{array}$ \\
\hline
\end{tabular}

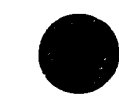




\begin{tabular}{|c|c|c|c|c|c|c|}
\hline \multirow{2}{*}{\multicolumn{2}{|c|}{$\begin{array}{l}\text { Test: Aug16_06 } \\
\text { Velocity } 1= \\
\text { Velocity } 2= \\
\text { Pressure drop } \\
\text { Tsat in = 268 } \\
\text { Phi inner = 11 } \\
\text { Phi outer = 11 } \\
\text { D inner = } 0 \\
\text { L inner = } 12\end{array}$}} & \multirow[b]{2}{*}{$\begin{array}{c}\text { suml } \\
3.4051 \text { । } \\
3.3290 \text { ! } \\
-4.786 \\
1245 \text { (deg } \\
1.1641 \text { (k) } \\
0.9578 \text { (k } \\
1961 \text { (ft) } \\
8610 \text { (ft) }\end{array}$} & \multirow[b]{2}{*}{$\begin{array}{l}\text { Date: Thu } \\
\text { t/sec) }(+/ \\
\text { t/sec) }(+/ \\
\text { (psi) } \\
\text { F) Tsat out } \\
\text { tu/hr-ft2) } \\
\text { tumr-ft2) } \\
\text { D outer - }\end{array}$} & \multirow{2}{*}{\multicolumn{2}{|c|}{$\begin{array}{l}\text { Aug } 1621: 37: 031990 \\
14.7580 \text { s) } \\
5.7766 \text { s) } \\
=274.4053 \text { (deg F) } \\
(+/-\quad 1.4116 \text { ) } \\
(+/-\quad 1.5426 \text { ) } \\
0.2413 \text { (ft) D hyd - } \\
12.8070 \text { (ft) A flow - }\end{array}$}} & \multirow{2}{*}{$\begin{array}{c}\text { Page: } 1 \\
0.0390 \text { (ft) } \\
0.0150 \text { (ft2) }\end{array}$} \\
\hline & & & & & & \\
\hline $\begin{array}{l}1 \\
1 \\
1 \\
2 \\
3 \\
4 \\
5 \\
6 \\
7 \\
8 \\
9 \\
10 \\
11 \\
12 \\
13 \\
14 \\
15 \\
16 \\
17 \\
18 \\
19 \\
20 \\
21 \\
22 \\
23 \\
24 \\
25 \\
26 \\
27 \\
28 \\
29 \\
30 \\
31 \\
32 \\
33 \\
34 \\
35 \\
36 \\
37 \\
38 \\
39 \\
40 \\
41 \\
42 \\
43 \\
44 \\
45\end{array}$ & 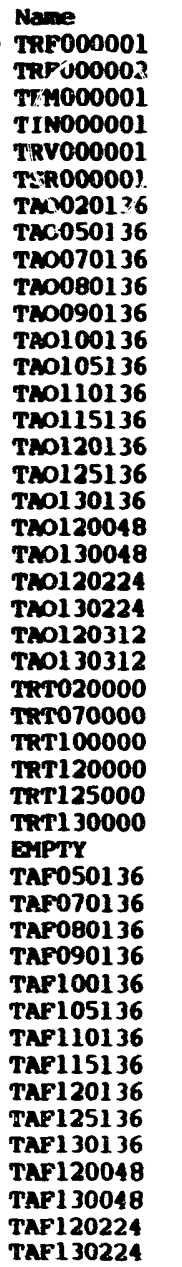 & $\begin{array}{r}\text { Min } \\
83.3996 \\
83.5252 \\
87.4401 \\
86.9973 \\
87.7520 \\
130.3257 \\
72.8630 \\
213.0646 \\
223.3035 \\
133.4003 \\
258.3138 \\
261.5568 \\
268.2841 \\
190.9881 \\
371.0782 \\
285.1337 \\
164.4123 \\
279.4313 \\
109.7182 \\
275.5790 \\
282.5840 \\
271.3044 \\
275.8713 \\
272.2268 \\
194.4634 \\
238.2822 \\
266.2819 \\
275.3937 \\
95.8642 \\
273.2855 \\
39.3761 \\
142.7799 \\
168.4567 \\
249.0549 \\
93.8148 \\
205.3120 \\
210.7029 \\
217.3607 \\
224.2040 \\
266.4738 \\
237.2571 \\
241.1812 \\
224.3290 \\
236.4146 \\
225.7050 \\
234.3385\end{array}$ & $\begin{array}{r}\text { Max } \\
83.8232 \\
84.1573 \\
88.4898 \\
87.4190 \\
88.4860 \\
132.2438 \\
74.6725 \\
214.3007 \\
228.9276 \\
135.1102 \\
259.4279 \\
263.4109 \\
269.4878 \\
208.4924 \\
273.1083 \\
286.1422 \\
165.4996 \\
280.5210 \\
110.7500 \\
276.4984 \\
283.5926 \\
272.6762 \\
276.8849 \\
273.0571 \\
196.0176 \\
239.5147 \\
268.0363 \\
276.6822 \\
96.5926 \\
273.9308 \\
40.0646 \\
144.2836 \\
170.9101 \\
250.9141 \\
94.99639 \\
207.5271 \\
213.2678 \\
219.6425 \\
225.8158 \\
268.3173 \\
239.1245 \\
243.1502 \\
227.0987 \\
238.2030 \\
228.3576 \\
235.9505\end{array}$ & $\begin{array}{r}\text { Ave } \\
83.5436 \\
83.8329 \\
87.9458 \\
87.1806 \\
88.0936 \\
131.2246 \\
73.6827 \\
213.7329 \\
225.4390 \\
134.1396 \\
258.9130 \\
262.2412 \\
268.7231 \\
200.4266 \\
272.0696 \\
285.6269 \\
164.9794 \\
279.8608 \\
110.1741 \\
276.0145 \\
283.1582 \\
271.8622 \\
276.4113 \\
272.6675 \\
195.2504 \\
238.8835 \\
267.3915 \\
275.9808 \\
96.1617 \\
273.5454 \\
39.7755 \\
143.5716 \\
169.7893 \\
249.9338 \\
94.4017 \\
206.1926 \\
211.7691 \\
218.1009 \\
224.8280 \\
267.4006 \\
238.1560 \\
241.9521 \\
225.6377 \\
237.2555 \\
226.4767 \\
235.1067\end{array}$ & $\begin{array}{l}\text { SD } \\
1.7350 e-01 \\
1.2918 e-01 \\
2.5936 e-01 \\
1.2912 e-01 \\
1.9261 e-01 \\
5.2983 e-01 \\
4.6726 e-01 \\
2.8296 e-01 \\
9.6030 e-01 \\
3.3509 e-01 \\
2.6872 e-01 \\
4.2816 e-01 \\
2.8422 e-01 \\
3.7045 e+00 \\
5.0551 e-01 \\
1.8971 e-01 \\
2.4682 e-01 \\
2.6017 e-01 \\
2.5182 e-01 \\
1.9190 e-01 \\
2.1825 e-01 \\
3.1073 e-01 \\
2.2650 e-01 \\
1.9281 e-01 \\
3.9444 e-01 \\
2.7716 e-01 \\
3.4415 e-01 \\
2.5419 e-01 \\
1.7354 e-01 \\
1.7637 e-01 \\
2.1655 e-01 \\
3.6095 e-01 \\
5.5189 e-01 \\
4.4883 e-01 \\
2.8981 e-01 \\
4.5954 e-01 \\
6.0797 e-01 \\
4.5576 e-01 \\
3.7423 e-01 \\
4.051 e-01 \\
4.3222 e-01 \\
4.2326 e-01 \\
4.9954 e-01 \\
4.0267 e-01 \\
5.0419 e-01 \\
3.6658 e-01\end{array}$ & 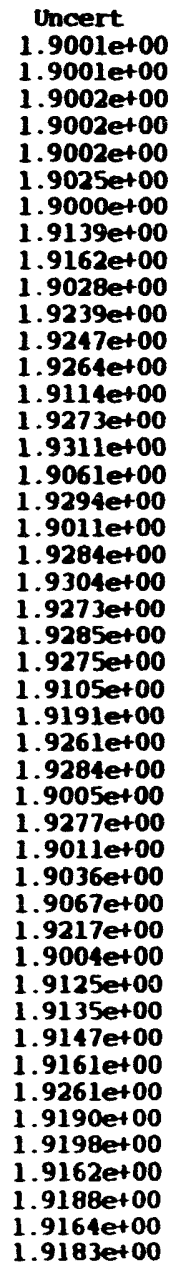 \\
\hline
\end{tabular}

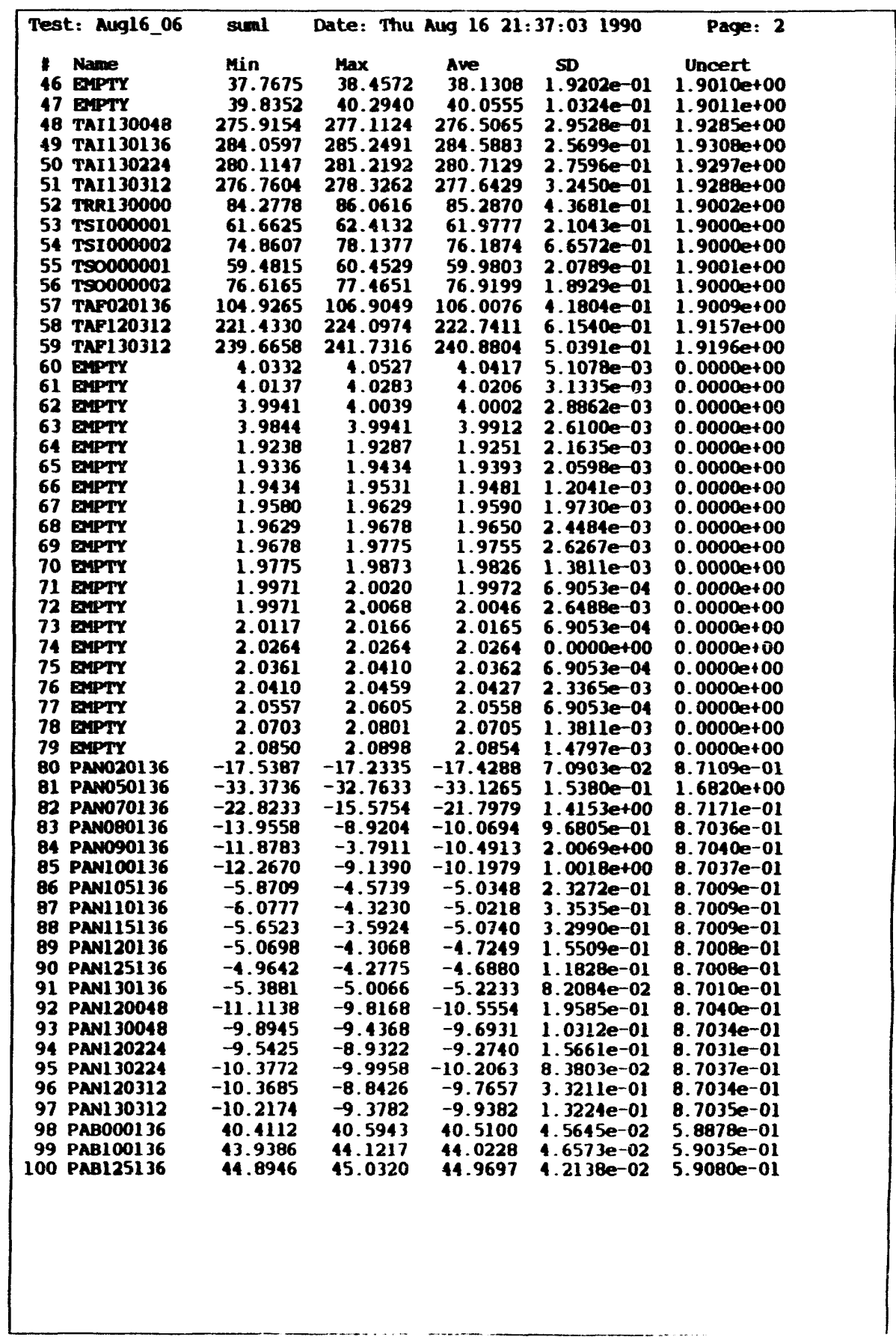



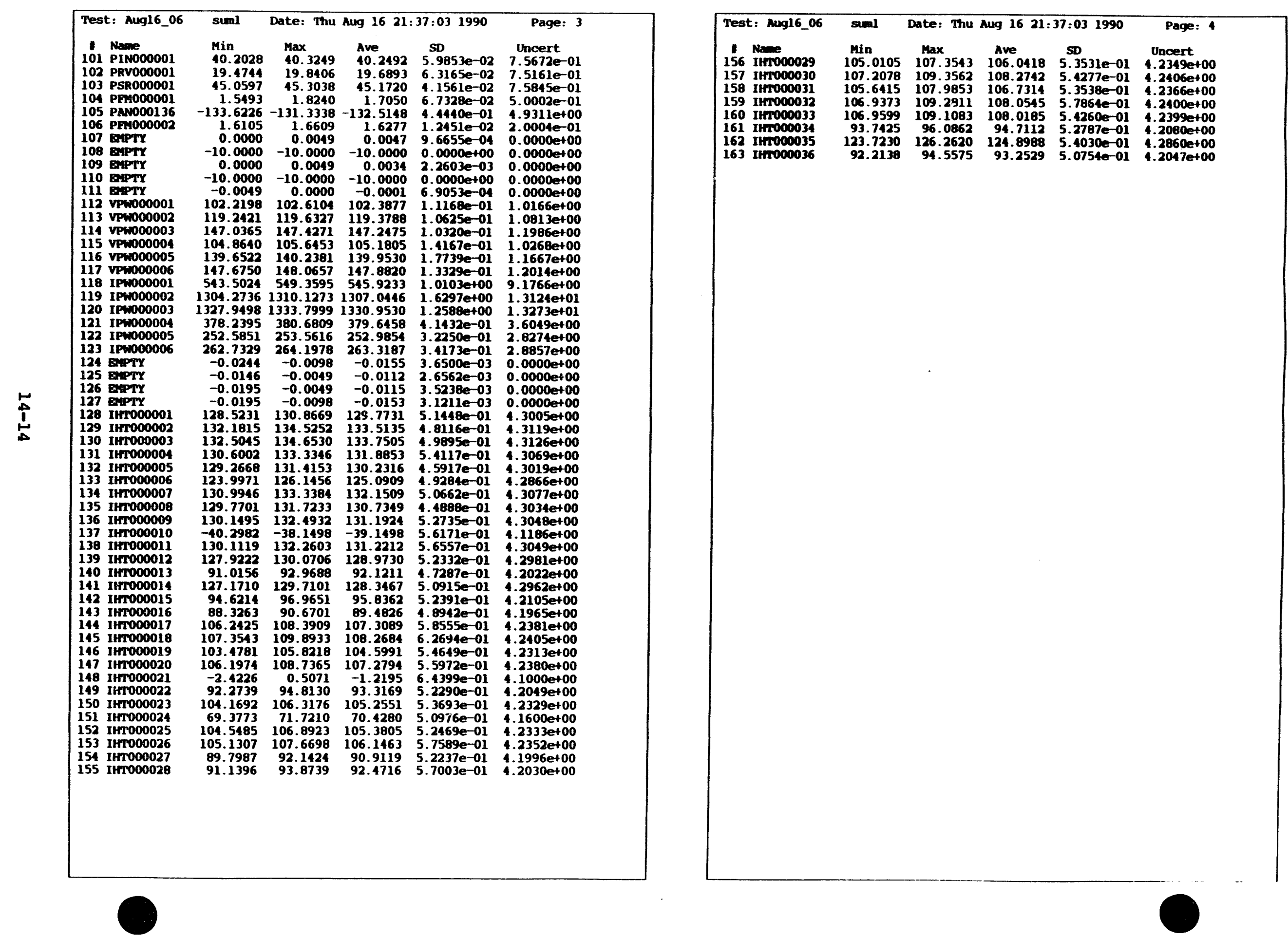


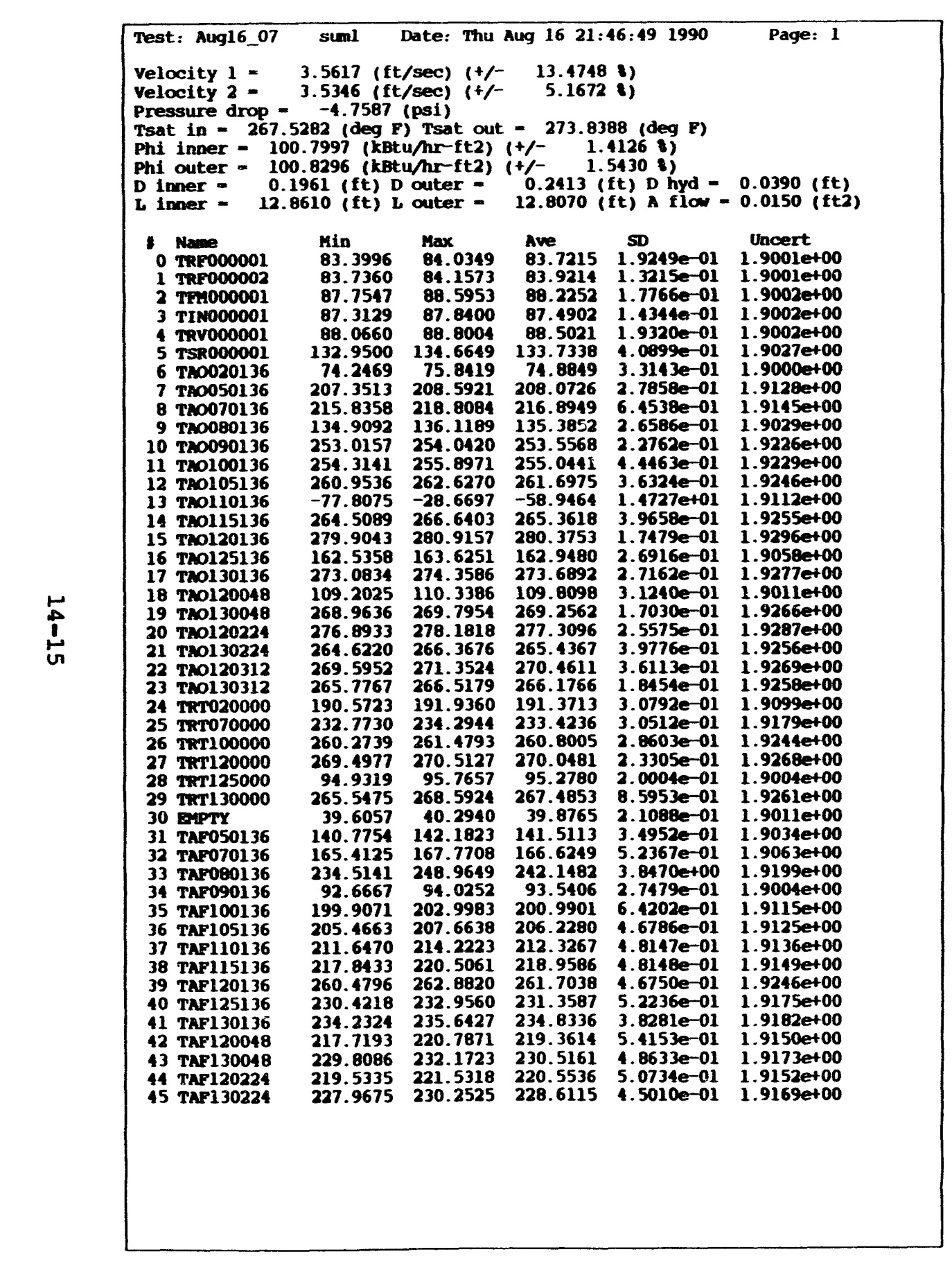

\begin{tabular}{|c|c|c|c|c|c|}
\hline 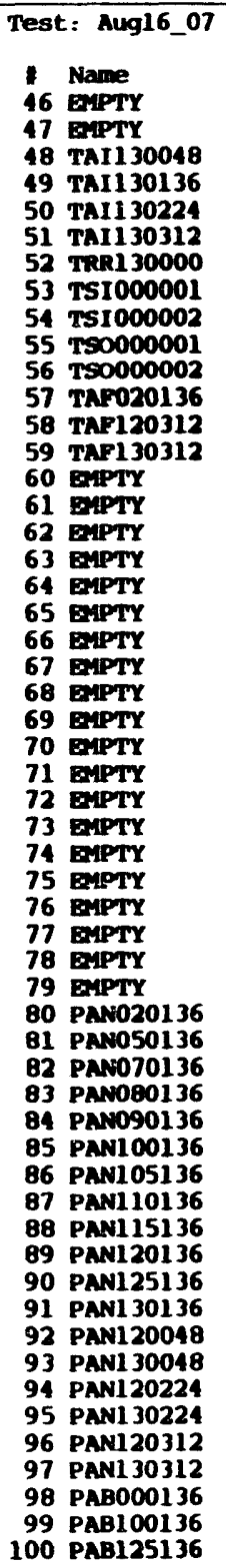 & 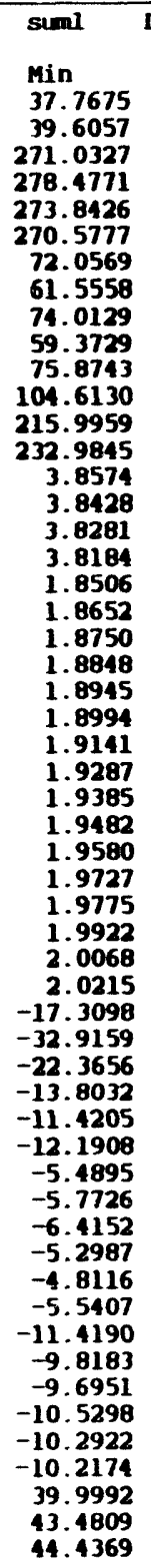 & 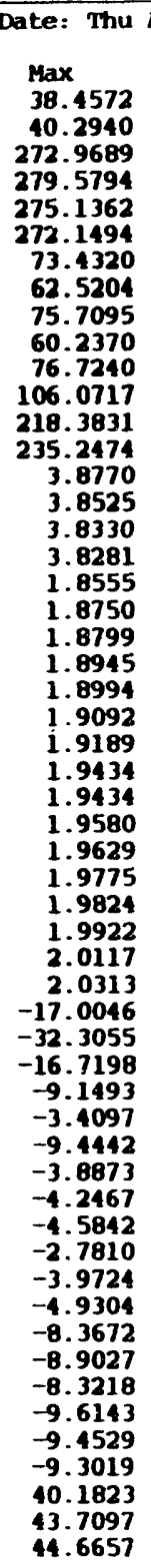 & 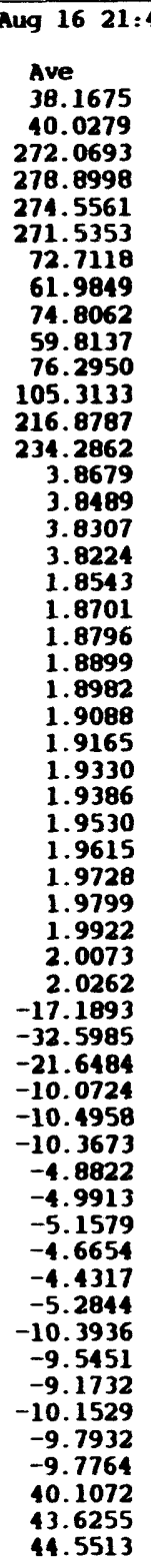 & 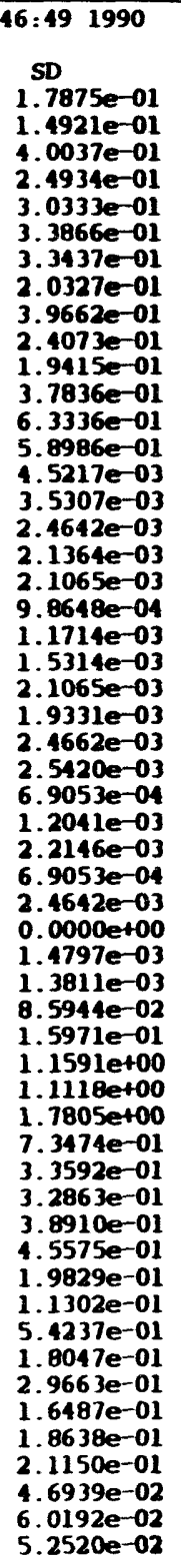 & 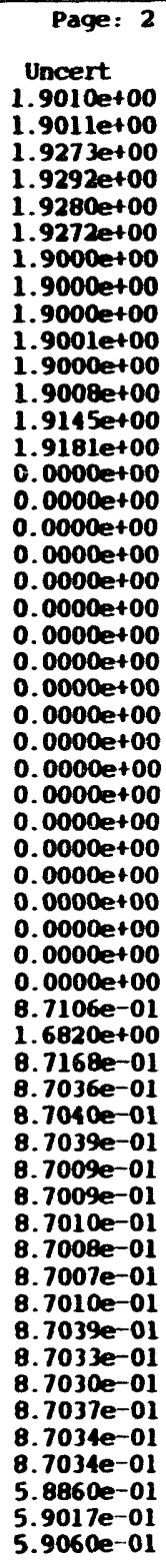 \\
\hline
\end{tabular}




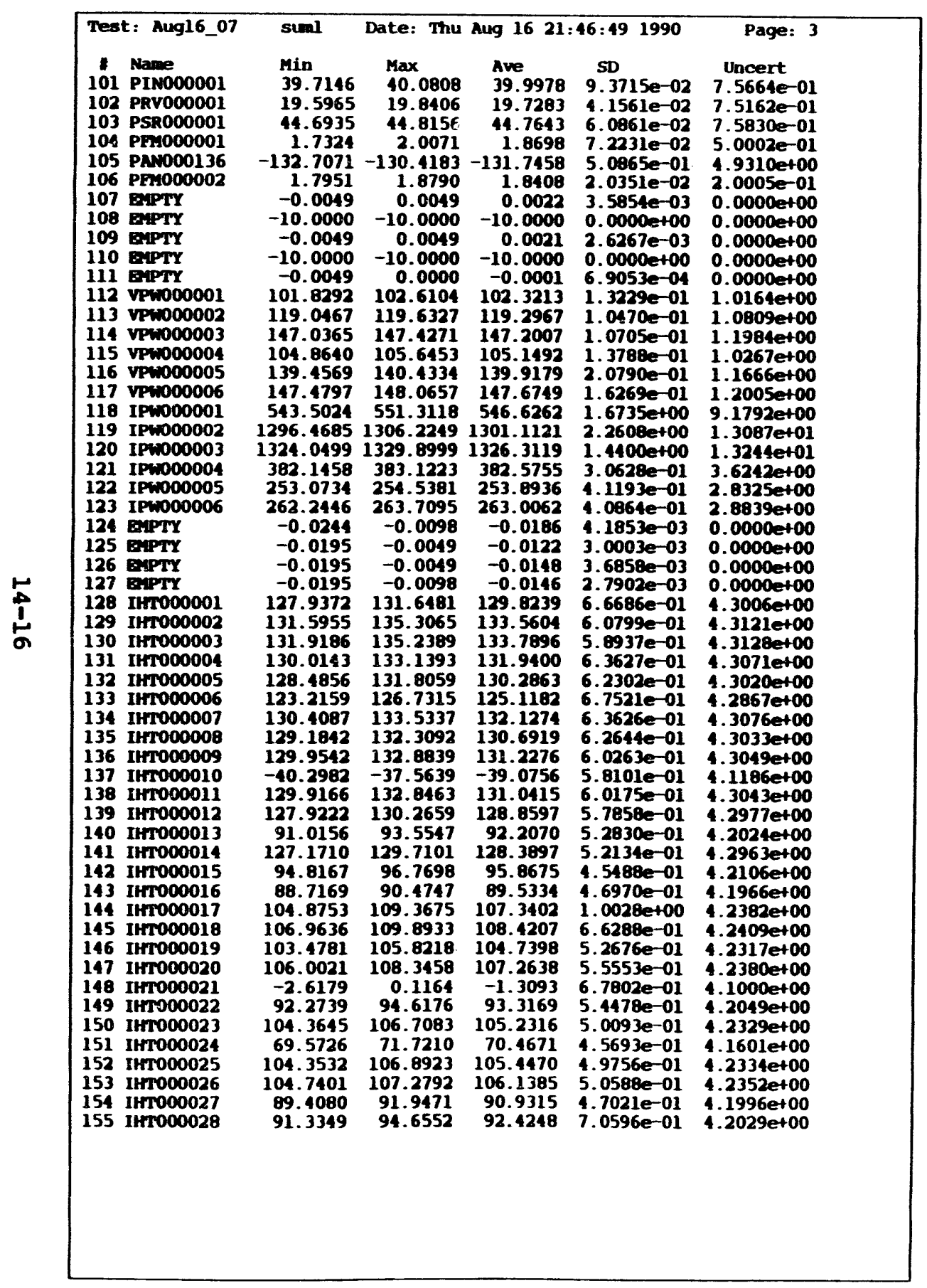

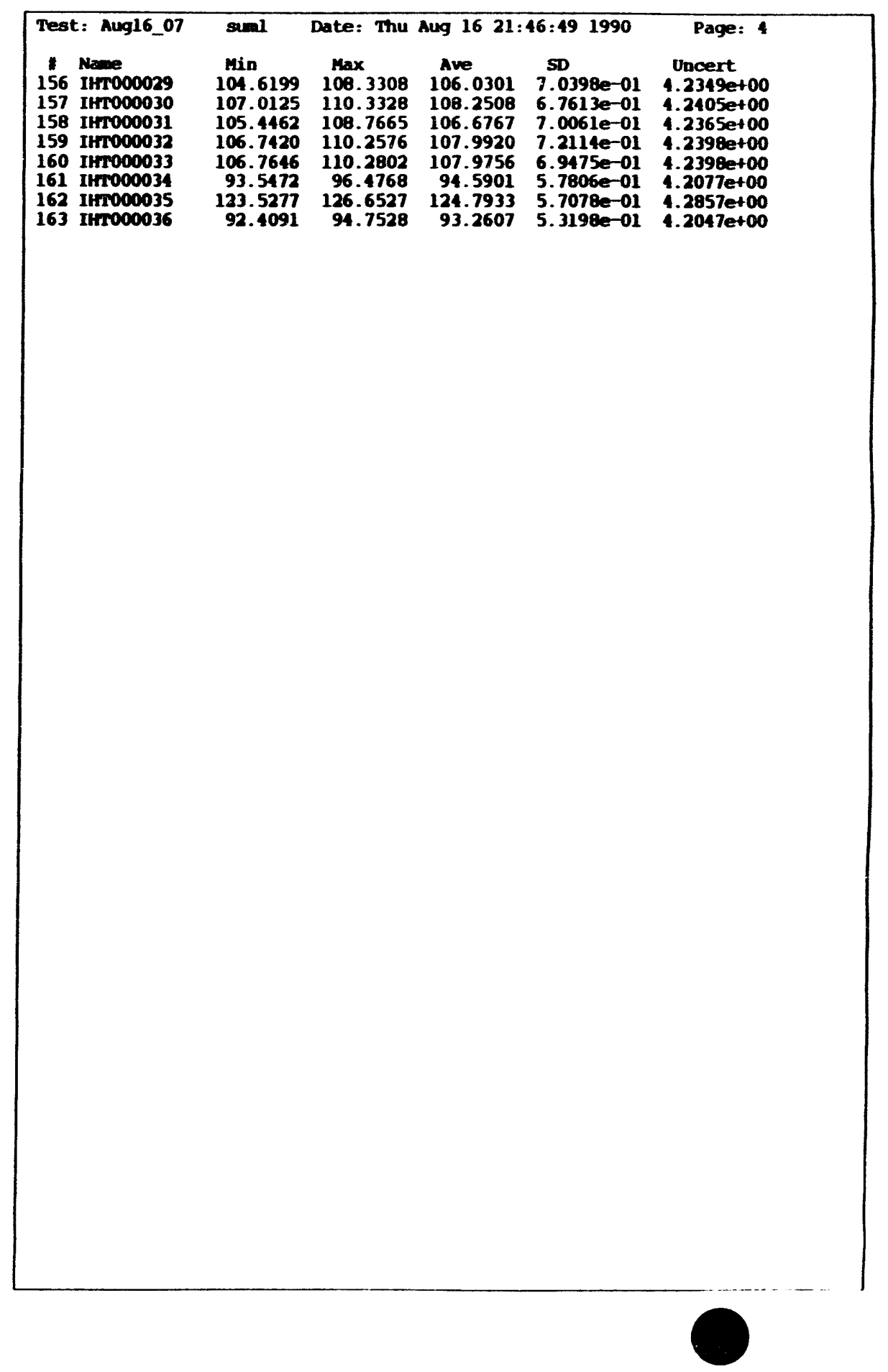


DEFECTIVE INSTRUNENTS FOR THE RIBBED GEOMETRY TEST SERIES

\begin{tabular}{|c|c|ll|}
\hline Series & \multicolumn{1}{|c|}{ Tests } & \multicolumn{1}{|c|}{ Defective Instruments } \\
\hline 5 & Aug16_01 to Aug16_07 & TAO120048 TAF070136 TRT120000 \\
& & TAO020136 TAF080136 TRT125000 & TAO070136 TAF090136 TRR130000 \\
& & TAO080136 TAF120136 & \\
& & TAO110136 & \\
& & & \\
& & & \\
& & IHTO000125136 & IHT000021 IHT000024 \\
& & IHT00033 & \\
\hline
\end{tabular}


Chapter 15

NUCLEATE BOILING PRESSURE DROP IN AN ANNULUS

Test Geometry: Ribbed (4)

Test Series: 6

Test Date: August 17, 1990 


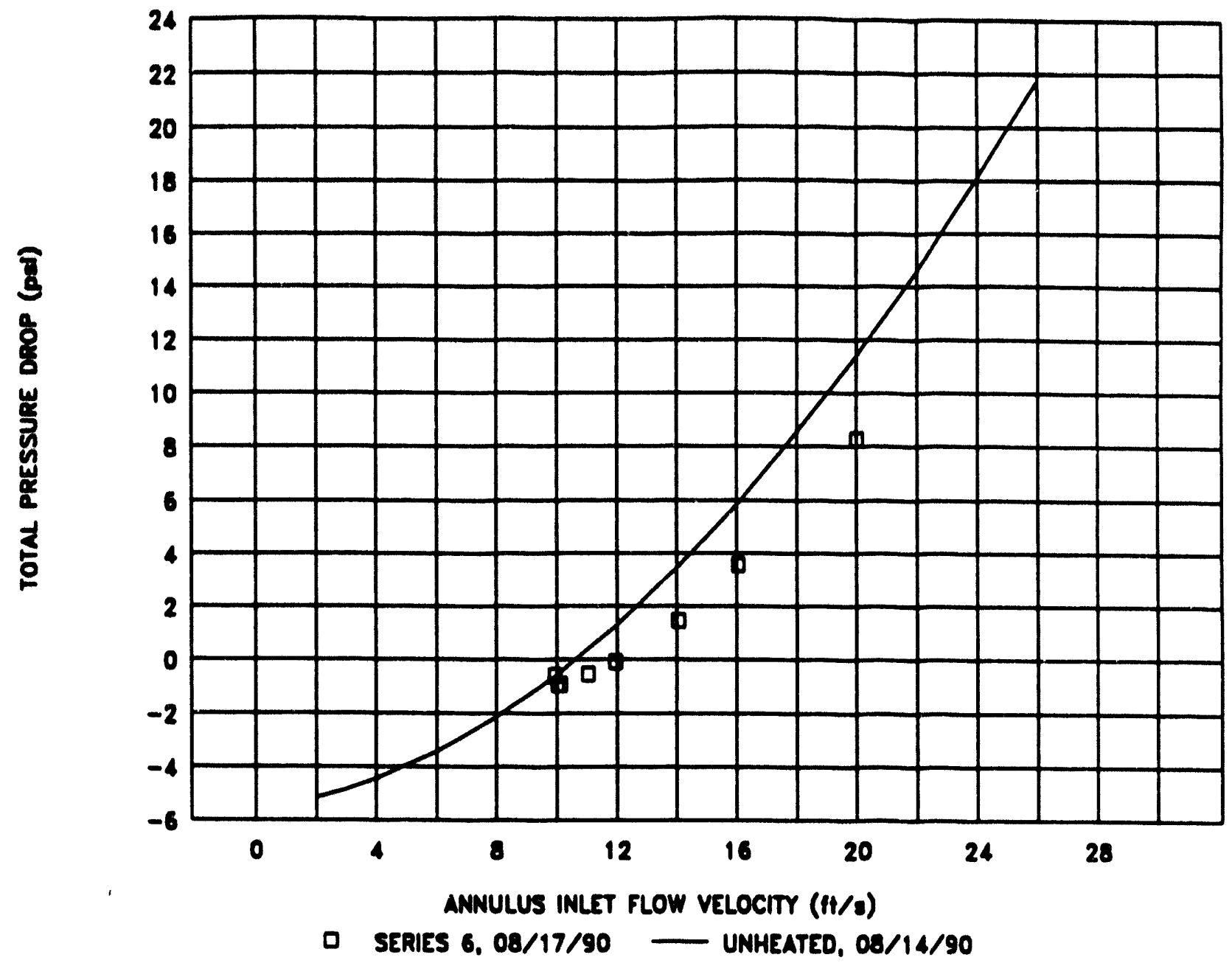

\begin{tabular}{|c|c|c|}
\hline $\begin{array}{c}\text { TEST } \\
\text { NUMBER }\end{array}$ & $\begin{array}{c}\text { INLET } \\
\text { VELOCITY(a) }\end{array}$ & $\begin{array}{c}\text { PRESSURE } \\
\text { DROP(b) }\end{array}$ \\
\hline & (ft/s) & (psi) \\
\hline Aug17_01 & 20.00 & 8.28 \\
\hline Aug17_02 & 16.04 & 3.57 \\
\hline Aug17_03 & 14.05 & 1.46 \\
\hline Aug17_04 & 11.96 & -0.09 \\
\hline Aug17_05 & 11.08 & -0.54 \\
\hline Aug17_08 & 10.14 & -0.94 \\
\hline$\% \cdot 1 \%$ & $10 \% 6$ & 0.9 \\
\hline Aug17_07 & 9.93 & -0.58 \\
\hline
\end{tabular}

\begin{tabular}{|l|l|}
\hline \multicolumn{2}{|c|}{ NOMINAL TEST CONDITIONS } \\
\hline Annulus geometry & ribbed (4) \\
Inlet temperature & $86^{\circ} \mathrm{F}$ \\
Inlet pressure & $40 \mathrm{psia}$ \\
Wall heat thux & $300 \mathrm{kBtu} / \mathrm{hr}-\mathrm{ft} 2$ \\
Flux distribution & uniform \\
He saturation pressure & $5 \mathrm{psig}$ \\
\hline NOTES: \\
(a) Inlot volocity calculated from PFMO00051 \\
(b) Preseure drop calculated trom PANO00136 \\
(c) Shading notes teat at minimum pressure drop
\end{tabular}

DEMAND CURVE AND DATA FOR SERIES 6 - RIBBED ANNULUS 

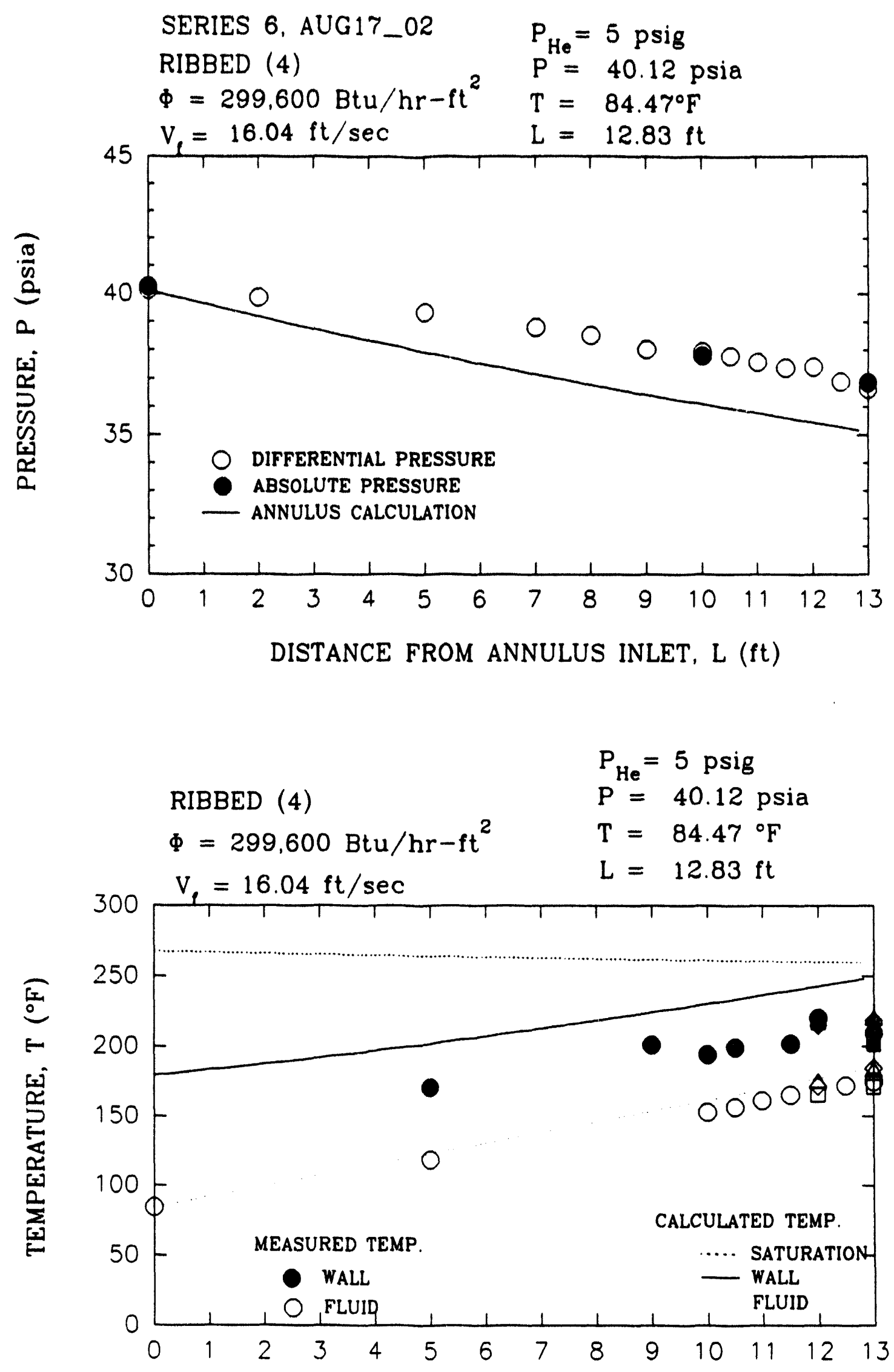

DISTANCE FROM ANNULUS INLET, L $(\mathrm{ft})$ 

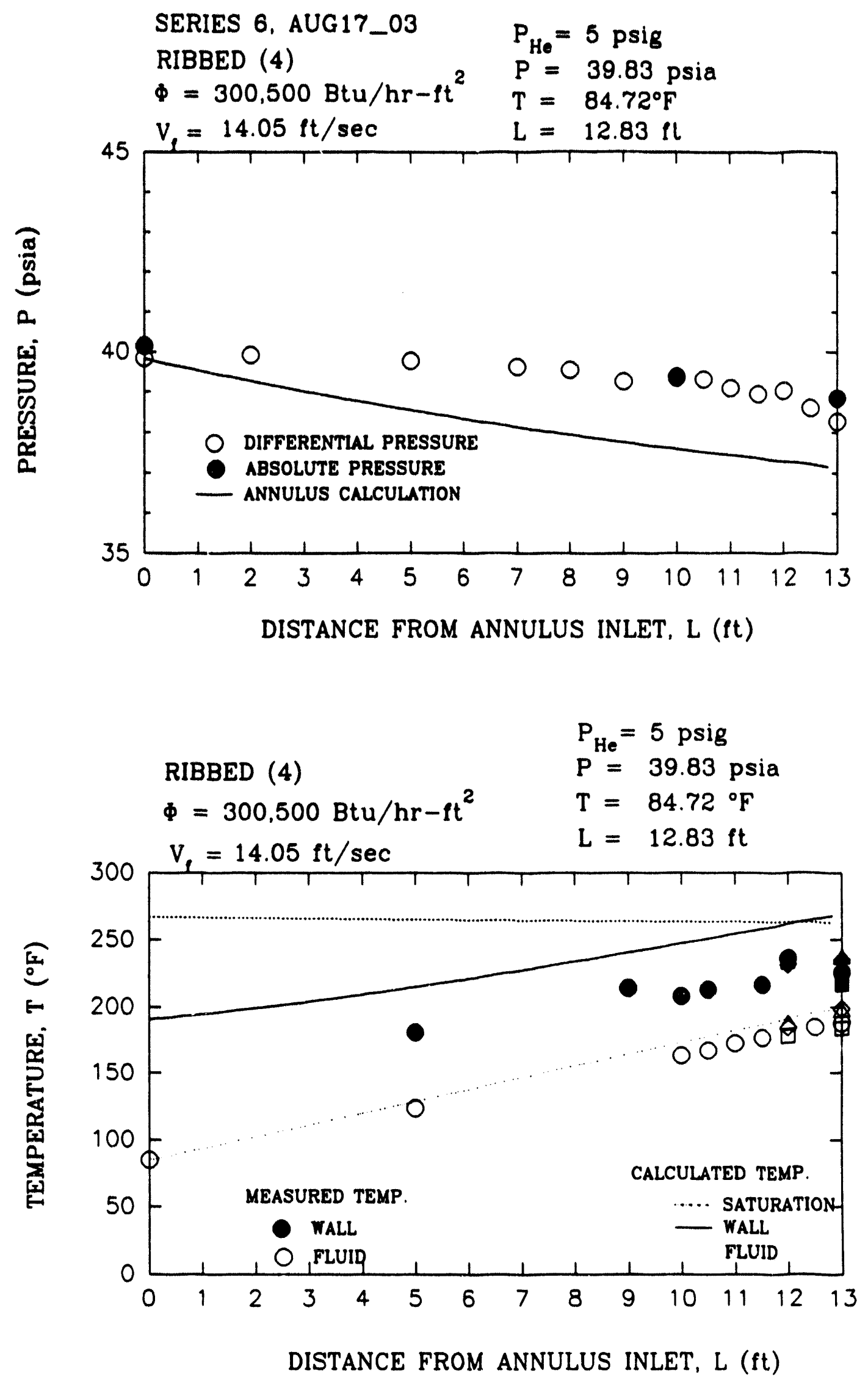

AUG17_03 

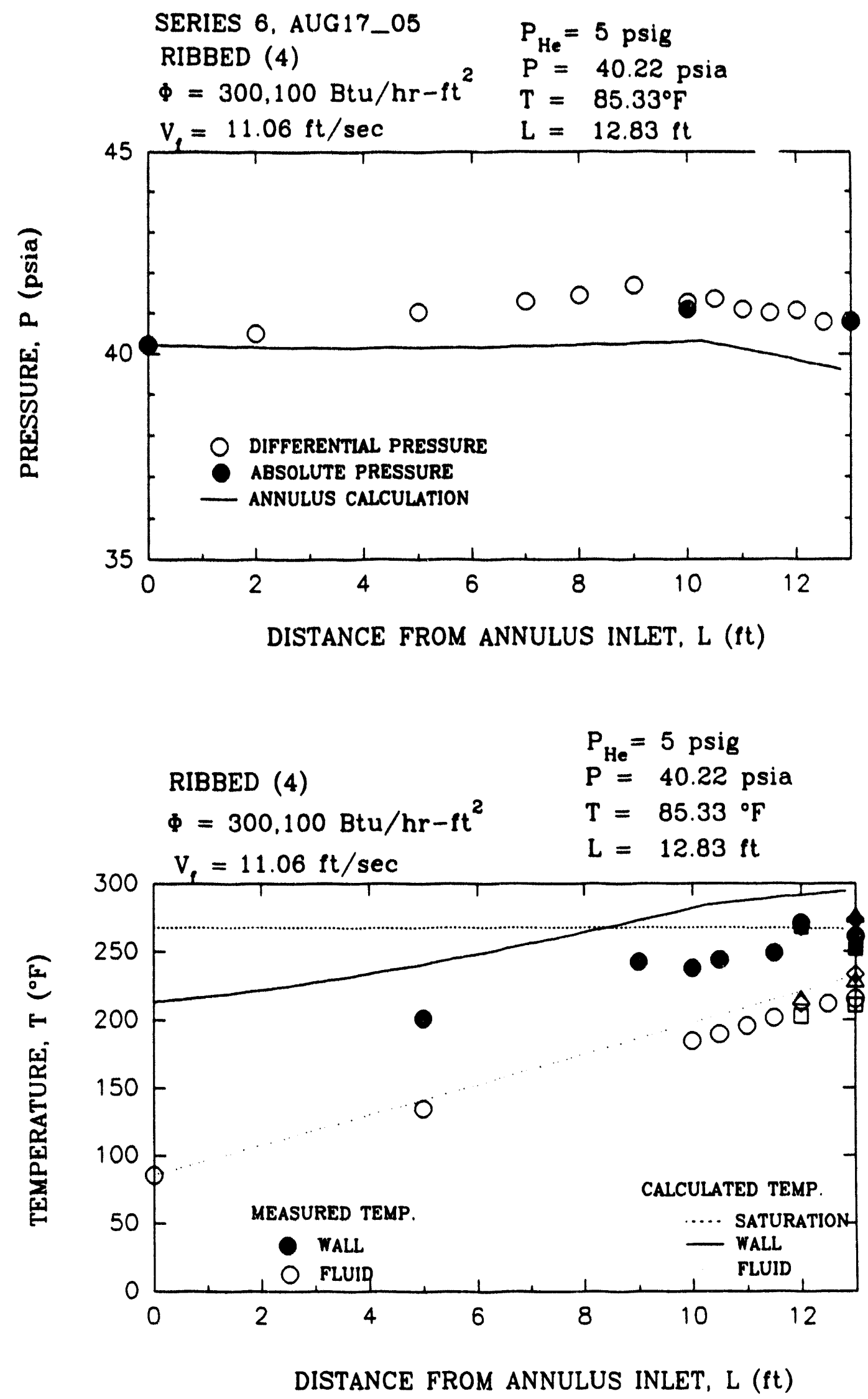

AUG17_05 

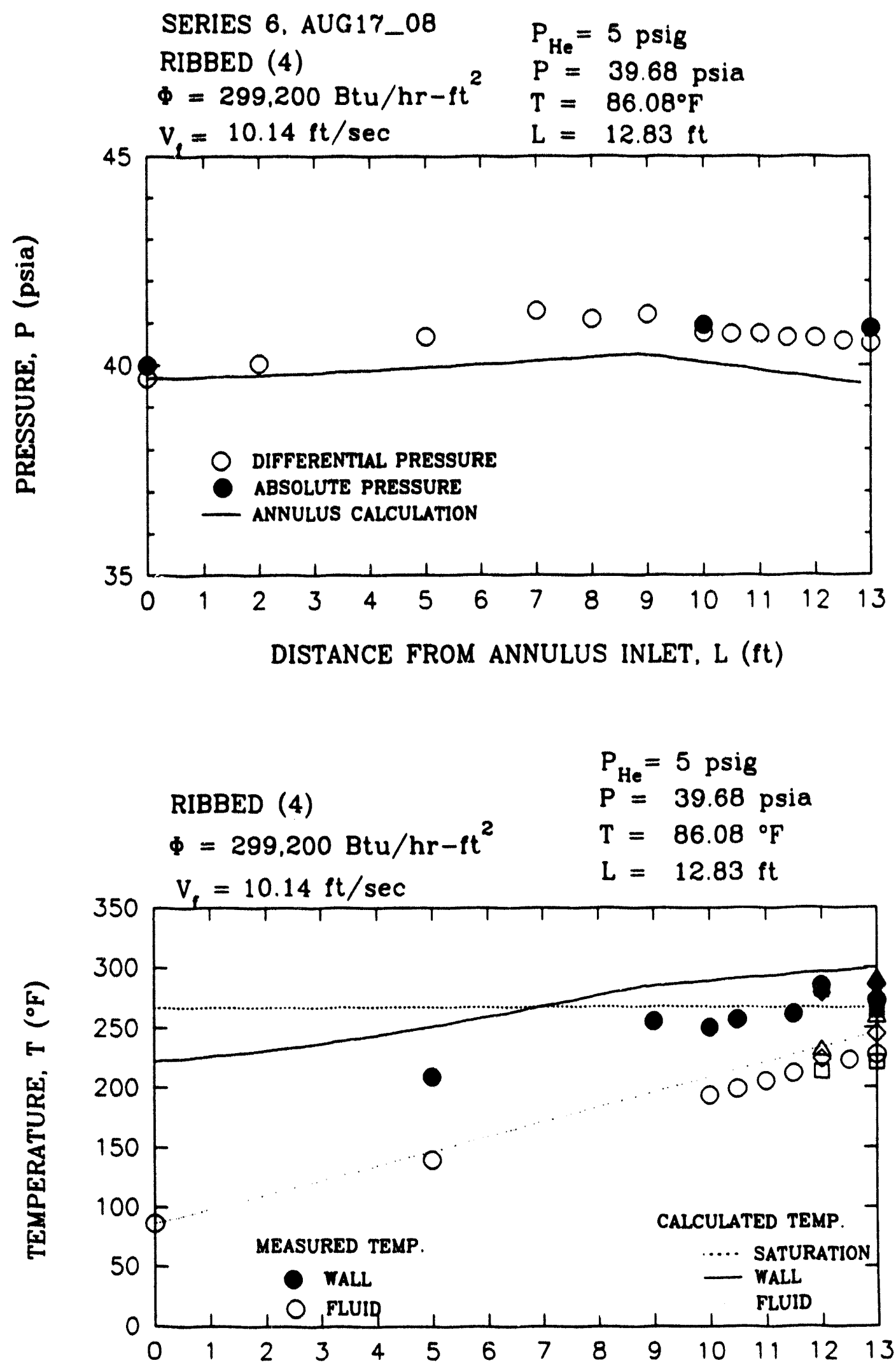

DISTANCE FROM ANNULUS INLET, L ( $\mathrm{ft}$ )

AUG17_08 

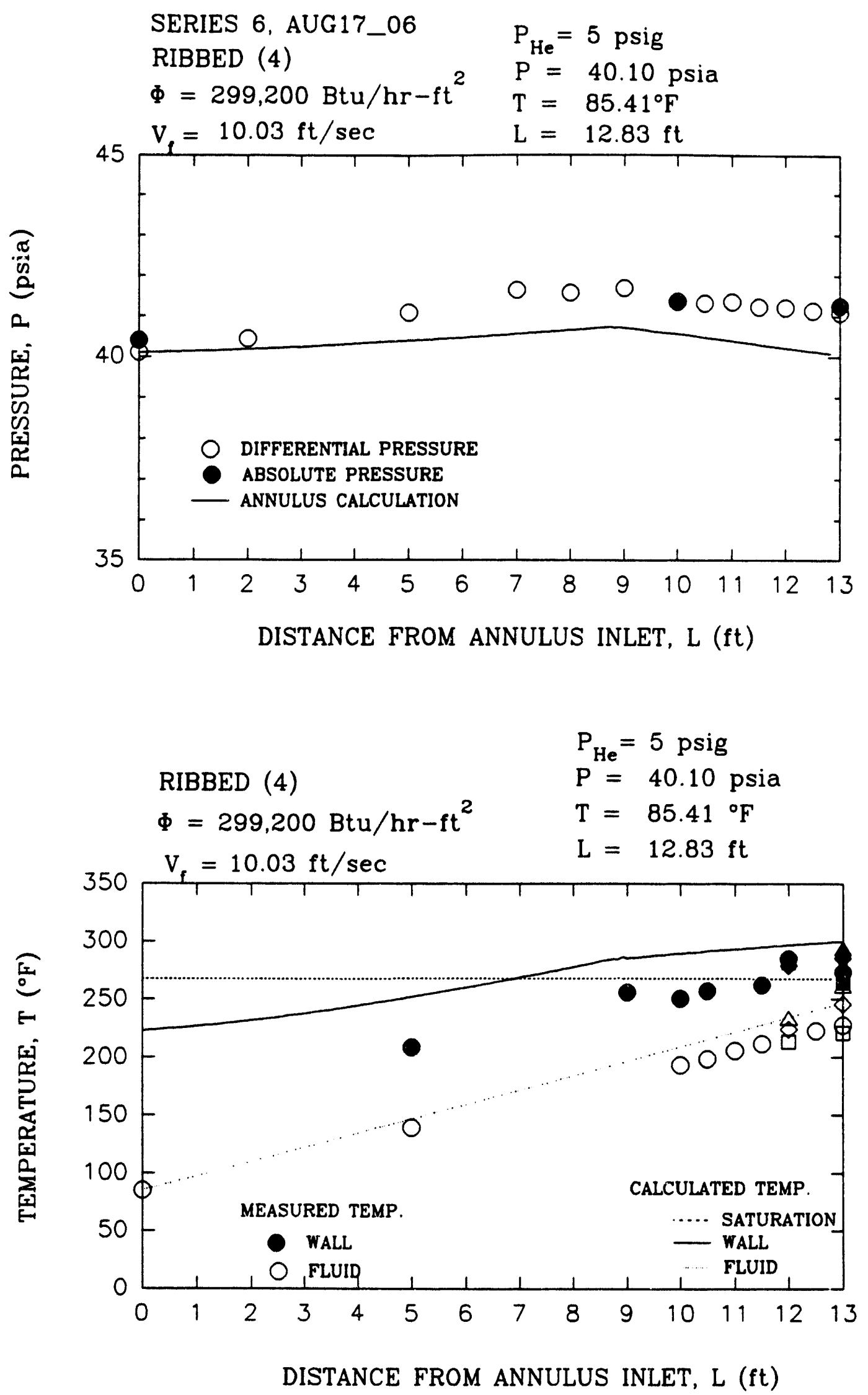

AUG17_06 

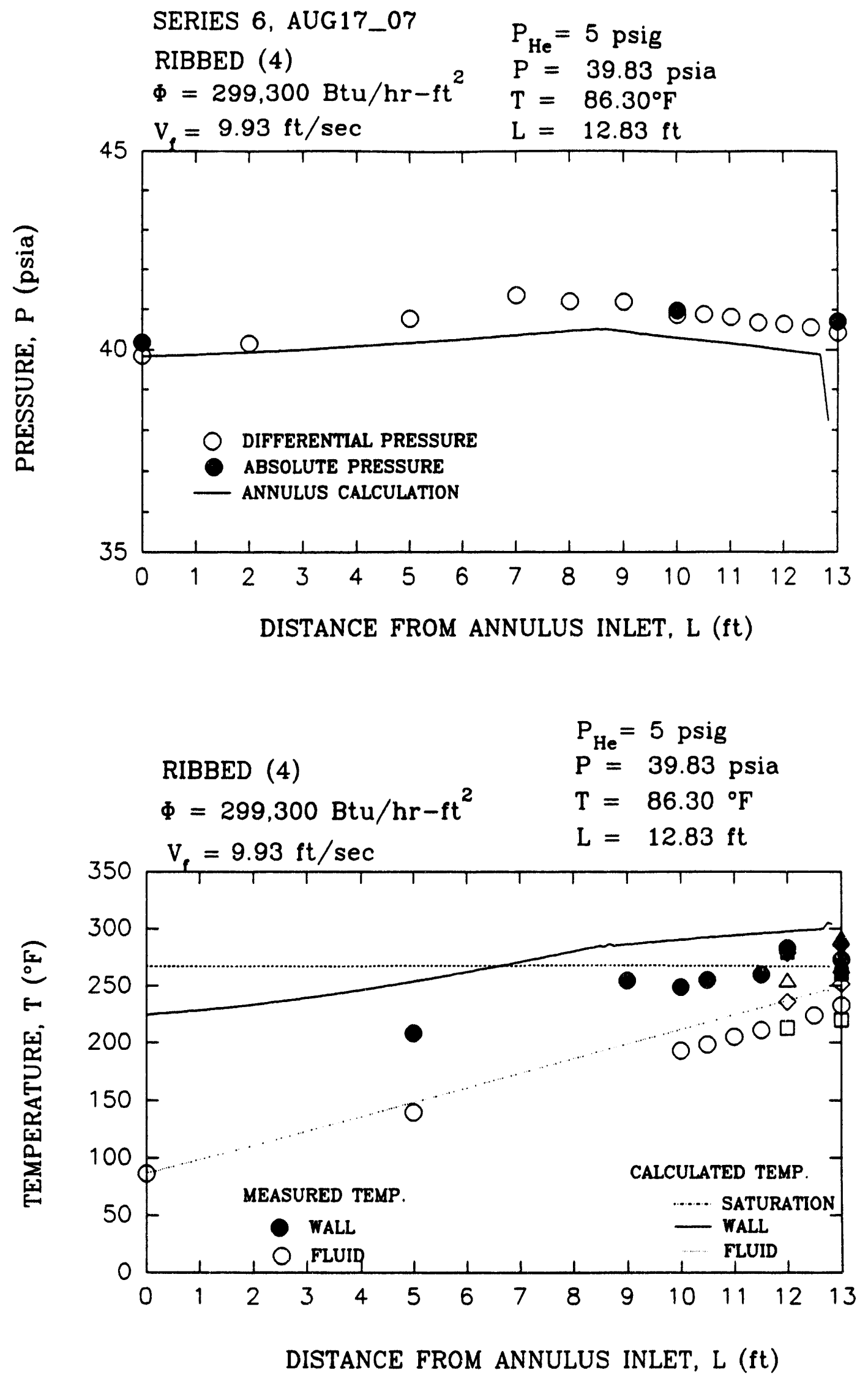

AUG17_07 


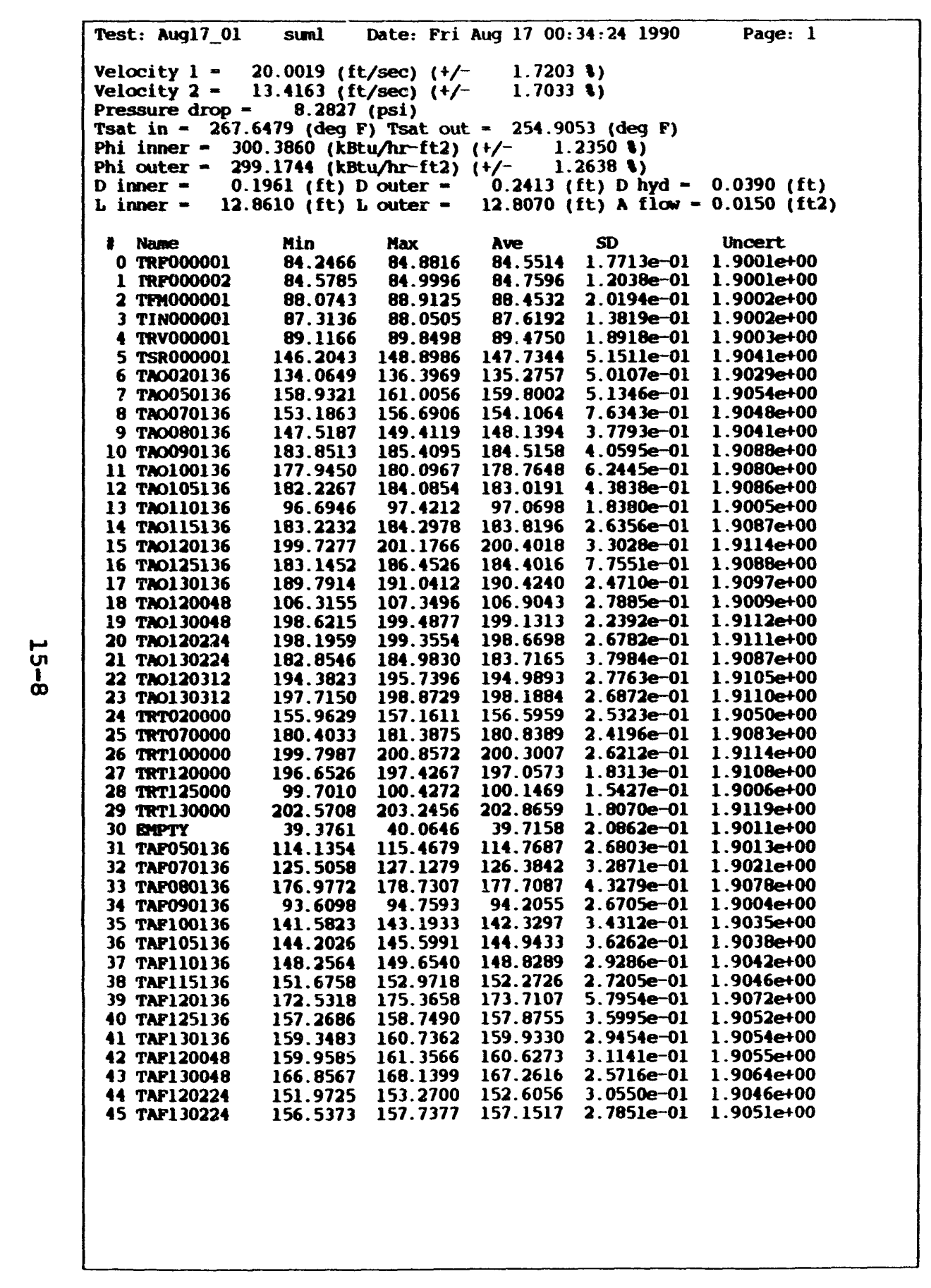

\begin{tabular}{|c|c|c|c|c|c|}
\hline 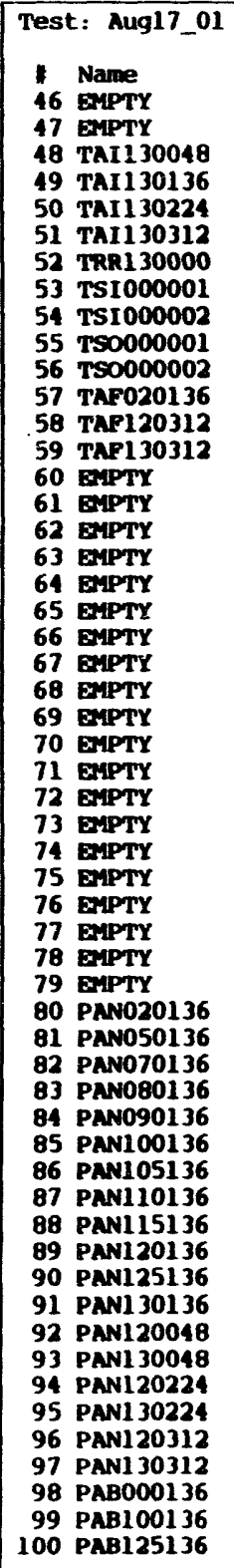 & 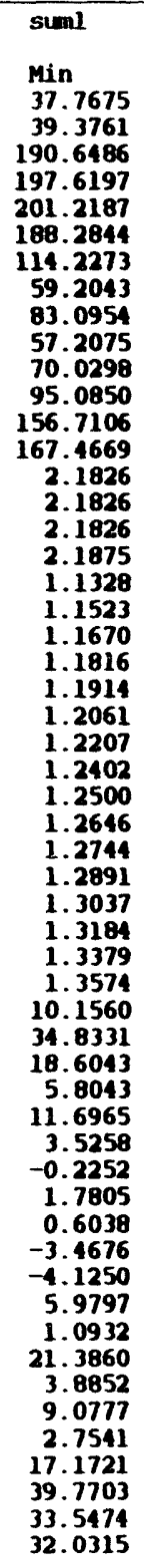 & 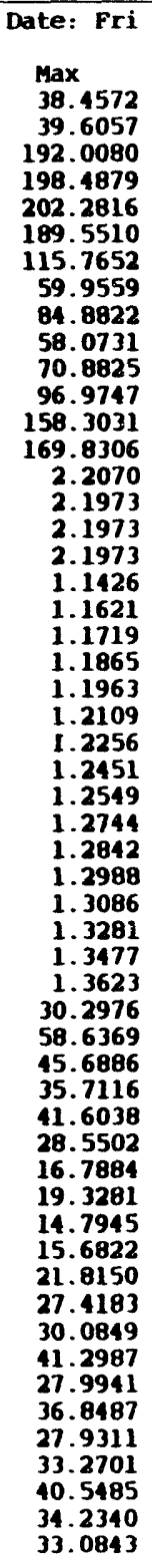 & 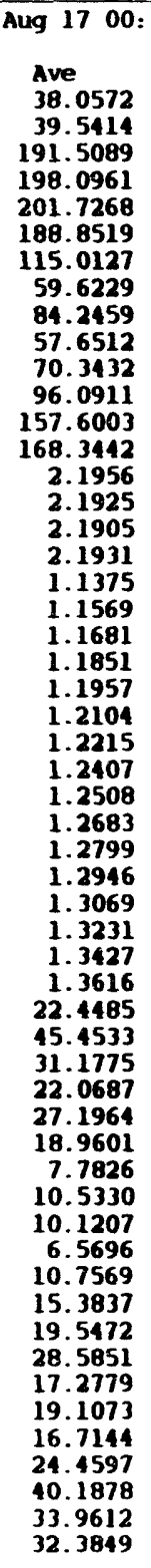 & 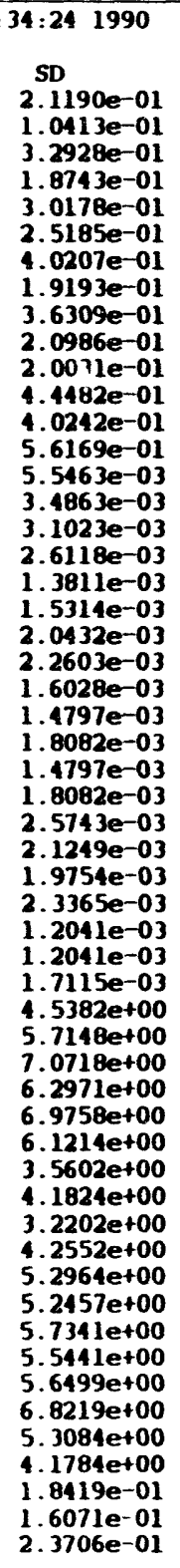 & 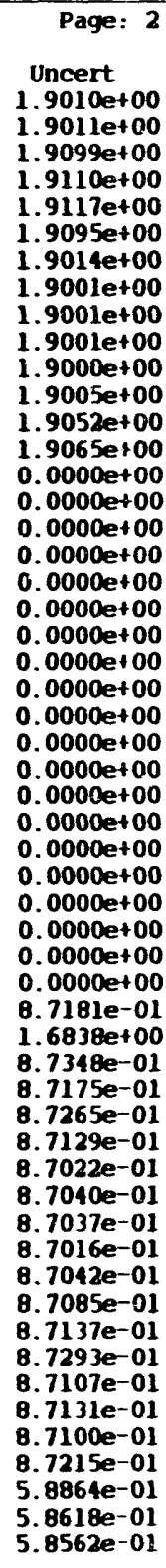 \\
\hline
\end{tabular}




\begin{tabular}{|c|c|c|c|c|c|}
\hline 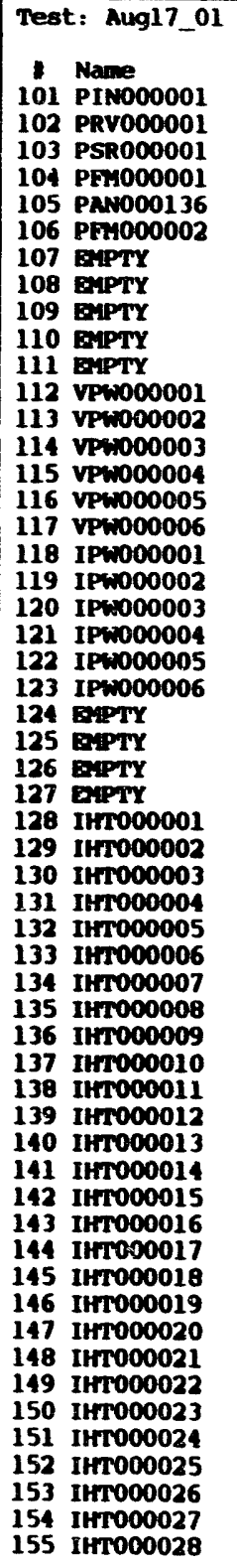 & $\begin{array}{l}\text { suml } \\
\text { Min } \\
39.1042 \\
19.5965 \\
29.4347 \\
60.8755 \\
215.1933 \\
27.5689 \\
0.0049 \\
-10.0000 \\
0.0049 \\
-10.0000 \\
0.0000 \\
177.0245 \\
206.9374 \\
255.6303 \\
182.0125 \\
242.1913 \\
257.4407 \\
939.4262 \\
2217.4678 \\
2267.8499 \\
652.1653 \\
432.7429 \\
149.7446 \\
-0.0244 \\
-0.0195 \\
-0.0195 \\
-0.0244 \\
220.7106 \\
2227.4940 \\
229.1842 \\
224.9361 \\
222.4309 \\
216.3799 \\
225.7212 \\
223.1295 \\
223.7042 \\
55.0143 \\
224.0572 \\
220.1097 \\
158.5938 \\
219.9444 \\
164.1526 \\
152.7794 \\
183.3909 \\
184.5027 \\
179.0640 \\
184.1271 \\
-3.3992 \\
158.8754 \\
180.3411 \\
144.9632 \\
179.5486 \\
181.6932 \\
155.0331 \\
156.9599\end{array}$ & $\begin{aligned} & \text { Date: } \text { Fri } \\
& \text { Max } \\
& 40.8132 \\
& 19.7196 \\
& 199.8009 \\
& 62.0657 \\
& 242.2014 \\
& 27.5689 \\
& 0.0098 \\
&-0.5225 \\
& 0.0098 \\
&-10.0000 \\
& 0.0049 \\
& 177.4151 \\
& 207.5233 \\
& 256.2162 \\
& 182.4031 \\
& 243.1678 \\
& 257.8313 \\
& 945.6852 \\
& 2223.3215 \\
& 2271.7498 \\
& 653.6301 \\
& 434.6958 \\
& 451.6978 \\
&-0.0098 \\
&-0.0049 \\
&-0.0098 \\
&-0.0098 \\
& 223.0544 \\
& 229.8377 \\
& 231.3326 \\
& 227.6705 \\
& 225.1653 \\
& 218.9190 \\
& 227.6743 \\
& 225.2779 \\
& 225.8526 \\
& 57.3580 \\
& 226.4010 \\
& 222.4534 \\
& 161.1328 \\
& 221.8976 \\
& 166.6917 \\
& 155.1232 \\
& 185.7347 \\
& 187.2371 \\
& 181.9937 \\
& 187.0568 \\
& 0.1164 \\
& 166.6098 \\
& 183.4661 \\
& 1147.5023 \\
& 191.8923 \\
& 118.2323 \\
& 155.7674 \\
& 160.4755\end{aligned}$ & $\begin{aligned} \text { Aug } 1700: \\
\text { Ave } \\
39.9831 \\
19.6844 \\
29.6324 \\
61.5603 \\
229.3107 \\
27.5689 \\
0.0096 \\
-7.3491 \\
0.0052 \\
-10.0000 \\
00.0002 \\
177.1963 \\
207.2499 \\
256.0482 \\
182.1493 \\
242.6298 \\
257.6319 \\
942.7570 \\
2220.0439 \\
2270.6575 \\
652.7808 \\
433.6412 \\
450.8283 \\
-0.0176 \\
-0.0122 \\
-0.0134 \\
-0.0168 \\
221.8825 \\
228.4941 \\
230.0380 \\
226.0495 \\
223.5362 \\
217.4346 \\
226.6898 \\
224.1256 \\
224.7119 \\
56.1314 \\
225.1003 \\
221.1018 \\
160.0000 \\
220.9444 \\
165.2504 \\
1153.9161 \\
184.6371 \\
186.2098 \\
180.6422 \\
185.7677 \\
-1.4070 \\
160.2973 \\
181.7472 \\
116.1430 \\
180.7126 \\
182.9980 \\
156.4706 \\
158.8232\end{aligned}$ & 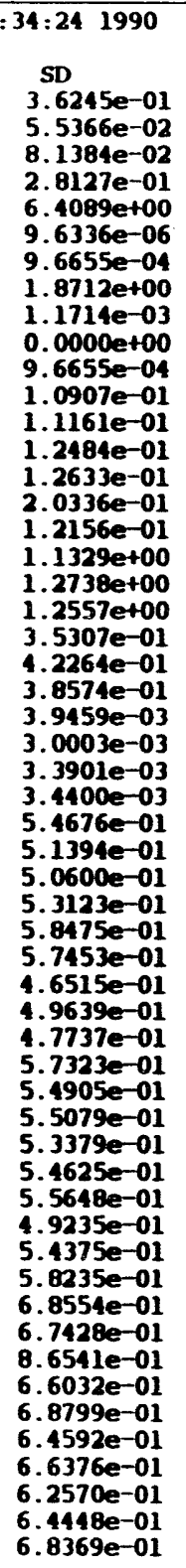 & 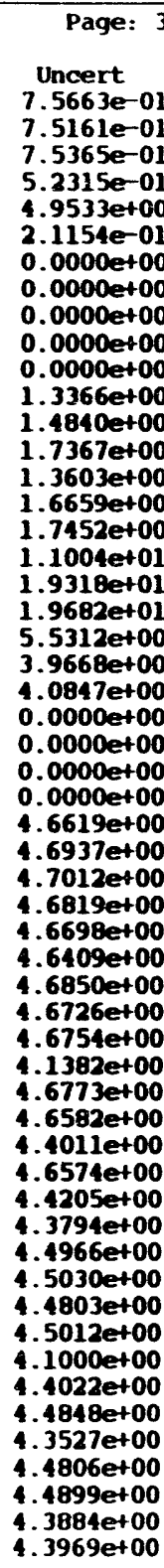 \\
\hline
\end{tabular}

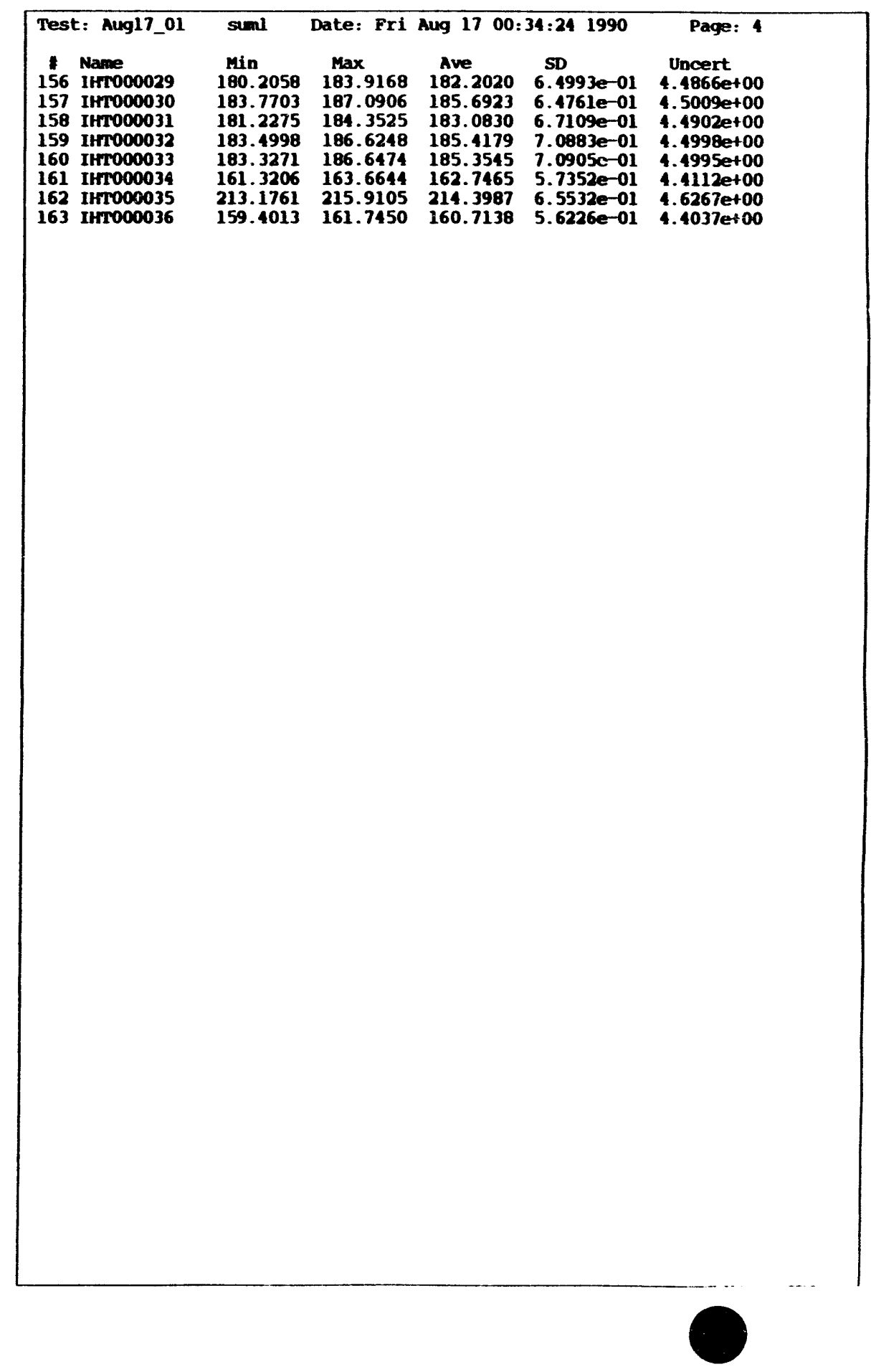




\begin{tabular}{|c|c|c|c|c|c|}
\hline \multicolumn{3}{|c|}{ 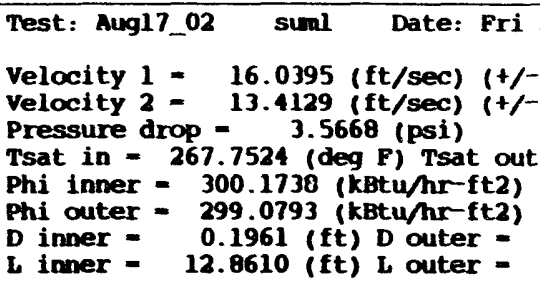 } & \multicolumn{2}{|c|}{ 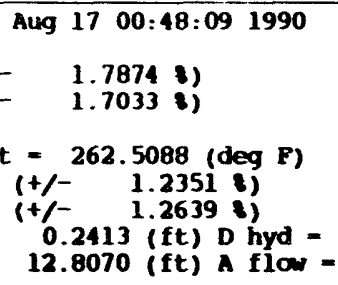 } & $\begin{array}{r}\text { Page: } 1 \\
\\
-0.0390 \text { (ft) }\end{array}$ \\
\hline 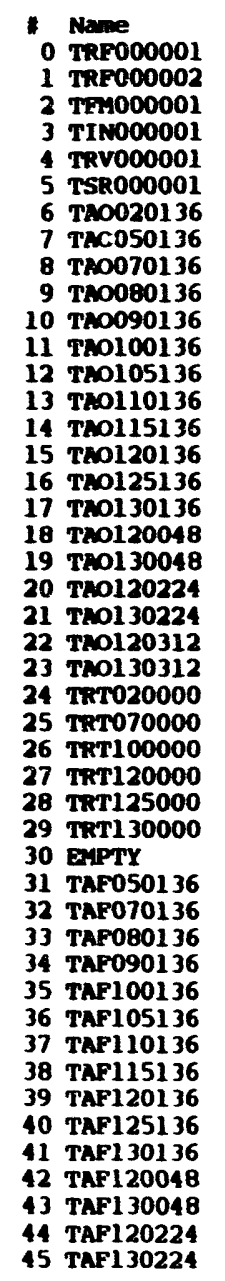 & 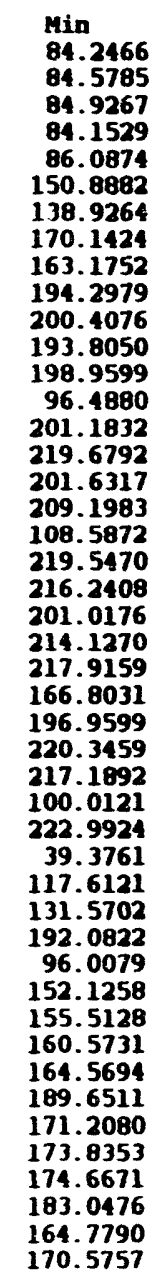 & 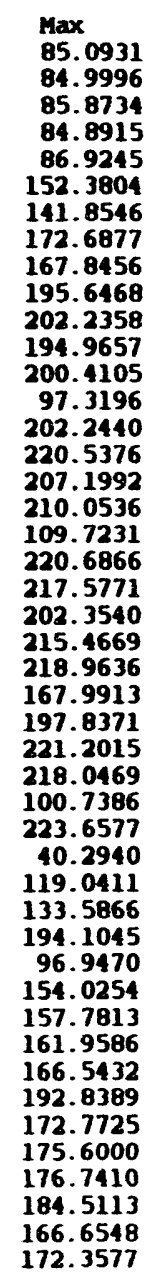 & $\begin{array}{l}\text { Ave } \\
84.6361 \\
84.8648 \\
85.3685 \\
84.4698 \\
84.4698 \\
86.4972 \\
151.5935 \\
140.5103 \\
170.9118 \\
165.0490 \\
194.04733 \\
201.0666 \\
194.3080 \\
199.4283 \\
996.8996 \\
201.6846 \\
220.1446 \\
203.4302 \\
209.6026 \\
109.1407 \\
220.3211 \\
217.0305 \\
201.6646 \\
214.9041 \\
218.2856 \\
167.5239 \\
197.3580 \\
220.6280 \\
217.6199 \\
100.3439 \\
223.3347 \\
39.7526 \\
118.4372 \\
132.4162 \\
192.9162 \\
96.4214 \\
153.0860 \\
156.5278 \\
161.1632 \\
165.2324 \\
191.0272 \\
172.0326 \\
174.6557 \\
175.5879 \\
183.6531 \\
165.6537 \\
171.2845\end{array}$ & 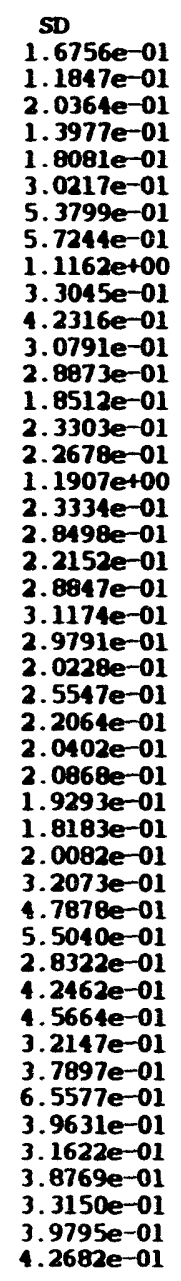 & 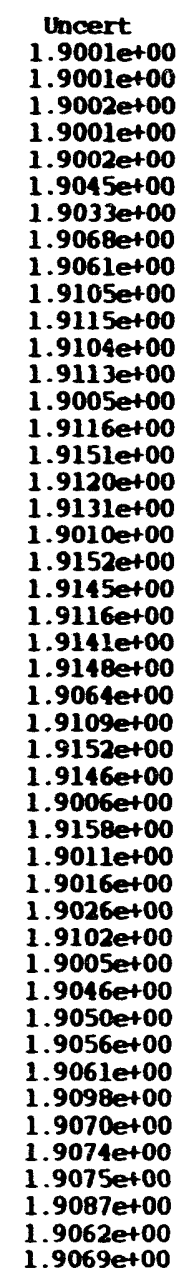 \\
\hline
\end{tabular}

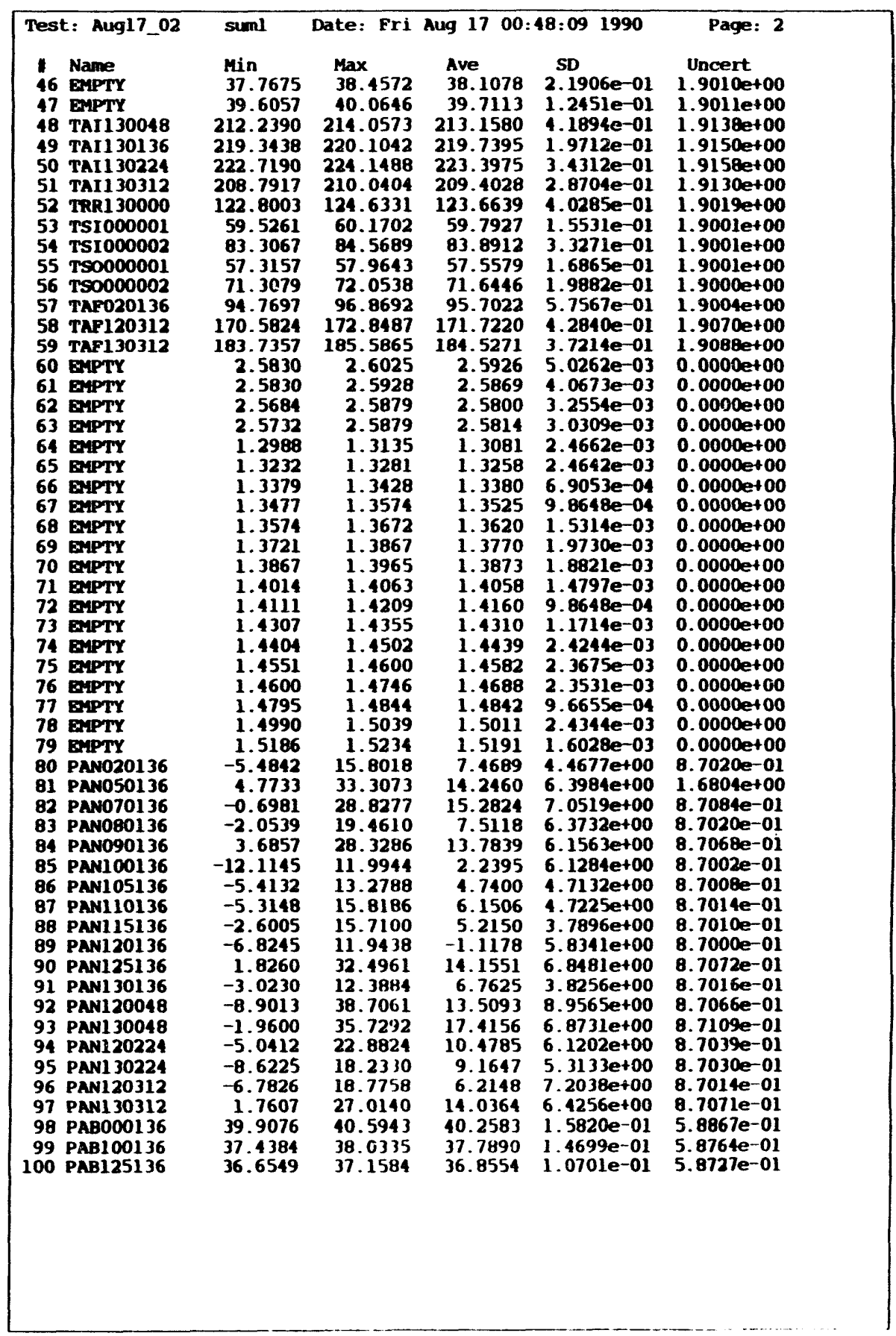




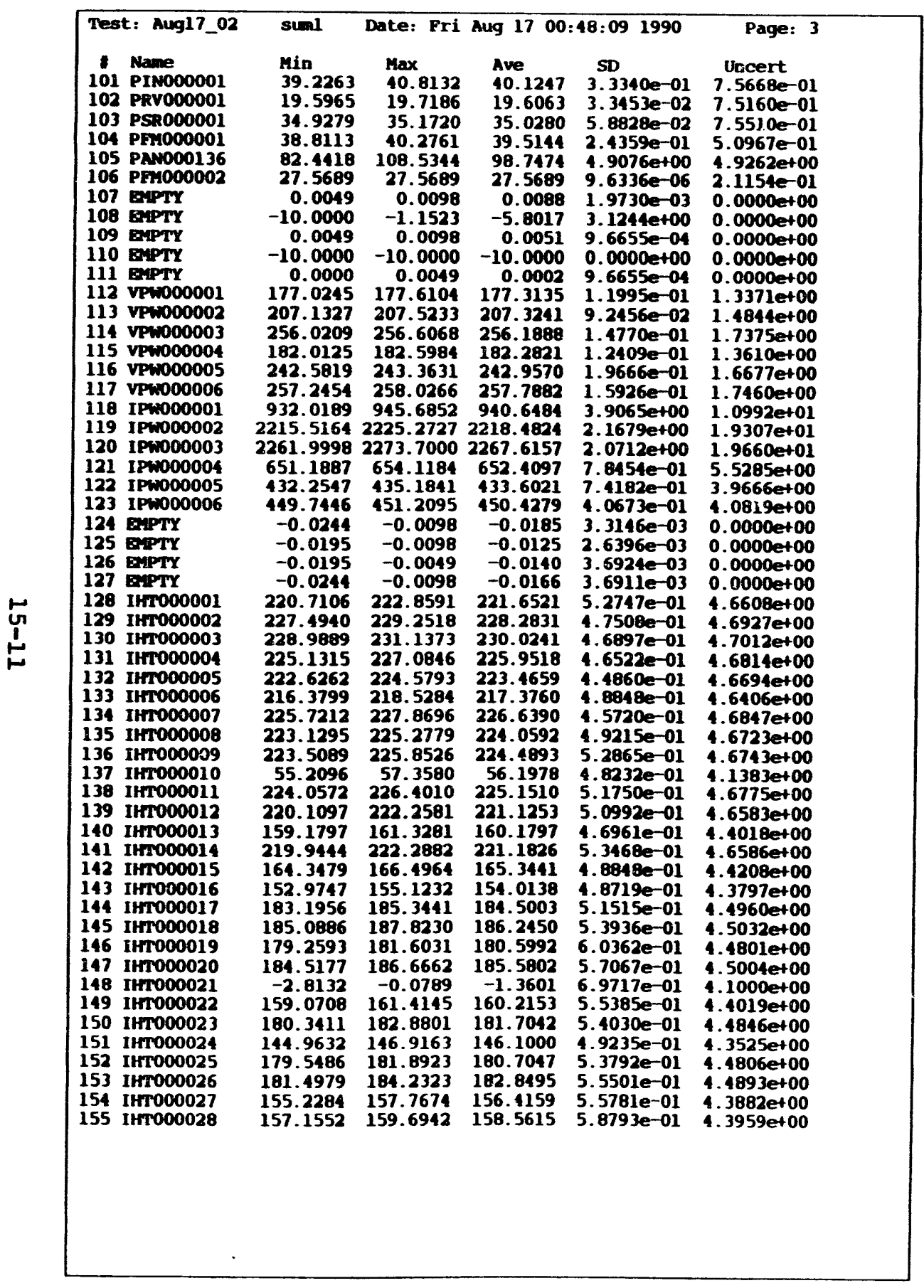

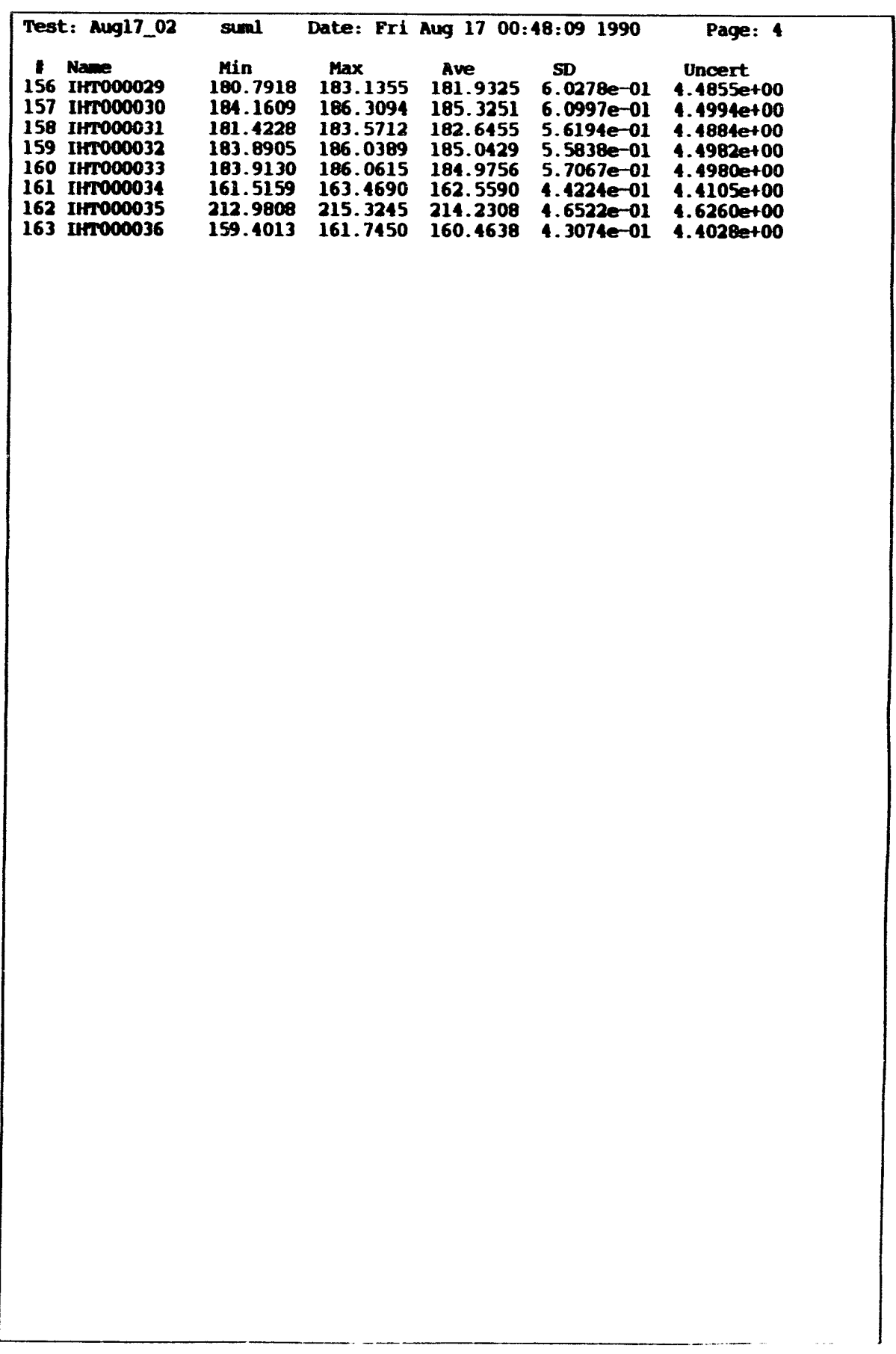




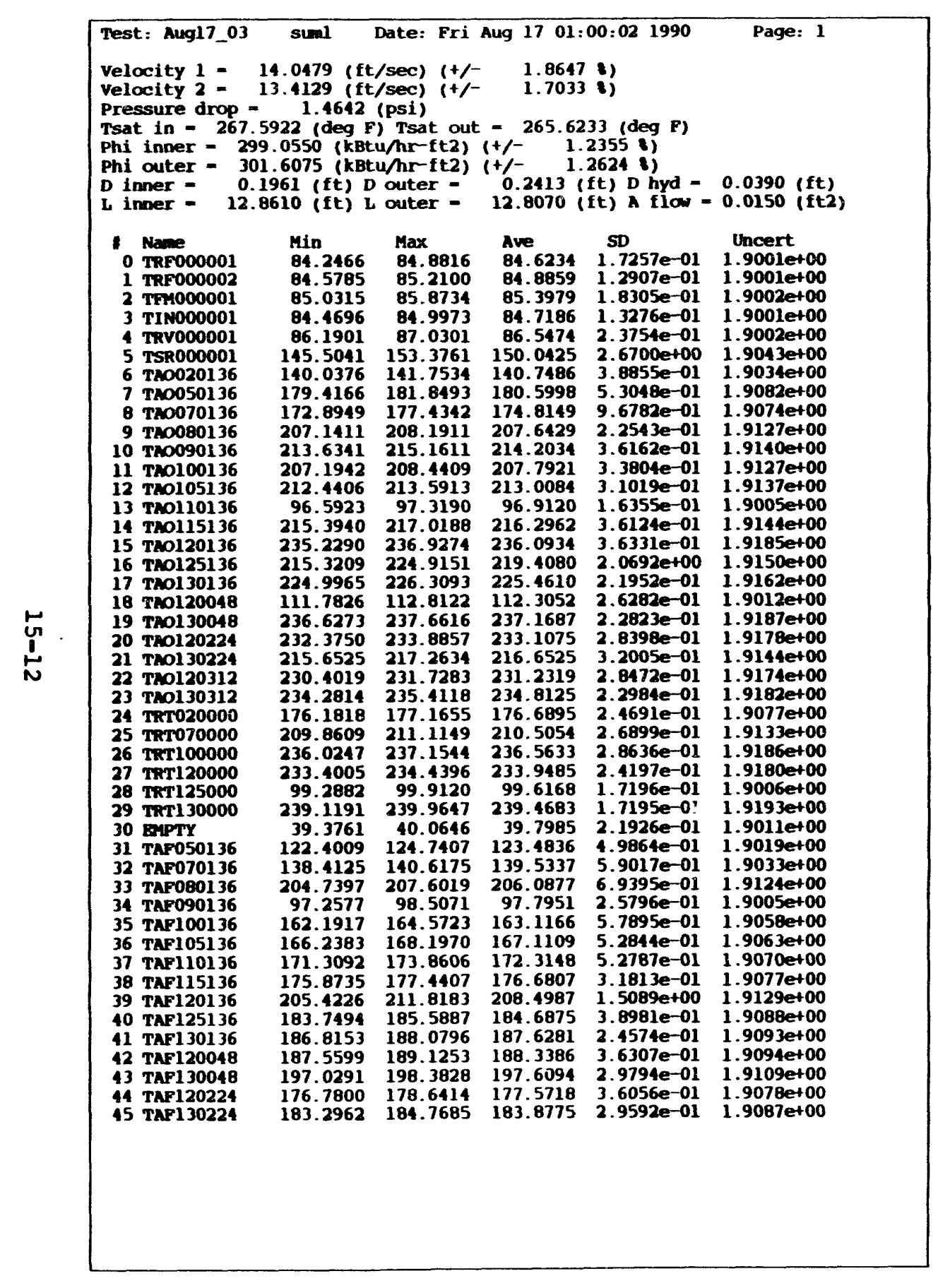

\begin{tabular}{|c|c|c|c|c|c|}
\hline 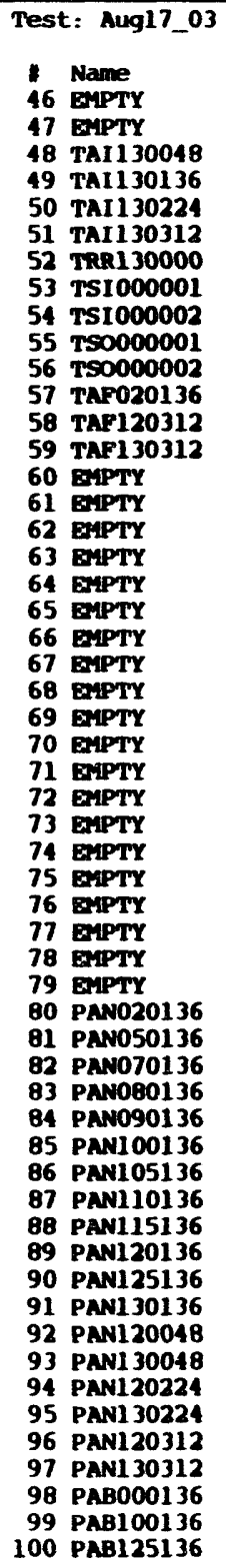 & $\begin{array}{l}\text { suml } \\
\text { Min } \\
37.7675 \\
39.6057 \\
228.6102 \\
235.6948 \\
239.5931 \\
224.3698 \\
129.0927 \\
59.5261 \\
83.9370 \\
56.7750 \\
72.3714 \\
96.5551 \\
183.3420 \\
197.8923 \\
2.9346 \\
2.9248 \\
2.9150 \\
2.9150 \\
1.4502 \\
1.4746 \\
1.4844 \\
1.4990 \\
1.5088 \\
1.5234 \\
1.5283 \\
1.5479 \\
1.5527 \\
1.5723 \\
1.5820 \\
1.5918 \\
1.6064 \\
1.6211 \\
1.6357 \\
11.6553 \\
-9.9856 \\
-8.1966 \\
-9.3193 \\
-8.66152 \\
-0.2053 \\
-12.1908 \\
-7.7020 \\
-7.0696 \\
-7.4071 \\
-6.7482 \\
-0.6917 \\
3.3857 \\
-3.1792 \\
4.0672 \\
3.2749 \\
-11.9031 \\
-8.9189 \\
-9.2256 \\
39.7703 \\
39.0405 \\
38.6233 \\
\end{array}$ & $\begin{array}{l}\text { Date: } \text { Pri } \\
\text { Max } \\
38.4572 \\
40.0646 \\
230.3135 \\
236.9175 \\
241.1957 \\
226.3658 \\
130.5099 \\
60.2711 \\
85.8283 \\
57.7485 \\
73.1172 \\
99.0708 \\
18.9049 \\
199.5329 \\
2.9492 \\
2.9395 \\
2.9297 \\
2.9248 \\
1.4648 \\
1.4795 \\
1.4893 \\
1.5039 \\
1.5137 \\
1.5234 \\
1.5381 \\
1.5527 \\
1.5625 \\
1.5771 \\
1.5869 \\
1.6016 \\
1.6113 \\
1.6260 \\
1.6406 \\
1.6602 \\
7.0280 \\
16.3700 \\
17.6125 \\
15.0359 \\
20.3941 \\
9.0953 \\
13.6603 \\
16.3527 \\
15.7863 \\
8.5868 \\
35.8531 \\
19.7889 \\
45.5726 \\
30.9990 \\
31.1985 \\
18.4619 \\
26.1763 \\
22.2075 \\
40.5485 \\
39.8645 \\
39.2641\end{array}$ & $\begin{array}{c}\text { Aug } 1701: \\
\text { Ave } \\
38.0986 \\
39.7709 \\
229.5647 \\
236.1919 \\
240.4266 \\
225.5868 \\
129.9225 \\
59.9513 \\
84.8157 \\
57.3847 \\
72.7679 \\
97.8090 \\
184.1663 \\
198.5449 \\
2.9417 \\
2.9330 \\
2.9234 \\
2.9227 \\
1.4594 \\
1.4757 \\
1.4876 \\
1.5001 \\
1.5091 \\
1.5234 \\
1.5332 \\
1.5497 \\
1.5584 \\
1.5729 \\
1.5854 \\
1.5971 \\
1.6066 \\
1.6213 \\
1.6402 \\
1.6563 \\
-2.2601 \\
4.2759 \\
3.9177 \\
2.3574 \\
7.9002 \\
-2.8188 \\
1.1603 \\
6.4299 \\
4.2186 \\
-2.2835 \\
11.8617 \\
8.5508 \\
17.7726 \\
9.9343 \\
15.8252 \\
3.5952 \\
10.3514 \\
7.7803 \\
40.1502 \\
39.3839 \\
38.8439\end{array}$ & 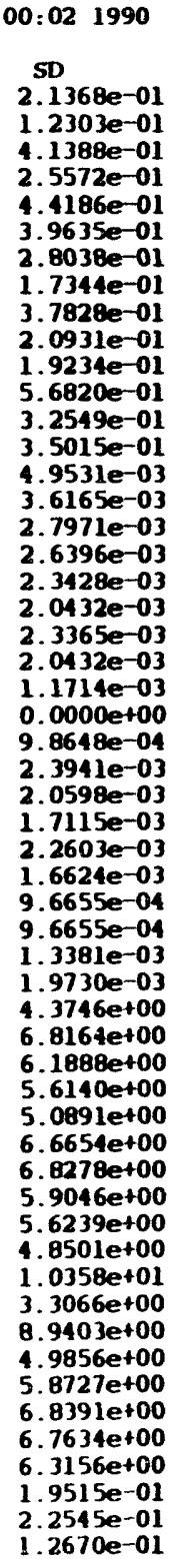 & 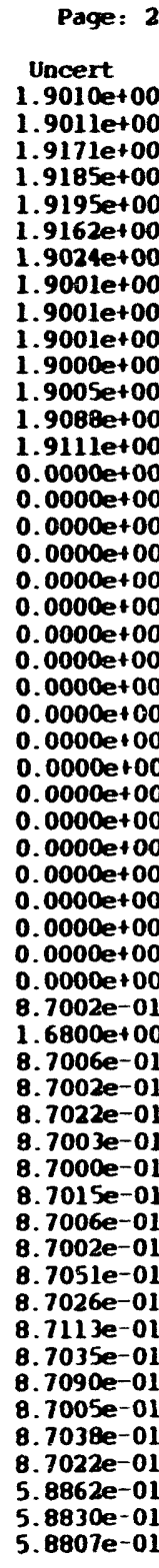 \\
\hline
\end{tabular}




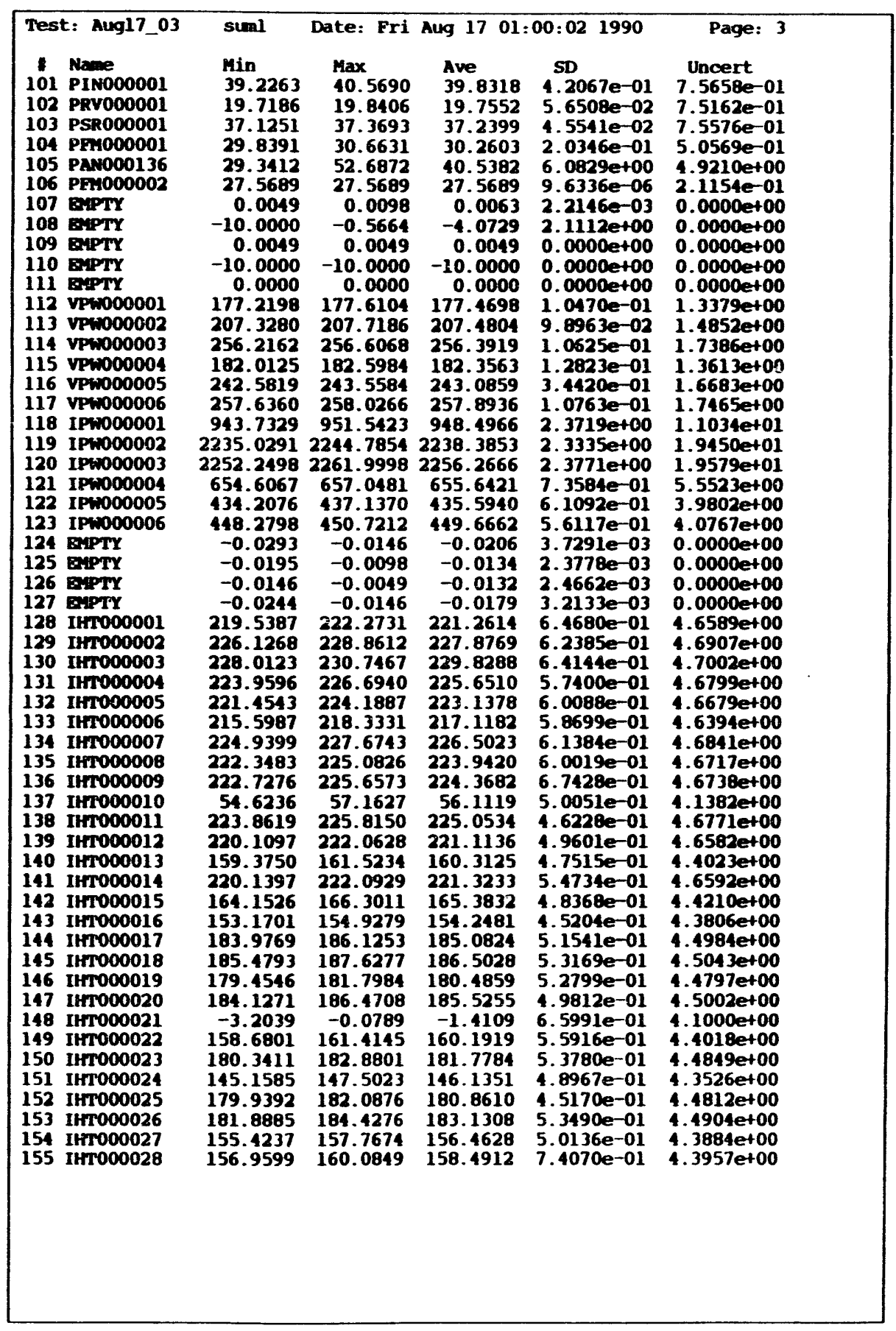

\begin{tabular}{|c|c|c|c|c|c|}
\hline Test: & suml & Date: & Aug 17 01: & $00: 02$ & Page: \\
\hline $\begin{array}{ll}c & \text { Name } \\
156 & \text { Ifrro00029 } \\
157 & \text { Ifrro00030 } \\
158 & \text { Ifrro00031 } \\
159 & \text { Ifrro00032 } \\
160 & \text { Ifrro00033 } \\
161 & \text { Ifrro00034 } \\
162 & \text { Ifro00035 } \\
163 & \text { Ifrro00036 }\end{array}$ & $\begin{array}{l}\text { Min } \\
180.4012 \\
183.9656 \\
181.2275 \\
183.4998 \\
183.7177 \\
161.3206 \\
212.9800 \\
159.4013\end{array}$ & $\begin{array}{l}\operatorname{Max} \\
183.5262 \\
187.0906 \\
184.3525 \\
187.0155 \\
187.0380 \\
164.0550 \\
215.5199 \\
161.7450\end{array}$ & $\begin{array}{c}\text { Ave } \\
181.9403 \\
185.3251 \\
182.5283 \\
185.0585 \\
185.0107 \\
162.4535 \\
214.2542 \\
160.4951\end{array}$ & $\begin{array}{l}\text { SD } \\
7.4727 e-01 \\
7.4133 e-01 \\
6.6335 e-01 \\
7.4502 e-01 \\
7.2640 e-01 \\
5.4391 e-01 \\
5.6115 e-01 \\
5.2497 e-01\end{array}$ & $\begin{array}{c}\text { Uncert } \\
4.4856 \mathrm{e}+00 \\
4.4994 \mathrm{e}+00 \\
4.4879 \mathrm{e}+00 \\
4.4983 \mathrm{e}+00 \\
4.4981 \mathrm{e}+00 \\
4.4101 \mathrm{e}+00 \\
4.6261 \mathrm{e}+00 \\
4.4029 \mathrm{e}+00\end{array}$ \\
\hline
\end{tabular}




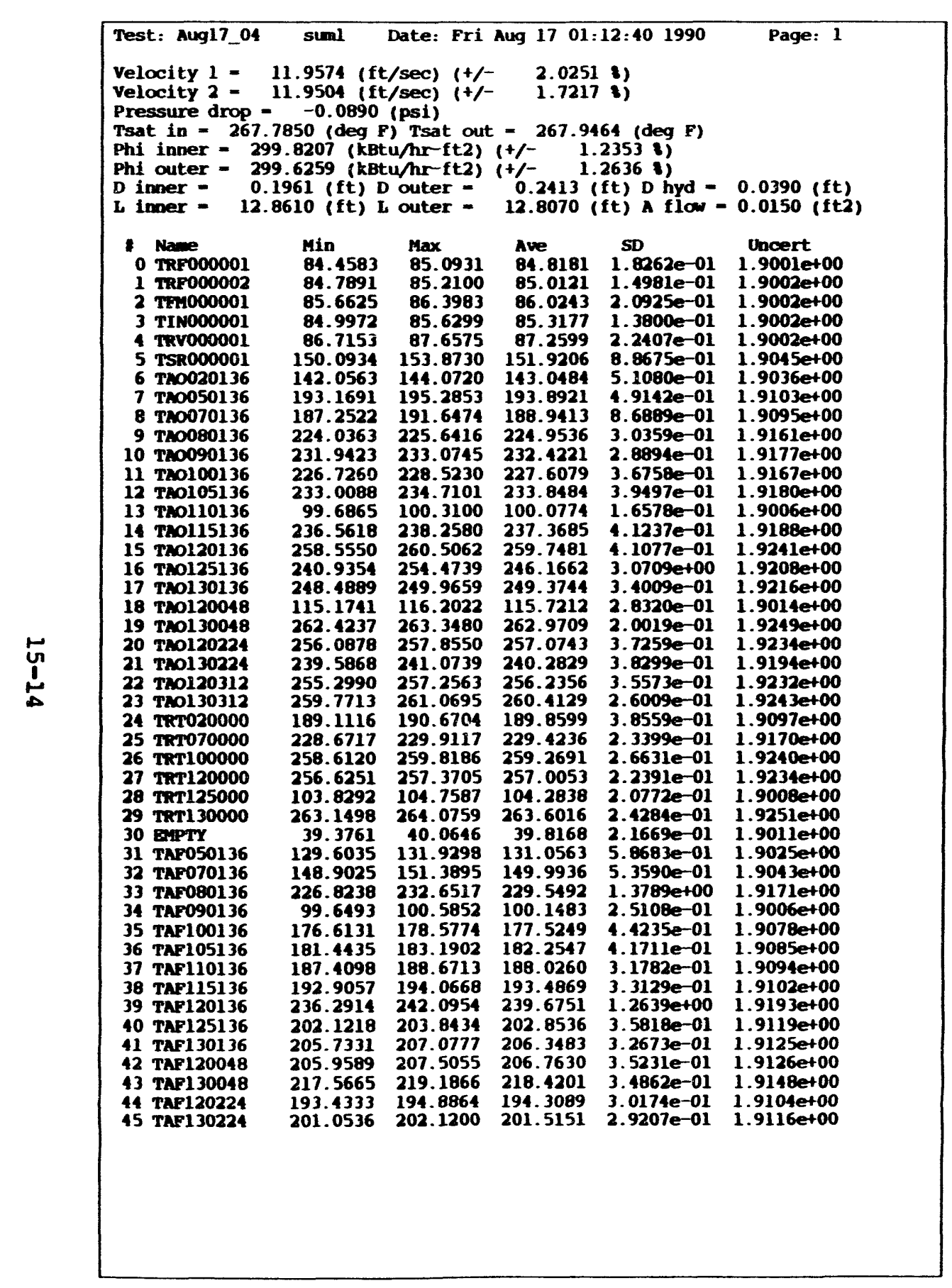

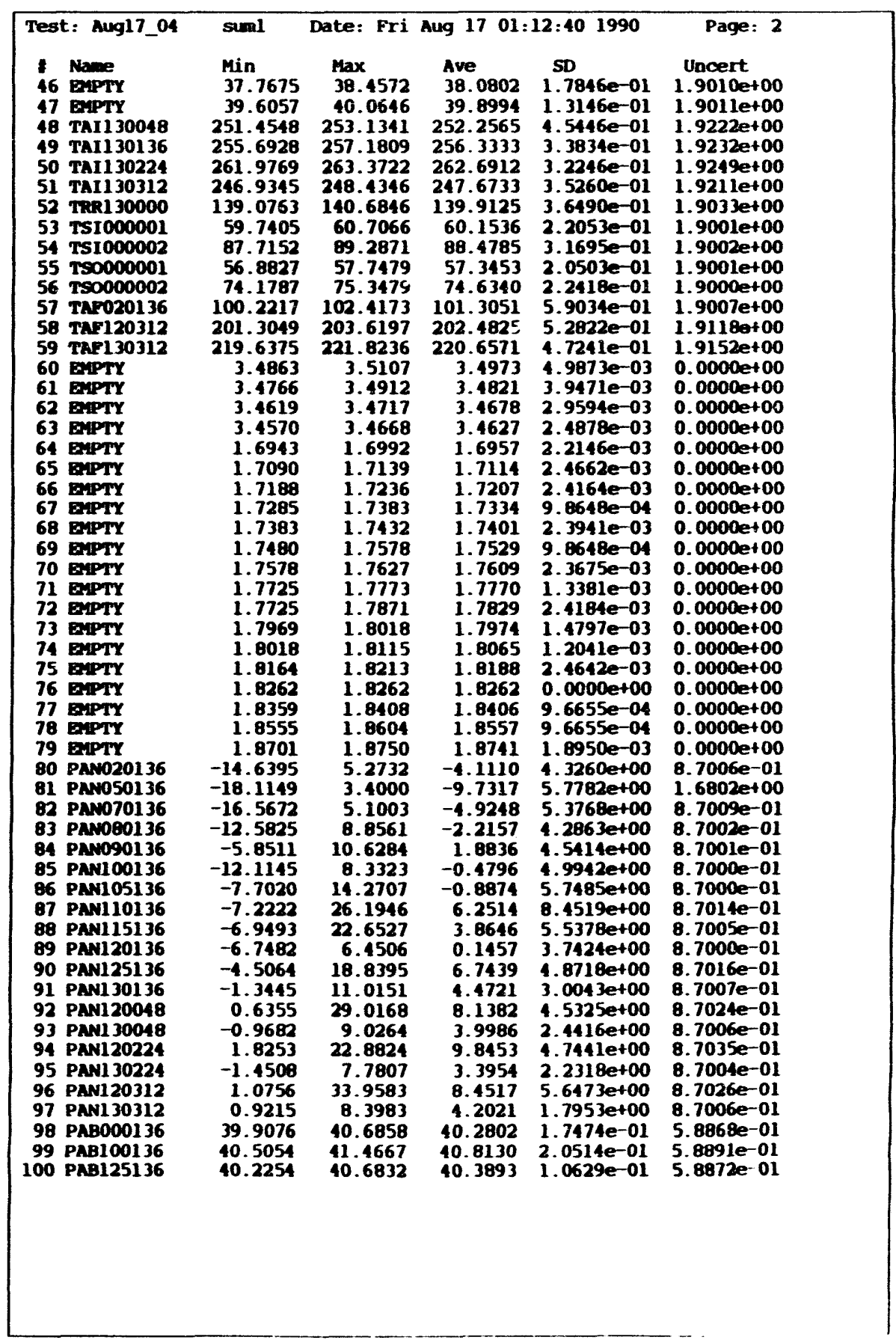




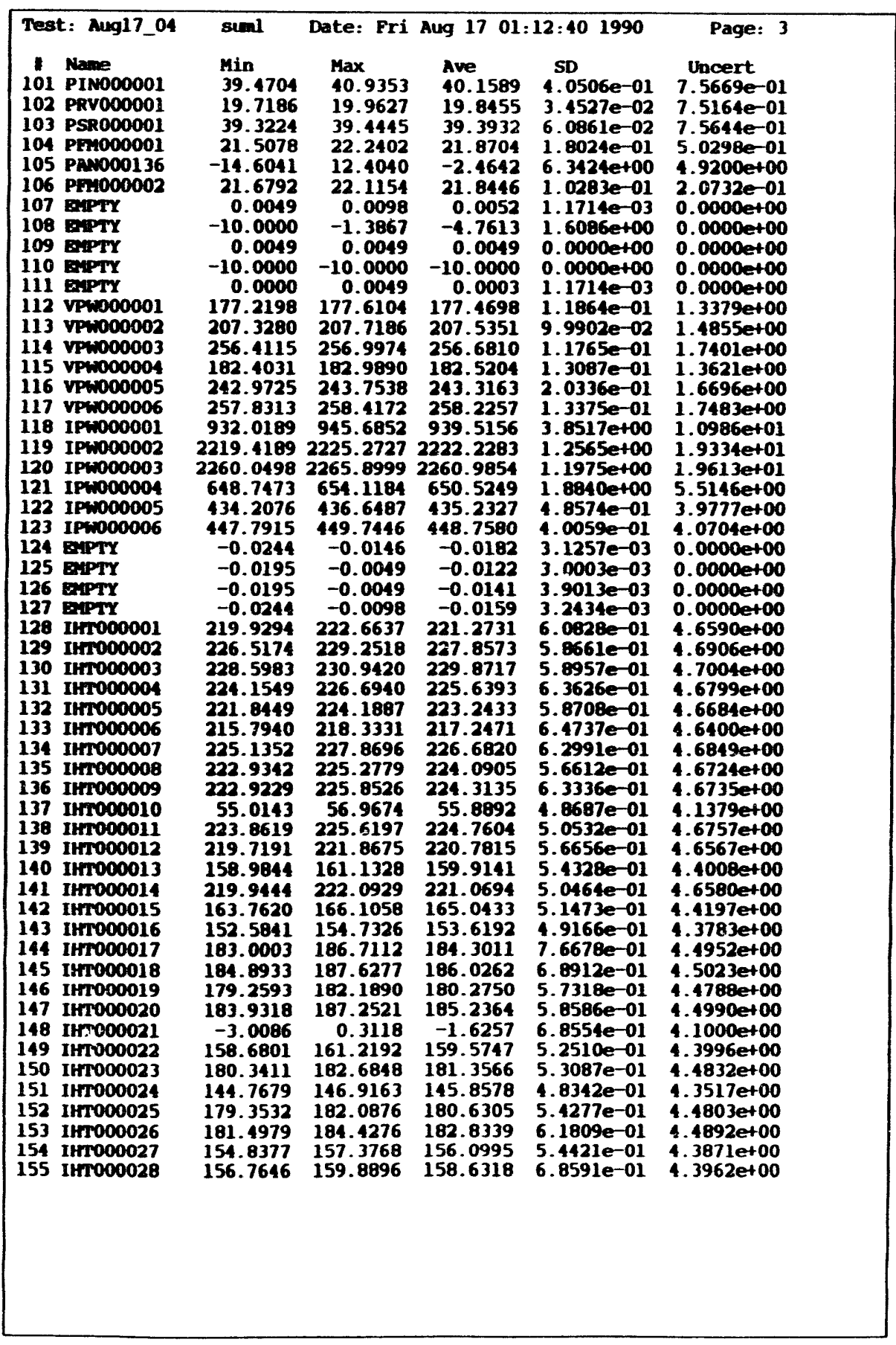

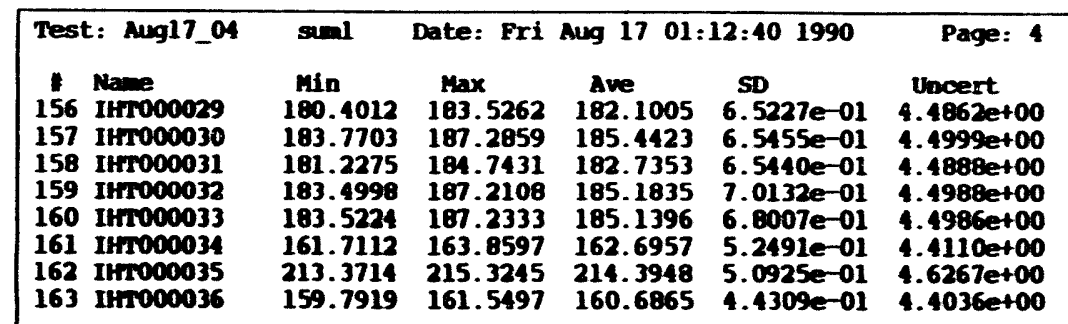




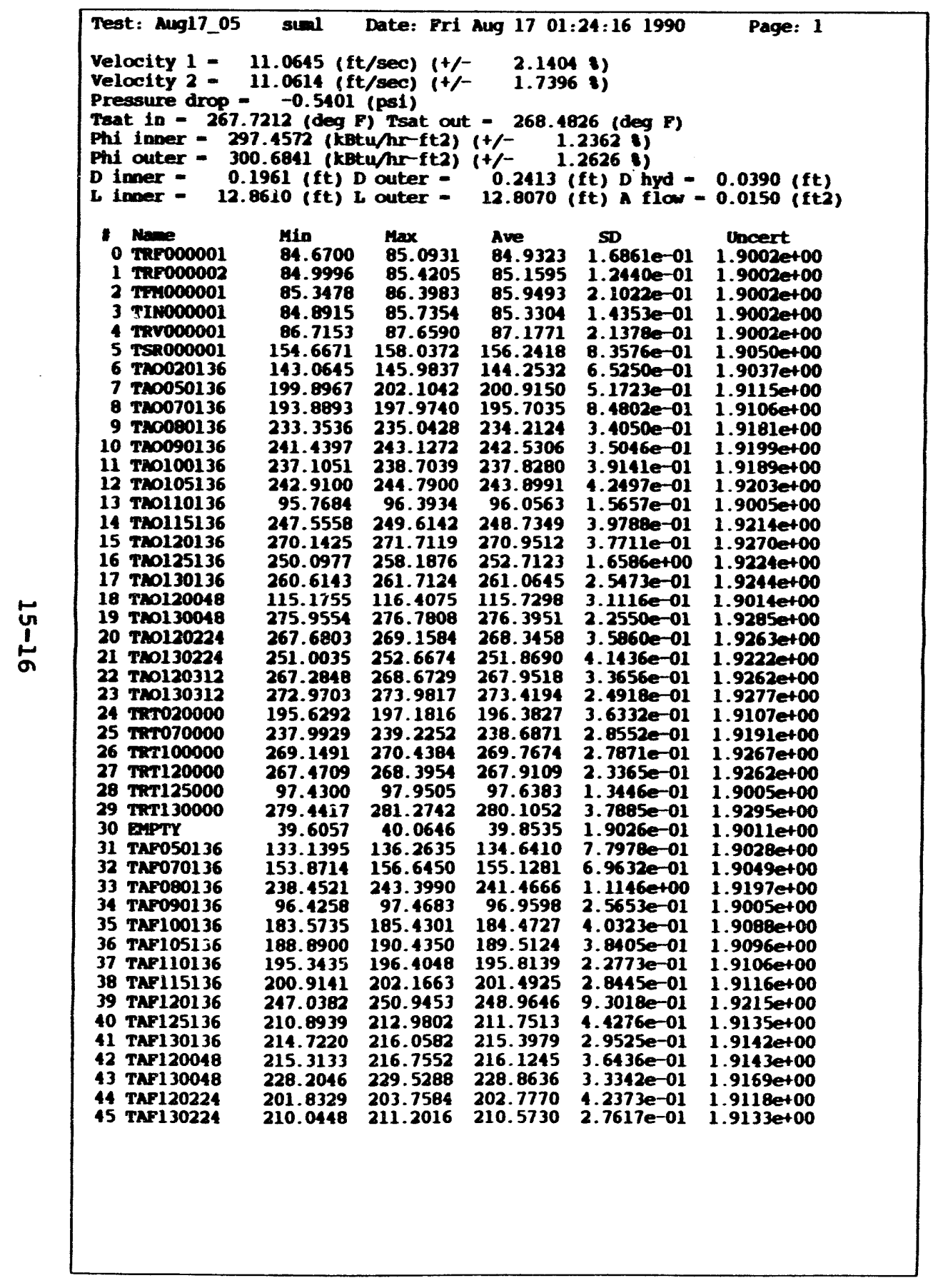

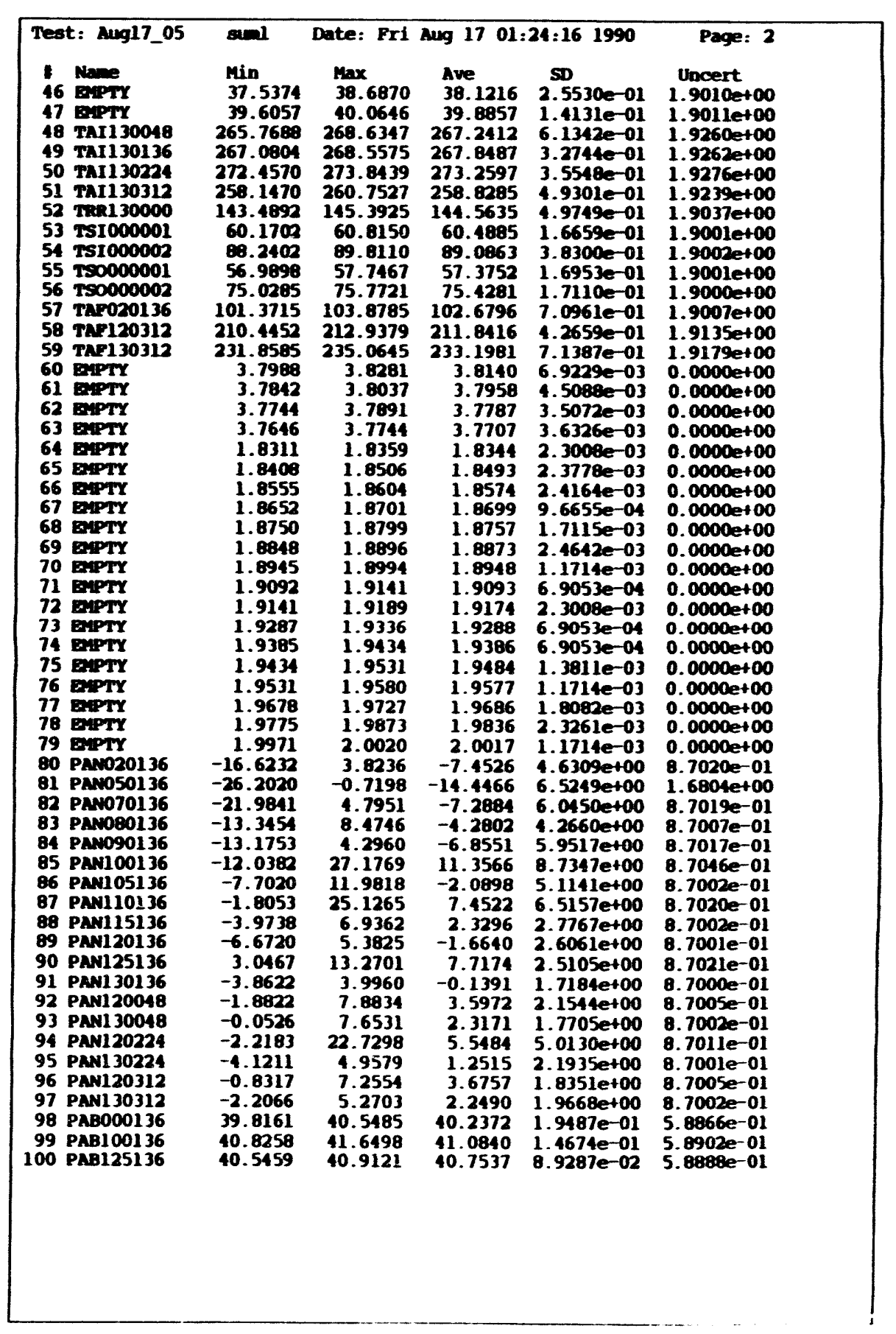




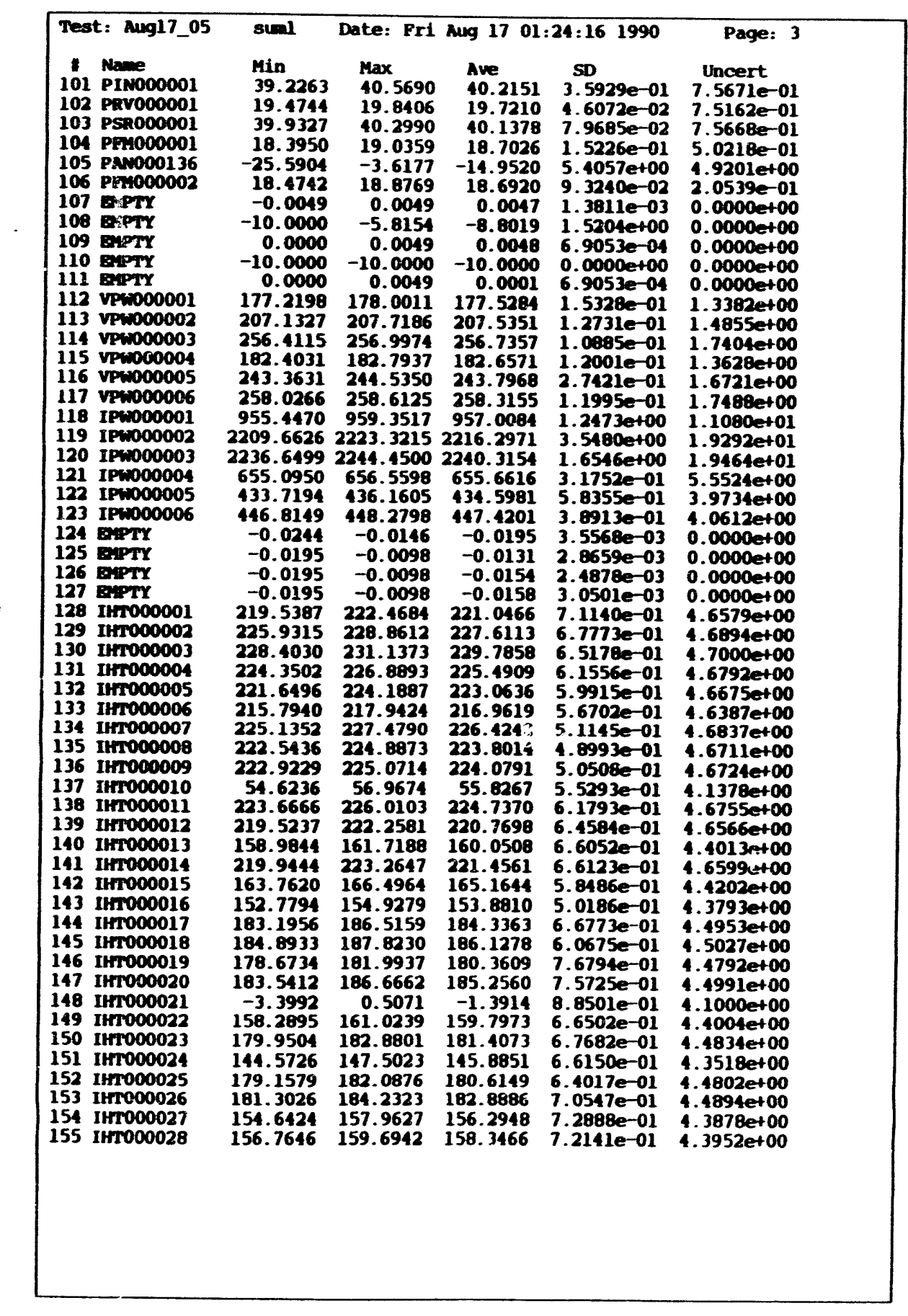

\begin{tabular}{|c|c|c|c|c|c|}
\hline Test: Mug17_05 & $\sin$ & Date: Fri & $\operatorname{sing} 1701$ & $24: 16 \quad 1990$ & Page: 4 \\
\hline 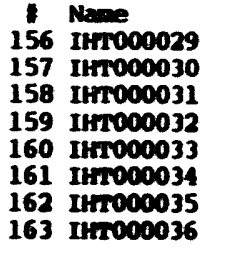 & $\begin{array}{l}\text { Min } \\
180.2050 \\
183.7703 \\
181.0322 \\
183.4998 \\
183.3271 \\
161.3206 \\
213.1761 \\
159.2060\end{array}$ & $\begin{array}{c}\operatorname{Max} \\
183.1355 \\
186.5047 \\
183.3759 \\
185.8436 \\
185.8661 \\
163.6644 \\
215.9105 \\
161.5497\end{array}$ & $\begin{array}{l}\text { Ave } \\
181.7762 \\
185.0712 \\
182.3408 \\
184.7929 \\
184.7217 \\
162.4066 \\
214.4339 \\
160.5029\end{array}$ & $\begin{array}{l}\text { SD } \\
6.8681 e-01 \\
7.0433 e-01 \\
7.2033 e-01 \\
6.9575 e-01 \\
7.0256 e-01 \\
5.8148 e-01 \\
6.8704 e-01 \\
5.8362 e-01\end{array}$ & $\begin{array}{l}\text { Uncert } \\
4.4849 \mathrm{e}+00 \\
4.4983 \mathrm{e}+00 \\
4.4872 \mathrm{e}+00 \\
4.4972 \mathrm{e}+00 \\
4.4969 \mathrm{e}+00 \\
1.4099 \mathrm{e}+00 \\
4.6269 \mathrm{e}+00 \\
4.4030 \mathrm{e}+00\end{array}$ \\
\hline
\end{tabular}




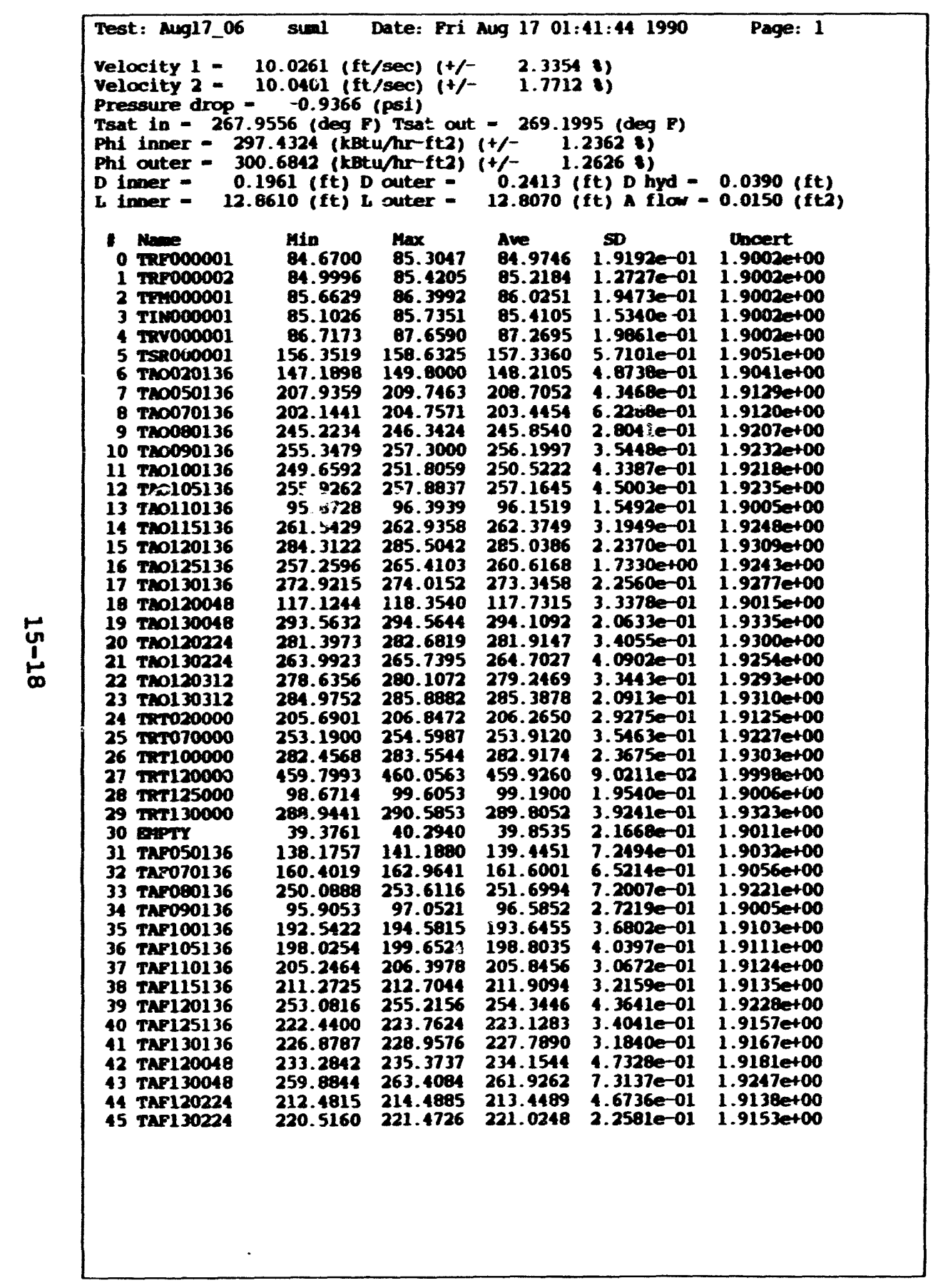

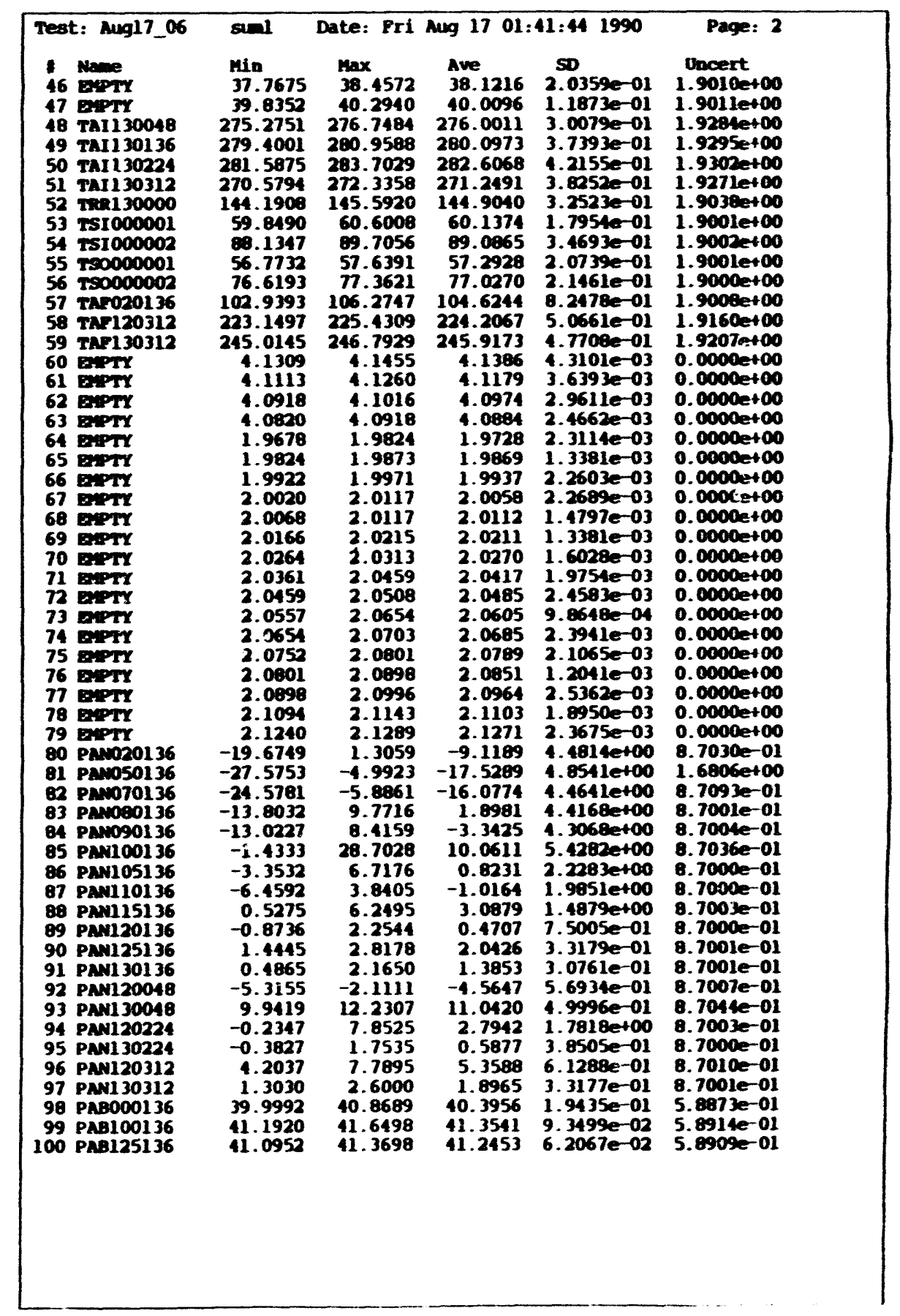




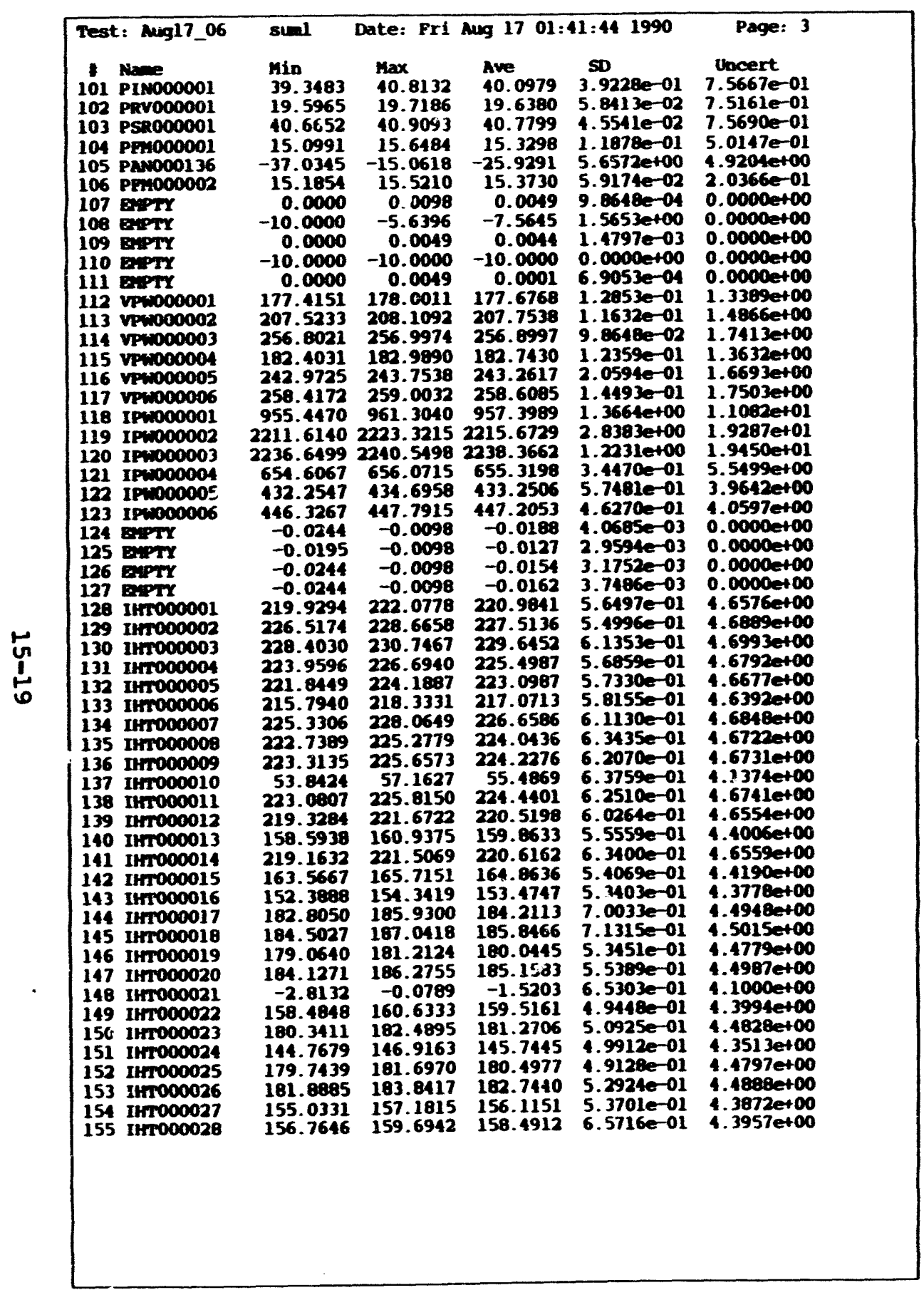

\begin{tabular}{|c|c|c|c|c|c|}
\hline 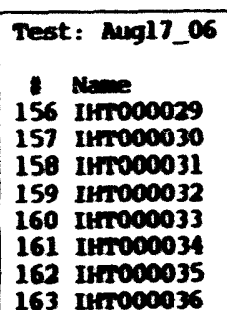 & $\begin{array}{l}\text { siml } \\
\text { min } \\
180.2058 \\
183.1844 \\
180.8368 \\
183.4998 \\
183.3271 \\
161.1253 \\
212.5902 \\
159.4013\end{array}$ & $\begin{array}{c}\text { Date: Fri } \\
\max \\
182.9402 \\
186.7000 \\
183.7665 \\
186.2342 \\
186.2568 \\
163.4690 \\
215.1292 \\
161.7450\end{array}$ & $\begin{array}{l}\text { Aug } 1701: \\
\text { Ave } \\
181.9130 \\
185.2821 \\
182.4814 \\
184.8905 \\
184.8193 \\
162.4183 \\
213.8206 \\
160.5615\end{array}$ & $\begin{array}{l}50 \\
6.3357 e-01 \\
6.8551 e-01 \\
6.3392 e-01 \\
6.5985 e-01 \\
6.5081 e-01 \\
5.3071 e-01 \\
6.1162 e-01 \\
5.1510 e-01\end{array}$ & $\begin{array}{l}\text { Page: } 1 \\
\text { cert } \\
854 e+00 \\
992 e+00 \\
878 e+00 \\
976 e+00 \\
973 e+00 \\
100 e+\infty 0\end{array}$ \\
\hline
\end{tabular}



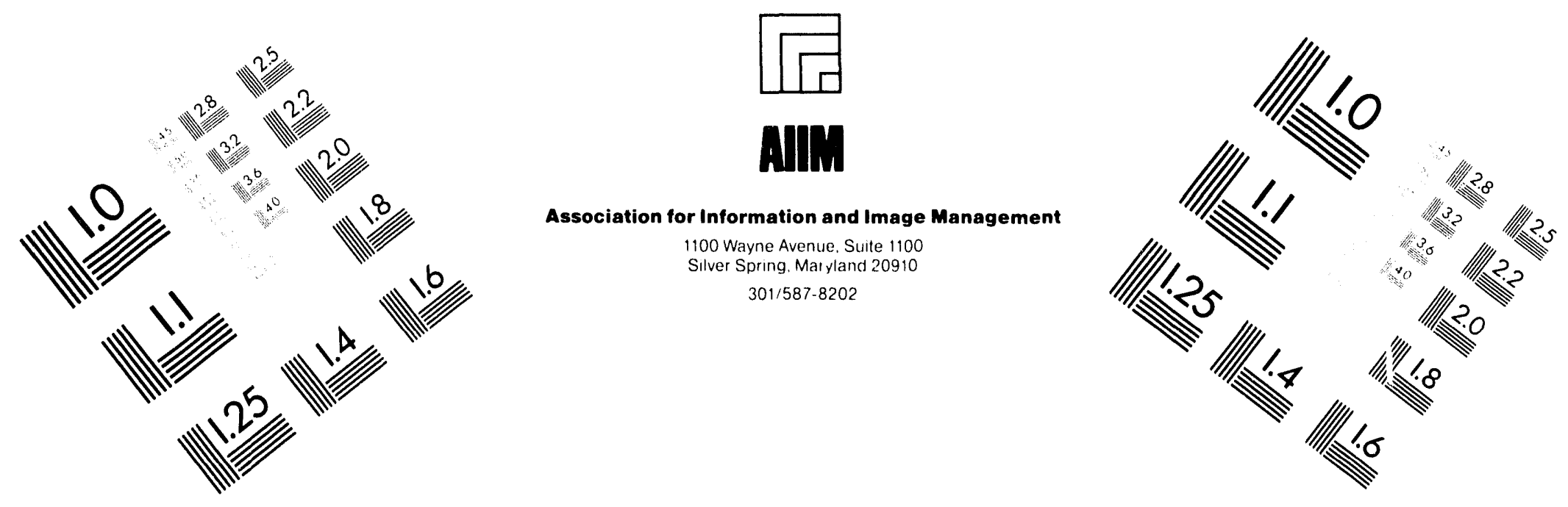

\section{Centimeter}

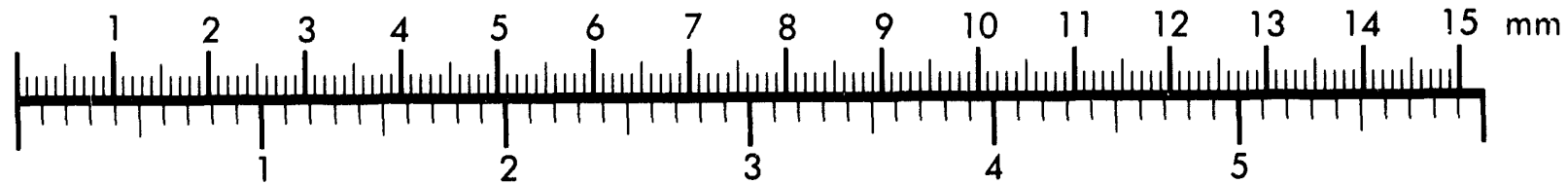

Inches
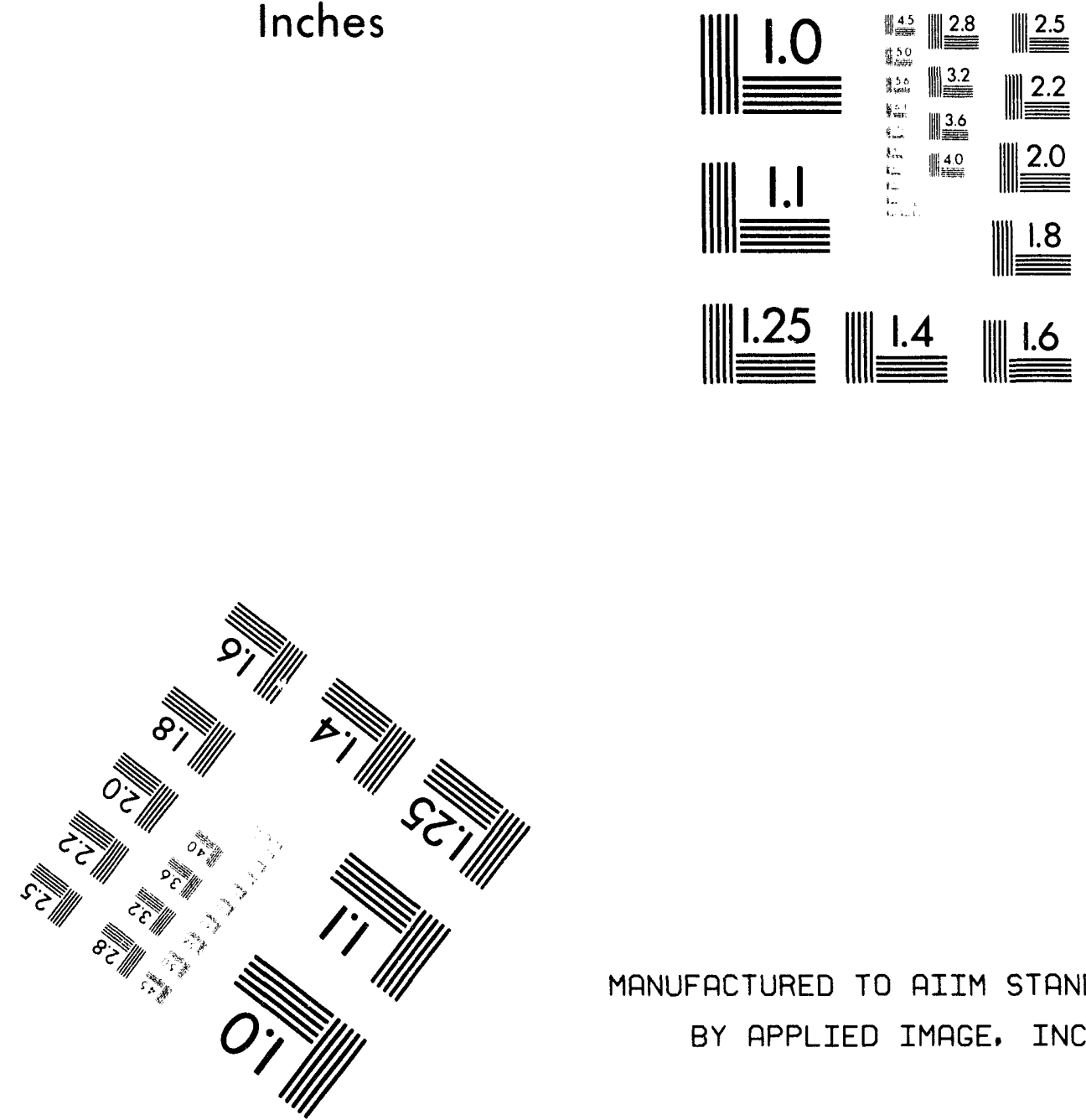

MANUFACTURED TO AIIM STANDARDS

BY APPLIED IMAGE, INC.

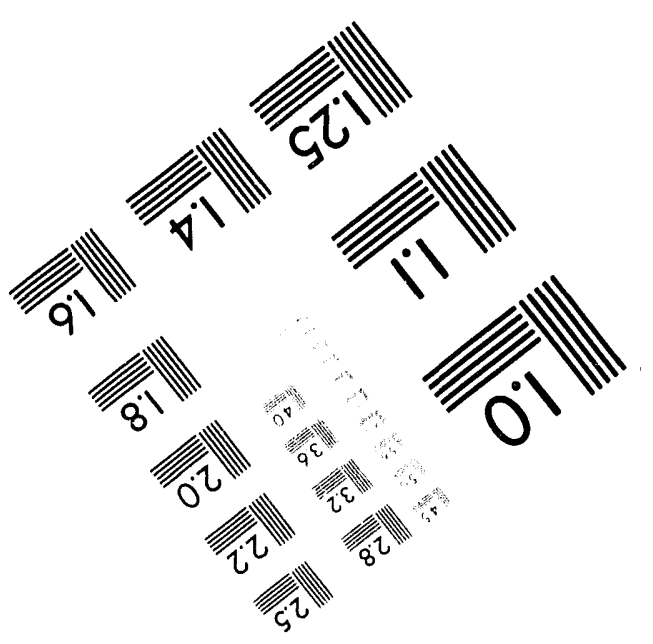



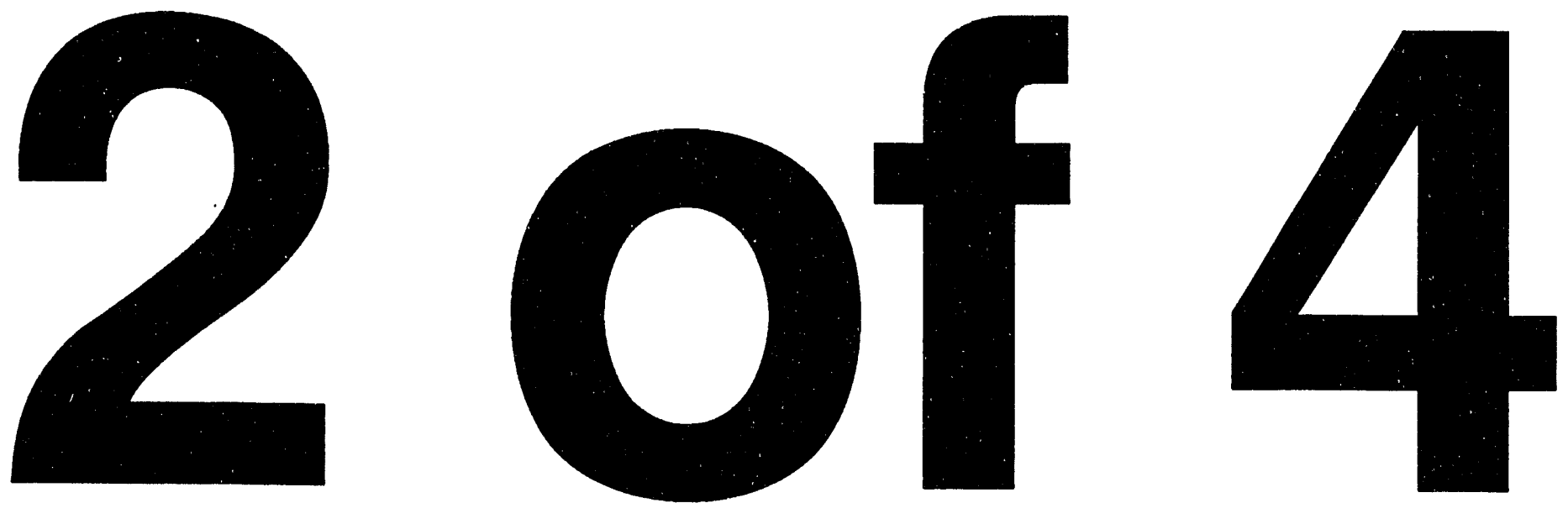


\begin{tabular}{|c|c|c|c|c|c|}
\hline \multicolumn{6}{|c|}{ 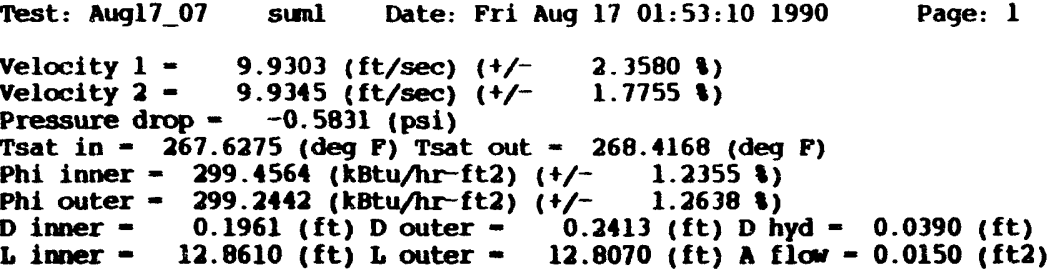 } \\
\hline 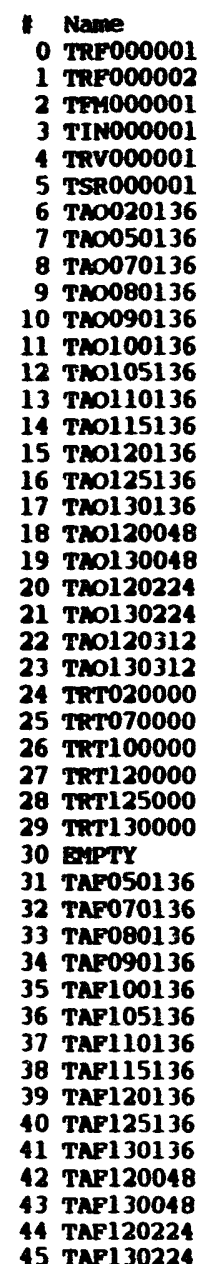 & $\begin{array}{l}\text { Min } \\
84.6700 \\
84.9996 \\
86.3977 \\
85.9456 \\
87.8660 \\
159.5216 \\
147.9939 \\
206.5978 \\
201.9503 \\
243.0825 \\
253.5811 \\
247.8857 \\
253.8730 \\
97.7313 \\
259.6846 \\
282.2952 \\
261.8961 \\
272.0164 \\
120.6042 \\
291.0203 \\
278.4599 \\
260.3196 \\
277.7159 \\
285.2484 \\
209.1575 \\
260.6906 \\
287.7520 \\
459.7993 \\
101.9762 \\
288.8533 \\
39.3761 \\
138.5791 \\
160.4995 \\
248.4224 \\
97.7794 \\
192.0558 \\
197.2579 \\
204.4795 \\
210.4099 \\
251.2262 \\
221.9698 \\
231.1268 \\
251.4293 \\
266.8333 \\
212.0031 \\
219.3659\end{array}$ & $\begin{array}{l}\operatorname{Max} \\
85.3047 \\
85.4205 \\
87.3423 \\
86.6833 \\
88.5999 \\
164.2595 \\
151.4034 \\
208.9843 \\
204.1769 \\
244.4796 \\
255.1640 \\
249.8472 \\
255.8337 \\
98.4573\end{array}$ & $\begin{array}{r}\text { Ave } \\
85.0000 \\
85.2816 \\
86.9211 \\
86.2975 \\
88.2502 \\
162.5688 \\
149.6372 \\
207.9635 \\
202.8782 \\
243.6503 \\
254.4728 \\
248.7397 \\
255.0997 \\
98.0661 \\
260.1924 \\
282.7449 \\
267.1101 \\
272.9702 \\
121.1101 \\
291.5569 \\
279.2340 \\
260.8256 \\
278.5792 \\
285.9449 \\
210.0516 \\
261.3132 \\
288.4981 \\
459.9619 \\
102.3717 \\
290.6195 \\
39.8122 \\
139.9131 \\
161.5313 \\
250.2441 \\
98.3768 \\
192.9209 \\
198.2041 \\
205.0965 \\
211.0287 \\
252.2498 \\
223.5716 \\
233.0338 \\
255.1404 \\
267.2497 \\
212.8051 \\
219.8164\end{array}$ & $\begin{array}{l}\text { SD } \\
1.6642 e-01 \\
1.2475 e-01 \\
1.9501 e-01 \\
1.5296 e-01 \\
1.7893 e-01 \\
1.1670 e+00 \\
6.7522 e-01 \\
5.0288 e-01 \\
4.7260 e-01 \\
2.8936 e-01 \\
3.5578 e-01 \\
4.7298 e-01 \\
4.1312 e-01 \\
1.6930 e-01 \\
2.4290 e-01 \\
1.9822 e-01 \\
1.7318 e+00 \\
4.2353 e-01 \\
3.2161 e-01 \\
3.3589 e-01 \\
3.0389 e-01 \\
2.8939 e-01 \\
4.2984 e-01 \\
2.4685 e-01 \\
3.0106 e-01 \\
3.7656 e-01 \\
3.0813 e-01 \\
8.5082 e-02 \\
1.9109 e-01 \\
8.8295 e-01\end{array}$ & $\begin{array}{c}\text { Uncert } \\
1.9002 \mathrm{e}+00 \\
1.9002 \mathrm{e}+00 \\
1.9002 \mathrm{e}+00 \\
1.9002 \mathrm{e}+00 \\
1.9002 \mathrm{e}+00 \\
1.9058 \mathrm{e}+00 \\
1.9043 \mathrm{e}+00 \\
1.9128 \mathrm{e}+00 \\
1.9119 \mathrm{e}+00 \\
1.9202 \mathrm{e}+00 \\
1.9228 \mathrm{e}+00 \\
1.9214 \mathrm{e}+00 \\
1.9229 \mathrm{e}+00 \\
1.9005 \mathrm{e}+00\end{array}$ \\
\hline
\end{tabular}

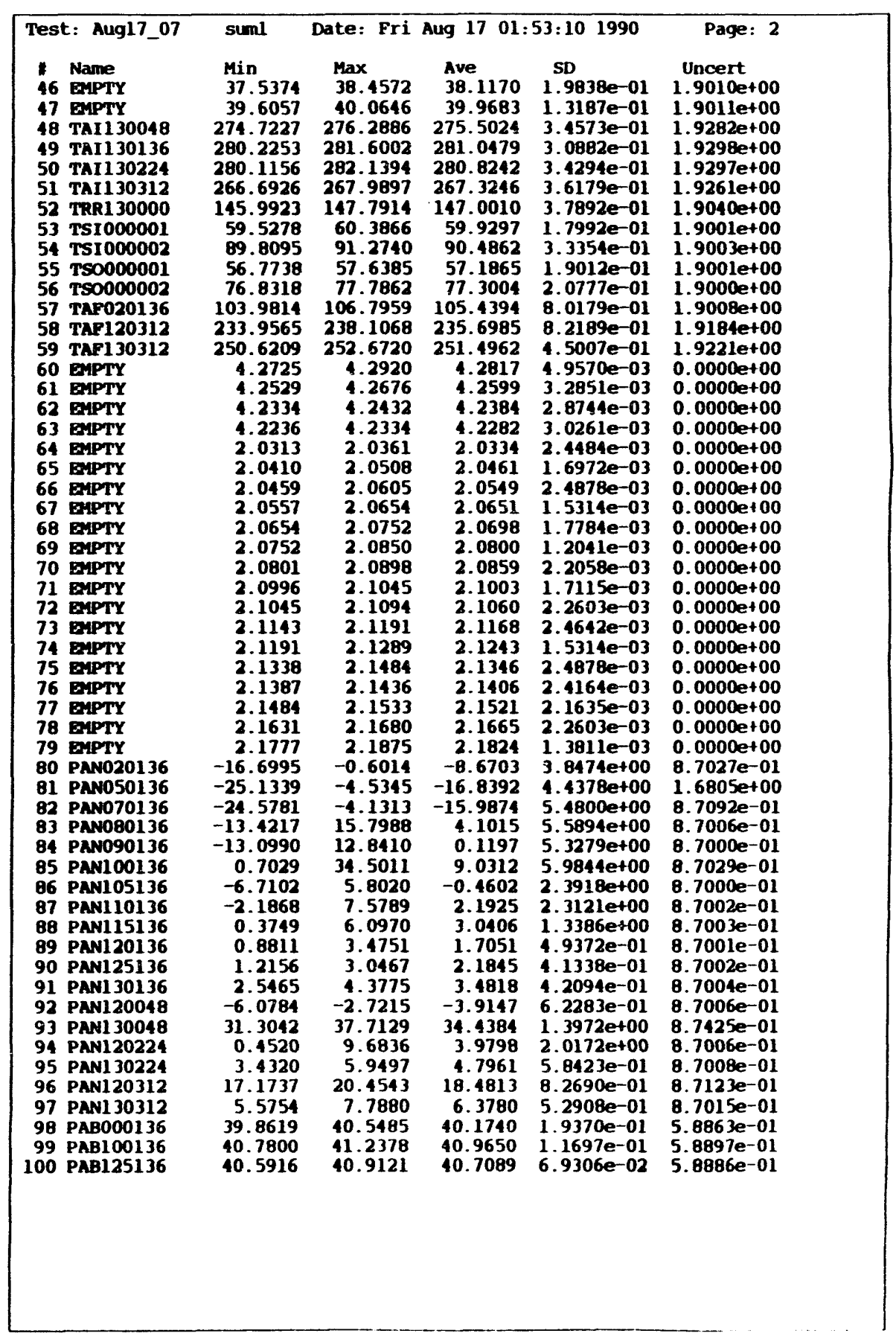




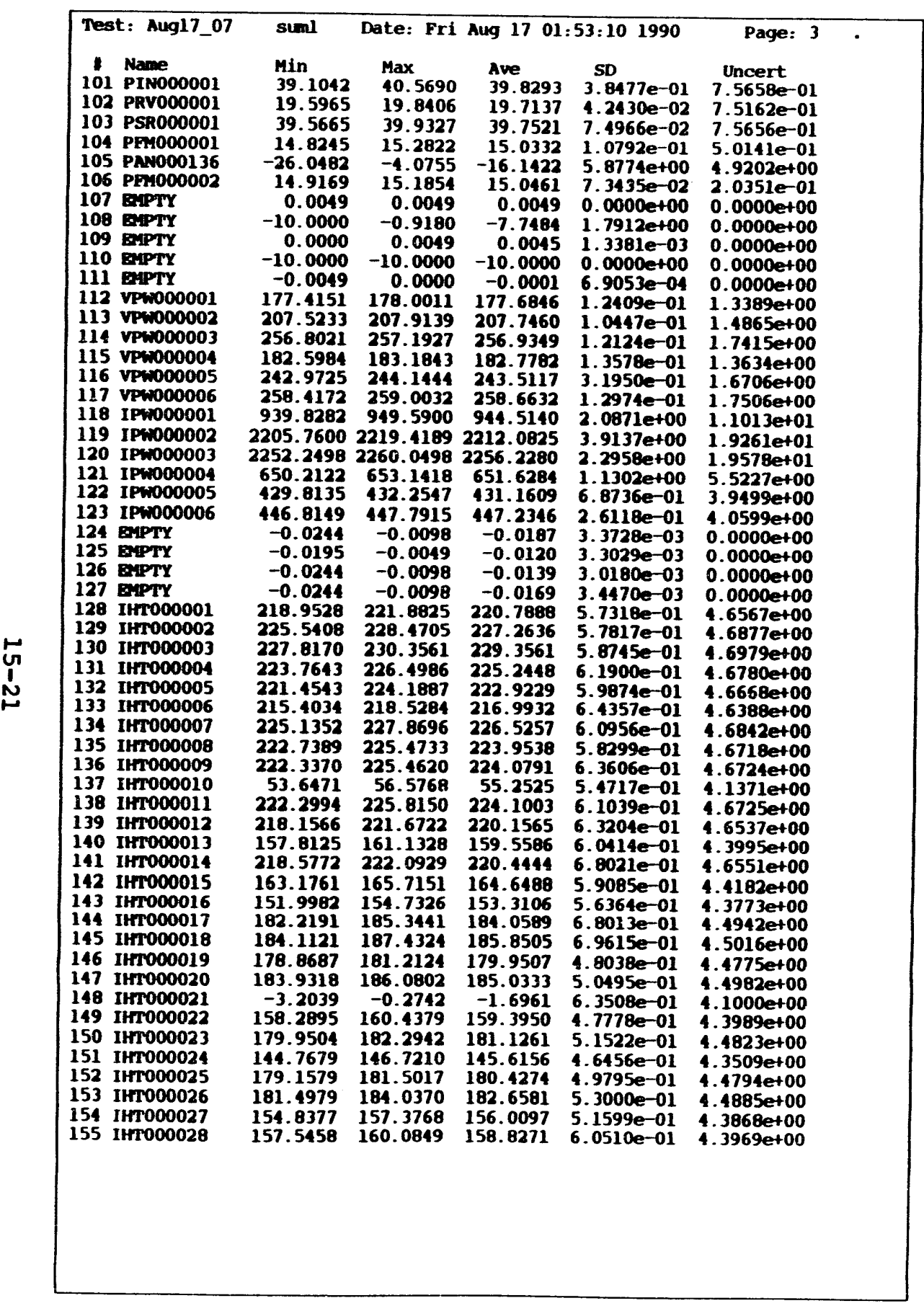

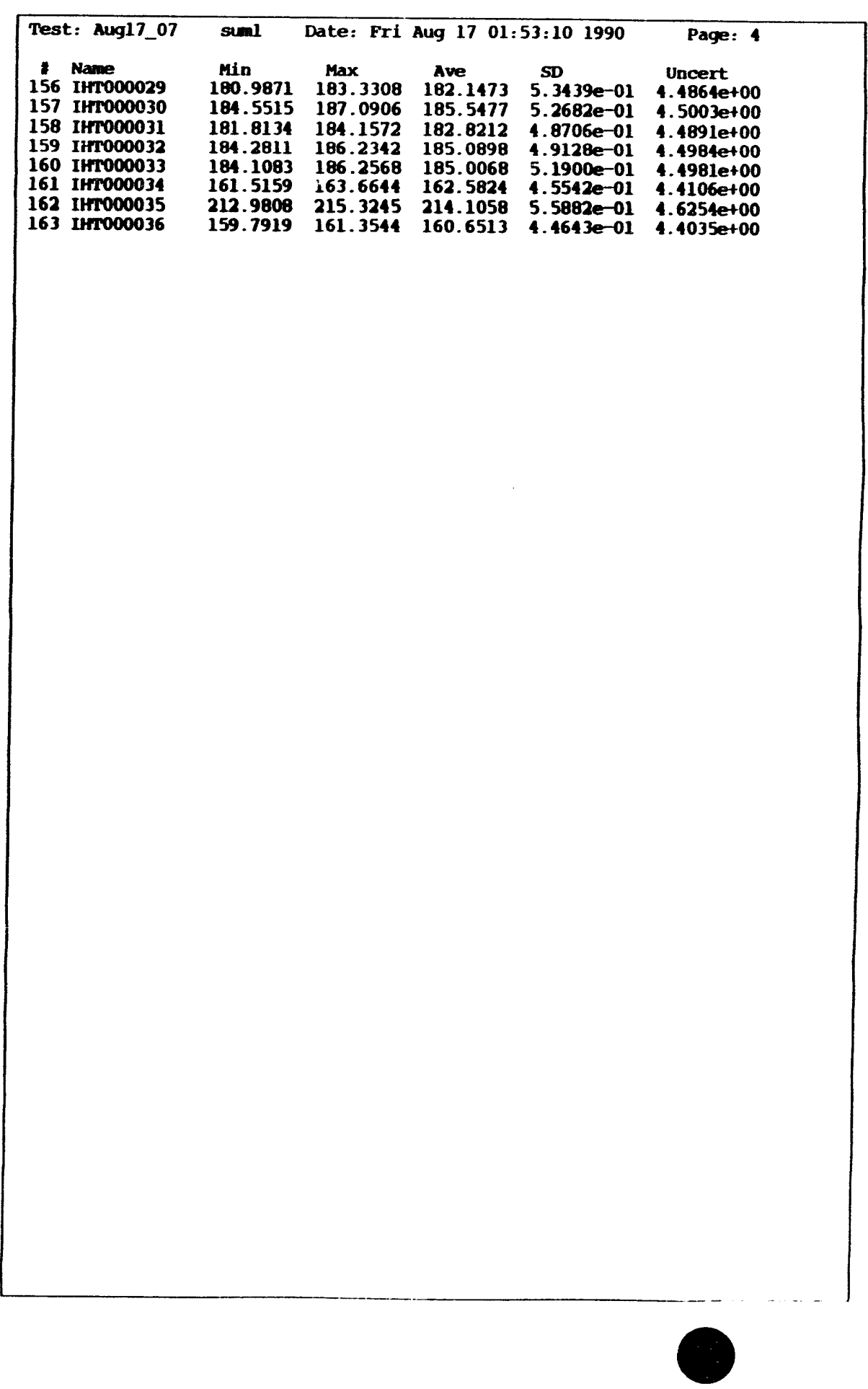




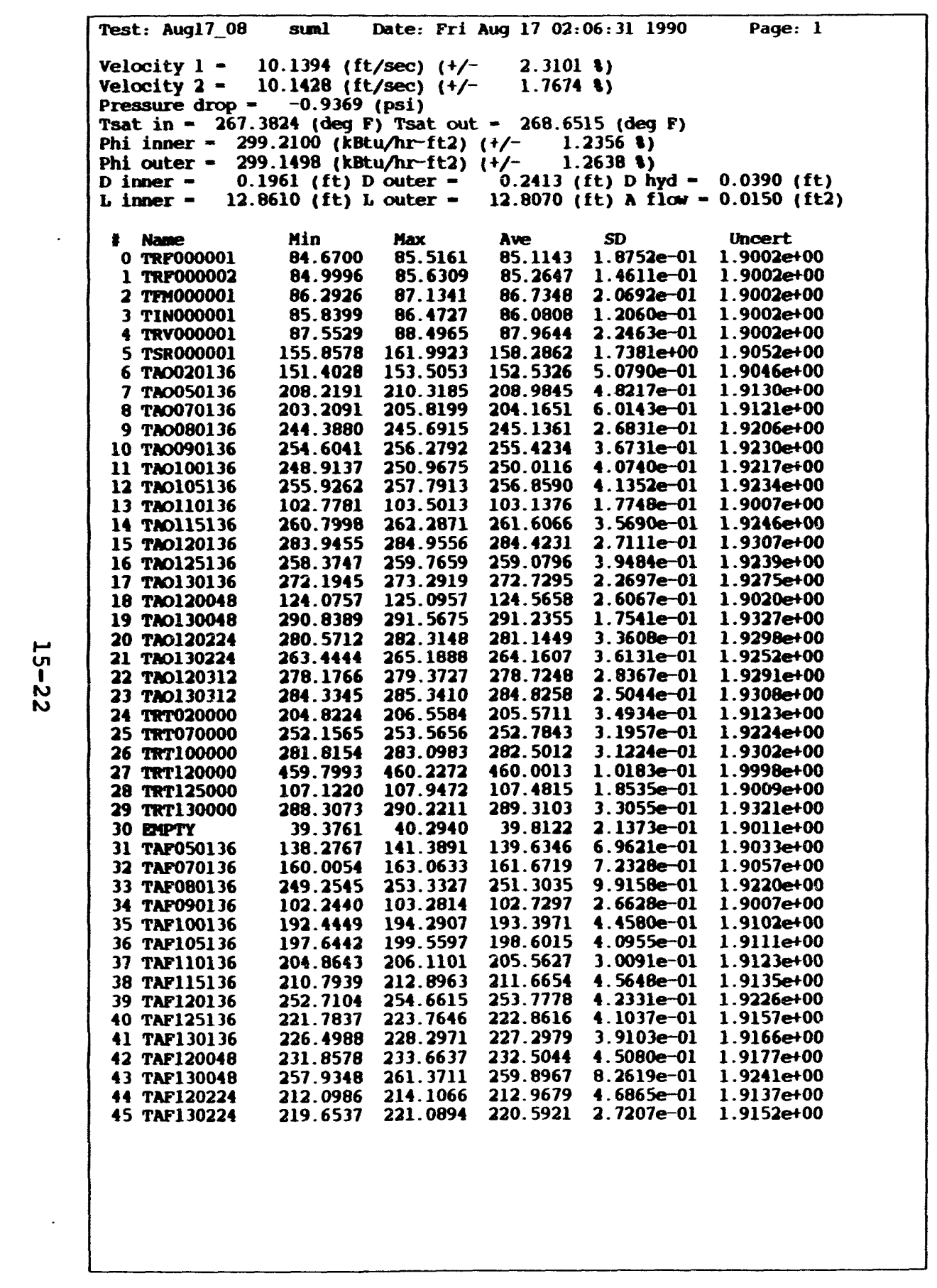

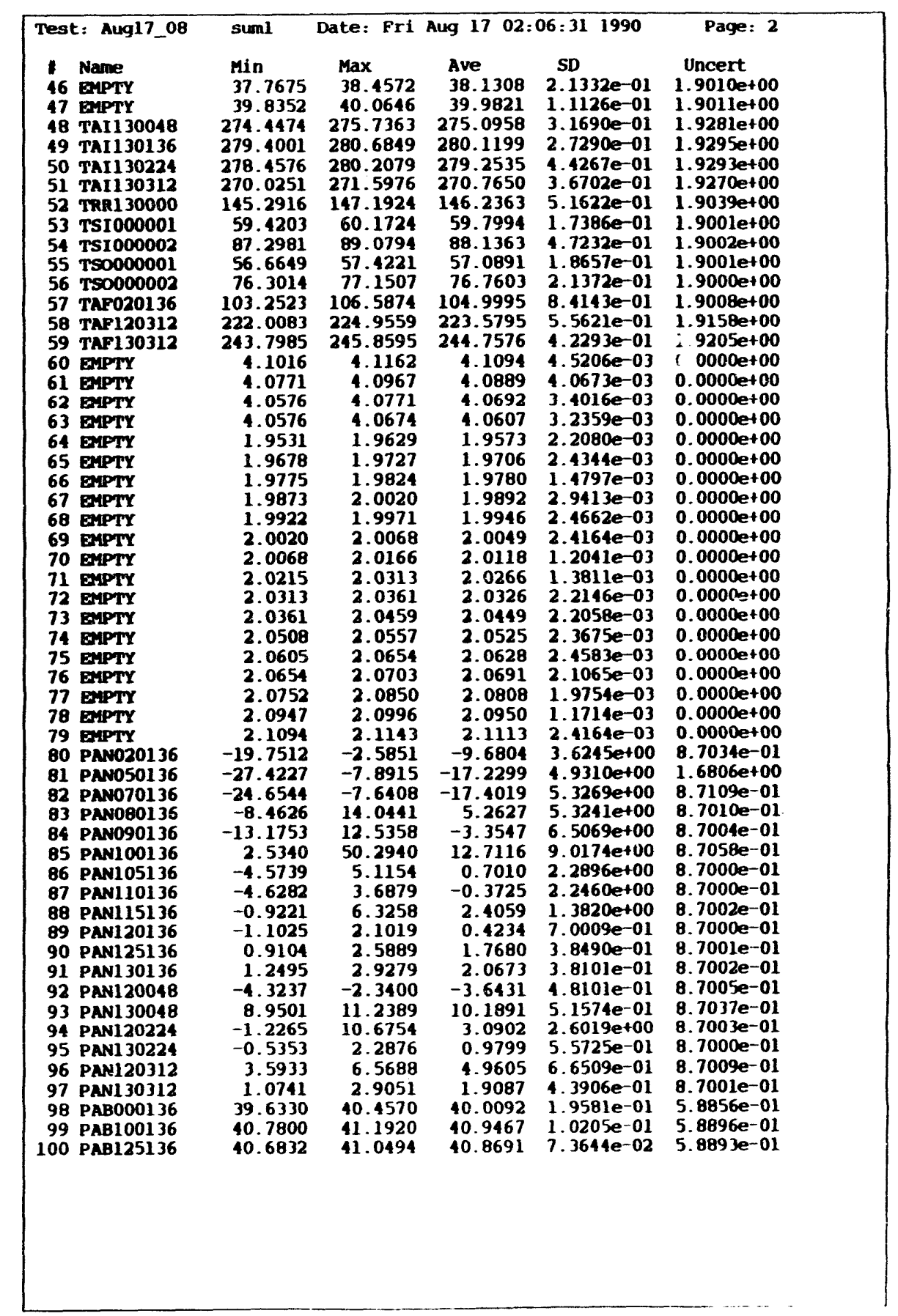




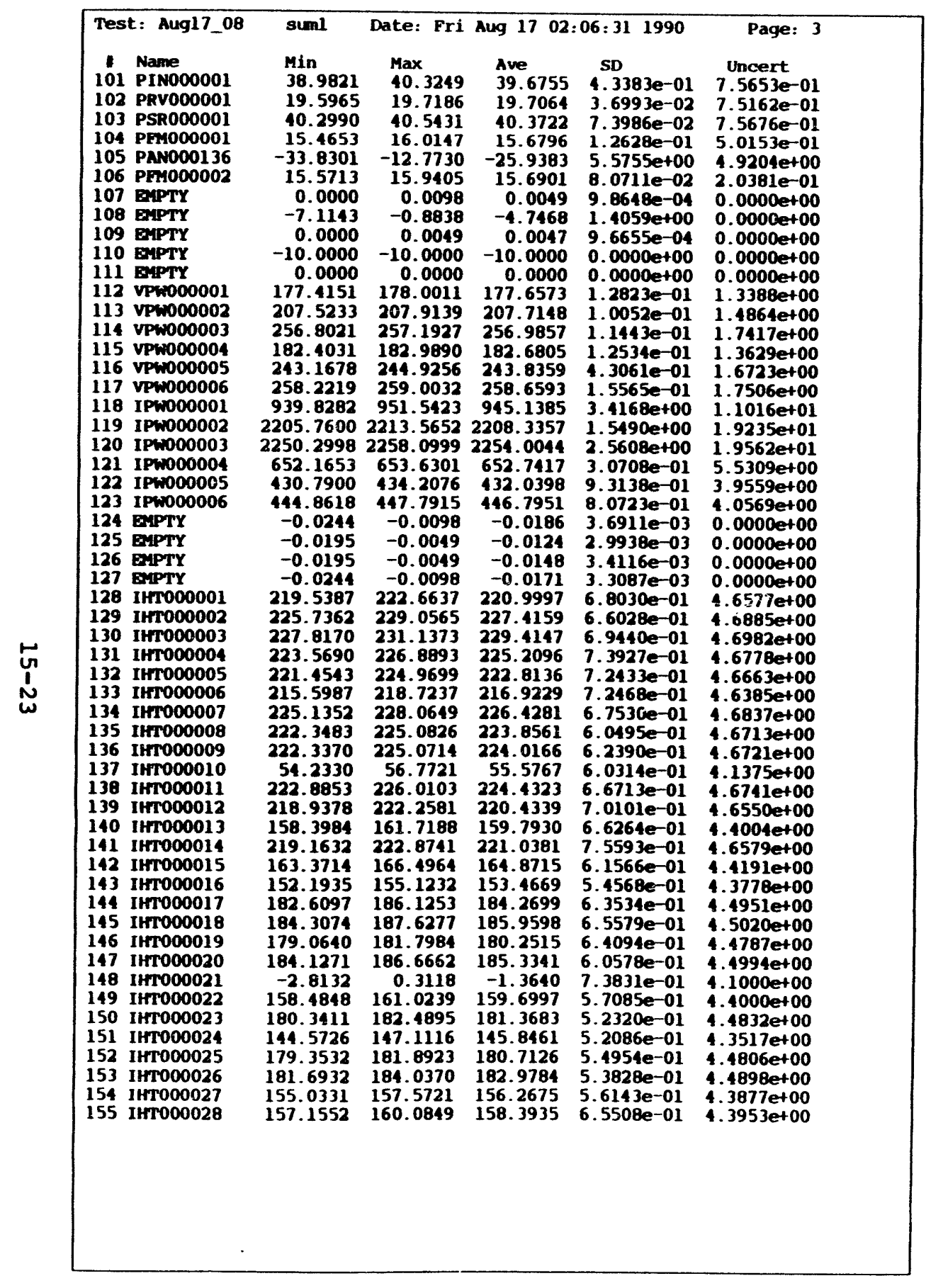

\begin{tabular}{|c|c|c|c|c|c|}
\hline st: $\mathbf{A t}$ & suml & Date: & Aug 17 & 990 & ge: \\
\hline $\begin{array}{cl}1 & \text { Name } \\
156 & \text { IHT000029 } \\
157 & \text { IHr000030 } \\
158 & \text { IHr000031 } \\
159 & \text { IHr000032 } \\
160 & \text { IHro! 0033 } \\
161 & \text { IHro00034 } \\
162 & \text { IHro00035 } \\
163 & \text { IHro00036 }\end{array}$ & $\begin{array}{l}\text { Min } \\
180.5965 \\
183.9656 \\
181.2275 \\
183.3045 \\
183.3271 \\
161.3206 \\
212.9808 \\
159.2060\end{array}$ & $\begin{array}{l}\operatorname{Max} \\
183.3308 \\
186.5047 \\
183.5712 \\
186.0389 \\
185.8661 \\
163.2737 \\
216.1058 \\
161.7450\end{array}$ & $\begin{array}{l}\text { Ave } \\
181.8192 \\
185.2040 \\
182.3564 \\
184.7851 \\
184.7295 \\
162.3051 \\
214.2073 \\
160.3974\end{array}$ & $\begin{array}{l}\text { SD } \\
6.5174 \mathrm{e}-01 \\
6.7383 \mathrm{e}-01 \\
6.5826 \mathrm{e}-01 \\
7.0043 \mathrm{e}-01 \\
6.9103 \mathrm{e}-01 \\
5.1593 \mathrm{e}-01 \\
7.0811 \mathrm{e}-01 \\
5.9353 \mathrm{e}-01\end{array}$ & $\begin{array}{l}\text { Uncert } \\
4.4851 \mathrm{e}+00 \\
4.4989 \mathrm{e}+00 \\
4.4872 \mathrm{e}+00 \\
4.4972 \mathrm{e}+00 \\
4.4969 \mathrm{e}+00 \\
4.4096 \mathrm{e}+00 \\
4.6258 \mathrm{e}+00 \\
4.4026 \mathrm{e}+00\end{array}$ \\
\hline
\end{tabular}


DEFECTIVE INSTRUMENTS FOR THE RIBBED GEOMETRY TEST SERIES

\begin{tabular}{|c|c|c|}
\hline Series & Tests & Defective Instruments \\
\hline 6 & Aug17_01 to Aug17_08 & $\begin{array}{lll}\text { TAO120048 } & \text { TAF070136 } & \text { TRT120000 } \\
\text { TAO020136 } & \text { TAF080136 } & \text { TRT125000 } \\
\text { TAO070136 TAF090136 } & \text { TRR130000 } \\
\text { TAO080136 } & \text { TAF120136 } & \\
\text { TAO110136 } & & \\
\text { TAO125136 } & & \\
& & \\
\text { IHT000010 } & \text { IHT000021 } & \text { IHT000024 } \\
\text { IHT000033 } & & \end{array}$ \\
\hline
\end{tabular}




\section{জ్Sreare}

\section{Chapter 16}

NUCLEATE BOILING PRESSURE DROP IN AN ANNULUS

Test Geometry: Ribbed (4)

Test Series: 7

Test Date: August 17, 1990 


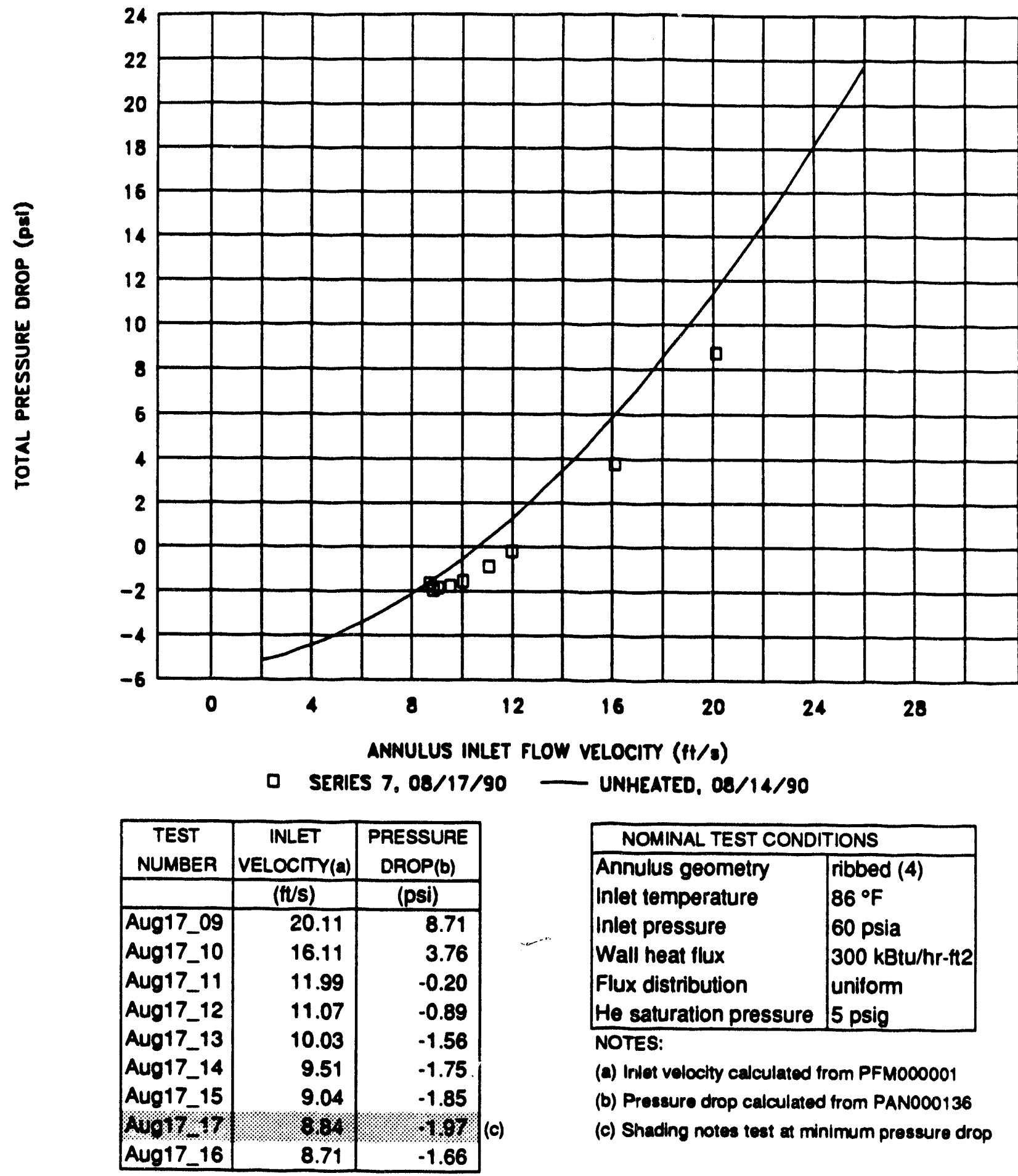

DEMAND CURVE AND DATA FOR SERIES 7 - RIBBED ANNULUS 

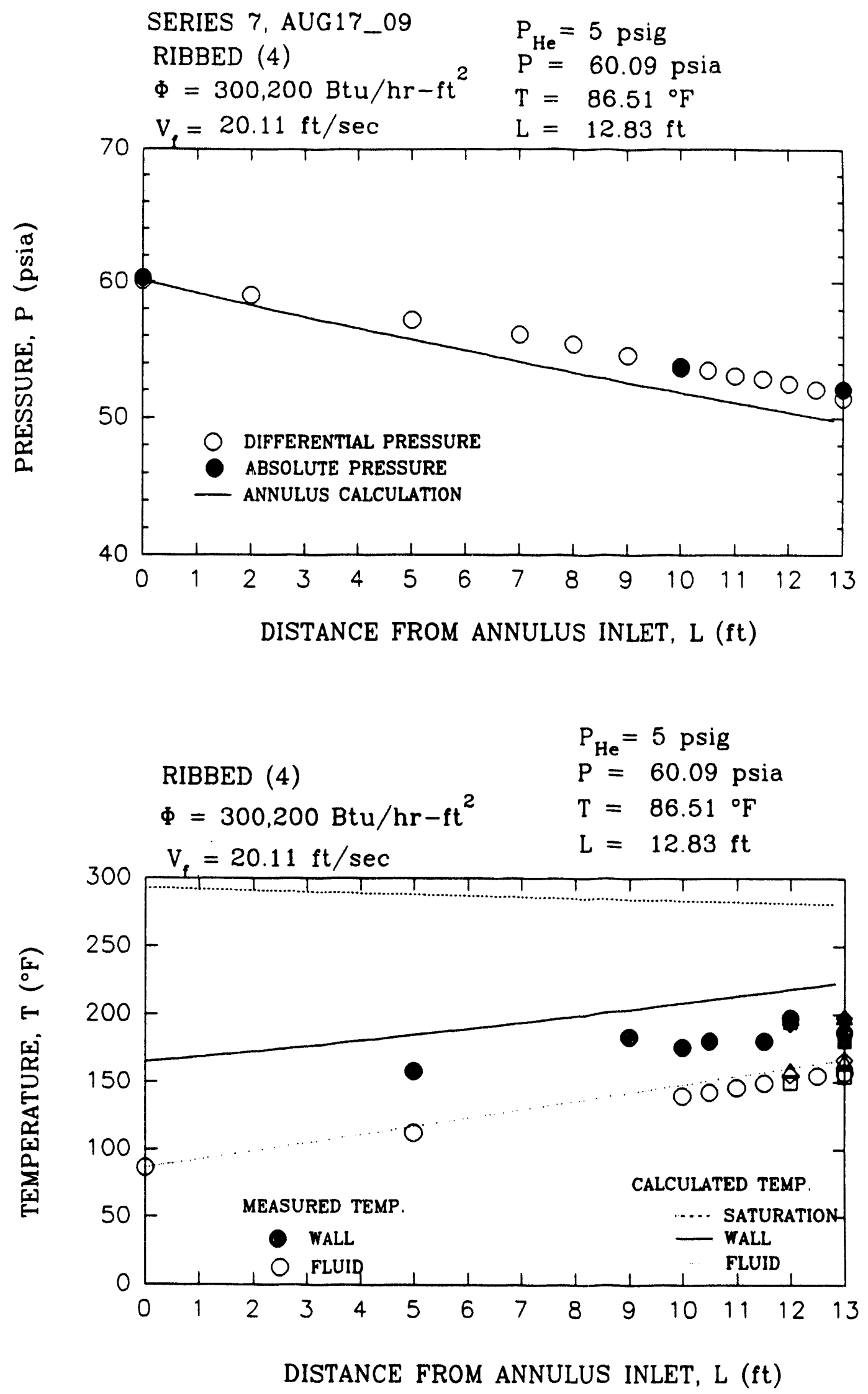

AUG17_09 

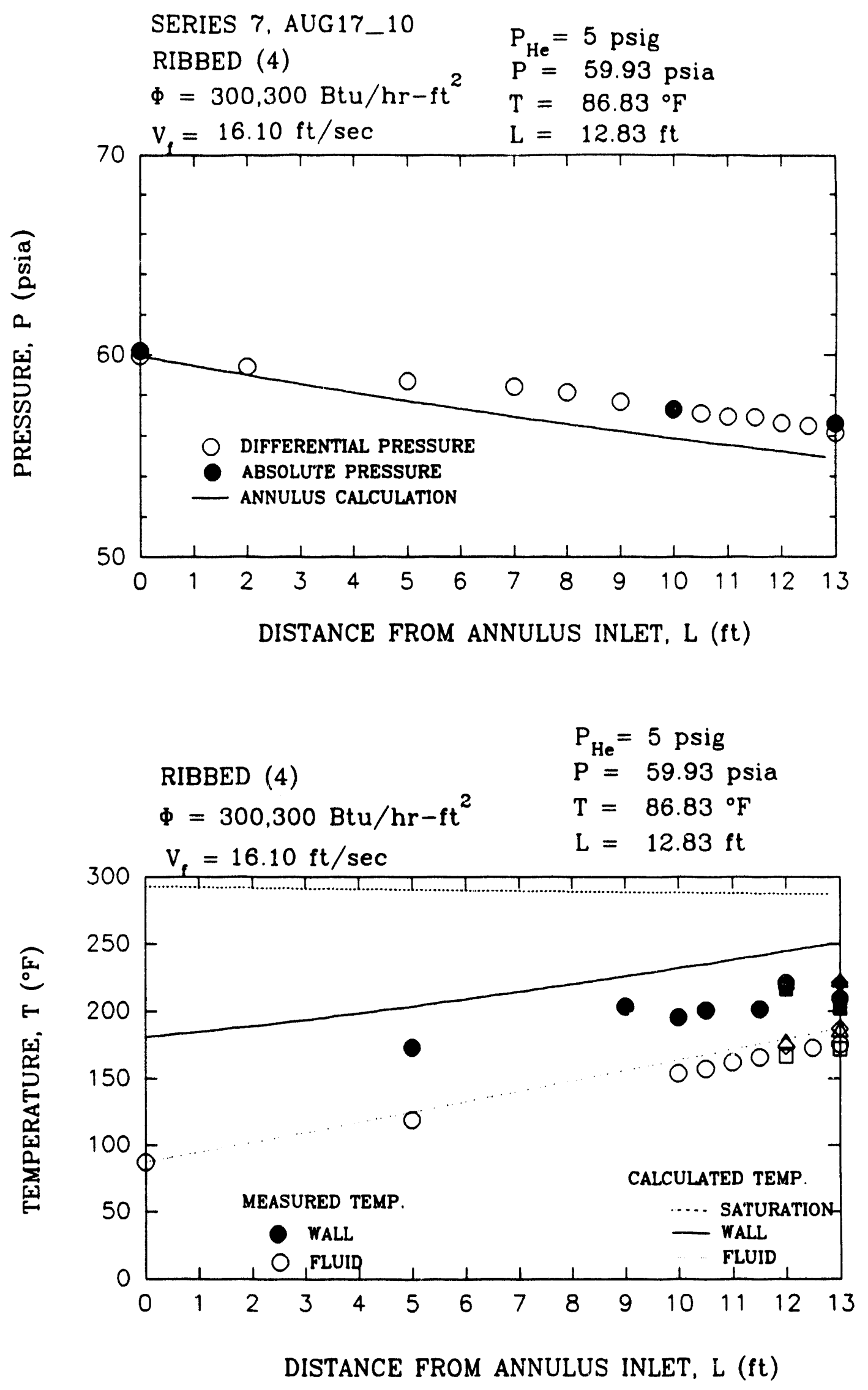

AUG17_10 

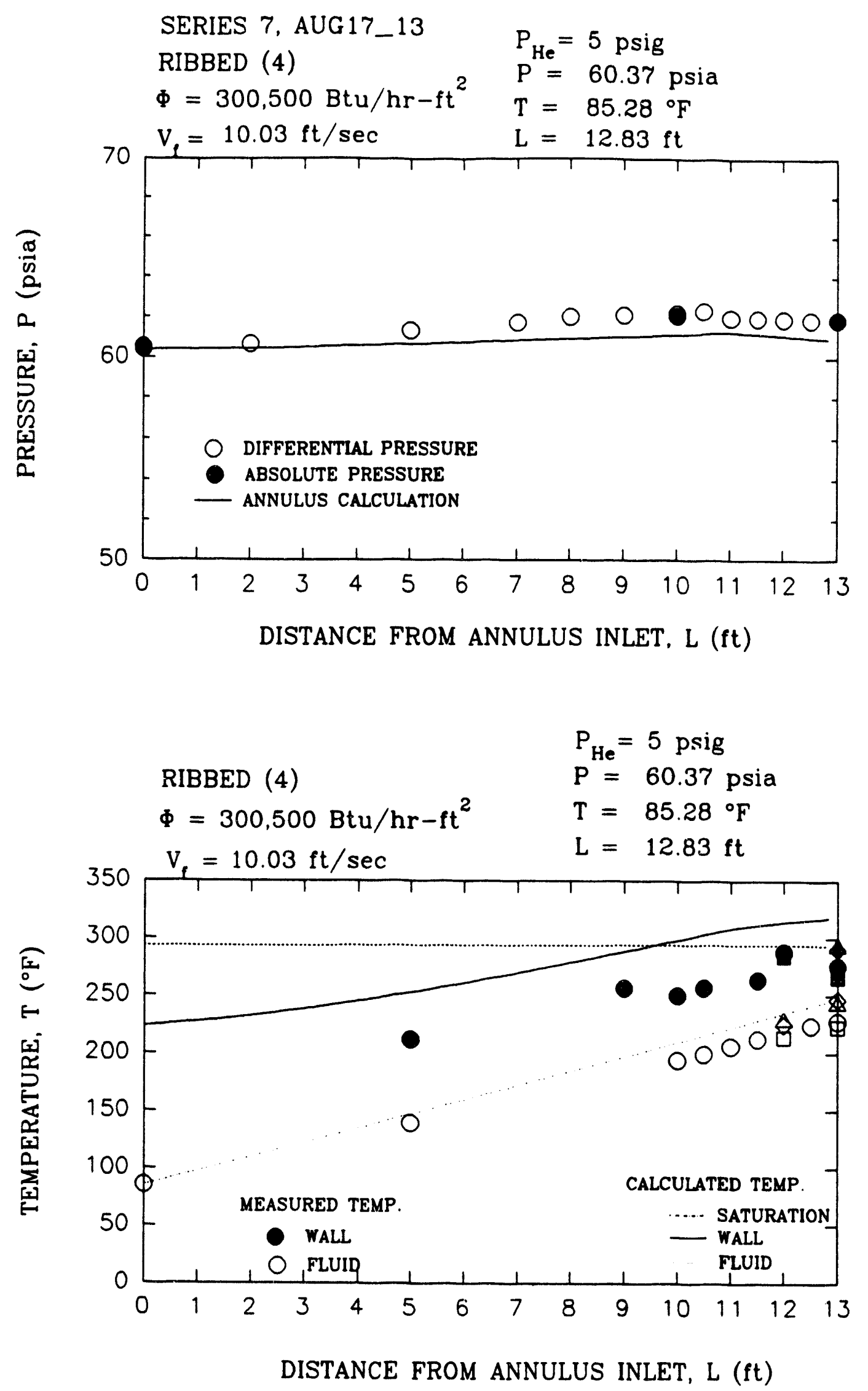

AUG17_13 

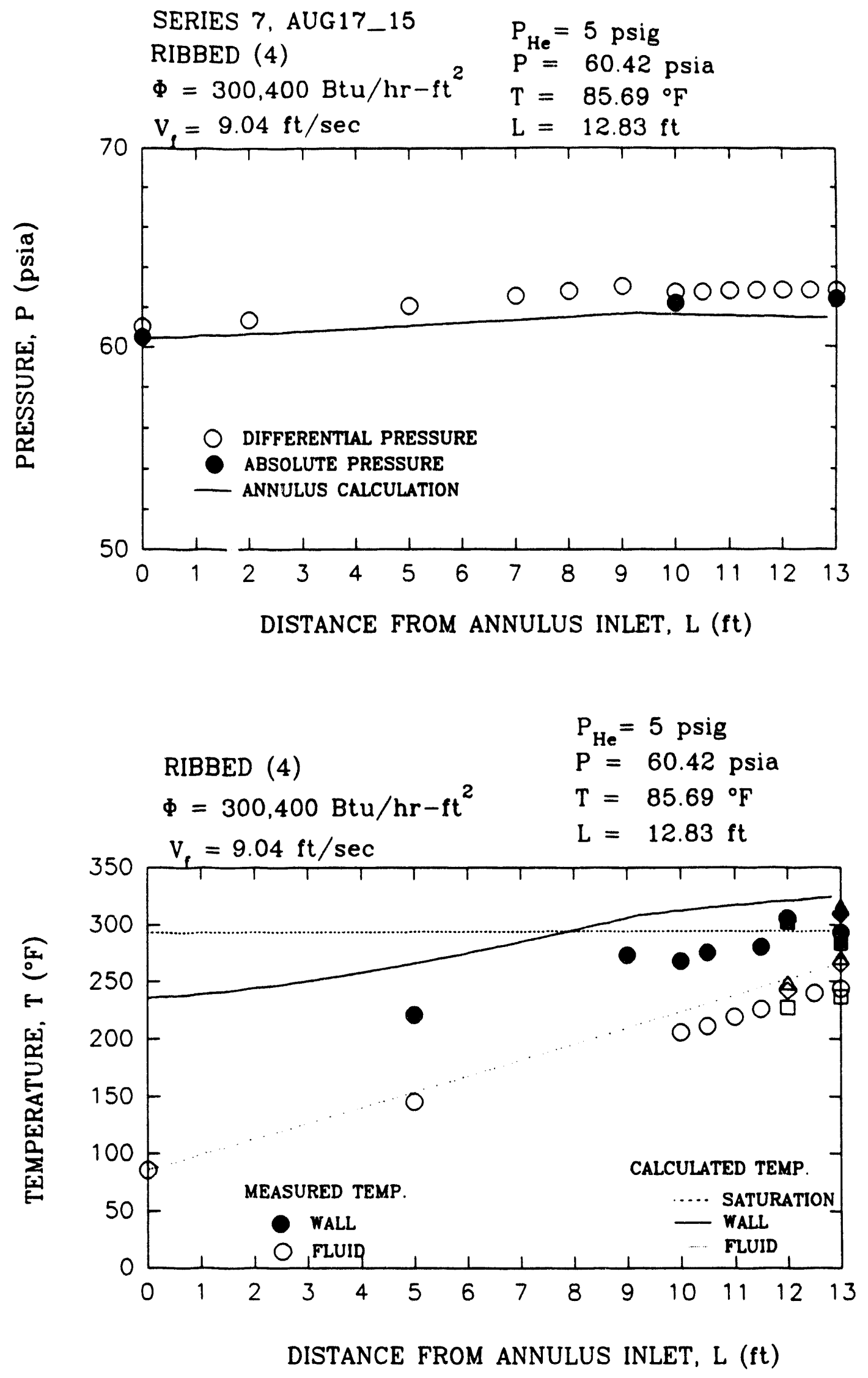

AUG17_15 

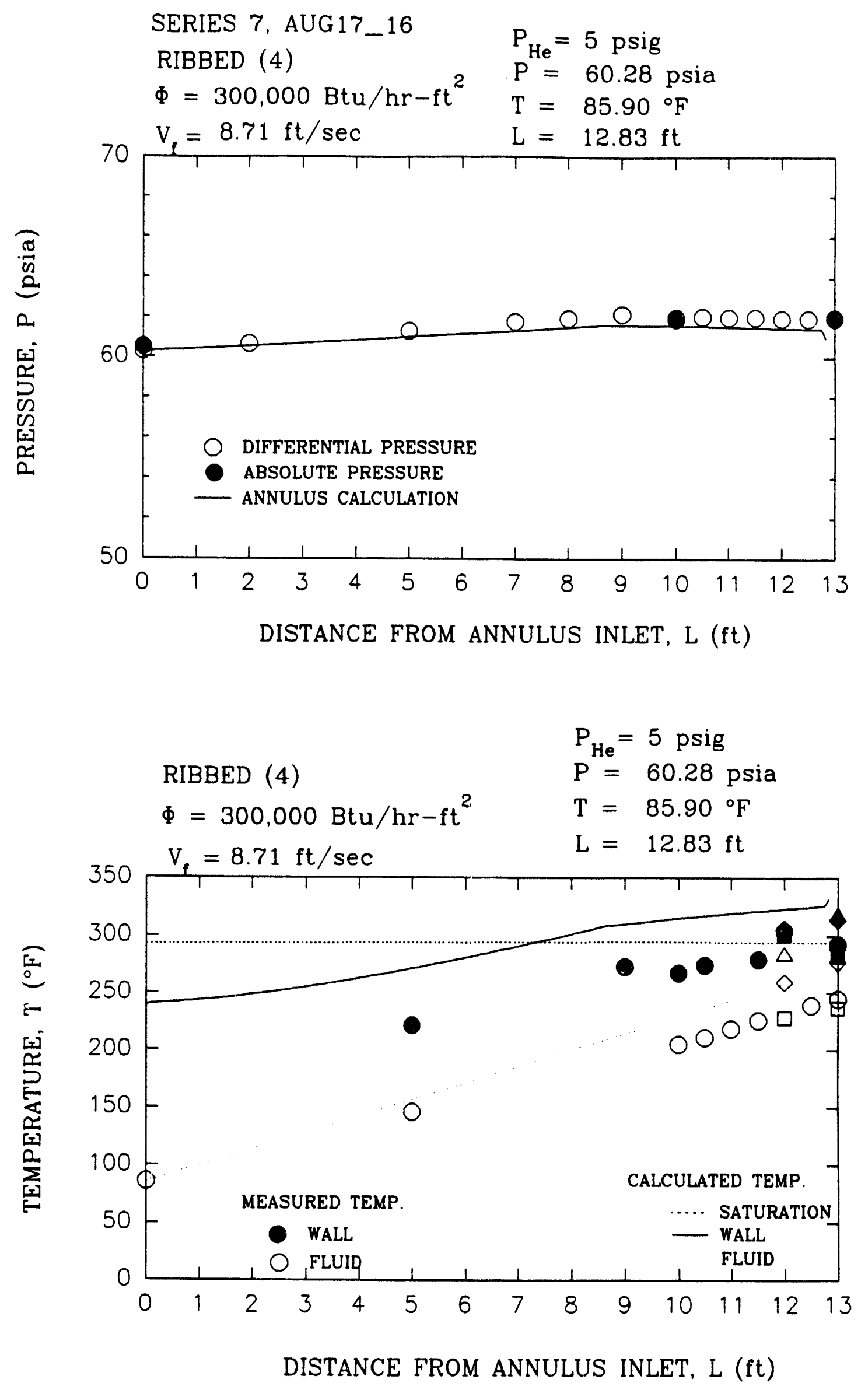

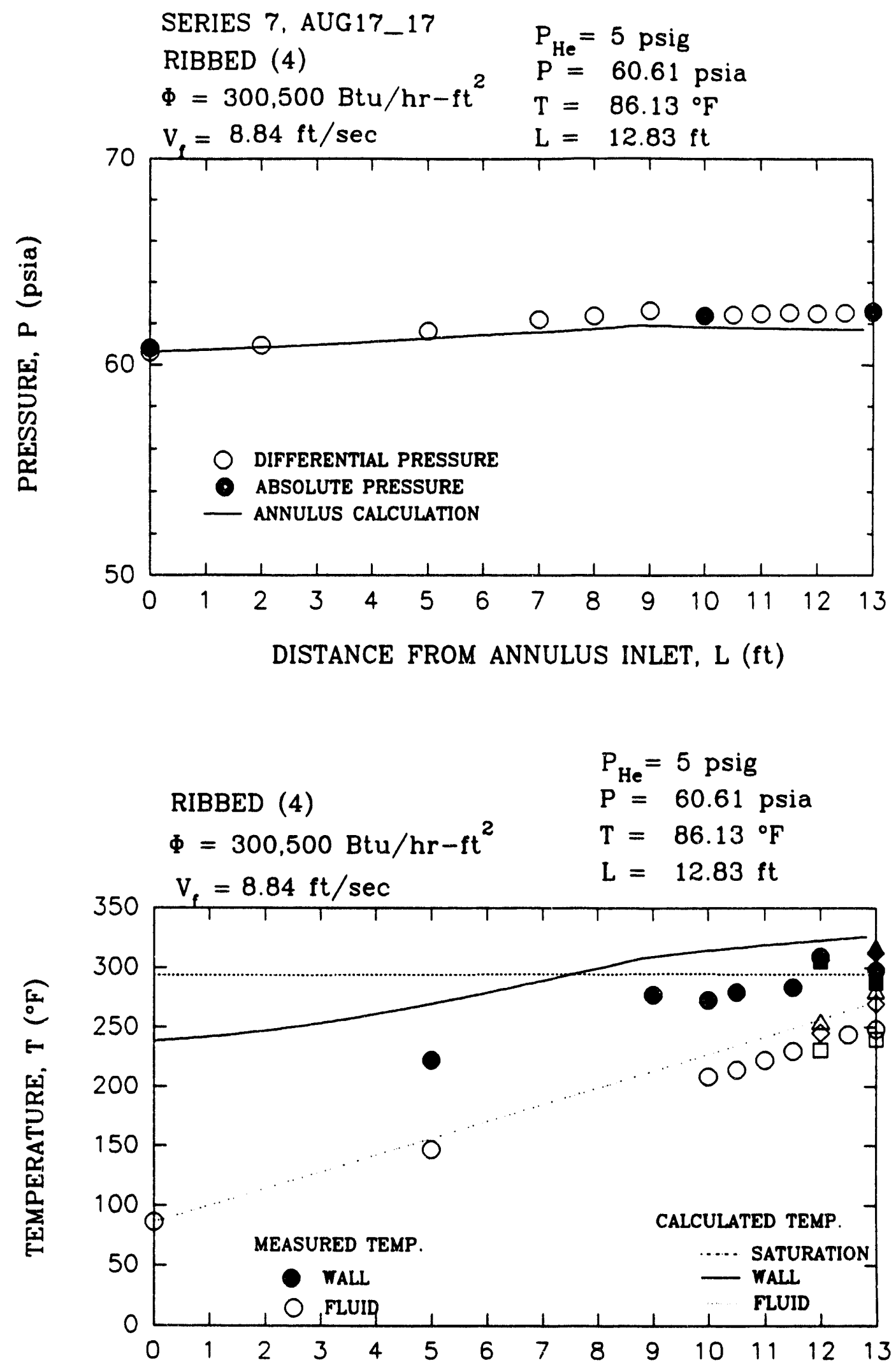

DISTANCE FROM ANNULUS INLET, L (ft) 


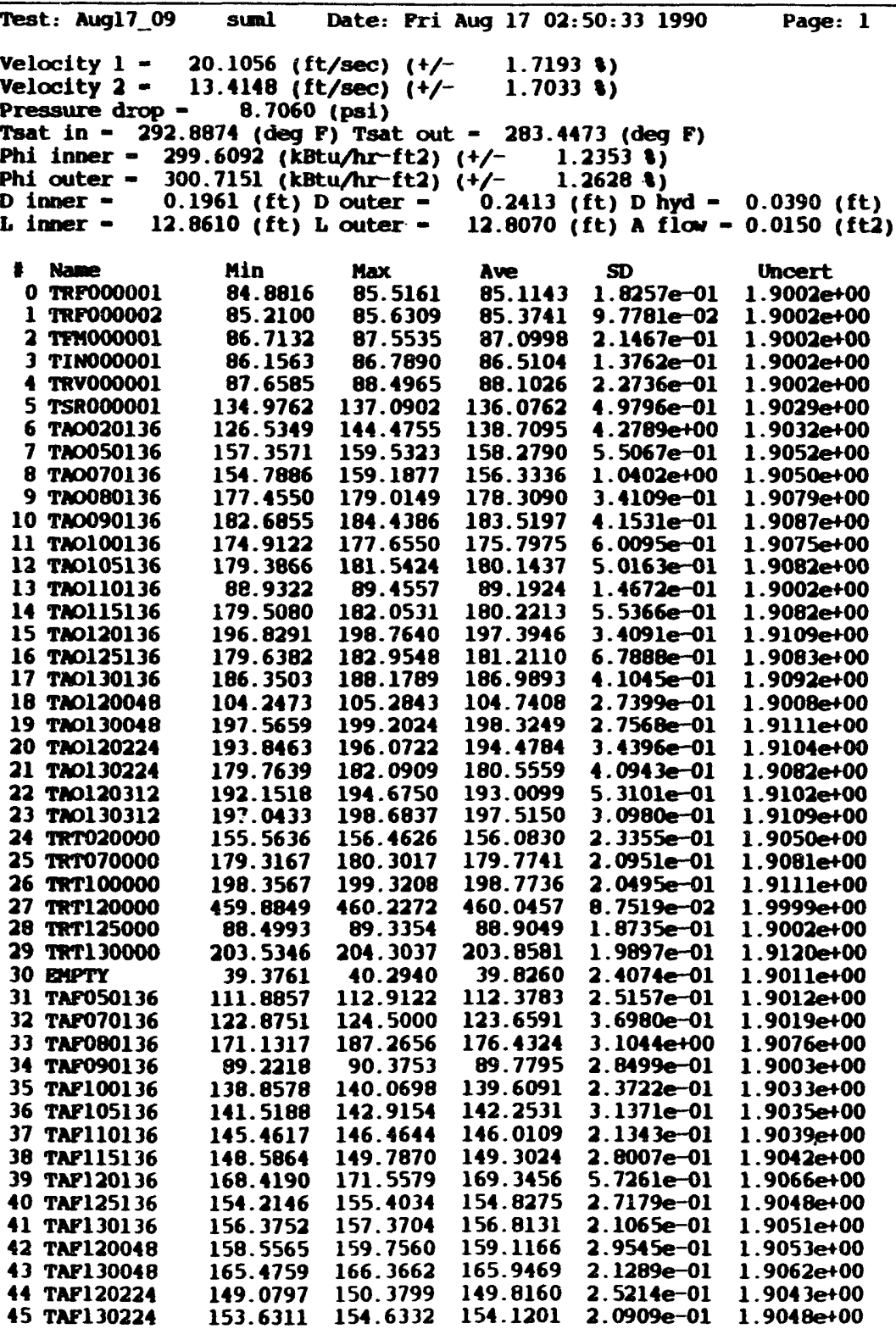

\begin{tabular}{|c|c|c|c|c|c|c|}
\hline Test & $t:$ Aug17_09 & suml & Date: Fri & Aug 17 02: & $50: 331990$ & Page: 2 \\
\hline 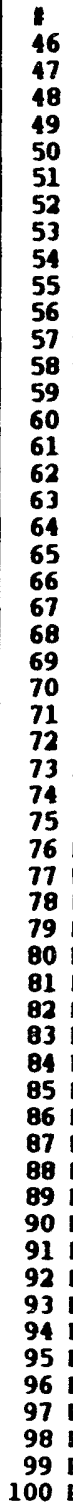 & 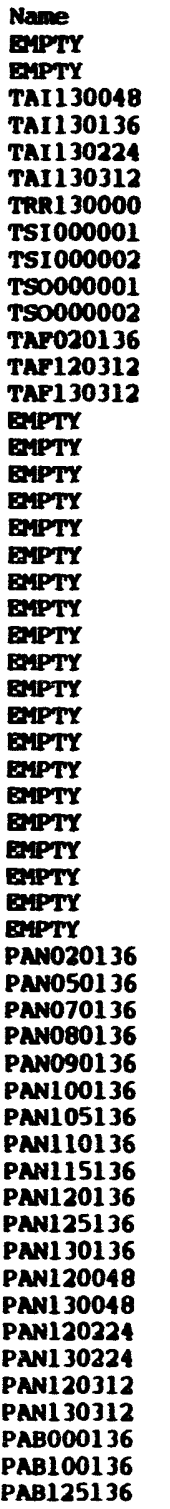 & $\begin{array}{r}\text { Min } \\
37.7675 \\
39.3761 \\
189.9708 \\
196.8506 \\
202.1850 \\
186.7266 \\
119.5449 \\
58.1344 \\
77.6170 \\
56.2316 \\
68.3245 \\
93.7162 \\
154.5182 \\
165.5957 \\
2.1240 \\
2.1289 \\
2.1240 \\
2.1289 \\
1.1084 \\
1.1279 \\
1.1426 \\
1.1572 \\
1.1670 \\
1.1816 \\
1.1963 \\
1.2158 \\
1.2256 \\
1.2451 \\
1.2549 \\
1.2744 \\
1.2842 \\
1.2988 \\
1.3184 \\
1.3379 \\
19.9216 \\
44.1410 \\
23.6397 \\
9.8479 \\
14.5720 \\
10.1634 \\
-4.3451 \\
-1.5764 \\
-5.1945 \\
-1.7991 \\
-1.9887 \\
5.4456 \\
5.8997 \\
6.5087 \\
1.9016 \\
16.6308 \\
5.2718 \\
11.2212 \\
59.8204 \\
53.2312 \\
51.5780\end{array}$ & $\begin{array}{r}\operatorname{Max} \\
38.6870 \\
39.8352 \\
191.5248 \\
197.9131 \\
203.7306 \\
188.1890 \\
132.0267 \\
58.8878 \\
83.8347 \\
57.2056 \\
69.3927 \\
94.7672 \\
156.1138 \\
168.0619 \\
2.1484 \\
2.1484 \\
2.1387 \\
2.1436 \\
1.1182 \\
1.1328 \\
1.1475 \\
1.1621 \\
1.1768 \\
1.1914 \\
1.2012 \\
1.2207 \\
1.2354 \\
1.2500 \\
1.2598 \\
1.2744 \\
1.2891 \\
1.3037 \\
1.3281 \\
1.3428 \\
40.8262 \\
54.8222 \\
36.2282 \\
28.6162 \\
35.9580 \\
36.7137 \\
19.6112 \\
24.3635 \\
18.3803 \\
23.3879 \\
26.2400 \\
26.5028 \\
38.7824 \\
33.2115 \\
22.1958 \\
32.2711 \\
33.5006 \\
34.9486 \\
60.7359 \\
53.9636 \\
52.2646\end{array}$ & $\begin{array}{r}\text { Ave } \\
38.0986 \\
39.6057 \\
190.6080 \\
197.2837 \\
202.9385 \\
187.4714 \\
123.4921 \\
58.5429 \\
79.8793 \\
56.7250 \\
68.8542 \\
94.1788 \\
155.1681 \\
166.4561 \\
2.1368 \\
2.1354 \\
2.1314 \\
2.1361 \\
1.1130 \\
1.1321 \\
1.1457 \\
1.1616 \\
1.1719 \\
1.1866 \\
1.1997 \\
1.2187 \\
1.2305 \\
1.2453 \\
1.2595 \\
1.2744 \\
1.2847 \\
1.3034 \\
1.3229 \\
1.3411 \\
30.3022 \\
49.8234 \\
30.3673 \\
19.1130 \\
23.0292 \\
21.4900 \\
7.7231 \\
12.0253 \\
5.6865 \\
10.6865 \\
12.0677 \\
17.0652 \\
23.8364 \\
18.8469 \\
15.5887 \\
23.2074 \\
20.3185 \\
22.0717 \\
60.2525 \\
53.6991 \\
52.0660\end{array}$ & $\begin{array}{c}\text { SD } \\
2.3756 e-01 \\
1.2270 e-01 \\
3.5441 e-01 \\
2.2273 e-01 \\
3.5503 e-01 \\
3.0891 e-01 \\
3.3317 e+00 \\
1.7909 e-01 \\
1.7811 e+00 \\
2.0537 e-01 \\
2.1464 e-01 \\
2.8568 e-01 \\
3.3544 e-01 \\
4.8532 e-01 \\
4.8216 e-03 \\
4.1196 e-03 \\
3.5895 e-03 \\
4.3270 e-03 \\
2.0716 e-03 \\
1.7115 e-03 \\
2.3675 e-03 \\
1.4797 e-03 \\
9.8648 e-04 \\
1.2041 e-03 \\
2.2603 e-03 \\
2.4344 e-03 \\
1.3951 e-03 \\
9.6655 e-04 \\
1.1714 e-03 \\
0.0000 e+00 \\
1.4797 e-03 \\
1.1719 e-03 \\
1.6624 e-03 \\
2.3365 e-03 \\
5.1040 e+00 \\
2.5675 e+00 \\
3.5237 e+00 \\
4.9962 e+00 \\
5.8108 e+00 \\
6.8101 e+00 \\
7.1518 e+00 \\
6.8774 e+00 \\
5.7770 e+00 \\
5.4254 e+00 \\
6.0559 e+00 \\
5.2617 e+00 \\
8.1708 e+00 \\
5.9769 e+00 \\
5.3925 e+00 \\
2.9160 e+00 \\
6.0183 e+00 \\
5.8903 e+00 \\
2.5699 e-01 \\
1.8778 e-01 \\
1.3131 e-01 \\
\end{array}$ & $\begin{array}{l}\text { Uncert } \\
1.9010 e+00 \\
1.9011 e+00 \\
1.9098 e+00 \\
1.9109 e+00 \\
1.9119 e+00 \\
1.9093 e+00 \\
1.9019 e+00 \\
1.9001 e+00 \\
1.9001 e+00 \\
1.9001 e+00 \\
1.9000 e+00 \\
1.9004 e+00 \\
1.9049 e+00 \\
1.9063 e+00 \\
0.0000 e+00 \\
0.0000 e+00 \\
0.0000 e+00 \\
0.0000 e+00 \\
0.0000 e+00 \\
0.0000 e+00 \\
0.0000 e+00 \\
0.0000 e+00 \\
0.0000 e+00 \\
0.0000 e+00 \\
0.0000 e+00 \\
0.0000 e+00 \\
0.0000 e+00 \\
0.0000 e+00 \\
0.0000 e+00 \\
0.0000 e+00 \\
0.0000 e+00 \\
0.0000 e+00 \\
0.0000 e+00 \\
0.0000 e+00 \\
8.7329 e-01 \\
1.6846 e+00 \\
8.7331 e-01 \\
8.7131 e-01 \\
8.7190 e-01 \\
8.7166 e-01 \\
8.7021 e-01 \\
8.7052 e-01 \\
8.7012 e-01 \\
8.7041 e-01 \\
8.7052 e-01 \\
8.7105 e-01 \\
8.7204 e-01 \\
8.7127 e-01 \\
8.7087 e-01 \\
8.7193 e-01 \\
8.7148 e-01 \\
8.7175 e-01 \\
5.9924 e-01 \\
5.9533 e-01 \\
5.9443 e-01\end{array}$ \\
\hline
\end{tabular}



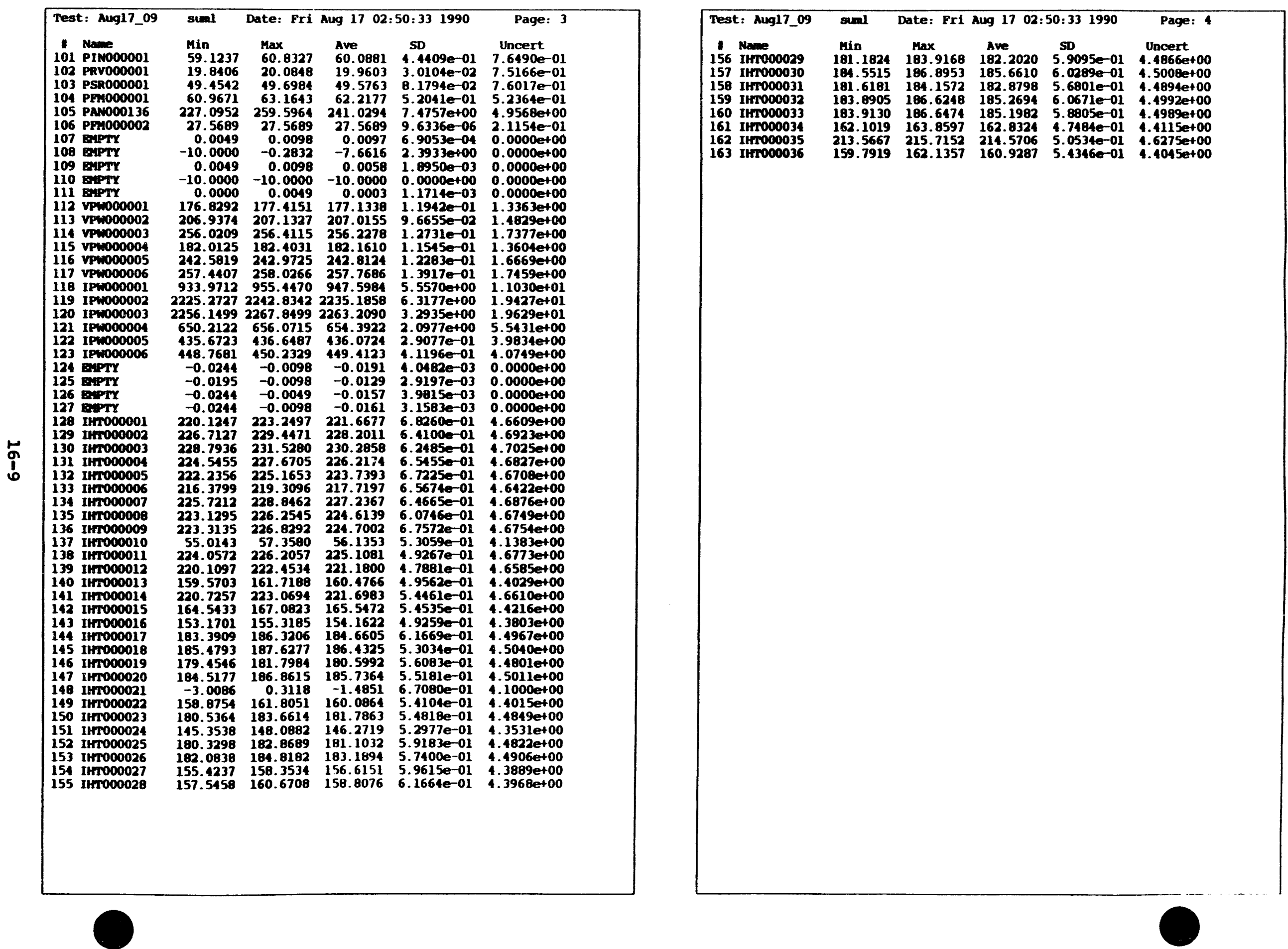


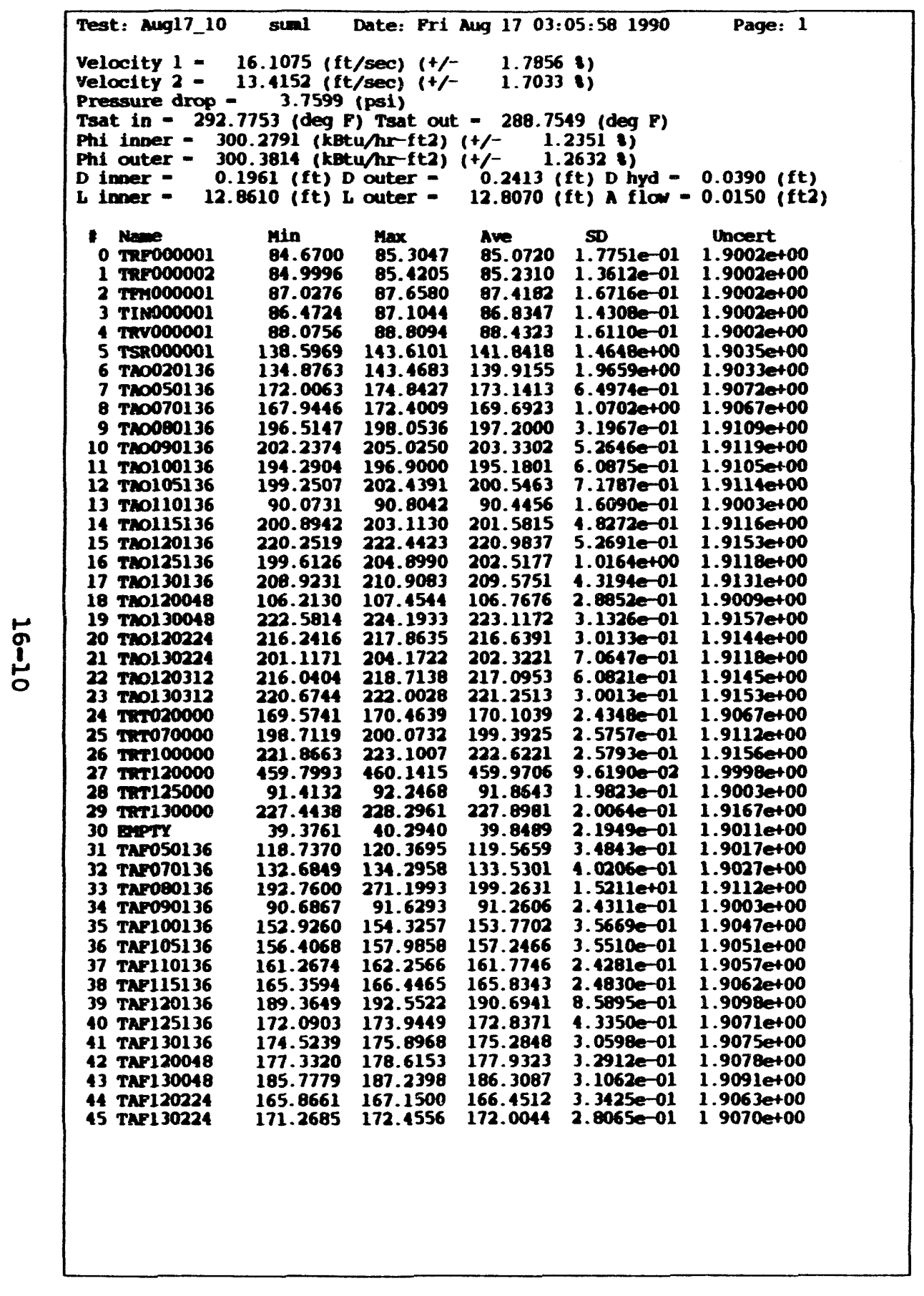

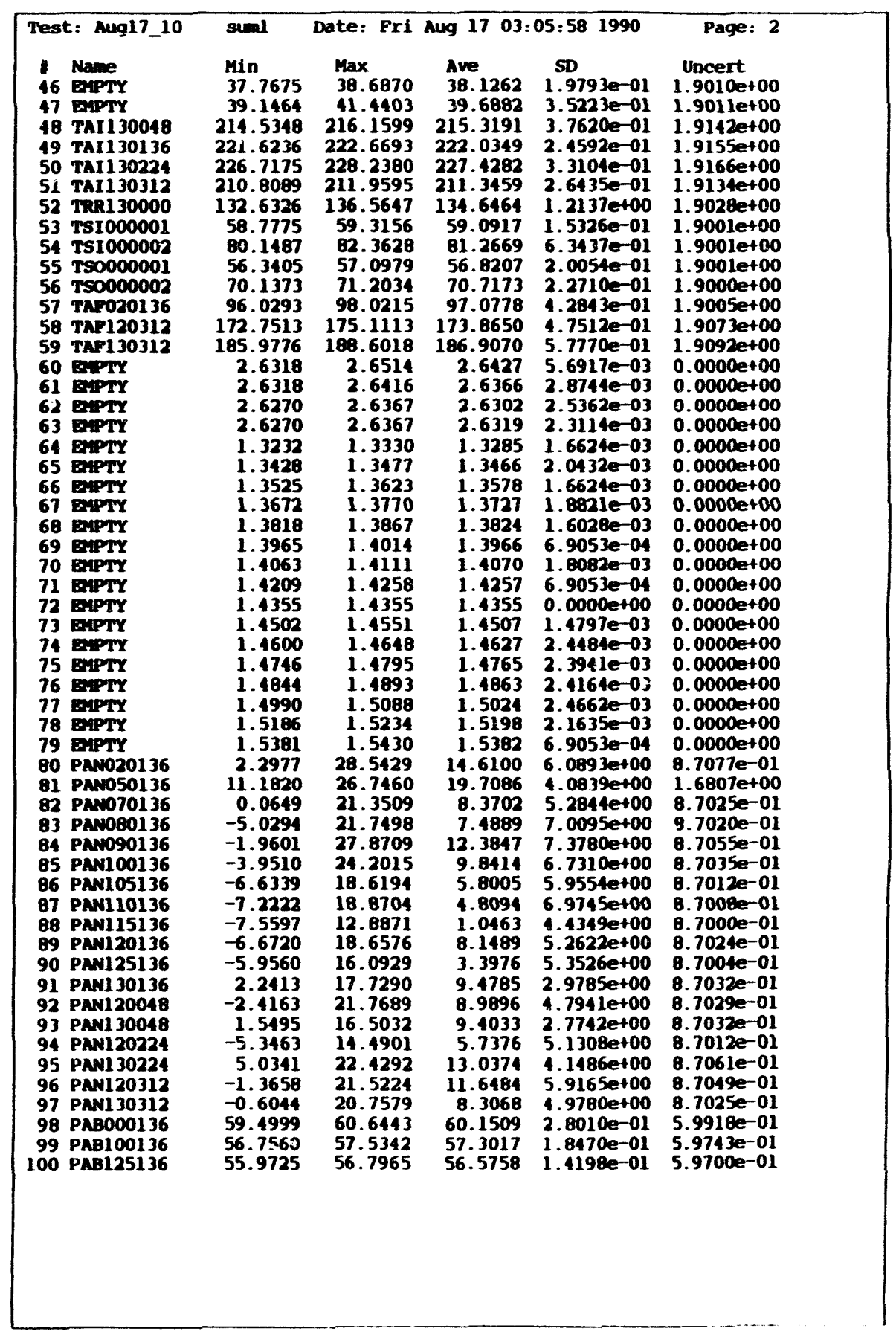




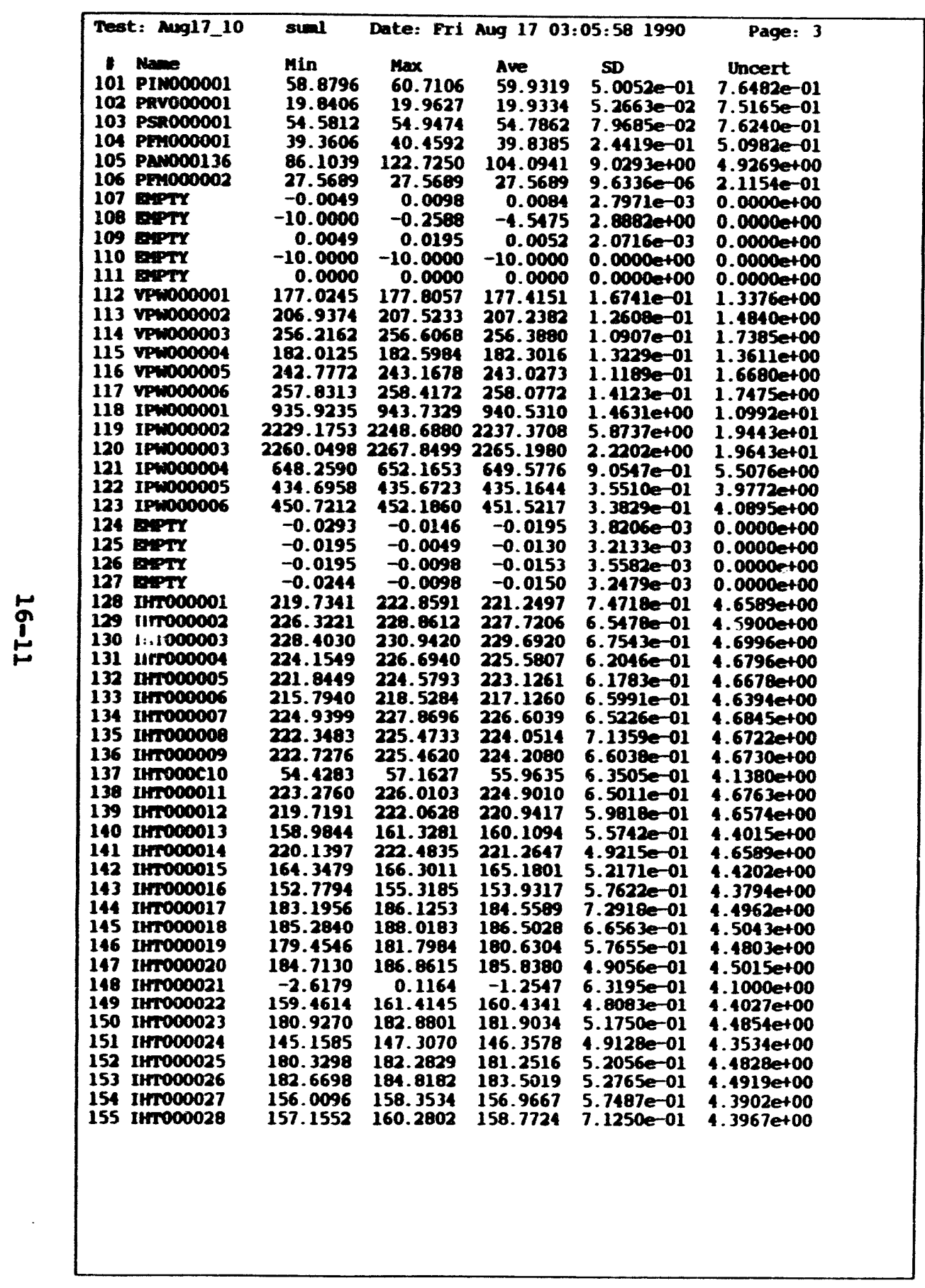

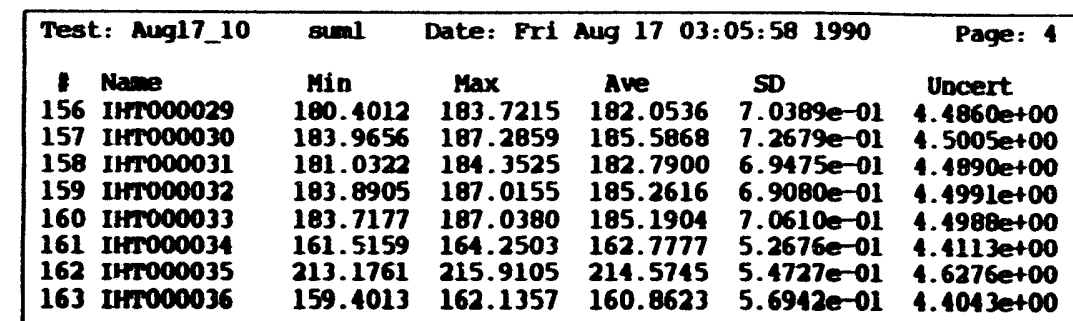




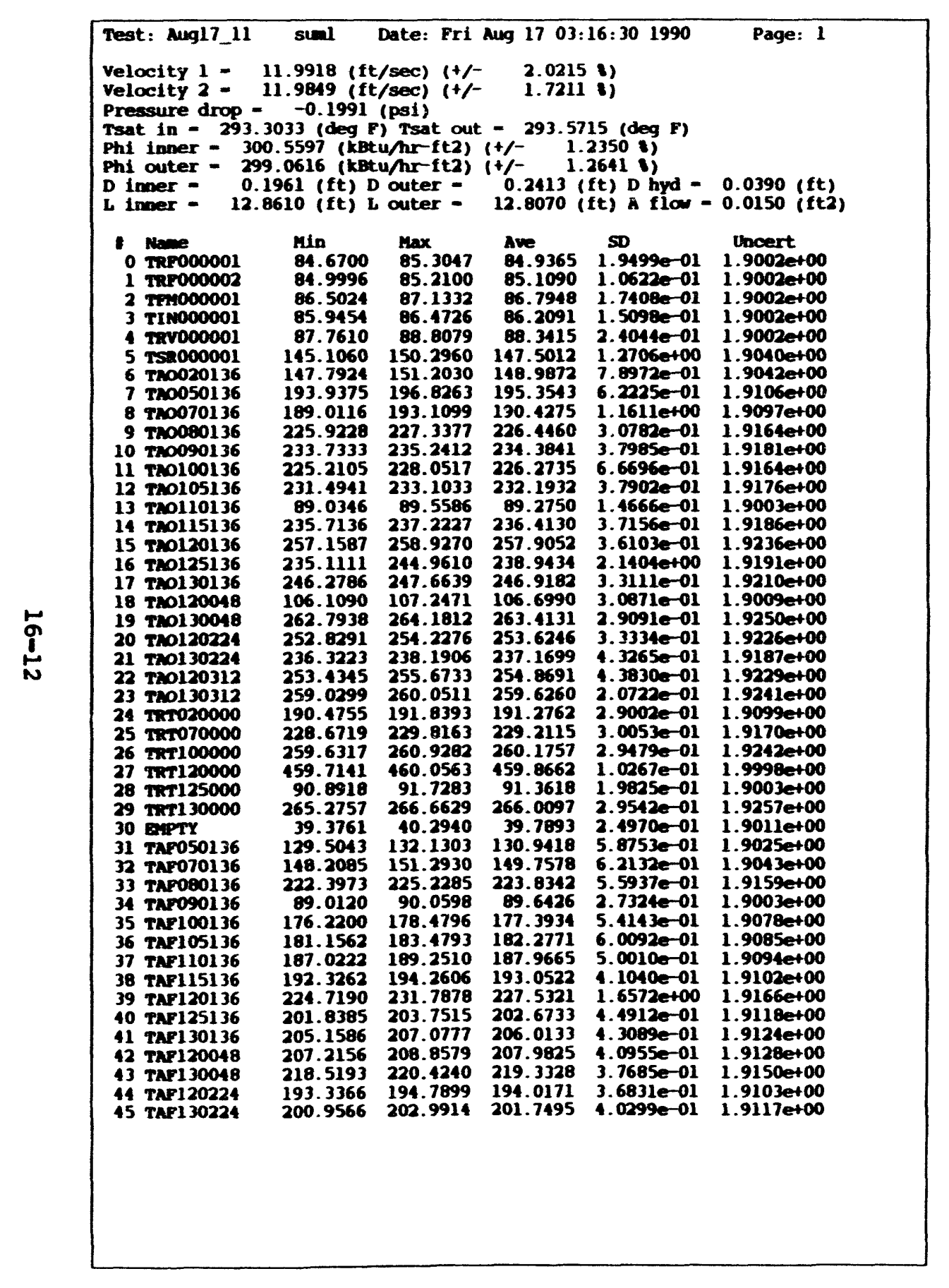

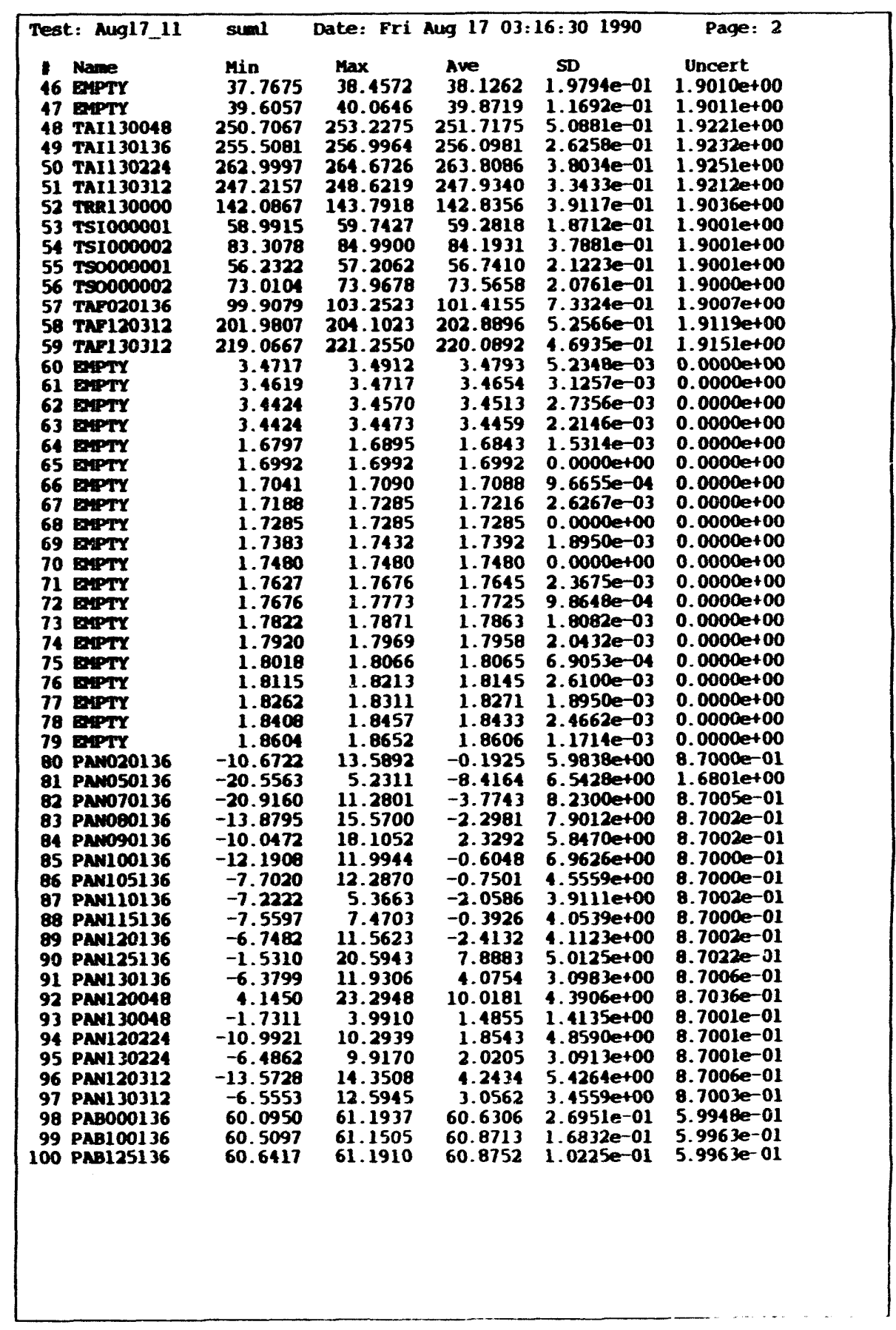




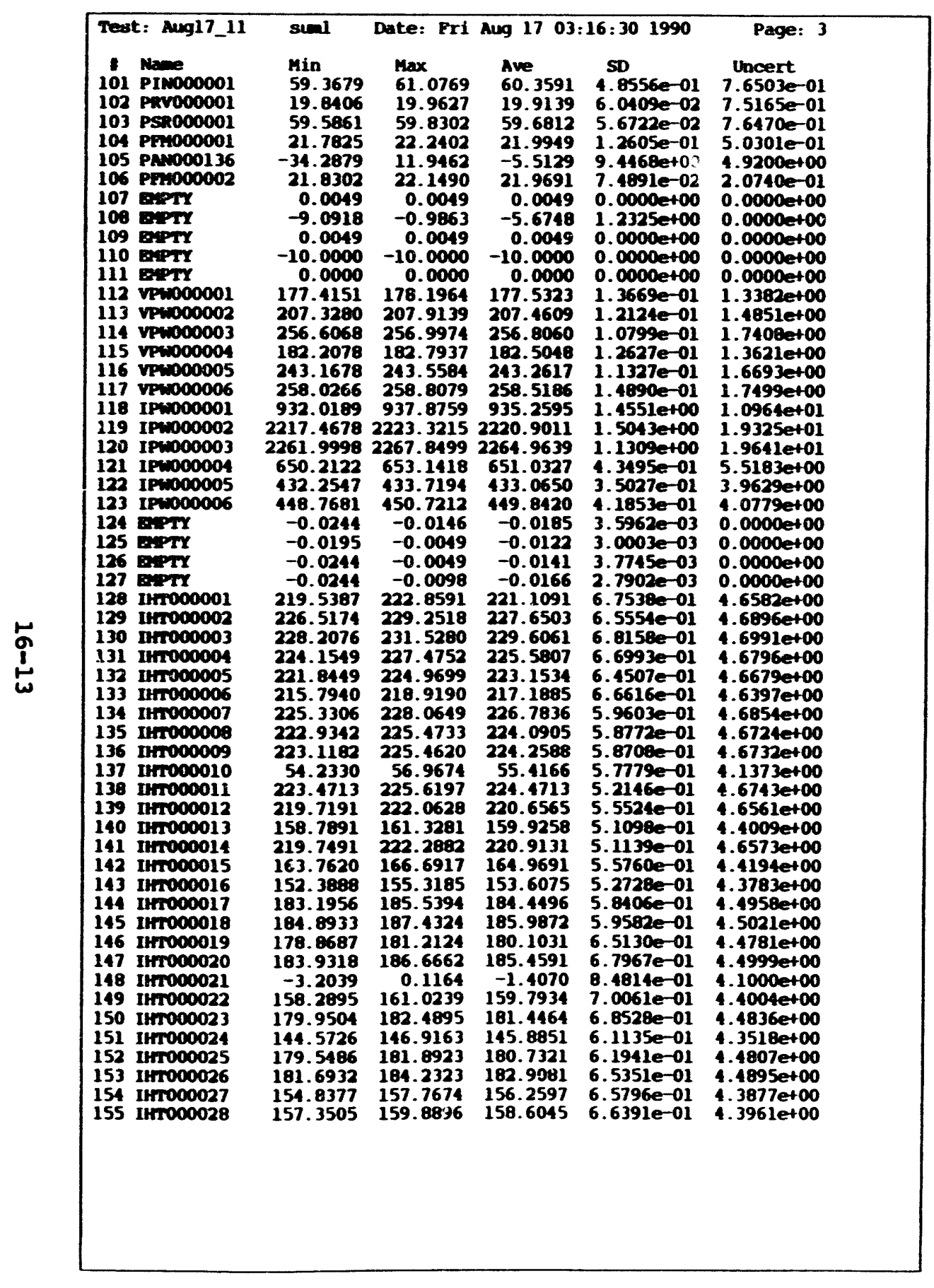

\begin{tabular}{|c|c|c|c|c|c|}
\hline Test: $\mathbf{M u}$ & 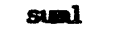 & ate: Fri & aug 17 & 30 & Page: \\
\hline 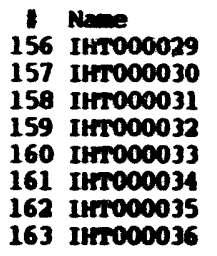 & $\begin{array}{l}\min \\
180.5965 \\
184.1609 \\
181.4228 \\
183.6951 \\
183.7177 \\
161.5159 \\
212.9808 \\
159.5966\end{array}$ & $\begin{array}{c}\max \\
182.9402 \\
186.7000 \\
183.9618 \\
186.2342 \\
186.2568 \\
163.2737 \\
215.1292 \\
161.5497\end{array}$ & $\begin{array}{c}\text { Ave } \\
181.9286 \\
185.4266 \\
182.5751 \\
184.9335 \\
184.8896 \\
162.3519 \\
214.0472 \\
160.5849\end{array}$ & $\begin{array}{l}\text { SD } \\
6.4600 e-01 \\
6.4264 e-01 \\
5.8160 e-01 \\
5.6305 e-01 \\
5.6082 e-01 \\
3.9467 e-01 \\
4.4154 e-01 \\
4.1649 e-01\end{array}$ & $\begin{array}{l}\text { 4.4855et } \\
4.4998 \mathrm{e} \\
4.4881 \mathrm{et} \\
4.4978 \mathrm{et} \\
4.4976 \mathrm{et} \\
4.4097 \mathrm{et} \\
4.6251 \mathrm{et} \\
4.403 \mathrm{et}\end{array}$ \\
\hline
\end{tabular}




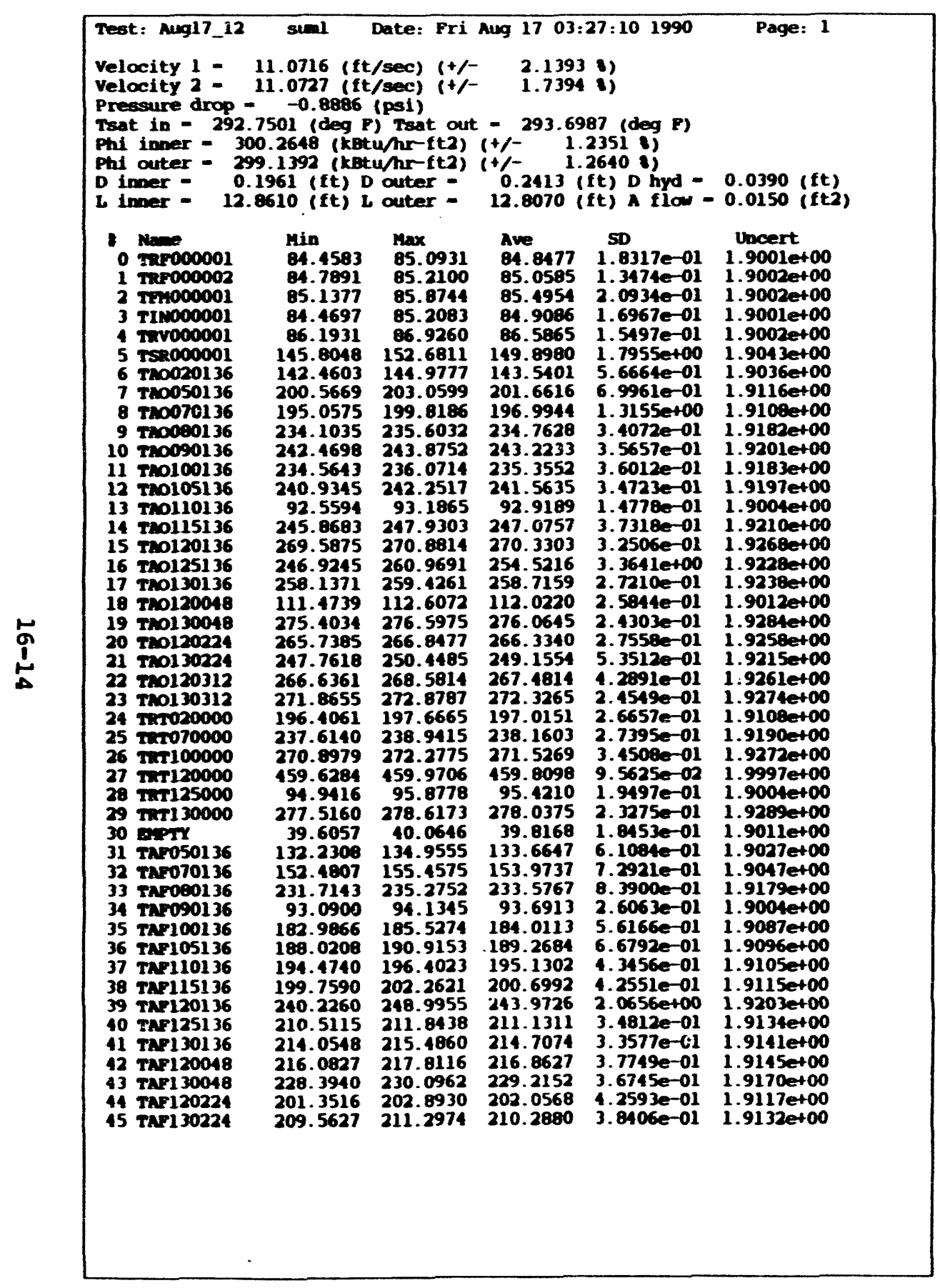

\begin{tabular}{|c|c|c|c|c|c|}
\hline 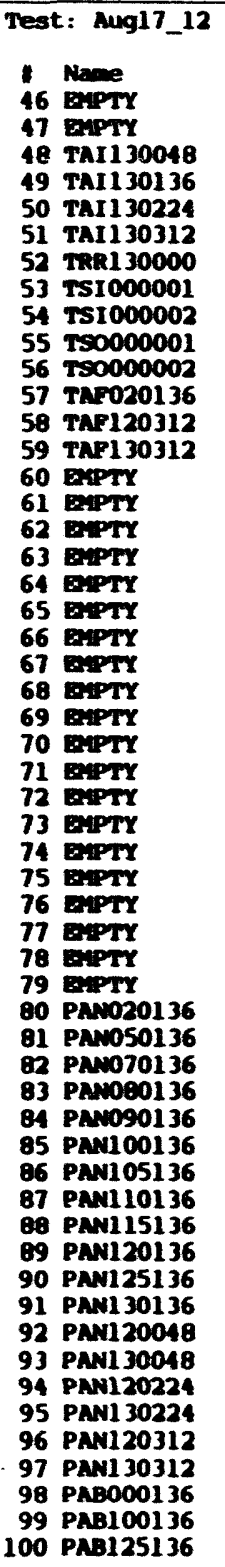 & $\begin{aligned} \text { san } \\
\text { Min } \\
37.7675 \\
39.6057 \\
262.0613 \\
265.7876 \\
274.1207 \\
258.1462 \\
149.3828 \\
59.2054 \\
85.9330 \\
56.1239 \\
74.1792 \\
100.2217 \\
210.1579 \\
229.2128 \\
3.7305 \\
3.7207 \\
3.7061 \\
3.7012 \\
1.7920 \\
1.8066 \\
1.8164 \\
1.8262 \\
1.8359 \\
1.8457 \\
1.8555 \\
1.8701 \\
1.8750 \\
1.8696 \\
1.8945 \\
1.9092 \\
1.9141 \\
1.9287 \\
1.9434 \\
1.9629 \\
-16.9283 \\
-23.1503 \\
-24.2729 \\
-13.8795 \\
-13.0990 \\
-12.1908 \\
-7.6257 \\
-4.2467 \\
-7.6359 \\
-6.8245 \\
-6.0323 \\
-2.4889 \\
0.0251 \\
-3.4859 \\
-9.1610 \\
-13.9630 \\
0.6179 \\
-2.4354 \\
59.6830 \\
60.7843 \\
60.7332\end{aligned}$ & $\begin{aligned} & \text { Date: } \text { Fri } \\
& \text { Max } \\
& 38.4572 \\
& 40.0646 \\
& 264.2867 \\
& 267.1739 \\
& 275.5987 \\
& 260.1018 \\
& 151.5773 \\
& 60.1713 \\
& 87.5077 \\
& 57.2062 \\
& 75.0295 \\
& 104.5046 \\
& 213.2246 \\
& 231.6707 \\
& 3.7549 \\
& 3.7354 \\
& 3.7207 \\
& 3.7109 \\
& 1.7969 \\
& 1.8115 \\
& 1.8213 \\
& 1.8359 \\
& 1.8408 \\
& 1.8506 \\
& 1.8604 \\
& 1.8750 \\
& 1.8799 \\
& 1.8945 \\
& 1.9043 \\
& 1.9141 \\
& 1.9238 \\
& 1.9336 \\
& 1.9482 \\
& 1.9678 \\
& 6.6465 \\
&-0.1095 \\
& 1.7188 \\
& 11.9079 \\
& 12.5358 \\
& 3.8310 \\
& 11.2189 \\
& 10.7832 \\
& 8.6910 \\
& 5.8403 \\
& 25.6297 \\
& 16.0505 \\
& 34.4337 \\
& 2.9991 \\
& 16.9315 \\
& 13.8079 \\
& 16.8685 \\
& 3.7444 \\
& 60.5070 \\
& 61.4710 \\
& 61.6488\end{aligned}$ & $\begin{aligned} \text { Aug } 1703: \\
\text { Ave } \\
38.1675 \\
39.8994 \\
263.0892 \\
266.4460 \\
274.7858 \\
259.0742 \\
150.2056 \\
59.7295 \\
86.6763 \\
56.7346 \\
74.7190 \\
101.8692 \\
211.4274 \\
230.3479 \\
3.7430 \\
3.7271 \\
3.7114 \\
3.7055 \\
1.7965 \\
1.8111 \\
1.8201 \\
1.8312 \\
1.8370 \\
1.8492 \\
1.8556 \\
1.8719 \\
1.8797 \\
1.8915 \\
1.9005 \\
1.9124 \\
1.9190 \\
1.9325 \\
1.9481 \\
1.9630 \\
-5.1928 \\
-12.4111 \\
-7.2792 \\
-3.1480 \\
-1.7281 \\
-4.2333 \\
-0.0970 \\
2.6640 \\
-0.7603 \\
-0.3106 \\
6.1930 \\
2.2687 \\
7.7338 \\
0.6310 \\
6.6410 \\
1.4712 \\
8.7920 \\
1.1199 \\
60.1280 \\
61.1560 \\
60.9914\end{aligned}$ & $\begin{array}{l}27.101990 \\
5 D \\
2.1189 e-01 \\
1.3146 e-01 \\
4.9451 e-01 \\
2.6640 e-01 \\
3.5604 e-01 \\
3.8127 e-01 \\
5.2178 e-01 \\
1.9604 e-01 \\
3.2637 e-01 \\
2.2483 e-01 \\
1.9660 e-01 \\
9.0576 e-01 \\
6.1595 e-01 \\
5.5360 e-01 \\
5.5066 e-03 \\
3.8762 e-03 \\
3.1583 e-03 \\
2.7267 e-03 \\
1.3381 e-03 \\
1.3381 e-03 \\
2.1065 e-03 \\
1.5566 e-03 \\
2.0432 e-03 \\
2.2146 e-03 \\
6.9053 e-04 \\
2.3675 e-03 \\
9.6655 e-04 \\
2.3941 e-03 \\
2.2689 e-03 \\
2.3365 e-03 \\
1.5566 e-03 \\
2.0432 e-03 \\
6.9053 e-04 \\
6.9053 e-04 \\
5.2474 e+00 \\
6.3463 e+00 \\
7.7350 e+00 \\
6.5308 e+00 \\
6.1675 e+00 \\
5.1538 e+00 \\
1.4343 e+00 \\
3.8939 e+00 \\
3.9777 e+00 \\
3.3620 e+00 \\
6.5448 e+00 \\
3.3240 e+00 \\
8.1452 e+00 \\
1.6165 e+00 \\
1.9676 e+00 \\
4.3181 e+00 \\
1.3620 e+00 \\
1.5427 e+00 \\
2.3140 e-01 \\
1.4787 e-01 \\
1.4079 e-01\end{array}$ & $\begin{array}{c}\text { Page: } 2 \\
\text { Uncert } \\
1.9010 e+00 \\
1.9011 e+00 \\
1.9250 e+00 \\
1.9258 e+00 \\
1.9280 e+00 \\
1.9239 e+00 \\
1.9043 e+00 \\
1.9001 e+00 \\
1.9002 e+00 \\
1.9001 e+00 \\
1.9000 e+00 \\
1.9007 e+00 \\
1.9134 e+00 \\
1.9172 e+00 \\
0.0000 e+00 \\
0.0000 e+00 \\
0.0000 e+00 \\
0.0000 e+00 \\
0.0000 e+00 \\
0.0000 e+00 \\
0.0000 e+00 \\
0.0000 e+00 \\
0.0000 e+00 \\
0.0000 e+00 \\
0.0000 e+00 \\
0.0000 e+00 \\
0.0000 e+00 \\
0.0000 e+00 \\
0.0000 e+00 \\
0.0000 e+00 \\
0.0000 e+00 \\
0.0000 e+00 \\
0.0000 e+00 \\
0.0000 e+00 \\
8.7010 e-01 \\
1.6803 e+00 \\
8.7019 e-01 \\
8.7004 e-01 \\
8.7001 e-01 \\
8.7006 e-01 \\
8.7000 e-01 \\
8.7003 e-01 \\
8.7000 e-01 \\
8.7000 e-01 \\
8.7014 e-01 \\
8.7002 e-01 \\
8.7021 e-01 \\
8.7000 e-01 \\
8.7016 e-01 \\
8.7001 e-01 \\
8.7028 e-01 \\
8.7000 e-01 \\
5.9916 e-01 \\
5.9981 e-01 \\
5.9971 e-01\end{array}$ \\
\hline
\end{tabular}




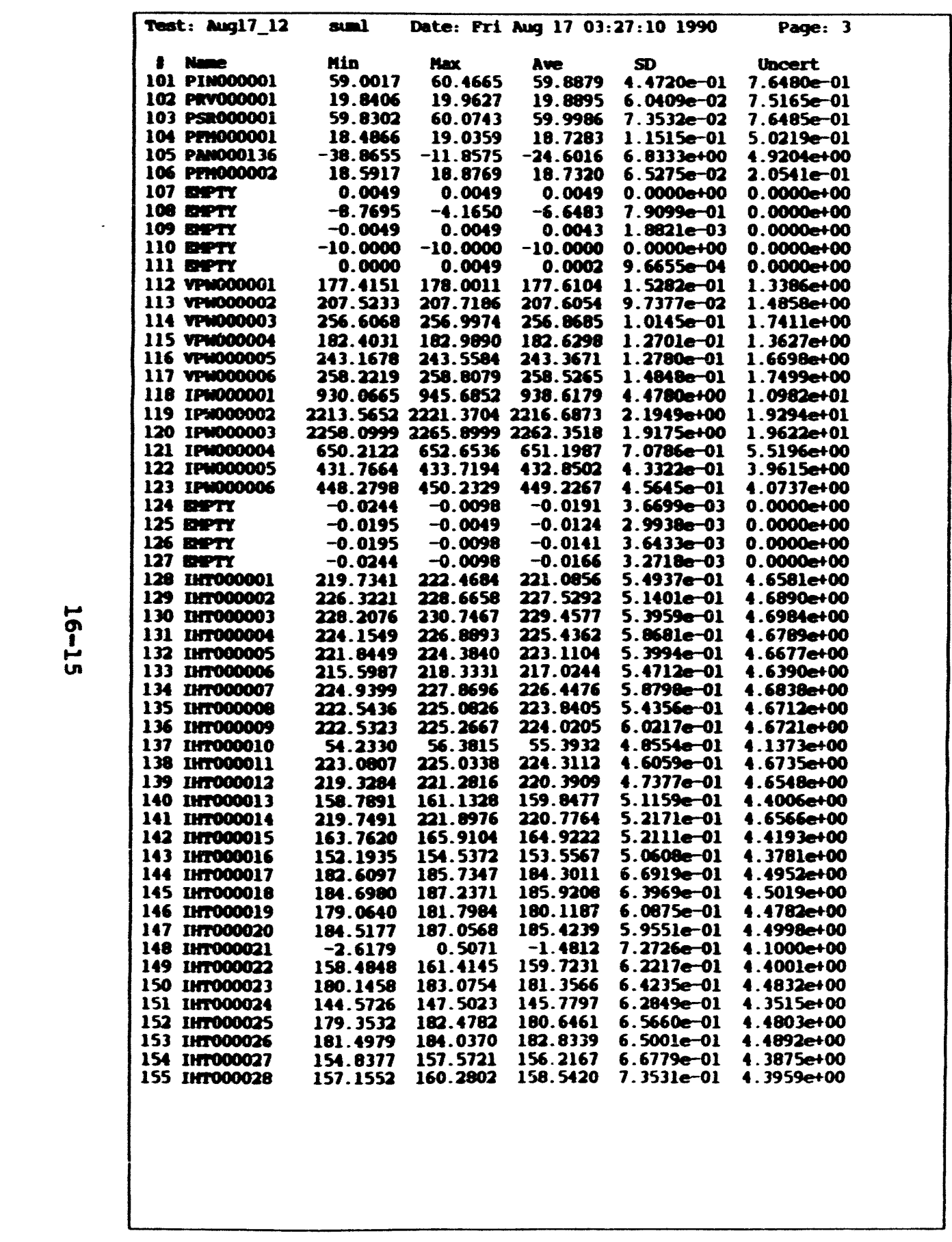

\begin{tabular}{|c|c|c|c|c|c|}
\hline 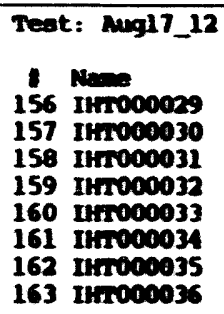 & $\begin{array}{l}\text { sand } \\
\text { min } \\
180.2058 \\
183.9656 \\
181.2275 \\
183.6951 \\
183.5204 \\
161.1253 \\
212.7855 \\
159.5966\end{array}$ & $\begin{array}{c}\text { Date: Fri } \\
\operatorname{Max} \\
183.3308 \\
186.7000 \\
183.5712 \\
186.0389 \\
186.0615 \\
163.2737 \\
214.9339 \\
161.5497\end{array}$ & $\begin{array}{l}\text { Ave } \\
181.8427 \\
185.3290 \\
182.4892 \\
184.8866 \\
184.8232 \\
162.4183 \\
213.9847 \\
160.5302\end{array}$ & $\begin{array}{l}50 \\
6.8333 e-01 \\
6.9000 e-01 \\
6.7221 e-01 \\
7.2033 e-01 \\
7.2931 e-01 \\
5.9437 e-01 \\
6.0619 e-01 \\
5.6261 e-01\end{array}$ & $\begin{array}{l}\text { Oncert } \\
4.4852 \\
4.49946 \\
4.48786 \\
4.4976 e \\
4.49736 \\
4.4100 \\
4.6248 \\
4.40316\end{array}$ \\
\hline
\end{tabular}

163 Inro00036

$\begin{array}{llllll}212.7855 & 214.9339 & 213.9847 & 6.06190-01 & 1.6248 e+\infty\end{array}$

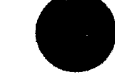




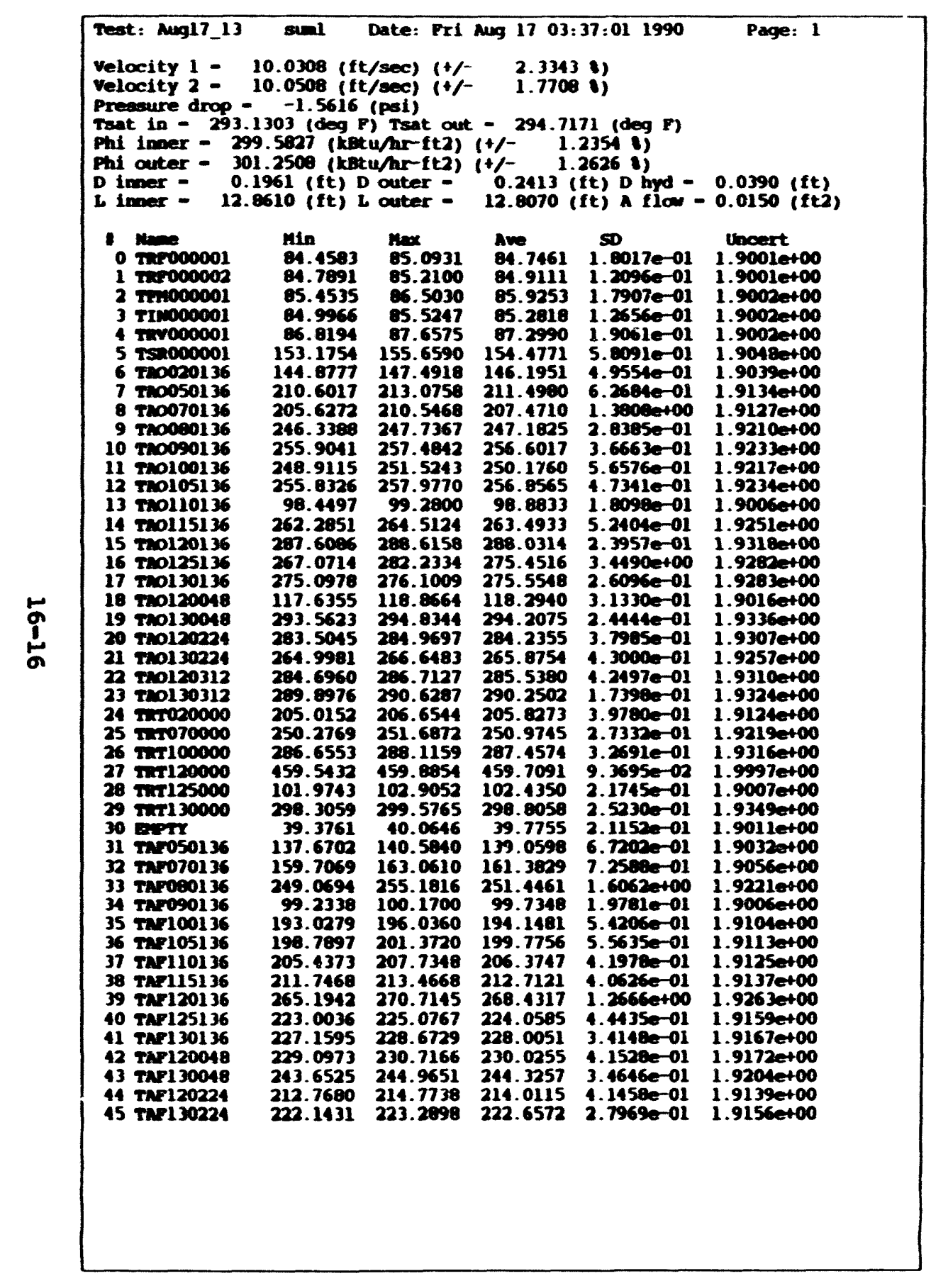

\begin{tabular}{|c|c|c|c|c|c|}
\hline 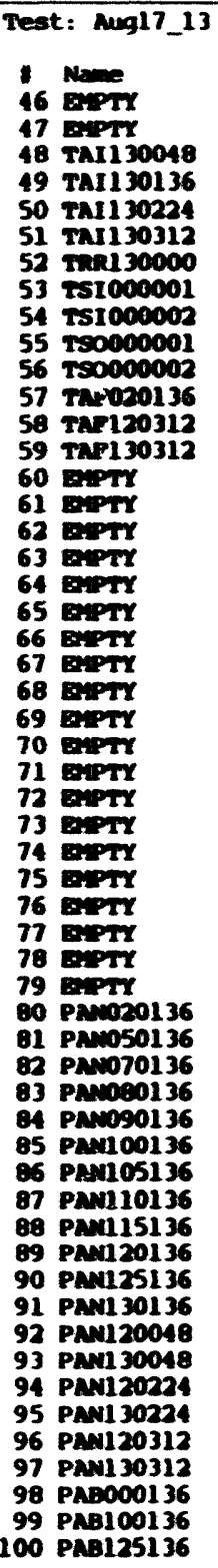 & 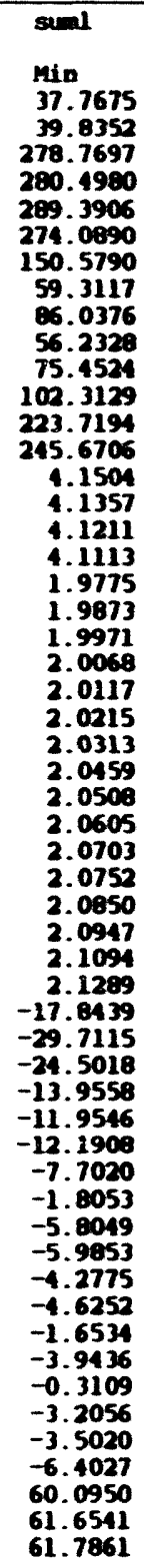 & 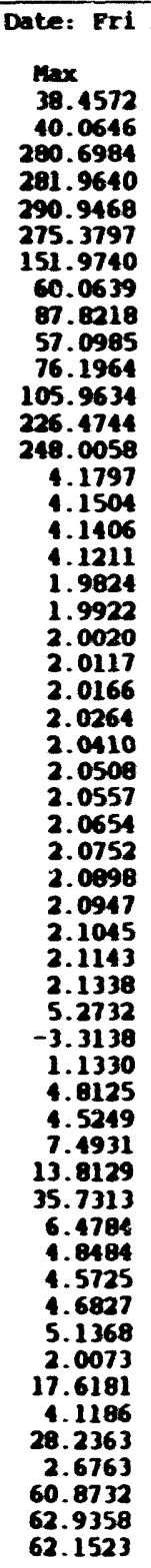 & 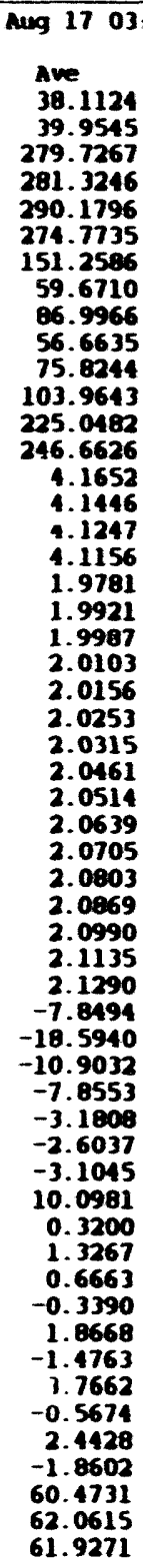 & 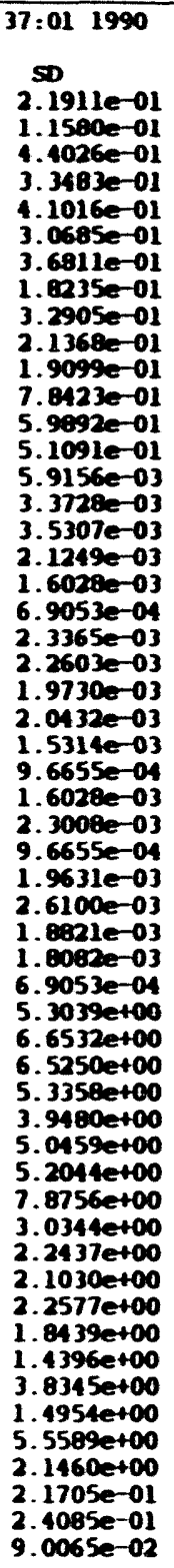 & 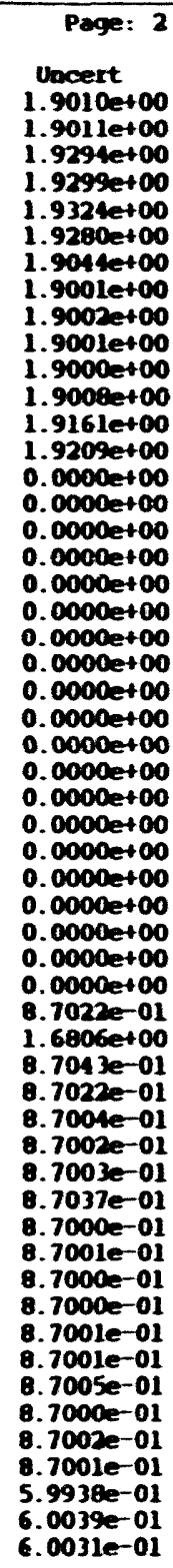 \\
\hline
\end{tabular}




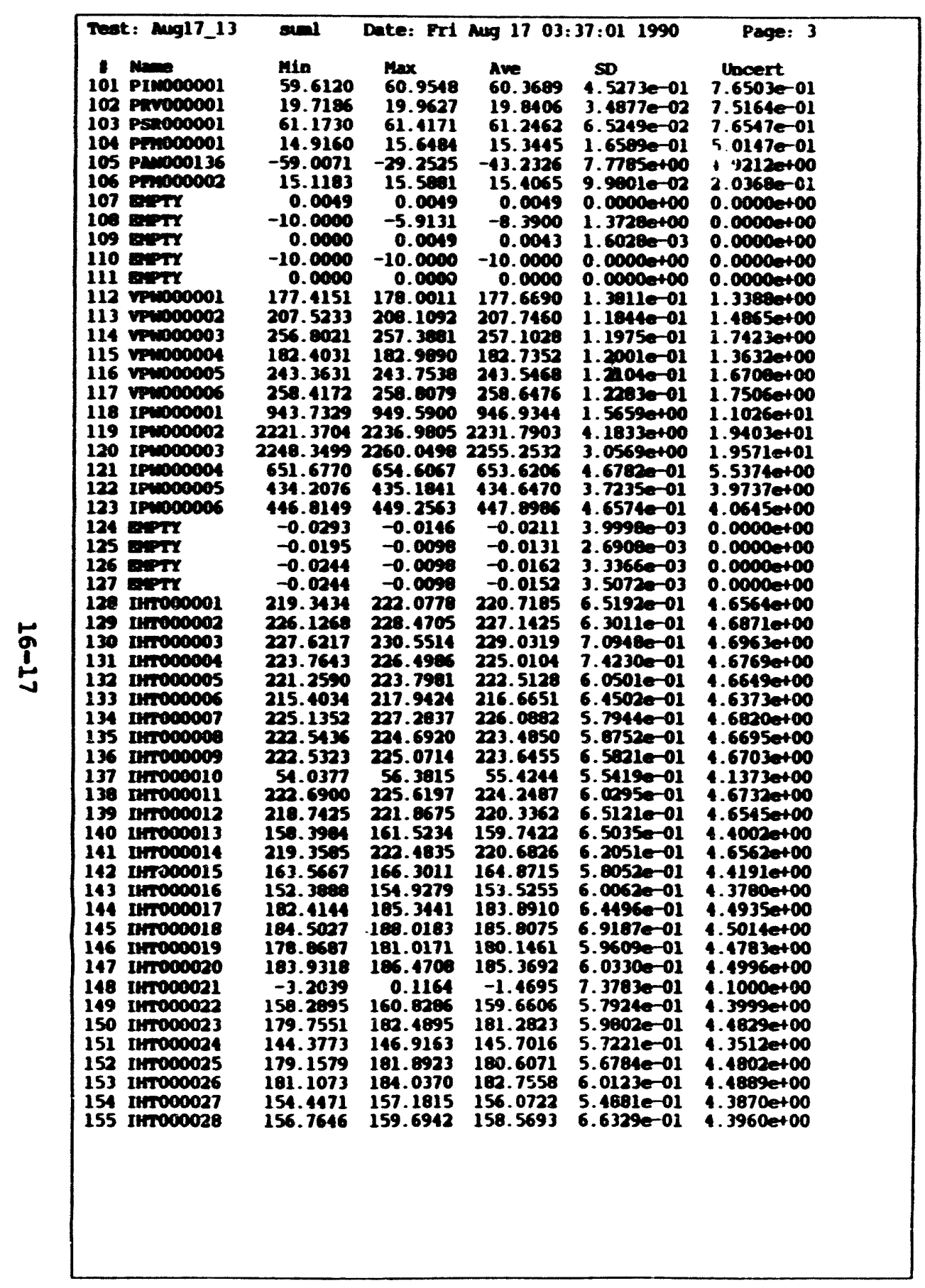

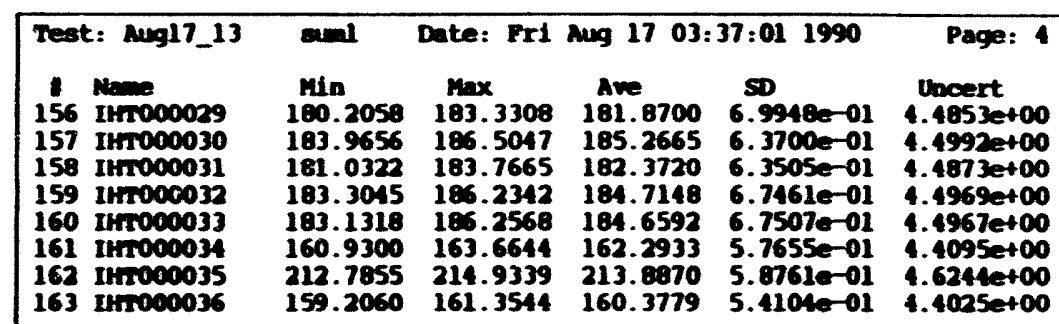




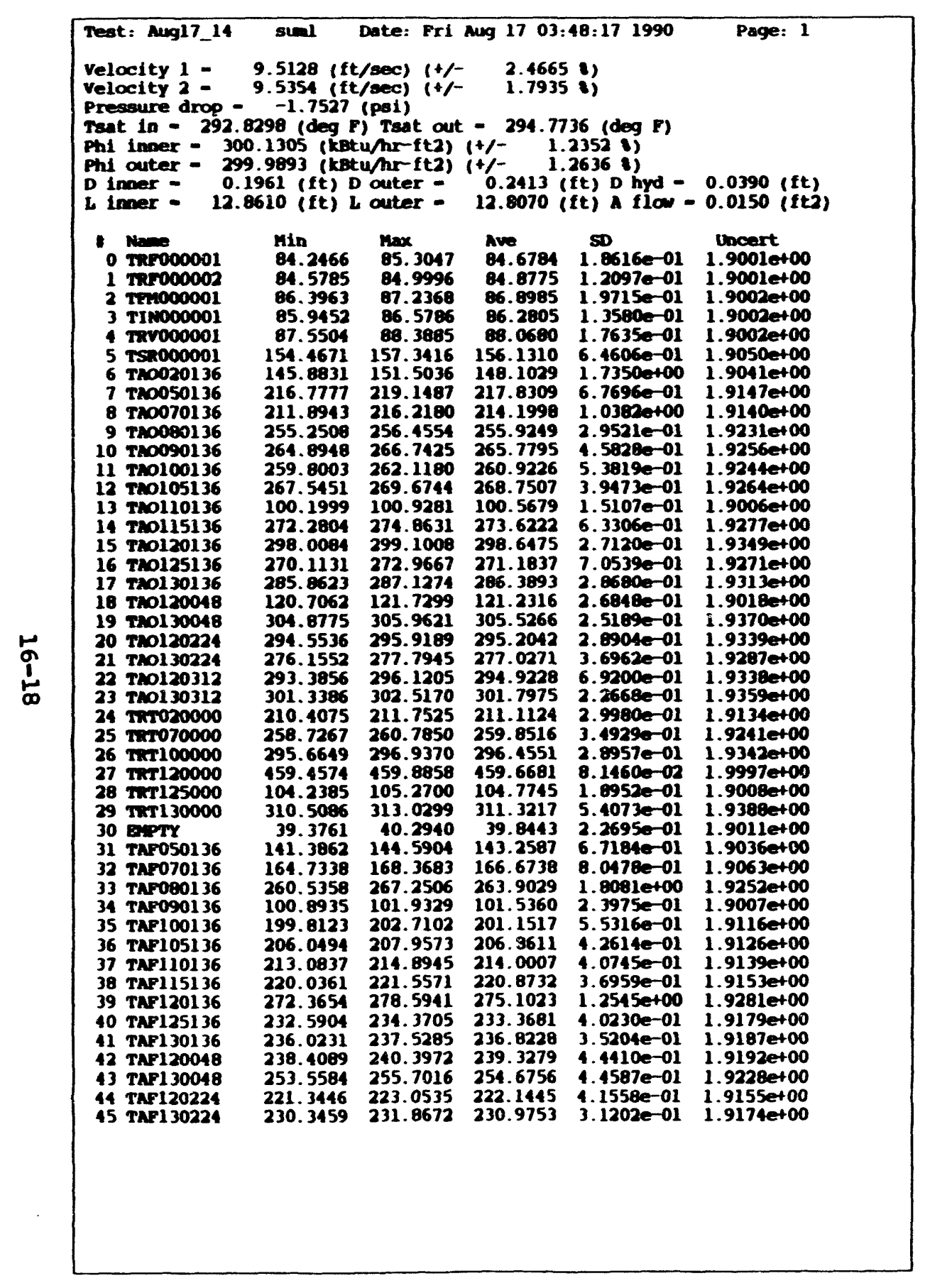

\begin{tabular}{|c|c|c|c|c|c|}
\hline Test: Aug17_14 & $\operatorname{sen}$ & Date: Pri & Aug 17 03: & $48: 17 \quad 1990$ & Page: 2 \\
\hline 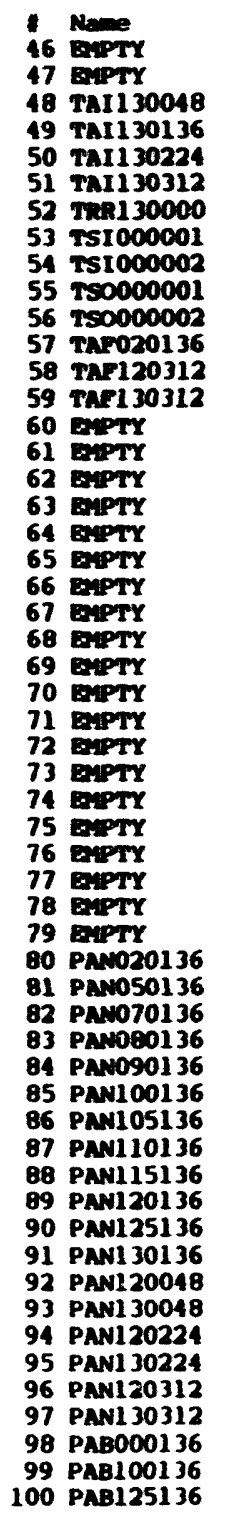 & 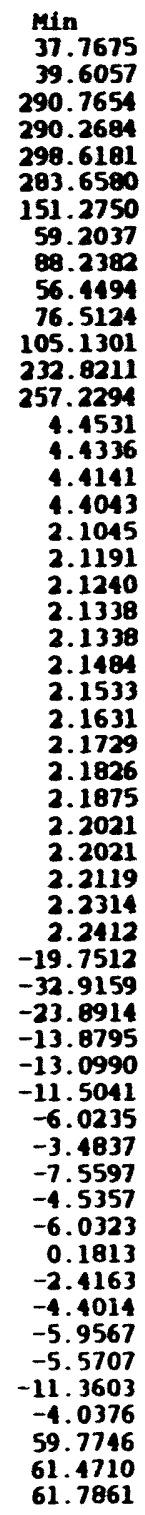 & 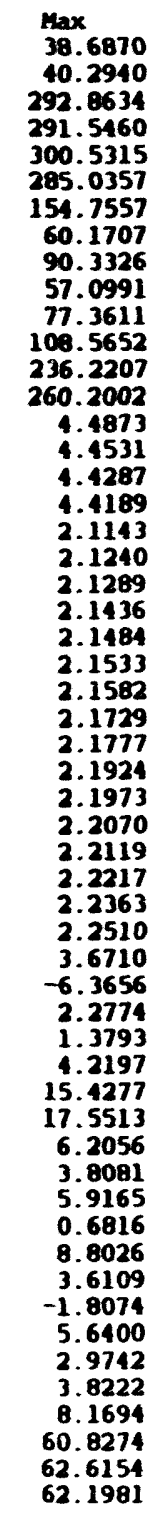 & 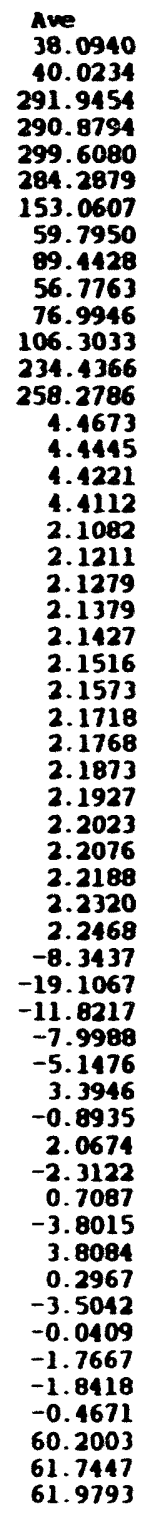 & 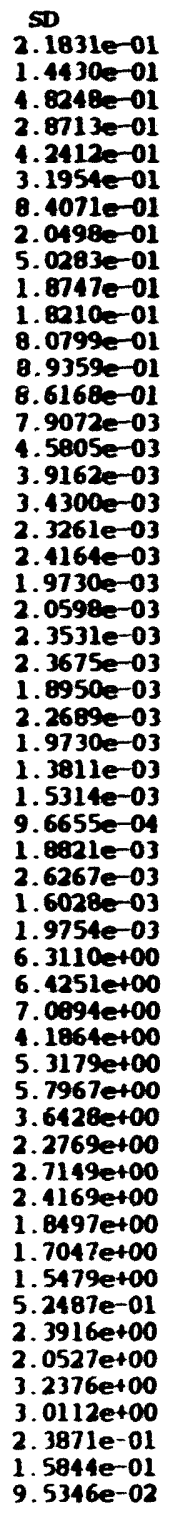 & 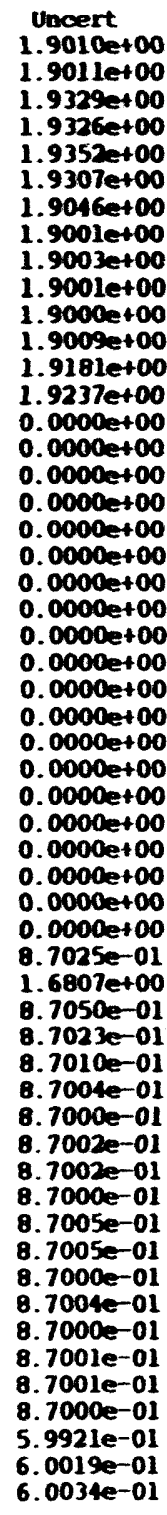 \\
\hline
\end{tabular}



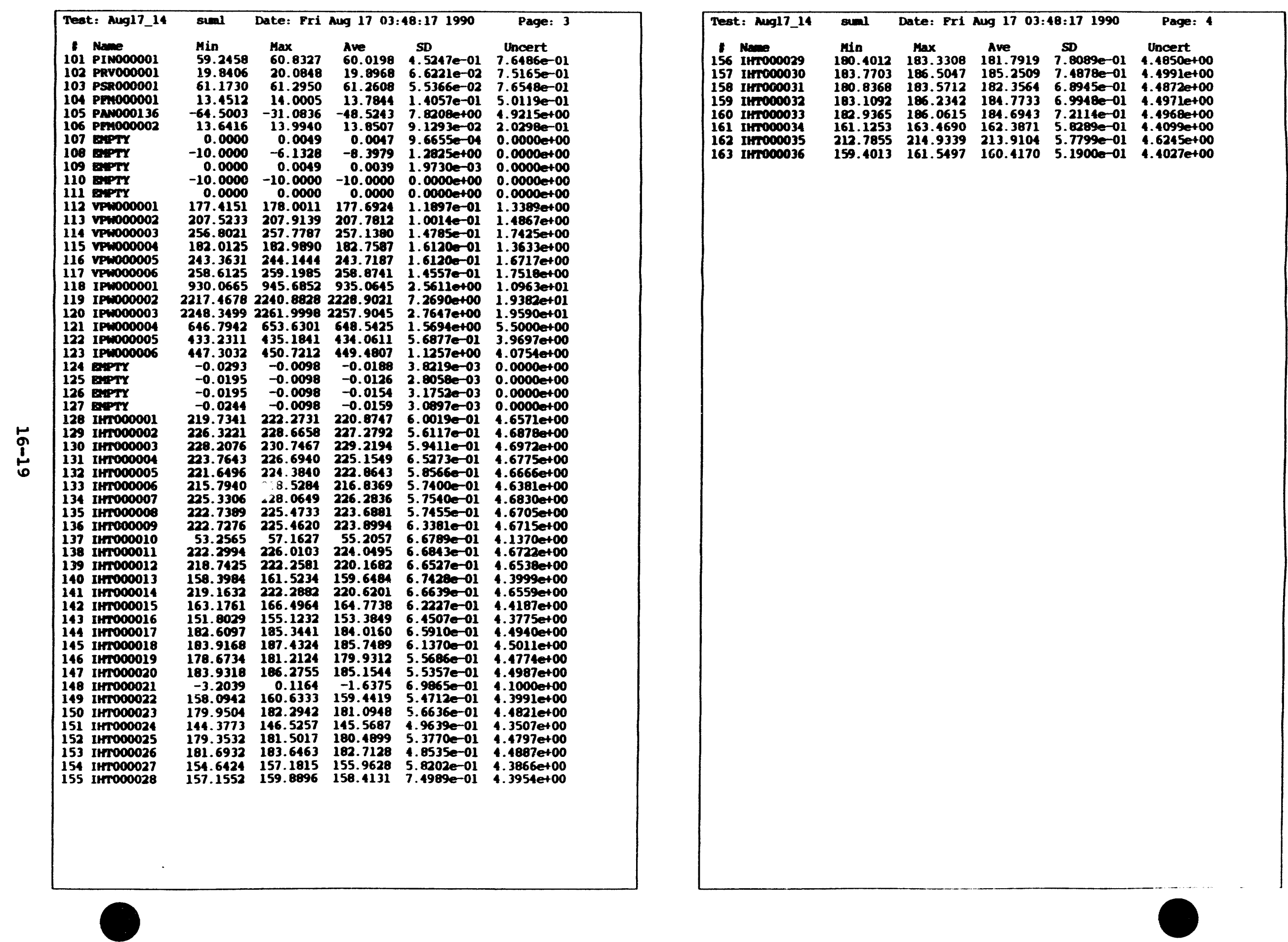


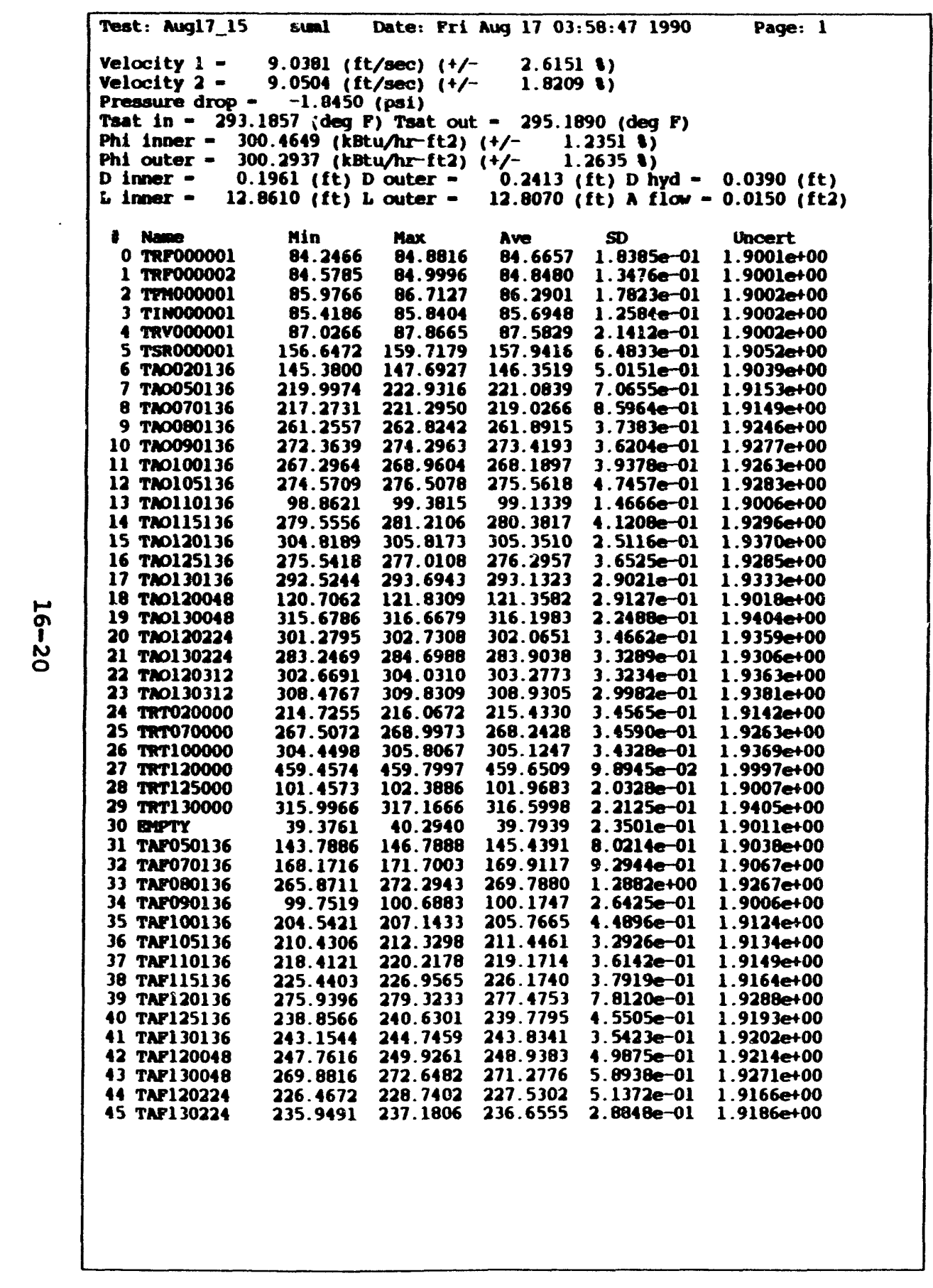

\begin{tabular}{|c|c|c|c|c|c|}
\hline 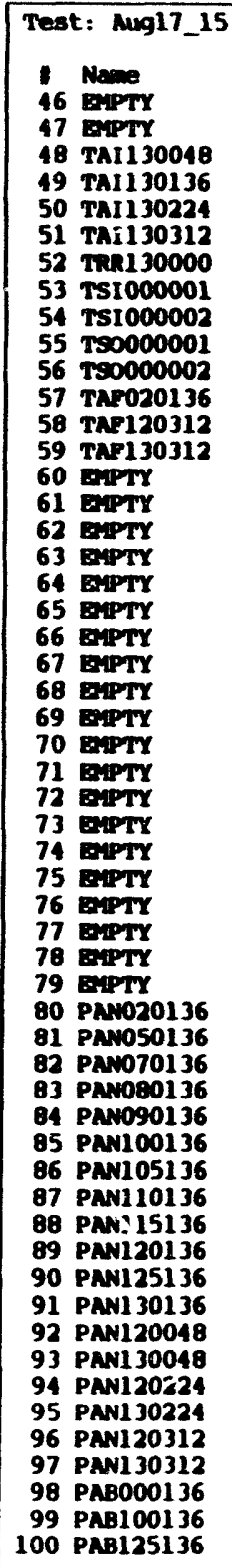 & 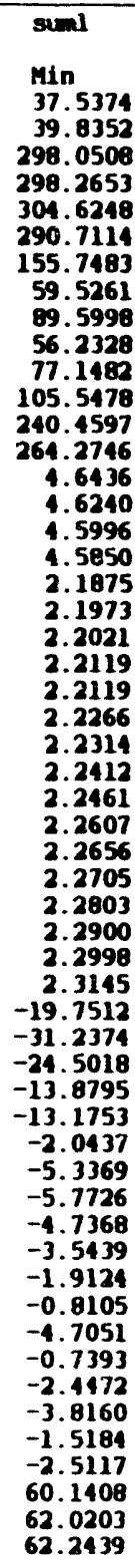 & 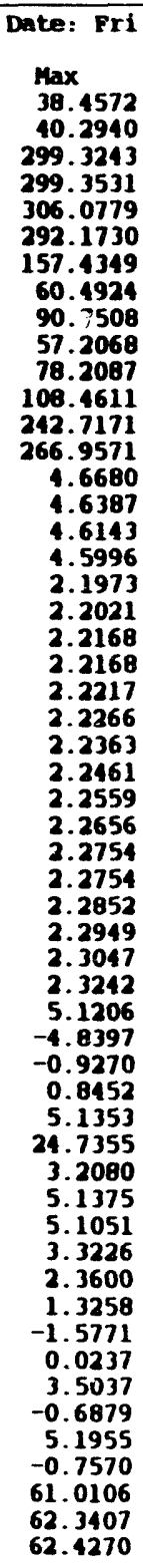 & 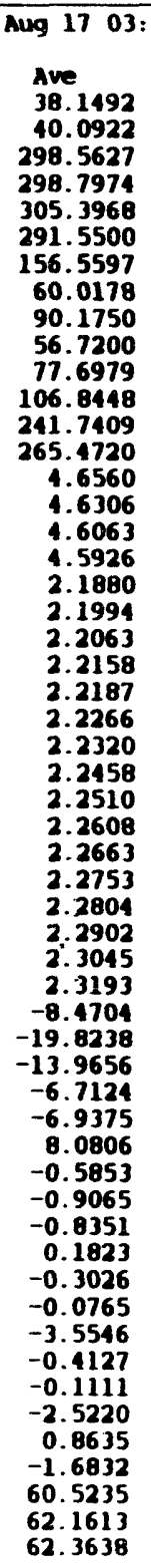 & 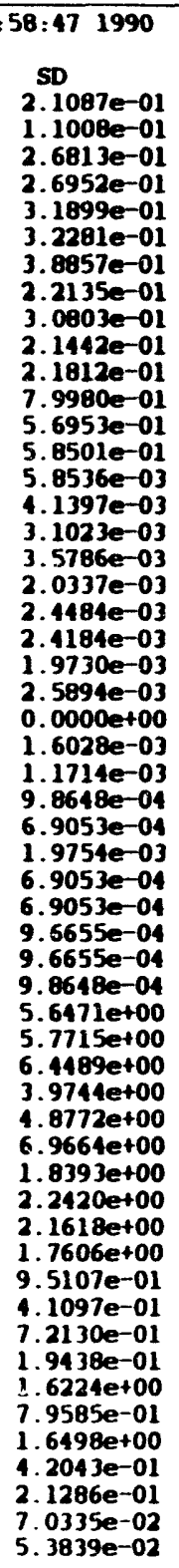 & 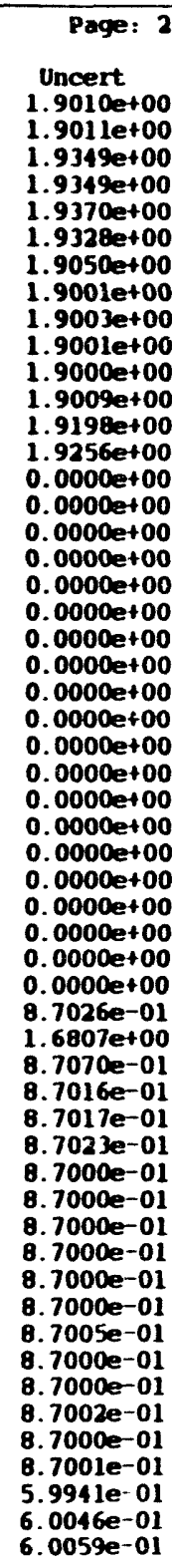 \\
\hline
\end{tabular}




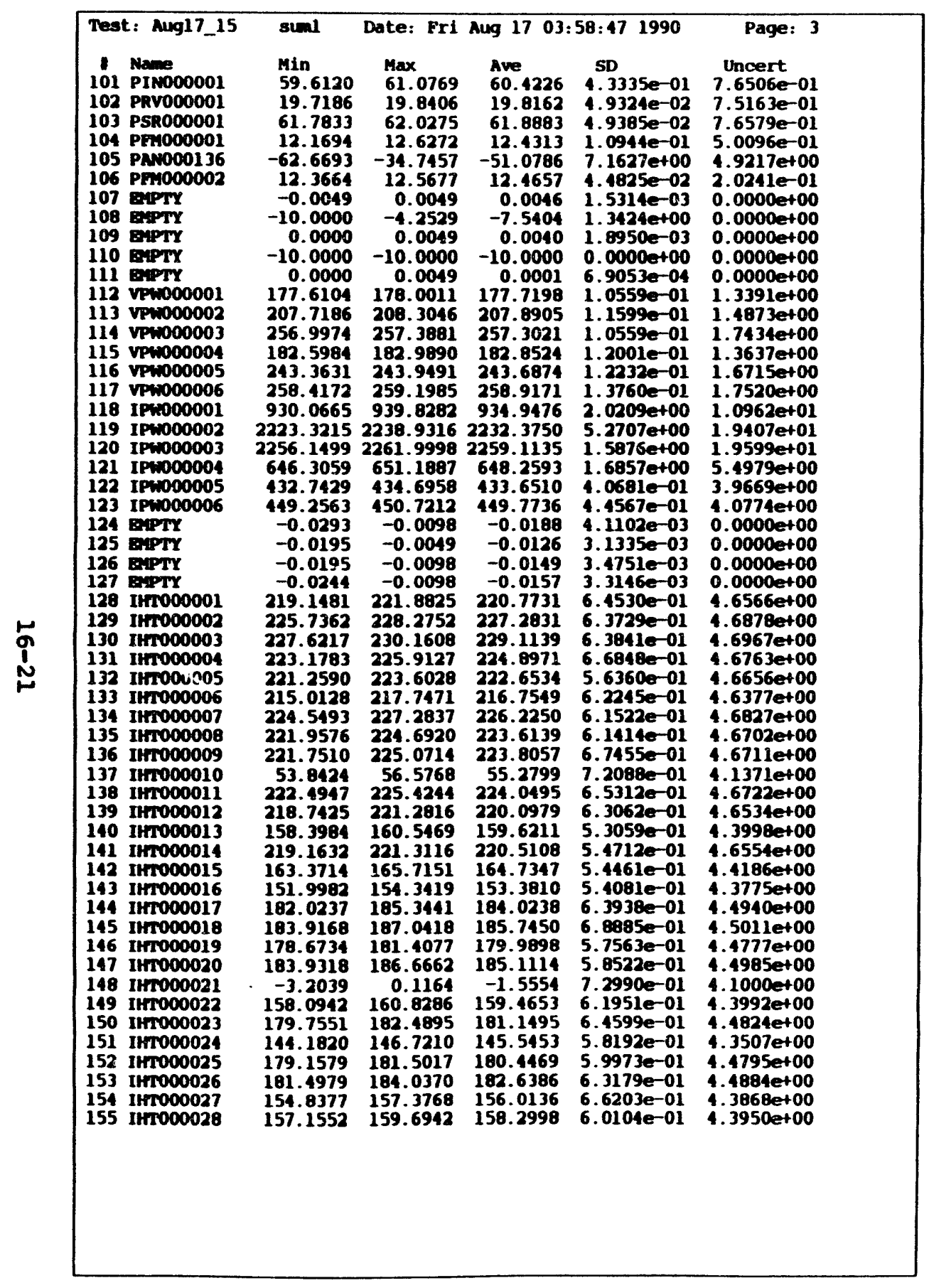

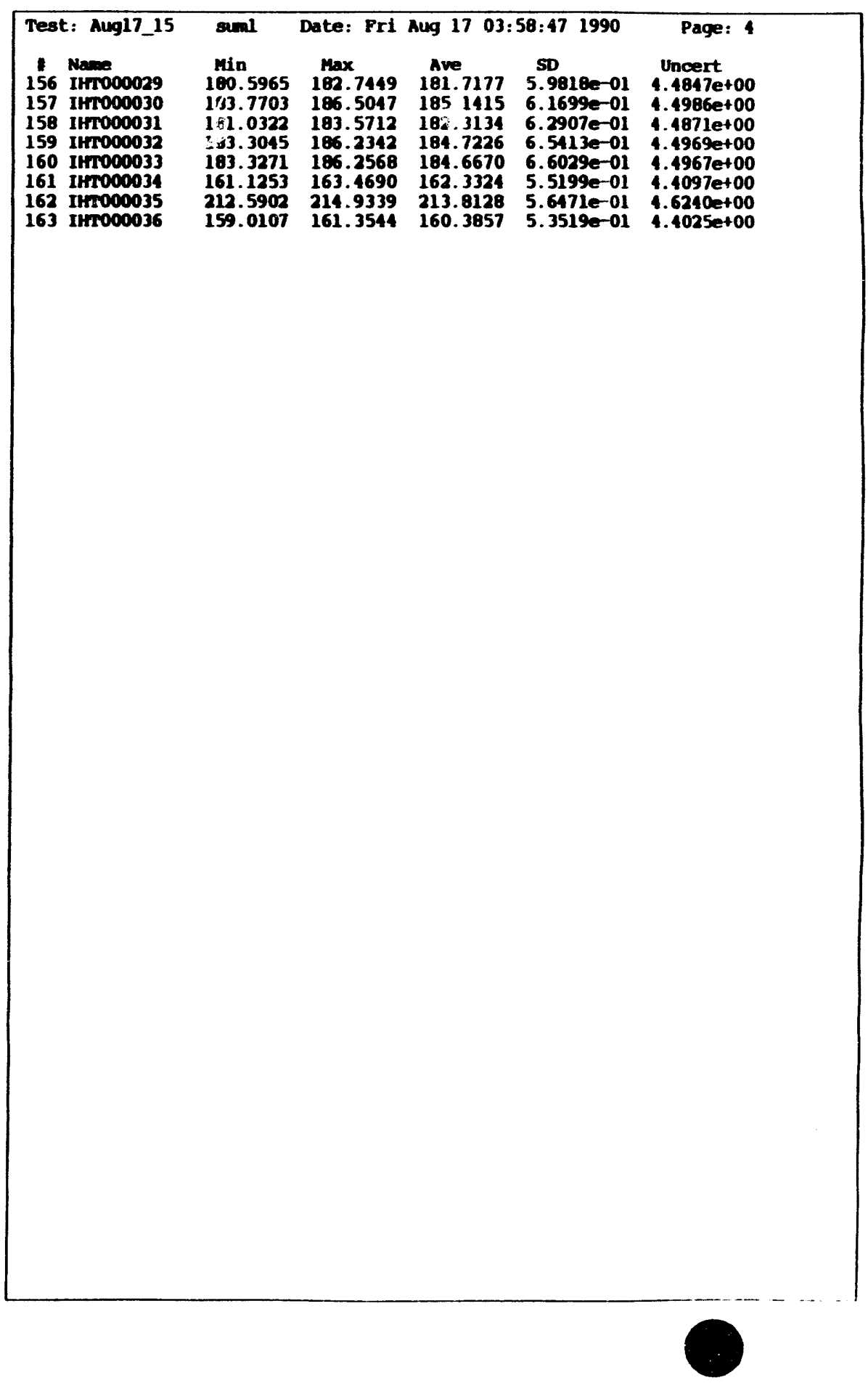




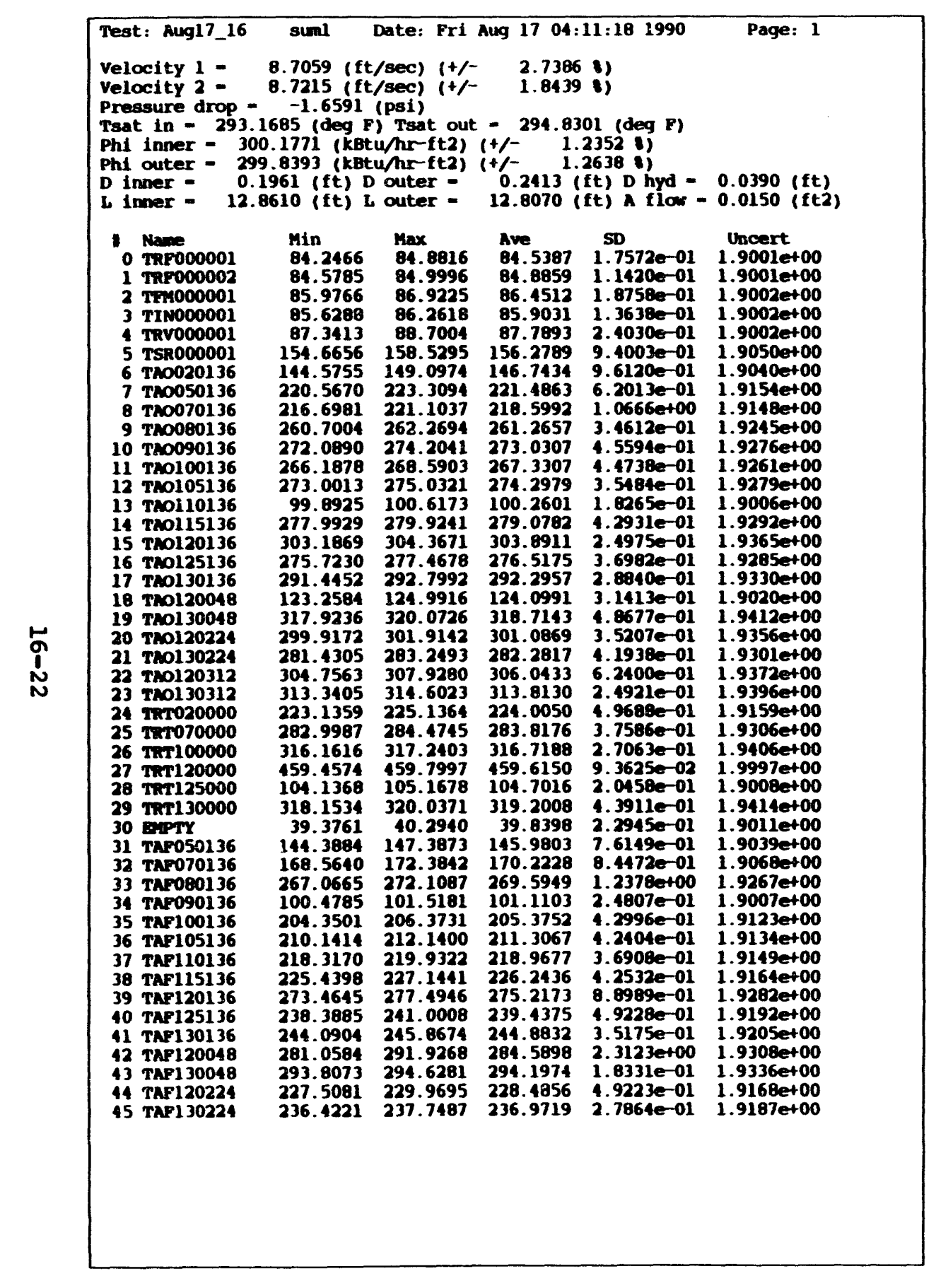

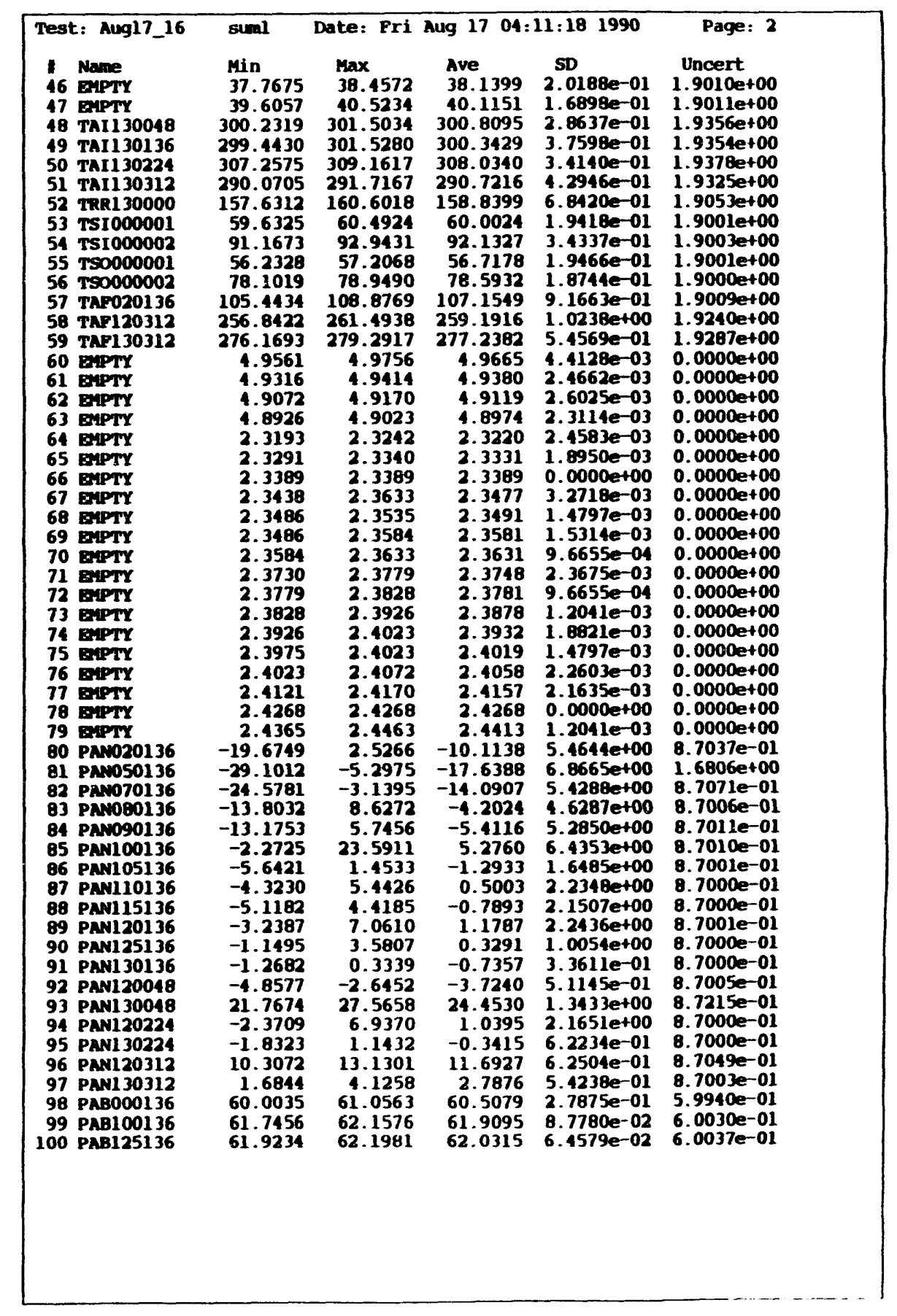




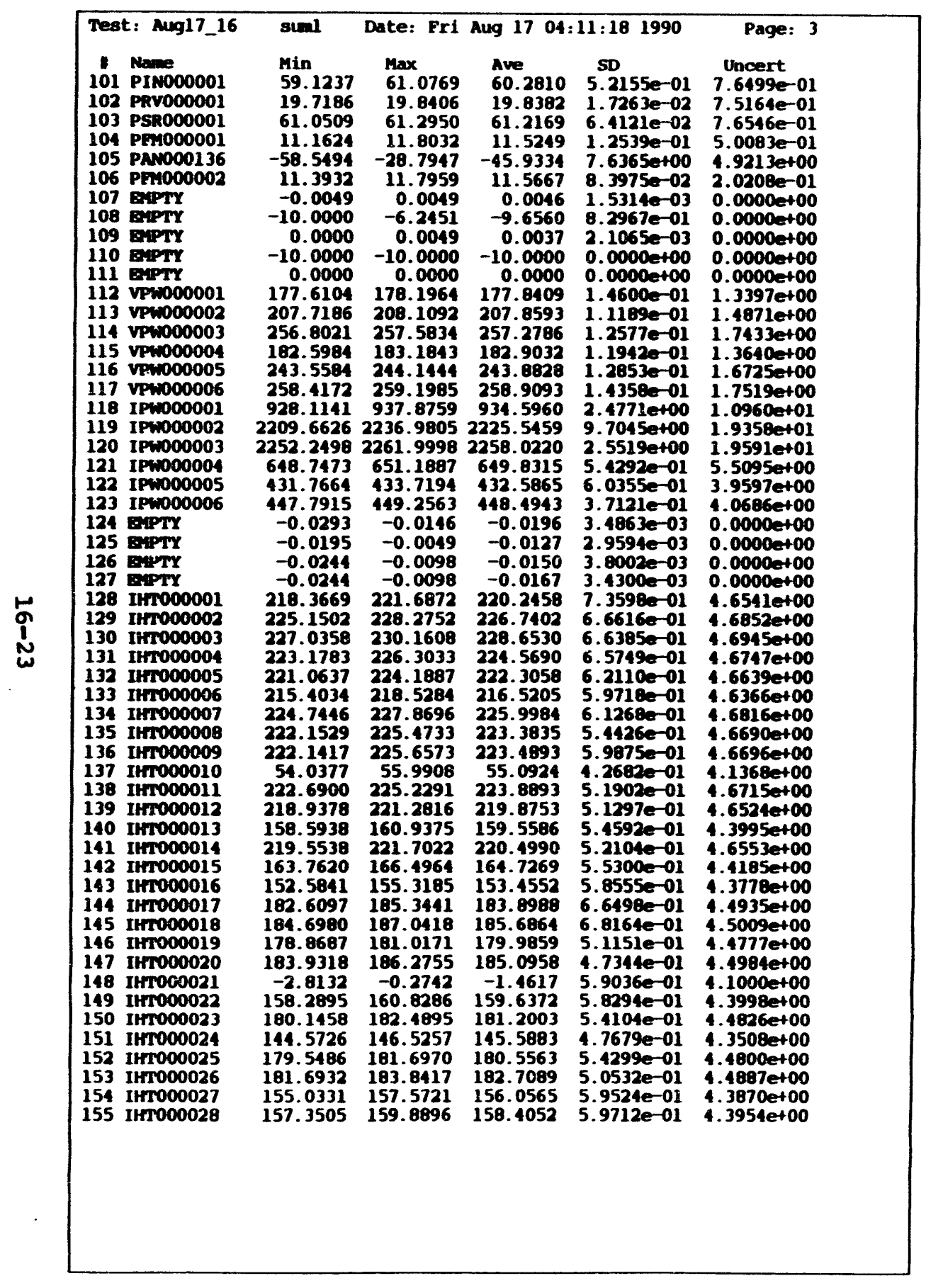

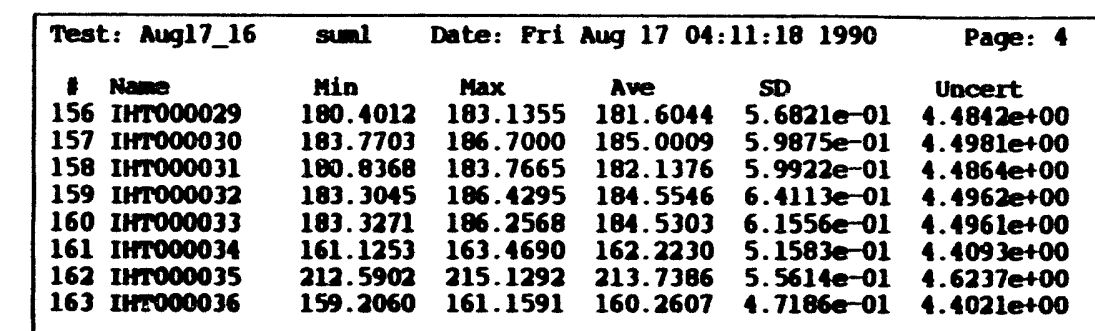




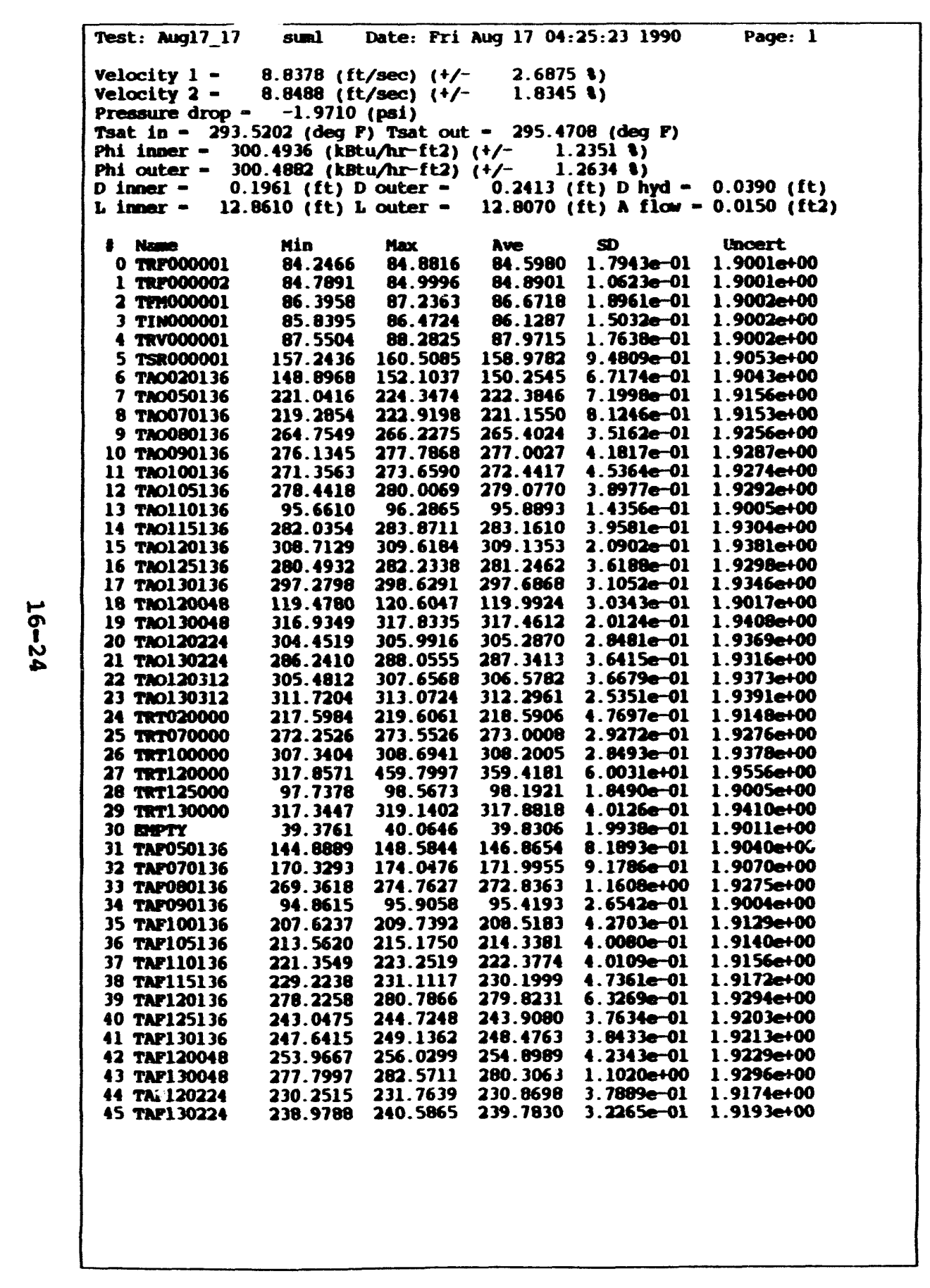

\begin{tabular}{|c|c|c|c|c|c|}
\hline 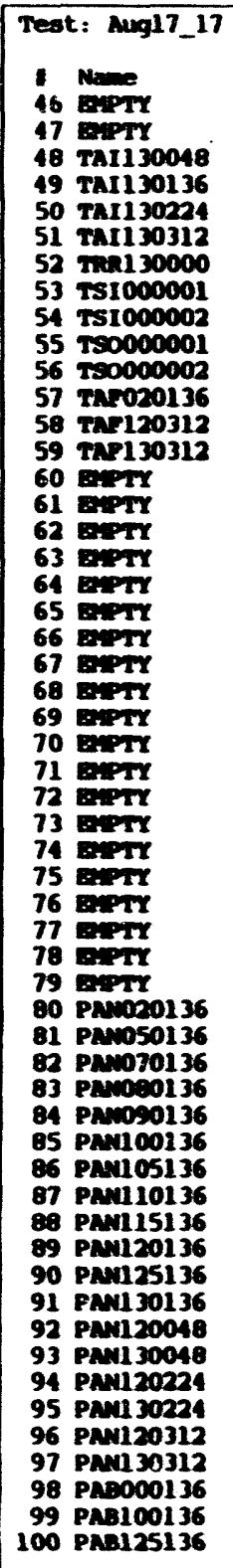 & 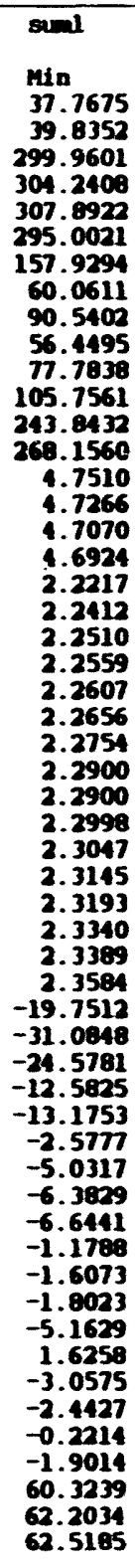 & 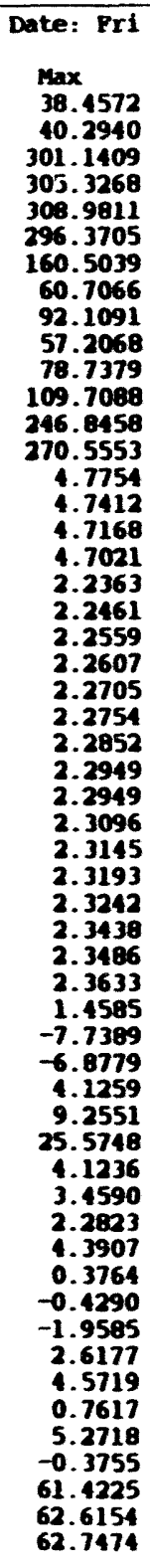 & 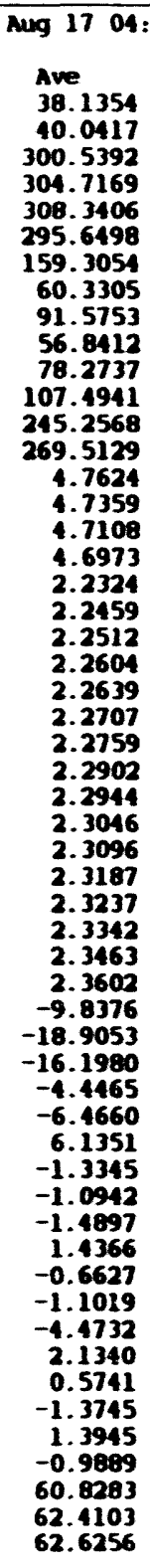 & 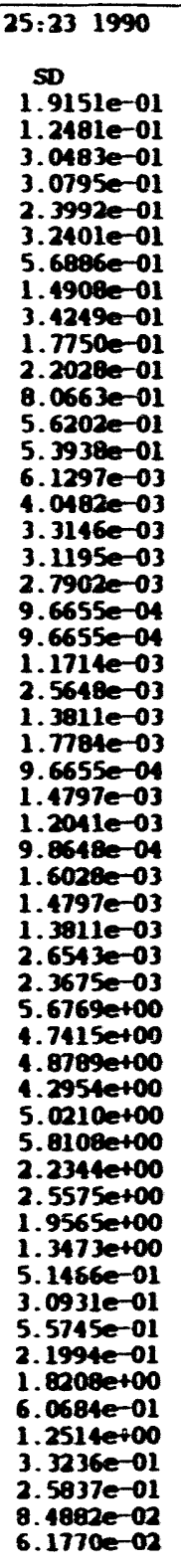 & 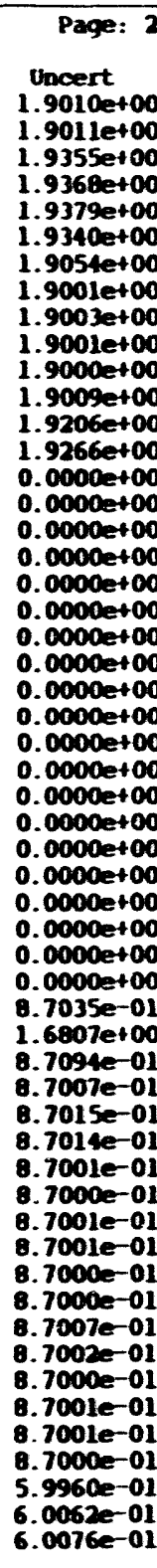 \\
\hline
\end{tabular}




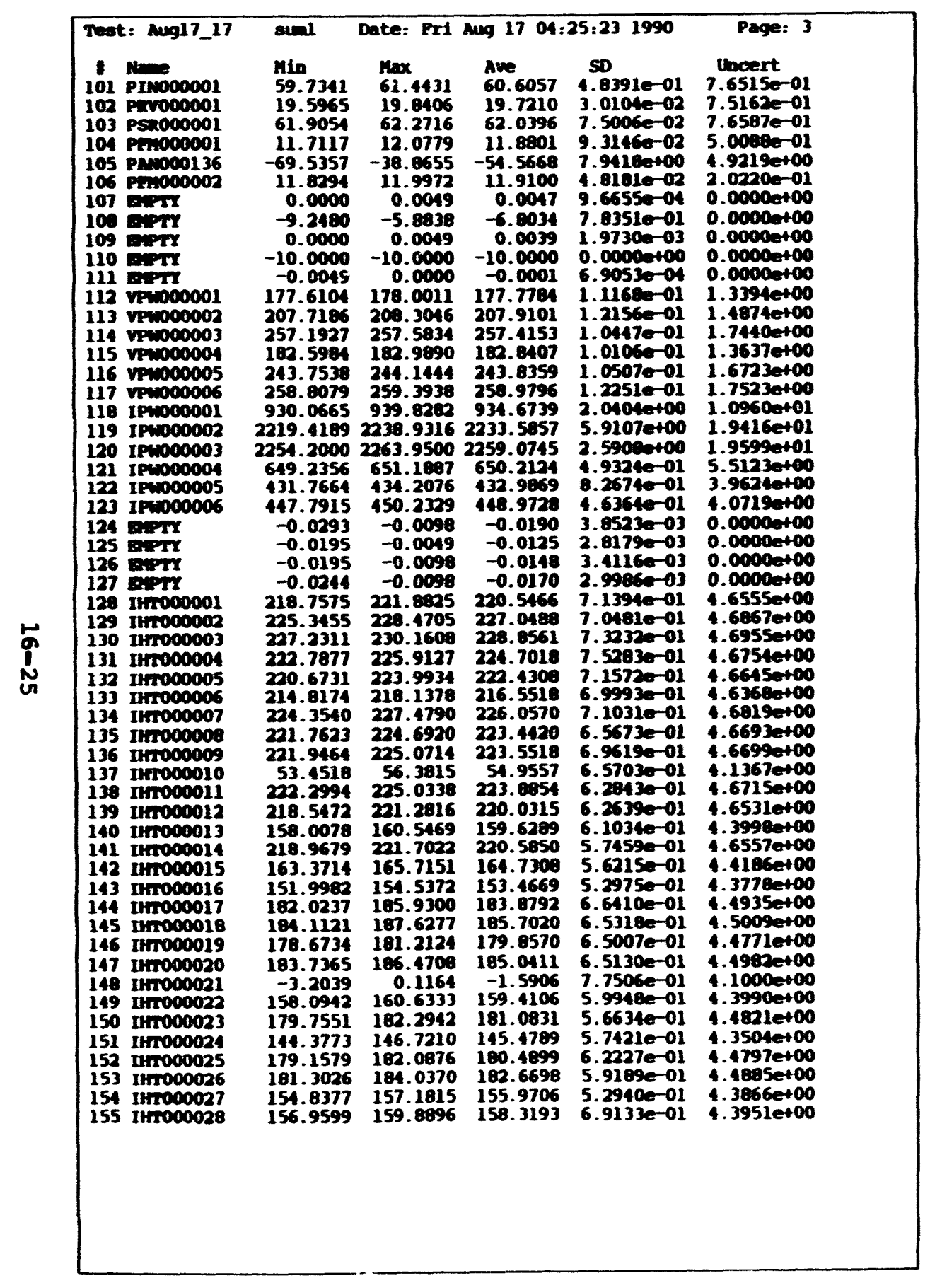

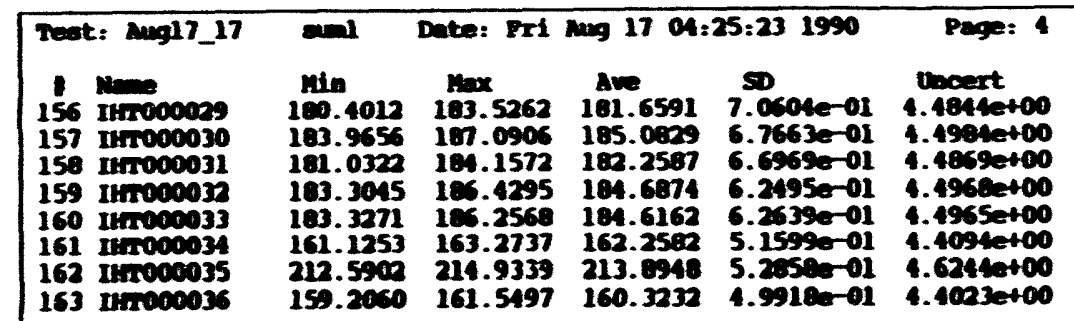


DEFECTIVE INSTRUMENTS FOR THE RIBBED GEOMEIRY TEST SERIES

\begin{tabular}{|c|c|c|}
\hline Series & Tests & Defective Instruments \\
\hline 7 & Aug17_09 to Aug17_17 & 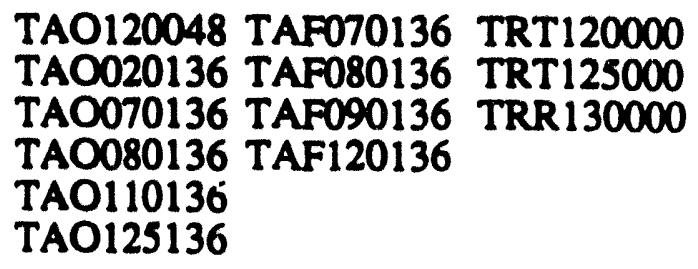 \\
\hline & & $\begin{array}{ll}\text { IHTO000110 IHTO00021 IHTO00024 } \\
\text { IHTO00033 }\end{array}$ \\
\hline
\end{tabular}




\section{Chapter 17}

NUCLEATE BOILING PRESSURE DROP IN AN ANNULUS

Test Geometry: Ribbed (4)

Test Series: 9

Test Date: August 17, 1990 


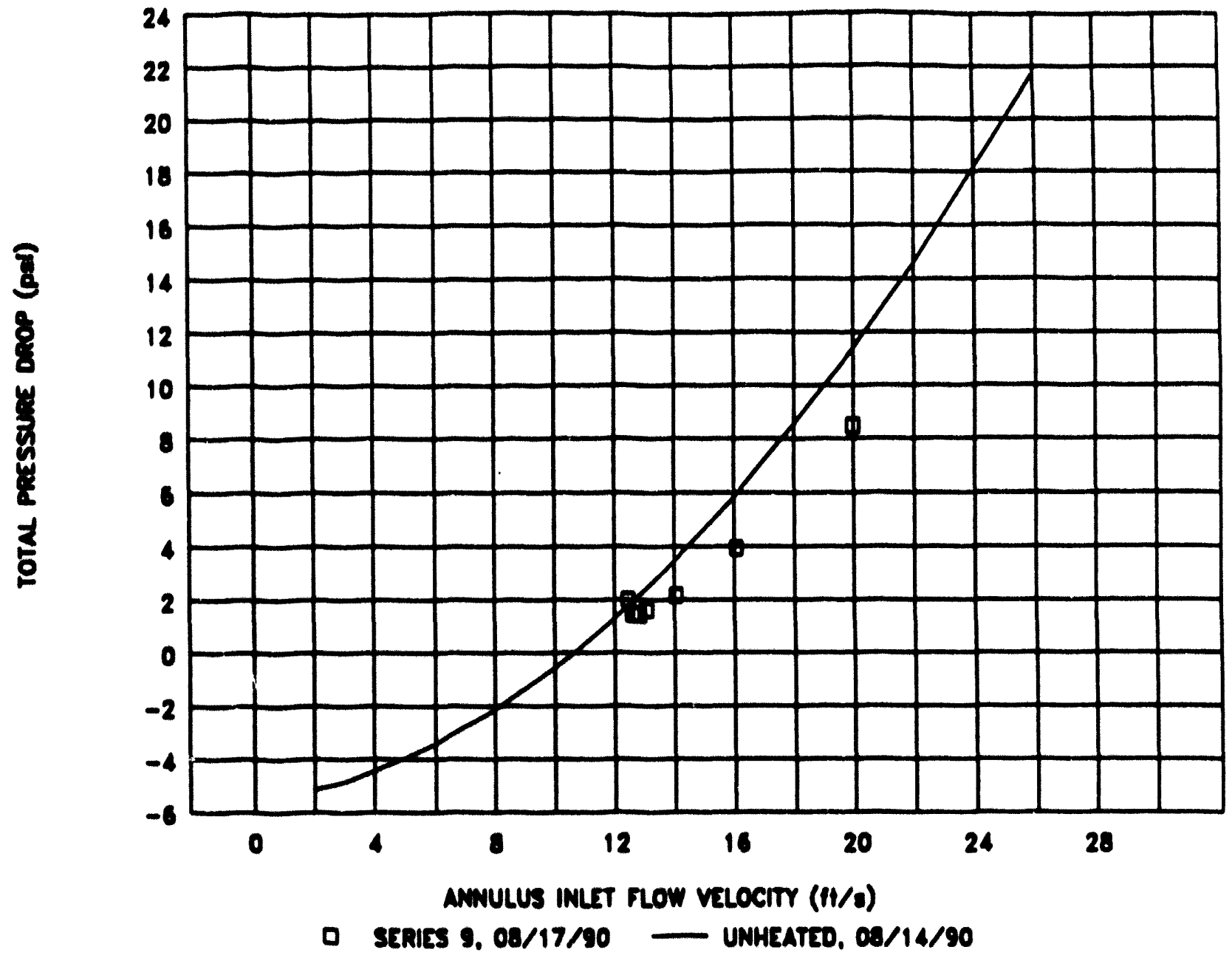

\begin{tabular}{|c|c|c|}
\hline $\begin{array}{c}\text { TEST } \\
\text { NUMBEA }\end{array}$ & $\begin{array}{c}\text { INLET } \\
\text { VELOCITY(o) }\end{array}$ & $\begin{array}{l}\text { PAESSUAE } \\
\text { DROP(b) }\end{array}$ \\
\hline & (w/s) & (pai) \\
\hline Aug17_18 & 19.97 & 8.48 \\
\hline Aug17_19 & 16.07 & 3.94 \\
\hline Aug17_20 & 14.05 & 2.17 \\
\hline Aug17_21 & 13.08 & 1.61 \\
\hline Aug17_22 & 12.82 & 1.44 \\
\hline Ayo17_24 & 12.70 & 1.47 \\
\hline$H^{\prime} \cdot 1 / 2$ & V. & X \\
\hline Aup17_25 & 12.46 & 2.00 \\
\hline
\end{tabular}

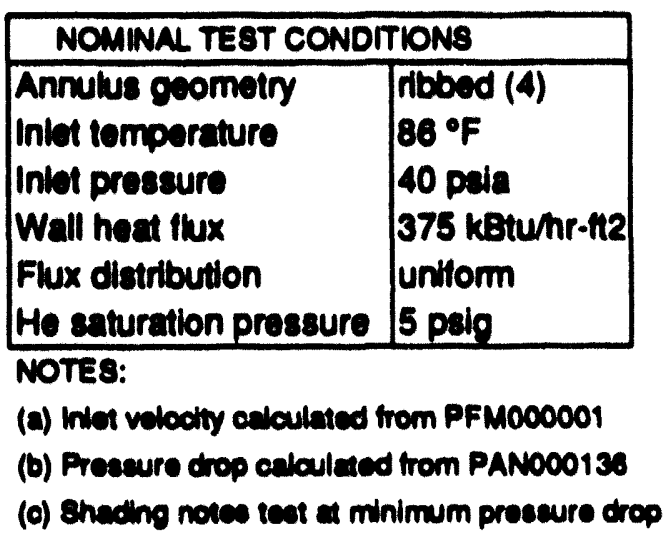

DEMAND CURVE AND DATA FOR SERIES 9 - RIBBED ANNULUS 

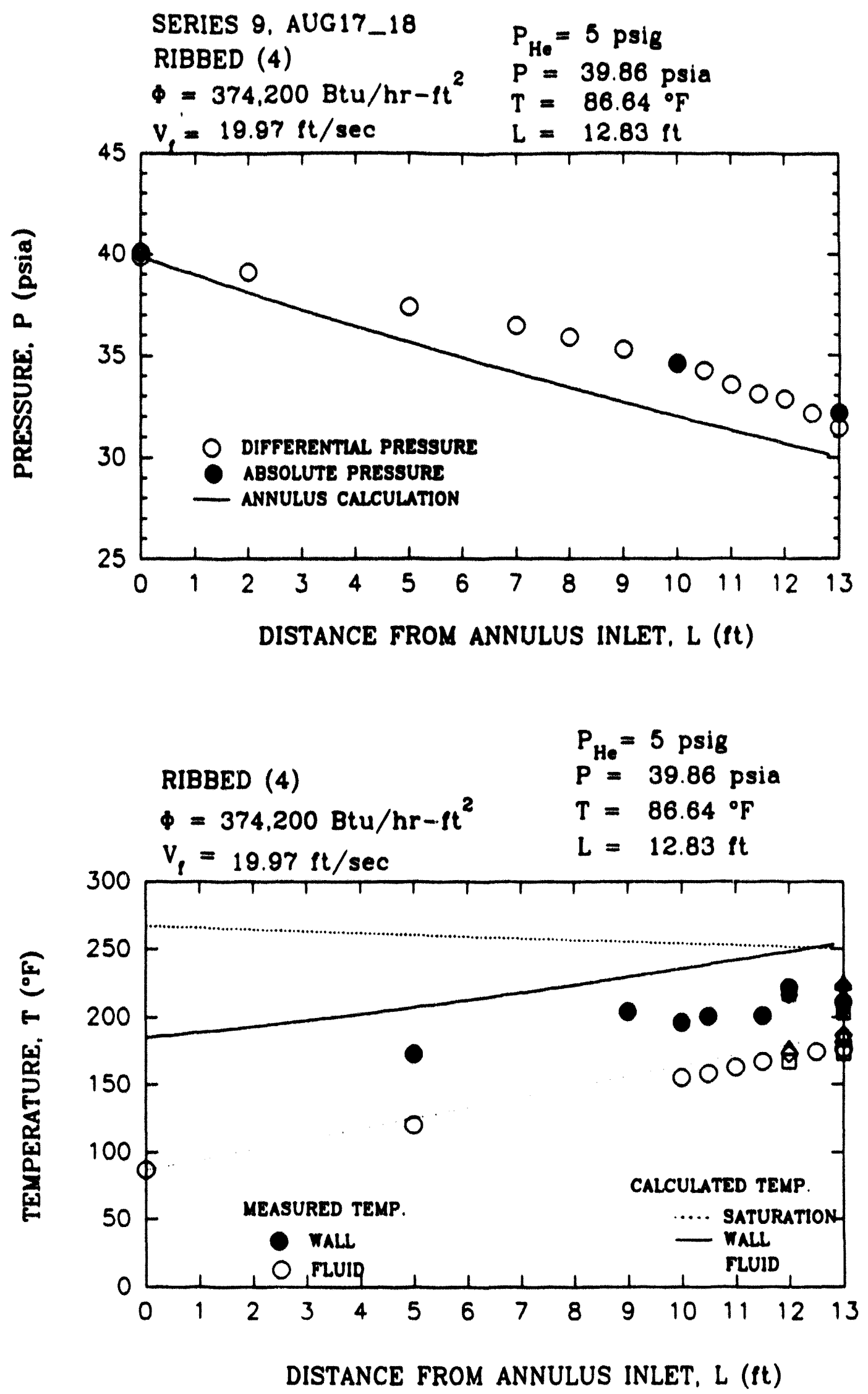

AUG17_18 

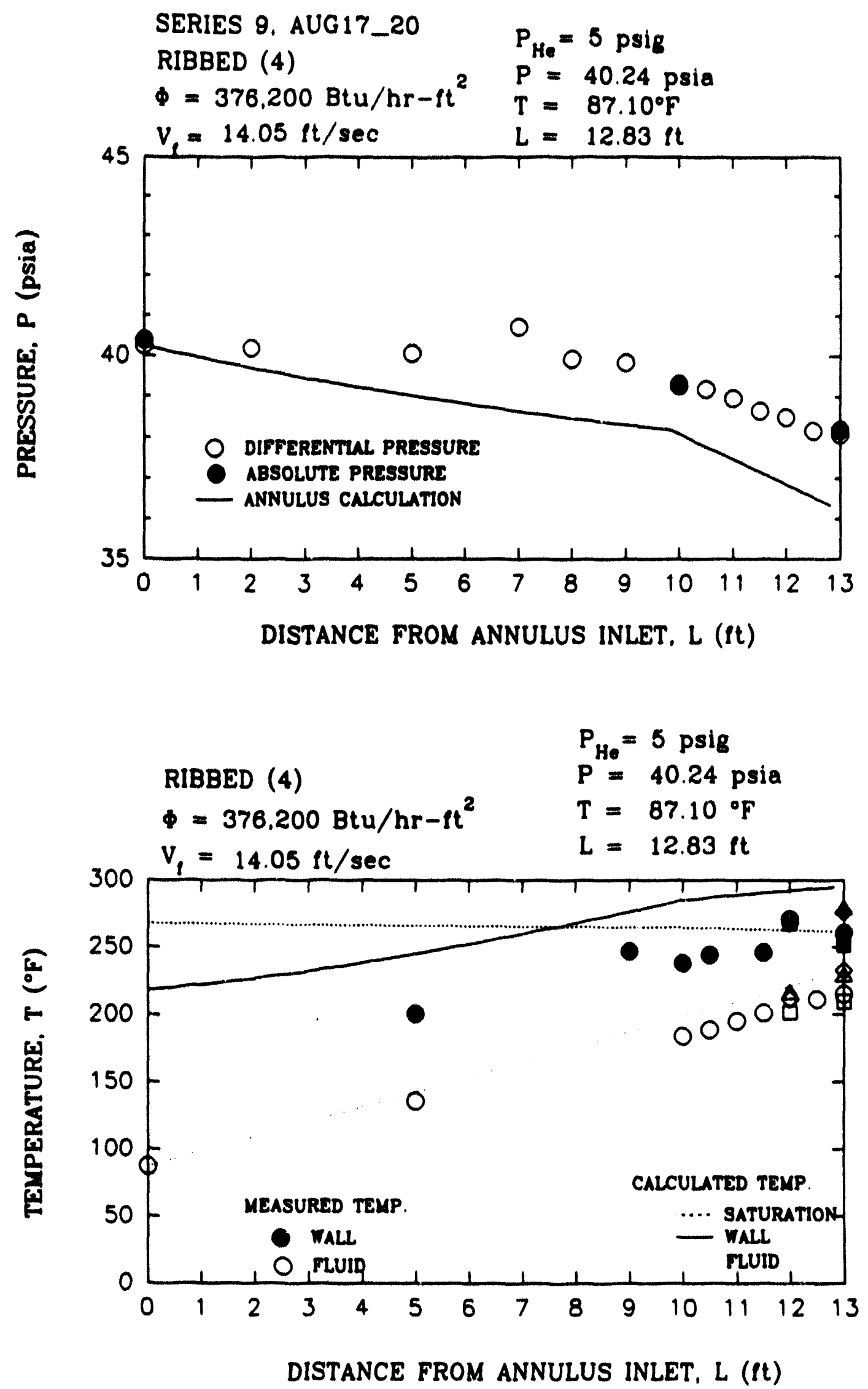

AUG17_20 

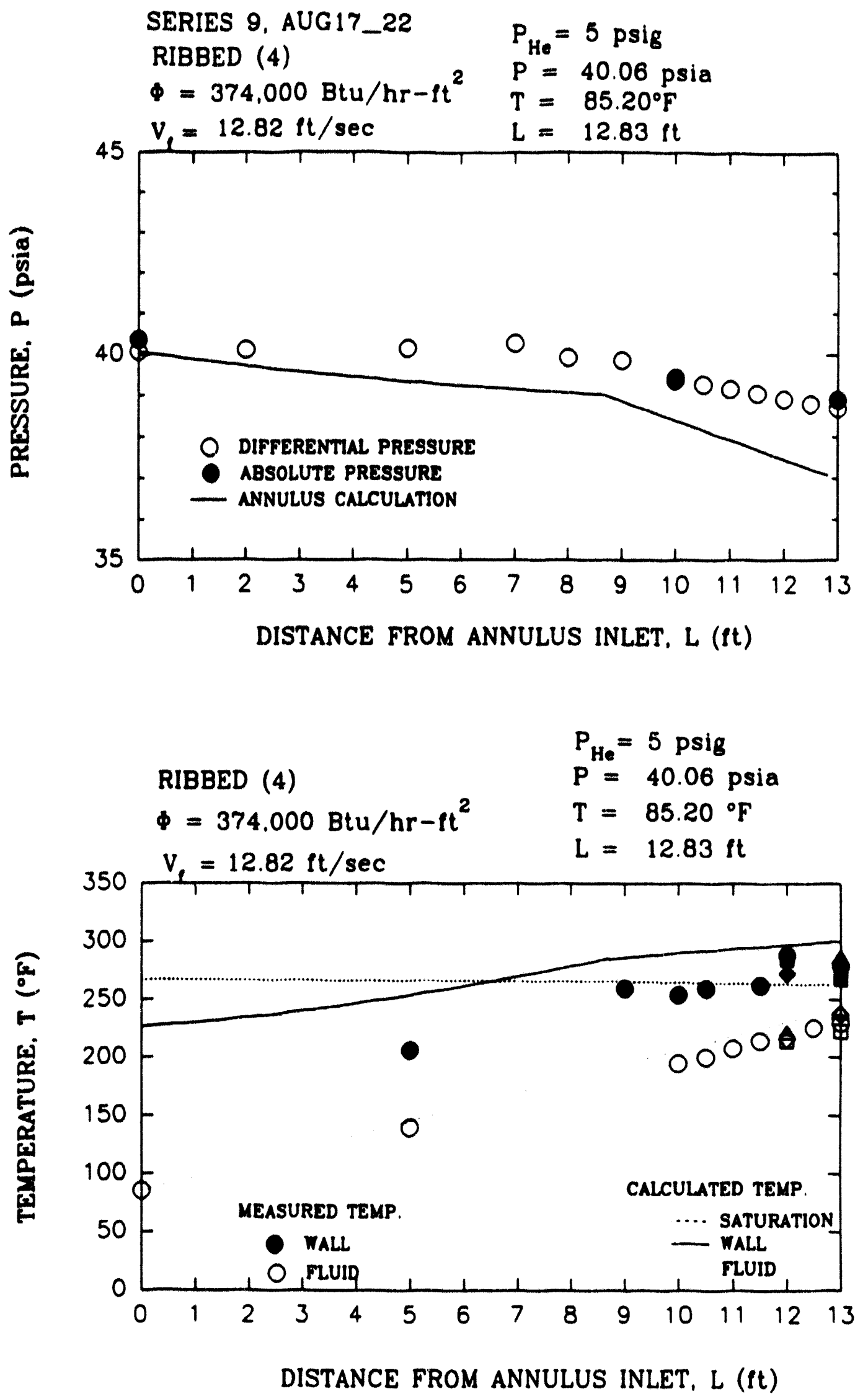

AUG17_22 

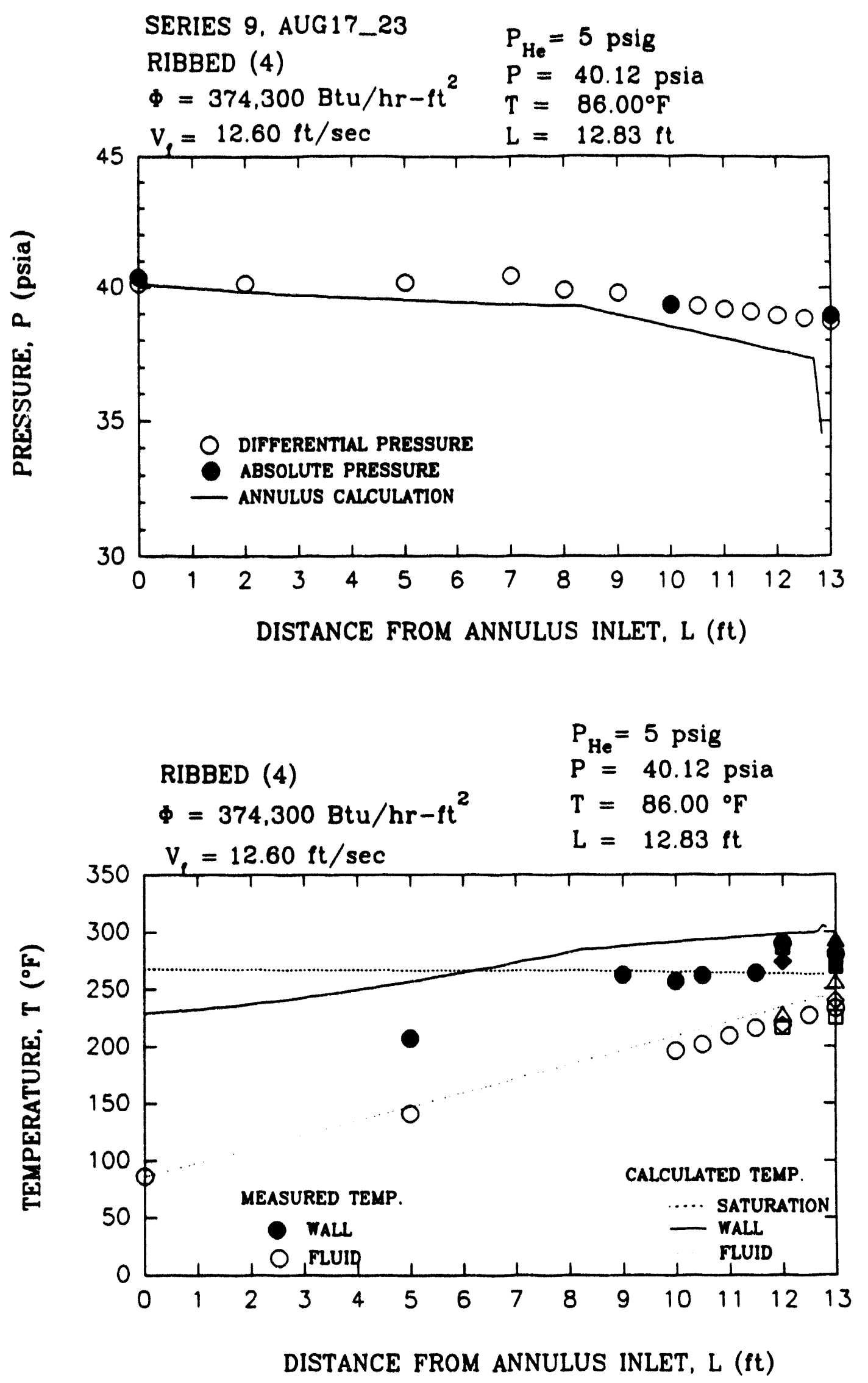

AUG17_23 

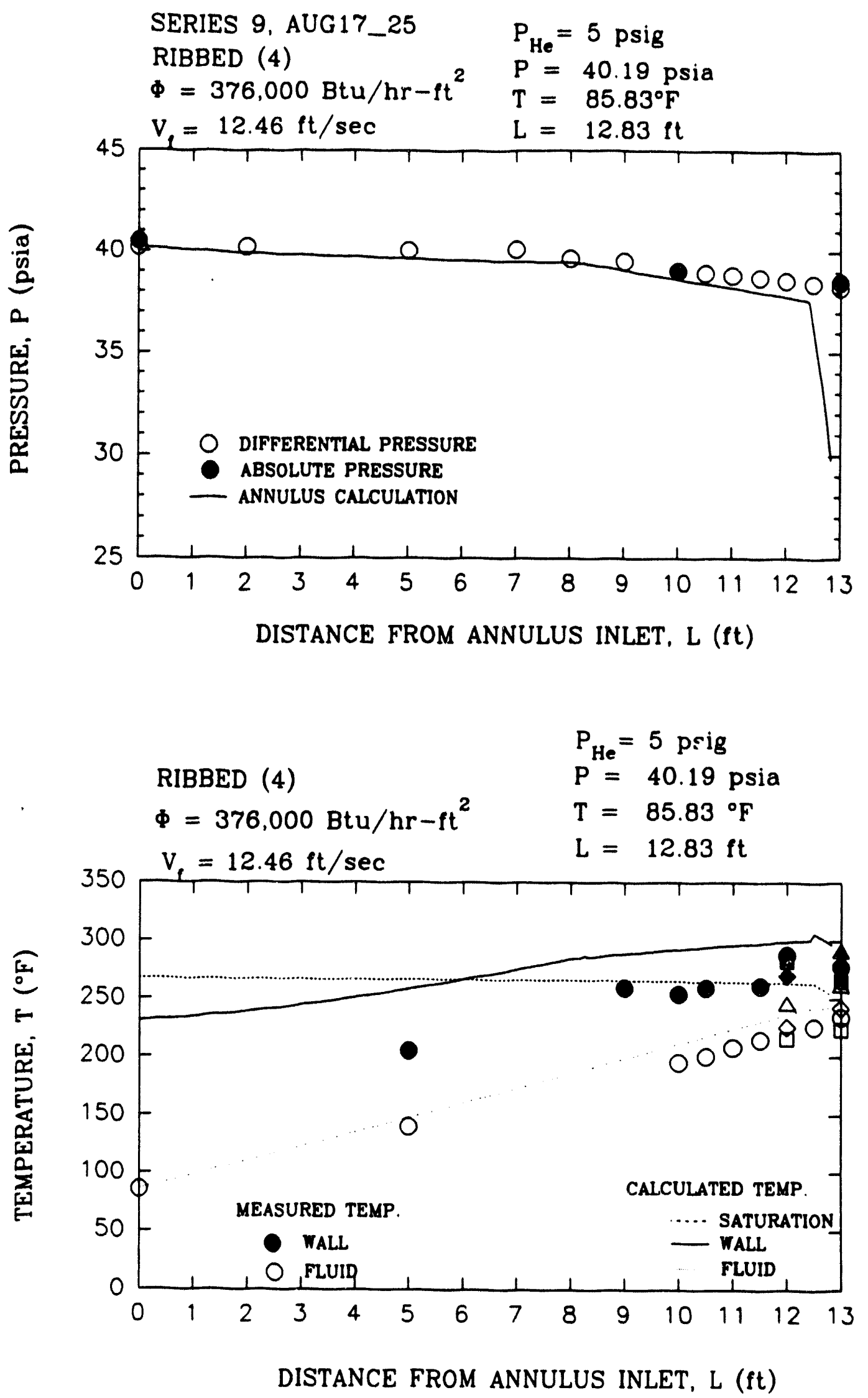

AUG17_25 


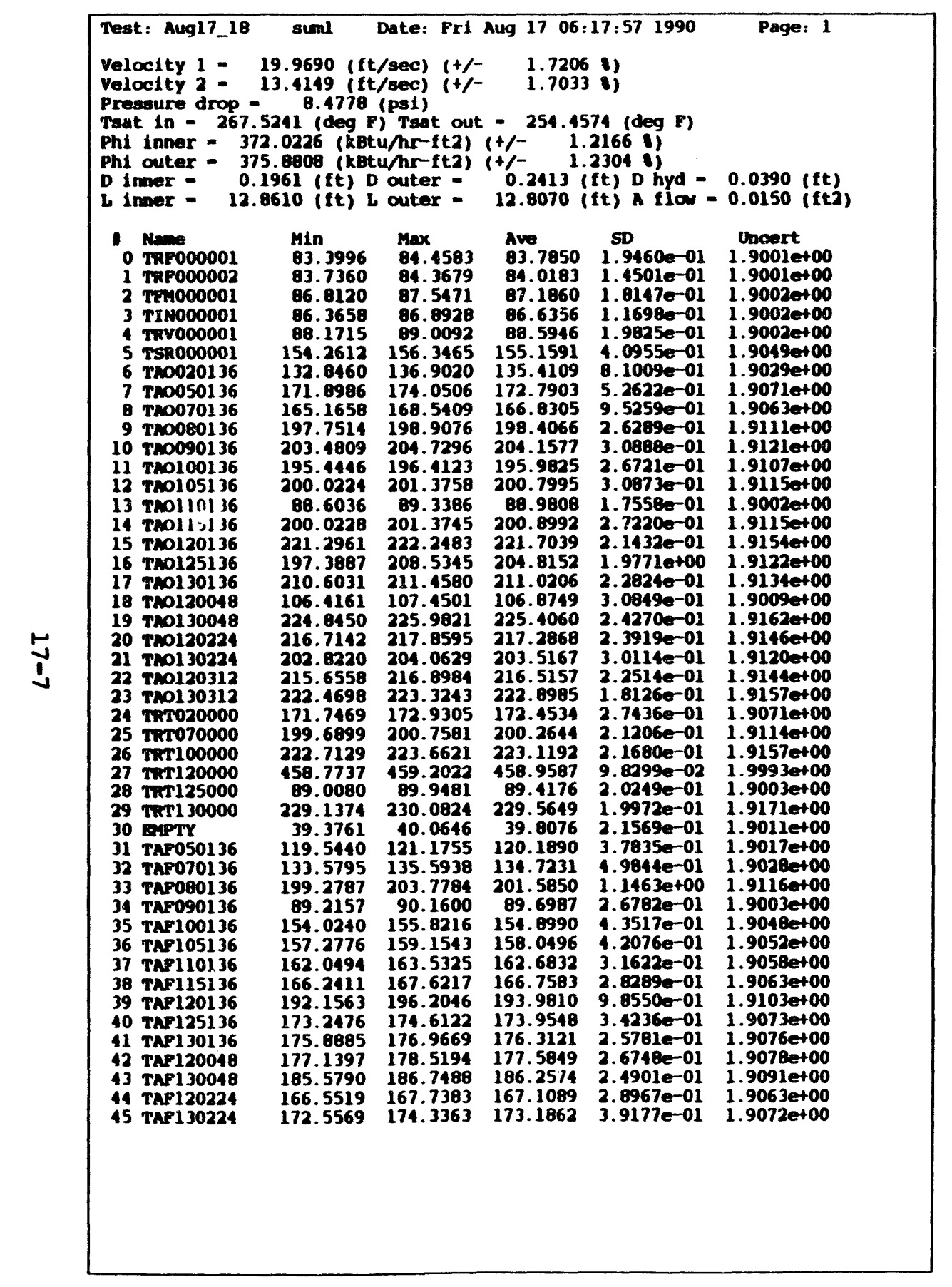

\begin{tabular}{|c|c|c|c|c|c|}
\hline 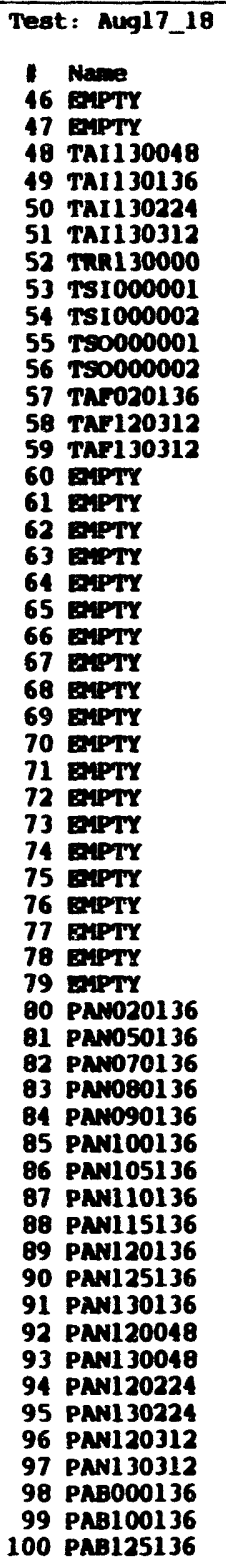 & 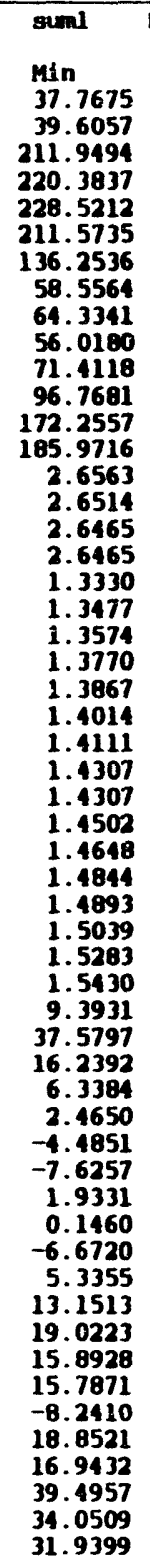 & 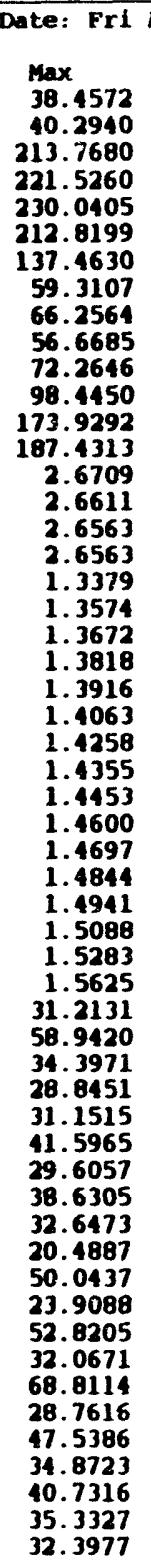 & 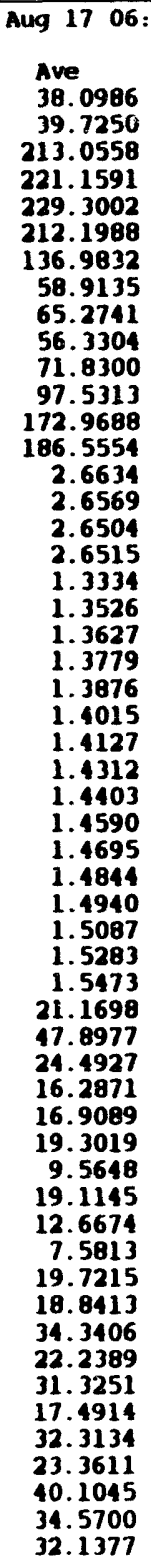 & 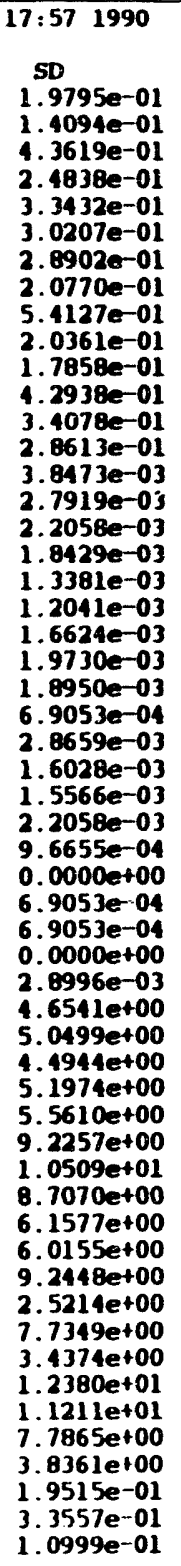 & 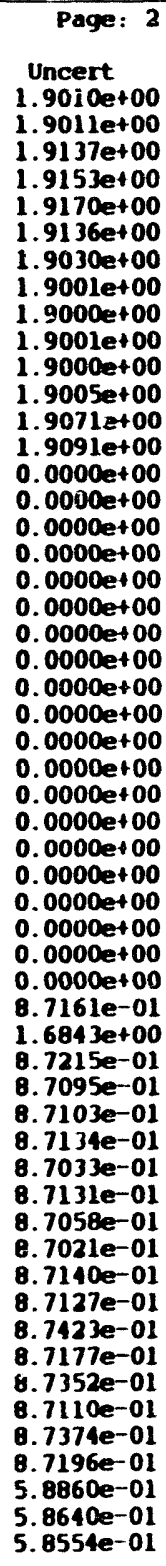 \\
\hline
\end{tabular}




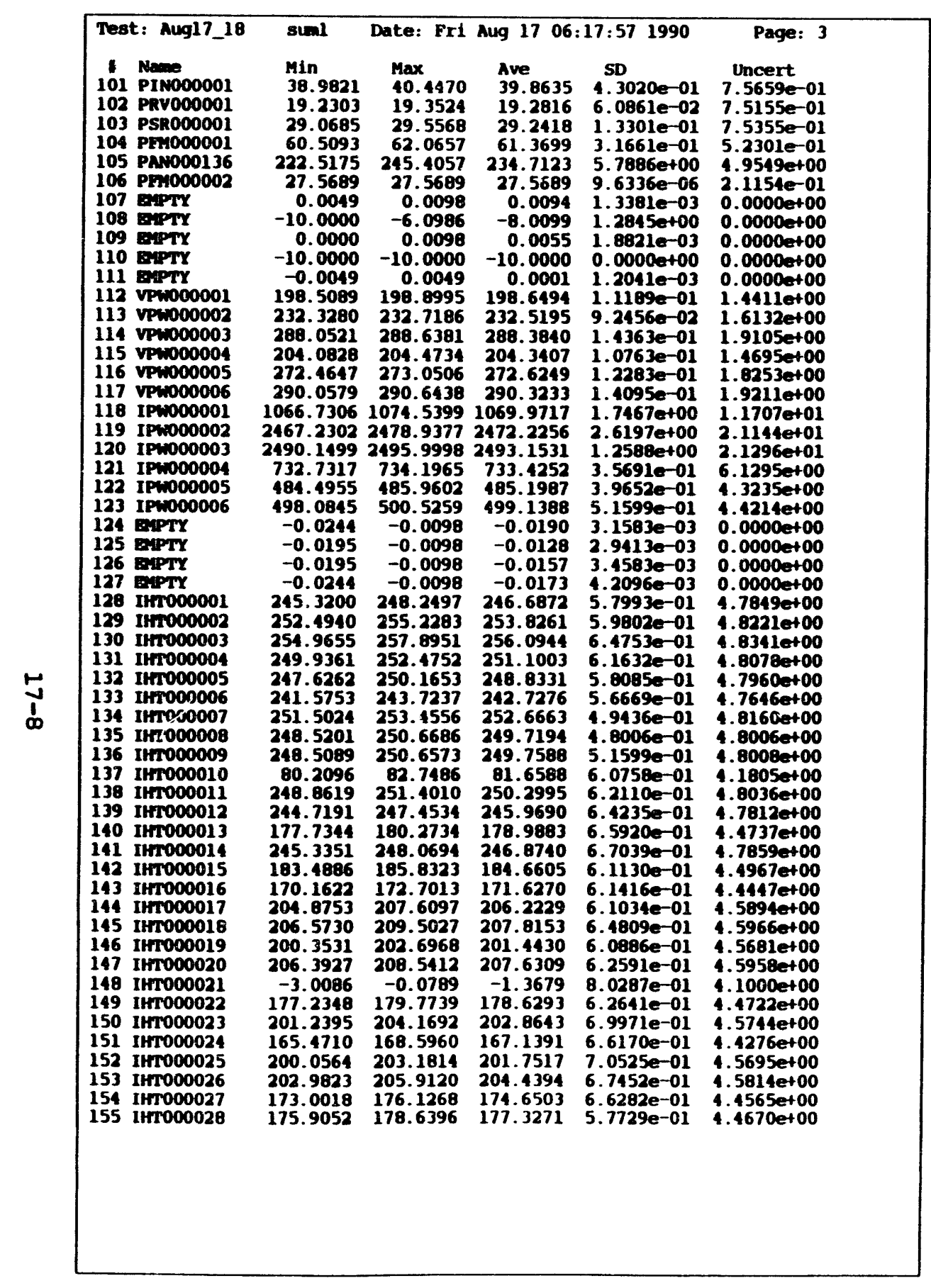

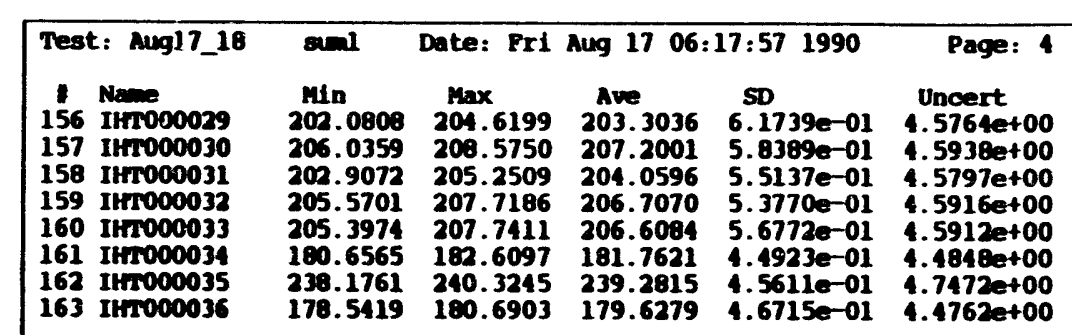


Test: Aug17_19 suml Date: Fri Aug 17 06:38:36 1990 Page: 1

Velocity 1 - $16.0692(\mathrm{ft} / \mathrm{sec})(t /-1.7866$ : $)$

Pressure drop - 13.41360 (Pe)

Tsat in - 267.7918 (deg F) Tsat out $=261.9453$ (deg F)

Phi inner = 375.6472 (kBtu/hr-ft2) $(+/-\quad 1.2157$ :

D inner - 0.1961 (ft) D outer - 0.2413 (ft) D hyd $=0.0390(\mathrm{ft})$ 6 inner = 12.8610 (ft) $L$ outer $=12.8070$ (ft) A flow $=0.0150$ (ft2)

o TRF000001

1 TRF000002

2 TFHOOOOO

TRVO00001

5 TSR000001

6 Th0020136

7 Tro050136

8 Tro070136

9 Th0080136

11 Tמ0100136

12 Tno105136

13 TRo110136

14 Tho115136

$15 \mathrm{Tn} 0120136$

16 Th0125136

18 TMO120048

19 TRO1 30048

20 TRO120224

21 T2013022

$23 \mathrm{THO130312}$

24 TRT020000

25 Tmr070000

26 TRT100000

27 TET120000

28 TRT125000

29 TRT1 30000

31 ExTY

32 TrFos 136

33 TrFo80136

34 TAF090136

35 TAF100136

36 TAF105136

37 TAF110136

38 TAP115136

39 TAF120136

10 TAF125136

41 TAF130136

42 TAP120048

43 TAF1 3004

44 TAF120224
$\operatorname{Min}_{83.1877} \operatorname{Max}_{83.8232}$

Ave SD Uncert

$\begin{array}{lll}1.6988 e-01 & 1.9001 \text { e+00 }\end{array}$

$\begin{array}{lllll} & 05.8676 & 85.5020 & 1.7932 \mathrm{e}-01 & 1.9002 \mathrm{e}+00\end{array}$

87.2297 86.6440 2.4869e-01 $1.9002 \mathrm{et00}$

$155.01537 \quad 1503242 \quad 156.7668 \quad 1.016004001 .9051 e+00$

$125.6153 \quad 134.7742 \quad 132.2874 \quad 2.436204001 .9026 \mathrm{e}+00$

$\begin{array}{lllllll}186.5883 & 188.4276 & 187.3901 & 4.9361 \mathrm{e}-01 & 1.9093 \mathrm{et} 0\end{array}$

$\begin{array}{lllllll}180.7822 & 184.0220 & 181.9921 & 6.3963 e-01 & 1.9084 e+00\end{array}$

$217.9720 \quad 219.3955 \quad 218.6173 \quad 3.3072 e-01 \quad 1.9148 e+00$

$\begin{array}{lllll}225.8902 & 227.1212 & 226.5108 & 3.1545 e-01 & 1.9164 e+00\end{array}$

$217.2234219 .2241 \quad 218.2829 \quad 3.5512 \mathrm{e}-01 \quad 1.9148 \mathrm{e}+00$

$22.5187224 .7558 \quad 224.10502 .6595 e-01 \quad 1.9159 e+00$

90.7807 $91.616091 .1864 \quad 1.5872 \mathrm{e}-01 \quad 1.9003 \mathrm{et} 00$

$223.9682 \quad 225.1098 \quad 224.4860$ 2.969se-01 1.9160e+00

$237.2780233 .3902237 .79002 .54320-011.9169 e 00$

109.9242 111.0509 110.5430 3.2129e-01 $1.91011 \mathrm{e} 00$

255.5640 $254.9515 \quad 2.45670-01 \quad 1.92290000$

$243.6609244 .8794 \quad 244.3287 \quad 2.73690-01 \quad 1.92040400$

$\begin{array}{lllllll}228.4461 & 230.3263 & 229.2520 & 3.8452 e-01 & 1.9170+00\end{array}$

$243.6951245 .0086 \quad 244.3408 \quad 3.0856 e-01 \quad 1.9204 e+00$

$\begin{array}{llllll}251.3117 & 252.2437 & 251.7333 & 2.1746 e-01 & 1.9221 e+00\end{array}$

187.1592 188.3310 $187.7334 \quad 2.7510 e-01 \quad 1.9093 e+00$

$220.9310222 .0803221 .4865 \quad 2.4709 e-01 \quad 1.9154$ et 00

$249.0307250 .1503249 .63102 .7445 e-01 \quad 1.9216 e+00$

$45.5175 \quad 458.8599 \quad 458.7228 \quad 8.9785 \mathrm{e}-02 \quad 1.9992 \mathrm{et} 00$ 261. 0146251.1305 91.7669 1.0616e-01 1.9003et00 $39.3761261 .8497261 .4042 \quad 1.76780-01 \quad 1.9245$ et 00 $126.1511 \quad 40.2940 \quad 127.0168$ 2.2638e-01 1.9011e+00 $143.4063146 .4027 \quad 144.0895 \quad 6.03610-01 \quad 1.9038 e 400$ $224.7419232 .1699229 .2585 \quad 1.537404001 .9170400$ $91.8301 \quad 92.9806 \quad 92.4498 \quad 2.35940-01 \quad 1.9003$ et00 $\begin{array}{llllll}169.1233 & 170.6046 & 169.7910 & 3.6598 e-01 & 1.9067 e+00\end{array}$ $179.1305 \quad 180.4972 \quad 179.6035 \quad 3.0826 e-01 \quad 1.9081 e+00$ 184.1655 185.5286 184.9135 2.7079e-01 1.9089et00 221.3996 227.1600 224.8588 1.378set00 1.9161e+00 192.9039 194.4443 193.8477 3.1050e-01 1.9103et00 196.3955197 .3614 196.8903 2.1764e-01 1.910eet00 197.32671 198.392 197.9182 2.6492e-01 1.9110et00 $184.4928 \quad 185.6623 \quad 185.0661 \quad 2.4081 \mathrm{e}-01 \quad 1.9089 \mathrm{e}+00$
05.

$223.1893249 .2244 \quad 248.6548 \quad 2.3993 \mathrm{e}-01 \quad 1.9214 \mathrm{e}+00$ $172.7743 \quad 174.9209 \quad 173.8718 \quad 4.0276 e-01 \quad 1.9073$ et 00

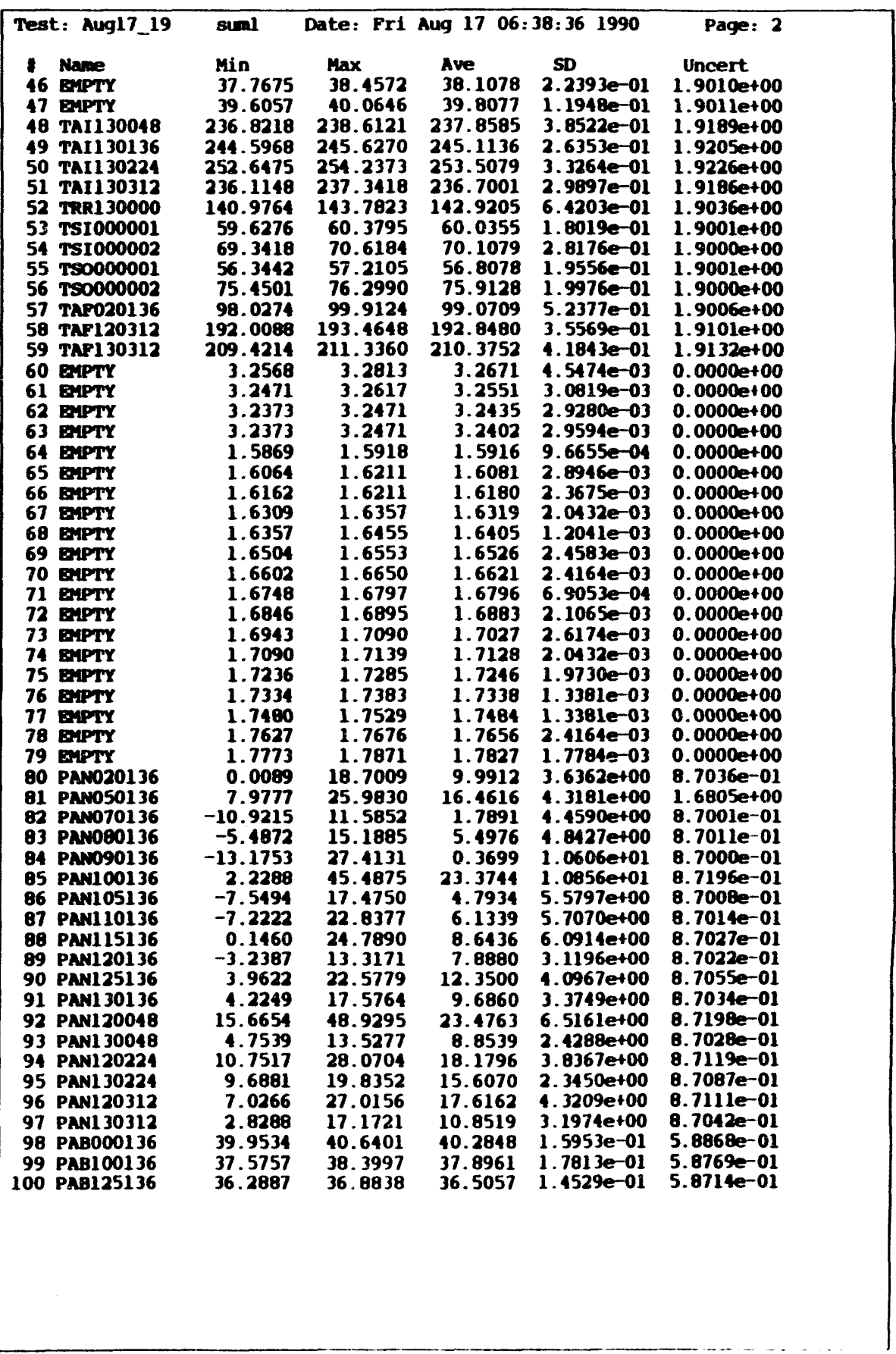




\begin{tabular}{|c|c|c|c|c|c|}
\hline 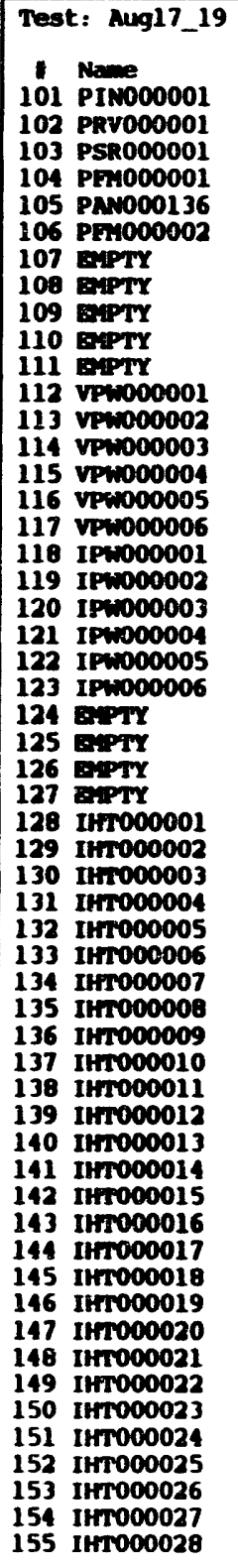 & 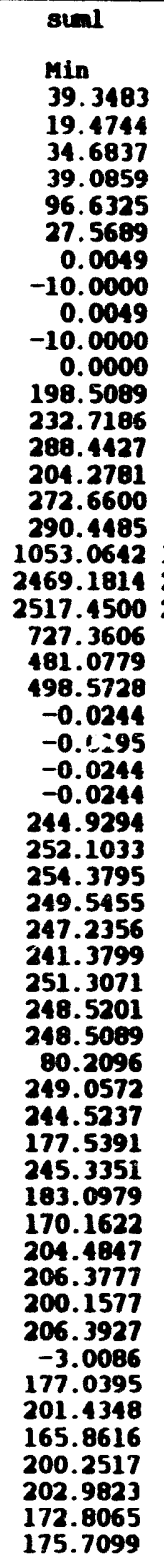 & 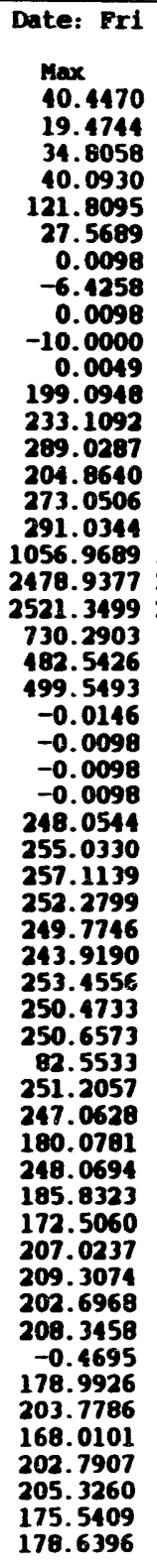 & 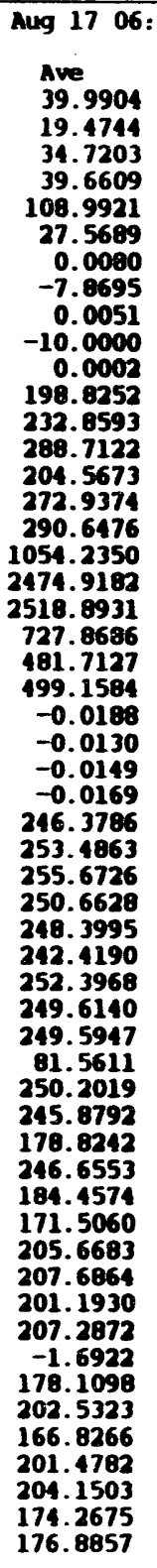 & 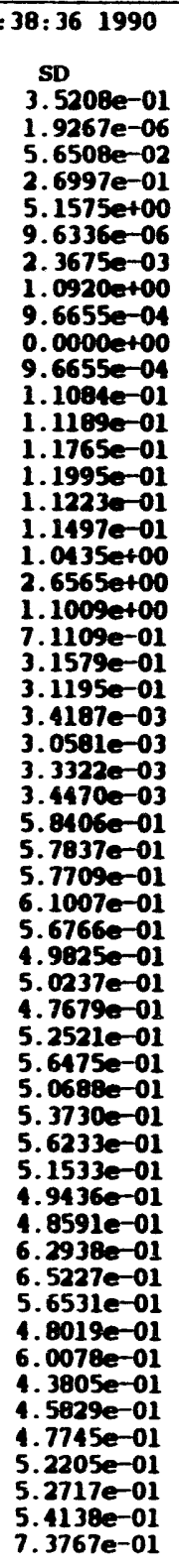 & 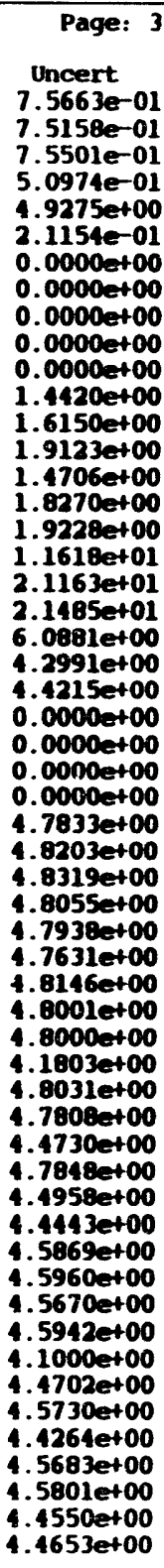 \\
\hline
\end{tabular}

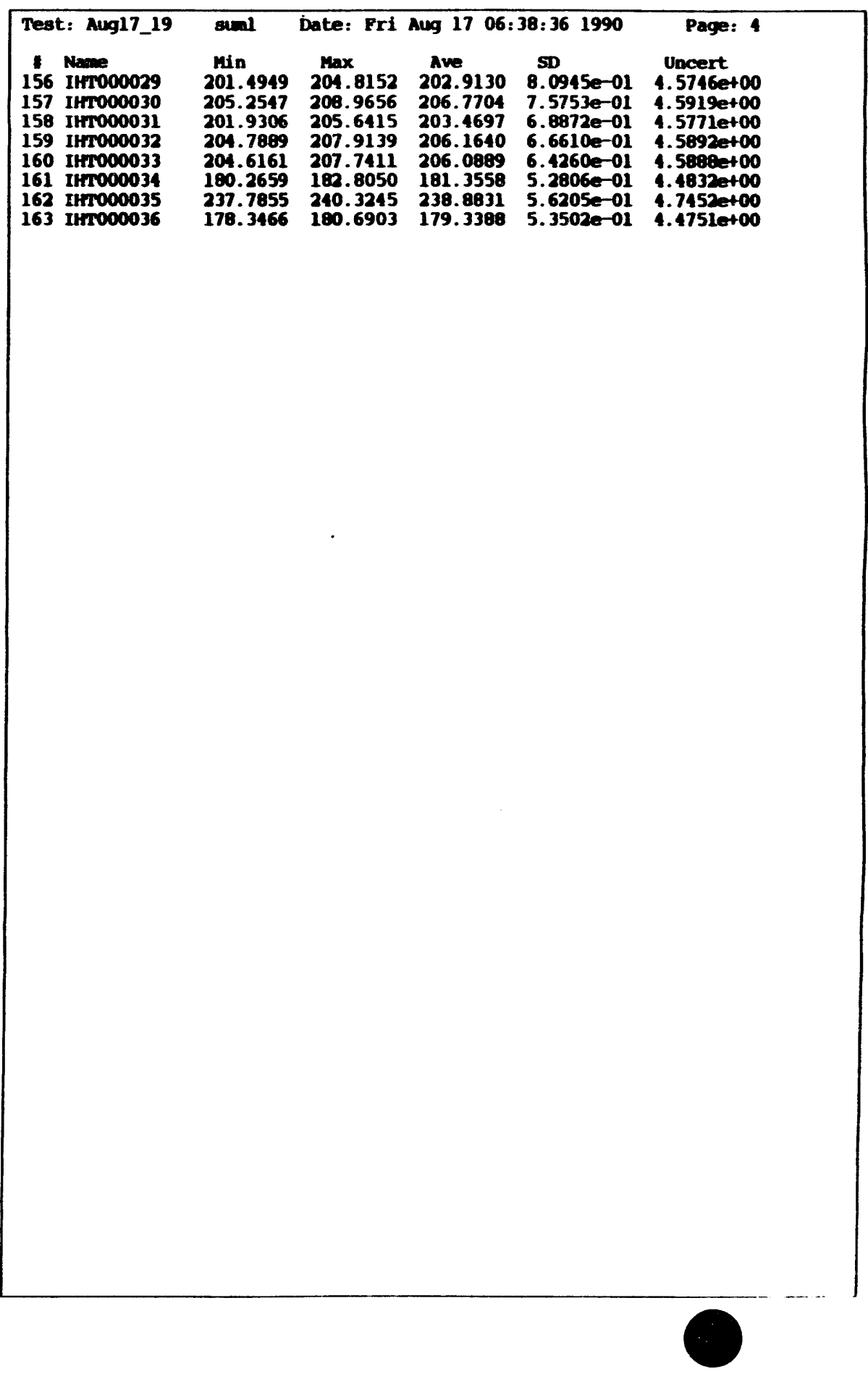




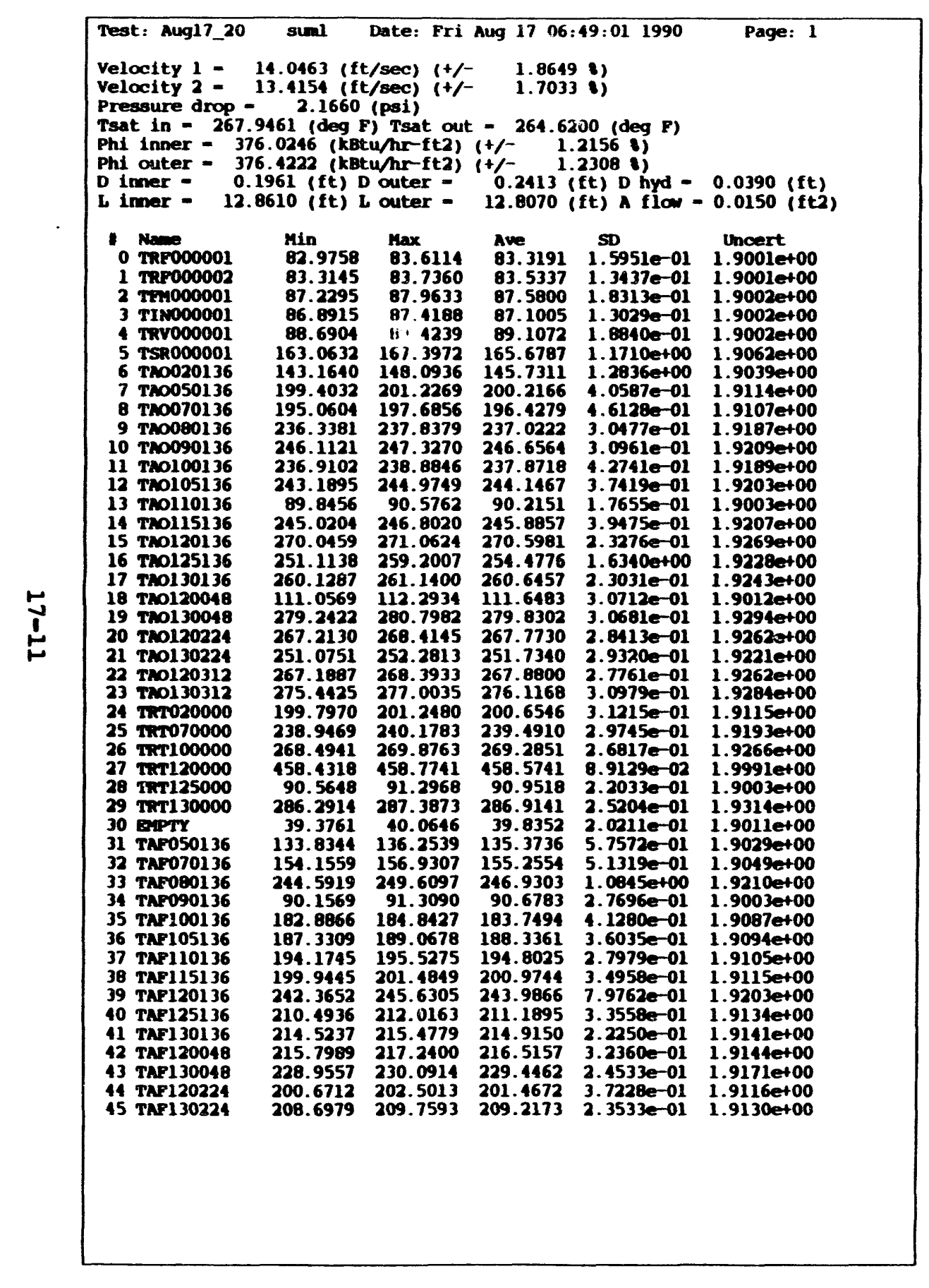

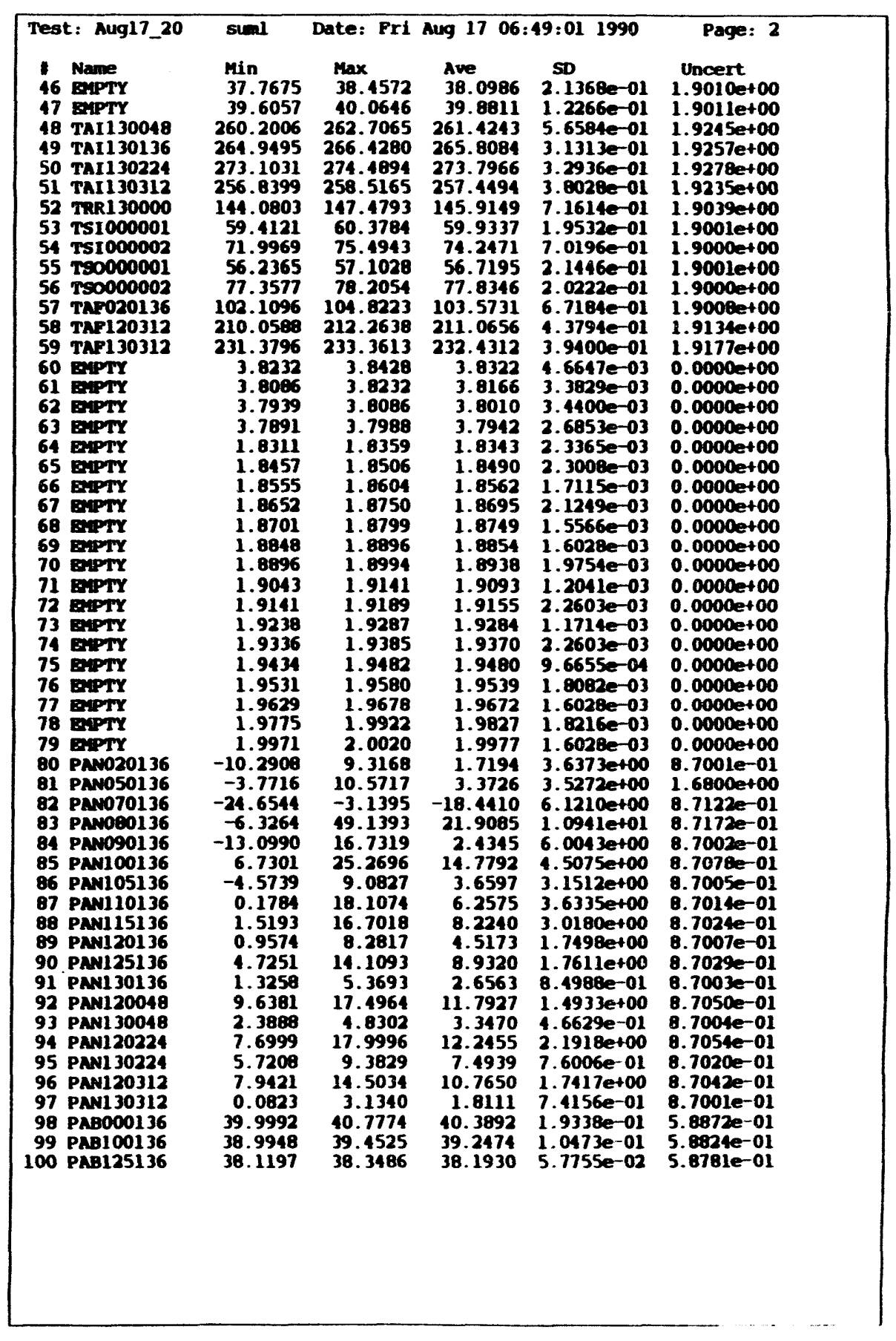




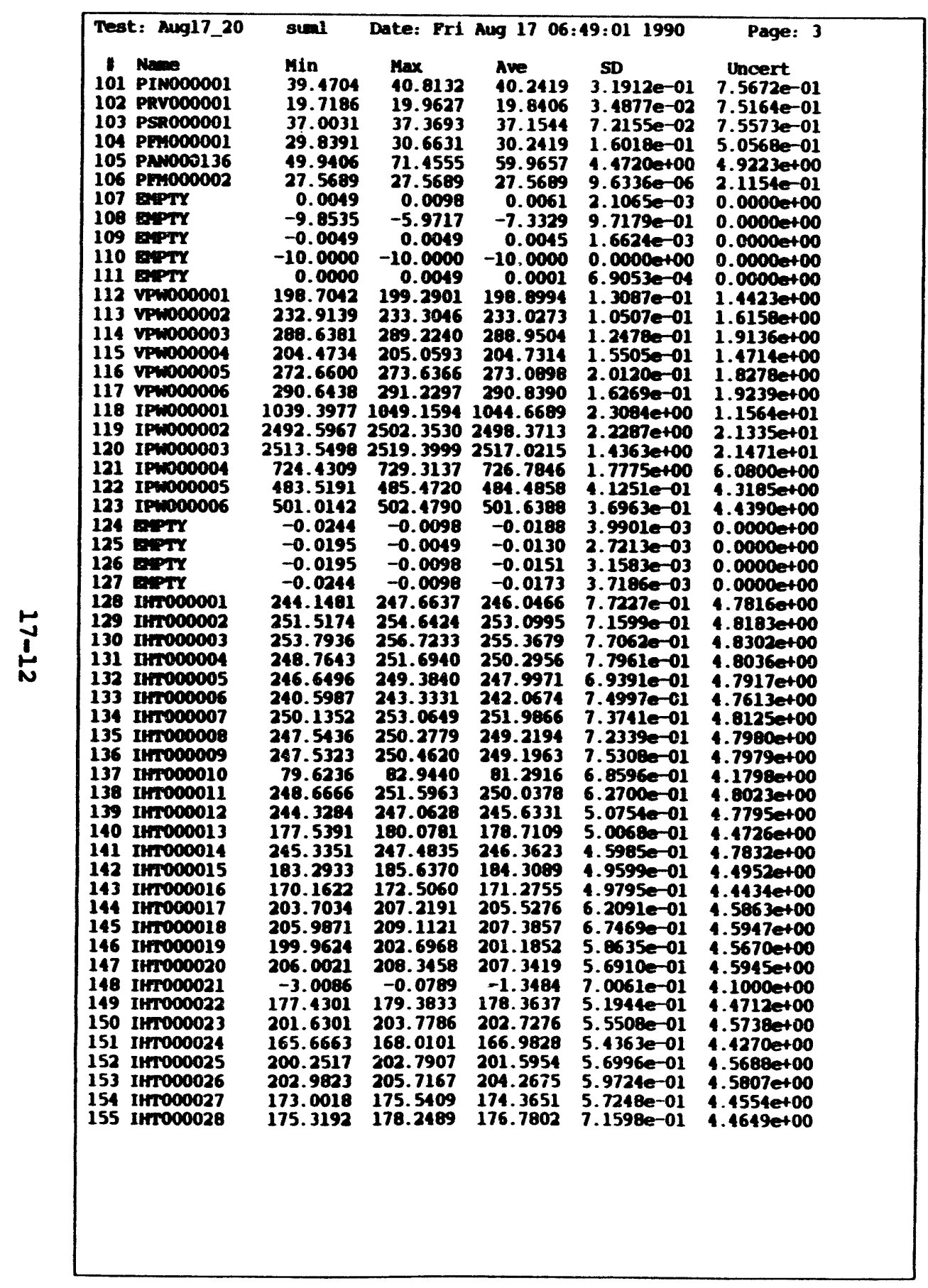

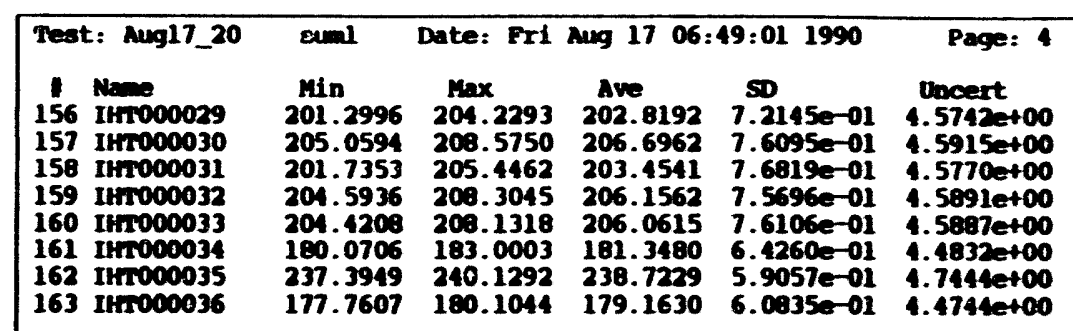




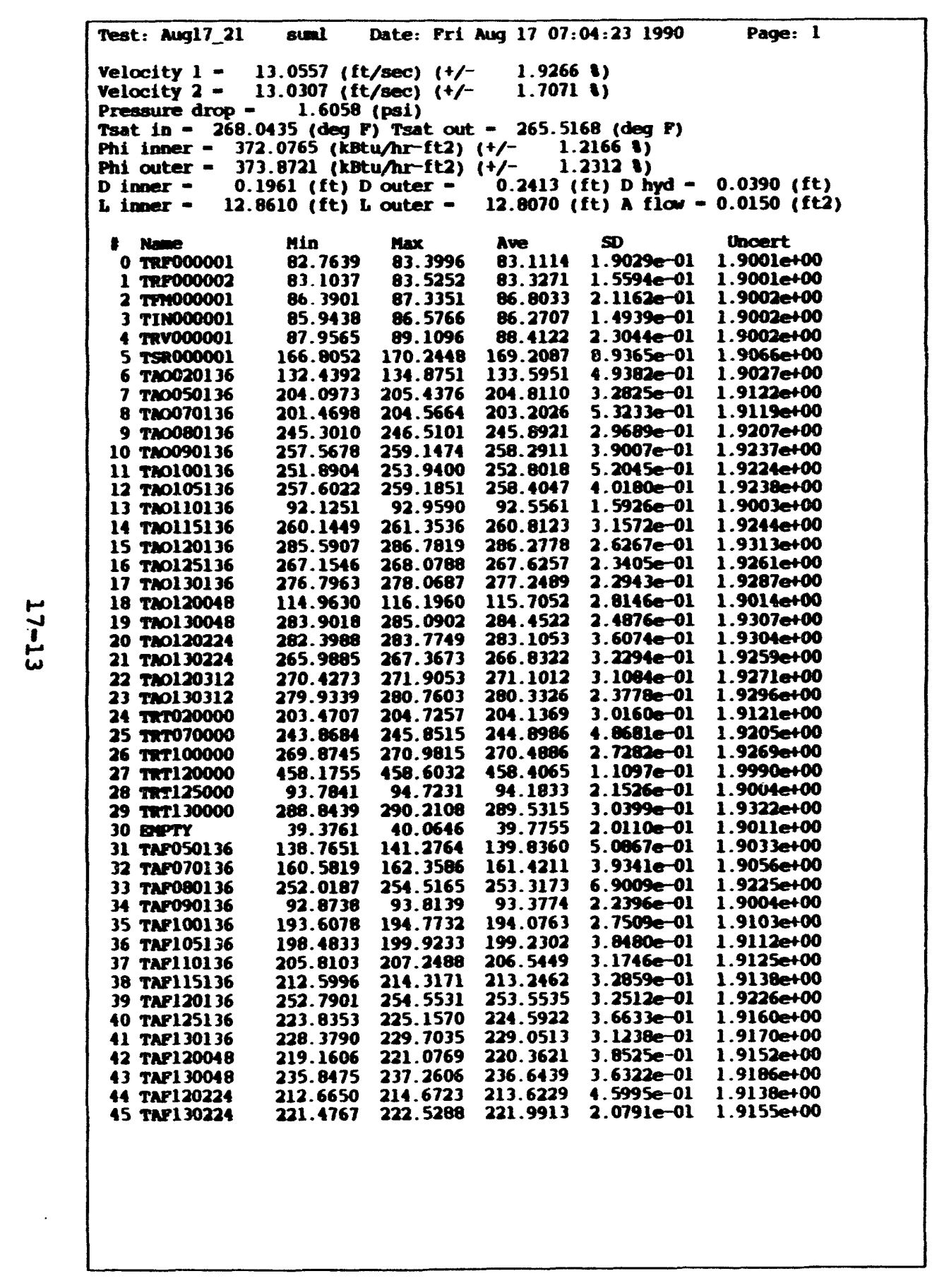

\begin{tabular}{|c|c|c|c|c|c|}
\hline 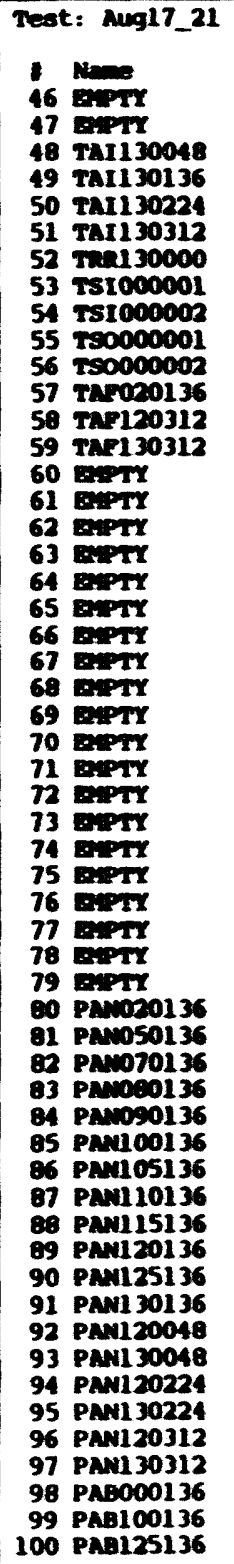 & 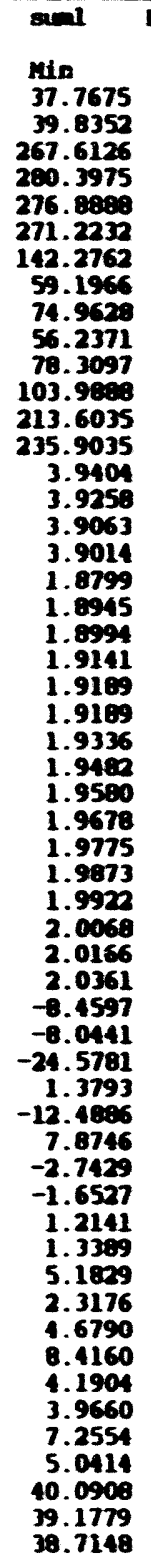 & 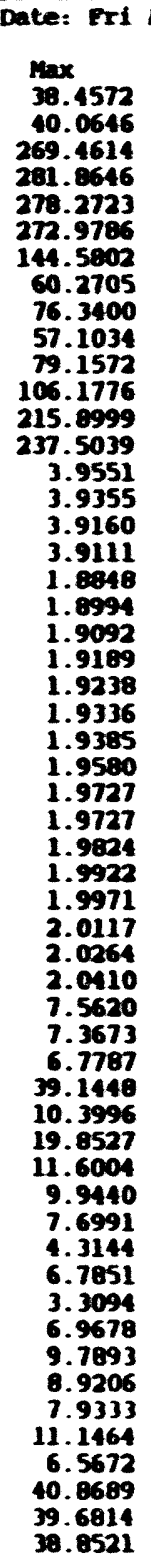 & 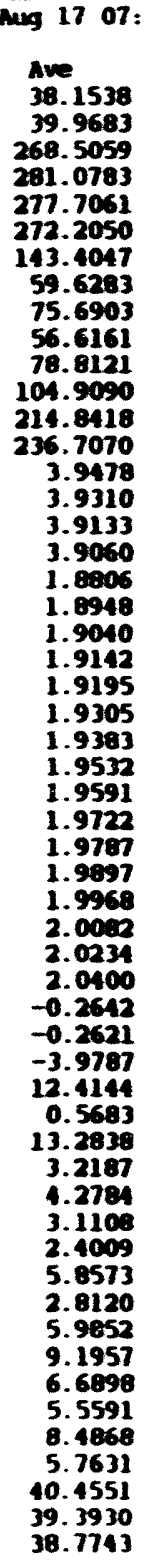 & 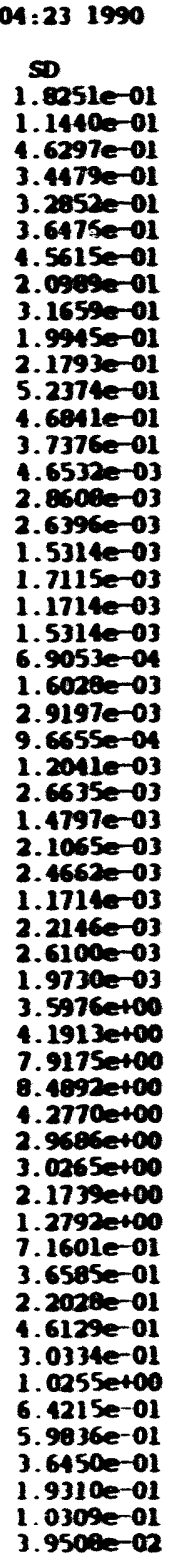 & 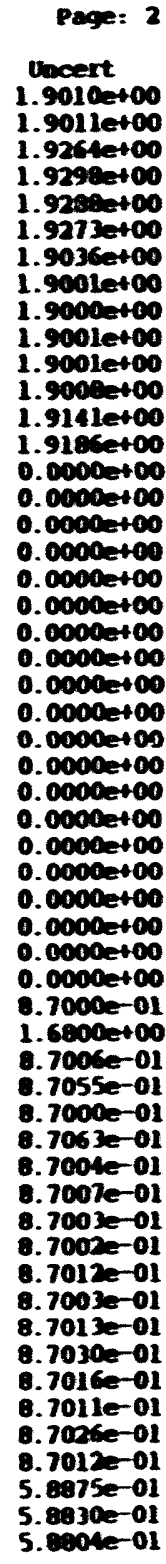 \\
\hline
\end{tabular}




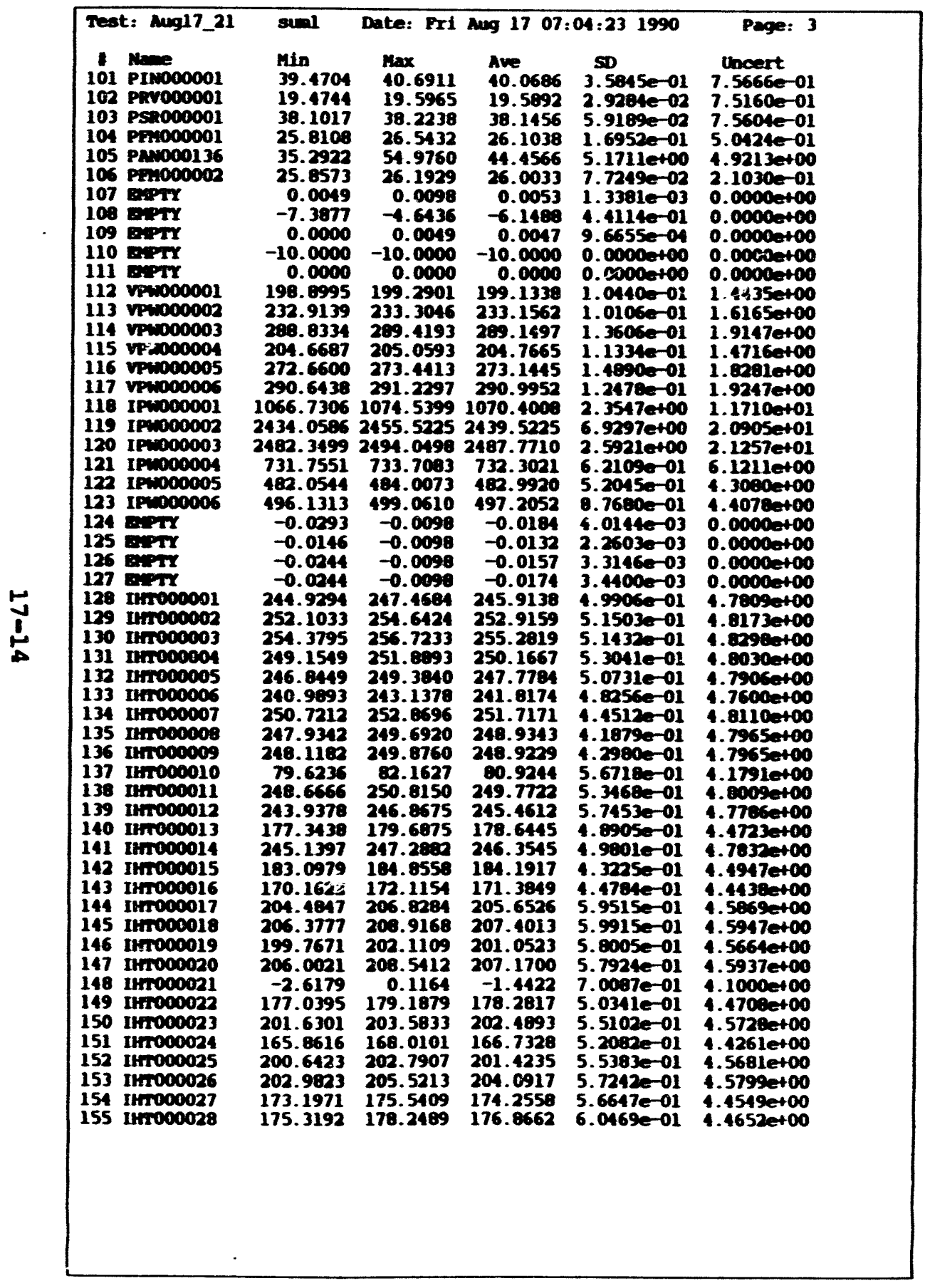

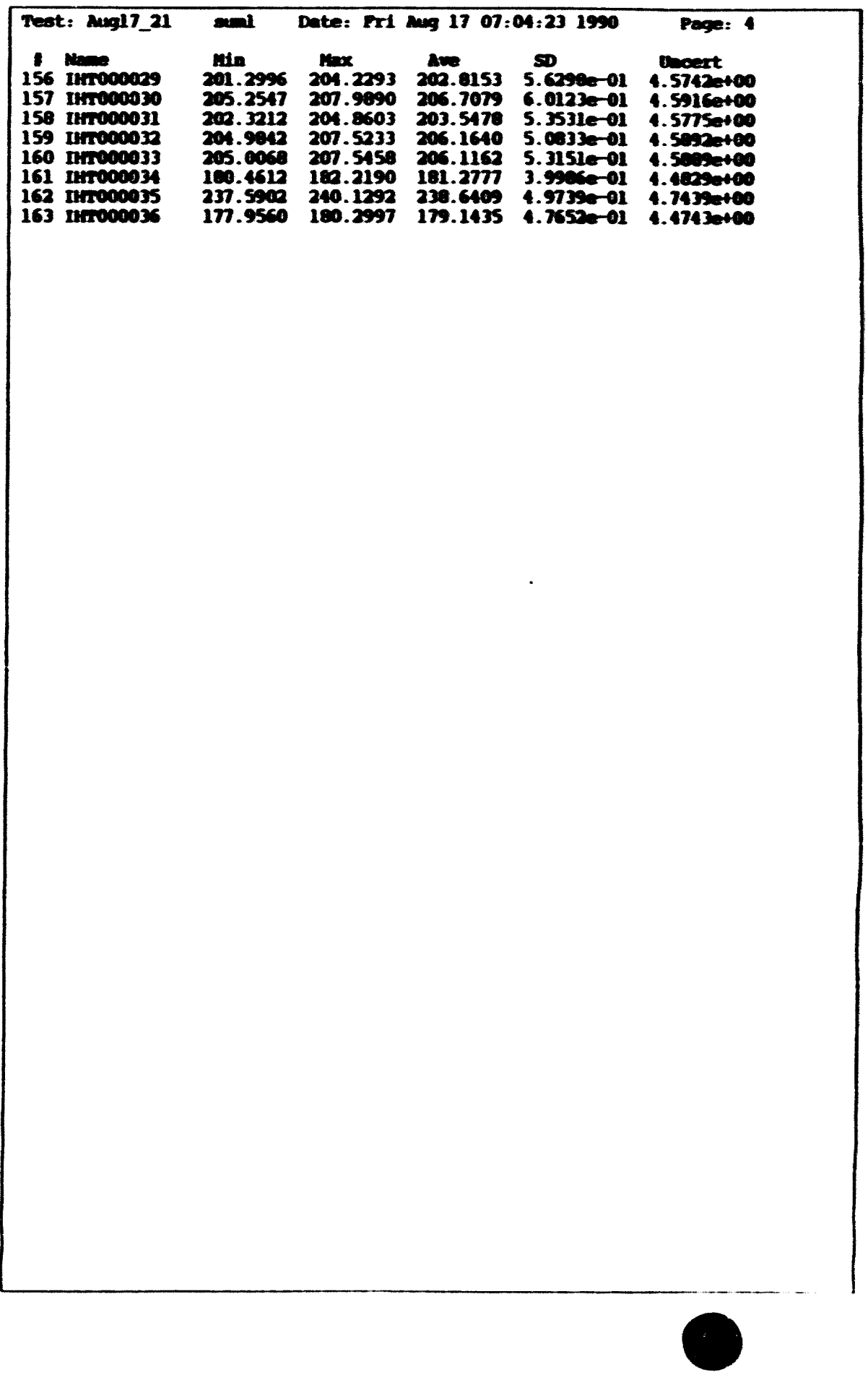




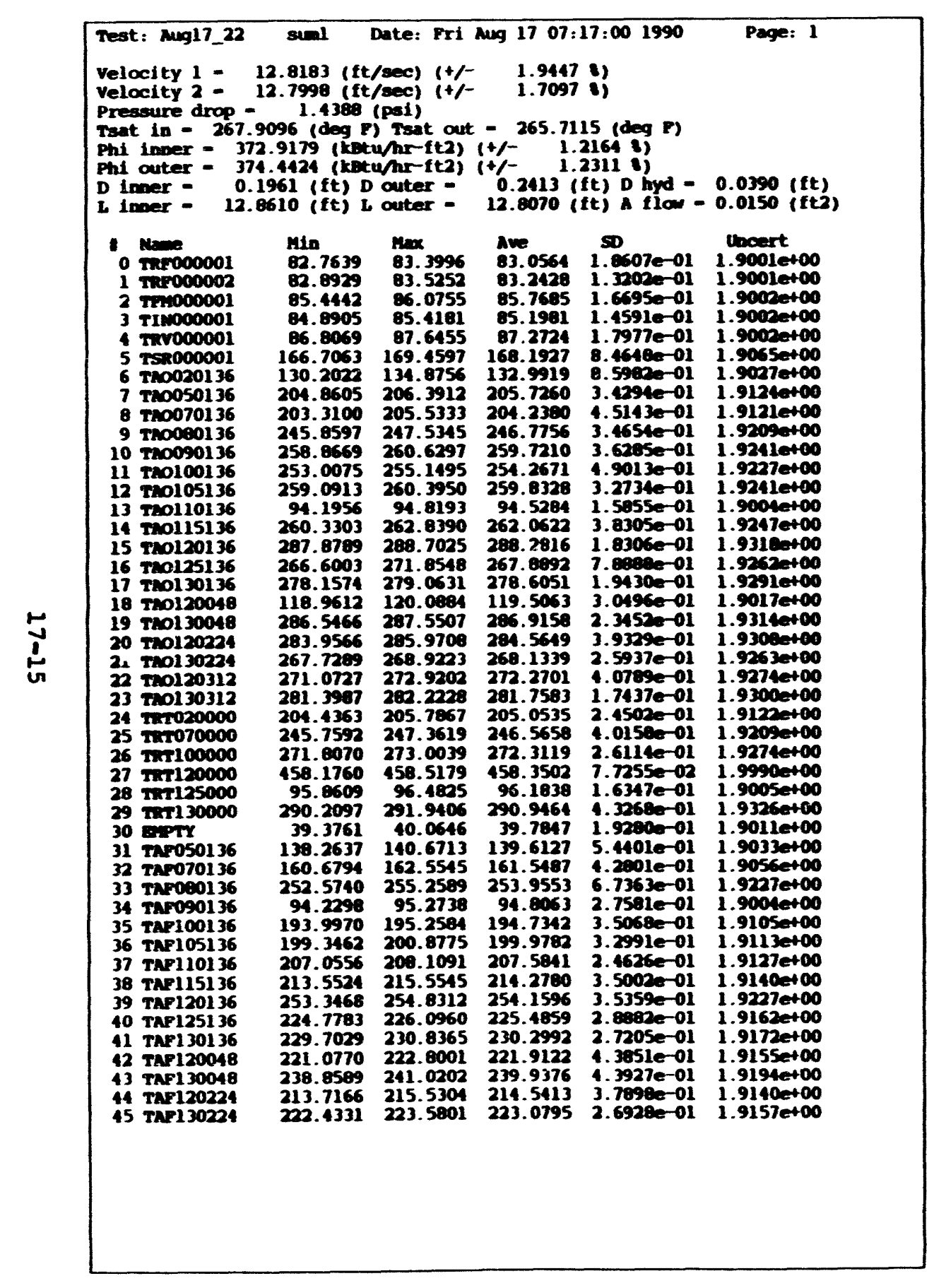

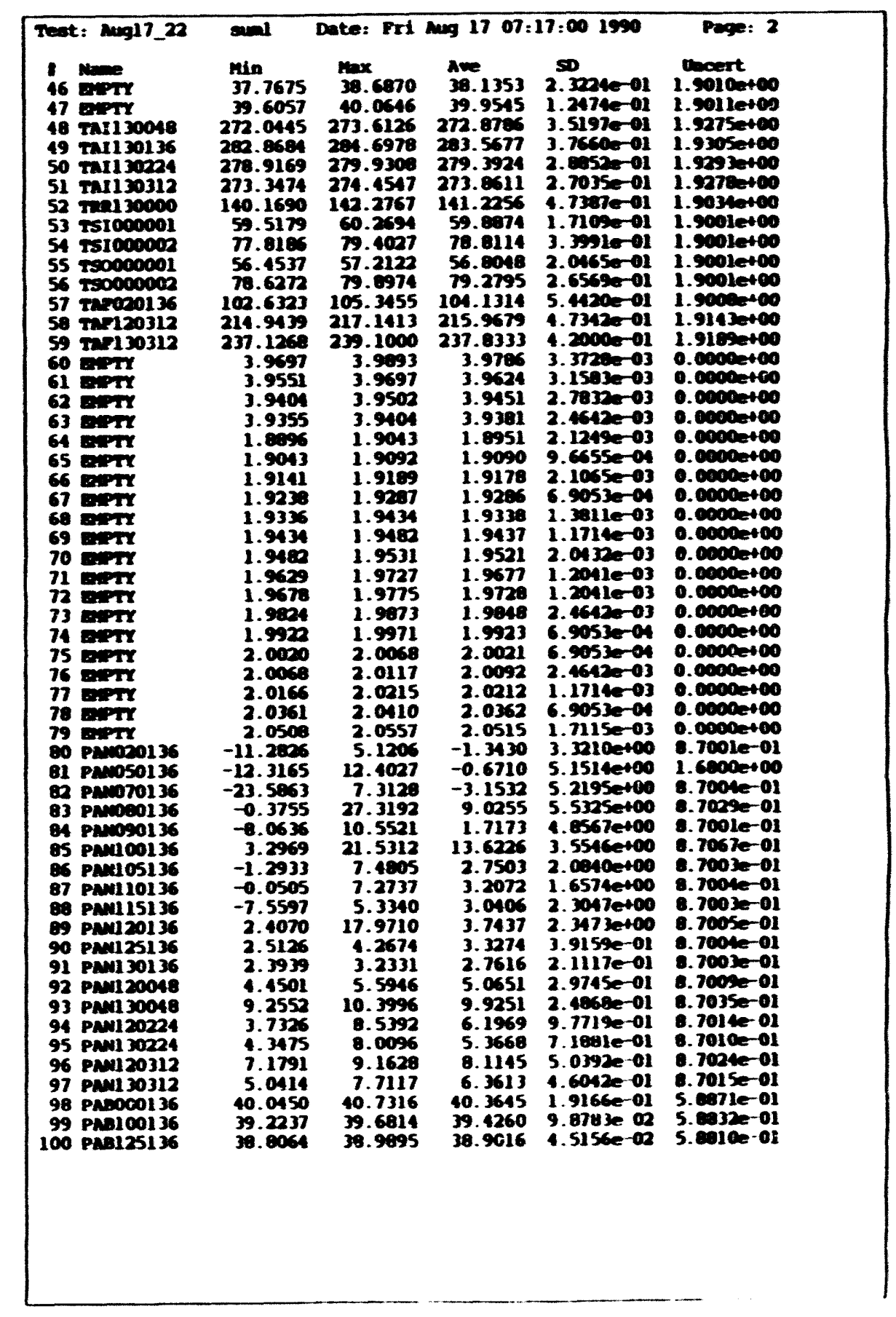




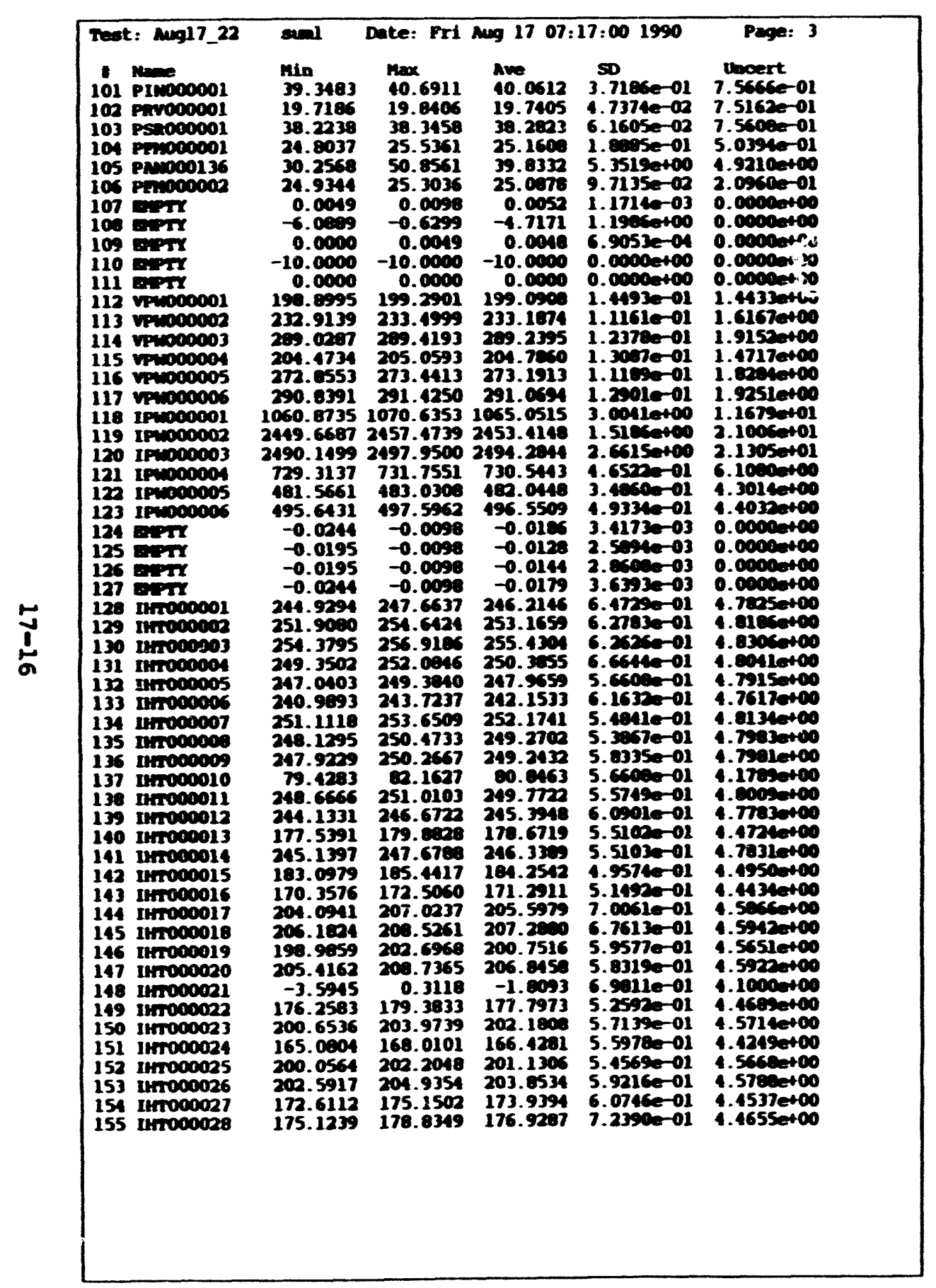

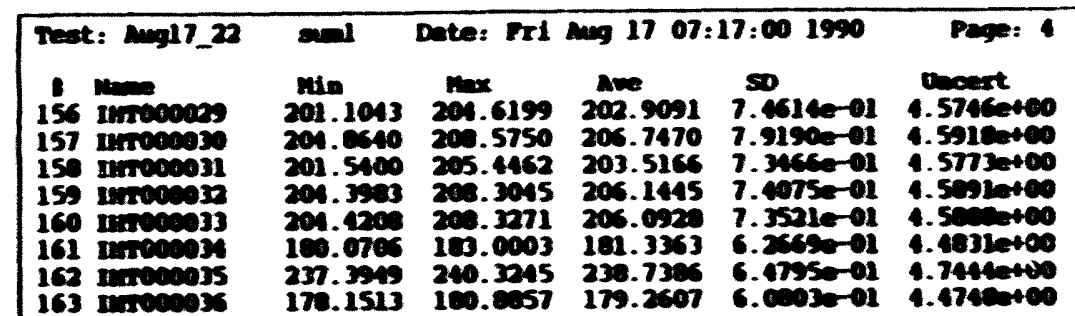


Test: Mug17_23 stal Date: Fri Mug 17 07:29:09 1990 Page:

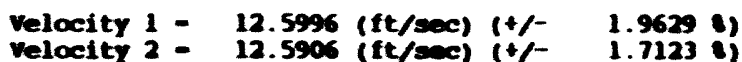
Velocity 2 - 12.5906 (ft/sac) (t/- 1.7123 e) Preasure drop - 1.4380 (poi) rast in - 267.9178 (dog F) Tant out - $265.7716(\mathrm{dog}$ F) Phi inner - 374.6482 (kotu/ni-ft2) $(+/-\quad 1.2160$ :

D inver - 0.1961 (ft) D outer - 0.2413 (ft) D bud - 0.0390 (ft) L inoer - 12.8610 (ft) $L$ outer - 12.0070 (ft) A fiow - 0.0150 (ft2)

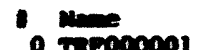

Intoscoor

Trimec0001

Trmosecol

invoc0001

$5 \operatorname{msn} 000001$

$7 \mathrm{rmo050136}$

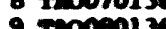

$10 \operatorname{mog} 0136$

$11 \mathrm{Tmol00136}$

12 molosi36

13 molloi 36

$15 \mathrm{~mol} 20136$

$16 \mathrm{~mol} 25136$

19 Tho1 30048

$20 \mathrm{~mol} 20202$

21 Thol 30224

23 Tno130312

24 Imropo000

25 $\mathrm{Tm}$

27 TnT120000

29 ImI 130000

30 Exry

31 Tr.050136

32 тnpo70136

33 TMP0e0136

35 TaP100136

36 TNF105136

37 TrP110136

38 TAF115136

40 TnF125136

11 mris 30136

12 TrF120048

13 TnPl 30049

44 Tar120024

\begin{tabular}{|c|c|c|c|c|c|}
\hline 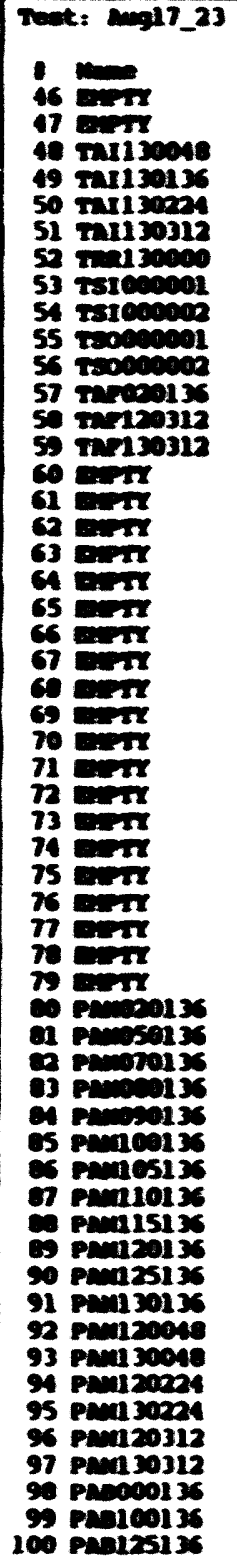 & 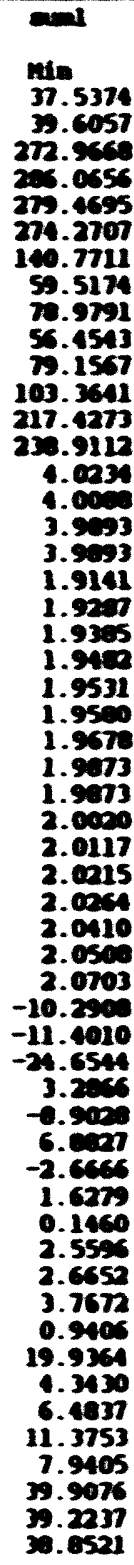 & 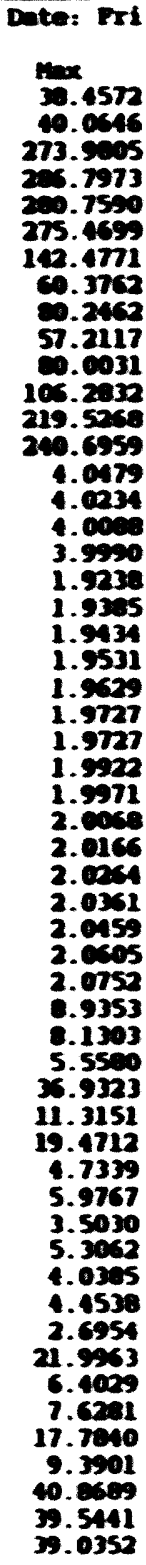 & 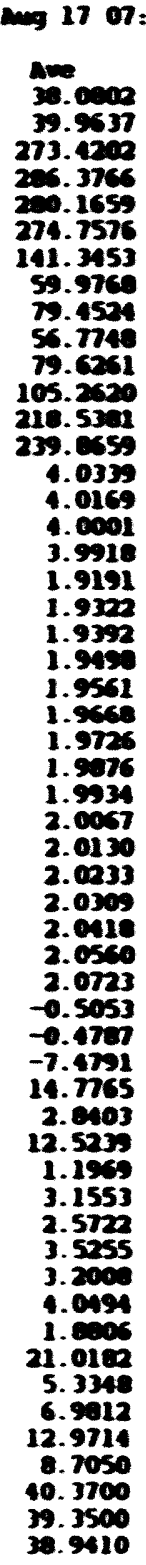 & 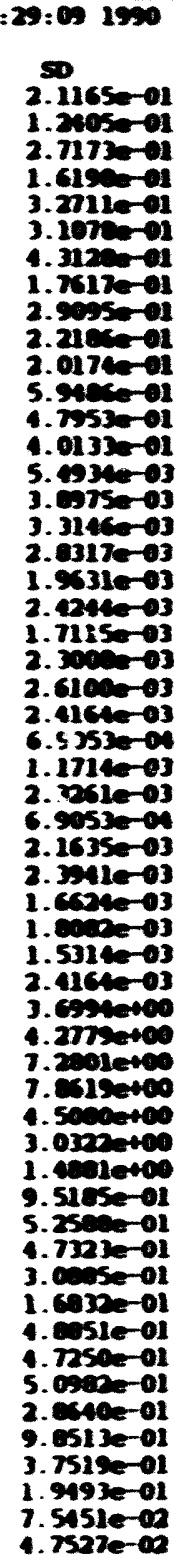 & 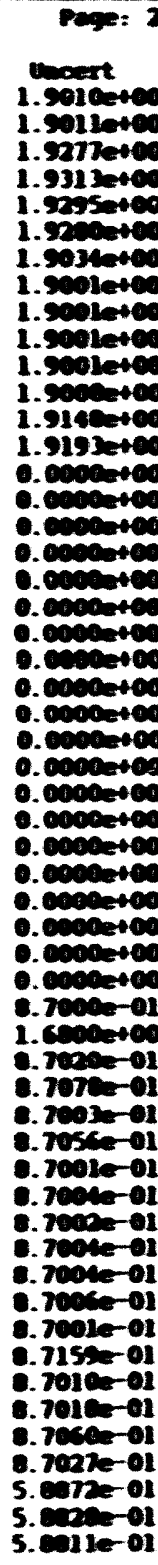 \\
\hline
\end{tabular}




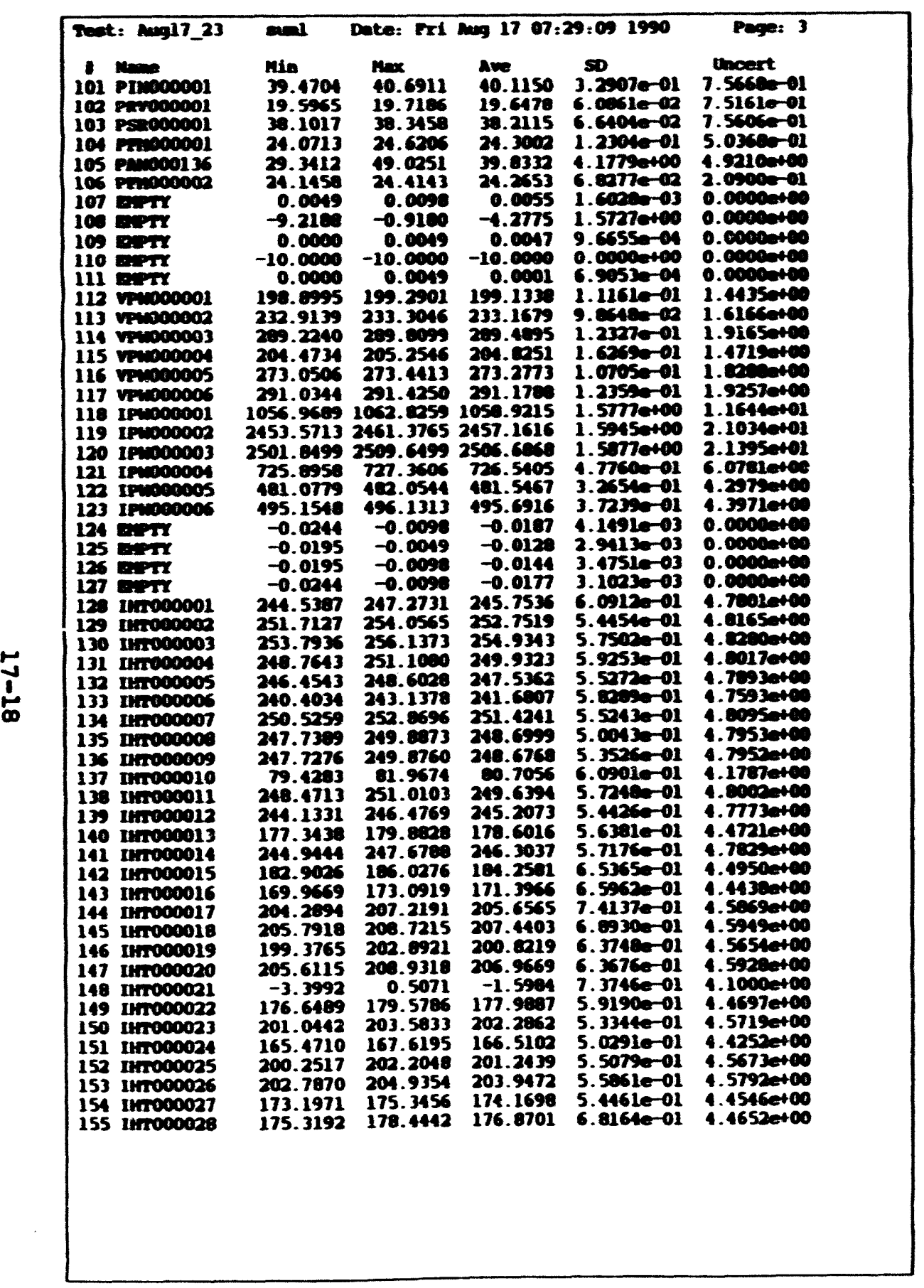

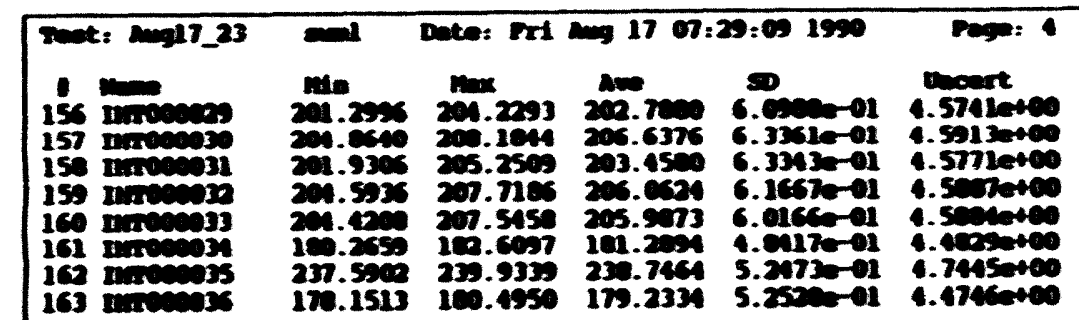




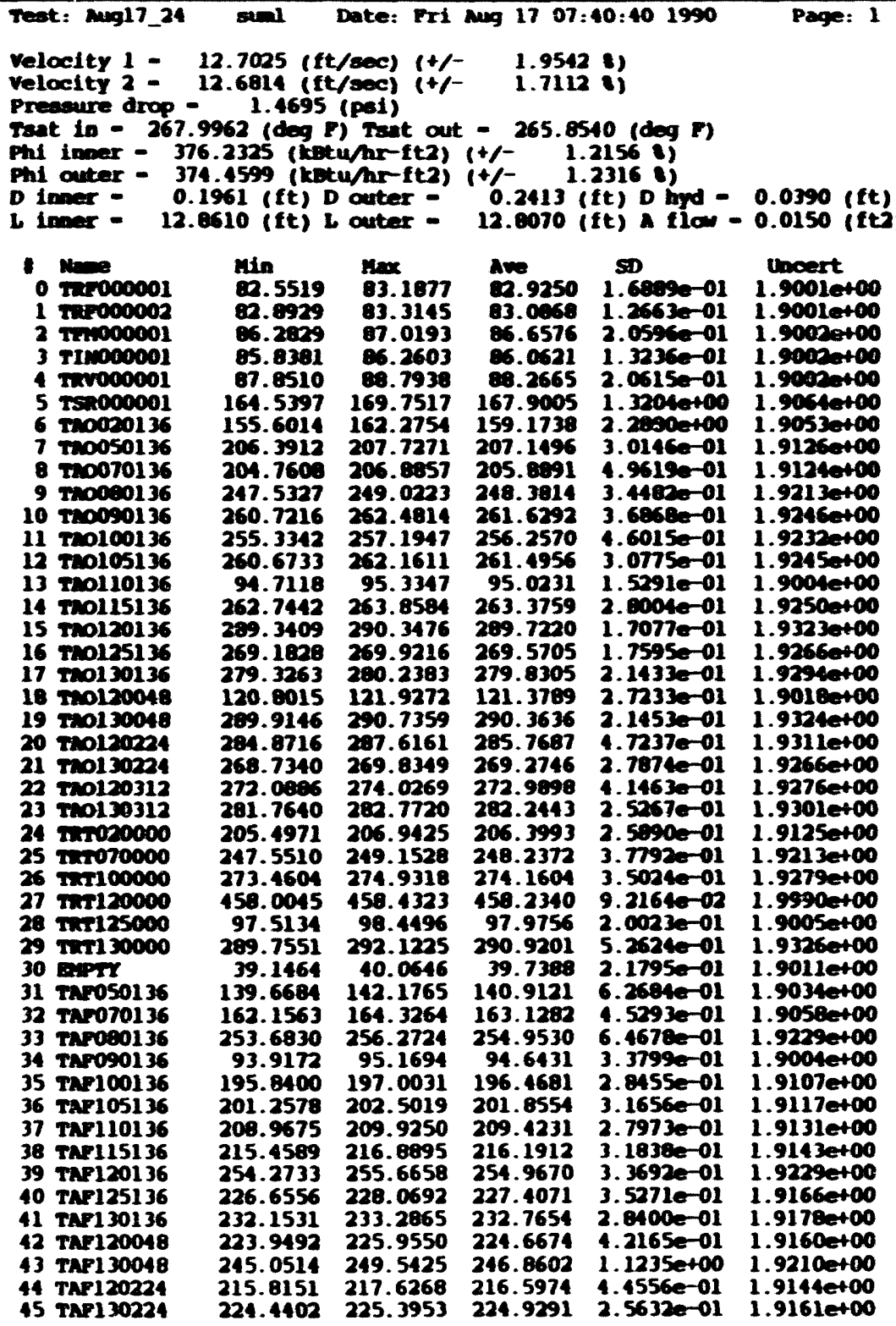

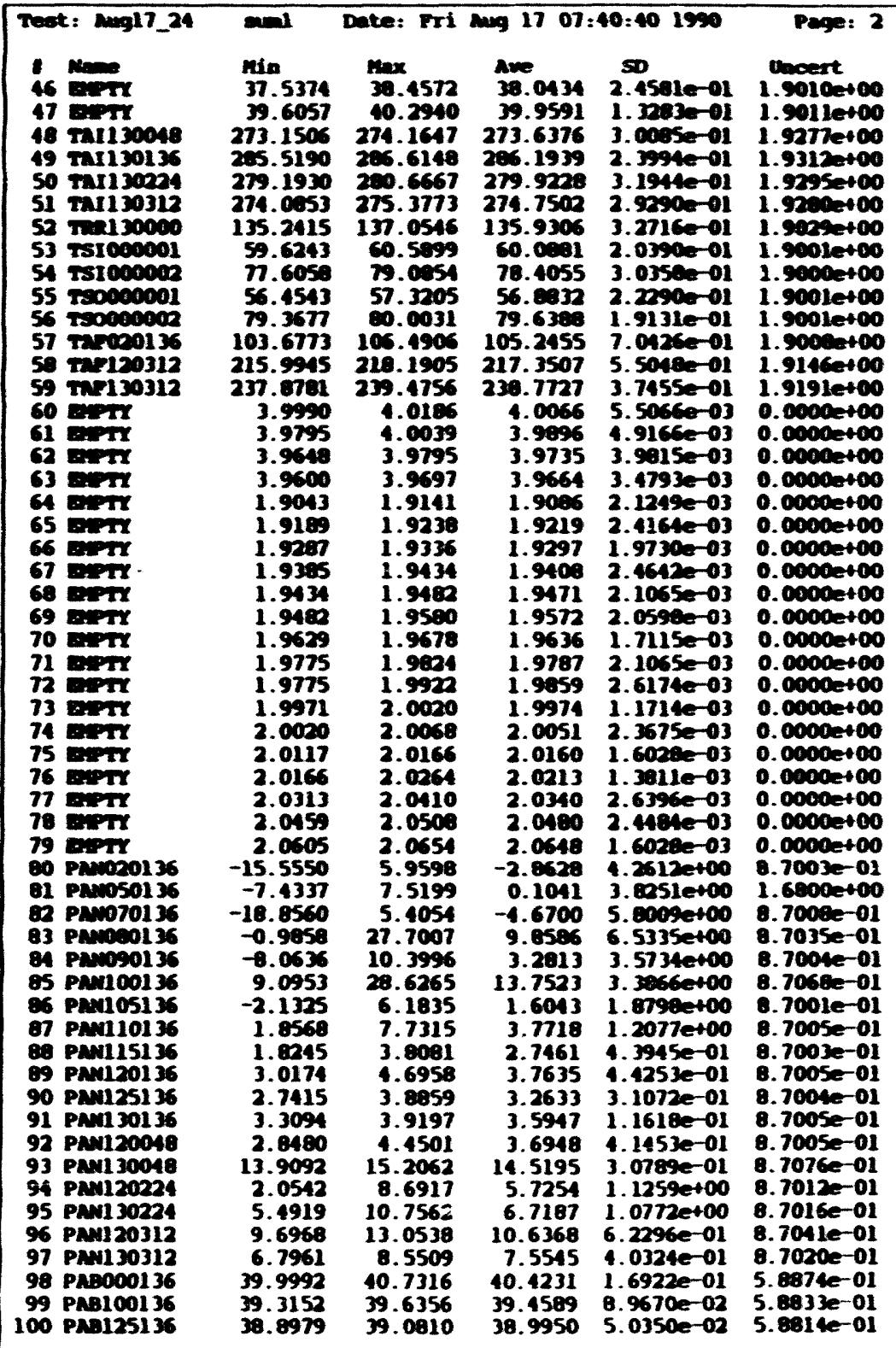




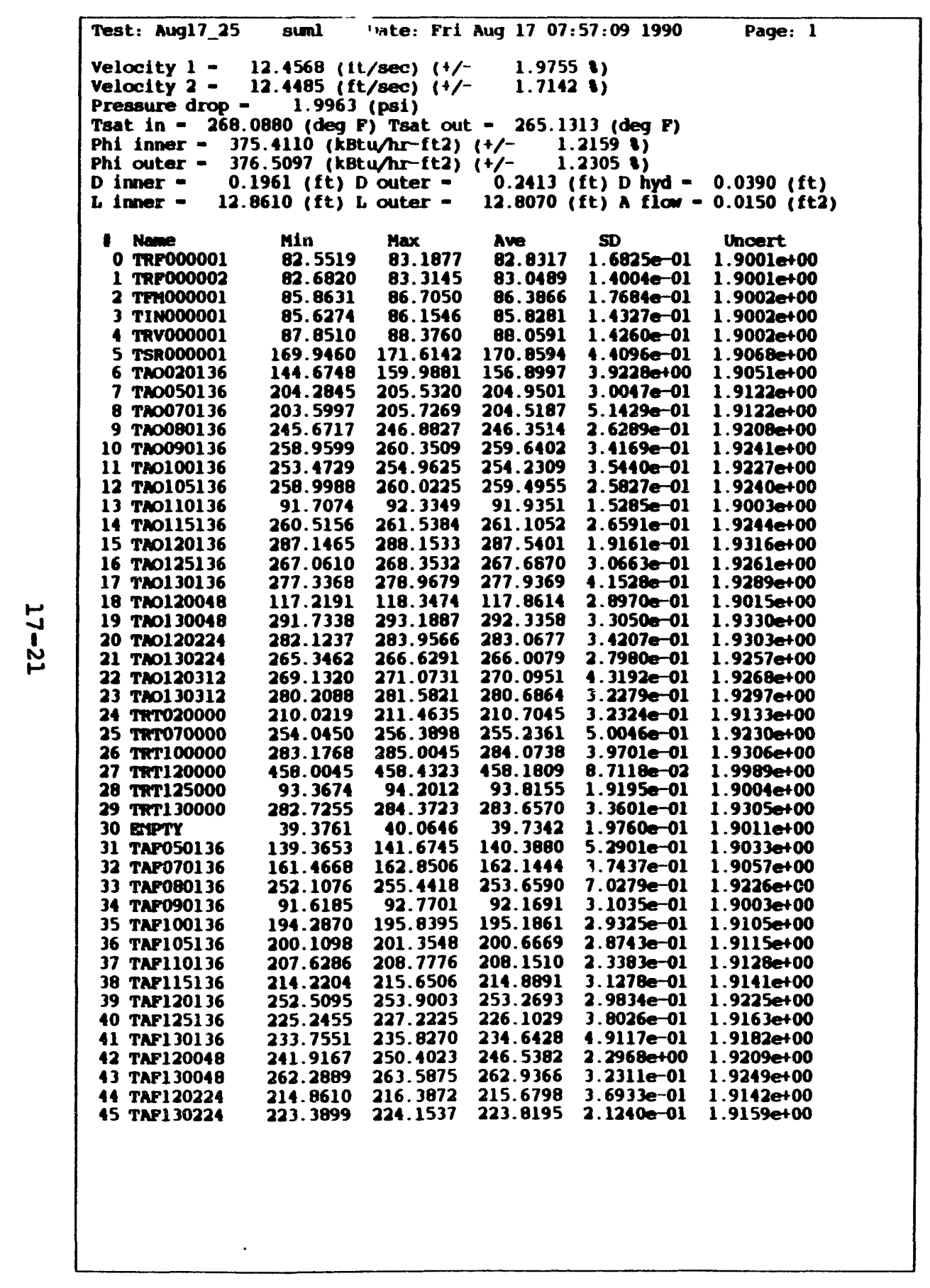

\begin{tabular}{|c|c|c|c|c|c|}
\hline 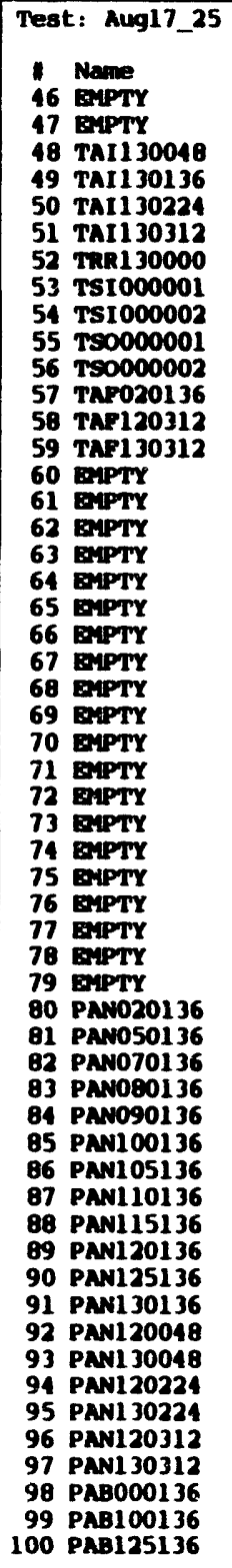 & 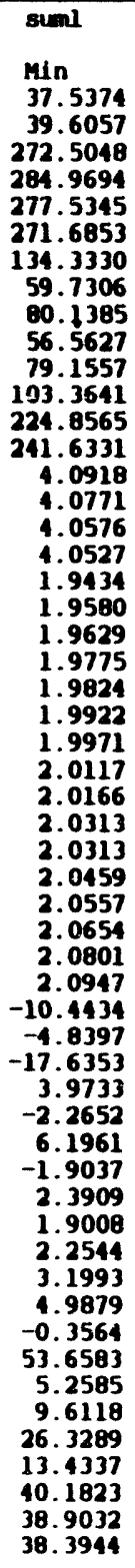 & 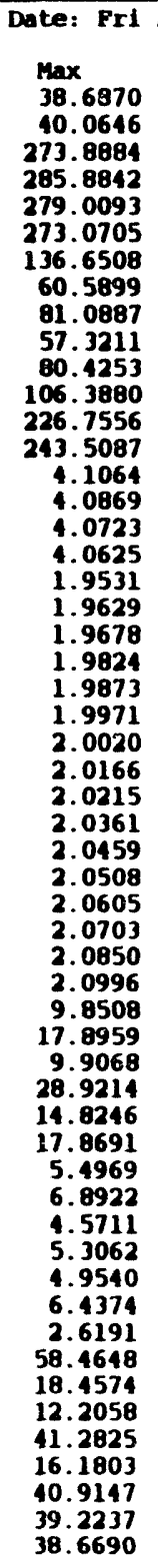 & 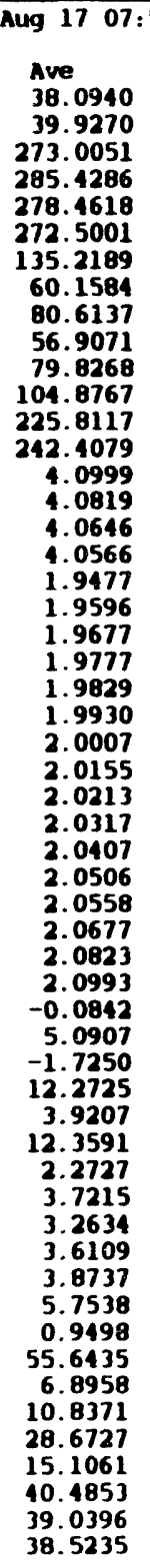 & 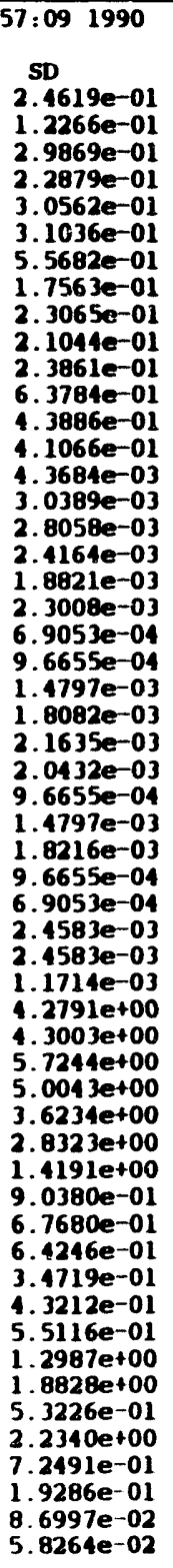 & 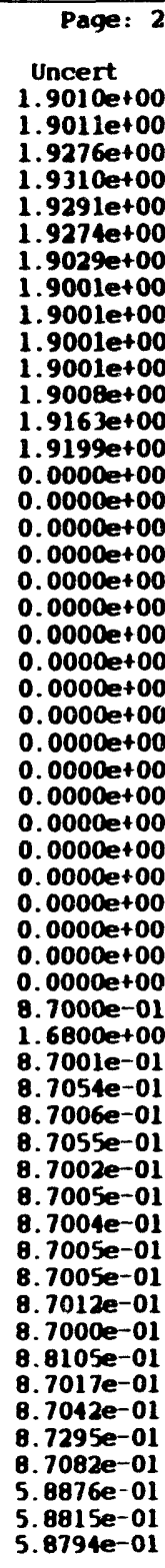 \\
\hline
\end{tabular}




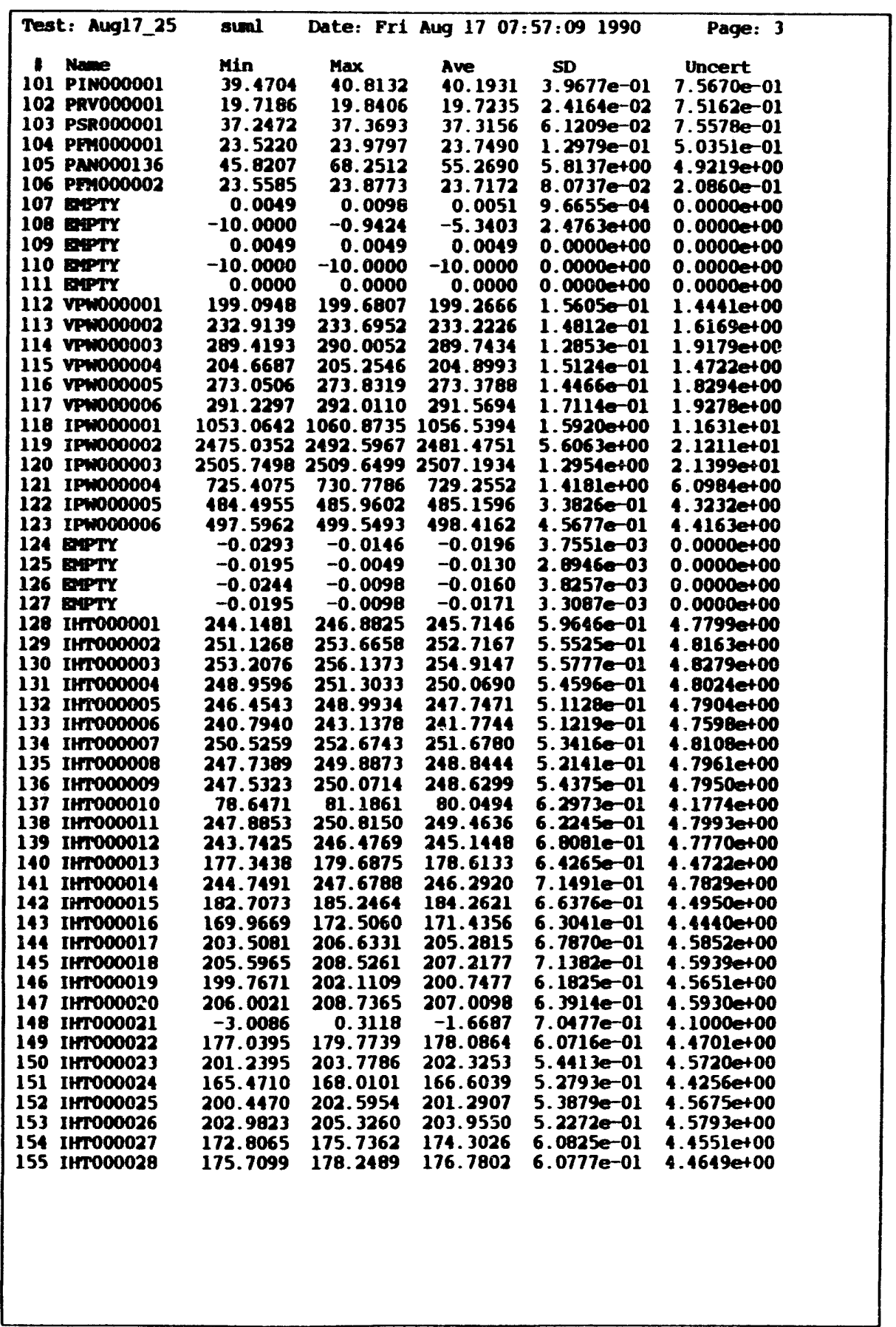

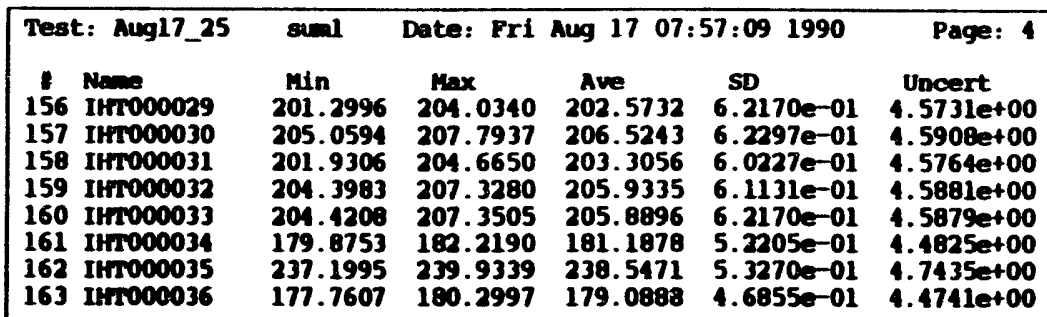


DEFECTIVE INSTRUAENTS FOR THE RIBBED GEONETRY TEST SERIES

\begin{tabular}{|c|c|c|}
\hline Series & Tests & Dufective Instruments \\
\hline 9 & Aug17_18 to Aug17_25 & 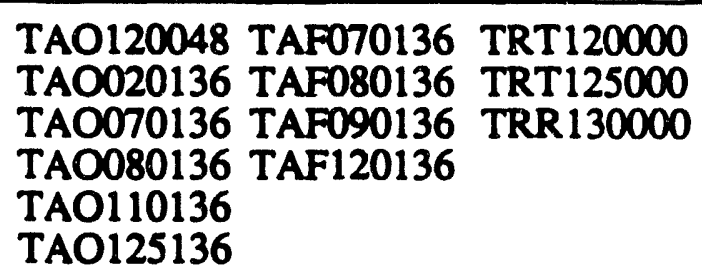 \\
\hline & & $\begin{array}{l}\text { IHTO00010 IHTO00021 IHTO00024 } \\
\text { IHT000033 }\end{array}$ \\
\hline
\end{tabular}




\section{জ5reare}

TN-499

\section{Chapter 18}

NUCLEATE BOILING PRESSURE DROP IN AN ANNULUS

Test Geometry: Ribbed (4)

Test Series: 13

Test Date: August 17, 1990 


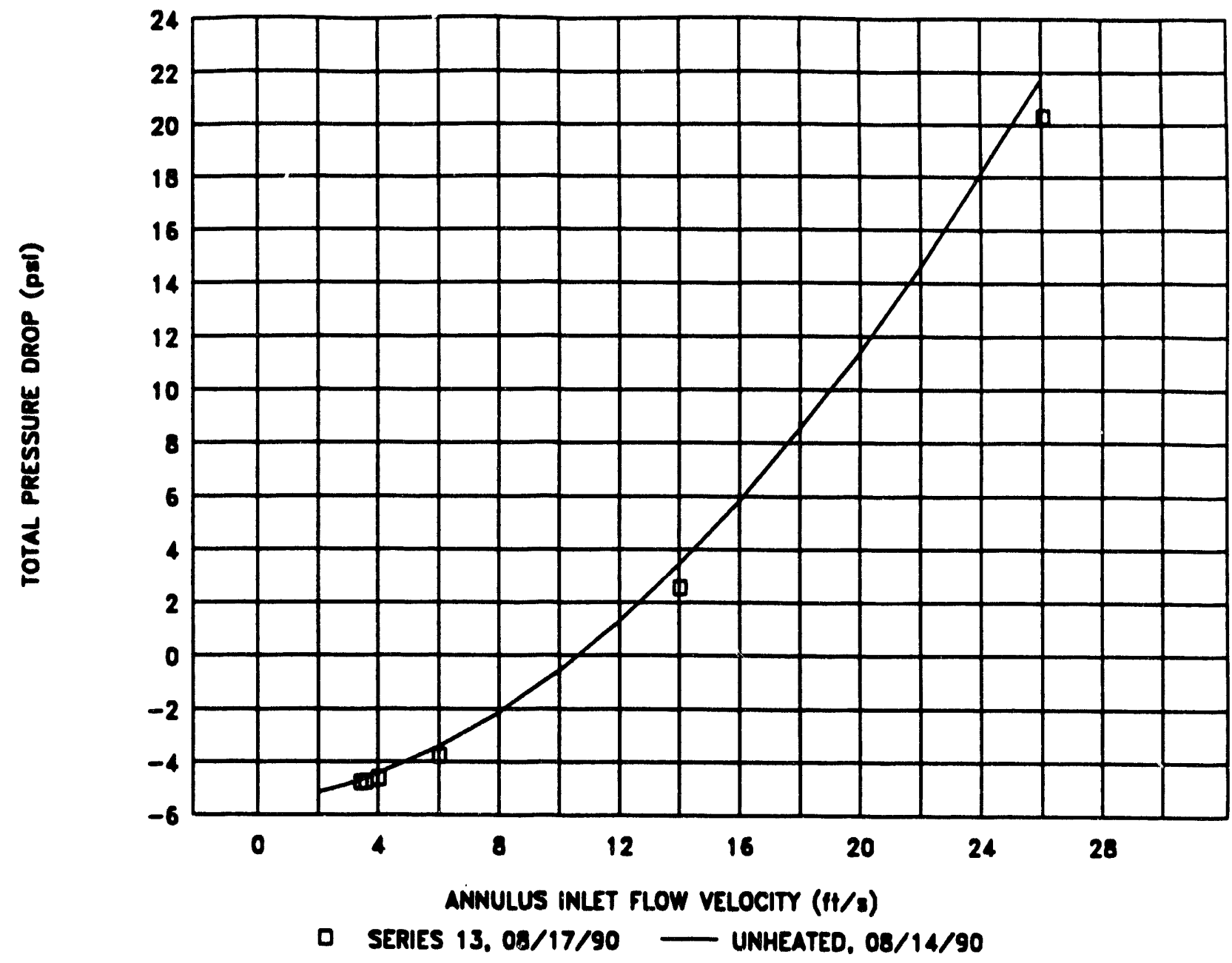

\begin{tabular}{|c|c|c|}
\hline $\begin{array}{c}\text { TEST } \\
\text { NUMBER }\end{array}$ & $\begin{array}{c}\text { INLET } \\
\text { VELOCITY(a) }\end{array}$ & $\begin{array}{l}\text { PRESSURE } \\
\text { DROP(b) }\end{array}$ \\
\hline & $(t / / 8)$ & $\overline{\text { (psi) }}$ \\
\hline Aug17_26 & 26.08 & 20.31 \\
\hline Aug17_27 & 14.02 & 2.56 \\
\hline Aug17_28 & 6.03 & -3.76 \\
\hline Aug17_29 & 4.01 & -4.63 \\
\hline Aug17_30 & 3.59 & -4.76 \\
\hline 2001751 & $8 \%$ & $\%$ \\
\hline
\end{tabular}

\begin{tabular}{|l|l|}
\hline \multicolumn{2}{|c|}{ NOMINAL TEST CONDITIONS } \\
\hline Annulus geometry & ribbed (4) \\
Inlet temperature & $86^{\circ} \mathrm{F}$ \\
Inlet pressure & 40 psia \\
Wall heat flux & $100 \mathrm{kBtu} / \mathrm{hr}-\mathrm{tt} 2$ \\
Flux distribution & uniform \\
He saturation pressure & $5 \mathrm{psig}$ \\
\hline
\end{tabular}
NOTES:
(a) Inlet velocity calculated from PFM000001
(b) Proseure drop calculated from PANO00136
(c) Shading notes test a minimum preseure drop 

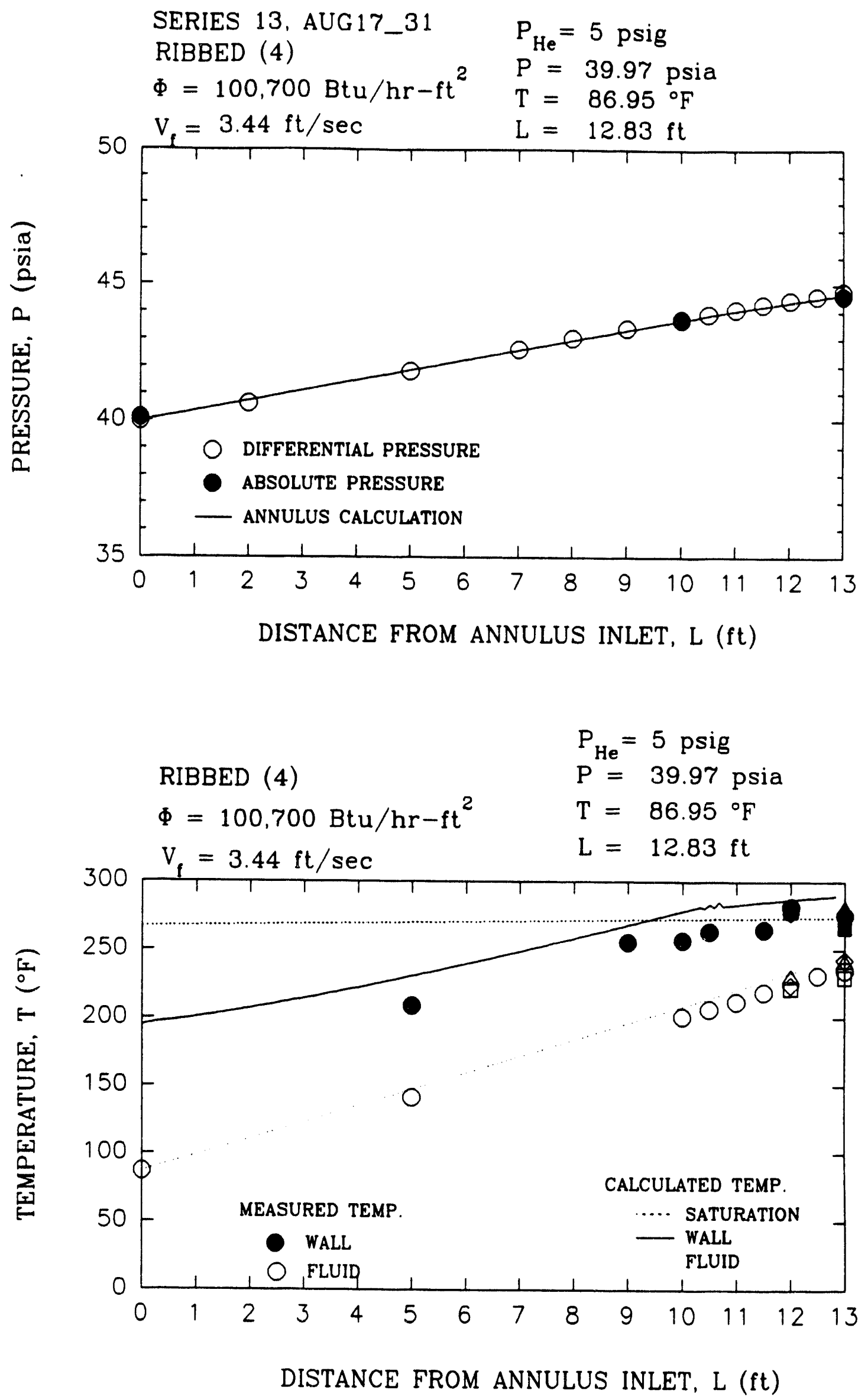
Test: Aug17_26 suml Date: Fri Aug 17 09:18:33 1990 Page: 1

Velocity $1=26.0797$ (ft/sec) $(t /-\quad 1.6886$ : $)$
Velocity $2=13.4155(\mathrm{ft} / \mathrm{sec})(t /-\quad 1.7033$ :

Pressure drop -20.3128 (psi)

9877 (deg F)

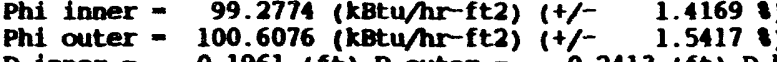

$D$ inner = 0.1961 (ft) D outer $=0.2413$ (ft) $D$ hyd $-0.0390(f t)$ L 12.8610 (ft) L outer = 12.8070 (ft) A flow $=0.0150$ (ft2)

\section{o TRF000001}

1 TRF000002

2 TFHO00001

4 TRV000001

5 TSR000001

6 Th0020136

7 Th0050136

9 Tro000136

10 TA0090136

11 T20100136

12 Tמ0105136

$13 \mathrm{T20110136}$

14 T20115136

$16 \mathrm{TM0125136}$

17 Tho1 30136

19 Tho1 30048

20 TMo12022

$\begin{array}{lllll} & & \end{array}$

$86.7854 \quad 87.3121 \quad 87.0320 \quad 1.2344$ e-01 1.9002 et00 $\min$

$81.7037 \quad 82$

$\begin{array}{lllll}1.8382 & 82.1279 & 81.9413 & 1.7479 e-01 & 1.9001 \text { et0o }\end{array}$

$07.5303 \quad 107.4700 \quad 107.0590$ 2.2712e-01 $1.9002 e+00$

$61.7360 \quad 63.7767 \quad 62.6537 \quad 4.90630-01 \quad 1.90000+00$

$106.6537 \quad 107.2725 \quad 106.9655 \quad 1.69600-01 \quad 1.90090+00$

106.4563108 .0145 107.2731 $3.6338 \mathrm{e}-01 \quad 1.9009 \mathrm{et} 00$

$92.6055 \quad 119.9361 \quad 105.3614 \quad 7.1171 \mathrm{et} 00 \quad 1.9008 \mathrm{et} 00$

$115.9741 \quad 116.5895 \quad 116.2466 \quad 1.4887 e-01 \quad 1.9014 e+00$

$\begin{array}{llllll}112.8052 & 113.5257 & 113.1409 & 1.5399 e-01 & 1.9013 \mathrm{et} 00\end{array}$

114.7318 115.6582115 .2240 2.1937e-01 1.9014 et00

78.9849 79.6158 79.3100 1.7045e-01 1.9001e+00

113.5904114 .5164 114.0410 2.1491e-01 $1.9013 \mathrm{et00}$

77.9255119 .959119 .6463 1.6625e-01 1.9017et00 $17.9255 \quad 79.0878 \quad 78.5279 \quad 2.4158 e-01 \quad 1.9000 e+00$ 118.5128 $83.5672 \quad 83.09712 .51150-0121.9001 \mathrm{et00}$ $118.0011218 .5138 \quad 118.2125 \quad 1.37890-01 \quad 1.9016 \mathrm{et00}$ $118.5185 \quad 119.2359 \quad 118.9632 \quad 1.7522001 \quad 1.9016 \mathrm{et} 00$ 113.7064 114.6265 114.1176 2.0469e-01 $1.9013 \mathrm{et} 00$ $116.9207 \quad 117.6400 \quad 117.3584 \quad 1.491 e-01$ 1.9015e+00 117.6678 118.4864 118.0873 1.6834e-01 $1.9016 \mathrm{et} 00$ $107.4614 \quad 108.1872 \quad 107.8410 \quad 1.7719 e-01 \quad 1.9010 e+00$ 113.5720 114.395 114.01691 .6224 e- 01 1.9013e+00 118.5644119 .3823118 .9917 1.9557e-01 $1.9016 e+00$ $\begin{array}{llllll}457.3205 & 457.5772 & 457.4388 & 7.9045 e-02 & 1.9986 e+00\end{array}$ $\begin{array}{llllll}78.7365 & 79.5793 & 79.1184 & 2.0937 e-01 & 1.9001 e+00\end{array}$ $119.5472120 .2635 \quad 119.8278 \quad 1.5579 e-01 \quad 1.9017$ et00 $\begin{array}{lllll}39.3761 & 40.0646 \quad 39.7388 & 2.2282 e-01 & 1.9011 e+00\end{array}$ $94.2044 \quad 94.8302 \quad 94.5114 \quad 1.4038001$ 1.9004et00 $112.0670113 .0909 \quad 112.5610 \quad 2.73930-01 \quad 1.90120400$ $112.0670 \quad 113.0909 \quad 112.5610 \quad 2.7383 e-01 \quad 1.9012 e+00$ $101.2394 \quad 101.9679101 .6371 \quad 1.7294 e-01 \quad 1.9007$ et00 $\begin{array}{lllll}101.9650 & 103.0997 \quad 102.5486 & 2.5093 \mathrm{e}-01 & 1.9007 \mathrm{e}+00\end{array}$ $102.9632 \quad 103.5849 \quad 103.3094 \quad 1.5881 \mathrm{e}-01 \quad 1.9007 \mathrm{e}+00$ 104.4965 105.2215 104.8590 1.5948e-01 1.90 109.0992 109.7171 109.3654 $1.5024 e-01$ 1.9010e+00 105.4360106 .5682106 .0473 2.8186e-01 1.9009e+00 $\begin{array}{lllll}106.3806 & 106.7948 & 106.5487 & 1.1992 e-01 & 1.9009 e+00\end{array}$ 105.8257 $106.5542 \quad 106.2065$ 1.6224e-01 1.9009 e+00 $107.1081 \quad 107.6258 \quad 107.3505 \quad 1.3485 e-01 \quad 1.9009$ et00 $\begin{array}{lllll}104.4426 & 105.2712 & 104.8589 & 2.2968 e-01 & 1.9008 e+00 \\ 105.2273 & 105.7471 & 105.4957 & 1.6822 e-01 & 1.9008 e+00\end{array}$

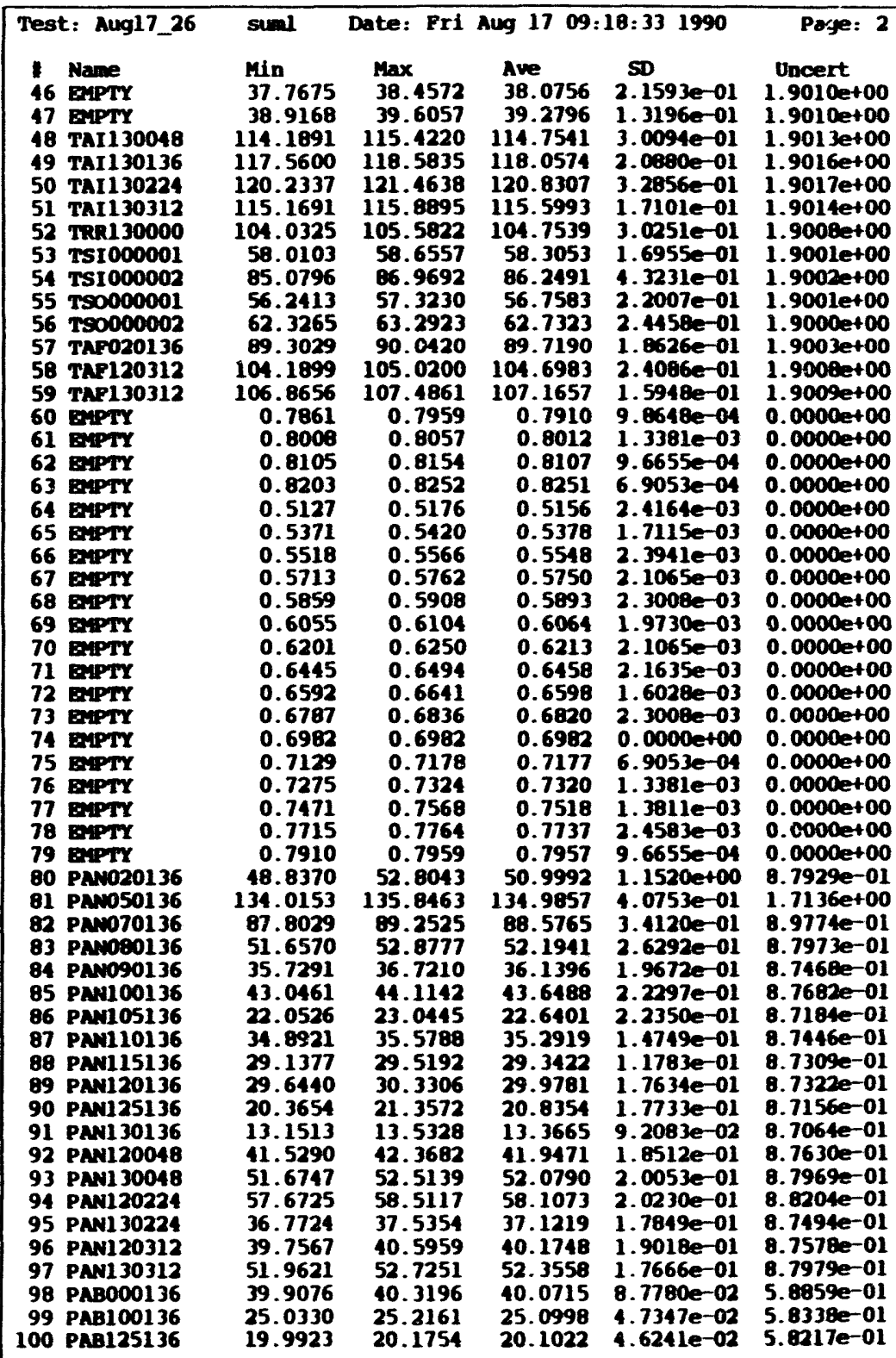

25.0330 


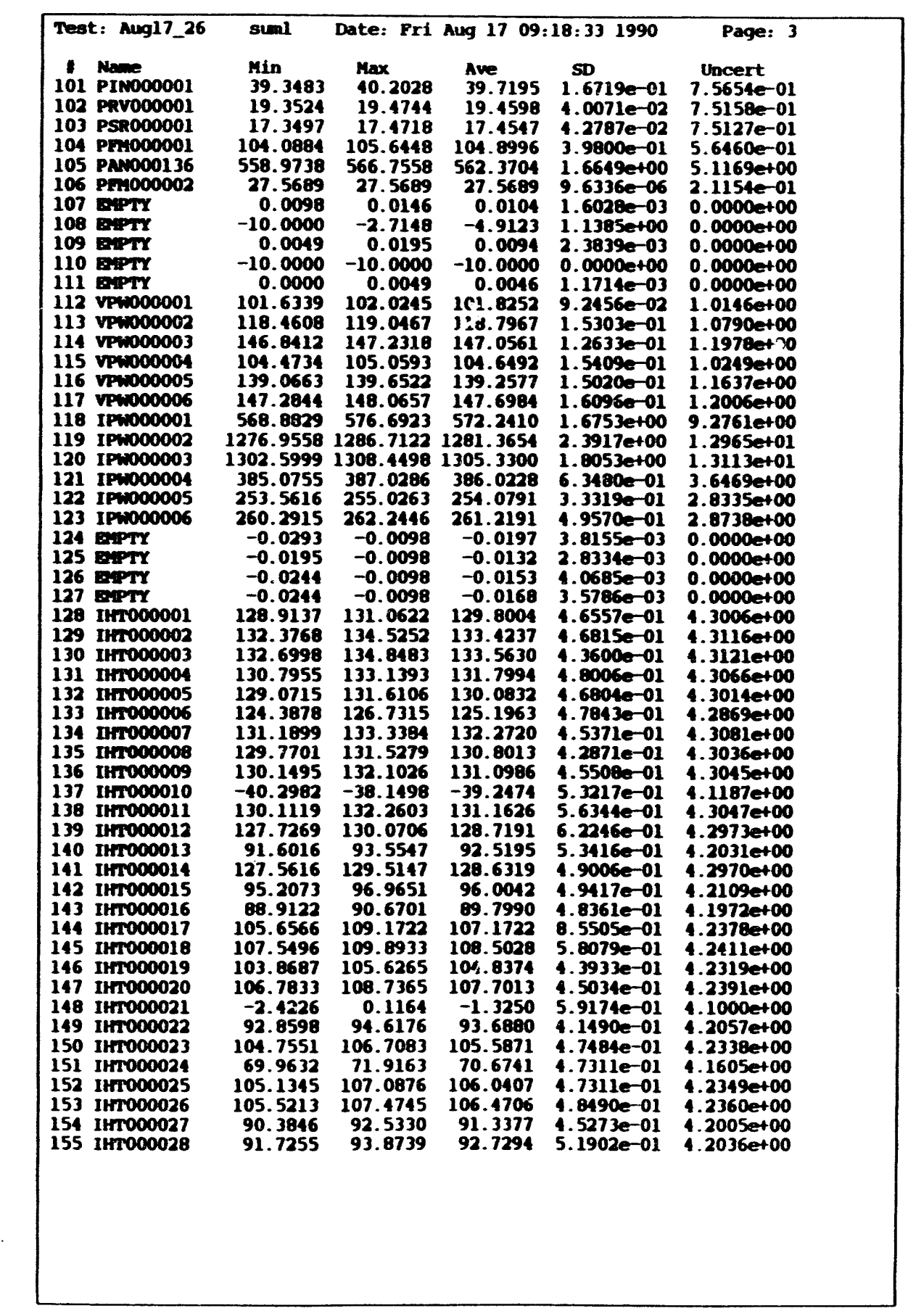

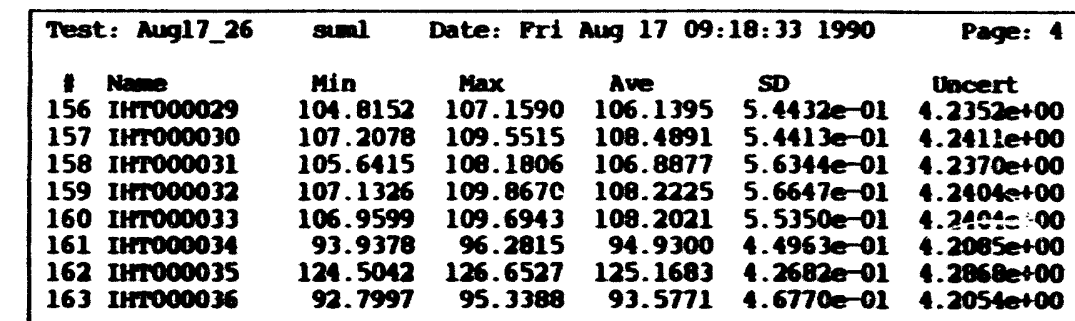

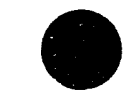




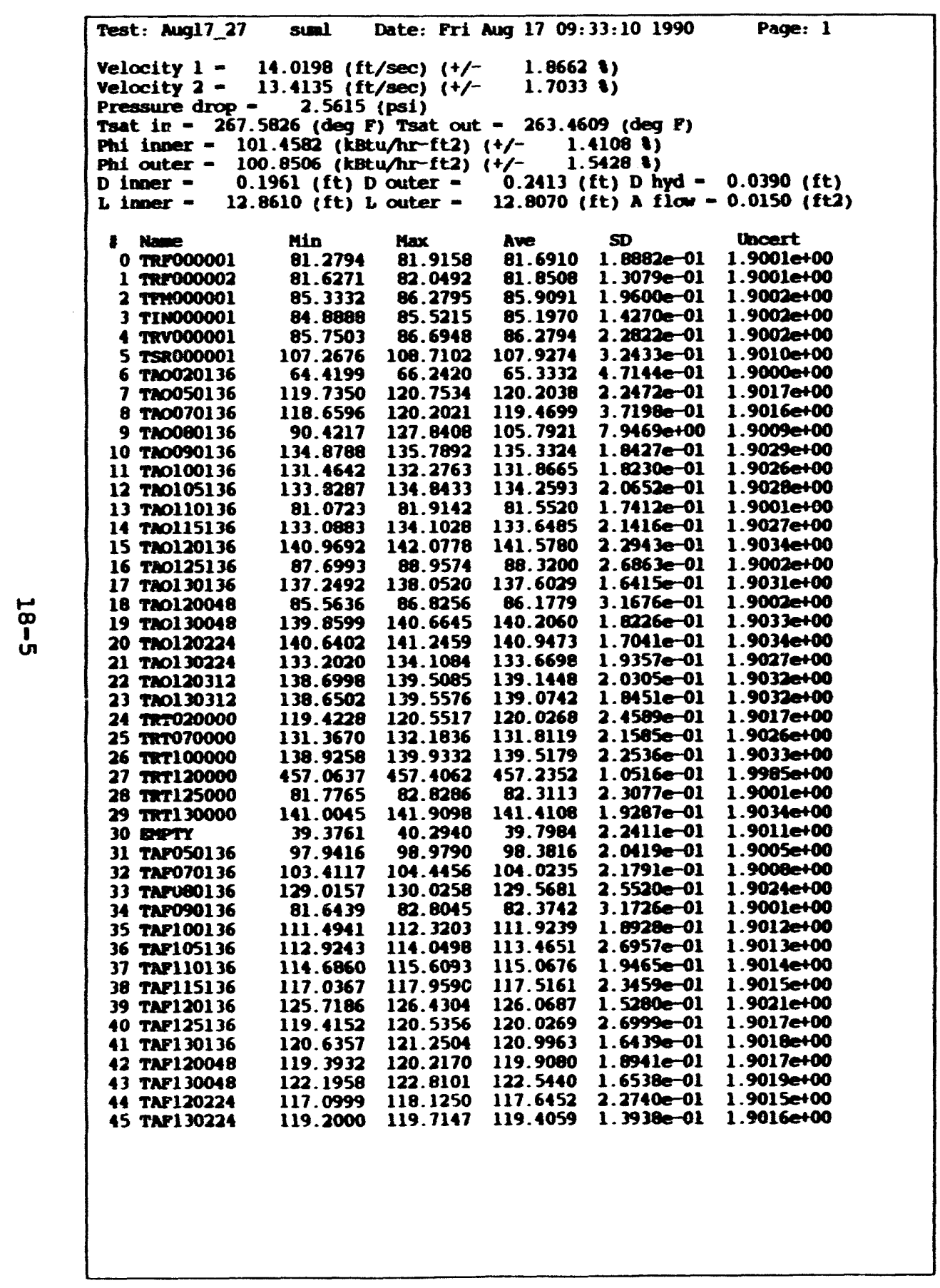

\begin{tabular}{|c|c|c|c|c|c|}
\hline 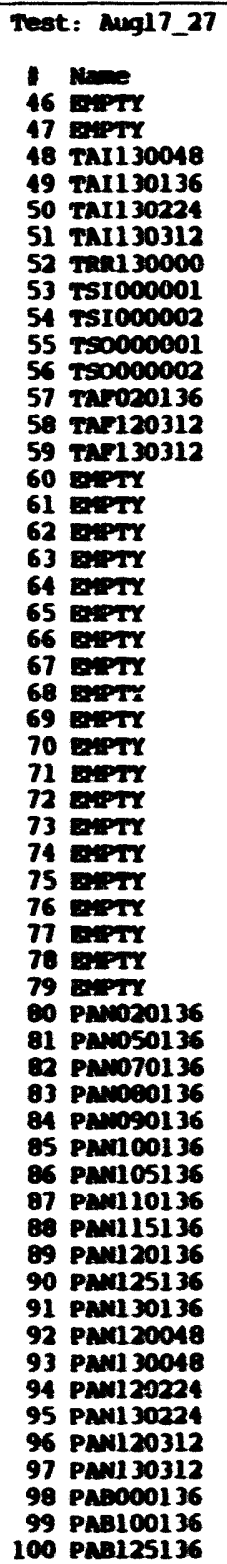 & 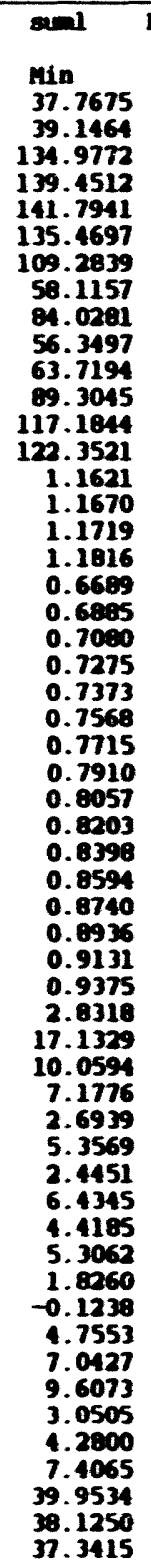 & 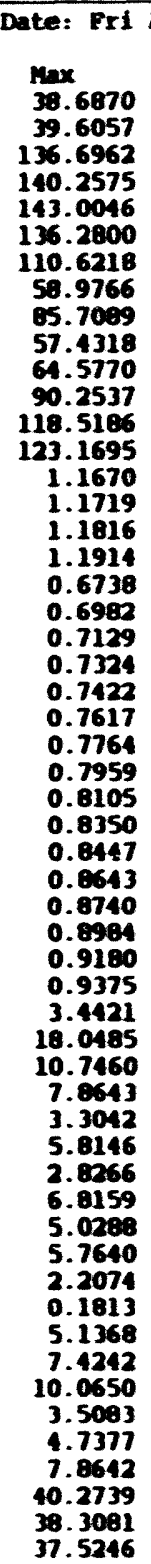 & 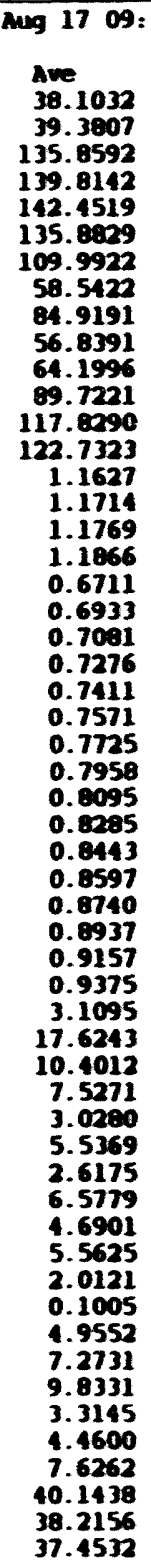 & 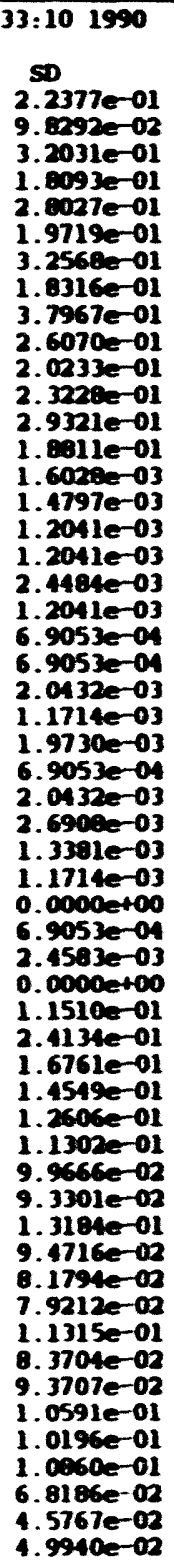 & 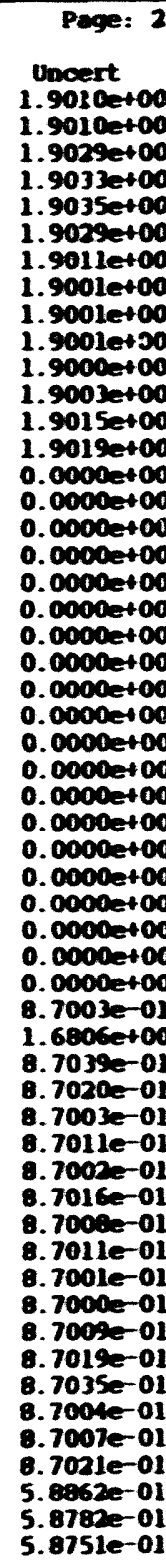 \\
\hline
\end{tabular}




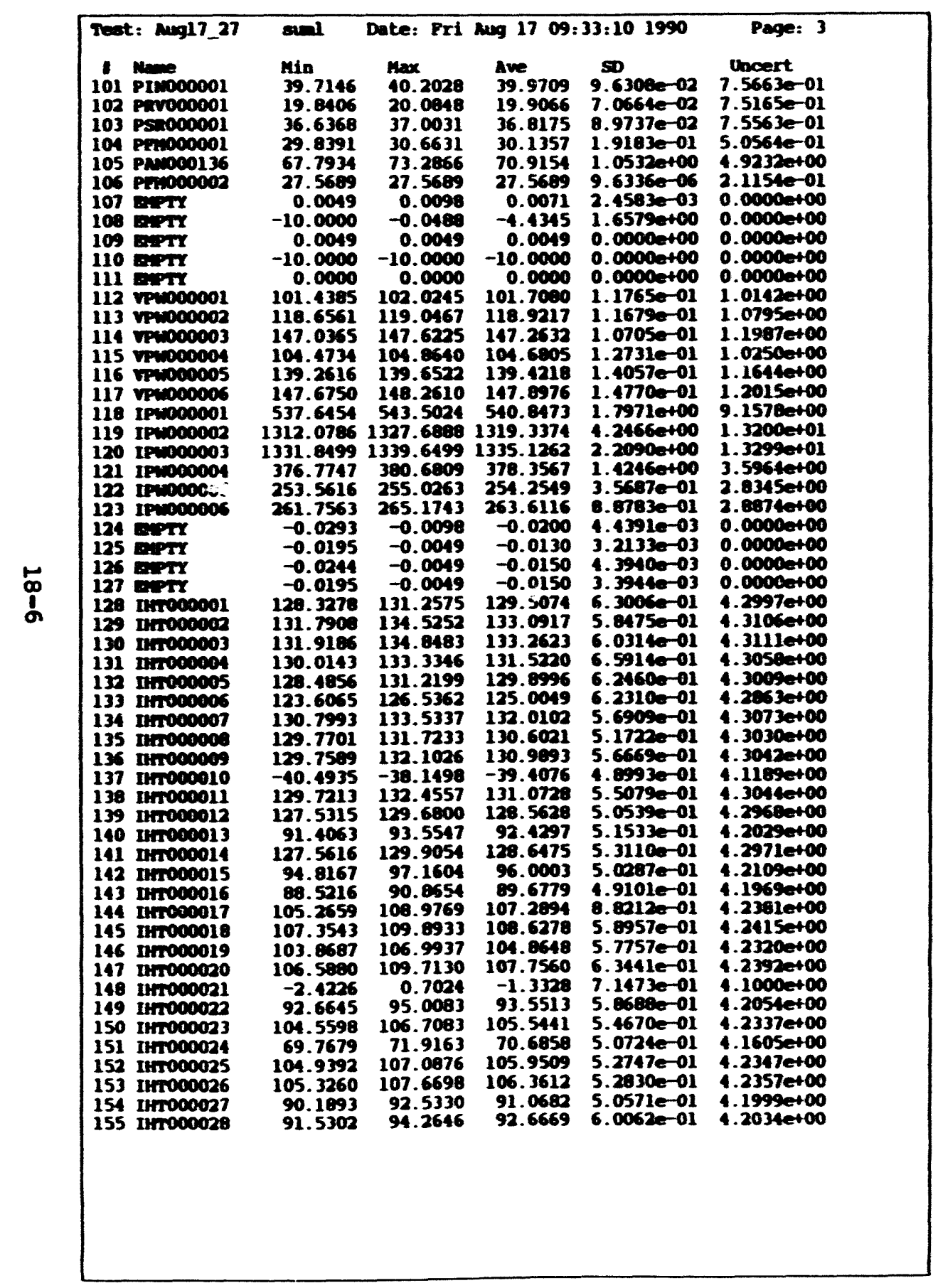

\begin{tabular}{|c|c|c|c|c|c|}
\hline 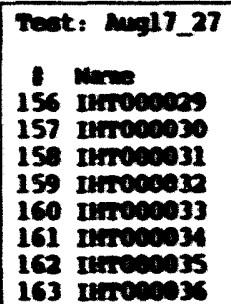 & $\begin{array}{c}105.0105 \\
107.4031 \\
105.6415 \\
107.1325 \\
105.9595 \\
93.9370 \\
124.1135 \\
9.4051\end{array}$ & $\begin{array}{c}n \times x \\
107.9402 \\
109.9422 \\
10 . .1006 \\
109.4764 \\
109.4990 \\
95.6956 \\
126.0567 \\
94.3622\end{array}$ & $\begin{array}{c}\text { ave } \\
105.1901 \\
100.5430 \\
105.9111 \\
109.1874 \\
109.1201 \\
94.9045 \\
125.1050 \\
93.4794\end{array}$ & $\begin{array}{l}5.9 \\
5.5 \\
5.5 \\
5.1 \\
5.5\end{array}$ & $\begin{array}{l}4 \\
4 \\
4 \\
4\end{array}$ \\
\hline
\end{tabular}




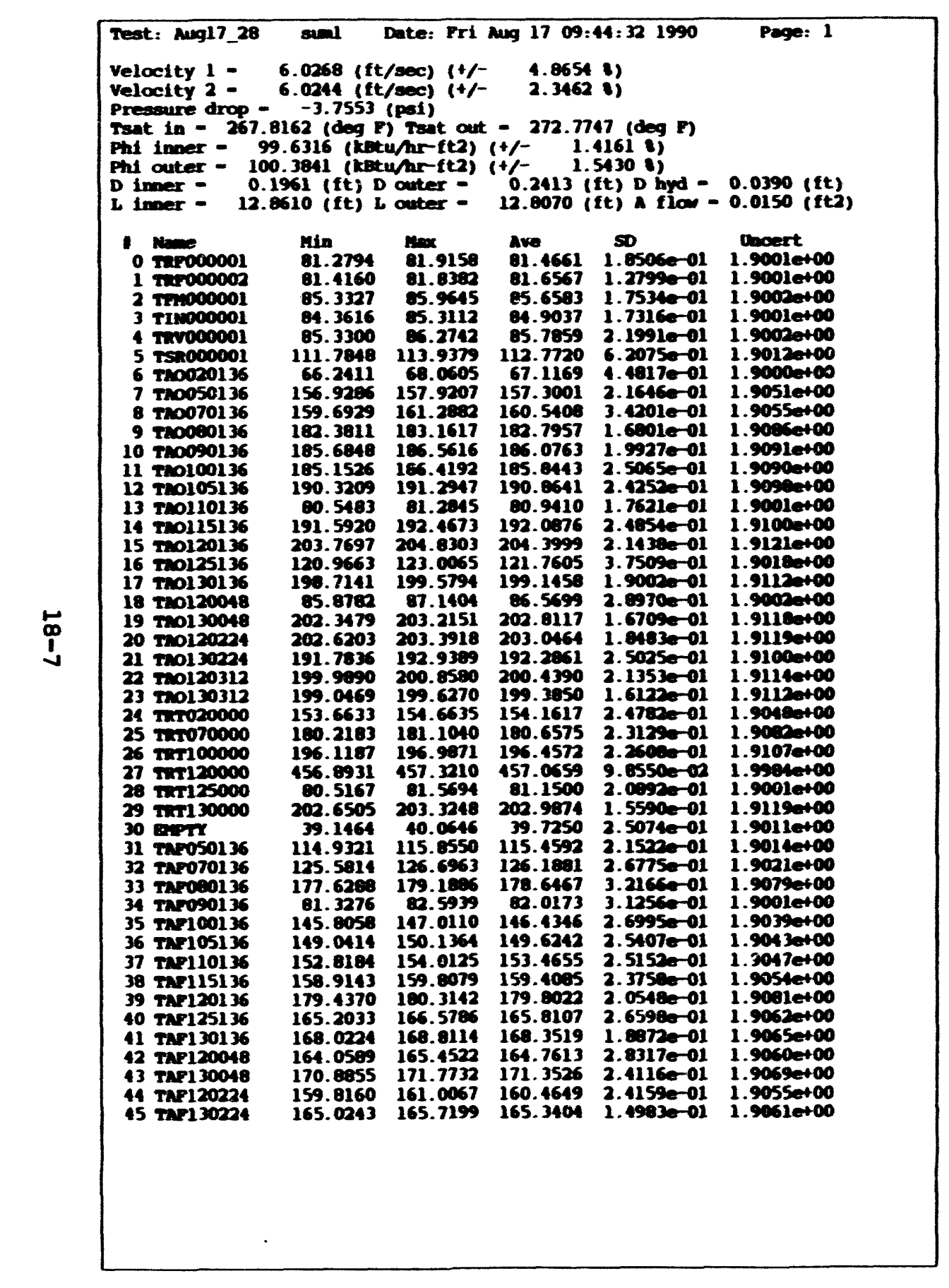

\begin{tabular}{|c|c|c|c|c|c|}
\hline 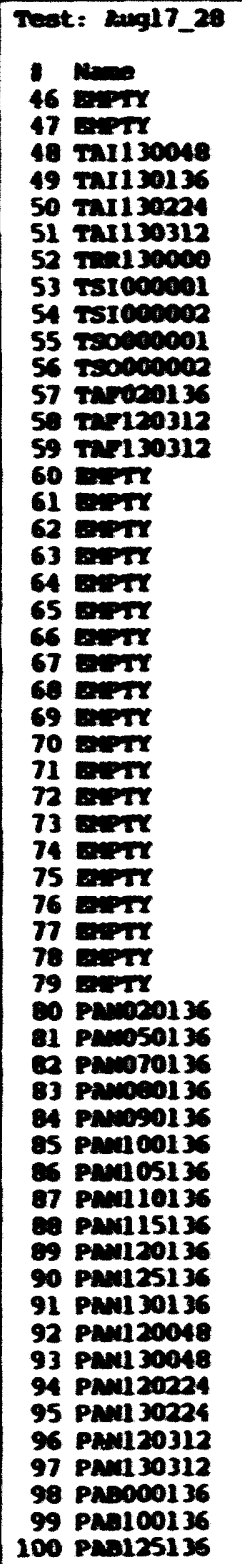 & 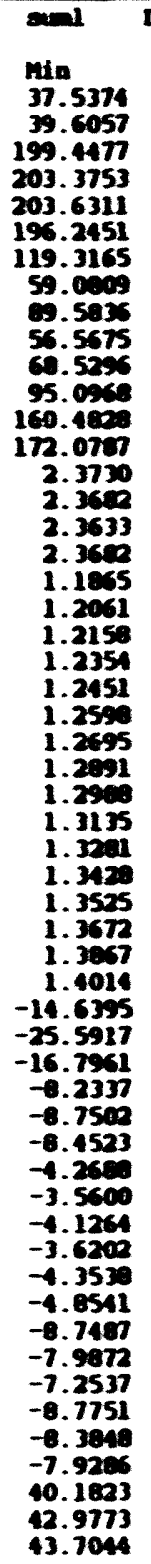 & 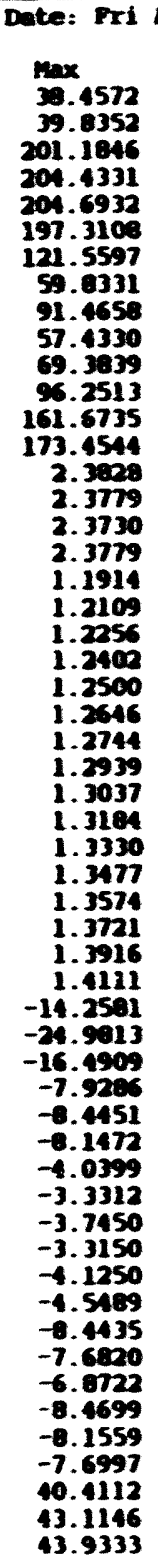 & 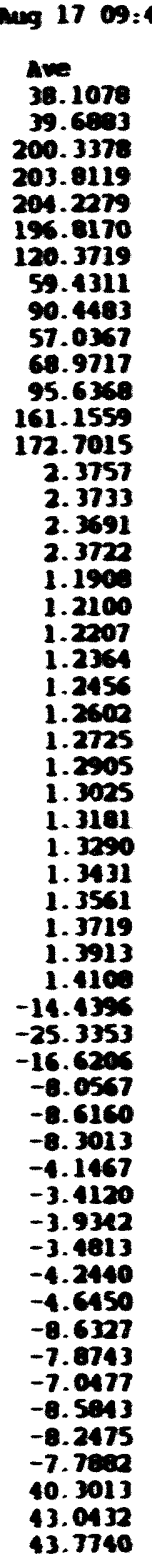 & 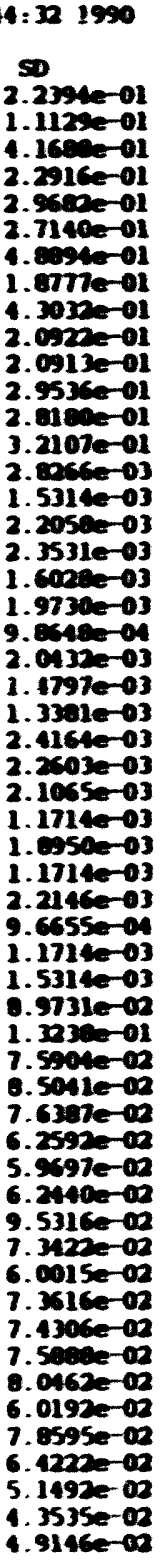 & 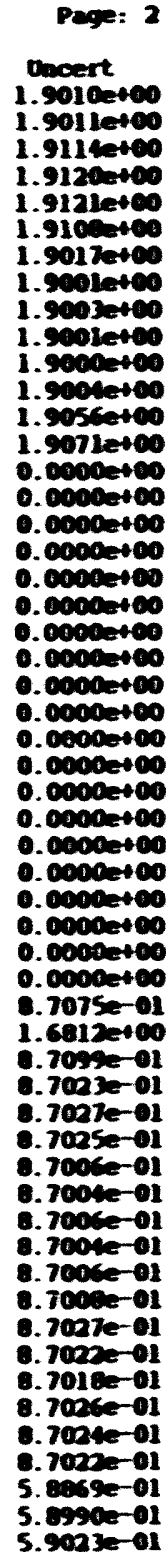 \\
\hline
\end{tabular}




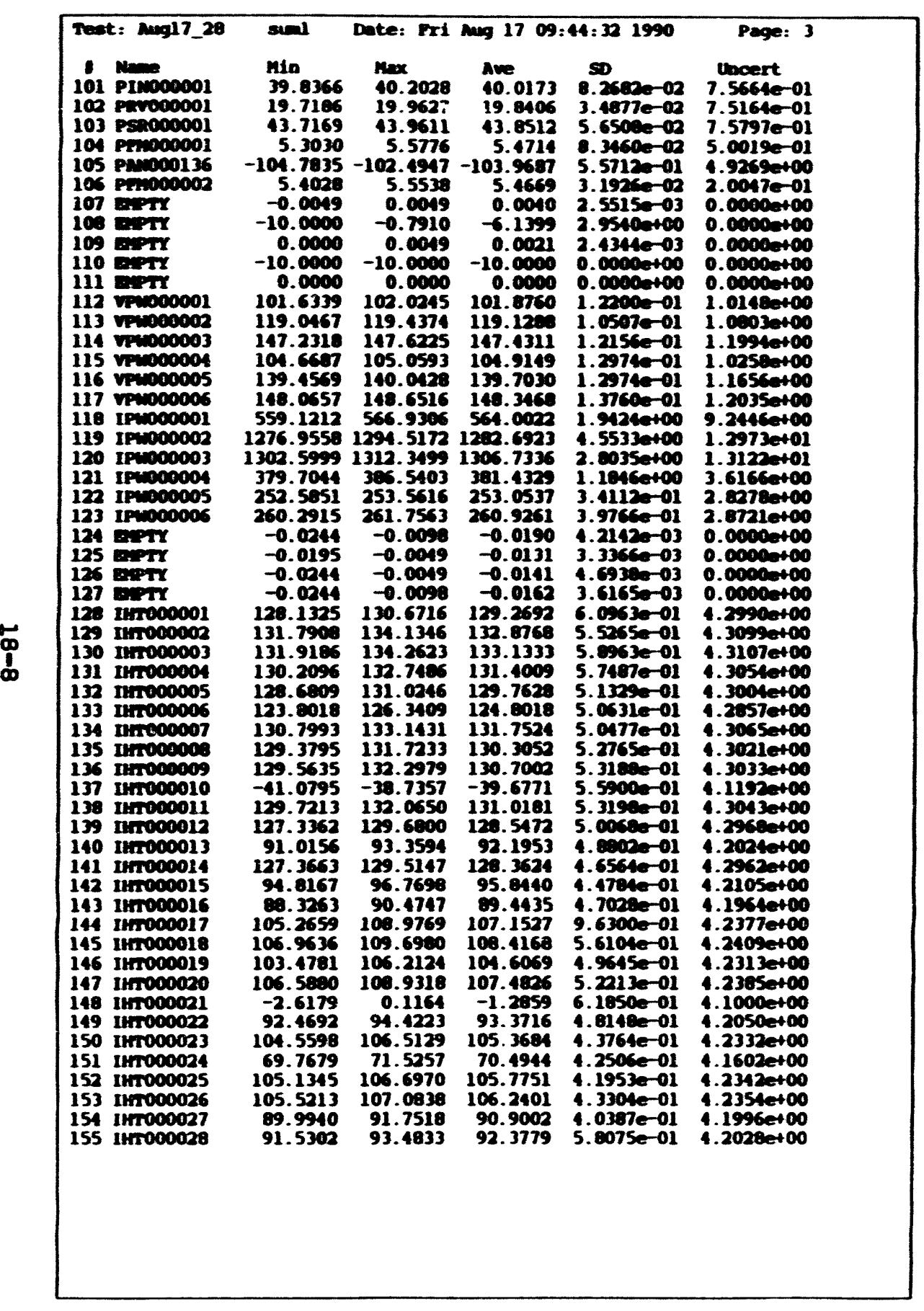

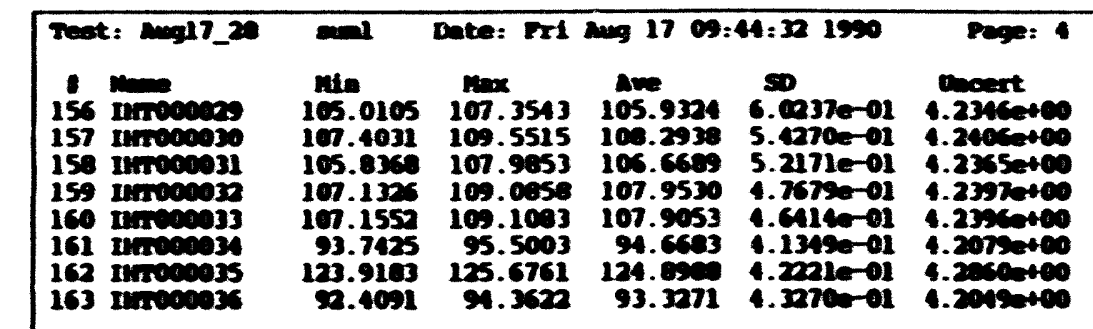

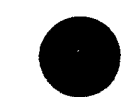


Test: Mug17_29 sin Date: Fri Mug 17 09:55:07 1990 Page: 1

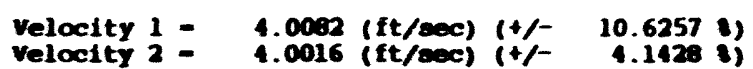

Preasure drop - -4.6340 (poi)

Trat in - 267.8853 (dog F) Trat out $-274.0029(\mathrm{dog} F)$

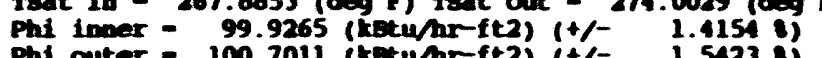

Phi couter = 100.7011 (ketwhr-ft2) $(+/-1.5423$ :)

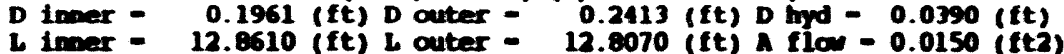

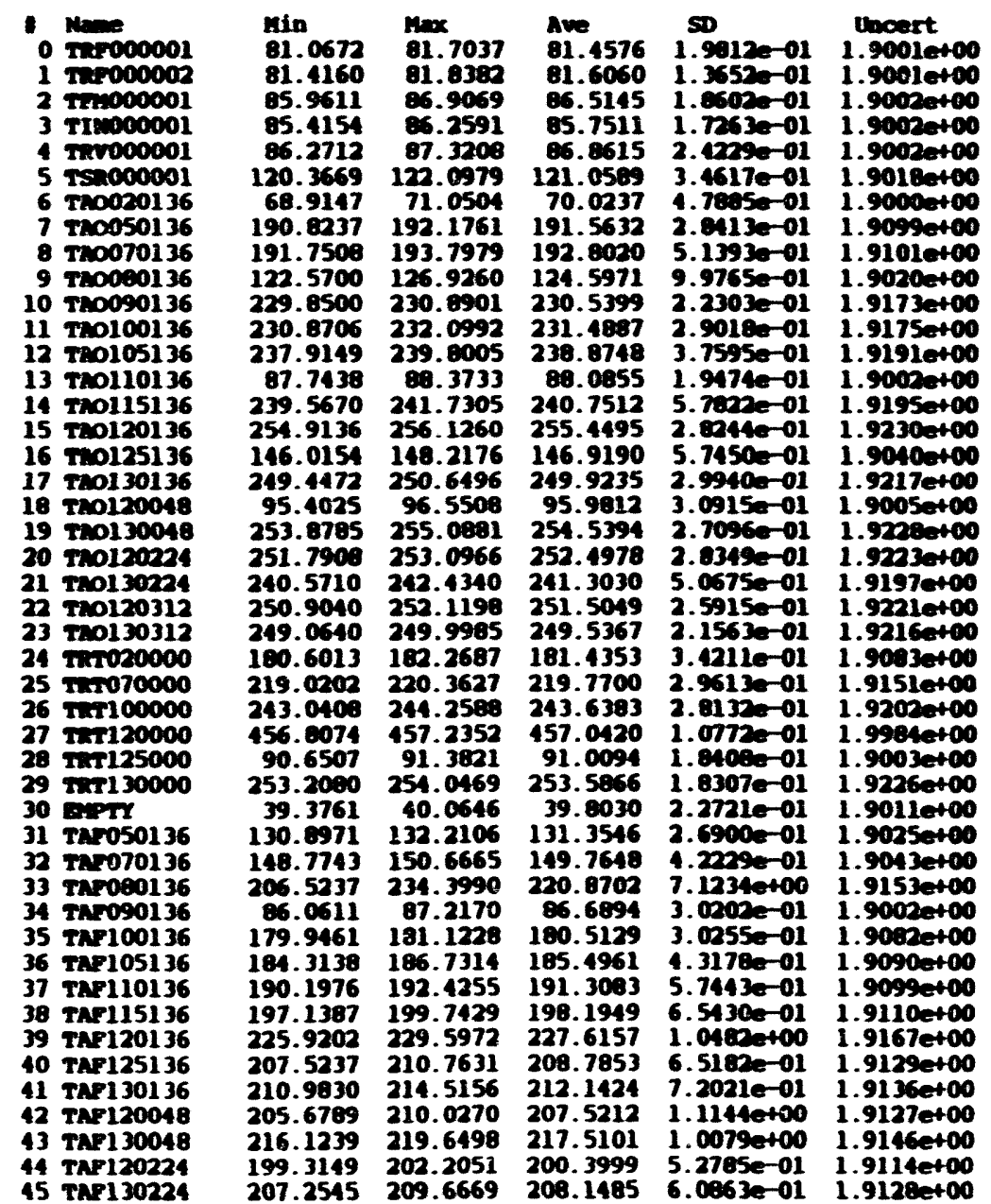

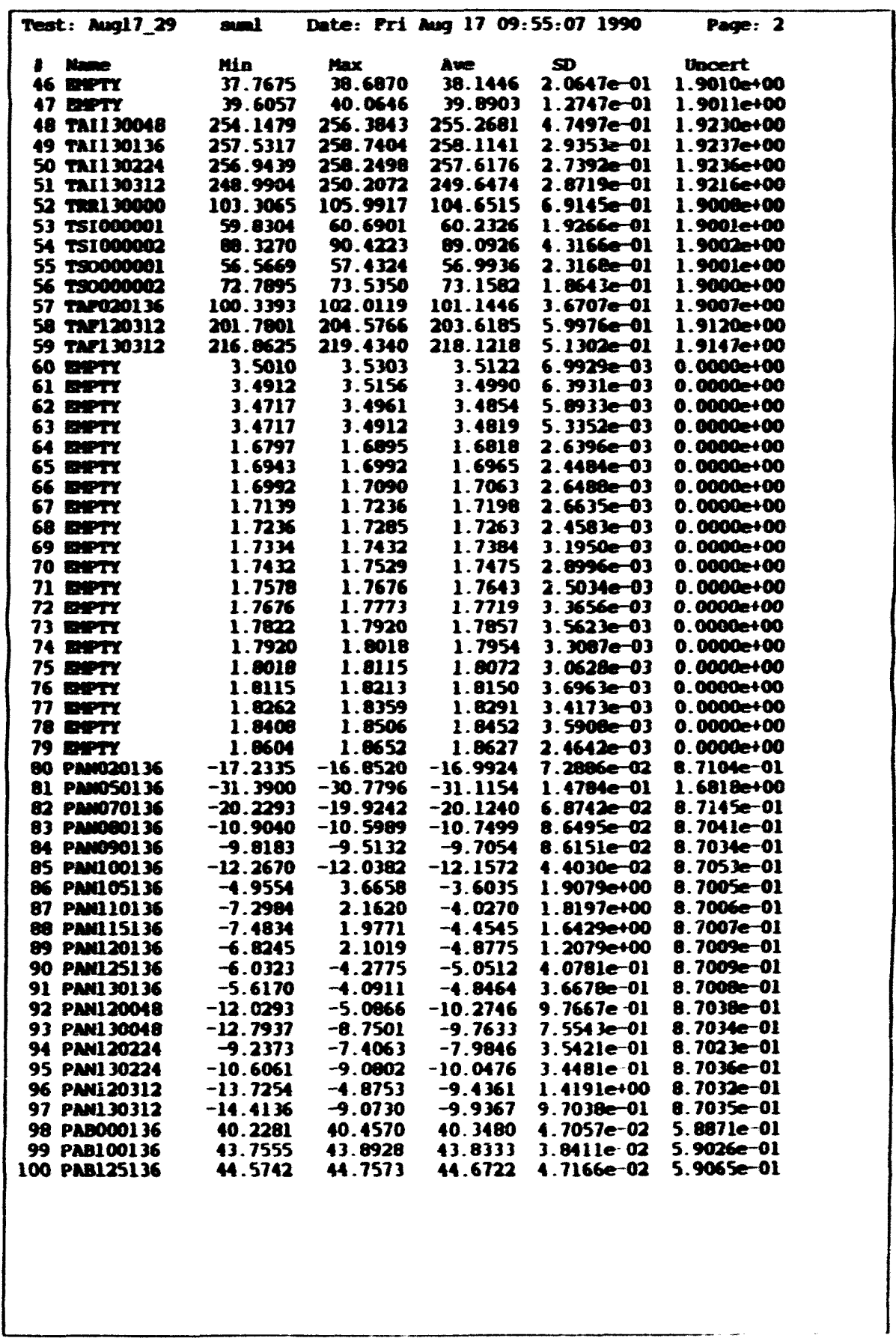



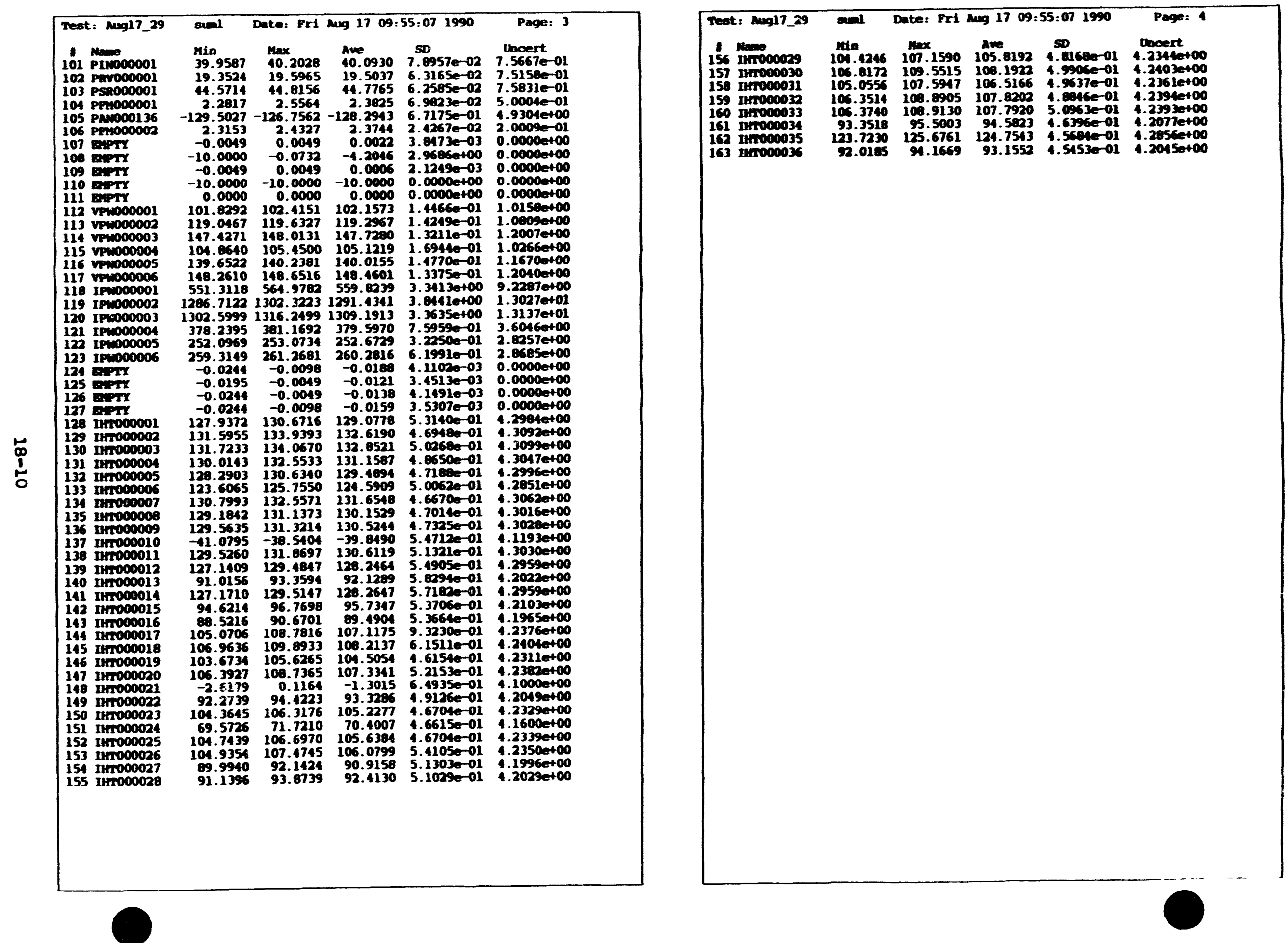


\begin{tabular}{|c|c|c|c|c|c|c|}
\hline \multicolumn{2}{|r|}{ est: Augl7_30 } & & \multicolumn{3}{|c|}{ ate: Fri Aug 17 10:07:33 } & Ige: \\
\hline \multicolumn{3}{|c|}{$\begin{array}{l}\text { Velocity } 1=3.5851 \\
\text { velocity } 2=3.5503 \text { ( } \\
\text { Pressure drop }=-4.762 \\
\text { Tsat in }=267.9691 \text { (deg } \\
\text { Phi inner }=101.377 \text { (k } \\
\text { Phi outer }=100.6743 \text { (k } \\
\text { D inner = } 0.1961 \text { (ft) } \\
\text { L inner }=12.8610 \text { (ft) }\end{array}$} & $\begin{array}{l}\text { c) } \\
\text { c) } \\
\text { i) }\end{array}$ & 6 & $(\operatorname{deg} F)$ & 90 \\
\hline $\begin{array}{l}17.019 \\
1 \\
1 \\
1 \\
1 \\
1 \\
1 \\
1\end{array}$ & $\begin{array}{l}\text { Nane } \\
\text { TRFO00001 }\end{array}$ & $\begin{array}{r}\text { Min } \\
81.0672 \\
81.4160 \\
86.8003 \\
86.0481 \\
87.0040 \\
128.0853 \\
68.8080 \\
202.5494 \\
205.7296 \\
128.3388 \\
248.2507 \\
247.6827 \\
255.4543 \\
92.5187 \\
257.0716 \\
274.1008 \\
158.8610\end{array}$ & $\begin{array}{c}\operatorname{Max} \\
81.7037 \\
81.8382 \\
87.5365 \\
86.5752 \\
88.0516 \\
130.0083 \\
70.3038 \\
203.7021 \\
207.8531 \\
129.6537 \\
249.4657\end{array}$ & $\begin{array}{r}\text { Ave } \\
81.3473 \\
81.5596 \\
87.2041 \\
86.3640 \\
87.5991 \\
129.2338 \\
69.6969 \\
203.2330 \\
206.7685 \\
129.0234 \\
248.7668 \\
249.1929 \\
256.2979 \\
92.7938 \\
258.2104 \\
274.4959 \\
159.8174 \\
268.4863 \\
102.3161 \\
273.5767 \\
271.1363 \\
259.4314 \\
270.4748 \\
267.9097 \\
191.0861 \\
233.9039 \\
260.7506 \\
456.9786 \\
96.3879 \\
271.4534 \\
39.8489 \\
138.1027 \\
158.9765 \\
237.0289 \\
91.0196 \\
194.6971 \\
200.5879 \\
206.2331 \\
212.6970 \\
255.5300 \\
224.7643 \\
228.3376 \\
223.3525 \\
234.0727 \\
2156603\end{array}$ & $\begin{array}{l}3 . \\
1 . \\
1 . \\
3 . \\
2 . \\
2 . \\
2 . \\
3 . \\
2 . \\
2 . \\
2 . \\
2 . \\
1 . \\
2 .\end{array}$ & $\begin{array}{l}\text { Uncert } \\
1.9001 \mathrm{e}+00 \\
1.9001 \mathrm{e}+00 \\
1.9002 \mathrm{e}+00 \\
1.9002 \mathrm{e}+00 \\
1.9002 \mathrm{e}+00 \\
1.9024 \mathrm{et}+00 \\
1.9000 \mathrm{e} \\
1.90\end{array}$ \\
\hline
\end{tabular}

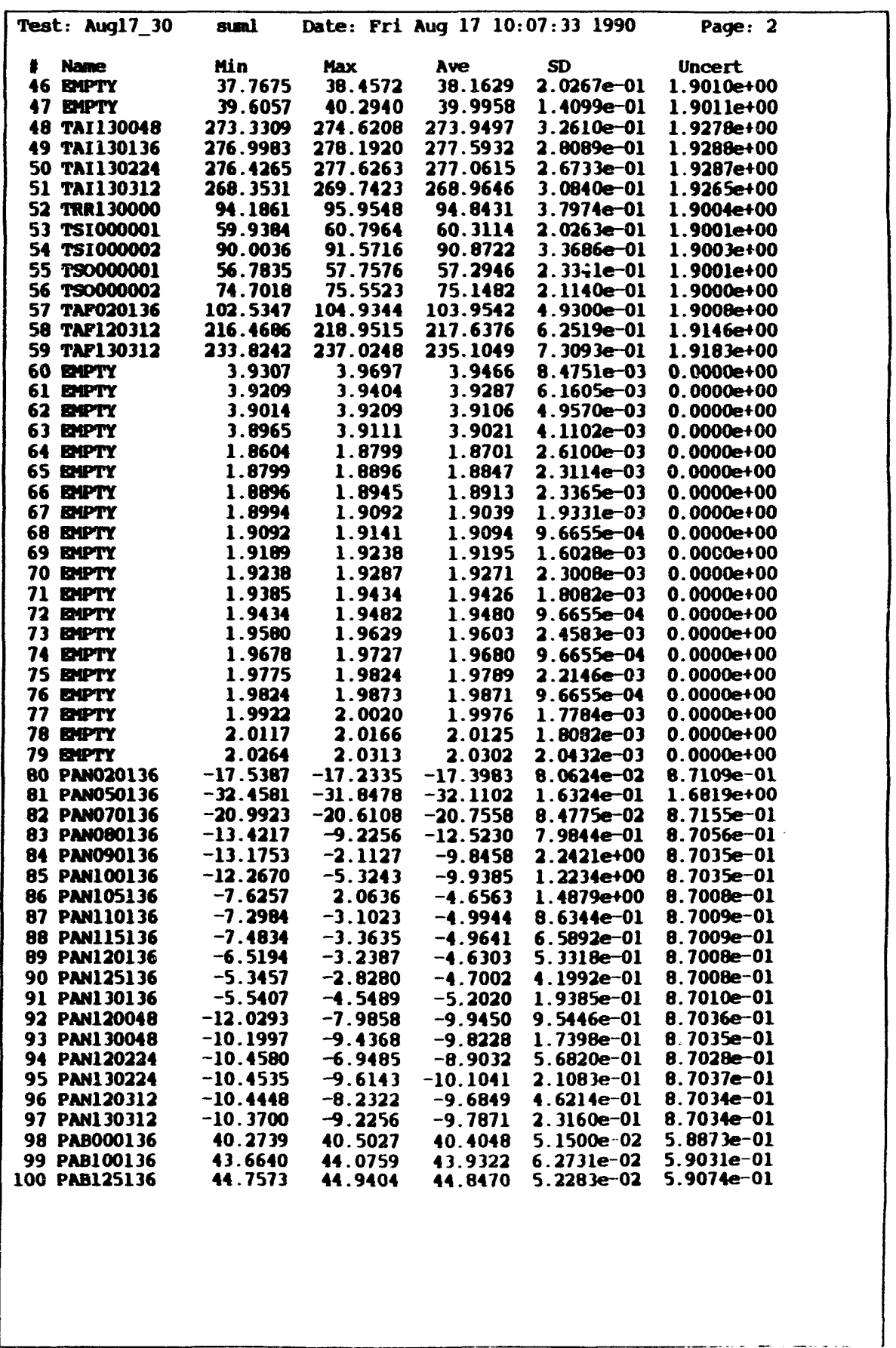




\begin{tabular}{|c|c|c|c|c|c|}
\hline 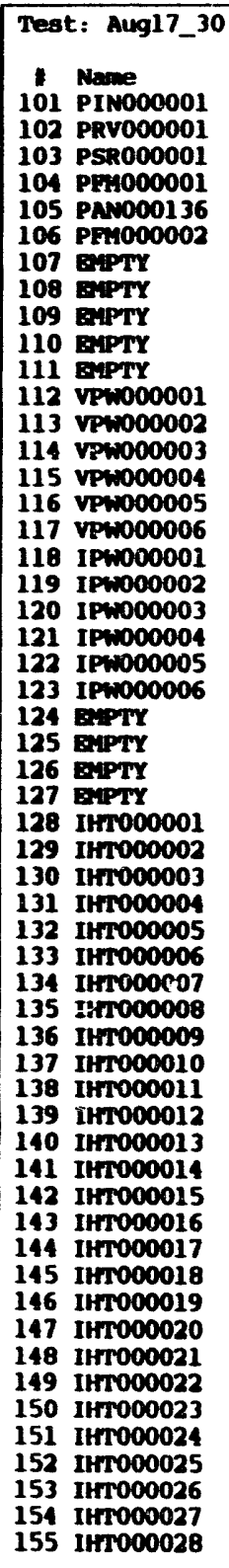 & \begin{aligned} \multicolumn{1}{c}{ suml } \\
Min \\
40.2028 \\
19.5965 \\
44.9376 \\
1.7324 \\
-133.1649 \\
1.8119 \\
-0.0049 \\
-10.0000 \\
-0.0049 \\
-10.0000 \\
0.0000 \\
102.0245 \\
119.0467 \\
147.6225 \\
104.8640 \\
139.6522 \\
148.4563 \\
533.7407 \\
1304.2736 \\
1324.0499 \\
374.8216 \\
252.5851 \\
261.2681 \\
-0.0244 \\
-0.0195 \\
-0.0195 \\
-0.0244 \\
128.1325 \\
131.2049 \\
131.9186 \\
130.0143 \\
128.6809 \\
123.8018 \\
131.1899 \\
129.7701 \\
129.7589 \\
-41.2748 \\
129.5260 \\
127.3362 \\
91.0156 \\
127.3663 \\
94.8167 \\
88.5216 \\
105.0706 \\
106.9636 \\
103.4701 \\
106.1974 \\
-2.4226 \\
92.4692 \\
104.3645 \\
69.5726 \\
104.7439 \\
105.1307 \\
89.9940 \\
91.1396\end{aligned} & $\begin{aligned} \text { Date: Fri } \\
\text { Max } \\
40.4470 \\
19.8406 \\
45.1818 \\
2.0071 \\
-130.8760 \\
1.9126 \\
0.0049 \\
-0.2246 \\
0.0049 \\
-10.0000 \\
0.0000 \\
102.4151 \\
119.4374 \\
148.0131 \\
105.4500 \\
140.4334 \\
148.8469 \\
547.4072 \\
1315.9812 \\
1331.8499 \\
380.1927 \\
254.0498 \\
262.7329 \\
-0.0098 \\
-0.0049 \\
0.0000 \\
-0.0098 \\
130.4762 \\
133.9393 \\
134.2623 \\
132.3580 \\
130.6340 \\
125.9503 \\
132.9477 \\
131.3326 \\
131.5167 \\
-38.9310 \\
131.2838 \\
129.0941 \\
92.9688 \\
129.1241 \\
96.3792 \\
90.0841 \\
108.5863 \\
109.3074 \\
105.4312 \\
108.3458 \\
-0.0799 \\
94.4223 \\
106.1223 \\
71.3304 \\
106.5017 \\
106.8885 \\
91.9471 \\
93.2880\end{aligned}$ & 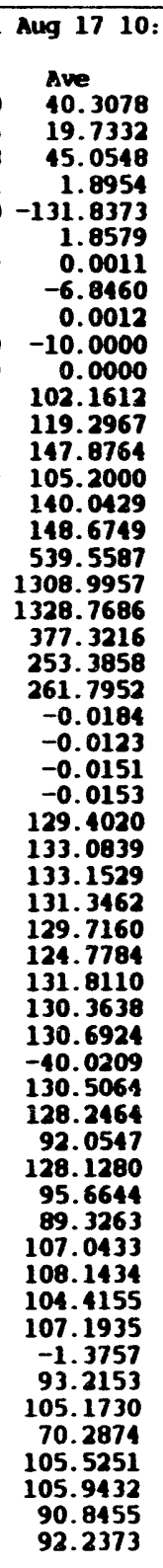 & 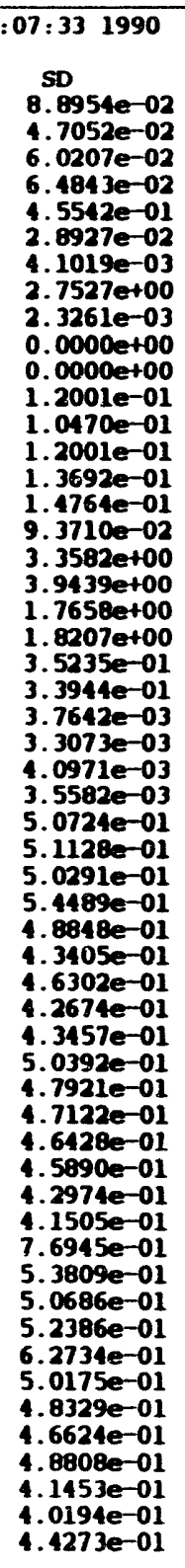 & 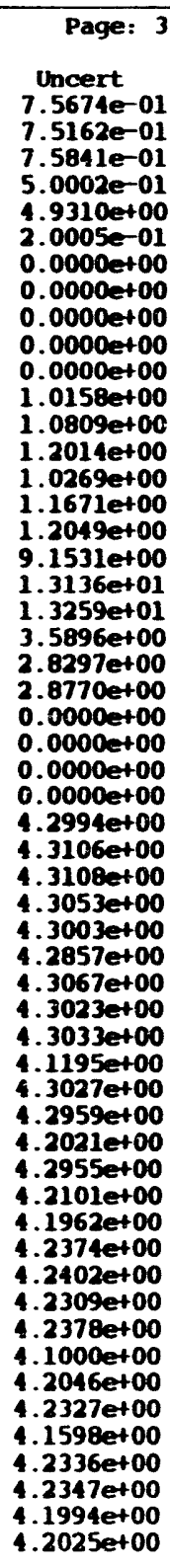 \\
\hline
\end{tabular}

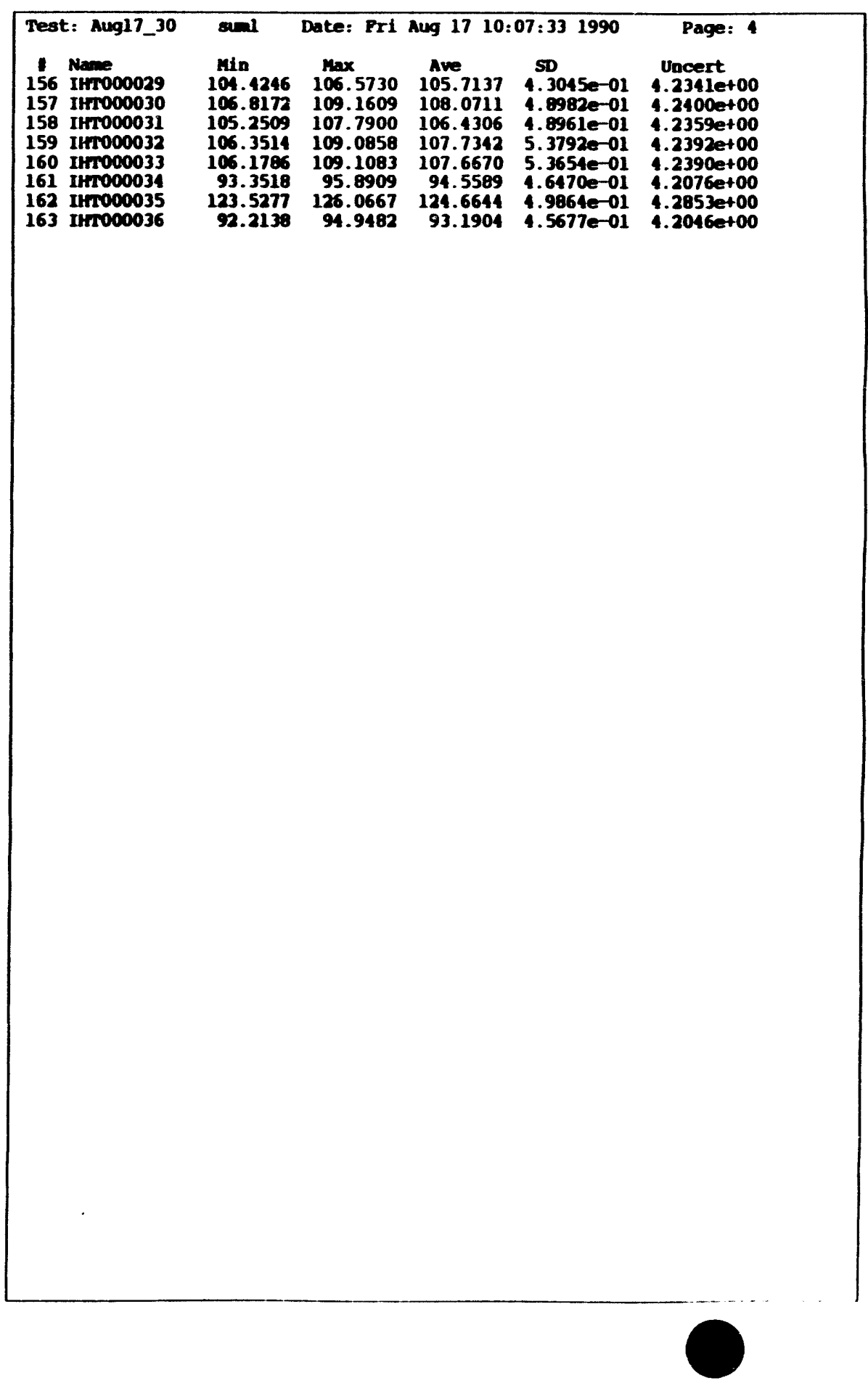




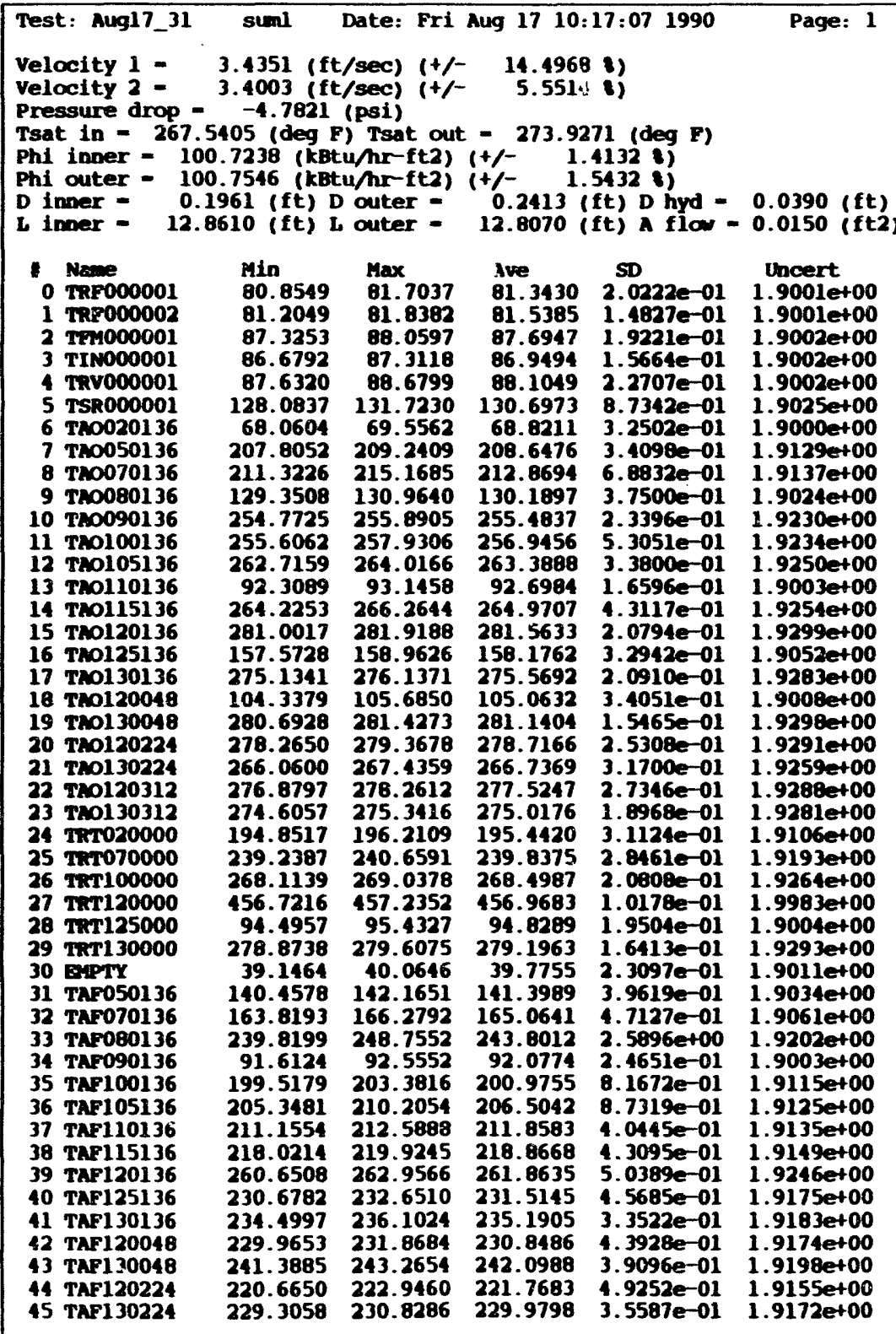

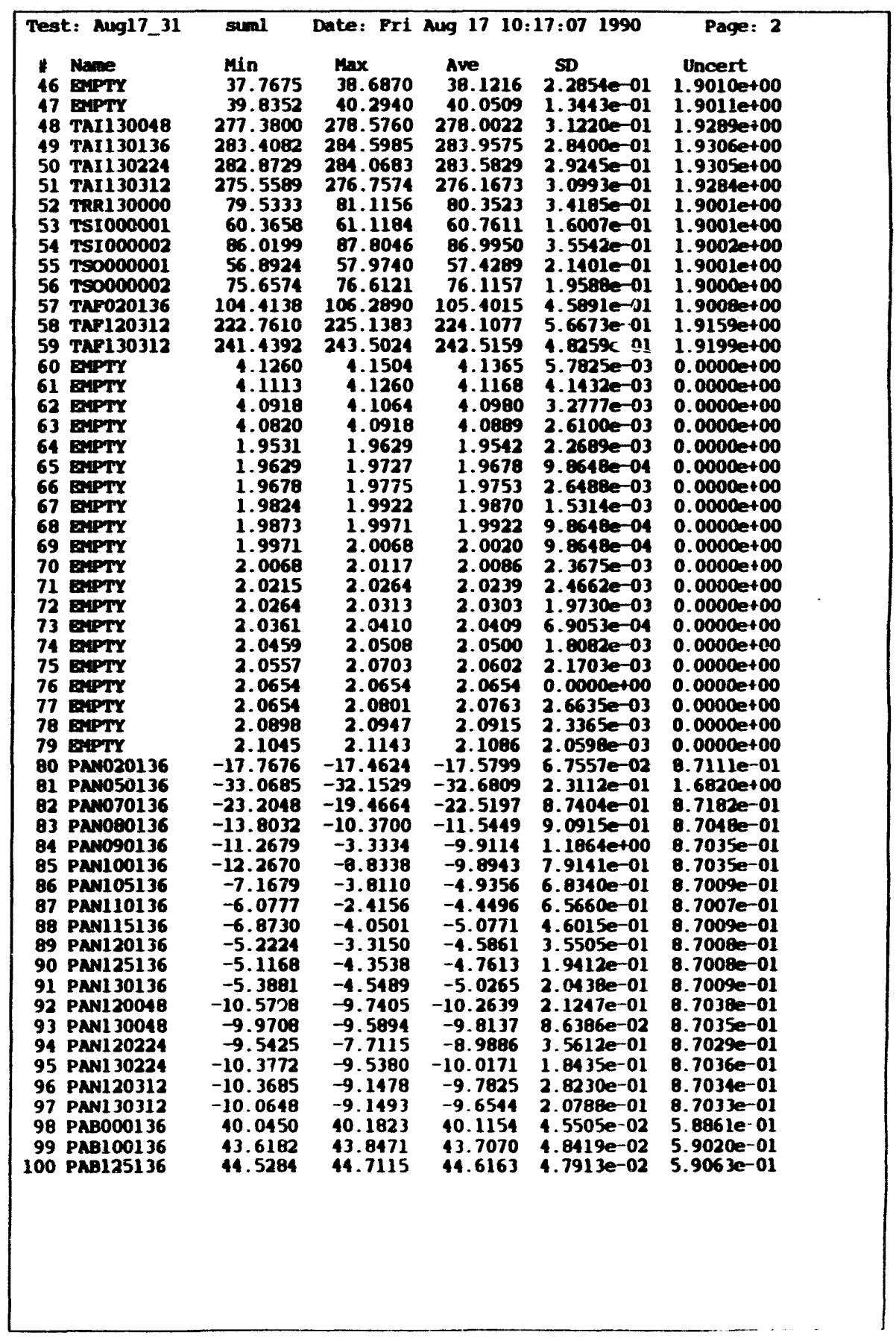



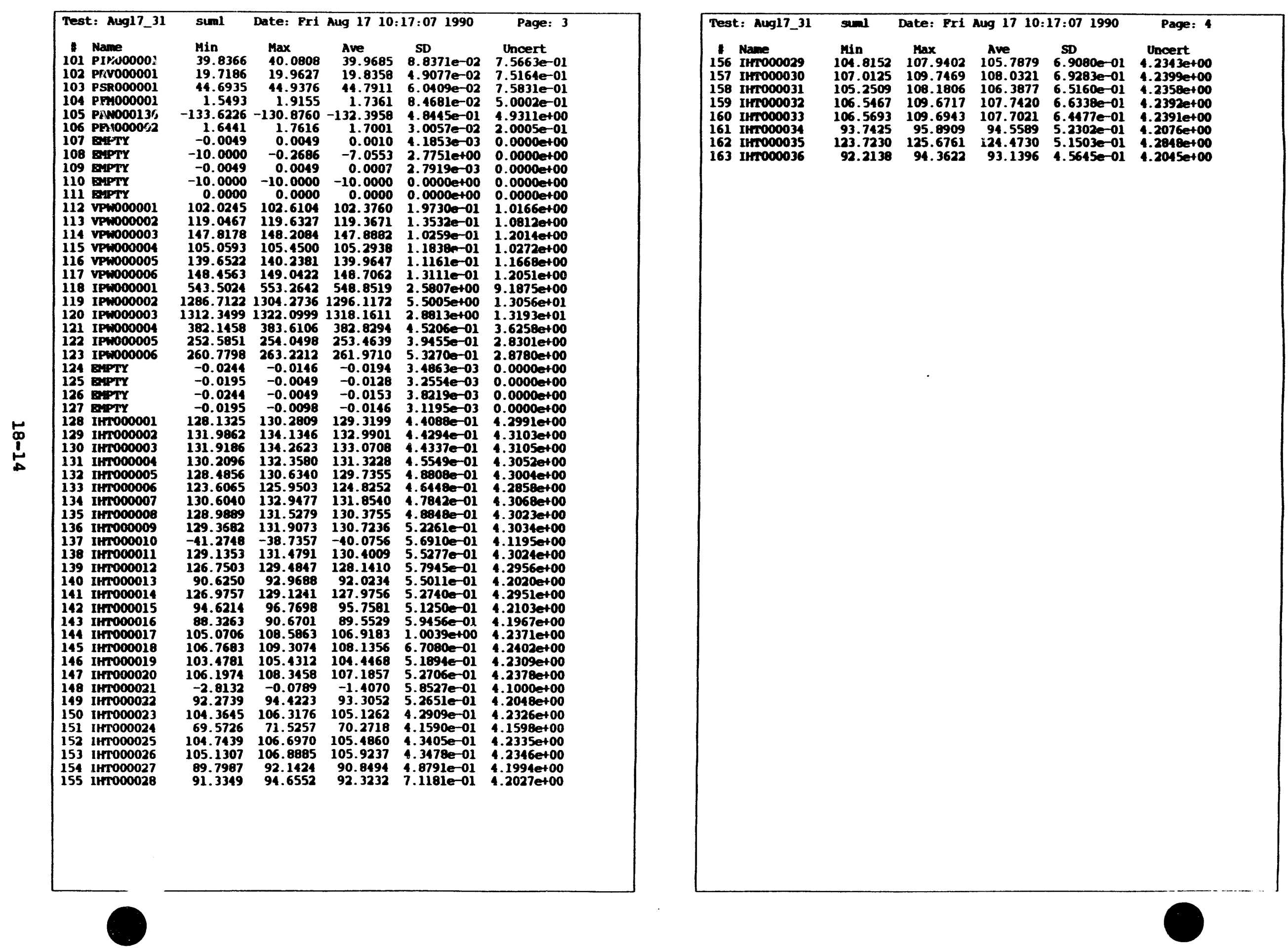
DEFECTIVE INSTRUMENTS FOR THE RIBBED GEOMETRY TEST SERIES

\begin{tabular}{|c|c|ll|}
\hline Series & Tests & \multicolumn{1}{|c|}{ Defective Instruments } \\
\hline 13 & Aug17_26 to Aug17_31 & TAO120048 TAF070136 & TRT120000 \\
& & TAO020136 TAF080136 & TRT125000 \\
& & TAO070136 TAF090136 & TRR130000 \\
& & TAO080136 TAF120136 & TAO110136 \\
& & TAO125136 & \\
& & IHT00010 IHT000021 IHTO00024 \\
& & IHT000033 & \\
\hline
\end{tabular}


(5)eare

TN-499

\section{Chapter 19}

NUCLEATE BOILING PRESSURE DROP IN AN ANNULUS

Test Geometry: Ribbed (4)

Test Series: $3 A$

Test Date: $\quad$ August 17, 1990 


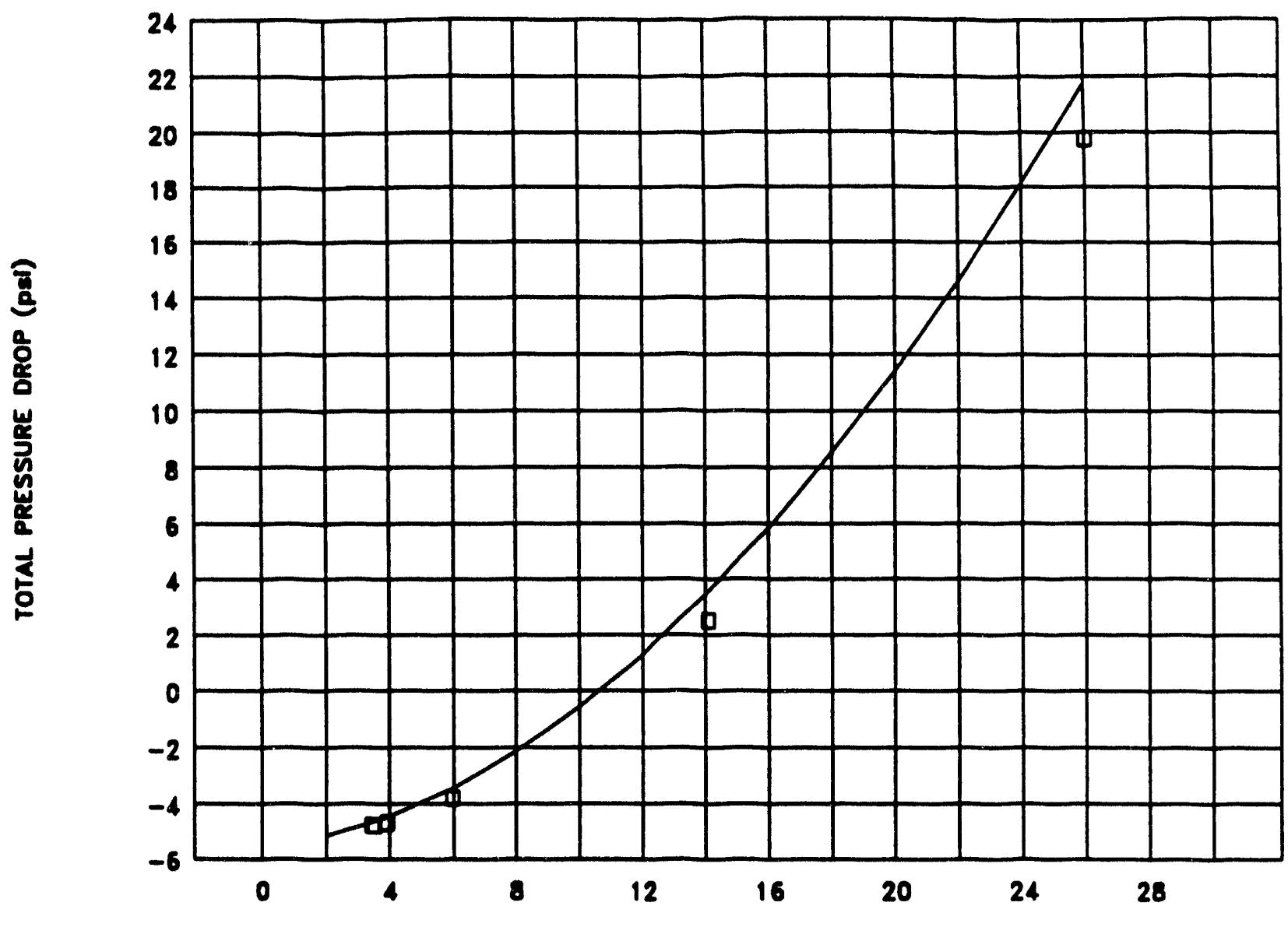

ANNULUS INLET FLOW VELOCITY ( $(1 / \mathrm{s})$

- SERIES 3A, 08/17/90 UNHEATED, 08/14/90

\begin{tabular}{|c|c|c|}
\hline $\begin{array}{c}\text { TEST } \\
\text { NUMBER }\end{array}$ & $\begin{array}{c}\text { INLET } \\
\text { VELOCITY(a) }\end{array}$ & $\begin{array}{c}\text { PRESSURE } \\
\text { DROP(b) }\end{array}$ \\
\hline & $(\mathrm{ft} / \mathrm{s})$ & (psi) \\
\hline Aug17_32 & 26.02 & 19.72 \\
\hline Aug17_33 & 14.09 & 2.51 \\
\hline Aug17_34 & 5.97 & -3.80 \\
\hline Aug17_35 & 3.91 & -4.69 \\
\hline Aug17_36 & 3.52 & -4.77 \\
\hline 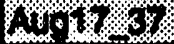 & 6,5 & 178 \\
\hline
\end{tabular}

\begin{tabular}{|l|l|}
\hline \multicolumn{2}{|c|}{ NOMINAL TEST CONDITIONS } \\
\hline Annulus geometry & ribbed (4) \\
Inlet temperature & $86^{\circ} \mathrm{F}$ \\
Inlet pressure & $40 \mathrm{psia}$ \\
Wall heat flux & $100 \mathrm{kBtu} / \mathrm{hr}-\mathrm{tt2}$ \\
Flux distribution & uniform \\
He saturation pressure & $15 \mathrm{psig}$ \\
\hline NOTES: \\
(a) Inlot velocity calculated from PFMO00001 \\
(b) Prosesure drop caloulated from PANO00136 \\
(c) Shading notes test at minimum pressure drop
\end{tabular}

DEMAND CURVE AND DATA FOR SERIES 3A - RIBBED ANNULUS 

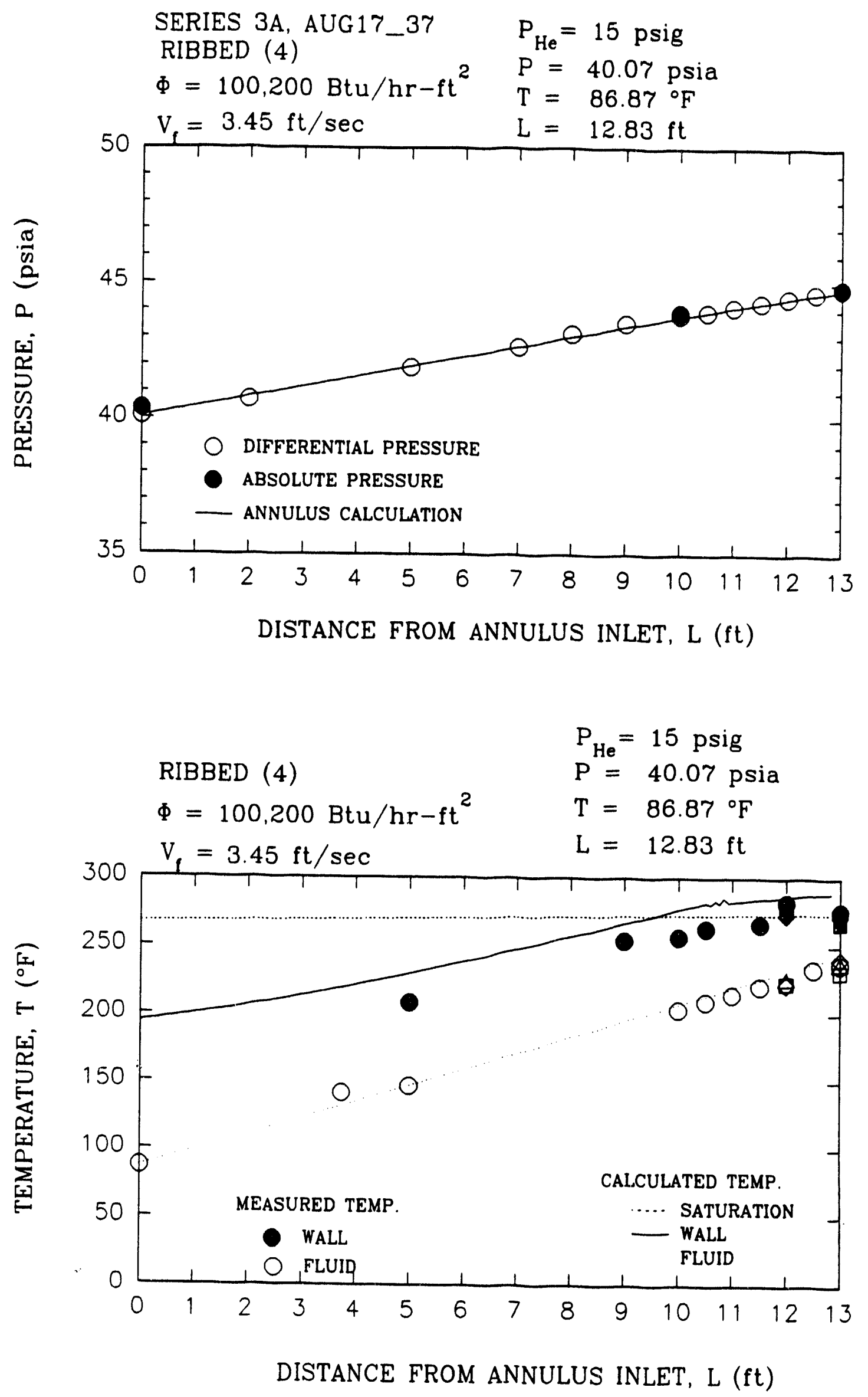
Test: Aug17_32 suml Date: Fri Aug 17 11:09:13 1990 Page: 1

Velocity $1=26.0223$ (ft/sec) $(+/-\quad 1.6887$ )

Pressure drop = 19.7212 (psi)

Tsat in $=268.0894(\mathrm{deg}$ F) Tsat out $=230.8713(\mathrm{deg}$ F)

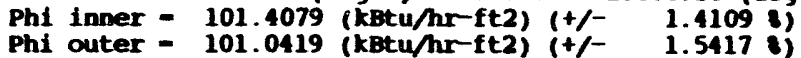

$\mathrm{D}$ inner $=0.1961$ (ft) D outer $=0.2413$ (ft) D hyd $=0.0390(\mathrm{ft})$

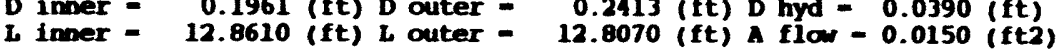

0 TRF000001

1 TRFO00002

3 TIN000001

4 TRro00001

5 TSR000001

7 TMO050136

- T20070136

9 T20080136

$10 \mathrm{Th0090136}$

11 T20100136

12 TM0105136

14 T20115136

15 T20120136

$16 \mathrm{TM0125136}$

$17 \mathrm{TMO} 30136$

19 TRO130048

20 Tho120224

21 Tho130224

23 TAO130312

24 TRT020000

25 TRT070000

26 TrT100000

(2)

29 Tre 130000

31 TAF050136

32 TNF070136

33 TAF080136

34 TAF090136

36 TAF105136

36 TAF105136

38 TAF115136

39 TAF120136

T TAF125136

11 Tri

13 TAF 30048

44 TAF12022

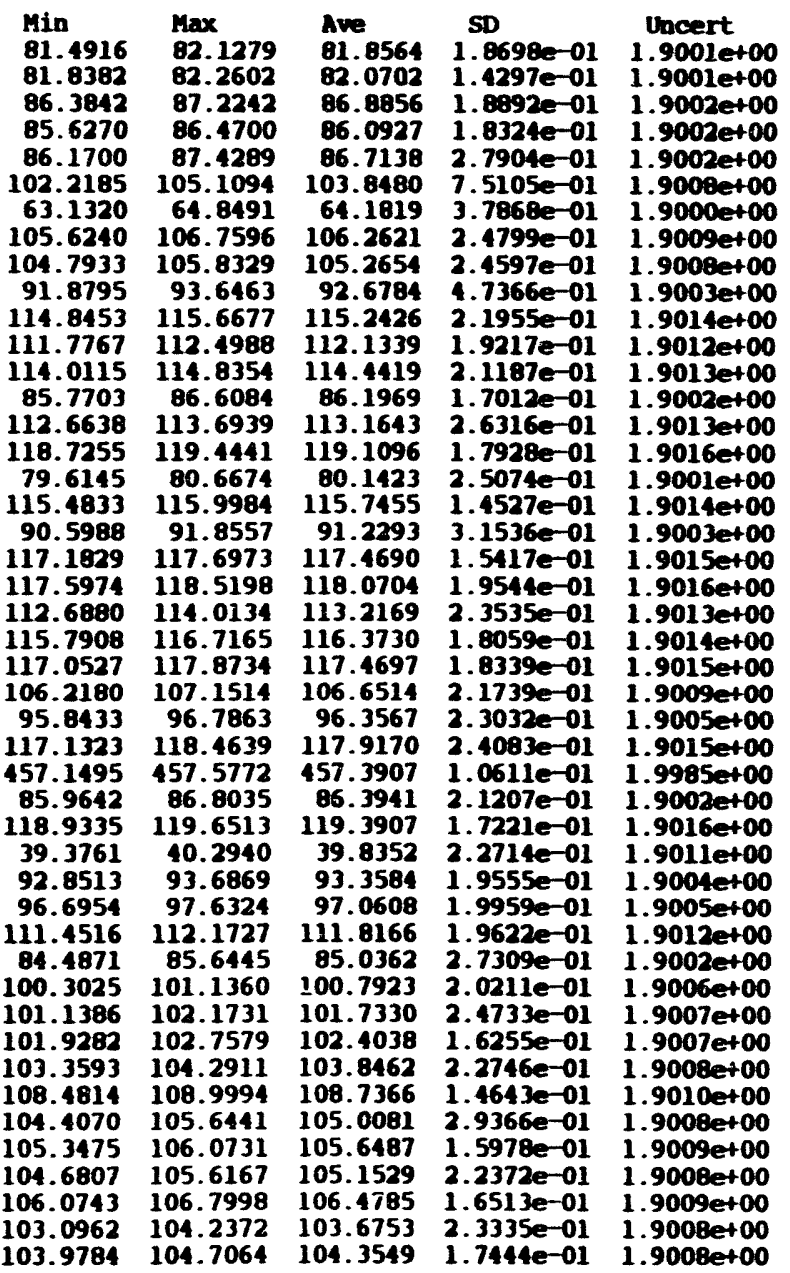

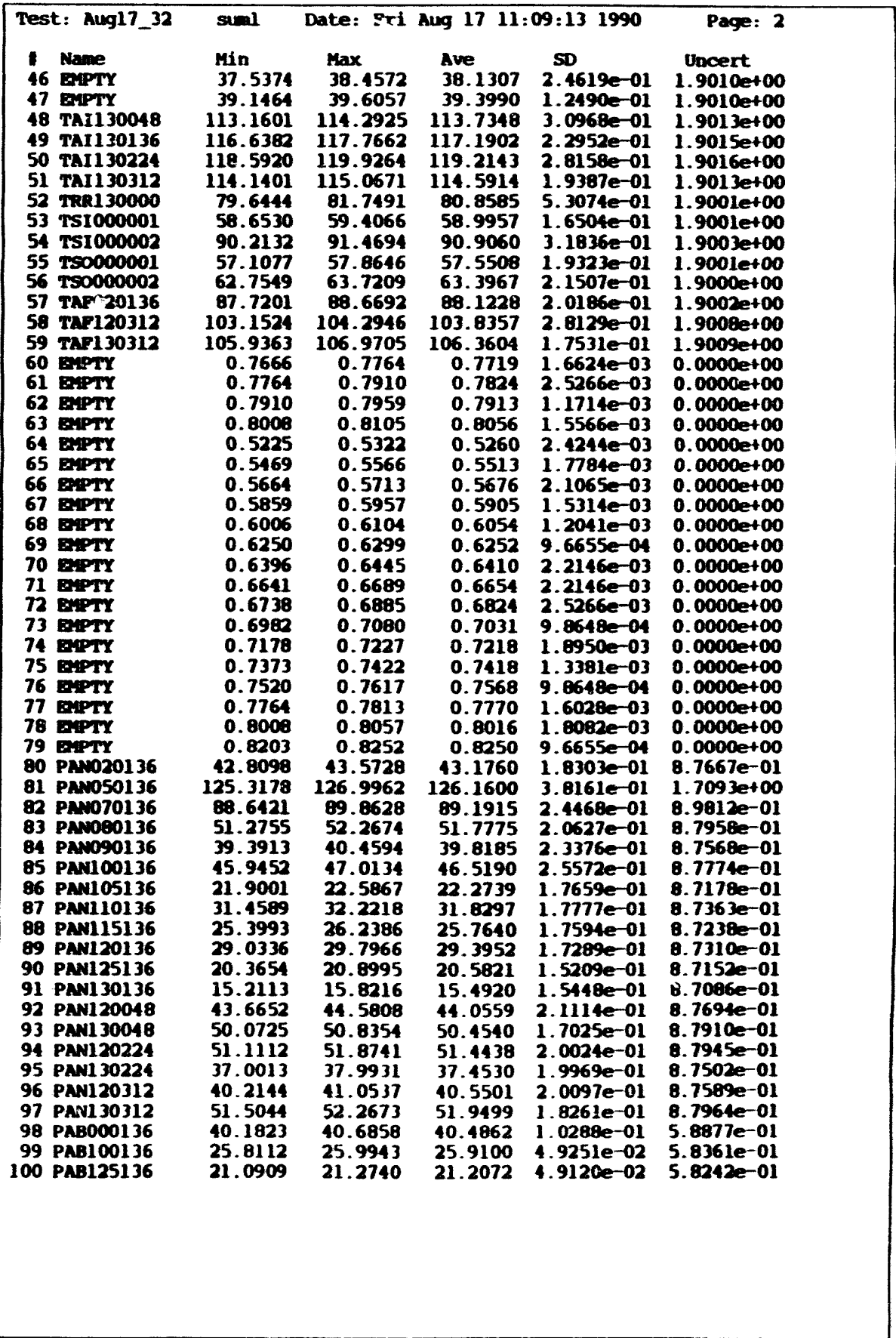




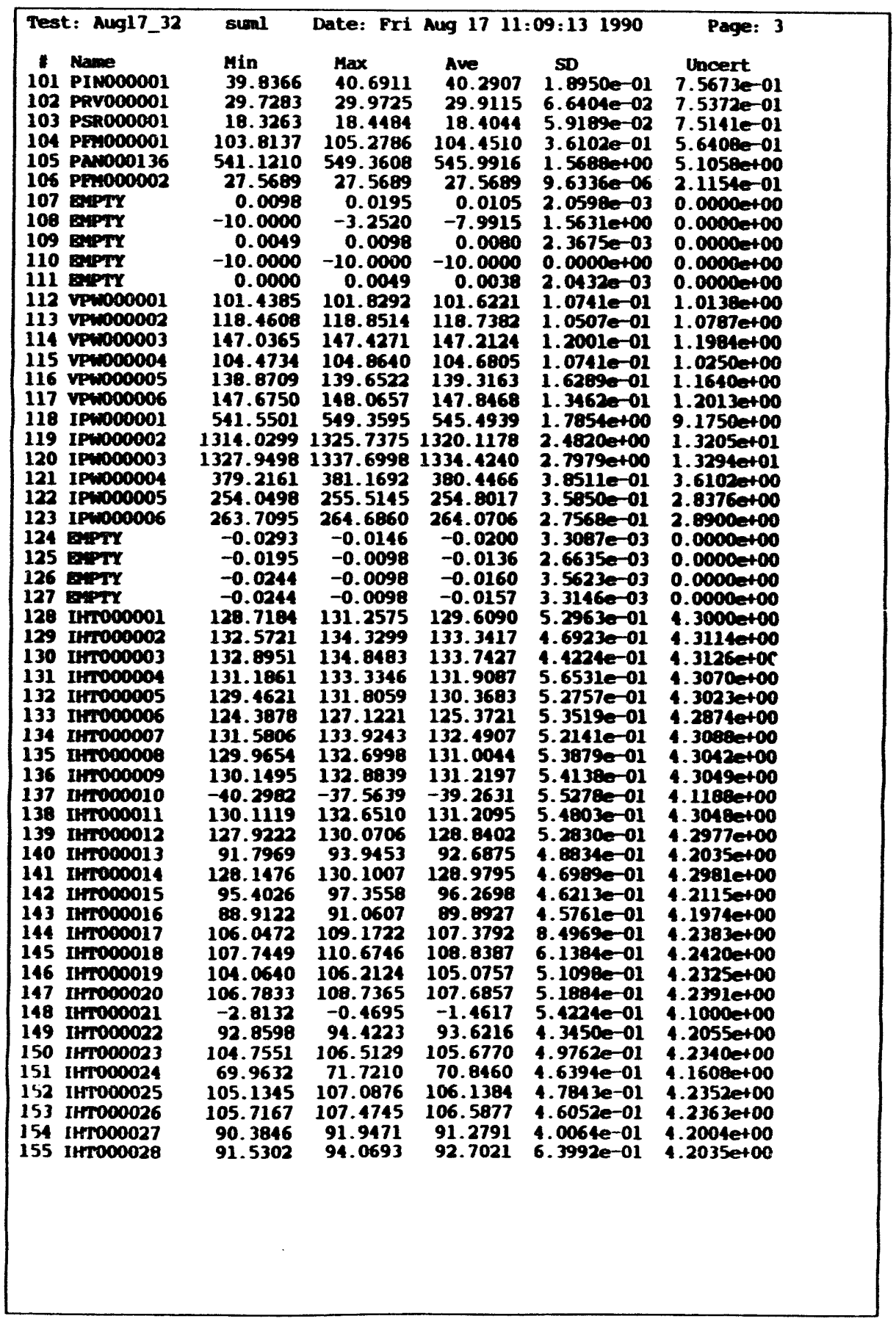

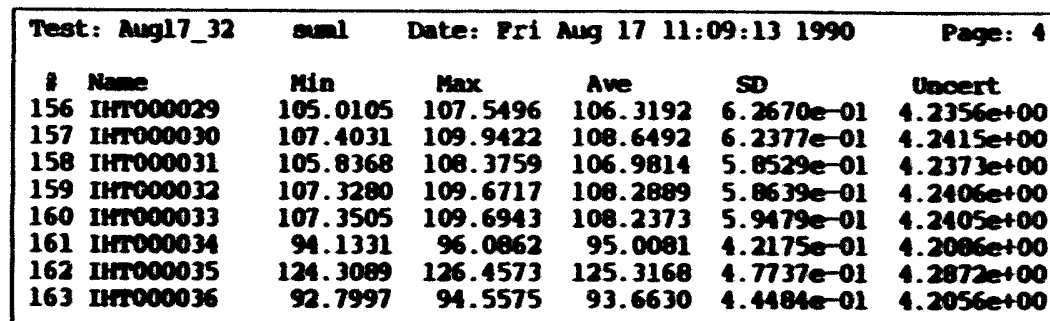




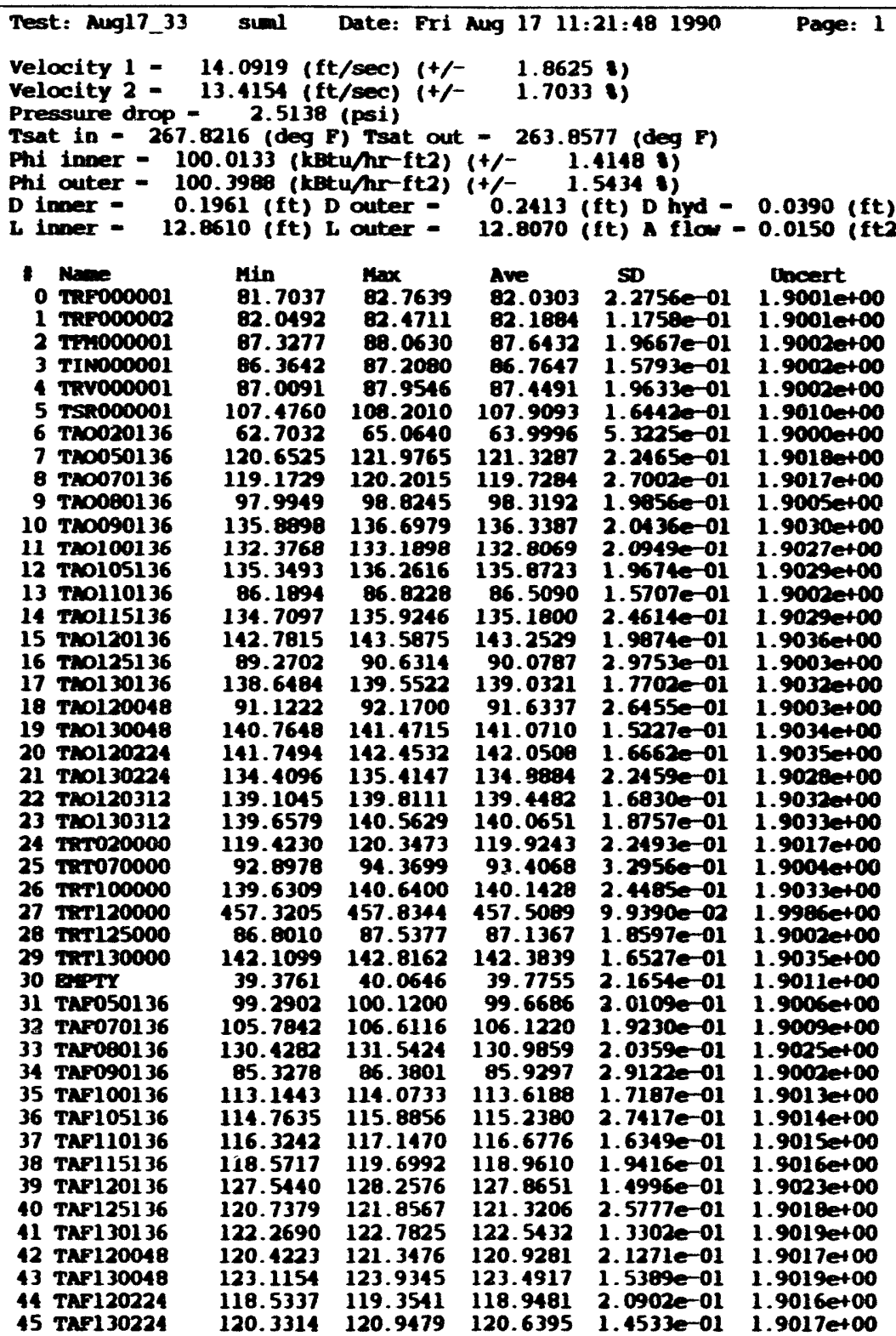

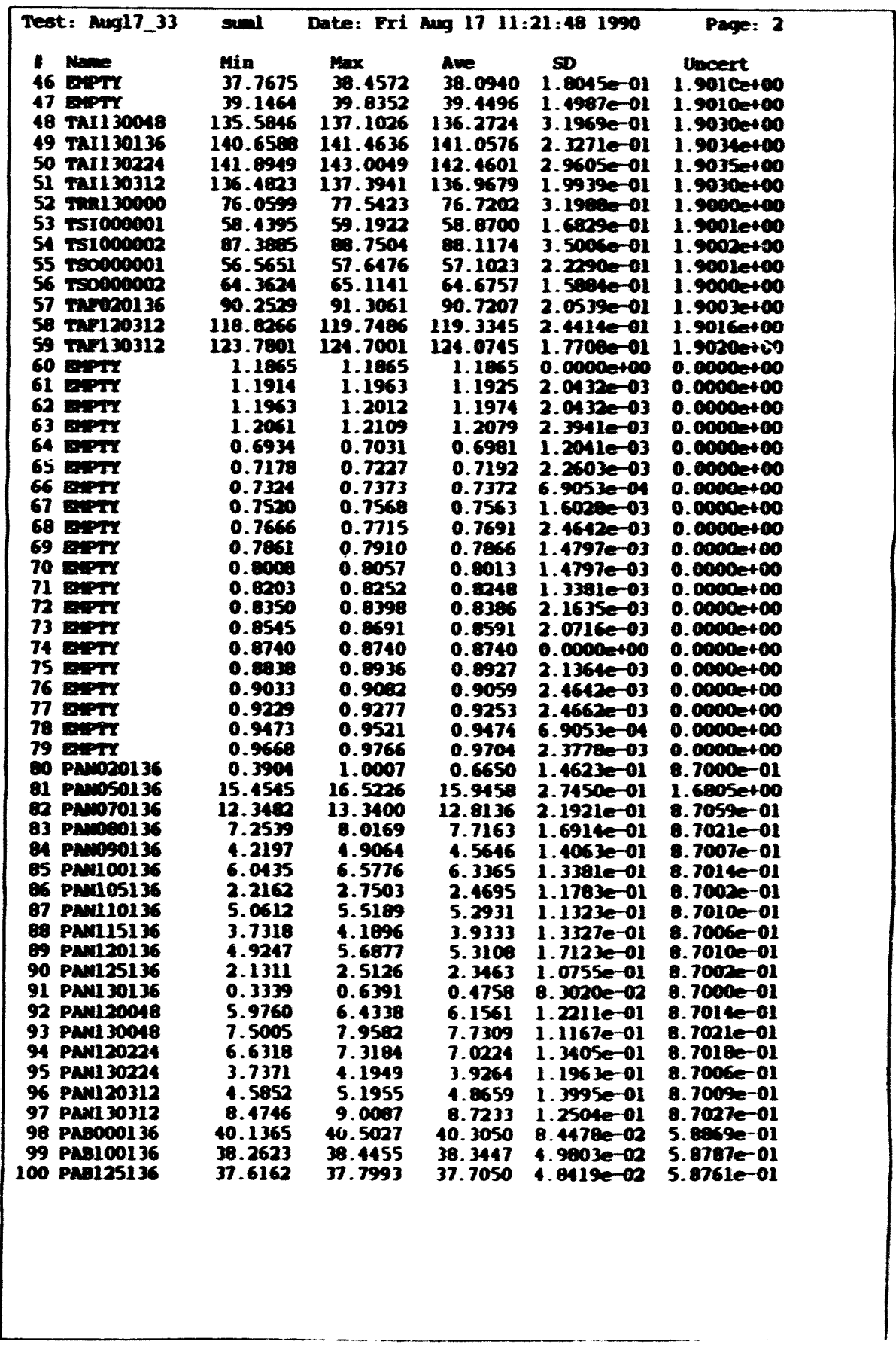




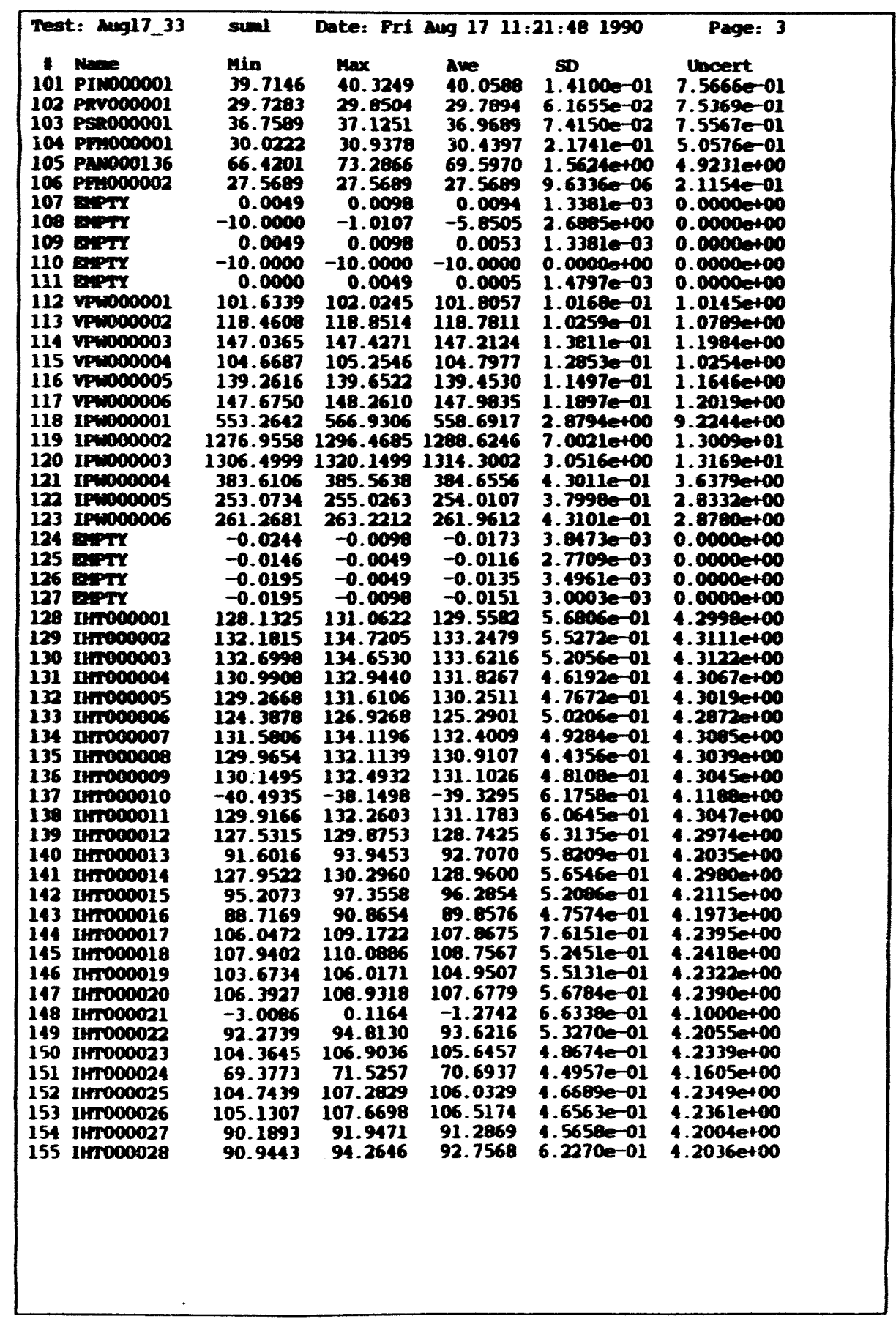
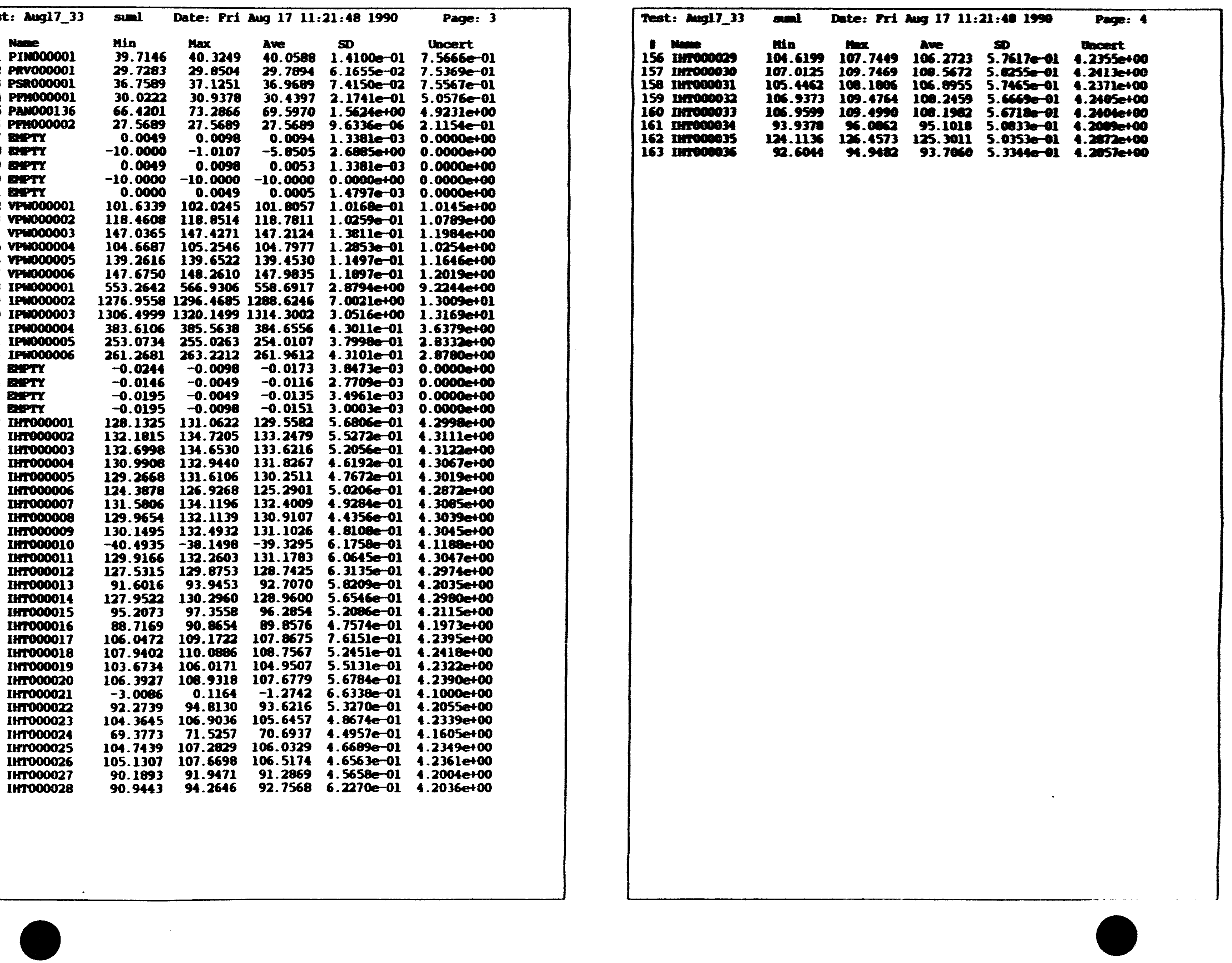
Test: Mugl7_34 sunl Date: Fri Mug 17 11:37:09 1990 Page: 1

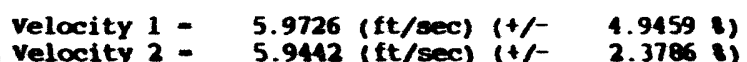
Pressure drop - -3.7960 (psi)

Trat in - 268.0205 (deg F) Tsat oui - $273.0343(\mathrm{deg} F)$

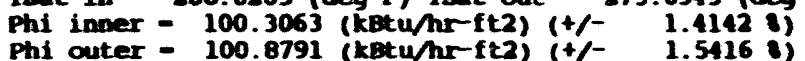

D inner - 0.1961 (ft) D outer $=0.2413$ (ft) D hyd $=0.0390$ (ft)

1 iner = 12.8610 (ft) I outer = 12.8070 (ft) A flow - 0.0150 (ft2)

- Mave Min Max Ave SD uncert

$\begin{array}{llllllll}0 & \text { TRF000001 } & 81.7037 & 82.5519 & 82.1448 & 1.8596 e-01 & 1.9001 \mathrm{e}+00 \\ 1 \text { TRP000002 } & 82.0492 & 82.8929 & 82.3783 & 1.4236 \mathrm{e}-01 & 1.9001 \mathrm{e}+00\end{array}$

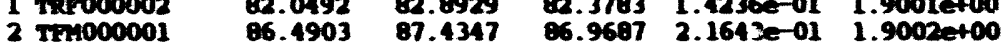

$\begin{array}{lllllll}3 \text { TINO00001 } & 85.8374 & 86.4702 & 86.2046 & 1.3999 e-01 & 1.9002 e+00\end{array}$

$\begin{array}{lllllll}4 \mathrm{TRV} 000001 & 86.6957 & 87.5349 & 87.1406 & 2.0480=01 & 1.9002 \mathrm{et} 00\end{array}$

$\begin{array}{llllllll}5 & \text { TSR000001 } & 116.0894 & 117.5206 & 117.0458 & 3.0591 e-01 & 1.9015 e+00\end{array}$

$\begin{array}{lllllll}6 & 130020136 & 70.6240 & 72.4366 & 71.4855 & 3.8764 e-01 & 1.90000+00\end{array}$

$\begin{array}{llllllll}7 \text { T20050136 } & 159.5021 & 160.4929 & 160.0456 & 2.2539 e-01 & 1.9055 e+00\end{array}$

8 mo070136 160.8088 162.3835 161.7895 3.62aje-01 1.9057 etco

9 Tno060136 $111.6785 \quad 112.6002 \quad 112.1161 \quad 2.33290-01 \quad 1.90120+00$

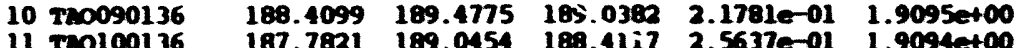

$11 \mathrm{TnO100136} \quad 187.7821 \quad 189.0454189 .41 \mathrm{il}$ 2.5637e-01 $1.9094 \mathrm{e}+00$

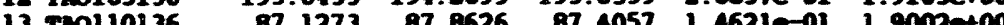

14 T20115136 $193.7290195 .0865194 .3495 \quad 2.7711 e-01$ 1.9104e+00

$\begin{array}{llllllll}15 & 700120136 & 207.3302 & 207.9081 & 207.7189 & 1.30260-01 & 1.9127 e+00\end{array}$

$\begin{array}{llllllll}16 & \mathrm{Tm} 0125136 & 121.0725 & 122.3989 & 121.6317 & 2.88720-01 & 1.90180+00\end{array}$

\begin{tabular}{lllllll}
16 & Tro125136 & 121.0725 & 122.3989 & 121.6317 & $2.85720-01$ & $1.9018 e+\infty 0$ \\
\hline
\end{tabular}

$\begin{array}{lllllll}18 & \text { T20120048 } & 93.8397 & 95.0945 & 94.4547 & 2.85150-01 & 1.9004 e+00\end{array}$

$\begin{array}{lllllll}19 & \mathrm{Tnol} 30048 & 203.1192 & 203.8996 & 203.4935 & 1.5749 e-01 & 1.9120 \mathrm{e}+00\end{array}$

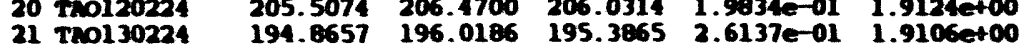

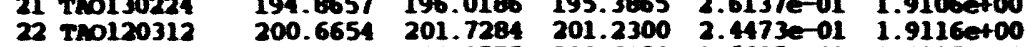

$\begin{array}{llllllll}23 & \text { T20130312 } & 200.3021 & 200.9775 & 200.6380 & 1.5605 \text { e-01 } & 1.9115 e+00\end{array}$

$\begin{array}{lllllll}24 & \text { TRT020000 } & 152.8637 & 153.9644 & 153.3638 & 2.3831 e-01 & 1.9047 e+00\end{array}$

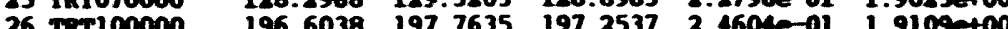

$\begin{array}{rrrrrrr}27 & \operatorname{Tr} 120000 & 457.4058 & 457.8340 & 157.6318 & 8.7749=-02 & 1.9987 e+00 \\ 28 & \operatorname{TrT} 125000 & 87.8469 & 88.6829 & 88.3270 & 1.72940-01 & 1.9002 \mathrm{e}+00\end{array}$

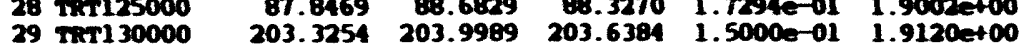

31 TAF050136

32 TNP070136

33 TAF080136

35 TAF100136

36 TNF105136

37 TAF110136

39 TAP120136

40 TAP125136

12 TAF120048

43 TAF130048

14 TAF12022 $\begin{array}{rrrrr}39.1464 & 40.2940 & 39.8260 & 2.4953 e-01 & 1.9011 e+00\end{array}$ $\begin{array}{llllll}115.9606 & 116.6807 & 116.3943 & 2.2702 e-01 & 1.9014 e+00\end{array}$ $\begin{array}{llllll}128.2178 & 129.2324 & 128.8823 & 2.5480 e-01 & 1.9023 e+00\end{array}$ $181.9106 \quad 182.7646182 .3293 \quad 2.06390-01$ 1.9005e+00 $\begin{array}{lllll}86.1683 \quad 87.6391 \quad 86.8413 & 3.0373 e-01 & 1.9002 e+00\end{array}$ $\begin{array}{lllll}148.9178 & 150.0207 & 149.2953 & 2.1493 e-01 & 1.9042 e+00\end{array}$ $\begin{array}{llllll}152.8167 & 153.8066 & 153.3547 & 2.5106 e-01 & 1.9047 e+00\end{array}$ $156.8927 \quad 157.8870157 .3984$ 1.8265e-01 1.9051 e+00 181.7771 182 9444102.45092 .7095001 1.9005e+00 $168.0530 \quad 169.2293 \quad 168.68772 .02530-01 \quad 1.90550+00$ $171.0747 \quad 171.7646 \quad 171.4722 \quad 1.7554 e-01 \quad 1.9069 e+00$ $164.5542 \quad 165.7487 \quad 165.2349 \quad 3.1610 e-01 \quad 1.9061 e+00$ $\begin{array}{llllll}171.4785 & 172.6597 & 171.9591 & 2.4244 e-01 & 1.9070 e+00\end{array}$ $\begin{array}{lllll}162.6928 & 163.8797 & 163.2381 & 2.6083 e-01 & 1.9058 e+00 \\ 167.5071 & 168.4003 & 168.0626 & 2.0041 e-01 & 1.9065 e+00\end{array}$

\begin{tabular}{|c|c|c|c|c|c|}
\hline 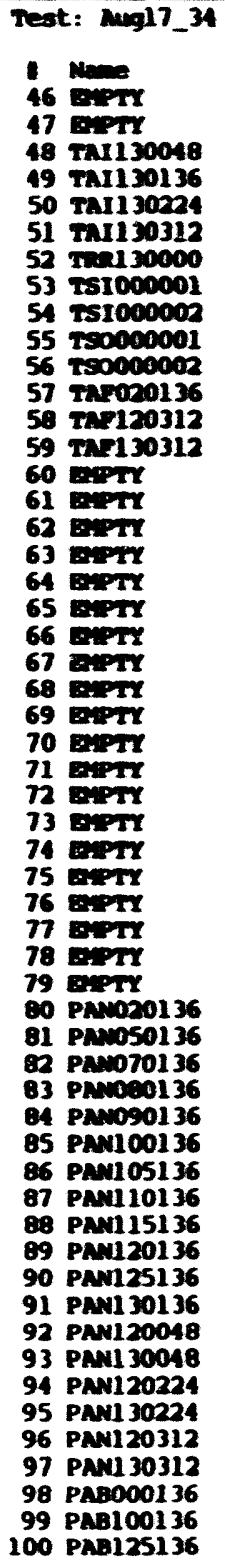 & 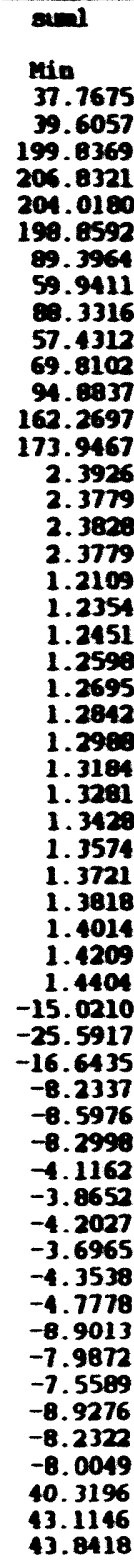 & 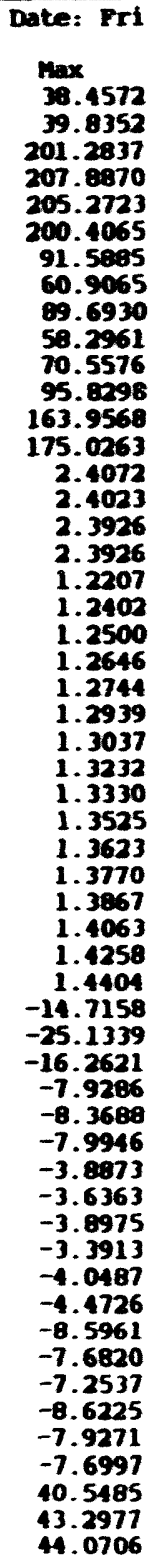 & $\begin{aligned} \text { Mug } 1711: \\
\text { Ave } \\
38.1446 \\
39.7067 \\
200.4770 \\
207.1967 \\
204.6083 \\
199.6607 \\
90.5865 \\
60.3626 \\
89.0311 \\
57.8290 \\
70.1097 \\
95.4129 \\
163.1948 \\
174.4457 \\
2.3992 \\
2.3939 \\
2.3892 \\
2.3894 \\
1.2161 \\
1.2356 \\
1.2484 \\
1.2646 \\
1.2742 \\
1.2891 \\
1.2995 \\
1.3168 \\
1.3300 \\
1.3473 \\
1.3606 \\
1.3747 \\
1.3862 \\
1.4015 \\
1.4211 \\
1.4404 \\
-14.8531 \\
-25.4208 \\
-16.4787 \\
-8.1178 \\
-8.4786 \\
-8.1365 \\
-4.0475 \\
-3.7828 \\
-4.0135 \\
-3.5363 \\
-4.2104 \\
-4.6328 \\
-8.7212 \\
-7.7934 \\
-7.4353 \\
-8.7415 \\
-8.0629 \\
-7.8065 \\
40.4395 \\
43.2044 \\
43.9626\end{aligned}$ & 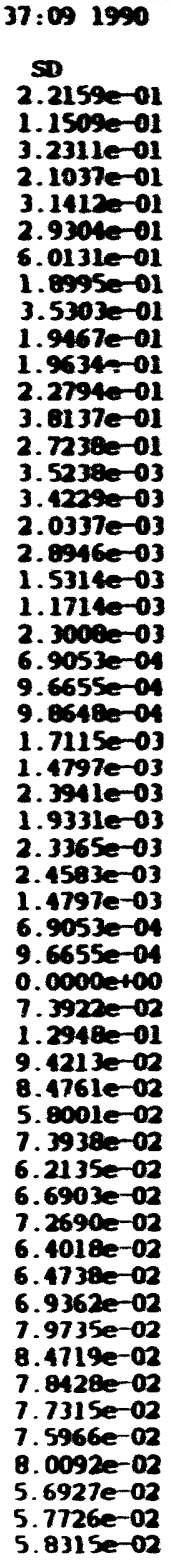 & 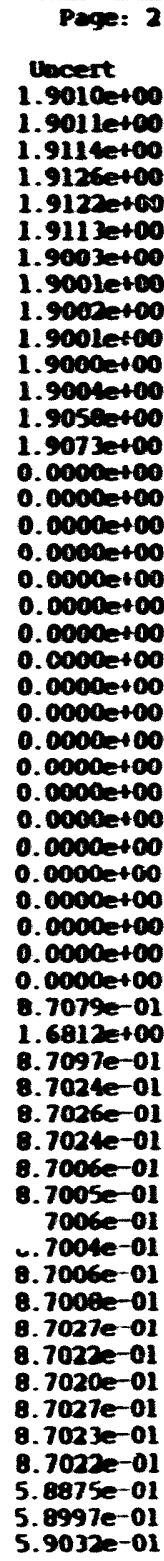 \\
\hline
\end{tabular}



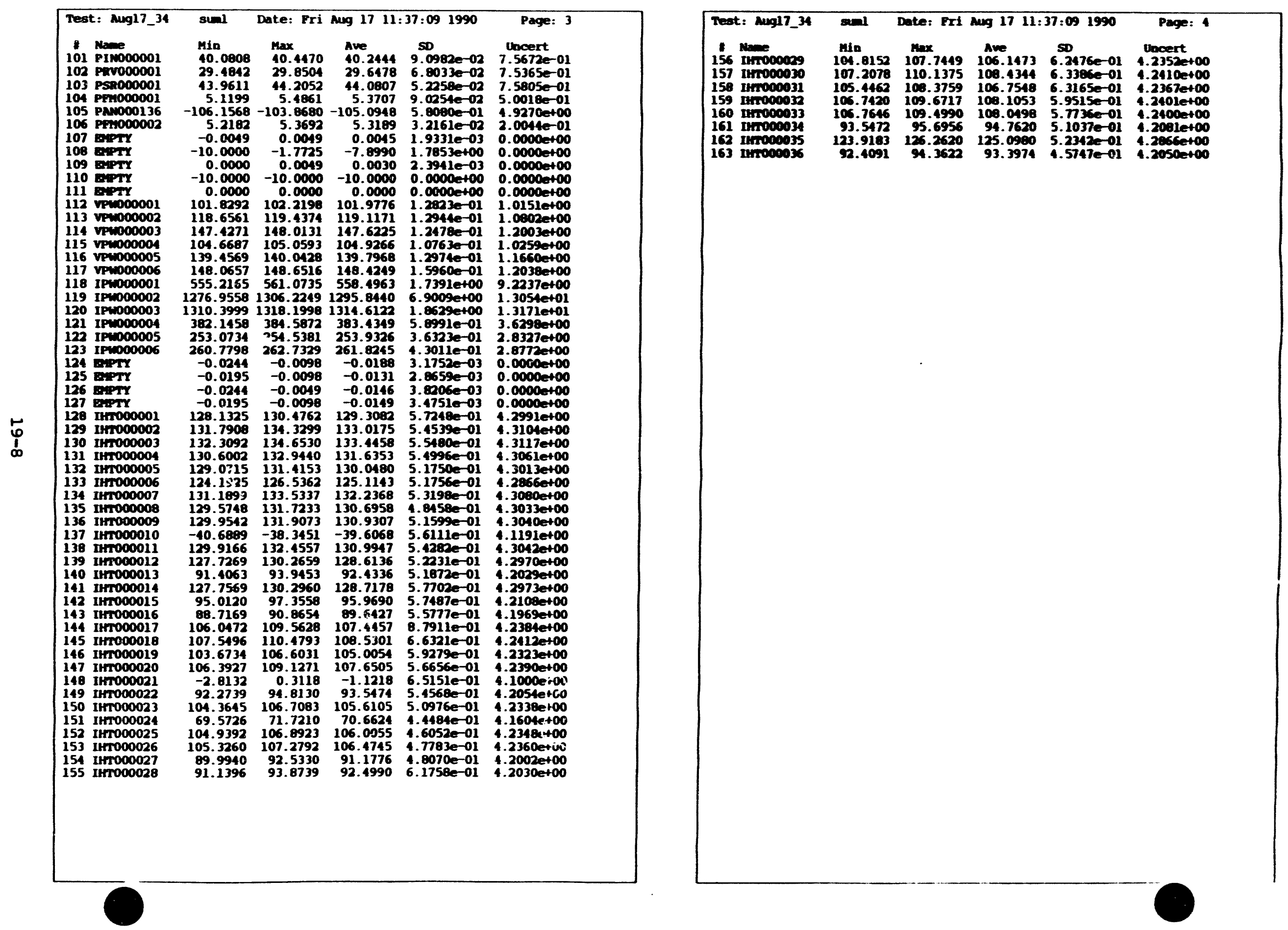
Test: Aug17 35 suml Date: Fri Aug 17 11.53.08 1990 Page: 1

Velocity $1=3.9095(\mathrm{ft} / \mathrm{sec})(+/-11.1709$ \%)

Pressure drop $=-4.6912$ (psi)

Tsat in = 267.7674 (deg F) Tsat out $=274.0140$ (deg F)

Phi inner $=100.9487$ (kBtu/hr-ft2) $(+/-\quad 1.4125$ ( )
Phi outer $=101.3060$ (kBtu/hr-ft2) $(+/-\quad 1.5410$ )

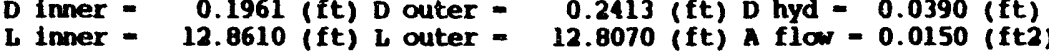

Name

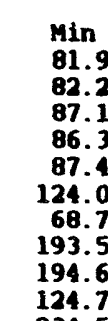

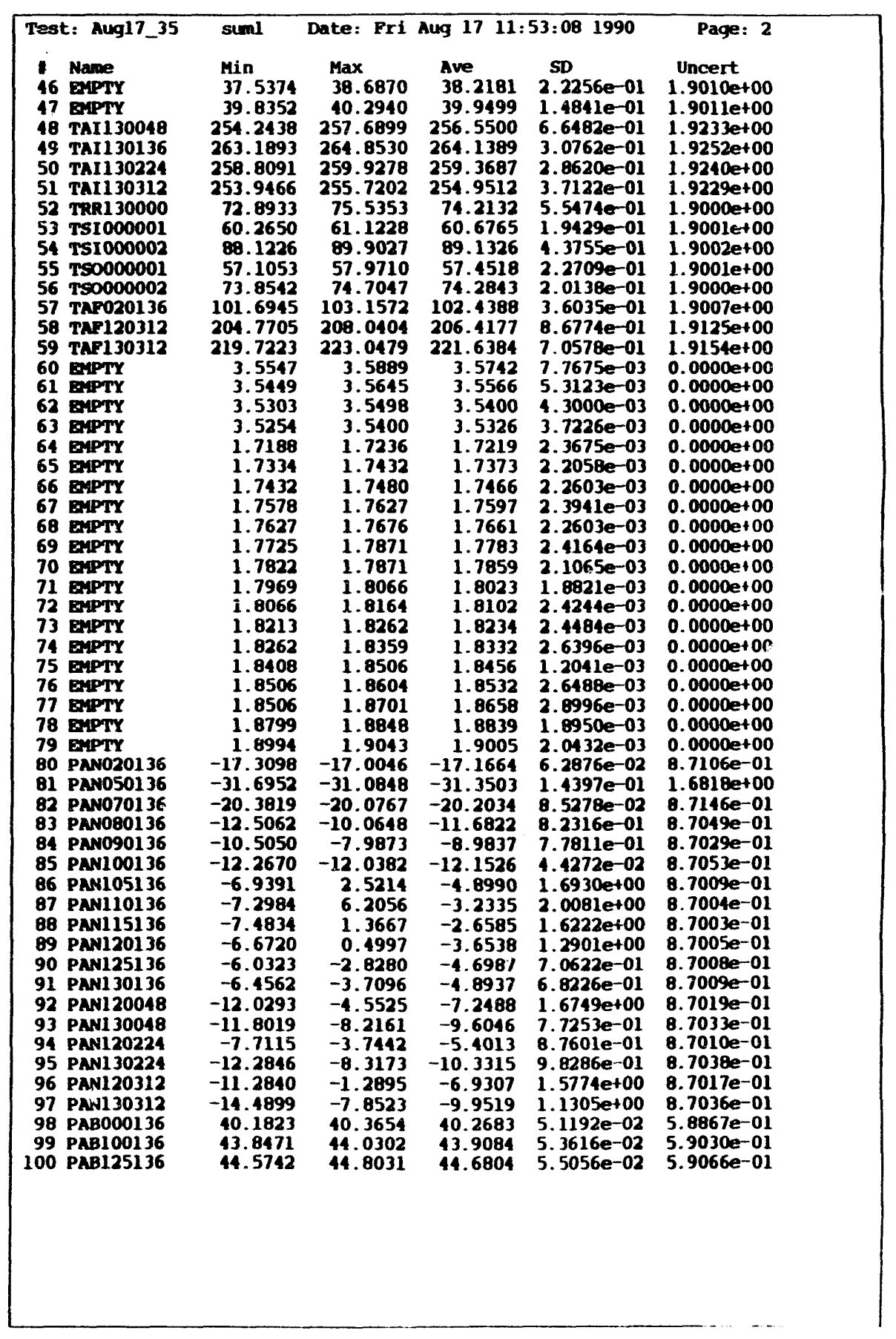



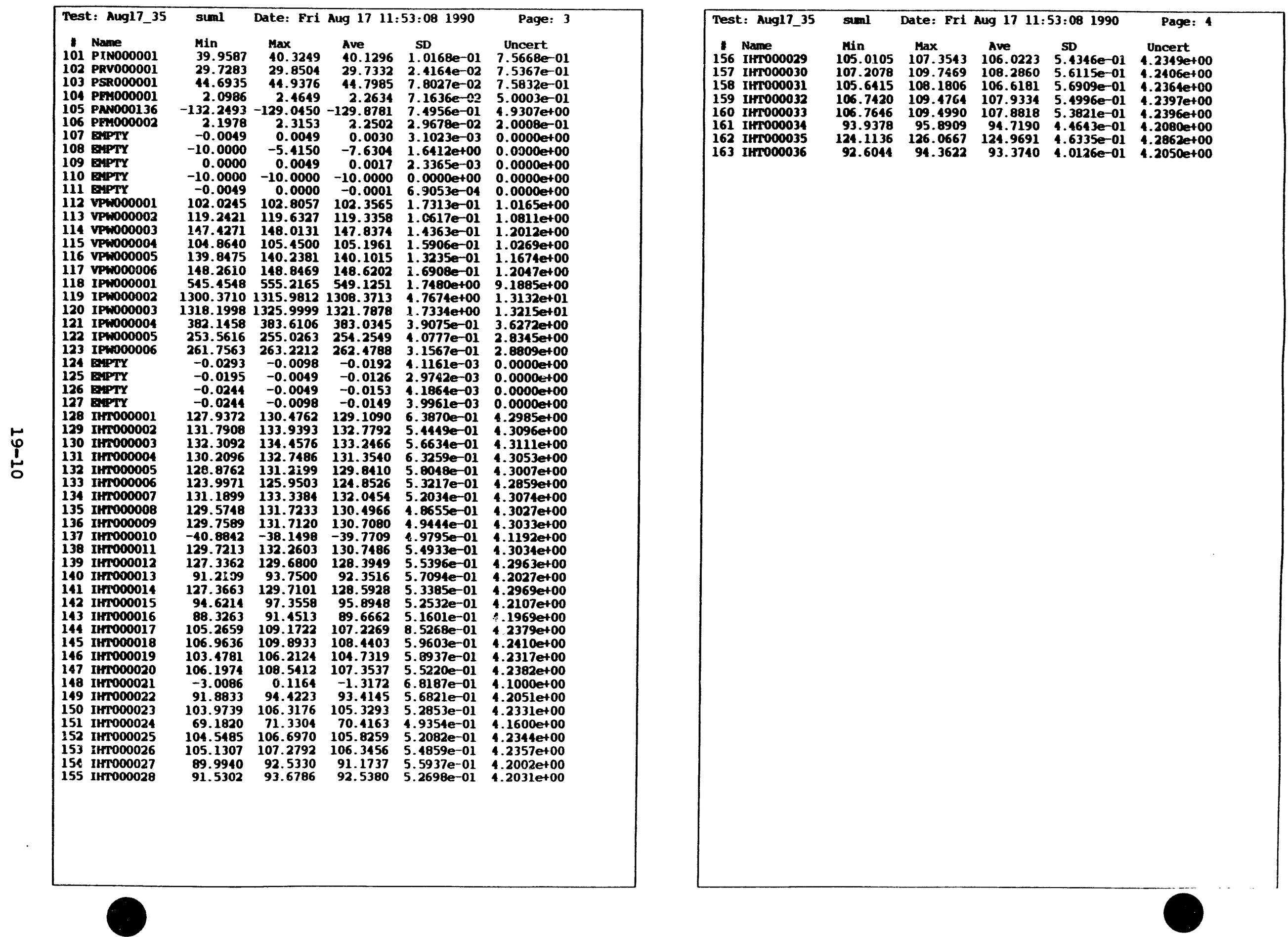


\begin{tabular}{|c|c|c|c|c|c|}
\hline \multicolumn{2}{|c|}{ 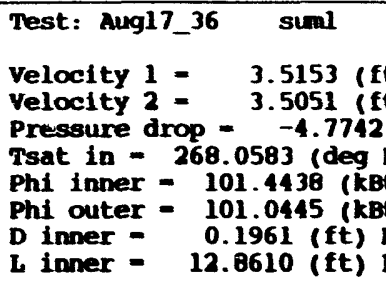 } & \multicolumn{3}{|c|}{ 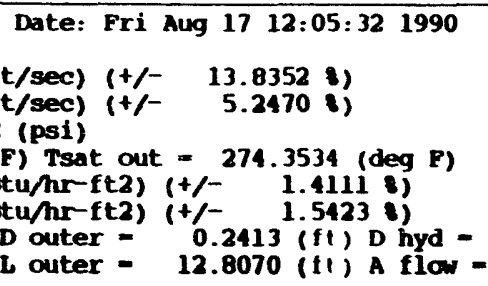 } & $\begin{array}{l}\text { Page: } 1 \\
\\
0.0390 \text { (ft) } \\
0.0150 \text { (ft2) }\end{array}$ \\
\hline 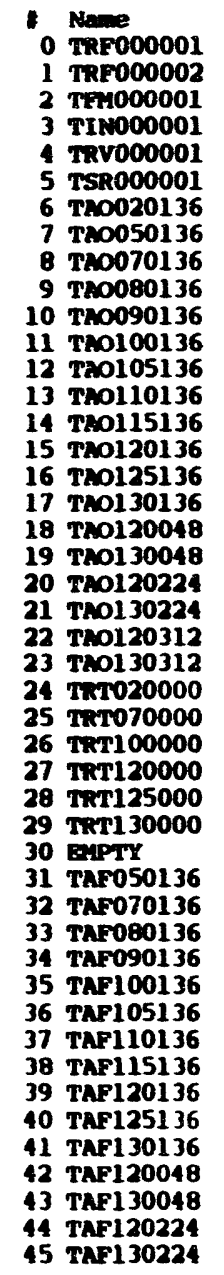 & $\begin{array}{l}\text { Min } \\
82.1279 \\
82.6820 \\
87.0169 \\
86.4697 \\
87.6389 \\
128.6000 \\
70.1975 \\
204.3795 \\
208.2370 \\
129.5619 \\
250.4957 \\
251.4223 \\
258.6251 \\
94.2955 \\
262.3725 \\
278.4310 \\
159.9583 \\
271.7984 \\
107.4445 \\
272.5444 \\
275.0500 \\
263.5083 \\
269.4081 \\
268.2634 \\
189.4030 \\
130.1372 \\
260.4529 \\
457.7478 \\
96.6833 \\
270.8881 \\
39.6057 \\
139.3634 \\
162.5510 \\
231.3158 \\
93.1837 \\
198.3584 \\
204.2182 \\
209.6344 \\
216.6943 \\
259.1794 \\
229.0038 \\
232.2455 \\
221.8449 \\
233.2047 \\
219.0532 \\
227.3032\end{array}$ & $\begin{array}{l}\text { Max } \\
82.9758 \\
83.1037 \\
87.9610 \\
87.3122 \\
88.3750 \\
130.6237 \\
71.7976 \\
206.0055 \\
212.5716 \\
130.9762 \\
251.4287 \\
253.7516 \\
260.2083 \\
95.1254 \\
264.2283 \\
279.3513 \\
235.0062 \\
273.4341 \\
108.5812 \\
273.3727 \\
276.3387 \\
264.7935 \\
270.7955 \\
269.1862 \\
191.1569 \\
131.5663 \\
261.6587 \\
158.1760 \\
97.4112 \\
271.6250 \\
40.2940 \\
140.8696 \\
165.7004 \\
246.5403 \\
94.3345 \\
202.2249 \\
208.4117 \\
214.5965 \\
219.4554 \\
261.0276 \\
231.2587 \\
235.0734 \\
224.7139 \\
234.9979 \\
221.7163 \\
229.3988\end{array}$ & $\begin{array}{l}\text { Ave } \\
82.5901 \\
82.8506 \\
87.5600 \\
86.8809 \\
87.8987 \\
129.6978 \\
70.9420 \\
205.3305 \\
209.9931 \\
130.2518 \\
250.9384 \\
252.4568 \\
259.3872 \\
94.7031 \\
263.2472 \\
278.8748 \\
167.5966 \\
272.8381 \\
108.0750 \\
273.0207 \\
275.7665 \\
264.0645 \\
270.1760 \\
268.7774 \\
190.3482 \\
130.9520 \\
261.0408 \\
458.0029 \\
97.0643 \\
271.2372 \\
39.8673 \\
140.0494 \\
163.9783 \\
238.5393 \\
93.7594 \\
199.8150 \\
205.8443 \\
211.1573 \\
217.7603 \\
260.0459 \\
230.1823 \\
233.6361 \\
223.1554 \\
234.2261 \\
220.5928 \\
228.2956\end{array}$ & $\begin{array}{l}\text { SD } \\
1.6964 e-01 \\
1.5359 e-01 \\
2.1369 e-01 \\
1.5376 e-01 \\
1.9285 e-01 \\
5.3961 e-01 \\
3.8936 e-01 \\
2.9359 e-01 \\
9.2456 e-01 \\
3.3774 e-01 \\
2.0891 e-01 \\
1.9739 e-01 \\
3.8714 e-01 \\
1.6852 e-01 \\
3.5081 e-01 \\
2.2284 e-01 \\
1.9065 e+01 \\
2.9278 e-01 \\
2.8317 e-01 \\
1.8375 e-01 \\
2.1201 e-01 \\
3.1409 e-01 \\
2.8836 e-01 \\
2.0074 e-01 \\
3.7045 e-01 \\
3.3924 e-01 \\
2.8857 e-01 \\
9.2220 e-02 \\
1.7507 e-01 \\
1.6161 e-01 \\
2.2239 e-01 \\
3.5475 e-01 \\
6.6981 e-01 \\
1.6959 e+00 \\
2.8639 e-01 \\
9.2563 e-01 \\
1.1326 e+00 \\
1.1453 e+00 \\
5.4506 e-01 \\
1.1622 e-01 \\
1.4950 e-01 \\
1.0244 e-01 \\
6.7671 e-01 \\
4.5405 e-01 \\
5.9462 e-01 \\
1.91332 e-01\end{array}$ & 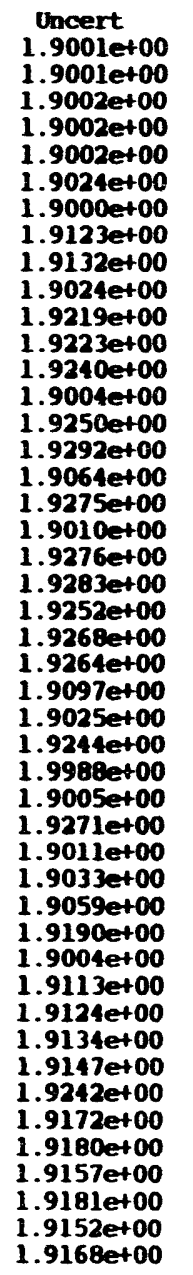 \\
\hline
\end{tabular}

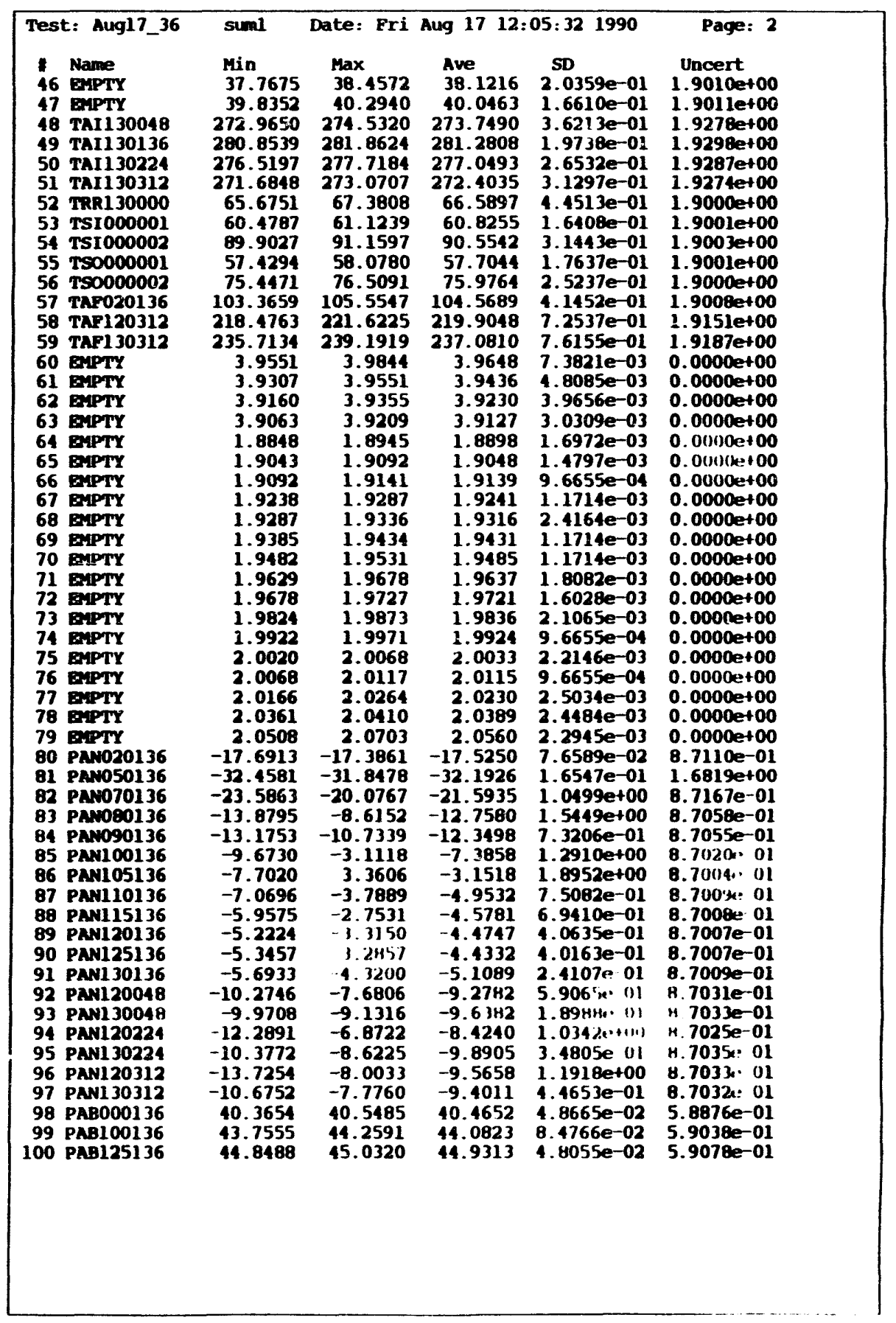




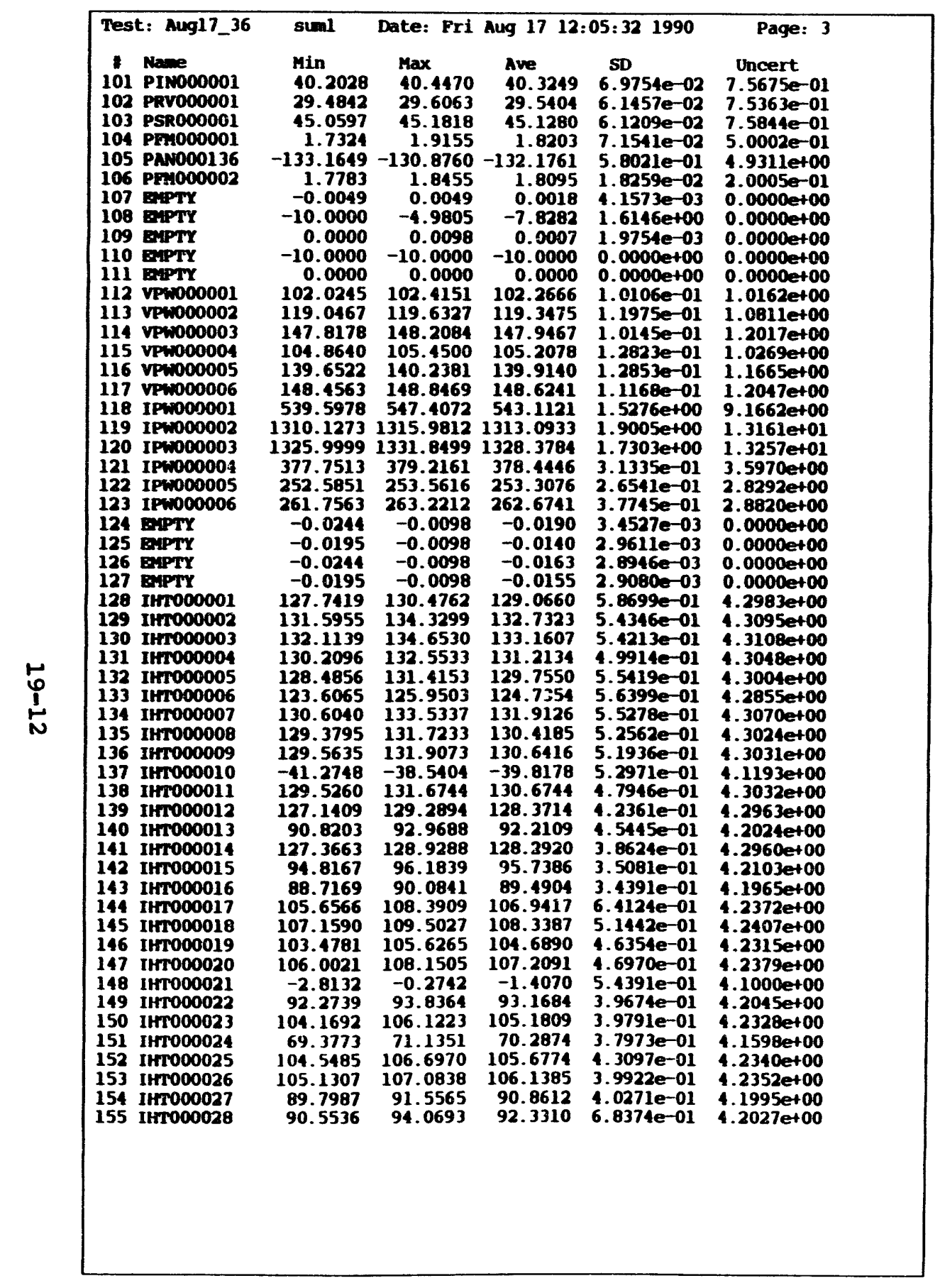

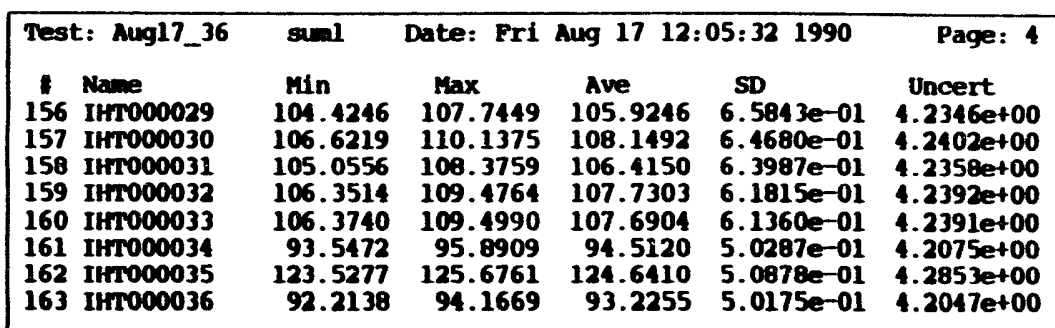




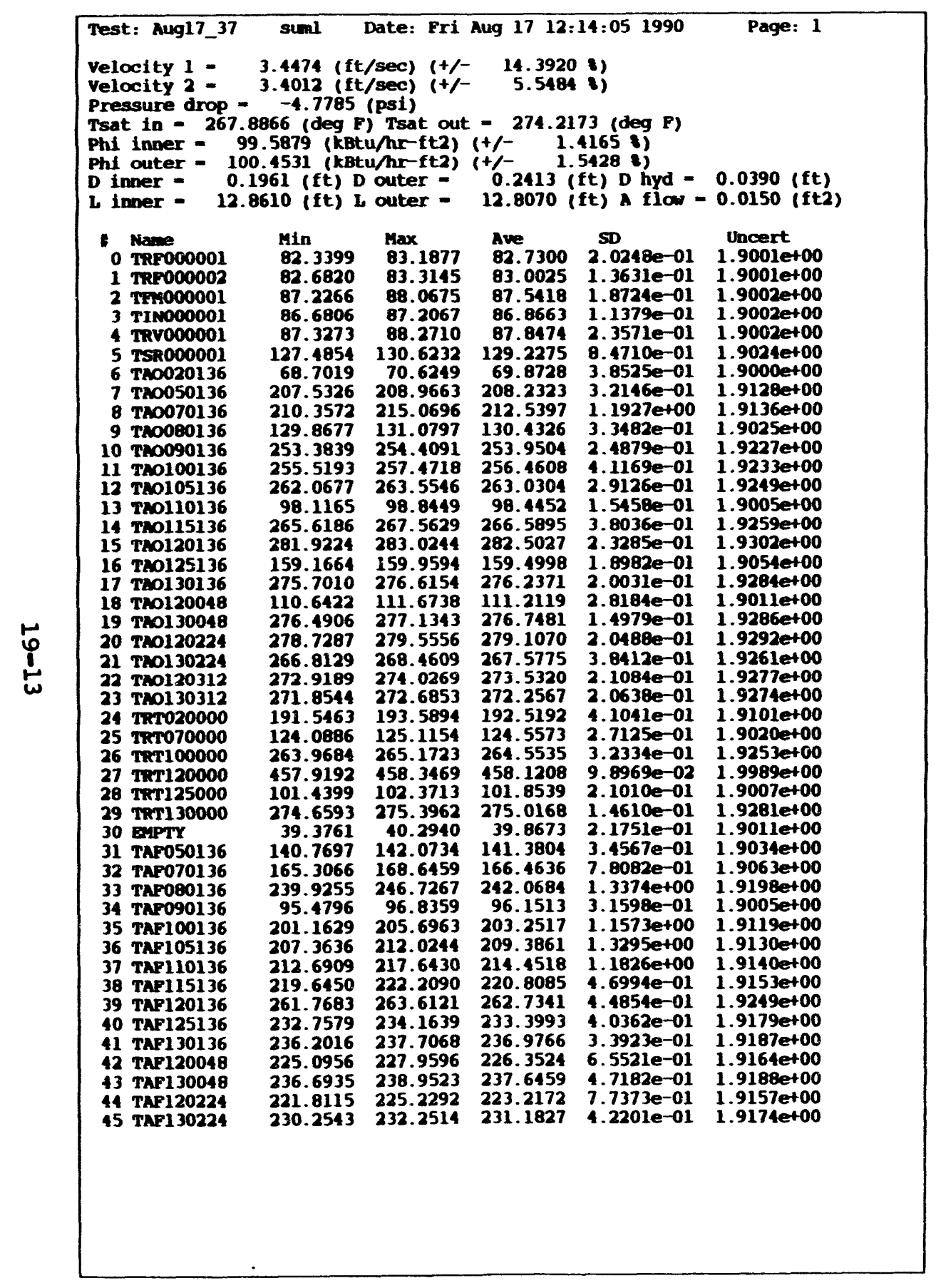

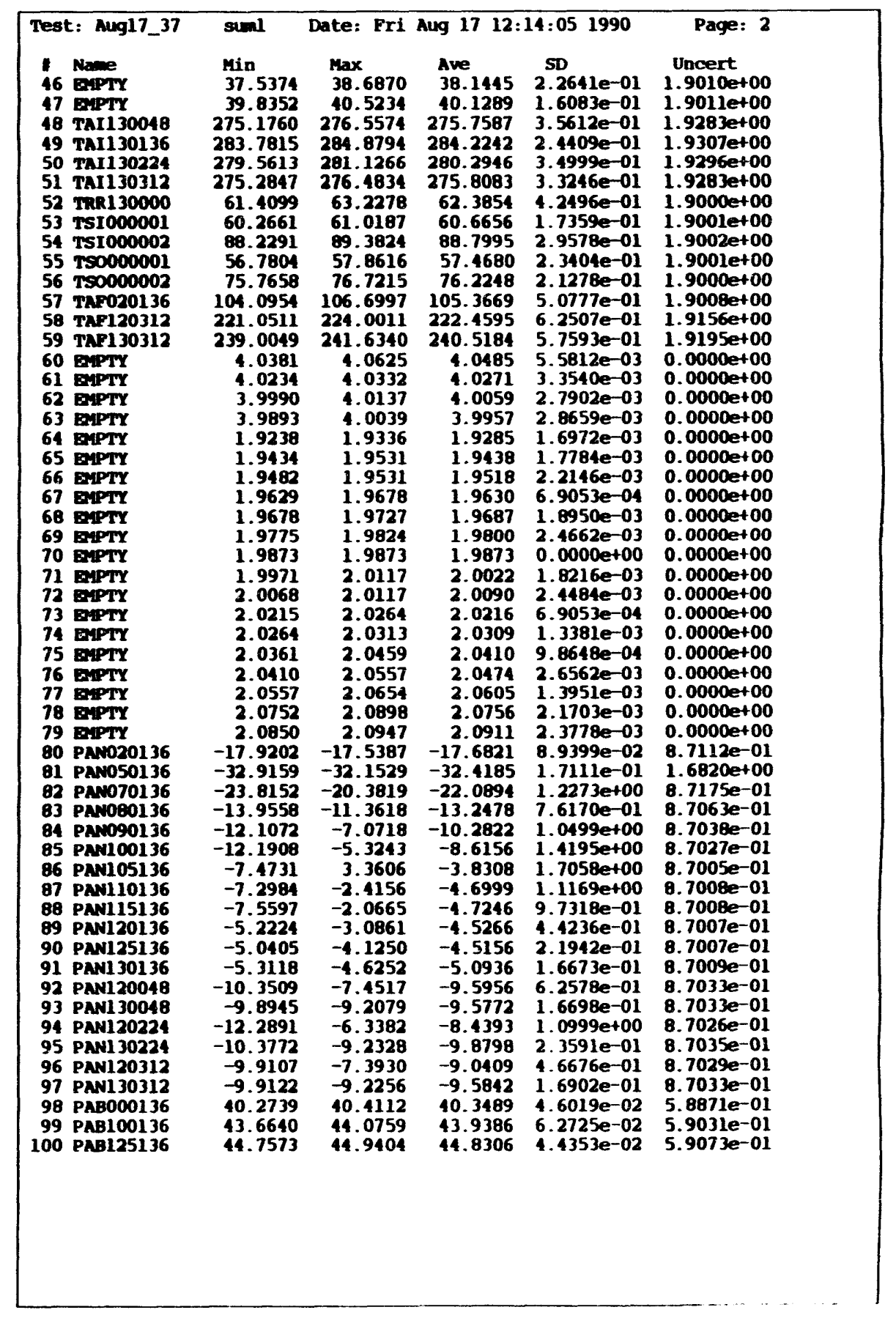



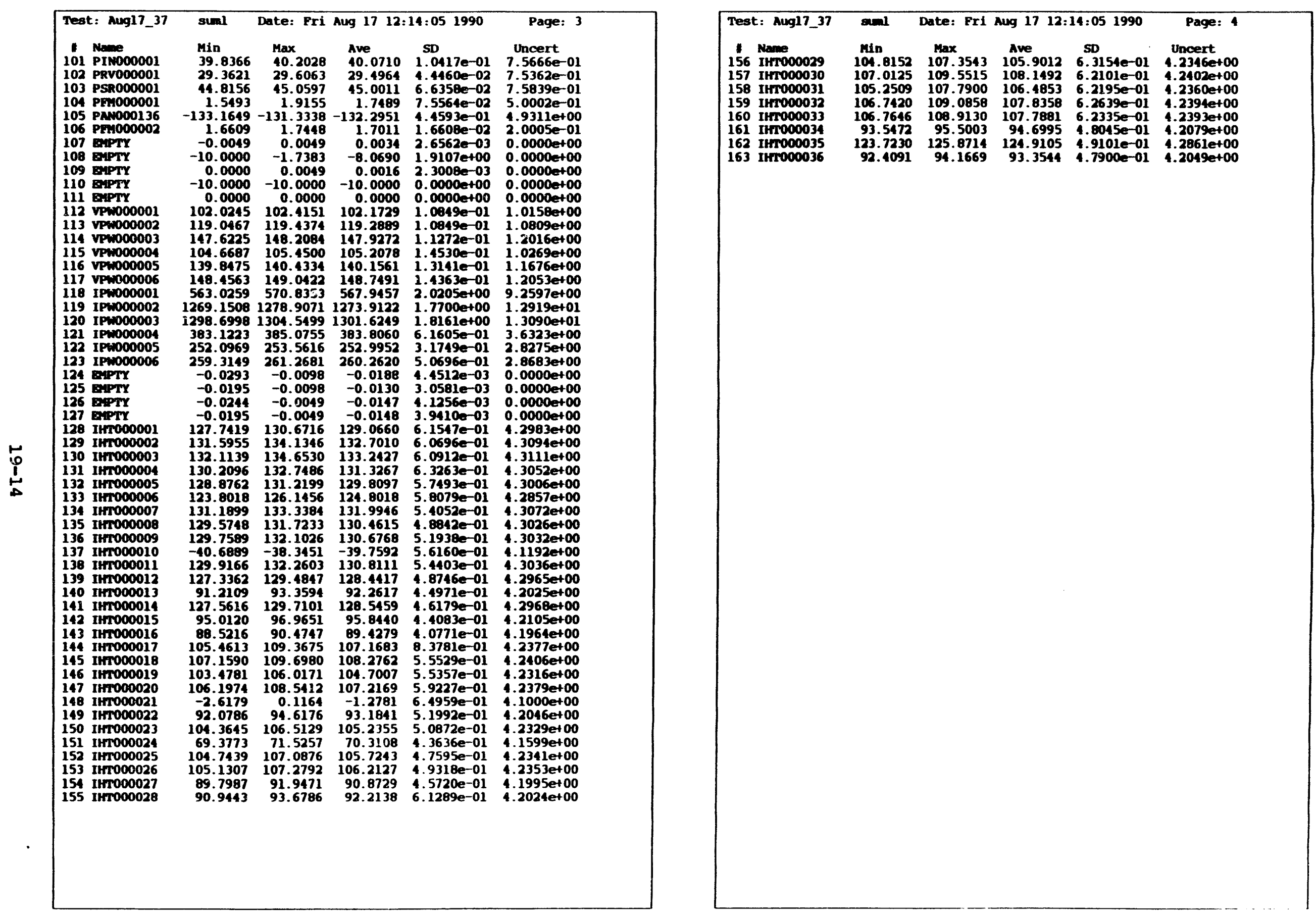
DEFECTIVE INSTRUMENTS FOR THE RIBBED GEOMETRY TEST SERIES

\begin{tabular}{|c|c|ll|}
\hline Series & \multicolumn{1}{|c|}{ Tests } & \multicolumn{1}{|c|}{ Defective Instruments } \\
\hline 3A & Aug17_32 to Aug17_37 & TAO120048 TAF070136 & TRT070000 \\
& & TAO020136 TAF080136 & TRT120000 \\
& & TAO070136 TAF090136 & TRT125000 \\
& & TAO080136 TAF120136 & TRR130000 \\
& & TAO110136 & \\
& & TAO125136 & \\
& & IHT000010 IHT000021 IHT000024 \\
& & IHT000033 & \\
\hline
\end{tabular}




\section{জ్reare}

TN-499

Chapter 20

PRESSURE DROP CURVES FOR NON-RIBBED AND RIBBED ANNULUS TEST SECTIONS 


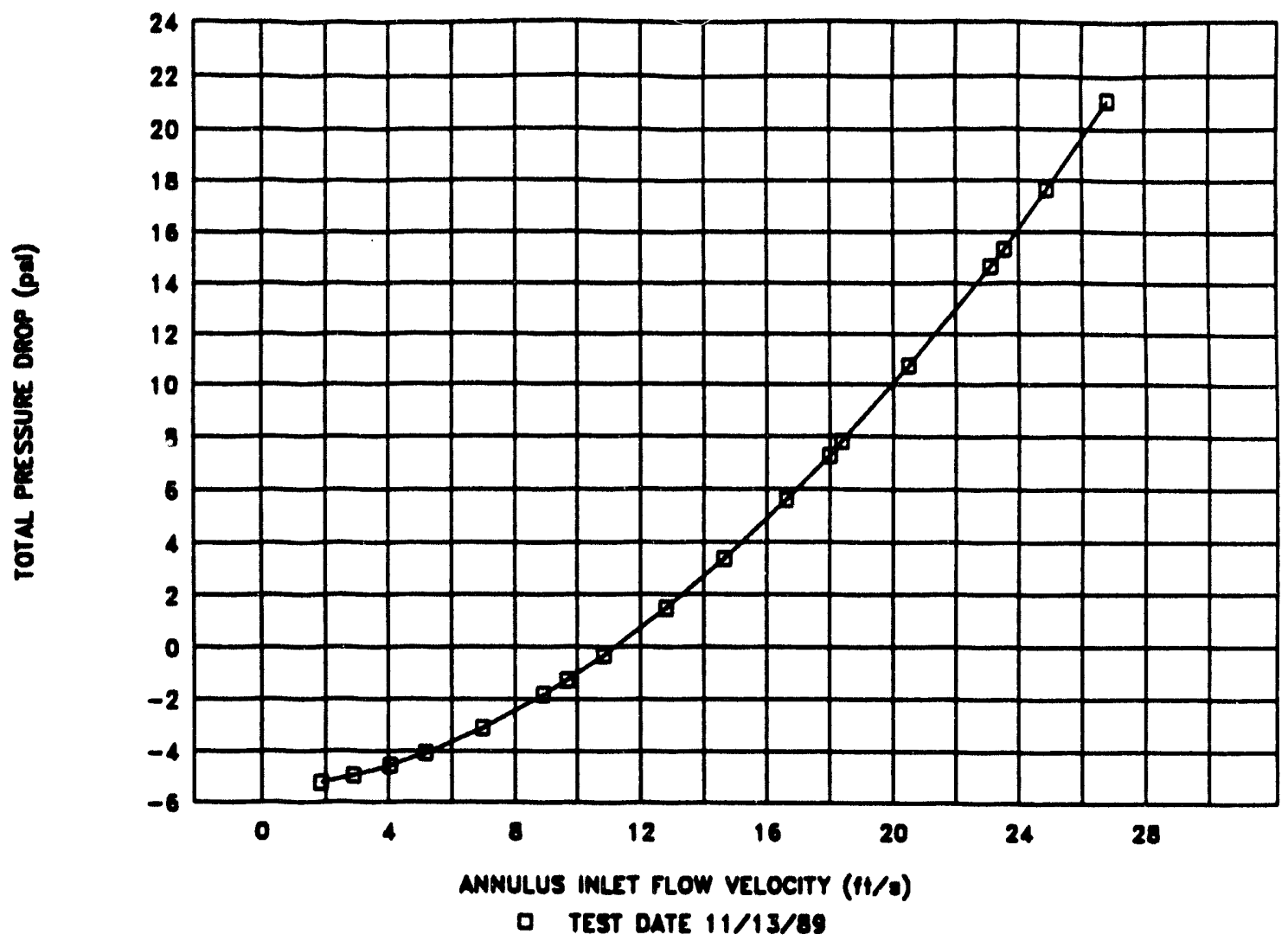

\begin{tabular}{|c|c|c|}
\hline $\begin{array}{c}\text { TEST } \\
\text { NUMBER }\end{array}$ & $\begin{array}{c}\text { INLET } \\
\text { VELOCITY(a) }\end{array}$ & $\begin{array}{c}\text { PRESSURE } \\
\text { DROP(b) }\end{array}$ \\
\hline & $(\mathrm{f} / \mathrm{s})$ & $(\mathrm{ps})$ \\
\hline Nov13_03 & 26.84 & 21.11 \\
Nov13_02 & 24.89 & 17.66 \\
Nov13_19 & 23.54 & 15.31 \\
Nov13_04 & 23.11 & 14.65 \\
Nov13_05 & 20.50 & 10.75 \\
Nov13_06 & 18.38 & 7.81 \\
Nov13_18 & 18.01 & 7.26 \\
Nov13_07 & 16.62 & 5.59 \\
Nov13_08 & 14.65 & 3.35 \\
Nov13_09 & 12.82 & 1.48 \\
Nov13_10 & 10.85 & -0.32 \\
Nov13_17 & 9.68 & -1.27 \\
Nov13_11 & 8.92 & -1.83 \\
Nov13_12 & 6.98 & -3.11 \\
Nov13_13 & 5.18 & -4.09 \\
Nov13_14 & 4.05 & -4.57 \\
Nov13_15 & 2.90 & -4.97 \\
Nov13_16 & 1.86 & -5.24 \\
\hline
\end{tabular}

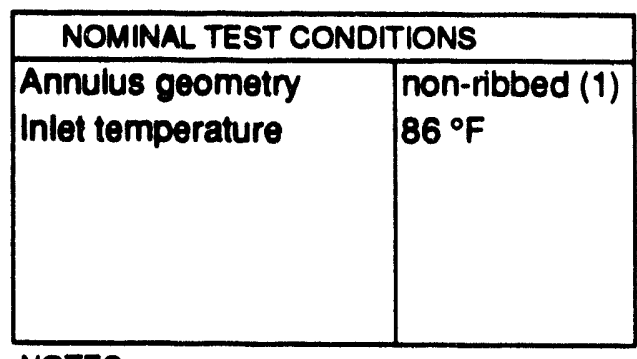

NOTES:
(a) Inlet veloci Jculated from PFM000001
(b) Prescure c jalculated from PANO00136 


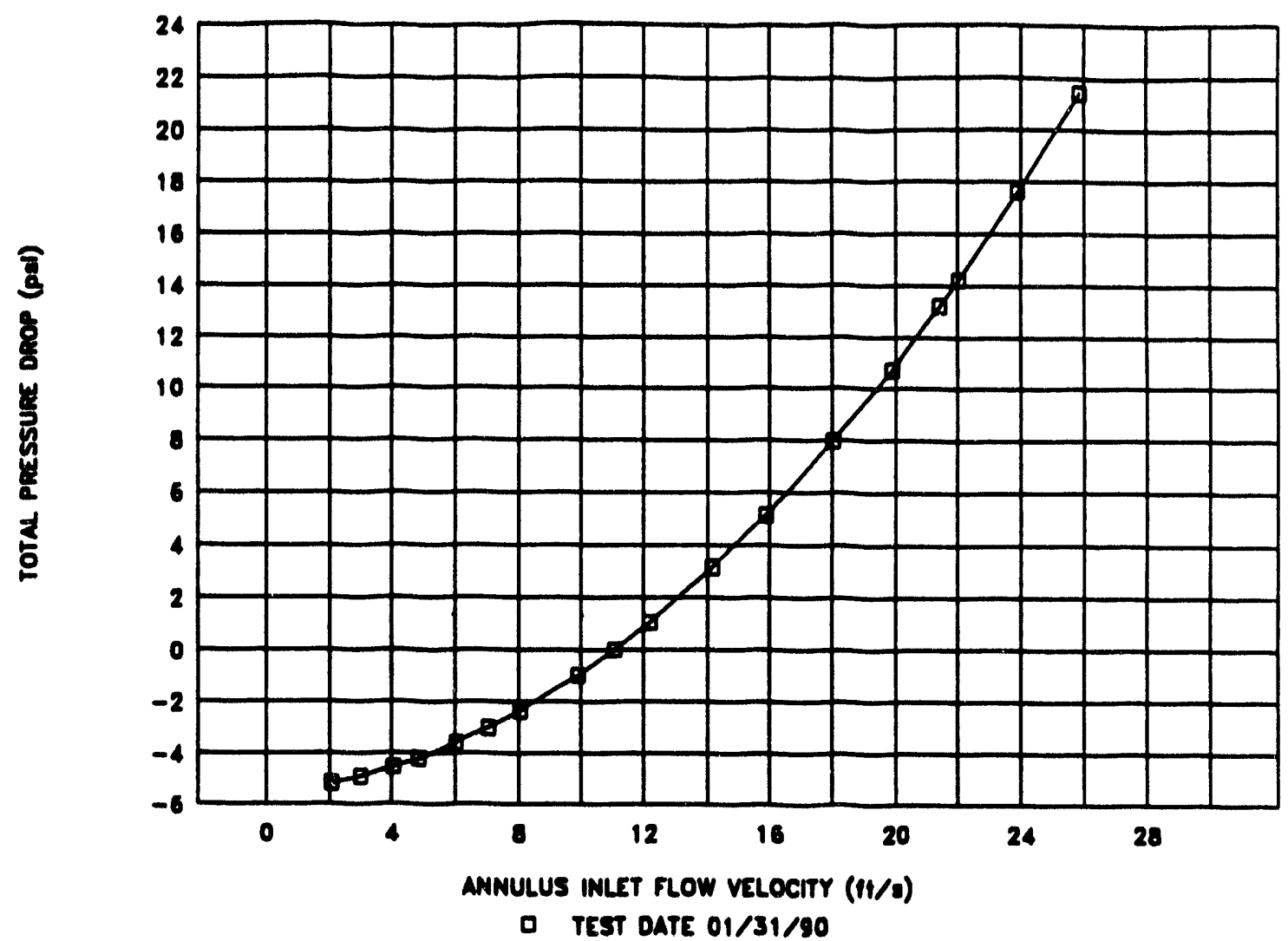

\begin{tabular}{|c|c|c|}
\hline $\begin{array}{c}\text { TEST } \\
\text { NUMBER }\end{array}$ & $\begin{array}{c}\text { INLET } \\
\text { VELOCITY(a) }\end{array}$ & $\begin{array}{c}\text { PRESSURE } \\
\text { DROP(b) }\end{array}$ \\
\hline & $(\mathrm{ft} / \mathbf{s})$ & $(\mathrm{P} 81)$ \\
\hline Jan31_01 & 25.86 & 21.44 \\
Jan31_02 & 23.89 & 17.62 \\
Jan31_03 & 22.01 & 14.22 \\
Jan31_15 & 21.42 & 13.22 \\
Jan31_04 & 19.89 & 10.72 \\
Jan31_05 & 18.03 & 7.96 \\
Jan31_06 & 15.89 & 5.12 \\
Jan31_07 & 14.17 & 3.15 \\
Jan31_08 & 12.20 & 1.05 \\
Jan31_14 & 11.06 & -0.01 \\
Jan31_09 & 9.91 & -0.99 \\
Jan31_10 & 8.06 & -2.37 \\
Jan31_16 & 7.07 & -3.03 \\
Jan31_11 & 6.03 & -3.61 \\
Jan31_17 & 4.84 & -4.23 \\
Jan31_12 & 4.04 & -4.56 \\
Jan31_18 & 3.02 & -4.92 \\
Jan31_13 & 2.06 & -5.19 \\
\hline
\end{tabular}

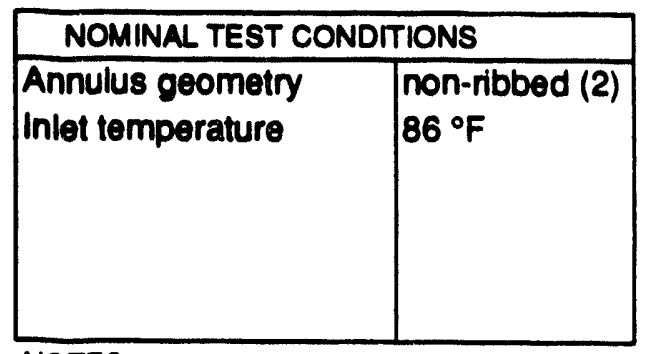

NOTES:

(a) Inlet velocity calculated from PFM000001

(b) Pressure drop calculated from PANO00136 


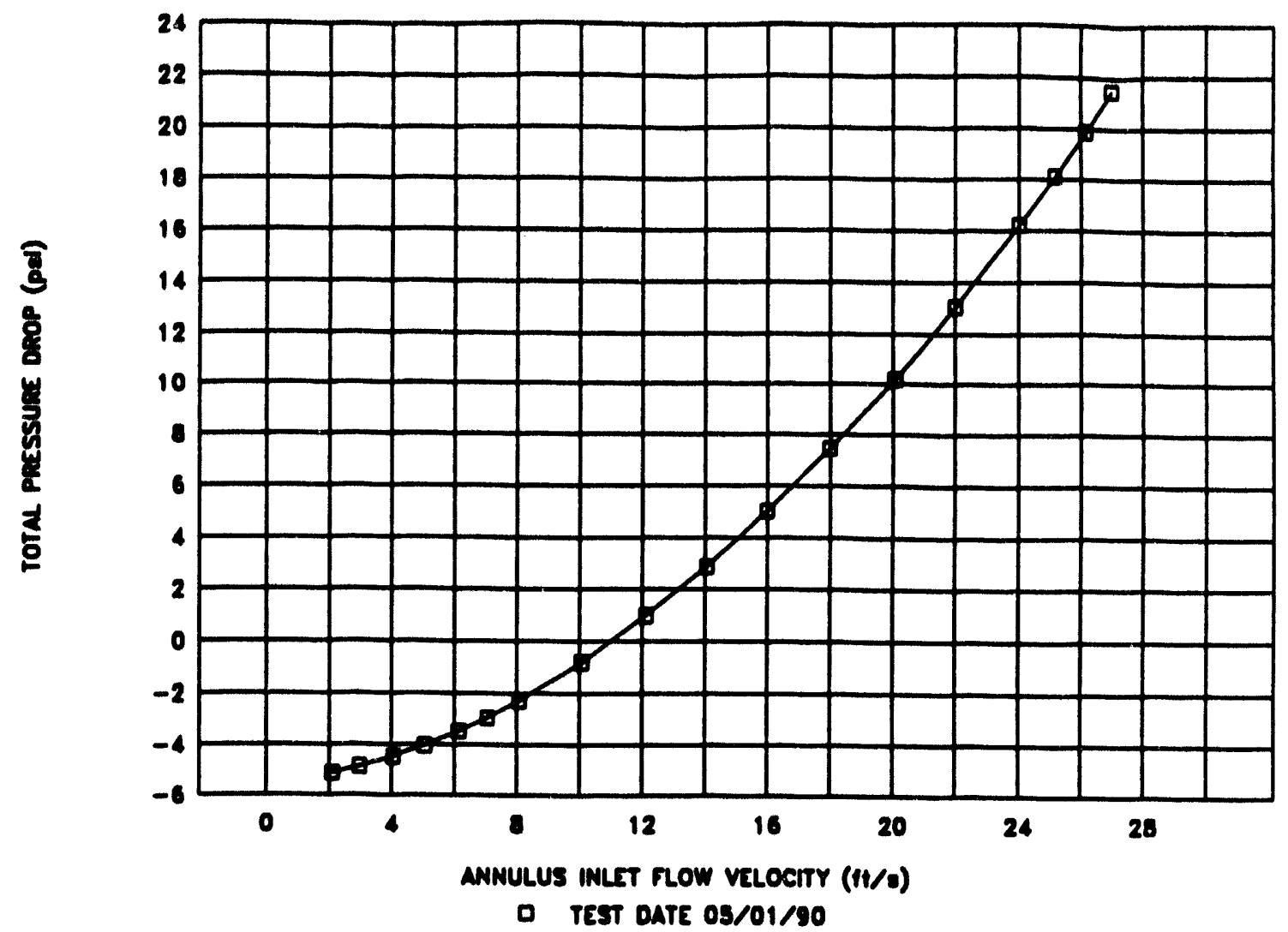

\begin{tabular}{|c|c|c|}
\hline $\begin{array}{c}\text { TEST } \\
\text { NUMBER }\end{array}$ & $\begin{array}{c}\text { INLET } \\
\text { VELOCITY(a) }\end{array}$ & $\begin{array}{c}\text { PRESSURE } \\
\text { DROP(b) }\end{array}$ \\
\hline & (tU/) & (psi) \\
\hline May01_01 & 27.00 & 21.47 \\
May01_02 & 26.16 & 19.86 \\
May01_18 & 25.17 & 18.10 \\
May01_03 & 24.01 & 16.22 \\
May01_04 & 22.00 & 13.04 \\
May01_05 & 20.07 & 10.19 \\
May01_06 & 17.98 & 7.45 \\
May01_07 & 15.97 & 5.00 \\
May01_08 & 14.06 & 2.86 \\
May01_09 & 12.12 & 0.98 \\
May01_10 & 10.06 & -0.81 \\
May01_11 & 8.10 & -2.28 \\
May01_17 & 7.05 & -2.96 \\
May01_12 & 6.14 & -3.49 \\
May01_16 & 5.05 & -4.05 \\
May01_13 & 4.04 & -4.49 \\
May01_14 & 2.96 & -4.88 \\
May01_15 & 2.10 & -5.13 \\
\hline
\end{tabular}

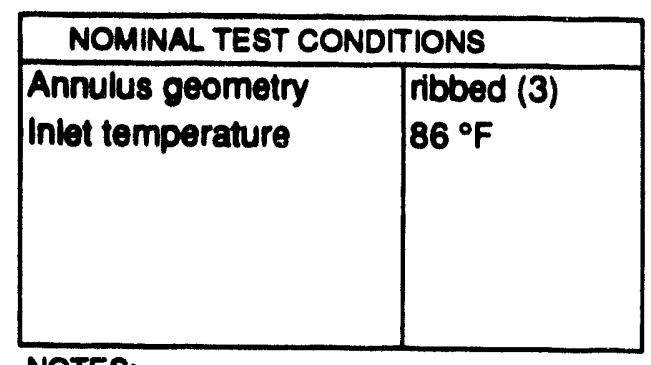

NOTES:

(a) Inlot volocity calculated from PFMO00001

(b) Pressure drop calculated from PANO00136 


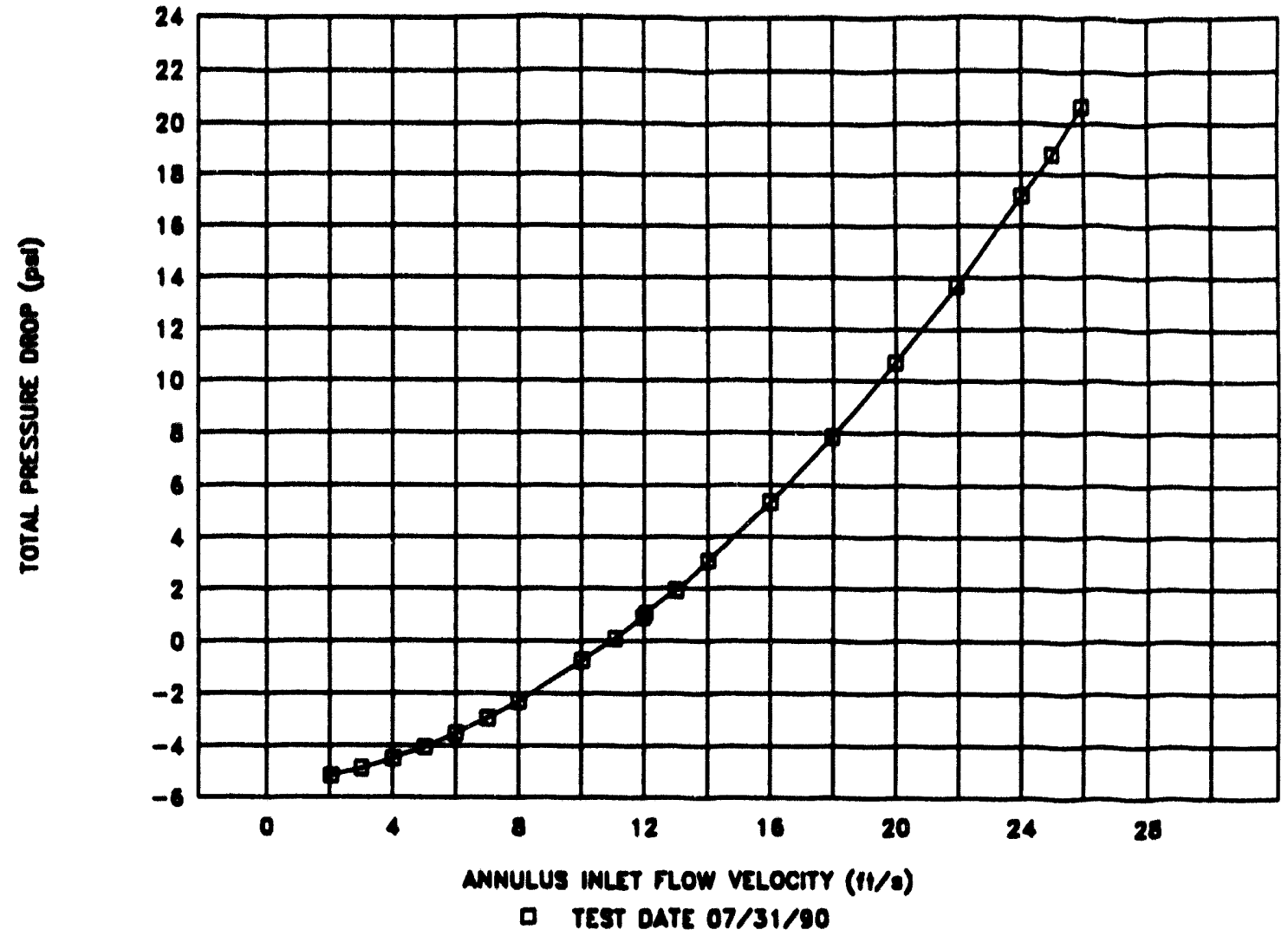

\begin{tabular}{|c|c|c|}
\hline $\begin{array}{c}\text { TEST } \\
\text { NUMBER }\end{array}$ & $\begin{array}{c}\text { INLET } \\
\text { VELOCIT(a) }\end{array}$ & $\begin{array}{c}\text { PRESSURE } \\
\text { DROP(b) }\end{array}$ \\
\hline & (tt/8) & (psi) \\
\hline Jul31_01 & 25.96 & 20.65 \\
Jul31_18 & 24.96 & 18.78 \\
Jul31_02 & 24.01 & 17.15 \\
Jul31_03 & 21.94 & 13.67 \\
Jul31_04 & 19.98 & 10.70 \\
Jul31_05 & 17.96 & 7.85 \\
Jul31_06 & 15.99 & 5.31 \\
Jul31_07 & 14.02 & 3.05 \\
Jul31_21 & 13.00 & 1.93 \\
Jul31_17 & 12.99 & 1.95 \\
Jul31_08 & 12.05 & 1.05 \\
Jul31_20 & 11.97 & 0.92 \\
Jul31_19 & 11.06 & 0.09 \\
Jul31_09 & 10.02 & -0.75 \\
Jul31_10 & 8.01 & -2.30 \\
Jul31_16 & 7.01 & -2.95 \\
Jul31_22 & 6.99 & -2.97 \\
Jul31_11 & 6.00 & -3.54 \\
Jul31_15 & 5.01 & -4.07 \\
Jul31_12 & 4.02 & -4.50 \\
Jul31_13 & 3.01 & -4.88 \\
Jul31_14 & 2.05 & -5.16 \\
\hline
\end{tabular}

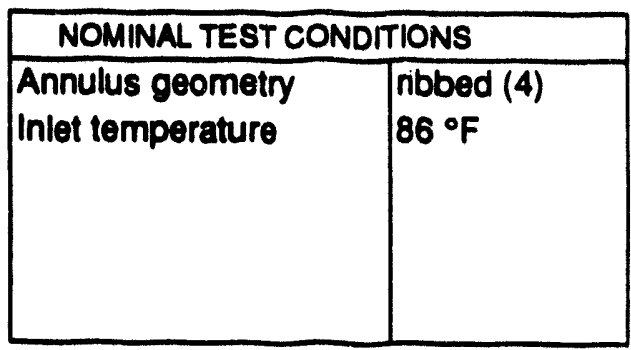

NOTES:

(a) Inlot veloolty calculated from PFMO00001

(b) Pressure drop calculated trom PAN000136

UNHEATED ANNULUS PRESSURE DROP - RIBBED GEOMETRY BUILD 4 


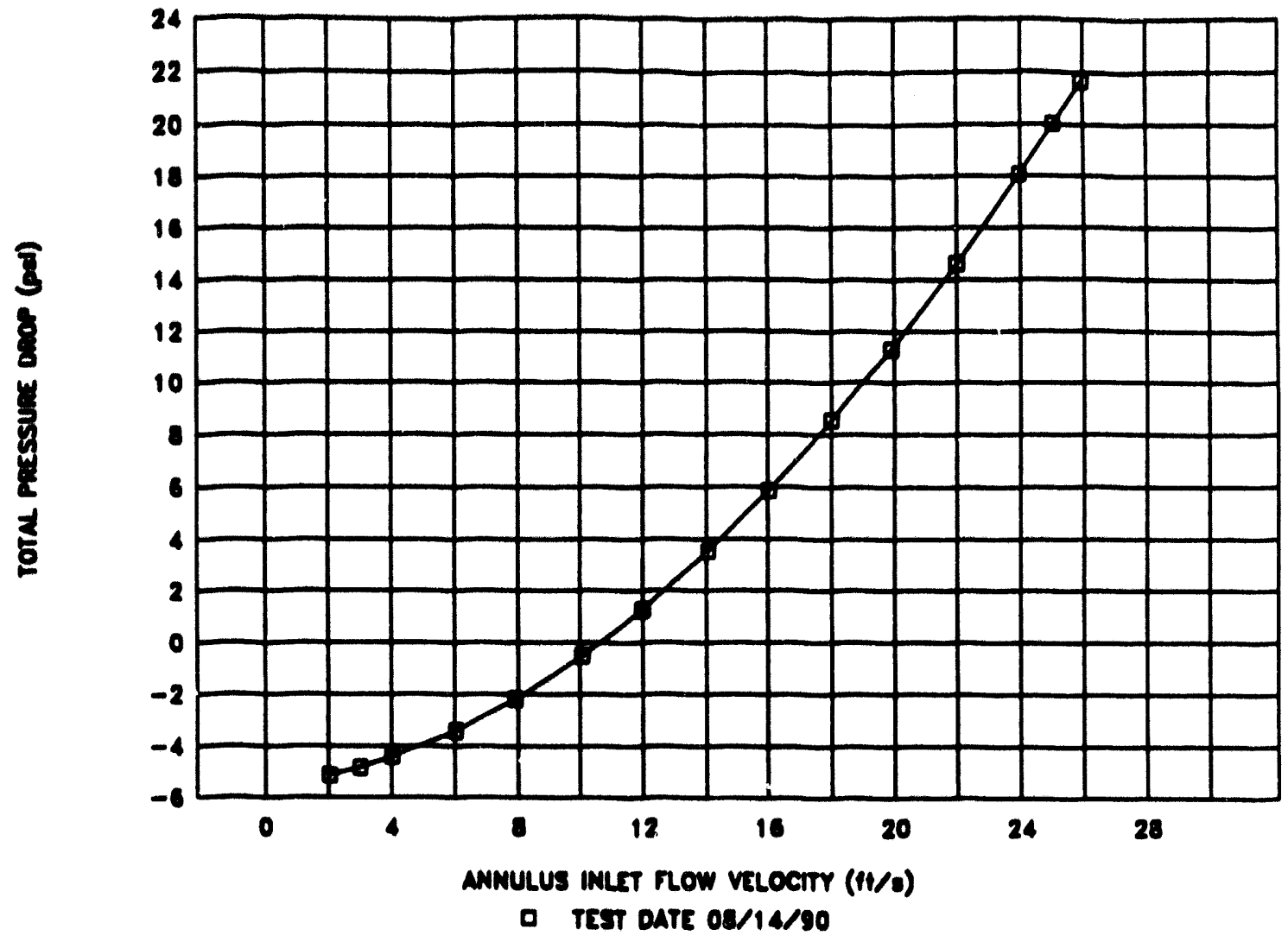

\begin{tabular}{|c|c|c|}
\hline $\begin{array}{c}\text { TEST } \\
\text { NUMBER }\end{array}$ & $\begin{array}{c}\text { INLET } \\
\text { VELOCTY(a) }\end{array}$ & $\begin{array}{c}\text { PRESSUAE } \\
\text { OROP(b) }\end{array}$ \\
\hline & $(\mathrm{H} / 8)$ & \multicolumn{1}{c|}{$(\mathrm{p}$ ( ) $)$} \\
\hline Aug14_01 & 25.95 & 21.68 \\
Aug14_16 & 25.03 & 20.02 \\
Aug14_02 & 23.96 & 18.06 \\
Aug14_03 & 21.99 & 14.62 \\
Aug14_04 & 19.91 & 11.31 \\
Aug14_05 & 18.00 & 8.56 \\
Aug14_06 & 16.02 & 5.87 \\
Aug14_07 & 14.06 & 3.52 \\
Aug14_09 & 12.02 & 1.29 \\
Aug14_08 & 11.97 & 1.25 \\
Aug14_10 & 10.06 & -0.52 \\
Aug14_11 & 7.93 & -2.19 \\
Aug14_12 & 6.02 & -3.43 \\
Aug14_13 & 4.01 & -4.42 \\
Aug14_14 & 2.99 & -4.84 \\
Aug14_15 & 2.04 & -5.13 \\
\hline
\end{tabular}

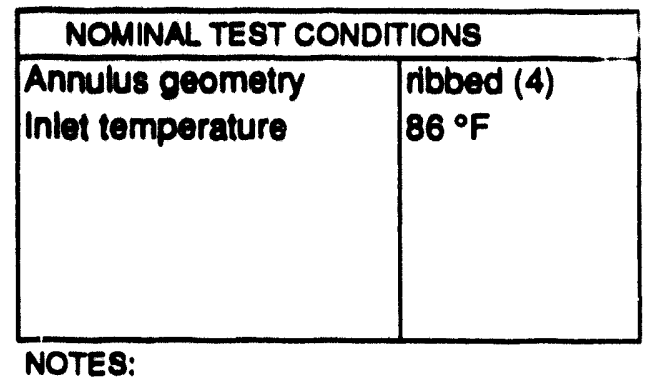
(a) Inlot velocity calculated from PFMO00001
(b) Pressure drop calculated from PANOOO136 


\begin{tabular}{|c|c|c|c|c|c|}
\hline \multicolumn{5}{|c|}{ 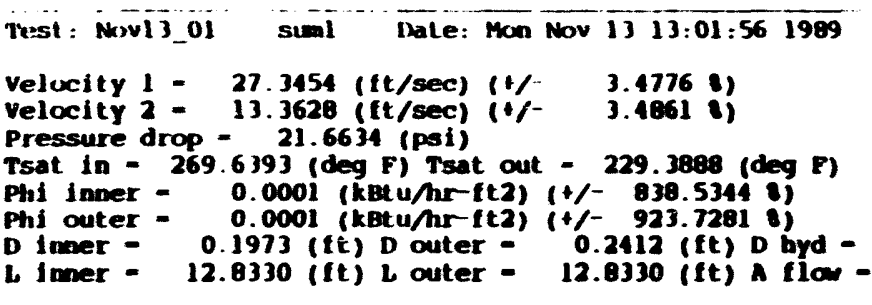 } & $\begin{array}{l}\text { Page: } 1 \\
0.0438 \text { (ft) } \\
0.0151 \text { (ft2) }\end{array}$ \\
\hline 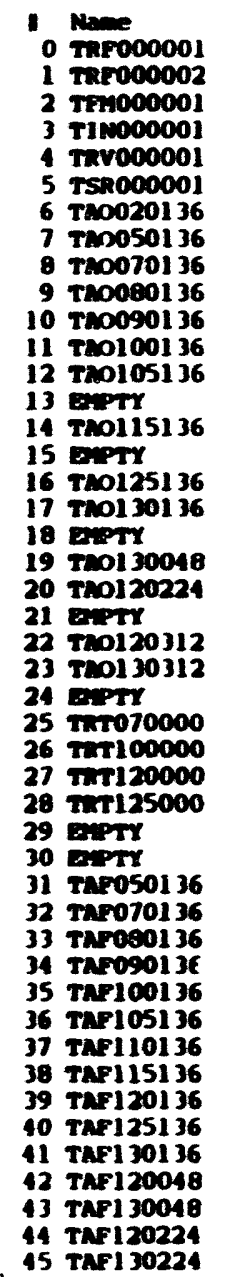 & 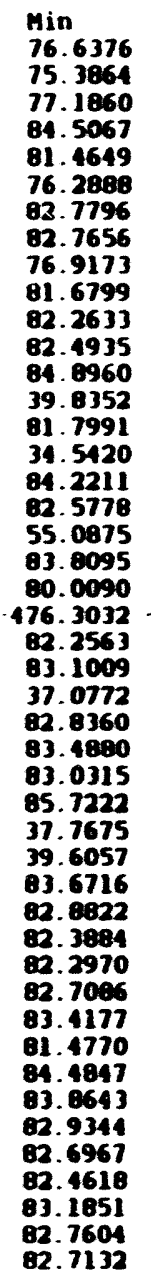 & 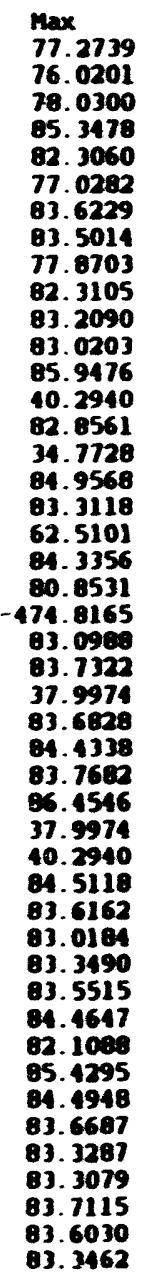 & 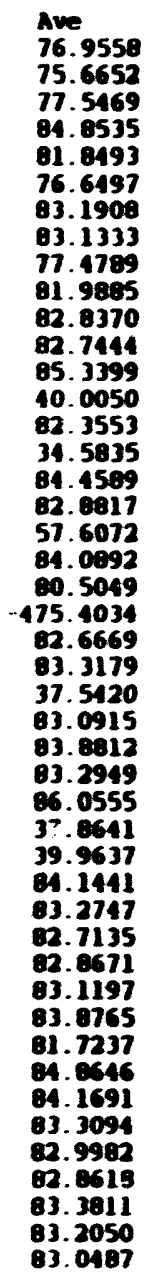 & 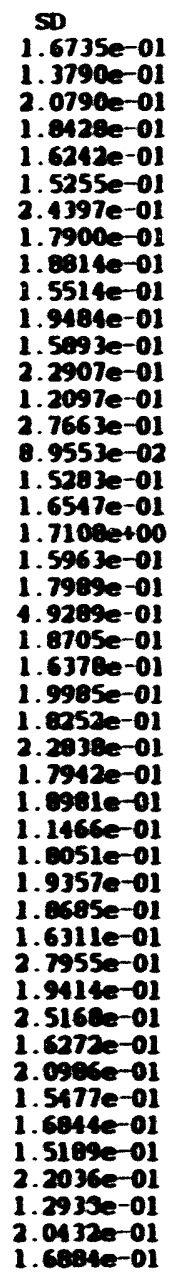 & 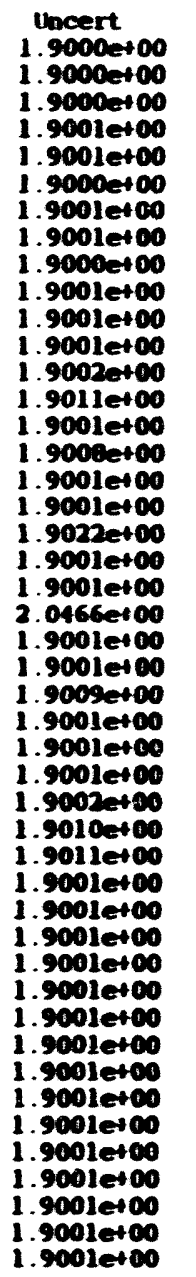 \\
\hline
\end{tabular}

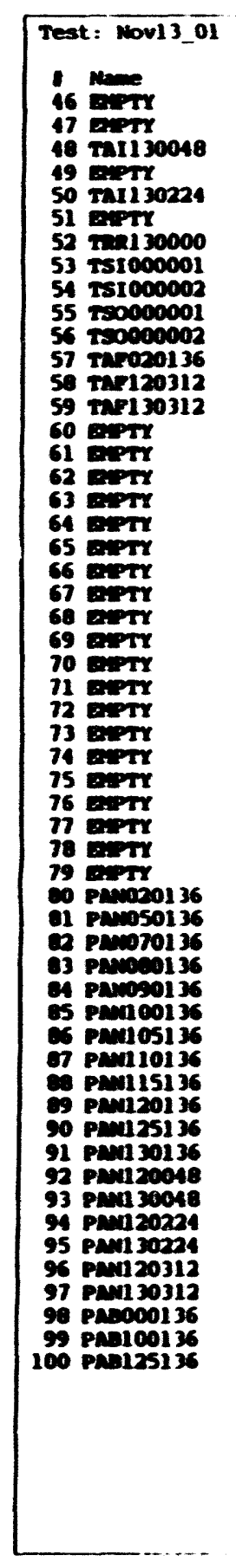

\begin{tabular}{|c|c|c|c|c|}
\hline & Daté & & 194 & me \\
\hline 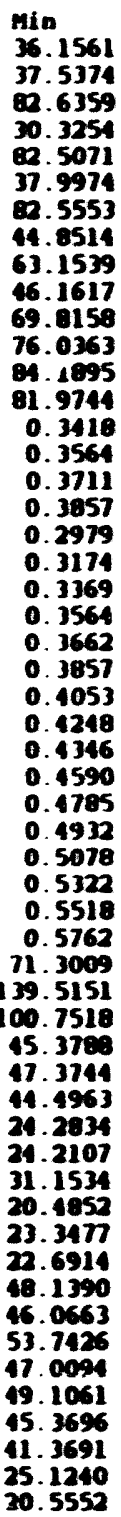 & 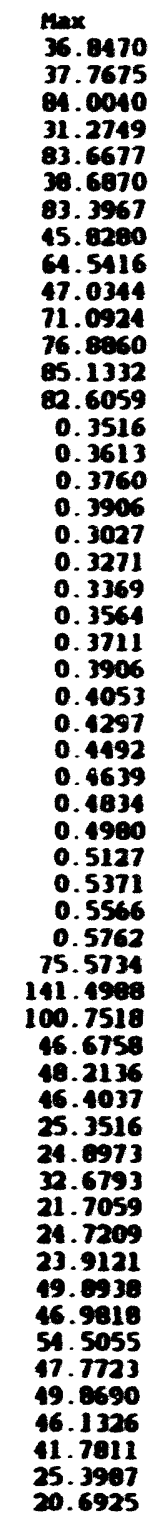 & 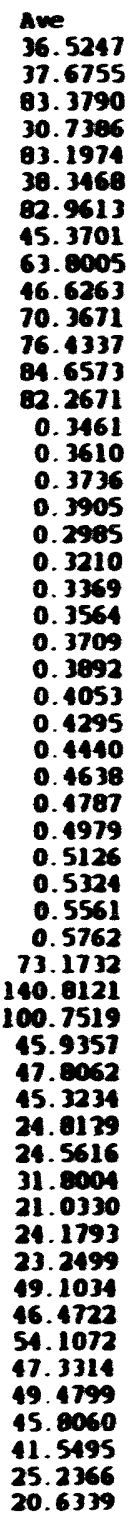 & 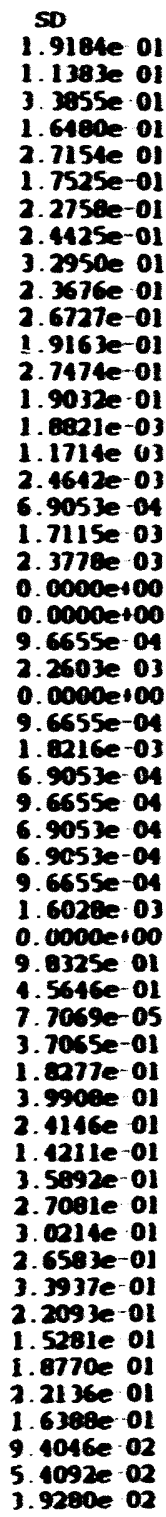 & 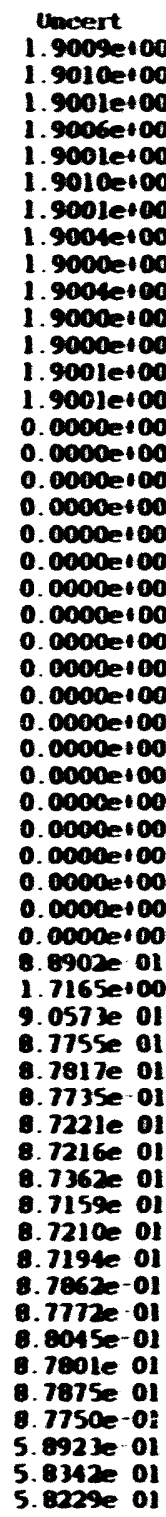 \\
\hline
\end{tabular}



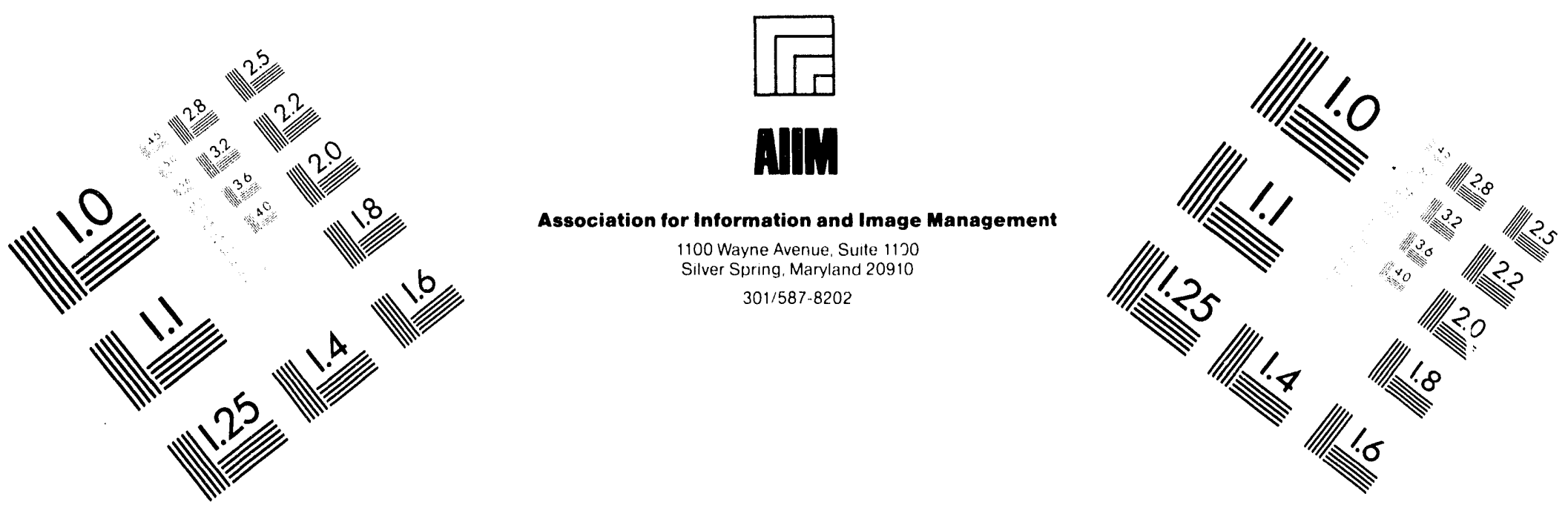

\section{Centimeter}

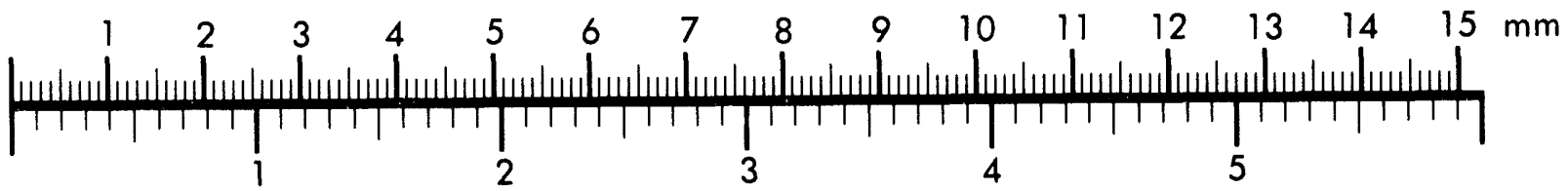

Inches
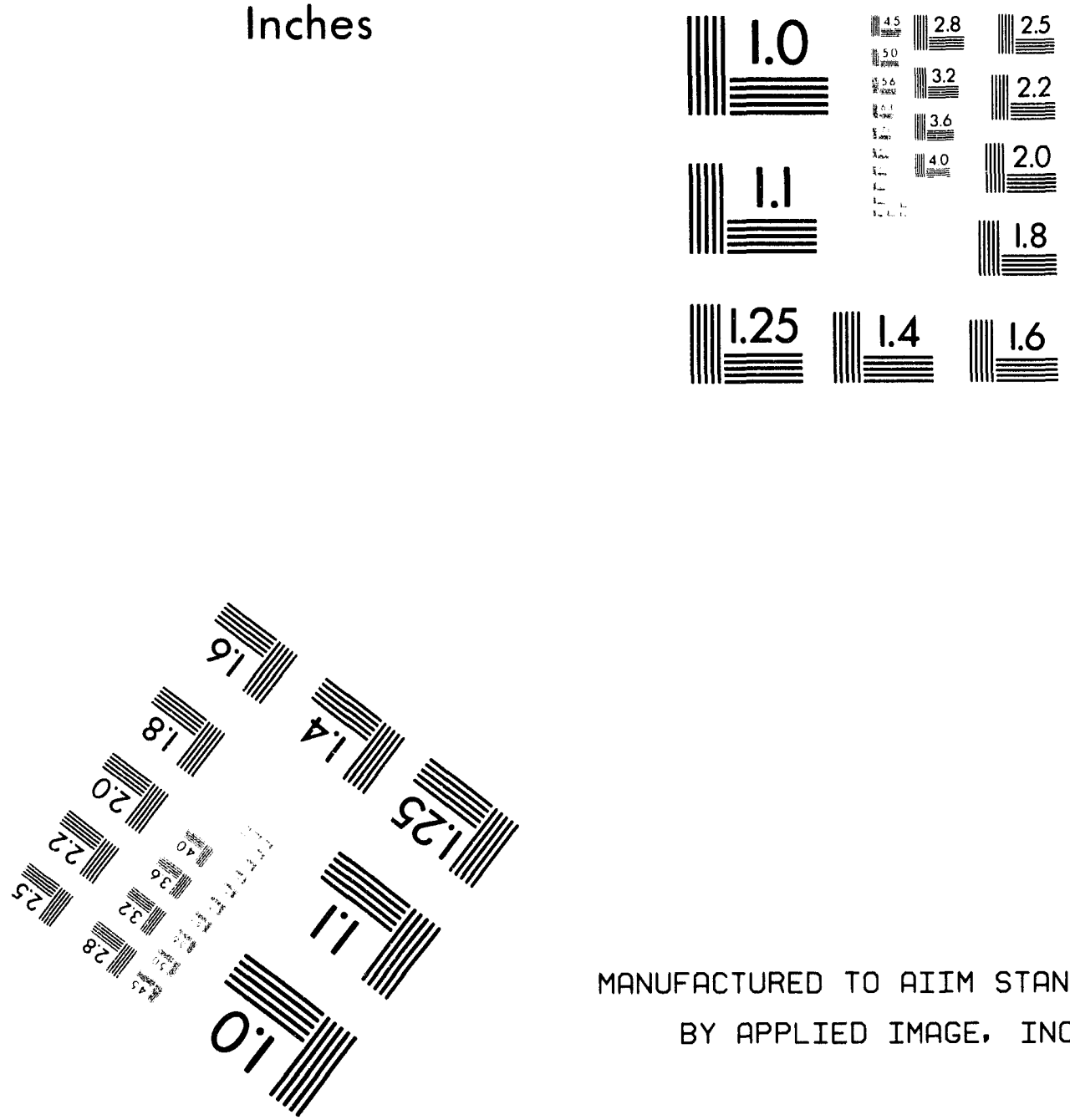

MANUFACTURED TO AIIM STANDARDS

BY APPLIED IMAGE, INC.

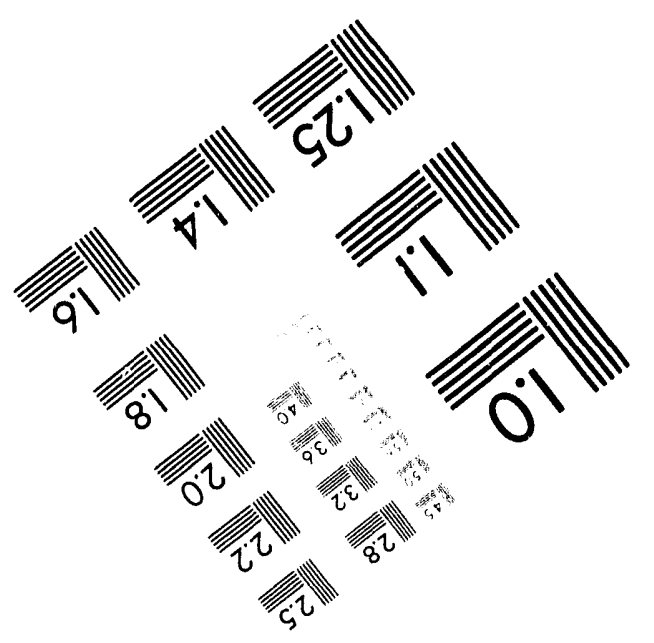



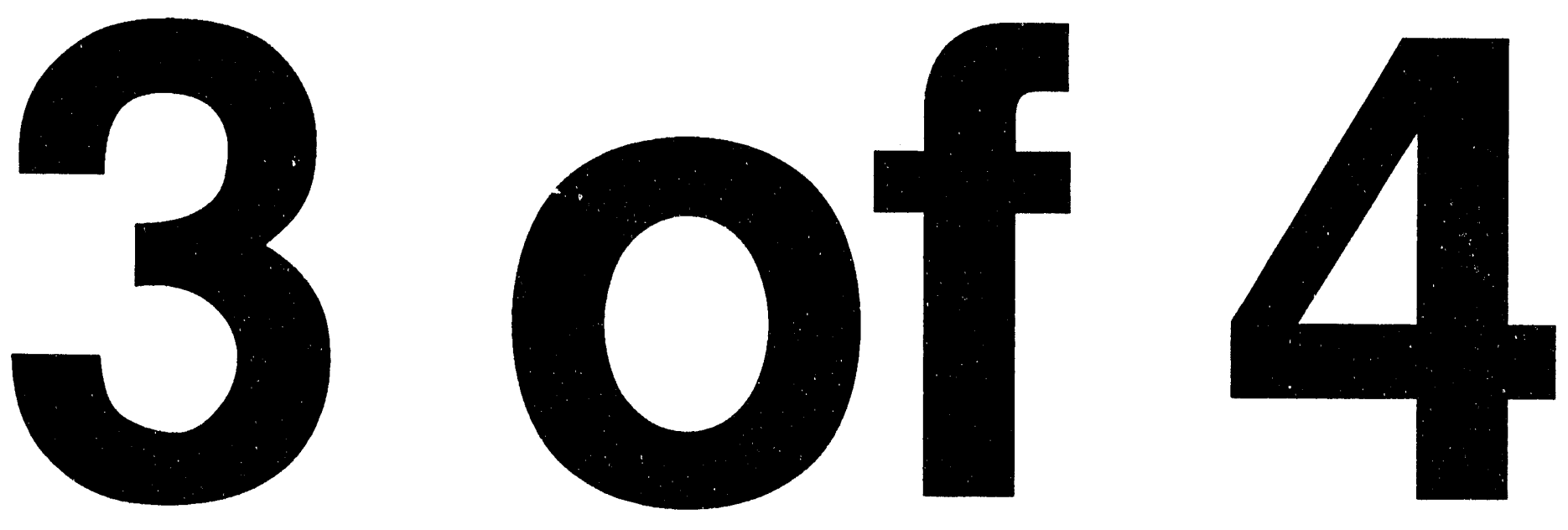


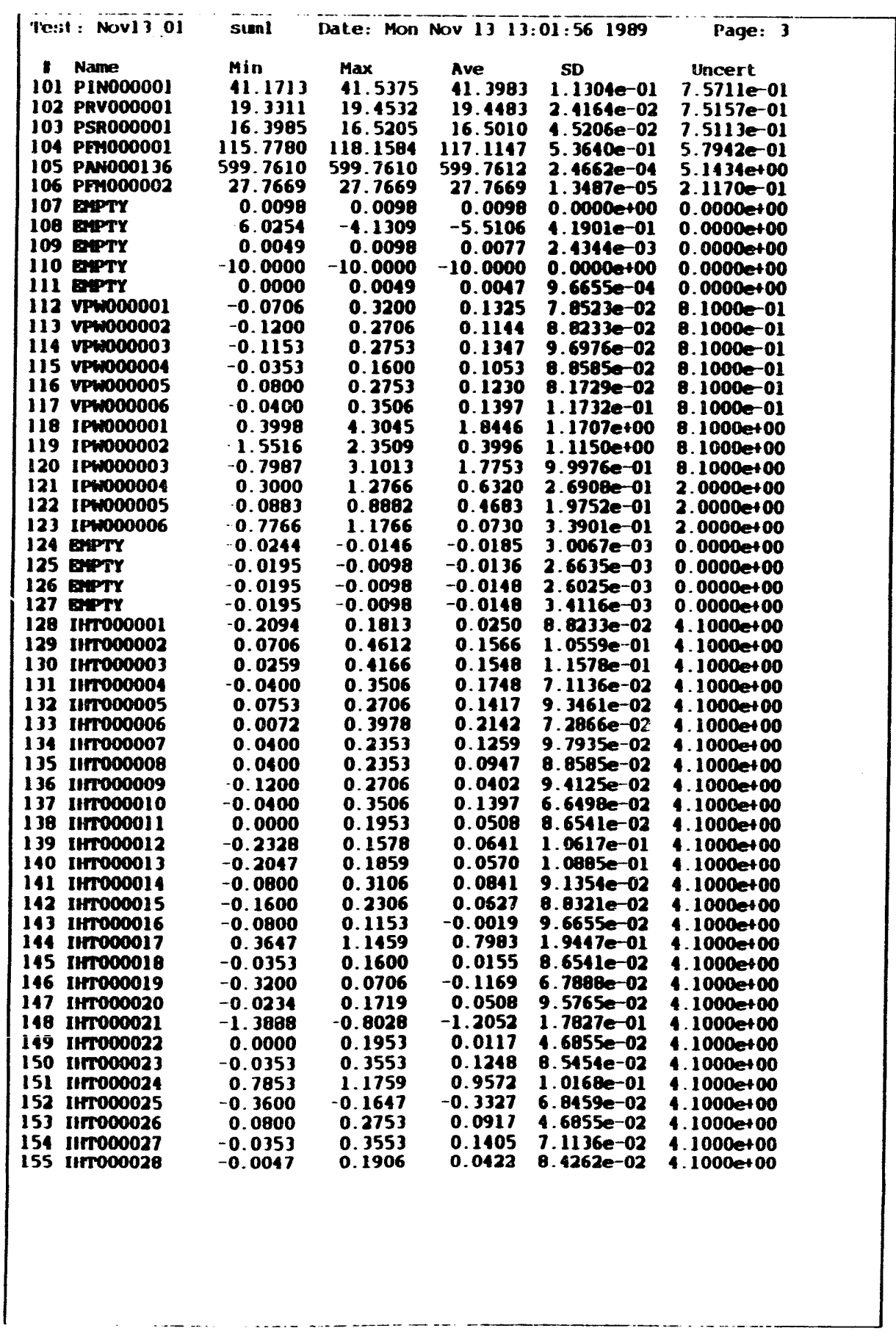

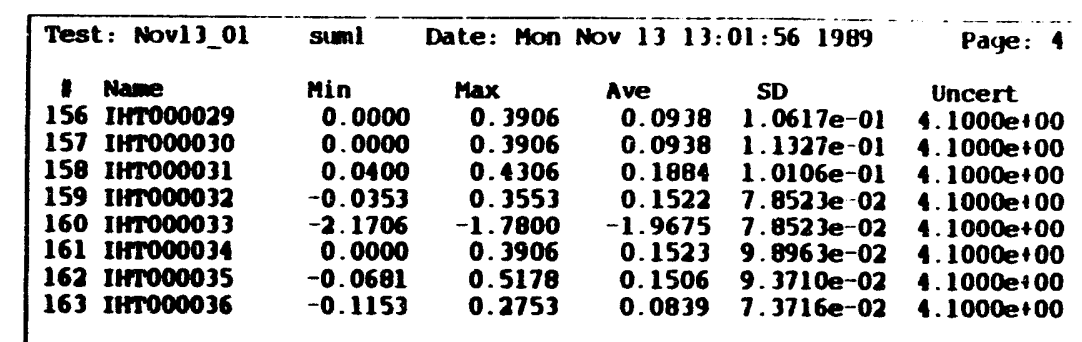




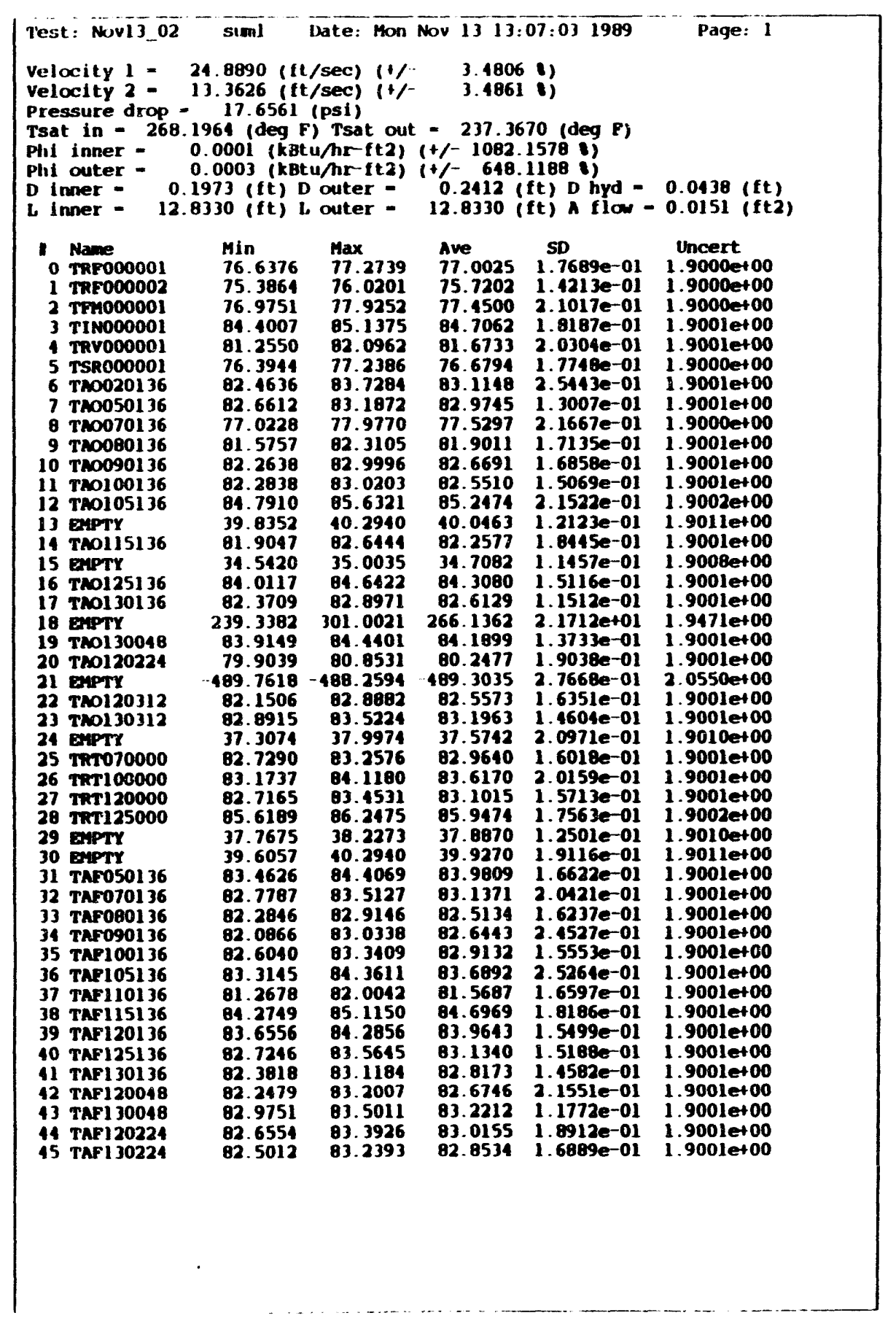

\begin{tabular}{|c|c|c|c|c|c|}
\hline 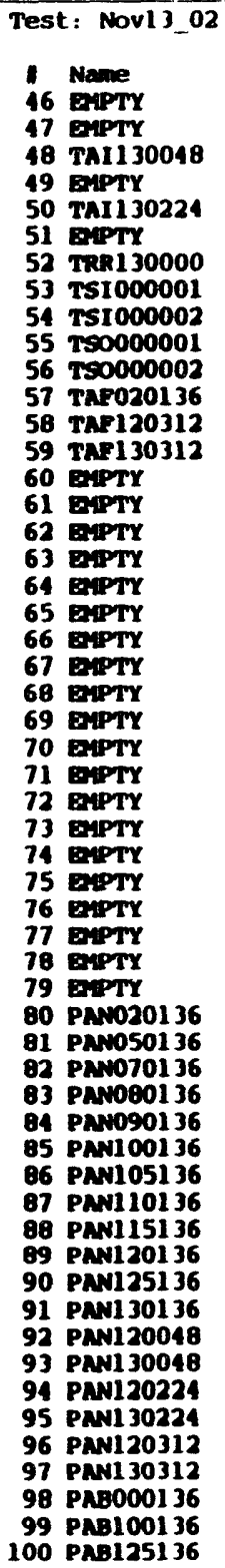 & $\begin{array}{l}\text { suml } \\
\text { Min } \\
36.1561 \\
37.5374 \\
82.7409 \\
30.3254 \\
82.4015 \\
37.9974 \\
82.1358 \\
15.6104 \\
63.5804 \\
16.7072 \\
69.6031 \\
76.1424 \\
83.9799 \\
81.7657 \\
0.3369 \\
0.3516 \\
0.3613 \\
0.3857 \\
0.2930 \\
0.3174 \\
0.3320 \\
0.3516 \\
0.3662 \\
0.3857 \\
0.4004 \\
0.4248 \\
0.4395 \\
0.4590 \\
0.4736 \\
0.4932 \\
0.5078 \\
0.5322 \\
0.5518 \\
0.5762 \\
57.6443 \\
11.7441 \\
82.5176 \\
36.7576 \\
37.7614 \\
35.8751 \\
19.1718 \\
19.2516 \\
25.4314 \\
16.1365 \\
19.6093 \\
18.0375 \\
38.4497 \\
36.9873 \\
43.4429 \\
37.7015 \\
39.4930 \\
36.4432 \\
40.3621 \\
27.4128 \\
23.8053\end{array}$ & $\begin{array}{l}\text { Date: Mon } \\
\text { Max } \\
37.0772 \\
37.7675 \\
83.8990 \\
31.0376 \\
83.5621 \\
38.6870 \\
83.1868 \\
16.3695 \\
64.8606 \\
17.7957 \\
70.5612 \\
76.8860 \\
85.0291 \\
82.3963 \\
0.3418 \\
0.3564 \\
0.3711 \\
0.3857 \\
0.2979 \\
0.3223 \\
0.3369 \\
0.3564 \\
0.3711 \\
0.3906 \\
0.4102 \\
0.4297 \\
0.4492 \\
0.4639 \\
0.4834 \\
0.5078 \\
0.5127 \\
0.5469 \\
0.5566 \\
0.5811 \\
61.1538 \\
113.2700 \\
83.9672 \\
37.8257 \\
38.5243 \\
36.7906 \\
19.9347 \\
19.7094 \\
26.3469 \\
16.8994 \\
20.3722 \\
18.5715 \\
39.6704 \\
37.9791 \\
44.0532 \\
38.3882 \\
40.0271 \\
37.2062 \\
10.7741 \\
27.6417 \\
23.9426\end{array}$ & $\begin{array}{l}\text { Nov } 1313: \\
\text { Ave } \\
36.5569 \\
37.6939 \\
83.2484 \\
30.7575 \\
82.9734 \\
38.2549 \\
82.7348 \\
45.9422 \\
64.1207 \\
17.3018 \\
70.1714 \\
76.4674 \\
84.5107 \\
82.0493 \\
0.3399 \\
0.3551 \\
0.3678 \\
0.3857 \\
0.2977 \\
0.3175 \\
0.3356 \\
0.3534 \\
0.3671 \\
0.3858 \\
0.4011 \\
0.4262 \\
0.4416 \\
0.4616 \\
0.1782 \\
0.4974 \\
0.5120 \\
0.5325 \\
0.5538 \\
0.5763 \\
59.6859 \\
112.5346 \\
83.2927 \\
37.4167 \\
39.1688 \\
36.3665 \\
19.4434 \\
19.5079 \\
25.8632 \\
16.5210 \\
19.9434 \\
18.3121 \\
39.1043 \\
37.5473 \\
43.7679 \\
37.9945 \\
39.7433 \\
36.8217 \\
10.5589 \\
27.5392 \\
23.8575\end{array}$ & 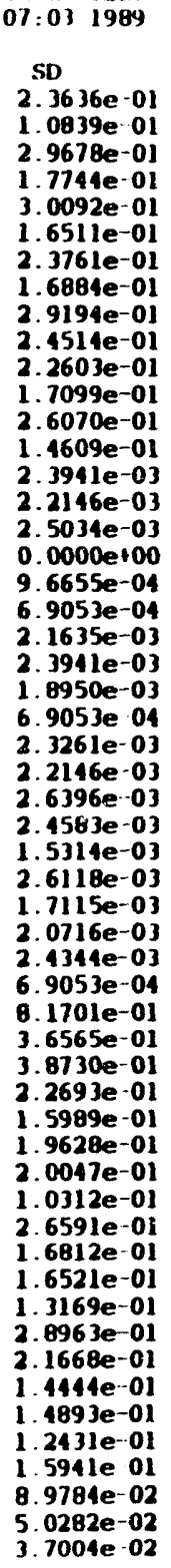 & 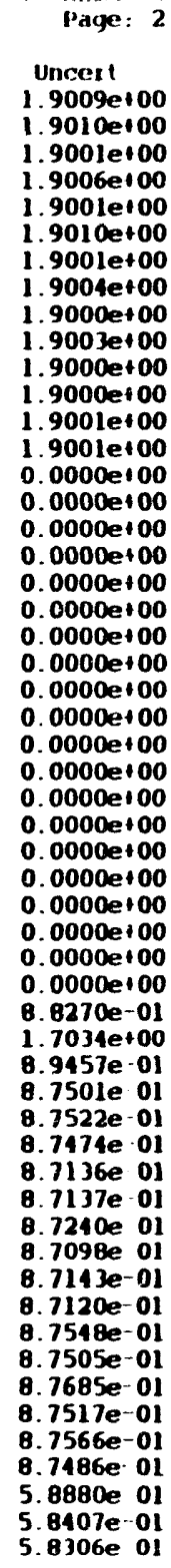 \\
\hline
\end{tabular}




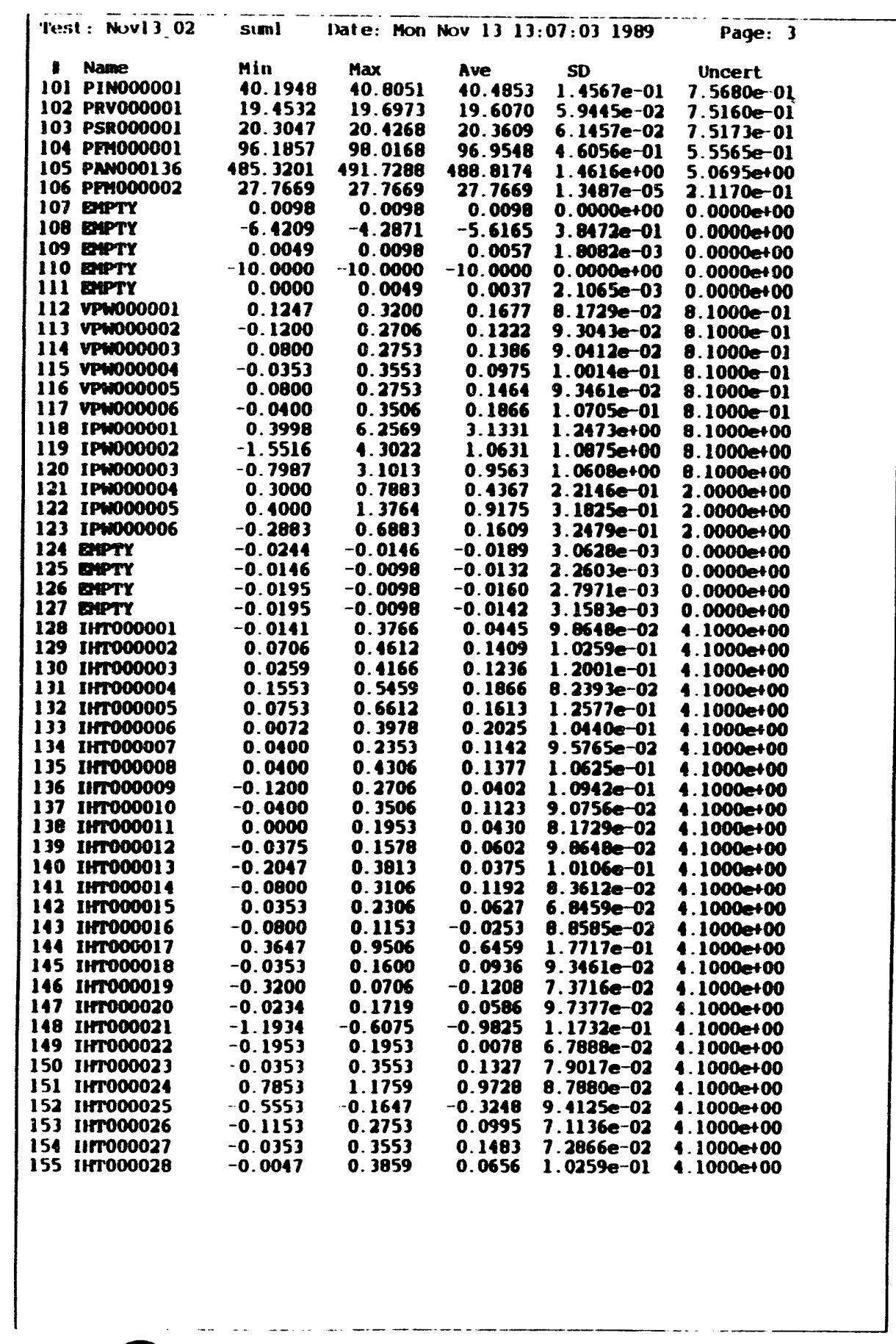

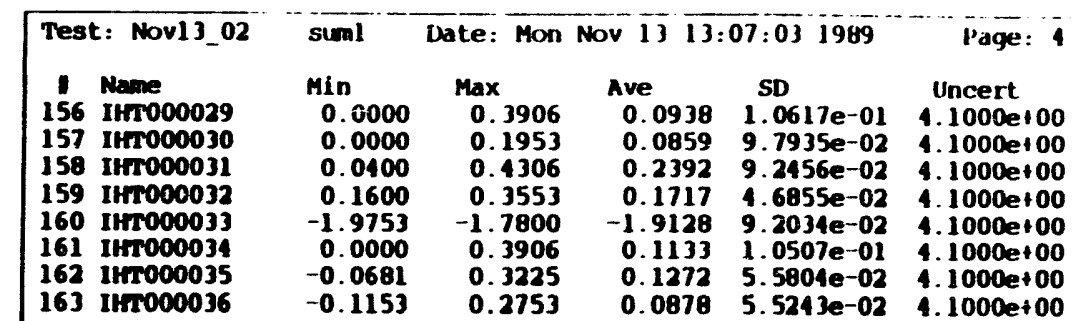




\begin{tabular}{|c|c|c|c|c|c|c|}
\hline \multicolumn{6}{|c|}{ 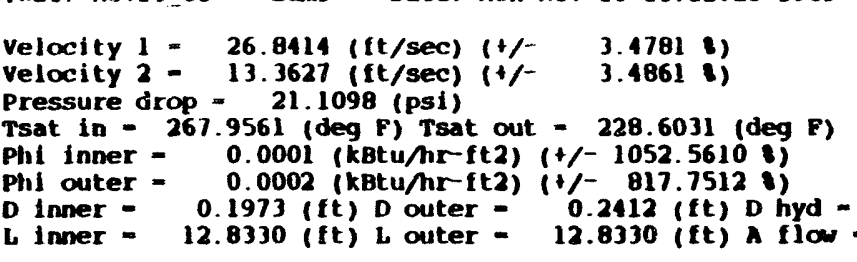 } & $\begin{array}{l}0.0438 \text { (ft) } \\
0.0151 \text { (fte) }\end{array}$ \\
\hline 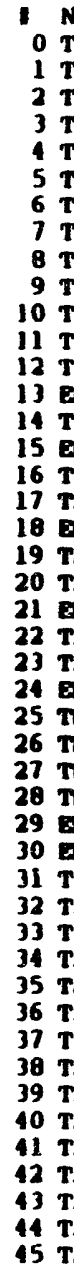 & 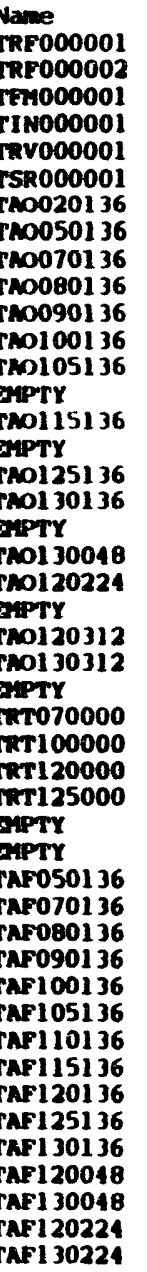 & $\begin{array}{r}\text { Min } \\
76.8497 \\
75.5976 \\
77.0806 \\
81.5061 \\
81.3607 \\
76.3944 \\
82.5677 \\
82.7668 \\
77.2338 \\
81.7850 \\
82.3683 \\
82.3888 \\
84.8958 \\
39.8352 \\
81.6929 \\
34.5420 \\
84.1166 \\
82.4760 \\
224.8616 \\
84.1244 \\
80.0092 \\
-499.9492 \\
82.3613 \\
82.9965 \\
37.3074 \\
82.7296 \\
83.3836 \\
82.9268 \\
85.5150 \\
37.7675 \\
39.6057 \\
83.7171 \\
82.6746 \\
82.2846 \\
82.1922 \\
82.6035 \\
83.2113 \\
81.1632 \\
84.3803 \\
83.9698 \\
82.9344 \\
82.5919 \\
82.3552 \\
83.0801 \\
82.7604 \\
82.6062\end{array}$ & $\begin{array}{r}\text { Max } \\
77.4860 \\
76.0201 \\
77.9253 \\
85.2423 \\
82.3060 \\
77.1331 \\
83.6230 \\
83.3965 \\
78.0830 \\
82.4155 \\
83.2101 \\
83.0208 \\
85.8422 \\
10.2940 \\
82.8561 \\
34.7728 \\
84.7476 \\
83.1050 \\
254.5360 \\
84.5456 \\
80.7481 \\
498.4349 \\
83.2038 \\
83.8372 \\
37.7675 \\
83.3631 \\
84.2246 \\
83.5596 \\
86.4566 \\
38.2273 \\
10.2940 \\
84.5118 \\
83.7217 \\
82.9152 \\
83.3490 \\
83.1464 \\
84.3616 \\
82.1088 \\
85.3252 \\
84.3910 \\
83.5651 \\
83.3292 \\
83.2019 \\
83.7115 \\
83.6030 \\
83.2393\end{array}$ & $\begin{array}{r}\text { Ave } \\
77.1000 \\
75.8089 \\
77.5346 \\
81.8324 \\
81.8415 \\
76.7725 \\
83.0682 \\
83.0839 \\
77.6058 \\
82.0189 \\
82.8290 \\
82.7068 \\
85.3398 \\
10.0463 \\
82.3207 \\
34.6112 \\
84.1467 \\
82.7719 \\
240.9162 \\
84.3453 \\
80.3575 \\
-499.2296 \\
82.7174 \\
83.3771 \\
37.5650 \\
83.0863 \\
83.8649 \\
83.2468 \\
86.0273 \\
37.8916 \\
39.8994 \\
84.1489 \\
83.2716 \\
82.6604 \\
82.7706 \\
83.0817 \\
83.8402 \\
81.6950 \\
84.8188 \\
84.1824 \\
83.2517 \\
82.9857 \\
82.7928 \\
83.3433 \\
83.1798 \\
82.9799\end{array}$ & $\begin{array}{c}\text { SD } \\
1.9957 e-01 \\
1.3495 e-01 \\
2.0812 e-01 \\
1.7429 e-01 \\
1.8399 e-01 \\
1.6675 e-01 \\
2.4826 e-01 \\
1.6095 e-01 \\
1.8324 e-01 \\
1.4457 e-01 \\
1.9922 e-01 \\
1.6674 e-01 \\
2.1478 e-01 \\
1.3782 e-01 \\
2.5043 e-01 \\
1.0682 e-01 \\
1.7637 e-01 \\
1.5926 e-01 \\
6.9302 e+00 \\
1.2256 e-01 \\
2.0135 e-01 \\
3.9927 e-01 \\
1.8786 e-01 \\
1.8014 e-01 \\
1.9521 e-01 \\
1.7585 e-01 \\
2.0034 e-01 \\
1.4641 e-01 \\
2.1477 e-01 \\
1.3312 e-01 \\
2.1265 e-01 \\
1.6468 e-01 \\
2.3307 e-01 \\
1.6572 e-01 \\
2.4721 e-01 \\
2.0219 e-01 \\
2.6333 e-01 \\
1.9400 e-01 \\
2.2321 e-01 \\
1.2296 e-01 \\
1.6082 e-01 \\
1.5017 e-01 \\
2.1502 e-01 \\
1.5525 e-01 \\
2.0569 e-01 \\
1.5117 e-01\end{array}$ & 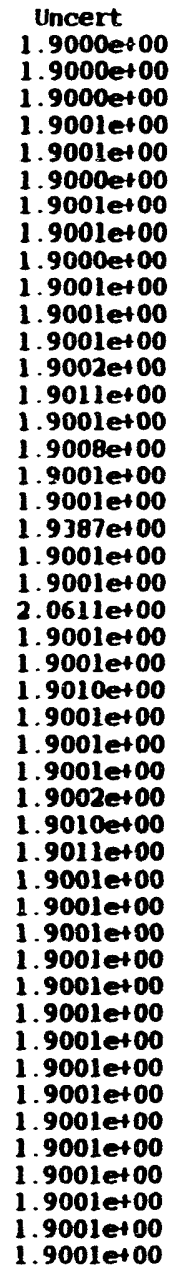 \\
\hline
\end{tabular}

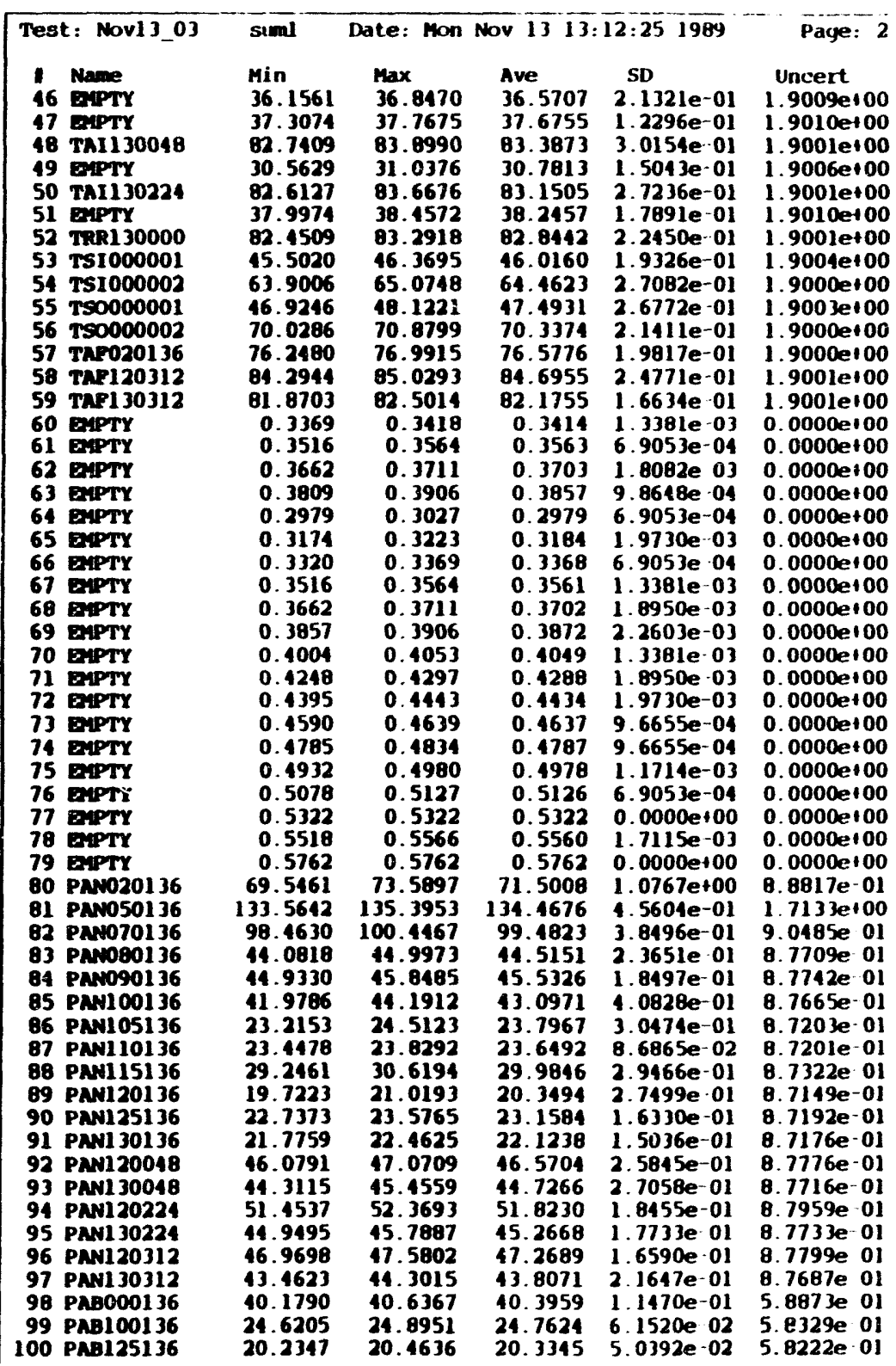




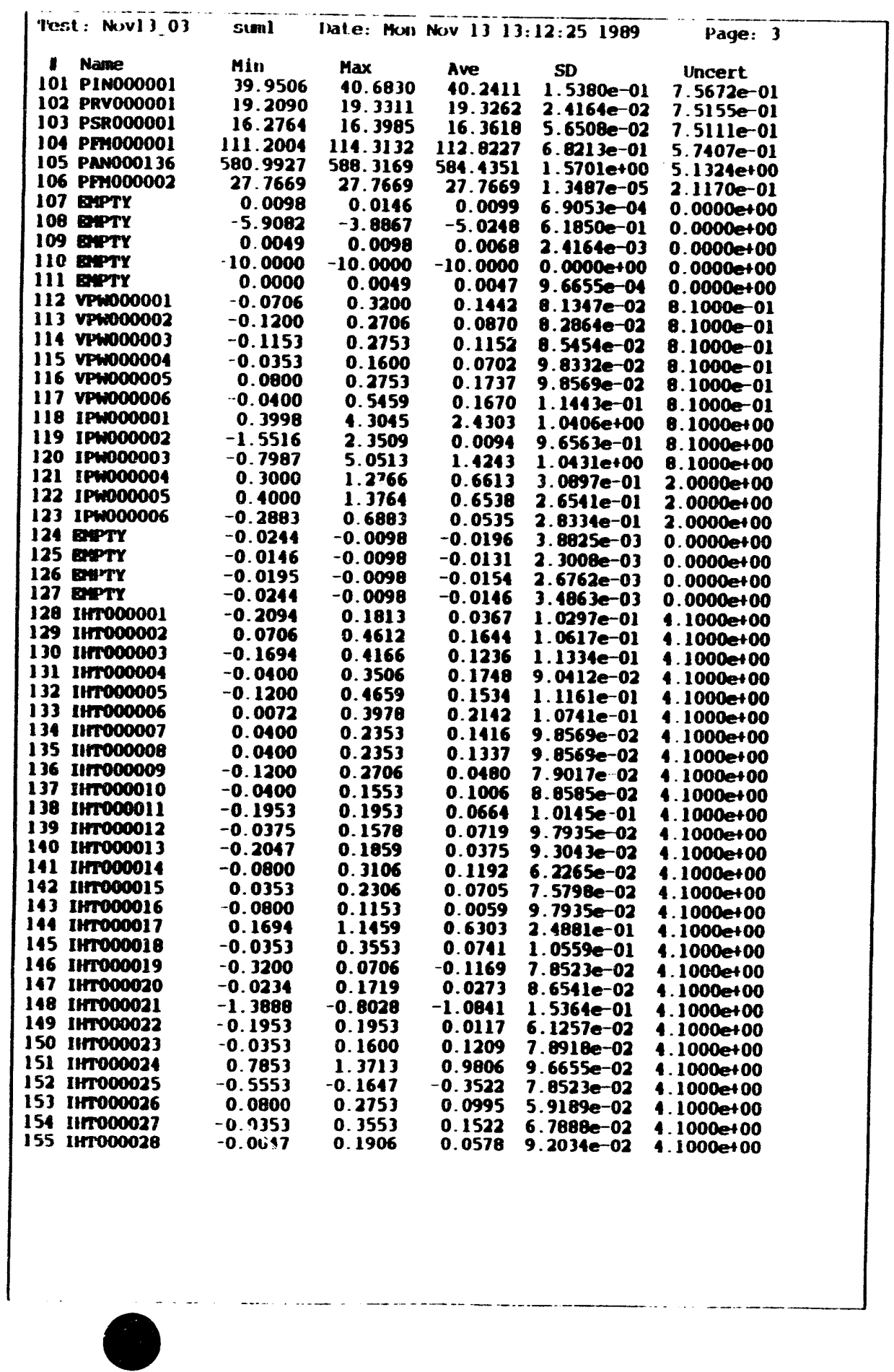

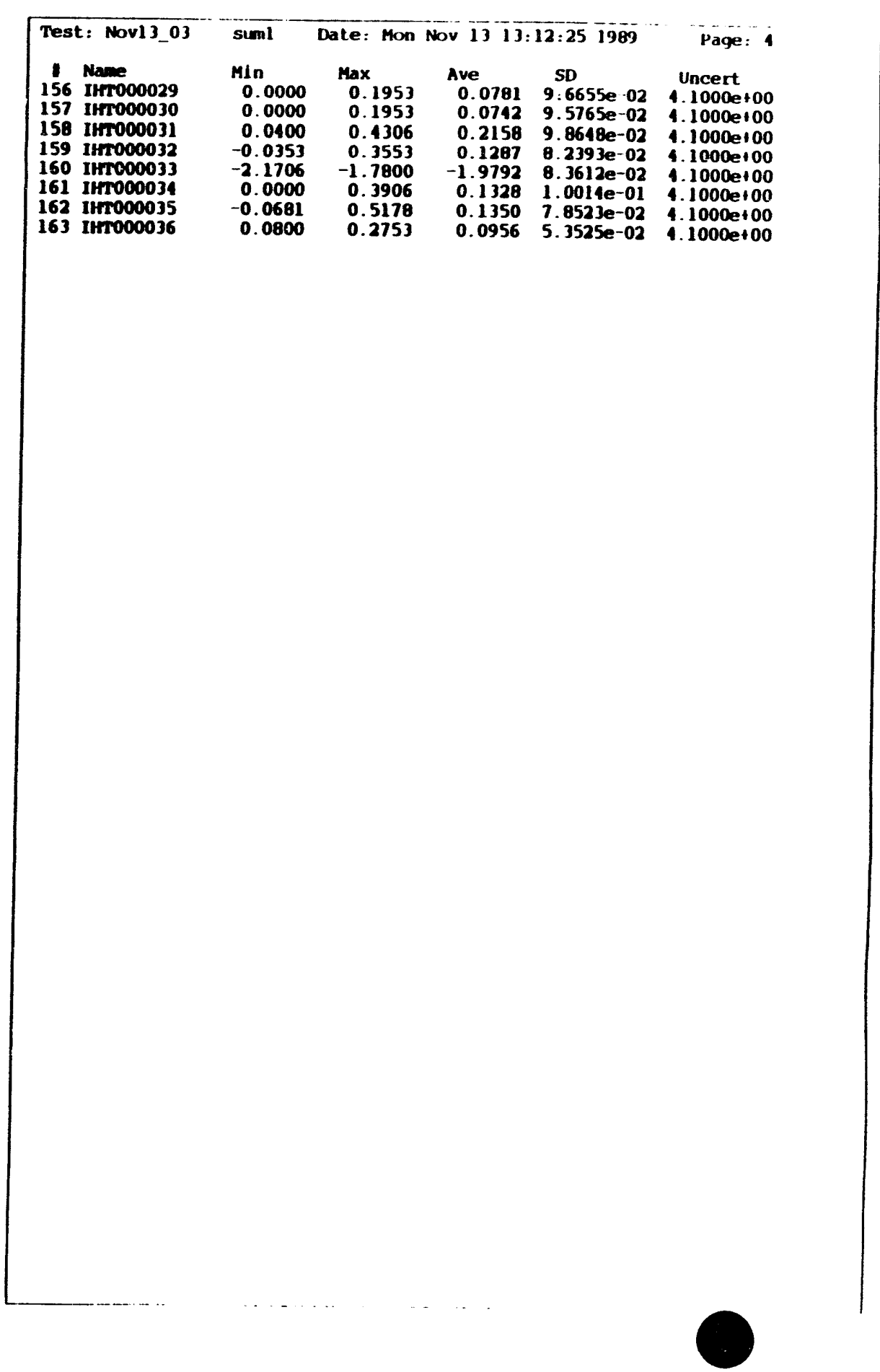




\begin{tabular}{|c|c|c|c|c|c|c|c|}
\hline \multicolumn{2}{|c|}{$\begin{array}{l}\text { rest: Niv13 } 04 \\
\text { velocity } 1= \\
\text { velocity } 2- \\
\text { Pressure drop } \\
\text { Tsat in - 268. } \\
\text { Ph1 inner - } \\
\text { Phi outer = } \\
\text { D inner = } 0 . \\
\text { L inner = } 12 .\end{array}$} & \multicolumn{2}{|c|}{ 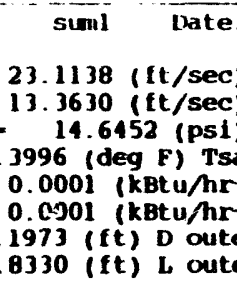 } & \multicolumn{3}{|c|}{ 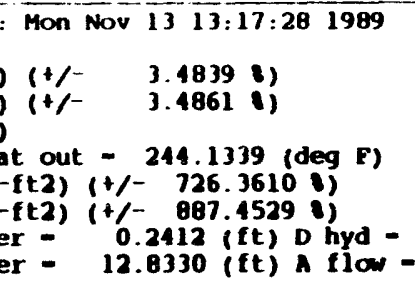 } & $\begin{array}{l}\text { Page: } 1 \\
0.0438 \text { (ft) } \\
0.0151 \text { (ft2) }\end{array}$ \\
\hline $\begin{array}{l}1 \\
0 \\
1 \\
2 \\
3 \\
1 \\
5 \\
6 \\
7 \\
8 \\
9 \\
10 \\
11 \\
12 \\
13 \\
14 \\
15 \\
16 \\
17 \\
18 \\
19 \\
20 \\
21 \\
22 \\
23 \\
24 \\
25 \\
26 \\
27 \\
28 \\
29 \\
30 \\
31 \\
32 \\
33 \\
34 \\
359 \\
369 \\
379 \\
389 \\
39 \\
109 \\
11 \\
12 \\
139 \\
14 \\
45\end{array}$ & 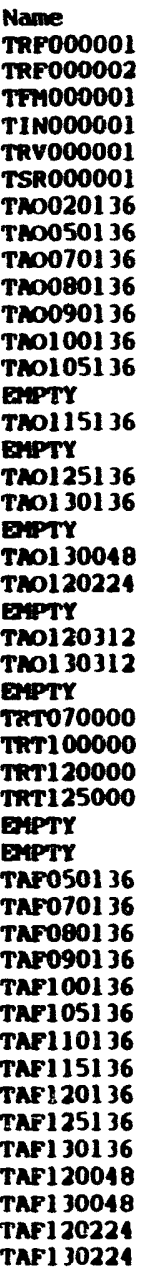 & $\begin{array}{r}\text { Min } \\
76.8497 \\
75.5976 \\
77.2915 \\
84.7164 \\
81.6757 \\
76.6055 \\
82.8843 \\
82.9761 \\
77.2349 \\
81.8901 \\
82.5790 \\
82.5995 \\
85.1062 \\
39.8352 \\
82.1154 \\
34.5420 \\
84.4314 \\
82.6866 \\
216.1143 \\
84.2293 \\
80.2200 \\
-502.9831 \\
82.5714 \\
83.2070 \\
37.0772 \\
83.0466 \\
83.5941 \\
83.0318 \\
85.8289 \\
37.7675 \\
39.6057 \\
83.9875 \\
82.9892 \\
82.5985 \\
82.4021 \\
82.9192 \\
83.5250 \\
81.4781 \\
84.5896 \\
84.0747 \\
83.0394 \\
82.8023 \\
82.5658 \\
83.3956 \\
82.9710 \\
82.9224\end{array}$ & $\begin{array}{l}\operatorname{Max} \\
77.4 \\
76.0 \\
78.2 \\
85.3 \\
82.3 \\
77.1 \\
83.6 \\
83.6 \\
77.9 \\
82.4 \\
83.1 \\
83.3 \\
85.8 \\
40.2 \\
83.0 \\
34.7 \\
84.8 \\
83.2 \\
232.9 \\
84.6 \\
81.0 \\
-501.1 \\
83.3 \\
83.8 \\
37.7 \\
83.5 \\
84.4 \\
83.8 \\
86.6 \\
37.9 \\
10.2 \\
84.6 \\
83.8 \\
83.2 \\
83.5 \\
83.6 \\
84.4 \\
82.2 \\
85.3 \\
84.4 \\
83.6 \\
83.4 \\
83.5 \\
83.9 \\
83.7 \\
83.3\end{array}$ & $\begin{array}{l}4860 \\
0201 \\
2419 \\
3477 \\
3065 \\
1342 \\
6230 \\
6069 \\
9763 \\
4161 \\
1195 \\
3359 \\
8427 \\
2940 \\
0666 \\
7728 \\
8525 \\
2100 \\
9711 \\
6504 \\
0640 \\
1652 \\
3093 \\
6377 \\
7675 \\
5745 \\
4343 \\
8737 \\
6645 \\
9974 \\
2940 \\
6167 \\
8266 \\
2289 \\
5597 \\
6570 \\
4665 \\
2138 \\
3252 \\
4959 \\
6700 \\
1342 \\
5184 \\
9219 \\
7085 \\
3462\end{array}$ & $\begin{array}{r}\text { Ave } \\
77.1933 \\
75.8638 \\
77.7858 \\
85.0574 \\
82.0202 \\
76.8508 \\
83.2221 \\
83.2456 \\
77.6034 \\
82.1680 \\
83.0245 \\
82.9277 \\
85.4891 \\
40.1014 \\
82.5766 \\
34.6851 \\
84.6210 \\
82.9701 \\
224.4527 \\
84.4315 \\
80.5578 \\
-502.1102 \\
82.9386 \\
83.5432 \\
37.5558 \\
83.3041 \\
84.0518 \\
83.4487 \\
86.2301 \\
37.9192 \\
39.9316 \\
84.3608 \\
83.4581 \\
82.9012 \\
82.9537 \\
83.2691 \\
84.0098 \\
81.8737 \\
85.0015 \\
84.2728 \\
83.4008 \\
83.1520 \\
82.9726 \\
83.6190 \\
83.3713 \\
83.1300\end{array}$ & $\begin{array}{l}\text { SD } \\
1.8123 e-01 \\
1.1136 e-01 \\
1.9939 e-01 \\
1.6536 e-01 \\
1.8617 e-01 \\
1.5000 e-01 \\
2.0544 e-01 \\
1.5471 e-01 \\
1.9065 e-01 \\
1.5414 e-01 \\
2.0870 e-01 \\
1.6001 e-01 \\
1.9901 e-01 \\
1.1689 e-01 \\
2.4048 e-01 \\
1.1314 e-01 \\
1.1640 e-01 \\
1.1443 e-01 \\
3.9139 e+00 \\
1.2169 e-01 \\
2.0098 e-01 \\
2.7906 e-01 \\
1.5819 e-01 \\
1.1405 e-01 \\
1.7291 e-01 \\
1.3184 e-01 \\
2.1713 e-01 \\
1.8887 e-01 \\
1.9285 e-01 \\
1.1004 e-01 \\
2.1290 e-01 \\
1.3274 e-01 \\
2.1387 e-01 \\
1.4310 e-01 \\
3.0691 e-01 \\
1.5847 e-01 \\
2.4057 e-01 \\
1.5841 e-01 \\
1.8933 e-01 \\
1.0978 e-01 \\
1.3607 e-01 \\
1.6394 e-01 \\
2.2373 e-01 \\
1.1386 e-01 \\
1.7016 e-01 \\
1.2237 e-01\end{array}$ & 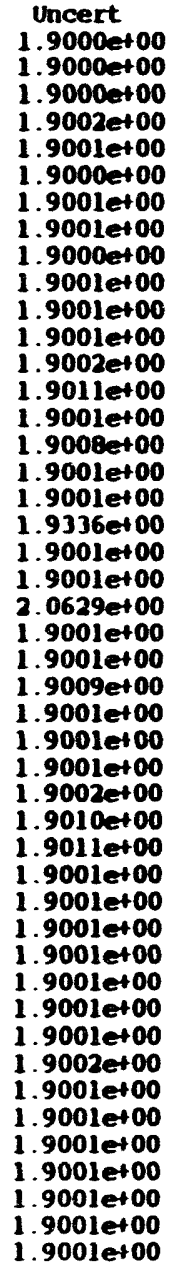 \\
\hline
\end{tabular}

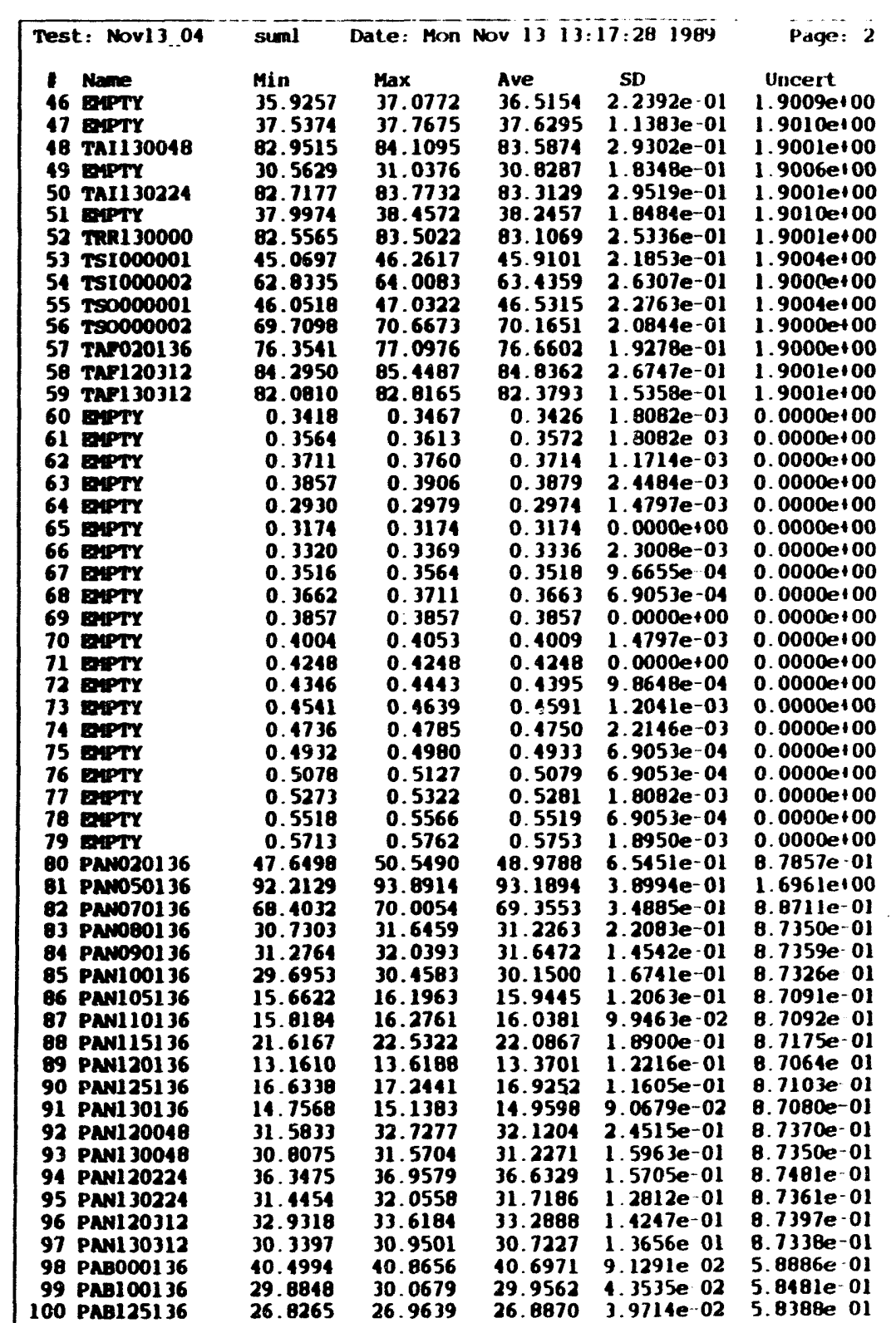




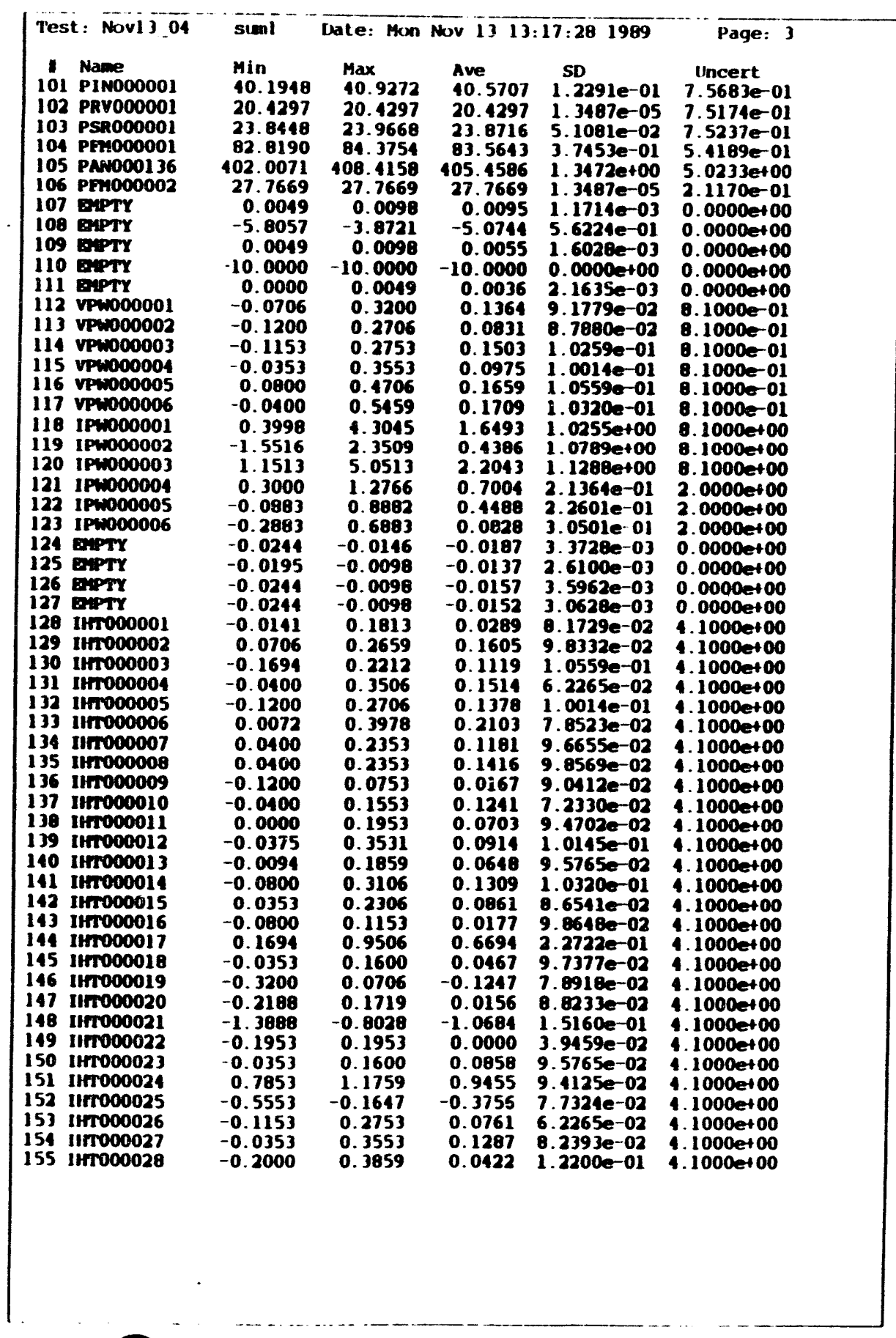

\begin{tabular}{|c|c|c|c|c|c|c|}
\hline & ti: & $\overline{1}$ & Date: Mon & Nov 1313 : & $17: 281989$ & Page: \\
\hline $\begin{array}{l}11 \\
156 \\
157 \\
158 \\
159 \\
160 \\
161 \\
162 \\
163\end{array}$ & 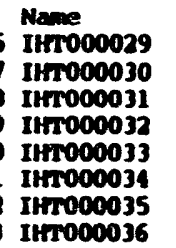 & $\begin{array}{r}\text { Min } \\
0.0000 \\
0.0000 \\
0.0400 \\
-0.0353 \\
-2.1706 \\
0.0000 \\
-0.0681 \\
-0.1153\end{array}$ & $\begin{array}{r}\operatorname{Max} \\
0.3906 \\
0.3906 \\
0.4306 \\
0.3553 \\
-1.7800 \\
0.1953 \\
0.3225 \\
0.4706\end{array}$ & $\begin{array}{l}\text { Ave } \\
0.0859 \\
0.1094 \\
0.2158 \\
0.1327 \\
-1.9675 \\
0.1133 \\
0.1428 \\
0.1191\end{array}$ & $\begin{array}{c}\text { SD } \\
1.0559 \mathrm{e}-01 \\
1.0559 \mathrm{e}-01 \\
9.0412 \mathrm{e}-02 \\
9.6735 \mathrm{e}-02 \\
7.8523 \mathrm{e}-02 \\
9.7377 \mathrm{e}-02 \\
8.6810 \mathrm{e}-02 \\
9.6655 e^{-02}\end{array}$ & 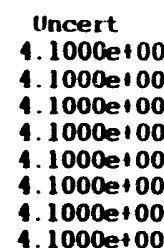 \\
\hline
\end{tabular}




\begin{tabular}{|c|c|c|c|c|c|c|}
\hline \multicolumn{4}{|c|}{ 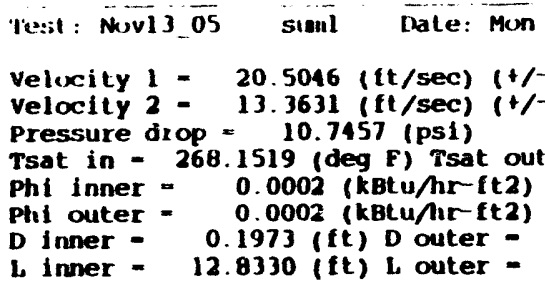 } & \multicolumn{2}{|c|}{$\begin{array}{l}\text { Nov } 1313: 21: 361989 \\
\quad 3.4918 \text { :) } \\
3.4861 \text { :) } \\
=251.3418 \text { (deg F) } \\
(1 /-707.7262:) \\
(+/-841.5335:) \\
0.2412 \text { (ft) D hyd }= \\
12.8330 \text { (ft) A flow - }\end{array}$} & $\begin{array}{l}0.0438 \text { (ft) } \\
0.0151 \text { (ft2) }\end{array}$ \\
\hline $\begin{array}{l}1 \\
0 \\
1 \\
2 \\
3 \\
1 \\
5 \\
6 \\
7 \\
8 \\
9 \\
10 \\
11 \\
12 \\
13 \\
14 \\
15 \\
16 \\
17 \\
18 \\
19 \\
20 \\
21 \\
22 \\
23 \\
24 \\
25 \\
26 \\
27 \\
28 \\
29 \\
30 \\
31 \\
32 \\
33 \\
34 \\
35 \\
36 \\
37 \\
38 \\
39 \\
40 \\
41 \\
42 \\
43 \\
44 \\
15 \\
\\
19 \\
\end{array}$ & 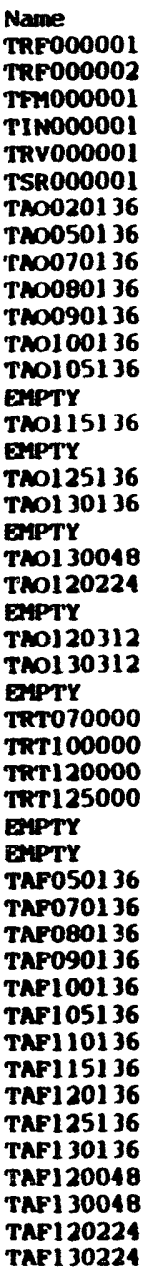 & $\begin{array}{r}\text { Min } \\
76.8497 \\
75.8089 \\
77.3975 \\
84.7164 \\
81.7798 \\
76.6060 \\
82.6737 \\
82.9779 \\
77.1283 \\
81.8901 \\
82.5790 \\
82.5995 \\
85.1062 \\
39.8352 \\
82.2211 \\
34.5420 \\
84.4314 \\
82.6860 \\
228.8300 \\
84.2293 \\
80.1150 \\
-499.5704 \\
82.6771 \\
83.3114 \\
37.3074 \\
83.0466 \\
83.8039 \\
83.2421 \\
85.9322 \\
37.7675 \\
39.6057 \\
83.8820 \\
83.0927 \\
82.5985 \\
82.5077 \\
83.0247 \\
83.7331 \\
81.5838 \\
84.5896 \\
84.0747 \\
83.2486 \\
82.9073 \\
82.5658 \\
83.3956 \\
82.9710 \\
82.9232\end{array}$ & $\begin{array}{r}\text { Max } \\
77.4860 \\
76.2313 \\
78.3467 \\
85.4526 \\
82.5157 \\
77.1342 \\
83.6225 \\
83.6069 \\
78.1884 \\
82.5211 \\
83.5250 \\
83.3359 \\
86.0523 \\
40.2940 \\
83.0666 \\
34.7728 \\
84.8525 \\
83.2100 \\
242.1349 \\
84.7543 \\
81.0640 \\
-498.4349 \\
83.3093 \\
83.8372 \\
37.7675 \\
83.6800 \\
84.5392 \\
83.8737 \\
86.5613 \\
38.2273 \\
40.2940 \\
84.7221 \\
84.1409 \\
83.2289 \\
83.4545 \\
83.5515 \\
84.5696\end{array}$ & $\begin{array}{r}\text { Ave } \\
77.2060 \\
75.9525 \\
77.8449 \\
\mathbf{8 5 . 1 4 5 6} \\
82.0791 \\
76.9057 \\
83.1966 \\
83.3379 \\
77.6753 \\
82.2561 \\
83.0834 \\
82.9655 \\
85.5647 \\
40.1059 \\
82.6482 \\
34.7405 \\
84.6840 \\
82.9998 \\
236.5051 \\
84.4903 \\
80.6210 \\
-499.0326 \\
83.0059 \\
83.5664 \\
37.5466 \\
83.3757 \\
84.1232 \\
83.4949 \\
86.2804 \\
37.9054 \\
39.9040 \\
84.4321 \\
83.4918 \\
82.9182 \\
82.9873 \\
83.3237 \\
84.0602 \\
81.9326 \\
85.0477 \\
84.3274 \\
83.5222 \\
83.2276 \\
83.0612 \\
83.6526 \\
83.4218 \\
83.2016\end{array}$ & $\begin{array}{r}\text { SD } \\
1.7893 e-01 \\
1.0829 e-01 \\
1.7905 e-01 \\
1.5735 e-01 \\
1.8669 e-01 \\
1.3887 e-01 \\
2.2341 e-01 \\
1.7379 e-01 \\
2.1136 e-01 \\
1.3125 e-01 \\
1.9465 e-01 \\
1.4619 e-01 \\
1.8729 e-01 \\
1.4429 e-01 \\
2.0812 e-01 \\
8.0882 e-02 \\
1.1449 e-01 \\
1.4204 e-01 \\
3.6723 e+00 \\
1.2609 e-01 \\
1.8815 e-01 \\
2.8703 e-01 \\
1.4683 e-01 \\
1.2778 e-01 \\
1.7365 e-01 \\
1.1422 e-01 \\
1.5148 e-01 \\
1.4264 e-01 \\
1.7360 e-01 \\
1.2292 e-01 \\
1.8689 e-01 \\
1.9948 e-01 \\
2.0297 e-01 \\
1.4678 e-01 \\
2.6516 e-01 \\
1.3851 e-01 \\
2.3945 e-01 \\
1.6526 e-01 \\
2.0316 e-01 \\
1.2209 e-01 \\
1.3750 e-01 \\
1.4883 e-01 \\
2.2476 e-01 \\
1.2795 e-01 \\
2.0068 e-01 \\
1.3617 e-01\end{array}$ & $\begin{array}{r}\text { Uncert } \\
1.9000 e+00 \\
1.9000 e+00 \\
1.9000 e+00 \\
1.9002 e+00 \\
1.9001 e+00 \\
1.9000 e+00 \\
1.9001 e+00 \\
1.9001 e+00 \\
1.9000 e+00 \\
1.9001 e+00 \\
1.9001 e+00 \\
1.9001 e+00 \\
1.9002 e+00 \\
1.9011 e+00 \\
1.9001 e+00 \\
1.900 B e+00 \\
1.9001 e+00 \\
1.9001 e+00 \\
1.9373 e+00 \\
1.9001 e+00 \\
1.9001 e+00 \\
2.0610 e+00 \\
1.9001 e+00 \\
1.9001 e+00 \\
1.9009 e+00 \\
1.9001 e+00 \\
1.9001 e+00 \\
1.9001 e+00 \\
1.9002 e+00 \\
1.9010 e+00 \\
1.9011 e+00 \\
1.9001 e+00 \\
1.9001 e+00 \\
1.9001 e+00 \\
1.9001 e+00 \\
1.9001 e+00 \\
1.9001 e+00 \\
1.9001 e+00 \\
1.9002 e+00 \\
1.9001 e+00 \\
1.9001 e+00 \\
1.9001 e+00 \\
1.9001 e+00 \\
1.9001 e+00 \\
1.9001 e+00 \\
1.9001 e+00\end{array}$ \\
\hline
\end{tabular}

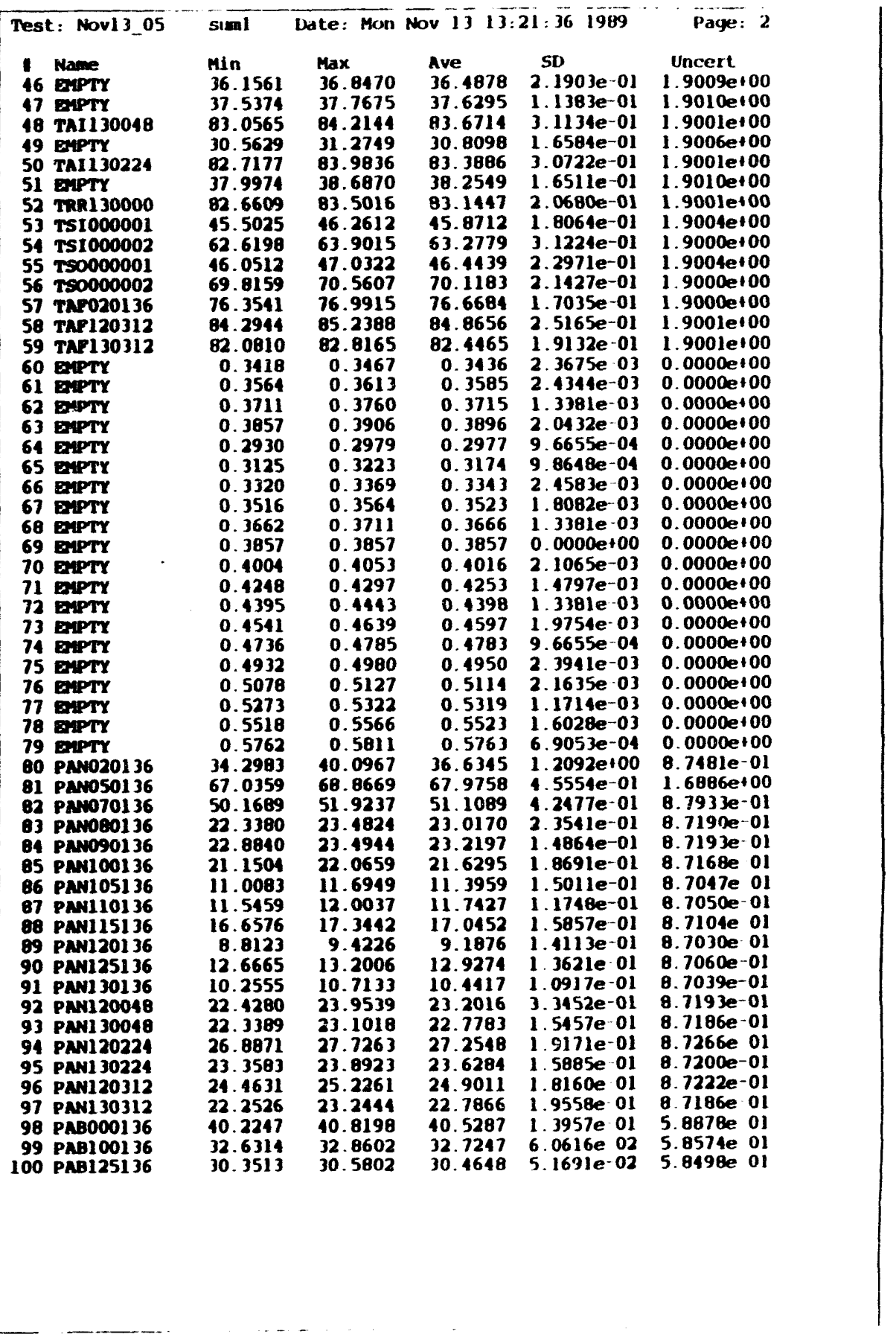




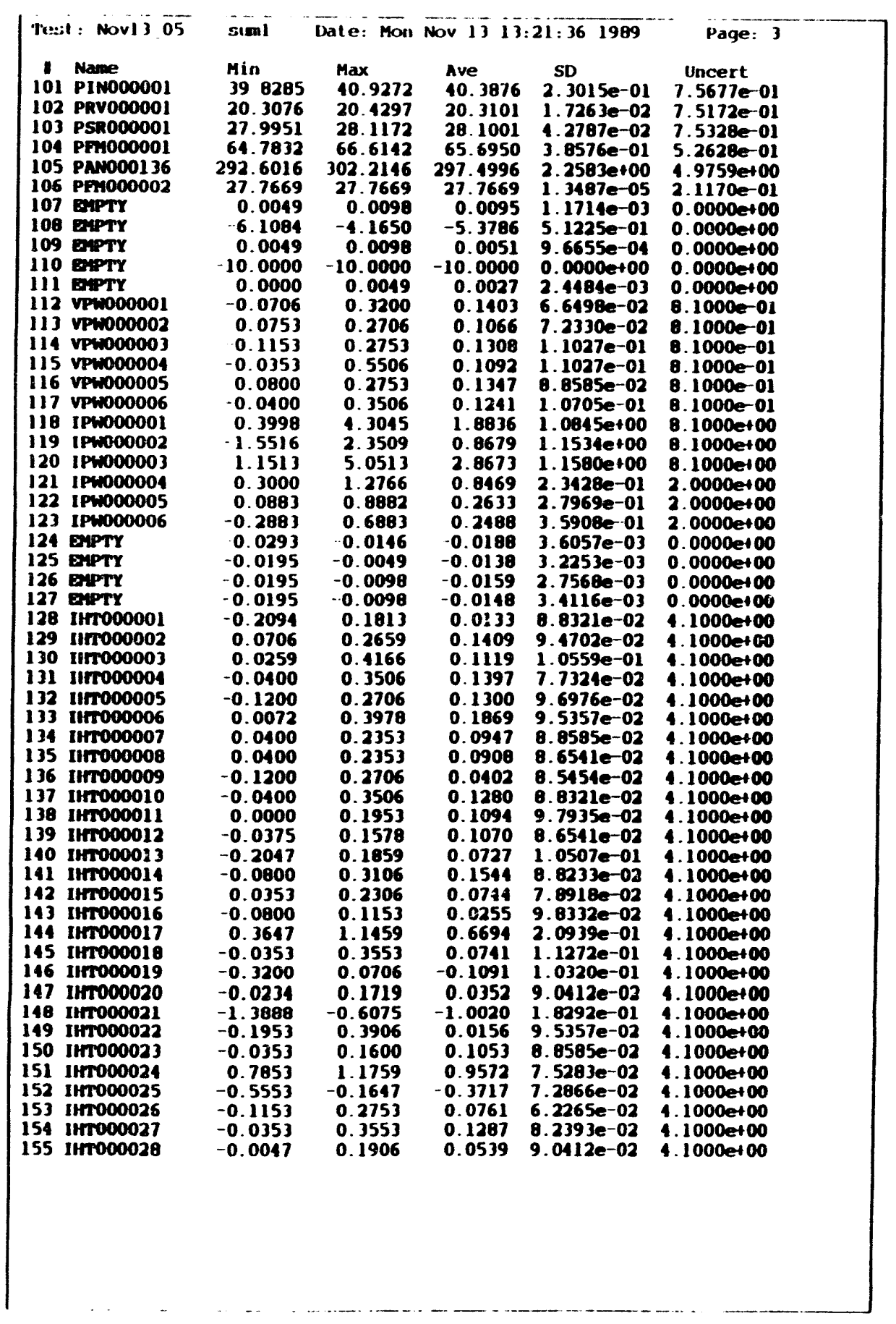

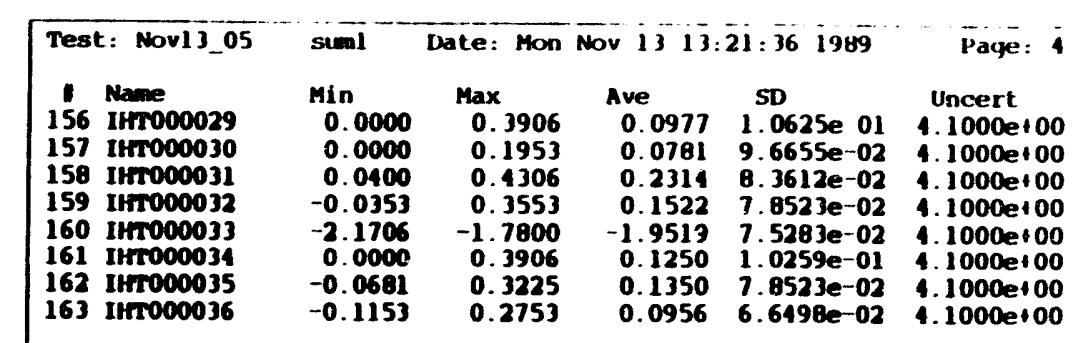




\begin{tabular}{|c|c|c|c|c|c|c|}
\hline \multicolumn{4}{|c|}{ 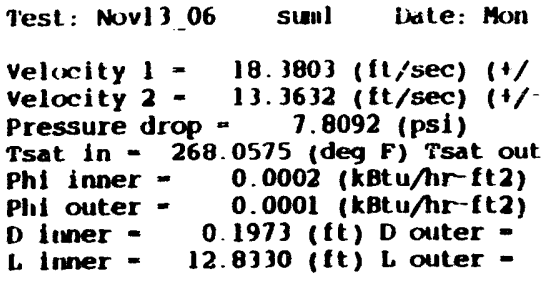 } & \multicolumn{2}{|c|}{ 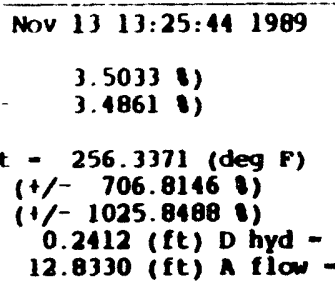 } & $\begin{array}{l}\text { Page: } 1 \\
0.0438 \text { (ft) } \\
0.0151 \text { (ft2) }\end{array}$ \\
\hline $\begin{array}{l}10 \\
1 \\
2 \\
3 \\
1 \\
5 \\
6 \\
7 \\
8 \\
9 \\
10 \\
11 \\
12 \\
13 \\
11 \\
15 \\
16 \\
17 \\
18 \\
19 \\
20 \\
21 \\
22 \\
23 \\
24 \\
25 \\
26 \\
27 \\
28 \\
29 \\
30 \\
31 \\
32 \\
33 \\
34 \\
35 \\
36 \\
37 \\
38 \\
39 \\
10 \\
11 \\
12 \\
13 \\
11 \\
15\end{array}$ & 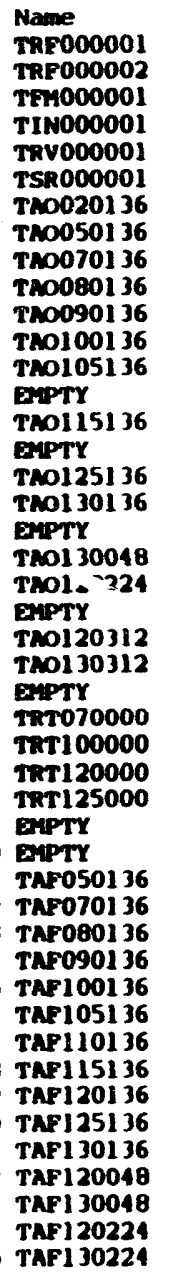 & $\begin{array}{r}\text { Min } \\
77.0619 \\
75.8089 \\
77.5030 \\
85.0321 \\
81.6757 \\
76.6060 \\
82.7792 \\
83.0817 \\
77.2343 \\
81.9957 \\
82.8940 \\
82.7045 \\
85.3163 \\
39.8352 \\
82.3261 \\
31.7728 \\
84.5368 \\
82.8939 \\
232.5953 \\
84.3347 \\
80.3258 \\
-489.0104 \\
82.7826 \\
83.4169 \\
37.3074 \\
83.1521 \\
83.8039 \\
83.3476 \\
86.0375 \\
37.7675 \\
39.6057 \\
84.1970 \\
83.1982 \\
82.8091 \\
82.6132 \\
83.0247 \\
83.6399 \\
81.7935 \\
84.6950 \\
84.1802 \\
83.1449 \\
83.1182 \\
82.7780 \\
83.5011 \\
83.0765 \\
83.0288\end{array}$ & $\begin{array}{r}\text { Max } \\
77.4860 \\
76.2313 \\
78.3473 \\
85.5579 \\
82.6213 \\
77.2397 \\
83.8329 \\
83.8162 \\
78.1894 \\
82.7302 \\
83.6300 \\
83.4409 \\
86.0522 \\
10.2940 \\
83.2782 \\
35.0035 \\
85.1670 \\
83.3150 \\
248.4163 \\
84.9616 \\
81.0640 \\
488.2594 \\
83.4144 \\
83.946 \\
37.9974 \\
83.7849 \\
84.6441 \\
83.9786 \\
86.7692 \\
37.9974 \\
10.2940 \\
84.9314 \\
84.0370 \\
83.1376 \\
83.5592 \\
83.8669 \\
84.7798 \\
82.3194 \\
85.4300 \\
84.8099 \\
83.9840 \\
83.7494 \\
83.7305 \\
83.9219 \\
84.0238 \\
83.6616\end{array}$ & $\begin{array}{r}\text { Ave } \\
77.2570 \\
76.0158 \\
77.9441 \\
85.2738 \\
82.1989 \\
76.9797 \\
83.3083 \\
83.4616 \\
77.7536 \\
82.4049 \\
83.2242 \\
83.1190 \\
85.6761 \\
40.0876 \\
82.7812 \\
34.7820 \\
84.8079 \\
83.0857 \\
240.2876 \\
64.5638 \\
80.7412 \\
-488.6649 \\
83.1048 \\
83.6714 \\
37.5420 \\
83.4834 \\
84.2681 \\
83.5980 \\
86.3997 \\
37.9054 \\
39.9178 \\
84.5475 \\
83.6573 \\
83.0711 \\
83.0988 \\
83.4395 \\
84.2212 \\
82.0482 \\
85.1212 \\
84.4762 \\
83.6209 \\
83.3560 \\
83.1647 \\
83.7094 \\
83.5375 \\
83.3389\end{array}$ & $\begin{array}{l}\text { SD } \\
1.7052 e-01 \\
1.3144 e-01 \\
1.8663 e-01 \\
1.5210 e-01 \\
1.9857 e-01 \\
1.4513 e-01 \\
2.8374 e-01 \\
1.7721 e-01 \\
2.0677 e-01 \\
1.6336 e-01 \\
1.7646 e-01 \\
1.7708 e-01 \\
1.8645 e-01 \\
1.0620 e-01 \\
2.3314 e-01 \\
1.5666 e-02 \\
1.7384 e-01 \\
1.2516 e-01 \\
3.5761 e+00 \\
1.5404 e-01 \\
1.6421 e-01 \\
2.4979 e-01 \\
1.6670 e-01 \\
1.4573 e-01 \\
1.8973 e-01 \\
1.6927 e-01 \\
2.1116 e-01 \\
1.4706 e-01 \\
1.5556 e-01 \\
1.1391 e-01 \\
1.8980 e-01 \\
1.7311 e-01 \\
1.9383 e-01 \\
1.1267 e-01 \\
2.7190 e-01 \\
1.7976 e-01 \\
2.8927 e-01 \\
1.5181 e-01 \\
1.9366 e-01 \\
1.5680 e-01 \\
1.5608 e-01 \\
1.5594 e-01 \\
2.2279 e-01 \\
1.2668 e-01 \\
1.8768 e-01 \\
1.5876 e-01\end{array}$ & 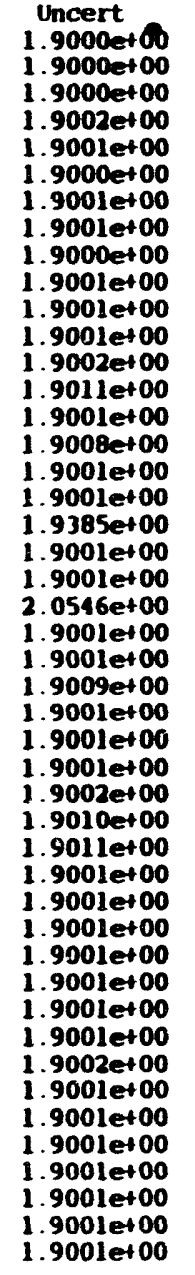 \\
\hline
\end{tabular}

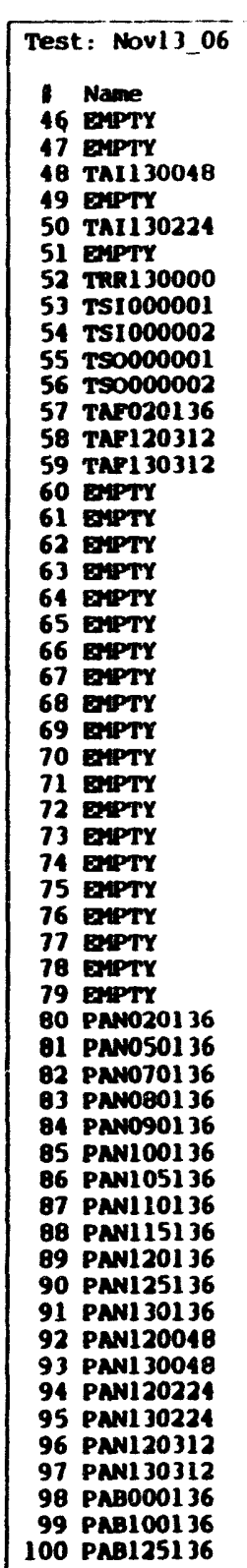

si $\operatorname{Min}$ $37.5374 \quad 37.7675$ 30. $3254 \quad 31.0376$ 82.8233 84.088 $37.9974 \quad 38.4572$
82.6615 $82.6615 \quad 83.8169$
45.2858
46.3699 $\begin{array}{lll}45.2858 & 46.3699 \\ 62.2992 & 63.7952\end{array}$ \begin{tabular}{l}
$46.0512 \quad 47.3589$ \\
\hline
\end{tabular} $69.7098 \quad 70.7733$ 76.354177 .2031 84.5047 85.5530 $82.2907 \quad 82.9216$ $\begin{array}{ll}0.3418 & 0.3467\end{array}$ $0.3564 \quad 0.3613$ 0.3711060 .3760 $0.2930 \quad 0.2979$ $0.3125 \quad 0.3174$ $0.3320 \quad 0.3369$ $0.3662 \quad 0.3662$ $\begin{array}{ll}0.3857 & 0.3857\end{array}$ 0.10040 .4053 $0.4248 \quad 0.4297$ $0.4395 \quad 0.4395$ $\begin{array}{ll}0.4590 & 0.4639\end{array}$ $0.4736 \quad 0.4785$ $\begin{array}{ll}0.1932 & 0.4980 \\ 0.5078 & 0.5127\end{array}$

0.5078

0.5273

0.5510

0.5713
24.8379

24.8379
49.1831

37.5804

15.8530

16.7805

15.1283

7.8040

12.7666

6. 2183

9. 6.9749

6.9749
16.4771

16.1591

19.8680

16.9496

16.54

40.3163

34.7371
33.0979
0.5322

0.5566

26.5164

50.5564

16. 3108

17.3909

15.8861
8.2617

0.9519
13.3007

6.6760
.9199

9.9199

7.5852
17.1637

17.1637
16.7694

20.3258

17.4836

18.6648
16.8357

40.6367

34.9660

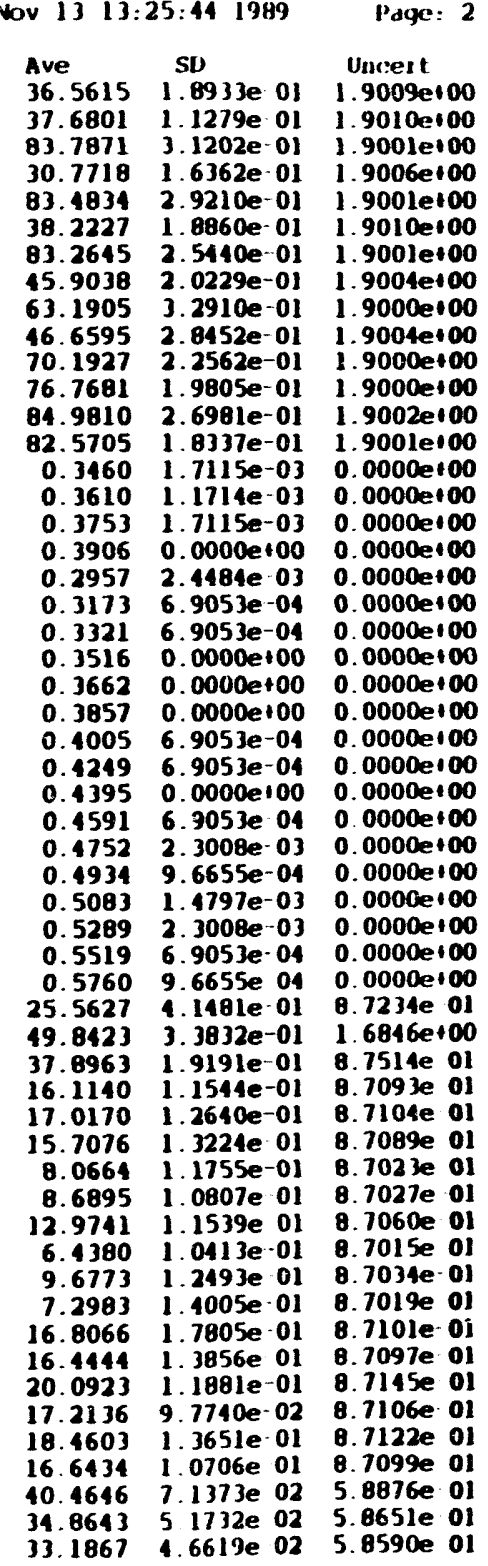




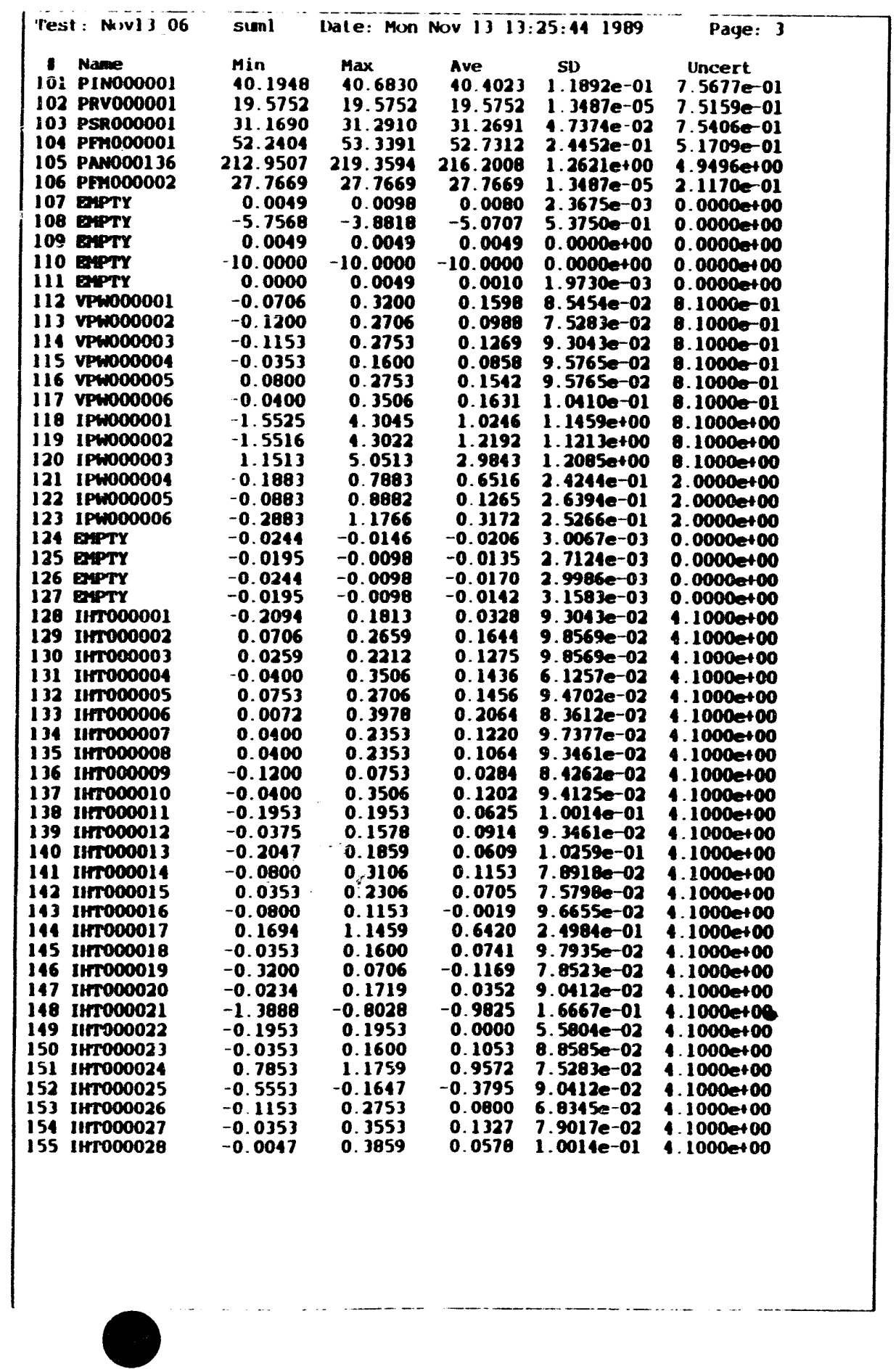

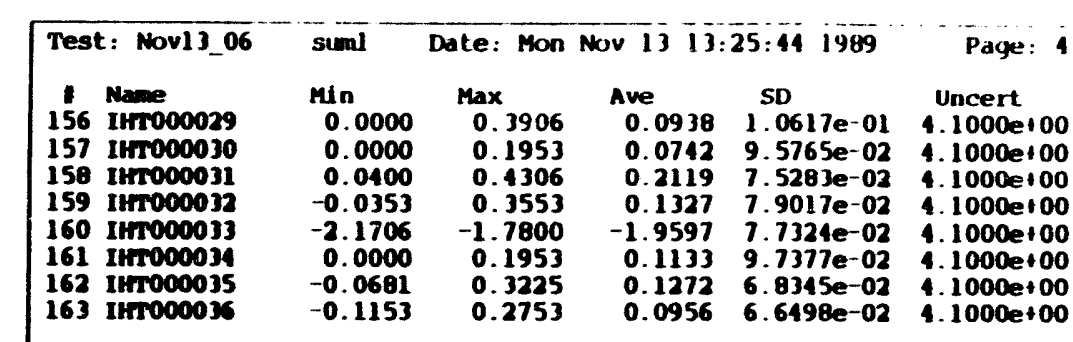




\begin{tabular}{|c|c|c|c|c|c|c|}
\hline \multirow{2}{*}{\multicolumn{4}{|c|}{ 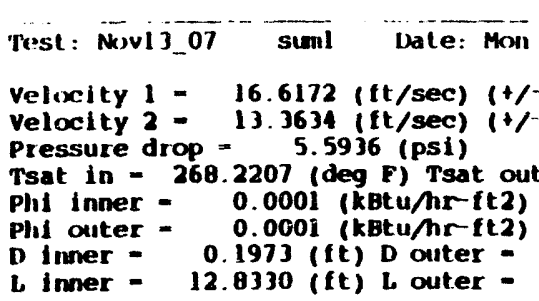 }} & \multirow{2}{*}{\multicolumn{2}{|c|}{ 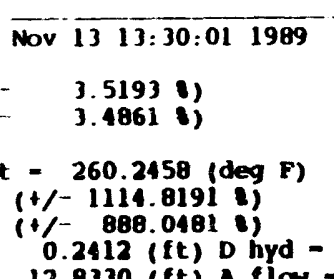 }} & \multirow{2}{*}{$\begin{array}{l}\text { Page: } 1 \\
-0.0438 \text { (ft) } \\
-0.0151 \text { (ft2) }\end{array}$} \\
\hline & & & & & & \\
\hline $\begin{array}{l}1 \\
0 \\
1 \\
3 \\
3 \\
1 \\
5 \\
6 \\
7 \\
8 \\
9 \\
10 \\
11 \\
12 \\
13 \\
14 \\
15 \\
16 \\
17 \\
18 \\
19 \\
20 \\
21 \\
22 \\
23 \\
21 \\
25 \\
26 \\
27 \\
28 \\
29 \\
30 \\
31 \\
32 \\
33 \\
34 \\
35 \\
36 \\
37 \\
38 \\
39 \\
10 \\
11 \\
42 \\
13 \\
14 \\
45\end{array}$ & 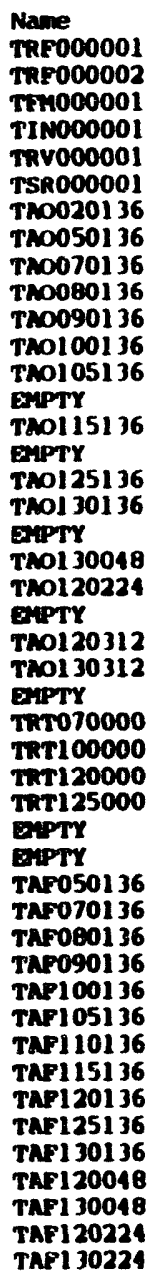 & $\begin{array}{r}\text { Min } \\
77.0619 \\
75.6089 \\
77.7144 \\
85.1375 \\
81.9906 \\
76.6060 \\
82.8843 \\
83.2910 \\
77.4458 \\
82.3099 \\
83.1040 \\
83.0199 \\
85.5265 \\
39.8352 \\
82.5389 \\
34.5420 \\
84.7466 \\
82.8939 \\
229.3957 \\
84.4396 \\
80.6418 \\
-456.7448 \\
82.8977 \\
83.4175 \\
37.3074 \\
83.1521 \\
84.1186 \\
83.4525 \\
86.1422 \\
37.7675 \\
39.6057 \\
84.4073 \\
83.5122 \\
82.9128 \\
82.9282 \\
83.2353 \\
83.9440 \\
81.8986 \\
84.9047 \\
84.3894 \\
83.4590 \\
83.1182 \\
82.8830 \\
83.7115 \\
83.2870 \\
83.2407\end{array}$ & $\begin{array}{r}\max \\
77.6980 \\
76.2313 \\
78.5580 \\
85.8728 \\
82.7263 \\
77.3457 \\
84.0437 \\
84.0248 \\
78.1884 \\
62.9392 \\
83.9453 \\
83.7565 \\
86.2624 \\
10.2940 \\
83.3832 \\
34.7728 \\
85.2724 \\
83.5222 \\
239.7115 \\
85.0699 \\
81.3798 \\
-456.0126 \\
83.7303 \\
84.2578 \\
37.7675 \\
83.9967 \\
84.7495 \\
84.0841 \\
86.8744 \\
37.9994 \\
10.2940 \\
85.0368 \\
84.2458 \\
83.5431 \\
83.7696 \\
84.0779 \\
84.8934 \\
82.5301 \\
85.7414 \\
84.9153 \\
84.0895 \\
83.9601 \\
83.7305 \\
84.2377 \\
84.1287 \\
83.8734\end{array}$ & $\begin{array}{r}\text { Ave } \\
77.3333 \\
76.0750 \\
78.1635 \\
85.5177 \\
82.3796 \\
77.0095 \\
83.3967 \\
83.7047 \\
77.8213 \\
82.5895 \\
83.4805 \\
83.2999 \\
85.8989 \\
10.0876 \\
82.9968 \\
34.7543 \\
85.0178 \\
83.2525 \\
234.7477 \\
84.7525 \\
80.9731 \\
-156.2394 \\
63.3070 \\
83.8310 \\
37.5236 \\
83.6948 \\
84.4318 \\
83.8292 \\
86.5169 \\
37.8594 \\
39.9316 \\
84.7572 \\
83.8333 \\
83.2762 \\
83.3049 \\
83.6922 \\
84.4262 \\
82.2490 \\
85.3604 \\
84.6690 \\
83.7844 \\
83.5537 \\
83.3721 \\
83.9829 \\
83.7185 \\
83.5165\end{array}$ & $\begin{array}{l}\text { SD } \\
1.7159 e-01 \\
1.3363 e-01 \\
1.9959 e-01 \\
1.7234 e-01 \\
2.0709 e-01 \\
1.7680 e-01 \\
2.1750 e-01 \\
1.6611 e-01 \\
2.0566 e-01 \\
1.4783 e-01 \\
1.9353 e-01 \\
1.5709 e-01 \\
1.8045 e-01 \\
9.5554 e-02 \\
1.9630 e-01 \\
6.3238 e-02 \\
1.2219 e-01 \\
1.6238 e-01 \\
2.3478 e+00 \\
1.6075 e-01 \\
1.8447 e-01 \\
2.5501 e-01 \\
1.7474 e-01 \\
1.6361 e-01 \\
1.5700 e-01 \\
1.8475 e-01 \\
1.7938 e-01 \\
1.3952 e-01 \\
1.6528 e-01 \\
1.1381 e-01 \\
2.0256 e-01 \\
1.7562 e-01 \\
1.9080 e-01 \\
1.6441 e-01 \\
2.3371 e-01 \\
1.6955 e-01 \\
2.1920 e-01 \\
1.3539 e-01 \\
2.1264 e-01 \\
1.2494 e-01 \\
1.7633 e-01 \\
1.6876 e-01 \\
1.9823 e-01 \\
1.1469 e-01 \\
1.5511 e-01 \\
1.2392 e-01\end{array}$ & 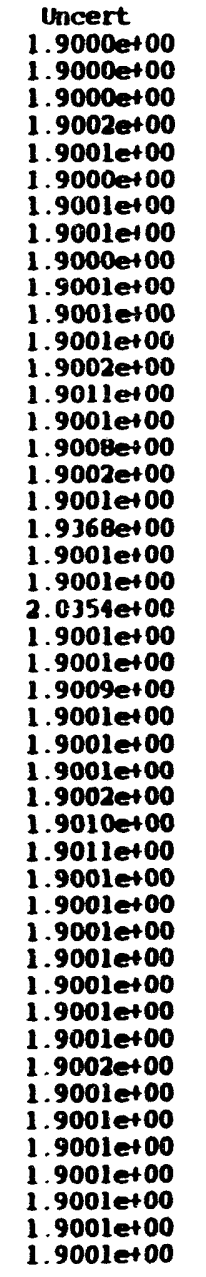 \\
\hline
\end{tabular}

\begin{tabular}{|c|c|c|c|c|c|}
\hline 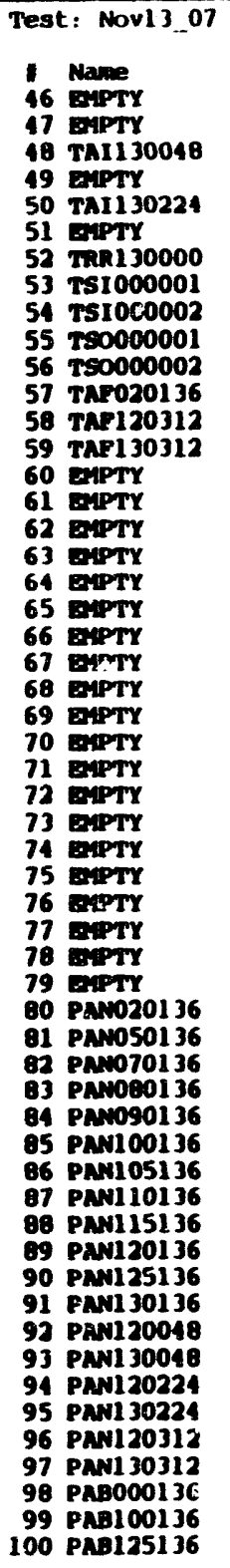 & $\begin{array}{l}\text { suml } \\
\text { Min } \\
36.1561 \\
37.5374 \\
83.2670 \\
30.3254 \\
93.2444 \\
37.7675 \\
83.0913 \\
15.8280 \\
62.2992 \\
16.0512 \\
69.8165 \\
76.4597 \\
84.7143 \\
82.3953 \\
0.3467 \\
0.3613 \\
0.3760 \\
0.3906 \\
0.2930 \\
0.3174 \\
0.3320 \\
0.3316 \\
0.3662 \\
0.3857 \\
0.4004 \\
0.4248 \\
0.4395 \\
0.4590 \\
0.4736 \\
0.4883 \\
0.5078 \\
0.5273 \\
0.5518 \\
0.5762 \\
17.5900 \\
34.5347 \\
26.7467 \\
10.7413 \\
11.8214 \\
11.3085 \\
5.5914 \\
5.9764 \\
9.1045 \\
1.3872 \\
6.3341 \\
5.0675 \\
11.8231 \\
11.5051 \\
14.1460 \\
11.6853 \\
12.4087 \\
11.4951 \\
40.4536 \\
36.5681 \\
35.3867\end{array}$ & $\begin{array}{l}\text { Date: Mun } \\
\text { Max } \\
36.8470 \\
37.7675 \\
84.6350 \\
31.2799 \\
84.2994 \\
38.4572 \\
83.9218 \\
16.4787 \\
63.4750 \\
17.1409 \\
70.6673 \\
77.3091 \\
85.7636 \\
03.2366 \\
0.3516 \\
0.3662 \\
0.3809 \\
0.4004 \\
0.2979 \\
0.3174 \\
0.3418 \\
0.3564 \\
0.3711 \\
0.3906 \\
0.4053 \\
0.4297 \\
0.4443 \\
0.4639 \\
0.4785 \\
0.4960 \\
0.5127 \\
0.5322 \\
0.5566 \\
0.5811 \\
18.9633 \\
35.6028 \\
27.8148 \\
11.7332 \\
12.4318 \\
11.8425 \\
6.0492 \\
6.5105 \\
9.7148 \\
1.9976 \\
7.0208 \\
5.8304 \\
12.7386 \\
11.9629 \\
14.6801 \\
12.4482 \\
13.4005 \\
11.8766 \\
10.5825 \\
36.7512 \\
35.5241\end{array}$ & $\begin{array}{l}\text { Nov } 1313: \\
\text { Ave } \\
36.5661 \\
37.7031 \\
84.0313 \\
30.8525 \\
83.7028 \\
38.2319 \\
83.4577 \\
16.1335 \\
62.9002 \\
16.5937 \\
70.2055 \\
76.8358 \\
85.2117 \\
82.7930 \\
0.3474 \\
0.3629 \\
0.3762 \\
0.3950 \\
0.2971 \\
0.3174 \\
0.3335 \\
0.3517 \\
0.3664 \\
0.3858 \\
0.4006 \\
0.4249 \\
0.4396 \\
0.4596 \\
0.4761 \\
0.4932 \\
0.5086 \\
0.5291 \\
0.5519 \\
0.5763 \\
18.3209 \\
35.1542 \\
27.2609 \\
11.2296 \\
12.1876 \\
11.6182 \\
5.7745 \\
6.2130 \\
9.3990 \\
1.6191 \\
6.7156 \\
5.4536 \\
12.3145 \\
11.7310 \\
14.3886 \\
12.0714 \\
13.0251 \\
11.6706 \\
10.5754 \\
36.6652 \\
35.4691 \\
\end{array}$ & 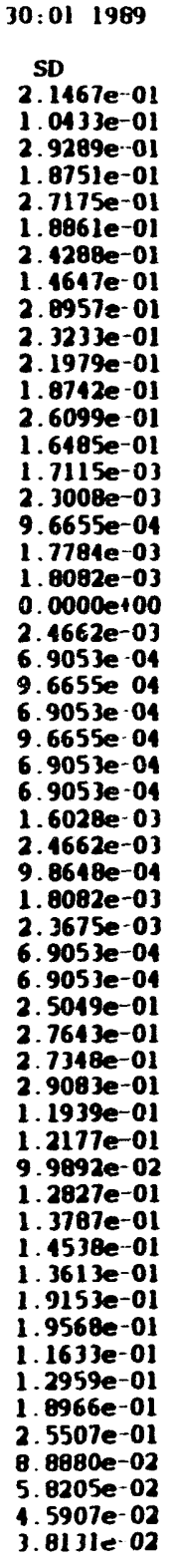 & 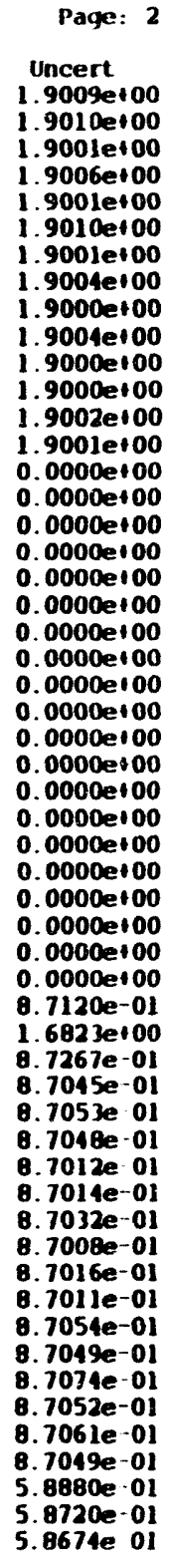 \\
\hline
\end{tabular}



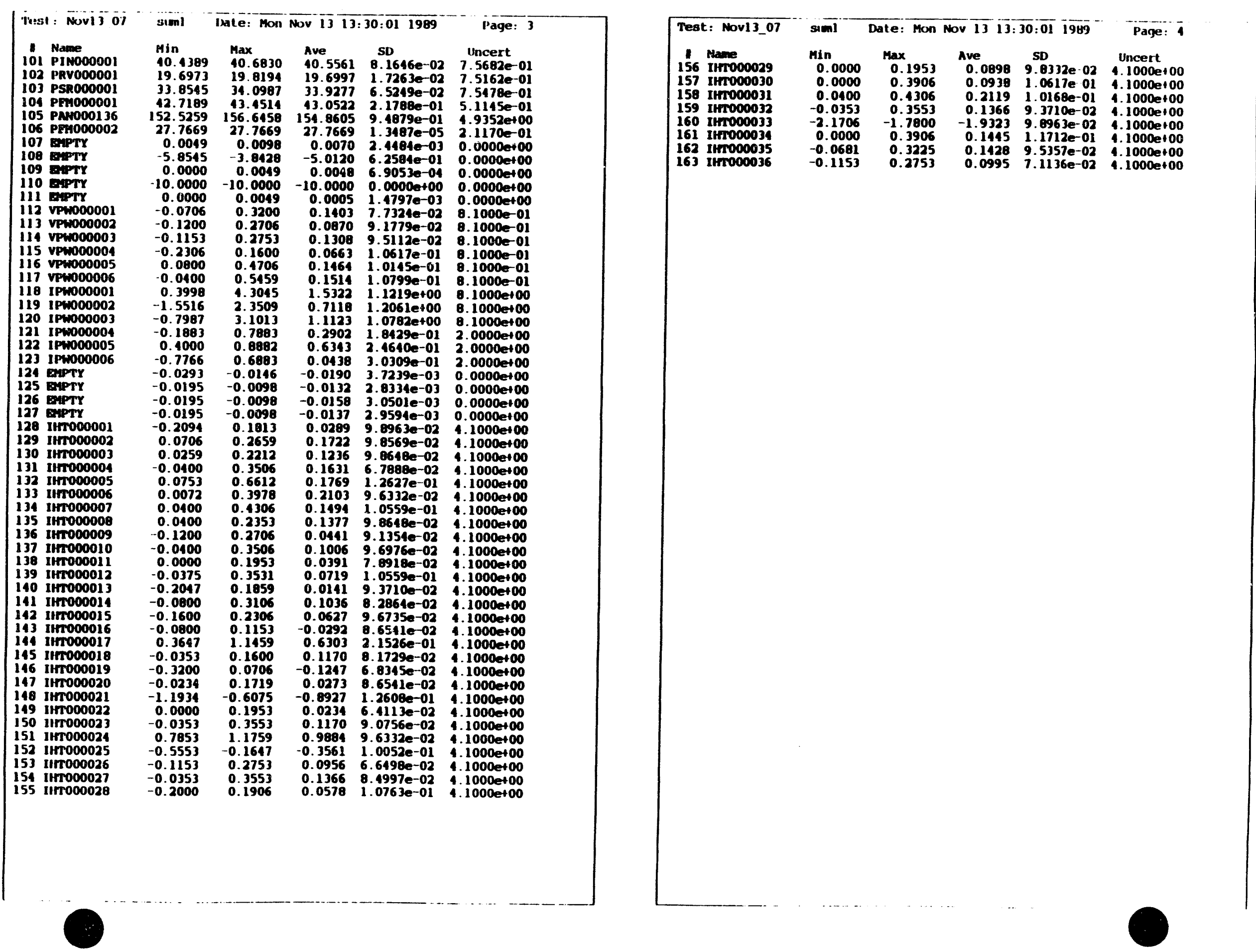


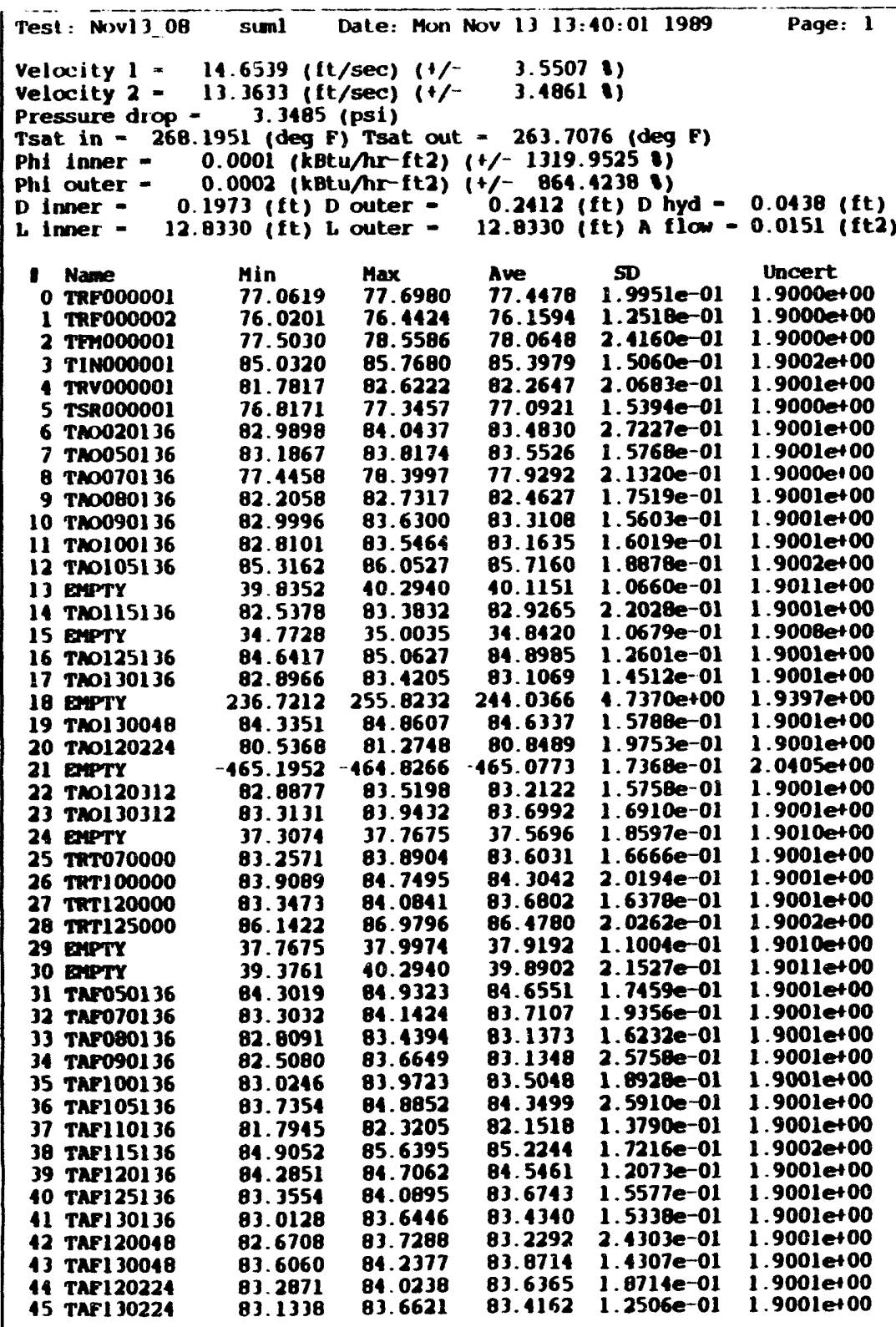

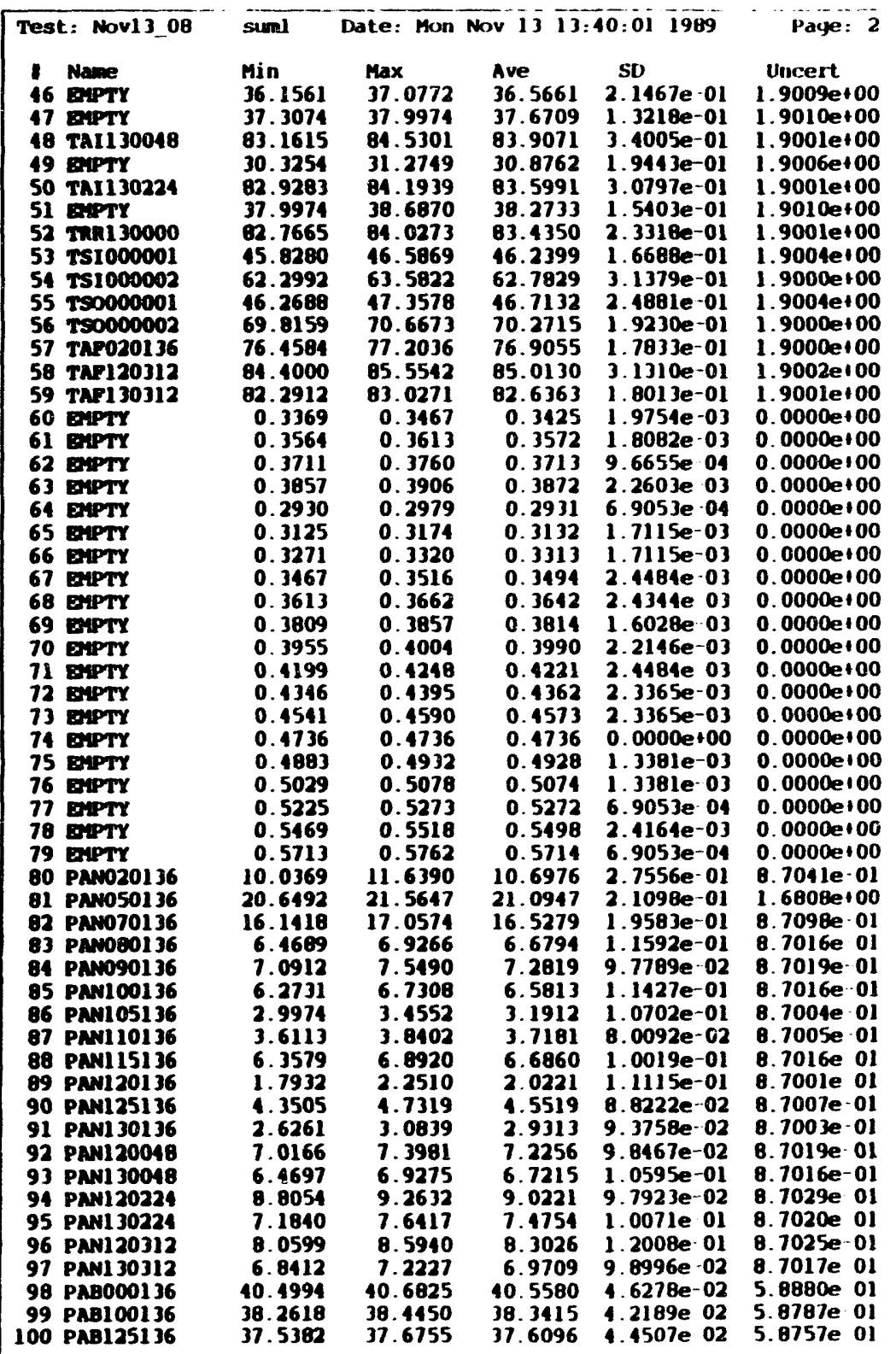



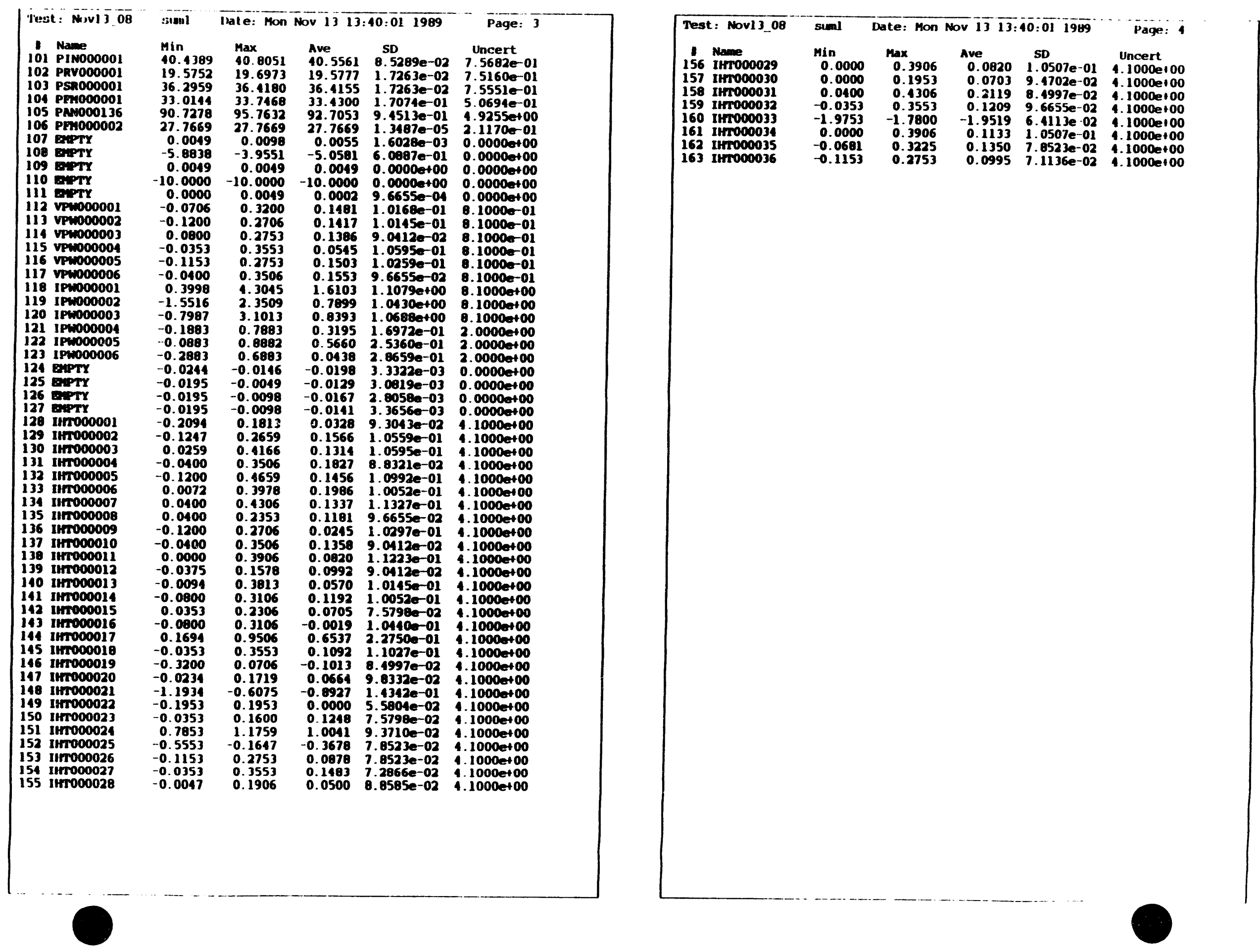


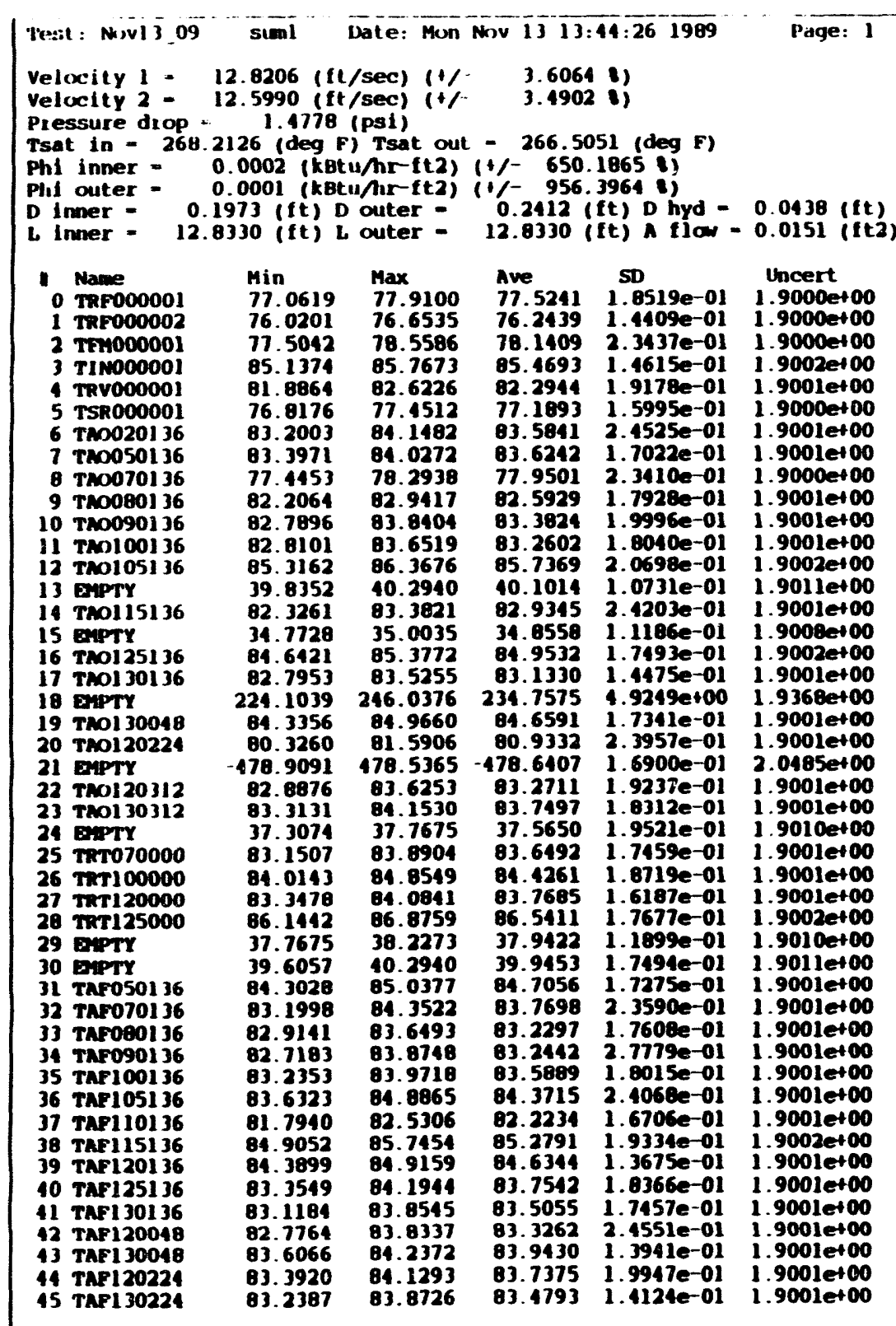

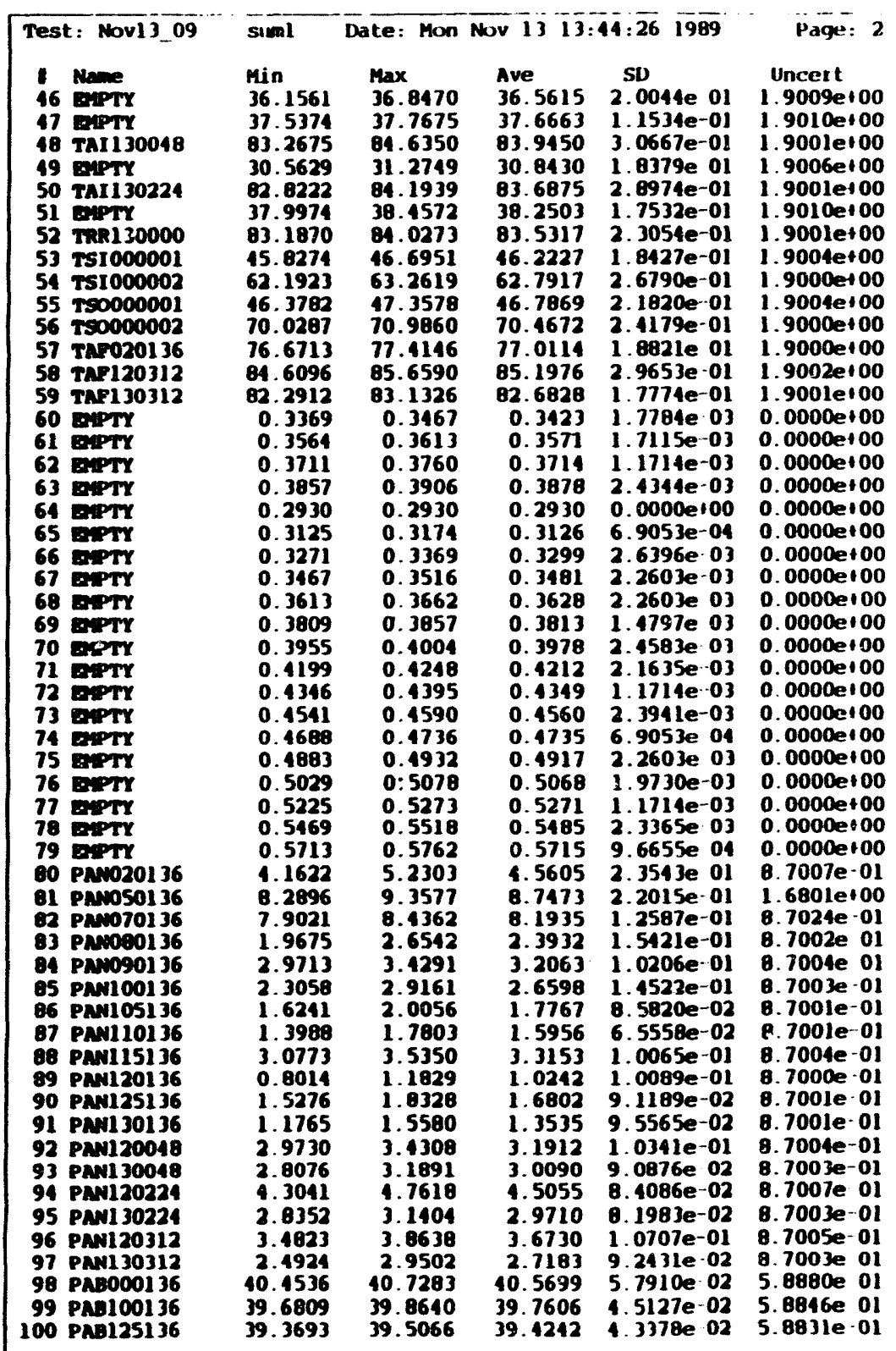




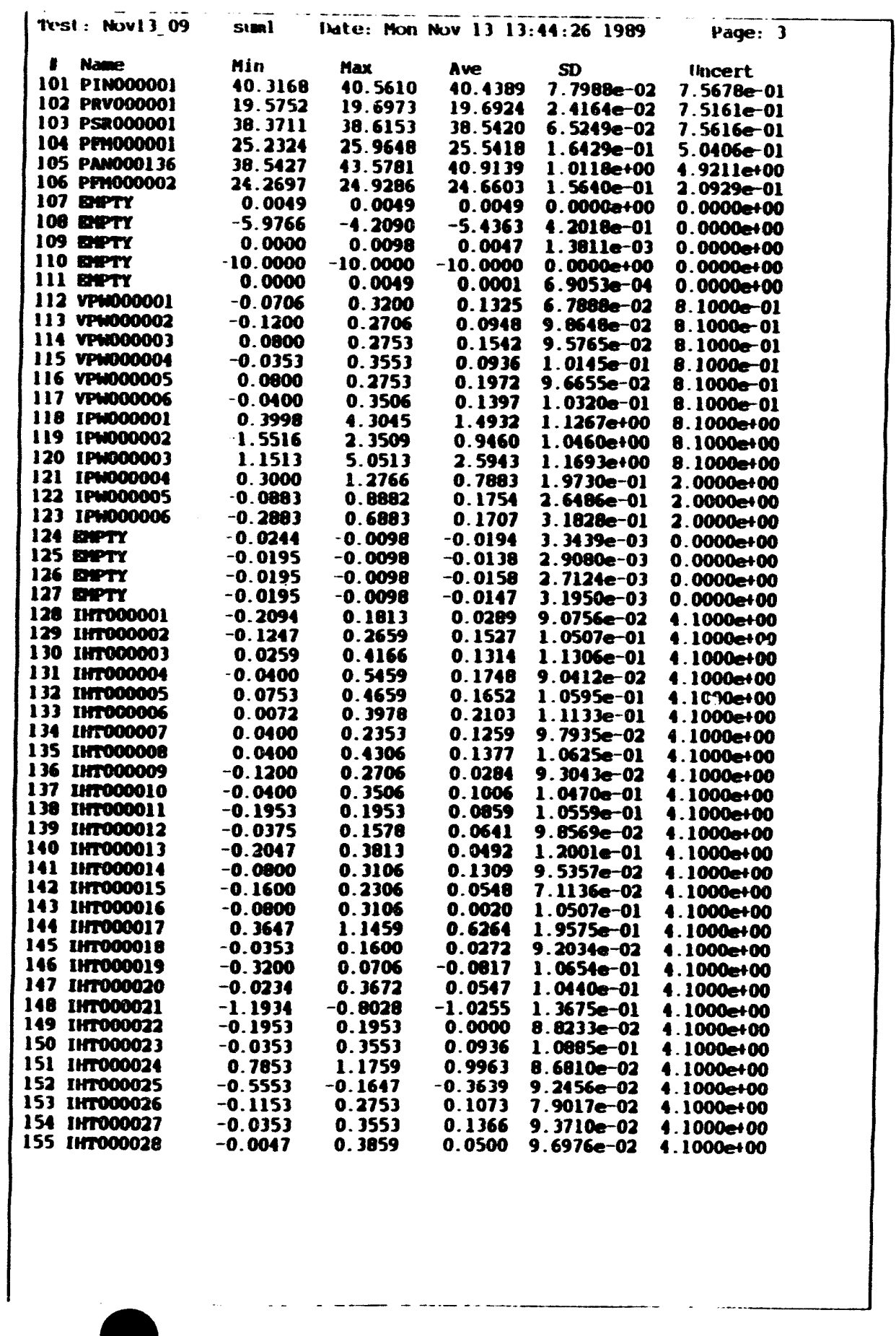

\begin{tabular}{|c|c|c|c|c|c|}
\hline Test: Nov13_09 & send & Date: Mon & Nov 131 & $14: 26 \quad 1949$ & Page: \\
\hline 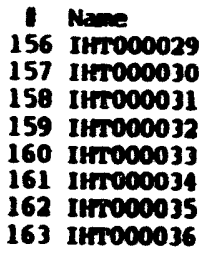 & $\begin{array}{l}\text { Min } \\
0.0000 \\
0.0000 \\
0.0400 \\
-0.0353 \\
-2.1706 \\
0.0000 \\
-0.0681 \\
-0.1153\end{array}$ & $\begin{array}{r}\operatorname{Max} \\
0.3906 \\
0.1953 \\
0.4306 \\
0.3553 \\
-1.7800 \\
0.1953 \\
0.3225 \\
0.2753\end{array}$ & $\begin{array}{r}\text { Ave } \\
0.1055 \\
0.0781 \\
0.2197 \\
0.1366 \\
-1.9480 \\
0.1055 \\
0.1194 \\
0.0917\end{array}$ & $\begin{array}{l}\text { SD } \\
1.0595 \mathrm{e}-01 \\
9.6655 \mathrm{e}-02 \\
7.7324 \mathrm{e}-02 \\
8.4997 \mathrm{e}-02 \\
8.8321 \mathrm{e}-02 \\
9.8332 \mathrm{e}-02 \\
8.7880 \mathrm{e}-02 \\
6.1257 \mathrm{e}-02\end{array}$ & 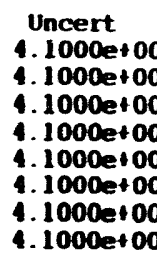 \\
\hline
\end{tabular}




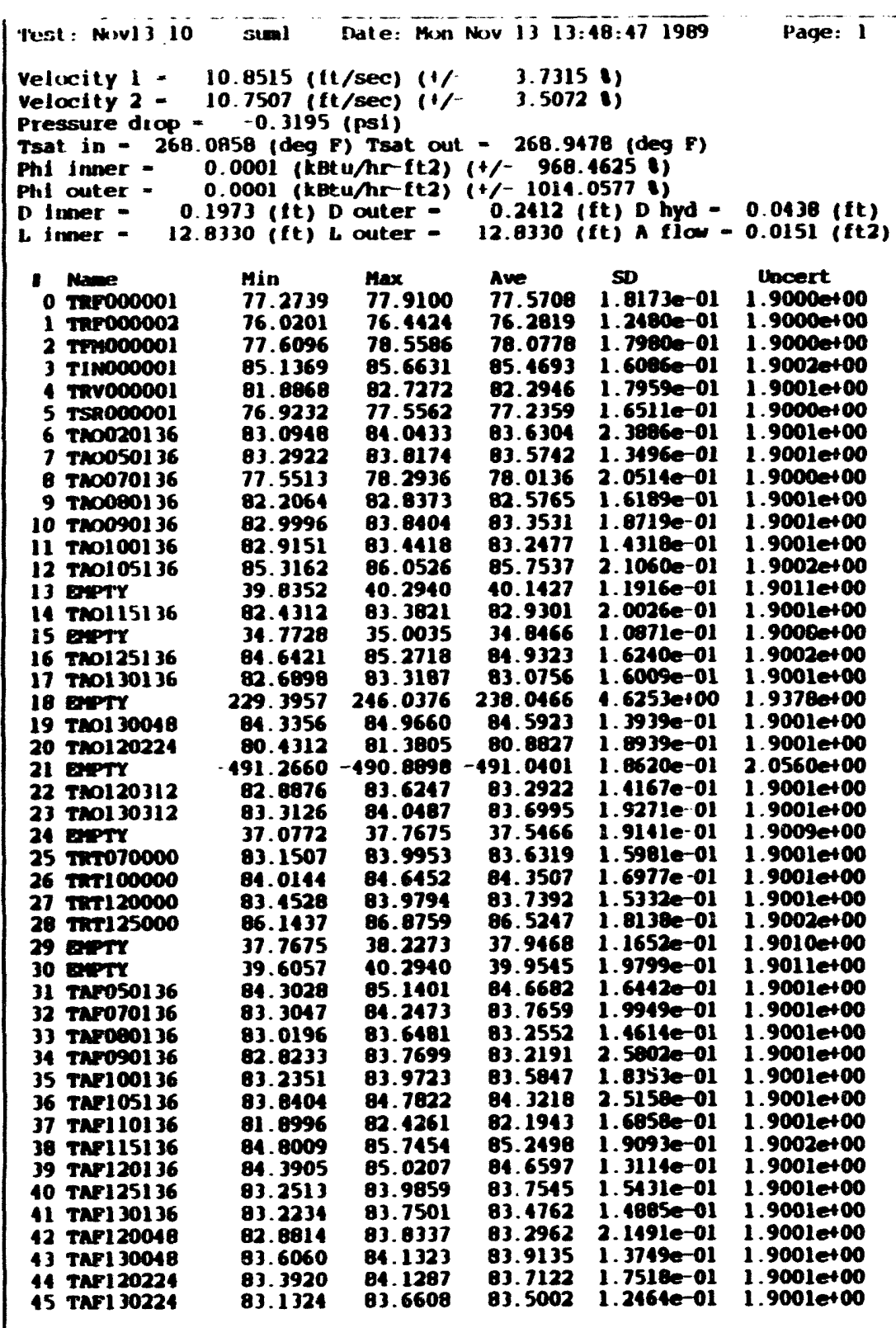

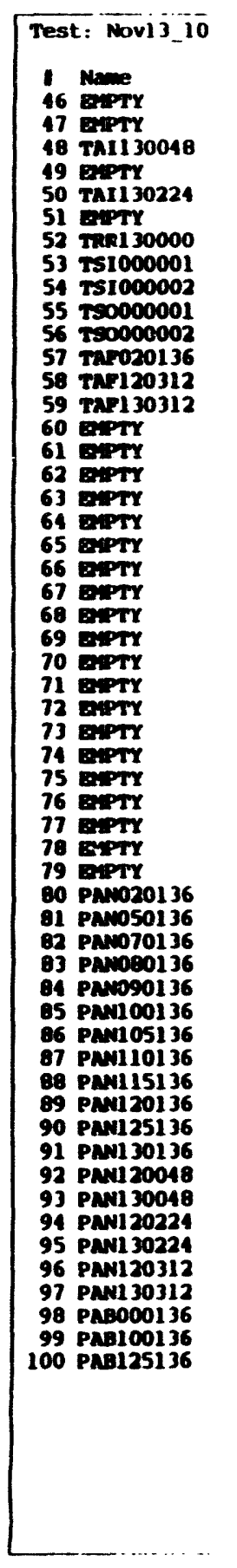

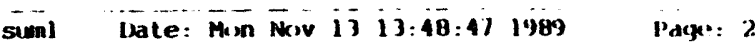

Min Max Ave Si Dncert

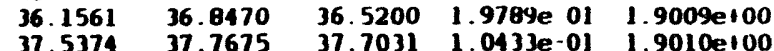
$83.3720 \quad 84.252 \quad 03.9576 \quad 3273801 \quad 1.90010100$

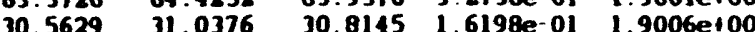
$83.1382 \quad 84.1939 \quad 83.7169 \quad 2.7148 \mathrm{e}-01 \quad 1.9001$ et00 $37.997438 .6870 \quad 38.2917 \quad 1.7402 e-01 \quad 1.9010 e+00$ $\begin{array}{lllll}82.9783 & 84.1322 & 83.4899 & 2.0522 \mathrm{e}-01 & 1.9001 \mathrm{et} 00\end{array}$

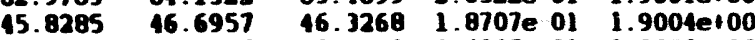
$\begin{array}{llllll}61.8732 & 62.8347 & 62.3431 & 2.4905 e-01 & 1.9000 e+00\end{array}$ $\begin{array}{llllll}46.2683 & 47.0300 & 46.6428 & 1.8019 e-01 & 1.9004 \text { to } 00\end{array}$ $\begin{array}{llllll}69.7098 & 70.7739 & 70.2971 & 2.4150 e-01 & 1.9000 e+00\end{array}$ $\begin{array}{lllllll}76.6713 & 77.4152 & 77.0282 & 2.0997 e-01 & 1.9000 e+00\end{array}$ $\begin{array}{llllll}84.6103 & 85.6590 & 85.1098 & 2.5010 e-01 & 1.9002 e+00\end{array}$ $\begin{array}{llllll}02.2917 & 83.0271 & 02.6662 & 1.7913 \mathrm{e}-01 & 1.9001 \mathrm{e}+00\end{array}$ $\begin{array}{lllll}0.3369 & 0.3467 & 0.3412 & 1.8821 e-03 & 0.0000 \text { t00 } \\ 0.3516 & 0.3564 & 0.3561 & 1.3381 e^{-03} & 0.0000+100\end{array}$ $0.3662 \quad 0.37110 .37021 .09500-030.00000100$ $0.3657 \quad 0.3906 \quad 0.38586 .9053 \mathrm{e}-040.0000100$ $\begin{array}{llllll}0.2801 & 0.2930 & 0.2923 & 1.7115 \mathrm{e}-03 & 0.0000 \mathrm{et} 00\end{array}$ $0.3125 \quad 0.3174 \quad 0.3127 \quad 9.6655$ e-04 0.0000 et 00 $\begin{array}{llllll}0.3271 & 0.3320 & 0.3288 & 2.3365 \text { e-03 } & 0.0000 \text { et } 00\end{array}$ $\begin{array}{llllll}0.3467 & 0.3516 & 0.3472 & 1.4797 e^{-03} & 0.0000 \text { et00 }\end{array}$ $\begin{array}{llllll}0.3613 & 0.3662 & 0.3618 & 1.4797 e-03 & 0.0000 t & 00\end{array}$ $\begin{array}{lllllll}0.3809 & 0.3857 & 0.3810 & 6.9053 e-04 & 0.0000 e r 00\end{array}$ $\begin{array}{lllll}0.3906 & 0.4004 & 0.3958 & 1.5314 e^{-03} & 0.0000400\end{array}$ $\begin{array}{lllll}0.4150 & 0.4248 & 0.4200 & 1.2041 \mathrm{e}-03 & 0.0000100\end{array}$ $0.43460 .4395 \quad 0.43476 .9053 e-04 \quad 0.0000$ eroo $0.45410 .4590 \quad 0.45451 .3381$ ( 0.430 .000400 $0.46880 .4736 \quad 0.4733$ 1.1714e-03 0.0000 e 00 $0.50290 .50780 .50962 .33659-030.0000100$ $0.52250 .52730 .52681 .60280-030.0000000$ $\begin{array}{llllll}0.5420 & 0.5518 & 0.5542 & 1.5314 \mathrm{e}-03 & 0.0000 \mathrm{e} 100\end{array}$ $\begin{array}{lllll}0.5713 & 0.5762 & 0.5715 & 9.6655 \mathrm{e}-04 & 0.0000 \mathrm{et00}\end{array}$ $\begin{array}{llllll}-1.7887 & -0.5680 & -1.2882 & 2.5382 \mathrm{e} \text { ol } & \mathbf{8 . 7 0 0 1 e - 0 1}\end{array}$ $\begin{array}{llllll}-3.4597 & -2.3916 & -3.0416 & 2.2823 e-01 & 1.6800 e+00\end{array}$ $\begin{array}{llllll}-0.4902 & 0.1201 & -0.1988 & 1.4840 e-01 & 8.7000 e-01\end{array}$ $\begin{array}{lllll}-1.6183 & -1.2368 & -1.4977 & 9.3872 e-02 & 8.7001 e-01\end{array}$ $\begin{array}{lllll}-0.9197 & -0.5382 & -0.6877 & 1.0103 e-01 & 8.7000 e-01\end{array}$ $-1.2800 \quad-0.0223 \quad-0.9703$ 9.3007e-02 8.7000e-01 $\begin{array}{llllll}-0.5884 & -0.2069 & -0.3946 & 0.4719 e-02 & 8.7000 e-01\end{array}$ $0.7005 \quad 0.2791 \quad-0.4109 \quad 7.2362202$ c.7000 01 $\begin{array}{llllll}-0.9534 & -0.7245 & -0.0359 & 7.5841 e^{-02} & 8.7000 \mathrm{e}-01\end{array}$ $\begin{array}{lllllll}-0.3798 & 0.0017 & -0.1432 & 7.7453 e-02 & 8.7000 e-01\end{array}$ $\begin{array}{llllll}-0.7308 & -0.5020 & -0.5813 & 6.1578 \mathrm{e}-02 & 8.7000 \mathrm{e}-01\end{array}$ $-1.1469-0.6891 \quad-0.8905 \quad 1.1613$ e $01 \quad 8.7000$ e -01 $\begin{array}{lllllll}-1.0071 & -0.5493 & -0.8057 & 1.0195 e & 01 & 8.7000 \mathrm{e} 01\end{array}$ $\begin{array}{llllll}0.1079 & 0.4694 & 0.3017 & 8.7479 e & 02 & 8.7000 e-01\end{array}$ $\begin{array}{lllllll}-1.0558 & -0.5217 & -0.8574 & 1.0679 e^{-01} & 0.7000 e-01\end{array}$ $\begin{array}{llllll}-0.5513 & -0.1798 & -0.3690 & 8.7519 \mathrm{e}-02 & 8.7000 \mathrm{e} & 01\end{array}$ $\begin{array}{llllll}-1.2460 & -0.7882 & -1.0644 & 9.489 e^{-02} & 8.7000 e & 01\end{array}$ $\begin{array}{lllll}10.1790 & 40.6825 & 40.4838 & 1.0809 \mathrm{e}-01 & 5.8876 \mathrm{e} \cdot 01\end{array}$

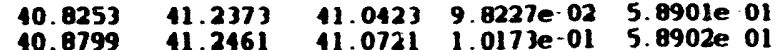




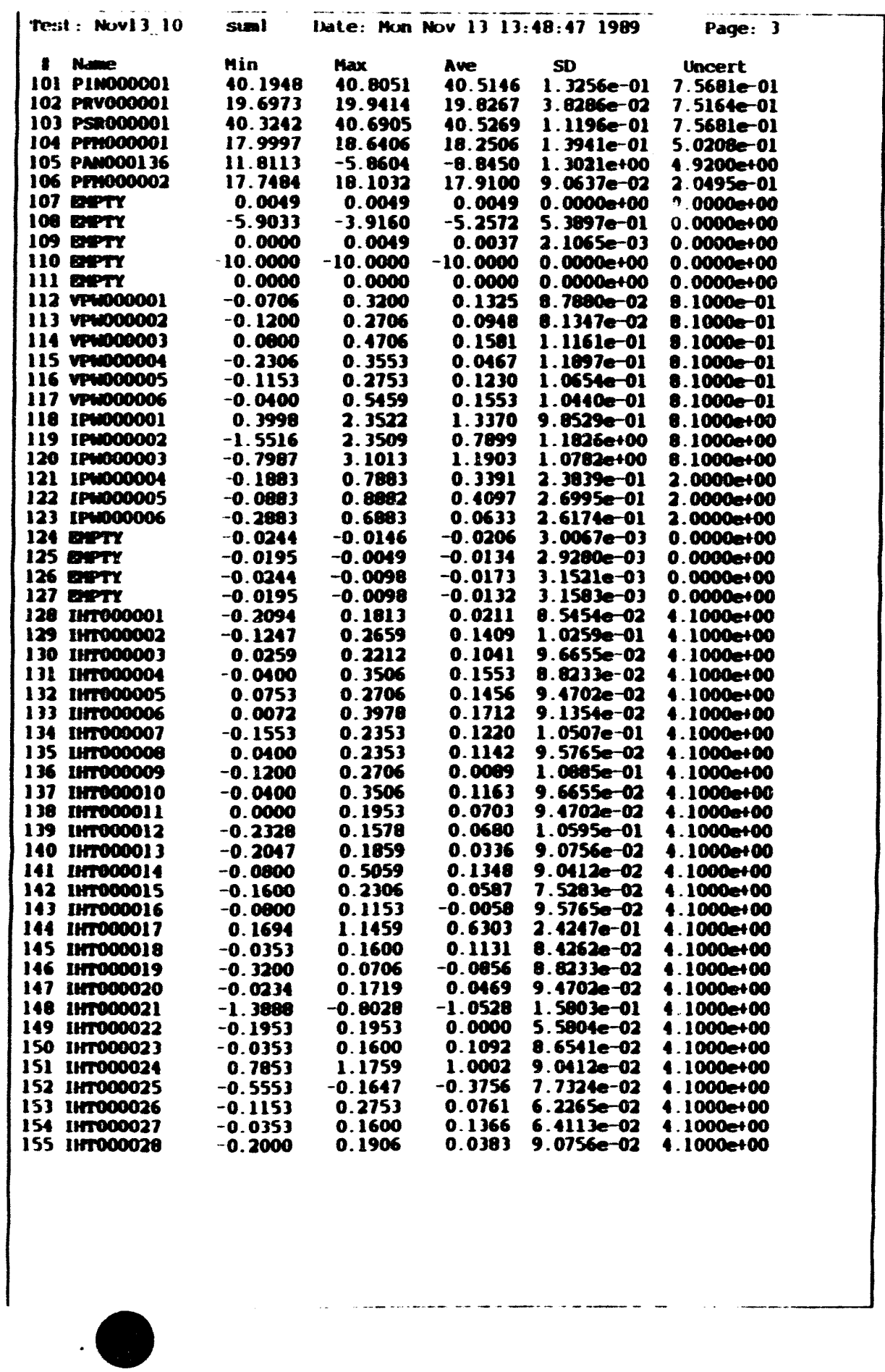

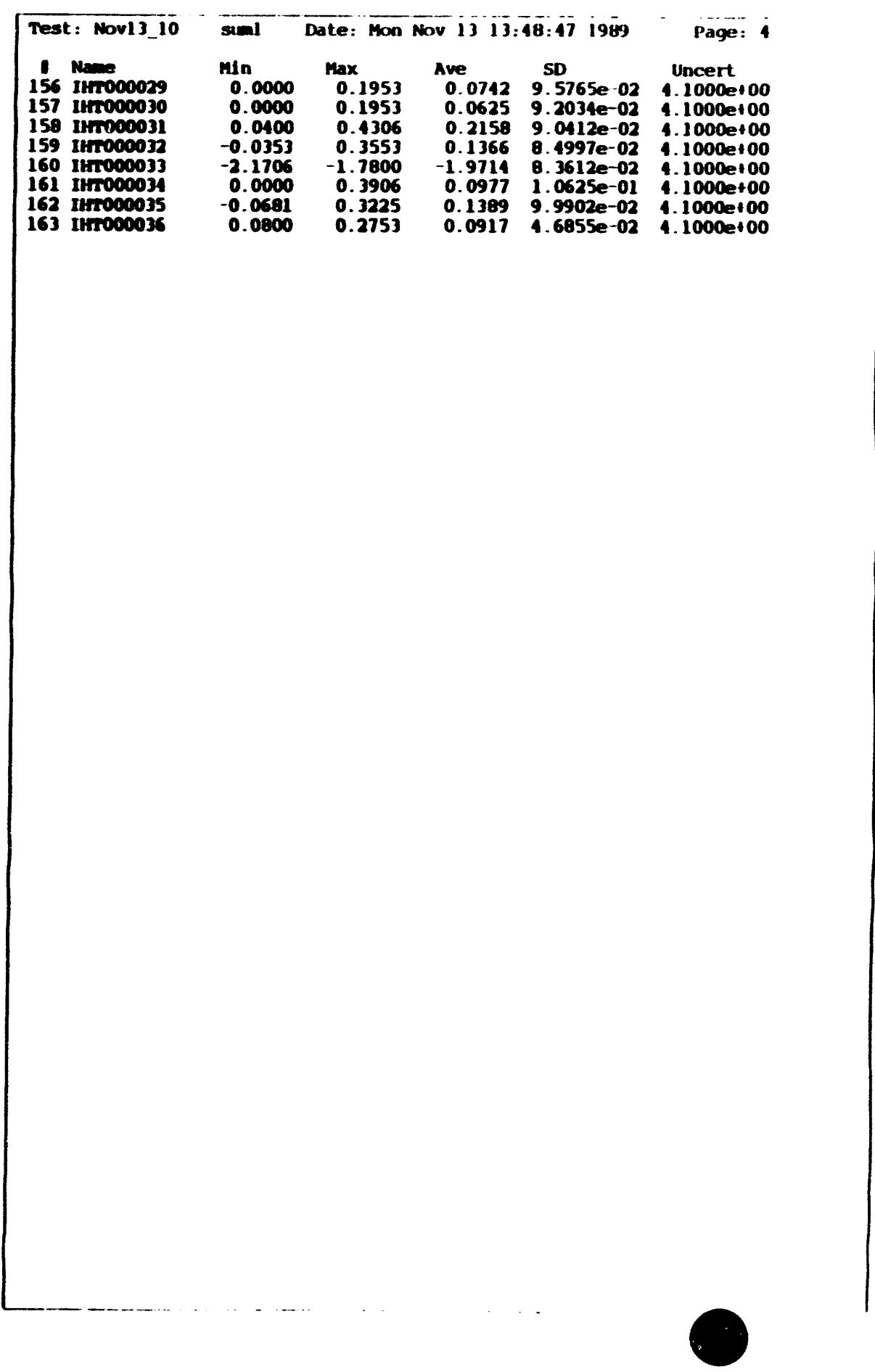




\begin{tabular}{|c|c|c|c|c|c|}
\hline $\begin{array}{l}\text { Tesil: Muvl } 3 \text { 11 } \\
\text { Velucity } 1= \\
\text { Velocity } 2= \\
\text { Pressure drop } \\
\text { Tsat in = } 268 \\
\text { Phi inmer = } \\
\text { Plil outer = } \\
\text { D inmer = } 0 \\
\text { L imer = } 12\end{array}$ & $\begin{array}{c}\text { sinl } \\
8.9236 \text { (tt } \\
8.8072 \text { ift } \\
-1.8307 \\
1493 \text { (deg F } \\
0.0002 \text { (kBt } \\
0.0002 \text { (kBt } \\
1973 \text { (tt) } \\
8330 \text { (ft) }\end{array}$ & $\begin{array}{l}\text { Wate: Mon } \\
\text { /sec) (1' } \\
\text { /sec) (1/ } \\
\text { (psi) } \\
\text { J Tset out } \\
\text { u/hr (12) } \\
\text { u/Mr ft2) } \\
\text { outer = } \\
\text { outer - }\end{array}$ & $\begin{array}{r}\text { Hov } 1313: \\
4.0227 \\
3.5486 \\
-\quad 271.07 \\
1+1-902 \\
1+9911 \\
0.2412 \\
12.8330\end{array}$ & $\begin{array}{l}16 \text { (deg F) } \\
2618 \text { i) } \\
7344 \text { \& } \\
(t) \text { D hyd - } \\
\text { f(t) A flow - }\end{array}$ & $\begin{array}{l}0.0438 \text { (ft) } \\
0.0151 \text { (ft2) }\end{array}$ \\
\hline 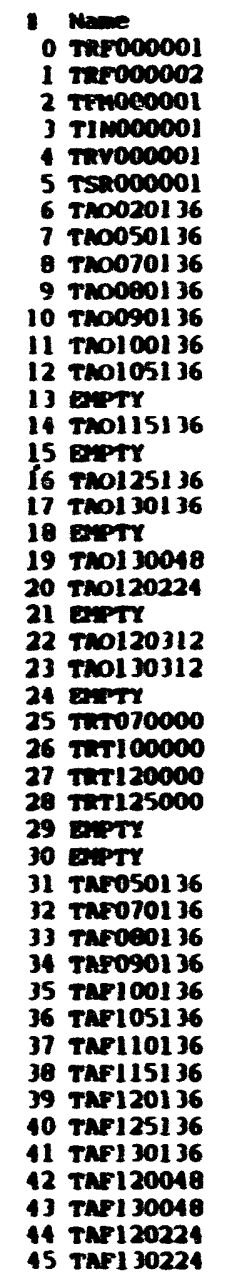 & $\begin{array}{l}\text { Min } \\
77.4860 \\
76.0201 \\
77.7150 \\
85.1374 \\
81.6775 \\
77.0292 \\
83.2003 \\
83.1864 \\
77.7633 \\
82.2079 \\
82.8951 \\
82.9156 \\
85.3167 \\
39.8352 \\
82.3250 \\
34.7728 \\
84.6427 \\
82.6930 \\
221.8276 \\
84.2317 \\
80.5369 \\
503.3629 \\
82.8891 \\
83.1032 \\
37.3074 \\
83.3612 \\
84.0149 \\
83.3481 \\
85.9357 \\
37.7675 \\
39.6057 \\
84.3033 \\
83.3062 \\
82.9164 \\
82.8236 \\
83.2351 \\
83.7372 \\
81.7956 \\
84.9057 \\
84.2867 \\
83.3568 \\
83.1186 \\
82.7747 \\
83.6066 \\
83.2871 \\
83.1329\end{array}$ & $\begin{array}{r}\operatorname{Max} \\
77.9100 \\
76.6535 \\
78.6640 \\
85.7679 \\
82.6231 \\
77.5572 \\
84.0427 \\
83.8186 \\
78.3990 \\
82.9423 \\
83.7360 \\
83.5468 \\
86.1573 \\
10.5234 \\
83.2761 \\
35.0035 \\
85.2723 \\
83.4237 \\
235.9722 \\
84.6523 \\
81.1693 \\
503.3629 \\
83.5198 \\
83.9438 \\
37.7675 \\
83.8689 \\
84.7495 \\
84.0943 \\
86.7727 \\
38.2273 \\
10.2940 \\
84.9332 \\
84.1439 \\
83.5443 \\
83.8754 \\
83.9722 \\
84.6786 \\
82.5311 \\
85.6406 \\
84.9164 \\
84.0900 \\
83.6448 \\
83.7272 \\
84.2377 \\
84.0238 \\
83.7651\end{array}$ & $\begin{array}{l}\text { Ave } \\
77.6556 \\
76.3537 \\
78.0717 \\
85.4924 \\
82.1800 \\
77.3015 \\
83.5902 \\
83.5893 \\
78.0076 \\
82.5666 \\
83.3008 \\
83.2206 \\
85.7852 \\
10.1748 \\
62.8641 \\
34.8281 \\
84.9136 \\
82.9918 \\
228.9000 \\
84.4817 \\
80.8907 \\
-503.3631 \\
83.2522 \\
83.5927 \\
37.5696 \\
83.6632 \\
84.3153 \\
83.6994 \\
86.5232 \\
37.9330 \\
39.9728 \\
84.6789 \\
83.7226 \\
83.2204 \\
83.2129 \\
83.5952 \\
84.3287 \\
82.1717 \\
85.2689 \\
84.5828 \\
83.7028 \\
83.4279 \\
83.2553 \\
83.8651 \\
83.6765 \\
83.1044\end{array}$ & $\begin{array}{r}\text { s0 } \\
1.5142 e-01 \\
1.3549 e-01 \\
2.1508 e-01 \\
1.5296 e-01 \\
2.1535 e-01 \\
1.3991 e-01 \\
2.5124 e-01 \\
1.2838 e-01 \\
2.0242 e-01 \\
1.6831 e-01 \\
1.9335 e-01 \\
1.4610 e-01 \\
1.9718 e-01 \\
1.4089 e-01 \\
2.1689 e-01 \\
9.9527 e-02 \\
1.1833 e-01 \\
1.5945 e-01 \\
3.3689 e+00 \\
1.2350 e-01 \\
1.4089 e-01 \\
2.4662 e-04 \\
1.4768 e-01 \\
1.5971 e-01 \\
1.8597 e-01 \\
1.4928 e-01 \\
1.7102 e-01 \\
1.4010 e-01 \\
1.8399 e-01 \\
1.1418 e-01 \\
2.4089 e-01 \\
1.5733 e-01 \\
2.0005 e-01 \\
1.8360 e-01 \\
2.3193 e-01 \\
1.8913 e-01 \\
2.1370 e-01 \\
1.6039 e-01 \\
1.6260 e-01 \\
1.4172 e-01 \\
1.5738 e-01 \\
1.3345 e-01 \\
2.5504 e-01 \\
1.2066 e-01 \\
2.0961 e-01 \\
1.1485 e-01\end{array}$ & 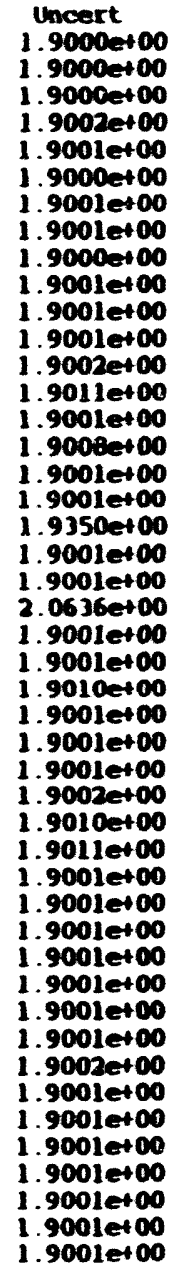 \\
\hline
\end{tabular}

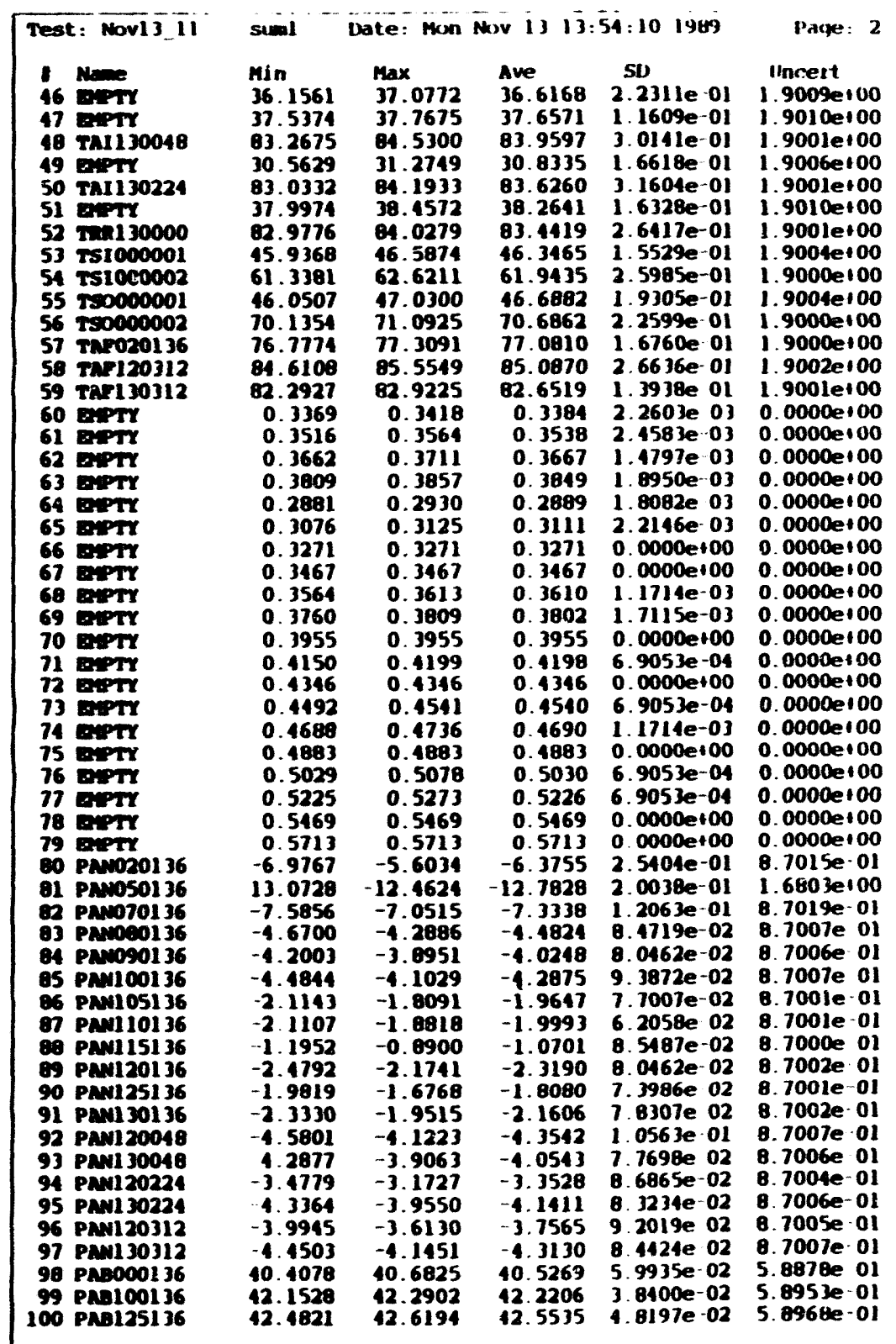




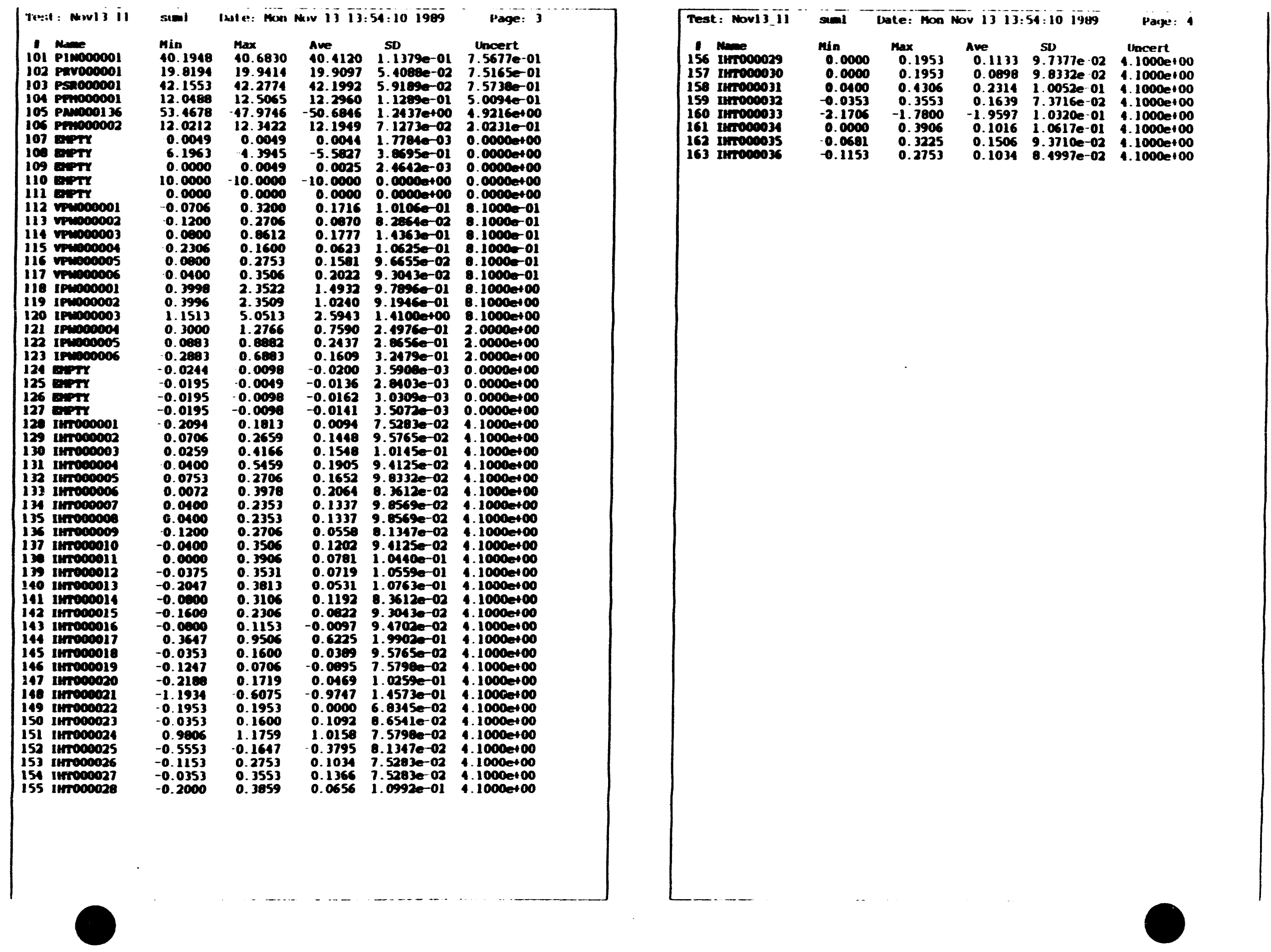




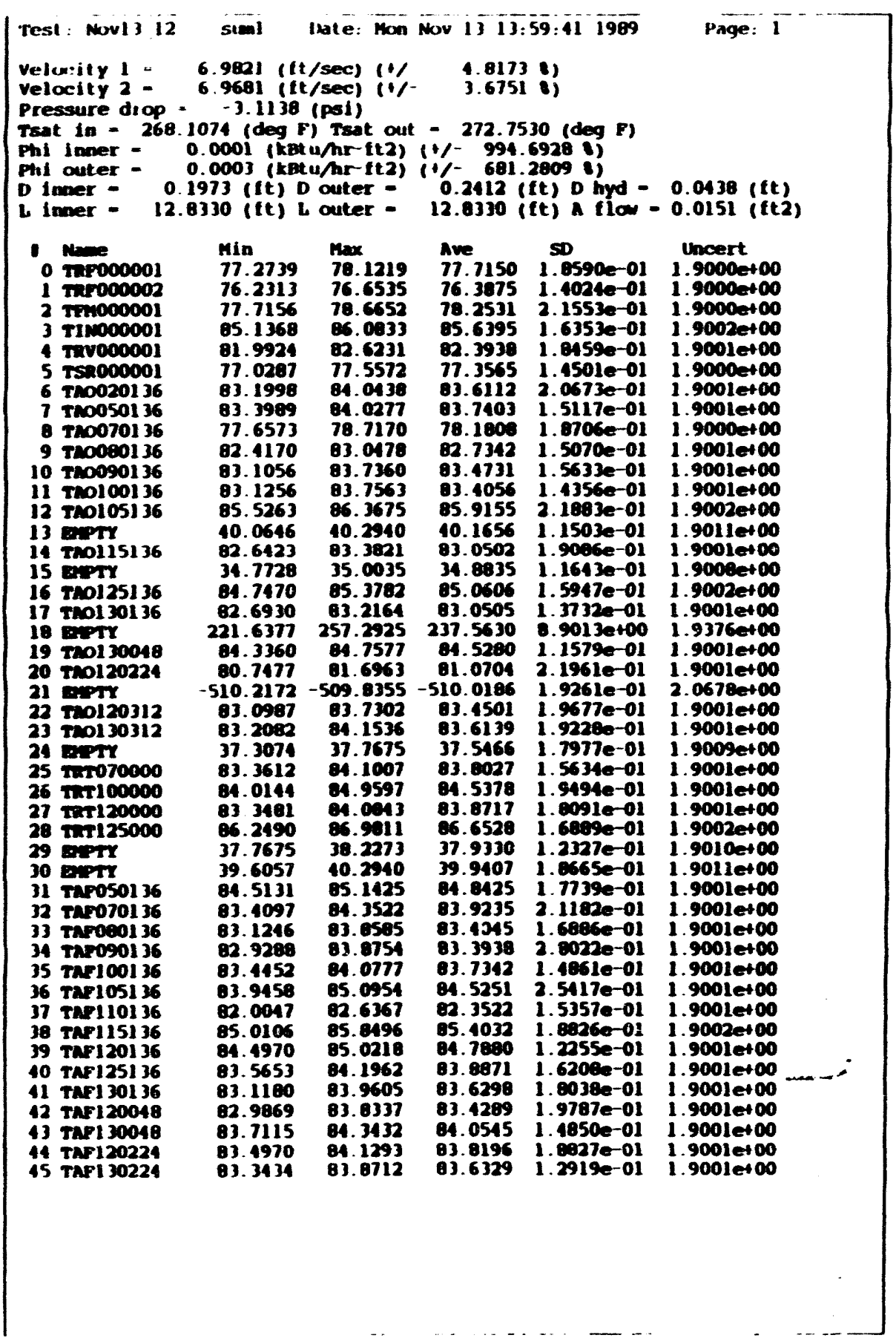

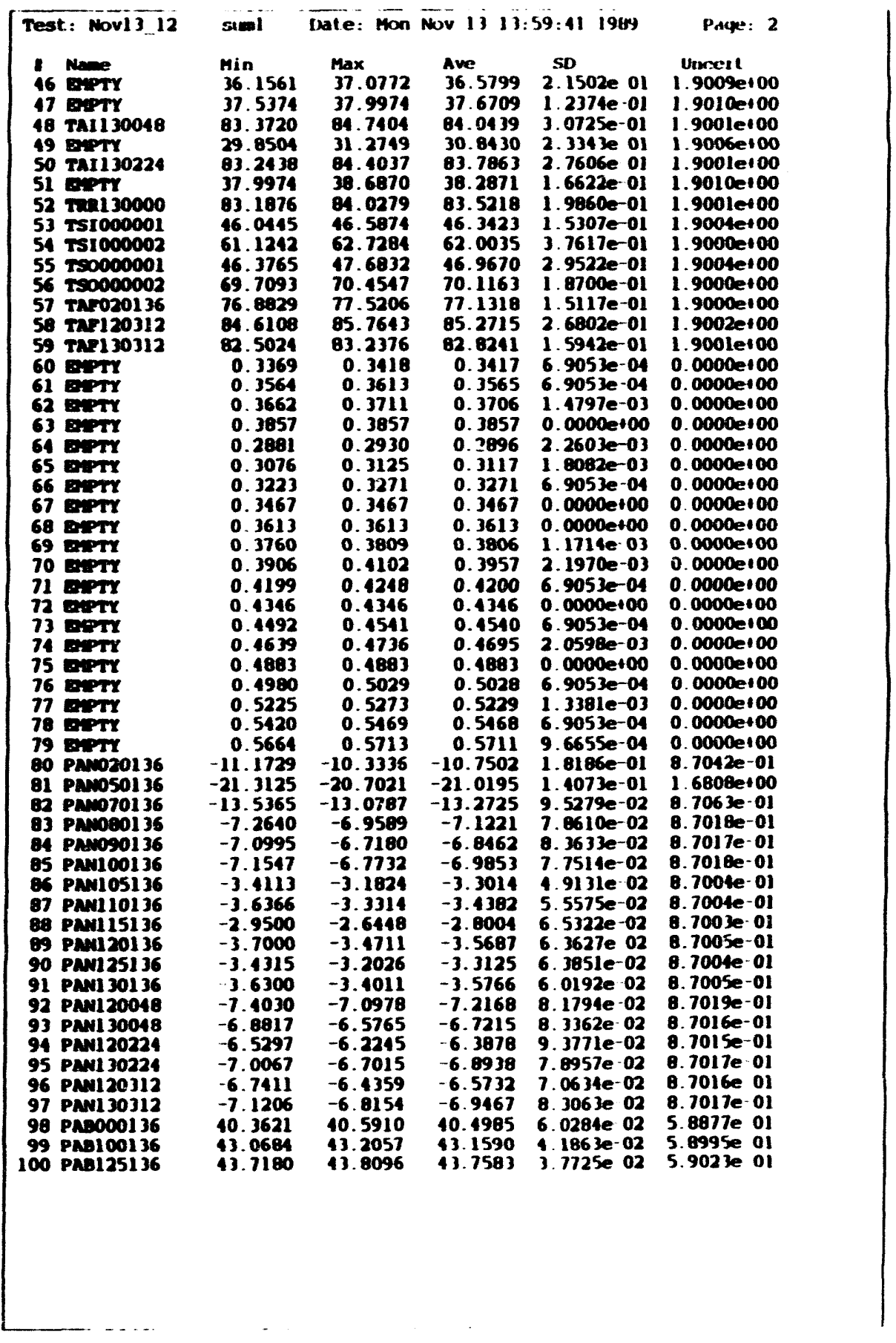




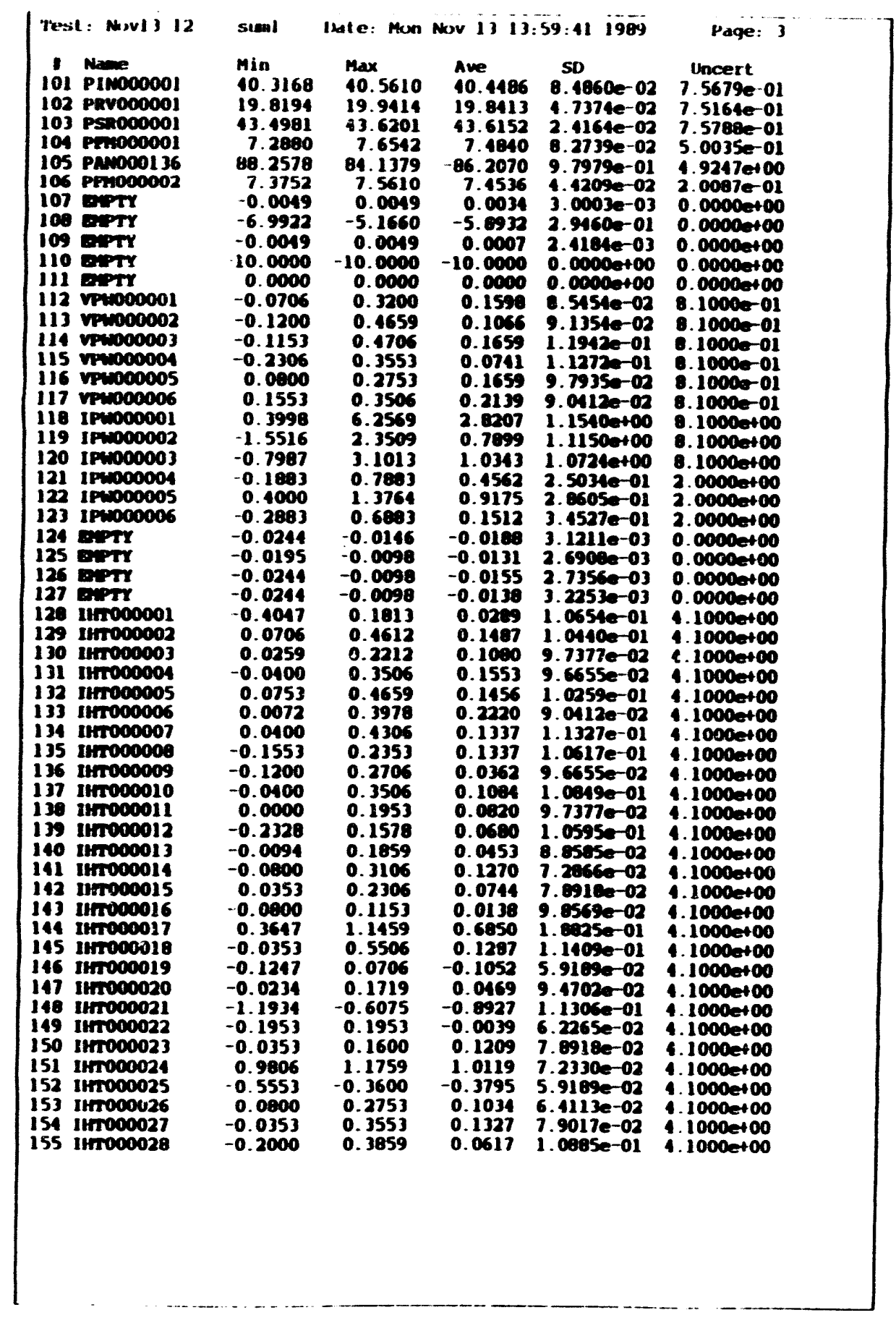

\begin{tabular}{|c|c|c|c|c|c|c|}
\hline & Nov1 3,12 & mi & Date: Mon & Nov is i3: & $59: 11 \quad 1989$ & Packe: \\
\hline $\begin{array}{l}1 \\
156 \\
157 \\
158 \\
159 \\
160 \\
161 \\
162 \\
163\end{array}$ & 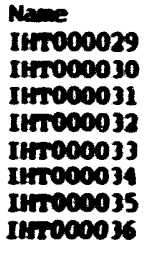 & $\begin{array}{l}\text { Min } \\
-0.1953 \\
0.0000 \\
0.0400 \\
-0.0353 \\
-2.1706 \\
0.0000 \\
-0.0681 \\
-0.1153\end{array}$ & $\begin{array}{r}\text { Max } \\
0.3906 \\
0.1953 \\
0.4306 \\
0.3553 \\
-1.7800 \\
0.3906 \\
0.3225 \\
0.2753\end{array}$ & $\begin{array}{l}\text { Ave } \\
0.1016 \\
0.0977 \\
0.2314 \\
0.1414 \\
-1.9362 \\
0.1367 \\
0.1506 \\
0.0995\end{array}$ & $\begin{array}{c}\text { so } \\
1.1995 \mathrm{e} 01 \\
9.8648 \mathrm{e}-02 \\
6.3612 \mathrm{e}-02 \\
1.0320 \mathrm{e}-01 \\
8.8233 \mathrm{e}-02 \\
1.2001 \mathrm{e}-01 \\
7.5283 \mathrm{e}-02 \\
8.1347 \mathrm{e}-02\end{array}$ & 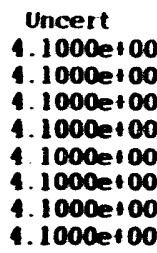 \\
\hline
\end{tabular}




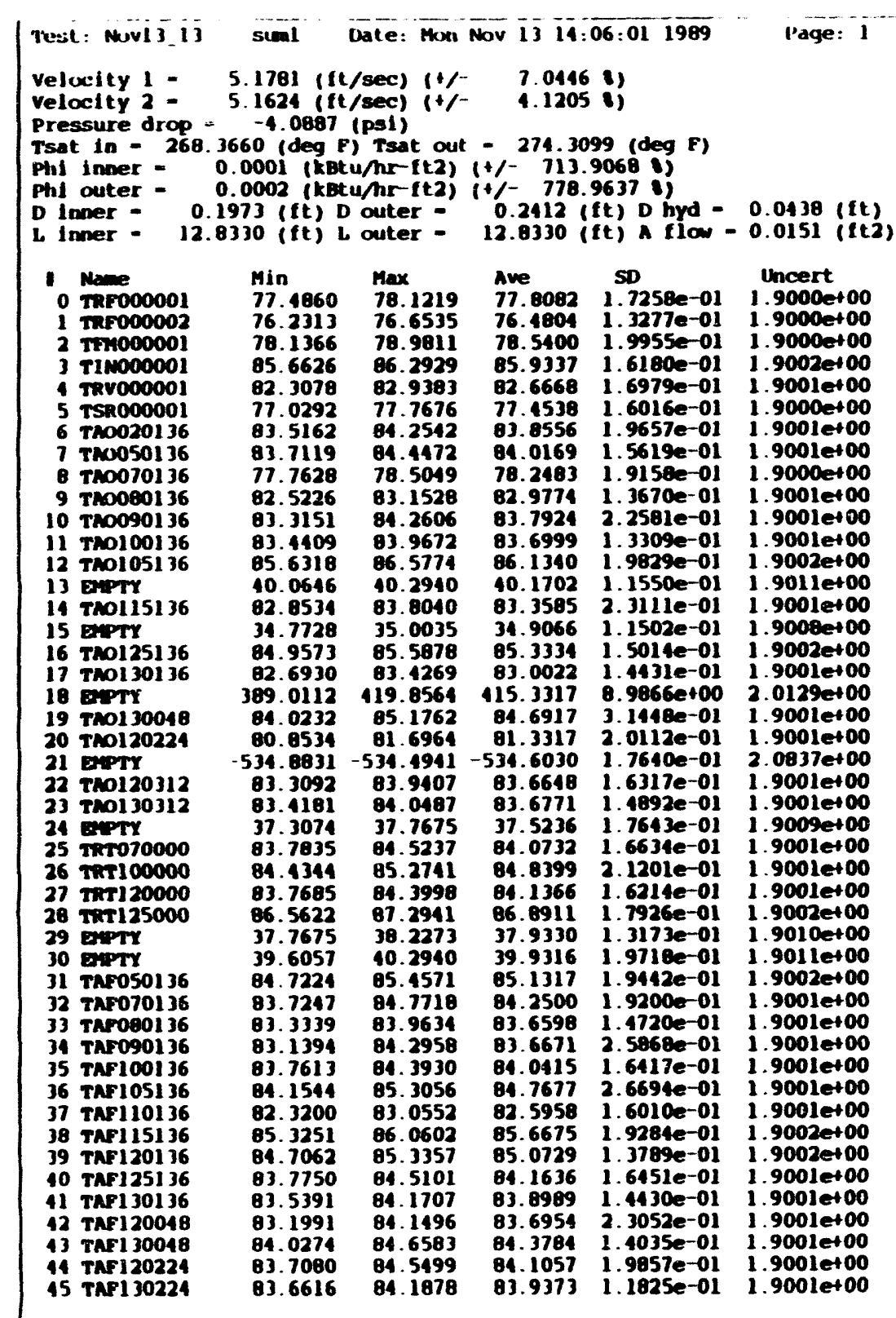

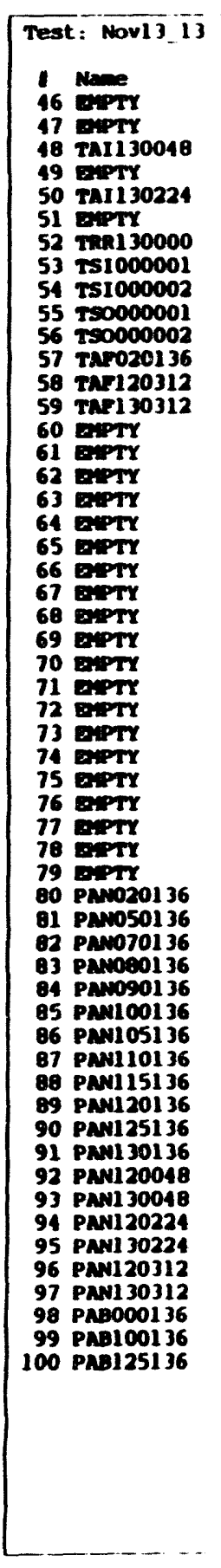

suml Date: Mon Nov 13 14:06:01 1989 Paqe: 2 Min Max Ave SD Uncert $\begin{array}{lllll}36.1561 & 37.0772 & 36.5937 & 2.3835 e-01 & 1.9009 e 100\end{array}$ $83.8989 \quad 85.0555 \quad 84.36792 .6474 \mathrm{e}-01 \quad 1.9001000$ $30.562931 .2749 \quad 30.09102 .176001$ $83.5604 \quad 04.6145 \quad 04.0567 \quad 300970-01 \quad 1.90010100$

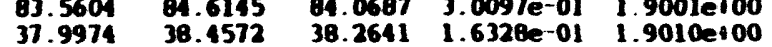
$83.3980 \quad 84.3425 \quad 83.8703$ 2.2461e-01 1.900let00 $\begin{array}{llll}4.6119 & 46.4797 \quad 46.0826 & 1.9048=-01 & 1.9004 e+00\end{array}$ $\begin{array}{llllll}60.6967 & 61.7666 & 61.2639 & 2.5226 e-01 & 1.9001 e+00\end{array}$ $\begin{array}{llllll}45.7226 & 46.9197 & 46.3735 & 2.0454 e \cdot 01 & 1.9004 e+00\end{array}$ $\begin{array}{lllllll}69.9227 & 70.6674 & 70.2653 & 1.9138 e-01 & 1.9000+00\end{array}$ $\begin{array}{lllllll}76.8629 & 77.6266 & 77.2632 & 1.6904 e \cdot 01 & 1.9000 e+00\end{array}$ $\begin{array}{llllll}85.0299 & 86.0790 & 85.5148 & 2.5829 e-01 & 1.9002 e+00\end{array}$ $\begin{array}{lllll}82.7120 & 83.5526 & 03.1136 & 1.7714 e-01 & 1.900 l e t 00\end{array}$ $\begin{array}{lllll}0.3418 & 0.3467 & 0.3465 & 9.6655 \text { e-04 } & 0.0000 \text { et } 00 \\ 0.3613 & 0.3613 & 0.3613 & 0.0000 e+00 & 0.0000 e 100\end{array}$ $0.37110 .3760 \quad 0.3757 \quad 1.17140-030.0000000$ $0.3657 \quad 0.3906 \quad 0.3905 \quad 6.9053 \mathrm{e}-04040.0000400$ $\begin{array}{llllll}0.2881 & 0.2930 & 0.2929 & 6.9053 \mathrm{e}-04 & 0.0000 \mathrm{et} 00\end{array}$ $\begin{array}{llllll}0.3076 & 0.3174 & 0.3126 & 1.2041 \text { e-03 } & 0.0000100\end{array}$ $\begin{array}{lllll}0.3271 & 0.3320 & 0.3292 & 2.4344 e-03 & 0.0000100\end{array}$ $\begin{array}{llllll}0.3467 & 0.3516 & 0.3475 & 1.8082 e-03 & 0.0000 e 100\end{array}$ $\begin{array}{llllll}0.3613 & 0.3662 & 0.3629 & 2.3008 \mathrm{e}-03 & 0.0000 \mathrm{t} 00\end{array}$ $\begin{array}{lllllll}0.3809 & 0.3857 & 0.3811 & 9.6655 \text { - } 04 & 0.0000 \text { t00 }\end{array}$ $\begin{array}{lllllll}0.3955 & 0.4004 & 0.3974 & 2.3941 \text { e } 03 & 0.0000 \text { et00 }\end{array}$ $\begin{array}{llllll}0.4199 & 0.4248 & 0.4214 & 2.2603 e-03 & 0.0000 e 100\end{array}$ 0.43460 .43950 .4351 1.4757e-03 0.0000 e100 $\begin{array}{llllll}0.4541 & 0.4590 & 0.4559 & 2.3675 e-03 & 0.0000 e+00\end{array}$

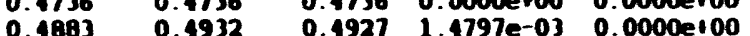
$0.50290 .50780 .50672 .0432 e-030.0000100$ 0.52250 .52730 .5271 1.0714e-03 0.00000100 $\begin{array}{lllll}0.5225 & 0.5273 & 0.5271 & 1.1714 e-03 & 0.0000 e+100 \\ 0.5469 & 0.5518 & 0.5494 & 2.4642 e-03 & 0.0000 \text { et00 }\end{array}$ $\begin{array}{llllll}0.5713 & 0.5762 & 0.5724 & 2.0432 e-03 & 0.0000 e 100\end{array}$ $-14.3772-13.7668-14.1529 \quad 1.2466 \mathrm{e}-01 \quad 8.7072 e-01$ $\begin{array}{lllll}-27.5686 & -26.9583 & -27.3153 & 1.4979 e-01 & 1.6814 e+c 0\end{array}$ $\begin{array}{lllll}-17.9615 & -17.5801 & -17.7632 & 9.3758 e-02 & 0.7113 e-01\end{array}$ $\begin{array}{lllll}-9.3240 & -9.0188 & -9.1165 & 8.0151 e-02 & 8.7030 e-01\end{array}$ $\begin{array}{lllllll}-9.0831 & -8.7780 & -8.9427 & 6.6046 e-02 & 0.7029 e-01\end{array}$ $-9.2146-8.9094-9.09416 .1828 e-02 \quad 8.7030 e-01$

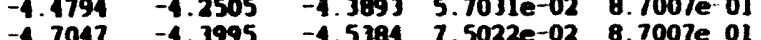

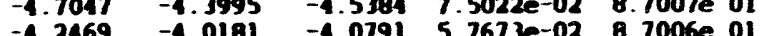
$-4.6918-4.3866-45226.59192-02$ 8.7007e. 01 $-4.4996-4.2708-438216.9293 \mathrm{e}-02 \quad 8.7007 \mathrm{e} 01$ $-4.6981-4.3929-4.5455$ i.2297e 02 B.7007e-01 $\begin{array}{lllllll}-9.6155 & -9.3866 & -9.4781 & 6.1655 e-02 & 8.7032 e-01\end{array}$ $\begin{array}{lllllll}-8.8654 & -8.5602 & -8.7662 & 5.8185 e-02 & 8.7028 e & 01\end{array}$ $\begin{array}{llllll}-8.8185 & -8.6659 & -8.7239 & 5.0089 e-02 & 0.7027 e-01\end{array}$ $\begin{array}{llllll}-9.1429 & -8.8378 & -9.0132 & 7.1054 e^{02} & 0.7029 & 01\end{array}$ $\begin{array}{llllll}-0.9536 & -0.6484 & -8.7873 & 9.0784 e .02 & 8.7028 e & 01\end{array}$

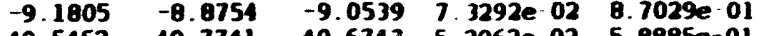
$40.5452 \quad 10.774 \quad 40.67435 .2062 e-02 \quad 5.8885 e^{-01}$ $\begin{array}{llllll}44.0297 & 14.1670 & 14.0791 & 3.7952 \mathrm{e}-02 & 5.9038 \mathrm{e} & 01 \\ 14.8167 & 14.9540 & 14.8991 & 4.4353 \mathrm{e}-02 & 5.9076 \mathrm{e} & 01\end{array}$ 


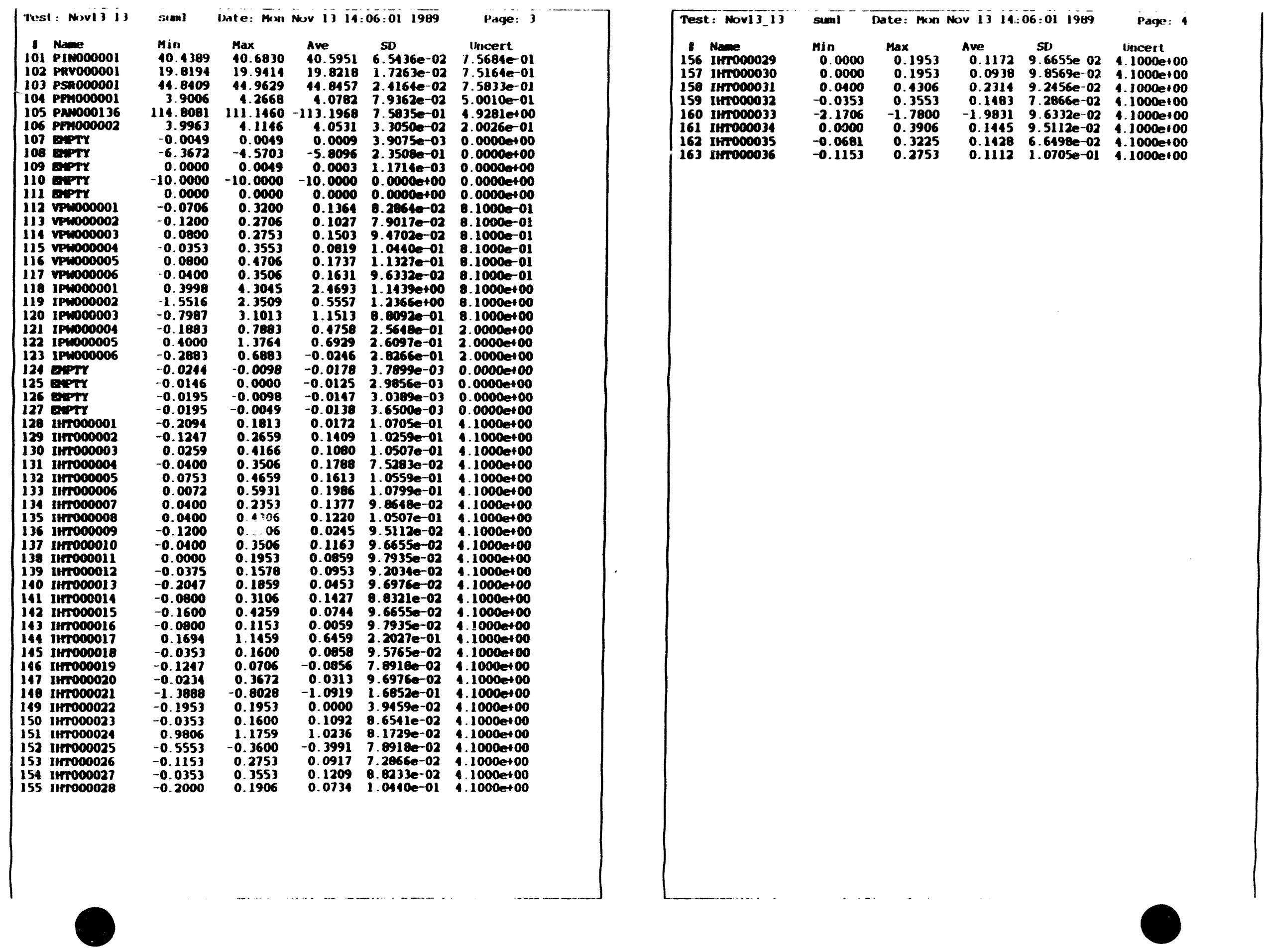




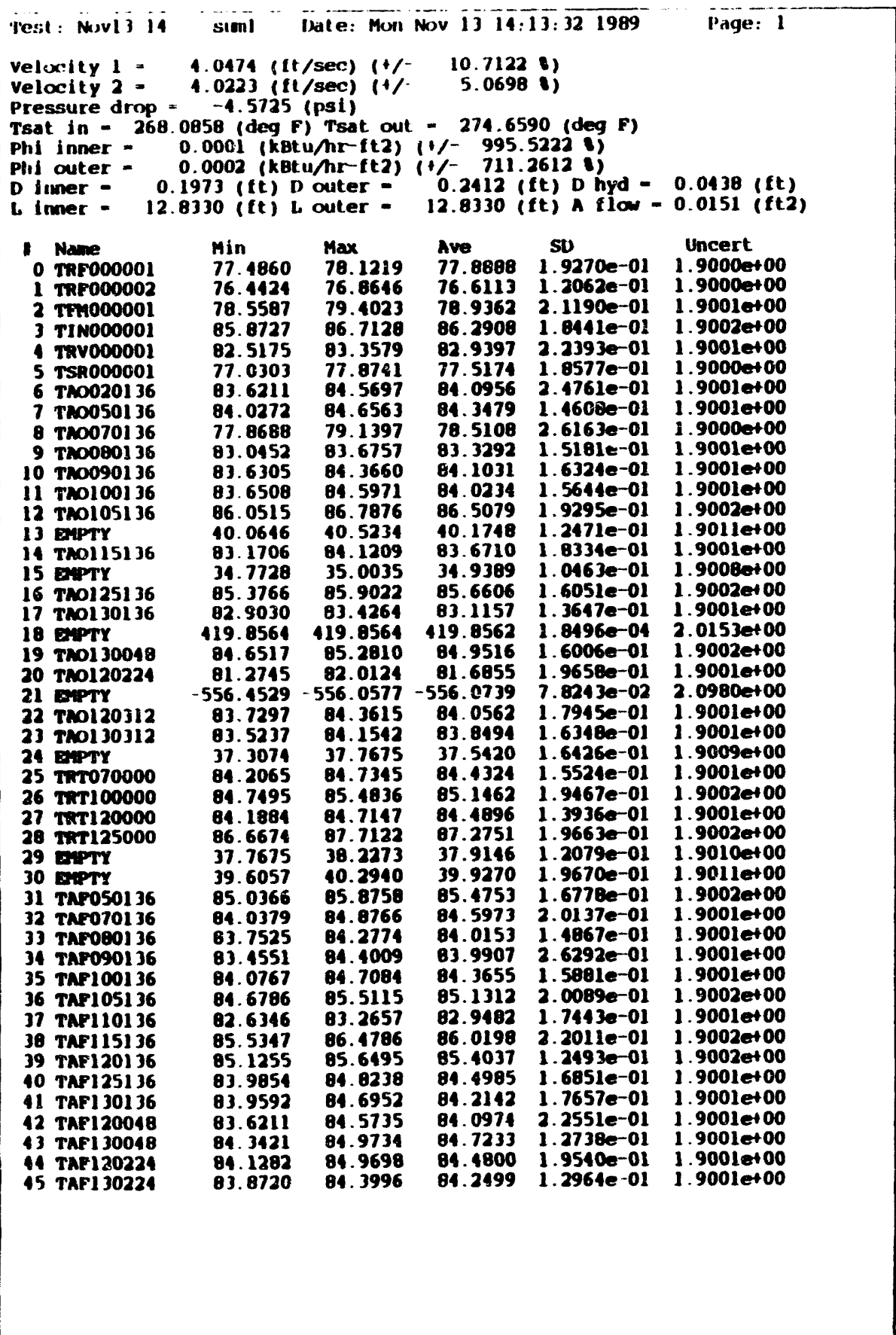

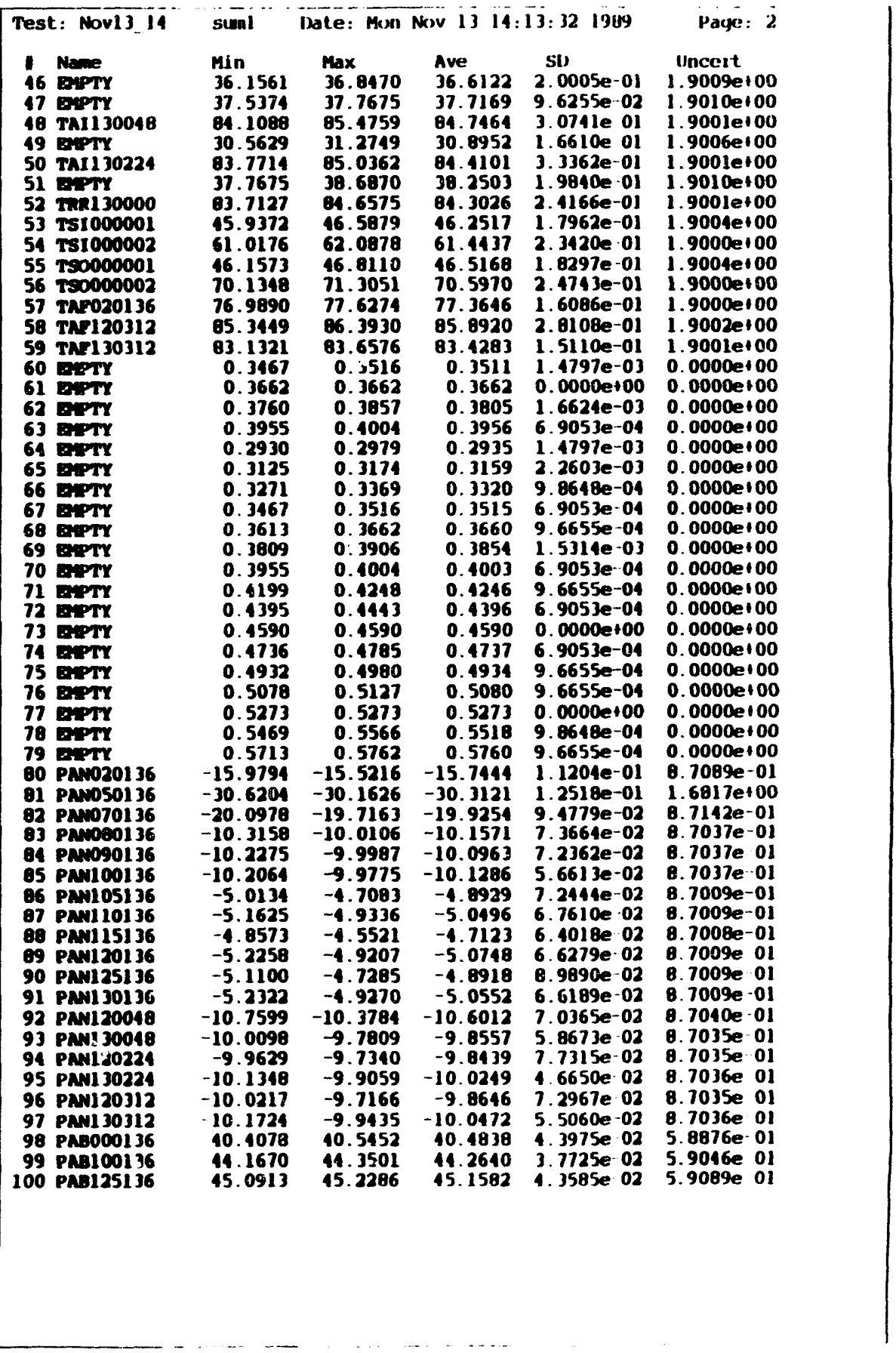




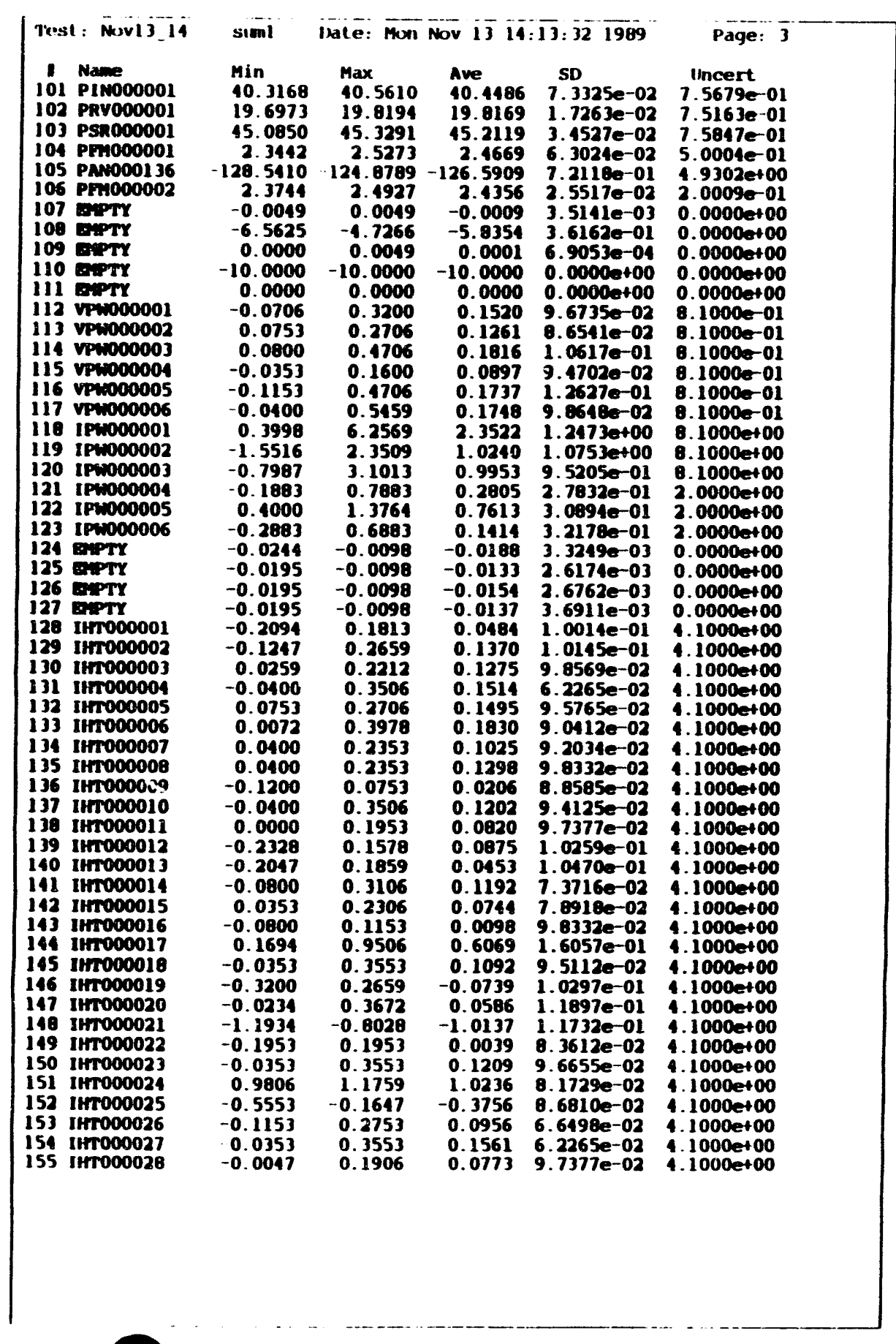

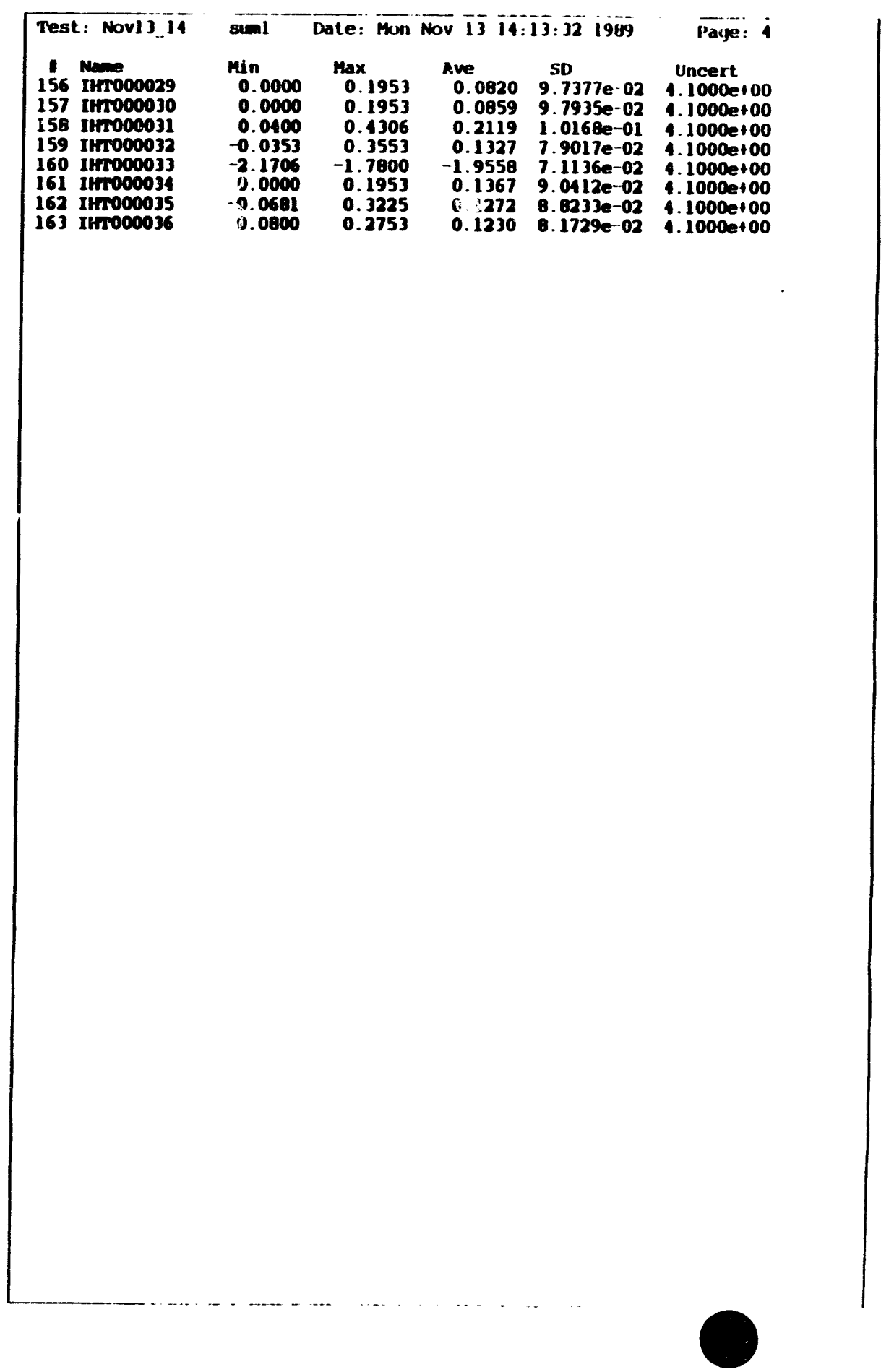




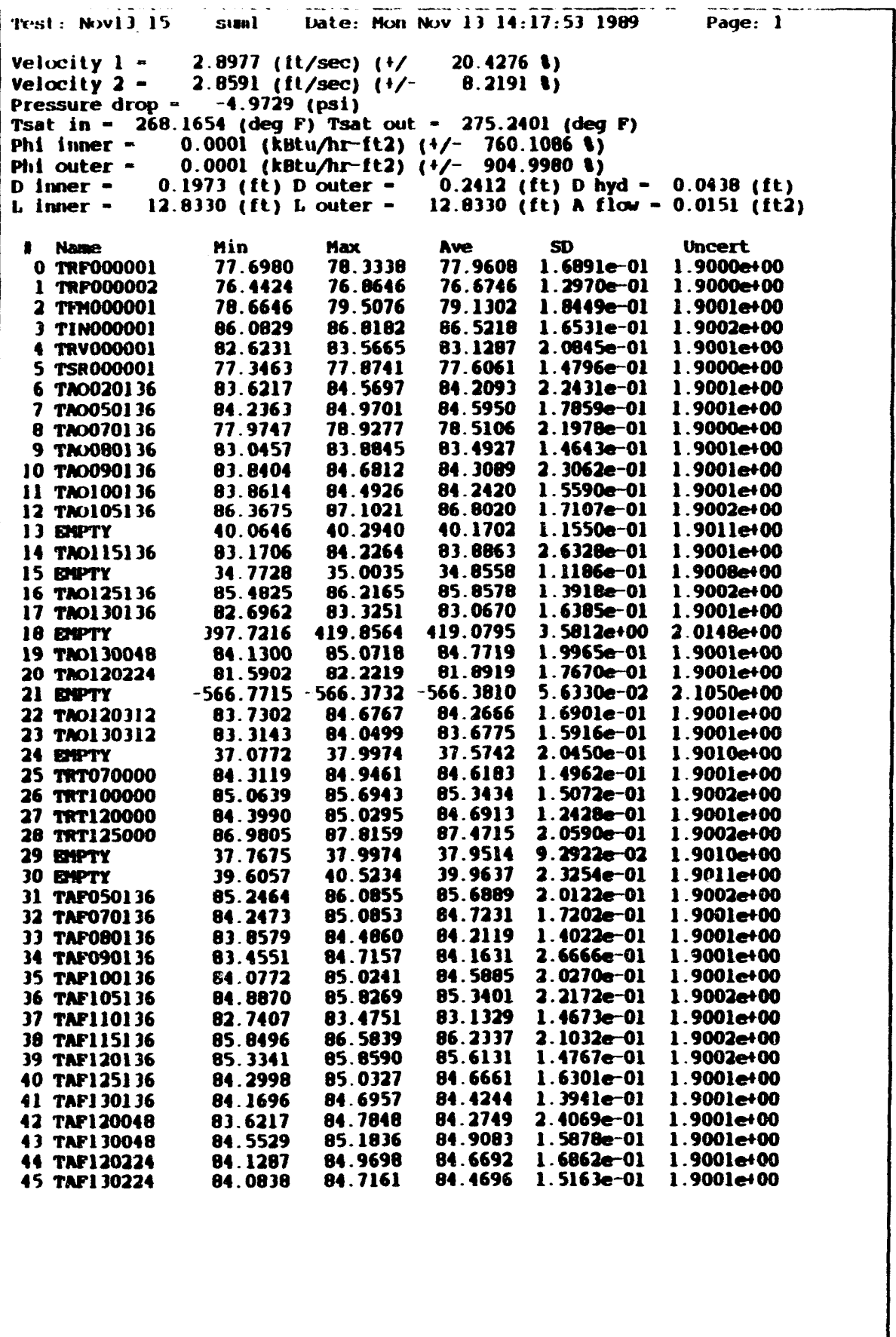

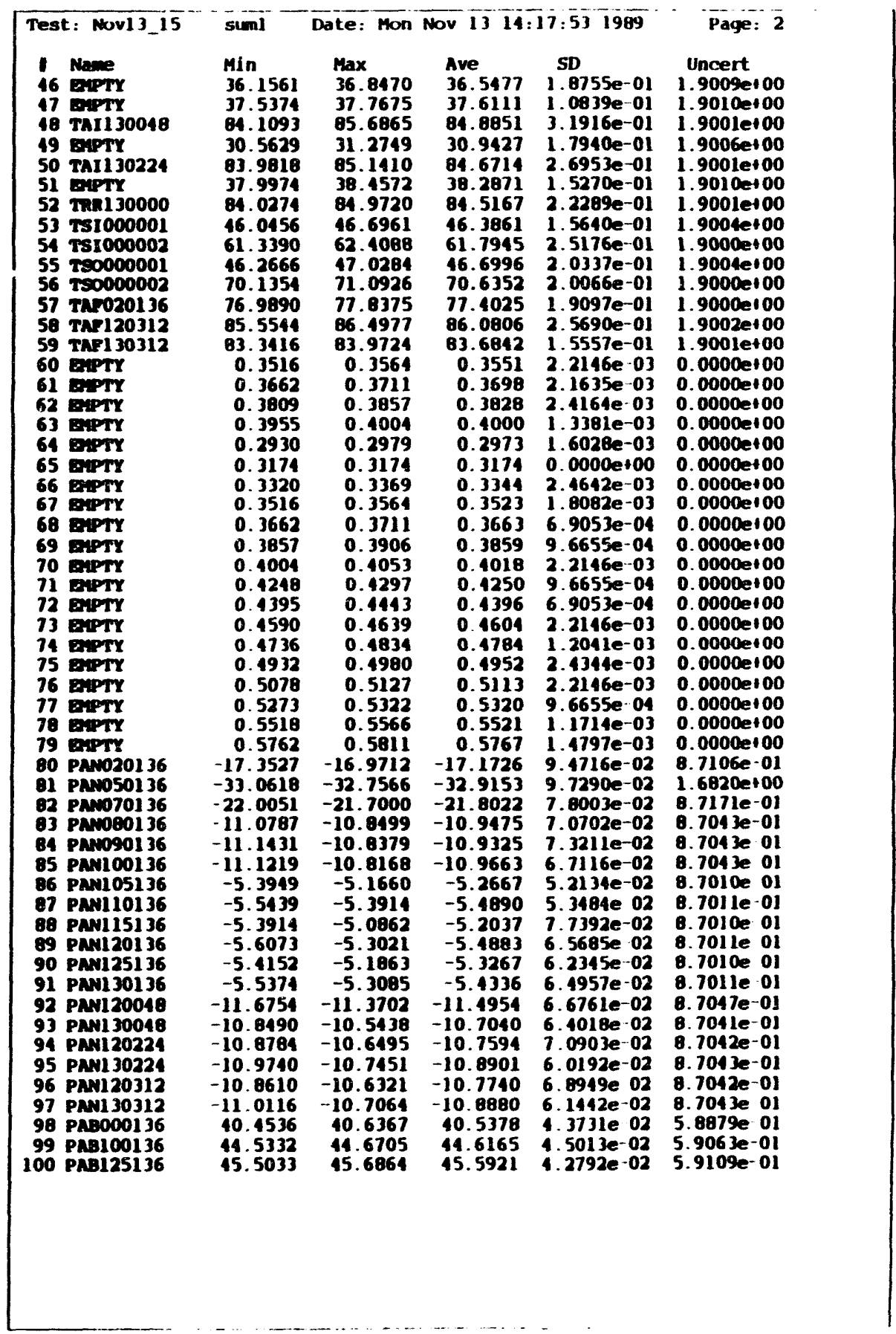




\begin{tabular}{|c|c|c|c|c|c|c|}
\hline \multirow[b]{2}{*}{$\begin{array}{l}10 s \\
11 \\
101 \\
102 \\
103 \\
104 \\
105 \\
106 \\
107 \\
109 \\
109 \\
110 \\
111 \\
112 \\
113 \\
114 \\
115 \\
116 \\
117 \\
118 \\
119 \\
120 \\
121 \\
122 \\
123 \\
124 \\
125 \\
126 \\
127 \\
128 \\
129 \\
130 \\
131 \\
132 \\
133 \\
134 \\
135 \\
136 \\
137 \\
138 \\
139 \\
140 \\
111 \\
112 \\
143 \\
144 \\
115 \\
116 \\
117 \\
118 \\
149 \\
150 \\
151 \\
152 \\
153 \\
154 \\
155\end{array}$} & \multirow[b]{2}{*}{ 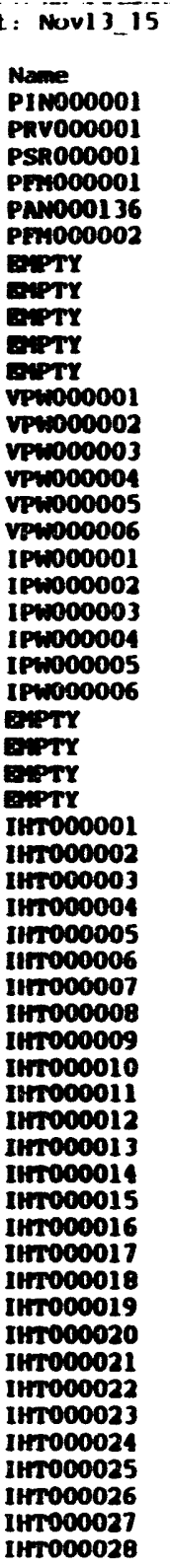 } & \multirow[b]{2}{*}{$\begin{array}{l}\text { Sinil } \\
\text { Hin } \\
40.4389 \\
19.8194 \\
45.6953 \\
1.1540 \\
138.6118 \\
1.1580 \\
-0.0049 \\
-6.5479 \\
0.0000 \\
-10.0000 \\
0.0000 \\
-0.0706 \\
-0.1200 \\
0.0800 \\
-0.0353 \\
0.0800 \\
-0.0400 \\
0.3998 \\
-1.5516 \\
1.1513 \\
-0.1883 \\
-0.0883 \\
-0.2893 \\
-0.0293 \\
-0.0195 \\
-0.0244 \\
-0.0244 \\
-0.2094 \\
0.0706 \\
0.0259 \\
-0.0400 \\
0.0753 \\
0.0072 \\
0.0400 \\
0.0400 \\
-0.1200 \\
-0.0400 \\
-0.1953 \\
-0.0375 \\
-0.4000 \\
-0.0800 \\
-0.1600 \\
-0.0800 \\
0.1694 \\
-0.0353 \\
-0.1247 \\
-0.0234 \\
-1.3888 \\
-0.1953 \\
-0.0353 \\
0.7853 \\
0.5553 \\
0.08000 \\
-0.0353 \\
-0.2000\end{array}$} & Date: Mon & \multirow{2}{*}{\multicolumn{2}{|c|}{ 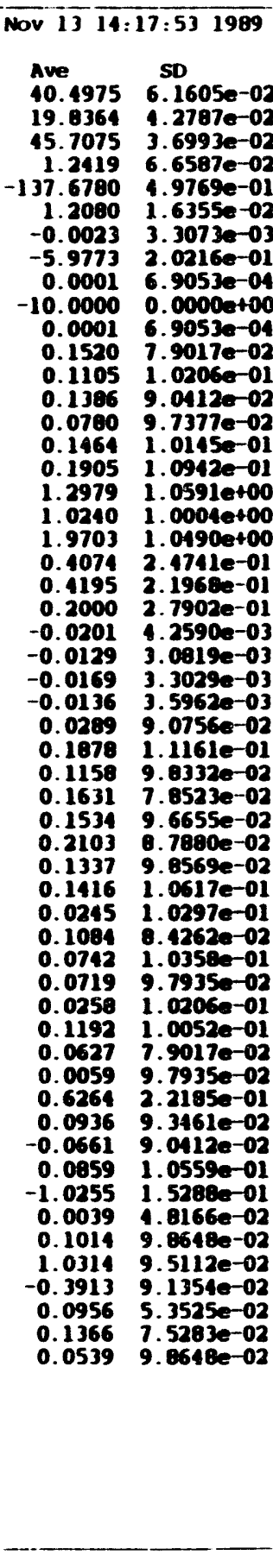 }} & \multirow[b]{2}{*}{ 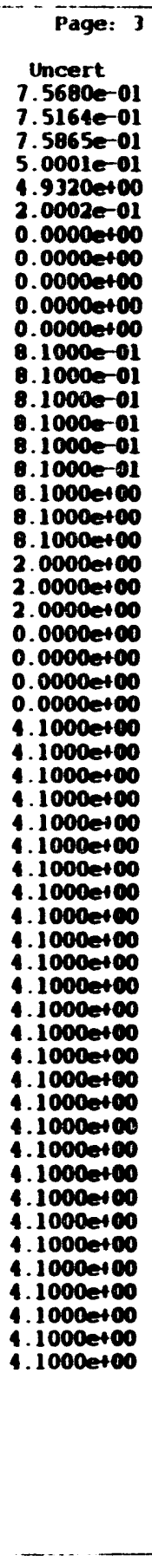 } \\
\hline & & & $\begin{array}{r}M a x \\
10.5610 \\
19.9414 \\
15.8174 \\
1.3371 \\
136.3230 \\
1.2425 \\
0.0049 \\
-5.6348 \\
0.0049 \\
-10.0000 \\
0.0049 \\
0.3200 \\
0.1659 \\
0.2753 \\
0.1600 \\
0.1706 \\
0.3506 \\
4.3045 \\
2.3509 \\
5.0513 \\
0.7883 \\
0.8682 \\
0.6883 \\
-0.0098 \\
-0.0098 \\
-0.0098 \\
-0.0098 \\
0.1813 \\
0.1612 \\
0.2212 \\
0.3506 \\
0.2706 \\
0.3978 \\
0.2353 \\
0.4306 \\
0.3706 \\
0.1553 \\
0.1953 \\
0.1570 \\
0.1859 \\
0.3106 \\
0.2306 \\
0.1153 \\
1.1459 \\
0.1600 \\
0.0706 \\
0.3672 \\
-0.8028 \\
0.1953 \\
0.3553 \\
1.1759 \\
-0.1647 \\
0.2753 \\
0.3553 \\
0.1906\end{array}$ & & & \\
\hline
\end{tabular}

\begin{tabular}{|c|c|c|c|c|c|}
\hline at: No & simal & Date: Mon & bov 1314 & $17: 531969$ & Page: 4 \\
\hline 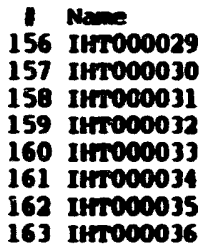 & $\begin{aligned} \text { Min } \\
0.0000 \\
-0.1953 \\
0.0400 \\
-0.0353 \\
-2.1705 \\
0.0000 \\
-0.0681 \\
-0.1153\end{aligned}$ & $\begin{array}{r}\operatorname{Max} \\
0.1953 \\
0.1953 \\
0.4306 \\
0.3553 \\
-1.7800 \\
0.3906 \\
0.3225 \\
0.2753\end{array}$ & $\begin{array}{r}\text { Ave } \\
0.1055 \\
0.0859 \\
0.2275 \\
0.1522 \\
-1.9636 \\
0.1172 \\
0.1389 \\
0.0995\end{array}$ & $\begin{array}{l}\text { SD } \\
9.8332 \mathrm{e}-02 \\
1.0559 \mathrm{e}-01 \\
7.8523 \mathrm{e}-02 \\
6.7886 \mathrm{e}-02 \\
6.1257 \mathrm{e}-02 \\
1.0410 \mathrm{e}-01 \\
9.1779 \mathrm{e}-02 \\
7.1136 \mathrm{e}-02\end{array}$ & $\begin{array}{l}\text { Uncert } \\
4.1000 \text { et } 00 \\
4.1000 \text { et } 00 \\
4.1000 \mathrm{e}+00 \\
1.1000 \mathrm{e}+00 \\
1.1000 \mathrm{e} 100 \\
4.1000 \mathrm{e}+00 \\
4.1000 \mathrm{e}+00 \\
4.1000 \mathrm{e}+00\end{array}$ \\
\hline
\end{tabular}




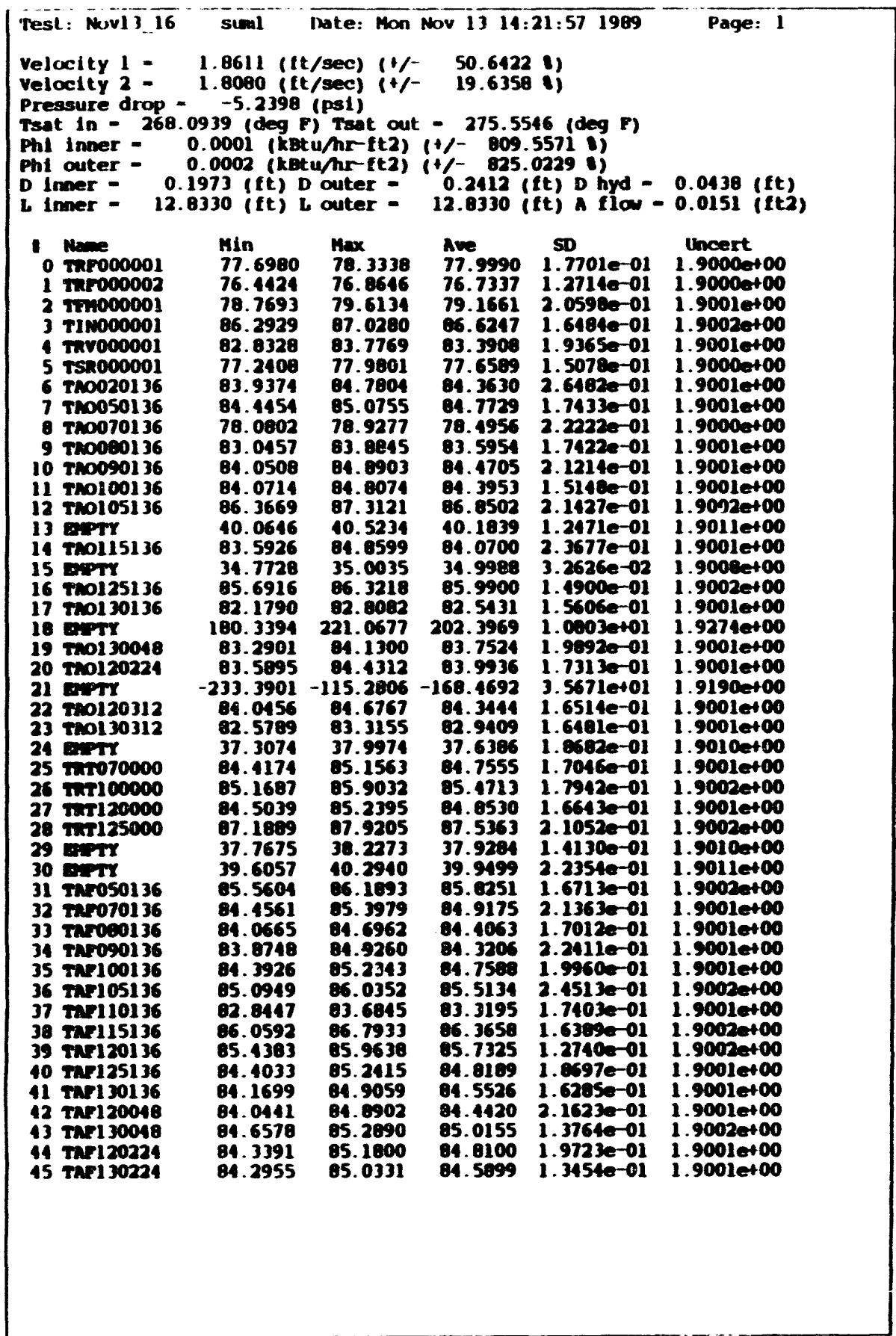

\begin{tabular}{|c|c|c|c|c|c|}
\hline 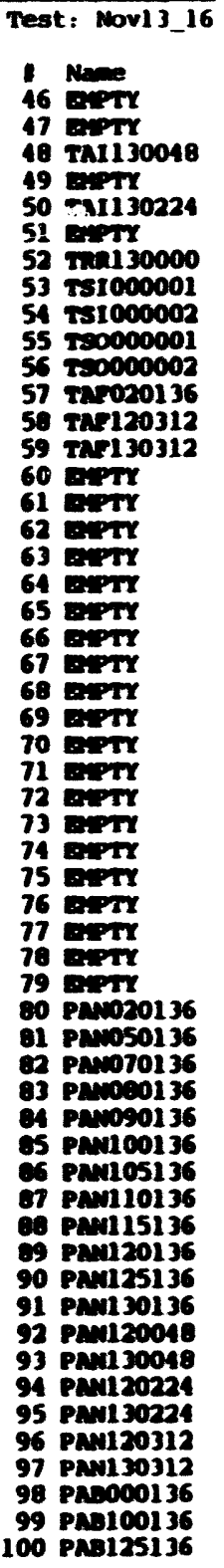 & 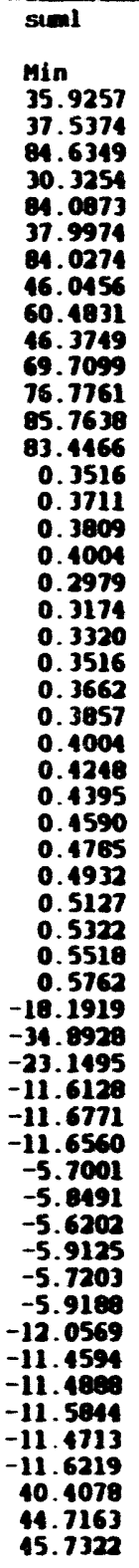 & $\begin{array}{l}\text { Date: Mon } \\
\text { Max } \\
37.0772 \\
37.7675 \\
85.7913 \\
31.2749 \\
65.4571 \\
38.4572 \\
85.1815 \\
46.6961 \\
61.6606 \\
17.1371 \\
70.8901 \\
77.9435 \\
86.8122 \\
64.0764 \\
0.3613 \\
0.3760 \\
0.3857 \\
0.4053 \\
0.2979 \\
0.3223 \\
0.3369 \\
0.3564 \\
0.3711 \\
0.3906 \\
0.4053 \\
0.4297 \\
0.4143 \\
0.4689 \\
0.1785 \\
0.4980 \\
0.5127 \\
0.5371 \\
0.5566 \\
0.5911 \\
-17.8104 \\
-34.4351 \\
-22.9207 \\
-11.4602 \\
-11.4482 \\
-11.1271 \\
-5.4712 \\
-5.6202 \\
-5.3914 \\
-5.6836 \\
-5.4915 \\
-5.5374 \\
-11.9043 \\
-11.1512 \\
-11.2599 \\
-11.2792 \\
-11.2424 \\
-11.3168 \\
40.5452 \\
44.8536 \\
45.9153\end{array}$ & 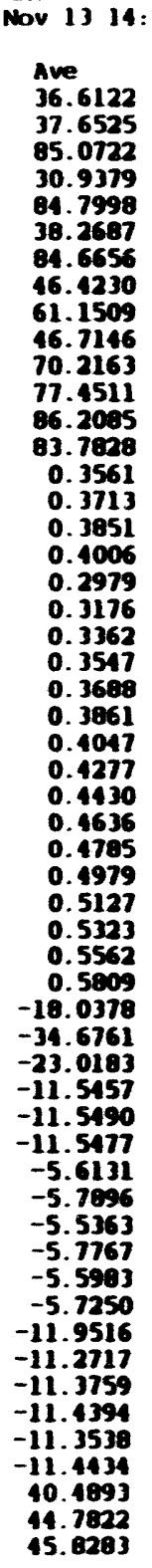 & 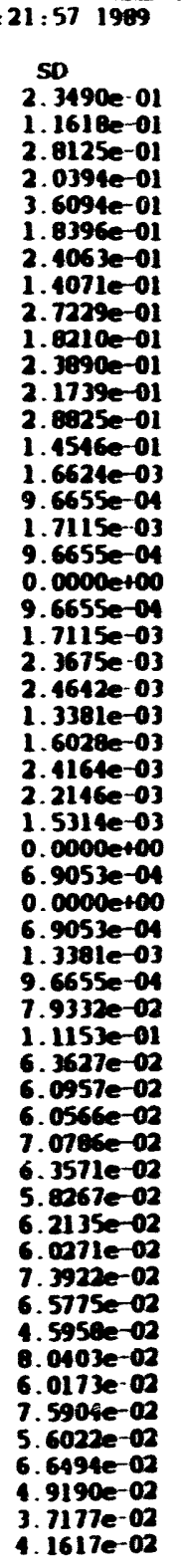 & 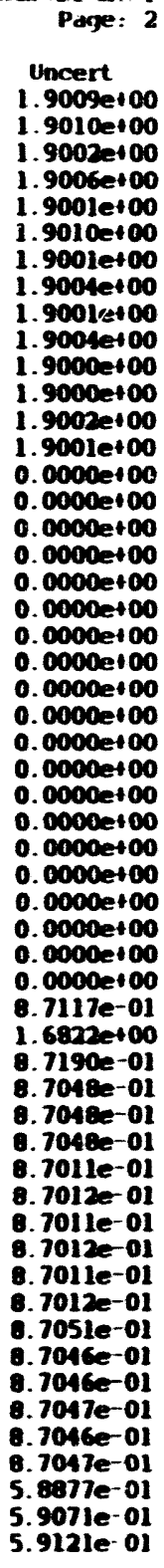 \\
\hline
\end{tabular}




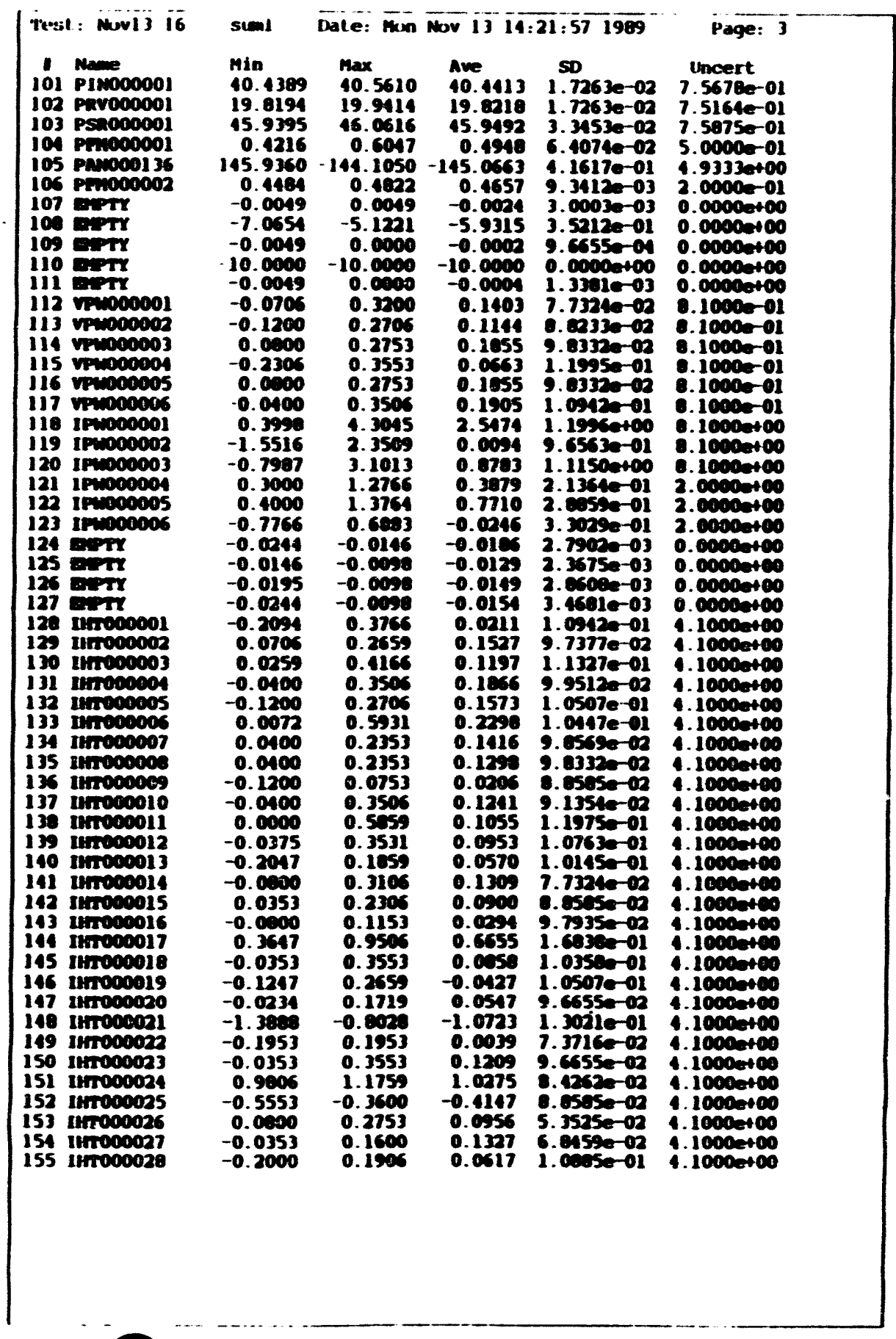

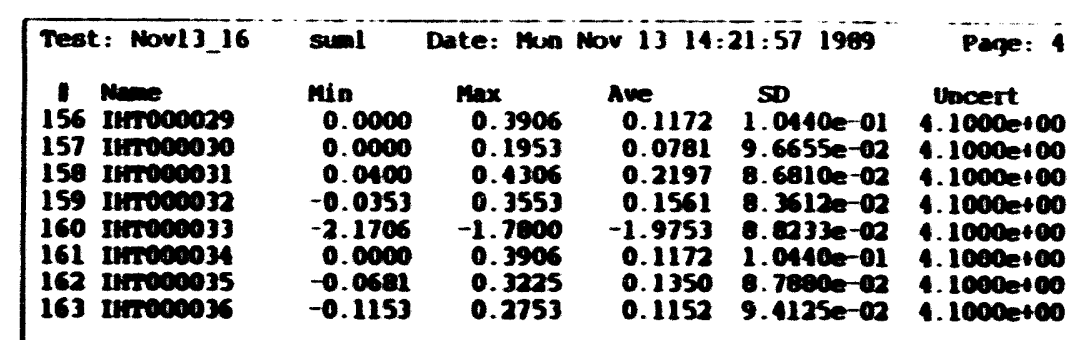




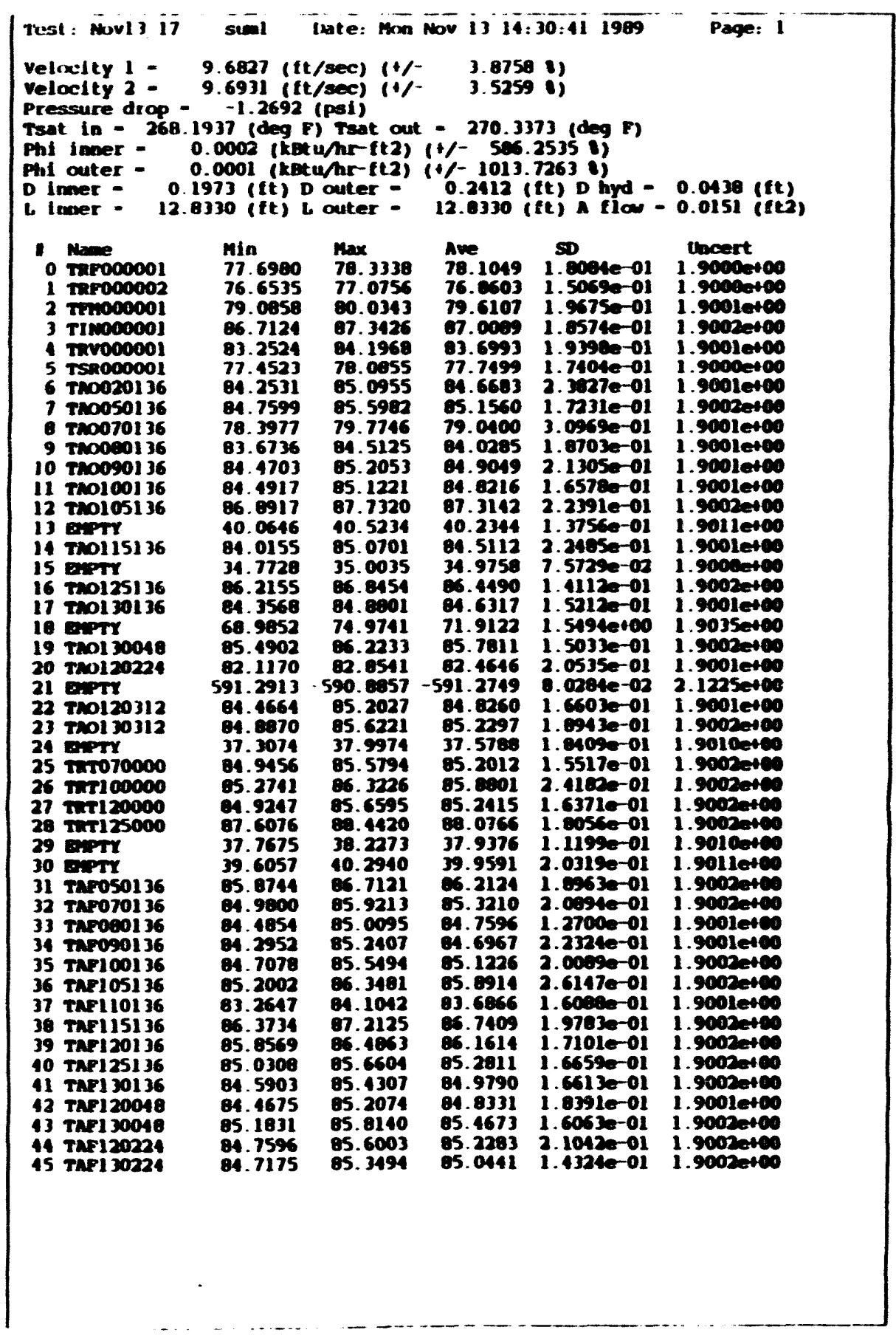

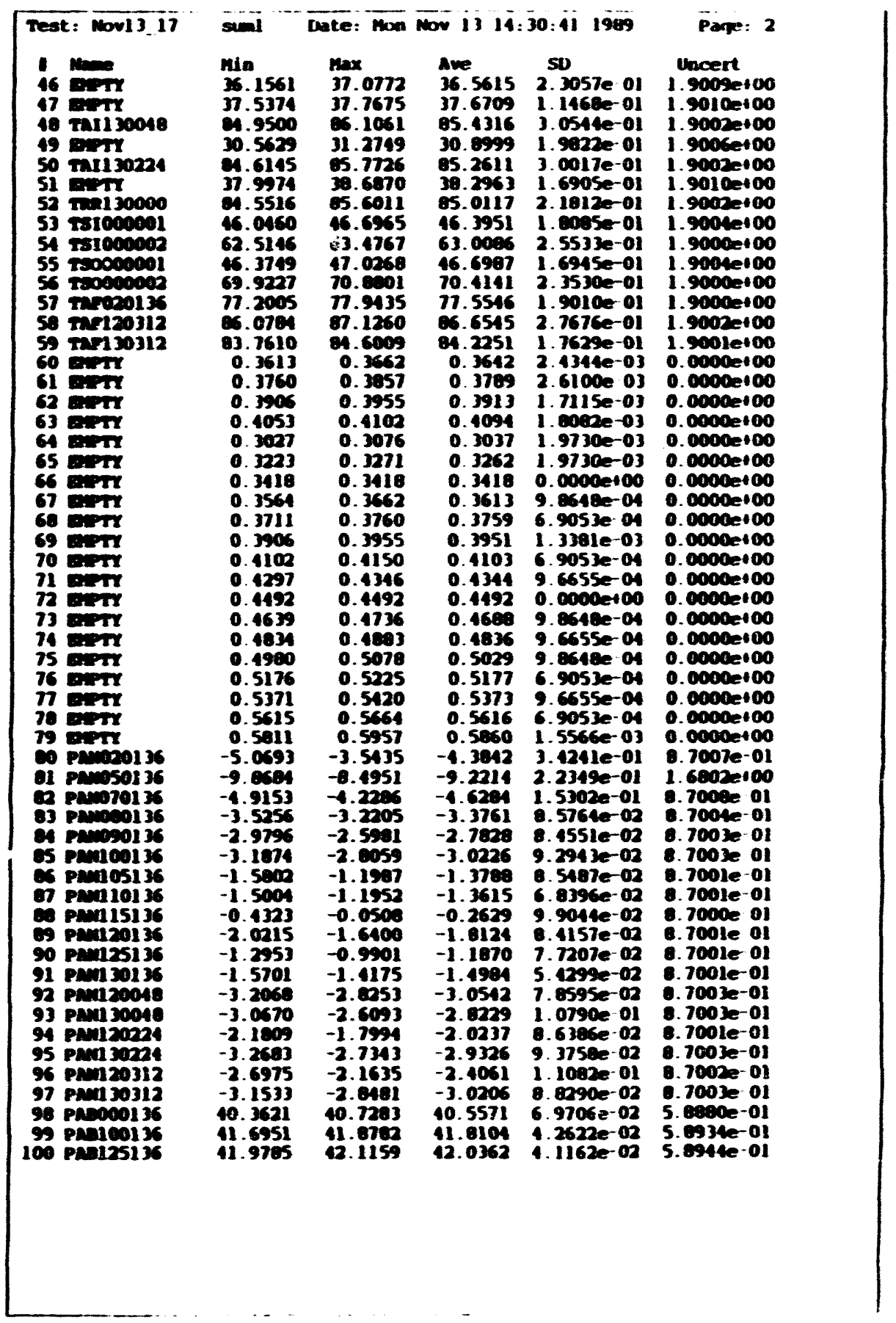




\begin{tabular}{|c|c|c|c|c|c|c|}
\hline $\begin{array}{l}\text { Tesi } \\
11 \\
101 \\
102 \\
103 \\
101 \\
105 \\
105 \\
107 \\
106 \\
109 \\
110 \\
111 \\
112 \\
113 \\
114 \\
115 \\
116 \\
117 \\
110 \\
119 \\
120 \\
121 \\
122 \\
123 \\
124 \\
125 \\
126 \\
127 \\
129 \\
129 \\
130 \\
131 \\
132 \\
133 \\
134 \\
135 \\
136 \\
137 \\
130 \\
139 \\
140 \\
141 \\
142 \\
143 \\
141 \\
145 \\
146 \\
117 \\
140 \\
149 \\
150 \\
151 \\
152 \\
153 \\
154 \\
155\end{array}$ & 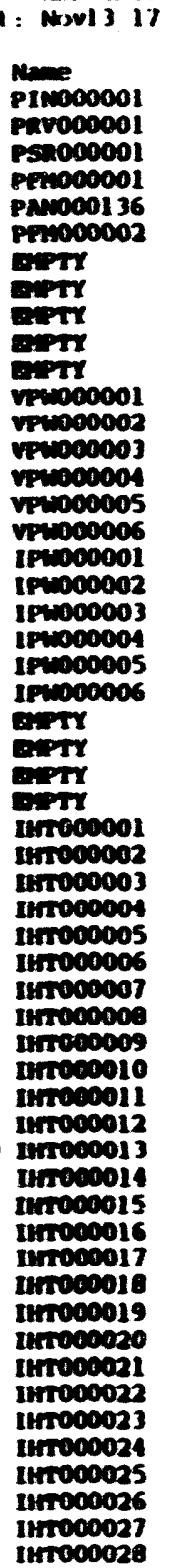 & $\begin{array}{l}\text { simi } \\
\text { Min } \\
40.3168 \\
19.5752 \\
11.5450 \\
14.2460 \\
37.9038 \\
14.4033 \\
-0.0049 \\
-7.9834 \\
0.0000 \\
10.0000 \\
0.0000 \\
0.1247 \\
-0.1200 \\
0.0000 \\
-0.0353 \\
0.0000 \\
-0.0400 \\
-1.5525 \\
0.3996 \\
1.1513 \\
0.3000 \\
-0.0963 \\
0.2803 \\
-0.0244 \\
-0.0195 \\
-0.0195 \\
-0.0195 \\
-0.2094 \\
0.0706 \\
0.0259 \\
-0.0400 \\
-0.1200 \\
0.0072 \\
0.0900 \\
0.0400 \\
0.1200 \\
-0.0900 \\
0.0000 \\
-0.0375 \\
-0.2047 \\
-0.0000 \\
-0.1600 \\
-0.0800 \\
0.1694 \\
-0.0353 \\
-0.1247 \\
-0.0234 \\
-1.3060 \\
-0.1953 \\
-0.0353 \\
0.7953 \\
-0.5553 \\
-0.1153 \\
-0.0353 \\
-0.2000\end{array}$ & 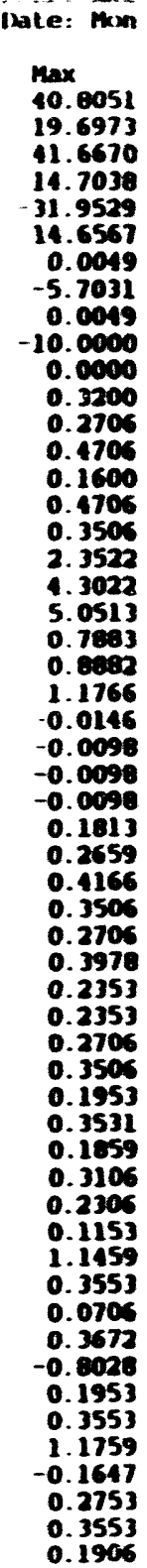 & 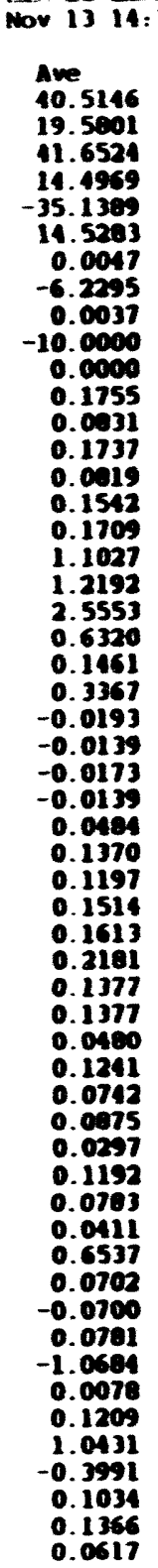 & 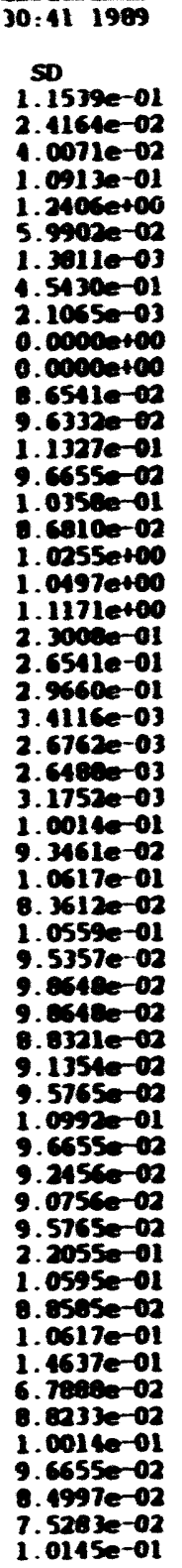 & 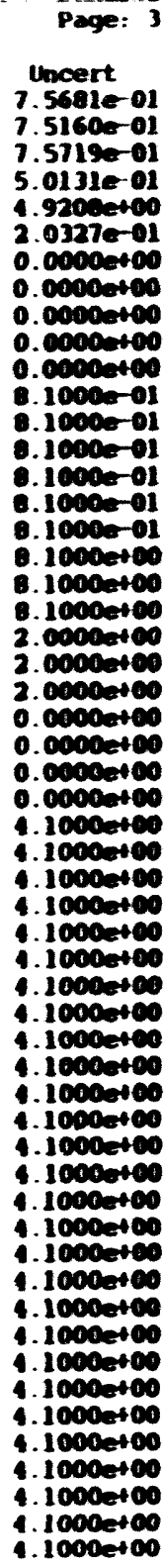 \\
\hline
\end{tabular}

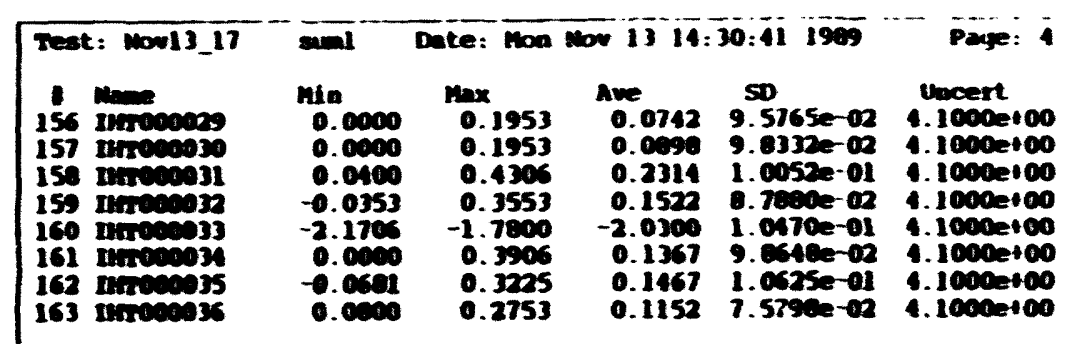




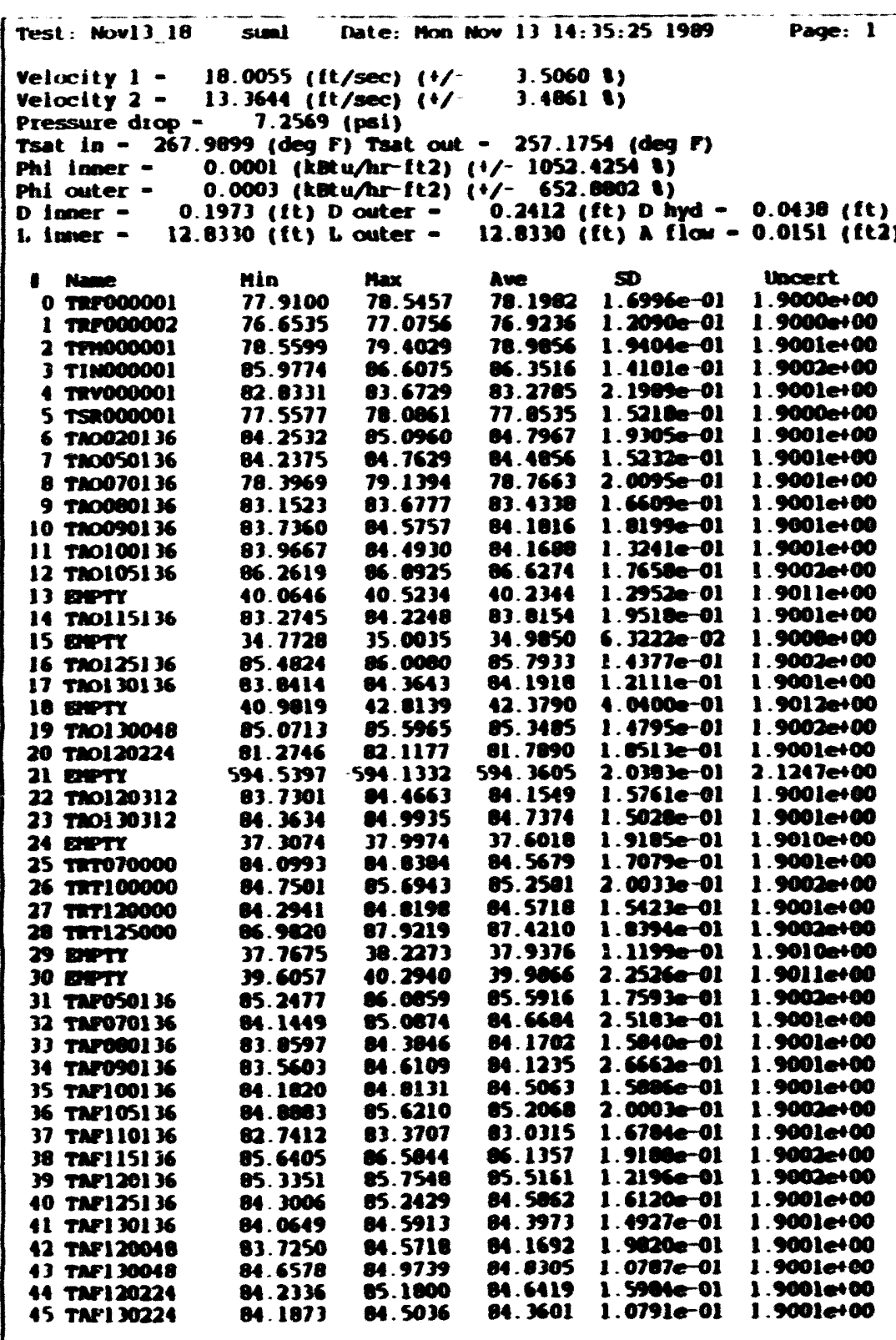

\begin{tabular}{|c|c|c|c|c|c|}
\hline 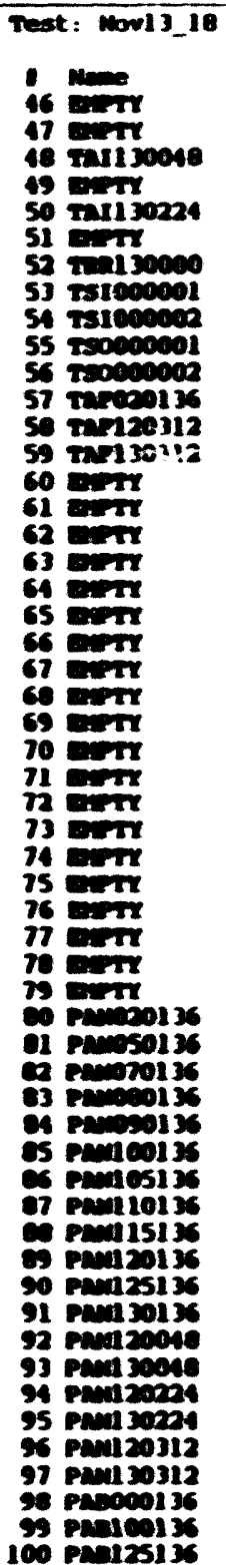 & 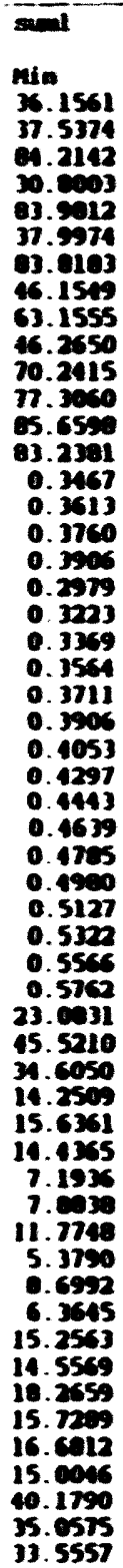 & 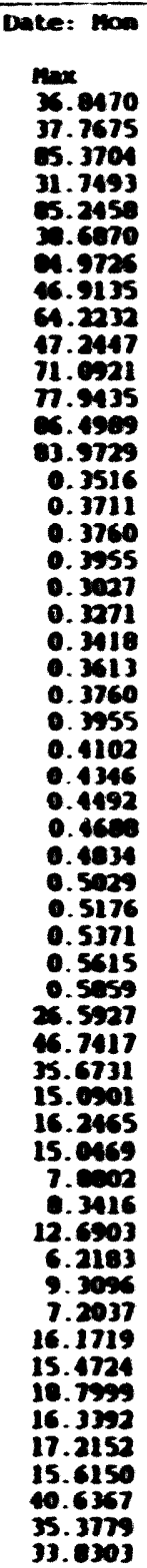 & 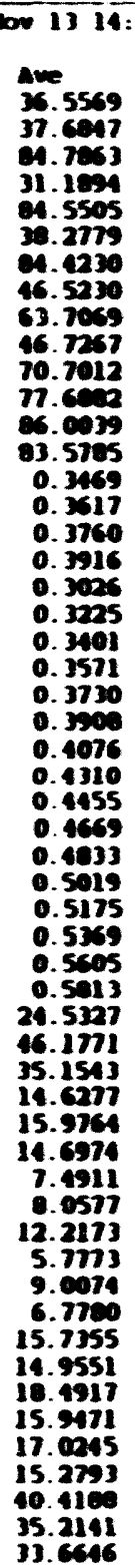 & 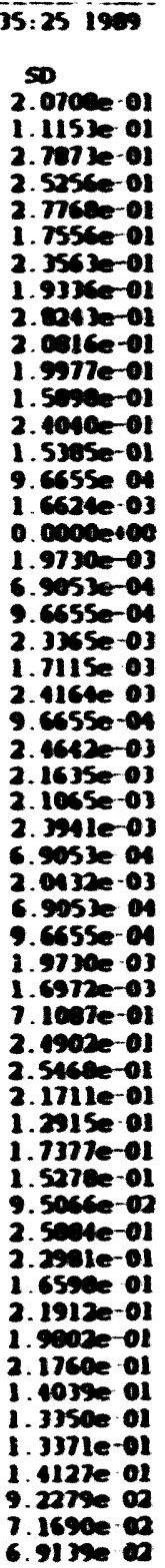 & $\begin{array}{l}01 \\
01 \\
01 \\
01 \\
01 \\
01 \\
01 \\
01 \\
01 \\
01 \\
01 \\
01 \\
01 \\
01 \\
\text { o1 } \\
01\end{array}$ \\
\hline
\end{tabular}




\begin{tabular}{|c|c|c|c|c|c|}
\hline 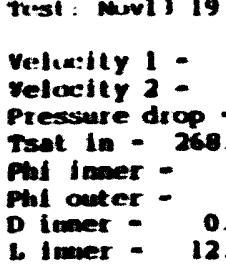 & 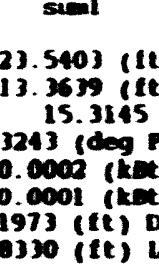 & $\begin{array}{l}/(s e c)(1 / \\
/ s e c)(1 /) \\
(p e i)\end{array}$ & $\begin{array}{r}-242.53 \\
10 /-604 \\
1 \%-940 \\
0.2412 \\
12.8330\end{array}$ & () & $\begin{array}{l}\text { it) } \\
\text { ite }\end{array}$ \\
\hline 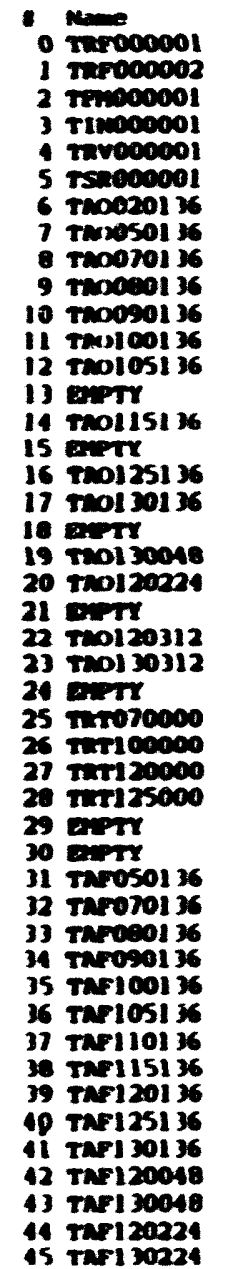 & 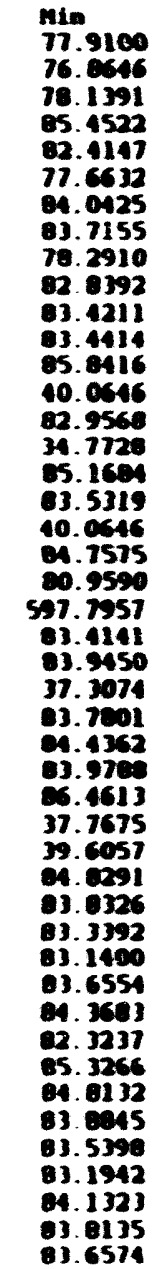 & 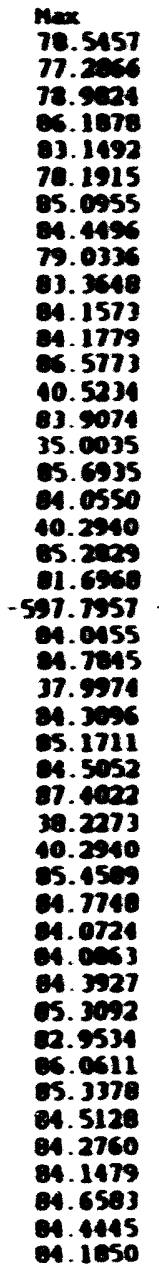 & 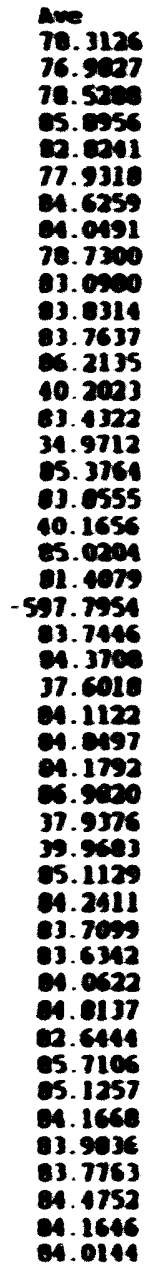 & 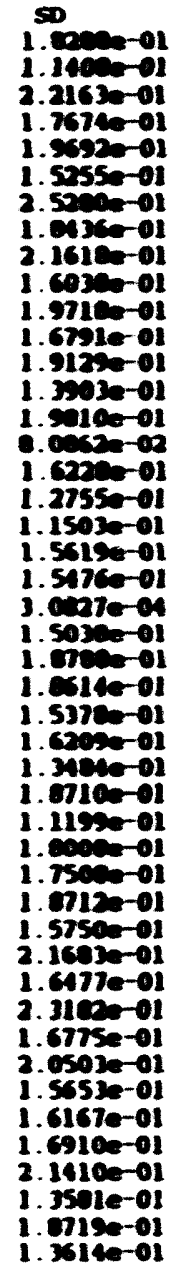 & $\begin{array}{l}1 \\
1\end{array}$ \\
\hline
\end{tabular}

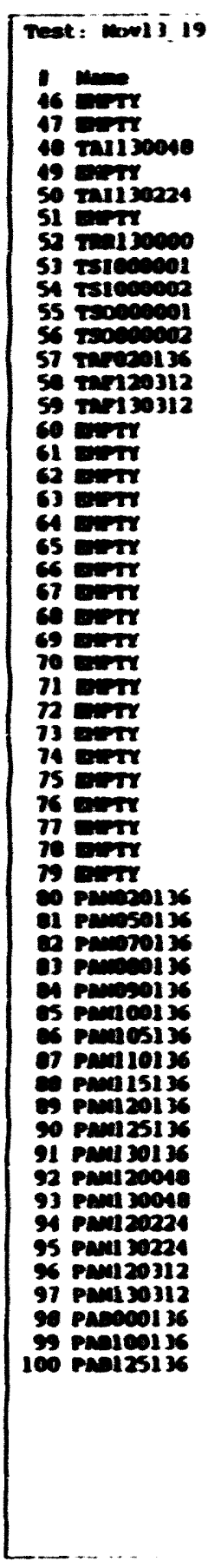

\begin{tabular}{|c|c|c|c|c|}
\hline 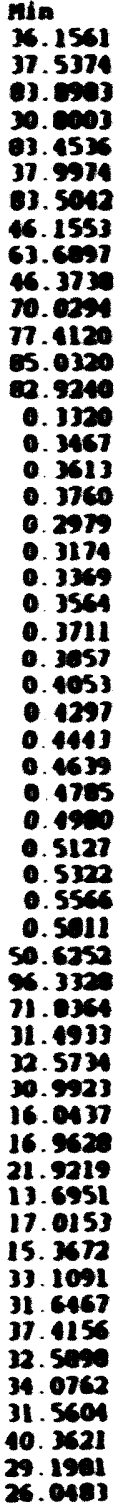 & 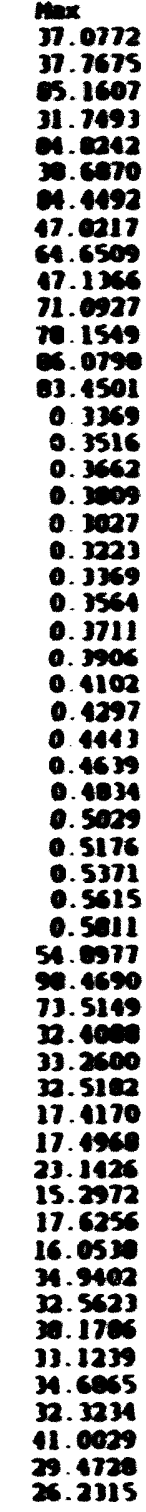 & 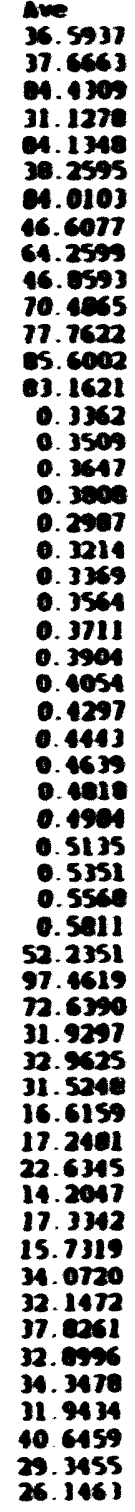 & 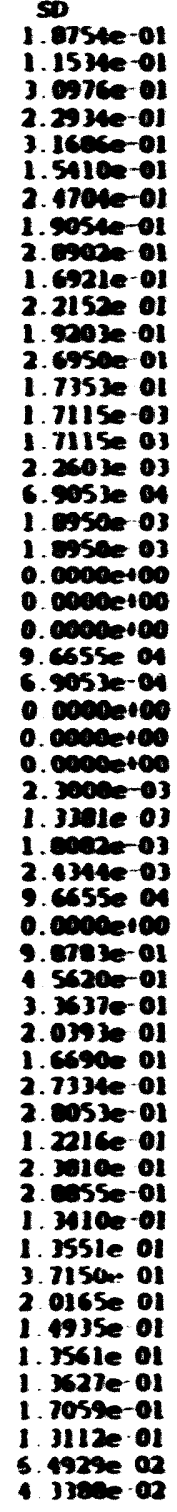 & 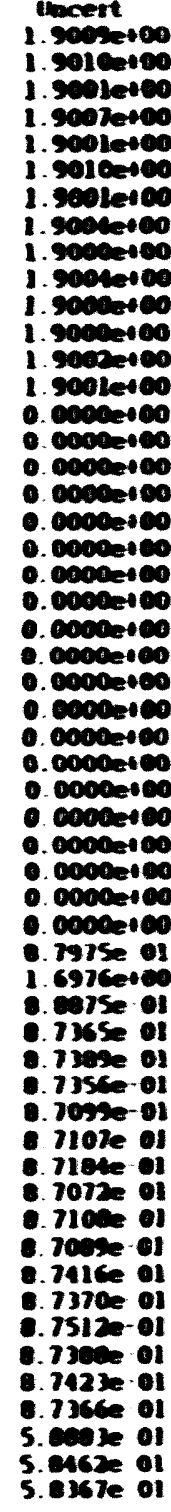 \\
\hline
\end{tabular}




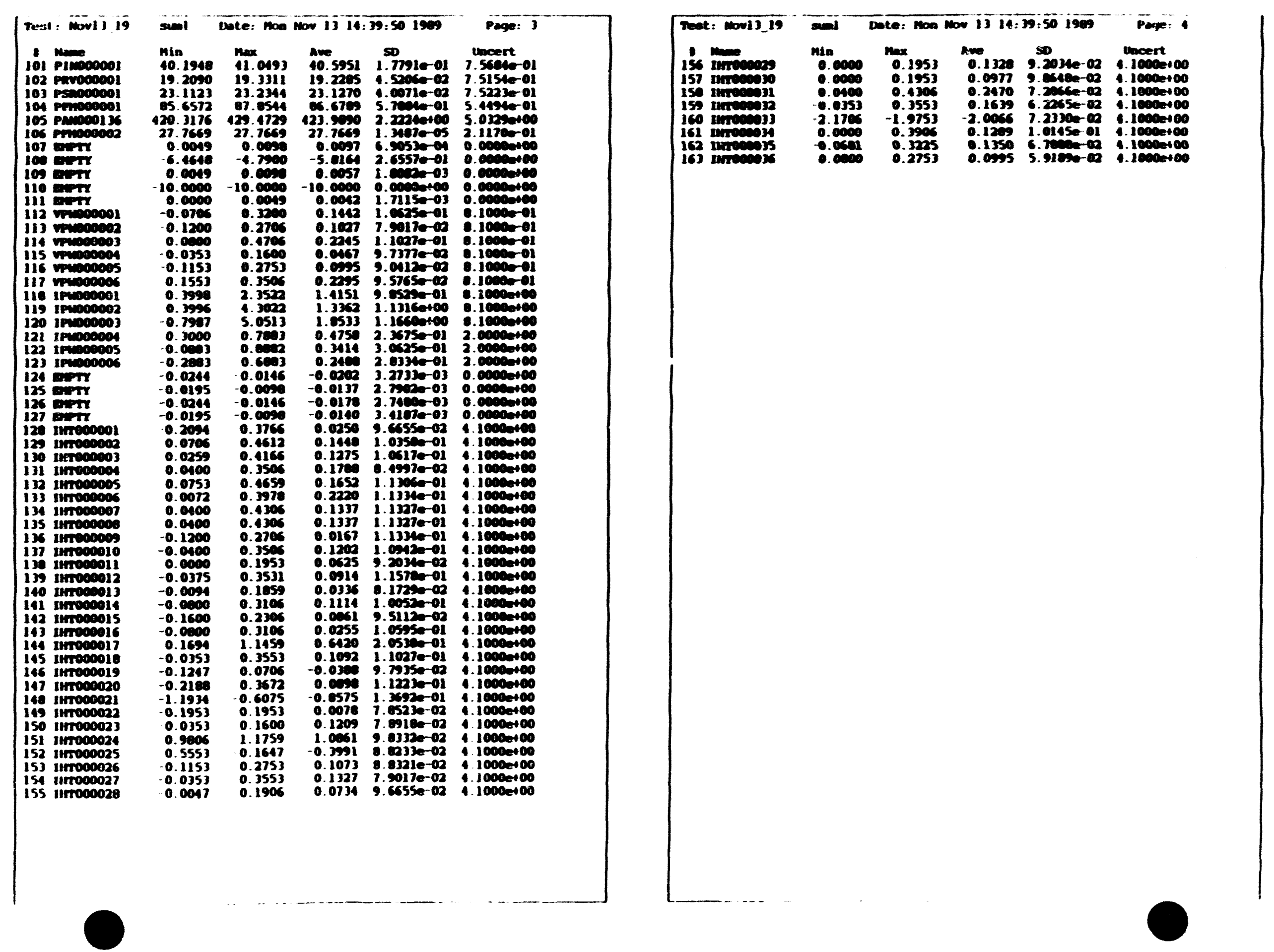




\begin{tabular}{|c|c|c|c|c|c|c|}
\hline \multicolumn{6}{|c|}{ 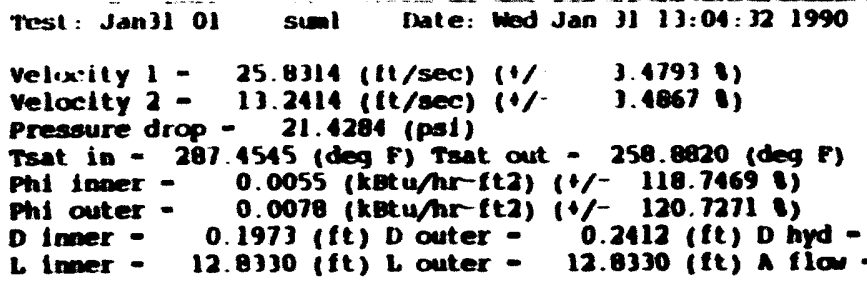 } & $\begin{array}{l}0.0430 \text { (ft) } \\
0.0151 \text { (ft2) }\end{array}$ \\
\hline $\begin{array}{l}1 \\
1 \\
1 \\
3 \\
1 \\
5 \\
6 \\
7 \\
8 \\
9 \\
10 \\
11 \\
12 \\
13 \\
14 \\
15 \\
16 \\
17 \\
18 \\
19 \\
20 \\
21 \\
22 \\
23 \\
24 \\
25 \\
26 \\
27 \\
26 \\
29 \\
30 \\
31 \\
32 \\
33 \\
31 \\
35 \\
36 \\
37 \\
36 \\
39 \\
10 \\
11 \\
12 \\
43 \\
11 \\
15\end{array}$ & 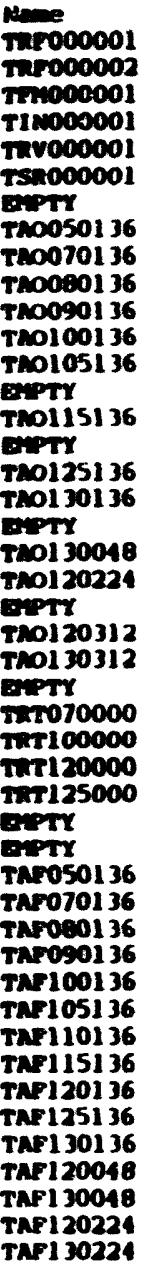 & $\begin{array}{r}\text { Min } \\
74.1838 \\
74.9393 \\
87.0794 \\
87.2565 \\
86.8377 \\
87.4509 \\
601.0594 \\
86.8680 \\
88.0549 \\
87.7349 \\
87.5065 \\
87.8177 \\
87.9663 \\
40.7527 \\
87.2809 \\
37.5374 \\
87.6087 \\
87.6617 \\
11.2111 \\
87.5696 \\
87.2711 \\
38.6870 \\
87.5505 \\
87.3422 \\
38.9168 \\
87.2403 \\
87.8416 \\
87.7526 \\
87.8338 \\
39.3761 \\
39.3761 \\
87.6858 \\
88.2798 \\
87.5771 \\
88.0689 \\
87.4203 \\
87.6927 \\
87.4834 \\
88.1606 \\
87.7306 \\
88.0038 \\
87.8078 \\
88.0322 \\
87.6273 \\
87.7423 \\
87.2704\end{array}$ & 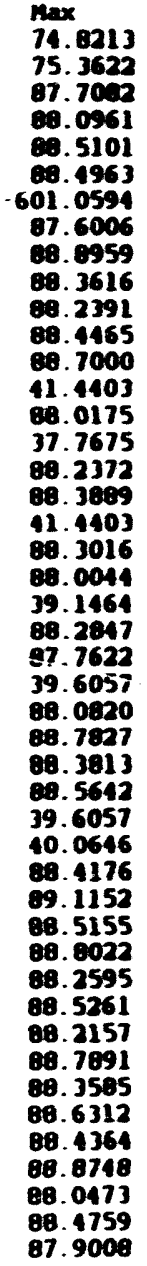 & 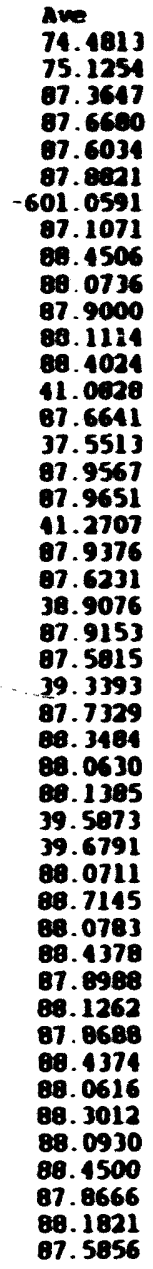 & 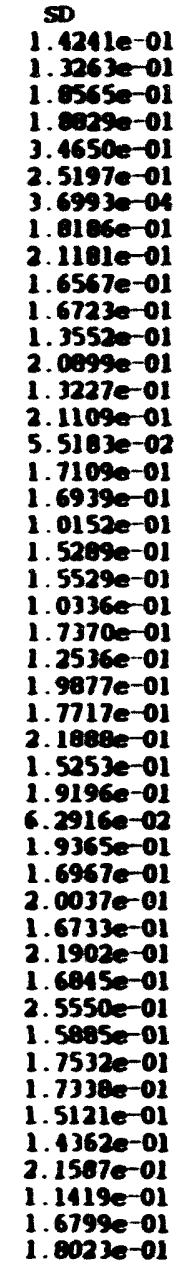 & 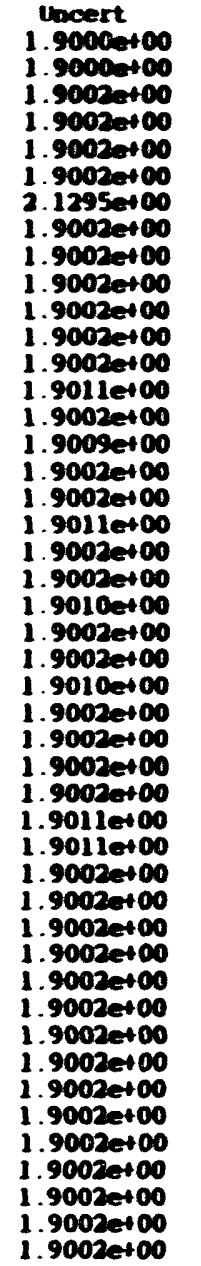 \\
\hline
\end{tabular}

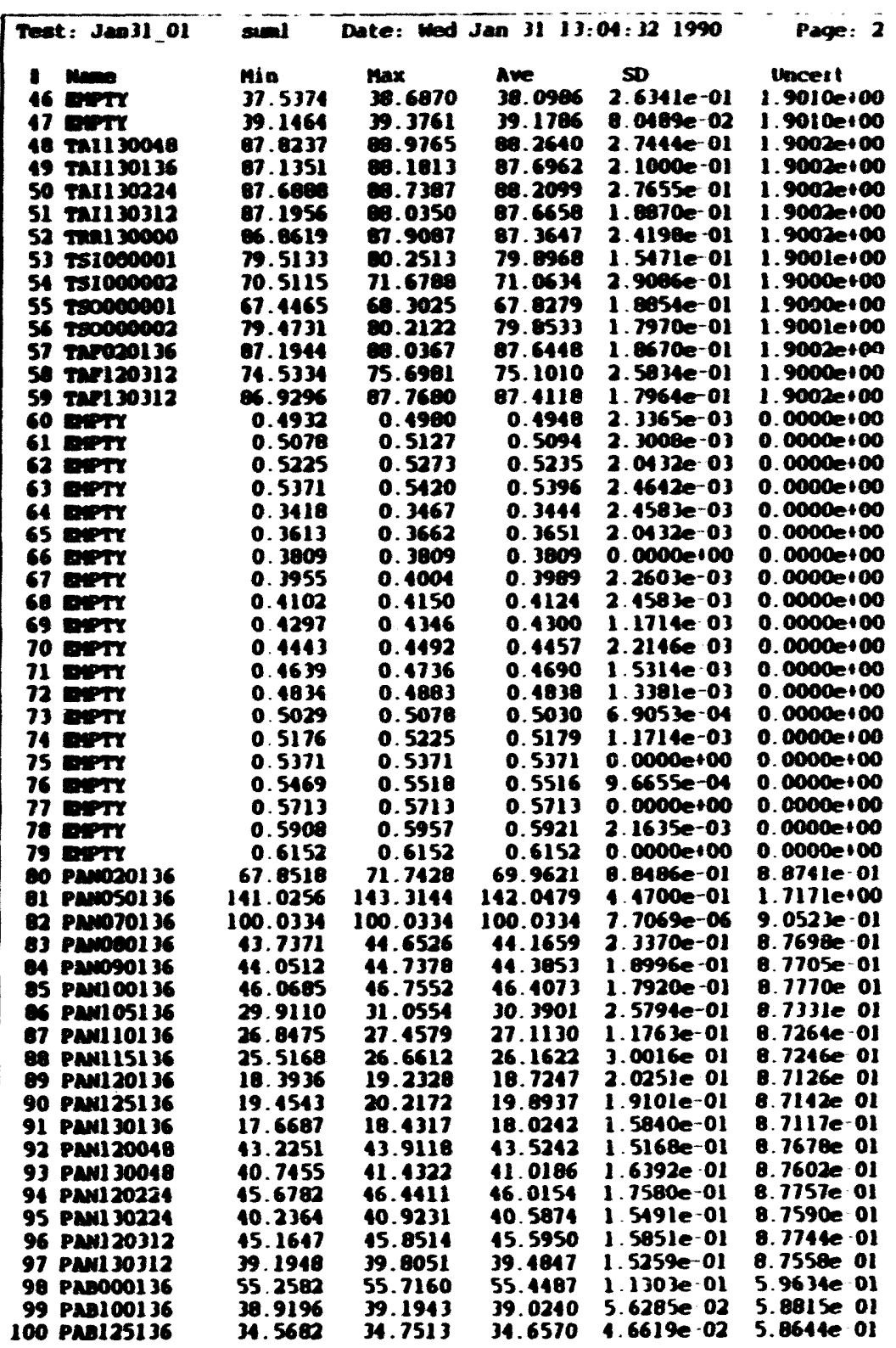




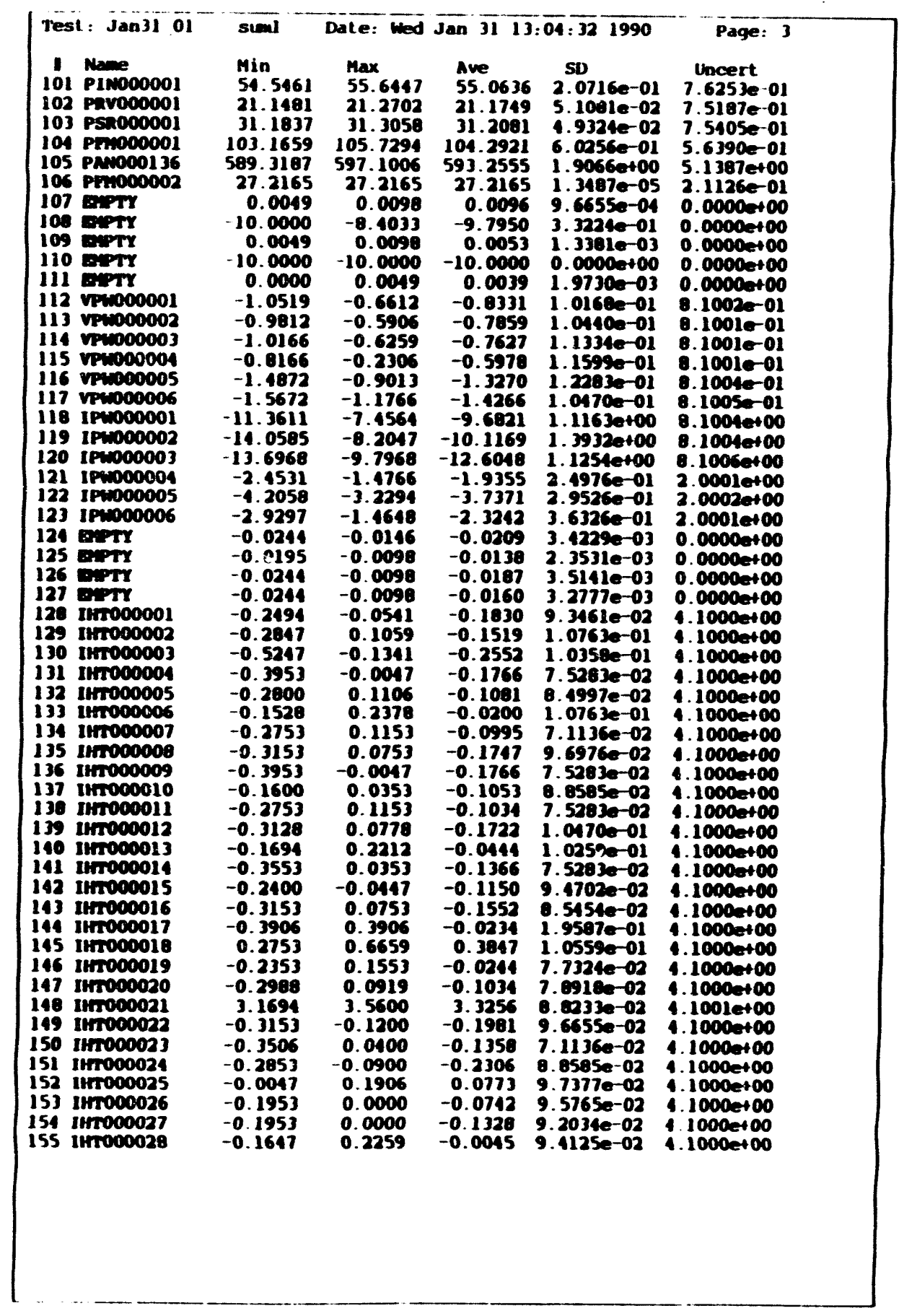

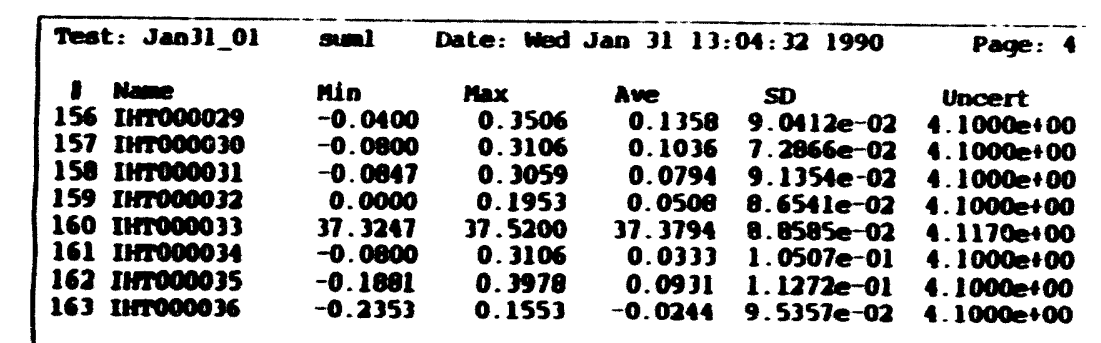

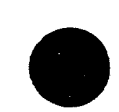




\begin{tabular}{|c|c|c|c|c|c|}
\hline \multicolumn{5}{|c|}{ 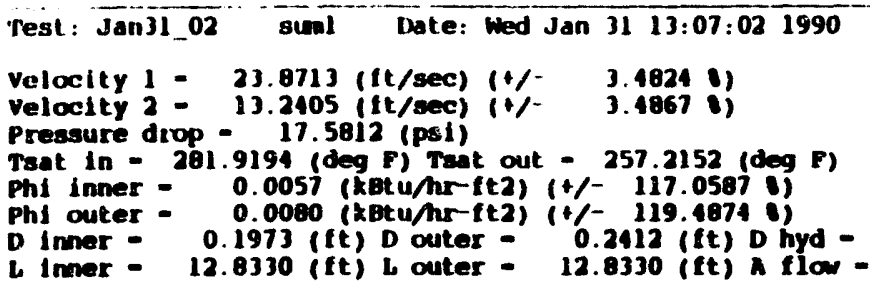 } & $\begin{array}{l}0.0438 \text { (ft) } \\
0.0151 \text { (ftz) }\end{array}$ \\
\hline 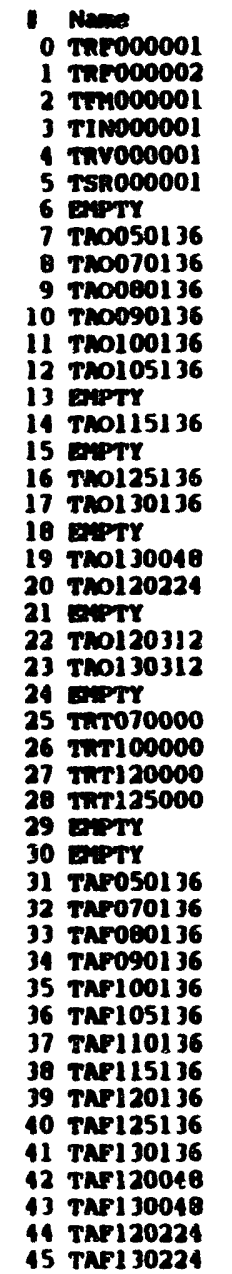 & $\begin{array}{l}\text { Min } \\
74.1838 \\
74.9393 \\
86.0328 \\
86.4155 \\
65.3752 \\
87.5560 \\
601.0594 \\
86.2418 \\
87.6338 \\
87.3188 \\
87.0081 \\
87.3991 \\
87.6514 \\
40.7527 \\
86.7546 \\
37.5374 \\
87.1915 \\
87.2486 \\
11.2111 \\
87.2564 \\
86.9567 \\
38.6870 \\
87.1311 \\
86.8201 \\
38.9168 \\
87.0269 \\
87.6336 \\
87.4390 \\
87.3139 \\
39.3761 \\
39.3761 \\
87.0602 \\
87.6567 \\
87.1631 \\
87.3364 \\
87.0003 \\
87.1734 \\
87.0669 \\
87.3241 \\
87.3146 \\
87.3800 \\
87.2848 \\
87.3988 \\
87.2082 \\
87.2185 \\
86.6371\end{array}$ & 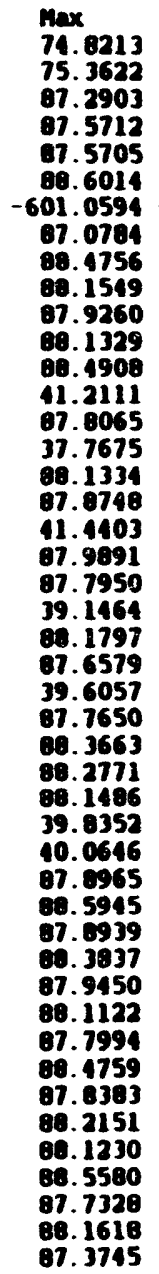 & $\begin{array}{l}\text { Ave } \\
74.5493 \\
75.1423 \\
86.5233 \\
86.9111 \\
86.1786 \\
89.0285 \\
-601.0591 \\
86.6939 \\
88.0295 \\
87.6793 \\
87.4986 \\
87.7178 \\
87.9972 \\
11.0599 \\
87.2603 \\
37.5466 \\
87.6016 \\
87.5486 \\
41.2799 \\
87.5703 \\
87.3132 \\
39.8938 \\
87.5339 \\
87.1845 \\
39.3485 \\
87.3442 \\
87.9976 \\
87.7407 \\
87.7810 \\
39.5552 \\
39.7020 \\
87.4951 \\
88.1696 \\
87.4758 \\
87.8854 \\
87.3196 \\
87.6110 \\
87.3806 \\
87.9314 \\
87.5532 \\
87.8104 \\
87.6117 \\
87.9474 \\
87.4097 \\
87.6378 \\
87.0752\end{array}$ & 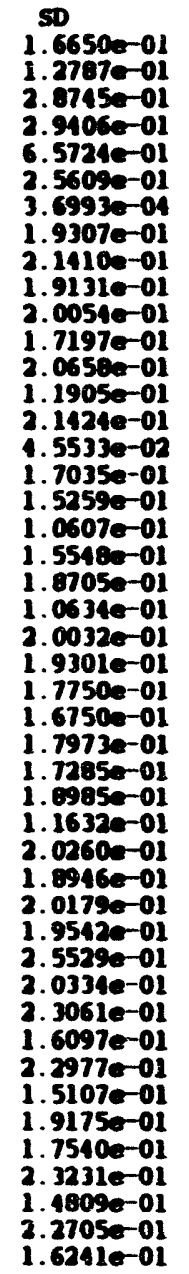 & $\begin{array}{c}\text { Uncert } \\
1.9000 e+00 \\
1.9000+00 \\
1.9002 e+00 \\
1.9002 e+00 \\
1.9002 e+00 \\
1.9002 e+00 \\
2.1295 e+00 \\
1.9002 e+00 \\
1.9002 e+00 \\
1.9002 e+00 \\
1.9002 e+00 \\
1.9002 e+00 \\
1.9002 e+00 \\
1.9011 e+00 \\
1.9002 e+00 \\
1.9009 e+00 \\
1.9002 e+00 \\
1.9002 e+00 \\
1.9011 e+00 \\
1.90020+00 \\
1.9002 e+00 \\
1.9010 e+00 \\
1.9002 e+00 \\
1.9002 e+00 \\
1.9010 e+00 \\
1.9002 e+00 \\
1.9002 e+00 \\
1.9002 e+00 \\
1.9002 e+00 \\
1.9011 e+00 \\
1.9011 e+00 \\
1.9002 e+00 \\
1.9002 e+00 \\
1.9002 e+00 \\
1.9002 e+00 \\
1.9002 e+00 \\
1.90020+00 \\
1.9002 e+00 \\
1.9002 e+00 \\
1.9002 e+00 \\
1.9002 e+00 \\
1.9002 e+00 \\
1.9002 e+00 \\
1.9002 e+00 \\
1.9002 e+00 \\
1.9002 e+00\end{array}$ \\
\hline
\end{tabular}

\begin{tabular}{|c|c|c|c|c|c|}
\hline 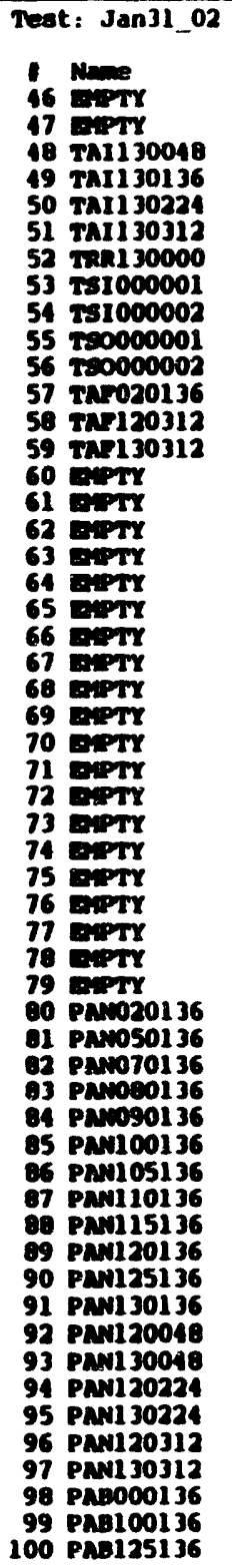 & 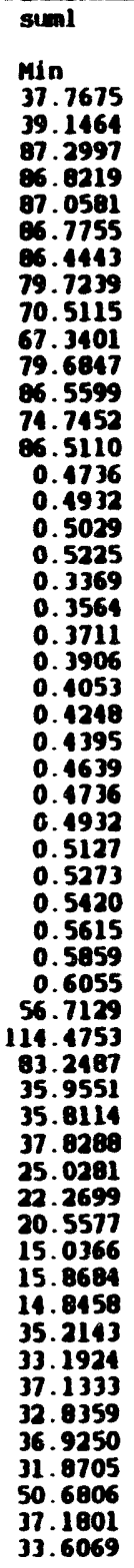 & 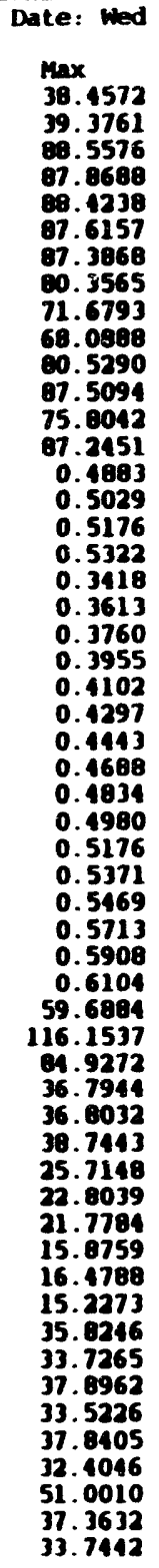 & $\begin{array}{c}\text { Jan } 3113: \\
\text { Ave } \\
38.0802 \\
39.1694 \\
87.8155 \\
87.2702 \\
87.8779 \\
87.2922 \\
87.0220 \\
79.9474 \\
71.1017 \\
67.7635 \\
80.0349 \\
87.0070 \\
75.2027 \\
86.8884 \\
0.4821 \\
0.4973 \\
0.5109 \\
0.5278 \\
0.3377 \\
0.3578 \\
0.3750 \\
0.3920 \\
0.4058 \\
0.4249 \\
0.1407 \\
0.4641 \\
0.4786 \\
0.4978 \\
0.5130 \\
0.5311 \\
0.5457 \\
0.5652 \\
0.5881 \\
0.6101 \\
58.4951 \\
115.2474 \\
84.0849 \\
36.3870 \\
36.3623 \\
38.2835 \\
25.3226 \\
22.5293 \\
21.0063 \\
15.4608 \\
16.0470 \\
15.0167 \\
35.4904 \\
33.4106 \\
37.1641 \\
33.1014 \\
37.2790 \\
32.0537 \\
50.8133 \\
37.2735 \\
33.6874\end{array}$ & $\begin{array}{c}07.021990 \\
50 \\
2.2641 e-01 \\
6.9589 e-02 \\
3.4072 e-01 \\
2.1143 e-01 \\
3.0316 e-01 \\
2.0760 e-01 \\
2.0643 e-01 \\
1.5014 e-01 \\
2.7893 e-01 \\
1.8066 e-01 \\
1.8762 e-01 \\
2.0825 e-01 \\
2.8015 e-01 \\
2.0117 e-01 \\
3.3901 e-03 \\
3.4681 e-03 \\
3.0919 e-03 \\
3.3087 e-03 \\
1.6082 e-03 \\
2.2146 e-03 \\
1.9730 e-03 \\
2.2146 e-03 \\
1.4797 e-03 \\
6.9053 e-04 \\
2.1635 e-03 \\
9.6655 e-04 \\
1.2041 e-03 \\
1.1714 e-03 \\
1.1714 e-03 \\
2.3261 e-03 \\
2.1065 e-03 \\
2.3261 e-03 \\
2.4484 e-03 \\
1.1714 e-03 \\
7.6554 e-01 \\
3.9407 e-01 \\
3.3521 e-01 \\
2.1280 e-01 \\
2.4676 e-01 \\
2.2493 e-01 \\
1.4785 e-01 \\
1.1739 e-01 \\
2.5822 e-01 \\
1.9628 e-01 \\
1.3403 e-01 \\
9.0614 e-02 \\
1.3516 e-01 \\
1.3873 e-01 \\
1.4913 e-01 \\
1.3010 e-01 \\
1.8545 e-01 \\
1.2427 e-01 \\
7.6403 e-02 \\
5.2283 e-02 \\
3.7635 e-02\end{array}$ & 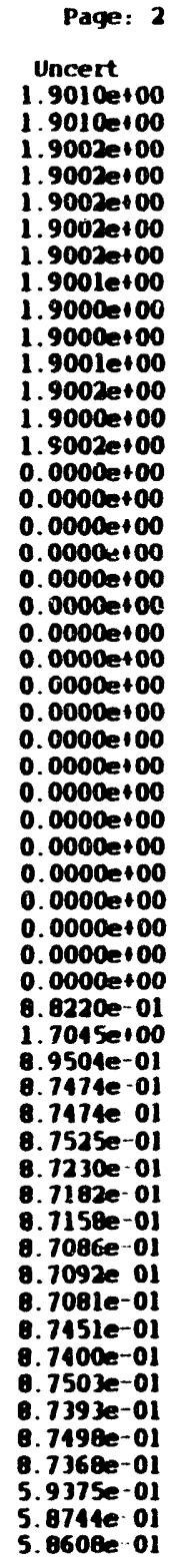 \\
\hline
\end{tabular}




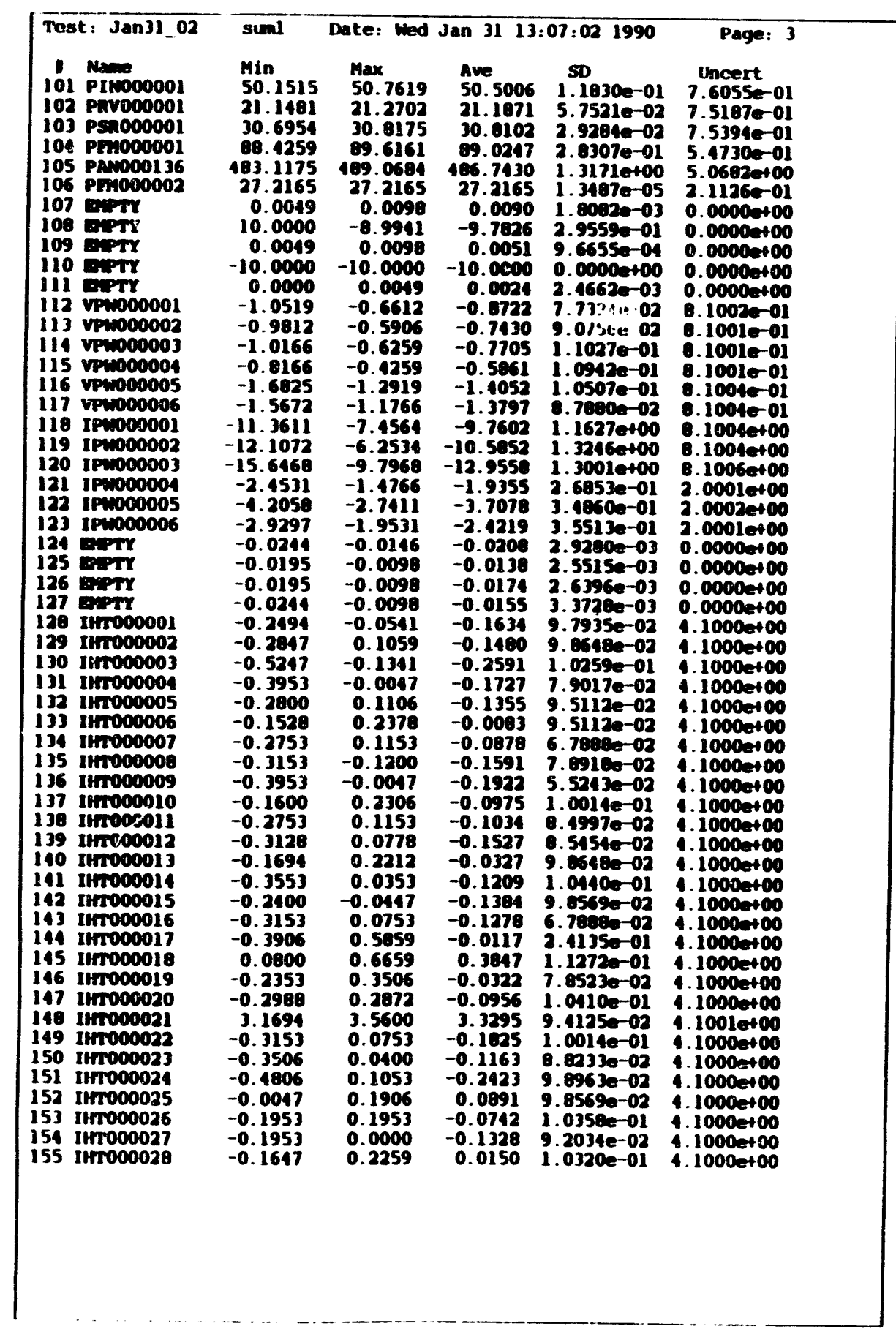

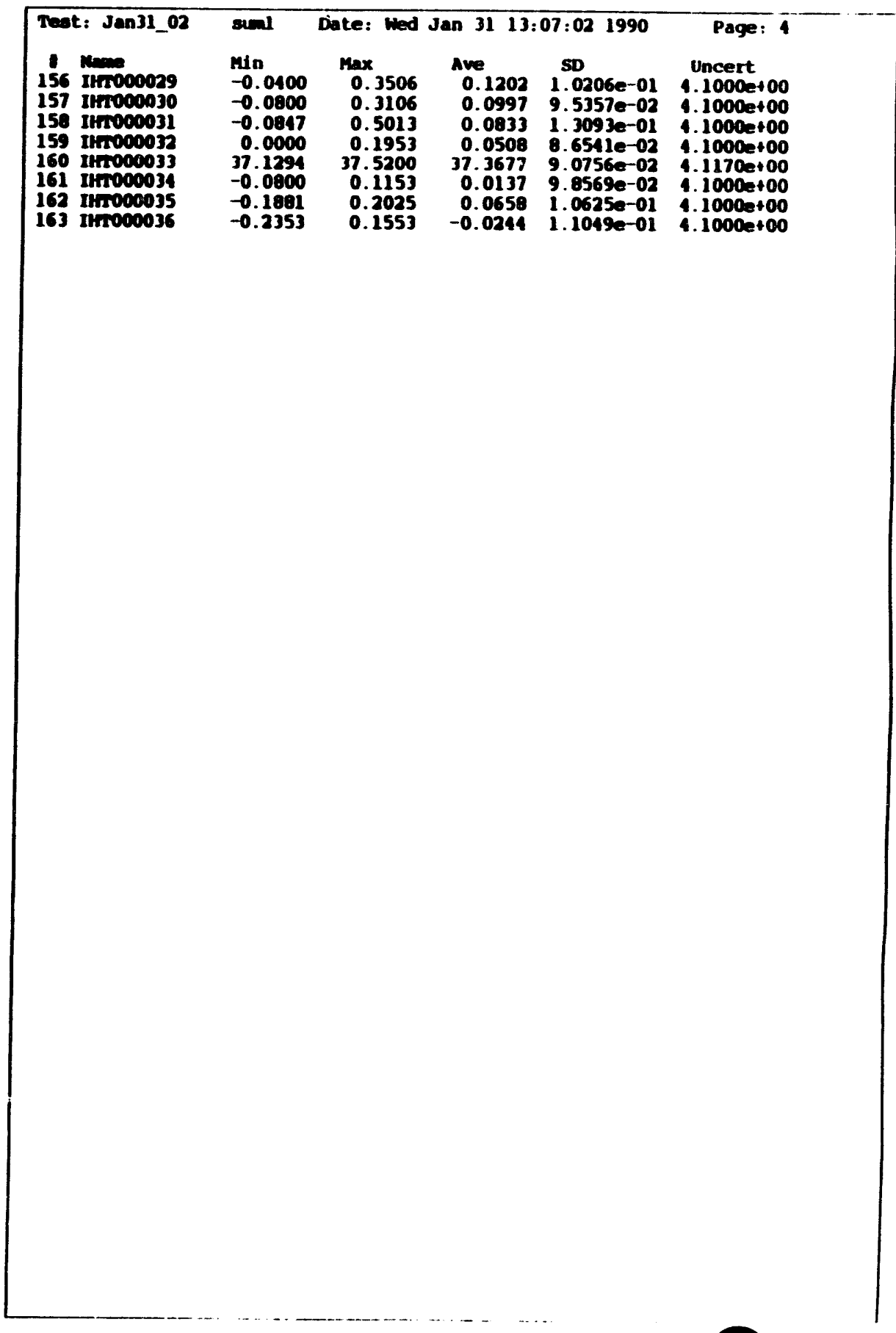




\begin{tabular}{|c|c|c|c|c|c|}
\hline $\begin{array}{l}\text { Test : Jan31 } 03 \\
\text { Velucity } 1= \\
\text { Velocity } 2- \\
\text { Pressure drop } \\
\text { Tsat in - } 276 . \\
\text { Phi Inner = } \\
\text { Phl outer = } \\
\text { D inner = } 0 . \\
\text { L Inner - } 12 .\end{array}$ & $\begin{array}{l}\text { siml } \\
22.0243 \text { (ft } \\
13.2409 \text { (ft } \\
14.2177 \\
8835 \text { (deg F } \\
0.0056 \text { (kBt } \\
0.0076 \text { (kBt } \\
1973 \text { (ft) } \\
0330 \text { (ft) }\end{array}$ & $\begin{array}{l}\text { Date: Wed } \\
\text { t/sec) }(1 /- \\
\text { t/sec) }(1 /- \\
\text { (psi) } \\
\text { F) Tsat out } \\
\text { tu/hr-ft2) } \\
\text { tumr ft2) } \\
\text { b outer - } \\
\text { L outer - }\end{array}$ & $\begin{array}{r}\text { Jan } 31 \text { 13: } \\
3.4867 \\
3.4867 \\
=255.96 \\
1+/-118 . \\
1+/-121 \\
0.2412 \\
12.8330\end{array}$ & $\begin{array}{l}09: 171990 \\
(1) \\
\text { I) } \\
40 \text { (deg F) } \\
8403 \text { :) } \\
8712 \text { :) } \\
\text { ft) D hyd - } \\
\text { (t) A flow - }\end{array}$ & $\begin{array}{l}0.0438 \text { (ft) } \\
0.0151 \text { (ft2) }\end{array}$ \\
\hline 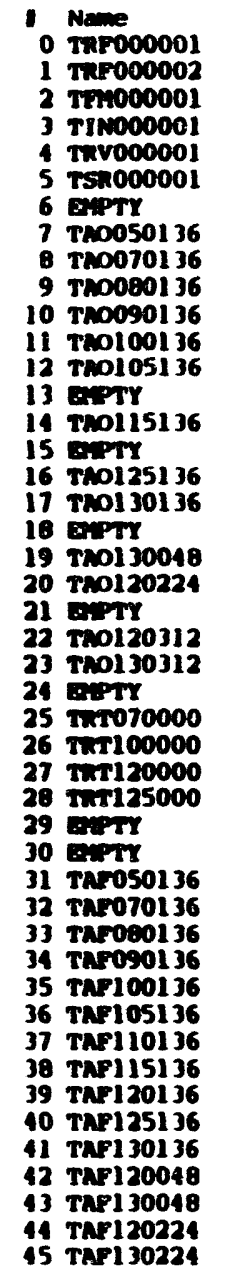 & $\begin{array}{l}\text { Min } \\
71.1838 \\
74.9393 \\
86.5599 \\
86.8362 \\
86.9429 \\
87.7647 \\
601.0594 \\
86.2418 \\
87.4240 \\
87.2136 \\
86.7736 \\
87.1893 \\
87.4115 \\
10.9819 \\
86.6492 \\
37.5314 \\
87.0866 \\
86.9369 \\
11.2111 \\
87.0465 \\
86.7467 \\
39.6870 \\
86.9213 \\
85.7149 \\
39.1464 \\
86.8179 \\
87.4251 \\
87.1244 \\
87.1041 \\
39.3761 \\
39.3761 \\
87.1644 \\
87.6567 \\
87.1612 \\
87.2313 \\
87.0003 \\
87.0687 \\
87.0658 \\
87.5339 \\
87.2101 \\
87.3808 \\
87.1796 \\
87.3993 \\
86.9983 \\
87.0086 \\
86.6376\end{array}$ & $\begin{array}{r}\operatorname{Max} \\
74.8213 \\
75.3622 \\
87.3944 \\
87.6761 \\
87.6751 \\
88.2882 \\
-601.0594 \\
86.9747 \\
88.1756 \\
87.8420 \\
87.8209 \\
87.8189 \\
88.2806 \\
41.2111 \\
87.3856 \\
37.7675 \\
87.7154 \\
87.7697 \\
11.4403 \\
87.6756 \\
87.3765 \\
39.1464 \\
87.6553 \\
87.3438 \\
39.6057 \\
87.4506 \\
88.2612 \\
87.8585 \\
87.9405 \\
39.8352 \\
40.0646 \\
87.8965 \\
88.5960 \\
87.7992 \\
88.2790 \\
87.6305 \\
88.0076 \\
97.6947 \\
88.2668 \\
87.7336 \\
88.1121 \\
87.8091 \\
88.3483 \\
87.6281 \\
88.0567 \\
87.2689\end{array}$ & $\begin{array}{l}\text { Ave } \\
74.5111 \\
75.1465 \\
86.9336 \\
87.2563 \\
87.3070 \\
88.0033 \\
-601.0591 \\
86.5434 \\
87.9538 \\
87.5281 \\
87.3521 \\
87.5586 \\
87.8025 \\
41.0920 \\
87.0536 \\
37.5743 \\
87.3925 \\
87.3254 \\
41.2707 \\
87.3738 \\
87.0700 \\
38.8092 \\
87.3282 \\
87.0089 \\
39.3393 \\
97.2049 \\
87.8136 \\
87.5606 \\
87.5643 \\
39.5701 \\
39.6883 \\
87.5159 \\
80.1611 \\
87.4964 \\
87.7973 \\
87.3531 \\
87.5983 \\
87.3387 \\
87.8769 \\
87.5197 \\
87.7178 \\
87.5614 \\
87.8085 \\
87.3635 \\
87.5832 \\
87.0457\end{array}$ & $\begin{array}{r}\text { SD } \\
1.5003 e-01 \\
1.4460 e-01 \\
1.8939 e-01 \\
1.6703 e-01 \\
1.8687 e-01 \\
1.5099 e-01 \\
3.6993 e-04 \\
1.6054 e-01 \\
2.0474 e-01 \\
1.6230 e-01 \\
2.1395 e-01 \\
1.5893 e-01 \\
1.8248 e-01 \\
1.1567 e-01 \\
1.8570 e-01 \\
8.5185 e-02 \\
1.6371 e-01 \\
1.8326 e-01 \\
1.0152 e-01 \\
1.5637 e-01 \\
1.5976 e-01 \\
1.3642 e-01 \\
1.6502 e-01 \\
1.4967 e-01 \\
1.6309 e-01 \\
1.7719 e-01 \\
1.9151 e-01 \\
1.5472 e-01 \\
1.9773 e-01 \\
9.9904 e-02 \\
2.2122 e-01 \\
1.6472 e-01 \\
1.9594 e-01 \\
1.5509 e-01 \\
2.3278 e-01 \\
1.9406 e-01 \\
2.4536 e-01 \\
1.3875 e-01 \\
1.7060 e-01 \\
1.4338 e-01 \\
1.7939 e-01 \\
1.5954 e-01 \\
1.9707 e-01 \\
1.3610 e-01 \\
2.2922 e-01 \\
1.5847 e-01\end{array}$ & $\begin{array}{l}\text { Uhcert } \\
1.9000 e+00 \\
1.9000 e+00 \\
1.9002 e+00 \\
1.9002 e+00 \\
1.9002 e+00 \\
1.9002 e+00 \\
2.1295 e+00 \\
1.9002 e+00 \\
1.9002 e+00 \\
1.9002 e+00 \\
1.9002 e+00 \\
1.9002 e+00 \\
1.9002 e+00 \\
1.9011 e+00 \\
1.9002 e+00 \\
1.9010 e+00 \\
1.9002 e+00 \\
1.9002 e+00 \\
1.90110+00 \\
1.9002 e+00 \\
1.9002 e+00 \\
1.9010 e+00 \\
1.9002 e+00 \\
1.9002 e+00 \\
1.9010 e+00 \\
1.9002 e+00 \\
1.9002 e+00 \\
1.9002 e+00 \\
1.9002 e+00 \\
1.9011 e+00 \\
1.90110+00 \\
1.9002 e+00 \\
1.9002 e+00 \\
1.9002 e+00 \\
1.9002 e+00 \\
1.9002 e+00 \\
1.9002 e+00 \\
1.9002 e+00 \\
1.9002 e+00 \\
1.9002 e+00 \\
1.9002 e+00 \\
1.90022+00 \\
1.9002 e+00 \\
1.9002 e+00 \\
1.9002 e+00 \\
1.9002 e+00\end{array}$ \\
\hline
\end{tabular}

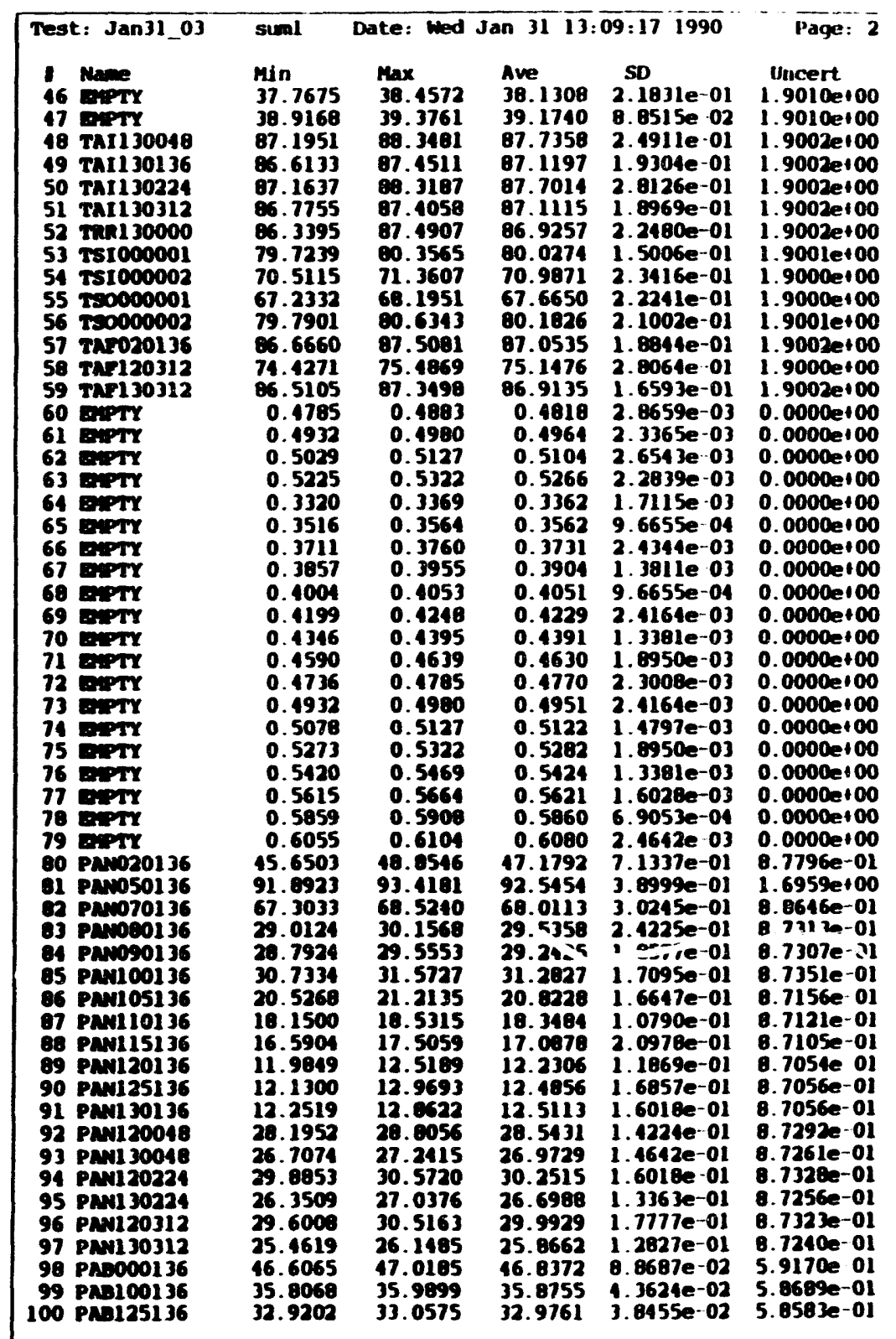



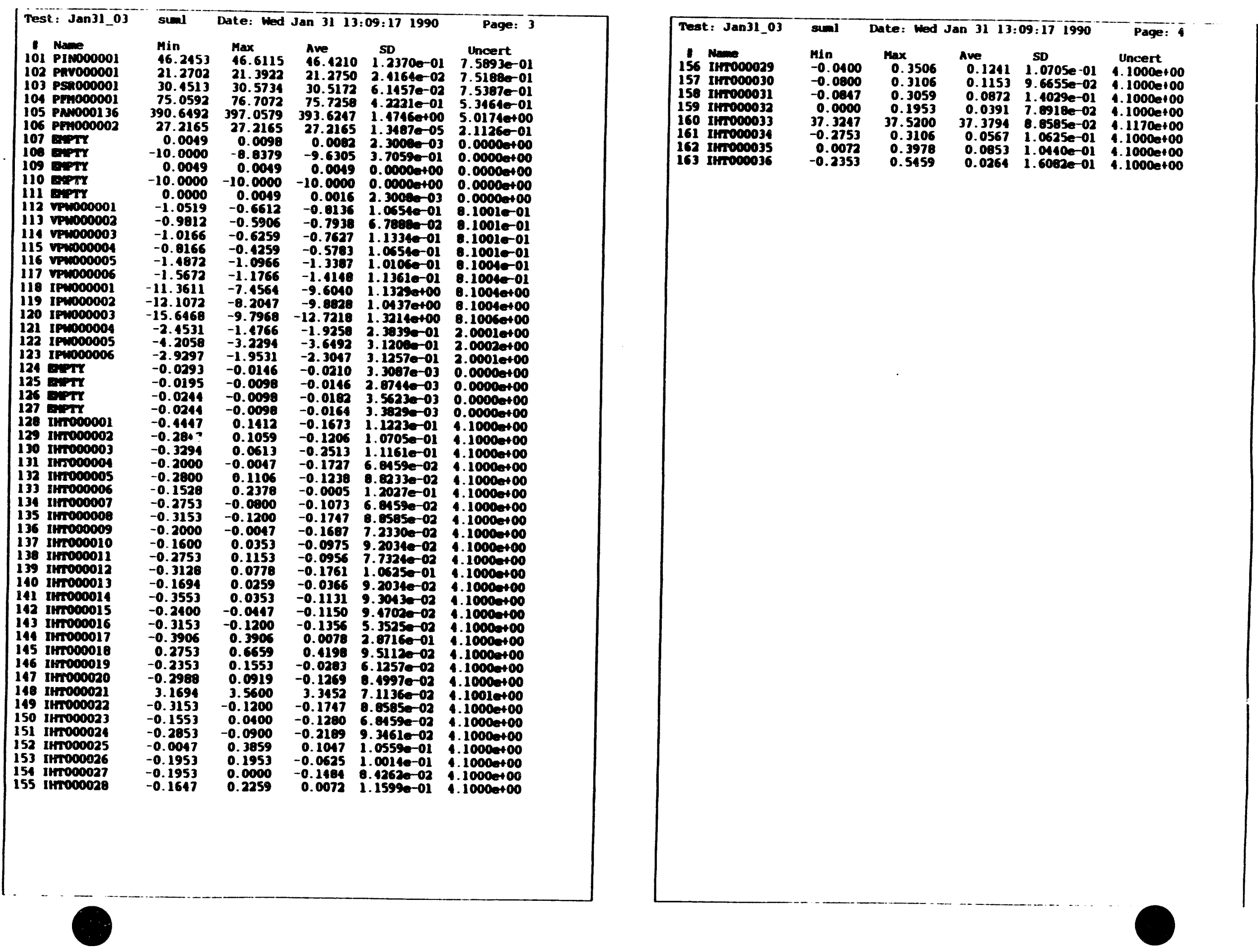


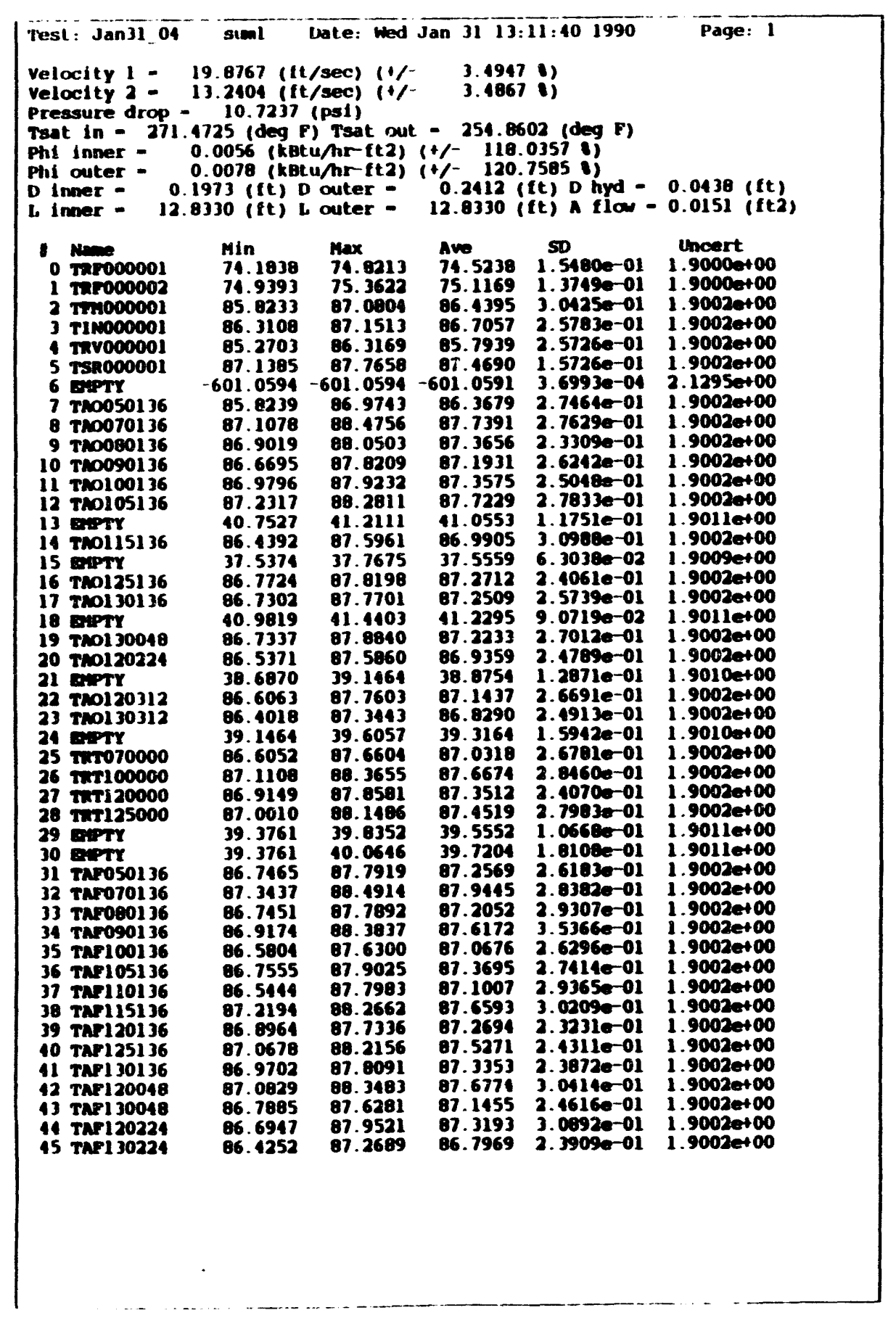

\begin{tabular}{|c|c|c|c|c|c|}
\hline 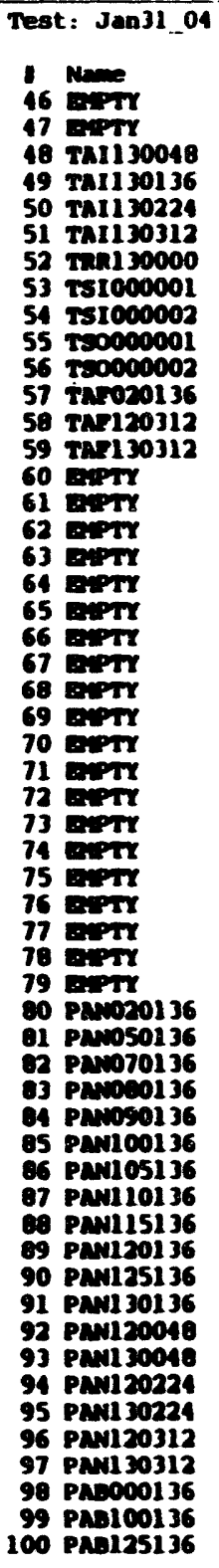 & 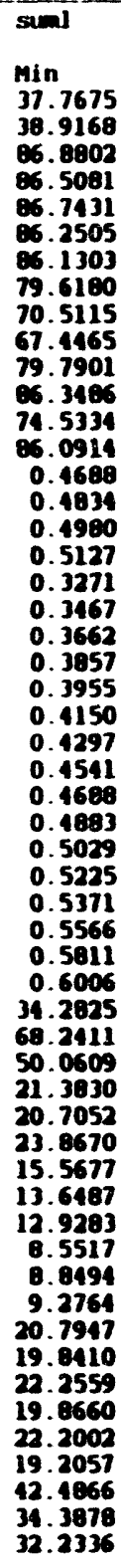 & 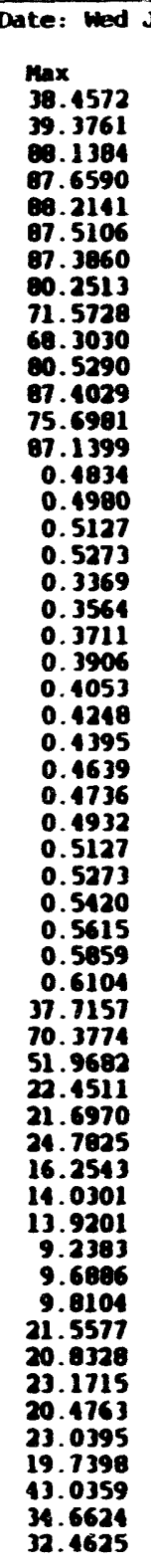 & 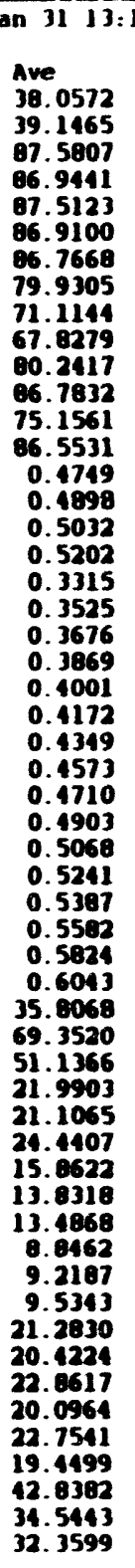 & 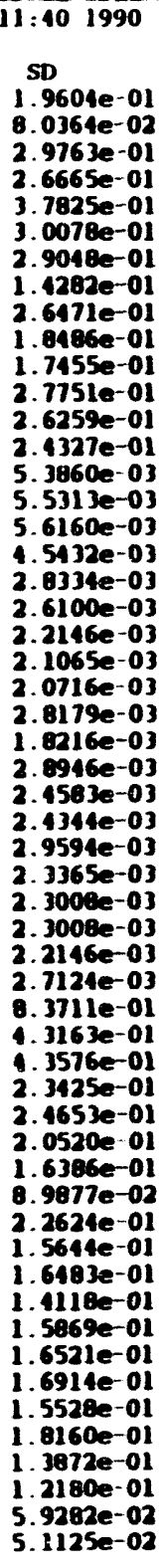 & 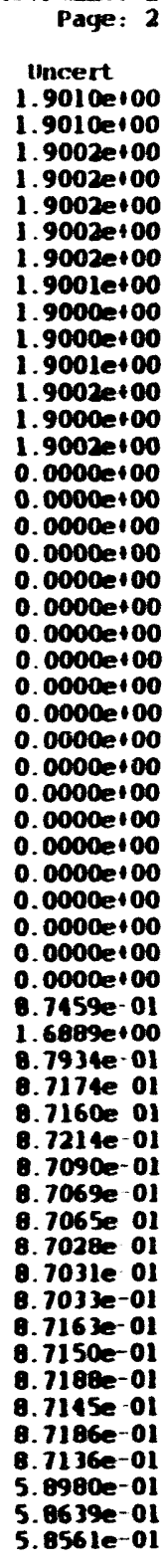 \\
\hline
\end{tabular}




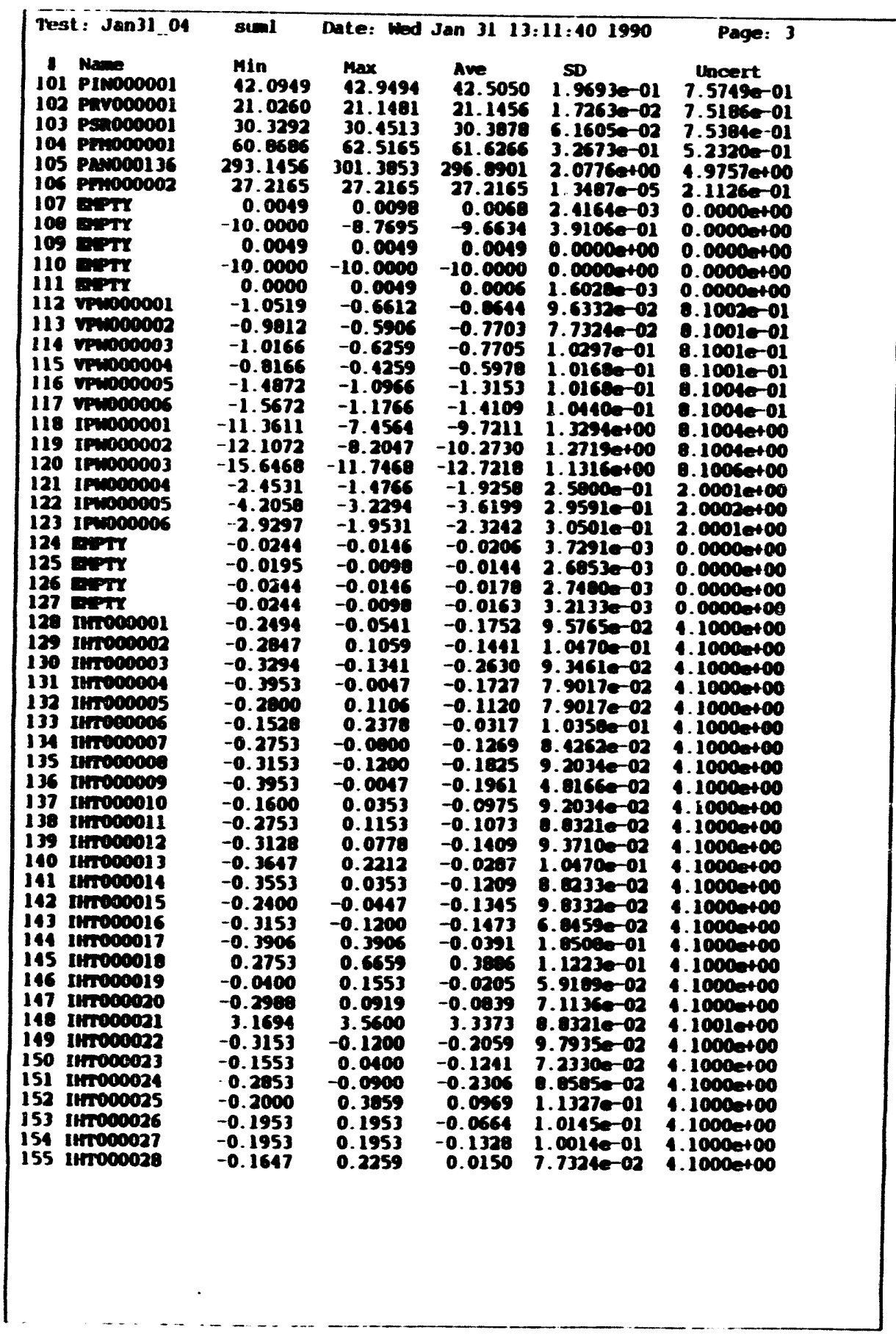

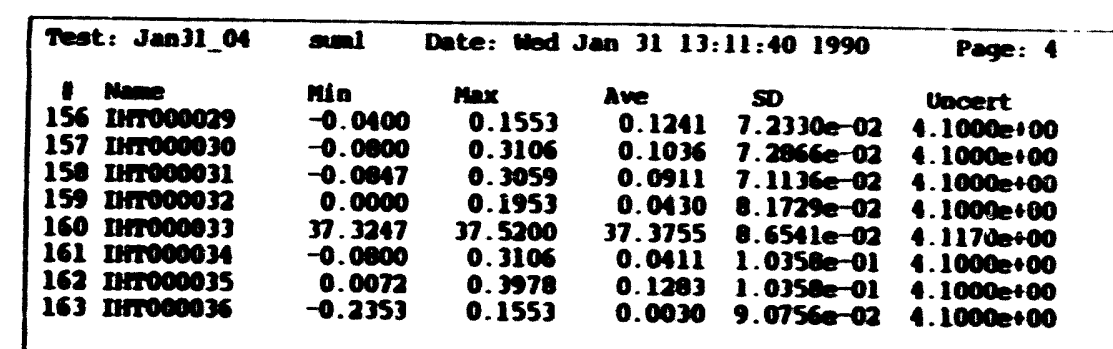




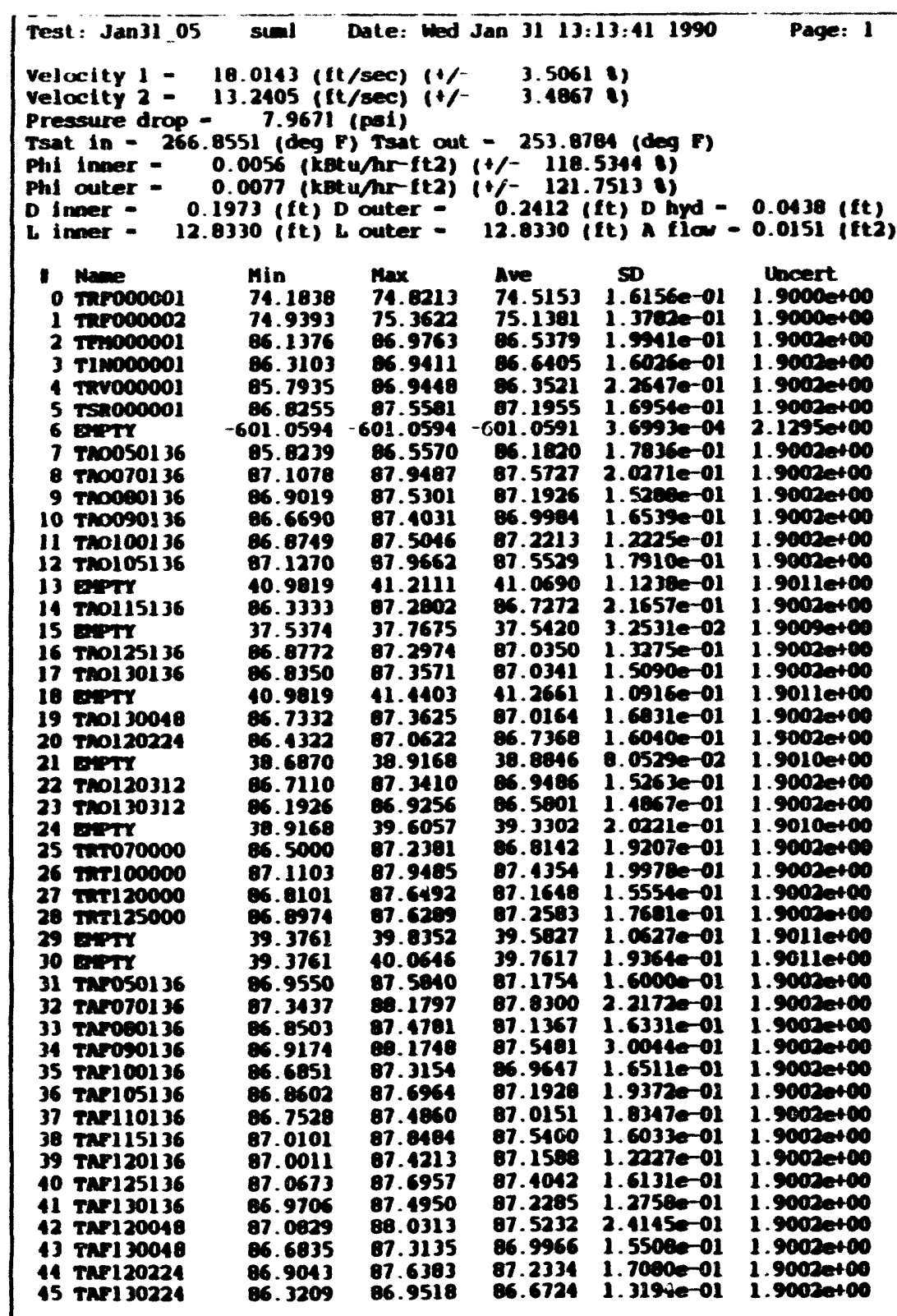

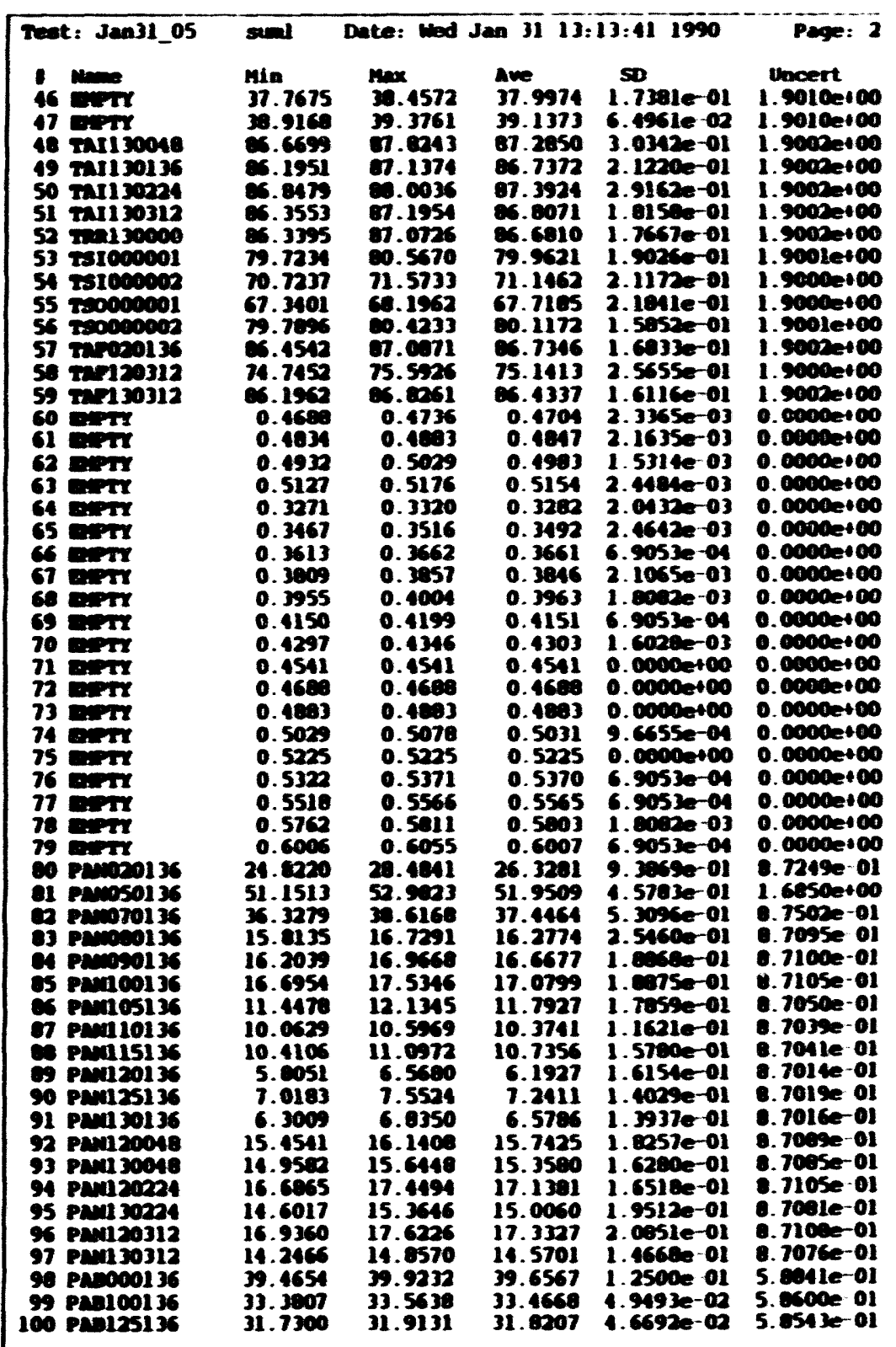




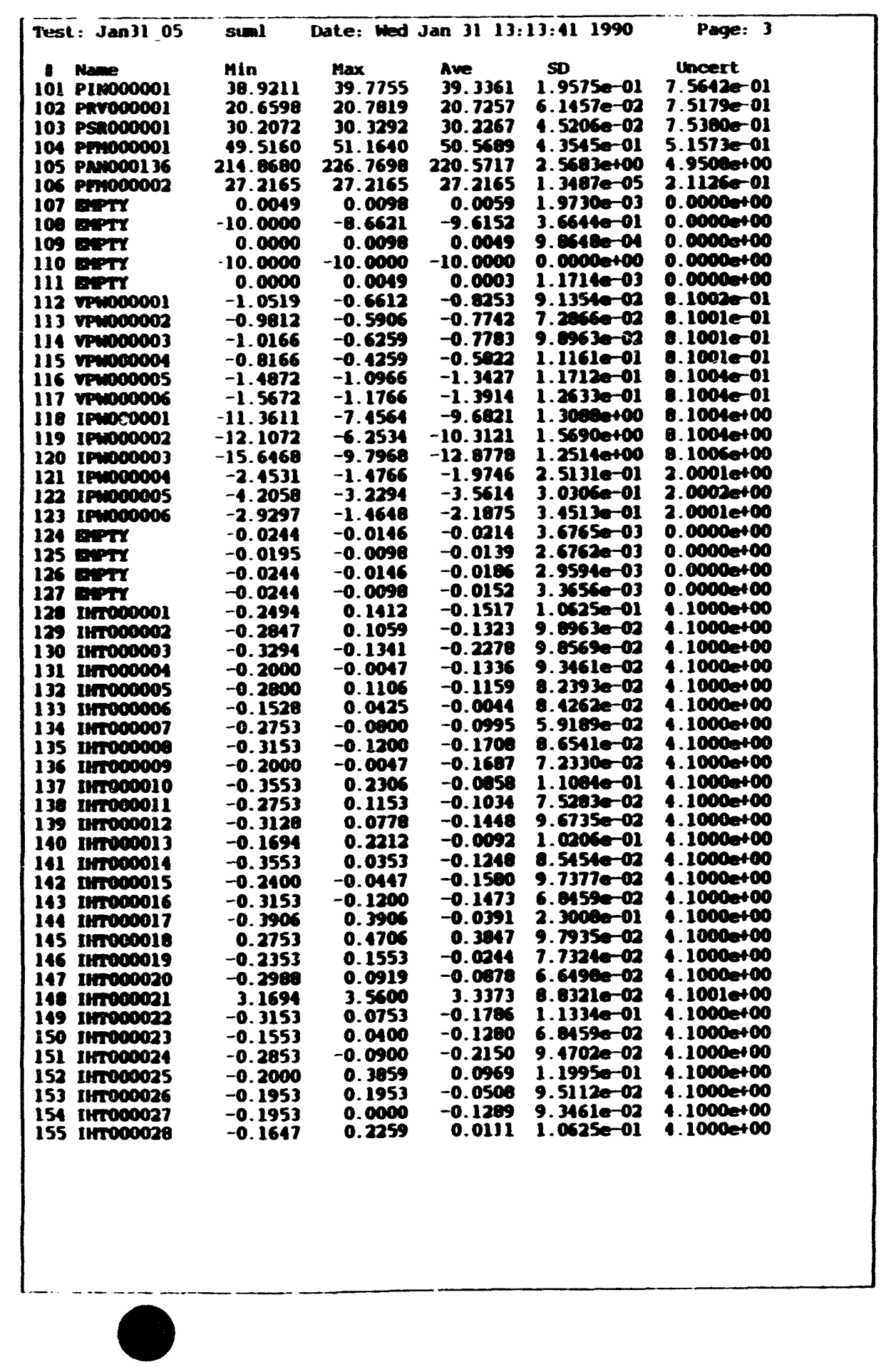

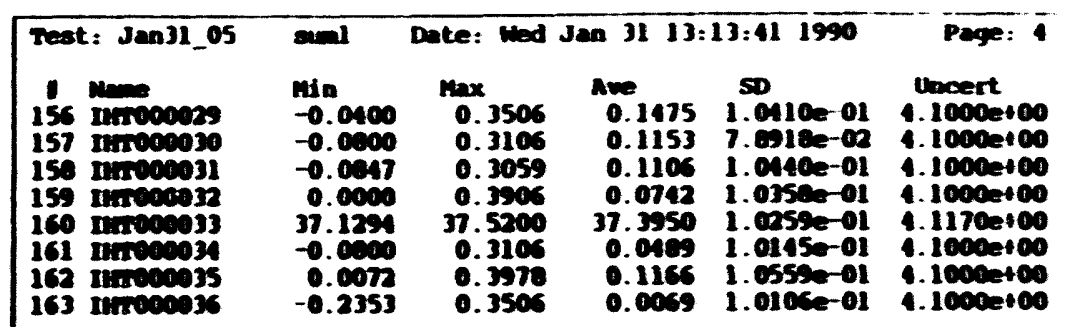




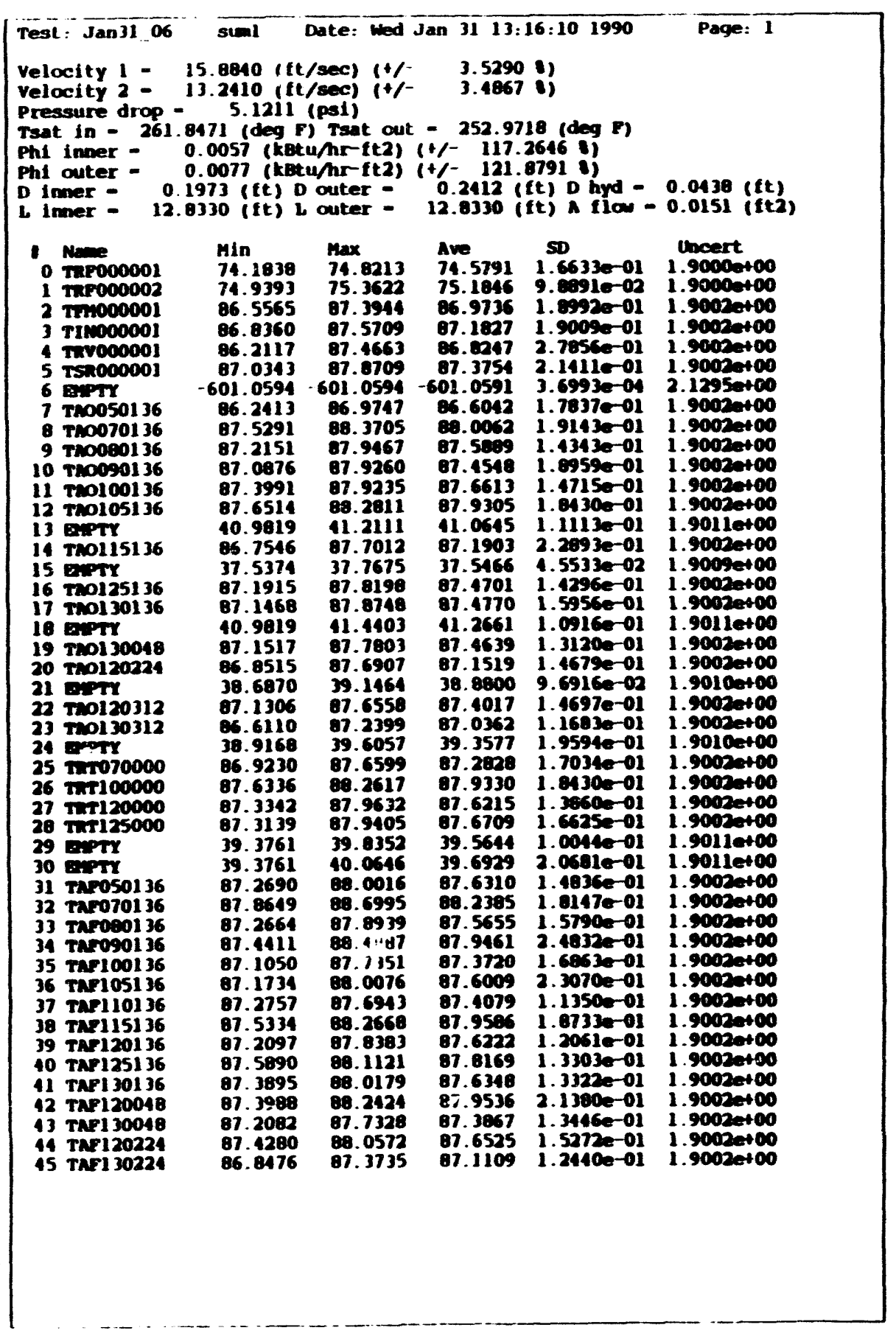

\begin{tabular}{|c|c|c|c|c|c|}
\hline 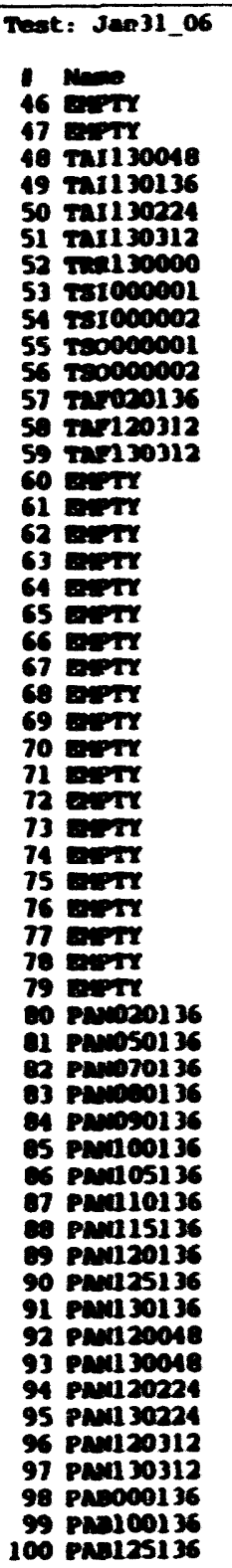 & 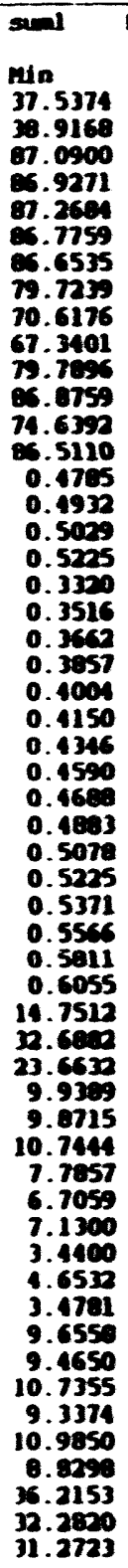 & 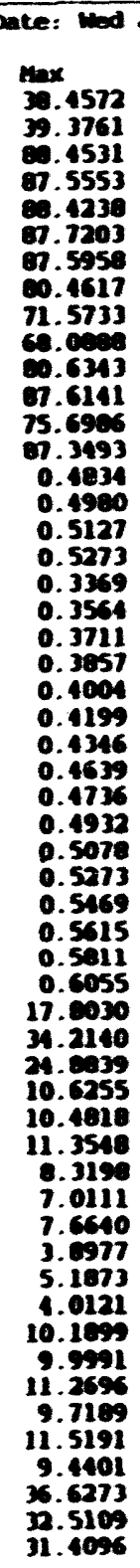 & 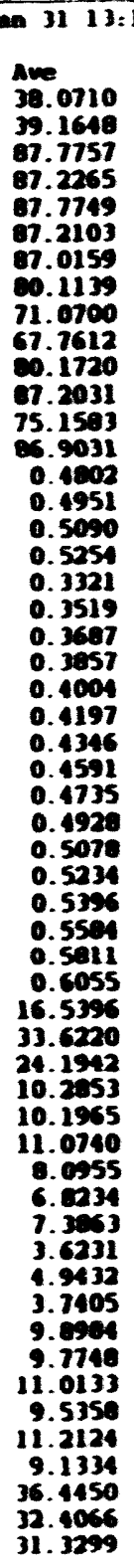 & 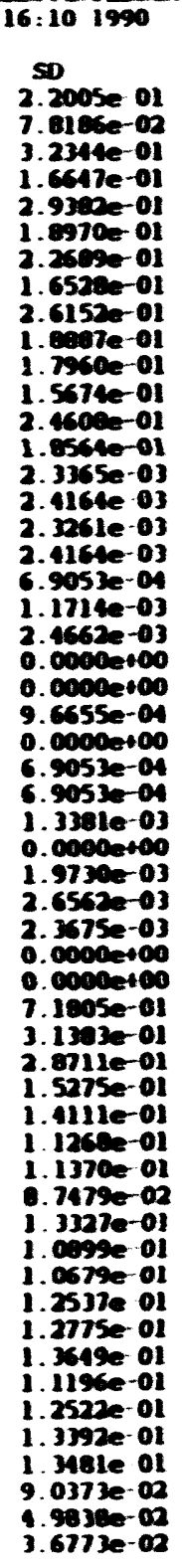 & 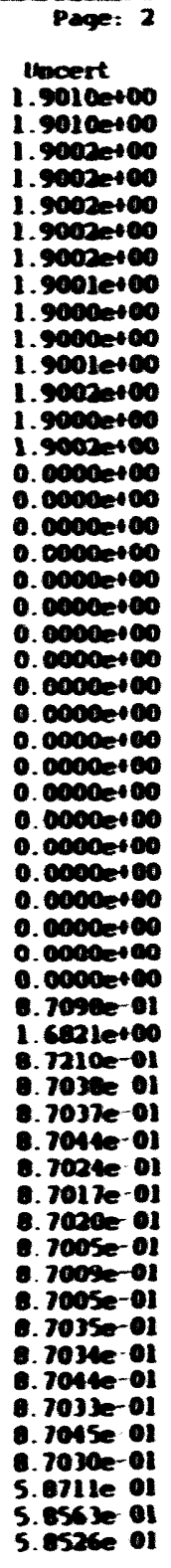 \\
\hline
\end{tabular}




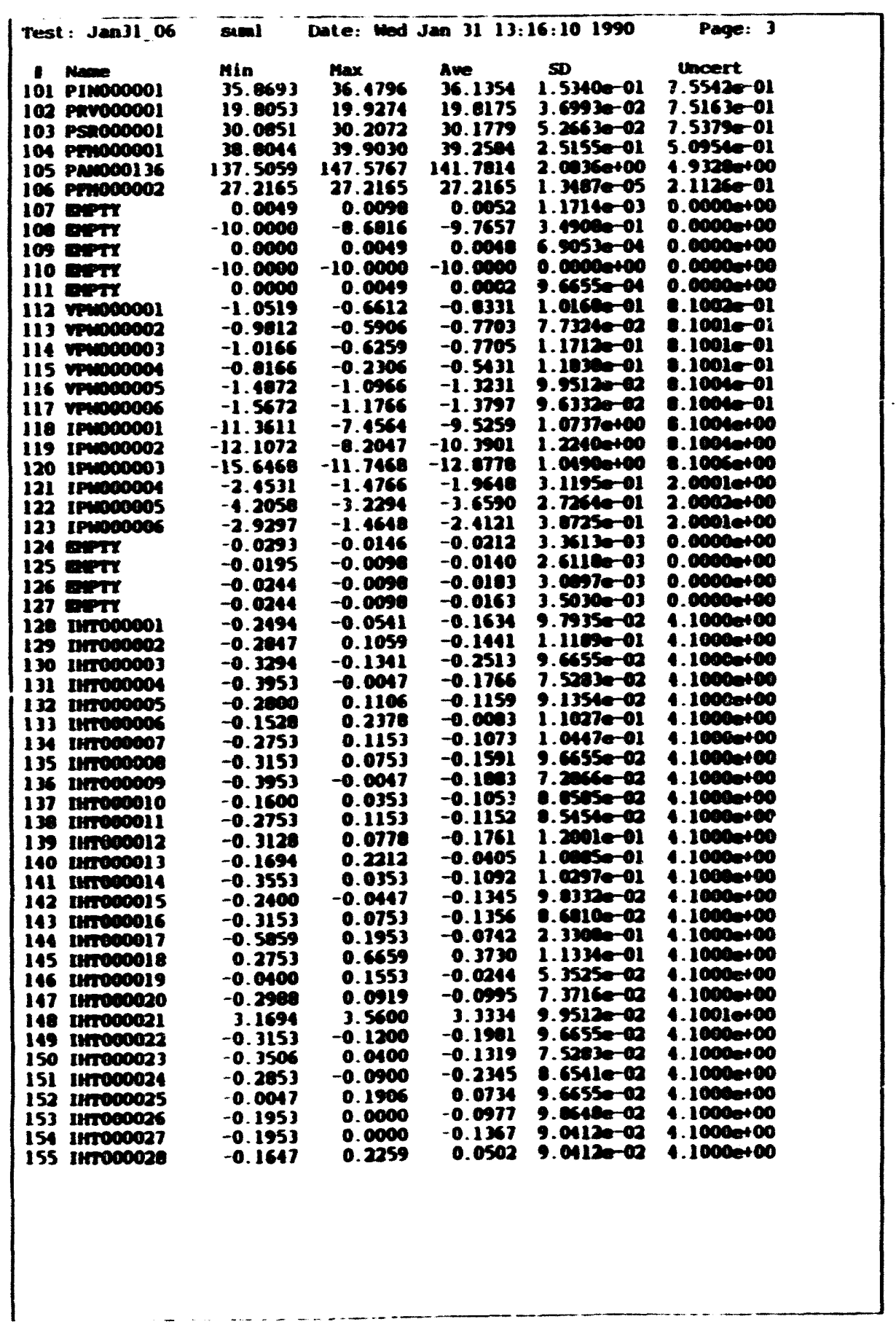

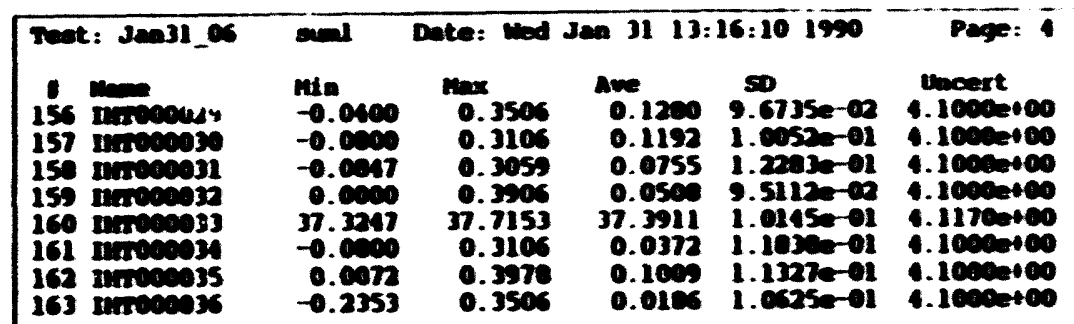




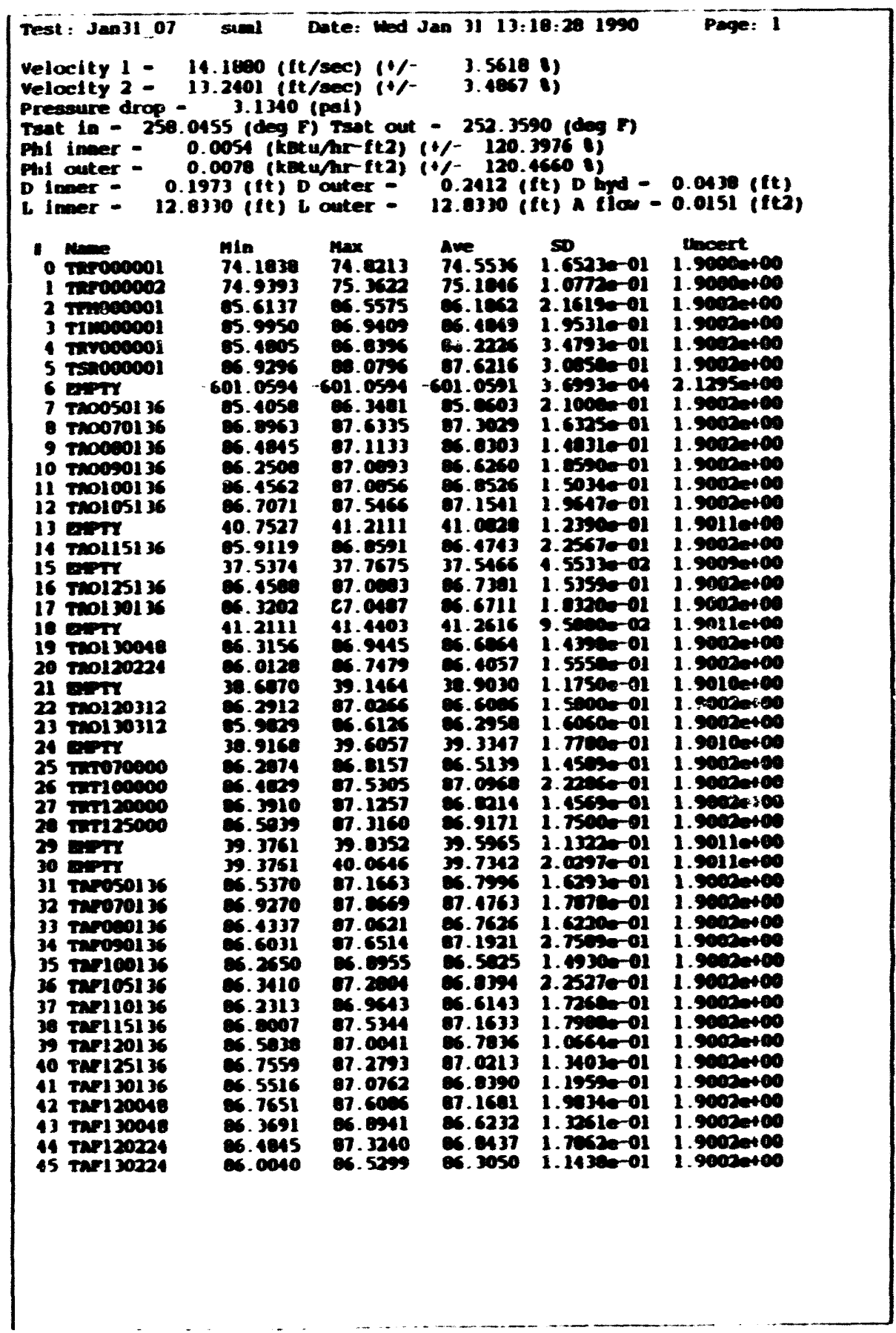

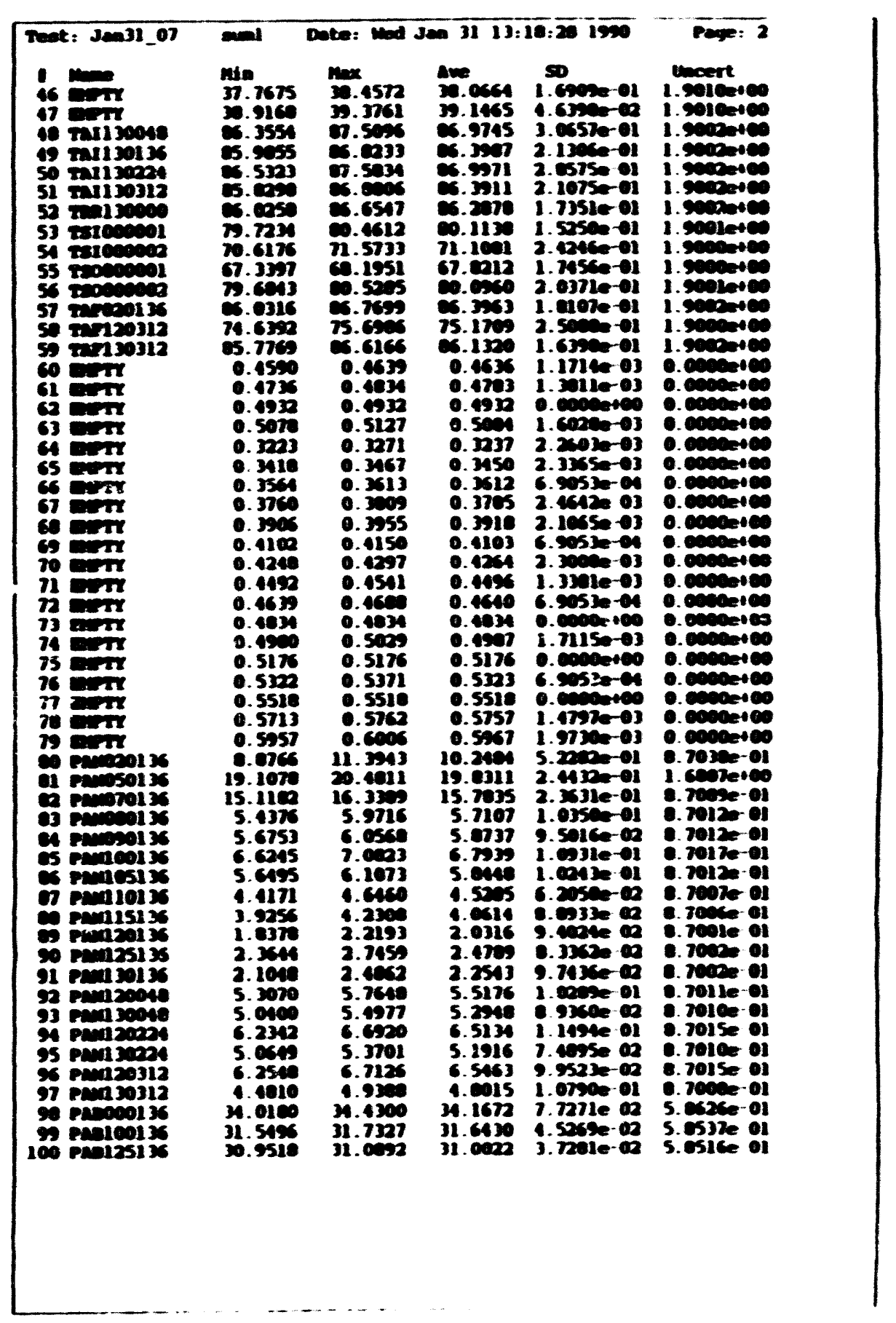




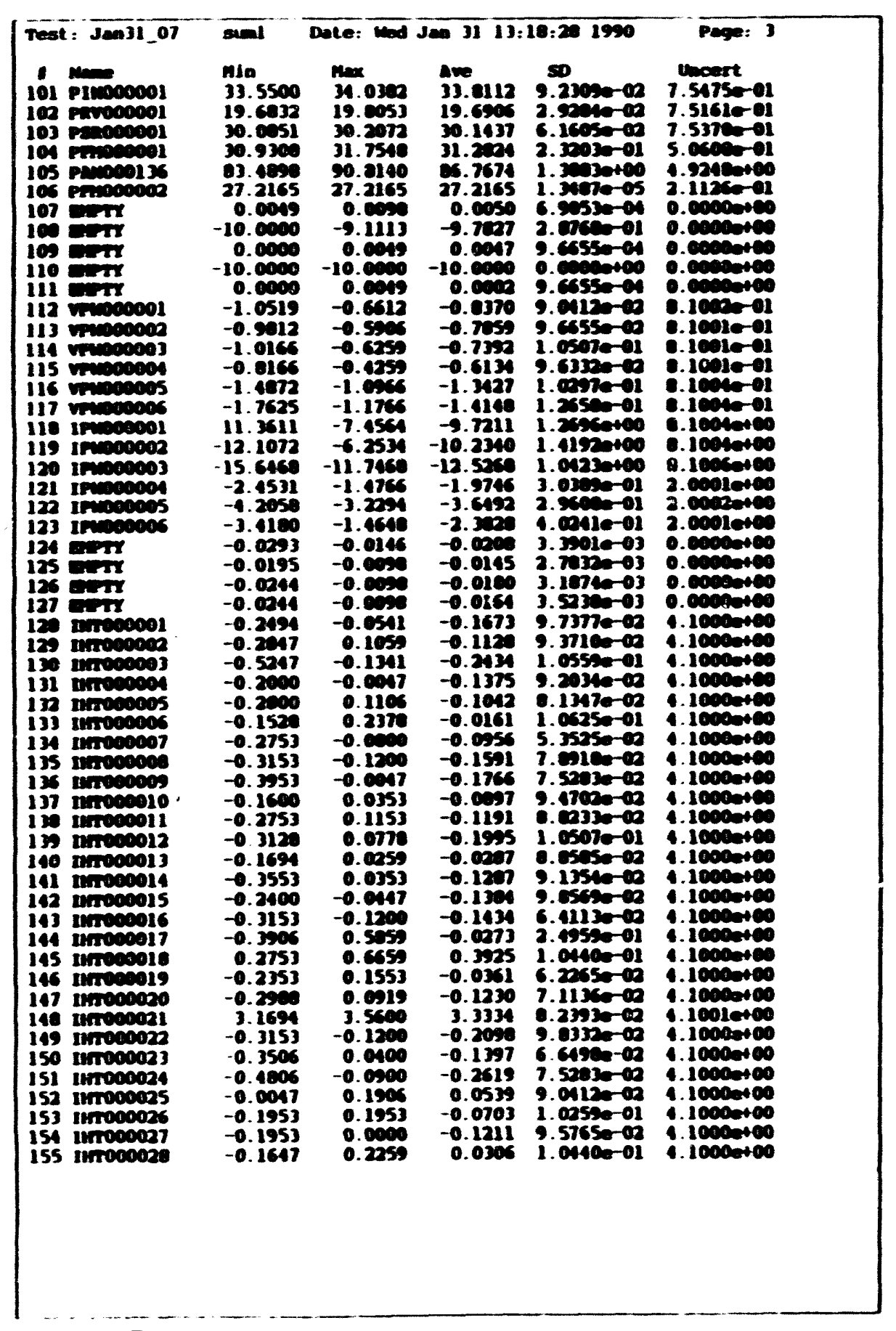

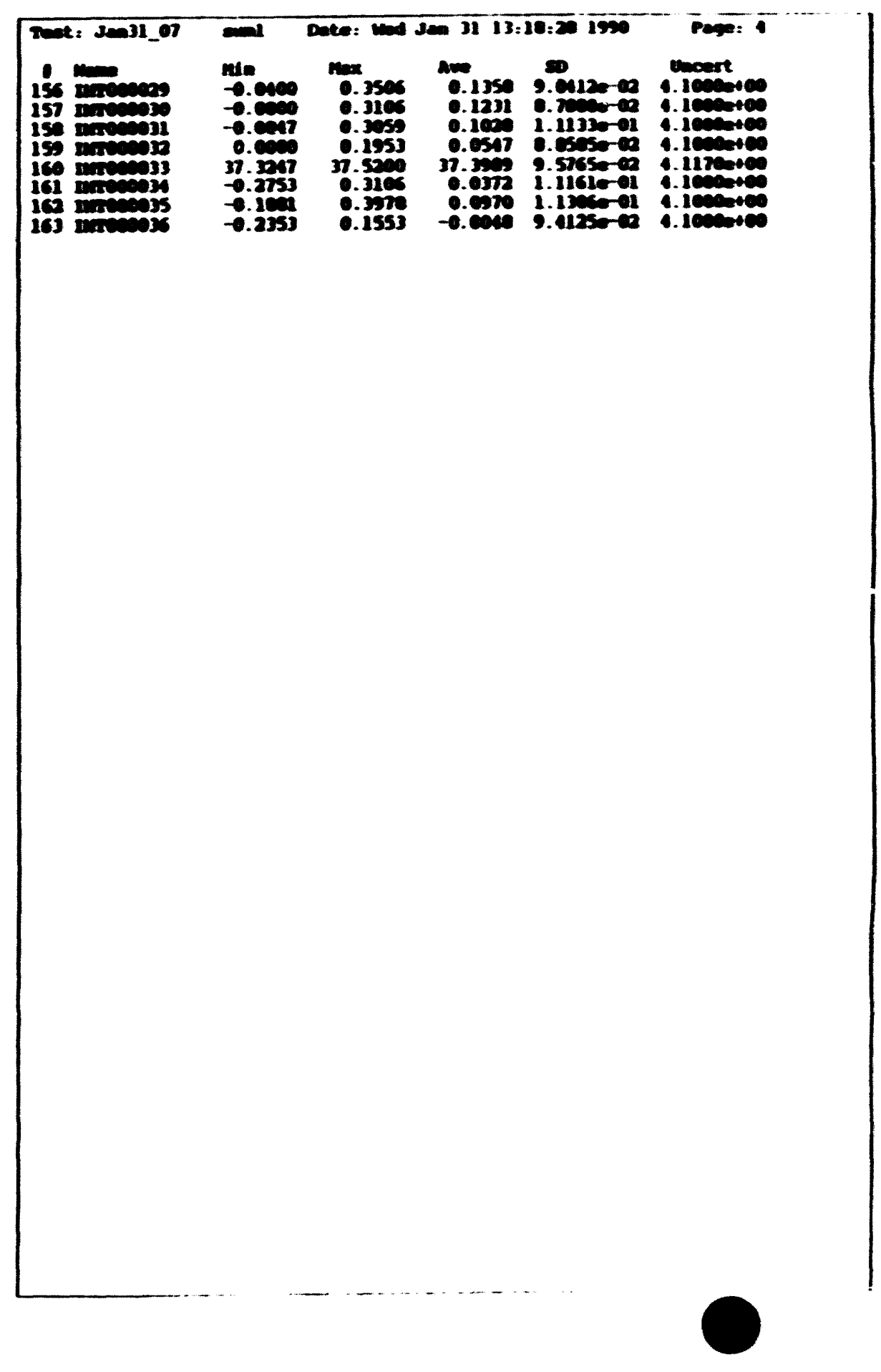




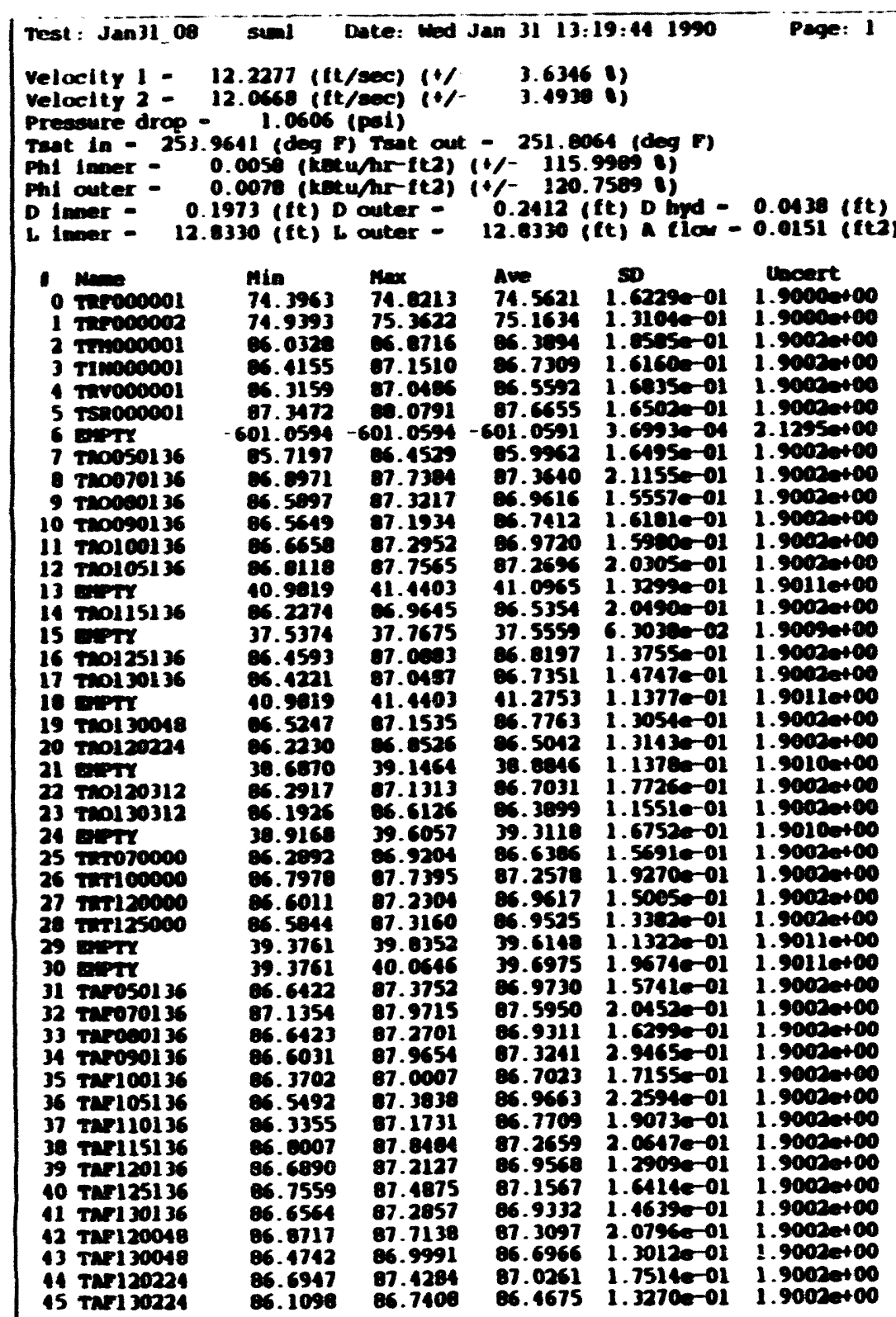

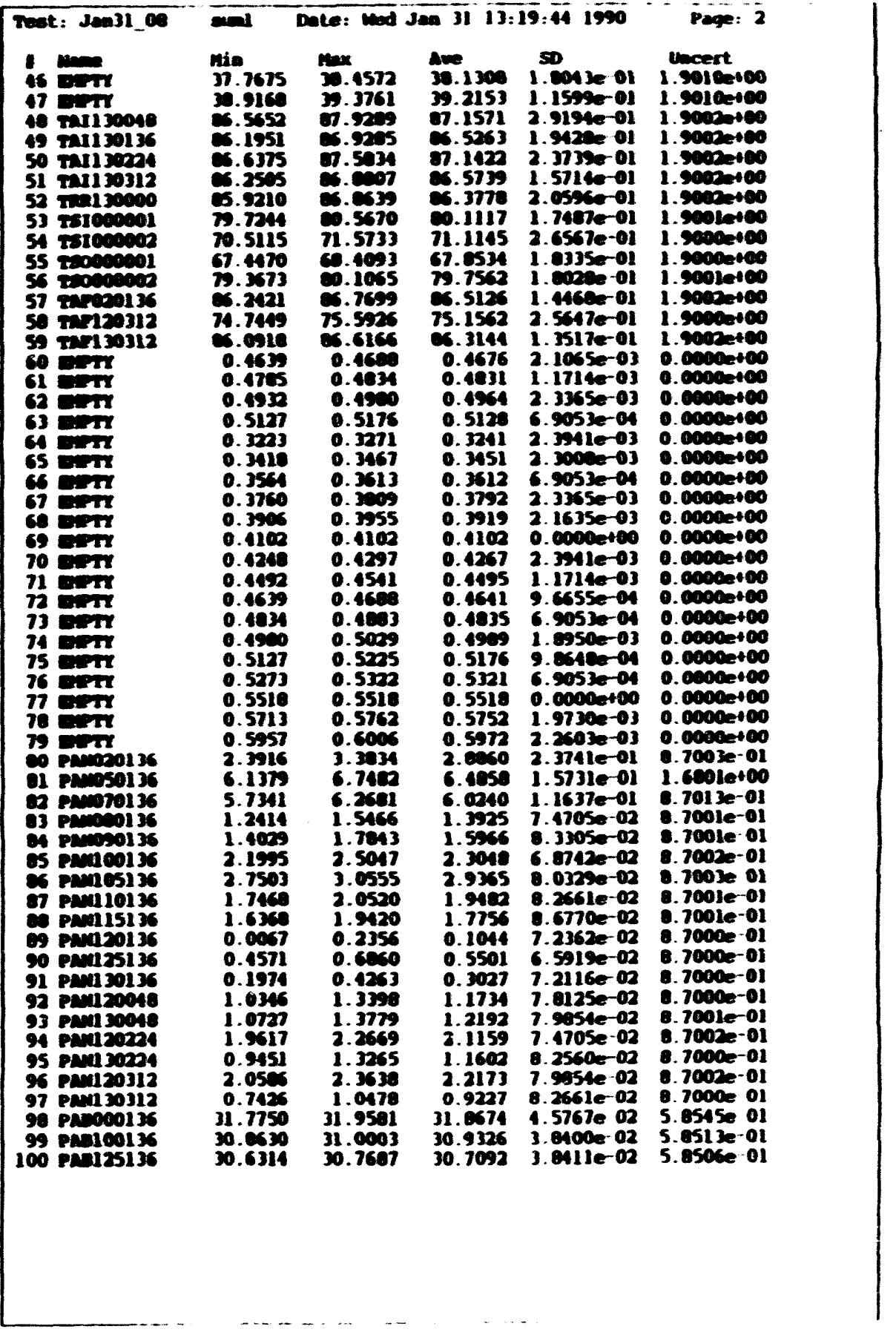




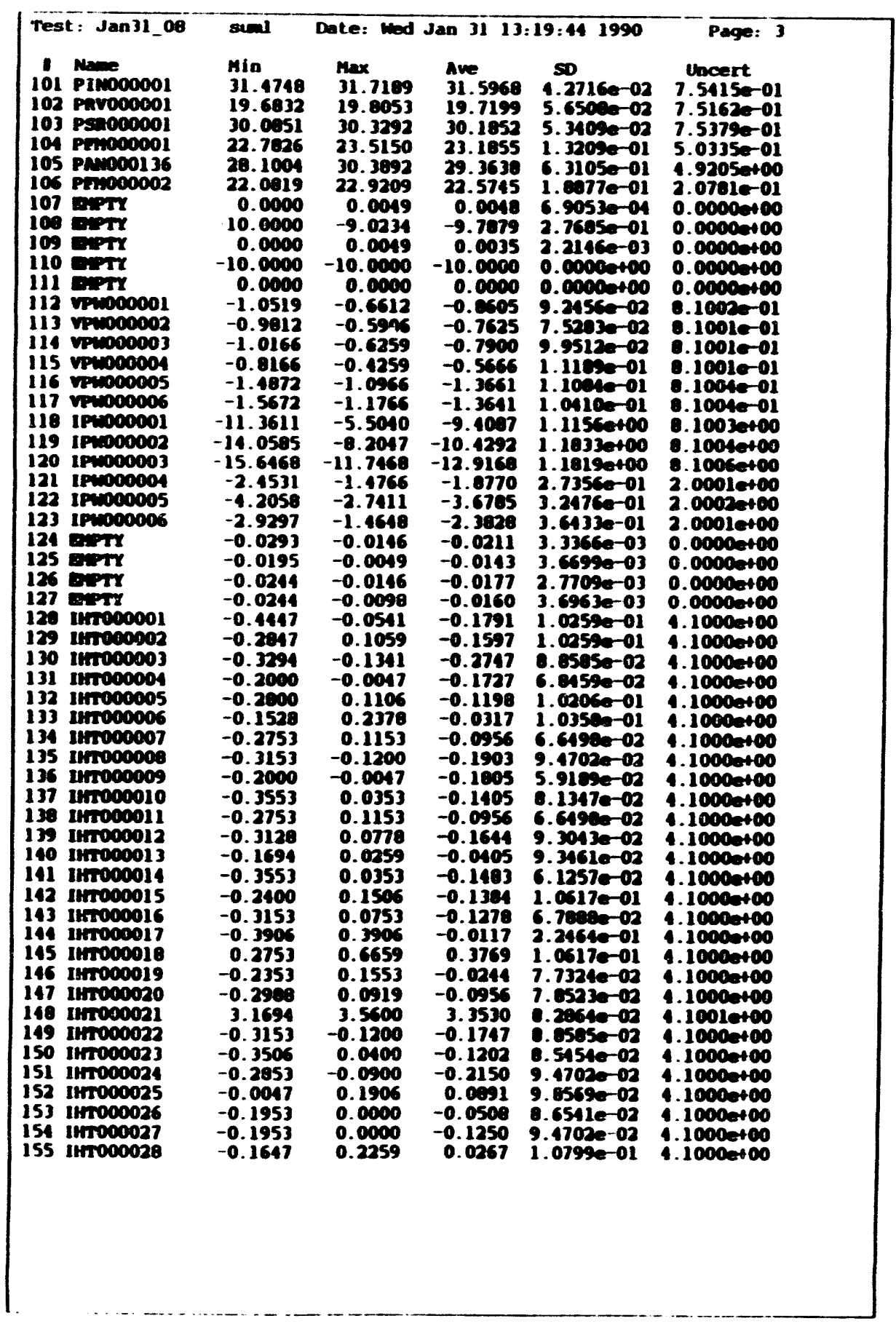

\begin{tabular}{|c|c|c|c|c|c|}
\hline 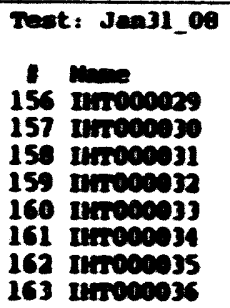 & 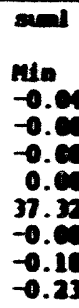 & $\begin{array}{c}\text { Date: }=0 d \\
\text { Max } \\
0.3506 \\
0.3106 \\
0.3059 \\
0.1953 \\
37.5200 \\
0.3106 \\
0.3978 \\
0.1553\end{array}$ & $\begin{array}{l}\text { an } 3113 \\
\text { Ave } \\
0.1202 \\
0.0950 \\
0.0033 \\
0.0391 \\
37.3755 \\
0.0294 \\
0.0970 \\
-0.0000\end{array}$ & 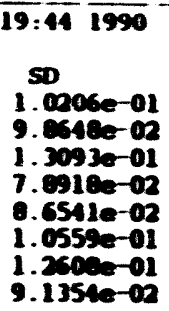 & $\begin{array}{l}\text { Page: } \\
\text { uncert } \\
1.1000=+100 \\
1.1000=+00 \\
4.1000=+00 \\
1.1000=+00 \\
1.1170=+00 \\
1.1000=+00 \\
1.1000=+00 \\
4.1000=+00\end{array}$ \\
\hline
\end{tabular}




\begin{tabular}{|c|c|c|c|c|c|}
\hline 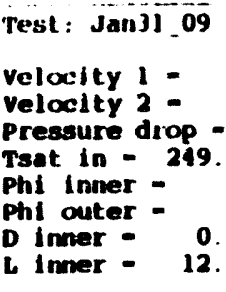 & $\begin{array}{l}\text { sionl } \\
9.9004 \text { (ft } \\
9.8064 \text { ift } \\
-0.9933 \\
1171 \text { (deg } \\
0.0001 \text { (kBt } \\
0.0000 \text { (kBt } \\
1973 \text { (ft) D } \\
8330 \text { (ft) }\end{array}$ & $\begin{array}{l}\text { Date: Wed } \\
\text { /sec) ( }+/- \\
\text { (sec) (t/- } \\
\text { (psi) } \\
\text { Tsat out } \\
\text { u/mr-ft2) } \\
\text { u/mr-ft2) } \\
\text { outer - } \\
\text { outer - }\end{array}$ & $\begin{array}{r}\text { Jan } 3113: \\
3.8424 \\
3.5235 \\
-250.73 \\
1+1-6769 \\
1+1-14263 \\
0.2412 ! \\
12.8330 !\end{array}$ & $\begin{array}{l}21: 221990 \\
\text { () } \\
\text { i) } \\
08 \text { (deg F) } \\
1182 \text { (I) } \\
6.8125 \text { (I) } \\
\text { (t) D hyd - } \\
\text { (t) A t iow - }\end{array}$ & $\begin{array}{l}0.0438 \text { (ft) } \\
0.0151 \text { (ft2) }\end{array}$ \\
\hline 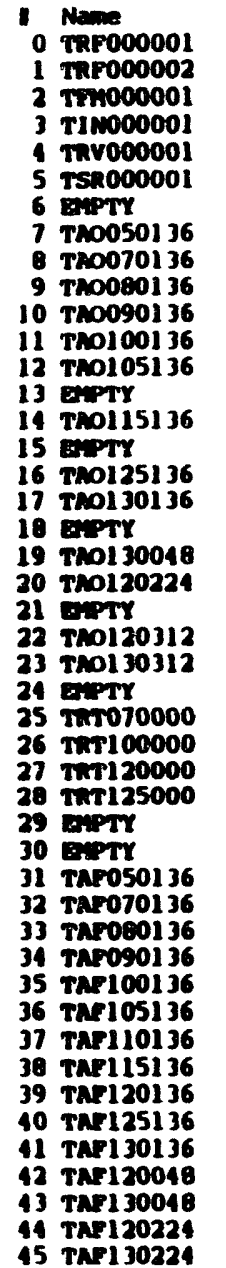 & $\begin{array}{l}\text { Min } \\
74.3963 \\
74.9393 \\
86.3471 \\
86.6258 \\
86.5249 \\
87.3472 \\
-601.0594 \\
85.9287 \\
87.2125 \\
86.9019 \\
86.6695 \\
86.9801 \\
86.9171 \\
10.9819 \\
86.3333 \\
37.5374 \\
86.8776 \\
86.6288 \\
11.2111 \\
86.7337 \\
86.4377 \\
38.6870 \\
86.7115 \\
86.4018 \\
38.9168 \\
86.5005 \\
86.9017 \\
86.8106 \\
87.0010 \\
39.3761 \\
39.3761 \\
86.8512 \\
87.4464 \\
86.8503 \\
86.9174 \\
86.6855 \\
86.6526 \\
86.6492 \\
87.0101 \\
87.0015 \\
87.0678 \\
86.8659 \\
87.0829 \\
86.6840 \\
86.9043 \\
86.5319\end{array}$ & $\begin{array}{l}\text { Max } \\
75.0338 \\
75.3622 \\
87.0815 \\
87.3616 \\
87.2579 \\
67.8709 \\
601.0594 \\
86.5570 \\
88.1592 \\
87.5301 \\
67.5078 \\
87.5051 \\
87.9663 \\
11.4403 \\
87.1751 \\
37.7675 \\
87.4018 \\
87.3571 \\
11.4403 \\
87.3625 \\
87.1656 \\
39.1464 \\
87.3410 \\
87.0308 \\
39.6057 \\
87.2381 \\
87.8434 \\
87.4403 \\
87.6289 \\
39.8352 \\
10.0646 \\
87.5840 \\
88.2833 \\
87.4781 \\
88.0701 \\
87.5253 \\
87.7997 \\
87.3819 \\
87.9529 \\
87.4217 \\
87.7993 \\
87.4955 \\
88.1359 \\
87.3140 \\
87.7430 \\
86.9522\end{array}$ & $\begin{array}{r}\text { Ave } \\
74.5918 \\
75.2015 \\
86.7077 \\
87.0011 \\
86.8979 \\
87.6489 \\
-601.0591 \\
86.3014 \\
87.6545 \\
87.2202 \\
87.0217 \\
87.2528 \\
87.5340 \\
41.1195 \\
86.7830 \\
37.5559 \\
87.1001 \\
87.0290 \\
41.2799 \\
87.0314 \\
86.7809 \\
38.8708 \\
87.0094 \\
86.6745 \\
39.3301 \\
86.9046 \\
87.4620 \\
87.1880 \\
87.2900 \\
39.6057 \\
39.7066 \\
87.2280 \\
87.8993 \\
87.1976 \\
87.5922 \\
87.0298 \\
87.2787 \\
87.0175 \\
87.5925 \\
87.2072 \\
87.4527 \\
87.2852 \\
87.6347 \\
87.0448 \\
87.3404 \\
86.7544\end{array}$ & 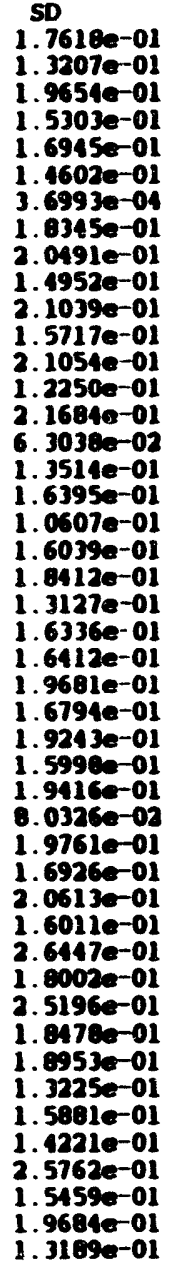 & $\begin{array}{r}\text { Uncert } \\
1.9000 e+00 \\
1.9000+00 \\
1.9002 e+00 \\
1.9002 e+00 \\
1.9002 e+00 \\
1.90020+00 \\
2.1295 e+00 \\
1.9002 e+00 \\
1.9002 e+00 \\
1.9002 e+00 \\
1.9002 e+00 \\
1.9002 e+00 \\
1.9002 e+00 \\
1.9011 e+00 \\
1.9002 e+00 \\
1.9009 e+00 \\
1.9002 e+00 \\
1.9002 e+00 \\
1.9011 e+00 \\
1.9002 e+00 \\
1.9002 e+00 \\
1.9010 e+00 \\
1.9002 e+00 \\
1.9002 e+00 \\
1.9010 e+00 \\
1.9002 e+00 \\
1.9002 e+00 \\
1.9002 e+00 \\
1.9002 e+00 \\
1.9011 e+00 \\
1.9011 e+00 \\
1.9002 e+00 \\
1.9002 e+00 \\
1.9002 e+00 \\
1.9002 e+00 \\
1.90020+00 \\
1.9002 e+00 \\
1.9002 e+00 \\
1.9002 e+00 \\
1.9002 e+00 \\
1.9002 e+00 \\
1.9002 e+00 \\
1.9002 e+00 \\
1.9002 e+00 \\
1.9002 e+00 \\
1.9002 e+00\end{array}$ \\
\hline
\end{tabular}

\begin{tabular}{|c|c|c|c|c|c|}
\hline 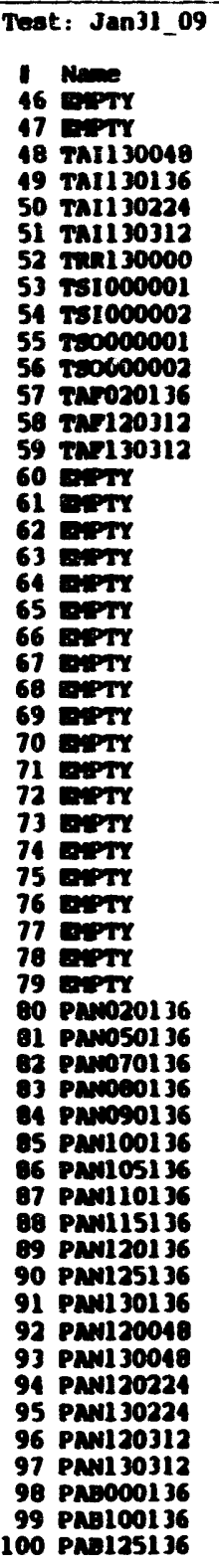 & 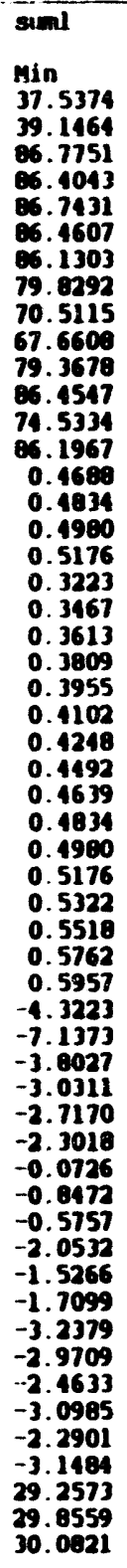 & $\begin{array}{l}\text { Date: } \\
\text { Max } \\
38.6870 \\
39.3761 \\
89.1385 \\
97.2421 \\
87.7935 \\
87.1958 \\
87.0726 \\
80.5670 \\
71.7853 \\
68.4093 \\
80.2122 \\
87.2970 \\
75.5926 \\
8.8266 \\
0.1785 \\
0.4932 \\
0.5029 \\
0.5225 \\
0.3320 \\
0.3467 \\
0.3662 \\
0.3809 \\
0.3955 \\
0.4150 \\
0.4297 \\
0.4541 \\
0.4686 \\
0.4883 \\
0.5029 \\
0.5225 \\
0.5371 \\
0.5518 \\
0.5762 \\
0.6006 \\
-3.7119 \\
-6.5269 \\
-3.4212 \\
-2.8022 \\
-2.4118 \\
-1.9967 \\
0.2326 \\
-0.5420 \\
-0.2706 \\
-1.7480 \\
-1.2977 \\
-1.4811 \\
-3.0090 \\
-2.7420 \\
-2.1581 \\
-2.7934 \\
-2.0512 \\
-2.9195 \\
29.3946 \\
30.0390 \\
30.2652\end{array}$ & $\begin{array}{l}\text { Ian } 3113 \\
\text { Ave } \\
38.0572 \\
39.2291 \\
87.1129 \\
86.8065 \\
87.3986 \\
86.8127 \\
86.6332 \\
80.1214 \\
71.2079 \\
68.0203 \\
79.8238 \\
86.8419 \\
75.1180 \\
86.5910 \\
0.1728 \\
0.1879 \\
0.5016 \\
0.5181 \\
0.3268 \\
0.3467 \\
0.3622 \\
0.3809 \\
0.3955 \\
0.1132 \\
0.4295 \\
0.4535 \\
0.4662 \\
0.4846 \\
0.5026 \\
0.5181 \\
0.5323 \\
0.5518 \\
0.5762 \\
0.6005 \\
-1.0735 \\
-6.8718 \\
-3.5645 \\
-2.9090 \\
-2.5782 \\
-2.1233 \\
0.1121 \\
-0.6854 \\
-0.4643 \\
-1.9372 \\
-1.4091 \\
-1.5894 \\
-3.1112 \\
-2.8382 \\
-2.3016 \\
-2.9566 \\
-2.1803 \\
-3.0507 \\
29.3186 \\
29.9374 \\
30.1462\end{array}$ & $\begin{array}{l}21.221990 \\
50 \\
2.3598 e-01 \\
1.1134 e-01 \\
3.1839 e-01 \\
2.0339 e-01 \\
2.1715 e-01 \\
1.7721 e-01 \\
1.9513 e-01 \\
1.7563 e-01 \\
2.9337 e-01 \\
1.7511 e-01 \\
2.0732 e-01 \\
1.6549 e-01 \\
2.6138 e-01 \\
1.6359 e-01 \\
2.1364 e-03 \\
1.6624 e-03 \\
2.2146 e-03 \\
1.4797 e-03 \\
1.6624 e-03 \\
0.0000 e+00 \\
1.6950 e-03 \\
0.0000 e-00 \\
0.0000 e+00 \\
2.3941 e-03 \\
9.6655 e-04 \\
1.6028 e-03 \\
2.1642 e-03 \\
2.1065 e-03 \\
1.1714 e-03 \\
1.1797 e-03 \\
6.9053 e-04 \\
0.0000 e+00 \\
0.0000 e+00 \\
6.9053 e-04 \\
1.3421 e-01 \\
1.3370 e-01 \\
8.5934 e-02 \\
7.2297 e-02 \\
9.0784 e-02 \\
8.3874 e-02 \\
6.5558 e-02 \\
8.1036 e-02 \\
7.4258 e-02 \\
7.4306 e-02 \\
5.3860 e-02 \\
6.7346 e-02 \\
6.2819 e-02 \\
7.0314 e-02 \\
7.8049 e-02 \\
7.2313 e-02 \\
7.1178 e-02 \\
6.1732 e-02 \\
1.0954 e-02 \\
3.9552 e-02 \\
1.5307 e-02\end{array}$ & 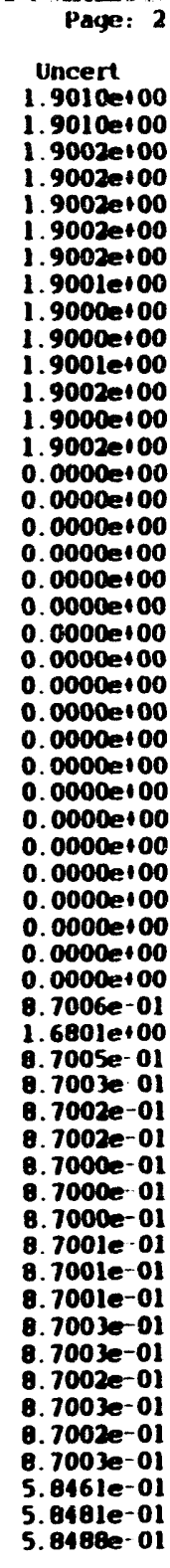 \\
\hline
\end{tabular}




\begin{tabular}{|c|c|c|c|c|c|c|}
\hline 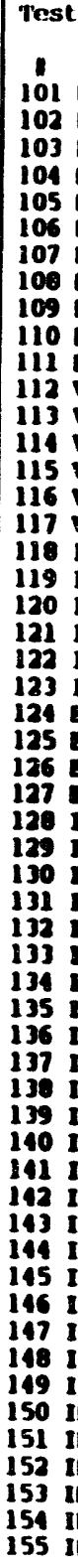 & 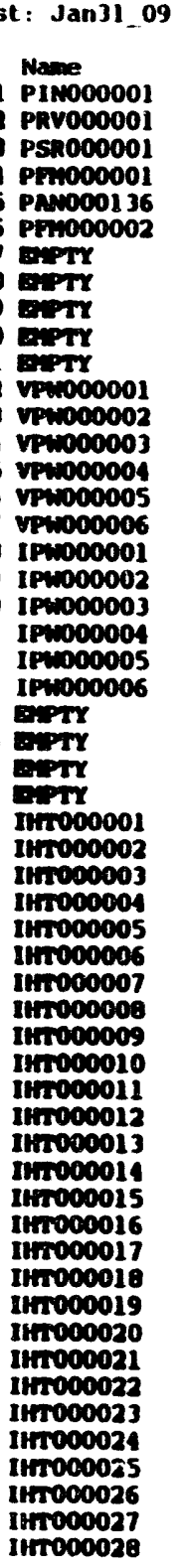 & $\begin{array}{l}\text { suml } \\
\text { Min } \\
28.9113 \\
20.1715 \\
29.8409 \\
14.9091 \\
-28.2045 \\
14.2457 \\
-0.0049 \\
-10.0000 \\
0.0000 \\
-10.0000 \\
0.0000 \\
-0.2706 \\
-0.2000 \\
-0.0400 \\
-0.2306 \\
-0.1200 \\
-0.2000 \\
-11.3611 \\
-37.4737 \\
-15.6468 \\
-2.1531 \\
-1.2058 \\
-2.9297 \\
-0.0293 \\
-0.0195 \\
-0.0244 \\
-0.0195 \\
-0.2494 \\
-0.2847 \\
-0.5247 \\
-0.2000 \\
-0.2000 \\
-0.1528 \\
-0.2753 \\
-0.3153 \\
-0.2000 \\
-0.1600 \\
-0.2753 \\
-0.3128 \\
-0.1694 \\
-0.3553 \\
-0.2400 \\
-0.3153 \\
-0.3906 \\
0.2753 \\
-0.0900 \\
-0.2989 \\
3.1694 \\
-0.3153 \\
-0.3506 \\
-0.2853 \\
-0.2000 \\
-0.3906 \\
-0.1953 \\
-0.1647\end{array}$ & $\begin{array}{c}\text { Date: Wed } \\
\text { Max } \\
29.0334 \\
20.2936 \\
29.9630 \\
15.3669 \\
-26.3735 \\
15.5545 \\
0.0049 \\
-8.0184 \\
0.0049 \\
-10.0000 \\
0.0000 \\
0.1200 \\
0.1906 \\
0.1553 \\
0.1600 \\
0.2706 \\
0.1906 \\
15.9717 \\
-8.2047 \\
-9.7968 \\
-1.4766 \\
-3.2294 \\
-1.4648 \\
-0.0146 \\
-0.0098 \\
-0.0098 \\
-0.0098 \\
0.1112 \\
0.1059 \\
-0.1311 \\
-0.0047 \\
0.1106 \\
0.2378 \\
0.1153 \\
0.0753 \\
-0.0047 \\
0.2306 \\
0.1153 \\
0.2731 \\
0.2212 \\
0.2306 \\
0.1506 \\
0.0753 \\
0.3906 \\
0.6659 \\
0.1553 \\
0.0919 \\
3.5600 \\
-0.1200 \\
0.0400 \\
-0.0900 \\
0.3859 \\
0.0000 \\
0.0000 \\
0.2259\end{array}$ & $\begin{array}{c}\text { Jan } 3113: \\
\text { Ave } \\
28.9552 \\
20.2001 \\
29.8800 \\
15.1691 \\
-27.4996 \\
14.8555 \\
0.0041 \\
-9.8616 \\
0.0016 \\
-10.0000 \\
0.0000 \\
-0.1027 \\
0.0031 \\
-0.0048 \\
0.0467 \\
-0.0067 \\
-0.0516 \\
-1.3260 \\
-19.0928 \\
-12.6438 \\
-1.9746 \\
-3.6785 \\
-2.2754 \\
-0.0203 \\
-0.0136 \\
-0.0183 \\
-0.0157 \\
-0.1634 \\
-0.1245 \\
-0.2473 \\
-0.1531 \\
-0.1277 \\
0.0073 \\
-0.0917 \\
-0.1591 \\
-0.1766 \\
-0.0975 \\
-0.0956 \\
-0.1448 \\
-0.0327 \\
-0.1053 \\
-0.1502 \\
-0.1395 \\
0.0000 \\
0.3964 \\
-0.0205 \\
-0.1230 \\
3.3412 \\
-0.1942 \\
-0.1241 \\
-0.2111 \\
0.0695 \\
-0.0430 \\
-0.1269 \\
0.0502 \\
0\end{array}$ & 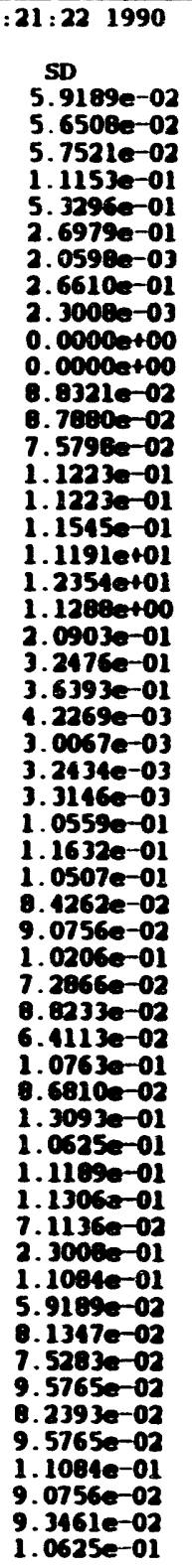 & 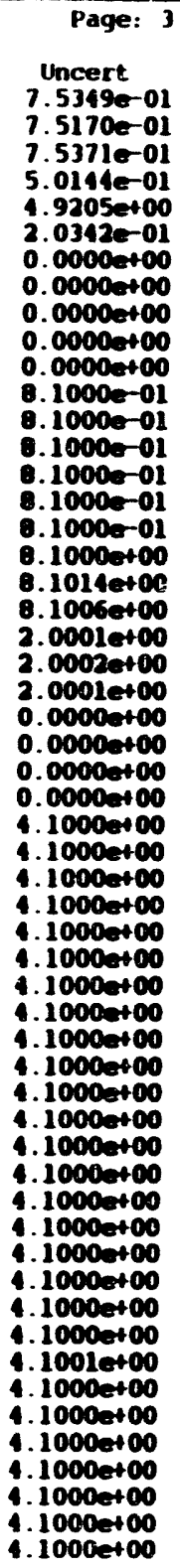 \\
\hline
\end{tabular}

\begin{tabular}{|c|c|c|c|c|c|}
\hline Test: Jan31_09 & $\operatorname{sinl}$ & Date: Wed & $\operatorname{Tan} 3113=$ & $\overline{1: 22} 1990$ & Page: 4 \\
\hline 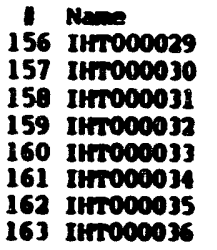 & $\begin{array}{l}\text { Min } \\
-0.0400 \\
-0.0800 \\
-0.0847 \\
-0.1953 \\
37.3247 \\
-0.0800 \\
-0.1601 \\
-0.2353\end{array}$ & $\begin{array}{r}\operatorname{Max} \\
0.3506 \\
0.3106 \\
0.3059 \\
0.1953 \\
37.5200 \\
0.3106 \\
0.3978 \\
0.3506\end{array}$ & $\begin{array}{c}\text { Ave } \\
0.1280 \\
0.0958 \\
0.0989 \\
0.0586 \\
37.3872 \\
0.0411 \\
0.0092 \\
-0.0205\end{array}$ & $\begin{array}{l}\text { SD } \\
1.0447 e-01 \\
9.8648 e-02 \\
9.9902 e-02 \\
9.8648 e-02 \\
9.2034 e-02 \\
1.2409 e-01 \\
1.1897 e-01 \\
9.0412 e-02\end{array}$ & 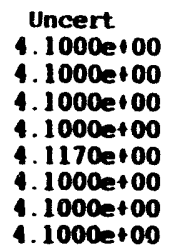 \\
\hline
\end{tabular}




\begin{tabular}{|c|c|c|c|c|c|c|}
\hline \multirow{2}{*}{\multicolumn{2}{|c|}{ 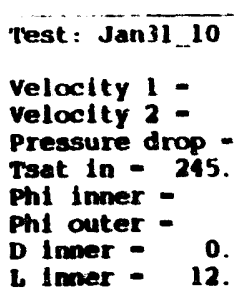 }} & \multirow{2}{*}{\multicolumn{4}{|c|}{ 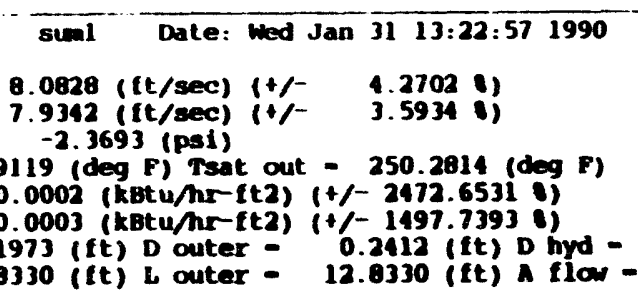 }} & \multirow[b]{2}{*}{$\begin{array}{l}\text { Page: } 1 \\
0.0438 \text { (ft) } \\
0.0151 \text { (ft2 }\end{array}$} \\
\hline & & & & & & \\
\hline $\begin{array}{l}1 \\
2 \\
3 \\
4 \\
5 \\
6 \\
7 \\
8 \\
9 \\
10 \\
11 \\
12 \\
13 \\
14 \\
15 \\
16 \\
17 \\
18 \\
19 \\
20 \\
21 \\
22 \\
23 \\
24 \\
25 \\
26 \\
27 \\
28 \\
29 \\
30 \\
31 \\
32 \\
33 \\
34 \\
35 \\
36 \\
37 \\
38 \\
39 \\
40 \\
11 \\
12 \\
13 \\
14\end{array}$ & 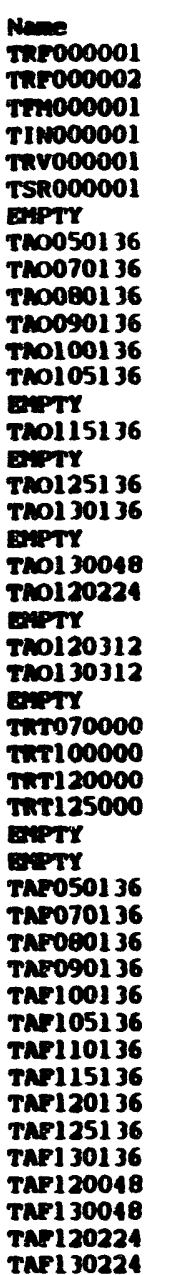 & $\begin{array}{l}\text { Min } \\
74.1838 \\
74.9393 \\
86.4518 \\
86.8362 \\
86.5249 \\
87.3472 \\
601.0594 \\
86.1376 \\
87.5283 \\
87.0052 \\
86.8788 \\
87.0849 \\
87.1270 \\
40.9819 \\
86.5440 \\
37.5374 \\
86.8776 \\
86.9369 \\
10.9819 \\
86.8375 \\
86.6423 \\
38.6870 \\
86.9213 \\
86.5058 \\
39.1464 \\
86.0178 \\
87.1108 \\
87.0201 \\
86.6958 \\
39.3761 \\
39.3761 \\
86.9554 \\
87.5520 \\
67.0584 \\
87.3364 \\
86.7904 \\
87.0687 \\
86.7533 \\
87.3236 \\
87.1050 \\
87.3792 \\
87.1796 \\
87.2942 \\
86.9983 \\
87.2182 \\
86.6376\end{array}$ & $\begin{array}{l}\text { Max } \\
74.8213 \\
75.3622 \\
87.2903 \\
87.5712 \\
87.3626 \\
87.9755 \\
601.0594 \\
87.0795 \\
88.3697 \\
87.9467 \\
87.6123 \\
87.9235 \\
88.2806 \\
11.4403 \\
87.4907 \\
37.7675 \\
87.7154 \\
87.5636 \\
11.4403 \\
87.5714 \\
87.2717 \\
39.1464 \\
87.6558 \\
87.2399 \\
39.6057 \\
87.5539 \\
88.1574 \\
87.8585 \\
87.8370 \\
39.8352 \\
10.0646 \\
87.7928 \\
88.5960 \\
87.8939 \\
88.3841 \\
87.5253 \\
88.0071 \\
87.5907 \\
89.1621 \\
87.6298 \\
88.0070 \\
87.7044 \\
88.2424 \\
87.5232 \\
87.8478 \\
87.1627\end{array}$ & $\begin{array}{r}\text { Ave } \\
74.5663 \\
75.1930 \\
86.8512 \\
87.2100 \\
86.8936 \\
87.6238 \\
\mathbf{6 0 1 . 0 5 9 1} \\
86.5227 \\
87.8946 \\
87.4743 \\
87.2727 \\
87.4958 \\
87.7186 \\
11.0920 \\
86.9945 \\
37.5835 \\
87.3383 \\
87.2725 \\
11.2982 \\
87.2403 \\
86.9989 \\
38.8800 \\
87.2066 \\
86.8710 \\
39.3302 \\
87.1370 \\
87.7177 \\
87.4686 \\
87.4698 \\
39.5919 \\
39.6745 \\
87.4493 \\
88.0740 \\
87.3929 \\
87.7949 \\
87.2356 \\
87.4989 \\
87.2596 \\
87.7640 \\
87.4157 \\
87.6649 \\
87.4778 \\
87.7995 \\
87.2671 \\
87.4744 \\
86.9527\end{array}$ & 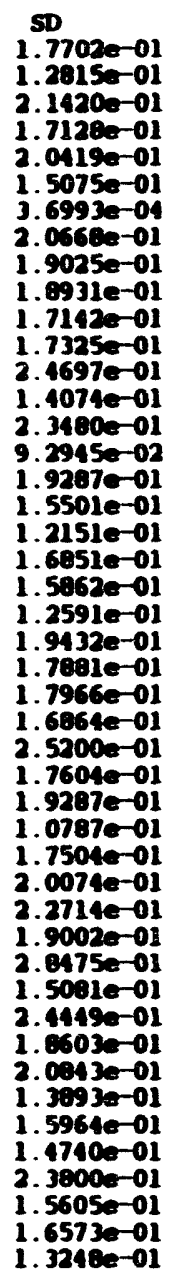 & $\begin{array}{c}\text { Uncert } \\
1.9000=+00 \\
1.9000+00 \\
1.9002 e+00 \\
1.9002 e+00 \\
1.9002 e+00 \\
1.9002 e+00 \\
2.1295 e+00 \\
1.9002 e+00 \\
1.9002 e+00 \\
1.90020+00 \\
1.9002 e+00 \\
1.9002 e+00 \\
1.9002 e+00 \\
1.9011 e+\infty 0\end{array}$ \\
\hline
\end{tabular}

\begin{tabular}{|c|c|c|c|c|c|c|}
\hline \multicolumn{2}{|c|}{ Teat: Jan31_10 } & $\operatorname{san} 1$ & \multicolumn{3}{|c|}{ Date: Wed Jan 31 13:22:51 1990} & age: \\
\hline $\begin{array}{l}5 \\
5 \\
5 \\
5 \\
5 \\
5 \\
5 \\
5 \\
5 \\
5 \\
5 \\
6 \\
6 \\
6 \\
6 \\
6 \\
6\end{array}$ & 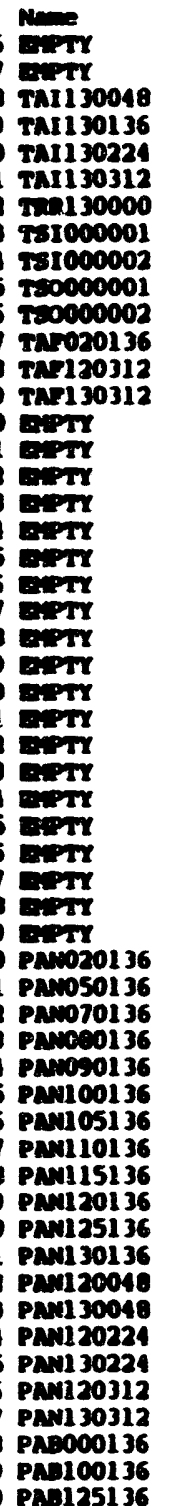 & 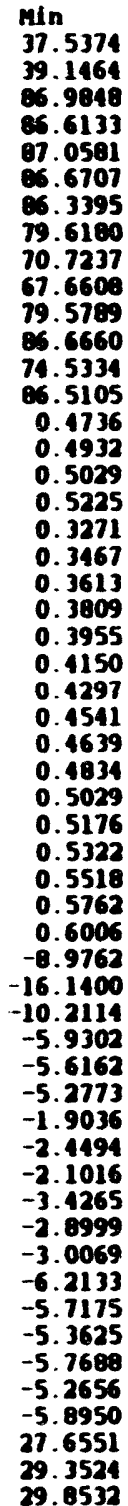 & $\begin{array}{l}\max \\
38.4572 \\
39.3761 \\
88.1385 \\
87.4511 \\
8 . .2137 \\
87.5105 \\
87.3868 \\
80.3565 \\
71.7853 \\
68.4093 \\
80.6343 \\
07.4029 \\
75.8042 \\
07.1404 \\
0.4834 \\
0.4980 \\
0.5078 \\
0.5273 \\
0.3320 \\
0.3516 \\
0.3662 \\
0.3857 \\
0.4004 \\
0.4150 \\
0.4346 \\
0.4541 \\
0.4688 \\
0.4883 \\
0.5029 \\
0.5225 \\
0.5371 \\
0.5566 \\
0.5811 \\
0.6055 \\
-8.5947 \\
-15.5296 \\
-9.8299 \\
-5.6251 \\
-5.2347 \\
-5.0484 \\
-1.6747 \\
-2.2205 \\
-1.8727 \\
-3.1213 \\
-2.7473 \\
-2.7018 \\
-5.9845 \\
-5.4886 \\
-5.0573 \\
-5.5399 \\
-5.0367 \\
-5.7424 \\
27.7924 \\
29.4897 \\
29.9905\end{array}$ & $\begin{array}{l}\text { Ave } \\
38.1307 \\
39.1924 \\
87.6142 \\
87.0698 \\
87.6971 \\
87.0989 \\
86.0172 \\
80.0443 \\
71.2036 \\
67.9818 \\
80.1066 \\
87.0532 \\
75.1350 \\
86.8080 \\
0.4785 \\
0.4933 \\
0.5077 \\
0.5230 \\
0.3273 \\
0.3469 \\
0.3658 \\
0.3821 \\
0.3956 \\
0.4150 \\
0.4299 \\
0.4541 \\
0.4687 \\
0.4891 \\
0.5029 \\
0.5218 \\
0.5367 \\
0.5560 \\
0.5770 \\
0.6008 \\
-8.7351 \\
-15.8592 \\
-9.9428 \\
-5.7868 \\
-5.1239 \\
-5.1354 \\
-1.7327 \\
-2.3670 \\
-2.0101 \\
-3.2586 \\
-2.8343 \\
-2.8437 \\
-6.0806 \\
-5.6351 \\
-5.2129 \\
-5.6437 \\
-5.1710 \\
-5.8110 \\
27.7338 \\
29.4302 \\
29.9136\end{array}$ & $\begin{array}{l}\text { SD } \\
2.4619 e-01 \\
9.2786 e-02 \\
2.0738 e-01 \\
2.1338 e-01 \\
2.6148 e-01 \\
1.9765 e-01 \\
2.1281 e-01 \\
1.6221 e-01 \\
2.5210 e-01 \\
2.1274 e-01 \\
2.1748 e-01 \\
1.7341 e-01 \\
2.9216 e-01 \\
1.6739 e-01 \\
9.8648 e-04 \\
6.9053 e-04 \\
6.9053 e-04 \\
1.6028 e-03 \\
9.6655 e-04 \\
9.6655 e-04 \\
1.3381 e-03 \\
2.1635 e-03 \\
6.9053 e-04 \\
0.0000 e+00 \\
9.6655 e-04 \\
0.0000 e+00 \\
6.9053 e-04 \\
9.6655 e-04 \\
0.0000 e+00 \\
1.7115 e-03 \\
1.3381 e-03 \\
1.7115 e-03 \\
1.8082 e-03 \\
9.6655 e-04 \\
6.7682 e-02 \\
1.2844 e-01 \\
9.7777 e-02 \\
7.8049 e-02 \\
7.2690 e-02 \\
7.5527 e-02 \\
6.4591 e-02 \\
6.6903 e-02 \\
6.3552 e-02 \\
6.7187 e-02 \\
5.5596 e-02 \\
6.8949 e-02 \\
6.6850 e-02 \\
6.5103 e-02 \\
6.7116 e-02 \\
5.9218 e-02 \\
6.4591 e-02 \\
6.0192 e-02 \\
3.8176 e-02 \\
3.1011 e-07 \\
\end{array}$ & 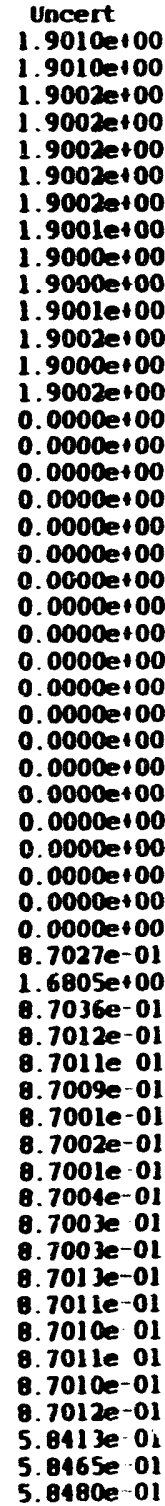 \\
\hline
\end{tabular}




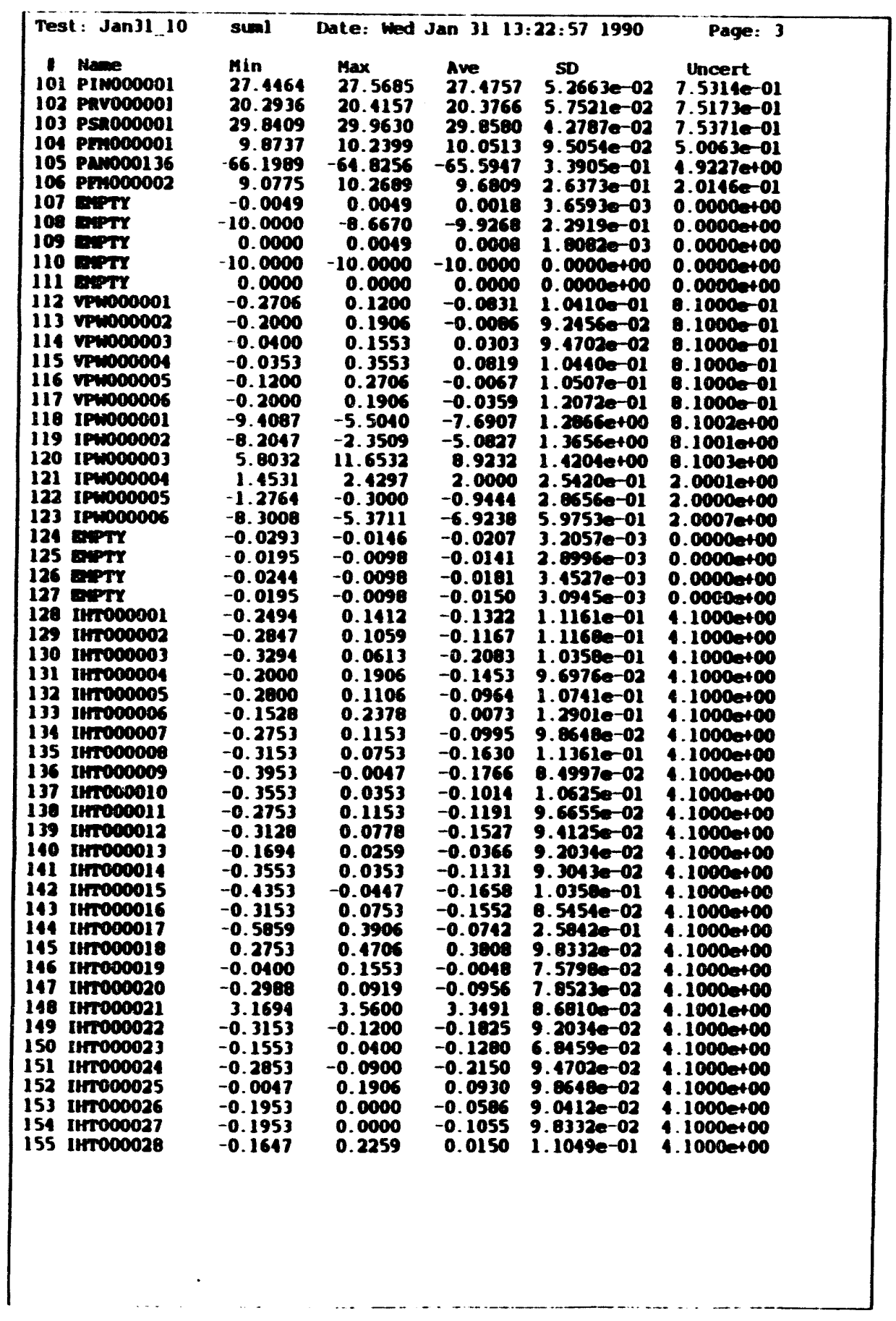

\begin{tabular}{|c|c|c|c|c|c|}
\hline 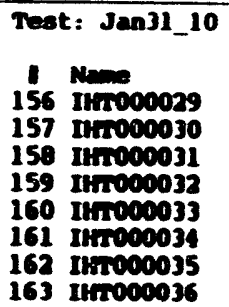 & $\begin{array}{l}\text { sini } \\
\text { min } \\
-0.0400 \\
-0.0000 \\
-0.0847 \\
0.0000 \\
37.3247 \\
-0.2753 \\
-0.1801 \\
-0.2353\end{array}$ & $\begin{array}{l}\text { Date: Wed } \\
\text { Max } \\
0.3506 \\
0.3106 \\
0.3059 \\
0.1953 \\
37.5200 \\
0.3106 \\
0.3978 \\
0.3506\end{array}$ & $\begin{array}{l}\text { an } 3113 \\
\text { Ave } \\
0.1084 \\
0.1153 \\
0.0872 \\
0.0508 \\
37.3638 \\
0.0216 \\
0.0931 \\
-0.0205\end{array}$ & $\begin{array}{l}22: 571990 \\
50 \\
1.0106 e-01 \\
8.6233 e-02 \\
1.2251 e-01 \\
8.6541 e-02 \\
7.8918 e-02 \\
1.1327 e-01 \\
1.3182 e-01 \\
1.1334 e-01\end{array}$ & 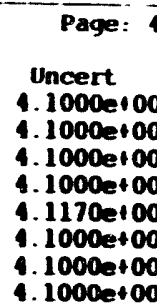 \\
\hline
\end{tabular}




\begin{tabular}{|c|c|c|c|c|c|c|}
\hline \multirow{2}{*}{\multicolumn{3}{|c|}{ 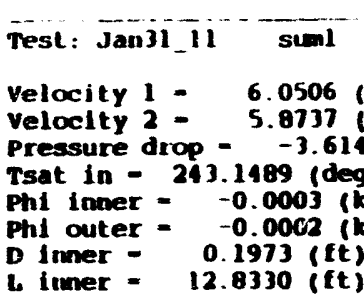 }} & Date & $\operatorname{Jan} 3$ & $24: 40 \quad 1990$ & \multirow{2}{*}{$\begin{array}{l}\text { Page: } 1 \\
0.0438 \text { (ft) } \\
0.0151 \text { (ft2) }\end{array}$} \\
\hline & & & $\begin{array}{l}t / \mathrm{sec})(+/- \\
t / \mathrm{sec})(+/- \\
\text { (psi) } \\
\text { f) Tsat out } \\
\text { tumr-ft2) } \\
\text { tumr-ft2) } \\
\text { o outer = } \\
\text { outer - }\end{array}$ & $\begin{array}{r}5.6618 \\
\quad 3.8695 \\
-\quad 250.04 \\
1+1-3016 \\
1+0.2412 \\
0.2412 \\
12.8330\end{array}$ & $\begin{array}{l}148 \text { (deg F) } \\
1479 \text { ह) } \\
8580 \text { 8) } \\
\text { f() D hyd - } \\
\text { (t) A flow - }\end{array}$ & \\
\hline $\begin{array}{l}1 \\
0 \\
1 \\
2 \\
3 \\
1 \\
5 \\
6 \\
7 \\
8 \\
9 \\
10 \\
11 \\
12 \\
13 \\
14 \\
15 \\
16 \\
17 \\
16 \\
19 \\
20 \\
21 \\
22 \\
23 \\
24 \\
25 \\
26 \\
27 \\
28 \\
29 \\
30 \\
31 \\
32 \\
339 \\
34 \\
35 \\
36 \\
37 \\
38 \\
39 \\
40 \\
11 \\
12 \\
43 \\
14 \\
45\end{array}$ & 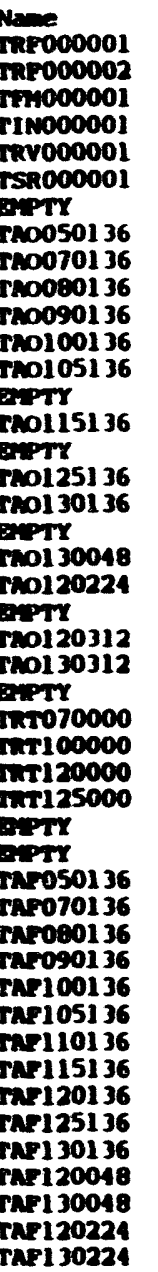 & $\begin{array}{r}\text { Min } \\
74.1838 \\
74.9393 \\
86.5565 \\
86.9409 \\
86.6297 \\
87.4519 \\
-601.0594 \\
86.1376 \\
87.6338 \\
87.2151 \\
86.9835 \\
87.1896 \\
87.3364 \\
10.9819 \\
86.5440 \\
37.5374 \\
87.0863 \\
87.0416 \\
10.9819 \\
86.9423 \\
86.7471 \\
38.6870 \\
86.9200 \\
86.6105 \\
38.9168 \\
86.9225 \\
87.3194 \\
87.1248 \\
87.1057 \\
39.3761 \\
39.3761 \\
87.1639 \\
87.8649 \\
87.1631 \\
87.3360 \\
86.8956 \\
87.0683 \\
87.0669 \\
87.1283 \\
87.2097 \\
87.3800 \\
87.2843 \\
87.3988 \\
87.1030 \\
87.1133 \\
86.7424\end{array}$ & $\begin{array}{r}\operatorname{Max} \\
74.8213 \\
75.3622 \\
87.3944 \\
87.5712 \\
87.2579 \\
87.8709 \\
-601.0594 \\
87.0795 \\
89.3697 \\
87.9467 \\
87.8214 \\
87.8189 \\
88.1760 \\
11.4103 \\
87.4907 \\
37.7675 \\
87.7154 \\
87.6683 \\
11.4403 \\
87.9891 \\
87.4812 \\
39.1464 \\
87.5506 \\
87.2399 \\
39.6057 \\
87.5539 \\
88.1574 \\
87.0505 \\
87.8370 \\
39.8352 \\
10.0646 \\
87.7928 \\
88.4910 \\
87.8939 \\
88.2795 \\
87.7351 \\
87.9043 \\
87.6943 \\
88.2668 \\
87.8393 \\
88.1121 \\
87.9137 \\
88.3470 \\
87.5232 \\
87.9524 \\
87.2689\end{array}$ & $\begin{array}{r}\text { Ave } \\
74.5578 \\
75.2100 \\
86.9463 \\
87.2898 \\
86.9772 \\
87.6571 \\
-601.0591 \\
86.5561 \\
87.9263 \\
87.5035 \\
87.3522 \\
87.5293 \\
87.7773 \\
41.1011 \\
87.0366 \\
37.5697 \\
87.3500 \\
87.2767 \\
11.2570 \\
87.3155 \\
87.0575 \\
38.8754 \\
87.2653 \\
86.9128 \\
39.3164 \\
87.2006 \\
87.7386 \\
87.4695 \\
87.5401 \\
39.5927 \\
39.7150 \\
87.5036 \\
88.1448 \\
87.4927 \\
87.7974 \\
87.3405 \\
87.5239 \\
87.3223 \\
87.8770 \\
87.4574 \\
87.7107 \\
87.5071 \\
87.8795 \\
87.3174 \\
87.5933 \\
86.9821\end{array}$ & 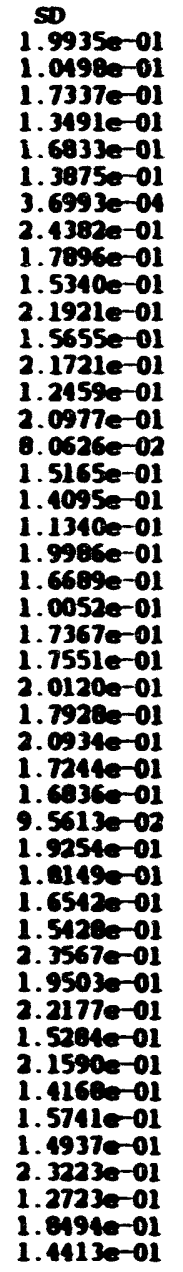 & 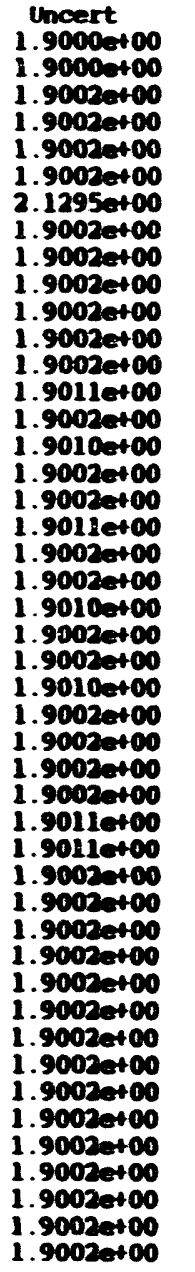 \\
\hline
\end{tabular}

\begin{tabular}{|c|c|c|c|c|c|}
\hline 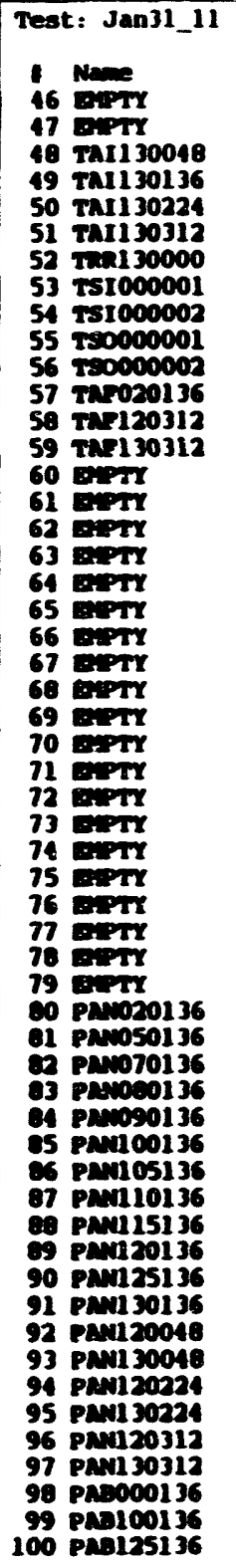 & 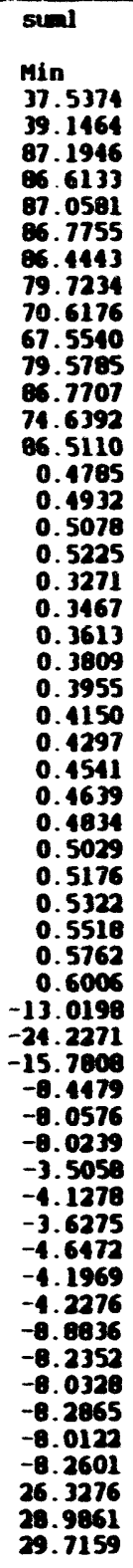 & 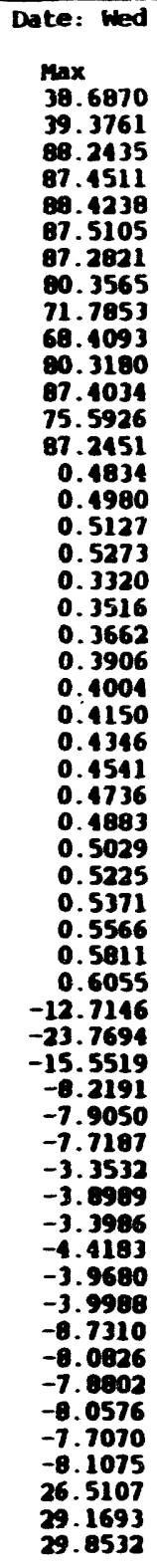 & 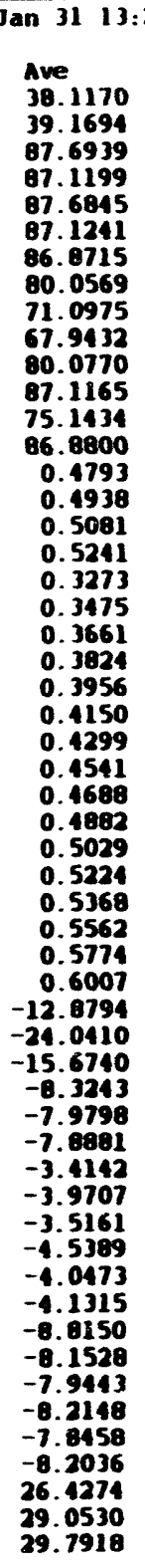 & $\begin{array}{l}24: 401990 \\
50 \\
2.5118 e-01 \\
6.9589 e-02 \\
2.6676 e-01 \\
2.0549 e-01 \\
3.2813 e-01 \\
2.1155 e-01 \\
2.2475 e-01 \\
1.6826 e-01 \\
2.6805 e-01 \\
2.0350 e-01 \\
1.9205 e-01 \\
1.6765 e-01 \\
2.2703 e-01 \\
1.9101 e-01 \\
1.8082 e-03 \\
1.6028 e-03 \\
1.1714 e-03 \\
2.3365 e-03 \\
9.6655 e-04 \\
1.6082 e-03 \\
6.9053 e-04 \\
2.5034 e-03 \\
6.9053 e-04 \\
0.0000 e+00 \\
9.6655 e-04 \\
0.0000 e+00 \\
9.8648 e-04 \\
6.9053 e-04 \\
0.0000 e+00 \\
6.9053 e-04 \\
1.1714 e-03 \\
1.4797 e-03 \\
2.1635 e-03 \\
6.9053 e-04 \\
6.4222 e-02 \\
1.2442 e-01 \\
8.0092 e-02 \\
6.8742 e-02 \\
1.7483 e-02 \\
7.2772 e-02 \\
5.7673 e-02 \\
5.2065 e-02 \\
6.0113 e-02 \\
7.2414 e-02 \\
5.9617 e-02 \\
5.2970 e-02 \\
5.6107 e-02 \\
5.3037 e-02 \\
6.2345 e-02 \\
5.6414 e-02 \\
9.2560 e-02 \\
1.8277 e-02 \\
3.6898 e-02 \\
4.4555 e-02 \\
1.1985 e-02\end{array}$ & 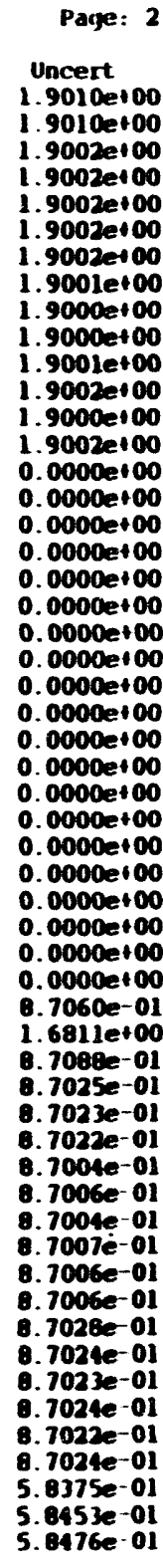 \\
\hline
\end{tabular}




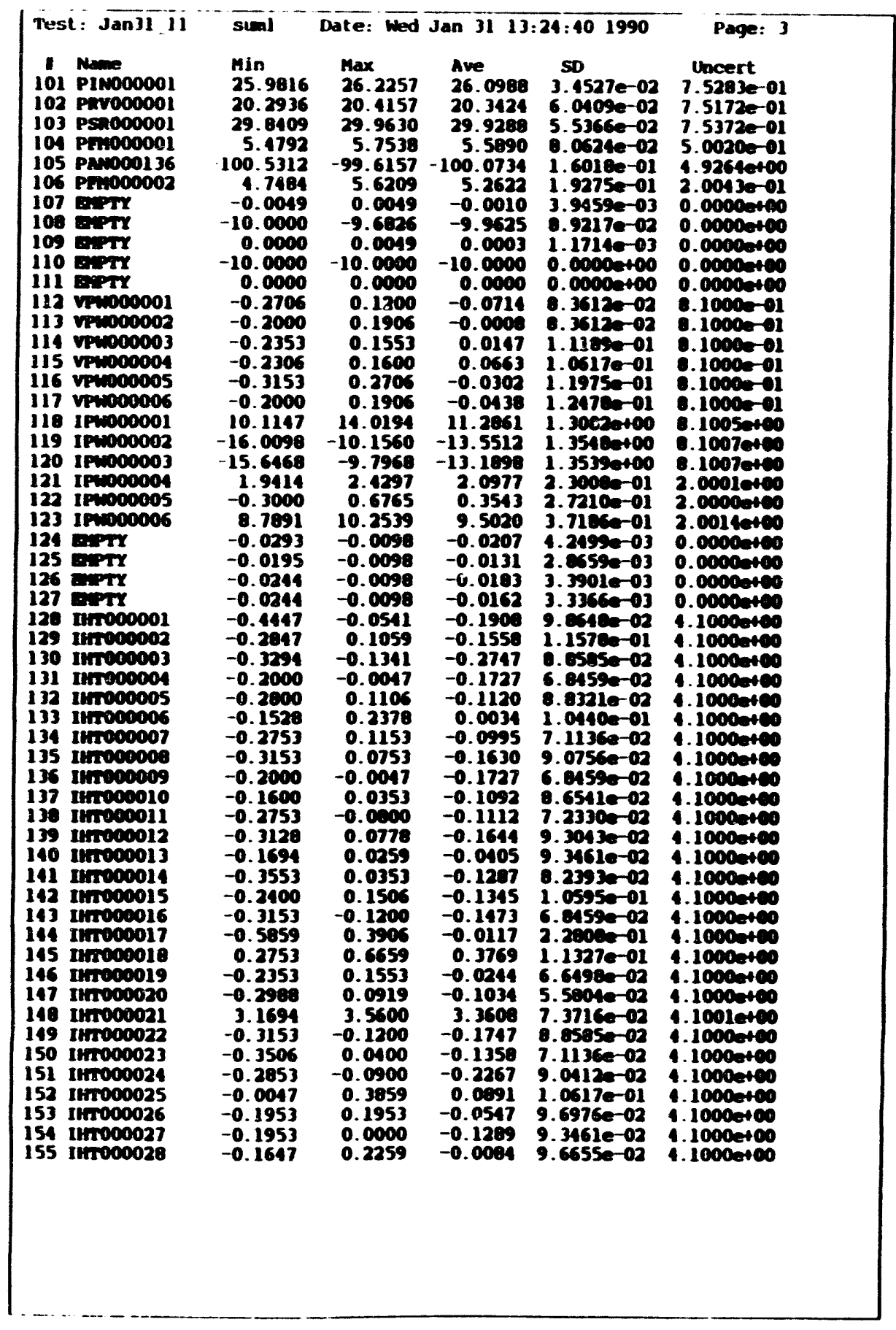

\begin{tabular}{|c|c|c|c|c|c|}
\hline at: & & ate: Med & n & 30 & age \\
\hline 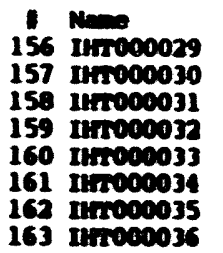 & $\begin{array}{l}\min _{-0.0100} \\
-0.0000 \\
-0.0947 \\
0.0000 \\
37.3217 \\
-0.0000 \\
-0.1001 \\
-0.2353\end{array}$ & $\begin{array}{l}\max \\
0.3506 \\
0.3106 \\
0.3059 \\
0.1953 \\
37.5200 \\
0.3106 \\
0.3978 \\
0.1553\end{array}$ & $\begin{array}{l}\text { Ave } \\
0.1084 \\
0.0958 \\
0.0833 \\
0.0391 \\
37.3677 \\
0.0333 \\
0.1009 \\
-0.0127\end{array}$ & $\begin{array}{l}\text { SD } \\
9.3043 e-02 \\
8.1347 e-02 \\
9.6735 e-02 \\
7.8918 e-02 \\
8.1729 e-02 \\
1.2534 e-01 \\
1.1327 e-01 \\
9.6735 e-02\end{array}$ & $\begin{array}{l}\text { Unc } \\
1.10 \\
4.10 \\
4.10 \\
4.10 \\
4.11 \\
4.10 \\
4.10 \\
4.10\end{array}$ \\
\hline
\end{tabular}




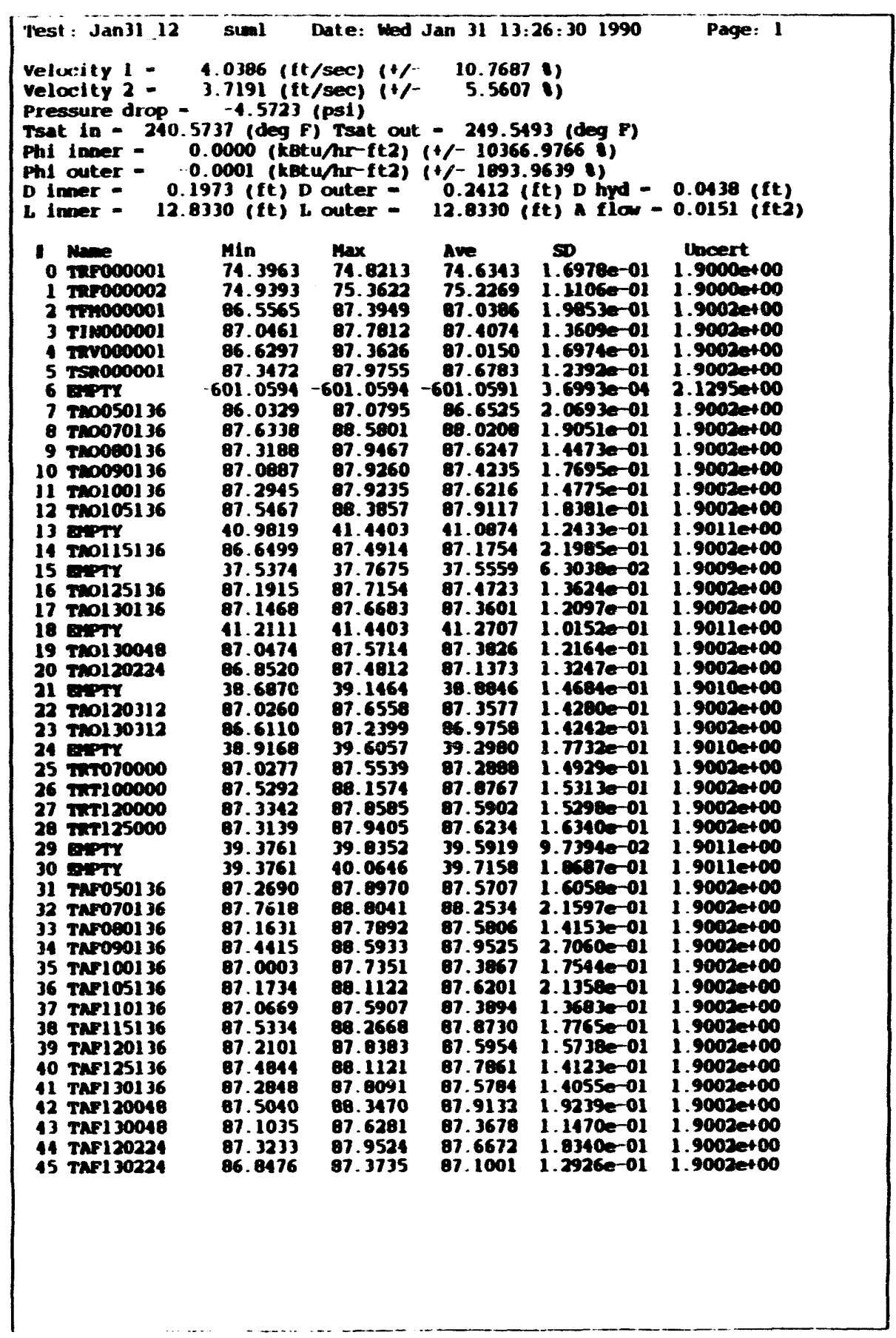

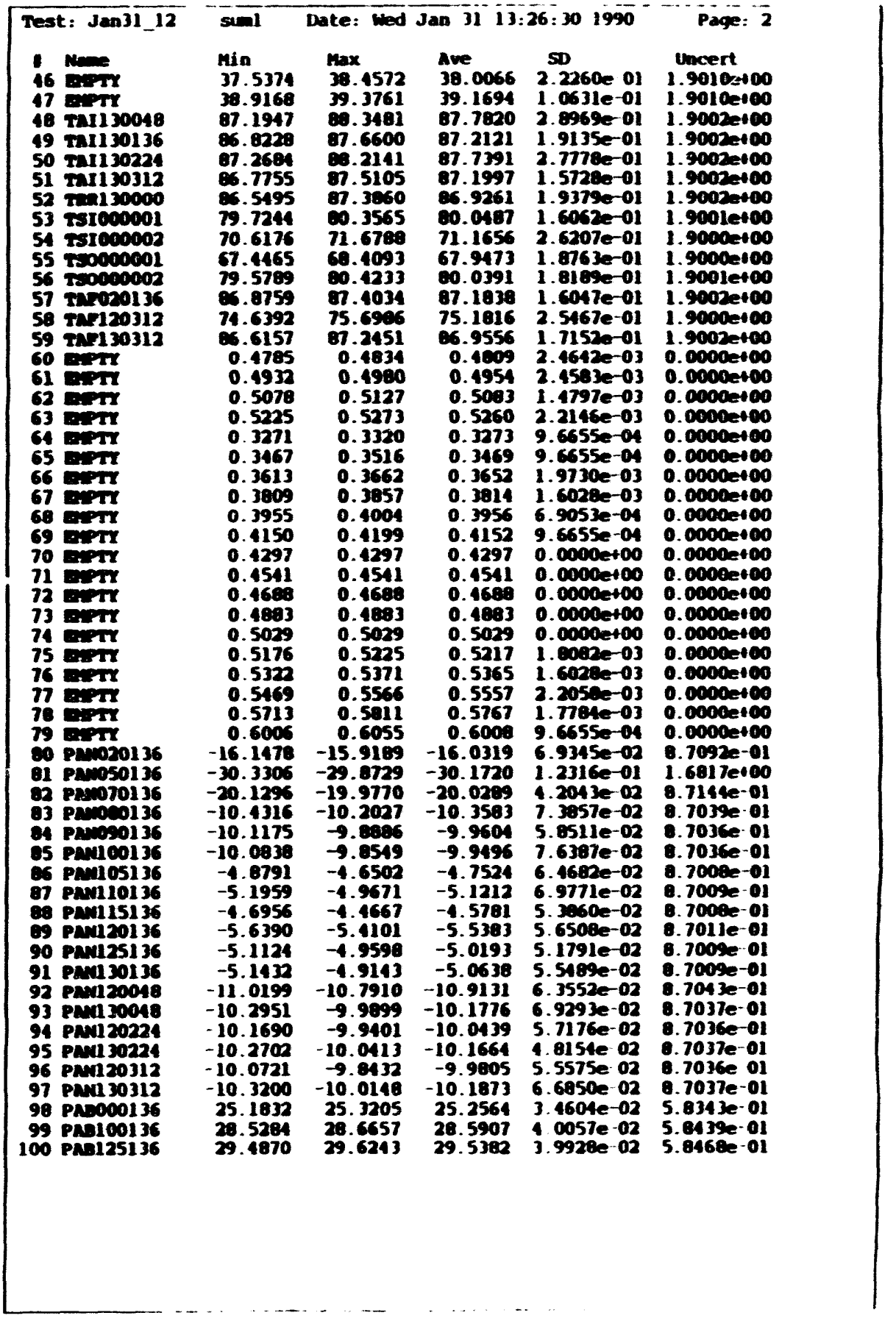




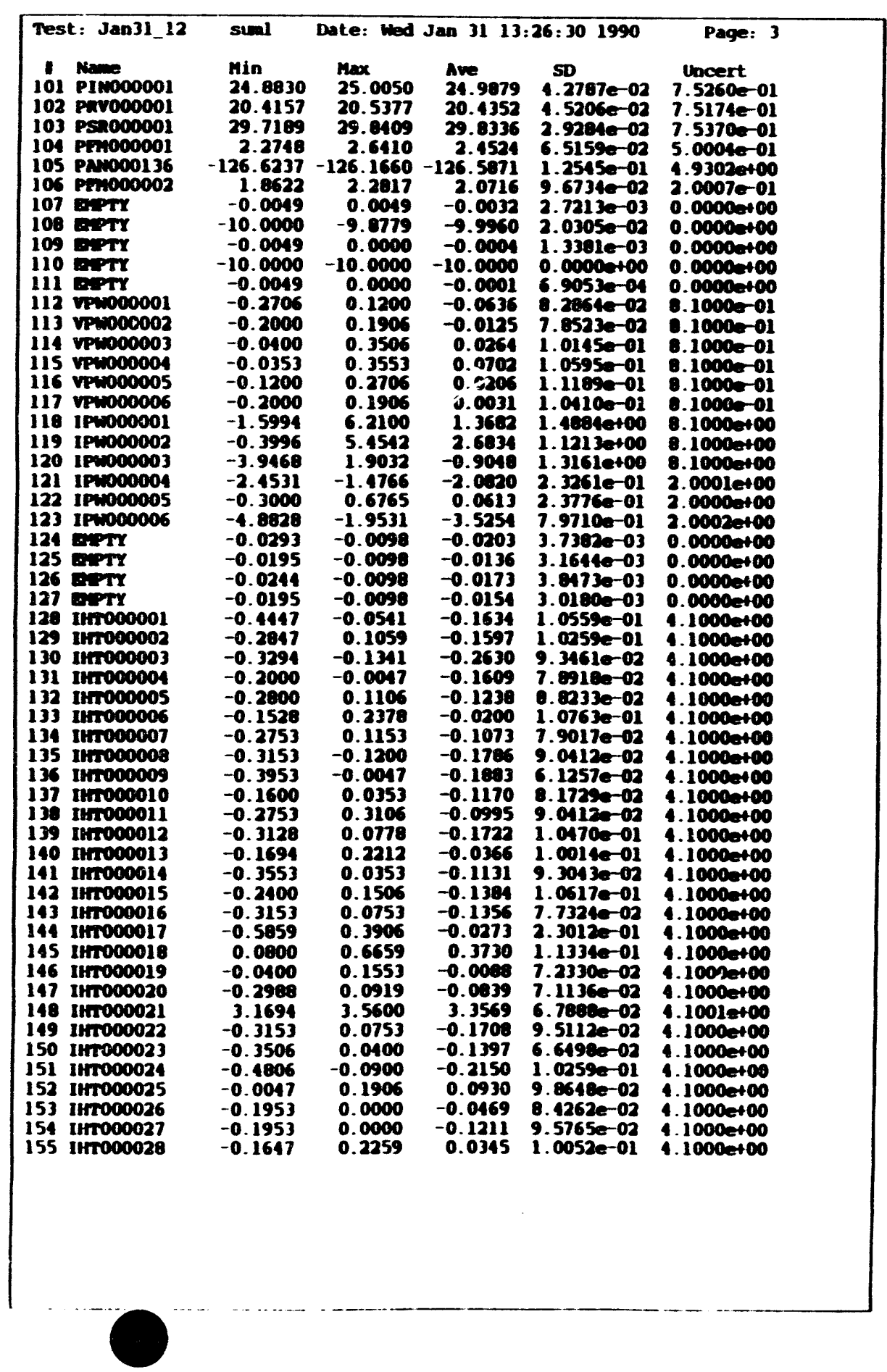

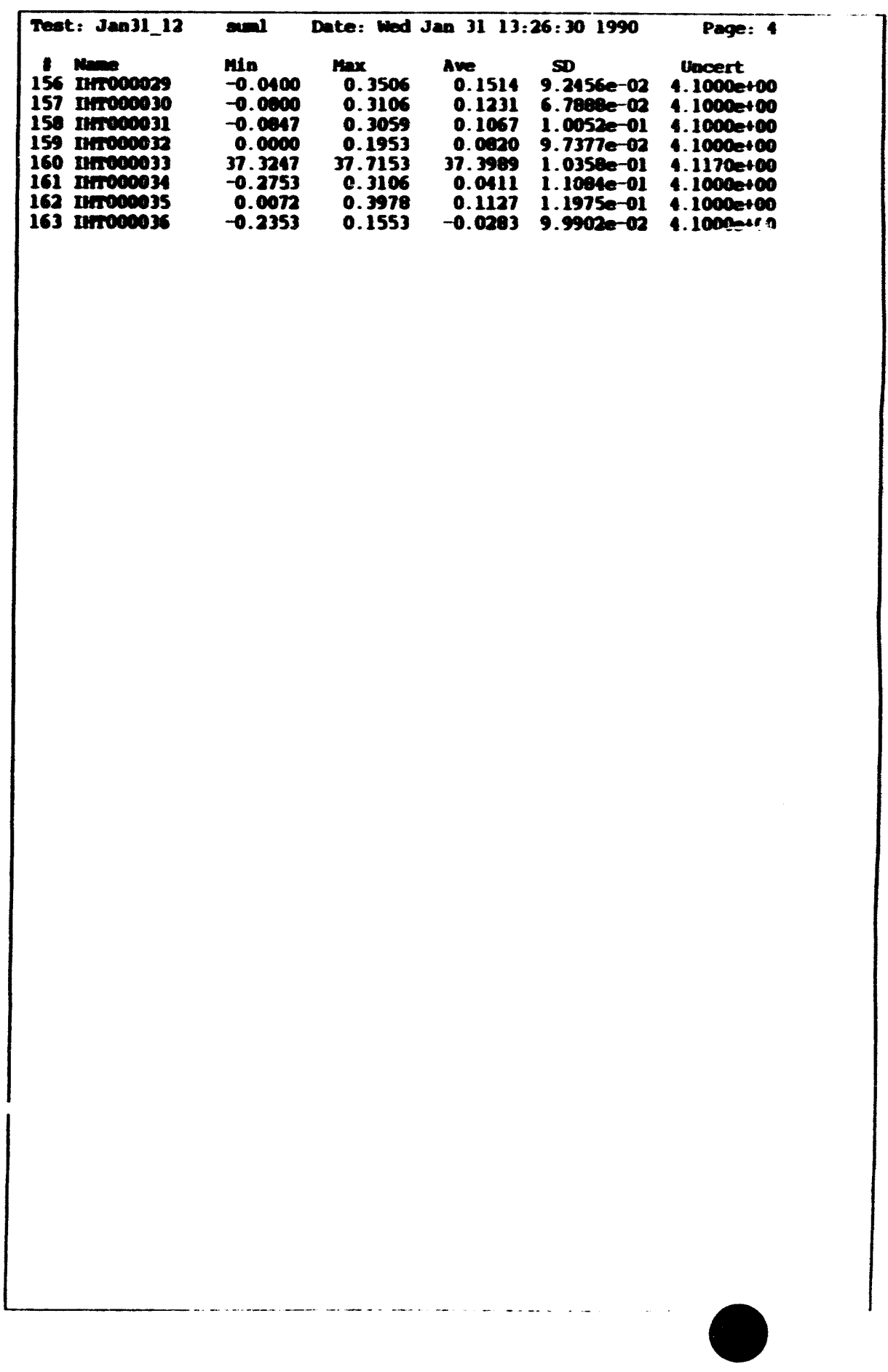




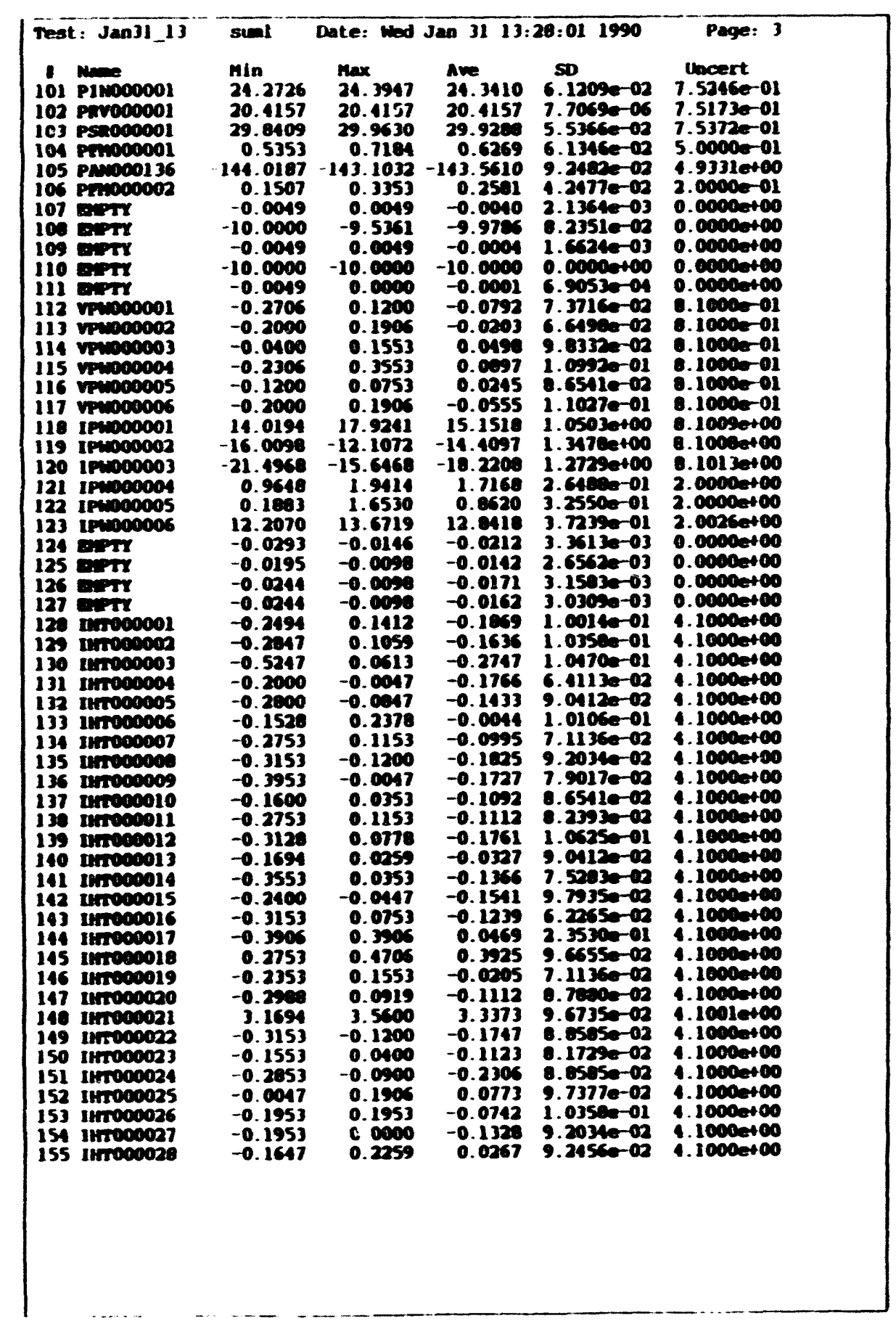

\begin{tabular}{|c|c|c|c|c|c|}
\hline at: $\mathbf{J}$ & $\operatorname{sen} 1$ & Date: Wod & $\operatorname{an} 3113:$ & $20: 011990$ & ege: \\
\hline 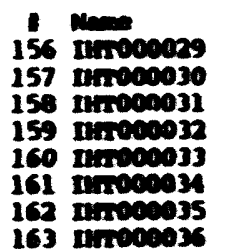 & $\begin{array}{l}\text { niv } \\
-0.0400 \\
-0.0000 \\
-0.0947 \\
-0.1953 \\
37.3217 \\
-0.2753 \\
-0.1801 \\
-0.2353\end{array}$ & $\begin{array}{l}n \times x \\
0.3506 \\
0.3105 \\
0.3059 \\
0.3906 \\
37.5200 \\
0.1153 \\
0.2025 \\
0.1553\end{array}$ & $\begin{array}{r}\text { Ave } \\
0.1202 \\
0.1153 \\
0.0598 \\
0.0273 \\
37.3716 \\
0.0333 \\
0.0558 \\
-0.0244\end{array}$ & $\begin{array}{l}50 \\
9.4125 e-02 \\
7.0918=-02 \\
9.51120-02 \\
6.01210-02 \\
. .12520-02 \\
1.0507 e-01 \\
9.05600-02 \\
7.7324=-02\end{array}$ & 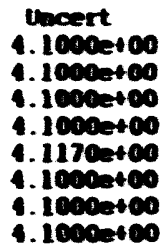 \\
\hline
\end{tabular}




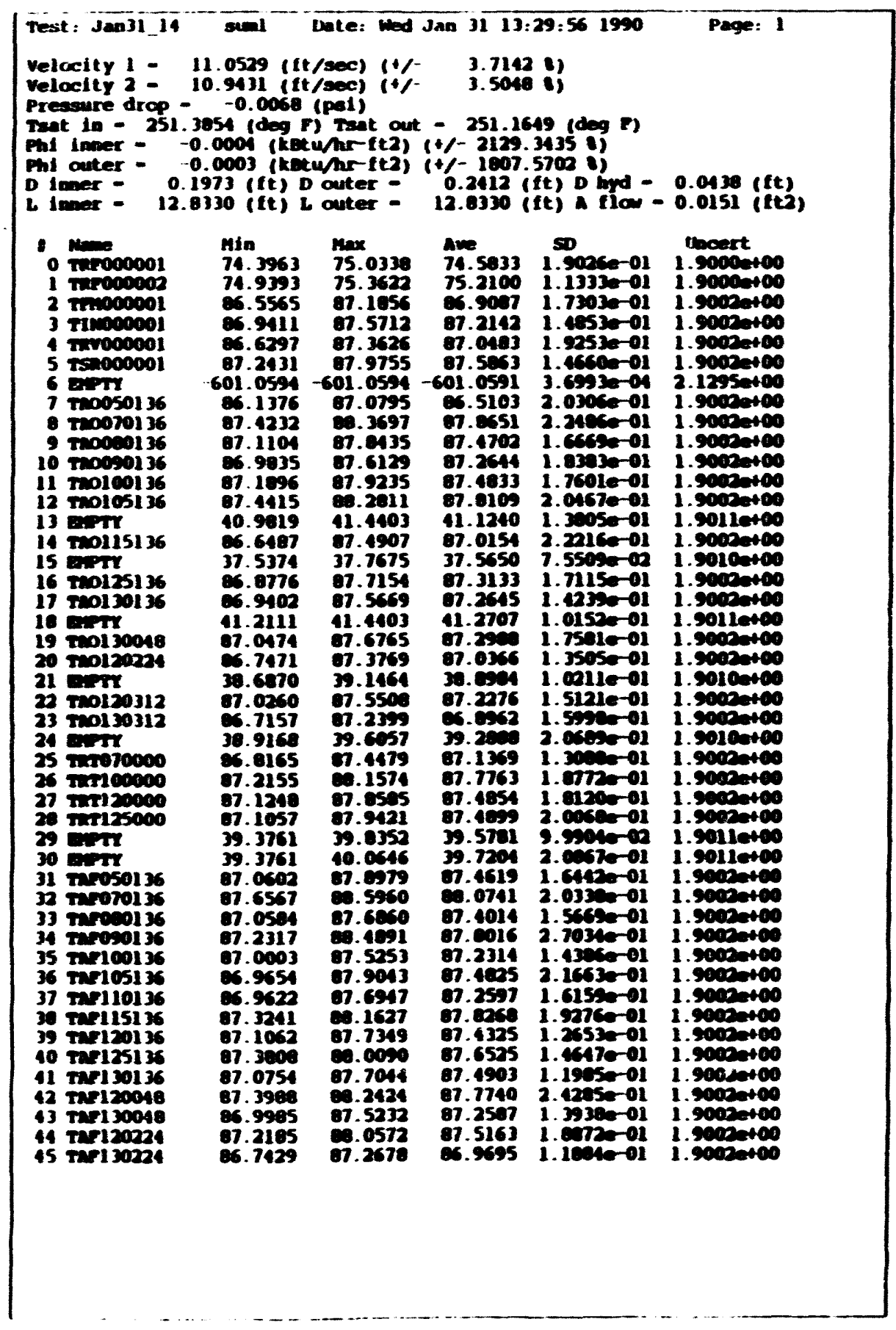

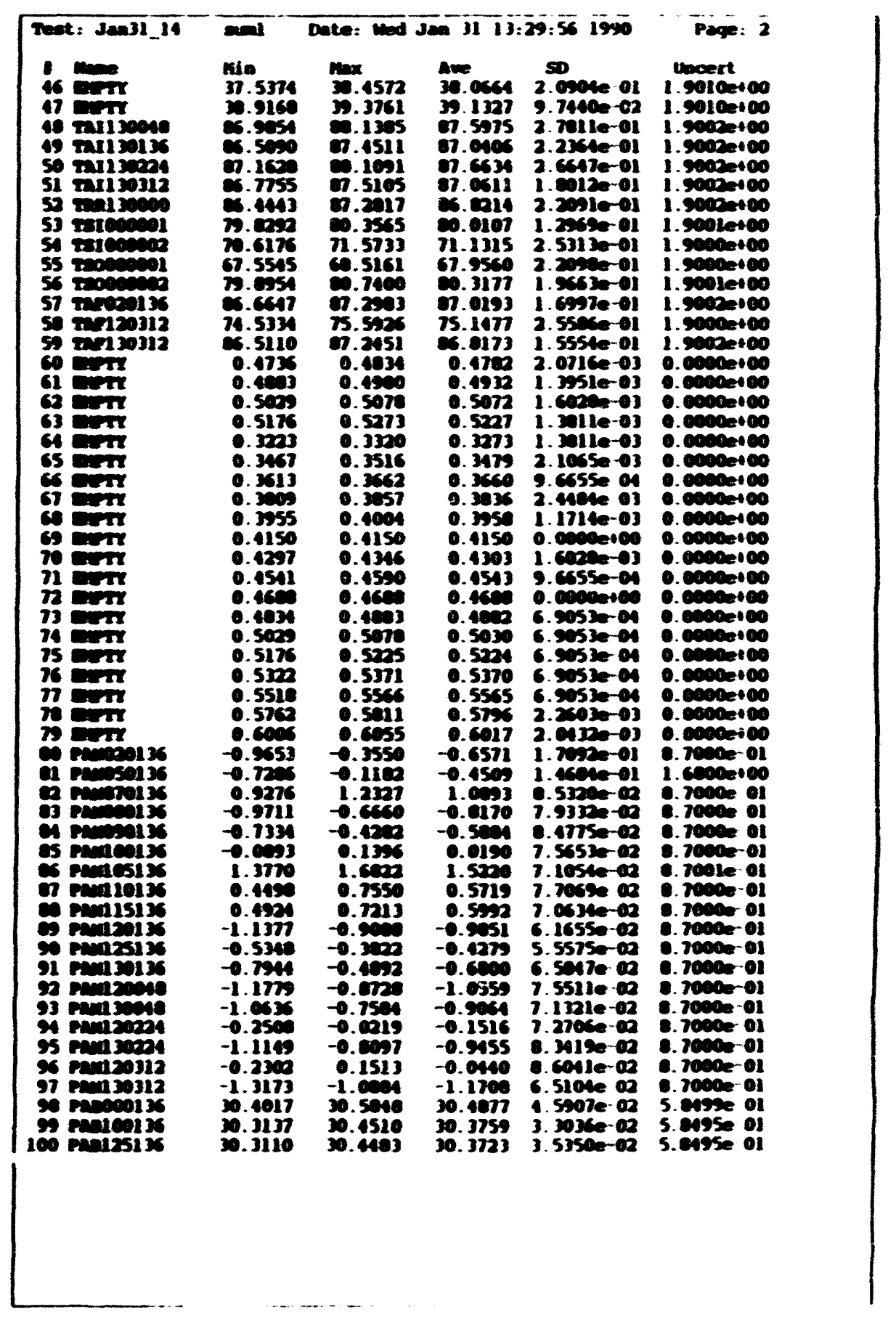




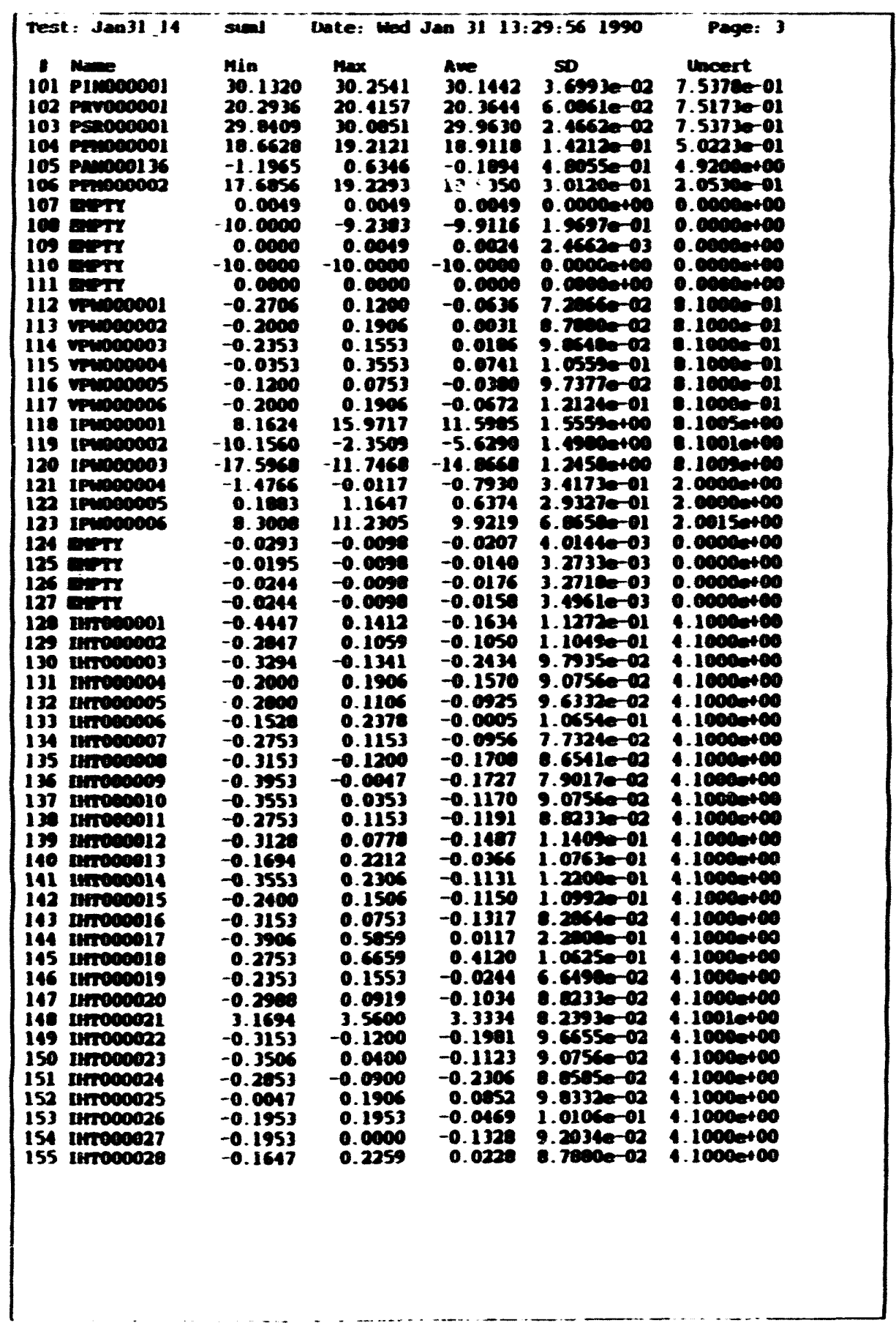

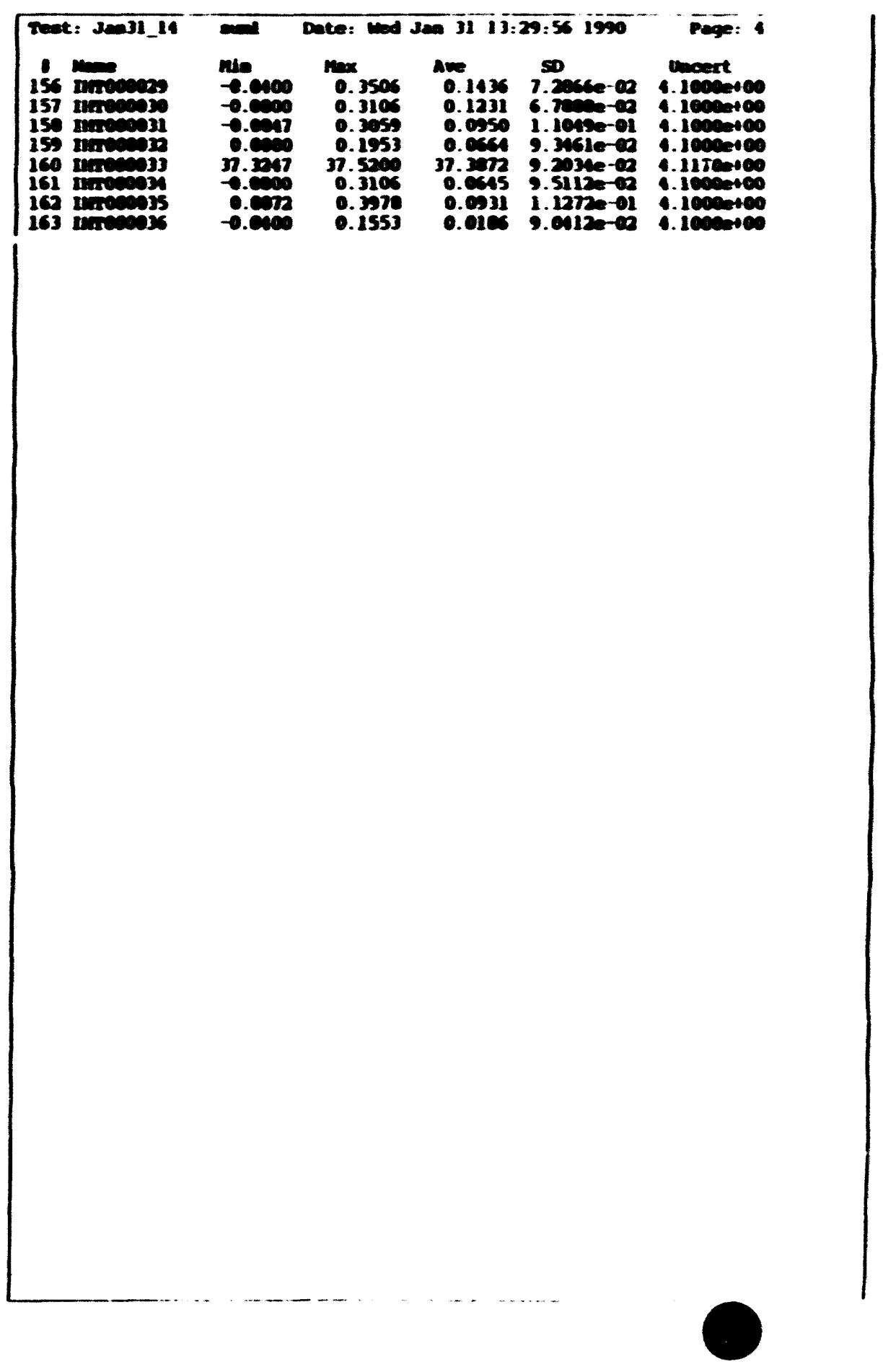




\begin{tabular}{|c|c|c|c|c|c|}
\hline \multicolumn{5}{|c|}{ 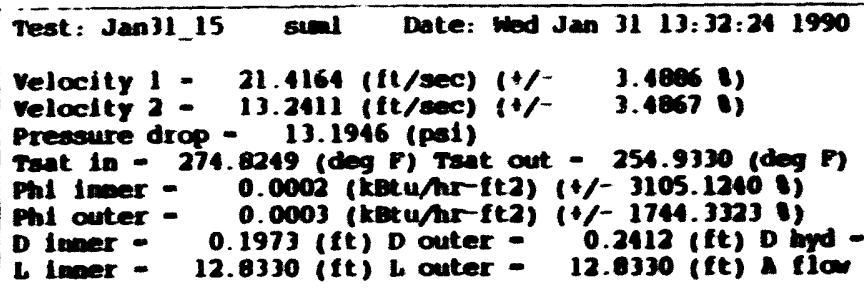 } & $\begin{array}{l}0.0430 \text { (ft) } \\
0.0151 \text { (ft2) } \\
0.01\end{array}$ \\
\hline 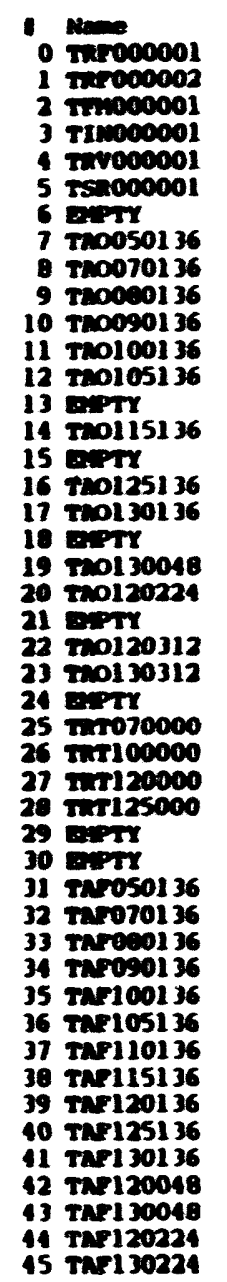 & 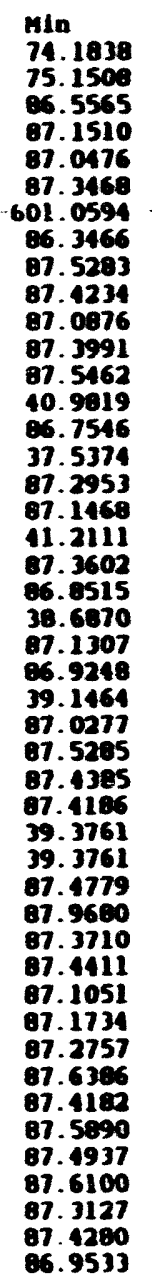 & 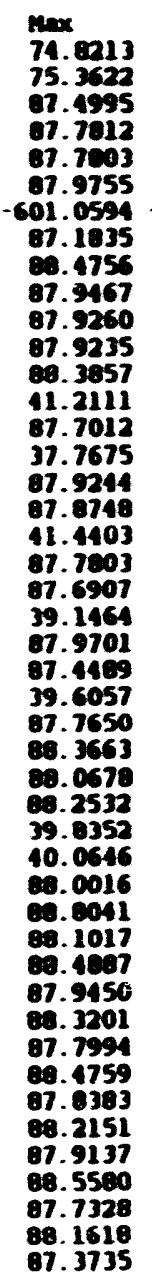 & 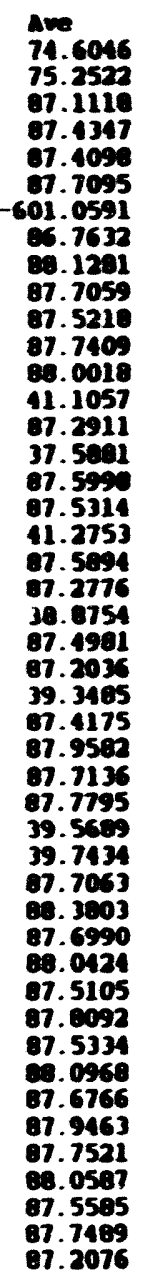 & 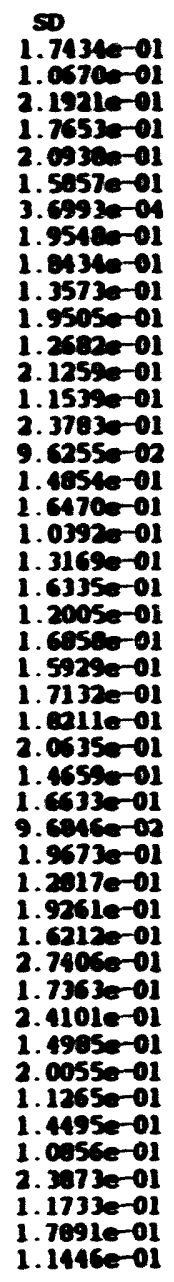 & 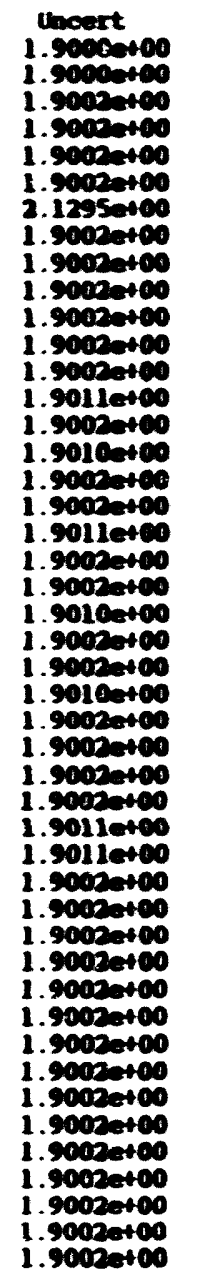 \\
\hline
\end{tabular}

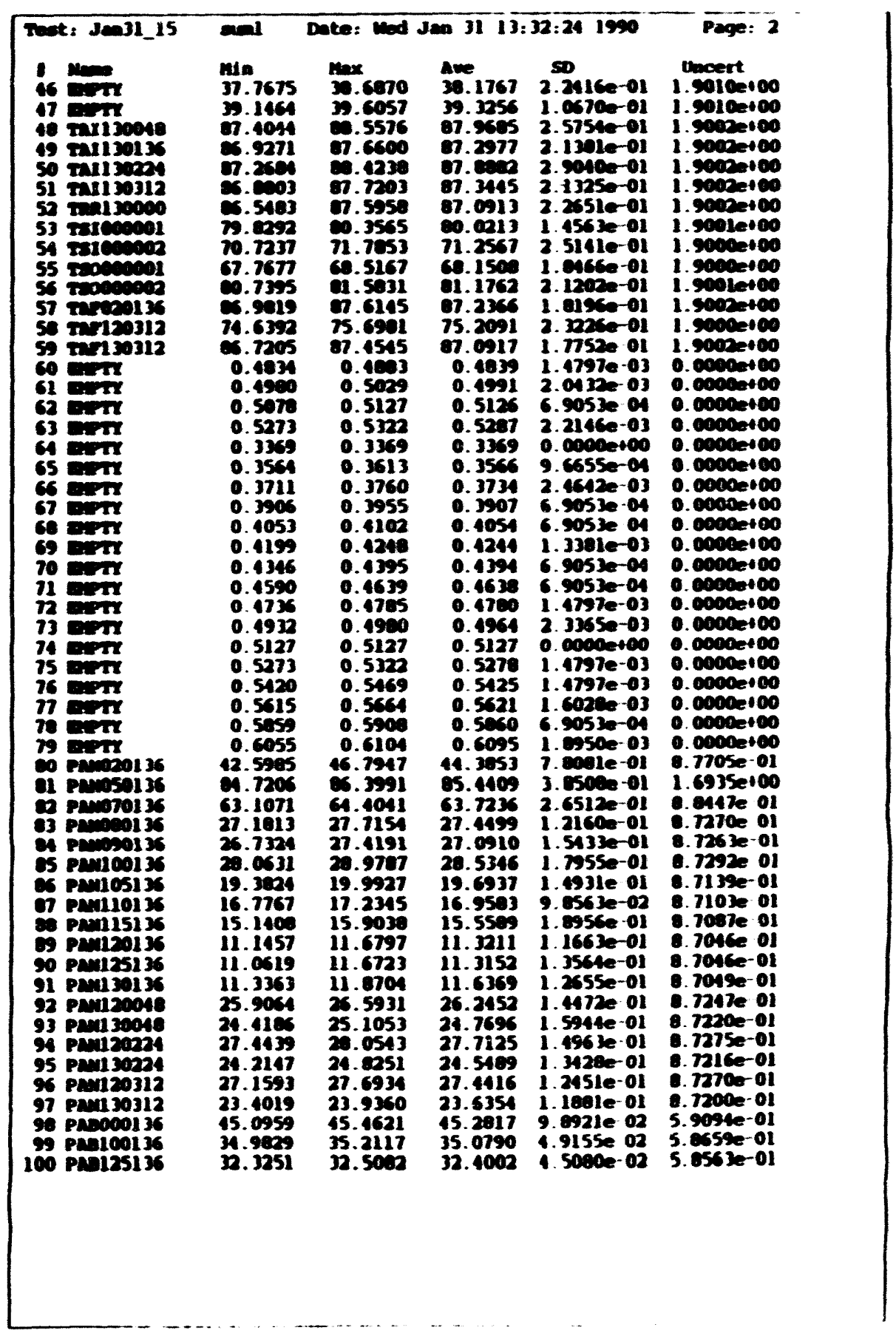




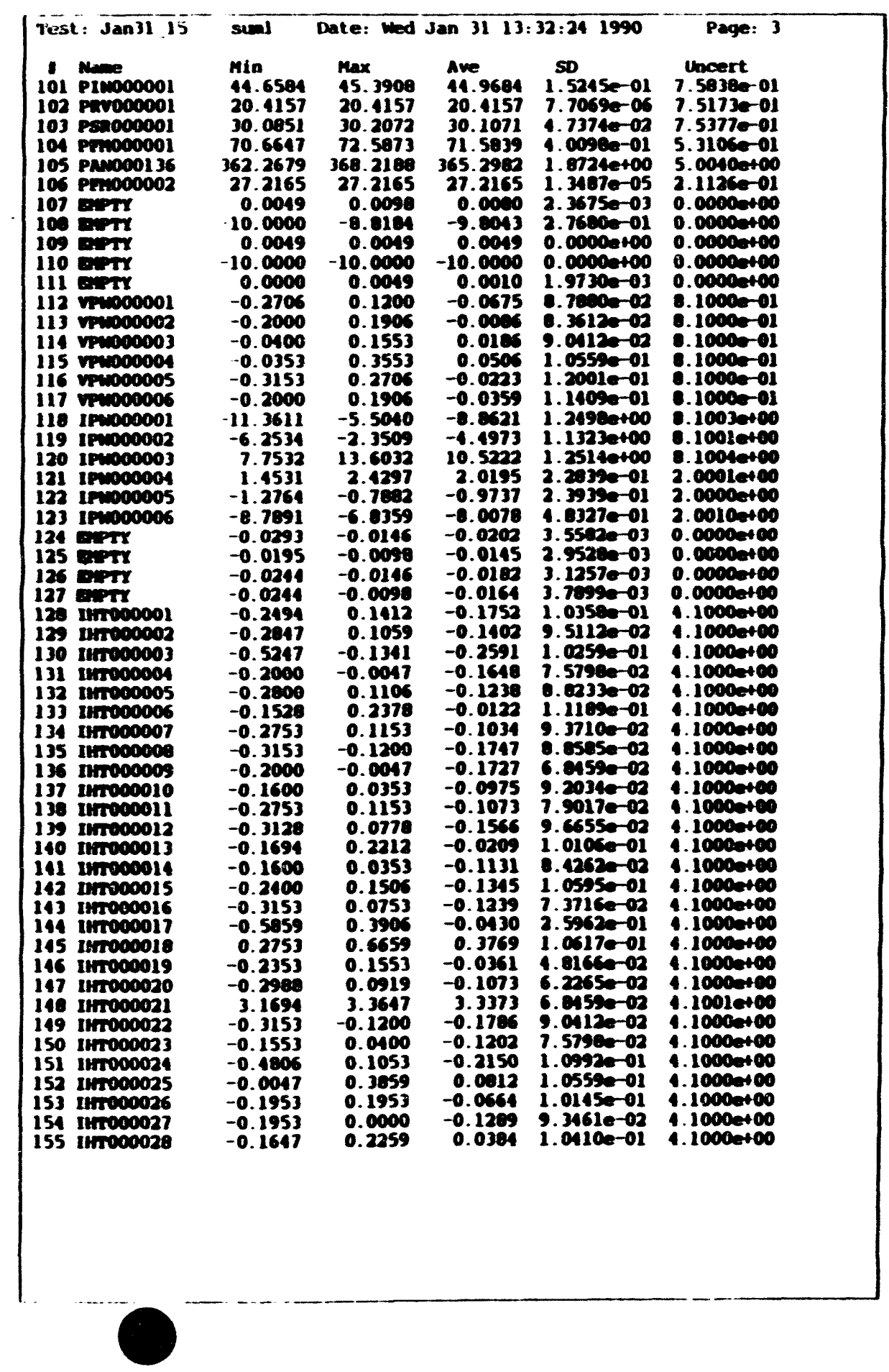

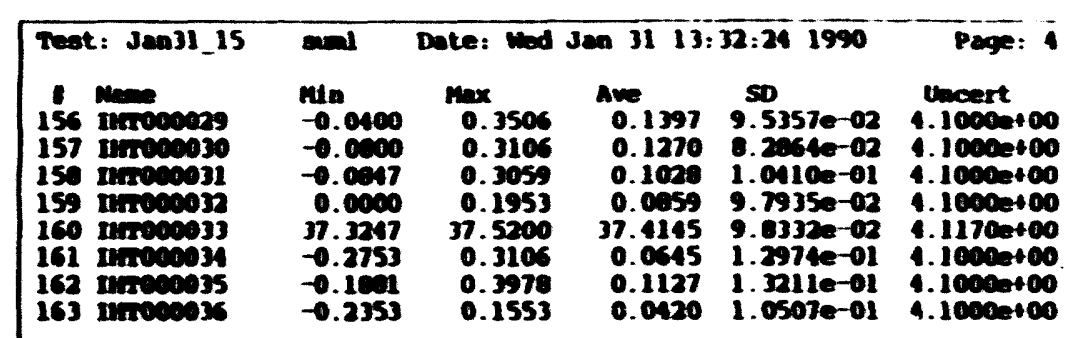




\begin{tabular}{|c|c|c|c|c|c|}
\hline \multicolumn{3}{|c|}{ 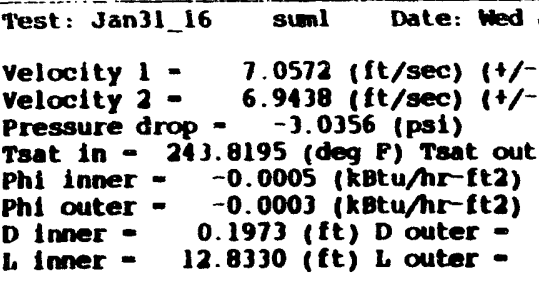 } & \multicolumn{2}{|c|}{$\begin{array}{l}\text { Jan } 3113: 34: 331990 \\
1.7714 \text { :) } \\
3.6785 \text { :) } \\
-249.5421 \text { (deg F) } \\
(+/-1432.7872 \text { ह) } \\
(+/-1345.0460 \text { 8) } \\
0.2412 \text { (ft) D hyd - } \\
12.8330 \text { (ft) A tlow - }\end{array}$} & $\begin{array}{l}\text { Page: } 1 \\
0.0438 \text { (ft) } \\
0.0151 \text { (ft2) }\end{array}$ \\
\hline 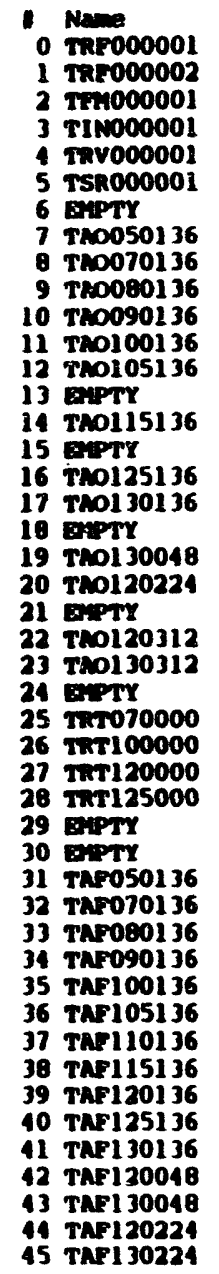 & $\begin{array}{r}\text { Min } \\
74.3963 \\
74.9393 \\
86.9752 \\
87.3614 \\
86.9439 \\
87.4519 \\
-601.0594 \\
86.4507 \\
87.9695 \\
87.6318 \\
87.2973 \\
87.5040 \\
87.7565 \\
10.9819 \\
86.8605 \\
37.3074 \\
87.4005 \\
87.3533 \\
11.2111 \\
87.3606 \\
87.0615 \\
38.6870 \\
87.3408 \\
87.0292 \\
38.9168 \\
87.2389 \\
87.8426 \\
87.5436 \\
87.5221 \\
39.3761 \\
39.1464 \\
87.5821 \\
88.0731 \\
87.4743 \\
87.7553 \\
87.2103 \\
87.3815 \\
87.3798 \\
87.7427 \\
87.6272 \\
87.7972 \\
87.4942 \\
87.7165 \\
87.4179 \\
87.5332 \\
86.9538\end{array}$ & $\begin{array}{r}\text { Max } \\
75.0338 \\
75.5736 \\
87.9180 \\
87.9912 \\
87.6756 \\
87.9755 \\
-601.0594 \\
87.2882 \\
88.7906 \\
88.2600 \\
88.1351 \\
88.3422 \\
88.7004 \\
11.2111 \\
87.9116 \\
37.7675 \\
88.0295 \\
88.0912 \\
11.4403 \\
88.0942 \\
87.7958 \\
39.1464 \\
88.0752 \\
87.6579 \\
39.6057 \\
87.9761 \\
88.5744 \\
88.3822 \\
88.3503 \\
39.8352 \\
90.0616 \\
88.2104 \\
89.0121 \\
88.2068 \\
88.8026 \\
88.0501 \\
88.5279 \\
88.0001 \\
88.7900 \\
88.1518 \\
88.4252 \\
88.1230 \\
88.7699 \\
87.9424 \\
88.2669 \\
87.6995\end{array}$ & $\begin{array}{r}\text { Ave } \\
74.7023 \\
75.2564 \\
87.3423 \\
87.6658 \\
87.3517 \\
87.7683 \\
-601.0591 \\
86.9724 \\
88.3512 \\
87.9646 \\
87.7187 \\
87.9631 \\
88.2284 \\
11.0782 \\
87.1763 \\
37.5466 \\
87.7682 \\
87.7140 \\
11.2691 \\
87.7442 \\
87.4747 \\
38.8616 \\
87.7038 \\
87.3544 \\
39.3623 \\
87.6116 \\
88.2134 \\
87.9357 \\
87.9465 \\
39.6011 \\
39.7066 \\
87.9196 \\
89.5598 \\
87.8658 \\
88.3189 \\
87.7079 \\
87.9179 \\
87.7132 \\
88.2727 \\
87.9064 \\
88.1341 \\
87.9156 \\
88.2822 \\
87.6761 \\
67.9292 \\
87.4352\end{array}$ & 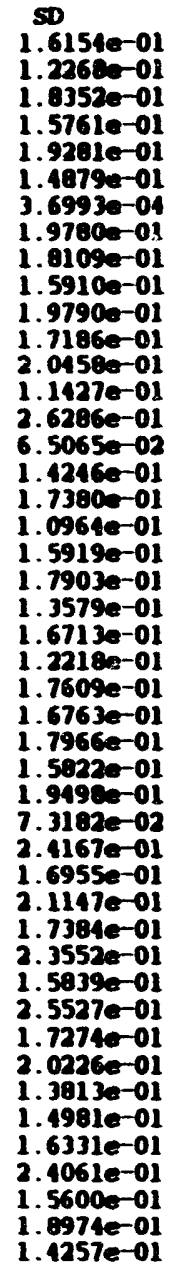 & 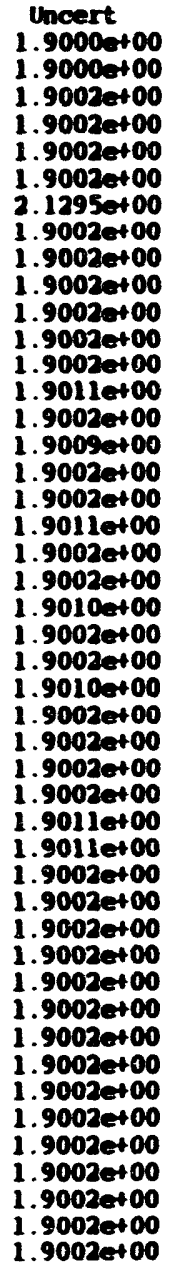 \\
\hline
\end{tabular}

\begin{tabular}{|c|c|c|c|c|c|}
\hline 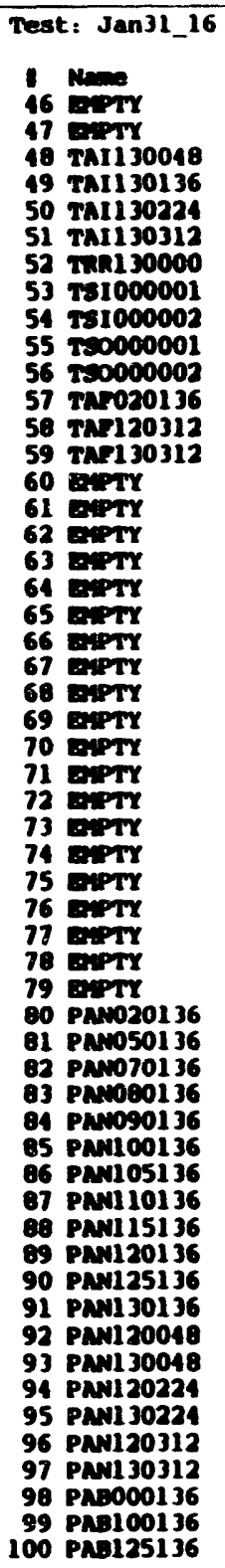 & 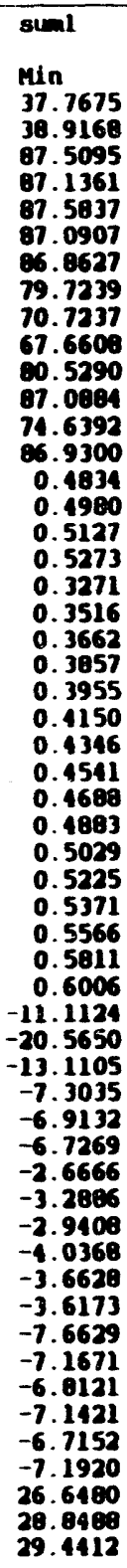 & 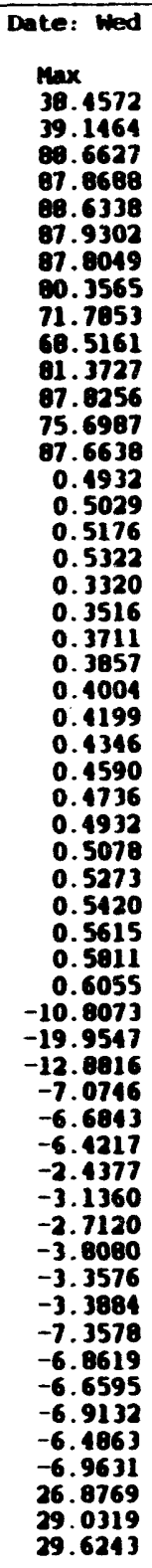 & $\begin{array}{l}\text { Jan } 3113: \\
\text { Ave } \\
38.0710 \\
39.1327 \\
80.1196 \\
87.5237 \\
88.1067 \\
87.5671 \\
87.2965 \\
80.0720 \\
71.3672 \\
68.1292 \\
80.9570 \\
87.4940 \\
75.2643 \\
87.2678 \\
0.4869 \\
0.5026 \\
0.5153 \\
0.5321 \\
0.3314 \\
0.3516 \\
0.3668 \\
0.3857 \\
0.4001 \\
0.4186 \\
0.4346 \\
0.4581 \\
0.4721 \\
0.4912 \\
0.5075 \\
0.5229 \\
0.5378 \\
0.5575 \\
0.5811 \\
0.6054 \\
-10.9766 \\
-20.2598 \\
-13.0403 \\
-7.1937 \\
-6.7667 \\
-6.5545 \\
-2.6055 \\
-3.2153 \\
-2.8264 \\
-3.9102 \\
-3.5072 \\
-3.5028 \\
-7.5226 \\
-7.0038 \\
-6.7236 \\
-7.0612 \\
-6.6328 \\
-7.0897 \\
26.7395 \\
28.9175 \\
29.5346\end{array}$ & $\begin{array}{l}34.331990 \\
50 \\
2.0481 e-01 \\
5.5101 e-02 \\
2.6332 e-01 \\
1.7590 e-01 \\
2.7293 e-01 \\
2.0360 e-01 \\
2.1046 e-01 \\
1.6120 e-01 \\
2.9194 e-01 \\
1.7262 e-01 \\
2.3086 e-01 \\
1.7660 e-01 \\
2.3946 e-01 \\
1.6982 e-01 \\
2.1214 e-03 \\
1.1714 e-03 \\
2.1583 e-03 \\
6.9053 e-04 \\
1.6028 e-03 \\
0.0000 e+00 \\
1.6028 e-03 \\
0.0000 e-00 \\
1.1714 e-03 \\
2.2146 e-03 \\
0.0000 e+00 \\
1.6950 e-03 \\
2.3006 e-03 \\
2.4164 e-03 \\
1.1714 e-03 \\
1.3381 e-03 \\
1.7115 e-03 \\
1.8950 e-03 \\
0.0000 e+00 \\
6.9053 e-04 \\
7.4386 e-02 \\
1.2331 e-01 \\
6.3253 e-02 \\
7.0903 e-02 \\
6.3253 e-02 \\
7.5212 e-02 \\
5.3395 e-02 \\
5.7590 e-02 \\
7.2706 e-02 \\
6.8257 e-02 \\
7.2231 e-02 \\
6.2135 e-02 \\
7.2886 e-02 \\
6.3571 e-02 \\
5.8410 e-02 \\
6.9635 e-02 \\
6.5104 e-02 \\
6.8257 e-02 \\
5.9935 e-02 \\
5.4900 e-02 \\
1.7121 e-02\end{array}$ & 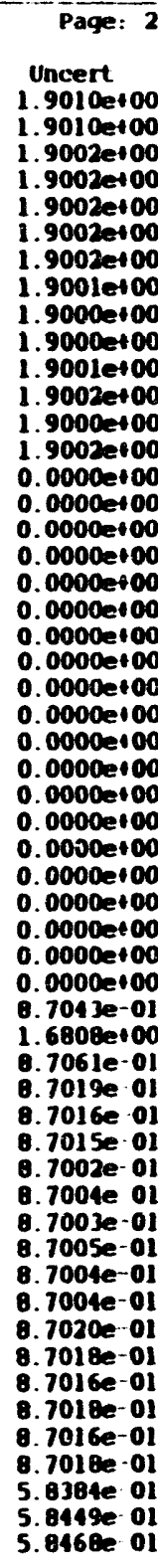 \\
\hline
\end{tabular}




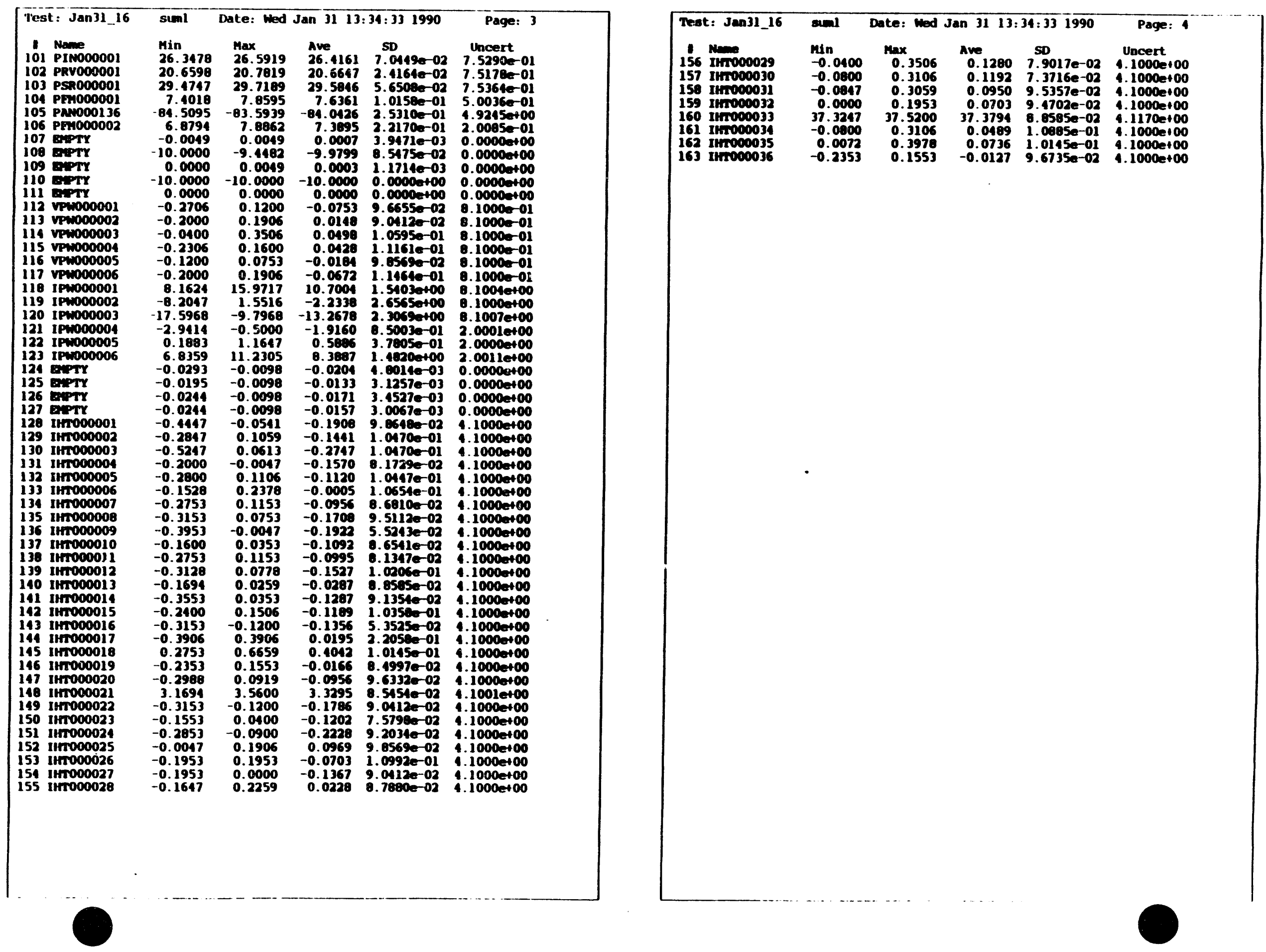




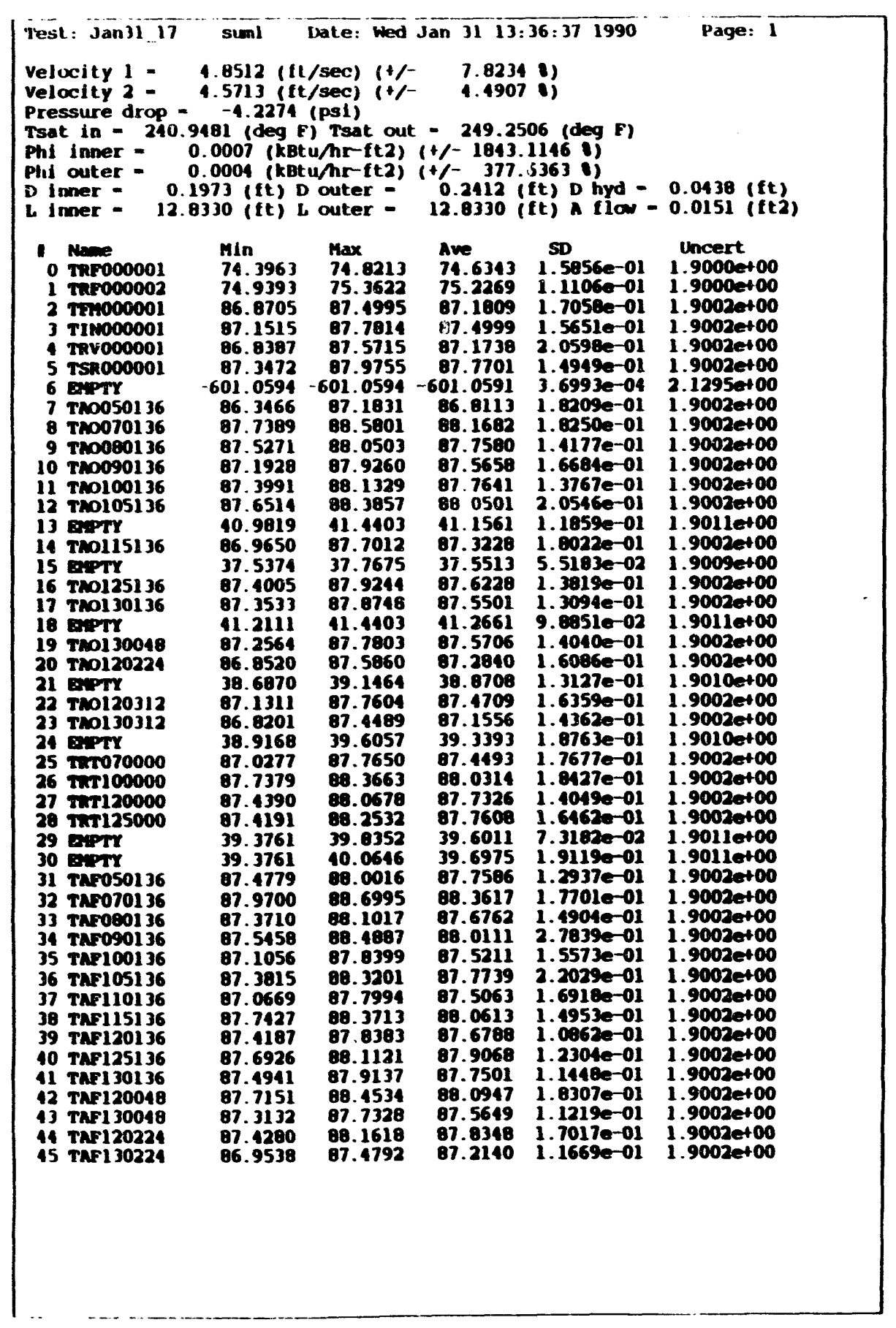

\begin{tabular}{|c|c|c|c|c|c|}
\hline 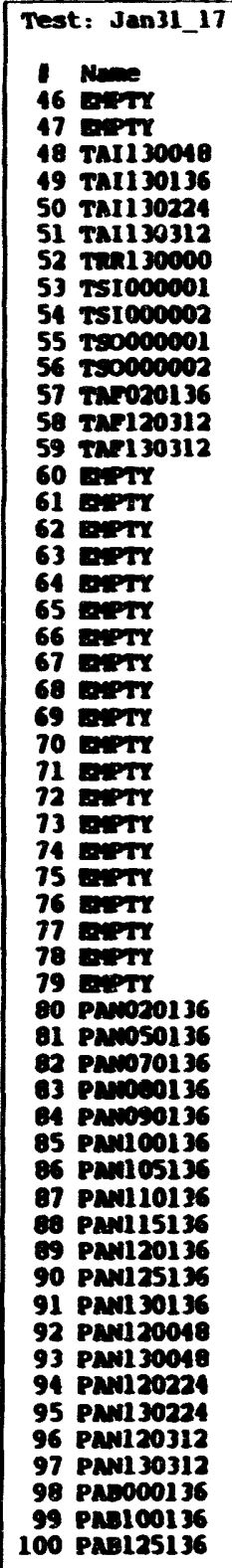 & 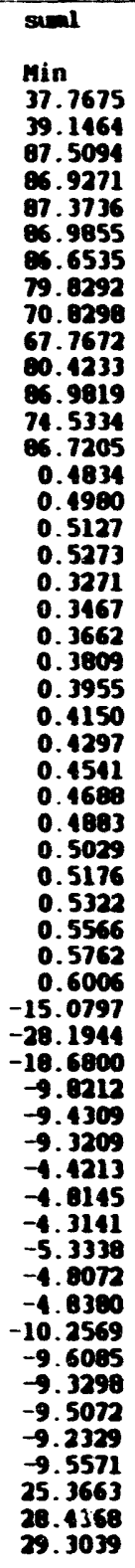 & 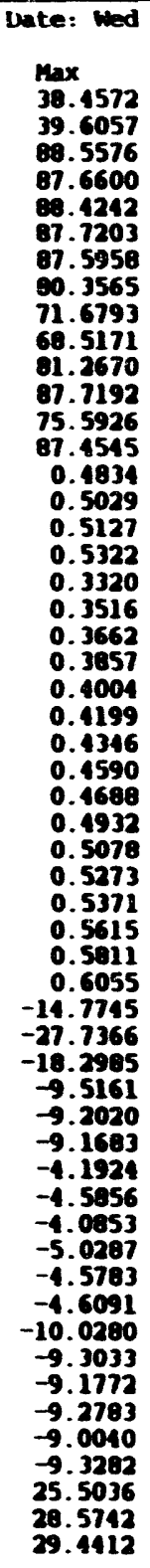 & 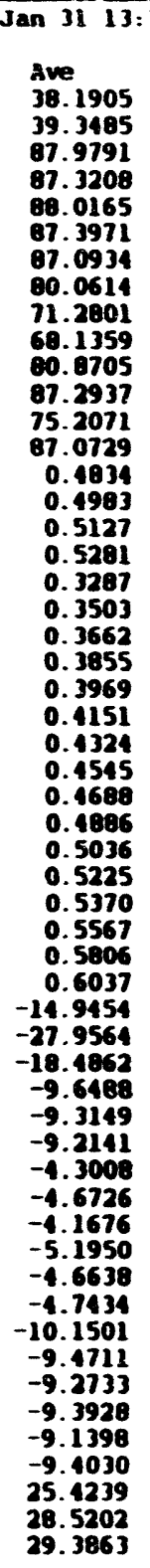 & 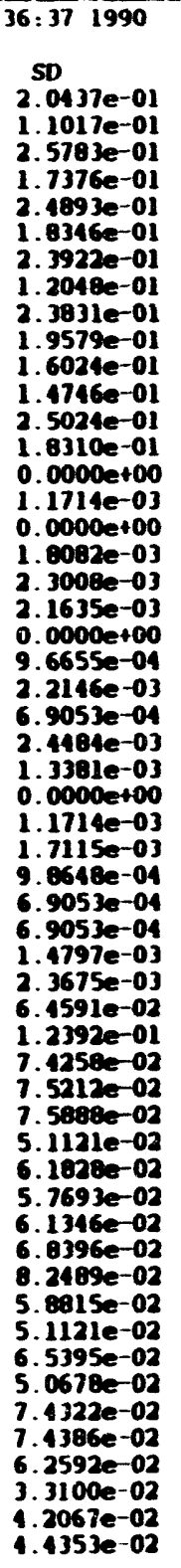 & 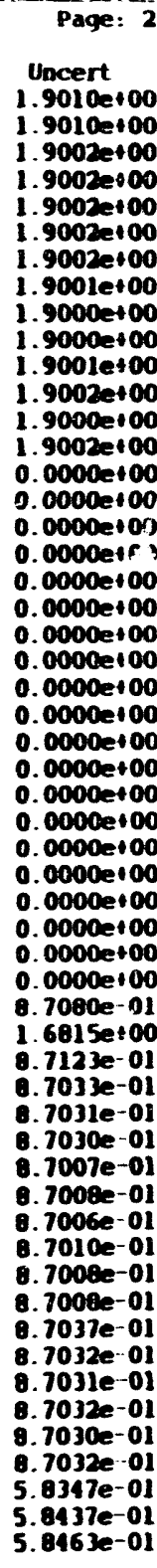 \\
\hline
\end{tabular}




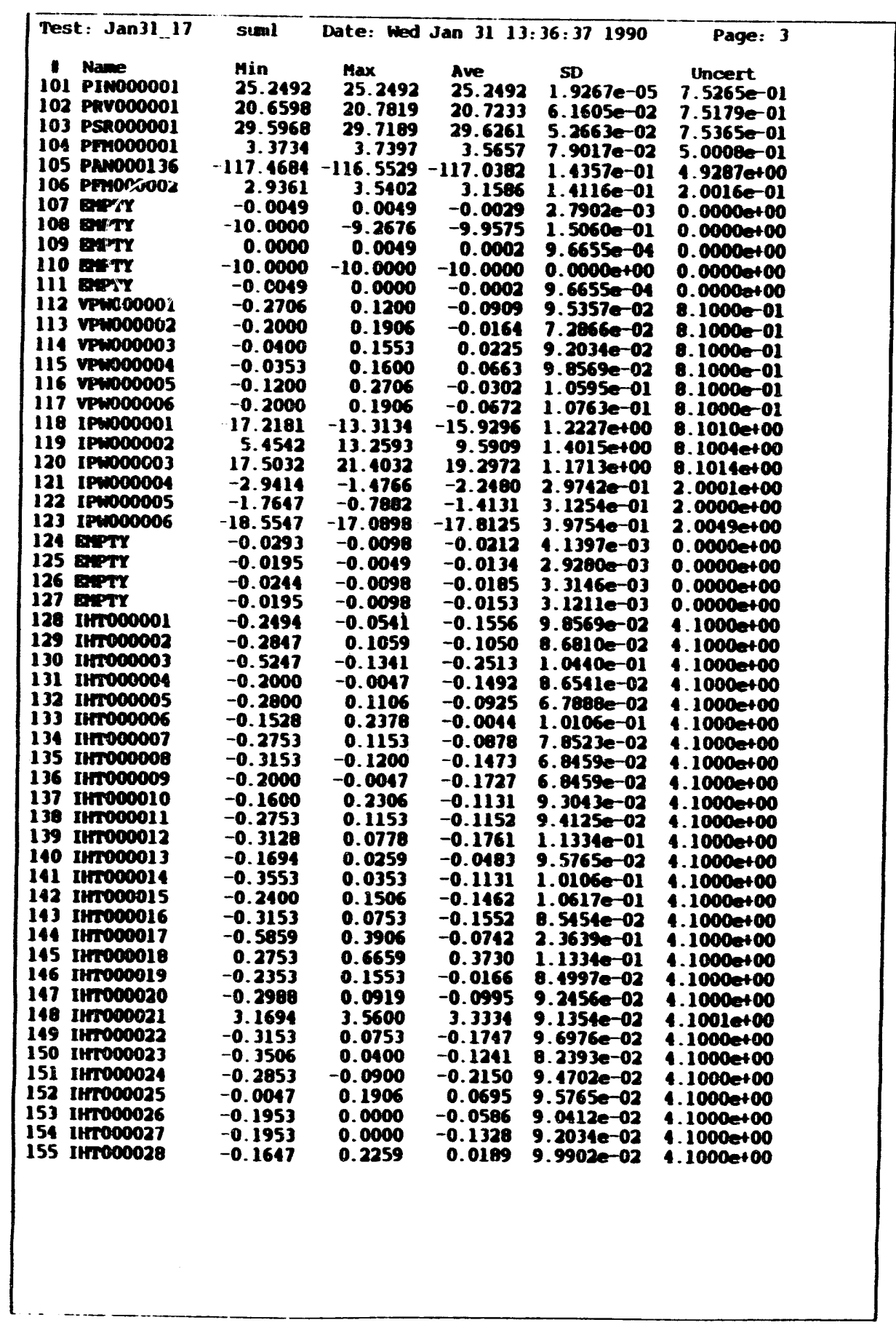

\begin{tabular}{|c|c|c|c|c|c|}
\hline 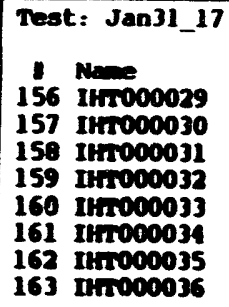 & $\begin{array}{l}\text { ond } \\
\text { nin } \\
-0.0900 \\
-0.0000 \\
-0.0847 \\
0.0000 \\
37.3247 \\
-0.0000 \\
-0.1891 \\
-0.2353\end{array}$ & $\begin{array}{l}\text { Date: Wed } \\
\text { Max } \\
0.3506 \\
0.3106 \\
0.3059 \\
0.1953 \\
37.5200 \\
0.3106 \\
0.3978 \\
0.1553\end{array}$ & $\begin{array}{l}\text { an } 3113 \\
\text { Ave } \\
0.1241 \\
0.1153 \\
0.1106 \\
0.0391 \\
37.3833 \\
0.0450 \\
0.1009 \\
-0.0009\end{array}$ & $\begin{array}{l}36: 371990 \\
\text { SD } \\
9.1354 e-02 \\
6.8345 e-02 \\
1.0490 e-01 \\
7.8918 e-02 \\
9.0412 e-02 \\
1.0992 e-01 \\
1.2627 e-01 \\
8.8233 e-02\end{array}$ & $\begin{array}{c}\text { Page: } \\
\text { Uncert } \\
1.1000=+00 \\
1.1000=+00 \\
1.1000=+00 \\
1.1000=+00 \\
1.11700+00 \\
1.1000=+00 \\
1.1000=+00 \\
1.1000=+00\end{array}$ \\
\hline
\end{tabular}

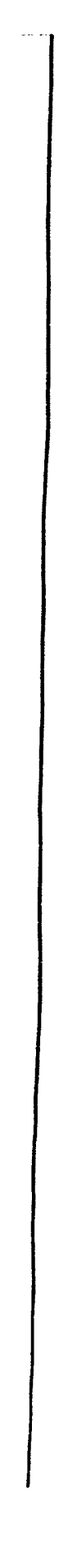




\begin{tabular}{|c|c|c|c|c|c|c|}
\hline \multicolumn{2}{|c|}{ 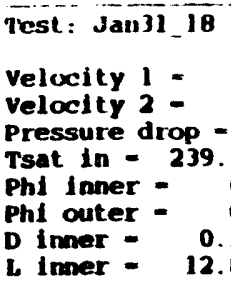 } & $\begin{array}{r}\text { suml } \\
3.0097 \text { । } \\
2.5852 \text { ! } \\
-4.925 \\
3214 \text { (deg } \\
.0008 \text { (k } \\
.0004 \text { (k } \\
1973 \text { (ft) } \\
3330 \text { (ft) }\end{array}$ & 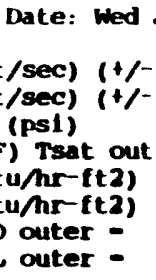 & $\begin{array}{r}\text { Jan } 3113: \\
18.9635 \\
9.8211\end{array}$ & $\begin{array}{l}38: 161990 \\
\because) \\
?\end{array}$ & $\begin{array}{l}\text { Page: } 1 \\
0.0438 \text { (ft) } \\
0.0151 \text { (ft2 }\end{array}$ \\
\hline 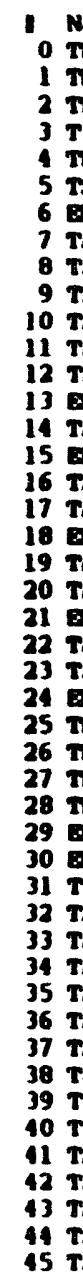 & 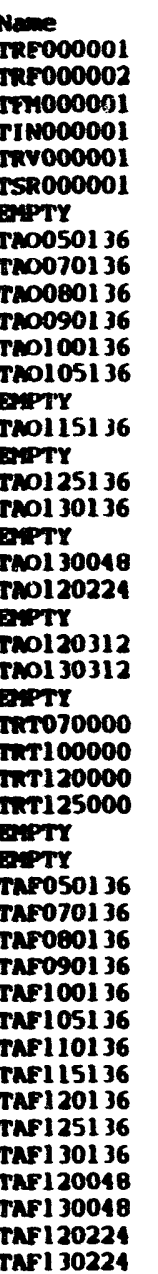 & $\begin{array}{l}\text { Min } \\
74.3963 \\
74.9393 \\
86.7659 \\
87.1515 \\
86.8387 \\
87.3472 \\
601.0594 \\
86.2418 \\
87.7397 \\
87.3188 \\
86.9835 \\
87.3991 \\
87.6514 \\
10.9819 \\
86.8598 \\
37.5374 \\
87.2958 \\
87.1468 \\
41.2111 \\
87.2564 \\
86.8520 \\
38.6870 \\
87.1311 \\
86.8201 \\
38.9168 \\
87.0277 \\
87.6336 \\
87.1390 \\
87.3139 \\
39.3761 \\
39.1464 \\
87.1779 \\
87.9685 \\
87.2664 \\
87.5463 \\
87.1056 \\
87.2768 \\
87.1710 \\
87.6381 \\
87.1187 \\
87.4844 \\
87.4942 \\
87.6100 \\
87.2082 \\
87.1280 \\
86.9538\end{array}$ & 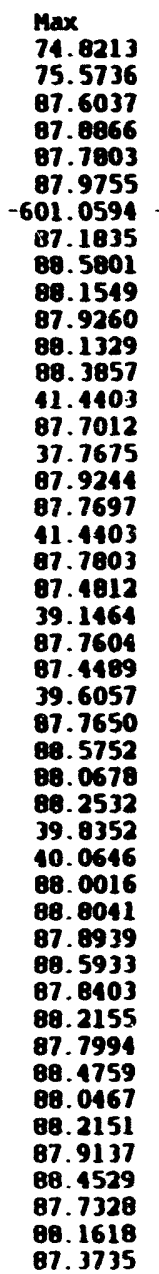 & $\begin{array}{r}\text { Ave } \\
74.6258 \\
75.2353 \\
87.2395 \\
87.5545 \\
87.2156 \\
87.7409 \\
-601.0591 \\
86.7736 \\
88.1261 \\
87.7330 \\
87.5365 \\
87.7682 \\
88.0878 \\
11.1286 \\
87.3059 \\
37.5513 \\
87.5936 \\
87.4922 \\
11.2982 \\
87.4870 \\
87.2881 \\
38.8692 \\
87.4667 \\
87.0971 \\
39.3531 \\
87.4197 \\
88.0063 \\
87.7242 \\
87.7233 \\
39.5609 \\
39.6745 \\
87.7711 \\
88.3700 \\
87.6762 \\
88.0781 \\
87.5252 \\
87.7864 \\
87.5313 \\
88.0236 \\
87.6913 \\
87.9234 \\
87.7501 \\
88.0693 \\
87.5607 \\
87.8347 \\
87.2097\end{array}$ & $\begin{array}{l}\text { SD } \\
1.7087 e-01 \\
1.2914 e-01 \\
1.9314 e-01 \\
1.8041 e-01 \\
1.9134 e-01 \\
1.5491 e-01 \\
3.6993 e-04 \\
2.3334 e-01 \\
2.0959 e-01 \\
1.8532 e-01 \\
2.1477 e-01 \\
1.5314 e-01 \\
2.0035 e-01 \\
1.3704 e-01 \\
2.2841 e-01 \\
5.5183 e-02 \\
1.4569 e-01 \\
1.4693 e-01 \\
1.1235 e-01 \\
1.6253 e-01 \\
1.3794 e-01 \\
1.1022 e-01 \\
1.5133 e-01 \\
1.4176 e-01 \\
1.8115 e-01 \\
1.5333 e-01 \\
1.9507 e-01 \\
1.5958 e-01 \\
1.9778 e-01 \\
1.0738 e-01 \\
1.9811 e-01 \\
1.5256 e-01 \\
1.7941 e-01 \\
1.6999 e-01 \\
2.6911 e-01 \\
1.7613 e-01 \\
2.2444 e-01 \\
1.4832 e-01 \\
1.9445 e-01 \\
1.3725 e-01 \\
1.5919 e-01 \\
1.2390 e-01 \\
2.2193 e-01 \\
1.3028 e-01 \\
2.1552 e-01 \\
1.2397 e-01\end{array}$ & 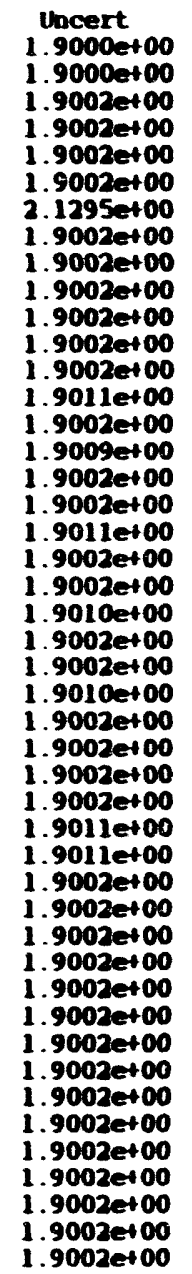 \\
\hline
\end{tabular}

\begin{tabular}{|c|c|c|c|c|c|}
\hline 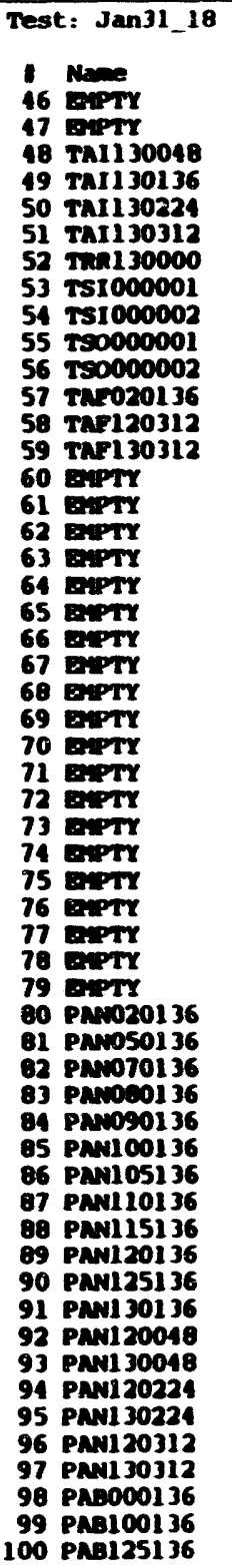 & $\begin{array}{l}\text { sual } \\
\text { Min } \\
37.7675 \\
39.1464 \\
87.5094 \\
87.0313 \\
87.4786 \\
86.9854 \\
86.3395 \\
79.7239 \\
70.9359 \\
67.8751 \\
60.2122 \\
86.8772 \\
74.7452 \\
86.6158 \\
0.1785 \\
0.4980 \\
0.5127 \\
0.5273 \\
0.3271 \\
0.3467 \\
0.3662 \\
0.3809 \\
0.3955 \\
0.1150 \\
0.4297 \\
0.1492 \\
0.4639 \\
0.4863 \\
0.5029 \\
0.5176 \\
0.5371 \\
0.5566 \\
0.5762 \\
0.6006 \\
-17.1448 \\
-32.7720 \\
-21.8080 \\
-11.1182 \\
-10.8805 \\
-10.8468 \\
-5.3368 \\
-5.6537 \\
-5.1534 \\
-6.0205 \\
-5.4939 \\
-5.5246 \\
-11.8591 \\
-10.9816 \\
-11.0082 \\
-10.9568 \\
-10.8350 \\
-11.0067 \\
24.5881 \\
28.2080 \\
29.2123\end{array}$ & $\begin{array}{l}\text { Date: Hed } \\
\text { Max } \\
38.4572 \\
39.6057 \\
89.1531 \\
87.6600 \\
88.3192 \\
87.7203 \\
87.5958 \\
80.3565 \\
71.7853 \\
68.6234 \\
81.0561 \\
87.7192 \\
75.8042 \\
87.4545 \\
0.4683 \\
0.5029 \\
0.5127 \\
0.5322 \\
0.3320 \\
0.3516 \\
0.3711 \\
0.3857 \\
0.4004 \\
0.4199 \\
0.4346 \\
0.4590 \\
0.4736 \\
0.4883 \\
0.5029 \\
0.5225 \\
0.5371 \\
0.5566 \\
0.5811 \\
0.6055 \\
-17.1396 \\
-32.3143 \\
-21.5792 \\
-10.8993 \\
-10.6516 \\
-10.6179 \\
-5.1843 \\
-5.3485 \\
-4.9245 \\
-5.7153 \\
-5.2650 \\
-5.2958 \\
-11.5539 \\
-10.8292 \\
-10.7031 \\
-10.7279 \\
-10.6824 \\
-10.7778 \\
24.8170 \\
28.4368 \\
29.4412\end{array}$ & $\begin{array}{l}\text { Jan } 3113: \\
\text { Ave } \\
38.1675 \\
39.3164 \\
87.9749 \\
87.3333 \\
87.9114 \\
87.3099 \\
87.1227 \\
80.0403 \\
71.3946 \\
68.1487 \\
80.6679 \\
87.3570 \\
75.1858 \\
87.1022 \\
0.4837 \\
0.4983 \\
0.5127 \\
0.5286 \\
0.3282 \\
0.3498 \\
0.3663 \\
0.3850 \\
0.3966 \\
0.4151 \\
0.4314 \\
0.4541 \\
0.4689 \\
0.4893 \\
0.5029 \\
0.5224 \\
0.5371 \\
0.5566 \\
0.5601 \\
0.6027 \\
-17.2678 \\
-32.5035 \\
-21.6936 \\
-11.0297 \\
-10.7538 \\
-10.7247 \\
-5.2514 \\
-5.5423 \\
-4.9947 \\
-5.9030 \\
-5.3947 \\
-5.4300 \\
-11.6745 \\
-10.8941 \\
-10.8679 \\
-10.9019 \\
-10.7572 \\
-10.8661 \\
24.7025 \\
28.3178 \\
29.3341\end{array}$ & 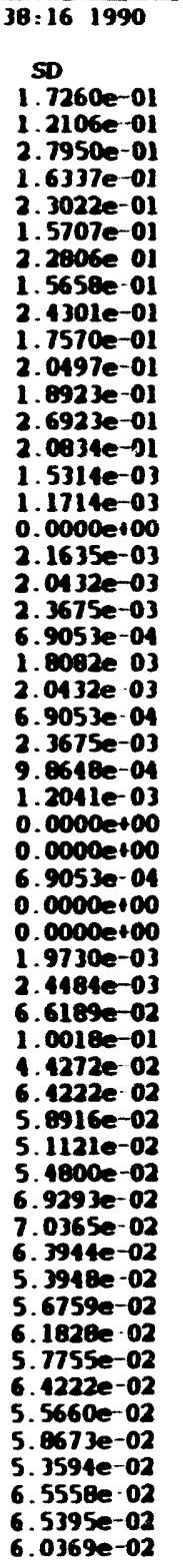 & 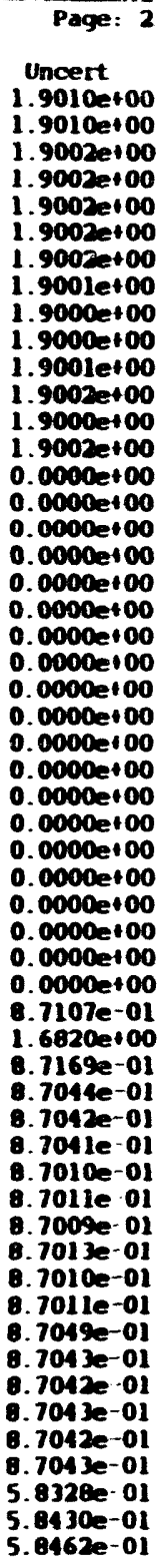 \\
\hline
\end{tabular}




\begin{tabular}{|c|c|c|c|c|c|c|}
\hline \multirow[b]{2}{*}{$\begin{array}{l}1 \text { lest } \\
1 \\
101 \\
102 \\
103 \\
104 \\
105 \\
106 \\
107 \\
100 \\
109 \\
110 \\
111 \\
112 \\
113 \\
114 \\
115 \\
116 \\
117 \\
118 \\
119 \\
120 \\
121 \\
122 \\
123 \\
124 \\
125 \\
126 \\
127 \\
128 \\
129 \\
130 \\
131 \\
132 \\
133 \\
134 \\
135 \\
136 \\
137 \\
139 \\
139 \\
140 \\
141 \\
142 \\
143 \\
144 \\
145 \\
146 \\
147 \\
148 \\
149 \\
150 \\
151 \\
152 \\
1531 \\
1541 \\
1551\end{array}$} & \multirow[b]{2}{*}{ 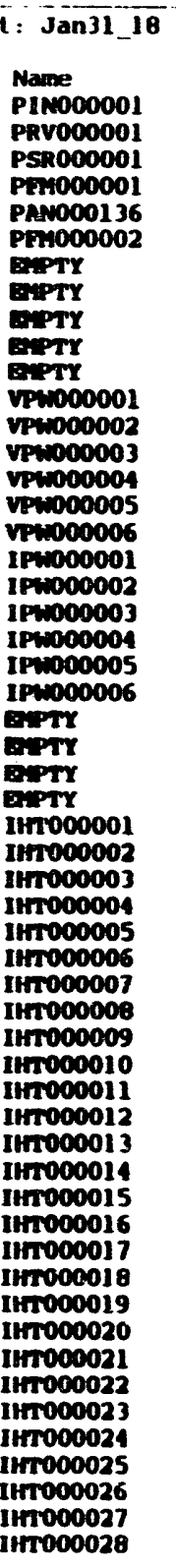 } & \multirow[b]{2}{*}{ 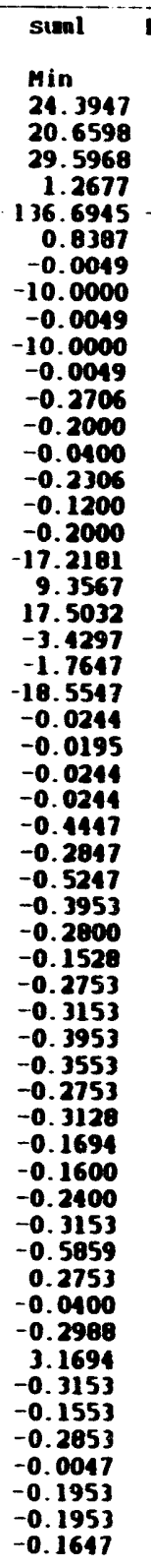 } & \multicolumn{3}{|c|}{ Date: Wed Jan 31 13:38:16 1990} & \multirow[b]{2}{*}{ 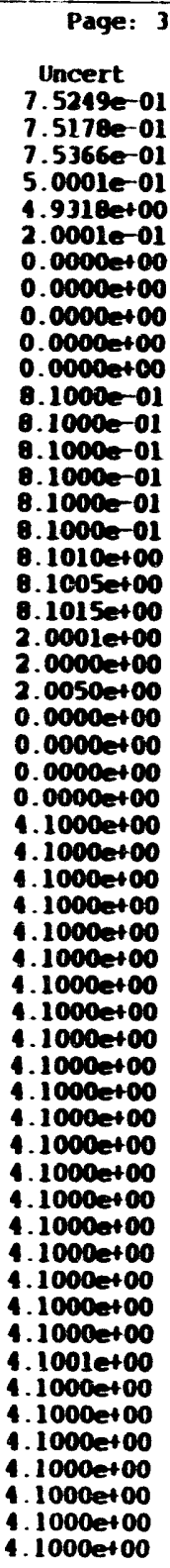 } \\
\hline & & & $\begin{array}{r}\text { Max } \\
24.5168 \\
20.7819 \\
29.7189 \\
1.1508 \\
-136.2368 \\
1.0904 \\
0.0049 \\
-9.1797 \\
0.0049 \\
-10.0000 \\
0.0000 \\
0.1200 \\
0.1906 \\
0.3506 \\
0.3553 \\
0.2706 \\
0.1906 \\
-13.3134 \\
13.2593 \\
21.4032 \\
-2.4531 \\
-0.7802 \\
-17.0098 \\
-0.0146 \\
-0.0098 \\
-0.0098 \\
-0.0098 \\
-0.0541 \\
0.1059 \\
-0.1341 \\
-0.0047 \\
0.1106 \\
0.2378 \\
0.1153 \\
0.0753 \\
-0.0047 \\
0.0353 \\
0.1153 \\
0.0778 \\
0.2212 \\
0.0353 \\
0.1506 \\
0.0753 \\
0.3906 \\
0.1706 \\
0.1553 \\
0.0919 \\
3.3647 \\
-0.1200 \\
0.0400 \\
-0.0900 \\
0.3859 \\
0.1953 \\
0.0000 \\
0.2259\end{array}$ & 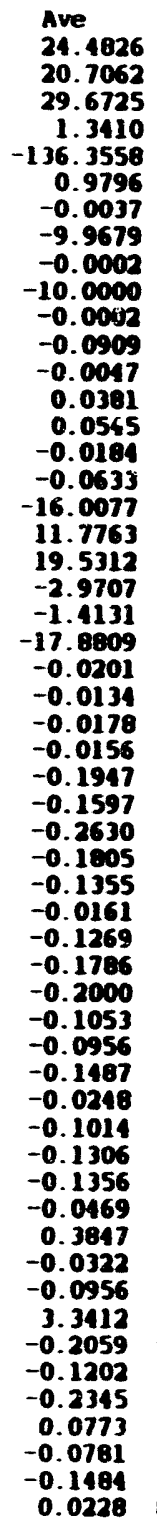 & 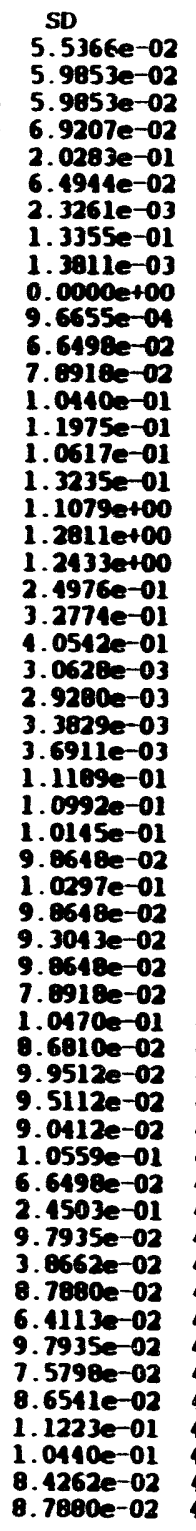 & \\
\hline
\end{tabular}

\begin{tabular}{|c|c|c|c|c|c|}
\hline Test: Jan31 18 & sumal & Date: Wed & an 31 13: & $38: 16 \quad 1990$ & Page: i \\
\hline 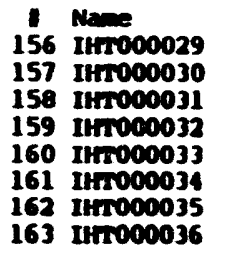 & $\begin{array}{l}\text { Min } \\
-0.0400 \\
-0.0800 \\
-0.0847 \\
0.0000 \\
37.3247 \\
-0.0600 \\
-0.1881 \\
-0.2353\end{array}$ & $\begin{array}{c}\operatorname{Max} \\
0.3506 \\
0.3106 \\
0.3059 \\
0.3906 \\
37.7153 \\
0.3106 \\
0.3978 \\
0.1553\end{array}$ & $\begin{array}{c}\text { Ave } \\
0.1202 \\
0.1192 \\
0.1145 \\
0.0586 \\
37.3911 \\
0.0567 \\
0.1048 \\
-0.0048\end{array}$ & $\begin{array}{l}\text { SD } \\
1.0206 e-01 \\
9.2456 e-02 \\
1.1497 e-01 \\
9.8648 e-02 \\
1.0145 e-01 \\
9.8548 e-02 \\
1.3235 e-01 \\
6.5454 e-02\end{array}$ & 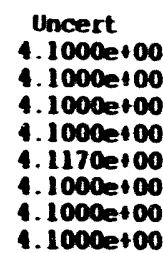 \\
\hline
\end{tabular}




\begin{tabular}{|c|c|c|c|c|c|c|}
\hline \\
\hline \multicolumn{7}{|c|}{ 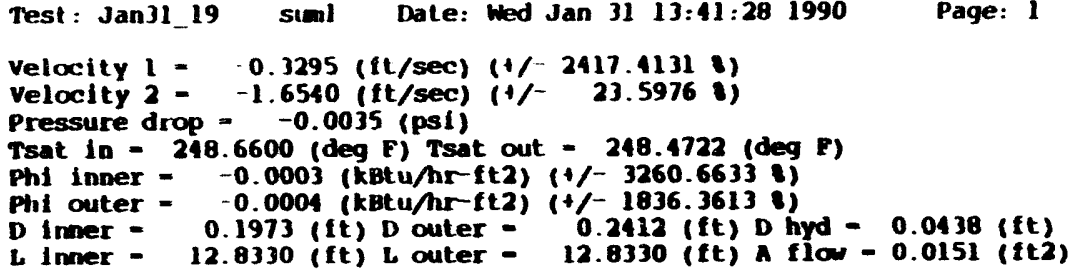 } \\
\hline $\begin{array}{l}1 \\
0 \\
1 \\
2 \\
3 \\
4 \\
5 \\
6 \\
7 \\
8 \\
9 \\
10 \\
11 \\
12 \\
13 \\
14 \\
15 \\
16 \\
17 \\
18 \\
19 \\
20 \\
21 \\
22 \\
23 \\
24 \\
25 \\
26 \\
27 \\
28 \\
29 \\
30 \\
31 \\
32 \\
33 \\
34 \\
35 \\
36 \\
37 \\
38 \\
39 \\
40 \\
11 \\
12 \\
43 \\
44 \\
45\end{array}$ & 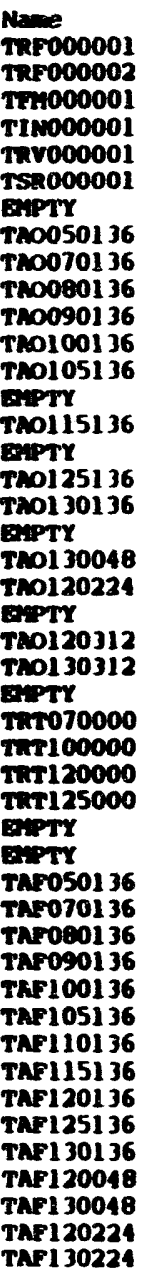 & $\begin{array}{r}\text { Min } \\
74.3963 \\
74.9393 \\
86.8711 \\
86.9411 \\
86.8387 \\
87.4519 \\
-601.0594 \\
86.5555 \\
87.8449 \\
87.4234 \\
87.2975 \\
87.5038 \\
87.7560 \\
40.9819 \\
86.7551 \\
37.5374 \\
87.4005 \\
87.1468 \\
41.2111 \\
87.0474 \\
86.9572 \\
38.6870 \\
87.2358 \\
86.7157 \\
39.1464 \\
87.2376 \\
87.6341 \\
87.5436 \\
87.5226 \\
39.3761 \\
39.3761 \\
87.4779 \\
87.9695 \\
87.3715 \\
87.5463 \\
87.2108 \\
87.3815 \\
87.2762 \\
87.5339 \\
87.5234 \\
87.6942 \\
86.9706 \\
87.7146 \\
87.2082 \\
87.4280 \\
86.1261\end{array}$ & $\begin{array}{r}\text { Max } \\
75.0338 \\
75.5736 \\
87.7088 \\
87.6763 \\
87.5715 \\
88.0001 \\
-601.0594 \\
87.2893 \\
88.7906 \\
88.2595 \\
88.0312 \\
88.2375 \\
88.4903 \\
11.2111 \\
87.8058 \\
37.7675 \\
87.9244 \\
87.7729 \\
11.6693 \\
87.8854 \\
87.7958 \\
39.1464 \\
87.8655 \\
87.3451 \\
39.6057 \\
87.8701 \\
88.5752 \\
88.1725 \\
88.1498 \\
39.8352 \\
10.0646 \\
88.1062 \\
88.9091 \\
87.9990 \\
88.5933 \\
87.8403 \\
88.3201 \\
87.7994 \\
88.4759 \\
88.0467 \\
88.2167 \\
87.6002 \\
88.6625 \\
87.6283 \\
88.2664 \\
87.0569\end{array}$ & $\begin{array}{r}\text { Ave } \\
74.6811 \\
75.2860 \\
87.3683 \\
87.3465 \\
87.2096 \\
87.6973 \\
-601.0591 \\
86.8972 \\
88.2711 \\
87.8438 \\
87.6392 \\
87.8458 \\
88.1109 \\
41.1011 \\
87.3542 \\
37.5651 \\
87.6711 \\
87.1414 \\
41.2645 \\
87.4267 \\
87.3867 \\
38.9076 \\
87.5779 \\
87.0241 \\
39.3853 \\
87.5397 \\
88.0880 \\
87.7724 \\
87.8590 \\
39.6057 \\
39.6929 \\
87.8111 \\
88.4724 \\
87.7744 \\
88.2101 \\
87.5903 \\
87.8222 \\
87.5504 \\
88.1221 \\
87.7437 \\
87.9925 \\
87.3419 \\
88.1302 \\
87.3867 \\
87.8746 \\
86.7982\end{array}$ & $\begin{array}{r}\text { sD } \\
1.5038 e-01 \\
1.3344 e-01 \\
1.8737 e-01 \\
1.6705 e-01 \\
1.7822 e-01 \\
1.5182 e-01 \\
3.6993 e-04 \\
1.9922 e-01 \\
1.9503 e-01 \\
1.6701 e-01 \\
1.7548 e-01 \\
1.5959 e-01 \\
1.9294 e-01 \\
1.1567 e-01 \\
2.3109 e-01 \\
7.5509 e-02 \\
1.5565 e-01 \\
1.5706 e-01 \\
1.1747 e-01 \\
1.6510 e-01 \\
1.6714 e-01 \\
1.2244 e-01 \\
1.6793 e-01 \\
1.5899 e-01 \\
1.7332 e-01 \\
1.6475 e-01 \\
2.0413 e-01 \\
1.5516 e-01 \\
1.4194 e-01 \\
9.2753 e-02 \\
1.9058 e-01 \\
1.3323 e-01 \\
2.1257 e-01 \\
1.3698 e-01 \\
2.5351 e-01 \\
1.4821 e-01 \\
2.3567 e-01 \\
1.4164 e-01 \\
2.1245 e-01 \\
1.2390 e-01 \\
1.1585 e-01 \\
1.4556 e-01 \\
1.9313 e-01 \\
1.2407 e-01 \\
2.0175 e-01 \\
1.4290 e-01\end{array}$ & 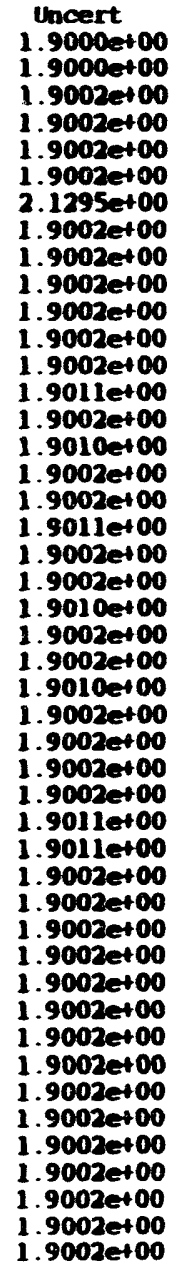 \\
\hline
\end{tabular}

\begin{tabular}{|c|c|c|c|c|c|}
\hline st & & & & & p: \\
\hline 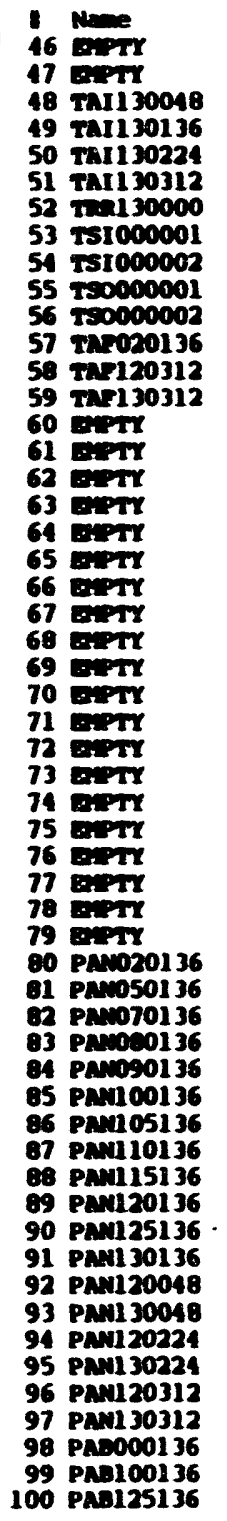 & 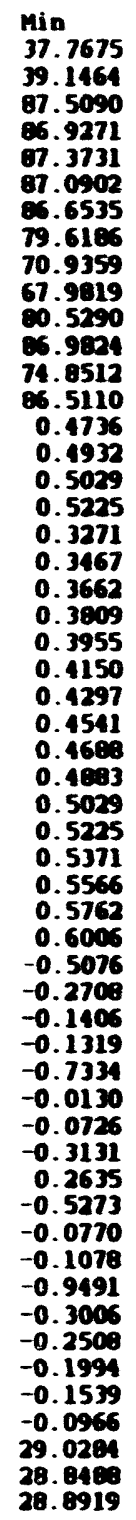 & 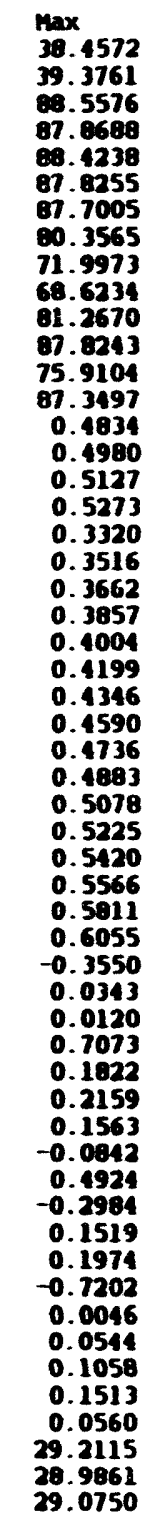 & 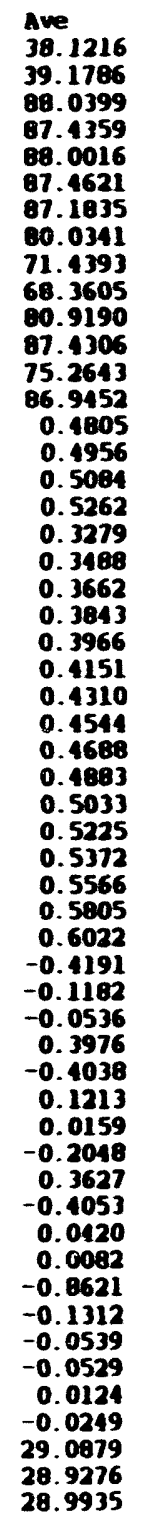 & $\begin{array}{r}50 \\
1.9 \\
0.0 \\
2.6 \\
1.9 \\
2.7 \\
1.8 \\
1.3 \\
1.9 \\
1.5 \\
1.9 \\
1.9 \\
2.0 \\
1.8 \\
2.6 \\
2.1 \\
2.7 \\
1.8 \\
2.4 \\
0.0 \\
2.2 .0 \\
2.0\end{array}$ & 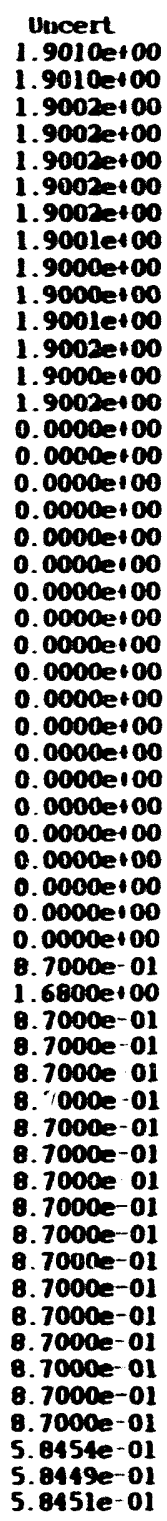 \\
\hline
\end{tabular}



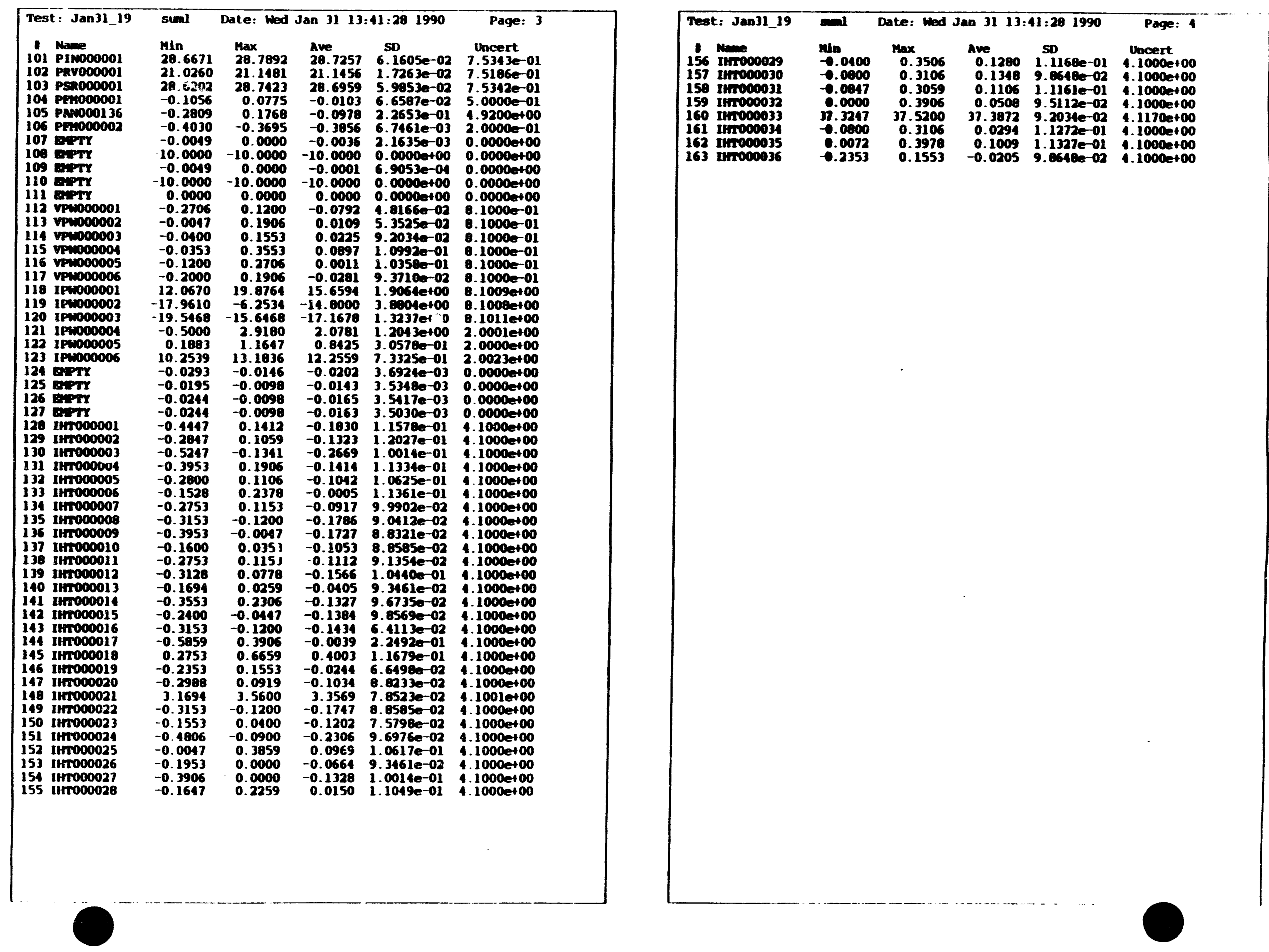


\begin{tabular}{|c|c|c|c|c|c|c|}
\hline \multirow{2}{*}{\multicolumn{2}{|c|}{$\begin{array}{l}\text { Test : May01 01 } \\
\text { Veloxity } 1= \\
\text { Velocity } 2= \\
\text { Pressure drop } \\
\text { Tsat in = 285 } \\
\text { Phi inner = } \\
\text { Phi outer = } \\
\text { D Inner = } \\
\text { C inner = } 12\end{array}$}} & & ate: Tue & \multicolumn{2}{|c|}{$\begin{array}{l}\operatorname{May} 111: 28: 531990 \\
(1.6864 \text { () }\end{array}$} & Page: 1 \\
\hline & & $\begin{array}{l}.0003 \text { (1t) } \\
.6779 \text { (ft } \\
21 \text {.4745 } \\
12 \text { (deg F } \\
0000 \text { (kBt } \\
0000 \text { (kBt } \\
62 \text { (ft) D } \\
50 \text { (ft) l. }\end{array}$ & $\begin{array}{l}\text { sec) }(1 /- \\
\text { sec) }(1 /- \\
\text { psi) } \\
\text { Tsat out } \\
\text { Mr-ft2) } \\
\text { Mrtt } \\
\text { outer - } \\
\text { outer - }\end{array}$ & $\begin{array}{r}1.6864 \\
1.7009 \\
=255.84 \\
1+1-4047 . \\
1+1-14868 \\
0.2413 \\
12.8070 !\end{array}$ & $\begin{array}{l}\text { 8) } \\
\text { 8) } \\
95 \text { (deg F) } \\
6887 \text { ! ! } \\
.3096 \text { is } \\
\text { ft) D hyd - } \\
\text { f() A tiou - }\end{array}$ & $\begin{array}{l}0.0389 \text { (ft) } \\
0.0150 \text { (ft2) }\end{array}$ \\
\hline $\begin{array}{l}1 \\
1 \\
1 \\
2 \\
3 \\
4 \\
5 \\
6 \\
7 \\
8 \\
9 \\
10 \\
11 \\
12 \\
13 \\
14 \\
15 \\
16 \\
17 \\
18 \\
19 \\
20 \\
21 \\
22 \\
23 \\
24 \\
25 \\
26 \\
27 \\
28 \\
29 \\
30 \\
31 \\
32 \\
33 \\
34 \\
35 \\
36 \\
37 \\
38 \\
39 \\
10 \\
11 \\
12 \\
13 \\
14 \\
15\end{array}$ & 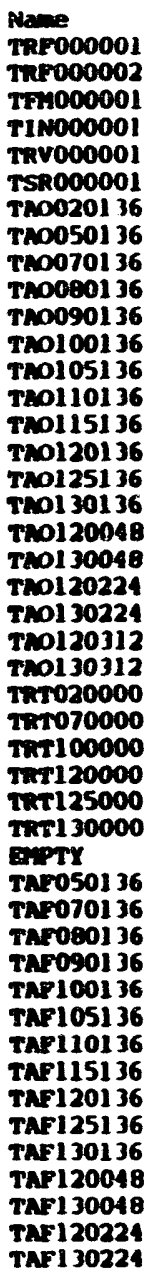 & $\begin{array}{l}\text { Min } \\
74.2113 \\
74.4388 \\
86.1010 \\
85.7259 \\
86.0357 \\
85.7521 \\
86.2113 \\
86.2866 \\
84.1966 \\
86.0737 \\
86.2539 \\
86.2228 \\
86.7143 \\
84.1291 \\
86.2733 \\
86.0060 \\
86.2956 \\
86.0333 \\
86.0341 \\
85.9675 \\
86.0640 \\
86.0722 \\
85.9898 \\
85.9244 \\
86.2892 \\
86.2552 \\
86.3042 \\
86.2149 \\
86.2876 \\
86.1947 \\
39.3761 \\
86.3923 \\
86.7069 \\
86.2292 \\
83.9447 \\
86.1924 \\
85.9068 \\
85.9561 \\
86.7000 \\
86.2074 \\
86.2882 \\
86.1880 \\
86.5527 \\
86.1171 \\
86.2943 \\
85.6667\end{array}$ & $\begin{array}{l}\text { Max } \\
74.8998 \\
74.8621 \\
86.8358 \\
86.3575 \\
86.7694 \\
86.2767 \\
87.0529 \\
86.9162 \\
85.0404 \\
86.7034 \\
87.1980 \\
86.8524 \\
87.4502 \\
84.7574 \\
87.3261 \\
86.7373 \\
86.9262 \\
86.6611 \\
86.7651 \\
86.1928 \\
86.5896 \\
86.7014 \\
86.7256 \\
86.4501 \\
87.2365 \\
86.8692 \\
87.2478 \\
87.0552 \\
87.0207 \\
86.7202 \\
40.0646 \\
87.0225 \\
87.5434 \\
86.9614 \\
84.8914 \\
86.7185 \\
66.9519 \\
66.5850 \\
87.2250 \\
86.7322 \\
67.3320 \\
67.0280 \\
87.3976 \\
86.9565 \\
87.0299 \\
86.5105\end{array}$ & $\begin{array}{l}\text { Ave } \\
74.4327 \\
74.5827 \\
86.4161 \\
86.0879 \\
86.1299 \\
86.0124 \\
66.6824 \\
86.6052 \\
84.6902 \\
86.4215 \\
86.7030 \\
86.4708 \\
87.0760 \\
84.1640 \\
86.6864 \\
86.3146 \\
86.6065 \\
86.3135 \\
86.4331 \\
86.2066 \\
86.3247 \\
86.3572 \\
86.2755 \\
86.2016 \\
86.7107 \\
86.5633 \\
66.8117 \\
86.5803 \\
86.6556 \\
86.1215 \\
39.6699 \\
86.7196 \\
87.1415 \\
86.5934 \\
84.3696 \\
86.4660 \\
86.5958 \\
86.3079 \\
86.9689 \\
86.4962 \\
86.7899 \\
86.6246 \\
86.9543 \\
86.5330 \\
86.6054 \\
86.0928\end{array}$ & $\begin{array}{r}\text { sD } \\
1.5481 e-01 \\
1.2425 e-01 \\
1.7725 e-01 \\
1.3635 e-01 \\
1.5768 e-01 \\
1.2573 e-01 \\
2.2205 e-01 \\
1.5833 e-01 \\
1.8503 e-01 \\
1.5751 e-01 \\
1.7981 e-01 \\
1.4503 e-01 \\
1.7407 e-01 \\
1.4042 e-01 \\
2.4141 e-01 \\
1.4496 e-01 \\
1.6146 e-01 \\
1.4510 e-01 \\
1.6702 e-01 \\
1.3742 e-01 \\
1.4273 e-01 \\
1.4339 e-01 \\
1.6143 e-01 \\
1.2335 e-01 \\
1.8785 e-01 \\
1.3607 e-01 \\
2.1115 e-01 \\
1.7780 e-01 \\
1.7956 e-01 \\
1.4692 e-01 \\
2.2259 e-01 \\
1.6909 e-01 \\
1.7078 e-01 \\
1.4976 e-01 \\
2.4566 e-01 \\
1.5609 e-01 \\
2.5459 e-01 \\
1.4944 e-01 \\
1.6845 e-01 \\
1.1149 e-01 \\
2.9064 e-01 \\
1.4748 e-01 \\
2.1208 e-01 \\
1.8723 e-01 \\
2.0579 e-01 \\
1.9644 e-01\end{array}$ & $\begin{array}{l}\text { Uncert } \\
1.9000 e+00 \\
1.9000 e+00 \\
1.9002 e+00 \\
1.9002 e+00 \\
1.9002 e+00 \\
1.9002 \text { et00 } \\
1.9002 \text { et00 } \\
1.9002 e+00 \\
1.9001 e+00 \\
1.9002 e+00 \\
1.9002 e+00 \\
1.9002 e+00 \\
1.9002 e+00 \\
1.9001 e+00 \\
1.9002 e+00 \\
1.9002 e+00 \\
1.9002 e+00 \\
1.9002 e+00 \\
1.9002 e+00 \\
1.9002 e+00 \\
1.9002 e+00 \\
1.9002 e+00 \\
1.9002 e+00 \\
1.9002 e+00 \\
1.9002 e+00 \\
1.9002 e+00 \\
1.9002 e+00 \\
1.9002 e+00 \\
1.9002 e+00 \\
1.9002 e+00 \\
1.9011 e+00 \\
1.9002 e+00 \\
1.9002 e+00 \\
1.9002 e+00 \\
1.9001 e+00 \\
1.9002 e+00 \\
1.9002 e+00 \\
1.9002 e+00 \\
1.9002 e+00 \\
1.9002 e+00 \\
1.9002 e+00 \\
1.9002 e+00 \\
1.9002 e+00 \\
1.9002 e+00 \\
1.9002 e+00 \\
1.9002 e+00\end{array}$ \\
\hline
\end{tabular}

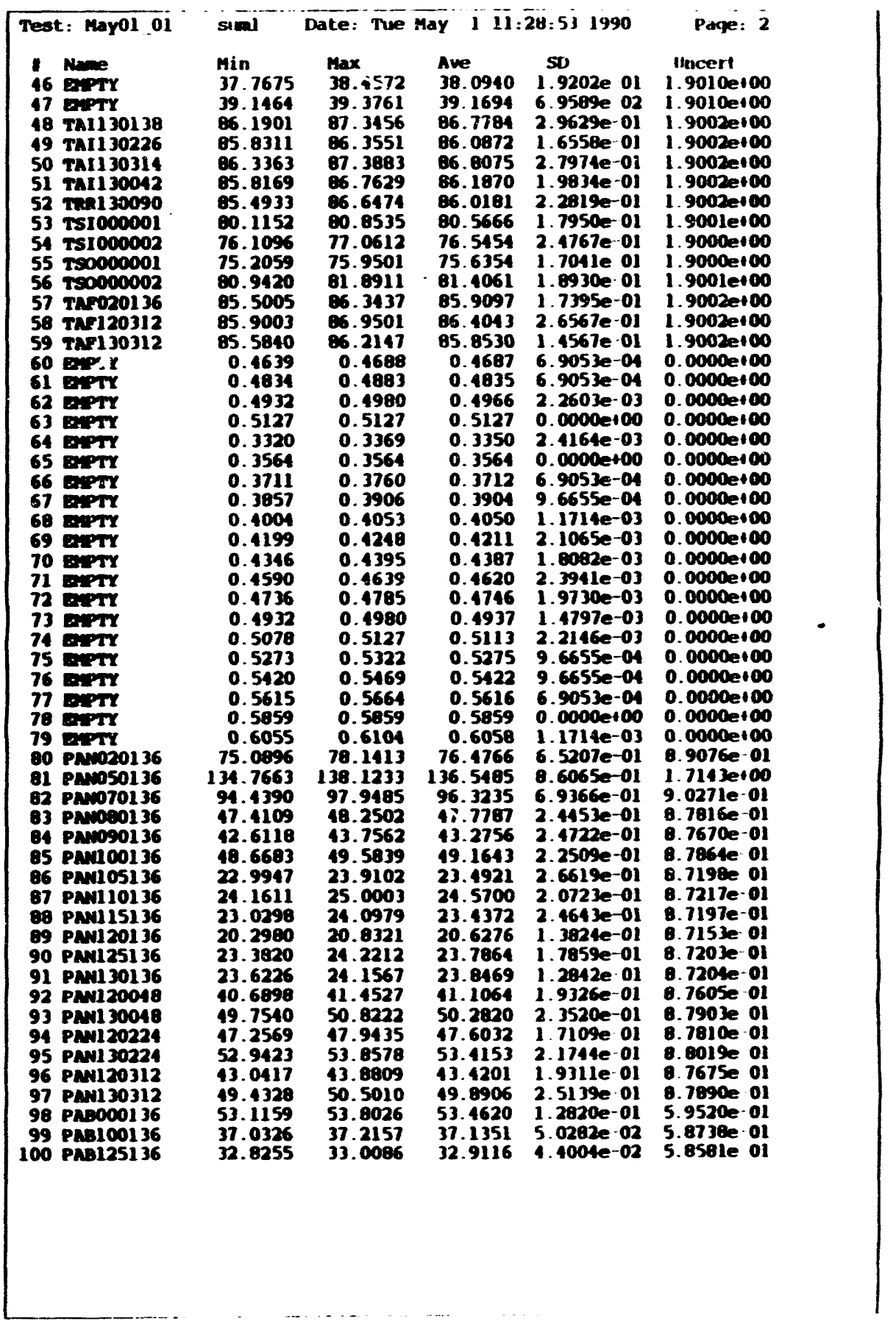




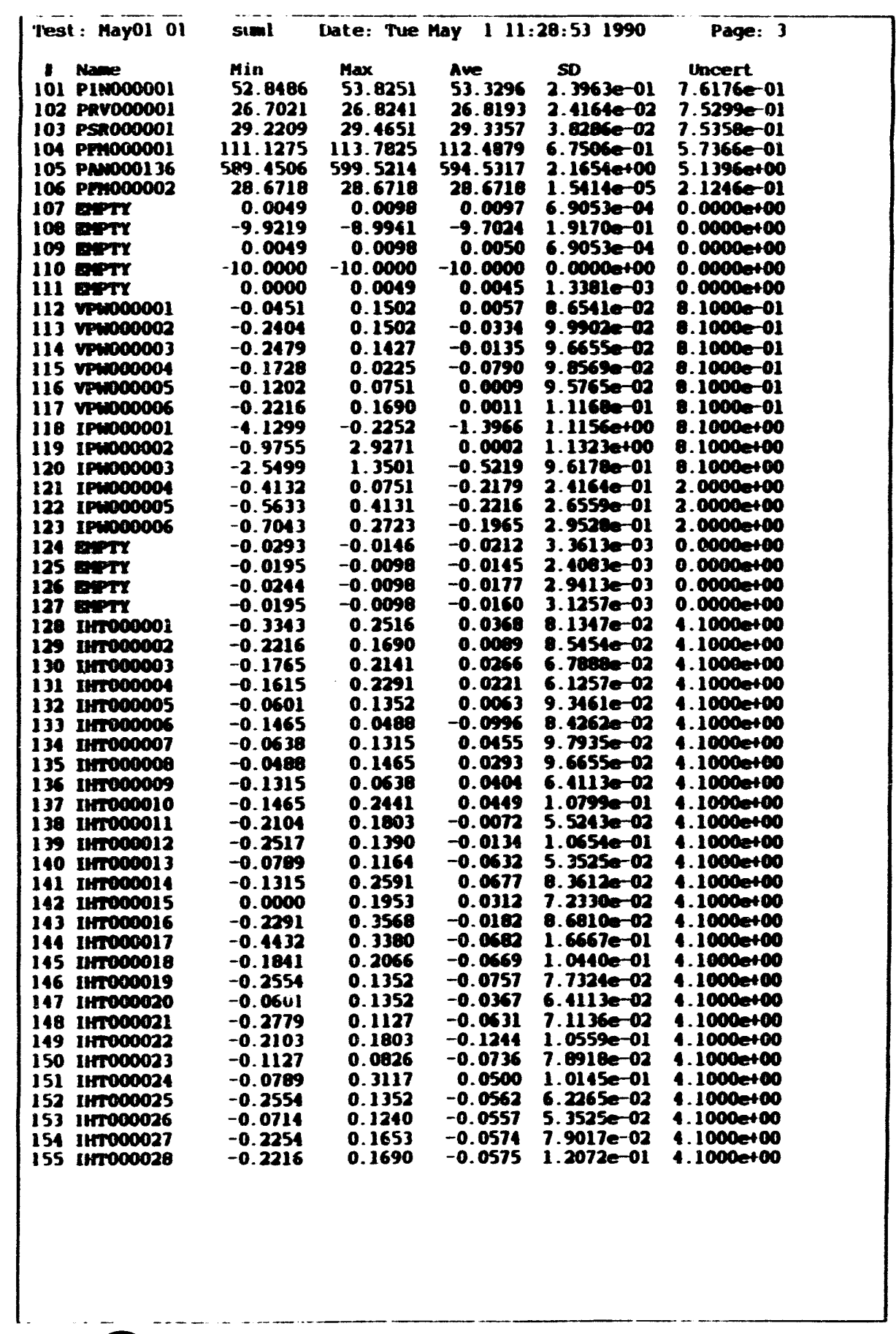

\begin{tabular}{|c|c|c|c|c|c|}
\hline t: & & Date: & ay & & age: \\
\hline 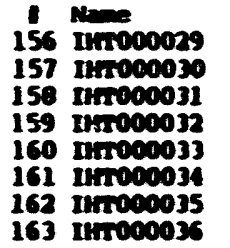 & $\begin{array}{l}\text { Mun } \\
-0.2141 \\
-0.1653 \\
-0.1352 \\
-0.2065 \\
-0.1953 \\
-0.0263 \\
-0.0375 \\
-0.1953\end{array}$ & $\begin{array}{l}\max \\
0.1765 \\
0.0300 \\
0.0501 \\
0.1840 \\
0.1953 \\
0.1690 \\
0.1578 \\
0.1953\end{array}$ & $\begin{array}{c}\text { Ave } \\
0.0007 \\
-0.0911 \\
-0.0610 \\
-0.0191 \\
0.0039 \\
0.0479 \\
0.0210 \\
0.0117\end{array}$ & $\begin{array}{l}\text { SD } \\
7.1136 e-02 \\
9.5765 e-02 \\
9.5765 e-02 \\
7.8523 e-02 \\
8.3512 e-02 \\
9.5765 e-02 \\
9.0412 e-02 \\
6.1257 e-02\end{array}$ & $\begin{array}{l}4 \\
4 \\
4 \\
4 \\
4 \\
4 \\
4\end{array}$ \\
\hline
\end{tabular}




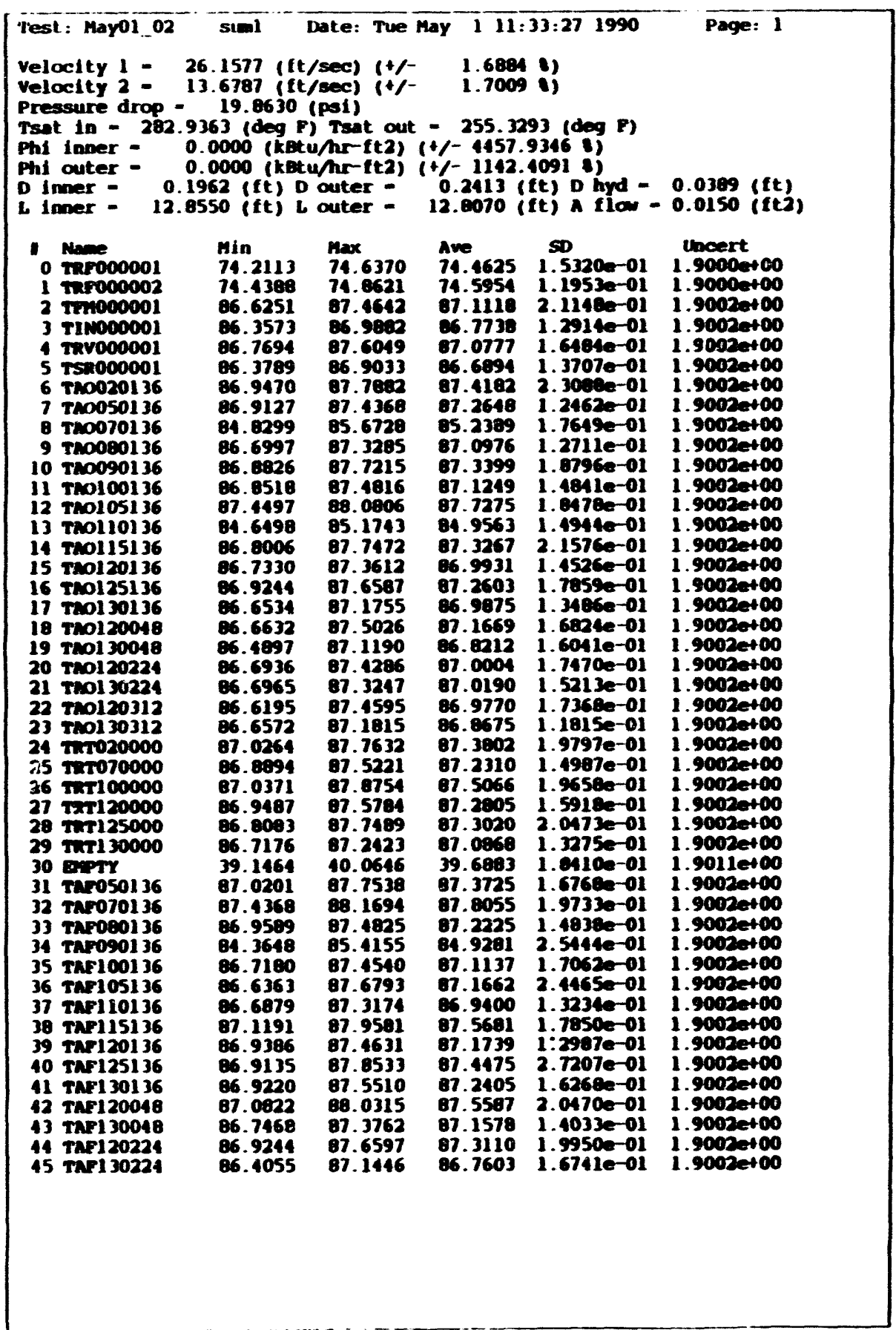

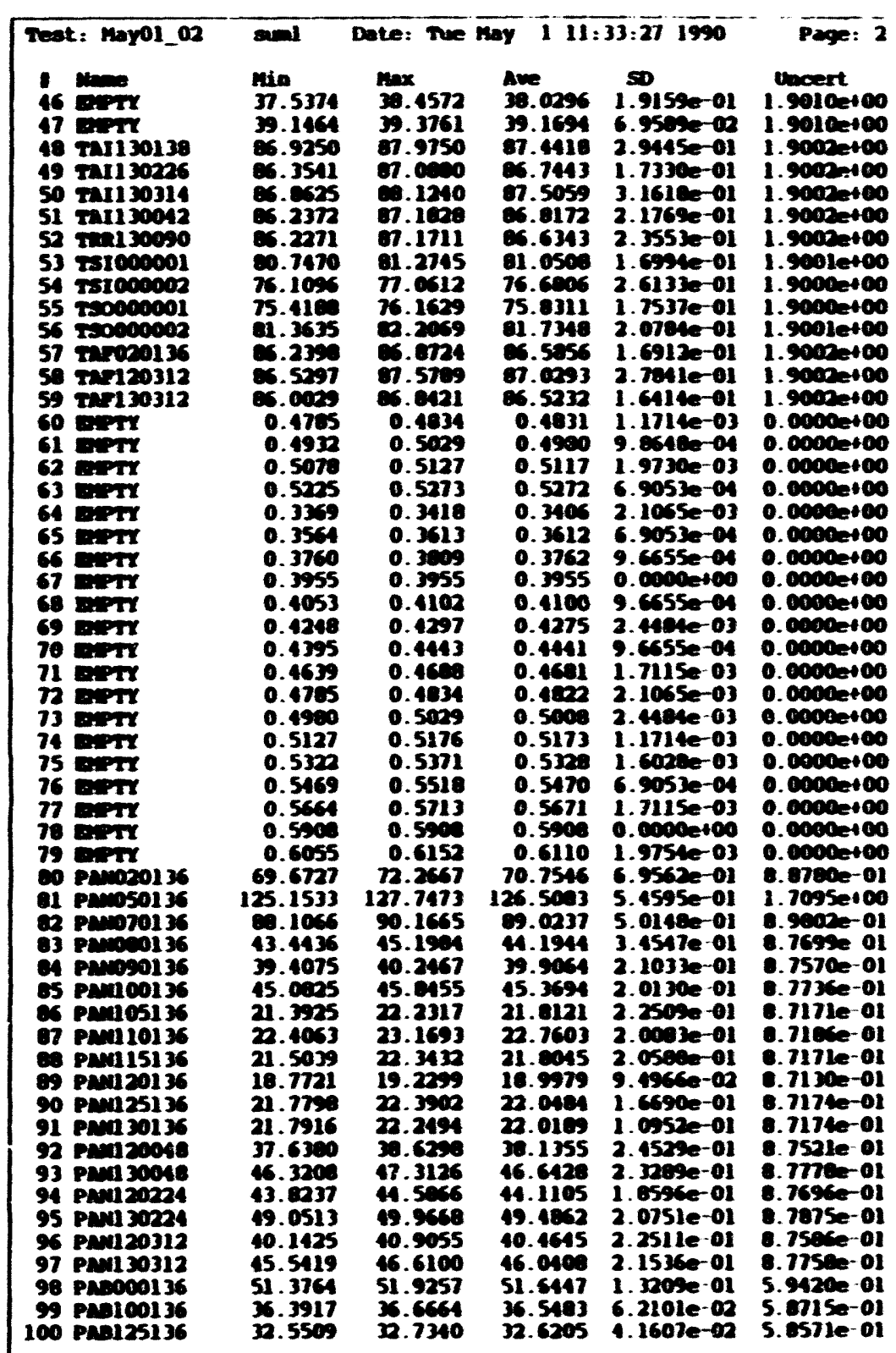




\begin{tabular}{|c|c|c|c|c|c|c|}
\hline $\begin{array}{l}1 e s t \\
1 \\
101 \\
102 \\
103 \\
104 \\
105 \\
106 \\
107 \\
100 \\
109 \\
110 \\
111 \\
112 \\
113 \\
114 \\
115 \\
116 \\
117 \\
118 \\
119 \\
120 \\
121 \\
122 \\
123 \\
124 \\
125 \\
126 \\
127 \\
128 \\
129 \\
130 \\
131 \\
132 \\
133 \\
134 \\
135 \\
136 \\
137 \\
130 \\
139 \\
140 \\
141 \\
142 \\
143 \\
144 \\
145 \\
146 \\
147 \\
140 \\
149 \\
150 \\
151 \\
152 \\
153 \\
154 \\
155\end{array}$ & 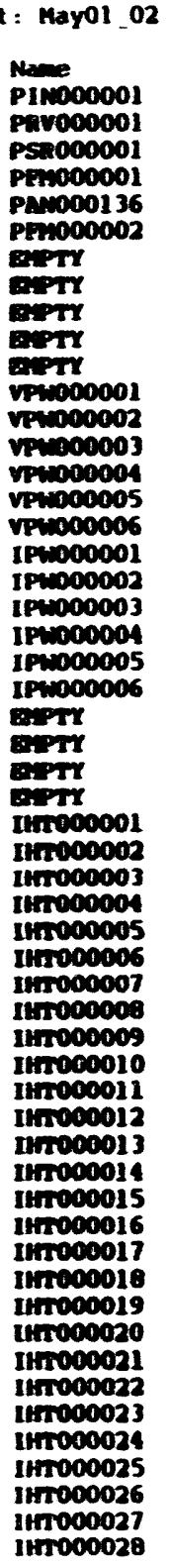 & $\begin{array}{l}\text { seal } \\
\text { Min } \\
51.0175 \\
26.0241 \\
29.2209 \\
104.6272 \\
545.9630 \\
28.6718 \\
0.0049 \\
10.0000 \\
0.0049 \\
-10.0000 \\
0.0049 \\
-0.0451 \\
-0.0451 \\
-0.0526 \\
-0.3681 \\
-0.1202 \\
-0.2216 \\
-2.1775 \\
-2.9267 \\
-2.5499 \\
0.4132 \\
-0.5633 \\
-0.7043 \\
-0.0244 \\
-0.0195 \\
-0.0244 \\
-0.0244 \\
-0.1390 \\
-0.2216 \\
-0.1765 \\
-0.1615 \\
-0.0601 \\
-0.3418 \\
-0.0638 \\
-0.0480 \\
-0.1315 \\
-0.1465 \\
-0.2104 \\
-0.0563 \\
-0.2742 \\
-0.1315 \\
-0.1953 \\
-0.2291 \\
-0.4432 \\
-0.1811 \\
-0.2554 \\
-0.2554 \\
-0.0626 \\
-0.2103 \\
-0.1127 \\
-0.0789 \\
-0.2554 \\
-0.2667 \\
-0.2254 \\
-0.2216\end{array}$ & 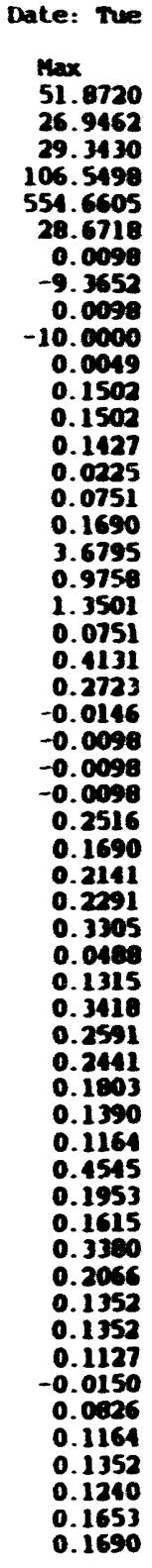 & $\begin{aligned} \text { May } 111: \\
\text { Ave } \\
51.1179 \\
26.8266 \\
29.2624 \\
105.5409 \\
549.9181 \\
28.6718 \\
0.0097 \\
-9.7842 \\
0.0050 \\
-10.0000 \\
0.0049 \\
0.0016 \\
-0.0334 \\
-0.0214 \\
-0.1064 \\
0.0007 \\
-0.0028 \\
-0.1595 \\
-0.1601 \\
-1.0679 \\
-0.1691 \\
0.0323 \\
-0.1965 \\
-0.0213 \\
-0.0146 \\
-0.0169 \\
-0.0163 \\
0.0524 \\
0.0245 \\
0.0227 \\
0.0455 \\
0.0024 \\
-0.1074 \\
0.0336 \\
0.0332 \\
0.0326 \\
0.0566 \\
-0.0033 \\
-0.0095 \\
-0.0711 \\
0.0716 \\
0.0312 \\
-0.0299 \\
-0.0917 \\
-0.0551 \\
-0.0974 \\
0.0024 \\
-0.0514 \\
-0.0931 \\
-0.0305 \\
0.0266 \\
-0.0562 \\
-0.0557 \\
-0.0457 \\
-0.0536\end{aligned}$ & 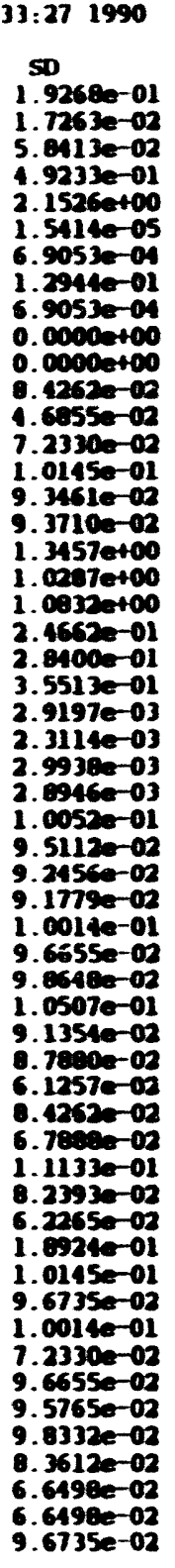 & 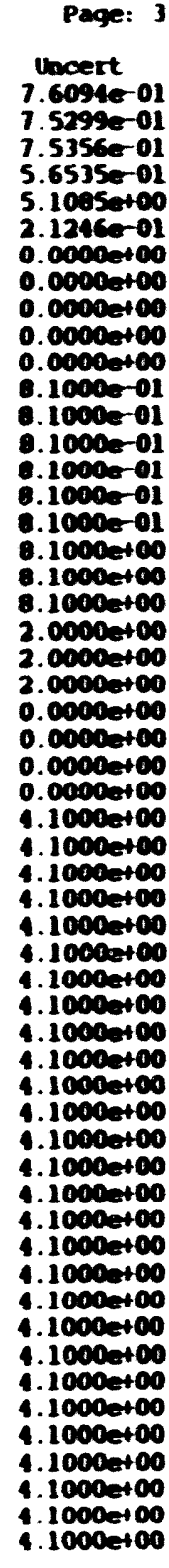 \\
\hline
\end{tabular}

\begin{tabular}{|c|c|c|c|c|c|}
\hline at: 1 & $=1$ & Date: The & ay 111: & $33: 271990$ & Page: \\
\hline 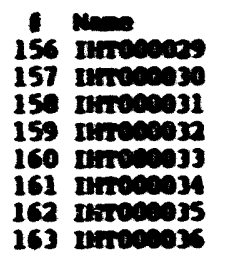 & $\begin{array}{l}\text { tin } \\
-0.2141 \\
-0.3605 \\
-0.3305 \\
-0.2065 \\
-0.1953 \\
-0.0253 \\
-0.0375 \\
-0.1953\end{array}$ & $\begin{array}{l}\max \\
0.1765 \\
0.2253 \\
0.0501 \\
0.1840 \\
0.1953 \\
0.1690 \\
0.1570 \\
0.1953\end{array}$ & $\begin{array}{l}\text { Ave } \\
-0.0012 \\
-0.0715 \\
-0.0512 \\
-0.0152 \\
0.0070 \\
0.0440 \\
0.0945 \\
0.0117\end{array}$ & $\begin{array}{l}s 0 \\
\text { 8.coloe- } \\
1.1995=-01 \\
1.0507 e-01 \\
1.00520-01 \\
9.6332=-02 \\
9.4702=02 \\
9.7377=02 \\
7.2066=-02\end{array}$ & 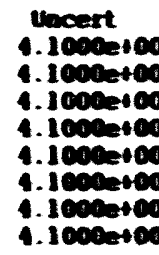 \\
\hline
\end{tabular}




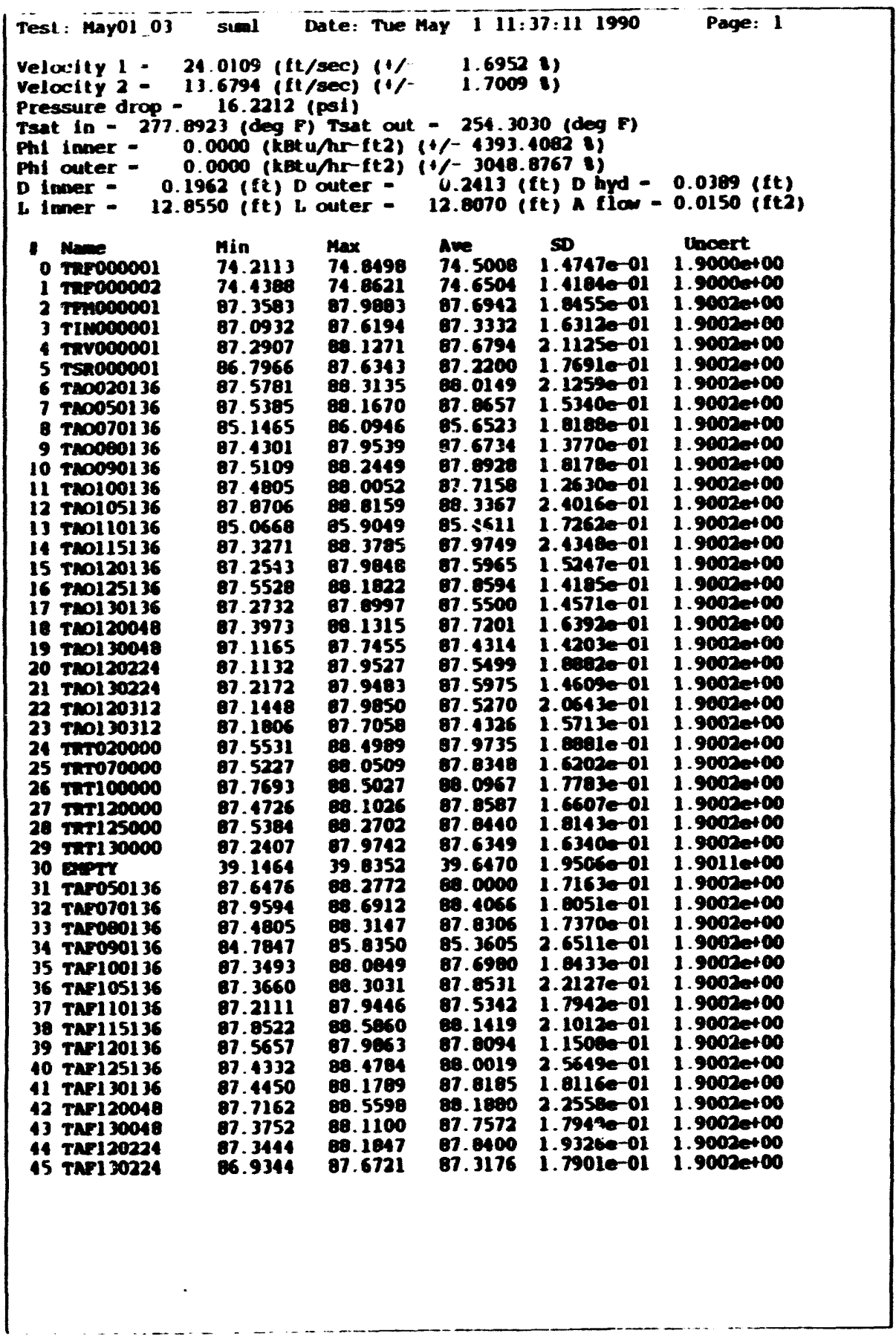

\begin{tabular}{|c|c|c|c|c|c|}
\hline 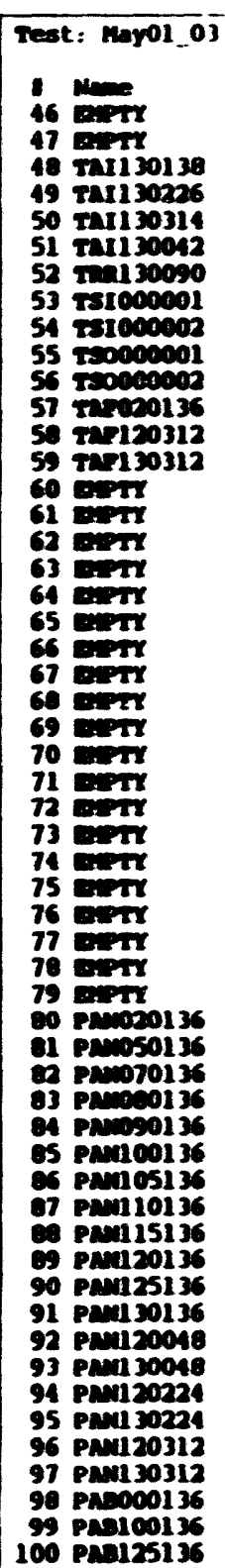 & 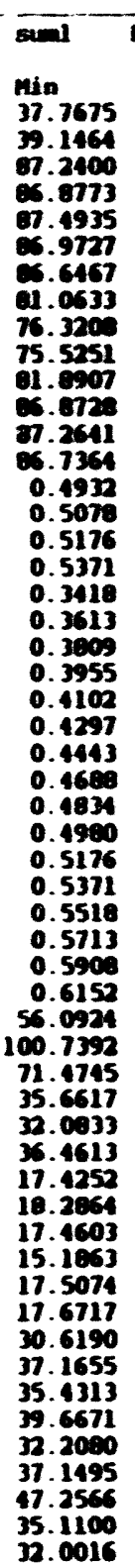 & 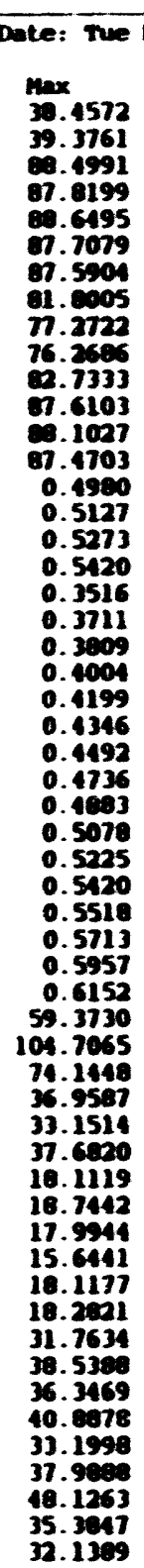 & 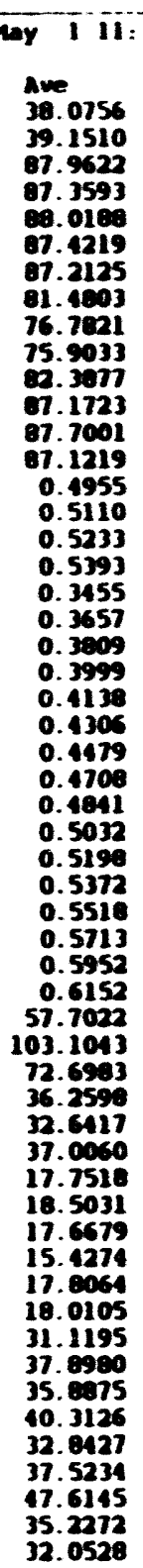 & 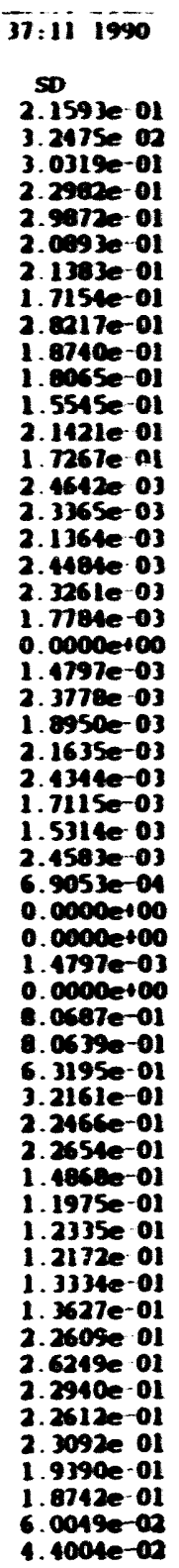 & 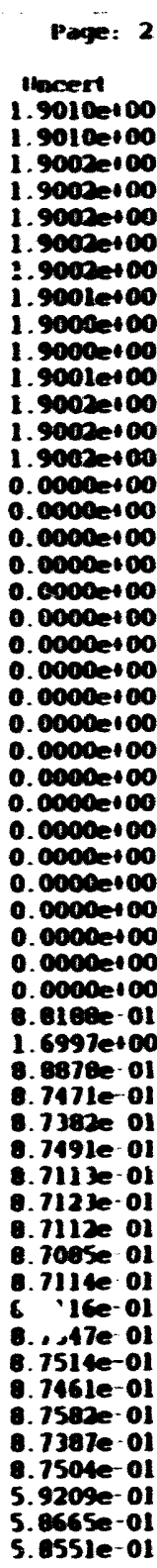 \\
\hline
\end{tabular}




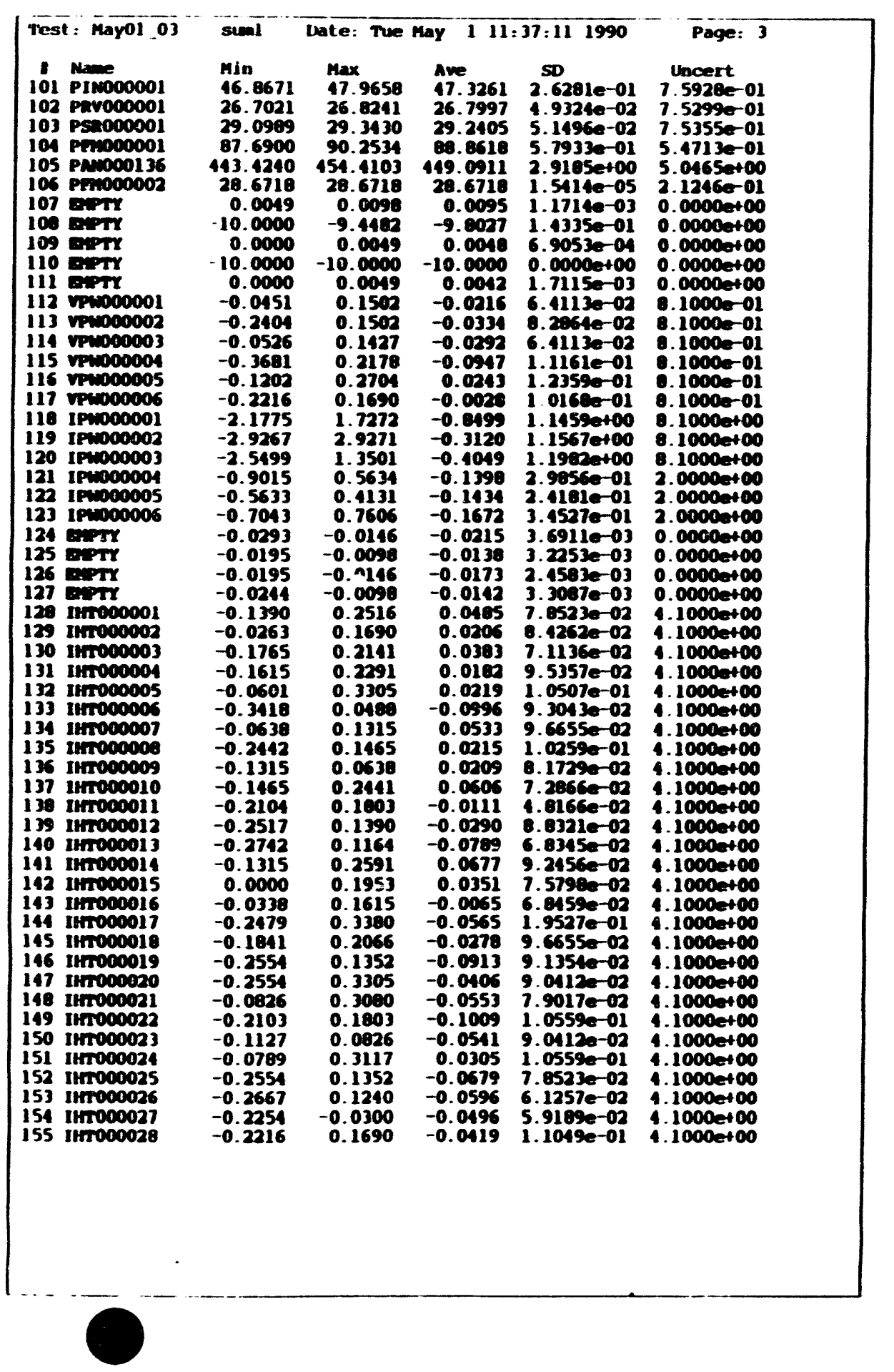

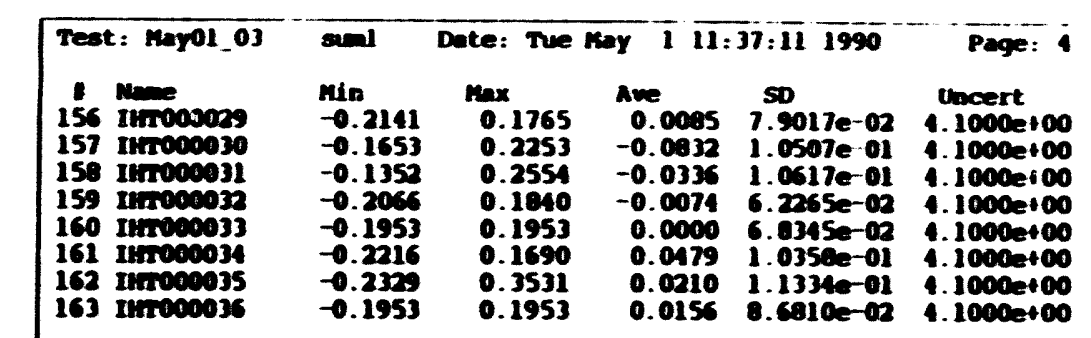




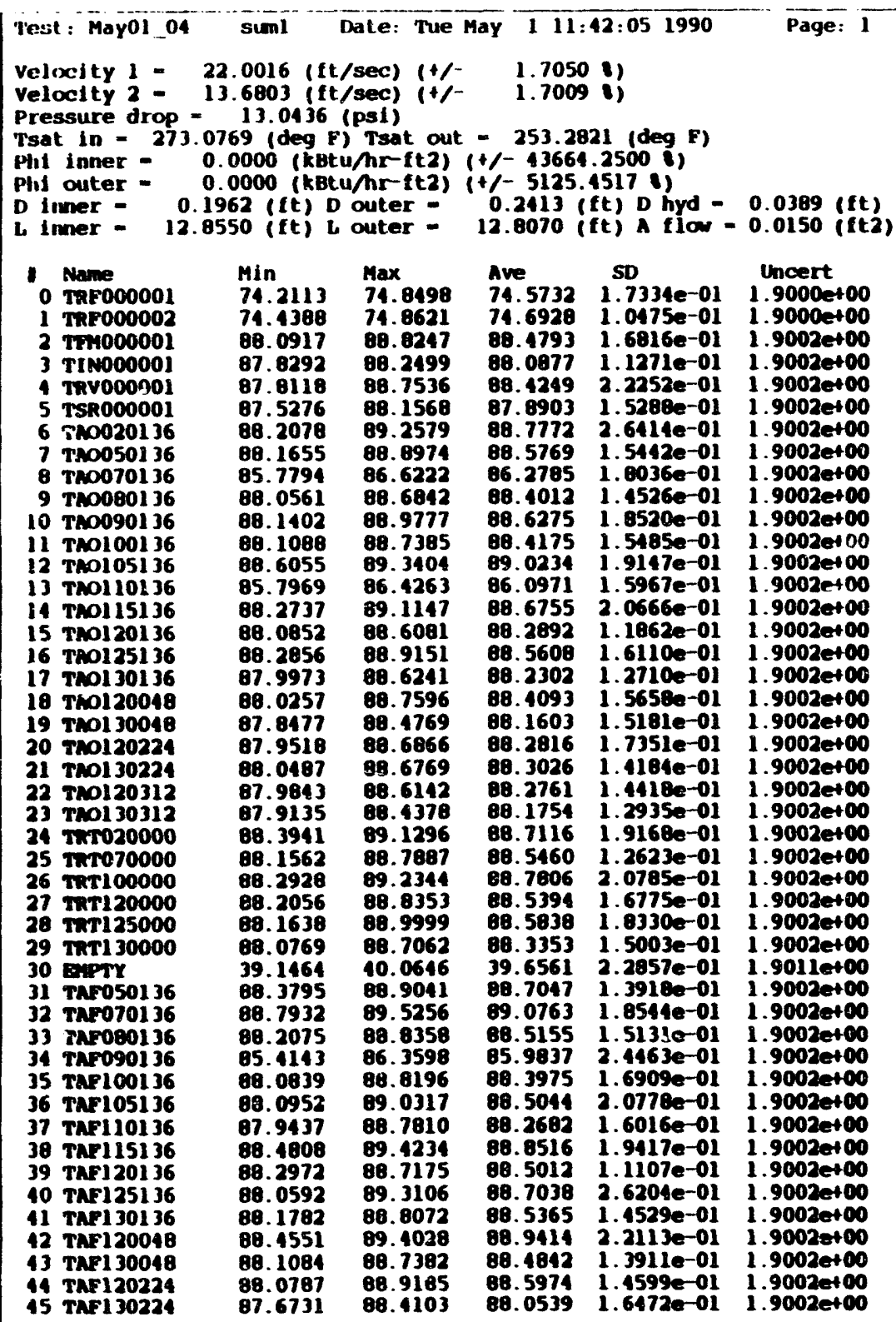

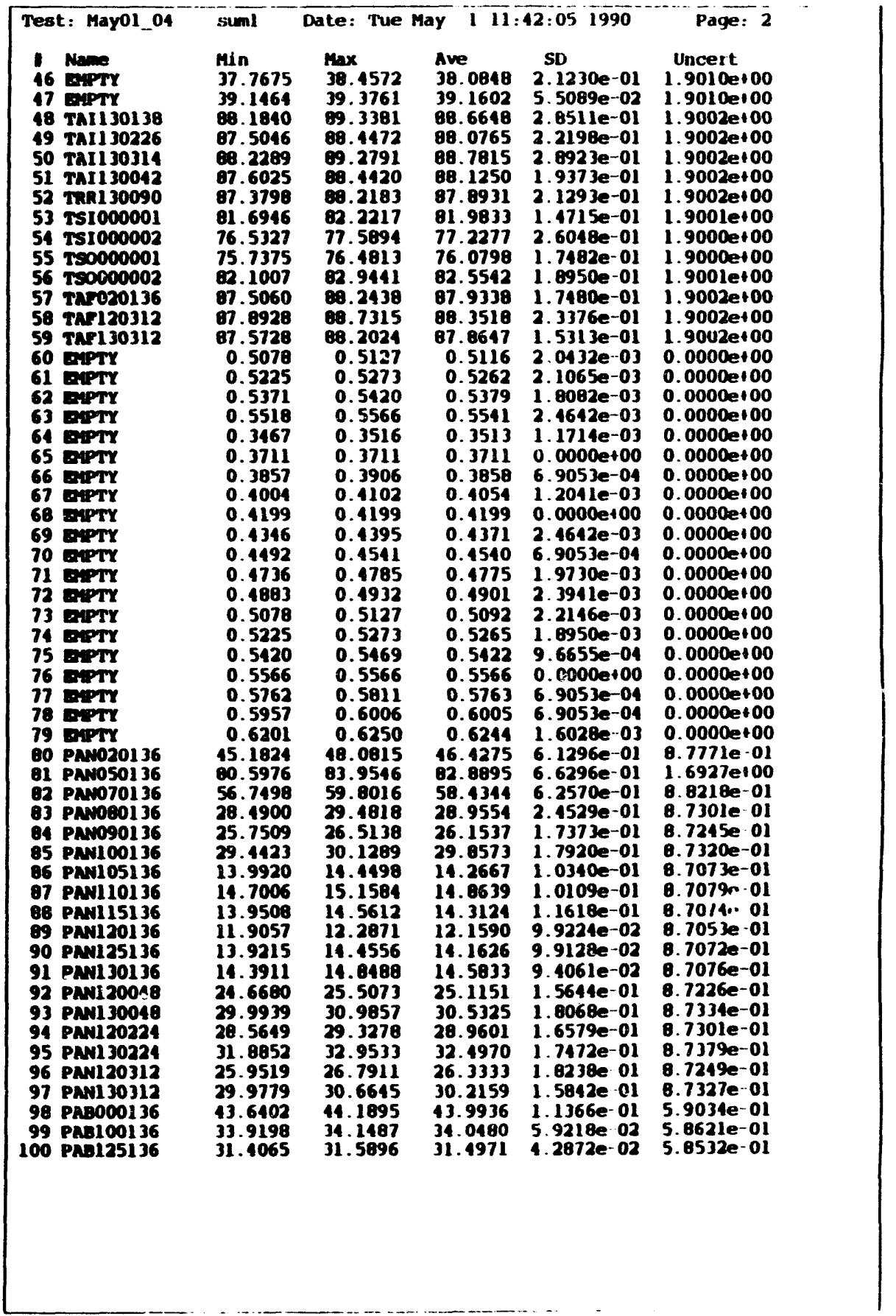




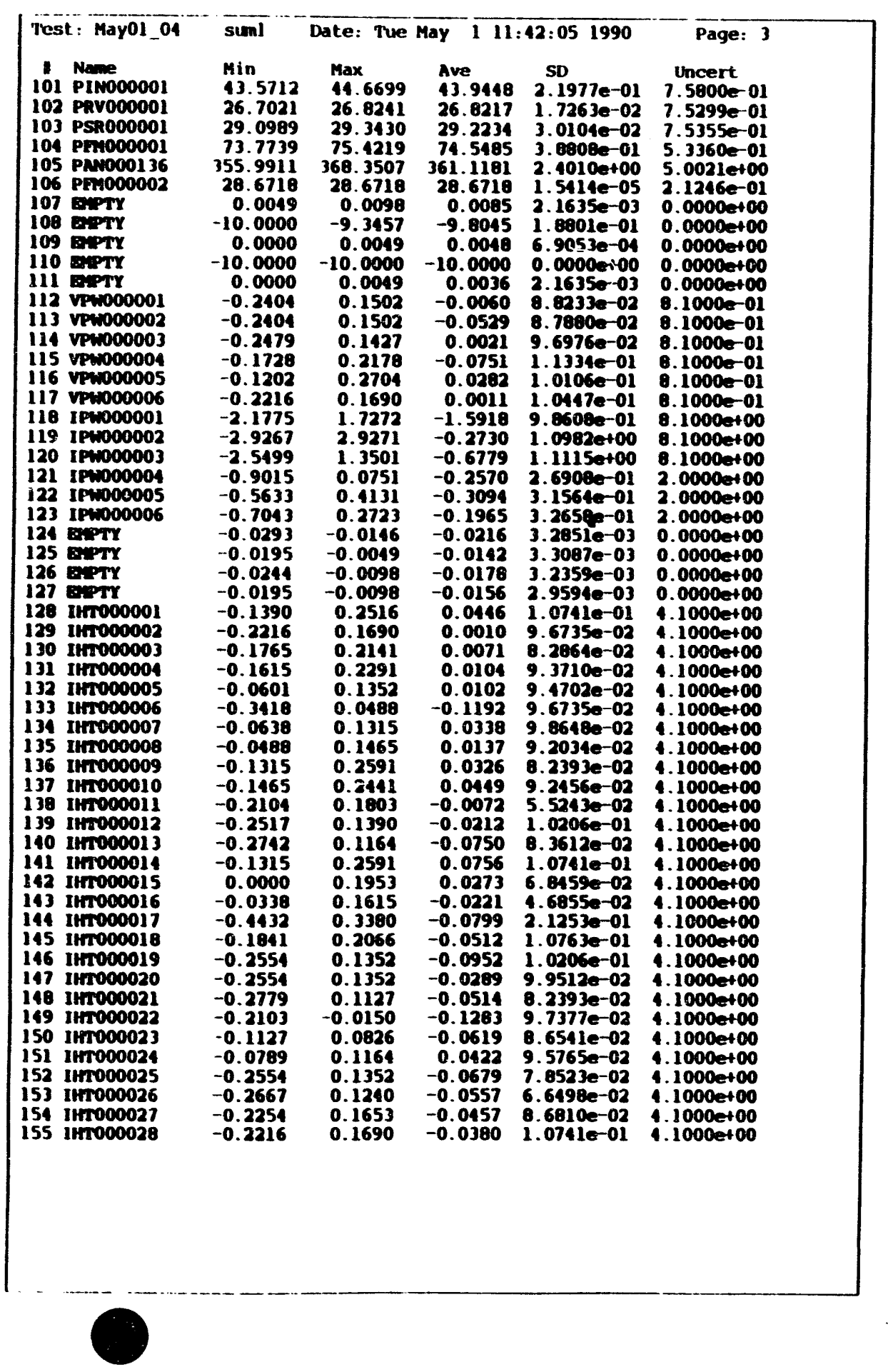

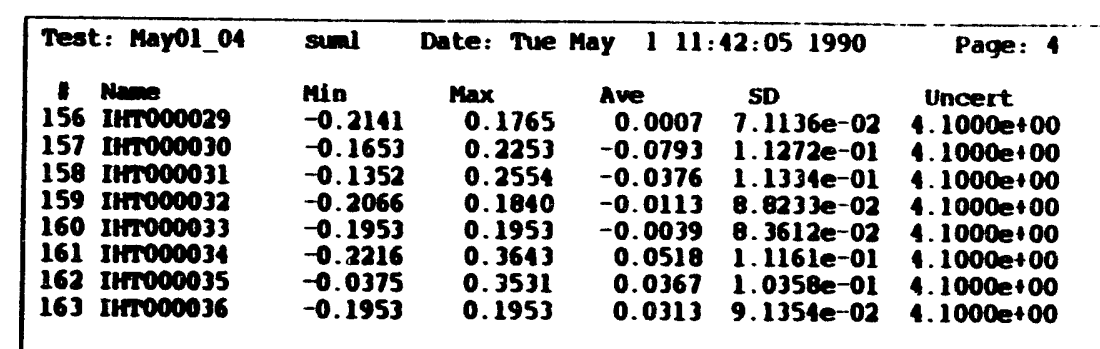




\begin{tabular}{|c|c|c|c|c|c|c|}
\hline \multicolumn{4}{|c|}{ 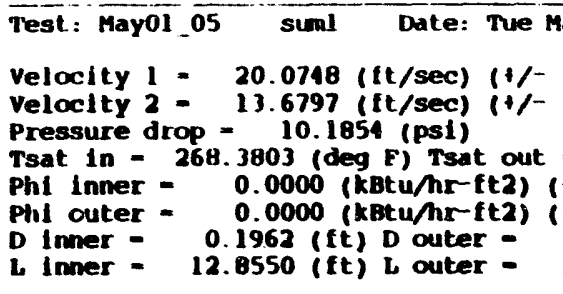 } & \multicolumn{2}{|c|}{$\begin{array}{l}\text { May } 111: 45: 551990 \\
1.7196 \text { ()) } \\
1.7009 \text { :) } \\
=252.1361 \text { (deg F) } \\
(+/-81821.3047 \text { (i) } \\
(+/-2380.1453 \text { (f) } \\
0.2413 \text { (ft) D hyd - } \\
12.8070 \text { (ft) A flow - }\end{array}$} & $\begin{array}{l}0.0389 \text { (ft) } \\
0.0150 \text { (ft2) }\end{array}$ \\
\hline 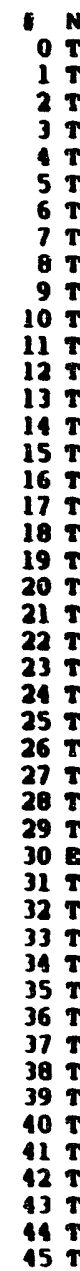 & 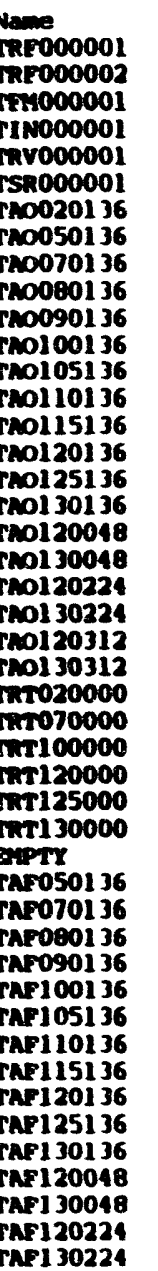 & $\begin{array}{l}\text { Min } \\
74.2113 \\
71.4388 \\
87.5683 \\
87.1985 \\
87.3948 \\
87.0416 \\
87.7883 \\
87.7486 \\
85.6734 \\
87.7439 \\
87.7203 \\
87.6899 \\
88.1854 \\
85.5885 \\
87.8529 \\
87.6695 \\
87.8675 \\
87.5844 \\
87.7115 \\
87.4307 \\
87.5330 \\
87.6331 \\
87.5646 \\
87.3896 \\
87.8683 \\
87.8391 \\
87.8746 \\
87.7873 \\
87.7467 \\
87.6589 \\
39.1464 \\
87.7529 \\
88.2728 \\
87.8967 \\
85.2050 \\
87.6643 \\
87.5740 \\
87.5255 \\
87.9575 \\
87.8794 \\
87.8515 \\
87.7591 \\
88.0309 \\
87.5847 \\
87.6596 \\
87.2504\end{array}$ & $\begin{array}{l}\operatorname{Max} \\
74.8498 \\
75.0738 \\
88.3023 \\
87.9343 \\
88.3358 \\
88.4687 \\
88.8381 \\
88.4807 \\
86.5163 \\
88.2676 \\
88.5593 \\
88.3198 \\
89.1304 \\
86.2180 \\
88.5889 \\
88.2973 \\
88.6007 \\
88.2114 \\
88.3403 \\
88.0595 \\
88.2675 \\
88.2614 \\
88.2995 \\
88.0196 \\
88.7090 \\
88.3655 \\
88.7126 \\
88.4166 \\
88.5836 \\
88.2884 \\
10.0646 \\
88.5900 \\
89.1082 \\
88.4199 \\
86.3593 \\
88.3997 \\
88.5130 \\
88.1536 \\
88.7953 \\
88.2999 \\
88.7911 \\
88.5975 \\
88.8761 \\
88.4241 \\
88.4991 \\
87.9878\end{array}$ & $\begin{array}{l}\text { Ave } \\
74.5945 \\
74.7690 \\
87.9918 \\
87.6610 \\
87.8178 \\
88.2139 \\
88.3214 \\
88.1541 \\
86.1413 \\
88.0116 \\
88.2280 \\
88.0553 \\
88.6517 \\
85.9496 \\
88.2482 \\
87.9385 \\
88.1778 \\
87.8739 \\
88.0303 \\
87.7744 \\
87.8897 \\
87.9228 \\
87.8963 \\
87.8012 \\
88.2930 \\
88.1340 \\
88.1149 \\
88.1689 \\
88.1781 \\
87.9864 \\
39.6102 \\
88.2889 \\
88.6742 \\
88.1852 \\
85.7929 \\
87.9962 \\
88.0828 \\
87.8566 \\
88.4268 \\
88.1275 \\
88.3566 \\
88.1663 \\
88.5463 \\
88.0884 \\
88.1422 \\
87.6421\end{array}$ & 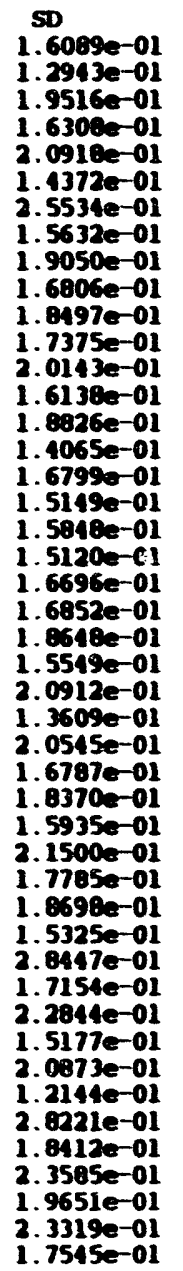 & 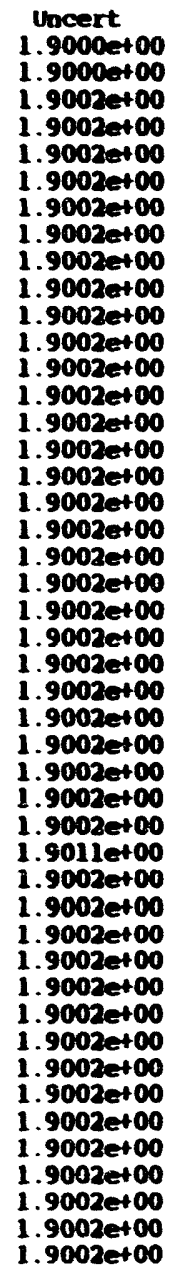 \\
\hline
\end{tabular}

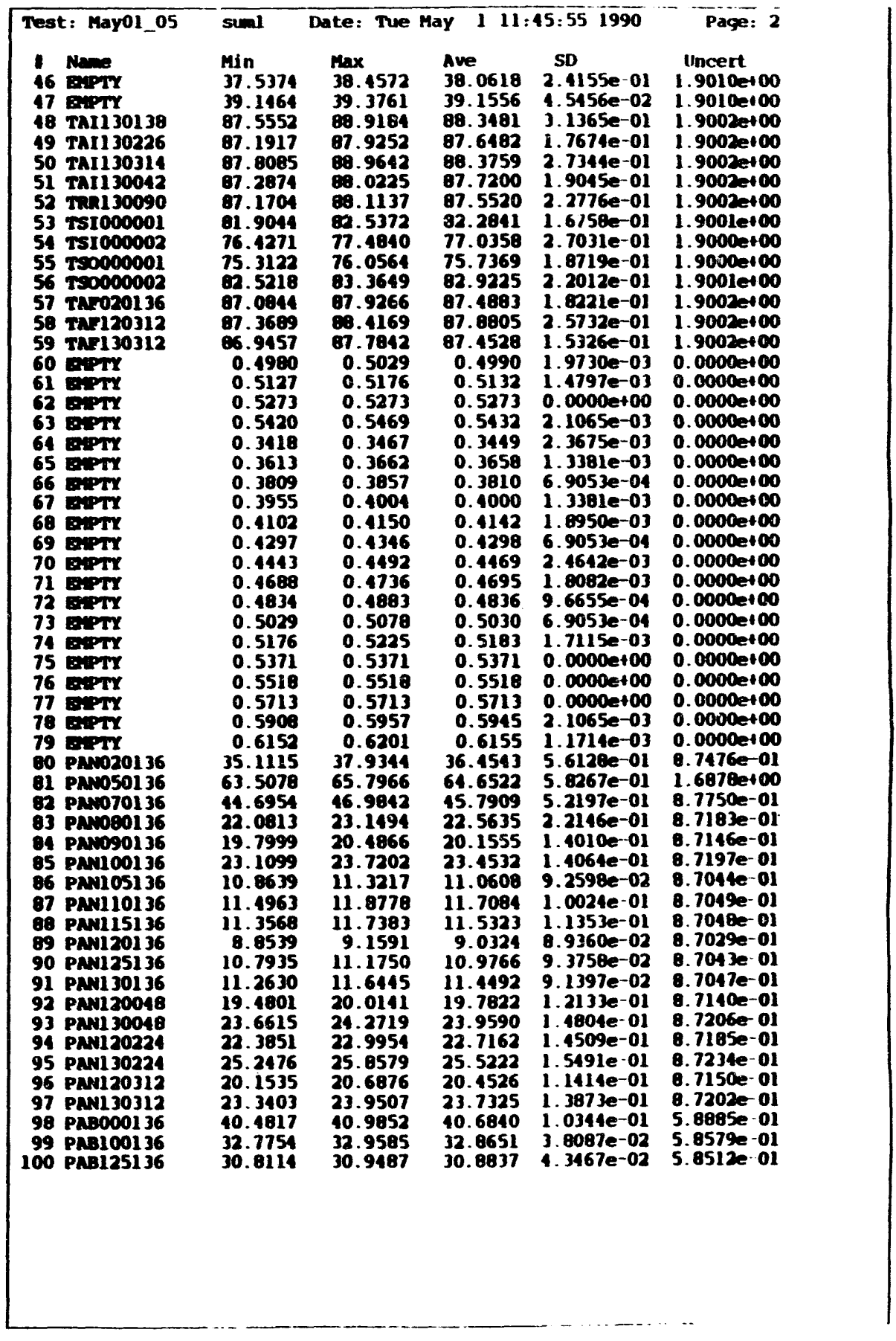




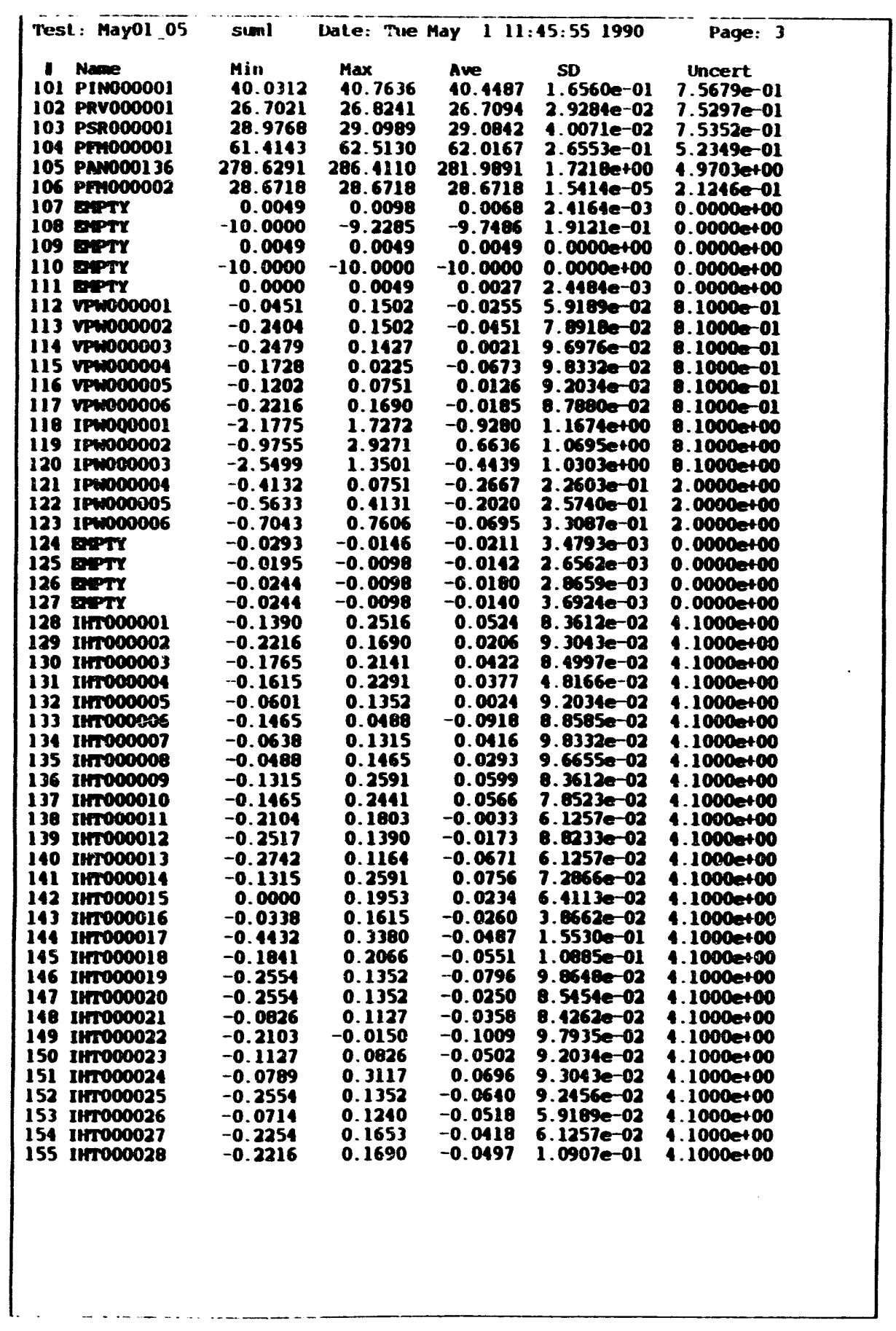

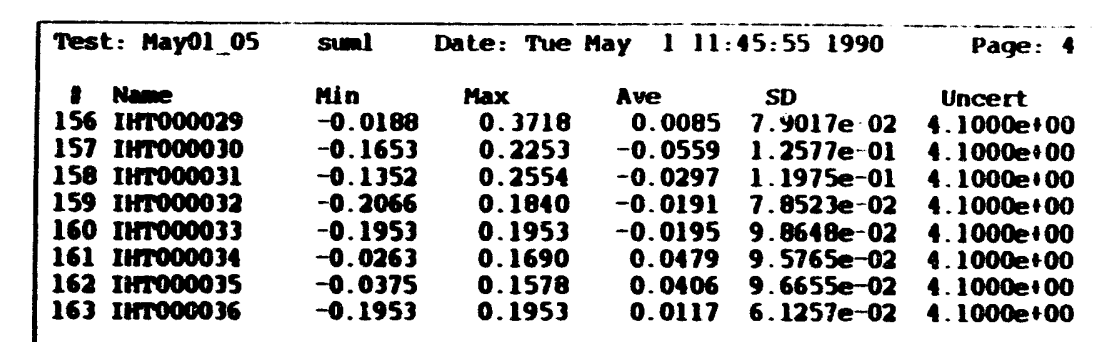




\begin{tabular}{|c|c|c|c|c|c|}
\hline $\begin{array}{l}\text { Tesl: May01_06 } \\
\text { velocity } 1= \\
\text { Velocity } 2 \text { - } \\
\text { Pressure drop } \\
\text { Tsat in = } 263 \\
\text { Phi inner = } \\
\text { Ph1 outer = } \\
\text { D inner = } 0 \\
\text { l. inner = } 12\end{array}$ & $\begin{array}{l}\text { suml } \\
.9844 \text { (ft } \\
.6779 \text { ift } \\
7.4500 \\
05 \text { (deg F } \\
0000 \text { (kBt } \\
0000 \text { (kBt } \\
62 \text { (ft) D } \\
50 \text { (ft) }\end{array}$ & $\begin{array}{l}\text { bate: Tue } \\
\text { (sec) }(1 /- \\
\text { sec) }(1 /- \\
\text { psi) } \\
\text { Tsat out } \\
\text { Mrtt(2) } \\
\text { (Mr-ft2) }\end{array}$ & $\begin{array}{r}\text { May 11: } \\
1.7456 \\
1.7009 \\
=251.15 \\
1+/-5945 . \\
1+/-3035 \\
0.2413 \\
12.8070 \text { i }\end{array}$ & $\begin{array}{l}302 \text { (deg F) } \\
4712 \text { : } \\
0711 \text { : } \\
\text { ft) D hyd } \\
\text { ft) A flow - }\end{array}$ & $\begin{array}{l}0.0389 \text { (ft) } \\
0.0150 \text { (ft2) }\end{array}$ \\
\hline 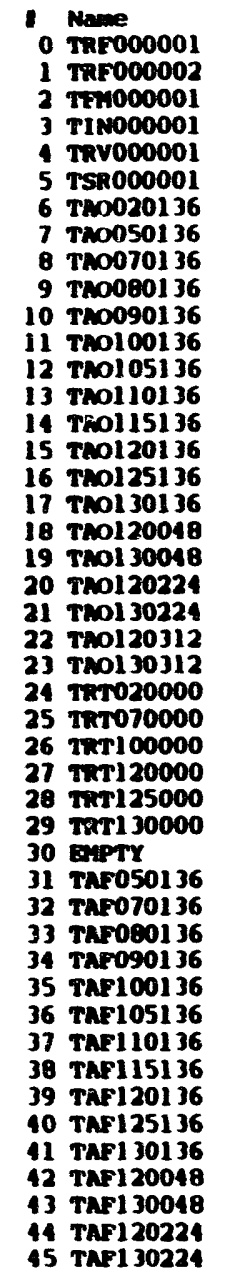 & $\begin{array}{l}\text { Min } \\
74.1242 \\
74.6505 \\
86.1017 \\
85.7257 \\
85.8279 \\
87.4243 \\
86.2112 \\
86.2880 \\
84.8294 \\
86.0752 \\
86.2545 \\
86.2234 \\
86.7142 \\
84.6518 \\
86.2733 \\
86.1109 \\
86.2963 \\
86.0369 \\
86.2443 \\
85.6639 \\
86.0644 \\
86.0744 \\
85.8846 \\
86.0299 \\
86.3946 \\
86.3601 \\
86.5144 \\
86.2155 \\
86.2893 \\
85.9870 \\
39.3761 \\
86.3932 \\
86.6020 \\
86.2312 \\
84.2604 \\
86.0874 \\
86.11171 \\
86.0609 \\
86.4910 \\
86.3122 \\
86.1846 \\
86.1887 \\
86.4466 \\
86.0127 \\
86.2944 \\
85.6656\end{array}$ & $\begin{array}{l}\text { Max } \\
75.0626 \\
74.8621 \\
86.8365 \\
86.4625 \\
86.7706 \\
88.0516 \\
87.1576 \\
87.0210 \\
85.9892 \\
86.7049 \\
87.0939 \\
86.7492 \\
87.4501 \\
85.3848 \\
87.0102 \\
86.7400 \\
87.0310 \\
86.7618 \\
86.8750 \\
86.5976 \\
86.5899 \\
86.7035 \\
86.8304 \\
86.5549 \\
87.2367 \\
86.8882 \\
87.2486 \\
86.9504 \\
87.1255 \\
86.8250 \\
40.0646 \\
87.1273 \\
87.7534 \\
87.0686 \\
85.4161 \\
86.8237 \\
87.1625 \\
86.6898 \\
87.3310 \\
86.8370 \\
87.3338 \\
86.9234 \\
87.3964 \\
86.7478 \\
87.0301 \\
86.4040\end{array}$ & $\begin{array}{l}\text { Ave } \\
74.6455 \\
74.7690 \\
86.4419 \\
86.1214 \\
86.3475 \\
87.7510 \\
86.8085 \\
86.6317 \\
85.1920 \\
86.4439 \\
86.6785 \\
86.4714 \\
87.1137 \\
84.9997 \\
86.7071 \\
86.4082 \\
86.6532 \\
86.3582 \\
86.5259 \\
86.2289 \\
86.3587 \\
86.4009 \\
86.3429 \\
86.2317 \\
86.7693 \\
86.6341 \\
86.8795 \\
86.6354 \\
86.6572 \\
86.4266 \\
39.6607 \\
86.6870 \\
87.1261 \\
86.6286 \\
84.8321 \\
86.4448 \\
86.5436 \\
86.3172 \\
86.9233 \\
86.5475 \\
86.7281 \\
86.5917 \\
86.9447 \\
86.5083 \\
86.6266 \\
86.0792\end{array}$ & $\begin{array}{l}\text { SD } \\
1.8222 e-01 \\
1.0614 e-01 \\
1.9147 e-01 \\
1.6272 e-01 \\
2.2492 e-01 \\
1.1457 e-01 \\
2.3792 e-01 \\
1.6809 e-01 \\
2.3157 e-01 \\
1.4409 e-01 \\
2.0222 e-01 \\
1.4365 e-01 \\
1.9350 e-01 \\
1.6997 e-01 \\
2.1657 e-01 \\
1.6445 e-01 \\
1.7620 e-01 \\
1.6713 e-01 \\
1.8789 e-01 \\
1.3957 e-01 \\
1.6495 e-01 \\
1.6214 e-01 \\
2.0009 e-01 \\
1.6421 e-01 \\
1.8436 e-01 \\
1.6076 e-01 \\
2.2148 e-01 \\
1.8790 e-01 \\
1.7847 e-01 \\
1.7737 e-01 \\
1.9433 e-01 \\
1.8723 e-01 \\
2.2862 e-01 \\
1.8079 e-01 \\
2.6607 e-01 \\
1.8283 e-01 \\
2.7495 e-01 \\
1.7113 e-01 \\
2.1576 e-01 \\
1.4779 e-01 \\
2.8613 e-01 \\
1.6066 e-01 \\
2.3069 e-01 \\
1.7110 e-01 \\
1.6221 e-01 \\
1.9037 e-01\end{array}$ & $\begin{array}{r}\text { Uncert } \\
1.9000 e+00 \\
1.9000 e+00 \\
1.9002 e+00 \\
1.9002 e+00 \\
1.9002 e+00 \\
1.9002 e+00 \\
1.9002 e+00 \\
1.9002 e+00 \\
1.9002 e+00 \\
1.9002 e+00 \\
1.9002 e+00 \\
1.9002 e+00 \\
1.9002 e+00 \\
1.9002 e+00 \\
1.9002 e+00 \\
1.9002 e+00 \\
1.9002 e+00 \\
1.9002 e+00 \\
1.9002 e+00 \\
1.9002 e+00 \\
1.9002 e+00 \\
1.9002 e+00 \\
1.9002 e+00 \\
1.9002 e+00 \\
1.9002 e+00 \\
1.9002 e+00 \\
1.9002 e+00 \\
1.9002 e+00 \\
1.9002 e+00 \\
1.9002 e+00 \\
1.9011 e+00 \\
1.9002 e+00 \\
1.9002 e+00 \\
1.9002 e+00 \\
1.9001 e+00 \\
1.9002 e+00 \\
1.9002 e+00 \\
1.9002 e+00 \\
1.9002 e+00 \\
1.9002 e+00 \\
1.9002 e+00 \\
1.9002 e+00 \\
1.9002 e+00 \\
1.9002 e+00 \\
1.9002 e+00 \\
1.9002 e+00\end{array}$ \\
\hline
\end{tabular}

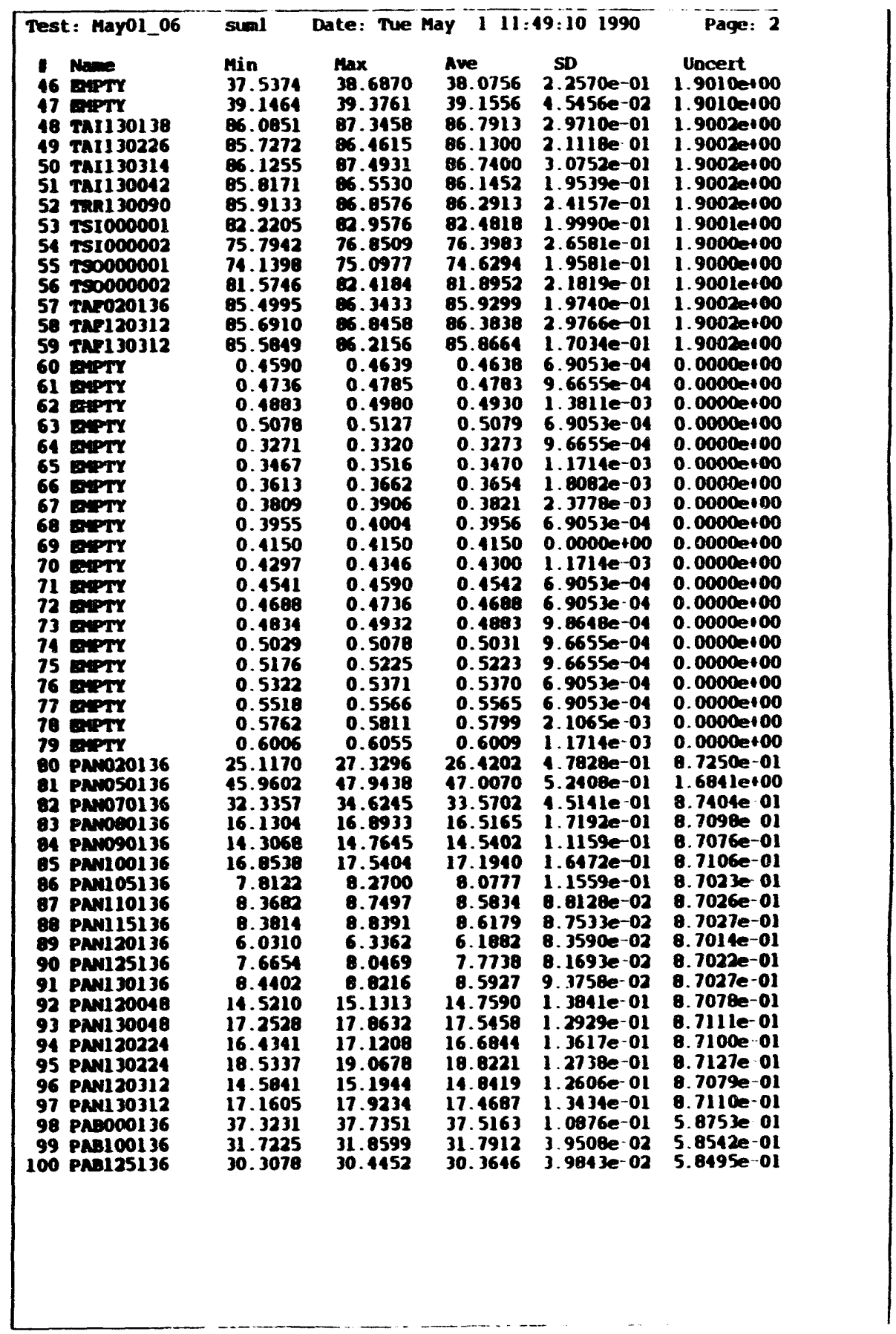




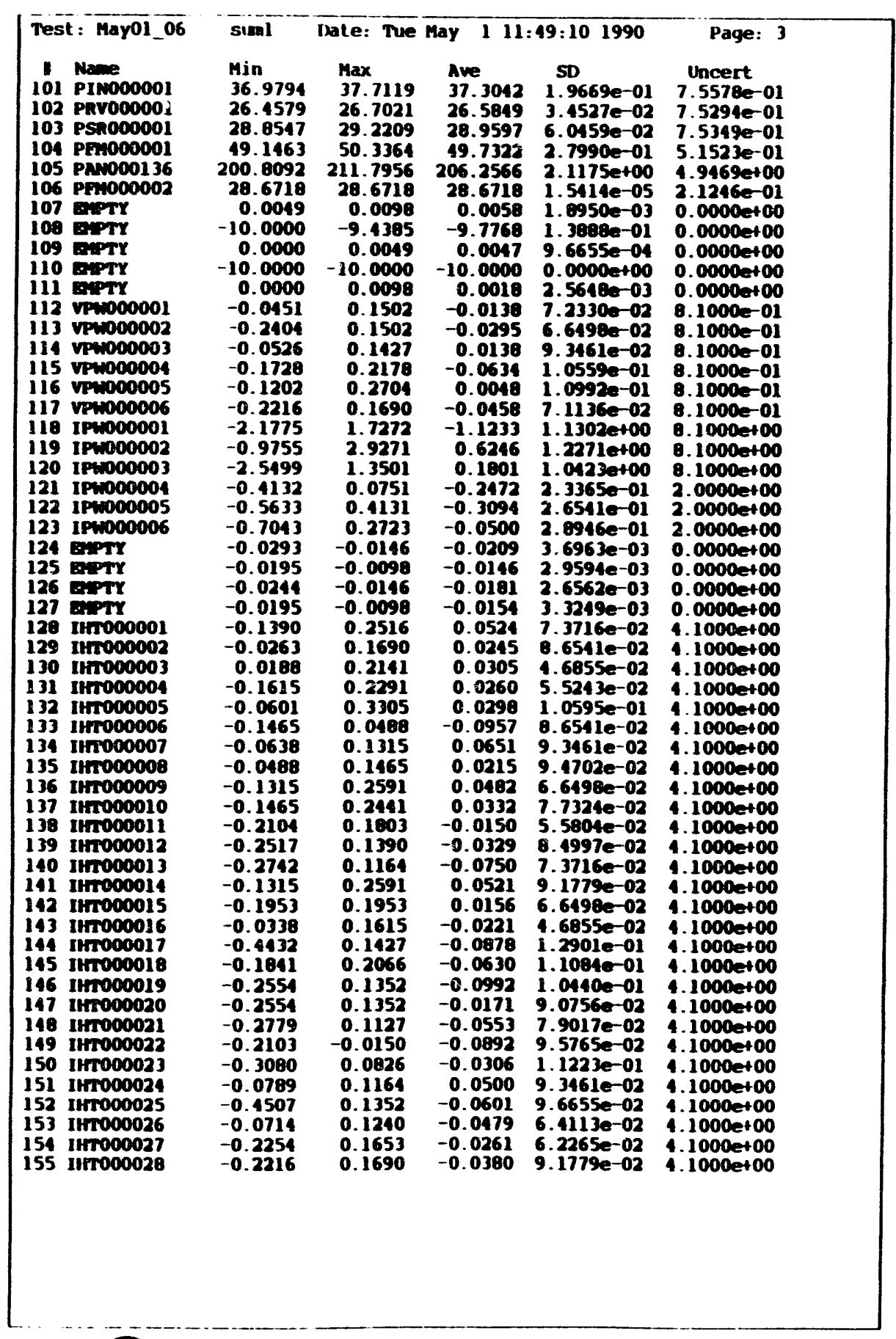

\begin{tabular}{|c|c|c|c|c|c|}
\hline st : & & Date & lay & 01990 & ge: \\
\hline 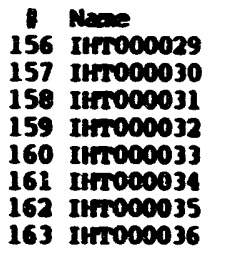 & $\begin{array}{l}\operatorname{Min} \\
-0.0168 \\
-0.1653 \\
-0.1352 \\
-0.2066 \\
-0.1953 \\
-0.0263 \\
-0.0375 \\
-0.1953\end{array}$ & $\begin{array}{l}\max \\
0.1765 \\
0.0300 \\
0.0601 \\
0.1840 \\
0.1953 \\
0.3643 \\
0.1578\end{array}$ & $\begin{array}{l}\text { Ave } \\
-0.0032 \\
-0.0754 \\
-0.0376 \\
-0.0113 \\
0.0000 \\
0.0518 \\
0.0406 \\
0.0195\end{array}$ & $\begin{array}{c}50 \\
5.3525 e-02 \\
9.8332 e-02 \\
9.8648 e-02 \\
6.8345 e-02 \\
5.5604 e-02 \\
1.0440 e-01 \\
9.6655 e-02 \\
7.1136 e-02\end{array}$ & $\begin{array}{l}\text { Un } \\
4.1 \\
4.1 \\
4.1 \\
4.1 \\
4.1 \\
4.1 \\
4.1 \\
4.1\end{array}$ \\
\hline
\end{tabular}




\begin{tabular}{|c|c|c|c|c|c|}
\hline \multicolumn{5}{|c|}{ 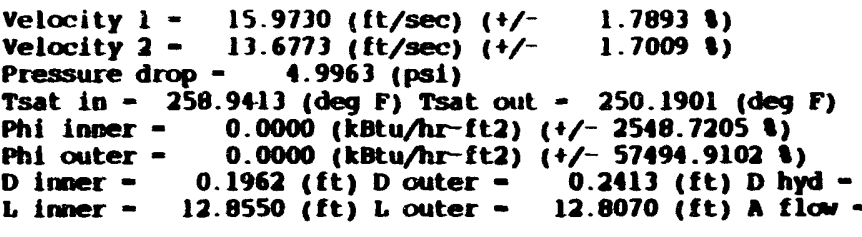 } & $\begin{array}{l}0.0389 \text { (ft) } \\
0.0150 \text { (ftz) }\end{array}$ \\
\hline 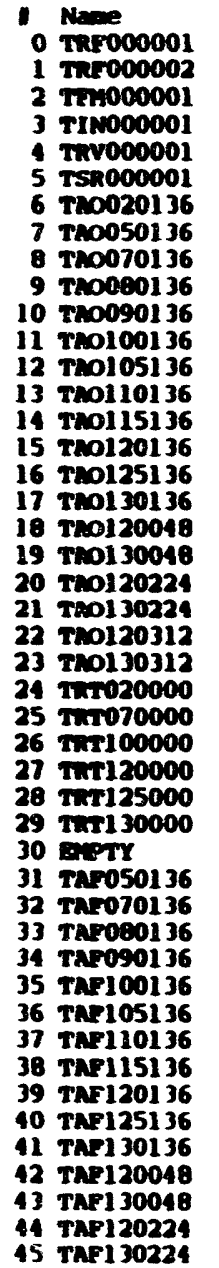 & $\begin{array}{l}\text { Min } \\
74.4242 \\
74.4388 \\
85.5714 \\
85.1996 \\
85.4096 \\
86.5691 \\
85.6855 \\
85.6616 \\
84.4072 \\
85.5528 \\
85.7303 \\
85.5941 \\
86.0831 \\
84.2347 \\
85.5360 \\
85.3814 \\
85.7721 \\
85.5184 \\
85.5101 \\
85.3407 \\
85.4344 \\
85.4499 \\
85.4642 \\
85.4014 \\
85.7625 \\
85.7257 \\
85.7812 \\
85.4812 \\
85.6623 \\
85.5673 \\
39.1464 \\
85.8696 \\
86.0801 \\
85.7095 \\
83.7352 \\
85.4561 \\
85.4922 \\
85.3278 \\
85.9668 \\
85.5796 \\
85.6626 \\
85.5597 \\
85.9168 \\
85.4891 \\
85.6640 \\
85.1362\end{array}$ & $\begin{array}{l}\text { Max } \\
74.8498 \\
75.0738 \\
86.4174 \\
85.8310 \\
86.2485 \\
87.4270 \\
86.7371 \\
86.3949 \\
85.2509 \\
86.2871 \\
86.4653 \\
86.2245 \\
86.8194 \\
84.9679 \\
86.5889 \\
86.1152 \\
86.4023 \\
85.9392 \\
86.2454 \\
85.9705 \\
86.0651 \\
86.0787 \\
85.9902 \\
85.9267 \\
86.8150 \\
86.5691 \\
86.6206 \\
86.3214 \\
86.5001 \\
86.1967 \\
40.0646 \\
86.4995 \\
86.9184 \\
86.3385 \\
84.9963 \\
86.2977 \\
86.4328 \\
86.2722 \\
86.7018 \\
86.2095 \\
86.7087 \\
86.3996 \\
87.0784 \\
86.3284 \\
86.4000 \\
85.9814\end{array}$ & $\begin{array}{l}\text { Ave } \\
74.6540 \\
74.7901 \\
85.9241 \\
85.5509 \\
85.8354 \\
87.0519 \\
86.1712 \\
86.0661 \\
84.7889 \\
85.8826 \\
86.1399 \\
85.9073 \\
86.5228 \\
84.6266 \\
85.0759 \\
85.8110 \\
86.0852 \\
85.7610 \\
85.9072 \\
85.6371 \\
85.7772 \\
85.8204 \\
85.7600 \\
85.6348 \\
86.2153 \\
86.0441 \\
86.2744 \\
86.0252 \\
86.0462 \\
85.8469 \\
39.6470 \\
86.1533 \\
86.5602 \\
86.0309 \\
84.3891 \\
85.8957 \\
86.0669 \\
85.7499 \\
86.3428 \\
85.9931 \\
86.1591 \\
86.0322 \\
86.3895 \\
85.9608 \\
86.0320 \\
85.5358\end{array}$ & $\begin{array}{l}\text { SD } \\
1.8161 e-01 \\
1.3929 e-01 \\
2.1964 e-01 \\
1.6001 e-01 \\
1.9561 e-01 \\
1.7590 e-01 \\
2.5139 e-01 \\
1.7599 e-01 \\
1.6762 e-01 \\
1.4527 e-01 \\
1.0154 e-01 \\
1.5533 e-01 \\
2.0893 e-01 \\
1.5811 e-01 \\
2.4354 e-01 \\
1.6563 e-01 \\
1.6085 e-01 \\
1.3908 e-01 \\
1.5055 e-01 \\
1.1191 e-01 \\
1.6422 e-01 \\
1.5992 e-01 \\
1.4829 e-01 \\
1.3143 e-01 \\
2.0543 e-01 \\
1.7502 e-01 \\
1.6971 e-01 \\
1.6258 e-01 \\
2.0152 e-01 \\
1.6247 e-01 \\
2.10940-01 \\
1.48880-01 \\
2.1265 e-01 \\
1.1263 e-01 \\
2.8028 e-01 \\
1.5433 e-01 \\
2.3233 e-01 \\
1.7165 e-01 \\
1.9791 e-01 \\
1.5206 e-01 \\
2.6515 e-01 \\
1.7656 e-01 \\
2.1707 e-01 \\
1.9367 e-01 \\
1.7676 e-01 \\
2.1138 e-01\end{array}$ & $\begin{array}{l}\text { Uncert } \\
1.9000 e+00 \\
1.90000+00 \\
1.9002 e+00 \\
1.9002 e+00 \\
1.9002 e+00 \\
1.9002 e+00 \\
1.9002 e+00 \\
1.9002 e+00 \\
1.9001 e+00 \\
1.9002 e+00 \\
1.9002 e+00 \\
1.9002 e+00 \\
1.9002 e+00 \\
1.9001 e+00 \\
1.9002 e+00 \\
1.9002 e+00 \\
1.9002 e+00 \\
1.9002 e+00 \\
1.9002 e+00 \\
1.9002 e+00 \\
1.9002 e+00 \\
1.9002 e+00 \\
1.9002 e+00 \\
1.9002 e+00 \\
1.9002 e+00 \\
1.9002 e+00 \\
1.9002 e+00 \\
1.9002 e+00 \\
1.9002 e+00 \\
1.9002 e+00 \\
1.9011 e+00 \\
1.9002 e+00 \\
1.9002 e+00 \\
1.9002 e+00 \\
1.9001 e+00 \\
1.9002 e+00 \\
1.9002 e+00 \\
1.9002 e+00 \\
1.9002 e+00 \\
1.9002 e+00 \\
1.9002 e+00 \\
1.9002 e+00 \\
1.9002 e+00 \\
1.9002 e+00 \\
1.9002 e+00 \\
1.9002 e+00\end{array}$ \\
\hline
\end{tabular}

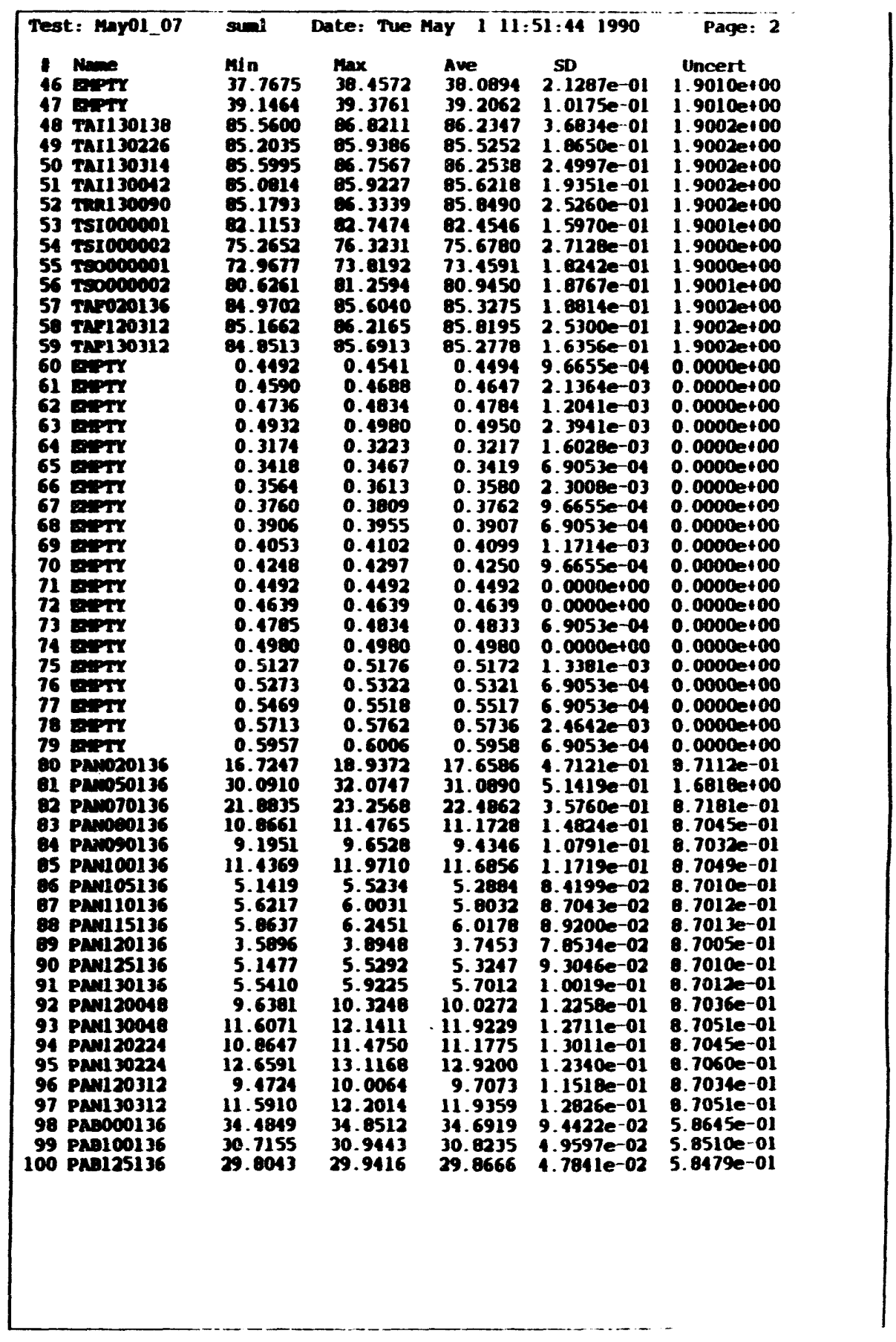




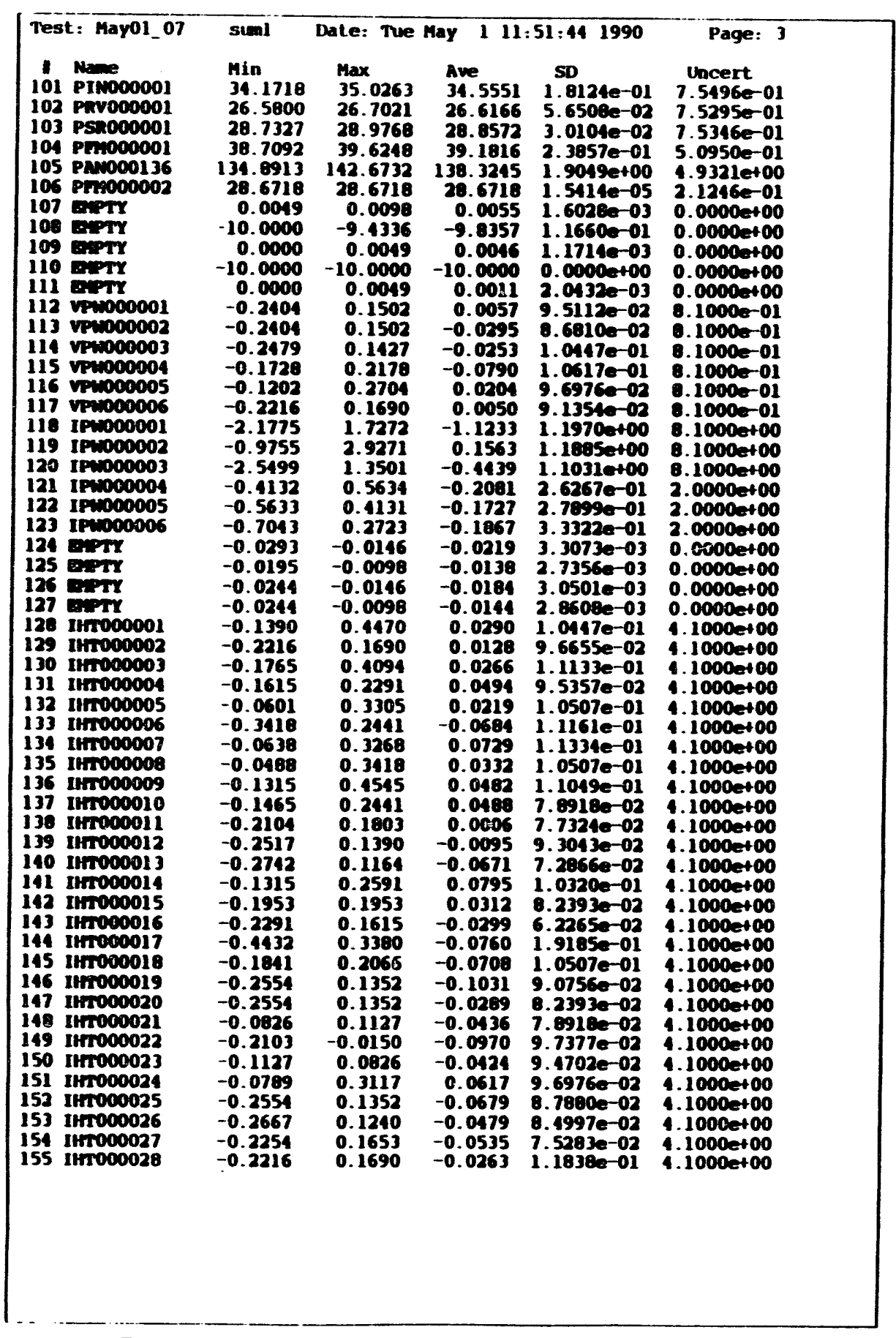

\begin{tabular}{|c|c|c|c|c|c|}
\hline Test: May01_07 & $\sin$ & Date: Tue & lay 111 & $51: 441990$ & Page: \\
\hline 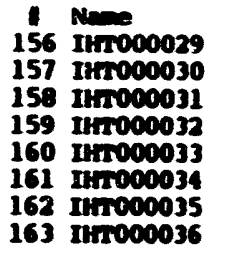 & $\begin{array}{l}\text { Min } \\
-0.0160 \\
-0.1653 \\
-0.1352 \\
-0.2066 \\
-0.1953 \\
-0.0263 \\
-0.2329 \\
-0.1953\end{array}$ & $\begin{array}{l}\max \\
0.3718 \\
0.2253 \\
0.2554 \\
0.1840 \\
0.1953 \\
0.3643 \\
0.1578 \\
0.1953\end{array}$ & $\begin{array}{l}\text { Ave } \\
0.0164 \\
-0.0872 \\
-0.0493 \\
-0.0113 \\
0.0039 \\
0.0440 \\
0.0328 \\
0.0195\end{array}$ & $\begin{array}{l}50 \\
8.5454 e-02 \\
1.0440 e-01 \\
1.0559 e-01 \\
6.8345 e-02 \\
7.3716 e-02 \\
1.0259 e-01 \\
1.0259 e-01 \\
8.1347 e-02\end{array}$ & 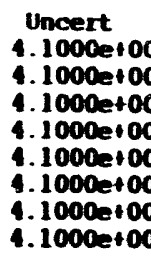 \\
\hline
\end{tabular}




\begin{tabular}{|c|c|c|c|c|c|}
\hline \multicolumn{3}{|c|}{ 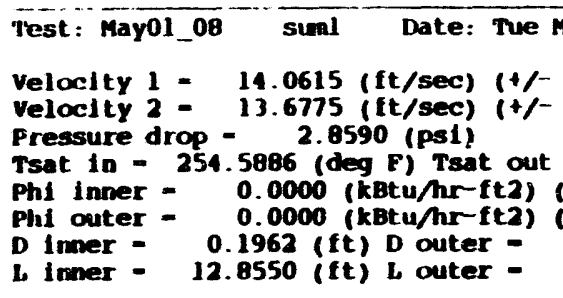 } & \multicolumn{2}{|c|}{$\begin{array}{l}\text { May } 111: 57: 341990 \\
1.8640 \text { :) } \\
1.7009 \text { :) } \\
=249.3634 \text { (deg F) } \\
(+/-17090.8477 \text { (t) } \\
(+/-3198.8848 \text { :) } \\
0.2413 \text { (ft) D hyd - } \\
12.8070 \text { (ft) A flow - }\end{array}$} & $\begin{array}{l}\text { Page: } 1 \\
0.0389 \text { (ft) } \\
0.0150 \text { (ft2) }\end{array}$ \\
\hline 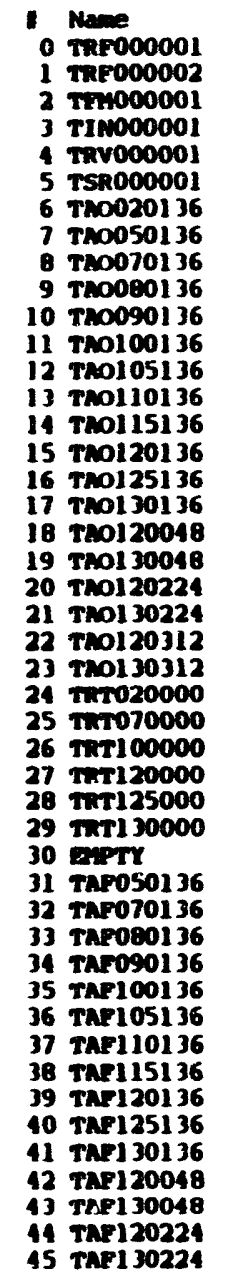 & $\begin{array}{l}\text { Min } \\
74.4242 \\
74.6505 \\
85.6823 \\
85.4095 \\
85.5151 \\
86.1713 \\
85.8952 \\
85.8704 \\
84.3016 \\
85.5543 \\
85.8352 \\
85.8039 \\
86.1885 \\
84.2347 \\
85.7463 \\
85.5912 \\
85.7727 \\
85.4165 \\
85.6150 \\
85.4456 \\
85.6445 \\
85.6581 \\
85.4642 \\
85.6109 \\
85.8679 \\
85.8311 \\
85.9915 \\
85.9609 \\
85.7672 \\
85.6722 \\
39.3761 \\
85.8699 \\
86.2898 \\
85.9193 \\
83.9453 \\
85.5610 \\
85.5977 \\
85.6422 \\
86.0717 \\
85.7899 \\
85.7679 \\
85.7694 \\
86.1284 \\
85.8029 \\
85.7689 \\
85.2428\end{array}$ & $\begin{array}{l}\operatorname{Max} \\
75.0626 \\
75.0738 \\
86.4180 \\
86.0413 \\
66.6557 \\
86.9060 \\
86.9473 \\
86.7085 \\
85.2509 \\
86.2876 \\
86.6749 \\
86.3299 \\
87.3447 \\
85.1784 \\
66.7991 \\
86.3239 \\
86.7173 \\
86.2513 \\
86.3508 \\
86.1808 \\
86.2750 \\
86.7030 \\
86.2005 \\
86.1370 \\
66.7102 \\
86.5697 \\
87.0401 \\
86.5317 \\
86.5001 \\
86.3027 \\
10.0646 \\
86.7088 \\
87.1285 \\
86.5488 \\
85.1013 \\
86.4031 \\
86.6430 \\
86.1683 \\
87.0167 \\
86.4198 \\
66.9188 \\
86.3996 \\
86.8683 \\
86.4338 \\
86.4000 \\
86.1917\end{array}$ & $\begin{array}{l}\text { Ave } \\
74.6891 \\
74.8706 \\
86.0899 \\
85.7213 \\
85.9757 \\
86.5280 \\
86.3415 \\
86.2146 \\
84.8415 \\
86.0144 \\
86.2595 \\
86.0899 \\
86.6804 \\
84.6750 \\
86.2728 \\
85.9260 \\
86.2132 \\
85.8963 \\
86.0563 \\
85.8026 \\
85.9263 \\
85.9520 \\
85.9016 \\
85.8298 \\
86.3394 \\
86.1770 \\
86.4400 \\
86.1868 \\
86.2073 \\
85.9958 \\
39.5699 \\
86.3356 \\
86.6670 \\
86.1876 \\
84.5676 \\
86.0071 \\
86.1445 \\
85.8020 \\
86.5295 \\
86.1210 \\
86.3200 \\
86.1266 \\
86.5552 \\
86.1300 \\
86.1392 \\
85.6560\end{array}$ & $\begin{array}{c}\text { SD } \\
1.8523 e-01 \\
1.4786 e-01 \\
1.9036 e-01 \\
1.7401 e-01 \\
2.0077 e-01 \\
1.5574 e-01 \\
2.2940 e-01 \\
1.8468 e-01 \\
2.2604 e-01 \\
1.8706 e-01 \\
1.8724 e-01 \\
1.5460 e-01 \\
2.2118 e-01 \\
1.9179 e-01 \\
2.3211 e-01 \\
1.6680 e-01 \\
1.9095 e-01 \\
1.7172 e-01 \\
1.5307 e-01 \\
1.7993 e-01 \\
1.7179 e-01 \\
2.0398 e-01 \\
1.7307 e-01 \\
1.3575 e-01 \\
1.8321 e-01 \\
1.6069 e-01 \\
2.3026 e-01 \\
1.6853 e-01 \\
1.8682 e-01 \\
1.7727 e-01 \\
2.0759 e-01 \\
1.86296 e-01 \\
2.2168 e-01 \\
1.6152 e-01 \\
3.0006 e-01 \\
1.6947 e-01 \\
2.5467 e-01 \\
1.4554 e-01 \\
2.3584 e-01 \\
1.4145 e-01 \\
2.6695 e-01 \\
1.5891 e-01 \\
2.1949 e-01 \\
1.8217 e-01 \\
1.8547 e-01 \\
2.1181 e-01\end{array}$ & 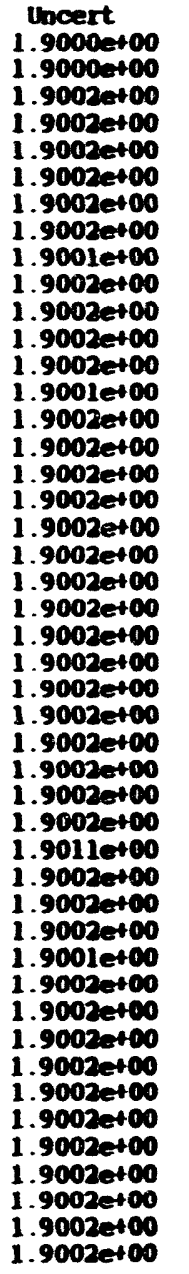 \\
\hline
\end{tabular}

\begin{tabular}{|c|c|c|c|c|c|}
\hline 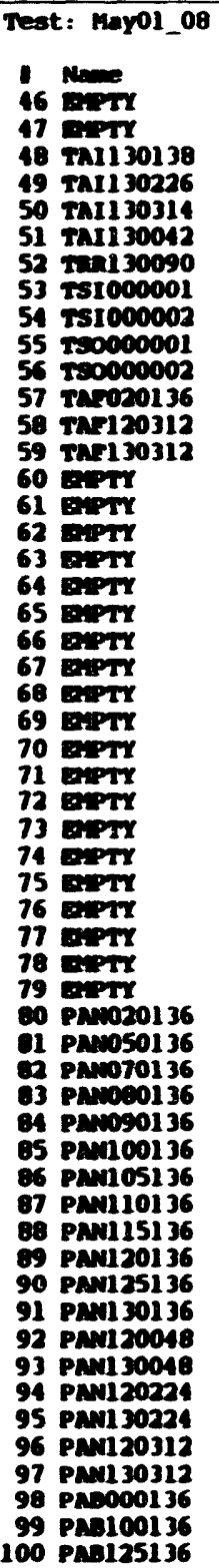 & 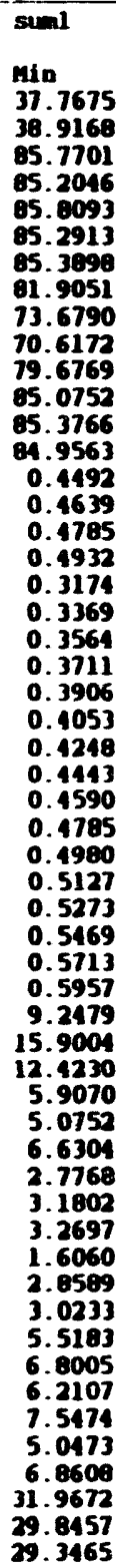 & $\begin{array}{l}\text { Date: Tue } \\
\text { Max } \\
38.4572 \\
39.3761 \\
86.9262 \\
86.1479 \\
67.0724 \\
86.2377 \\
86.6489 \\
89.6423 \\
74.7381 \\
71.3648 \\
80.5215 \\
85.9202 \\
86.5312 \\
85.6922 \\
0.4541 \\
0.1688 \\
0.4834 \\
0.4980 \\
0.3271 \\
0.3467 \\
0.3613 \\
0.3760 \\
0.3906 \\
0.4150 \\
0.4297 \\
0.4492 \\
0.4639 \\
0.4834 \\
0.5029 \\
0.5176 \\
0.5322 \\
0.5518 \\
0.5659 \\
0.6006 \\
11.3076 \\
18.1892 \\
14.2541 \\
6.5174 \\
5.5330 \\
7.1644 \\
3.1583 \\
3.4854 \\
3.6511 \\
1.9112 \\
3.2404 \\
3.4048 \\
5.9760 \\
7.3346 \\
6.6685 \\
7.9289 \\
5.5051 \\
7.3186 \\
32.1250 \\
30.0746 \\
29.5296\end{array}$ & $\begin{array}{l}\text { Aay } 111 \\
\text { Ave } \\
38.0802 \\
39.1602 \\
86.3040 \\
85.6824 \\
86.4368 \\
85.7795 \\
85.9603 \\
62.2802 \\
74.1791 \\
70.9505 \\
80.0488 \\
85.5069 \\
85.9601 \\
85.4435 \\
0.4519 \\
0.4662 \\
0.4795 \\
0.4969 \\
0.3222 \\
0.3418 \\
0.3571 \\
0.3758 \\
0.3906 \\
0.4098 \\
0.4250 \\
0.4490 \\
0.4638 \\
0.4833 \\
0.4981 \\
0.5170 \\
0.5321 \\
0.5517 \\
0.5727 \\
0.5959 \\
9.9757 \\
17.2676 \\
12.9845 \\
6.1527 \\
5.2675 \\
6.8959 \\
2.9736 \\
3.3130 \\
3.4802 \\
1.7189 \\
3.0359 \\
3.2400 \\
5.7288 \\
7.0264 \\
6.4289 \\
7.7503 \\
5.2808 \\
7.0973 \\
32.2099 \\
29.9849 \\
29.4436\end{array}$ & $\begin{array}{l}57: 341990 \\
50 \\
1.8441 e-01 \\
7.2025 e-02 \\
3.1469 e-01 \\
1.8744 e-01 \\
3.2381 e-01 \\
2.3251 e-01 \\
2.8451 e-01 \\
1.7181 e-01 \\
2.5048 e-01 \\
1.9833 e-01 \\
2.0595 e-01 \\
2.0090 e-01 \\
2.9174 e-01 \\
1.7845 e-01 \\
2.1583 e-03 \\
2.4642 e-03 \\
1.9730 e-03 \\
2.1065 e-03 \\
1.2041 e-03 \\
9.8648 e-04 \\
1.7115 e-03 \\
9.6655 e-04 \\
0.0000 e+00 \\
1.6624 e-03 \\
9.6655 e-04 \\
9.6655 e-04 \\
6.9053 e-04 \\
6.9053 e-04 \\
6.9053 e-04 \\
1.6028 e-03 \\
6.9053 e-04 \\
6.9053 e-04 \\
2.7971 e-03 \\
9.6655 e-04 \\
5.0094 e-01 \\
5.2312 e-01 \\
3.6865 e-01 \\
1.5589 e-01 \\
1.1033 e-01 \\
1.3721 e-01 \\
8.0225 e-02 \\
7.9810 e-02 \\
8.7953 e-02 \\
8.1910 e-02 \\
9.1760 e-02 \\
1.0032 e-01 \\
1.0404 e-01 \\
1.3345 e-01 \\
1.0900 e-01 \\
9.3262 e-02 \\
1.0944 e-01 \\
1.0707 e-01 \\
1.0014 e-01 \\
5.0621 e-02 \\
4.5907 e-02\end{array}$ & 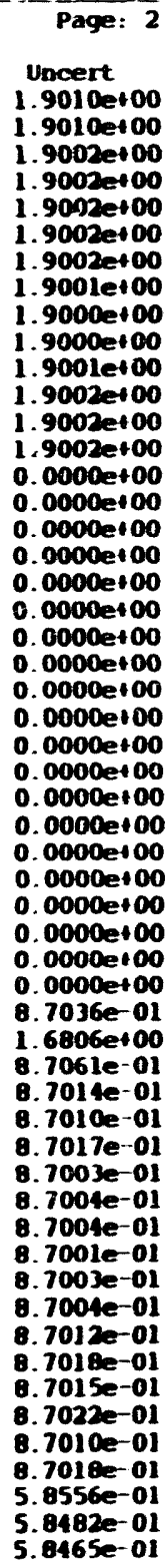 \\
\hline
\end{tabular}



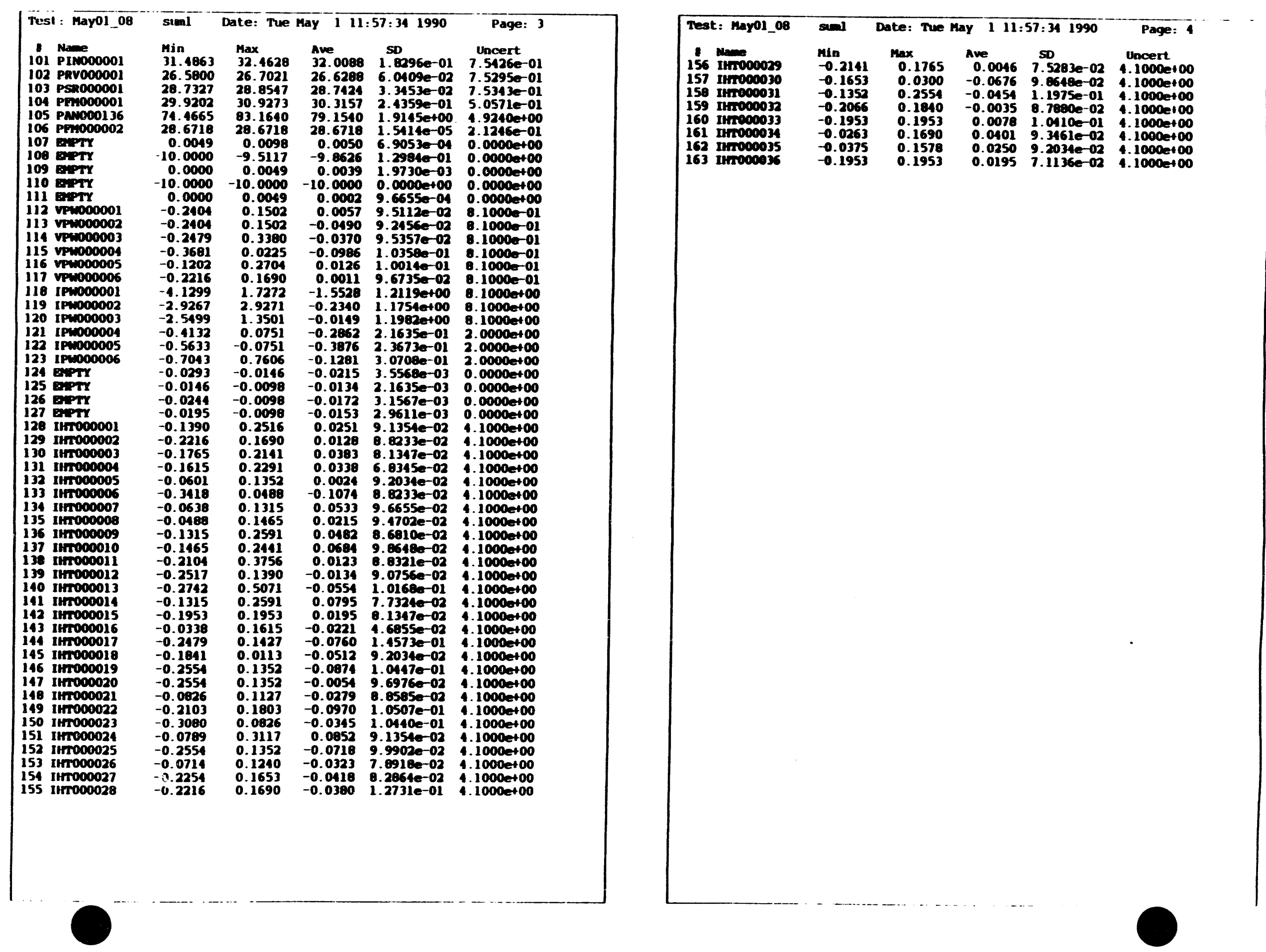


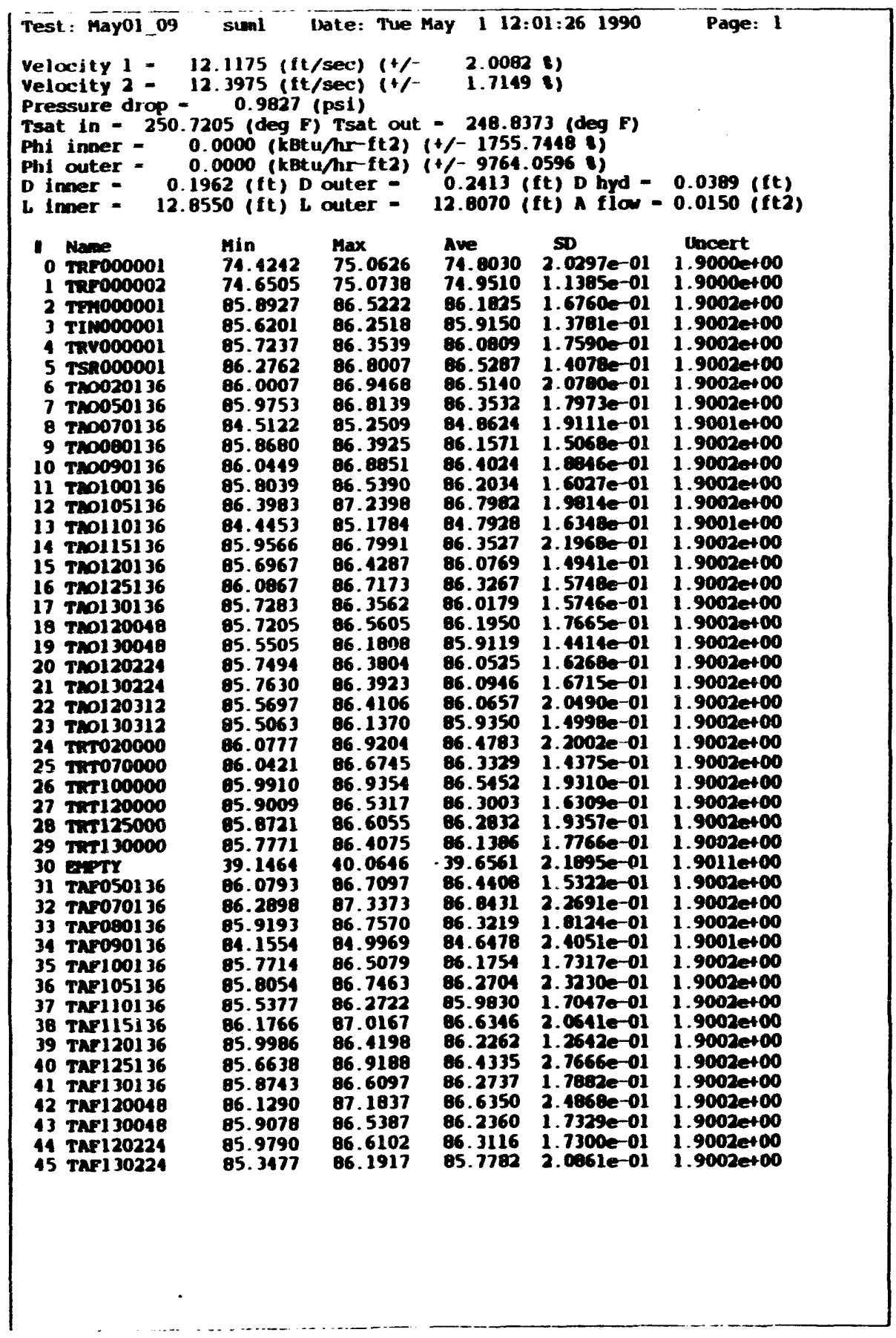

\begin{tabular}{|c|c|c|c|c|c|}
\hline 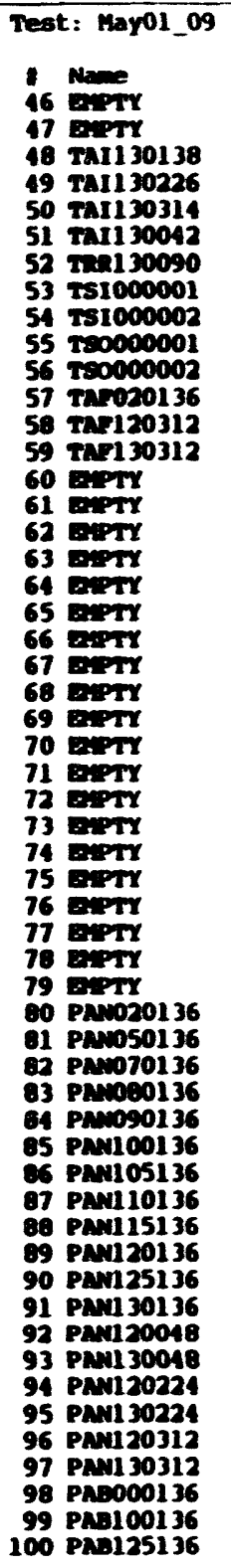 & $\begin{array}{l}\text { suil } \\
\text { Min } \\
37.7675 \\
38.9168 \\
85.9805 \\
85.4134 \\
85.8093 \\
85.5016 \\
65.5989 \\
81.9051 \\
73.0439 \\
69.4409 \\
79.2554 \\
85.2871 \\
65.6914 \\
85.2700 \\
0.1492 \\
0.4639 \\
0.1785 \\
0.4932 \\
0.3174 \\
0.3369 \\
0.3564 \\
0.3760 \\
0.3857 \\
0.4053 \\
0.4199 \\
0.4443 \\
0.4590 \\
0.4736 \\
0.4932 \\
0.5127 \\
0.5273 \\
0.5469 \\
0.5713 \\
0.5957 \\
2.5340 \\
4.1511 \\
3.3440 \\
1.5583 \\
1.1079 \\
2.4342 \\
0.7169 \\
1.0440 \\
1.2097 \\
-0.2251 \\
0.7227 \\
0.9633 \\
1.7799 \\
2.3755 \\
2.0146 \\
2.9697 \\
1.0037 \\
2.4358 \\
29.8615 \\
29.2964 \\
29.0719\end{array}$ & $\begin{array}{l}\text { Date: Tue } \\
\text { Max } \\
38.4572 \\
39.3761 \\
86.9262 \\
86.2533 \\
86.9674 \\
86.3431 \\
86.4393 \\
82.7490 \\
73.9978 \\
70.4024 \\
80.2057 \\
86.2379 \\
66.6365 \\
85.9016 \\
0.4541 \\
0.4688 \\
0.1834 \\
0.4980 \\
0.3223 \\
0.3418 \\
0.3613 \\
0.3760 \\
0.3906 \\
0.4102 \\
0.4248 \\
0.1492 \\
0.4639 \\
0.4834 \\
0.4980 \\
0.5176 \\
0.5322 \\
0.5510 \\
0.5762 \\
0.5957 \\
1.4414 \\
6.4399 \\
5.3277 \\
2.2449 \\
1.7183 \\
2.7394 \\
1.0983 \\
1.4255 \\
1.5912 \\
0.1564 \\
1.1042 \\
1.2685 \\
2.1613 \\
2.8333 \\
2.5486 \\
3.4275 \\
1.7667 \\
3.0461 \\
30.3651 \\
29.4337 \\
29.2550 \\
\end{array}$ & 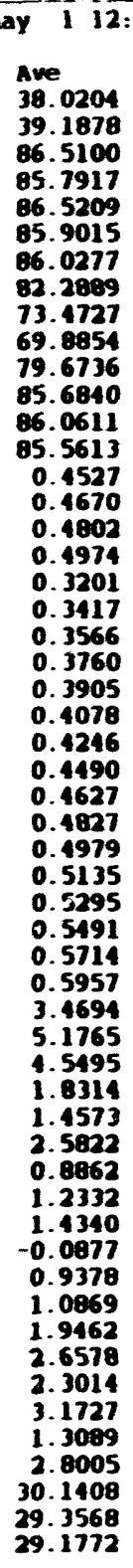 & $\begin{array}{l}01.261990 \\
51 \\
2.1911 e-01 \\
1.1067 e-01 \\
2.5456 e-01 \\
2.0752 e-01 \\
2.8964 e-01 \\
1.8157 e-01 \\
2.1520 e-01 \\
1.6871 e-01 \\
2.5676 e-01 \\
1.9589 e-01 \\
2.0123 e-01 \\
1.8033 e-01 \\
2.3736 e-01 \\
1.6653 e-01 \\
2.2146 e-03 \\
2.3675 e-03 \\
2.3365 e-03 \\
1.715 e-03 \\
2.4184 e-03 \\
6.9053 e-04 \\
9.6655 e-04 \\
0.0000 e+00 \\
6.9053 e-04 \\
2.4642 e-03 \\
9.6655 e-04 \\
9.6655 e-04 \\
2.1065 e-03 \\
1.9754 e-03 \\
9.6555 e-04 \\
1.8082 e-03 \\
2.4484 e-03 \\
2.4583 e-03 \\
6.9053 e-04 \\
0.0000 e+00 \\
1.9078 e-01 \\
5.7180 e-01 \\
3.9297 e-01 \\
1.4050 e-01 \\
1.2531 e-01 \\
9.1721 e-02 \\
8.3419 e-02 \\
9.1501 e-02 \\
8.3590 e-02 \\
8.7193 e-02 \\
6.1109 e-02 \\
6.6992 e-02 \\
1.1294 e-01 \\
1.0482 e-01 \\
1.1385 e-01 \\
1.1494 e-01 \\
1.2522 e-01 \\
1.2165 e-01 \\
1.0473 e-01 \\
1.1775 e-02 \\
4.3624 e-02\end{array}$ & 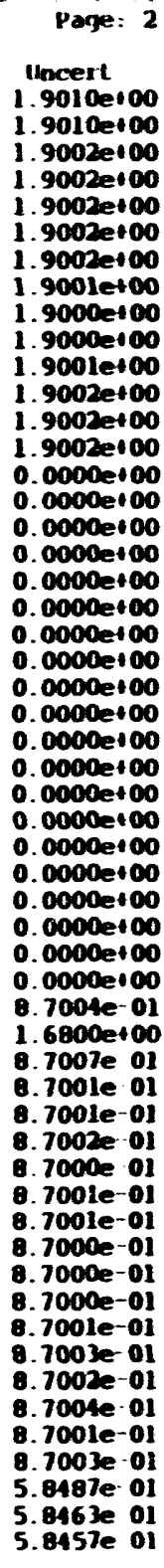 \\
\hline
\end{tabular}




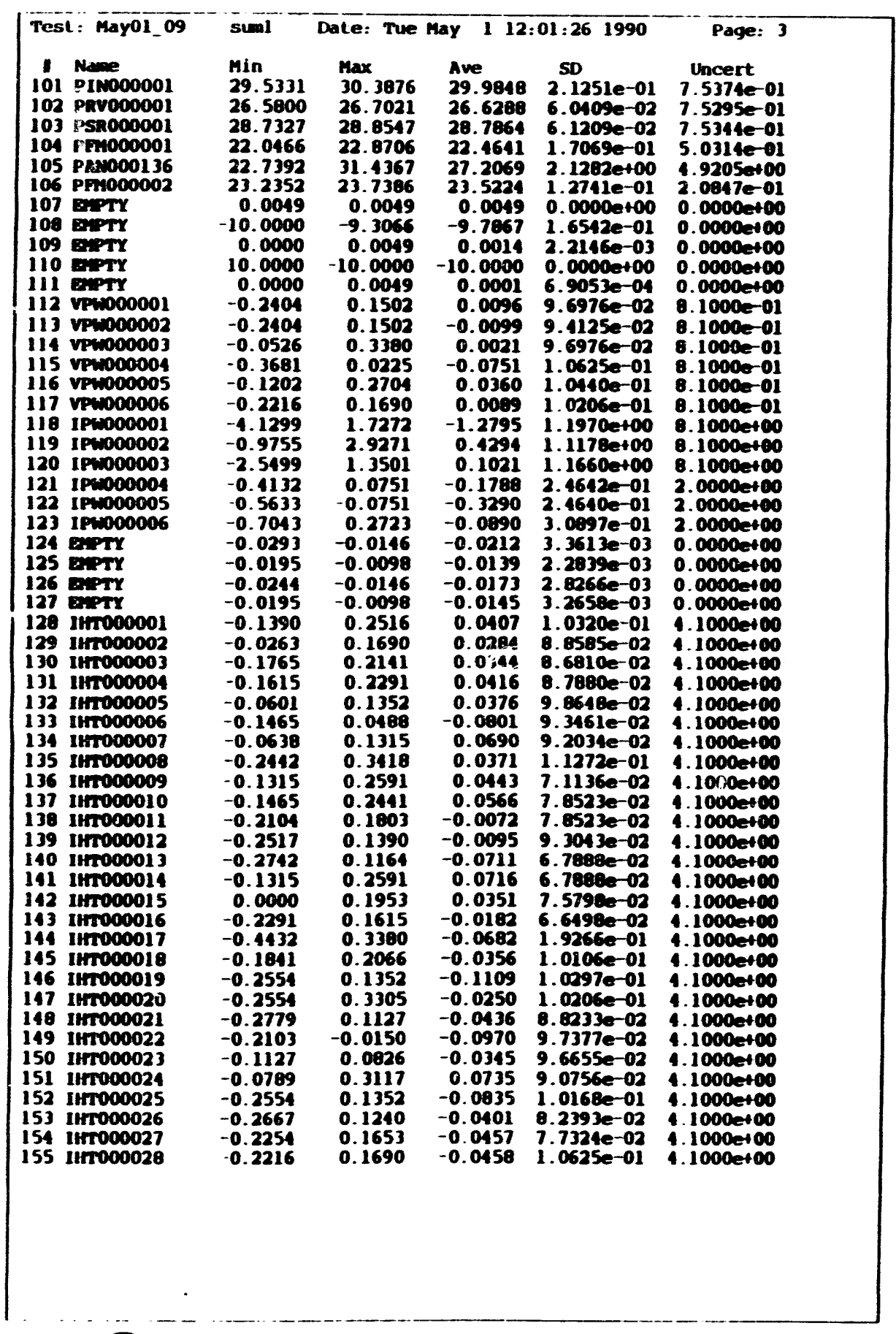

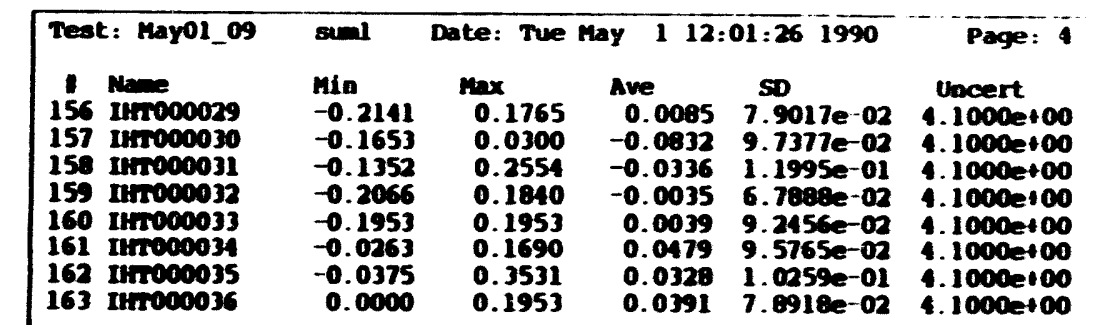

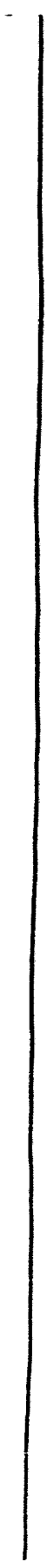




\begin{tabular}{|c|c|c|c|c|c|}
\hline \multicolumn{5}{|c|}{ 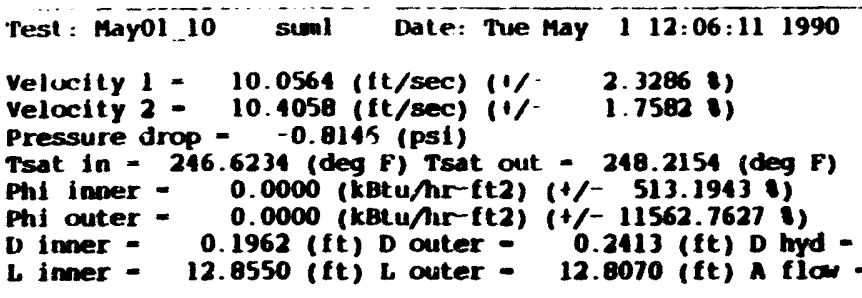 } & $\begin{array}{l}\text { Page: } 1 \\
0.0389 \text { (ft) } \\
0.0150 \text { (ft2) }\end{array}$ \\
\hline 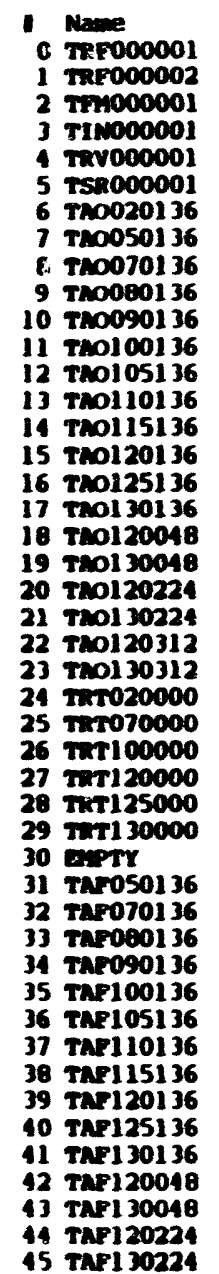 & 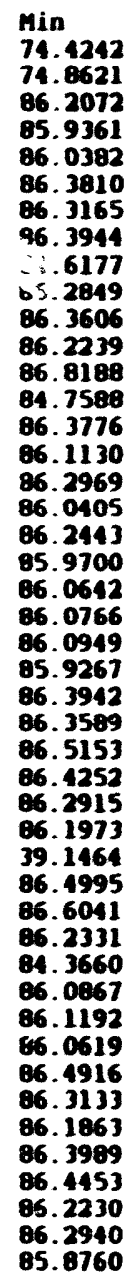 & $\begin{array}{l}\text { Max } \\
75.2754 \\
75.0738 \\
86.9425 \\
86.5672 \\
86.7719 \\
87.0114 \\
87.3677 \\
87.0225 \\
85.5668 \\
86.8098 \\
87.1999 \\
86.9594 \\
67.6602 \\
85.3868 \\
87.3257 \\
86.9501 \\
87.0316 \\
86.6682 \\
86.8755 \\
86.5988 \\
86.8001 \\
86.8003 \\
86.9360 \\
86.7652 \\
87.3414 \\
87.0972 \\
87.3548 \\
87.0563 \\
87.2326 \\
86.9313 \\
10.0646 \\
87.2335 \\
87.7547 \\
86.9652 \\
85.2068 \\
86.8236 \\
87.0592 \\
86.7961 \\
87.3304 \\
86.9434 \\
87.3355 \\
87.1335 \\
87.3951 \\
86.8537 \\
87.0303 \\
86.5089\end{array}$ & 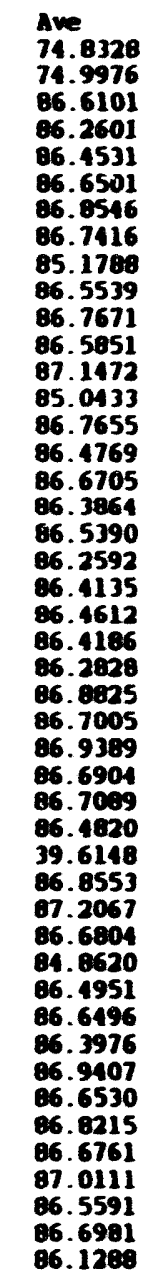 & $\begin{array}{c}50 \\
1.7110 e-01 \\
1.0261 e-01 \\
1.0279 e-01 \\
1.3857 e-01 \\
1.6107 e-01 \\
1.3420 e-01 \\
2.5105 e-01 \\
1.5162 e-01 \\
2.2988 e-01 \\
1.6690 e-01 \\
1.9151 e-01 \\
1.6590 e-01 \\
2.0110 e-01 \\
1.5282 e-01 \\
2.2356 e-01 \\
1.5450 e-01 \\
1.7386 e-01 \\
1.3776 e-01 \\
1.8498 e-01 \\
1.4160 e-01 \\
1.8312 e-01 \\
1.6032 e-01 \\
1.8616 e-01 \\
1.7990 e-01 \\
2.1660 e-01 \\
1.6714 e-01 \\
1.9404 e-01 \\
1.5766 e-01 \\
2.0596 e-01 \\
1.5871 e-01 \\
1.9654 e-01 \\
1.6221 e-01 \\
2.2218 e-01 \\
1.6386 e-01 \\
2.1433 e-01 \\
1.8364 e-01 \\
2.6220 e-01 \\
1.7734 e-01 \\
2.0549 e-01 \\
1.4313 e-01 \\
2.8749 e-01 \\
1.7711 e-01 \\
2.2745 e-01 \\
1.6971 e-01 \\
1.8430 e-01 \\
1.5362 e-01\end{array}$ & $\begin{array}{l}\text { Uncert } \\
1.9000 e+00 \\
1.9000 e+00 \\
1.9002 e+00 \\
1.9002 e+00 \\
1.9002 e+00 \\
1.9002 e+00 \\
1.9002 e+00 \\
1.9002 e+00 \\
1.9002 e+00 \\
1.9002 e+00 \\
1.9002 e+00 \\
1.9002 e+00 \\
1.9002 e+00 \\
1.9002 e+00 \\
1.9002 e+00 \\
1.9002 e+00 \\
1.9002 e+00 \\
1.9002 e+00 \\
1.9002 e+00 \\
1.9002 e+00 \\
1.9002 e+00 \\
1.9002 e+00 \\
1.9002 e+00 \\
1.9002 e+00 \\
1.9002 e+00 \\
1.9002 e+00 \\
1.9002 e+00 \\
1.9002 e+00 \\
1.9002 e+00 \\
1.9002 e+00 \\
1.9011 e+00 \\
1.9002 e+00 \\
1.9002 e+00 \\
1.9002 e+00 \\
1.9001 e+00 \\
1.9002 e+00 \\
1.9002 e+00 \\
1.9002 e+00 \\
1.9002 e+00 \\
1.9002 e+00 \\
1.9002 e+00 \\
1.9002 e+00 \\
1.9002 e+00 \\
1.9002 e+00 \\
1.9002 e+00 \\
1.9002 e+00\end{array}$ \\
\hline
\end{tabular}

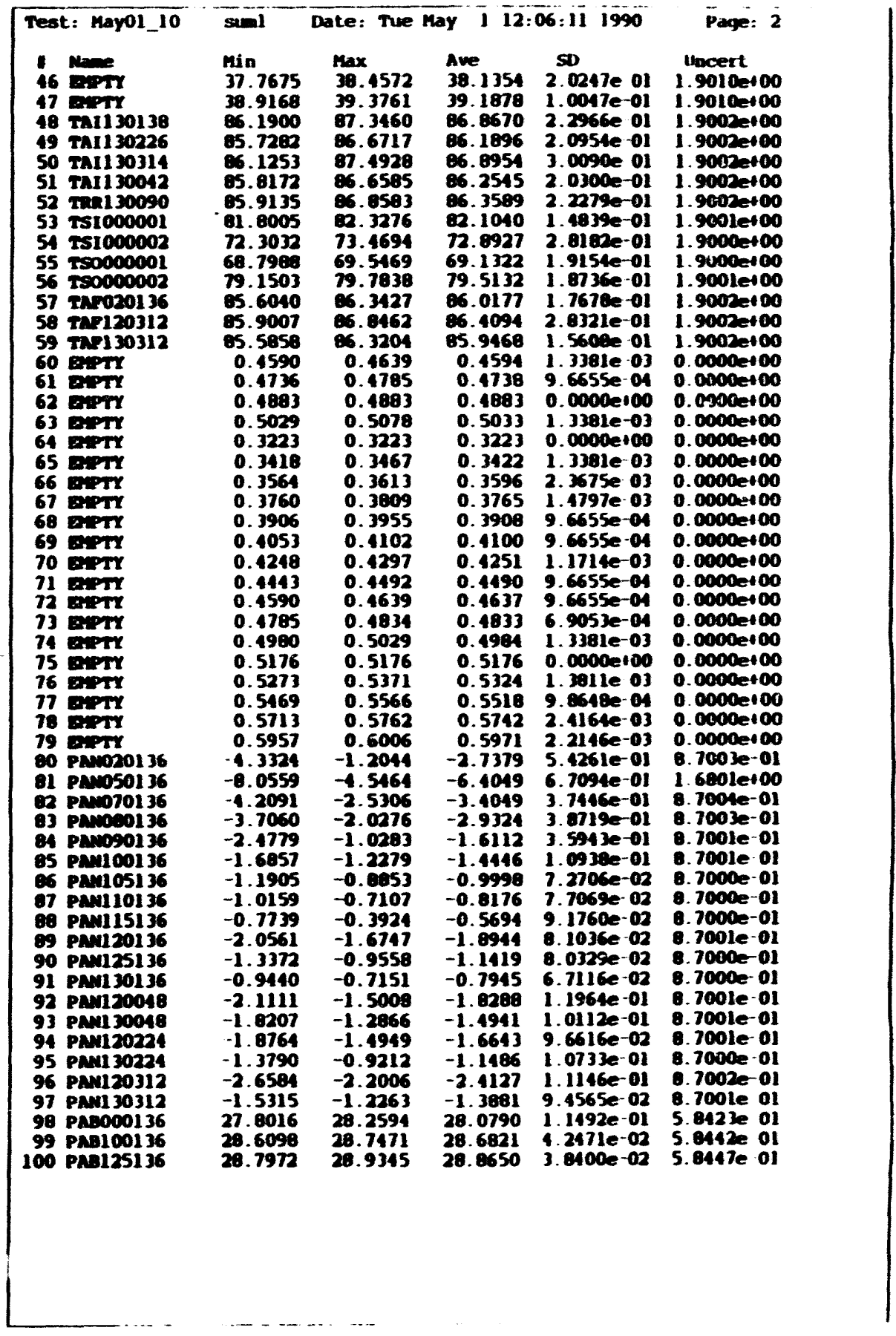




\begin{tabular}{|c|c|c|c|c|c|c|}
\hline $\begin{array}{l}\text { Test } \\
1 \\
101 \\
102 \\
103 \\
104 \\
105 \\
106 \\
107 \\
100 \\
109 \\
110 \\
111 \\
112 \\
113 \\
114 \\
115 \\
116 \\
117 \\
110 \\
119 \\
120 \\
121 \\
122 \\
123 \\
124 \\
125 \\
126 \\
127 \\
120 \\
129 \\
130 \\
131 \\
132 \\
133 \\
134 \\
135 \\
136 \\
137 \\
130 \\
139 \\
110 \\
111 \\
112 \\
143 \\
144 \\
145 \\
140 \\
147 \\
140 \\
149 \\
150 \\
151 \\
152 \\
153 \\
154 \\
155\end{array}$ & 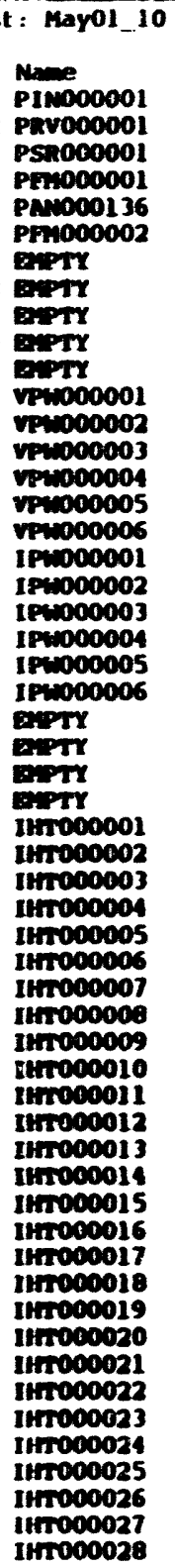 & $\begin{array}{l}\text { sull } \\
\text { Min } \\
27.5600 \\
26.5000 \\
24.1327 \\
15.1802 \\
26.6993 \\
16.2044 \\
0.0000 \\
10.0000 \\
0.0000 \\
10.0000 \\
0.0000 \\
-0.0451 \\
-0.2404 \\
-0.2479 \\
-0.3681 \\
-0.1202 \\
-0.2216 \\
-2.1775 \\
-2.9267 \\
-2.5499 \\
-0.9015 \\
-1.0516 \\
-0.7043 \\
-0.0244 \\
-0.0195 \\
-0.0195 \\
-0.0195 \\
-0.1390 \\
-0.0263 \\
0.0180 \\
-0.1615 \\
-0.0601 \\
-0.1465 \\
-0.0638 \\
-0.0480 \\
-0.1315 \\
-0.1465 \\
-0.2104 \\
-0.2517 \\
-0.2742 \\
-0.1315 \\
-0.1953 \\
-0.0338 \\
-0.2479 \\
-0.1941 \\
-0.2554 \\
-0.0601 \\
-0.2779 \\
-0.2103 \\
-0.1127 \\
-0.0789 \\
-0.2554 \\
-0.2667 \\
-0.2254 \\
-0.2216\end{array}$ & $\begin{array}{c}\text { Date: Tue } \\
\text { Max } \\
28.1345 \\
26.9462 \\
2 . .8517 \\
15.6380 \\
-19.3751 \\
16.8421 \\
0.0049 \\
-9.2529 \\
0.0049 \\
-10.0000 \\
0.0000 \\
0.1502 \\
0.1502 \\
0.1427 \\
0.0225 \\
0.2704 \\
0.1690 \\
-0.2252 \\
2.9271 \\
1.3501 \\
0.0751 \\
0.9014 \\
0.2723 \\
-0.0146 \\
-0.0098 \\
-0.0098 \\
-0.0098 \\
0.2516 \\
0.1690 \\
0.2141 \\
0.2291 \\
0.3305 \\
0.0488 \\
0.1315 \\
0.1465 \\
0.2591 \\
0.2141 \\
0.1803 \\
0.1390 \\
0.1164 \\
0.2591 \\
0.1953 \\
0.1615 \\
0.3360 \\
0.2066 \\
0.1352 \\
0.3305 \\
0.1127 \\
-0.0150 \\
0.2780 \\
0.3117 \\
0.1352 \\
0.1240 \\
0.1653 \\
0.1690\end{array}$ & $\begin{aligned} \text { May } 112: \\
\text { Ave } \\
27.9023 \\
26.6654 \\
28.7400 \\
15.4219 \\
-22.5520 \\
16.5226 \\
0.0046 \\
-9.5650 \\
0.0003 \\
-10.0000 \\
0.0000 \\
0.0057 \\
-0.0217 \\
-0.0096 \\
-0.0751 \\
0.0400 \\
-0.0185 \\
-1.3185 \\
0.5075 \\
0.0241 \\
-0.2667 \\
-0.3778 \\
-0.0793 \\
-0.0202 \\
-0.0140 \\
-0.0169 \\
-0.0147 \\
0.0524 \\
0.0516 \\
0.0383 \\
0.0377 \\
0.0259 \\
-0.1035 \\
0.0573 \\
0.0050 \\
0.0560 \\
0.0566 \\
-0.0111 \\
-0.0173 \\
-0.0554 \\
0.0795 \\
0.0273 \\
-0.0221 \\
-0.0643 \\
-0.0356 \\
-0.1070 \\
-0.0132 \\
-0.0514 \\
-0.1166 \\
-0.0580 \\
0.0656 \\
-0.0674 \\
-0.0518 \\
-0.0300 \\
-0.0693\end{aligned}$ & 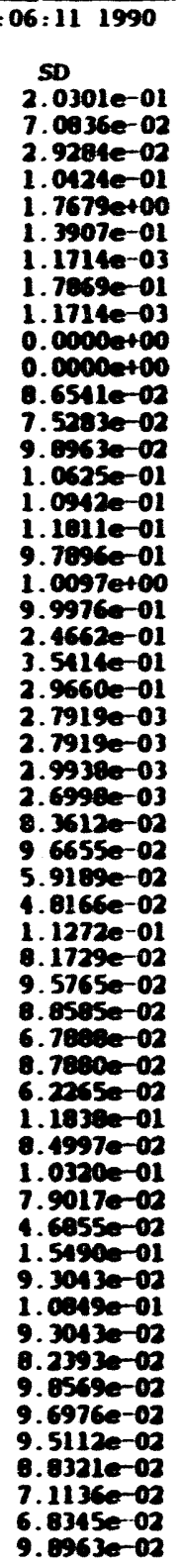 & 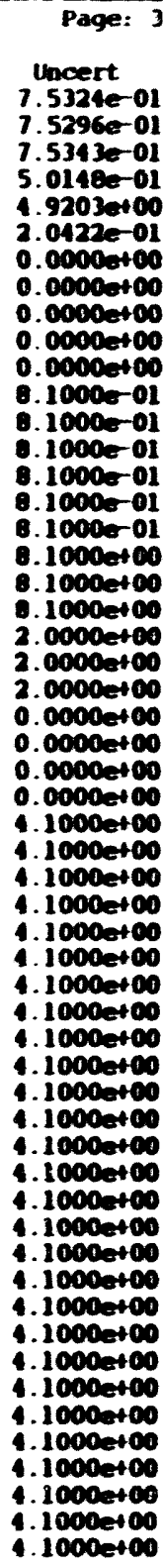 \\
\hline
\end{tabular}

\begin{tabular}{|c|c|c|c|c|c|}
\hline Test: Mayol_10 & $\sin$ & Date: Tue & ay 12: & $06: 11 \quad 1990$ & Page: 4 \\
\hline 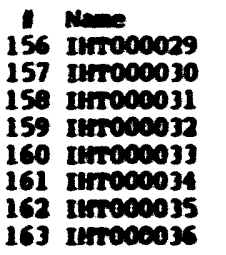 & $\begin{array}{l}\text { Min } \\
-0.2141 \\
-0.1653 \\
-0.1353 \\
-0.2066 \\
-0.1953 \\
-0.0263 \\
-0.2329 \\
-0.1953\end{array}$ & $\begin{array}{l}\operatorname{Max} \\
0.3718 \\
0.0300 \\
0.2554 \\
0.1840 \\
0.1953 \\
0.1690 \\
0.1578 \\
0.1953\end{array}$ & $\begin{array}{r}\text { Ave } \\
0.0164 \\
-0.0832 \\
-0.0415 \\
-0.0230 \\
-0.0117 \\
0.0128 \\
0.0289 \\
0.0156\end{array}$ & $\begin{array}{l}\text { So } \\
9.4125 e-02 \\
9.7377 e-02 \\
1.1327 e-01 \\
9.1779 e-02 \\
8.2064 e-02 \\
7.0916 e-02 \\
1.0145 e-01 \\
8.6010=-02\end{array}$ & $\begin{array}{l}\text { Uncert } \\
1.1000=+00 \\
1.1000=+00 \\
1.1000=+00 \\
1.1000=+00 \\
1.1000=+00 \\
1.1000=+00 \\
1.1000=+00 \\
4.1000=+00\end{array}$ \\
\hline
\end{tabular}



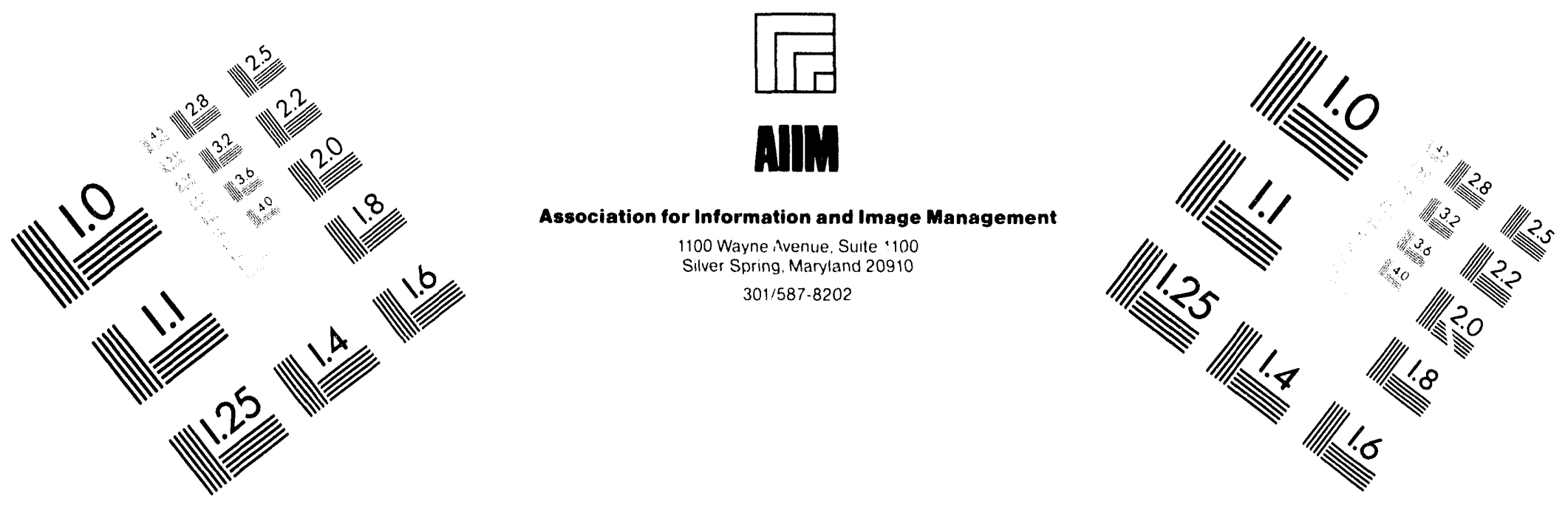

\section{Centimeter}

$\left.\right|_{1}$ Inches
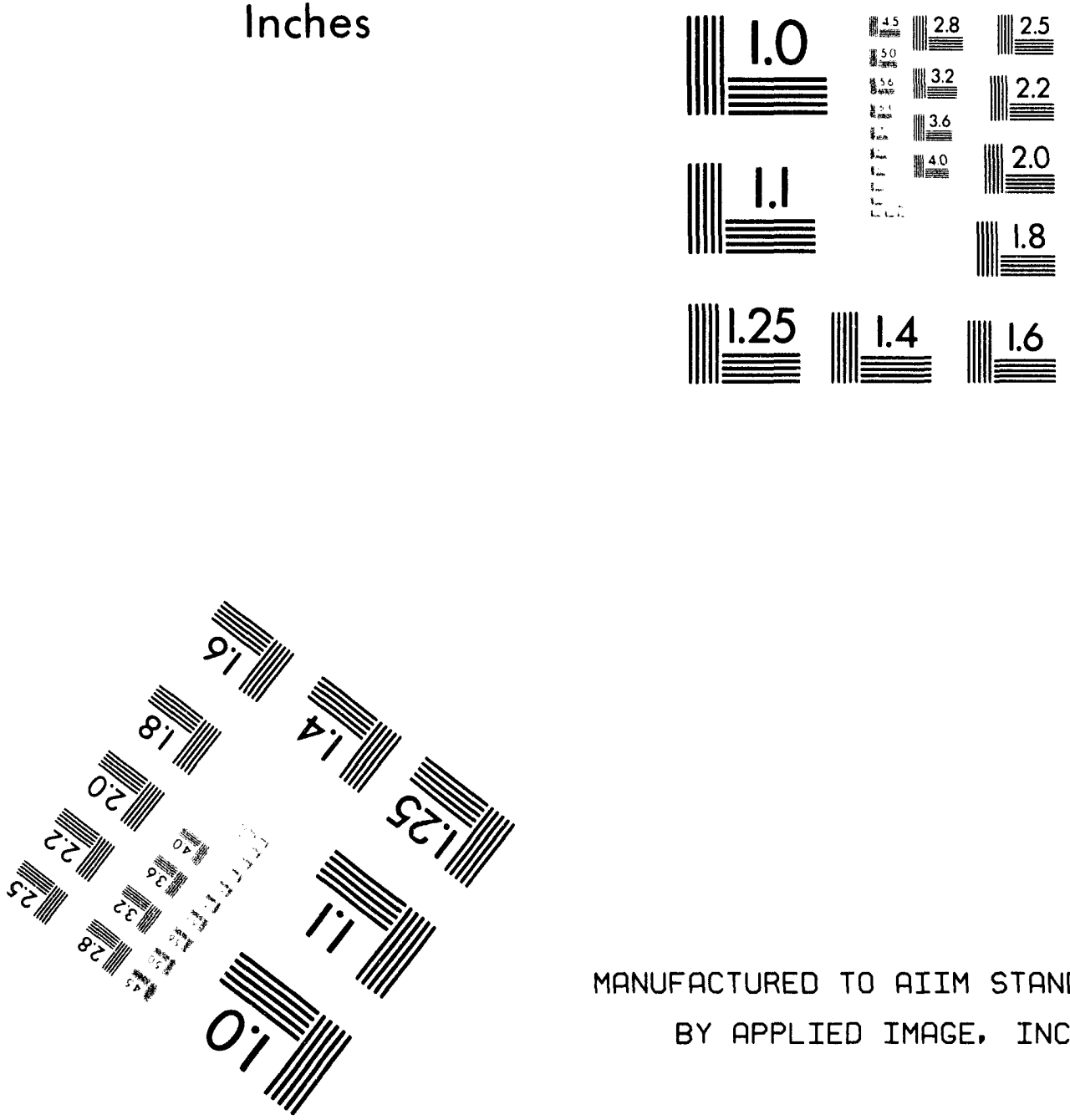

MANUFACTURED TO AIIM STANDARDS

BY APPLIED IMAGE, INC.

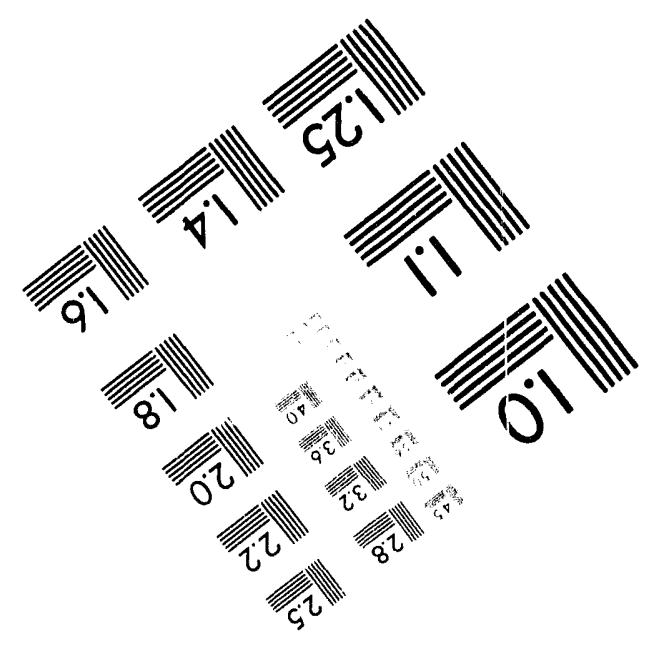



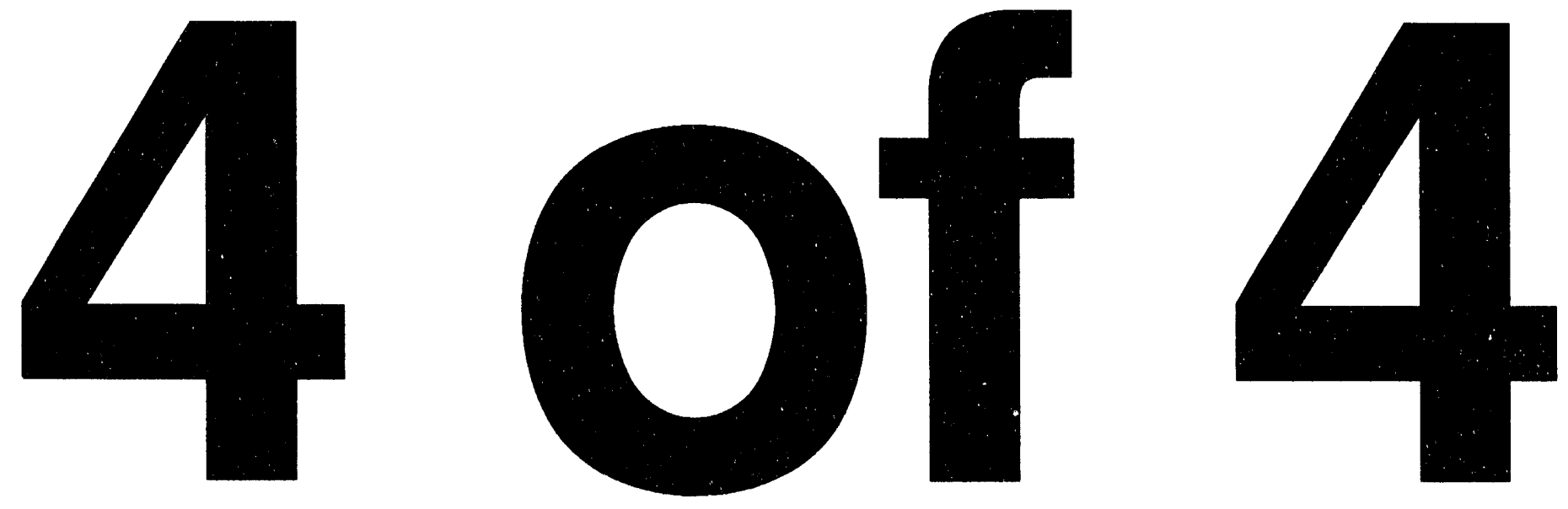


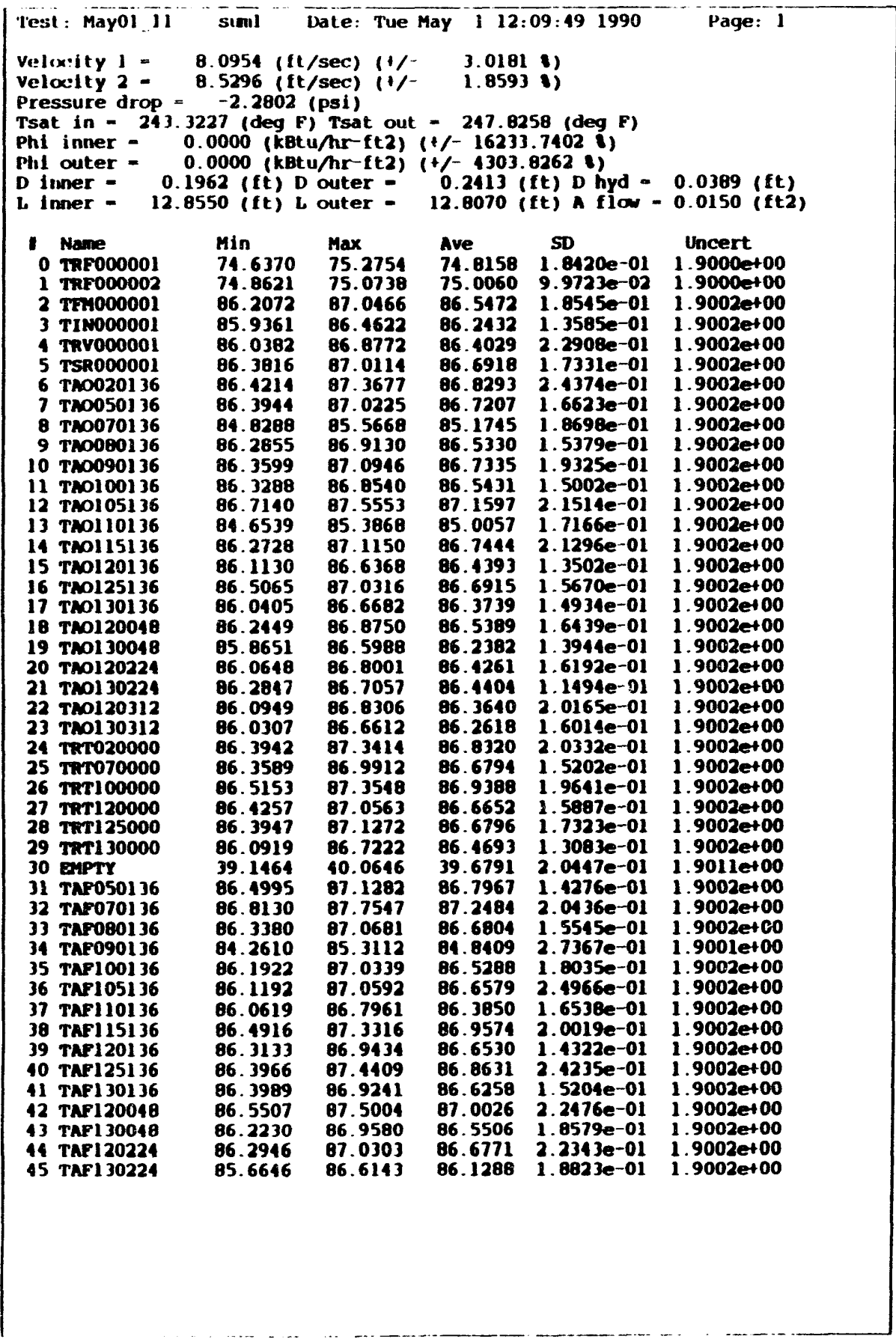

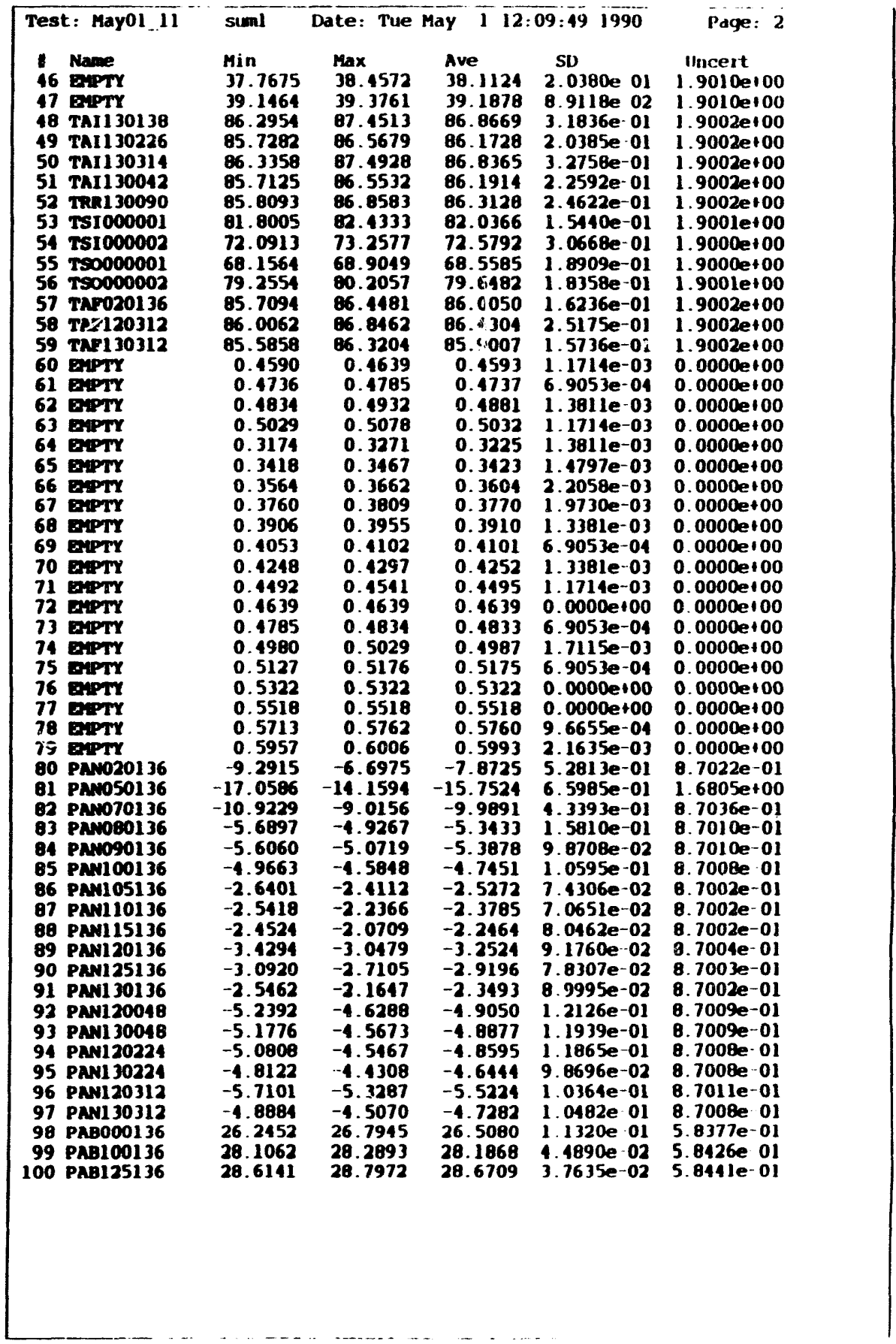




\begin{tabular}{|c|c|c|c|c|c|c|}
\hline \multicolumn{2}{|c|}{ Test : May01 11} & $\begin{array}{l}\text { sionl } \\
\text { Min }\end{array}$ & Date: Tue & \multicolumn{2}{|c|}{ May 1 12:09:49 1990} & $\begin{array}{c}\text { Page: } 3 \\
\text { Uncert }\end{array}$ \\
\hline $\begin{array}{l}10 \\
101 \\
102 \\
103 \\
104 \\
105 \\
106 \\
107 \\
108 \\
109 \\
110 \\
111 \\
112 \\
113 \\
114 \\
115 \\
116 \\
117 \\
118 \\
119 \\
120 \\
121 \\
122 \\
123 \\
124 \\
125 \\
126 \\
127 \\
128 \\
129 \\
130 \\
131 \\
132 \\
133 \\
134 \\
135 \\
136 \\
137 \\
138 \\
139 \\
140 \\
141 \\
142 \\
143 \\
144 \\
145 \\
146 \\
147 \\
148 \\
149 \\
150 \\
151 \\
152 \\
153 \\
154 \\
155 \\
\end{array}$ & 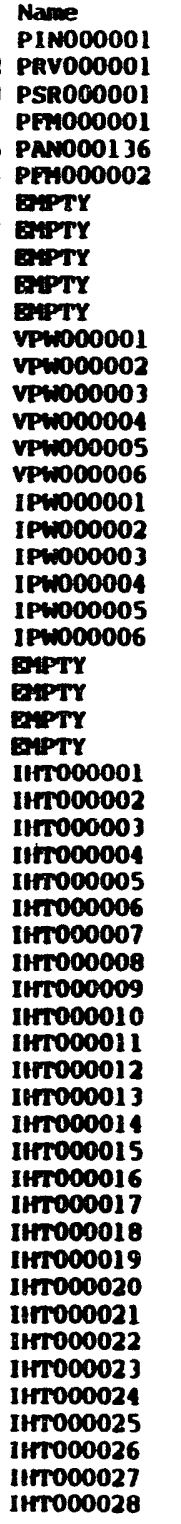 & $\begin{array}{r}\text { Min } \\
25.9931 \\
26.5800 \\
28.6106 \\
9.6870 \\
68.3558 \\
10.6671 \\
-0.0049 \\
10.0000 \\
0.0000 \\
-10.0000 \\
0.0000 \\
-0.2404 \\
-0.2404 \\
-0.2479 \\
-0.3681 \\
-0.1202 \\
-0.2216 \\
-1.1299 \\
-0.9755 \\
-2.5499 \\
-0.9015 \\
-0.5633 \\
-0.7043 \\
-0.0293 \\
-0.0195 \\
-0.0244 \\
-0.0195 \\
-0.1390 \\
-0.2216 \\
0.0188 \\
-0.1615 \\
-0.0601 \\
-0.1465 \\
-0.0638 \\
-0.0488 \\
-0.1315 \\
-0.1465 \\
-0.2104 \\
-0.2517 \\
-0.2742 \\
-0.1315 \\
-0.1953 \\
-0.2291 \\
-0.4432 \\
-0.3794 \\
-0.2554 \\
-0.0601 \\
-0.2779 \\
-0.2103 \\
-0.3080 \\
-0.0789 \\
-0.2554 \\
-0.2667 \\
-0.2254 \\
-0.2216\end{array}$ & $\begin{array}{r}\operatorname{Max} \\
26.8476 \\
26.7021 \\
28.8547 \\
10.2363 \\
-57.3695 \\
11.3886 \\
0.0049 \\
-9.1504 \\
0.0049 \\
-10.0000 \\
0.0000 \\
0.1502 \\
0.1502 \\
0.1427 \\
0.0225 \\
0.2704 \\
0.1690 \\
1.7272 \\
2.9271 \\
3.3001 \\
0.0751 \\
0.1131 \\
0.7606 \\
-0.0146 \\
-0.0098 \\
-0.0098 \\
-0.0098 \\
0.2516 \\
0.1690 \\
0.2111 \\
0.2291 \\
0.1352 \\
0.0488 \\
0.1315 \\
0.1465 \\
0.2591 \\
0.2441 \\
0.1803 \\
0.1390 \\
0.1169 \\
0.2591 \\
0.3906 \\
0.1615 \\
0.1427 \\
0.2066 \\
0.1352 \\
0.1352 \\
0.1127 \\
-0.0150 \\
0.0826 \\
0.3117 \\
0.1352 \\
0.1240 \\
-0.0300 \\
0.1690\end{array}$ & $\begin{array}{r}\text { Ave } \\
26.3251 \\
26.6215 \\
28.7620 \\
9.9489 \\
-63.1281 \\
11.0577 \\
0.0030 \\
-9.4775 \\
0.0002 \\
-10.0000 \\
0.0000 \\
0.0213 \\
-0.0295 \\
-0.0214 \\
-0.0634 \\
0.0360 \\
0.0323 \\
-1.4747 \\
0.2733 \\
0.0241 \\
-0.2001 \\
-0.2704 \\
-0.0305 \\
-0.0214 \\
-0.0141 \\
-0.0175 \\
-0.0146 \\
0.0485 \\
0.0167 \\
0.0383 \\
0.0494 \\
0.0102 \\
-0.1231 \\
0.0416 \\
0.0215 \\
000443 \\
00.0410 \\
-0.0072 \\
-0.0251 \\
-0.0632 \\
0.0560 \\
0.0091 \\
-0.0221 \\
-0.0917 \\
-0.0512 \\
-0.1226 \\
0.0024 \\
-0.0279 \\
-0.1088 \\
-0.0345 \\
0.0696 \\
-0.0992 \\
-0.0675 \\
-0.0652 \\
-0.0302\end{array}$ & $\begin{array}{l}\text { SD } \\
1.9268 e-01 \\
5.0413 e-02 \\
5.8152 e-02 \\
1.1088 e-01 \\
2.0179 e+00 \\
1.6639 e-01 \\
2.5894 e-03 \\
2.0959 e-01 \\
9.6655 e-04 \\
0.0000 e+00 \\
0.0000 e+00 \\
1.0145 e-01 \\
7.7334 e-02 \\
9.1354 e-02 \\
1.0559 e-01 \\
8.8233 e-02 \\
1.0625 e-01 \\
1.0988 e+00 \\
1.0250 e+00 \\
1.3325 e+00 \\
2.6267 e-01 \\
2.7899 e-01 \\
2.9413 e-01 \\
3.6765 e-03 \\
2.5420 e-03 \\
2.8058 e-03 \\
3.1195 e-03 \\
9.6332 e-02 \\
9.0756 e-02 \\
5.9189 e-02 \\
6.6498 e-02 \\
9.1702 e-02 \\
6.1113 e-02 \\
9.8332 e-02 \\
9.1702 e-02 \\
7.1136 e-02 \\
7.8523 e-02 \\
7.8523 e-02 \\
9.9512 e-02 \\
8.6810 e-02 \\
1.0410 e-01 \\
1.1161 e-01 \\
6.1257 e-02 \\
1.2478 e-01 \\
1.2750 e-01 \\
1.0763 e-01 \\
9.2034 e-02 \\
9.6976 e-02 \\
9.8569 e-02 \\
1.0440 e-01 \\
1.0106 e-01 \\
8.8233 e-02 \\
6.2265 e-02 \\
7.5798 e-02 \\
1.0052 e-01\end{array}$ & 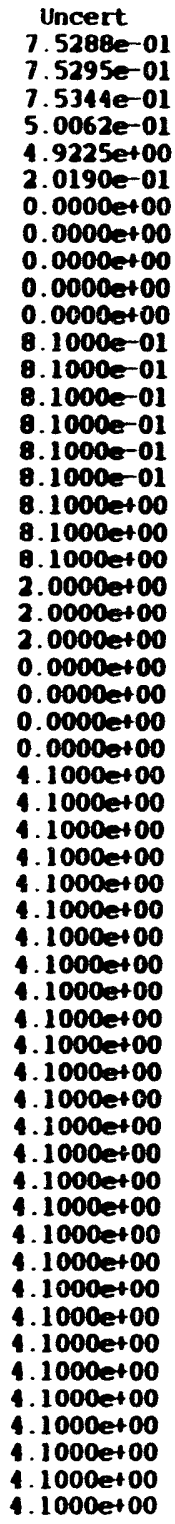 \\
\hline
\end{tabular}

\begin{tabular}{|c|c|c|c|c|c|}
\hline Test: May01_11 & sin & Date: Tue & $\operatorname{May} 112$ & $09: 491990$ & Page: \\
\hline 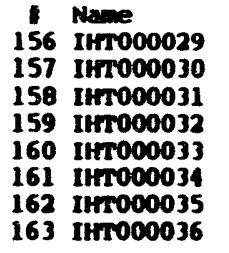 & $\begin{array}{l}\text { Min } \\
-0.2141 \\
-0.1653 \\
-0.1352 \\
-0.2066 \\
-0.1953 \\
-0.2216 \\
-0.2329 \\
0.0000\end{array}$ & $\begin{array}{l}\operatorname{Max} \\
0.3718 \\
0.2253 \\
0.2554 \\
0.1840 \\
0.1953 \\
0.3643 \\
0.1578 \\
0.1953\end{array}$ & $\begin{array}{l}\text { Ave } \\
0.0085 \\
-0.0598 \\
-0.0258 \\
-0.0152 \\
-0.0117 \\
0.0557 \\
0.0171 \\
0.0273\end{array}$ & $\begin{array}{l}\text { SD } \\
8.8321 e-02 \\
1.1975 \mathrm{e}-01 \\
1.1942 \mathrm{e}-01 \\
8.3612 \mathrm{e}-02 \\
9.9902 \mathrm{e}-02 \\
1.1897 \mathrm{e}-01 \\
9.6976 \mathrm{e}-02 \\
6.8459 \mathrm{e}-02\end{array}$ & $\begin{array}{l}\text { Uncert } \\
4.1000 \text { et } 00 \\
4.1000 \text { et } 00 \\
4.1000 \text { et } 00 \\
4.1000 \text { e t } 00 \\
4.1000 \text { e t } 00 \\
4.1000 \text { et } 00 \\
4.1000 \text { et } 00 \\
4.1000 \text { et } 00\end{array}$ \\
\hline
\end{tabular}




\begin{tabular}{|c|c|c|c|c|c|c|}
\hline $\begin{array}{l}\text { Test } \\
\text { Velu } \\
\text { Velo } \\
\text { Pres } \\
\text { Tsat } \\
\text { Phi } \\
\text { PhI } \\
\text { D in } \\
\text { L in }\end{array}$ & $\begin{array}{l}\text { May01 } 12 \\
\text { xity } 1= \\
\text { xity } 2= \\
\text { sure drop } \\
\text { in }=240 \\
\text { inner }= \\
\text { outer }= \\
\text { mer }=0 \\
\text { mer }=12 .\end{array}$ & $\begin{array}{l}\text { siml } \\
6.1398 \text { (ft } \\
6.7423 \text { ift } \\
-3.4908 \\
1684 \text { (deg } \\
0.0000 \text { (kBt } \\
0.0000 \text { (kBt } \\
1962 \text { (ft) } \\
8550 \text { (ft) }\end{array}$ & $\begin{array}{l}\text { Date: Tue } \\
\text { (sec) (1/ } \\
\text { (sec) (1/ } \\
\text { (psi) } \\
\text { Tsat out } \\
\text { (mr-ft2) } \\
\text { i/hr-ft2) } \\
\text { outer = } \\
\text { outer : }\end{array}$ & $\begin{array}{r}\text { May } 112 \text { : } 4.7077 \\
2.1240 \\
=247.30 \\
1+1 /-18071 \\
1+8019 \\
0.2413 ; \\
12.8070\end{array}$ & $\begin{array}{l}: 12: 281990 \\
(1) \\
0 \text { i) } \\
077 \text { (deg F) } \\
(.2715 \text { (s) } \\
9341 \text { () } \\
\text { (f() D hyd = } \\
\text { (ft) A flow - }\end{array}$ & $\begin{array}{l}0.0389 \text { (ft) } \\
0.0150 \text { (ft2) }\end{array}$ \\
\hline $\begin{array}{l}1 \\
0 \\
1 \\
2 \\
3 \\
4 \\
5 \\
6 \\
7 \\
8 \\
9 \\
10 \\
11 \\
12 \\
13 \\
14 \\
15 \\
16 \\
17 \\
18 \\
19 \\
20 \\
21 \\
22 \\
23 \\
24 \\
25 \\
26 \\
27 \\
28 \\
29 \\
30 \\
31 \\
32 \\
33 \\
34 \\
35 \\
36 \\
37 \\
38 \\
39 \\
40 \\
11 \\
12 \\
13 \\
14 \\
45\end{array}$ & 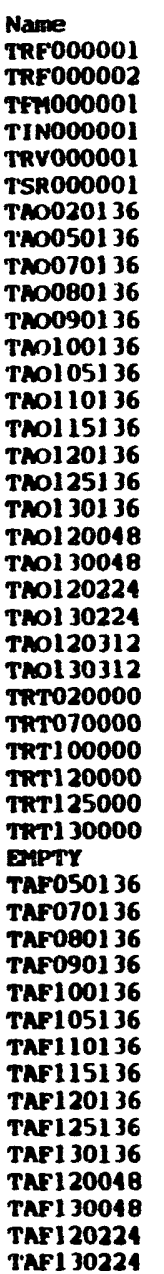 & $\begin{array}{l}\text { Min } \\
74.6370 \\
74.8621 \\
86.3120 \\
86.0409 \\
86.0382 \\
86.4870 \\
86.4214 \\
86.3944 \\
84.8288 \\
86.2855 \\
86.3599 \\
86.3288 \\
86.8194 \\
84.7588 \\
86.2728 \\
86.1130 \\
86.5065 \\
85.9386 \\
86.2449 \\
85.9700 \\
86.0648 \\
86.1844 \\
86.0949 \\
86.0307 \\
86.4990 \\
86.4638 \\
86.6201 \\
86.4257 \\
86.3947 \\
86.1967 \\
399.1464 \\
86.3941 \\
86.9178 \\
86.4114 \\
84.4709 \\
86.1922 \\
86.2240 \\
86.0619 \\
86.4916 \\
86.4181 \\
86.3966 \\
86.3989 \\
86.6561 \\
86.2230 \\
86.3994 \\
85.7695\end{array}$ & $\begin{array}{l}\text { Max } \\
75.0626 \\
75.2854 \\
87.0466 \\
86.6726 \\
86.8767 \\
87.2196 \\
87.3677 \\
87.2311 \\
85.6717 \\
86.9146 \\
87.0946 \\
86.8540 \\
87.6602 \\
85.3868 \\
87.3251 \\
86.8426 \\
87.0316 \\
86.6677 \\
86.9798 \\
86.5988 \\
86.9049 \\
86.7051 \\
86.8306 \\
86.5558 \\
87.4462 \\
86.9907 \\
87.3542 \\
87.0557 \\
87.0236 \\
86.8260 \\
40.0646 \\
87.2329 \\
87.7547 \\
87.0700 \\
85.5216 \\
87.0333 \\
87.1640 \\
86.7961 \\
87.5405 \\
86.9428 \\
87.3355 \\
87.0288 \\
87.3951 \\
86.8532 \\
87.1350 \\
86.5078\end{array}$ & $\begin{array}{l}\text { Ave } \\
74.8966 \\
75.0695 \\
86.7109 \\
86.3863 \\
86.4953 \\
86.7925 \\
86.8924 \\
86.8339 \\
85.2377 \\
86.6211 \\
86.7756 \\
86.6104 \\
87.2228 \\
85.1064 \\
86.8412 \\
86.5150 \\
86.7504 \\
86.4000 \\
86.5769 \\
86.3013 \\
86.4892 \\
86.4952 \\
86.4775 \\
86.3124 \\
86.9456 \\
86.7551 \\
87.0228 \\
86.7409 \\
86.7428 \\
86.5157 \\
39.6286 \\
86.8974 \\
87.2781 \\
86.7518 \\
84.9503 \\
86.5792 \\
86.7335 \\
86.1439 \\
86.9869 \\
86.7287 \\
86.8345 \\
86.6973 \\
87.0656 \\
86.6053 \\
86.7696 \\
86.2088\end{array}$ & $\begin{array}{l}\text { SD } \\
1.6810 e-01 \\
1.2457 e-01 \\
1.6940 e-01 \\
1.4730 e-01 \\
2.1231 e-01 \\
1.4645 e-01 \\
2.1890 e-01 \\
1.7954 e-01 \\
2.0390 e-01 \\
1.6661 e-01 \\
1.9869 e-01 \\
1.3160 e-01 \\
2.0188 e-01 \\
1.5494 e-01 \\
2.2409 e-01 \\
1.6011 e-01 \\
1.4918 e-01 \\
1.6882 e-01 \\
1.6208 e-01 \\
1.3646 e-01 \\
1.8252 e-01 \\
1.3728 e-01 \\
1.7603 e-01 \\
1.5365 e-01 \\
2.1638 e-01 \\
1.4206 e-01 \\
2.0137 e-01 \\
1.5150 e-01 \\
1.7222 e-01 \\
1.4685 e-01 \\
2.0969 e-01 \\
1.9412 e-01 \\
2.1040 e-01 \\
1.4485 e-01 \\
2.7352 e-01 \\
2.0092 e-01 \\
2.2647 e-01 \\
1.5400 e-01 \\
2.2525 e-01 \\
1.4382 e-01 \\
2.5040 e-01 \\
1.7011 e-01 \\
1.5618 e-01 \\
1.7842 e-01 \\
2.0050 e-01 \\
1.9097 e-01\end{array}$ & 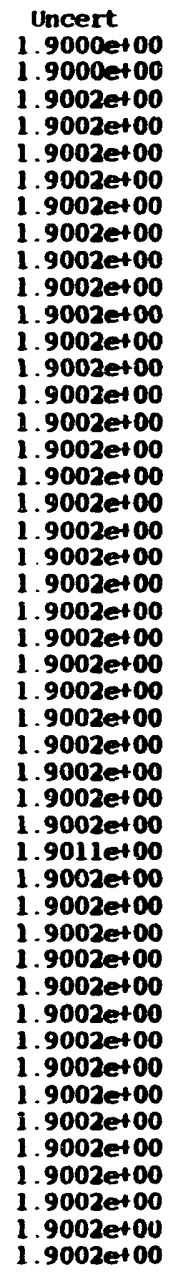 \\
\hline
\end{tabular}

\begin{tabular}{|c|c|}
\hline $\begin{array}{l}\text { 1est } \\
1 \\
46 \\
47 \\
48 \\
49 \\
50 \\
51 \\
52 \\
53 \\
54 \\
55 \\
56 \\
57 \\
58 \\
59 \\
60 \\
61 \\
62 \\
63 \\
64 \\
65 \\
66 \\
67 \\
68 \\
69 \\
70 \\
71 \\
72 \\
73 \\
74 \\
75 \\
76 \\
77 \\
76 \\
79 \\
80 \\
81 \\
82 \\
83 \\
84 \\
85 \\
86 \\
87 \\
88 \\
89 \\
90 \\
91 \\
92 \\
93 \\
94 \\
95 \\
96 \\
97 \\
98 \\
99 \\
00\end{array}$ & 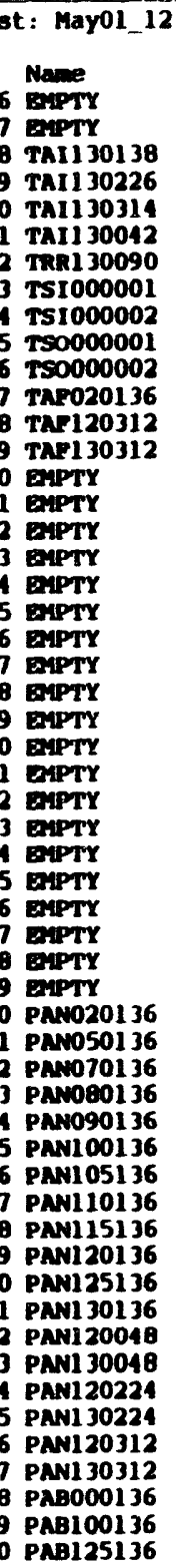 \\
\hline
\end{tabular}

suml Date: Tue May 1 12:12:28 1990 Page: 2

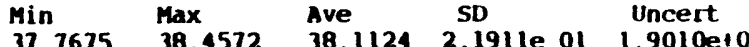
$\begin{array}{lllll}37.7675 & 38.4572 & 38.1124 & 2.1911 \text { e } 01 & 1.9010 e 100\end{array}$ $38.9168 \quad 39.3761 \quad 39.1786$ 9.2905e-02 $1.9010 \mathrm{e}+00$ $\begin{array}{llllll}86.2954 & 87.4507 & 86.9048 & 3.0146 e-01 & 1.9002 e 100\end{array}$ $\begin{array}{llllll}85.9376 & 86.7765 & 86.2652 & 1.9477 e-01 & 1.9002 e+00\end{array}$ $\begin{array}{llllll}86.3358 & 87.4928 & 86.9668 & 3.0205 e & 01 & 1.9002 e+00\end{array}$ $\begin{array}{lllll}85.9221 & 86.7632 & 86.3134 & 2.1133 e-01 & 1.9002 e+00\end{array}$ 91.5901 $02.4328 \quad 02.03691 .54400-01 \quad 1.90010100$

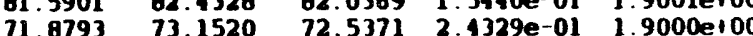
$68.0486 \quad 68.797268 .37831 .9782 \mathrm{e}-01 \quad 1.9000+00$ $79.2554 \quad 79.9948 \quad 79.5893 \quad 1.7119 e-01 \quad 1.9001 e t 00$ 85.8148 06.0062 85.5858 0.4590 0.4736
0.4834 0.1834
0.5029 0.3174 0.3418 0.3760 0.3906 0.4053 0.1248 0.1492 0.4639 0.4785 0.5127 0.5273 0.5713

014.0406

16.0346 -8.3600
-7.8948 $-7.8948$

$-7.6366$ $\begin{array}{llll}86.4389 & 2.6022 \mathrm{e}-01 & 1.9002 \mathrm{e}+00\end{array}$ $86.3204 \quad 86.0057 \quad 1.9198 \mathrm{e}-01 \quad 1.9002 \mathrm{e}+00$

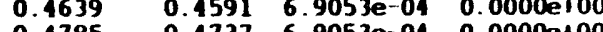
0.48530 .47376 .9053 e 040.0000 et 00 $0.48070 .48819 .6655 \mathrm{e}-04 \quad 0.0000 \mathrm{e} 100$ 0.32710 .5220 . 0.34670 .34196 .5314050 .040 .0000100 $0.36130 .3572 \quad 1.00820-030.00000400$ $0.38090 .3761 \quad 6.9053 \mathrm{e}-04 \quad 0.0000 \mathrm{e} 100$ $0.3955 \quad 0.3907 \quad 6.9053 e^{-04} \quad 0.0000 e+00$ $\begin{array}{llll}0.4099 & 1.1714 e^{-03} & 0.0000 e+00\end{array}$ $\begin{array}{llll}4248 & 0.4248 & 0.0000 \text { et00 } & 0.0000 \text { et } 00 \\ 0.1492 & 0.4492 & 0.0000 e+00 & 0.0000 e+00\end{array}$ $\begin{array}{llll}0.4639 & 0.4638 & 6.9053 e-04 & 0.0000 e+00\end{array}$ $0.4834 \quad 0.48329 .6655 e-040.0000 e+00$ 0.49000 .49800 .0000 eroo 0.0000 et00 $\begin{array}{llll}0.5322 & 0.5168 & 1.8082 e-03 & 0.0000 e t 00\end{array}$ $0.55180 .5516 \quad 0.6655 e^{-04} 0.0000100$ $0.5762 \quad 0.5723 \quad 1.9730030 .0000400$ 0.60060 .59641 .71150030 .0000100 $-11.5803-12.2090 \quad 3.4416 e .01 \quad 8.7054001$ $-22.7044-23.9098,1042 e \cdot 01,681$ let00 $\begin{array}{lllll}-14.5850 & -15.2808 & 2.8573 e-01 & 8.7084 e & 01\end{array}$

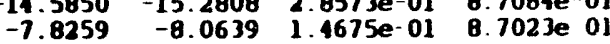

$-3.9914-3.7845$

$-3.8257$ -3.7625
-3.5205 -3.5205
-4.4212 $-4.4653 \quad-4.1601$ $\begin{array}{ll}-3.8432 & -3.5380 \\ -7.6043 & -7.2228\end{array}$

$-7.6953-7.3138$ 7.8273 $-7.3138$ $-7.6351-7.2536$ $-7.4061 \quad-7.1773$ -7.
24.8719 $\begin{array}{llllll}27.6027 & 27.7858 & 27.6942 & 4.7157 e 02 & 5.8412 e-0 \\ 28.3395 & 28.4768 & 28.4145 & 4.2138 e^{-02} & 5.8433 e^{-0}\end{array}$ -7.1773
25.2381
27.7858 $-7.76817 .1653 e^{-02}$ B.7022e 01 $-7.4398 \quad 8.8666 \mathrm{e}-02 \quad 8.7020 \mathrm{e} 0$ $\begin{array}{llll}-3.8898 & 6.3346 \mathrm{e}-02 & \mathbf{8} .7005 \mathrm{e}-0\end{array}$ $-3.8403 \quad 6.6279 e-02 \quad 8.7005 e-01$ $-3.6273 \quad 8.3005$ e- 02 B. 7005 e 01 $-4.3188 \quad 6.3253 \mathrm{e}-02 \quad 8.7007 \mathrm{e}-01$ $\begin{array}{lll}-3.7287 & 6.2135 e-02 & 8.7005 e-01\end{array}$ $-7.5412 \quad 0.64950 .02 \quad 0.70200-01$ -7.5786 9.0941e-02 8.7021e-01 $-7.4917 \quad 8.2489 e^{-02}$ B.7020e-01 $-8.21117 .7514 e^{-02} 8.7024 e^{01}$ $-7.2887 \quad 7.0987$ e $02 \quad 8.7019$ e 01 100 PABI25136

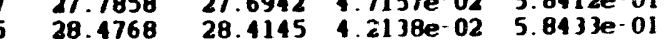




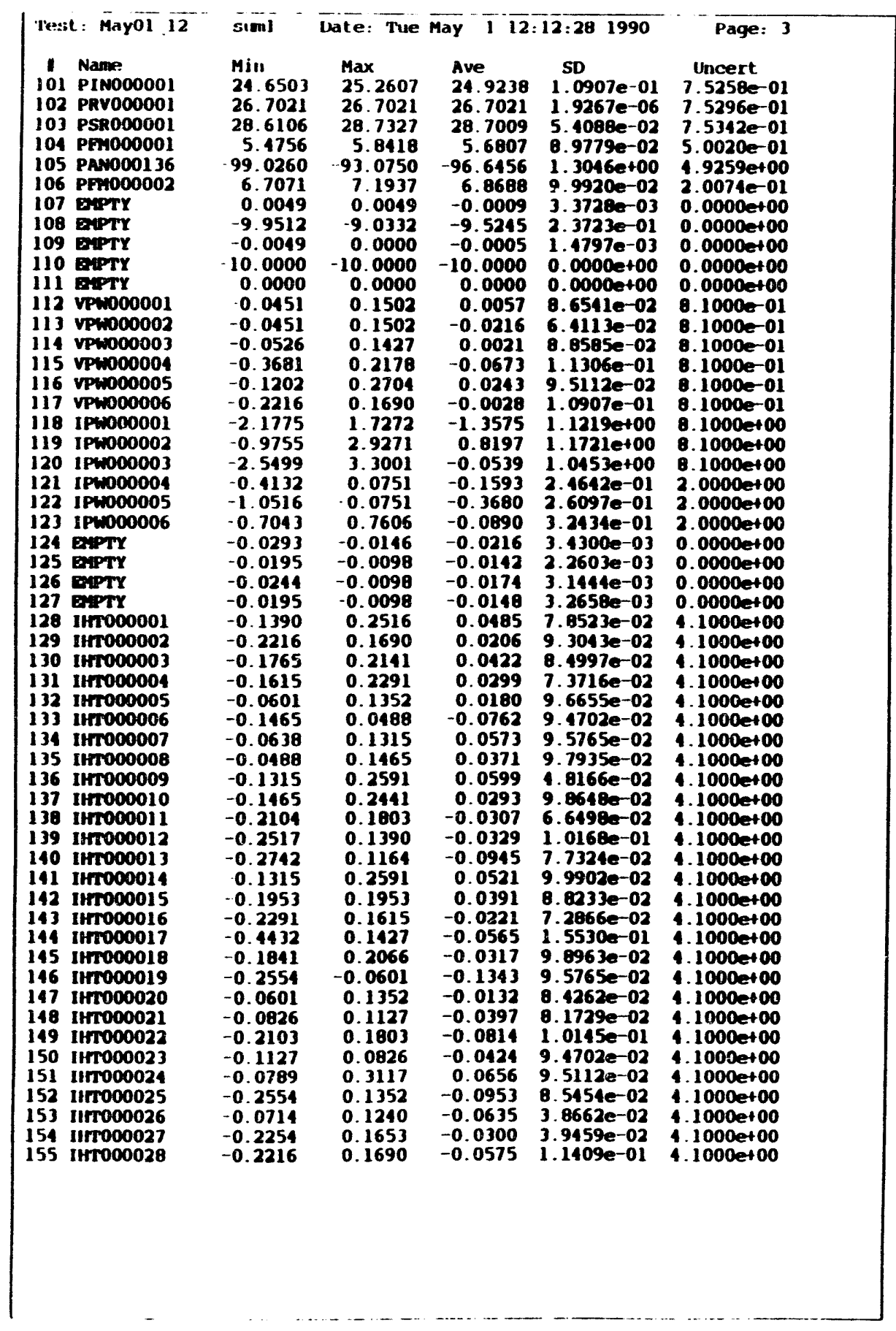

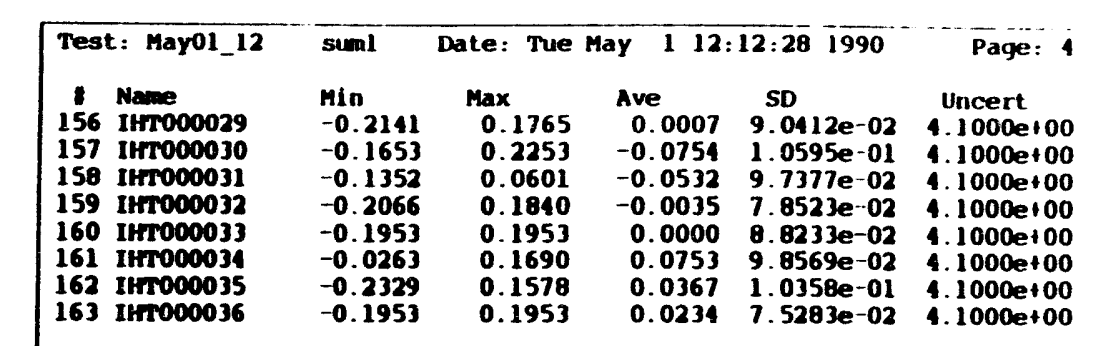




\begin{tabular}{|c|c|c|c|c|c|c|}
\hline \multicolumn{4}{|c|}{ 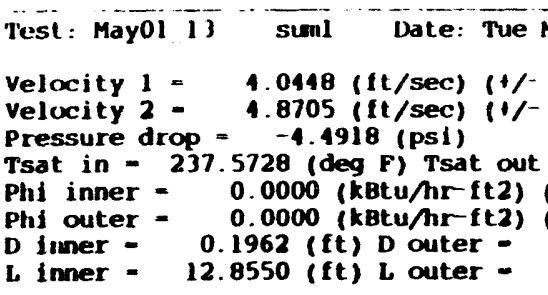 } & \multicolumn{2}{|c|}{$\begin{array}{l}\text { May } 112: 15: 291990 \\
10.4360 \text { \&) } \\
3.0389 \text { :) } \\
=246.9575 \text { (deg F) } \\
(+1 / 6786.3994 \text { : }) \\
(1-5073.3003 \text { : ) } \\
0.2413 \text { (ft) Dyd - } \\
12.8070 \text { (ft) A flow - }\end{array}$} & $\begin{array}{l}\text { Page: } 1 \\
0.0389 \text { (ft) } \\
0.0150 \text { (ft2) }\end{array}$ \\
\hline 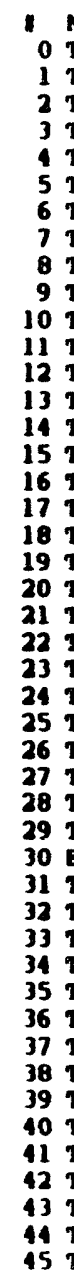 & 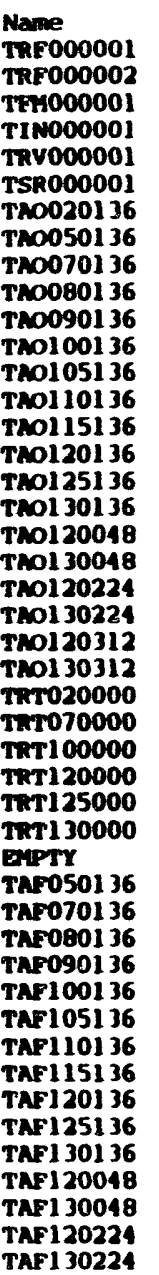 & $\begin{array}{l}\text { Min } \\
74.6370 \\
74.8621 \\
86.6264 \\
86.3570 \\
86.3527 \\
86.5918 \\
86.6316 \\
86.6037 \\
84.9338 \\
86.4942 \\
86.5695 \\
86.5385 \\
87.0291 \\
84.8624 \\
86.5884 \\
86.4260 \\
86.6119 \\
86.2472 \\
86.5594 \\
86.1790 \\
86.3795 \\
86.1814 \\
86.0949 \\
86.1356 \\
86.7096 \\
86.6745 \\
86.8294 \\
86.5306 \\
86.4995 \\
86.4060 \\
39.1464 \\
86.7082 \\
87.1266 \\
86.6516 \\
84.5759 \\
86.5075 \\
86.5376 \\
86.3761 \\
86.8060 \\
86.6273 \\
86.4997 \\
86.6092 \\
86.9718 \\
86.5376 \\
86.6095 \\
85.9814\end{array}$ & $\begin{array}{l}\operatorname{Max} \\
75.2754 \\
75.2854 \\
87.4662 \\
86.8829 \\
87.0861 \\
87.2201 \\
87.6826 \\
87.4412 \\
85.7772 \\
87.1237 \\
87.4093 \\
87.1690 \\
87.8702 \\
85.5972 \\
87.4309 \\
87.0549 \\
87.2417 \\
86.8748 \\
87.1899 \\
86.8090 \\
87.0103 \\
87.0185 \\
87.0408 \\
86.7660 \\
87.5519 \\
87.3073 \\
87.6687 \\
87.3706 \\
87.3373 \\
87.0361 \\
10.0646 \\
87.4421 \\
88.0601 \\
87.1754 \\
85.8368 \\
87.2435 \\
87.3721 \\
87.0063 \\
87.7499 \\
87.2572 \\
87.5456 \\
87.2383 \\
87.8164 \\
87.1682 \\
87.4503 \\
86.9303\end{array}$ & $\begin{array}{l}\text { Ave } \\
74.9562 \\
75.1034 \\
86.9583 \\
86.6346 \\
86.7547 \\
86.9098 \\
87.1994 \\
87.0262 \\
85.4064 \\
86.8635 \\
87.0189 \\
86.8454 \\
87.4286 \\
85.2361 \\
87.0434 \\
86.7319 \\
86.9475 \\
86.5827 \\
86.8328 \\
86.4914 \\
86.6950 \\
86.6705 \\
86.6540 \\
86.5094 \\
87.1392 \\
86.9366 \\
87.1486 \\
86.9339 \\
86.9641 \\
86.7168 \\
39.6332 \\
87.1320 \\
87.5373 \\
86.9481 \\
85.0763 \\
86.7980 \\
86.9754 \\
86.6618 \\
87.2678 \\
86.8753 \\
87.1225 \\
86.9237 \\
87.3317 \\
86.8697 \\
86.9460 \\
86.4410\end{array}$ & $\begin{array}{c}\text { SD } \\
1.7328 e-01 \\
1.2833 e-01 \\
1.8760 e-01 \\
1.3564 e-01 \\
1.9077 e-01 \\
1.4359 e-01 \\
2.3457 e-01 \\
1.7703 e-01 \\
2.0675 e-01 \\
1.4858 e-01 \\
2.1720 e-01 \\
1.6410 e-01 \\
2.0472 e-01 \\
1.6696 e-01 \\
2.2847 e-01 \\
1.6492 e-01 \\
1.6695 e-01 \\
1.5654 e-01 \\
1.6839 e-01 \\
1.5373 e-01 \\
1.6839 e-01 \\
1.7234 e-01 \\
1.9395 e-01 \\
1.3626 e-01 \\
1.9460 e-01 \\
1.5967 e-01 \\
1.8576 e-01 \\
1.4904 e-01 \\
1.8082 e-01 \\
1.4833 e-01 \\
2.1573 e-01 \\
1.5853 e-01 \\
1.9586 e-01 \\
1.2594 e-01 \\
3.1008 e-01 \\
1.5253 e-01 \\
2.1914 e-01 \\
1.6285 e-01 \\
1.9185 e-01 \\
1.7827 e-01 \\
2.3695 e-01 \\
1.6693 e-01 \\
2.0553 e-01 \\
1.8040 e-01 \\
1.9912 e-01 \\
1.9378 e-01\end{array}$ & 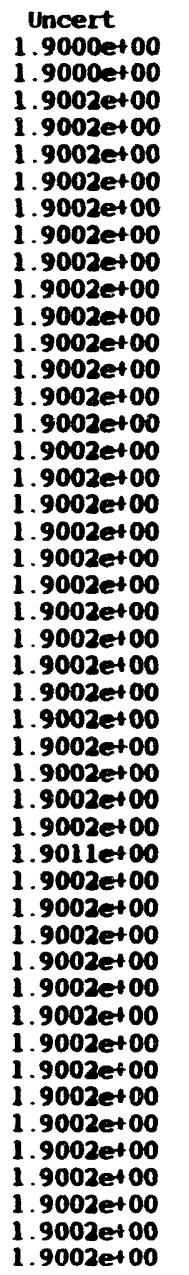 \\
\hline
\end{tabular}

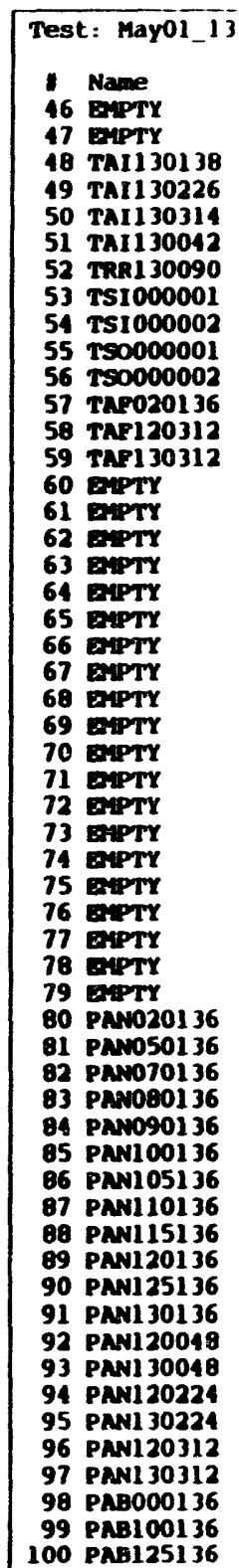

siml Date: Tue May 1 i2:15:29 1990

Min Max Ave SD Uncert

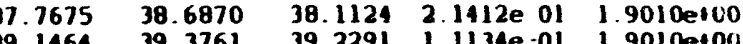

$\begin{array}{lllll}39.1464 & 39.3761 & 39.2291 & 1.1134 \text { e-01 } & 1.9010 e 100 \\ 86.5060 & 87.7658 & 87.3736 & 3.2211 \text { e-01 } & 1.9002 e 100\end{array}$

$\begin{array}{lllll}86.1479 & 06.8819 & 86.4998 & 1.8062 \mathrm{e}-01 & 1.9002 \mathrm{e} \\ 0 & 00\end{array}$

$86.5460 \quad 87.8084 \quad 87.1772$ 3. $3390 \mathrm{e}-01 \quad 1.9002 e t 00$

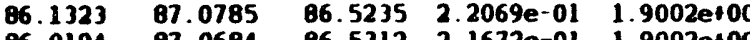

$\begin{array}{lllll}06.0194 & 87.0684 & 86.5312 & 2.1672 e-01 & 1.9002 e+00\end{array}$

$\begin{array}{lllll}81.5901 & 82.3282 & 82.0160 & 1.8678 \mathrm{e}-01 & 1.9001 \text { et } 00\end{array}$

11.985473 .0468 72.4356 2.7150 el $1.9000 \mathrm{el} 00$

$79.2554 \quad 80.2061 \quad 79.8342 \quad 20928 \mathrm{e}-01 \quad 1.9001 \mathrm{e} 00$

$85.9212 \quad 06.6593 \quad 86.3383 \quad 1.8187 e^{-01} 1.9002 e 100$

$\begin{array}{llllll}85.92160 & 86.6593 & 86.3383 & 1.8187 e-01 & 1.9002 e 100\end{array}$

$\begin{array}{lllll}85.7953 & 86.6346 & 86.2363 & 1.6150 e-01 & 1.9002 e+00\end{array}$

$\begin{array}{llllll}0.4590 & 0.4639 & 0.4638 & 6.9053 e-04 & 0.0000100\end{array}$

$\begin{array}{llllll}0.4785 & 0.4834 & 0.4788 & 1.1714 e^{-03} & 0.0000400\end{array}$

$\begin{array}{llllll}0.4883 & 0.4932 & 0.4931 & 6.9053 e-04 & 0.0000 e 100\end{array}$

$\begin{array}{lllll}0.5078 & 0.5078 & 0.5078 & 0.0000 \mathrm{e}+00 & 0.0000 \mathrm{e} \\ 0.00\end{array}$

$\begin{array}{lllll}0.3174 & 0.3223 & 0.3222 & 6.9053 e-04 & 0.0000 e+00\end{array}$

$\begin{array}{llllll}0.3418 & 0.3467 & 0.3421 & 1.1714 e^{-03} & 0.0000 \text { e100 }\end{array}$

$0.3564 \quad 0.3613 \quad 0.3601$ 2.1635e-03 0.0000 ero0

0.376060 .38590 .3766 1.6025e.04 0.0000100

$0.41020 .01102 \quad 0.11020 .0000+000.0000100$

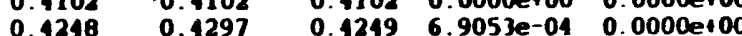

$\begin{array}{llllll}0.1492 & 0.1492 & 0.4492 & 0.0000 e 100 & 0.0000 e 100\end{array}$

$\begin{array}{llllll}0.4639 & 0.4639 & 0.4639 & 0.0000 e+00 & 0.0000 e+00\end{array}$

$\begin{array}{llllll}0.4785 & 0.4834 & 0.4833 & 6.9053 e-04 & 0.0000 e+00\end{array}$

$\begin{array}{llllll}0.4980 & 0.5029 & 0.4983 & 1.1714 e-03 & 0.0000 \text { et } 00\end{array}$

$\begin{array}{llllll}0.5176 & 0.5176 & 0.5176 & 0.0000 e+00 & 0.0000 e r 00\end{array}$

$\begin{array}{llllll}0.5273 & 0.5322 & 0.5321 & 6.9053 \mathrm{e}-04 & 0.0000 \mathrm{e} 00\end{array}$

$\begin{array}{lllll}0.5469 & 0.5566 & 0.5518 & 9.8548 \mathrm{e}-04 & 0.0000 \mathrm{e} 100\end{array}$

$\begin{array}{llllll}0.5713 & 0.5762 & 0.5750 & 2.1065 e^{-03} & 0.0000 \text { et00 } \\ 0.5957 & 0.6006 & 0.5983 & 2.4583 e-03 & 0.0000 e\end{array}$

$\begin{array}{llllll}0.5957 & 0.6006 & 0.5983 & 2.4583 e-03 & 0.0000 e & 00\end{array}$

$16.1580-15.2425-15.7567$ 2.2591e-0 0.7089

$-20.2308-19.3916-19.8127 \quad 1.0956 \mathrm{e}-01$ B. $7141 \mathrm{e}$ ol

$-10.5725-10.2673-10.4092 \quad 8.9890 \mathrm{e}-02 \quad 8.7039 \mathrm{e}-01$

$-9.8784-9.7258-9.8021 \quad 4.8742 \mathrm{e} .028 .7035 \mathrm{e}^{-01}$

$\begin{array}{lllllll}-9.8784 & -9.6202 & -9.7667 & 6.8656 e & 02 & 8.7034 e^{-01}\end{array}$

$\begin{array}{llllll}-5.1578 & -4.8526 & -1.9823 & 6.9362 e-02 & 8.7009 e-01\end{array}$

$\begin{array}{llllll}-5.0595 & -4.8306 & -1.9451 & 7.7453 e-02 & 8.7009 e-01\end{array}$

$\begin{array}{llllll}-4.7412 & -4.5886 & -4.6954 & 5.1121 e^{-02} & 8.7008 \mathrm{e}-01\end{array}$

$\begin{array}{llllll}-5.7945 & -5.5656 & -5.6480 & 6.3253 e-02 & 8.7011 \text { e } 01\end{array}$

$\begin{array}{llllll}-5.5334 & -5.3045 & -5.4113 & 6.3552 e \cdot 02 & 0.7011 e-01\end{array}$

$\begin{array}{llllll}-1.9113 & -4.7587 & -4.8136 & 5.3484 \text { e } 02 & 0.7008 \mathrm{e} 01\end{array}$

$\begin{array}{llllll}-9.9841 & -9.7552 & -9.8758 & 7.7207 e^{-02} & 8.7035 e-01\end{array}$

$\begin{array}{lllll}-9.0873 & -9.5821 & -9.7759 & 8.8826 e-02 & 8.7034 e-01\end{array}$

$10.4404-10.1352-10.3381 \quad 7.4321 e^{-02} 8.7035$ - 02 8.7038e-01

$\begin{array}{lllllll}-9.5424 & -9.3135 & -9.4813 & 6.5395 e-02 & 8.7032 e & 01\end{array}$

$\begin{array}{llllll}23.8191 & 24.0937 & 23.9454 & 6.1038 \mathrm{e} 02 & 5.8308 \mathrm{e} & 01\end{array}$

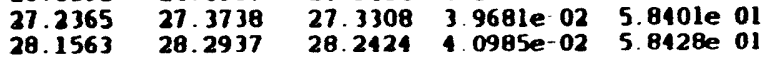




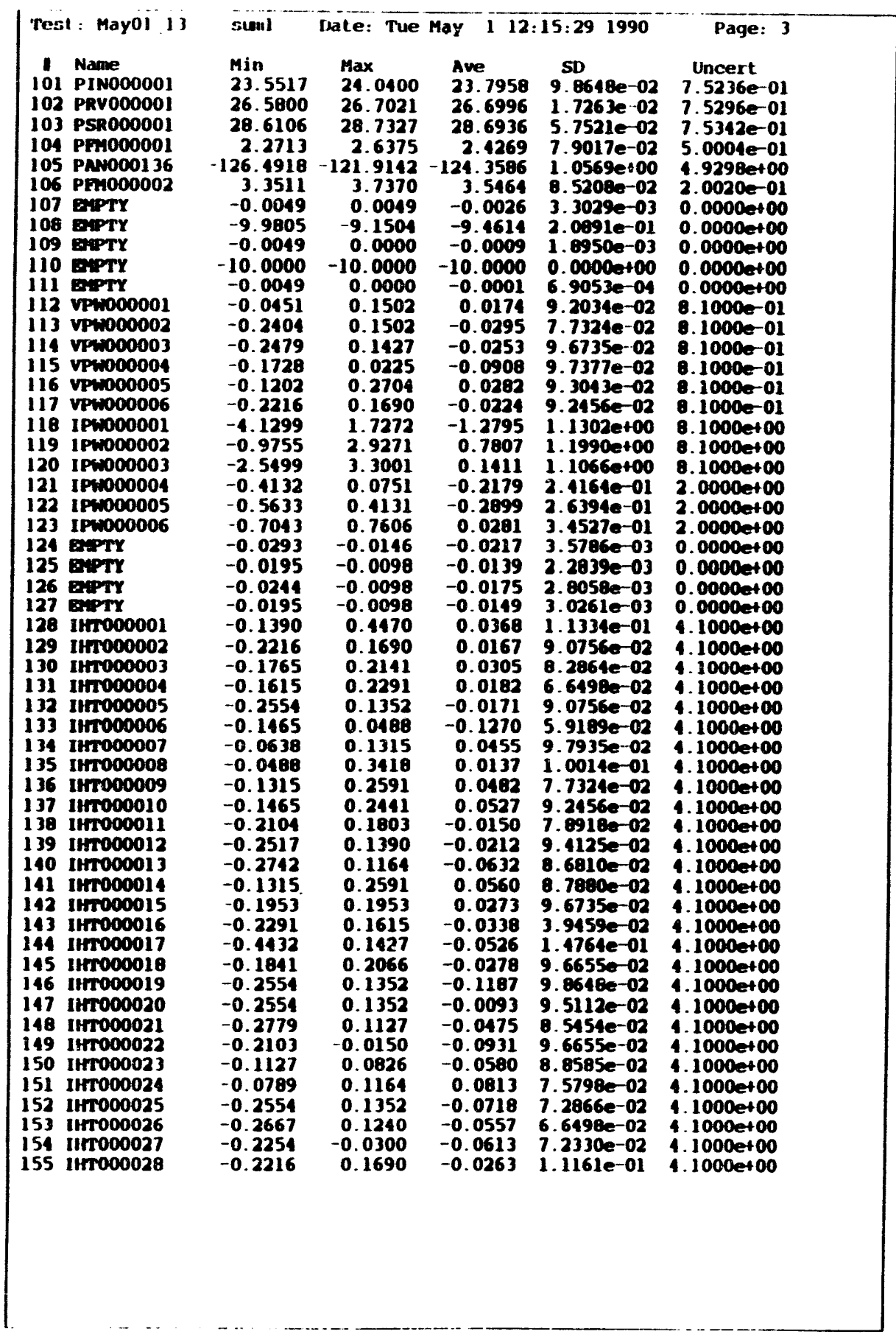

\begin{tabular}{|c|c|c|c|c|c|}
\hline Test: May01_13 & sumal & Date: Tue & May 112 & $15: 291990$ & Page: 1 \\
\hline 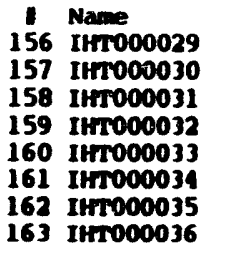 & $\begin{array}{l}\text { Min } \\
-0.0188 \\
-0.1653 \\
-0.3305 \\
-0.2066 \\
-0.1953 \\
-0.0263 \\
-0.2329 \\
0.0000\end{array}$ & $\begin{array}{l}\operatorname{Max} \\
0.1765 \\
0.0300 \\
0.0601 \\
0.1840 \\
0.1953 \\
0.1690 \\
0.3531 \\
0.1953\end{array}$ & $\begin{array}{l}\text { Ave } \\
0.0242 \\
-0.0715 \\
-0.0376 \\
-0.0191 \\
0.0000 \\
0.0479 \\
0.0484 \\
0.0313\end{array}$ & $\begin{array}{l}\text { SD } \\
8.1729 e-02 \\
9.8569 \mathrm{e}-02 \\
1.0625 \mathrm{e}-01 \\
6.7888 \mathrm{e}-02 \\
7.8918 \mathrm{e}-02 \\
9.5765 \mathrm{e}-02 \\
1.1272101 \\
7.2330 \mathrm{e} 02\end{array}$ & 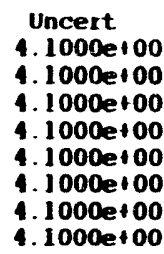 \\
\hline
\end{tabular}

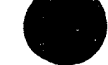




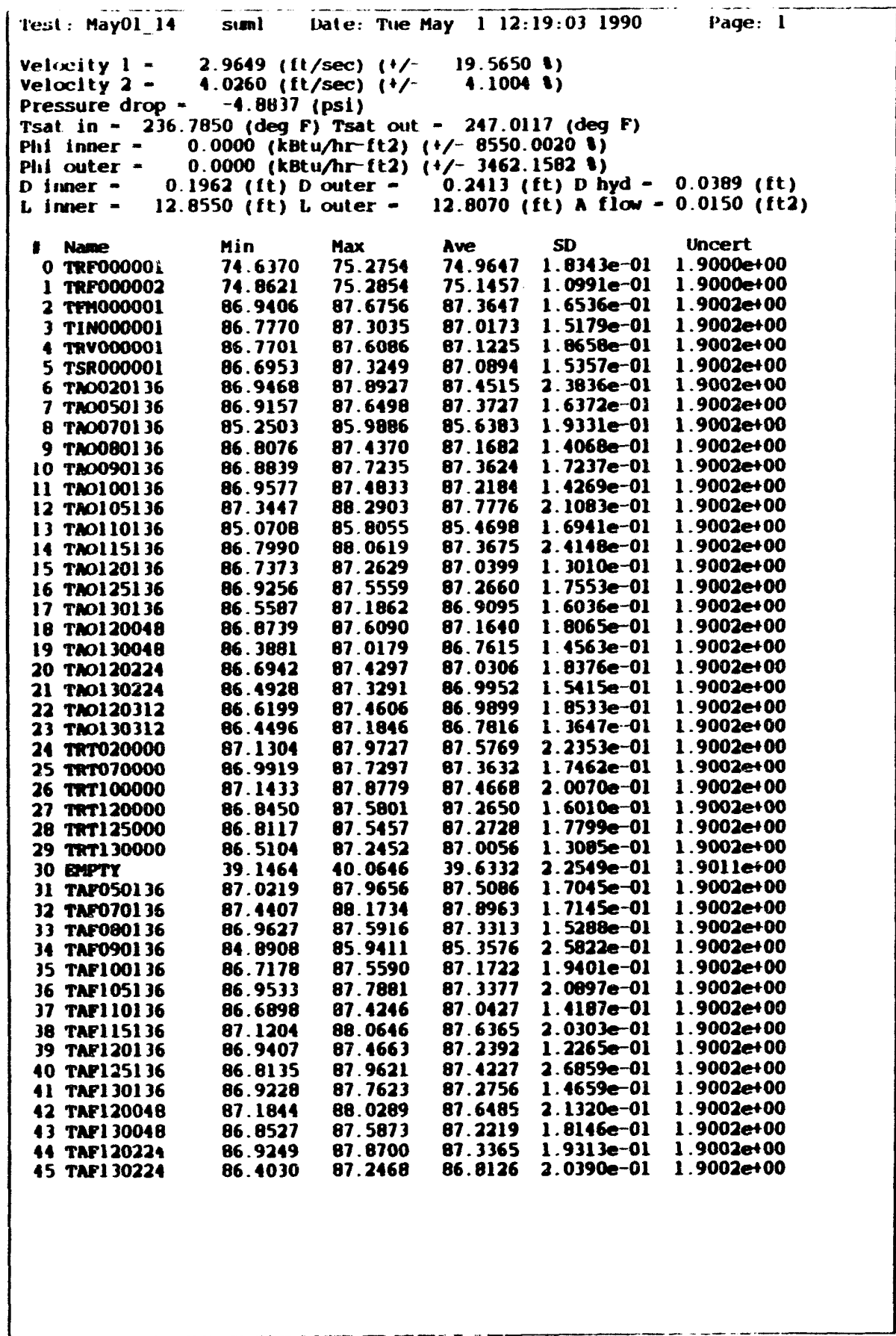

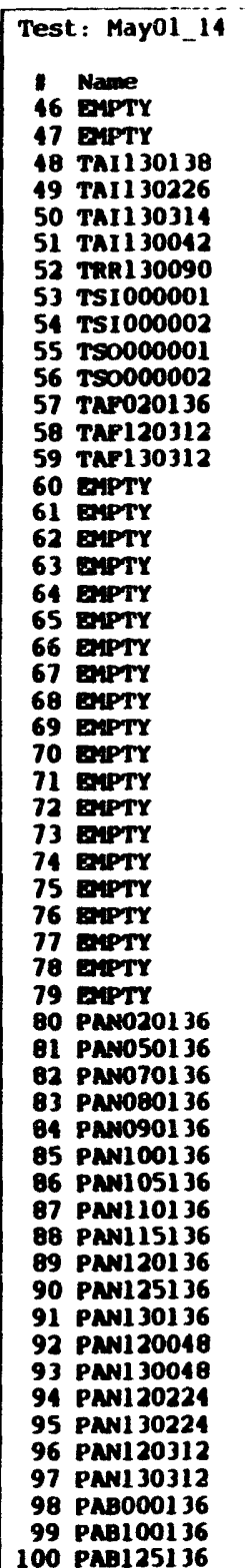

suml Date: Tue May 1 12:19:03 1990 Packe: 2

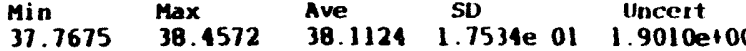
39.1878 8.9118e 02 1.9010et 00 $\begin{array}{lllllll}86.9256 & 80.1854 & 87.4800 & 3.1094 e & 01 & 1.9002 e 100\end{array}$

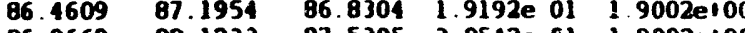
$\begin{array}{lllll}86.9669 & 80.1233 & 87.5305 & 3.0542 \mathrm{e}-01 & 1.9002 \mathrm{e} \\ 0600\end{array}$ $86.44767 .1833 \quad 86.8637$ 1.9102e ol $1.9002 \mathrm{et0}$ $\begin{array}{llllll}66.3339 & 87.3819 & 86.8329 & 2.6674 e-01 & 1.9002 e+00\end{array}$ $71.8795 \quad 72.8350 \quad 72.3425 \quad 2.7251 \mathrm{e}-01$ 1.9001et00 $67.513768 .797268 .24952 .1358 \mathrm{e} 01 \quad 1.9000 \mathrm{e} 00$ $\begin{array}{lllll}69.4668 & 80.4170 & 79.9481 & 2.2688 \mathrm{e}-01 & 1.9001 \mathrm{et} 00\end{array}$ $\begin{array}{llllll}86.3427 & 87.0806 & 86.6887 & 1.6768 e-01 & 1.9002 e t 00\end{array}$ $\begin{array}{llllll}86.5306 & 87.6852 & 87.0556 & 2.8744 e-01 & 1.9002 e t 00\end{array}$ $\begin{array}{llllll}86.2141 & 86.9492 & 86.5630 & 1.7918 e \text { o1 } & 1.9002 e+00\end{array}$ $\begin{array}{llllll}0.4688 & 0.4736 & 0.4710 & 2.4583 e & 03 & 0.0000 e 100\end{array}$ $\begin{array}{lllll}0.4834 & 0.4883 & 0.4845 & 2.0432 e 03 & 0.0000 \\ 0.00\end{array}$ $\begin{array}{lllll}0.4980 & 0.5029 & 0.4983 & 1.1714 \text { e } 03 & 0.0000100\end{array}$ $\begin{array}{lllll}0.5127 & 0.5176 & 0.5139 & 2.1065 e-03 & 0.0000+100\end{array}$ $\begin{array}{lllll}0.3223 & 0.3271 & 0.3260 & 2.1065 e-03 & 0.0000 \text { e100 } \\ 0.3467 & 0.3467 & 0.3467 & 0.0000 \mathrm{e} 100 & 0.0000 \mathrm{e}\end{array}$ $0.34670 .3467 \quad 0.3467 \quad 0.0002$ et00 0.0000 et 00

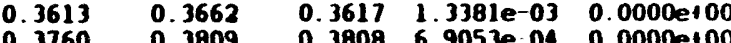
0.39530 .66550040 .00000400 $0.4102 \quad 0.4150 \quad 0.41312 .4164$ e-03 $0.0000+00$ $\begin{array}{llllll}0.4297 & 0.4297 & 0.4297 & 0.0000 e+00 & 0.0000 e+00\end{array}$ $\begin{array}{llllll}0.4492 & 0.4541 & 0.4536 & 1.4797 e-03 & 0.000010\end{array}$ $\begin{array}{llllll}0.4639 & 0.4688 & 0.4663 & 2.4662 e .03 & 0.0000 e+00\end{array}$ $\begin{array}{lllll}0.4834 & 0.4883 & 0.4853 & 2.3941 \text { e } 03 & 0.0000100\end{array}$

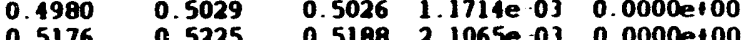
$\begin{array}{lllll}0.5176 & 0.5225 & 0.5188 & 2.1065 \text { e } 03 & 0.0000 \text { et } 00 \\ 0.5322 & 0.5371 & 0.5327 & 1.4797 e^{-03} & 0.0000 \text { to }\end{array}$ $\begin{array}{lllll}0.5322 & 0.5371 & 0.5327 & 1.4797 e-03 & 0.0000 \text { et } 00 \\ 0.5518 & 0.5566 & 0.5521 & 1.3381 \mathrm{e}-03 & 0.0000 \text { to }\end{array}$ $\begin{array}{lllll}0.5518 & 0.5566 & 0.5521 & 1.3381 \text { e-03 } & 0.0000 \text { t } 00 \\ 0.5713 & 0.5762 & 0.5761 & 6.9053 e^{-04} & 0.0000 e 100\end{array}$ $\begin{array}{lllll}0.5713 & 0.5762 & 0.5761 & 6.9053 e-04 & 0.0000 e \\ 0.6006 & 0.6055 & 0.6007 & 6.9053 e-04 & 0.0000 e 100\end{array}$ $\begin{array}{llllll}-17.6076 & -16.6920 & -17.1986 & 2.0142 e-01 & 8.7106 e & 01\end{array}$ $\begin{array}{llllll}-22.0619 & -21.1463 & -21.6071 & 2.3679 e-01 & 1.6820 e+00\end{array}$ $-11.7932-11.3354-11.5139 \quad 1.1073 e_{-01} \quad 8.7048 \mathrm{e} 01$ $-10.4125-10.1836-10.2965 \quad 6.7610 e-02 \quad 8.7038$ e 01 $\begin{array}{llllll}-10.8409 & -10.5358 & -10.6487 & 6.3999 e-02 & \mathbf{\theta} .7041 \mathrm{e} .0\end{array}$ $\begin{array}{lllll}-5.5392 & -5.2341 & -5.3653 & 6.9001 \mathrm{e}-02 & 8.7010 \mathrm{e}\end{array}$ $\begin{array}{lllll}-5.1410 & -5.2884 & -5.3799 & 5.5575 e-02 & 8.7010 e\end{array}$ $\begin{array}{llllll}-5.1990 & -4.9701 & -5.0555 & 6.4738 e^{02} & 0.7009 e-01\end{array}$ $\begin{array}{llllll}-6.0997 & -5.9471 & -6.0127 & 5.3417 e-02 & 6.7013 e-01\end{array}$ $\begin{array}{llllll}-5.9912 & -5.7623 & -5.8569 & 5.6759 e \cdot 02 & 0.7012 e-01\end{array}$ $\begin{array}{llllll}-5.3691 & -5.1402 & -5.2455 & 6.3346 e-02 & 0.7010 e-01\end{array}$ $10.4272-10.1983-10.2975$ 5.3946e-02 $0.7038 e$ ol $10.8028-10.6502-10.7265 \quad 5.7673$ e $02 \quad 8.7041$ e- 01 $-10.9158-10.6869-10.7693 \quad 6 \quad 8656 \mathrm{e}-02 \quad 8.7042 \mathrm{e} 01$ $-11.3559-11.0507-11.1880 \quad 6.5395 e 02$ 8.7045e-01 $\begin{array}{lllll}10.4579 & -10.3053 & -10.3389 & 5.1492 e-02 & 8.7038 e\end{array}$ $\begin{array}{llllll}23.4986 & 23.7733 & 23.6103 & 6.4843 e-02 & 5.8300 e & 0\end{array}$ $\begin{array}{llllll}27.1907 & 27.3738 & 27.2822 & 3.9237 e-02 & 5.8400 e & 01 \\ 28.2479 & 28.2937 & 28.2690 & 2.3046 e-02 & 5.8429 e & 01\end{array}$ 


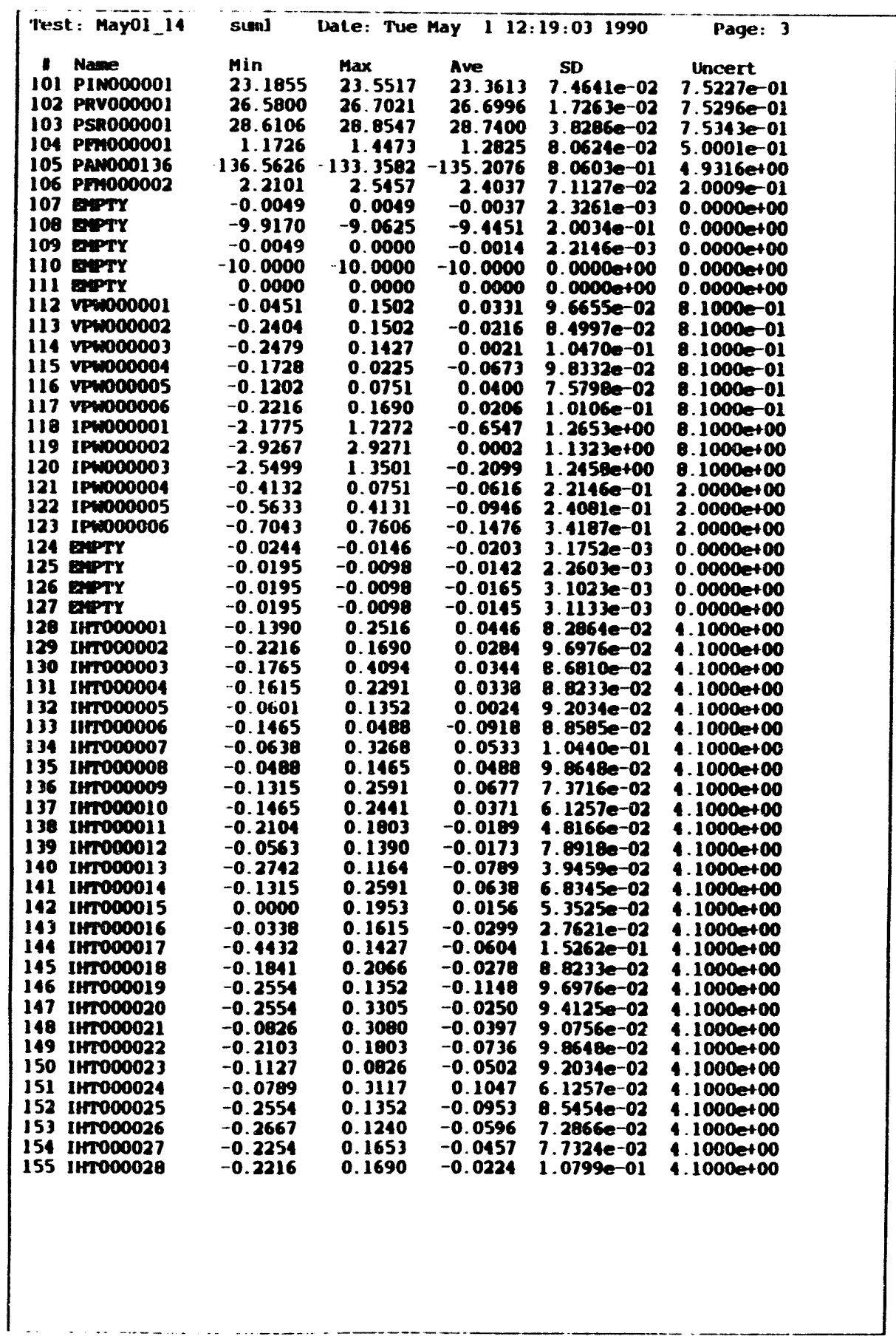

\begin{tabular}{|c|c|c|c|c|c|}
\hline Test: May01_14 & suml & Date: Tue & Iay 112 & $19: 031990$ & Page: 4 \\
\hline 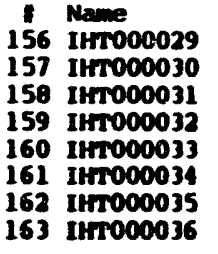 & $\begin{array}{l}\min \\
-0.2141 \\
-0.1653 \\
-0.1352 \\
-0.2066 \\
-0.1953 \\
-0.2216 \\
-0.0375 \\
0.0000\end{array}$ & $\begin{array}{l}\text { Max } \\
0.1765 \\
0.0300 \\
0.2554 \\
0.1840 \\
0.1953 \\
0.1690 \\
0.3531 \\
0.1953\end{array}$ & $\begin{array}{l}\text { Ave } \\
-0.0071 \\
-0.0872 \\
-0.0415 \\
-0.0152 \\
0.0078 \\
0.0440 \\
0.0289 \\
0.0352\end{array}$ & $\begin{array}{l}\text { SD } \\
7.2866 \mathrm{e}-02 \\
9.6655 \mathrm{e}-02 \\
1.0617 \mathrm{e}-01 \\
7.3716 \mathrm{e}-02 \\
7.8523 \mathrm{e}-02 \\
1.0259 \mathrm{e}-01 \\
1.0145 \mathrm{e}-01 \\
7.5798 \mathrm{e}-02\end{array}$ & $\begin{array}{l}\text { Uncert } \\
4.1000 \text { et } 00 \\
1.1000 \text { e } 00 \\
1.1000 \text { e } 00 \\
1.1000 \text { et } 00 \\
1.1000 e+00 \\
1.1000 \text { e } 00 \\
1.1000 \text { t } 00 \\
1.1000 \text { e } 00\end{array}$ \\
\hline
\end{tabular}

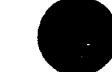




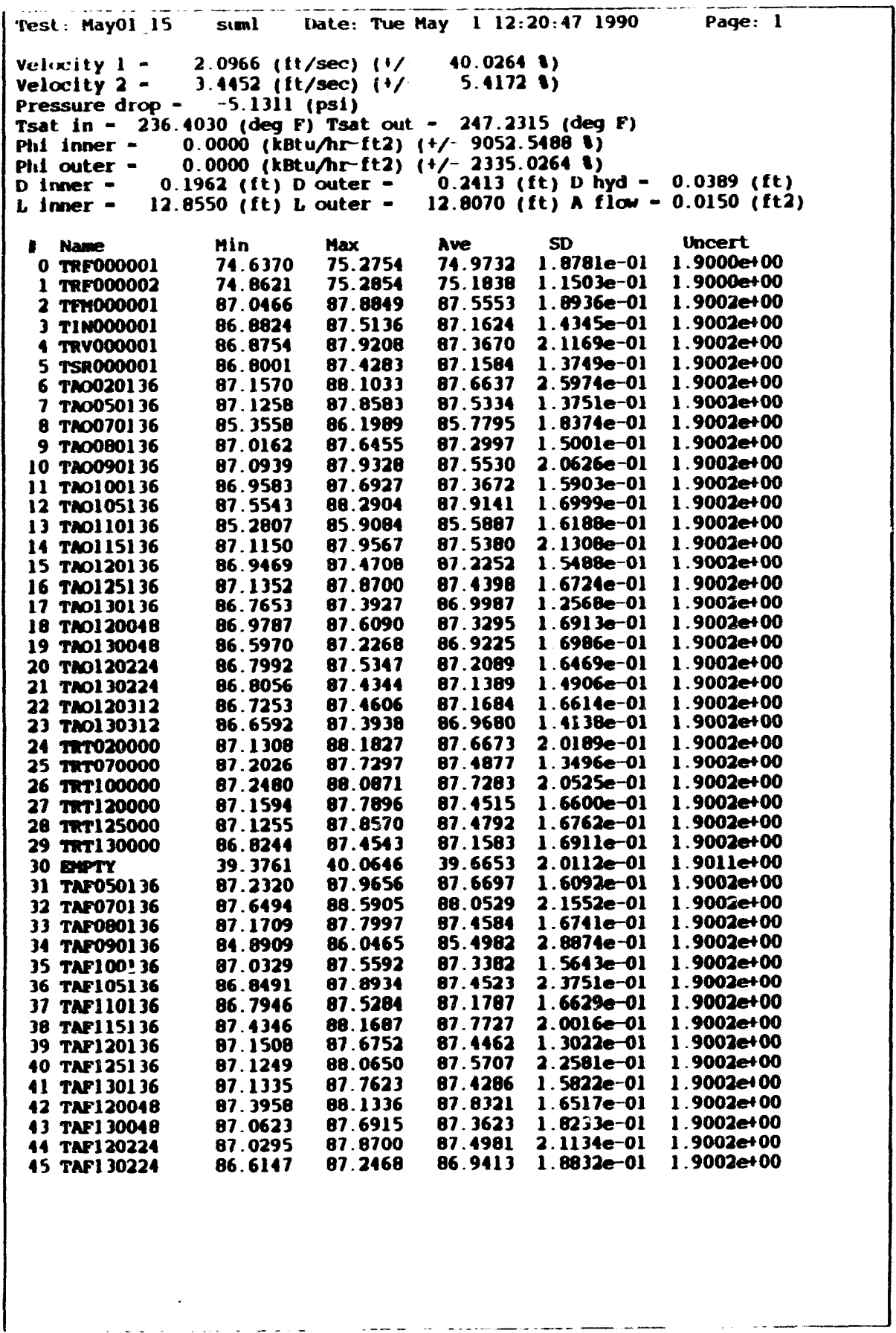

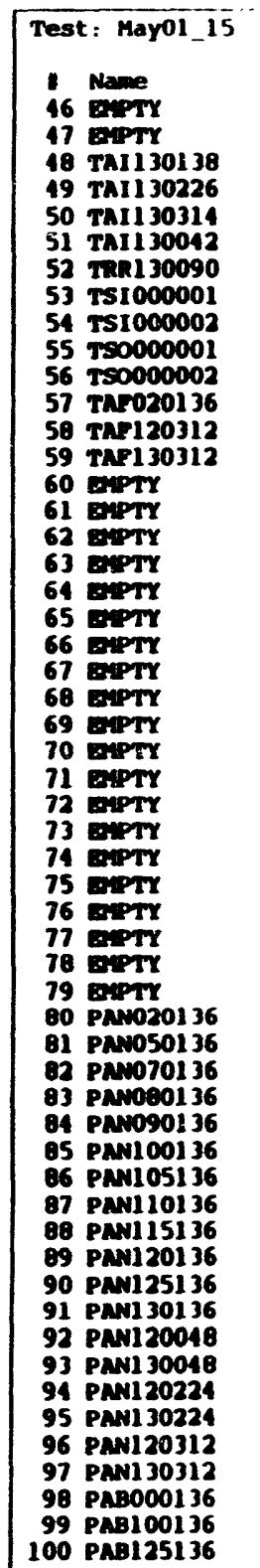

suml Date: Tue May 1 12:20:47 1990 Page: 2 Min Max Ave SD Uncert 37.7675 38.4572 38.0664 1.6909e $01 \quad 1.9010$ et 00 $\begin{array}{llllll}39.1464 & 39.3761 & 39.1648 & 6.2930 \mathrm{e} & 02 & 1.9010 \mathrm{et} 00\end{array}$ $\begin{array}{lllll}86.9254 & 89.1846 & 87.6541 & 3.0312 e-01 & 1.9002 e+00\end{array}$ $\begin{array}{llllll}86.5663 \quad 87.4044 & 86.9632 & 1.7874 e & 01 & 1.9002 e+00\end{array}$ $\begin{array}{llllll}87.0719 & 88.2282 & 07.7470 & 2.8344 e .01 & 1.9002 e+00\end{array}$ $86.7625 \quad 87.6031 \quad 87.0884 \quad 2.1671$ e-01 $1.9002 e+00$ $\begin{array}{llllll}86.3339 & 87.5913 & 86.9816 & 2.4852 e-01 & 1.9002 e+00\end{array}$ $\begin{array}{llll}01.4856 & 02.3282 & 81.9004 & 2.0017 e-01 \\ 71.9001 e+00\end{array}$ $67.9408 \quad 68.6900 \quad 68.35431 .6940001$ 79.5718 80.4170 BO. 3319 1.8201e-01 1.900let00 86.4485 87.2917 86.8596 1.7848-01 1.9002et00 $\begin{array}{llllll}86.6355 & 07.6852 & 07.2213 & 2.3037 \mathrm{e} 01 & 1.9002 \mathrm{e}+00\end{array}$ $86.3190 \quad 86.9492 \quad 86.6991 \quad 1.6545 \mathrm{e}-01 \quad 1.9002 e 100$

$\begin{array}{lllll}0.4688 & 0.4785 & 0.4736 & 9.8648 \mathrm{e}-04 & 0.0000 \mathrm{e} 100\end{array}$

$\begin{array}{llllll}0.4883 & 0.4932 & 0.4884 & 6.9053 \mathrm{e}-04 & 0.0000 \mathrm{e}+00\end{array}$

$\begin{array}{lllll}0.4960 & 0.5029 & 0.5028 & 6.9053 e^{-04} & 0.0000 e+00\end{array}$

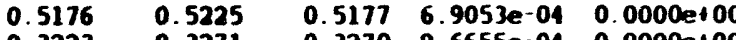
$\begin{array}{lllll}0.3223 & 0.3271 & 0.3270 & 9.6655 e-04 & 0.0000 e+00\end{array}$ $\begin{array}{lllll}0.3467 & 0.3516 & 0.3470 & 1.1714 \mathrm{e}-03 & 0.0000 \mathrm{e}+00\end{array}$ $0.3613 \quad 0.3662 \quad 0.36462 .3365$-03 0.000 et 00 $0.39550 .3955 \quad 0.39550 .000001000 .00000100$ $0.4150 \quad 0.4150 \quad 0.4150 \quad 0.0000+000.0000+00$ $\begin{array}{llllll}0.4297 & 0.4297 & 0.4297 & 0.0000 e+00 & 0.0000+100\end{array}$ $\begin{array}{lllllll}0.4541 & 0.4590 & 0.4542 & 6.9053 e-04 & 0.0000 e+00\end{array}$ $\begin{array}{lllll}0.4689 & 0.4688 & 0.4688 & 0.0000 e+00 & 0.0000 e t 00\end{array}$ $\begin{array}{llllll}0.4883 & 0.4932 & 0.4884 & 6.9053 e-04 & 0.0000 e+00\end{array}$ $\begin{array}{llllll}0.5029 & 0.5078 & 0.5031 & 9.6655 \text { e-04 } & 0.0000 \text { t } 00\end{array}$ $\begin{array}{llllll}0.5176 & 0.5273 & 0.5225 & 9.8648 e-04 & 0.0000 e+00\end{array}$ $\begin{array}{lllll}0.5322 & 0.5371 & 0.5370 & 6.9053 e^{-04} & 0.0000 e 100\end{array}$ $\begin{array}{lllll}0.5518 & 0.5664 & 0.5567 & 1.8429 e-03 & 0.0000 e+00\end{array}$ $0.57620 .5811 \quad 0.5783$ 2.4484e 030.0000100 0.5957 $-18.59507-17.6076$ - 34.2797 2.3681e-01 $1.6822 \mathrm{e}-00$ $\begin{array}{lllll}-22.3670 & -22.7226 & 1.3839 e-01 & \text { B } & 7185 e-01\end{array}$ 12.2509 - $11.8695-12.0465$ B 3633e-02 8.7052e-01 $-10.9465-10.6414-10.8077 \quad 7.9631 \mathrm{e}-02 \quad$ B.7042e-01 $\begin{array}{llllll}-11.2987 & -10.9935 & -11.1766 & 6.3552 e-02 & 0.7045 e-01\end{array}$ $\begin{array}{llllll}-5.7681 & -5.5392 & -5.6720 & 6.1288 \mathrm{e}-02 & 8.7012 \mathrm{e} \cdot 01\end{array}$ $\begin{array}{llllll}-5.8224 & -5.5173 & -5.6561 & 5.9077 e-02 & 8.7011 e-01\end{array}$ $\begin{array}{llllll}-5.5041 & -5.2753 & -5.3821 & 5.7673 \mathrm{e}-02 & 8.7010 e-01\end{array}$ $-6.0997 \quad-5.9471 \quad-6.0173 \quad 5.0748 e^{-02} \quad 0.7013 e^{-01}$ $\begin{array}{llllll}-6.1438 & -5.9912 & -6.0415 & 1.2521 e^{-02} & 0.7013 e-01\end{array}$ $-5.5979-5.3691-5.5010$ 5.5167e-02 8.7011e-01 $\begin{array}{lllll}-11.0375 & -10.7323 & -10.0910 & 7.0365 e-02 & 8.7043 e-01\end{array}$ $-11.4337-11.2048-11.2750 \quad 6.1346 e-02$ 8.7046e-01 $-11.3735-11.2209-11.289152588 \mathrm{e}-02$ 8.7046e-01 11.8:36 -11.5085 - 11.6565 6.7908e-02 8.7049e-01 $-11.1446-10.8394-11.01186 .1288 e^{-02}$ 8.7044e-01 $23.3613 \quad 23.544423 .44924 .1194 \mathrm{e} 02$ 5.8296e 01 $\begin{array}{llllll}27.3655 & 27.3738 & 27.3207 & 4.0692 e & 02 & 5.8401 \text { e } 01 \\ 28.2937 & 28.4310 & 28.3770 & 3.7782 e-02 & 5.8432 e-01\end{array}$ 100 PAB125136 


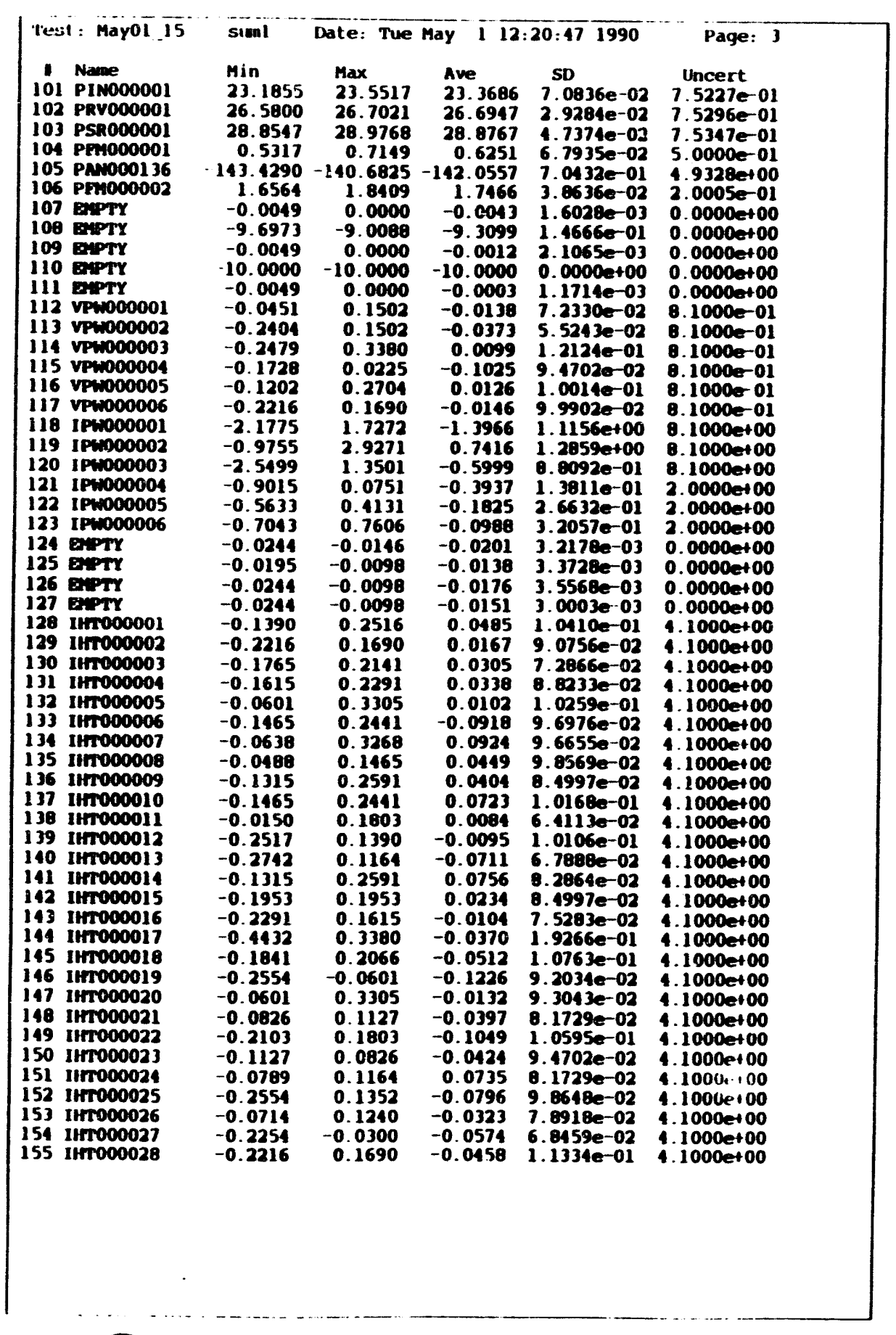

\begin{tabular}{|c|c|c|c|c|c|}
\hline Test: May & sumel & Date: Tue & May 112 & $20: 471990$ & Page: 4 \\
\hline 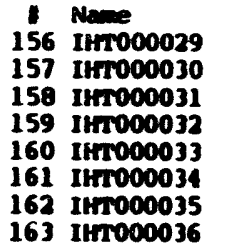 & $\begin{array}{l}\text { Min } \\
-0.0188 \\
-0.1653 \\
-0.3305 \\
-0.2066 \\
-0.1953 \\
-0.0263 \\
-0.0375 \\
-0.1953\end{array}$ & $\begin{array}{l}\max \\
0.1765 \\
0.2253 \\
0.2554 \\
0.1840 \\
0.1953 \\
0.1690 \\
0.1578 \\
0.1953\end{array}$ & $\begin{array}{r}\text { Ave } \\
0.0046 \\
-0.0793 \\
-0.0571 \\
-0.0035 \\
0.0000 \\
0.0167 \\
0.0523 \\
0.0156\end{array}$ & $\begin{array}{l}50 \\
6.1113 e 02 \\
1.0559 e-01 \\
1.1161 \mathrm{e}-01 \\
6.7888 \mathrm{e}-02 \\
5.5804 e^{-02} \\
6.1729 \mathrm{e}-02 \\
9.0332 \mathrm{e}-02 \\
6.6498 \mathrm{e}-02\end{array}$ & $\begin{array}{c}\text { Uncert } \\
1.1000 e+00 \\
1.1000 e+00 \\
1.1000 e+00 \\
1.1000 \text { et } 00 \\
1.1000 \text { e } 00 \\
1.1000=+00 \\
1.1000=+00 \\
1.1000 \text { e } 100\end{array}$ \\
\hline
\end{tabular}




\begin{tabular}{|c|c|c|c|c|c|c|}
\hline \multicolumn{6}{|c|}{ 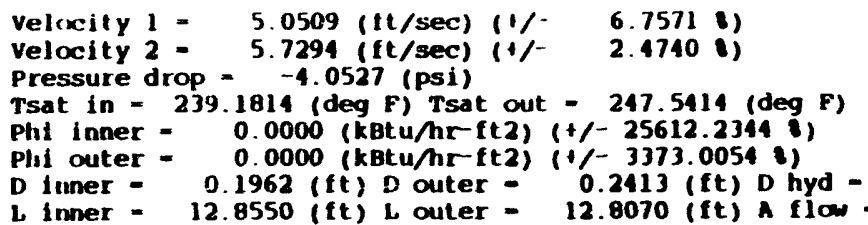 } & $\begin{array}{l}0.0389 \text { (ft) } \\
0.0150 \text { (ft2) }\end{array}$ \\
\hline $\begin{array}{r}9 \\
9 \\
10 \\
11 \\
12 \\
13 \\
14 \\
15 \\
16 \\
17 \\
18 \\
19 \\
20 \\
21 \\
22 \\
23 \\
21 \\
25 \\
26 \\
27 \\
28 \\
29 \\
30 \\
31 \\
32 \\
33 \\
34 \\
35 \\
36 \\
37 \\
38 \\
39 \\
40 \\
41 \\
12 \\
43\end{array}$ & 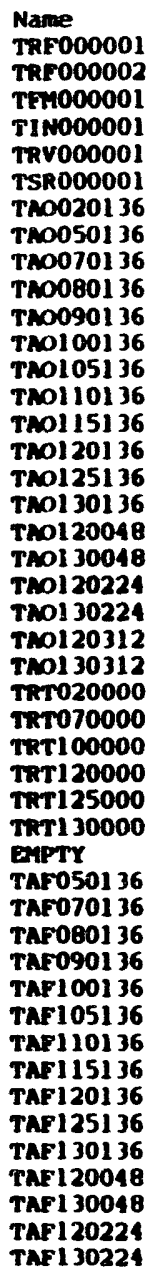 & $\begin{array}{l}\text { Min } \\
74.6370 \\
74.8621 \\
87.3602 \\
87.1982 \\
87.2932 \\
87.1129 \\
87.6827 \\
87.6462 \\
85.6723 \\
87.5386 \\
87.6175 \\
87.5869 \\
88.0799 \\
85.5926 \\
87.5362 \\
87.3633 \\
87.6588 \\
87.1785 \\
87.5031 \\
87.1202 \\
87.3241 \\
87.2295 \\
87.2498 \\
87.1823 \\
67.5518 \\
87.6256 \\
87.8757 \\
87.5784 \\
87.5417 \\
87.3480 \\
39.1464 \\
87.8594 \\
88.0668 \\
87.5872 \\
85.3118 \\
87.4539 \\
87.3700 \\
87.3183 \\
87.8534 \\
87.6726 \\
87.5438 \\
87.5511 \\
87.7124 \\
87.4815 \\
87.4494 \\
87.1425\end{array}$ & $\begin{array}{l}\text { Max } \\
75.2754 \\
75.2854 \\
88.3036 \\
87.9340 \\
88.2330 \\
87.6376 \\
88.6280 \\
88.2752 \\
86.4097 \\
88.0623 \\
88.4560 \\
88.1116 \\
88.8157 \\
86.2221 \\
88.4827 \\
88.0944 \\
88.2887 \\
87.9074 \\
88.2373 \\
87.7480 \\
88.0587 \\
87.9532 \\
88.0901 \\
87.7075 \\
88.6033 \\
88.3631 \\
88.5052 \\
88.2084 \\
88.2736 \\
87.9762 \\
10.0646 \\
88.4874 \\
89.0076 \\
88.2158 \\
86.4659 \\
88.1894 \\
88.3092 \\
88.0518 \\
88.6925 \\
88.0931 \\
88.5866 \\
88.1809 \\
88.6612 \\
88.2157 \\
88.2897 \\
87.7747\end{array}$ & $\begin{array}{l}\text { Ave } \\
75.0541 \\
75.1626 \\
87.9366 \\
87.6040 \\
87.7097 \\
87.4176 \\
88.1386 \\
88.0217 \\
86.1254 \\
87.8046 \\
88.0180 \\
87.8618 \\
88.3764 \\
85.9267 \\
88.0264 \\
87.7122 \\
87.9761 \\
87.6024 \\
87.8200 \\
87.4617 \\
87.6788 \\
87.6674 \\
87.6429 \\
87.1662 \\
88.1387 \\
87.9734 \\
88.1804 \\
87.9460 \\
87.9254 \\
87.6980 \\
39.6194 \\
88.1299 \\
88.5205 \\
87.9499 \\
85.8215 \\
87.8385 \\
87.9103 \\
87.6516 \\
88.2502 \\
87.9229 \\
88.0875 \\
87.9061 \\
88.2714 \\
87.8612 \\
87.9432 \\
87.3932\end{array}$ & $\begin{array}{c}\text { SD } \\
1.7702 e-01 \\
1.1383 e-01 \\
2.1194 e-01 \\
1.4710 e-01 \\
1.9402 e-01 \\
1.4987 e-01 \\
2.4711 e-01 \\
1.4671 e-01 \\
1.9766 e-01 \\
1.5714 e-01 \\
1.8663 e-01 \\
12872 e-01 \\
2.0968 e-01 \\
1.6074 e-01 \\
2.2648 e-01 \\
1.4294 e-01 \\
1.7277 e-01 \\
1.5269 e-01 \\
1.7287 e-01 \\
1.5380 e-01 \\
1.7344 e-01 \\
1.4164 e-01 \\
1.7598 e-01 \\
1.2768 e-01 \\
2.3469 e-01 \\
1.6515 e-01 \\
1.4395 e-01 \\
1.3108 e-01 \\
1.6586 e-01 \\
1.3348 e-01 \\
2.2912 e-01 \\
1.4055 e-01 \\
2.0645 e-01 \\
1.5408 e-01 \\
2.5620 e-01 \\
1.8701 e-01 \\
2.3983 e-01 \\
1.6084 e-01 \\
1.9781 e-01 \\
1.2167 e-01 \\
2.6760 e-01 \\
1.5112 e-01 \\
2.1961 e-01 \\
1.9293 e-01 \\
1.9232 e-01 \\
1.7145 e-01\end{array}$ & 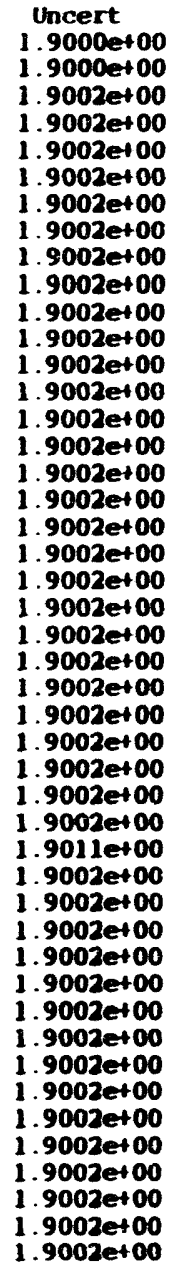 \\
\hline
\end{tabular}

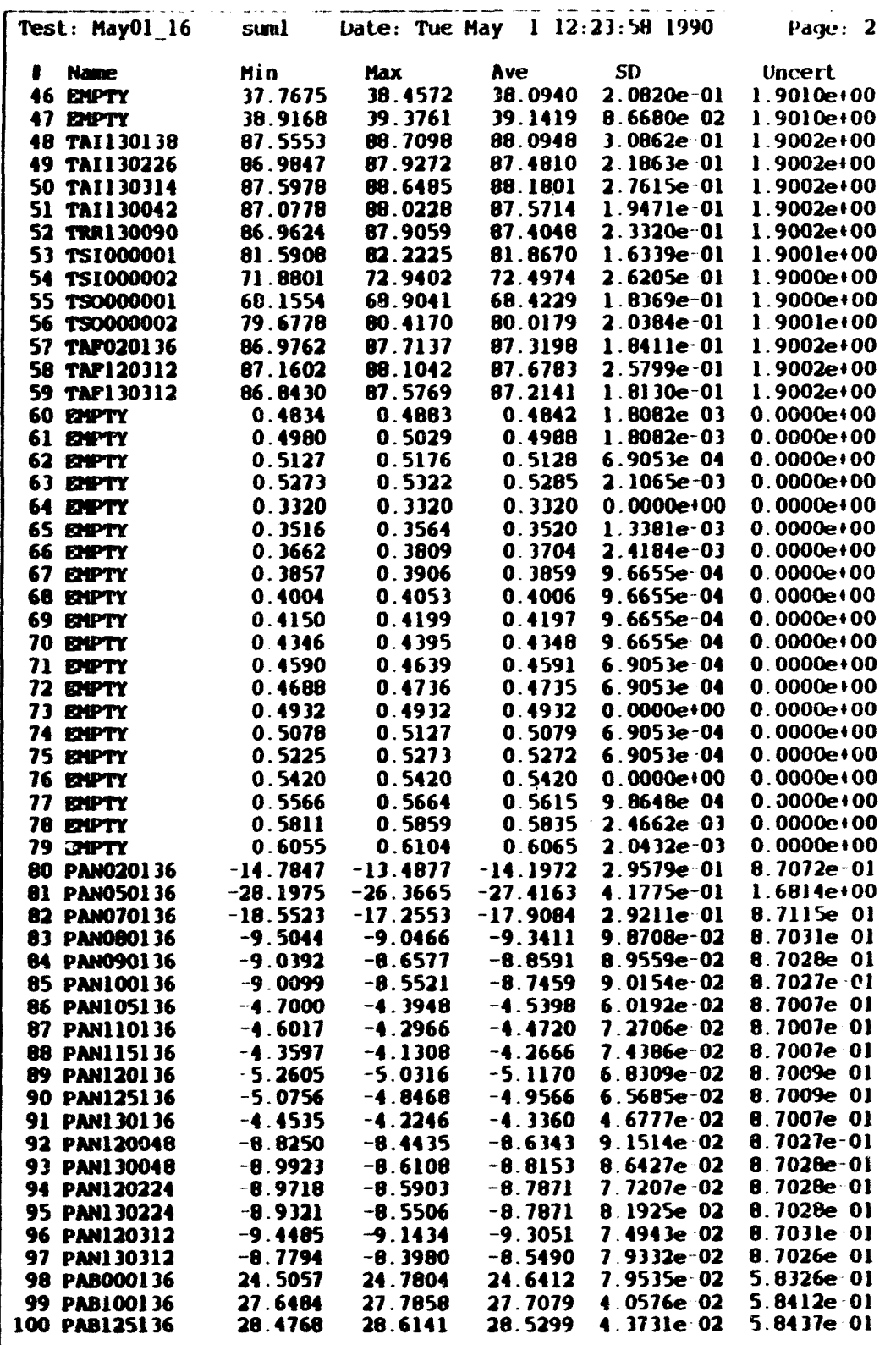




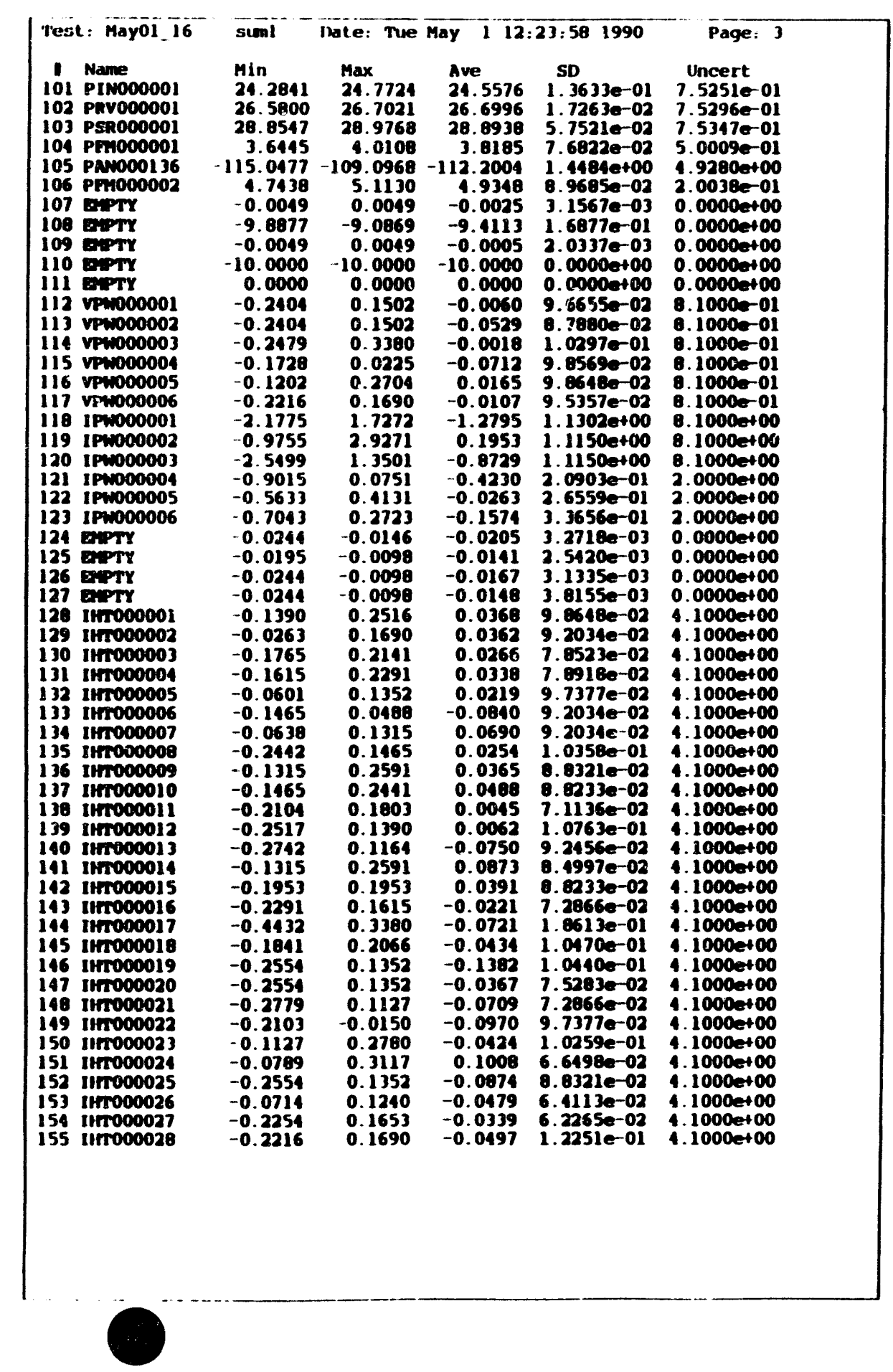

\begin{tabular}{|c|c|c|c|c|c|}
\hline Test: May01_16 & $\sin$ & Date: Tue & ay 12: & $23: 58 \quad 1990$ & Page: 4 \\
\hline 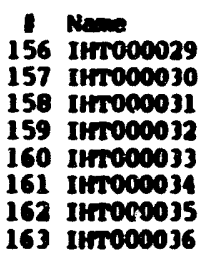 & $\begin{array}{l}\text { Min } \\
-0.0188 \\
-0.1653 \\
-0.1352 \\
-0.2065 \\
-0.1953 \\
-0.0263 \\
-0.0375 \\
-0.1953\end{array}$ & $\begin{array}{l}\operatorname{Max} \\
0.1765 \\
0.0300 \\
0.2554 \\
0.1840 \\
0.1953 \\
0.1690 \\
0.1578 \\
0.1953\end{array}$ & $\begin{array}{l}\text { Ave } \\
0.0046 \\
-0.0793 \\
-0.0376 \\
-0.0152 \\
0.0039 \\
0.0264 \\
0.0132 \\
0.0195\end{array}$ & $\begin{array}{l}\text { SD } \\
6.4113 e-02 \\
9.7935 e-02 \\
1.0625 e-01 \\
7.3716 e-02 \\
6.2265 e-02 \\
6.8585 e-02 \\
8.6541 e-02 \\
8.1347 e-02\end{array}$ & $\begin{array}{l}\text { Uncert } \\
4.1000 e+00 \\
4.1000 e+00 \\
1.1000 e+00 \\
1.1000 e+00 \\
1.1000 e+00 \\
1.1000=+00 \\
1.1000=+00 \\
1.1000=+00\end{array}$ \\
\hline
\end{tabular}


-

Veloxity $1=7.0456$ (ft/sec) $(1 /-\quad 3.7258$ : $)$
velocity $2=7.5856$ (ft/sec) $(1 /-1.9647$ :

Pressure drop - -2.9634 (psi)

Tsat in - 241.9549 (deg F) Tsat out - 247.8922 (deg F)

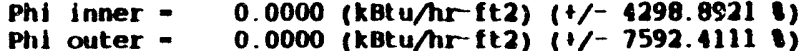

D Inner - 0.1962 (ft) D outer $=0.2413$ (ft) D hyd - 0.0389 (ft)

\begin{tabular}{|c|c|c|c|c|c|c|}
\hline $\begin{array}{r}1 \\
2 \\
5 \\
6 \\
9 \\
9 \\
9\end{array}$ & 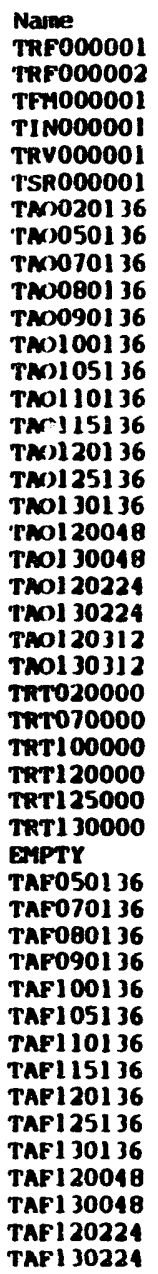 & $\begin{array}{l}\text { Min } \\
74.8498 \\
75.0738 \\
87.6744 \\
87.4084 \\
87.5020 \\
87.2182 \\
87.6827 \\
87.7515 \\
85.8832 \\
87.7471 \\
87.8269 \\
87.6922 \\
88.2899 \\
85.6980 \\
87.7467 \\
87.5713 \\
87.7641 \\
87.4903 \\
87.7126 \\
87.3291 \\
87.5338 \\
87.5348 \\
87.3551 \\
87.3924 \\
87.7623 \\
87.6256 \\
87.9810 \\
87.8932 \\
87.6470 \\
87.5571 \\
39.1464 \\
87.8594 \\
88.2754 \\
87.6925 \\
85.3112 \\
87.6640 \\
87.6834 \\
87.5274 \\
87.9587 \\
87.7778 \\
87.7515 \\
87.7617 \\
88.0284 \\
87.6910 \\
87.6600 \\
87.1425\end{array}$ & $\begin{array}{l}\text { Max } \\
75.2754 \\
75.4969 \\
88.5128 \\
87.9340 \\
88.1301 \\
87.8457 \\
88.6280 \\
88.4837 \\
86.6205 \\
88.2707 \\
88.4560 \\
88.3204 \\
88.9203 \\
86.4303 \\
88.6931 \\
88.0944 \\
88.2897 \\
88.0121 \\
88.2373 \\
87.8532 \\
88.2682 \\
88.1605 \\
88.0901 \\
87.9161 \\
88.7084 \\
88.3631 \\
88.7143 \\
88.4178 \\
88.4818 \\
88.1851 \\
10.0646 \\
88.5927 \\
89.0076 \\
88.4238 \\
86.4659 \\
88.2942 \\
88.6197 \\
88.0518 \\
88.6925 \\
88.3020 \\
88.7947 \\
88.5994 \\
88.8723 \\
88.4252 \\
88.4994 \\
87.8811\end{array}$ & $\begin{array}{l}\text { Ave } \\
75.0456 \\
75.2007 \\
88.0224 \\
87.6985 \\
87.8831 \\
87.5909 \\
88.2583 \\
88.1480 \\
86.2666 \\
87.9651 \\
88.1707 \\
87.9853 \\
88.6430 \\
86.1121 \\
88.1589 \\
87.8515 \\
88.0870 \\
87.7904 \\
87.9938 \\
87.6559 \\
87.8612 \\
87.8777 \\
87.8041 \\
87.6608 \\
88.2459 \\
88.1274 \\
88.3455 \\
88.1073 \\
88.1025 \\
87.8631 \\
39.6332 \\
88.2449 \\
88.6519 \\
88.1102 \\
85.9326 \\
87.9498 \\
88.0705 \\
87.7958 \\
88.4114 \\
88.0378 \\
88.2729 \\
88.0672 \\
88.3748 \\
88.0015 \\
88.1005 \\
87.5260\end{array}$ & $\begin{array}{l}\text { SD } \\
1.5991 e-01 \\
1.1310 e-01 \\
1.8385 e-01 \\
1.4643 e-01 \\
1.6464 e-01 \\
1.3244 e-01 \\
1.9364 e-01 \\
1.5955 e-01 \\
1.8648 e-01 \\
1.3339 e-01 \\
1.5119 e-01 \\
1.2883 e-01 \\
1.8582 e-01 \\
1.5238 e-01 \\
2.4583 e-01 \\
1.3097 e-01 \\
1.5098 e-01 \\
1.2737 e-01 \\
1.1443 e-01 \\
1.2456 e-01 \\
1.5081 e-01 \\
1.3199 e-01 \\
1.2734 e-01 \\
1.1833 e-01 \\
1.6997 e-01 \\
1.3724 e-01 \\
2.1898 e-01 \\
1.5558 e-01 \\
1.7735 e-01 \\
1.5094 e-01 \\
2.4818 e-01 \\
1.7372 e-01 \\
1.8386 e-01 \\
1.6871 e-01 \\
2.7297 e-01 \\
1.6311 e-01 \\
1.9840 e-01 \\
1.5289 e-01 \\
1.8139 e-01 \\
1.4698 e-01 \\
2.8072 e-01 \\
1.5819 e-01 \\
2.1307 e-01 \\
1.5421 e-01 \\
1.9657 e-01 \\
1.7403 e-01\end{array}$ & 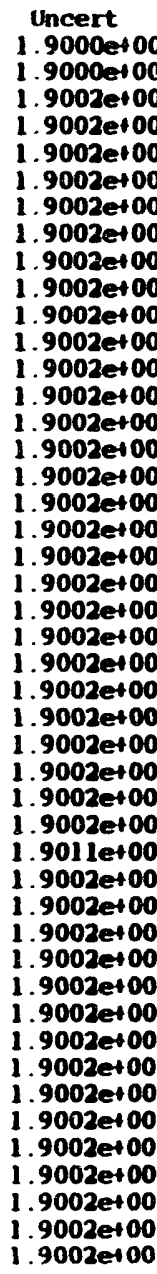 \\
\hline
\end{tabular}

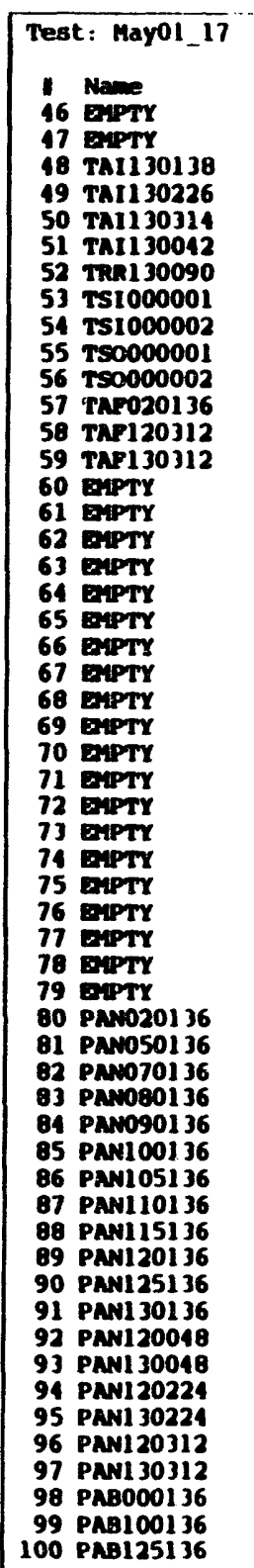

suml Date: Tue May 1 12:26:23 1990 Page: 2

Min Max Ave SD Uncert

$\begin{array}{lllll}37.7675 & 38.4572 & 38.0756 & 1.8931 \mathrm{e} .01 & 1.9010 \mathrm{e} 00\end{array}$

$\begin{array}{lllll}39.1464 & 39.3761 & 39.2291 & 1.1134 e-01 & 1.9010 e+00\end{array}$

$87.8701 \quad 80.9194 \quad 86.3360 \quad 3.0352 e .01 \quad 1.9002 e 100$

$87.2985 \quad 8.0309 \quad 87.6211$ 1.7159e-01 $1.9002 e+00$

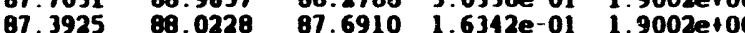

$87.0677 \quad 009907.99002 .0803 e-011.90022+00$

$81.5908 \quad 82.1180 \quad 81.8060$ 1.4432e.01 1.900010100

$\begin{array}{llllll}72.3039 & 73.0468 & 72.7410 & 2.2627 \mathrm{e}-01 & 1.9000 \mathrm{e} 00\end{array}$

$68.3698 \quad 69.1182 \quad 68.7675$ 1.7826e-01 $1.9000 e+00$

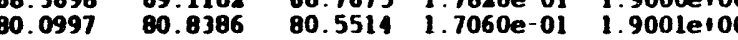

$87.0816 \quad 87.7147 \quad 07.4411 \quad 1.6646 \mathrm{e}-01 \quad 1.9002 e+00$

$\begin{array}{llllll}07.3698 & 88.4184 & 87.8396 & 2.2226 e & 01 & 1.9002 e t 00\end{array}$

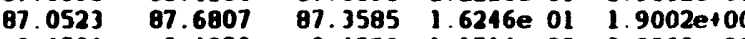

$\begin{array}{llllll}0.4834 & 0.4883 & 0.4880 & 1.1714 e-03 & 0.0000 e+00\end{array}$

$0.51290 .5029 \quad 0.50290 .0000$ eroo 0.0000100

$\begin{array}{llllll}0.5127 & 0.5176 & 0.5166 & 1.9730 \mathrm{e}-03 & 0.0000 \mathrm{e} 100\end{array}$

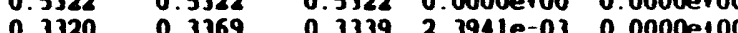

$0.35160 .35640 .35542 .041320-030.000000$

0.3516 0.3564 0.3511 0.00004000 .000010

$\begin{array}{llllll}0.3857 & 0.3906 & 0.3901 & 1.4797 e \cdot 03 & 0.0000 e t 00\end{array}$

$\begin{array}{lllllll}0.4004 & 0.4053 & 0.4037 & 2.3008 \text { 03 } 0.0000100\end{array}$

$\begin{array}{llllll}0.4199 & 0.4248 & 0.4201 & 9.6655 \mathrm{e}-04 & 0.0000 \mathrm{e} 00\end{array}$

$\begin{array}{llllll}0.4346 & 0.4395 & 0.4378 & 2.3365 \text { e }-03 & 0.0000 e 100\end{array}$

$\begin{array}{llllll}0.4590 & 0.4639 & 0.4604 & 2.2603 e^{-03} & 0.0000 e 100\end{array}$

$0.4736 \quad 0.47850 .474 \quad 1.7115$ e-03 0.0000 e 00

$\begin{array}{lllll}0.4932 & 0.4980 & 0.4934 & 9.6655 e-04 & 0.0000 e+00\end{array}$

$\begin{array}{llllll}0.5078 & 0.5127 & 0.5109 & 2.3675 \mathrm{e}-03 & 0.0000 \mathrm{e} 100\end{array}$

$\begin{array}{lllll}0.5273 & 0.5322 & 0.5275 & 9.6655 \mathrm{e}-04 & 0.0000 \mathrm{e} 100\end{array}$

$0.5420 \quad 0.54690 .54216 .9053 \mathrm{e} .040 .0000$ e 00

0.58590 .58590 .58590 .00001000 .0000100

$0.6055 \quad 0.6104 \quad 0.61020 .66550-040.0000100$

$11.1226-9.5204-10.3963 \quad 3.93950-0101.70390-01$

$-21.6362-19.5000-20.408 \quad 5.6191 \mathrm{e}-01 \quad 1.6808 \mathrm{e} 100$

$-13.6695-12.2199-12.9615 \quad 3.3947 e-01 \quad 8.7060 e-0$

$-7.2918 \quad-6.7578 \quad-7.0767 \quad 1.4016 \mathrm{e}-01 \quad 8.7018 \mathrm{e}-01$

$\begin{array}{llllll}-6.9030 & -6.4452 & -6.6802 & 9.9702 e-02 & 8.7016 e-01\end{array}$

$\begin{array}{lllllll}-6.4922 & -6.0344 & -6.2480 & 9.2482 e & 02 & 8.7014 e & 01\end{array}$

$\begin{array}{lllllll}-3.4793 & -3.1741 & -3.3099 & 6.9430 \mathrm{e}-02 & 8.7004 \text { e } 01\end{array}$

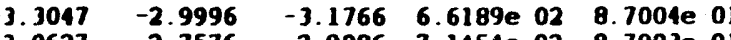

$\begin{array}{llllll}-3.0627 & -2.7576 & -2.9086 & 7.1454 \mathrm{e} .02 & \mathbf{8} .7003 \mathrm{e} 01\end{array}$

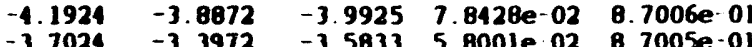

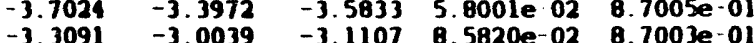

$-6.5362-6.0784-6.3021 \quad 1.10600010 .7015-01$

$-6.5509-6.1694-6.3739 \quad 1.03900010 .01015$

$-6.6066-6.1489-6.4052 \quad 1.0312 e .018 .7015 e-01$

$\begin{array}{llllll}-6.4144 & -6.0329 & -6.2099 & 1.1268 \mathrm{e} 01 & 8.7014 \mathrm{e}-01\end{array}$

$\begin{array}{llllll}-7.1597 & -6.7020 & -6.9018 & 1.0667 e-01 & 8.7017 e & 01\end{array}$

$\begin{array}{llllll}-6.4906 & -6.0329 & -6.2236 & 9.6567 \mathrm{e}-02 & \mathbf{8 . 7 0 1 4 e - 0 1}\end{array}$

$\begin{array}{lllllll}25.6959 & 26.1079 & 25.8790 & 9.6996 e & 02 & 5.8360 e \cdot 01\end{array}$

$\begin{array}{llllll}28.0147 & 28.2435 & 28.1007 & 5.0350 \mathrm{e} \cdot 02 & 5.8424 \mathrm{e}-01 \\ 28.6141 & 28.7514 & 28.7038 & 4.2340 \mathrm{e}-02 & 5.8442 \mathrm{e} \cdot 01\end{array}$ 


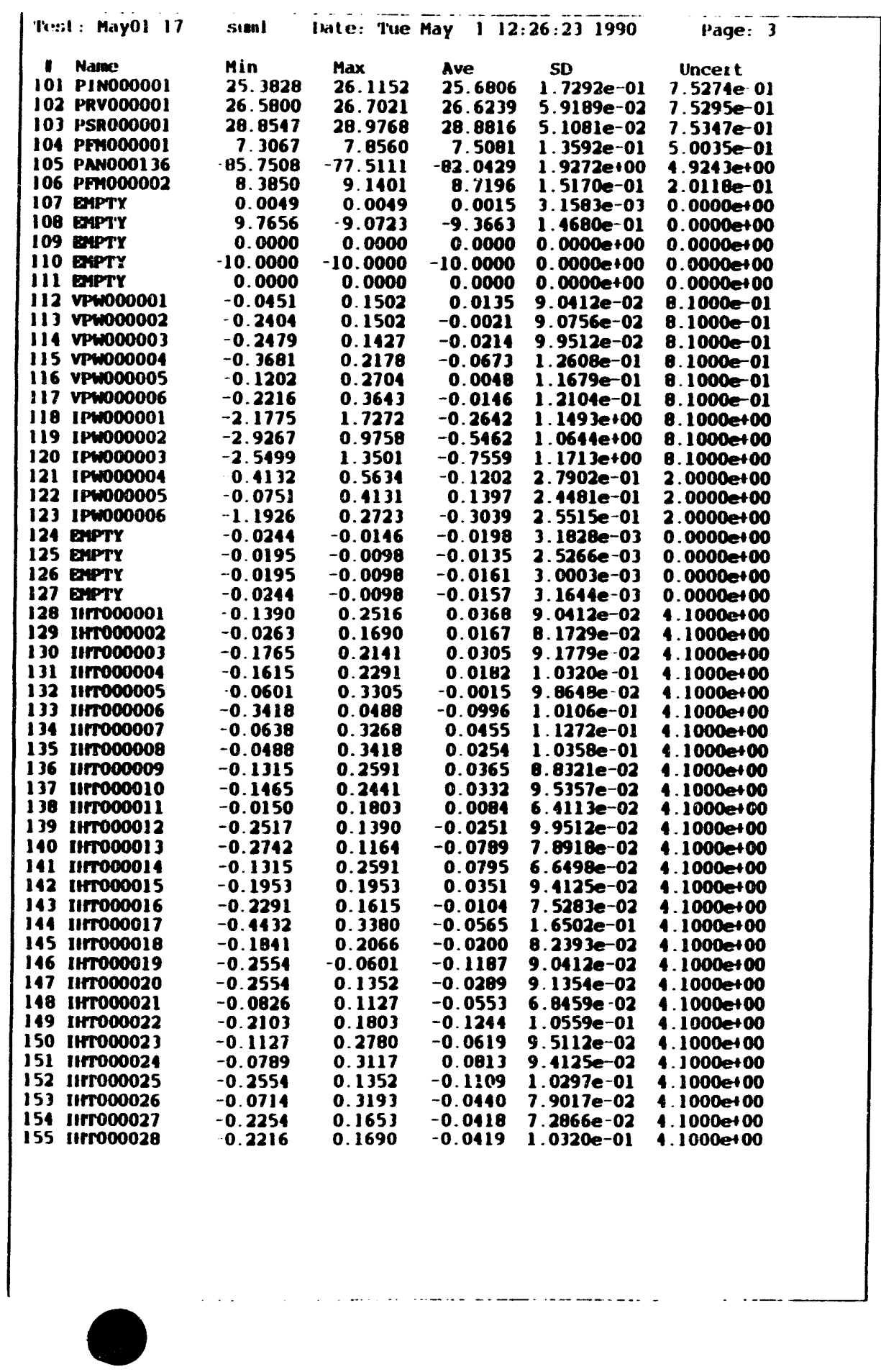

\begin{tabular}{|c|c|c|c|c|c|c|}
\hline Test & $t: M$ & 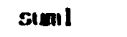 & Date: & tay 1 & $: 231990$ & tye: \\
\hline $\begin{array}{l}1 \\
156 \\
157 \\
158 \\
159 \\
160 \\
161 \\
162 \\
163\end{array}$ & 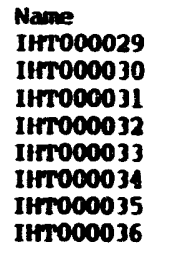 & $\begin{array}{l}\text { Min } \\
-0.0188 \\
-0.1653 \\
-0.1352 \\
-0.2066 \\
-0.1953 \\
-0.0263 \\
-0.2329 \\
-0.1953\end{array}$ & $\begin{array}{l}\operatorname{Max} \\
0.1765 \\
0.0300 \\
0.2554 \\
0.1840 \\
0.1953 \\
0.1690 \\
0.1578 \\
0.1953\end{array}$ & $\begin{array}{l}\text { Ave } \\
0.0164 \\
-0.0754 \\
-0.0376 \\
-0.0113 \\
0.0117 \\
0.0518 \\
0.0328 \\
0.0195\end{array}$ & $\begin{array}{l}\text { SD } \\
7.5798 \mathrm{e}-02 \\
9.8332 \mathrm{e}-02 \\
1.0625 \mathrm{e}-01 \\
7.8918 \mathrm{e}-02 \\
7.2866 \mathrm{e}-02 \\
9.6655 \mathrm{e}-02 \\
1.0259 \mathrm{e}-01 \\
9.0412 \mathrm{e}-02\end{array}$ & 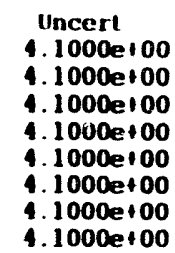 \\
\hline
\end{tabular}




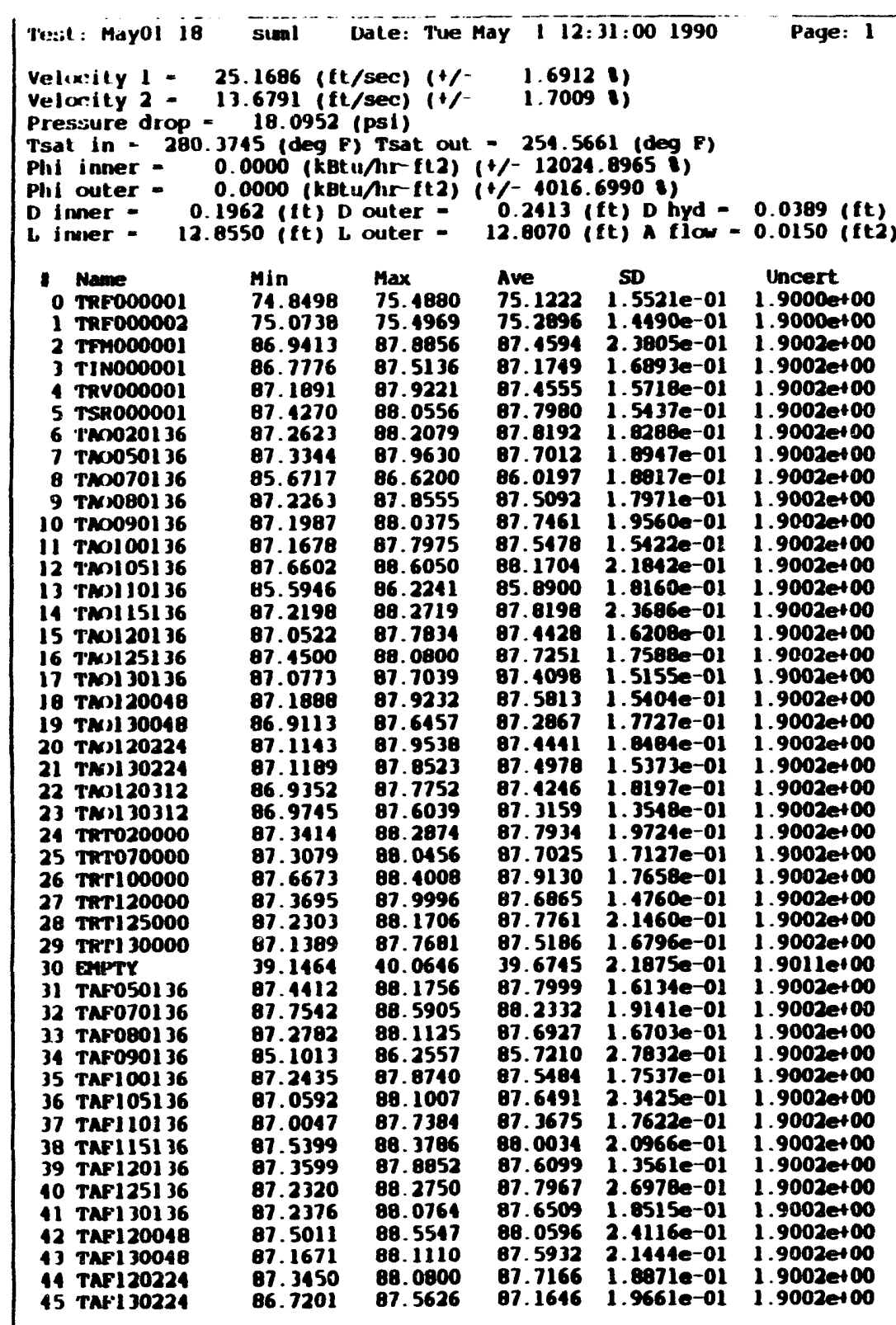

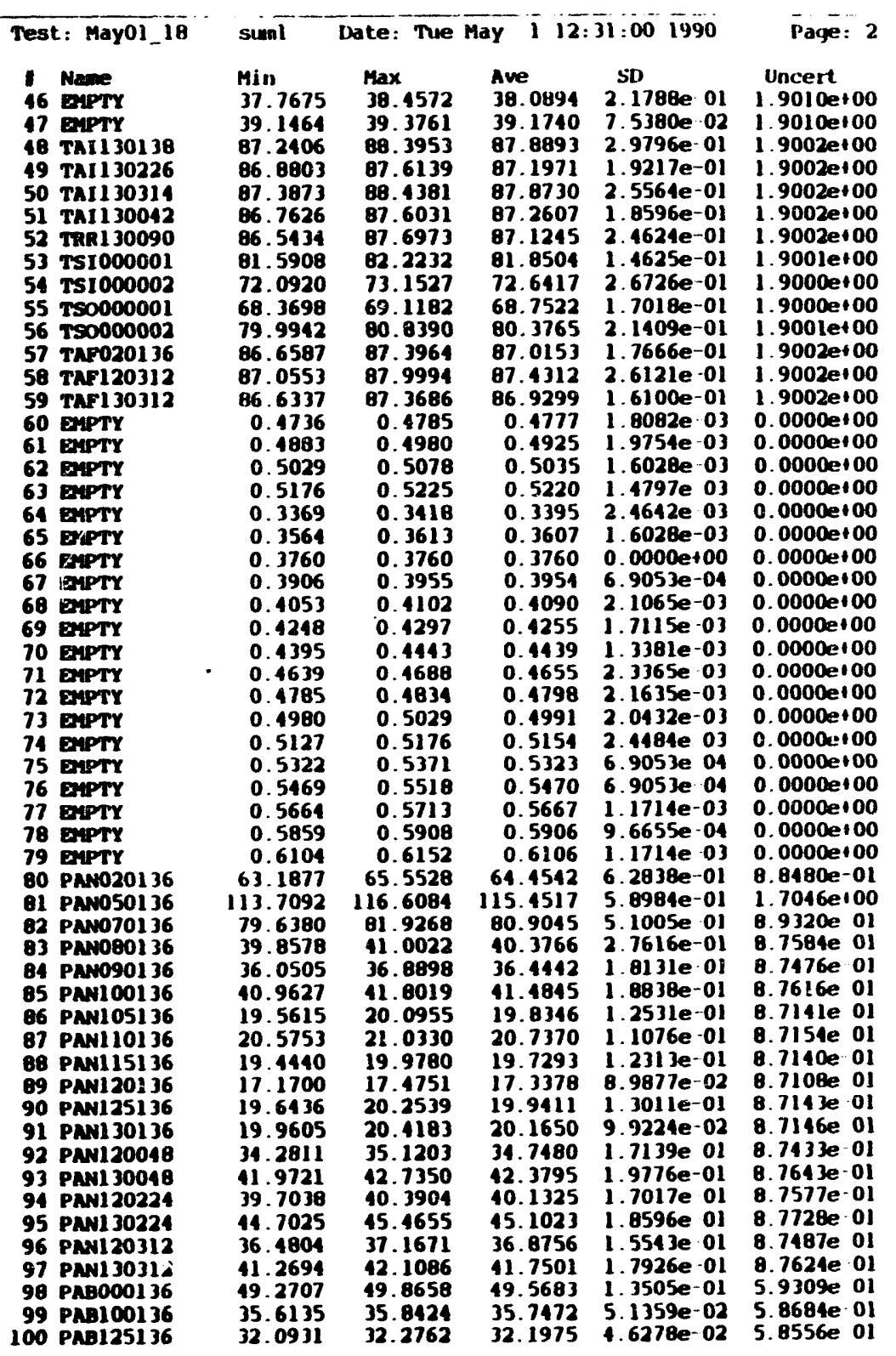




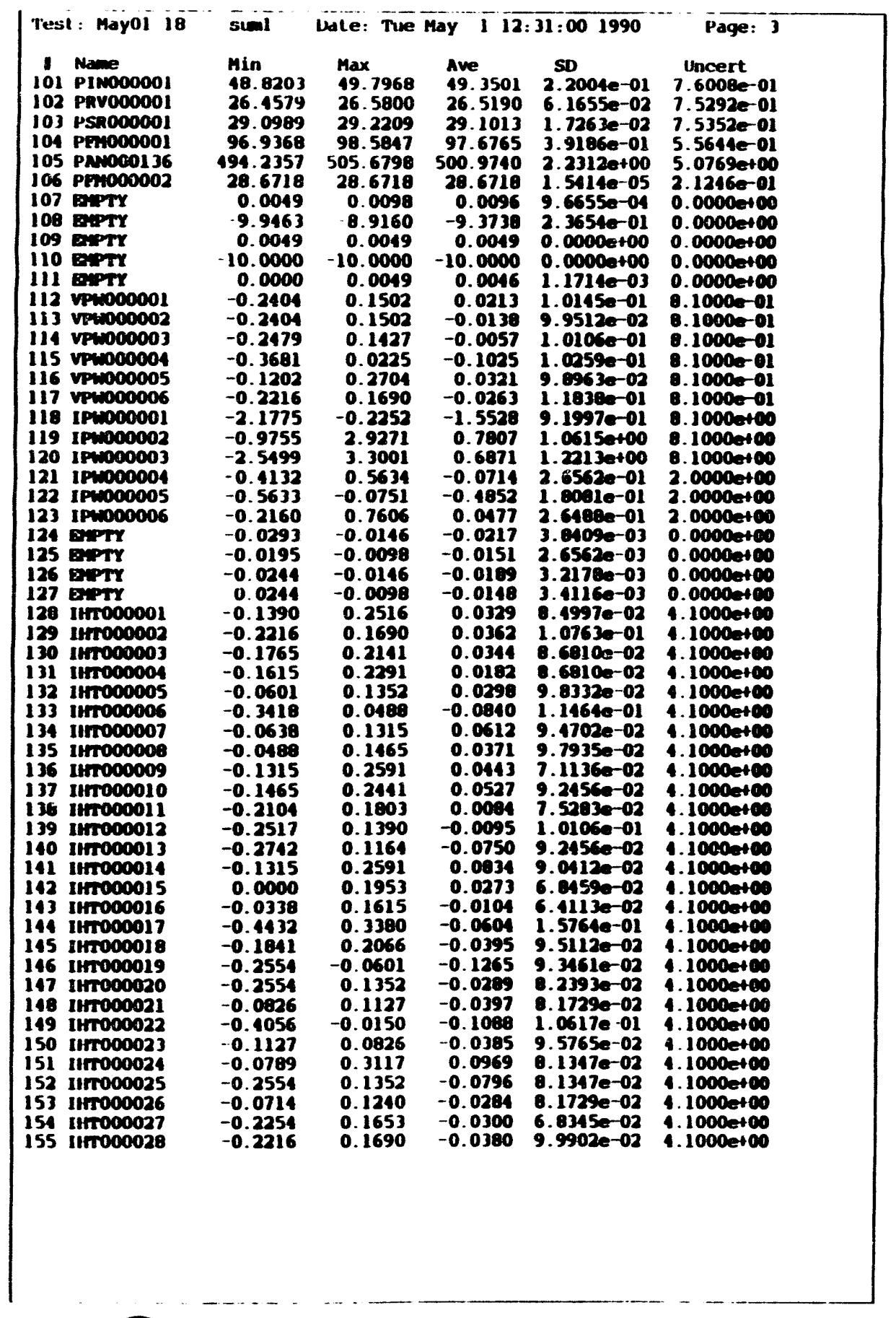

\begin{tabular}{|c|c|c|c|c|c|}
\hline 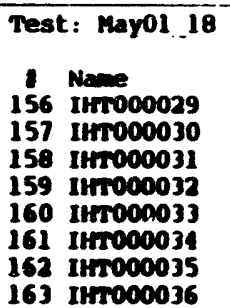 & $\begin{array}{l}\text { sial } \\
\text { Min } \\
-0.0188 \\
-0.1653 \\
-0.1352 \\
-0.2066 \\
-0.1953 \\
-0.0263 \\
-0.2329 \\
-0.1953\end{array}$ & $\begin{array}{l}\text { Date: Tue } \\
\text { Max } \\
0.1765 \\
0.2253 \\
0.2554 \\
0.1640 \\
0.1953 \\
0.3643 \\
0.3531 \\
0.1953\end{array}$ & $\begin{array}{l}\text { Ave } \\
0.0161 \\
-0.0715 \\
-0.0415 \\
-0.0035 \\
0.0117 \\
0.0675 \\
0.0328 \\
0.0273\end{array}$ & $\begin{array}{l}50 \\
7.5798 \mathrm{e}-02 \\
1.0617 \mathrm{e}-01 \\
1.0617 \mathrm{e}-01 \\
6.7808 \mathrm{e}-02 \\
8.2864 \mathrm{e}-02 \\
1.0617 \mathrm{e}-01 \\
1.0992 \mathrm{e}-01 \\
7.9017 \mathrm{e}-02\end{array}$ & 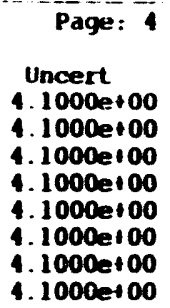 \\
\hline
\end{tabular}




\begin{tabular}{|c|c|c|c|c|c|}
\hline \multicolumn{6}{|c|}{ 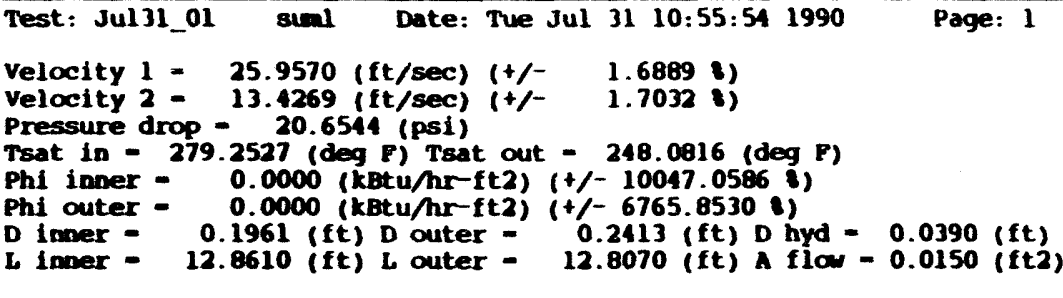 } \\
\hline 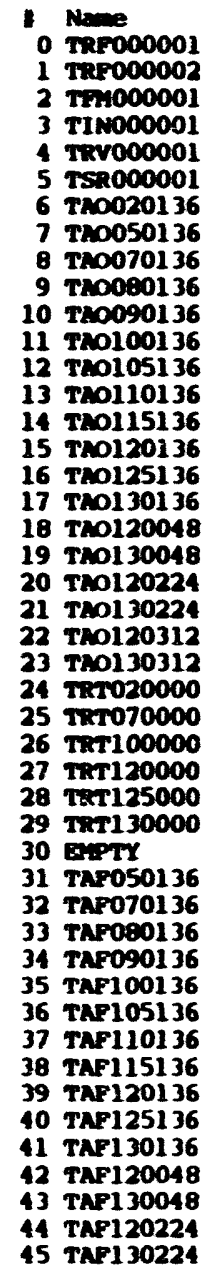 & $\begin{array}{l}\text { Min } \\
73.8393 \\
73.7731 \\
87.7874 \\
86.9990 \\
87.5752 \\
88.0985 \\
80.2648 \\
87.4371 \\
87.4575 \\
87.2338 \\
88.0158 \\
87.4741 \\
88.2964 \\
87.7400 \\
87.9081 \\
87.6301 \\
88.2663 \\
87.4815 \\
71.7958 \\
87.3540 \\
87.5697 \\
87.5219 \\
87.5520 \\
87.5278 \\
87.9318 \\
87.9196 \\
88.0795 \\
87.8859 \\
87.7649 \\
87.6877 \\
39.1464 \\
87.9224 \\
88.3312 \\
87.8832 \\
72.6689 \\
87.7398 \\
87.5334 \\
87.6706 \\
88.0605 \\
88.1001 \\
87.9722 \\
87.8628 \\
88.1365 \\
87.9383 \\
87.9288 \\
87.3532\end{array}$ & $\begin{array}{l}\text { Max } \\
74.4796 \\
74.1978 \\
88.5229 \\
87.6312 \\
88.3092 \\
88.7288 \\
81.2177 \\
88.2760 \\
88.1953 \\
88.0731 \\
88.7507 \\
88.1054 \\
89.3483 \\
88.4745 \\
88.8549 \\
88.1567 \\
88.8971 \\
88.2150 \\
72.5416 \\
87.9849 \\
88.3054 \\
88.3593 \\
88.2892 \\
88.1588 \\
89.0697 \\
88.1474 \\
89.0212 \\
88.5164 \\
88.6034 \\
88.2142 \\
40.0646 \\
88.7618 \\
89.1696 \\
88.7204 \\
73.5190 \\
88.5821 \\
88.6826 \\
88.3011 \\
88.8992 \\
89.6265 \\
89.0179 \\
88.9114 \\
89.1913 \\
88.5693 \\
88.7684 \\
88.1959\end{array}$ & $\begin{array}{l}\text { Ave } \\
74.1850 \\
73.9684 \\
88.2018 \\
87.3551 \\
87.9583 \\
88.3534 \\
80.6358 \\
87.8301 \\
87.9022 \\
87.6596 \\
88.4208 \\
87.7249 \\
88.7825 \\
88.1407 \\
88.3269 \\
87.8725 \\
88.5798 \\
87.7739 \\
72.1476 \\
87.6509 \\
87.9835 \\
88.0057 \\
88.0132 \\
87.8080 \\
88.3930 \\
88.1878 \\
88.4716 \\
88.2281 \\
88.2076 \\
87.9930 \\
39.6102 \\
88.3738 \\
88.7321 \\
89.2756 \\
73.0661 \\
88.1757 \\
88.0707 \\
87.9674 \\
88.5285 \\
88.3676 \\
88.4428 \\
88.2099 \\
88.6025 \\
88.2475 \\
88.2632 \\
87.7558\end{array}$ & 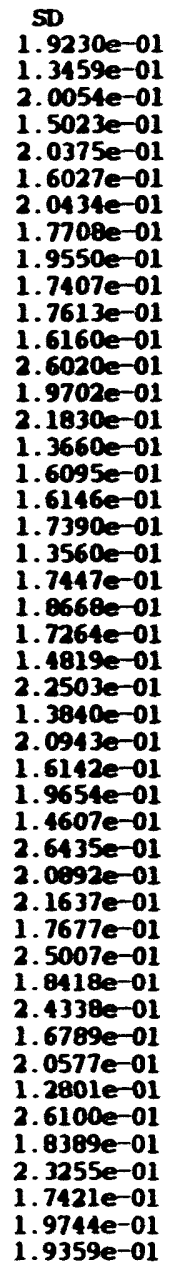 & $\begin{array}{c}\text { Uncert } \\
1.9000 e+00 \\
1.9000 e+00 \\
1.9002 e+00 \\
1.9002 e+00 \\
1.9002 e+00 \\
1.9002 e+00 \\
1.9001 e+00 \\
1.9002 e+00 \\
1.9002 e+00 \\
1.9002 e+00 \\
1.9002 e+00 \\
1.9002 e+00 \\
1.9002 e+00 \\
1.9002 e+00 \\
1.9002 e+00 \\
1.9002 e+00 \\
1.9002 e+00 \\
1.9002 e+00 \\
1.9000 e+00 \\
1.9002 e+00 \\
1.9002 e+00 \\
1.9002 e+00 \\
1.9 n 02 e+00 \\
1.9002 e+00 \\
1.9002 e+00 \\
1.9002 e+00 \\
1.9002 e+00 \\
1.9002 e+00 \\
1.9002 e+00 \\
1.9002 e+00 \\
1.9011 e+00 \\
1.9002 e+00 \\
1.9002 e+00 \\
1.9002 e+00 \\
1.9000 e+00 \\
1.9002 e+00 \\
1.9002 e+00 \\
1.9002 e+00 \\
1.9002 e+00 \\
1.9002 e+00 \\
1.9002 e+00 \\
1.9002 e+00 \\
1.9002 e+00 \\
1.9002 e+00 \\
1.9002 e+00 \\
1.9002 e+00\end{array}$ \\
\hline
\end{tabular}

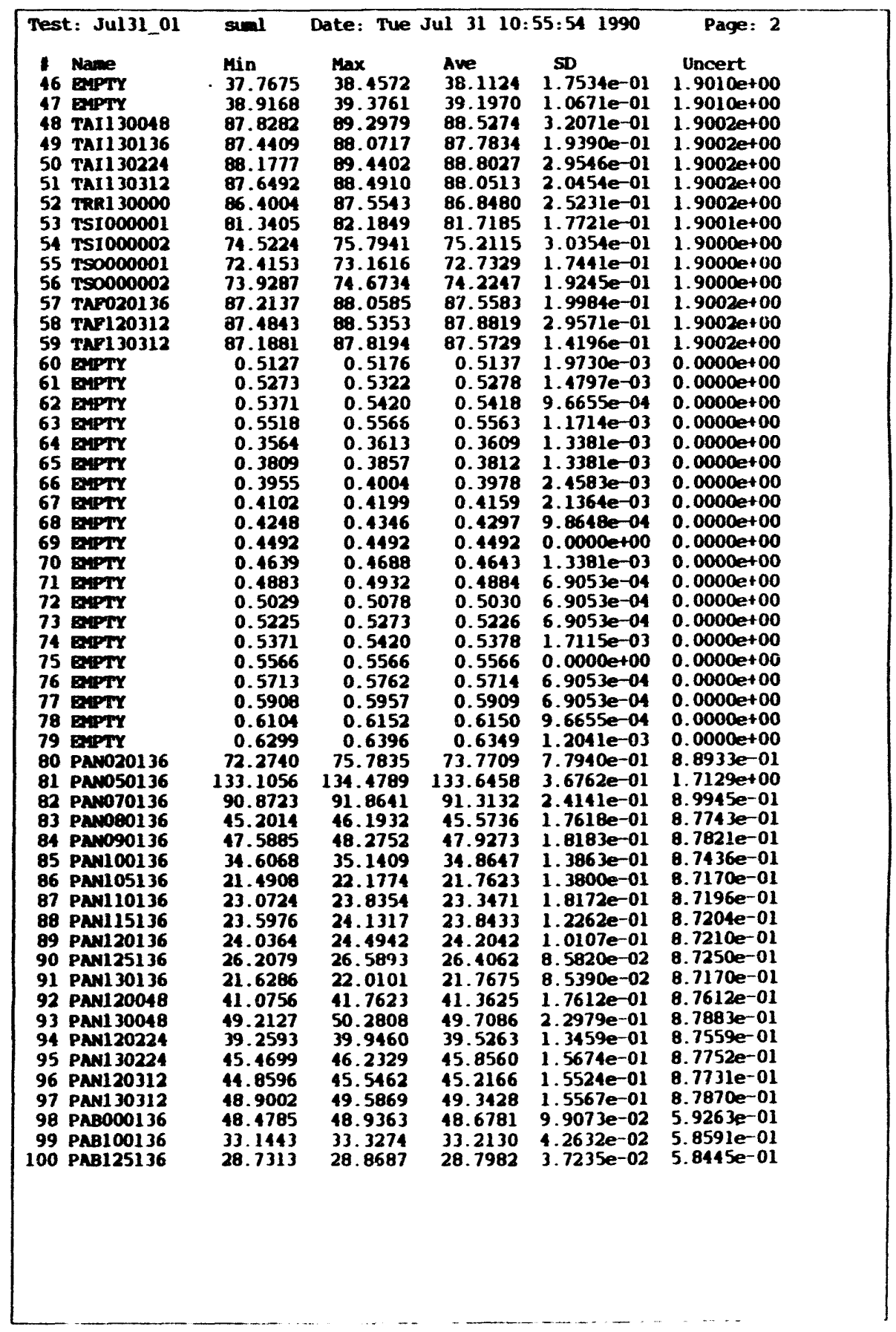




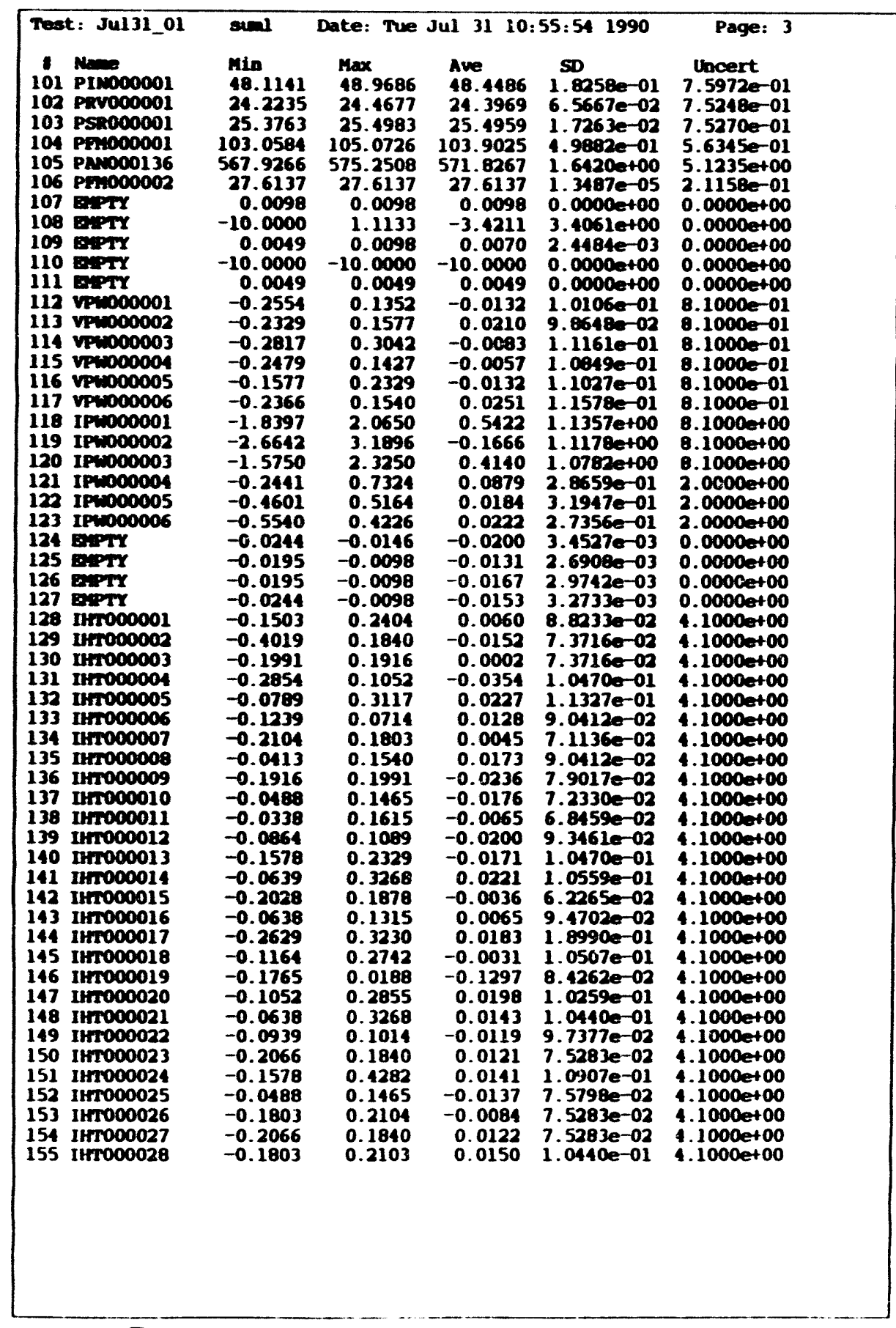

\begin{tabular}{|c|c|c|c|c|c|}
\hline Test: J & $\sin 1$ & Date: Tu & 31 & 541990 & Page: \\
\hline 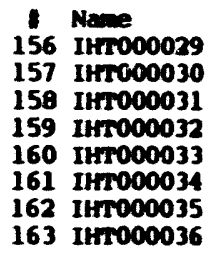 & $\begin{array}{l}\text { Min } \\
-0.2366 \\
-0.1878 \\
-0.2442 \\
-0.2404 \\
-0.2329 \\
-0.1765 \\
-0.2216 \\
-0.0939\end{array}$ & $\begin{array}{l}\operatorname{Max} \\
0.1540 \\
0.2028 \\
0.1465 \\
0.1502 \\
0.1578 \\
0.2141 \\
0.1690 \\
0.1014\end{array}$ & $\begin{array}{r}\text { Ave } \\
-0.0101 \\
0.0388 \\
-0.0020 \\
0.0057 \\
0.0054 \\
-0.0086 \\
0.0011 \\
0.0233\end{array}$ & $\begin{array}{l}\text { SD } \\
8.2393 e-02 \\
9.9512 e-02 \\
1.1545 e-01 \\
9.5112 e-02 \\
9.0756 e-02 \\
8.8321 e-02 \\
9.6735 e-02 \\
9.6655 e-02\end{array}$ & $\begin{array}{l}\text { Uncert } \\
4.1000 \text { et00 } \\
4.1000 \mathrm{e}+00 \\
4.1000 \mathrm{e}+00 \\
1.1000 \mathrm{e}+00 \\
4.1000 \mathrm{e}+00 \\
4.1000 \mathrm{e}+00 \\
4.1000 \mathrm{e}+00 \\
4.1000 \mathrm{e}+00\end{array}$ \\
\hline
\end{tabular}

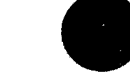




\begin{tabular}{|c|c|c|c|c|c|}
\hline \multicolumn{3}{|c|}{ 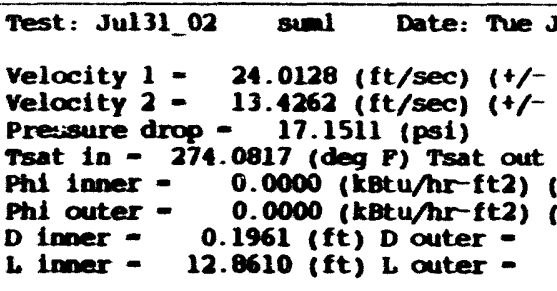 } & \multicolumn{2}{|c|}{$\begin{array}{l}\text { Jul } 3111: 00: 151990 \\
1.6952 \text { :) } \\
1.7032 \text { : } \\
-246.8510 \text { (deg F) } \\
(+/-5380.4985 \text { ) } \\
(+/-10507.3291 \text { ( ) } \\
0.2413 \text { (ft) D hyd }= \\
12.8070 \text { (ft) A flow - }\end{array}$} & $\begin{array}{l}\text { Page: } 1 \\
0.0390 \text { (ft) } \\
0.0150 \text { (ft2) }\end{array}$ \\
\hline 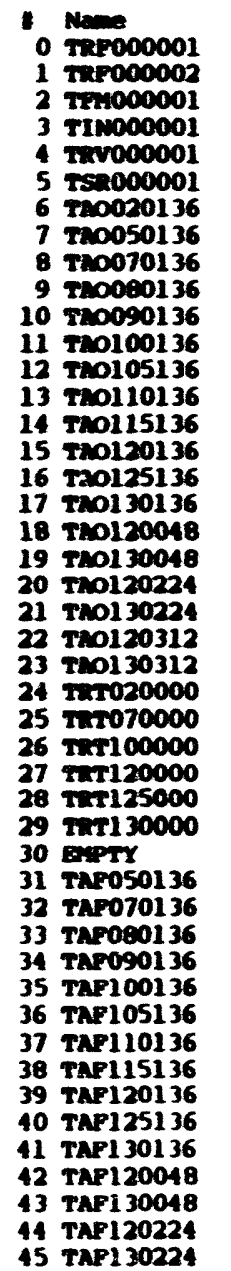 & $\begin{array}{l}\text { Min } \\
73.8393 \\
73.7731 \\
87.1589 \\
86.2626 \\
86.8427 \\
87.4712 \\
80.1592 \\
86.9156 \\
86.9293 \\
86.7110 \\
87.3870 \\
86.8447 \\
87.6654 \\
87.2211 \\
87.1721 \\
87.0000 \\
87.7426 \\
86.7590 \\
72.5398 \\
86.7260 \\
87.0443 \\
87.0033 \\
87.0269 \\
87.0044 \\
87.0890 \\
87.2859 \\
87.3471 \\
87.2567 \\
87.2439 \\
87.0590 \\
39.1464 \\
87.5042 \\
87.7051 \\
87.3633 \\
72.9870 \\
87.2138 \\
86.8033 \\
87.0434 \\
87.5368 \\
87.3691 \\
87.3481 \\
87.2364 \\
87.5026 \\
87.2045 \\
87.1962 \\
86.8260\end{array}$ & $\begin{array}{l}\text { Max } \\
74.4796 \\
74.1978 \\
88.1041 \\
86.9999 \\
87.7862 \\
88.2069 \\
81.2182 \\
87.5459 \\
87.7733 \\
87.3418 \\
88.2272 \\
87.5813 \\
88.7178 \\
88.0585 \\
88.2243 \\
87.6312 \\
88.2687 \\
87.4893 \\
73.0729 \\
87.4622 \\
87.7809 \\
87.7355 \\
87.8681 \\
87.5306 \\
88.2479 \\
87.9193 \\
89.5004 \\
88.0972 \\
87.9779 \\
87.6903 \\
39.8352 \\
88.1348 \\
88.5434 \\
87.9932 \\
74.1551 \\
87.9509 \\
87.8500 \\
87.8832 \\
88.3770 \\
87.9998 \\
88.1856 \\
88.1800 \\
88.4525 \\
88.0448 \\
88.0364 \\
87.5635\end{array}$ & $\begin{array}{l}\text { Ave } \\
74.2106 \\
73.9303 \\
87.5996 \\
86.7427 \\
87.3667 \\
87.8288 \\
80.6676 \\
87.2429 \\
87.3434 \\
87.0728 \\
87.8321 \\
87.1775 \\
88.2085 \\
87.5601 \\
87.6940 \\
87.2656 \\
87.9406 \\
87.1468 \\
72.7789 \\
87.0838 \\
87.3519 \\
87.3589 \\
87.3760 \\
87.1943 \\
87.7843 \\
87.5982 \\
87.8334 \\
87.6307 \\
87.6173 \\
87.4085 \\
39.5781 \\
87.7948 \\
88.1084 \\
87.6655 \\
73.5646 \\
87.5927 \\
87.4648 \\
87.3759 \\
87.9322 \\
87.7262 \\
87.8204 \\
87.6050 \\
87.9837 \\
87.5959 \\
87.6496 \\
87.1422\end{array}$ & 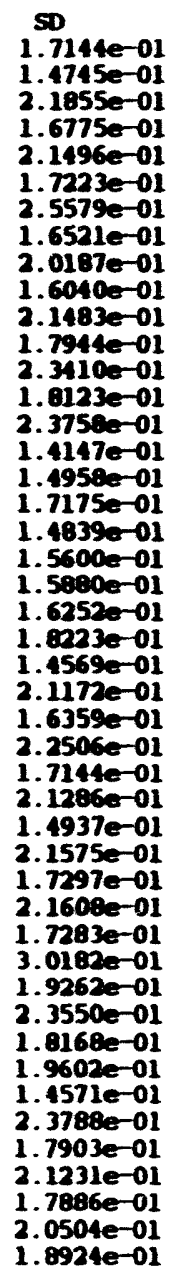 & $\begin{array}{l}\text { Uncert } \\
1.9000 e+00 \\
1.9000 e+00 \\
1.9002 e+00 \\
1.9002 e+00 \\
1.9002 e+00 \\
1.9002 e+00 \\
1.9001 e+00 \\
1.9002 e+00 \\
1.9002 e+00 \\
1.9002 e+00 \\
1.9002 e+00 \\
1.9002 e+00 \\
1.9002 e+00 \\
1.9002 e+00 \\
1.9002 e+00 \\
1.9002 e+00 \\
1.90020+00 \\
1.9002 e+00 \\
1.9000 e+00 \\
1.9002 e+00 \\
1.9002 e+00 \\
1.9002 e+00 \\
1.9002 e+00 \\
1.9002 e+00 \\
1.9002 e+00 \\
1.9002 e+00 \\
1.9002 e+00 \\
1.9002 e+00 \\
1.9002 e+00 \\
1.9002 e+00 \\
1.9011 e+00 \\
1.9002 e+00 \\
1.9002 e+00 \\
1.9002 e+00 \\
1.9000 e+00 \\
1.9002 e+00 \\
1.9002 e+00 \\
1.9002 e+00 \\
1.9002 e+00 \\
1.9002 e+00 \\
1.9002 e+00 \\
1.9002 e+00 \\
1.9002 e+00 \\
1.9002 e+00 \\
1.9002 e+00 \\
1.9002 e+00\end{array}$ \\
\hline
\end{tabular}

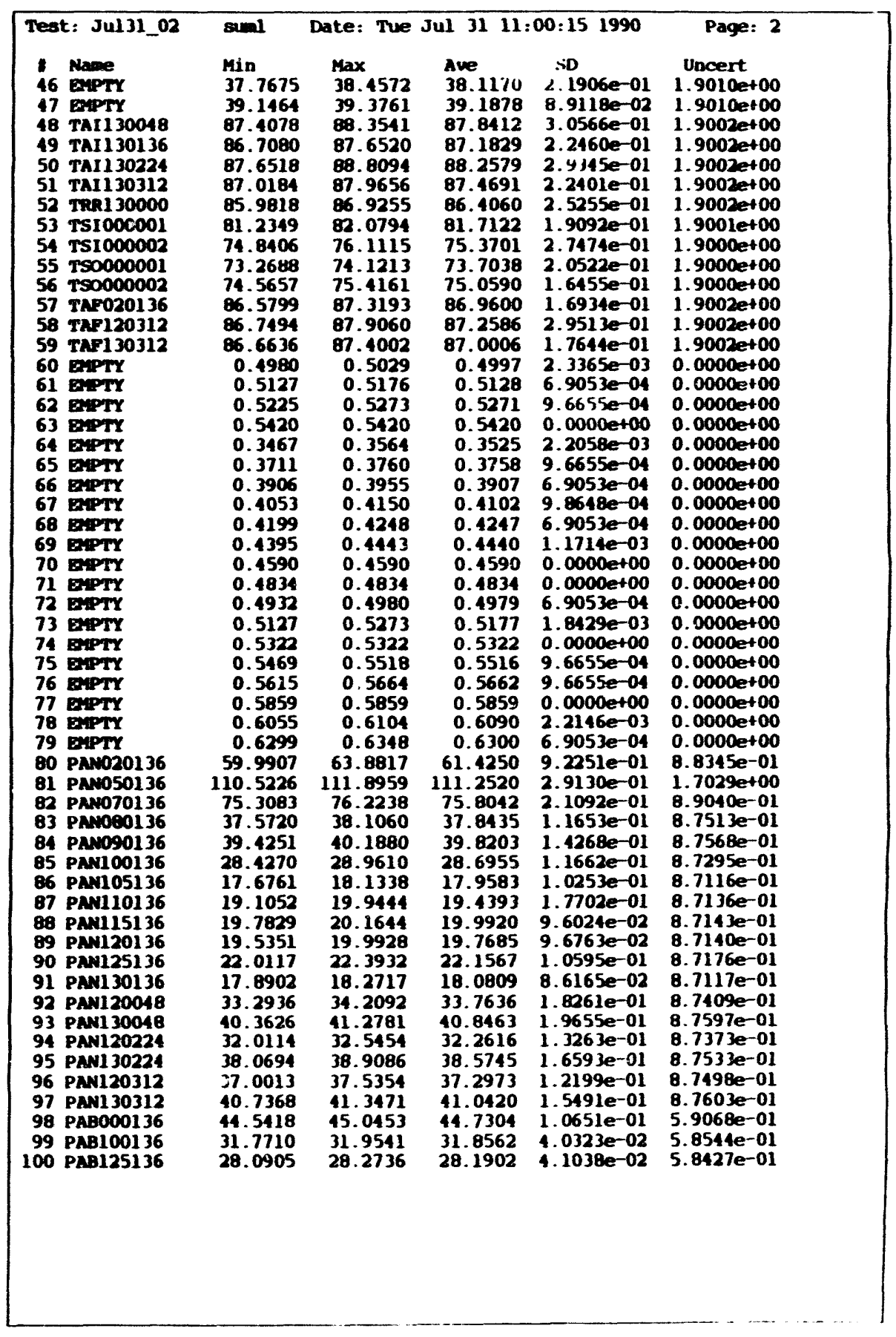




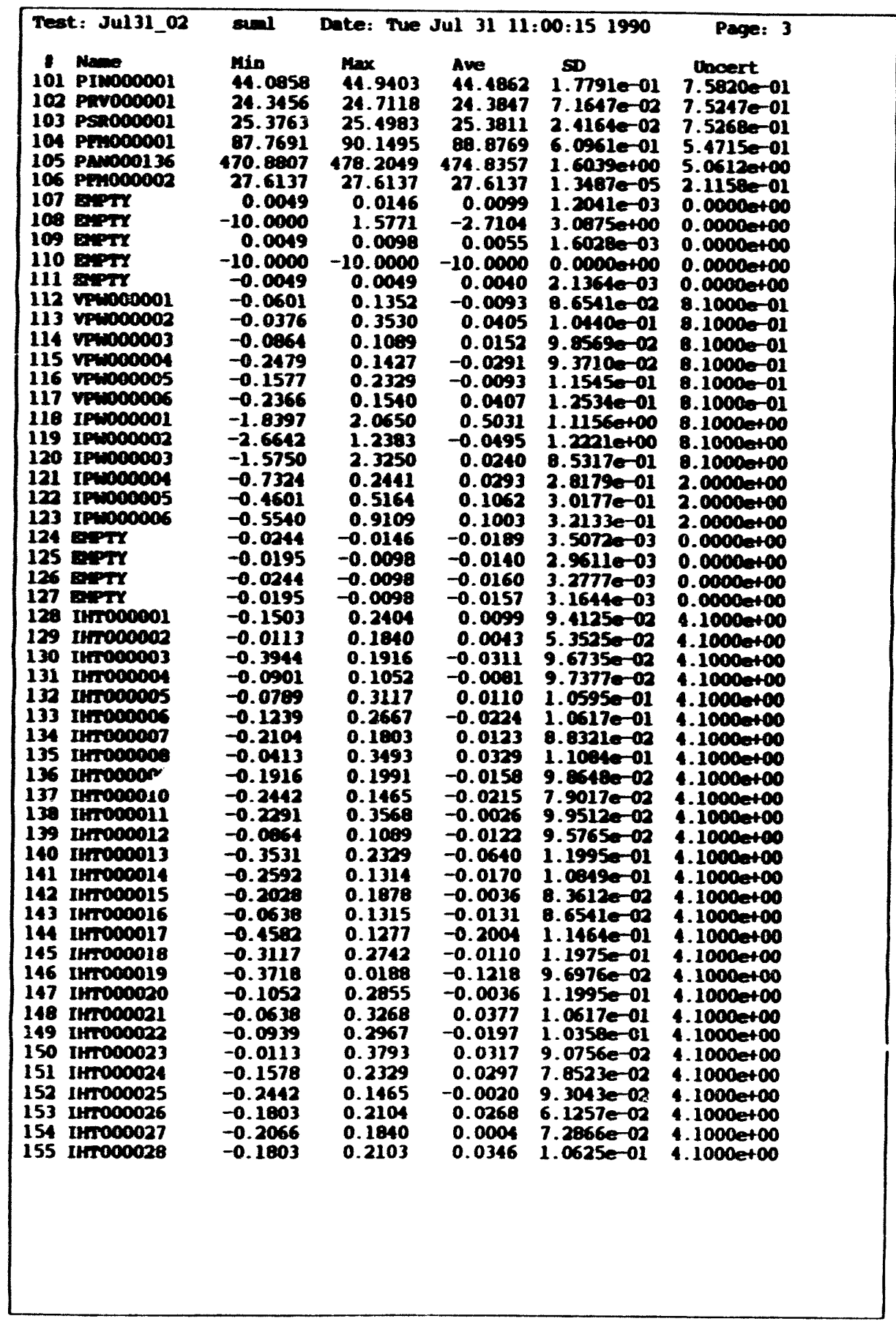

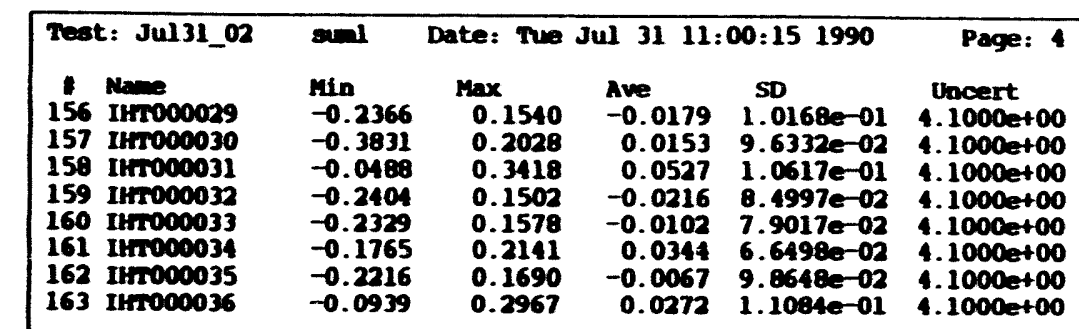




\begin{tabular}{|c|c|c|c|c|c|}
\hline \multicolumn{3}{|c|}{ 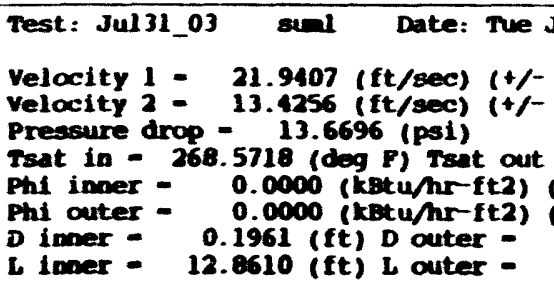 } & \multicolumn{2}{|c|}{ 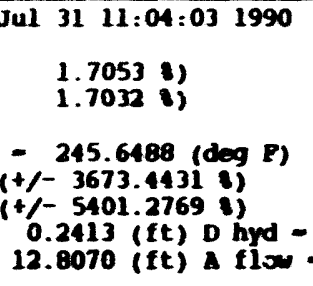 } & $\begin{array}{l}\text { Page: } 1 \\
0.0390 \text { (ft) } \\
0.0150 \text { (ft2) }\end{array}$ \\
\hline 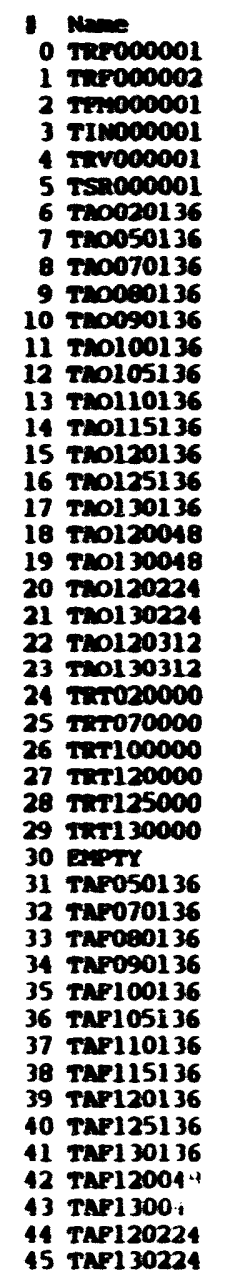 & $\begin{array}{l}\text { Min } \\
73.8393 \\
73.7731 \\
86.6333 \\
85.9463 \\
86.4257 \\
86.9493 \\
80.2648 \\
86.3934 \\
86.5078 \\
86.2948 \\
86.9672 \\
86.3201 \\
87.3495 \\
86.8041 \\
86.7507 \\
86.4746 \\
87.1136 \\
86.3447 \\
72.8596 \\
86.2029 \\
86.5199 \\
86.5871 \\
86.4997 \\
86.3757 \\
86.7724 \\
86.7565 \\
86.9289 \\
86.7329 \\
86.8265 \\
86.5356 \\
39.1464 \\
86.9817 \\
86.9755 \\
86.7386 \\
73.4113 \\
86.7920 \\
86.4921 \\
86.3123 \\
87.0141 \\
86.9510 \\
86.7230 \\
86.7113 \\
87.0795 \\
86.6905 \\
86.7764 \\
86.4034\end{array}$ & 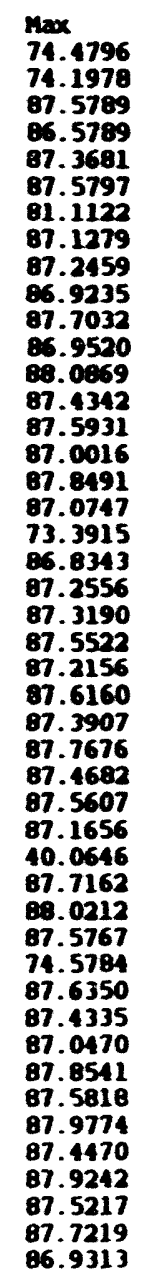 & $\begin{array}{l}\text { Ave } \\
74.1680 \\
73.9642 \\
787.0915 \\
86.2372 \\
86.8520 \\
87.2766 \\
80.6337 \\
86.7243 \\
86.8328 \\
86.5671 \\
87.3080 \\
86.6402 \\
87.7036 \\
87.1062 \\
87.1638 \\
86.7276 \\
87.4586 \\
86.6828 \\
73.1272 \\
86.5310 \\
86.8773 \\
86.8902 \\
86.9424 \\
86.7374 \\
87.2490 \\
87.0965 \\
87.3182 \\
87.0768 \\
87.1289 \\
86.9053 \\
39.6791 \\
87.2593 \\
87.5781 \\
87.1903 \\
73.8784 \\
87.0958 \\
86.9688 \\
86.8321 \\
87.4421 \\
877.2494 \\
87.3954 \\
87.0771 \\
87.5187 \\
87.1178 \\
87.1298 \\
86.6484\end{array}$ & 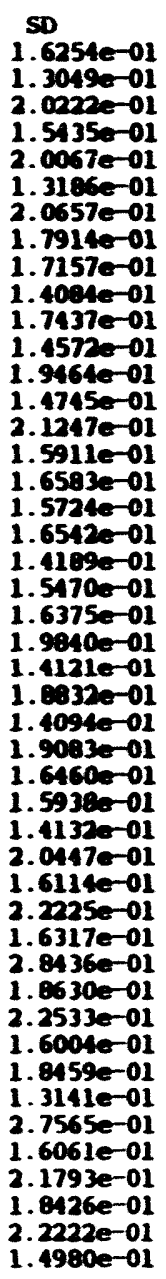 & 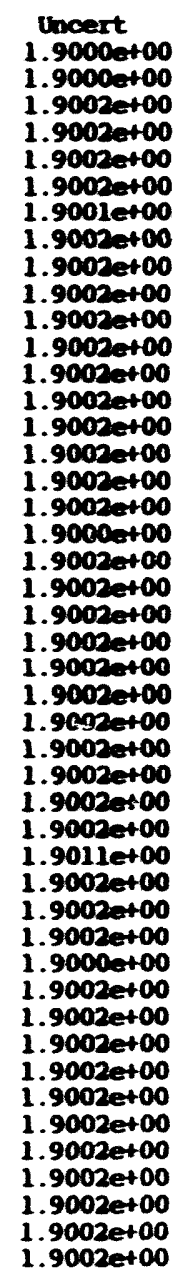 \\
\hline
\end{tabular}

\begin{tabular}{|c|c|c|c|c|c|}
\hline Test: Jul31_03 & simal & Date: Tue & u1 31 11: & $04: 031990$ & Page: 2 \\
\hline 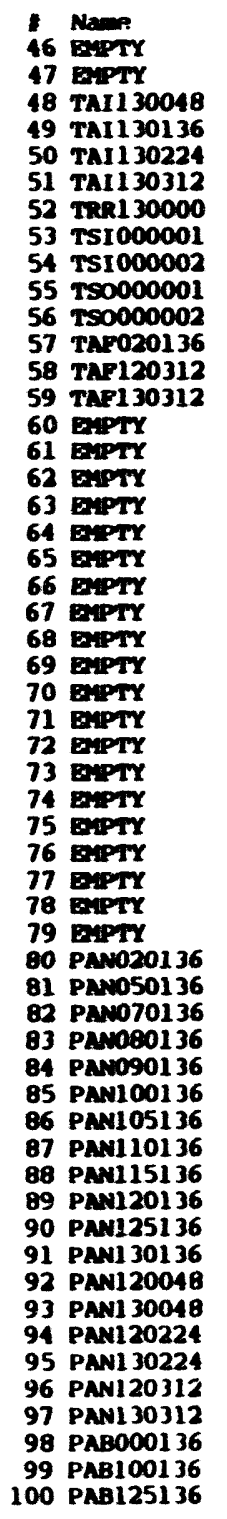 & $\begin{array}{l}\operatorname{Min} \\
37.7675 \\
38.9168 \\
86.7783 \\
86.1854 \\
87.2302 \\
86.4923 \\
85.5621 \\
81.1305 \\
75.0521 \\
73.9089 \\
74.8846 \\
86.0507 \\
86.2243 \\
86.1399 \\
0.4834 \\
0.4932 \\
0.5127 \\
0.5273 \\
0.3467 \\
0.3662 \\
0.3857 \\
0.4053 \\
0.4150 \\
0.4346 \\
0.4541 \\
0.4736 \\
0.4883 \\
0.5127 \\
0.5225 \\
0.5469 \\
0.5615 \\
0.5811 \\
0.6006 \\
0.6250 \\
47.4022 \\
87.7870 \\
59.8969 \\
30.0951 \\
30.9564 \\
22.1709 \\
14.0139 \\
14.9090 \\
16.0145 \\
15.1963 \\
18.0444 \\
14.1518 \\
25.7405 \\
31.5125 \\
24.7635 \\
31.3555 \\
28.9141 \\
32.4970 \\
40.5592 \\
30.4893 \\
27.5411 \\
\end{array}$ & $\begin{array}{l}\text { Max } \\
38.4572 \\
39.3761 \\
88.1442 \\
87.0257 \\
88.1778 \\
87.3346 \\
86.5078 \\
81.9745 \\
76.2167 \\
74.7608 \\
75.7343 \\
86.8954 \\
87.3812 \\
86.9809 \\
0.4932 \\
0.5078 \\
0.5176 \\
0.5322 \\
0.3467 \\
0.3711 \\
0.3857 \\
0.4053 \\
0.4248 \\
0.4395 \\
0.4541 \\
0.4785 \\
0.4932 \\
0.5273 \\
0.5322 \\
0.5469 \\
0.5615 \\
0.5811 \\
0.6104 \\
0.6299 \\
50.4539 \\
89.6181 \\
60.9650 \\
30.7818 \\
31.8720 \\
22.8575 \\
14.4717 \\
15.8245 \\
16.5023 \\
15.7204 \\
18.4259 \\
14.6096 \\
26.8087 \\
33.0384 \\
25.3738 \\
31.9659 \\
29.6771 \\
33.1074 \\
41.1085 \\
30.6266 \\
27.6785 \\
\end{array}$ & $\begin{array}{l}\text { Ave } \\
38.1262 \\
39.2337 \\
87.3751 \\
86.6553 \\
87.7486 \\
86.9643 \\
85.9868 \\
81.5059 \\
75.7378 \\
74.2581 \\
75.3432 \\
86.4436 \\
86.8008 \\
86.4552 \\
0.4881 \\
0.5026 \\
0.5154 \\
0.5319 \\
0.3467 \\
0.3694 \\
0.3857 \\
0.4053 \\
0.4198 \\
0.4392 \\
0.4541 \\
0.4783 \\
0.4930 \\
0.5130 \\
0.5273 \\
0.5469 \\
0.5615 \\
0.5811 \\
0.6043 \\
0.6252 \\
48.8335 \\
88.6782 \\
60.4112 \\
30.3850 \\
31.4127 \\
22.5081 \\
14.2062 \\
15.3698 \\
16.2536 \\
15.4716 \\
18.2229 \\
14.3273 \\
26.2212 \\
32.2281 \\
25.0656 \\
31.6424 \\
29.3078 \\
32.8114 \\
40.8146 \\
30.5497 \\
27.6071 \\
\end{array}$ & 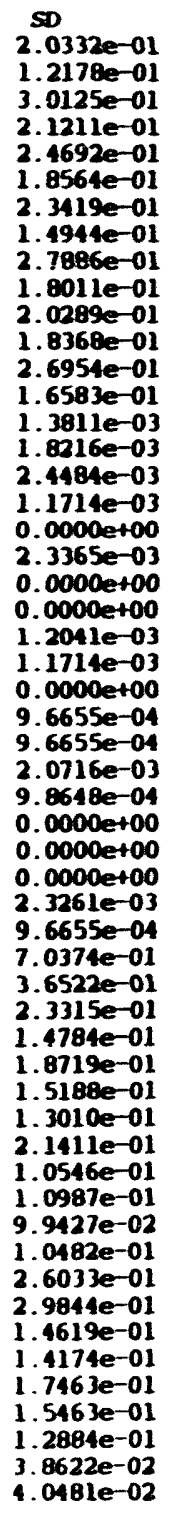 & 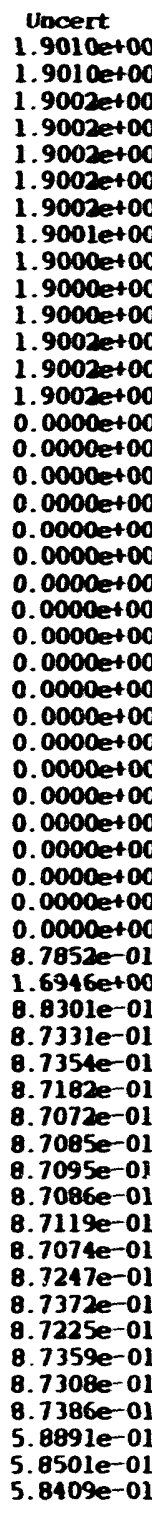 \\
\hline
\end{tabular}




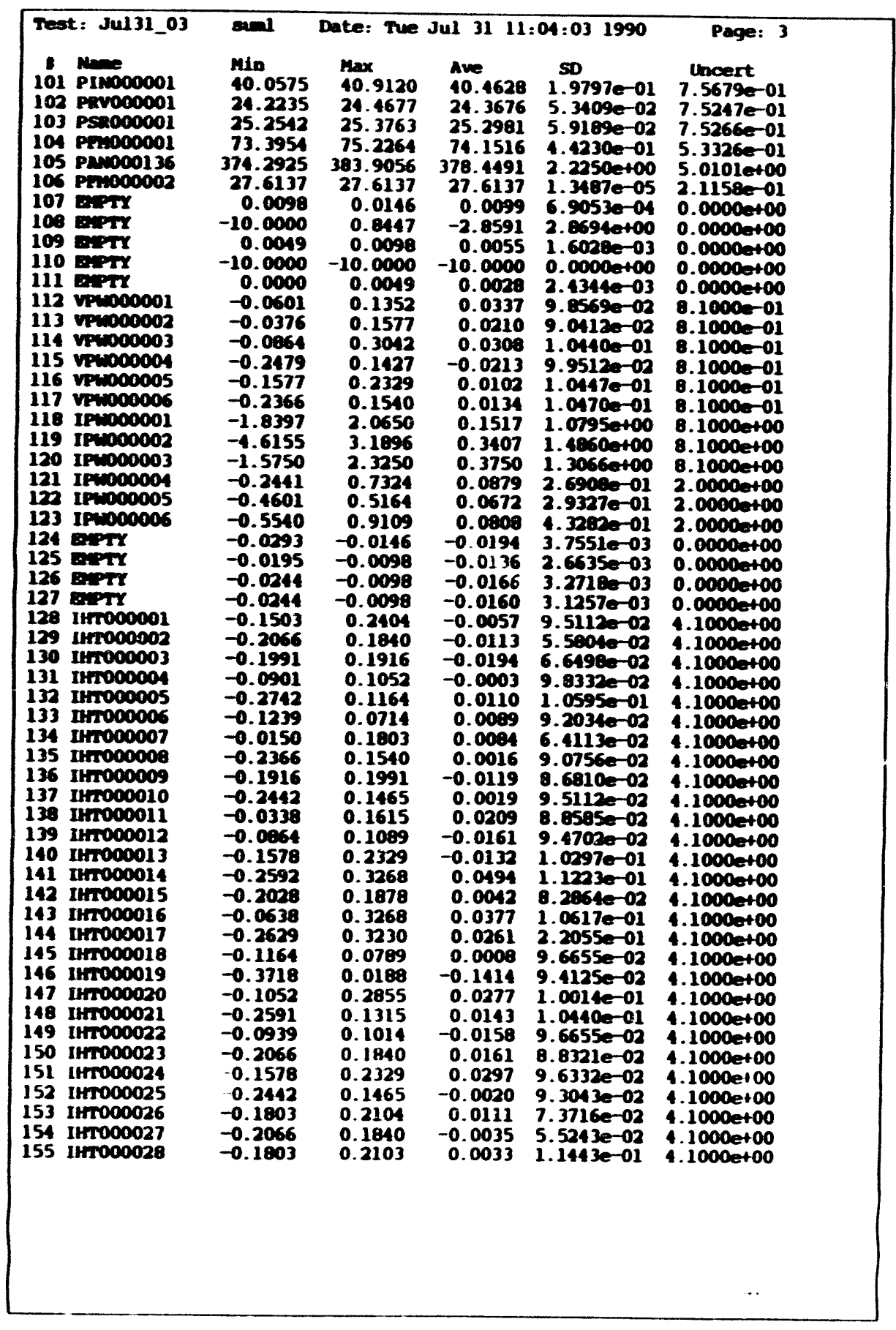

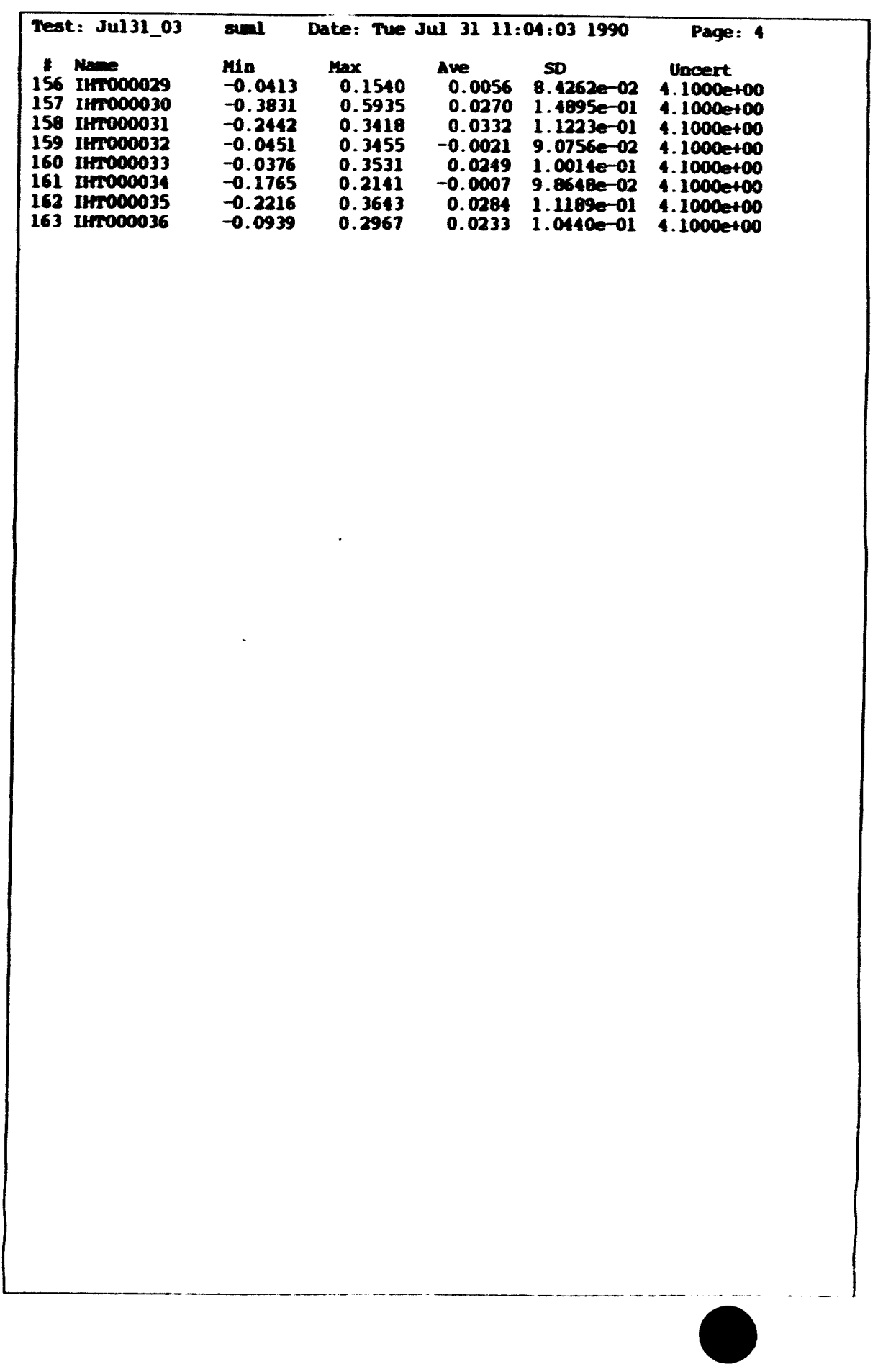




\begin{tabular}{|c|c|c|c|c|c|c|}
\hline \multicolumn{6}{|c|}{ 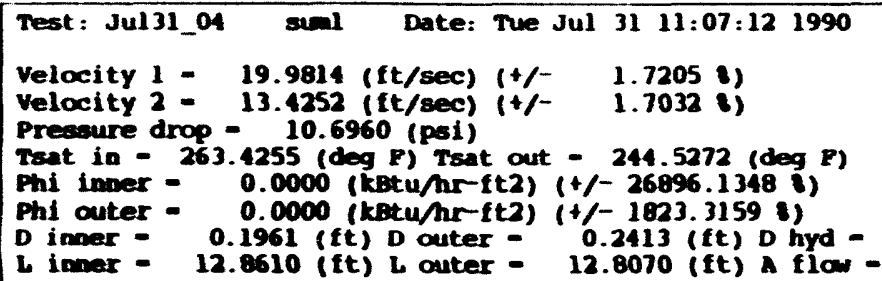 } & $\begin{array}{r}0.0390 \text { (ft) } \\
-0.0150 \text { (ft. }\end{array}$ \\
\hline 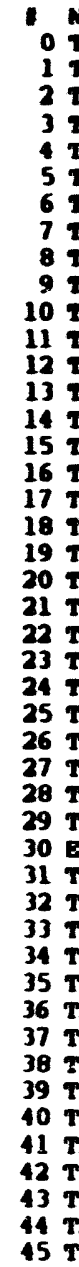 & 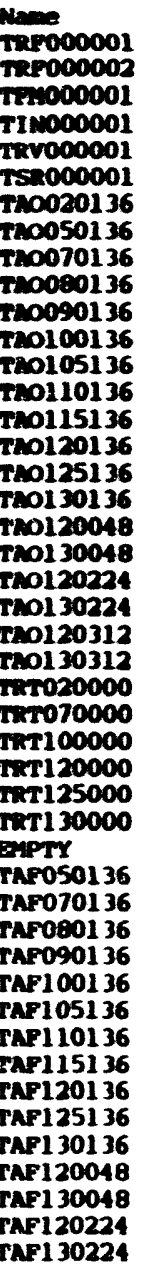 & $\begin{array}{l}\text { Min } \\
73.8393 \\
73.7731 \\
86.3188 \\
85.5247 \\
86.0056 \\
86.5302 \\
80.0531 \\
85.9734 \\
85.9802 \\
85.8750 \\
86.6523 \\
85.9002 \\
86.9285 \\
86.2624 \\
86.3301 \\
85.9490 \\
86.7988 \\
85.9298 \\
72.9648 \\
85.8682 \\
86.2041 \\
86.1707 \\
86.2892 \\
86.0609 \\
86.3508 \\
86.3342 \\
86.5080 \\
86.3117 \\
86.3038 \\
86.2202 \\
38.9168 \\
86.5637 \\
86.7661 \\
86.5296 \\
73.5166 \\
86.3707 \\
85.9717 \\
86.1025 \\
86.5951 \\
86.1275 \\
86.5141 \\
86.3966 \\
86.5510 \\
86.1557 \\
86.2519 \\
85.8758\end{array}$ & $\begin{array}{l}\text { Max } \\
74.4796 \\
74.1978 \\
87.0540 \\
86.2626 \\
86.8441 \\
87.1614 \\
81.1122 \\
86.7098 \\
86.9295 \\
86.7146 \\
87.2639 \\
86.5323 \\
87.7712 \\
87.0178 \\
87.1726 \\
86.6864 \\
87.3257 \\
86.5591 \\
73.7100 \\
86.4155 \\
86.7313 \\
86.8004 \\
86.8166 \\
86.5881 \\
87.1945 \\
86.9678 \\
87.1539 \\
87.0487 \\
87.1433 \\
86.8520 \\
10.0646 \\
87.1943 \\
87.7093 \\
87.1600 \\
74.4723 \\
87.1083 \\
87.1219 \\
86.7338 \\
87.5398 \\
87.1637 \\
87.4531 \\
87.0281 \\
87.6074 \\
86.9958 \\
87.0932 \\
86.7203\end{array}$ & $\begin{array}{l}\text { Ave } \\
74.1552 \\
73.9727 \\
86.7114 \\
85.8728 \\
86.4942 \\
86.9188 \\
80.5299 \\
86.3499 \\
86.4887 \\
86.2222 \\
86.9116 \\
86.2477 \\
87.3269 \\
86.6750 \\
86.7912 \\
86.3514 \\
87.0748 \\
86.2581 \\
73.2780 \\
86.1351 \\
86.4593 \\
86.4701 \\
86.5024 \\
86.3077 \\
86.8632 \\
86.6867 \\
87.0062 \\
86.7012 \\
86.7176 \\
85.5299 \\
39.6148 \\
86.9015 \\
87.2210 \\
86.8089 \\
74.0204 \\
86.6892 \\
86.5874 \\
86.4450 \\
87.0462 \\
86.8458 \\
86.8975 \\
86.7063 \\
87.0853 \\
86.6920 \\
86.7126 \\
86.2495\end{array}$ & $\begin{array}{l}50 \\
1.6271 e-01 \\
1.3161 e-01 \\
1.6149 e-01 \\
1.7454 e-01 \\
1.9015 e-01 \\
1.3624 e-01 \\
2.5058 e-01 \\
1.7632 e-01 \\
1.7925 e-01 \\
1.8144 e-01 \\
1.6736 e-01 \\
1.3667 e-01 \\
1.9293 e-01 \\
1.5818 e-01 \\
2.0600 e-01 \\
1.4844 e-01 \\
1.3422 e-01 \\
1.6208 e-01 \\
1.6289 e-01 \\
1.3204 e-01 \\
1.5480 e-01 \\
1.3891 e-01 \\
1.4036 e-01 \\
1.1773 e-01 \\
2.0975 e-01 \\
1.5176 e-01 \\
2.0308 e-01 \\
1.4609 e-01 \\
1.9564 e-01 \\
1.6092 e-01 \\
2.3630 e-01 \\
1.4112 e-01 \\
2.1830 e-01 \\
1.7783 e-01 \\
2.4601 e-01 \\
1.8734 e-01 \\
2.9192 e-01 \\
1.5854 e-01 \\
2.0034 e-01 \\
1.6634 e-01 \\
2.3942 e-01 \\
1.5803 e-01 \\
2.3118 e-01 \\
1.5784 e-01 \\
1.6691 e-01 \\
1.8850 e-01\end{array}$ & $\begin{array}{c}\text { Uncert } \\
1.9000 e+00 \\
1.9000 e+00 \\
1.9002 e+00 \\
1.9002 e+00 \\
1.9002 e+00 \\
1.9002 e+00 \\
1.9001 e+00 \\
1.9002 e+00 \\
1.9002 e+00 \\
1.9002 e+00 \\
1.9002 e+00 \\
1.9002 e+00 \\
1.9002 e+00 \\
1.9002 e+00 \\
1.9002 e+00 \\
1.9002 e+00 \\
1.9002 e+00 \\
1.9002 e+00 \\
1.9000 e+00 \\
1.9002 e+00 \\
1.9002 e+00 \\
1.9002 e+00 \\
1.9002 e+00 \\
1.9002 e+00 \\
1.9002 e+00 \\
1.9002 e+00 \\
1.9002 e+00 \\
1.9002 e+00 \\
1.9002 e+00 \\
1.9002 e+00 \\
1.9011 e+00 \\
1.9002 e+00 \\
1.9002 e+00 \\
1.9002 e+00 \\
1.9000 e+00 \\
1.9002 e+00 \\
1.9002 e+00 \\
1.9002 e+00 \\
1.9002 e+00 \\
1.9002 e+00 \\
1.9002 e+00 \\
1.9002 e+00 \\
1.9002 e+00 \\
1.9002 e+00 \\
1.9002 e+00 \\
1.9002 e+00\end{array}$ \\
\hline
\end{tabular}

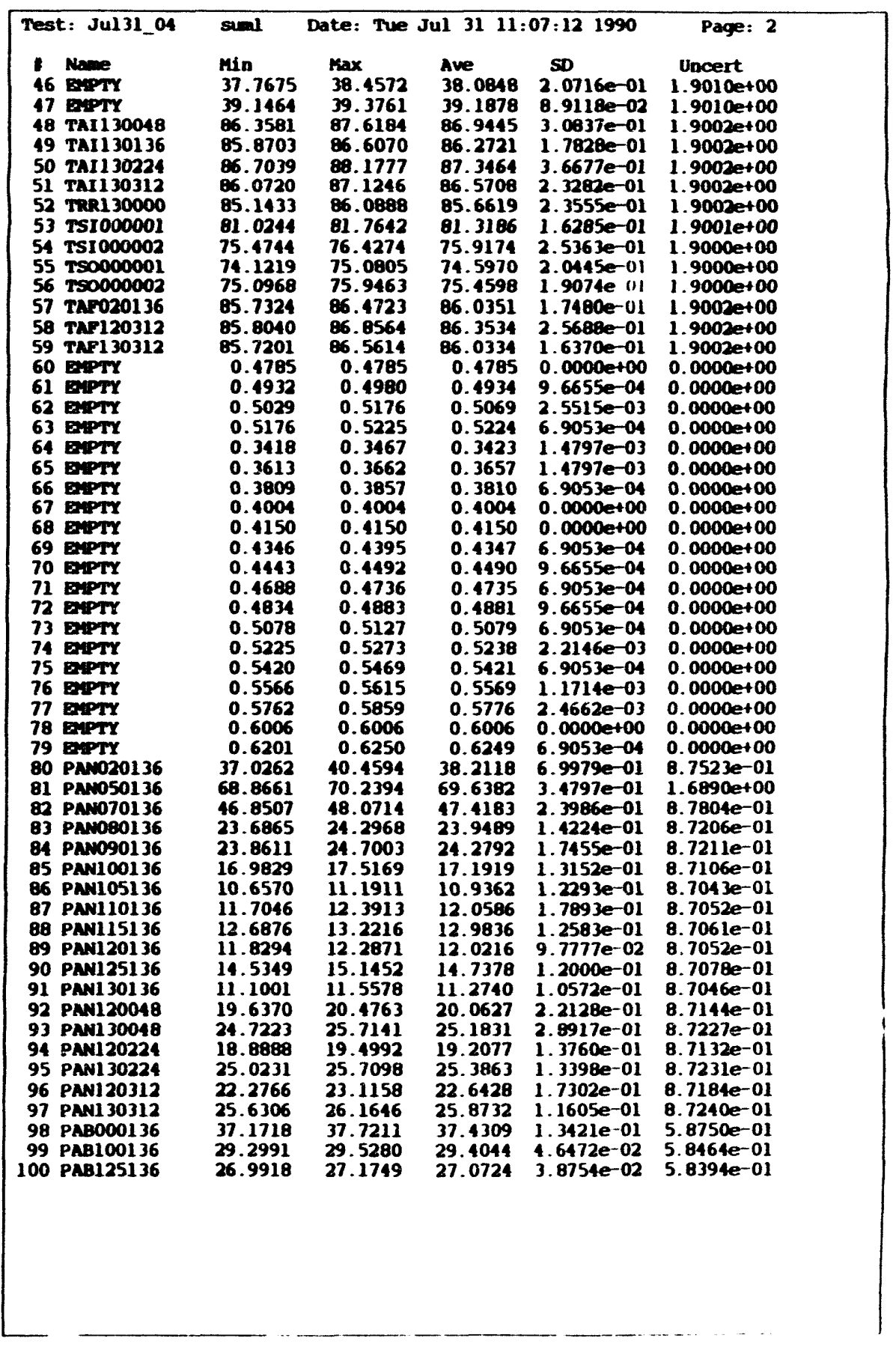




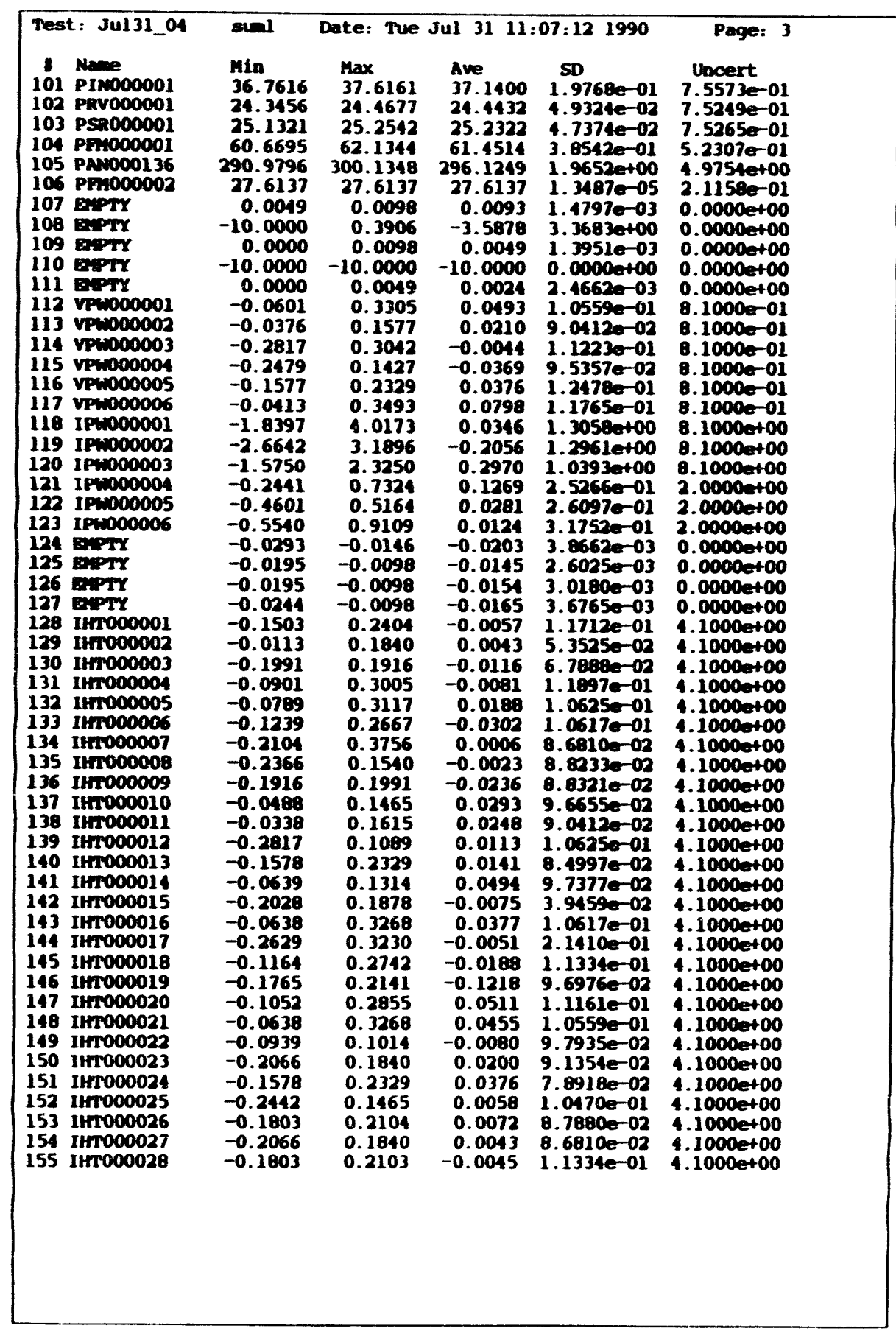

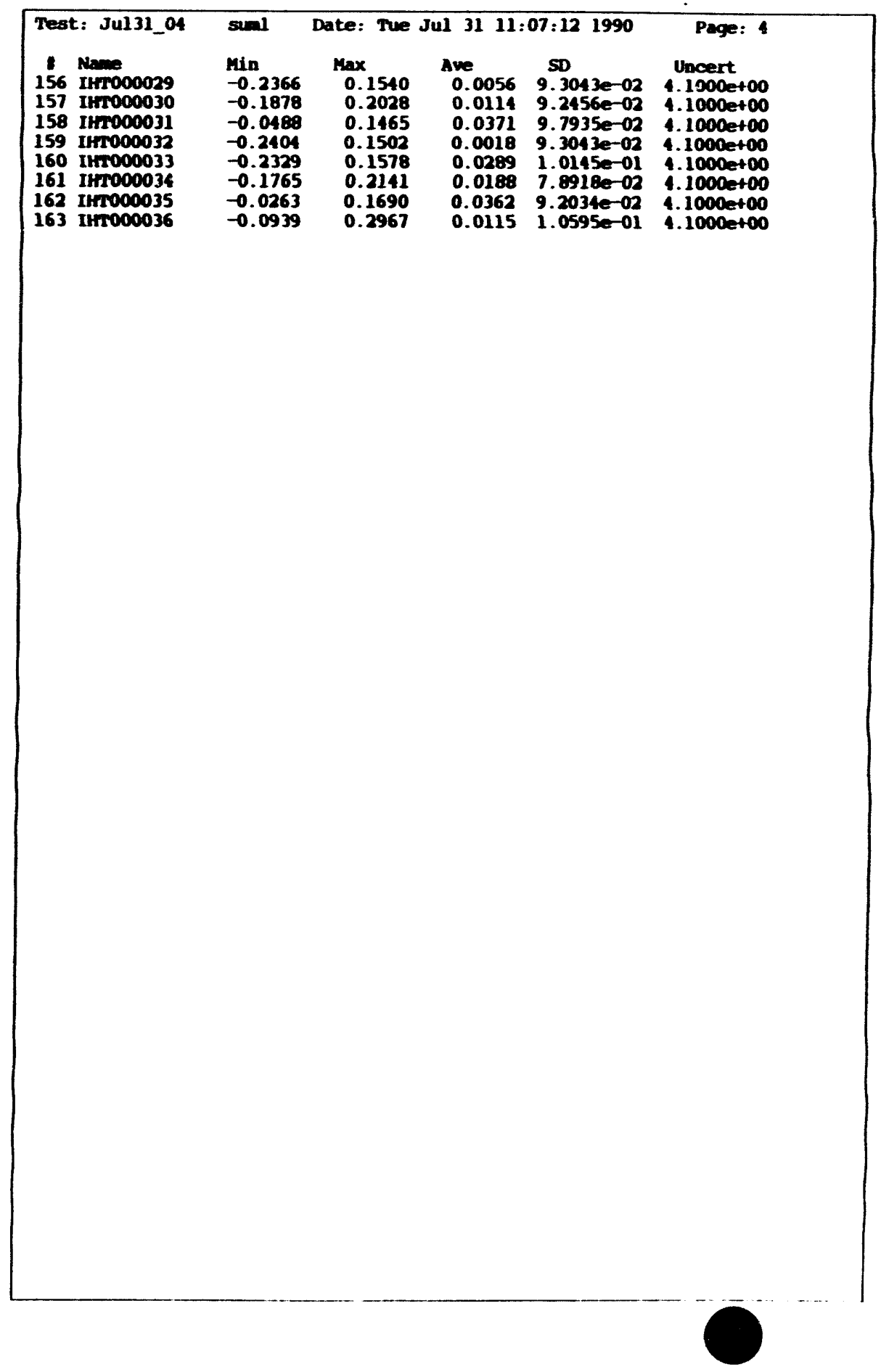




\begin{tabular}{|c|c|c|c|c|c|c|}
\hline \multicolumn{4}{|c|}{ 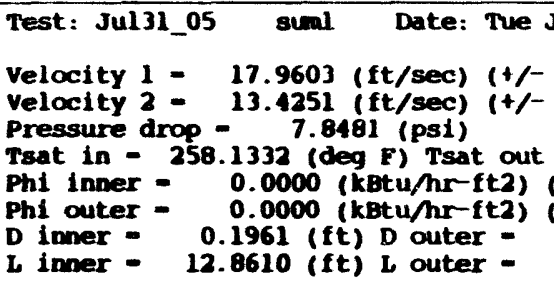 } & \multicolumn{2}{|c|}{ 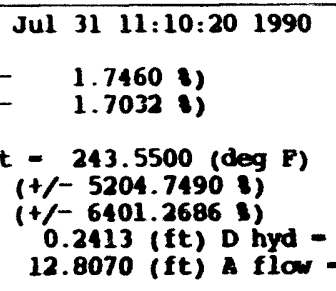 } & $\begin{array}{l}\text { Page: } 1 \\
0.0390 \text { (ft) } \\
0.0150 \text { (ft2) }\end{array}$ \\
\hline 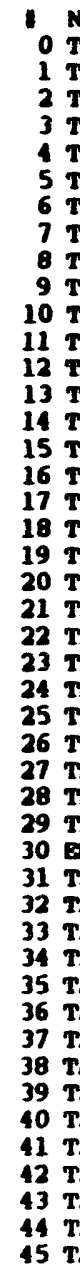 & 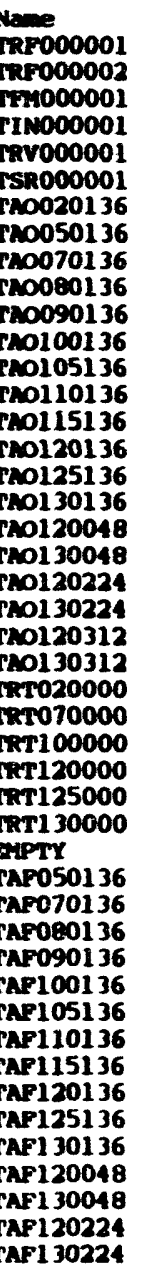 & $\begin{array}{l}\text { Min } \\
73.8393 \\
73.7731 \\
86.1075 \\
85.4192 \\
85.7956 \\
86.3209 \\
80.0536 \\
85.7641 \\
85.8741 \\
85.7716 \\
86.2327 \\
85.6907 \\
86.5079 \\
86.0740 \\
86.1196 \\
85.8438 \\
86.5890 \\
85.7231 \\
73.0711 \\
85.6787 \\
85.9940 \\
86.0650 \\
85.8685 \\
85.6414 \\
86.2450 \\
86.1226 \\
86.4036 \\
86.2069 \\
86.0949 \\
86.0104 \\
39.1464 \\
86.3538 \\
86.6610 \\
86.1126 \\
73.4107 \\
86.0548 \\
85.8660 \\
85.9973 \\
86.3850 \\
86.1275 \\
86.2095 \\
85.9774 \\
86.5504 \\
86.1567 \\
86.1473 \\
85.7701\end{array}$ & $\begin{array}{l}\text { Max } \\
74.4796 \\
74.1978 \\
86.9991 \\
85.9471 \\
86.7406 \\
86.9502 \\
80.9005 \\
86.4990 \\
86.8239 \\
86.5056 \\
87.0743 \\
86.4272 \\
87.4552 \\
86.9122 \\
87.0670 \\
86.3711 \\
87.0110 \\
86.4540 \\
73.8156 \\
86.3104 \\
86.6262 \\
86.6953 \\
86.6061 \\
86.4825 \\
86.9837 \\
86.7562 \\
87.1395 \\
86.8380 \\
6.8290 \\
86.6423 \\
40.0646 \\
87.0887 \\
87.5005 \\
87.0544 \\
74.4723 \\
86.8982 \\
87.0163 \\
86.6287 \\
87.2248 \\
86.8495 \\
87.2477 \\
86.8187 \\
87.2902 \\
86.7876 \\
86.8831 \\
86.4036\end{array}$ & $\begin{array}{l}\text { Ave } \\
74.1594 \\
73.9854 \\
86.5728 \\
85.7168 \\
86.2765 \\
86.5882 \\
80.4071 \\
86.1868 \\
86.3114 \\
86.1094 \\
86.7355 \\
86.0419 \\
87.0785 \\
86.4668 \\
86.6228 \\
86.1538 \\
86.8525 \\
86.0552 \\
73.1437 \\
85.9885 \\
86.2702 \\
86.2829 \\
86.2960 \\
86.1107 \\
86.6903 \\
86.4709 \\
86.7213 \\
86.4955 \\
86.5590 \\
86.3076 \\
39.5827 \\
86.6965 \\
87.0373 \\
86.5922 \\
73.9653 \\
86.4828 \\
86.4290 \\
86.2819 \\
86.7991 \\
86.6911 \\
86.7267 \\
86.5009 \\
86.8950 \\
86.4823 \\
86.5407 \\
86.0426\end{array}$ & $\begin{array}{l}\text { SD } \\
1.8420 e-01 \\
1.4862 e-01 \\
2.0829 e-01 \\
1.4400 e-01 \\
2.1206 e-01 \\
1.5768 e-01 \\
2.4357 e-01 \\
1.6229 e-01 \\
2.1107 e-01 \\
1.6350 e-01 \\
2.0940 e-01 \\
1.7740 e-01 \\
2.1285 e-01 \\
1.8006 e-01 \\
2.3624 e-01 \\
1.8111 e-01 \\
1.3826 e-01 \\
1.8121 e-01 \\
1.5233 e-01 \\
1.3182 e-01 \\
1.5182 e-01 \\
1.6186 e-01 \\
1.6540 e-01 \\
1.5511 e-01 \\
2.0789 e-01 \\
1.7620 e-01 \\
1.8795 e-01 \\
1.6018 e-01 \\
1.7477 e-01 \\
1.4058 e-01 \\
2.4650 e-01 \\
1.5715 e-01 \\
2.2393 e-01 \\
1.7976 e-01 \\
2.6537 e-01 \\
1.8119 e-01 \\
2.9780 e-01 \\
1.7026 e-01 \\
1.9486 e-01 \\
1.2464 e-01 \\
2.8049 e-01 \\
1.7827 e-01 \\
1.9875 e-01 \\
1.7296 e-01 \\
1.8846 e-01 \\
1.7326 e-01\end{array}$ & $\begin{array}{c}\text { Uncert } \\
1.9000 e+00 \\
1.9000 e+00 \\
1.9002 e+00 \\
1.9002 e+00 \\
1.9002 e+00 \\
1.9002 e+00 \\
1.9001 e+00 \\
1.9002 e+00 \\
1.9002 e+00 \\
1.9002 e+00 \\
1.9002 e+00 \\
1.9002 e+00 \\
1.9002 e+00 \\
1.9002 e+00 \\
1.9002 e+00 \\
1.9002 e+00 \\
1.9002 e+00 \\
1.9002 e+00 \\
1.9000 e+00 \\
1.9002 e+00 \\
1.9002 e+00 \\
1.9002 e+00 \\
1.9002 e+00 \\
1.9002 e+00 \\
1.9002 e+00 \\
1.9002 e+00 \\
1.9002 e+00 \\
1.9002 e+00 \\
1.9002 e+00 \\
1.9002 e+00 \\
1.9011 e+00 \\
1.9002 e+00 \\
1.9002 e+00 \\
1.9002 e+00 \\
1.9000 e+00 \\
1.9002 e+00 \\
1.9002 e+00 \\
1.9002 e+00 \\
1.9002 e+00 \\
1.9002 e+00 \\
1.9002 e+00 \\
1.9002 e+00 \\
1.9002 e+00 \\
1.9002 e+00 \\
1.9002 e+00 \\
1.9002 e+00\end{array}$ \\
\hline
\end{tabular}

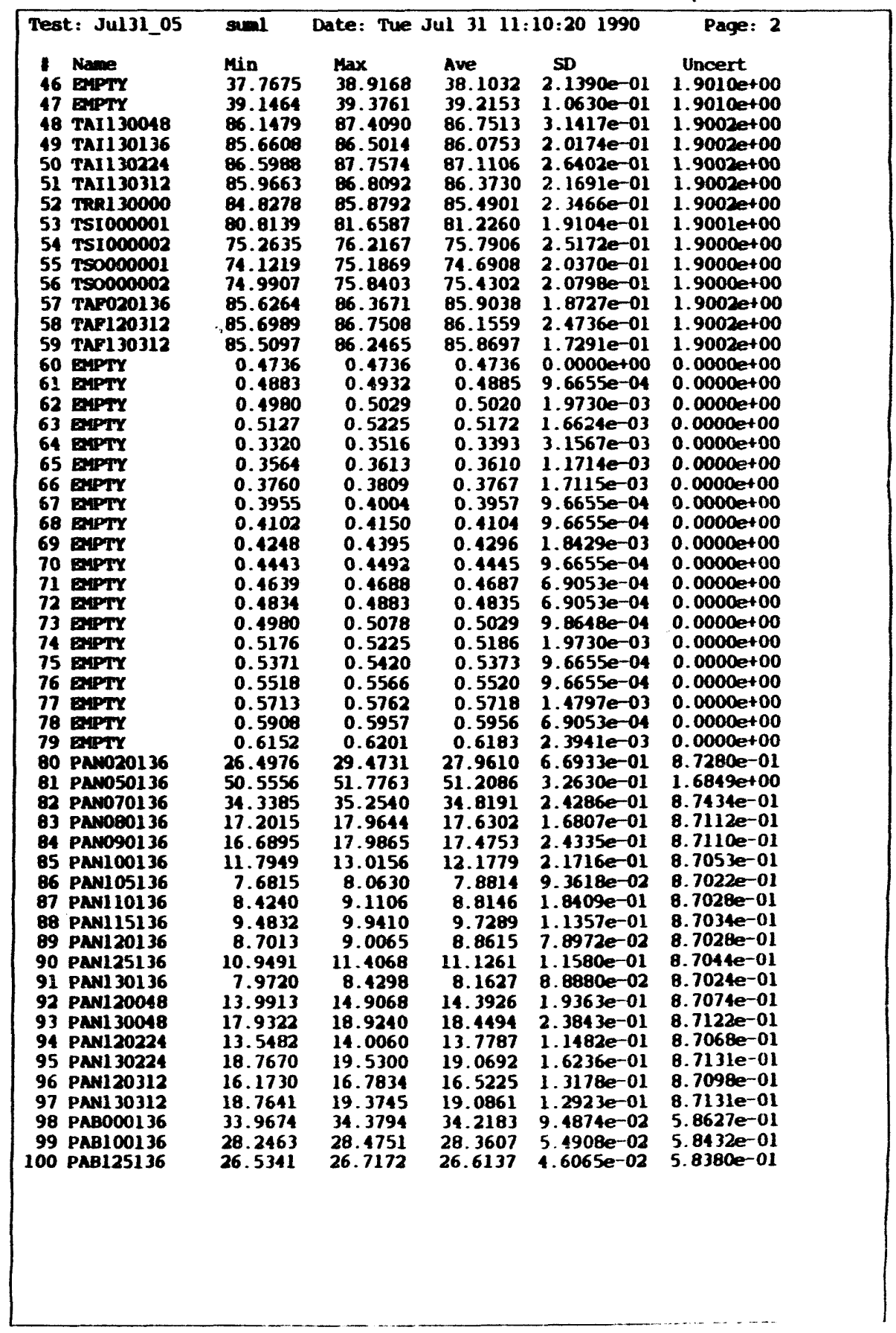




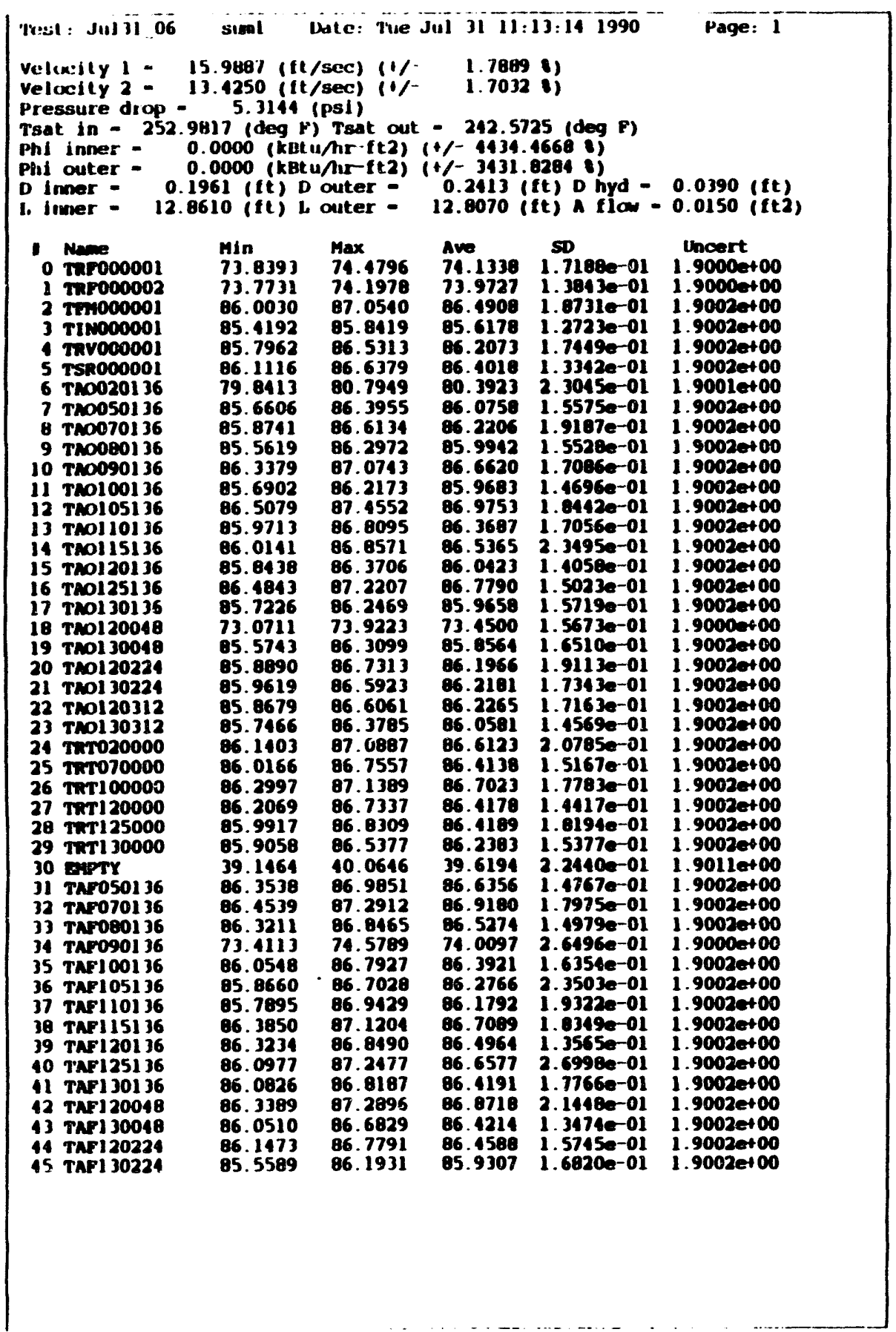

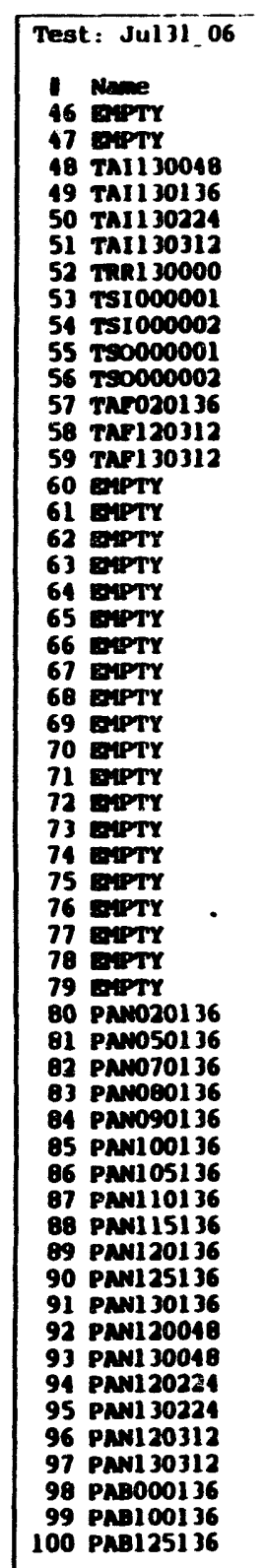

suml Date: Tive Jul ii $11: 11: 14$ 1990 Pauge: 2 Min Max Ave SD Hincert

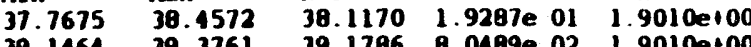
$\begin{array}{llllll}39.1464 & 39.3761 & 39.1786 & 8.0469 e & 02 & 1.9010 e 100\end{array}$

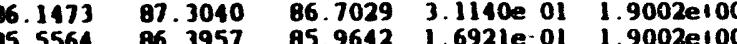
65. 85.8614 86.5989 05.2614 1.7672e-01 1.9002e+00 $\begin{array}{lllllll}85.0389 & 85.8787 & 85.3579 & 2.0991 e^{-01} & 1.9002 e 100\end{array}$ $80.7090 \quad 01.4483$ 81.0849 1.5551e-01 1.9001e100 $75.4744 \quad 76.4280 \quad 75.9110 \quad 2.7499 e-01 \quad 1.9000 e+00$ $\begin{array}{llllll}24.1413 & 75.2938 & 74.8990 & 1.8134 \mathrm{e}-01 & 1.9000 e+100\end{array}$ $\begin{array}{llllll}75.2035 & 75.9464 & 75.5850 & 2.0452 e-01 & 1.9000 e+00\end{array}$ $\begin{array}{llllll}05.4149 & 86.1555 & 85.7663 & 1.9400 e-01 & 1.9002 e 100\end{array}$ $\begin{array}{llllll}85.5938 & 86.5413 & 86.1159 & 2.7771 e^{-01} & 1.9002 e 100\end{array}$ $\begin{array}{llllll}05.5097 & 86.1418 & 85.7836 & 1.573 e^{-01} & 1.9002 e+00\end{array}$ $\begin{array}{llllll}0.4688 & 0.4736 & 0.4739 & 1.8082 e-03 & 0.0000 e+00\end{array}$

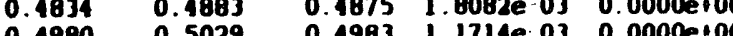
$0.4980 \quad 0.5029 \quad 0.4963$ 1.1714e.03 0.00002100 $0.3369 \quad 0.3418 \quad 0.3370 \quad 6.90532-040.00000100$ $\begin{array}{lllllll}0.3564 & 0.3613 & 0.3604 & 1.8950 e^{-03} & 0.0000 e 100\end{array}$ $0.3760 \quad 0.3760 \quad 0.3760 \quad 0.0000$ e $100 \quad 0.0000$ e 100 $\begin{array}{llllll}0.3955 & 0.4004 & 0.3957 & 9.6655 e^{-04} & 0.0000 e 100\end{array}$ $\begin{array}{llllll}0.4053 & 0.4150 & 0.4100 & 1.3811 e^{-03} & 0.0000 e 100\end{array}$ $\begin{array}{llllll}0.4248 & 0.4297 & 0.4292 & 1.4797 e .03 & 0.0000 e 100\end{array}$ $\begin{array}{llllll}0.4395 & 0.4143 & 0.4142 & 6.9053 e^{-04} & 0.0000 e 100\end{array}$

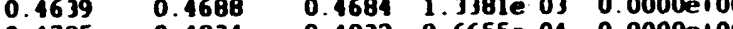
$\begin{array}{llllll}0.4785 & 0.4834 & 0.4832 & 1.6635 e-04 & 0.0000 e 100\end{array}$ $\begin{array}{llllll}0.4980 & 0.5078 & 0.5029 & 9.8548 e-04 & 0.0000 e 100\end{array}$ $0.5127 \quad 0.51760 .51950 .9053 e-040.0000100$ $0.53220 .54200 .53169 .0540-040.00000100$ $0.5654 \quad 0.5713 \quad 0.5710 \quad 1.17140-03 \quad 0.00000100$ $\begin{array}{llllll}0.5908 & 0.5957 & 0.5938 & 2.3941 e^{-03} & 0.0000 e 100\end{array}$ $\begin{array}{llllll}0.6152 & 0.6201 & 0.6154 & 9.6655 e^{-04} 0.0000100\end{array}$ $\begin{array}{lllllll}17.4949 & 19.6312 & 18.4883 & 4.8092 e-01 & 8.7123 e-0\end{array}$ $\begin{array}{llllll}34.2287 & 35.2968 & 34.8787 & 2.3841 \text { le-01 } & 1.6823 e 100\end{array}$ $23.1995 \quad 24.1151 \quad 23.7962 \quad 1.9233 \mathrm{e}-01 \quad 8.7203 \mathrm{e}-0$ $\begin{array}{lllllll}12.3950 & 12.0547 & 1.1659 e & 01 & 0.7052 e-0\end{array}$ $\begin{array}{llllll}11.1200 & 11.7304 \quad 11.4557 & 1.2804 e^{-01} & 8.7047 e-0\end{array}$ $\begin{array}{llllll}7.4461 & 7.9802 & 7.7178 & 1.1343 e^{-01} & 8.7021 e^{-0}\end{array}$

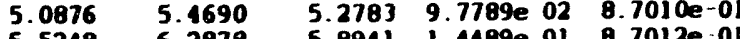
$5.5240 \quad 6.207015 .0941$ 1.4469e o1 0.701280

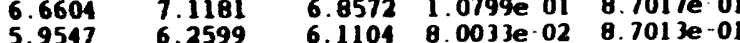
$7.9684 \quad 6.2599 \quad 6.8622 \quad 9.27520020 .70220-01$ $\begin{array}{llllll}5.3017 & 5.6832 & 5.520 & 8.5556 e & 02 & 8.7011 \mathrm{e} 01\end{array}$

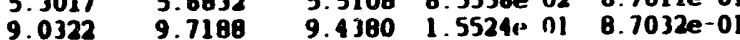
$\begin{array}{lllllll}12.1338 & 12.9731 & 12.6252 & 2.0400 & \text { I } & 8.7057 \text { e }\end{array}$ $\begin{array}{llllll}8.6654 & 9.1995 & 0.9370 & 1.3002 \% & \text { ol } & 8.7029 e .0\end{array}$

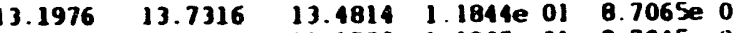
$\begin{array}{llllll}10.8325 & 11.4428 & 11.1758 & 1.1865 e & 01 & 8.7045 e \\ 01 & 01\end{array}$

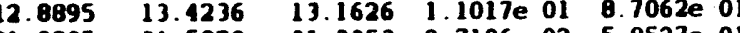
$\begin{array}{llllll}31.0835 & 31.5870 & 31.3353 & 9.7106 e & 02 & 5.8527 e \\ 0\end{array}$ $\begin{array}{lllllll}27.2850 & 27.4681 & 27.3847 & 4.5013 e & 02 & 5.8403 \mathrm{e} & 01 \\ 26.0763 & 26.2594 & 26.1614 & 4.3388 \mathrm{e} & 02 & 5.8368 \mathrm{e} & 01\end{array}$ 


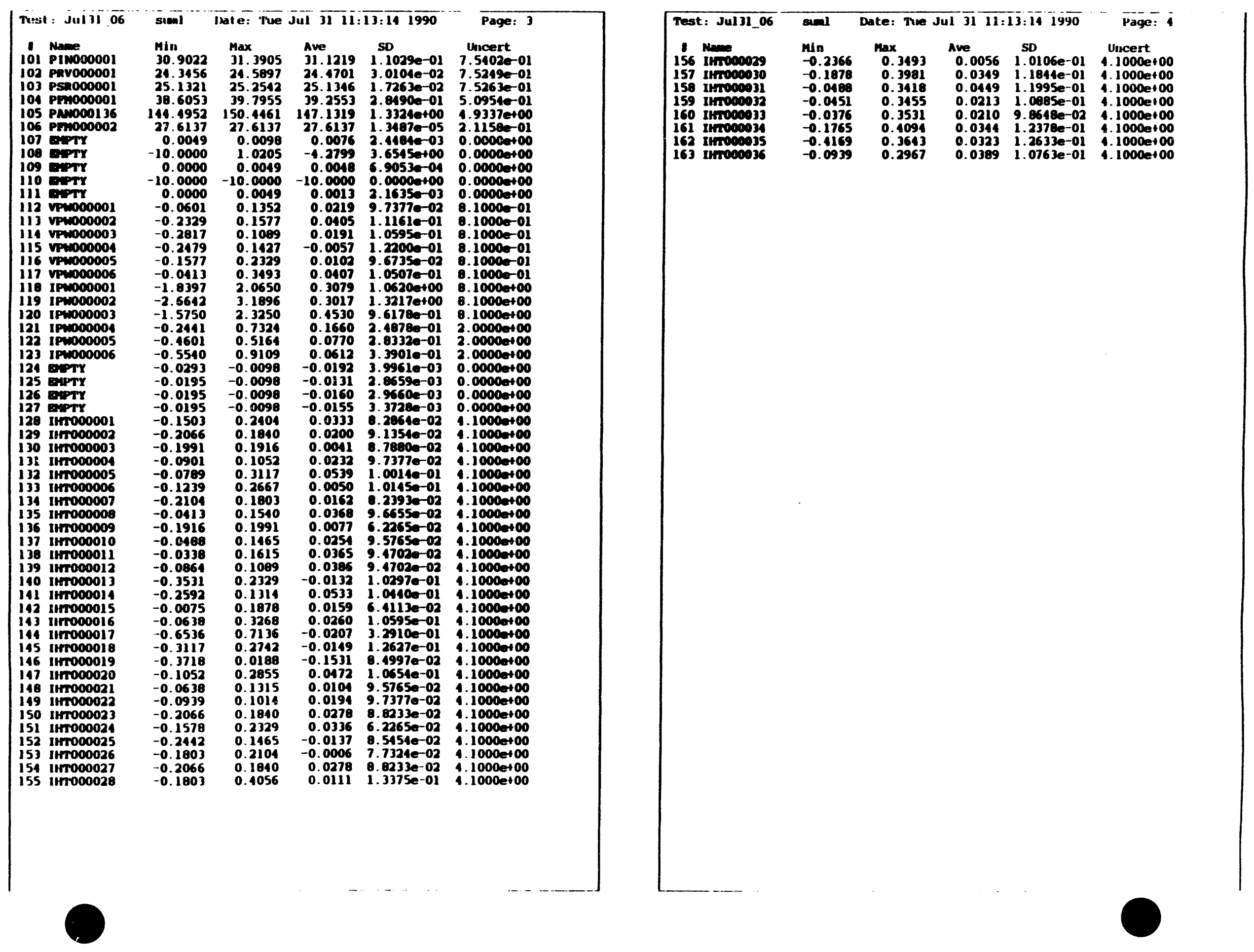




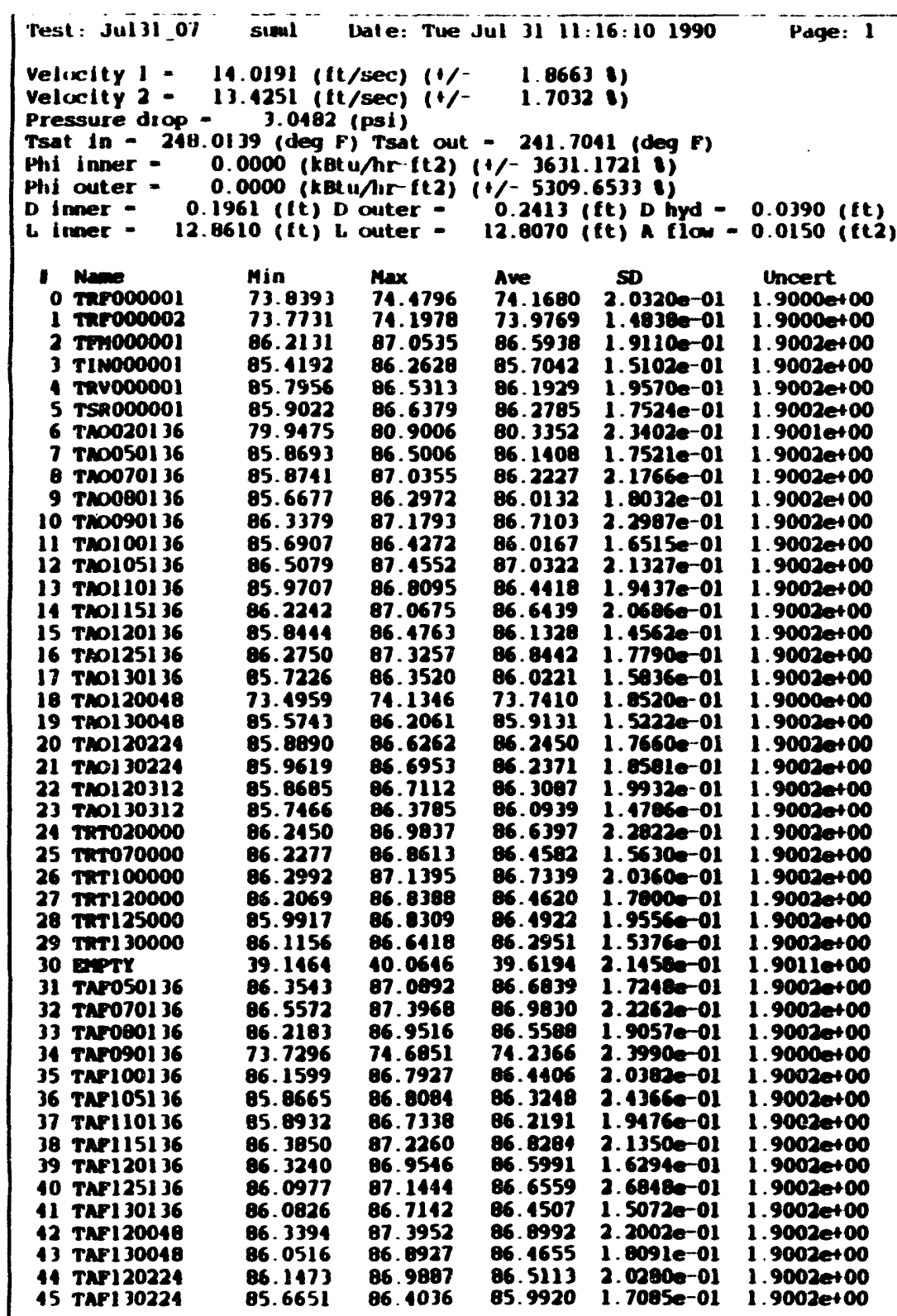

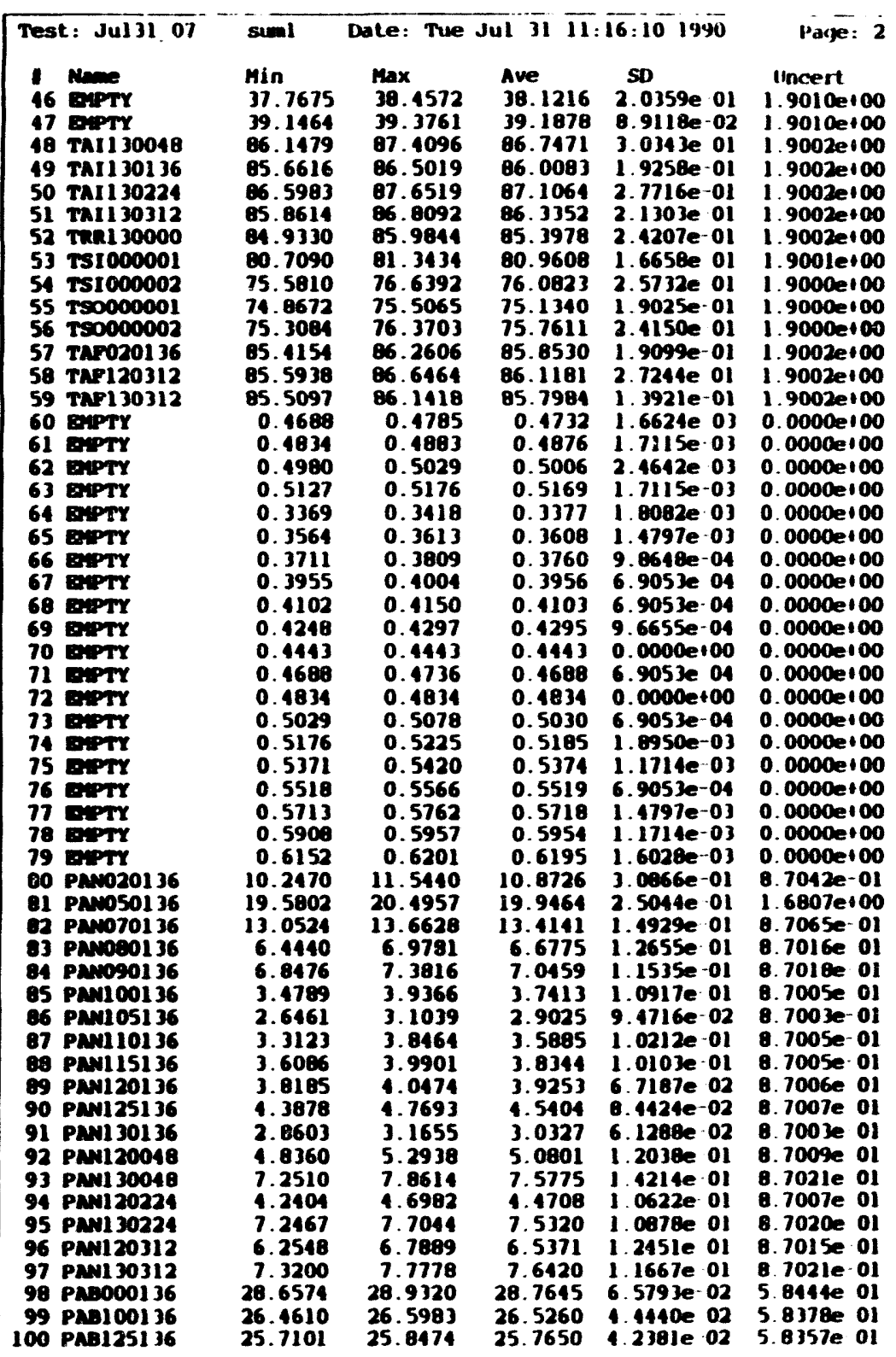




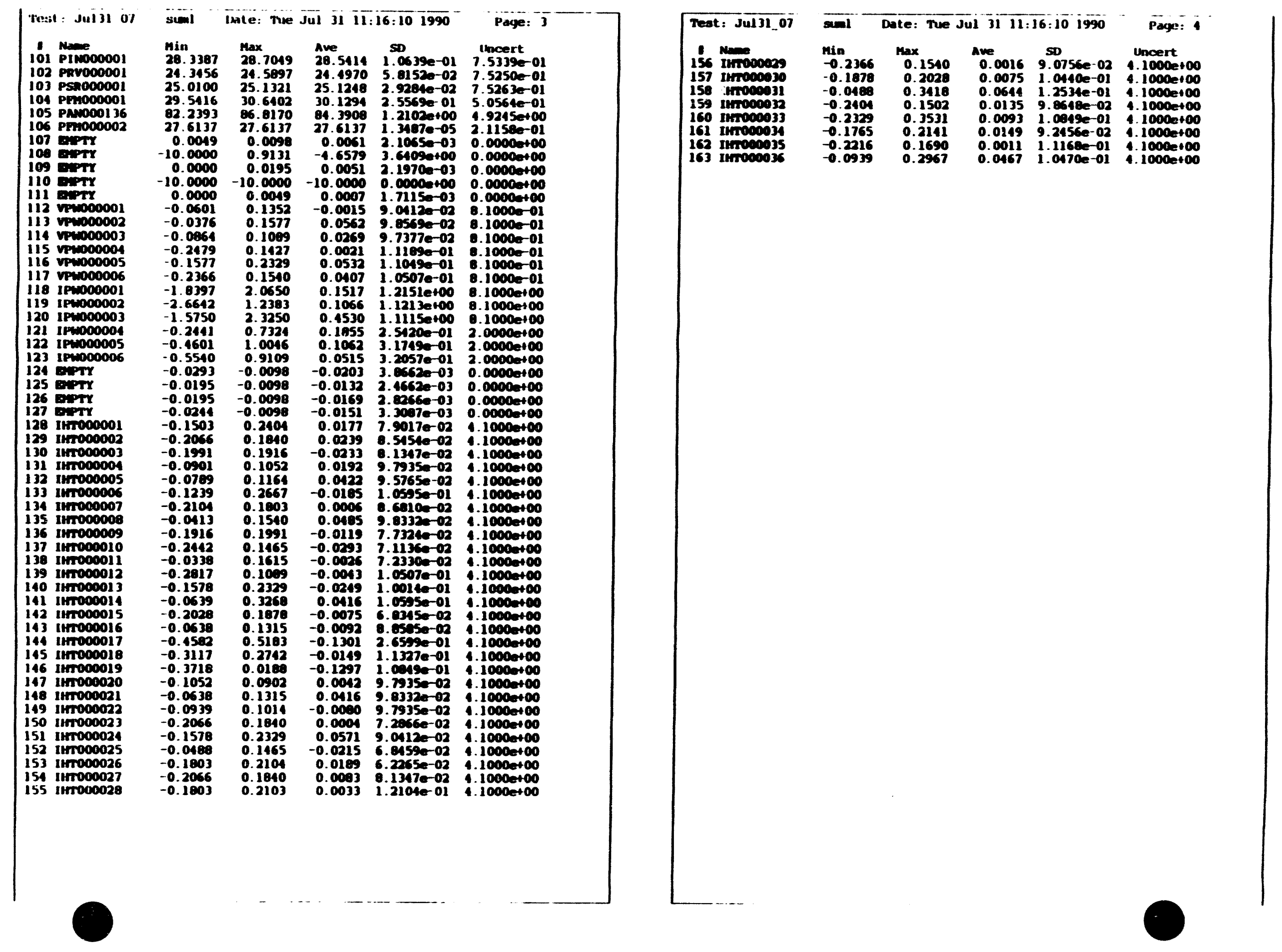




\begin{tabular}{|c|c|c|c|c|c|}
\hline 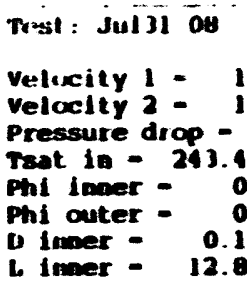 & $\begin{array}{l}\text { suel } \\
12.0548 \text { (t } \\
11.9100 \text { it } \\
1.0502 \\
1324 \text { (deg } \\
0.0000 \text { (kt } \\
0.0000 \text { (kt } \\
1961 \text { (ti) } \\
6610 \text { (ft) }\end{array}$ & $\begin{array}{l}\text { e: } \\
\text { c) } \\
\text { c) } \\
\text { i) }\end{array}$ & 11 & D:08 & ge: 1 \\
\hline 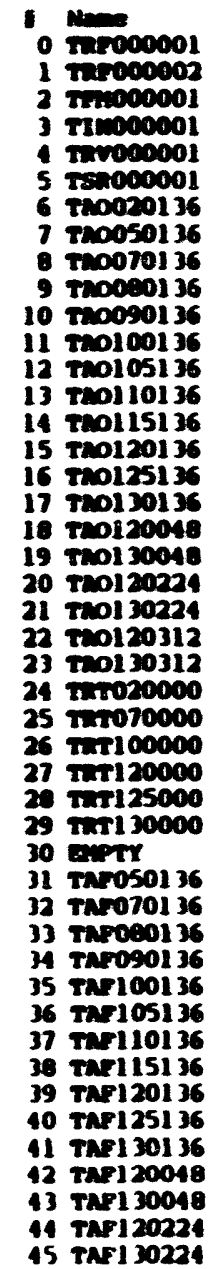 & 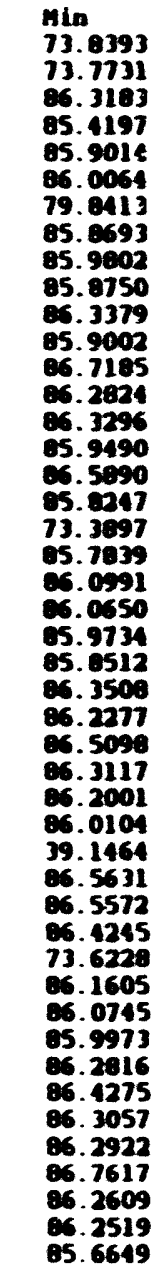 & 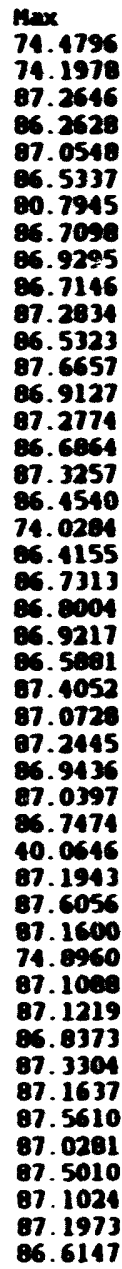 & 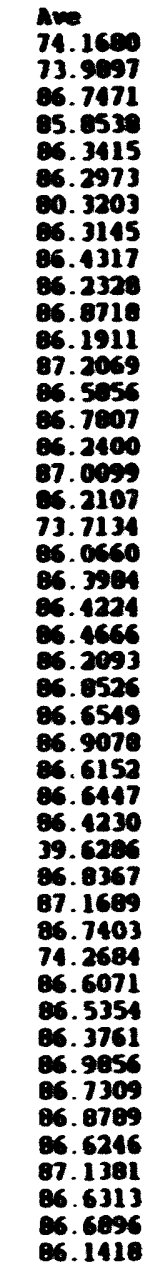 & 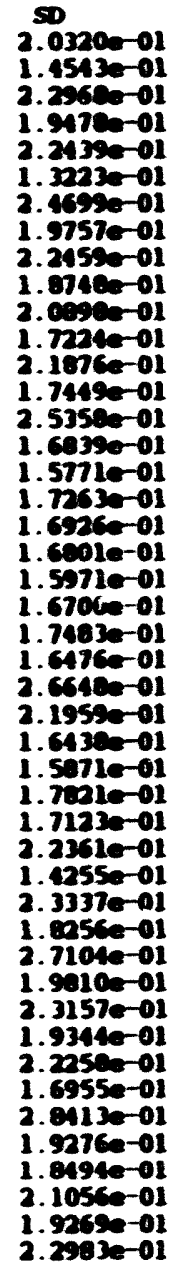 & $\begin{array}{l}1 \\
1 \\
1 \\
1 \\
1 \\
1\end{array}$ \\
\hline
\end{tabular}

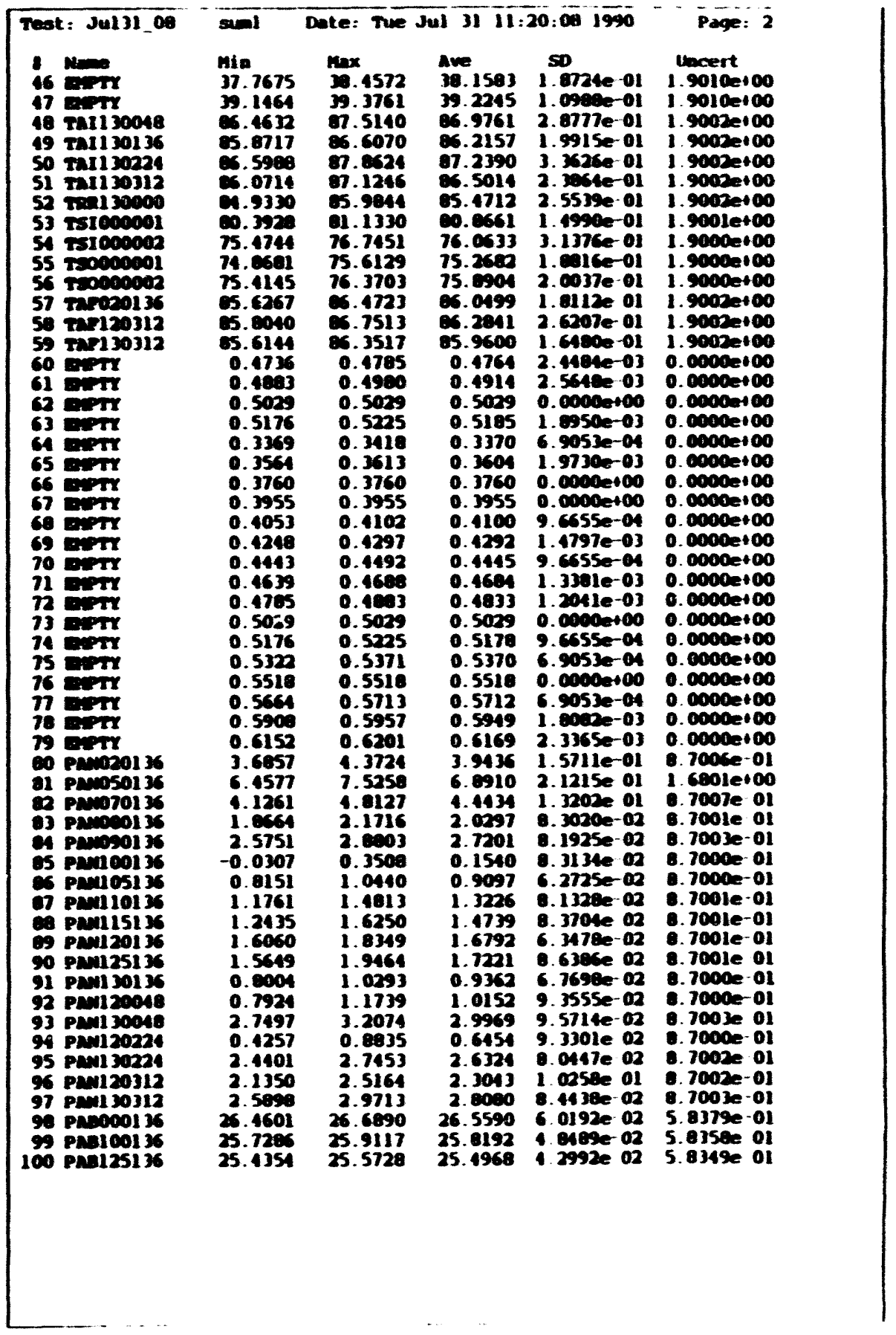




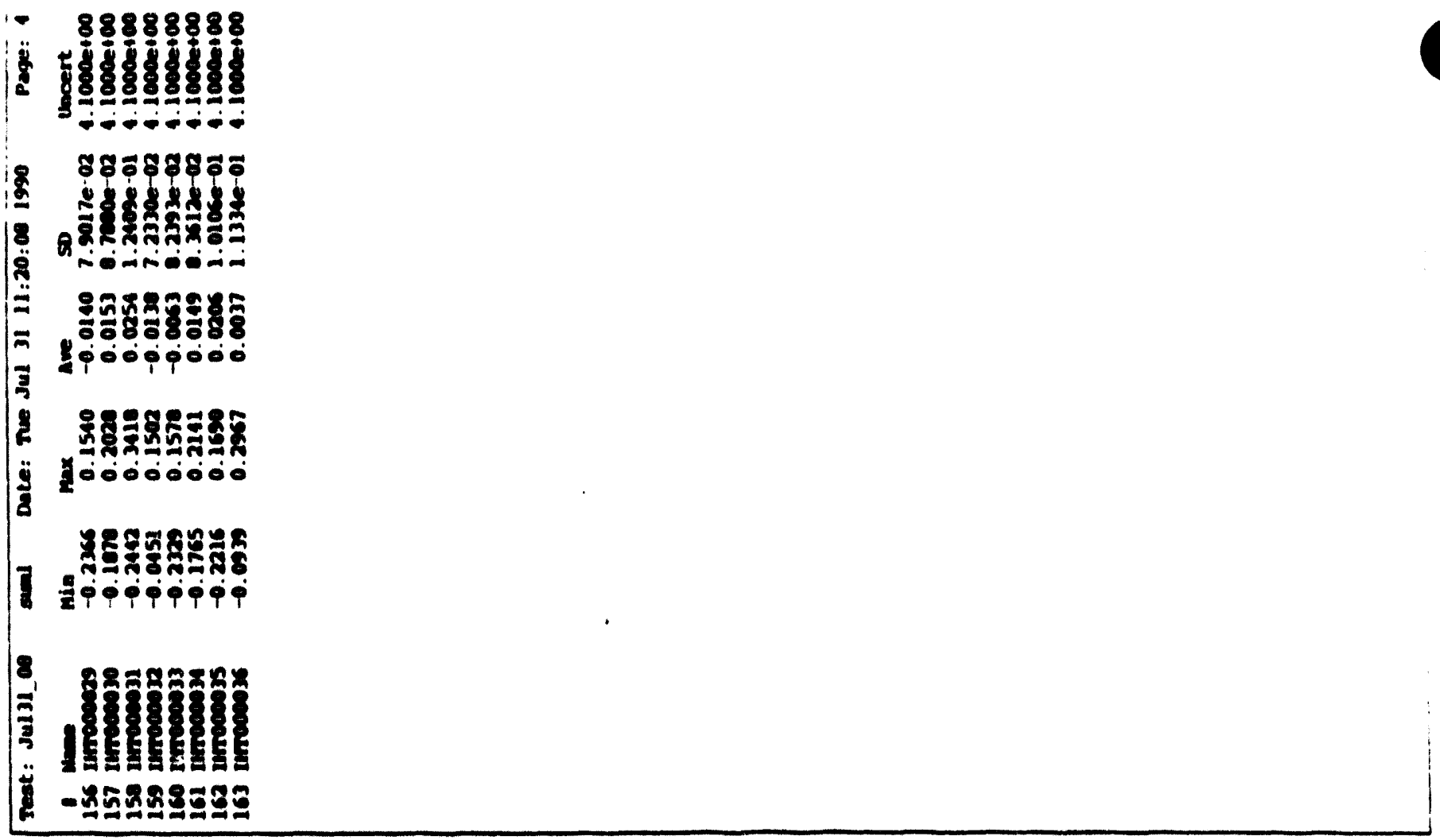

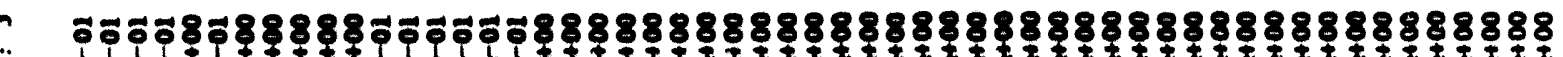
\%

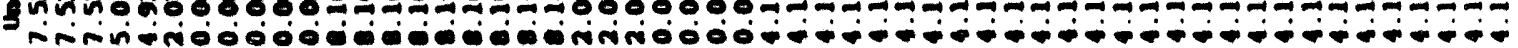

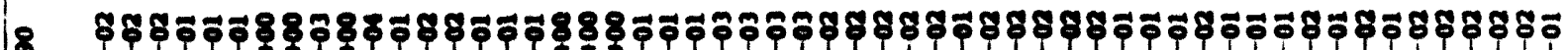

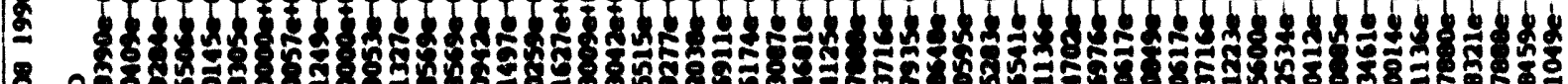

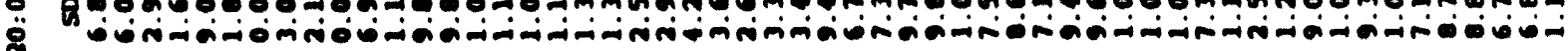

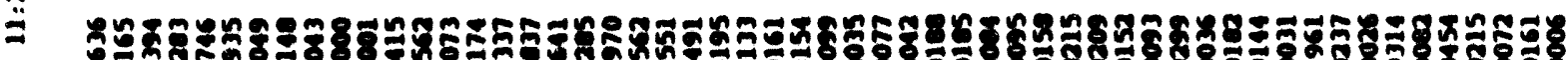
im liñ

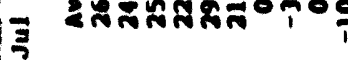

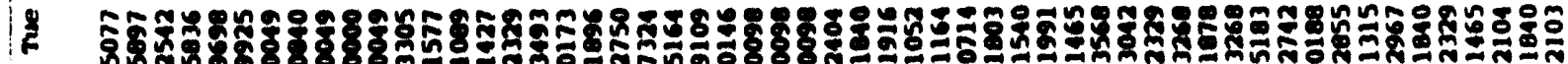

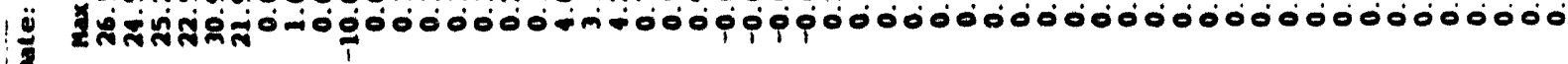

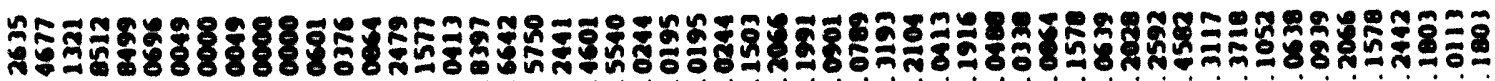

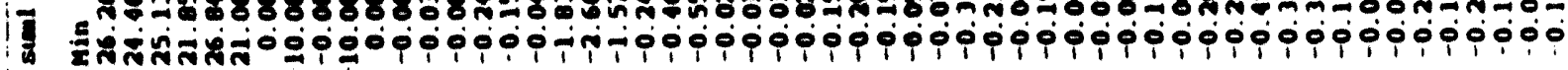

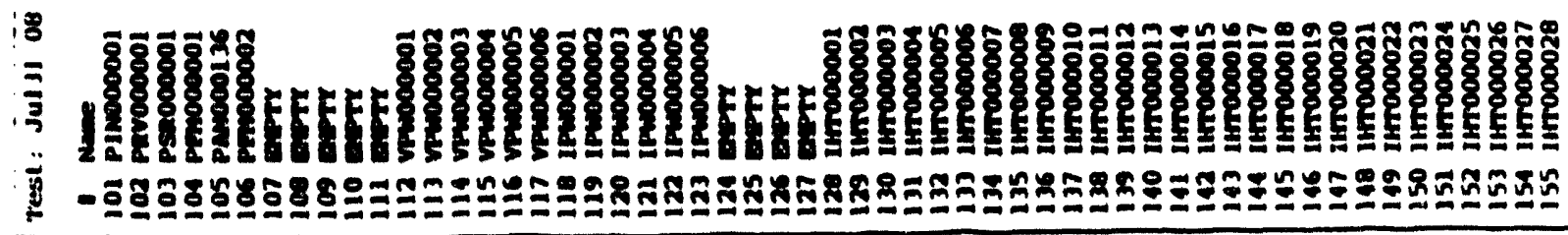




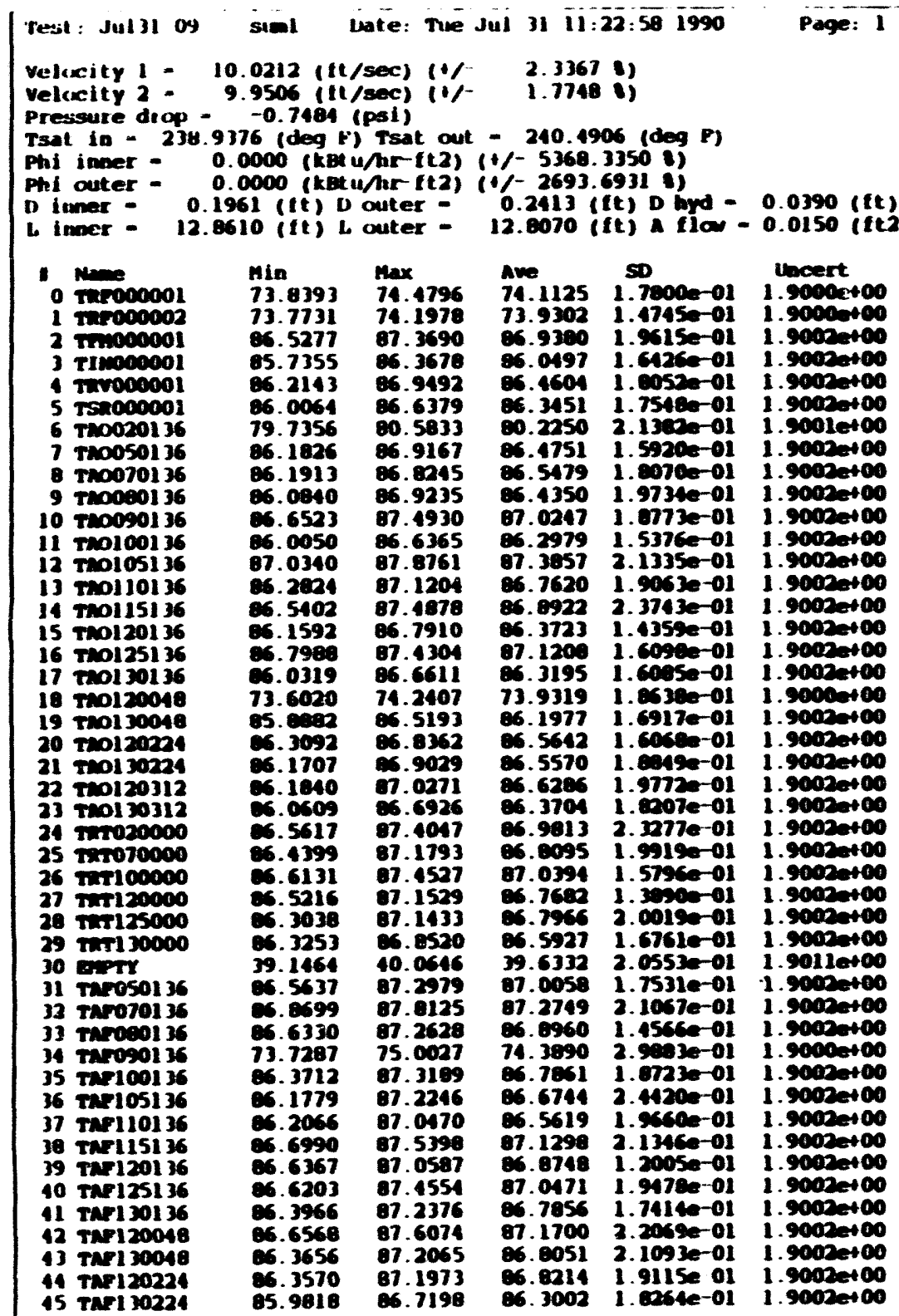

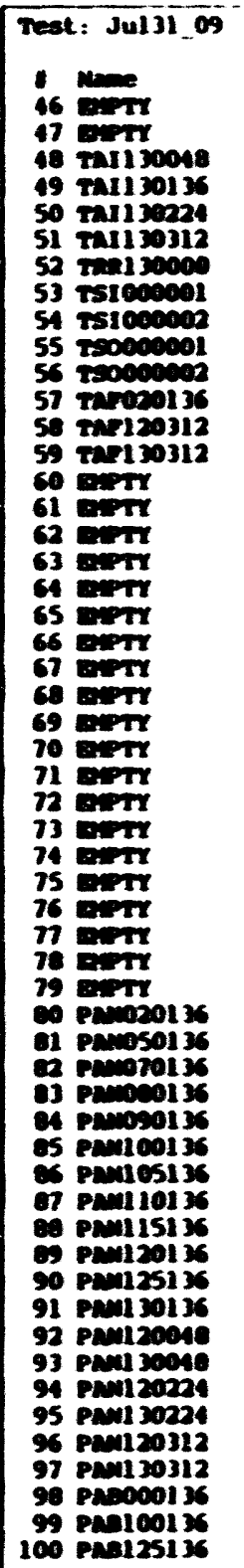

simi Trte: Tue Jul 11 11:22:s4 1990 Page: 2

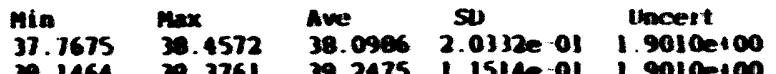
39.146439 .376139 .2475 1.1514e ol $1.9010=100$

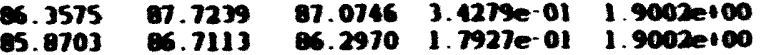
$6.703987 .9674 \quad 07.3843$ j.zejle.01 $1.9002=100$

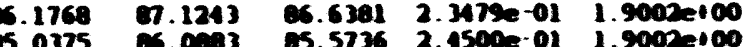
$5.0375 \% 6.063 \quad 85.57362 .45002 .01 \quad 1.9002=100$ $75.5797 \quad 76.7439 \quad 76.16652 .97930 .01 \quad 1.0000100$ 74.974575 .05075 .002 1915e- ol $19000+00$ $75.6266 \quad 76.2644$ 75.9603 1.6500e-01 $1.9000=100$ $6.7333 \quad 5.5702 \quad 6.1962$ 1.8506e ol $1.9002=100$ $05.01420 .9600 \quad 86.48772 .6000 \mathrm{e}-01 \quad 1.9002 \mathrm{el} 00$ $05.8243 \quad 6.4563 \quad 6.1633 \quad 1.6512=01 \quad 1.9002=100$

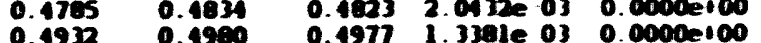

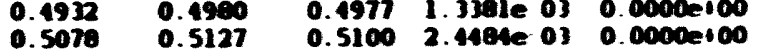
$\begin{array}{lllll}0.5078 & 0.5127 & 0.5100 & 2.4464 e^{0} 03 & 0.0000=100 \\ 0.5225 & 0.5273 & 0.5264 & 1.97302-01 & 0.0000=100\end{array}$ $0.3369 \quad 0.3418 \quad 0.3417$ 6.9053e-04 $0.0000=100$

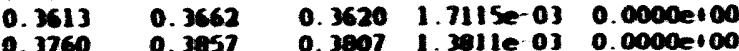
$0.3955 \quad 0.4004 \quad 0.4001$ i.1714e o3 $0.0000=100$ $0.4100 \quad 0.41990 .4147$ i.5314e 03 0.0000etco

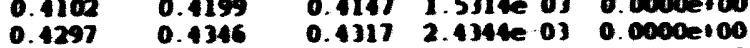
$\begin{array}{lllll}0.4413 & 0.4492 & 0.4486 & 1.4028=03 & 0.0000=100\end{array}$ $\begin{array}{lllllll}0.4639 & 0.4736 & 0.4730 & 1.0021 e^{0} & 03 & 0.0000=100\end{array}$ $\begin{array}{llllll}0.4834 & 0.4833 & 0.4860 & 2.4503 e^{-03} & 0.0000 \mathrm{e} 100\end{array}$ $\begin{array}{llllll}0.5029 & 0.5078 & 0.5076 & 9.6655 e-04 & 0.0000=100\end{array}$ $\begin{array}{llllll}0.5225 & 0.5273 & 0.5226 & 6.5053=-04 & 0.00000100\end{array}$ $\begin{array}{llllll}0.5420 & 0.5120 & 0.5420 & 0.0000 \text { t00 } & 0.00600100\end{array}$ $0.5566 \quad 0.5615 \quad 0.5567 \quad 6.90532-040.00001000$ $0.5713 \quad 0.5762 \quad 0.5761 \quad 6.90531040 .00000+00$ $0.6201 \quad 0.6250 \quad 0.6232 \quad 2.36750-03 \quad 0.00000000$ $-2.4941-2.1809-2.3506 .240002 \quad 07002=01$ $\begin{array}{lllll}-5.1390 & -2.5297 & -2.3565 & 1.2462=-02 & 1.6000-100\end{array}$ $\begin{array}{lllll}-3.5796 & -3.2745 & -3.4123 & 0.5000-02 & 0.7004 e^{-01}\end{array}$ $\begin{array}{llllll}-2.0246 & -1.7194 & -1.0659 & 7.0365 e & 02 & 0.7001 e^{-01}\end{array}$ $-1.5446-1.2396-1.4319 \quad 6.7610$ e 02 e.700le-01 $-3.3076-3.0051-3.1740$. sa77e 02 8.7004e or

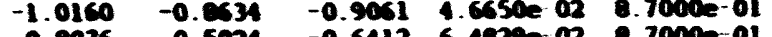

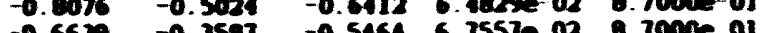
$-0.6639-0.3587-0.5464$ c.7557e 02 . $7000 \mathrm{e}$ ol $-0.5302-0.3014 \quad-0.40215 .65000 .020 .7000=01$ $0.0357-0.7255-0.0795$ 5.9771e 02 .

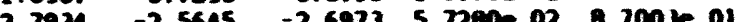
$-1.2939-0.9607-1.1352$ B 13200202.7000 o1 $-3.0075-2.7707-2.6931$ i 60790.028 .7003 ol $\begin{array}{llllll}-1.5271 & -1.2220 & -1.3746 & \text { T.5511e } 02 & 8.7001 \text { e } 01\end{array}$ $\begin{array}{lllllll}-1.8323 & -1.4509 & -1.6000 & 0.2157 e & 02 & \text { B.7001e ol }\end{array}$

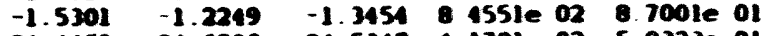
24.445924 .629024 .5347 . 1701 e 02 5.8323e o1

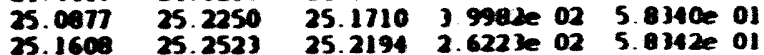




\begin{tabular}{|c|c|c|c|c|c|c|}
\hline 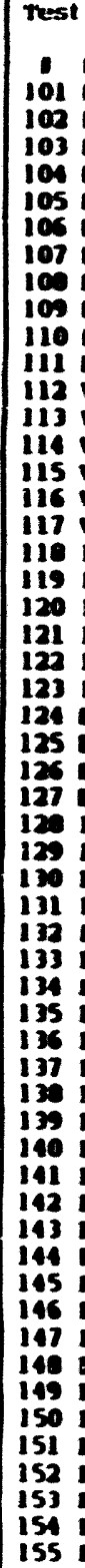 & 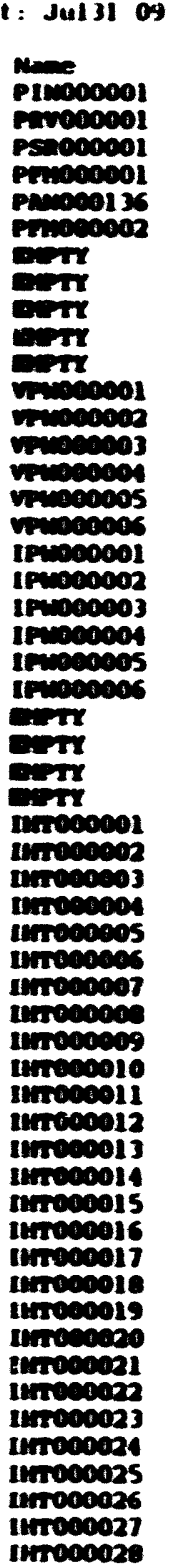 & 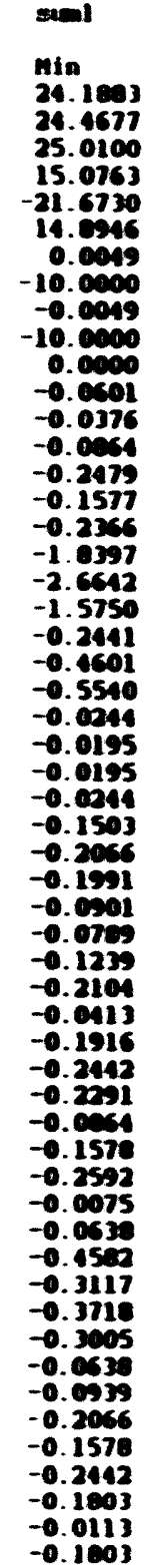 & 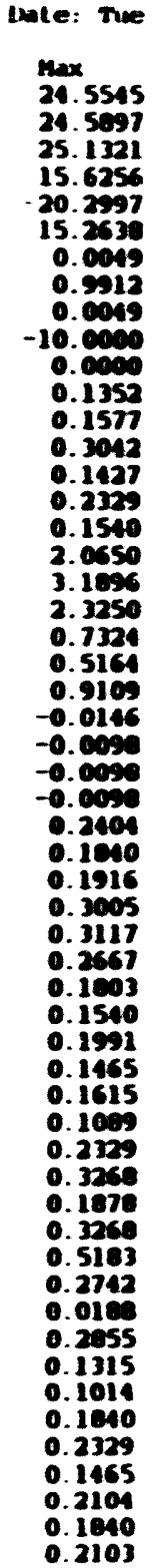 & 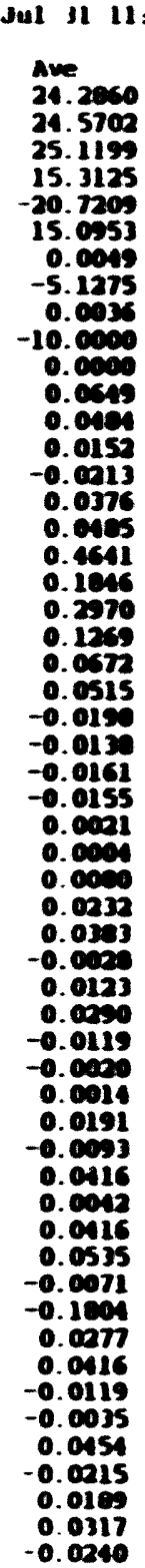 & 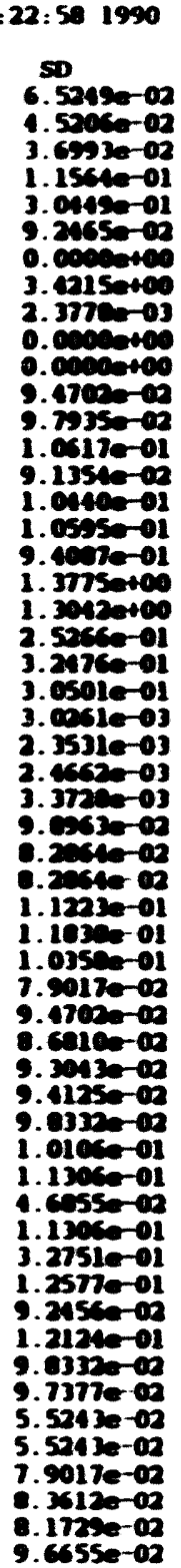 & 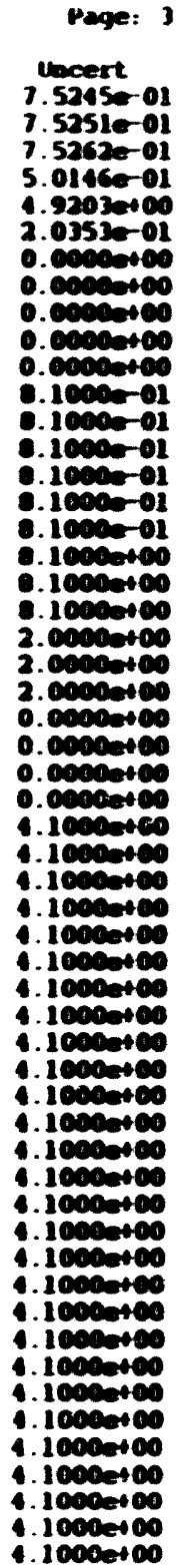 \\
\hline
\end{tabular}

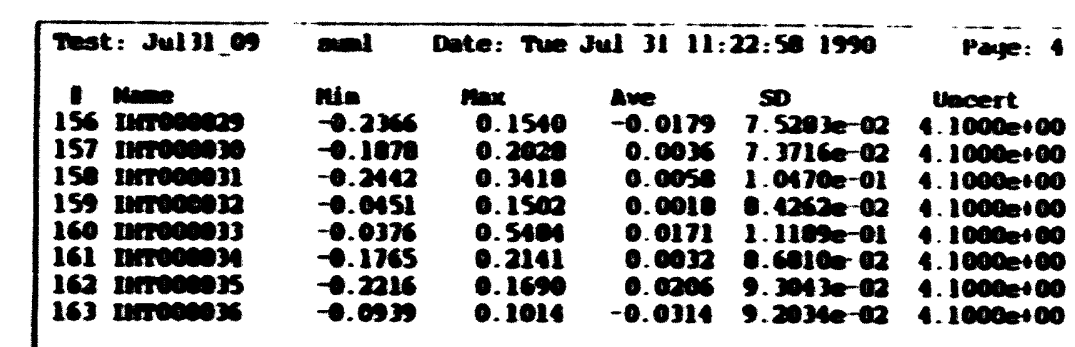




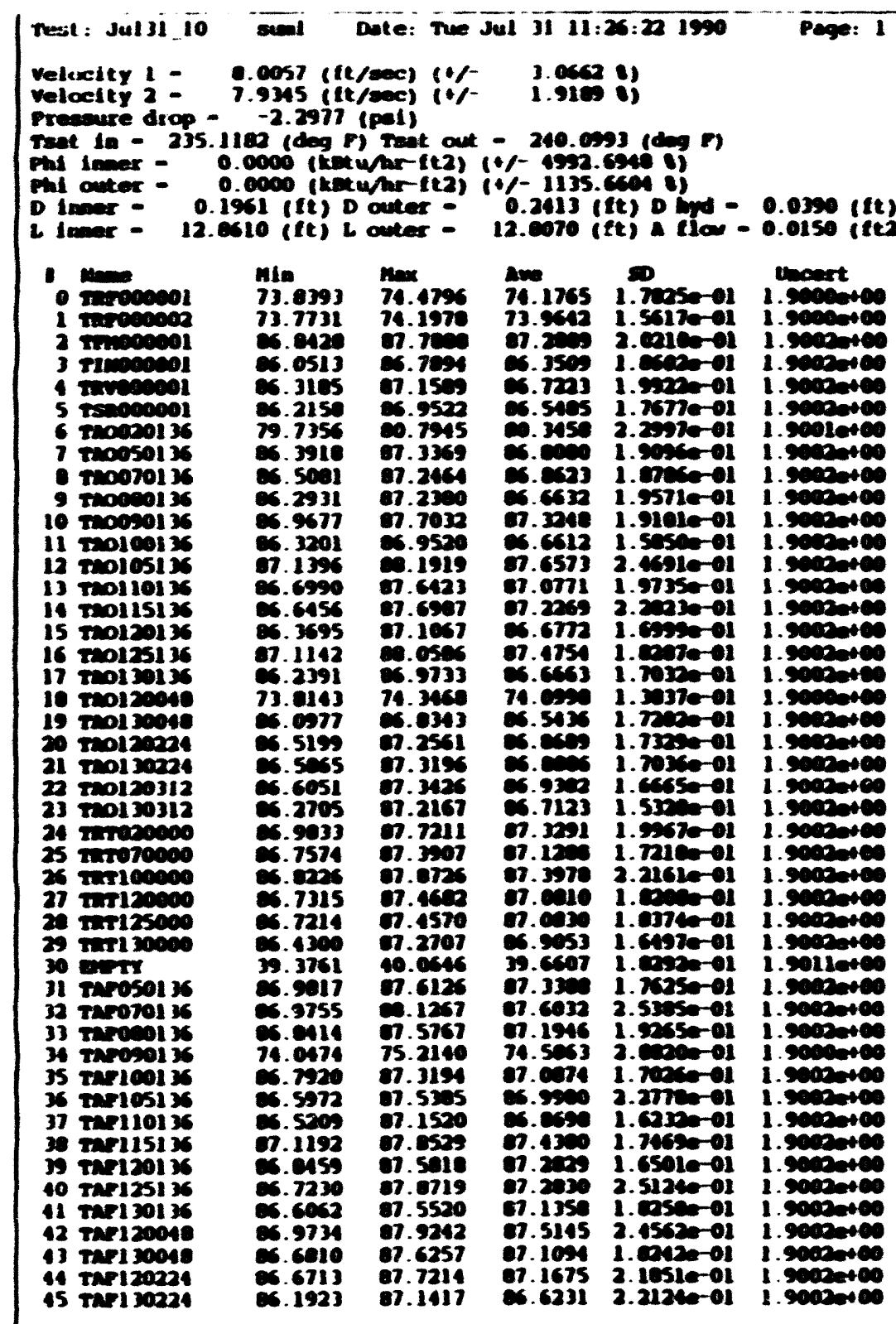

\begin{tabular}{|c|c|c|c|c|c|}
\hline 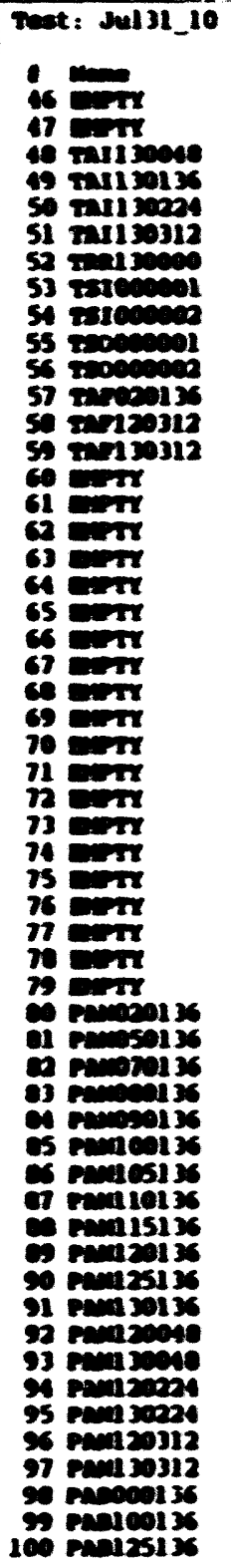 & 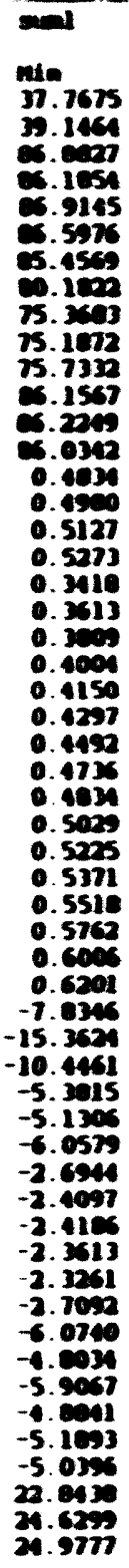 & 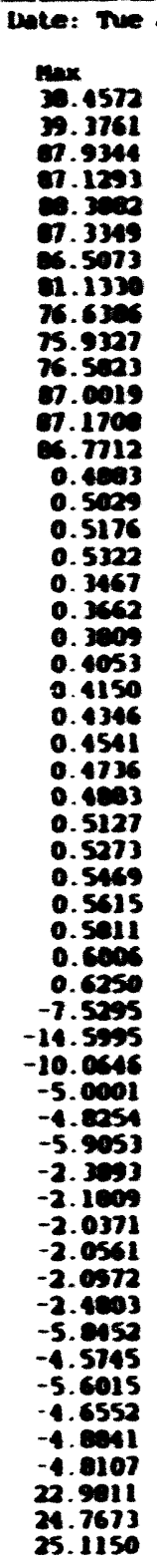 & 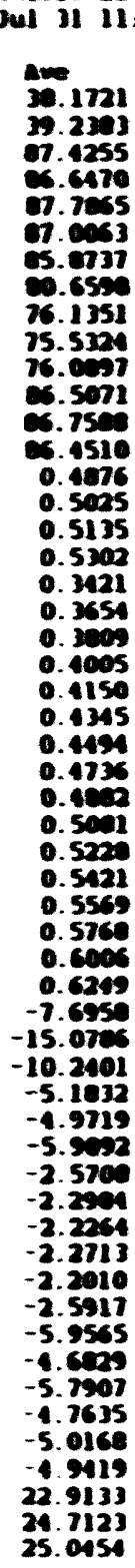 & 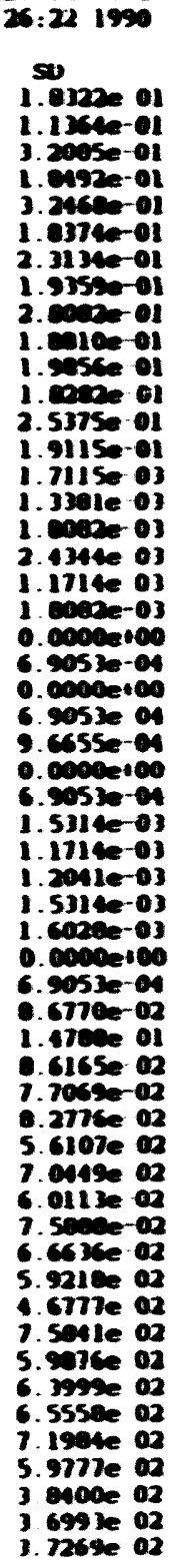 & 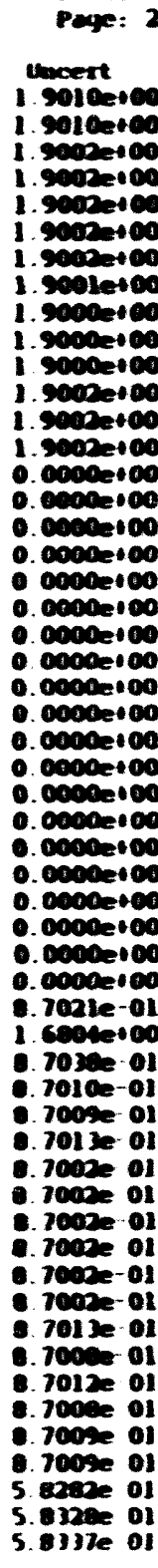 \\
\hline
\end{tabular}




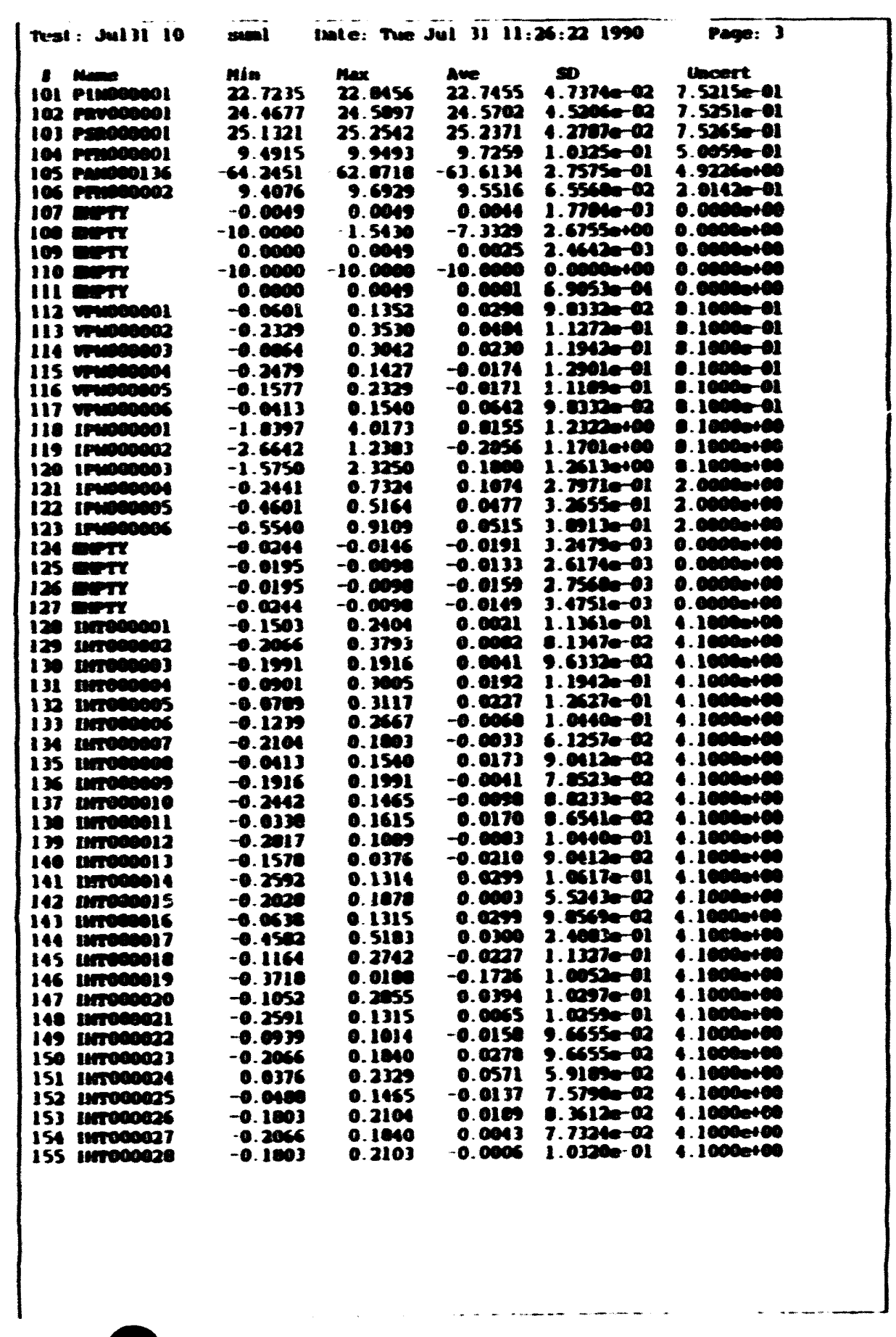

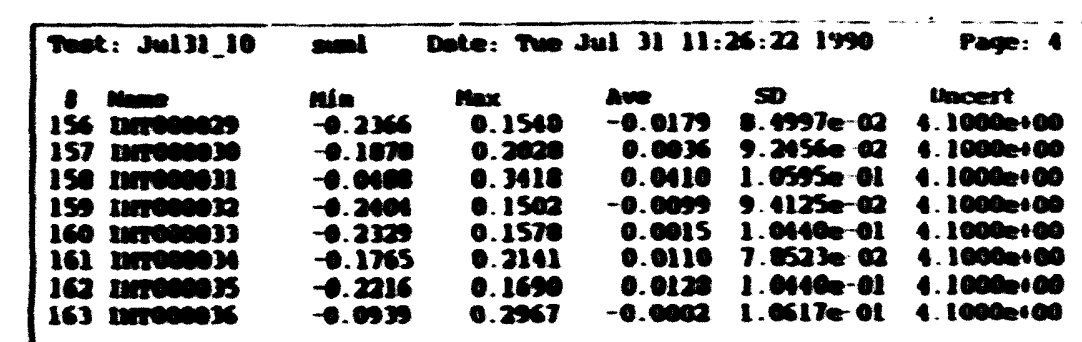




\begin{tabular}{|c|c|c|c|c|c|}
\hline 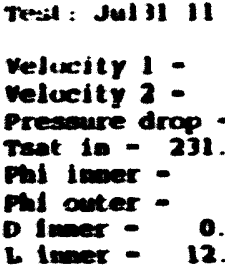 & $\begin{array}{l}\text { sual } \\
6.0009 \text { (It) } \\
5.9727 \text { ift } \\
-3.5305 \\
650 \text { ideg f } \\
.0000 \text { (t) }\end{array}$ & 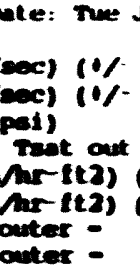 & $\begin{array}{r}\text { sut } 11 \text { 11: } \\
1.9023 \\
2.3671\end{array}$ & : 011990 & $\begin{array}{l}(t+) \\
(f t 2)\end{array}$ \\
\hline 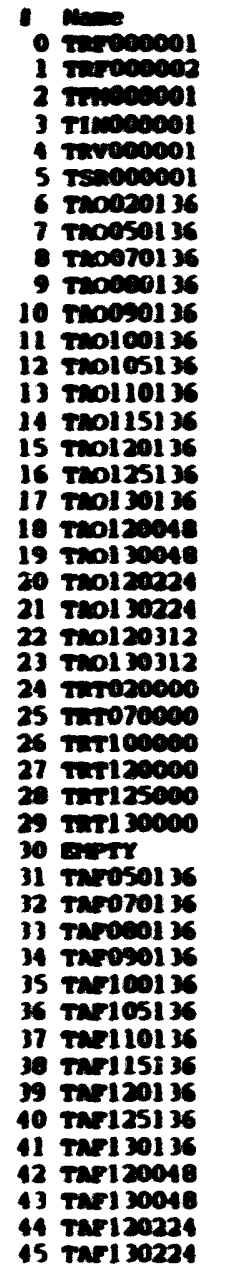 & 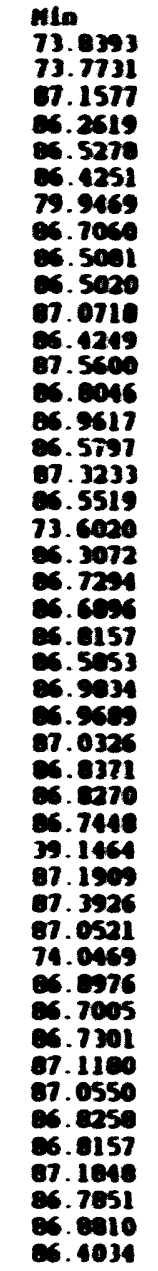 & 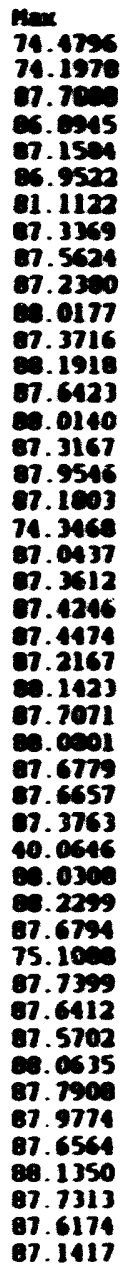 & 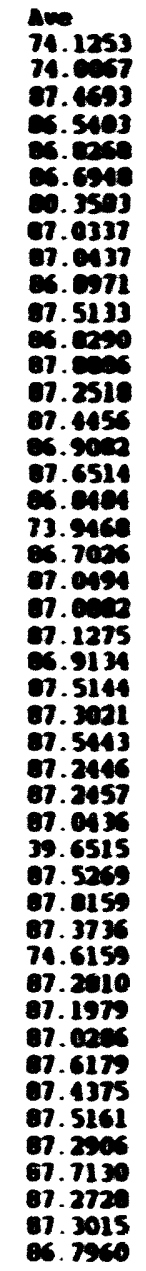 & 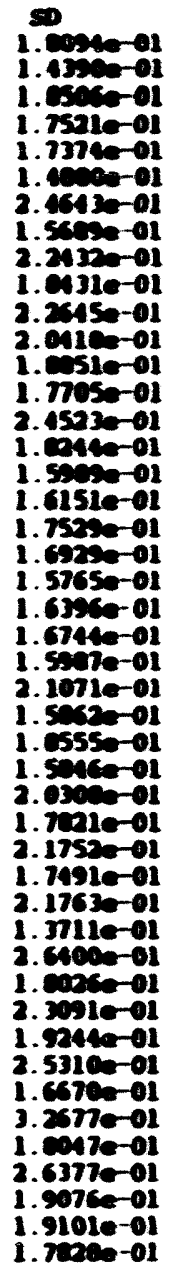 & \\
\hline
\end{tabular}

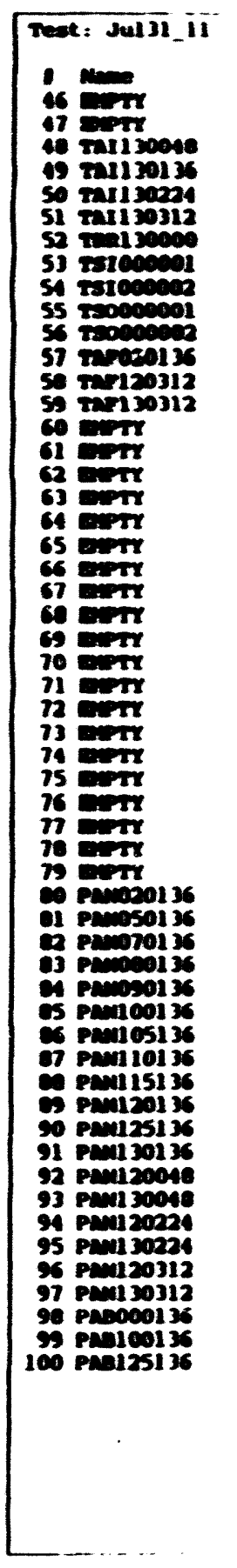

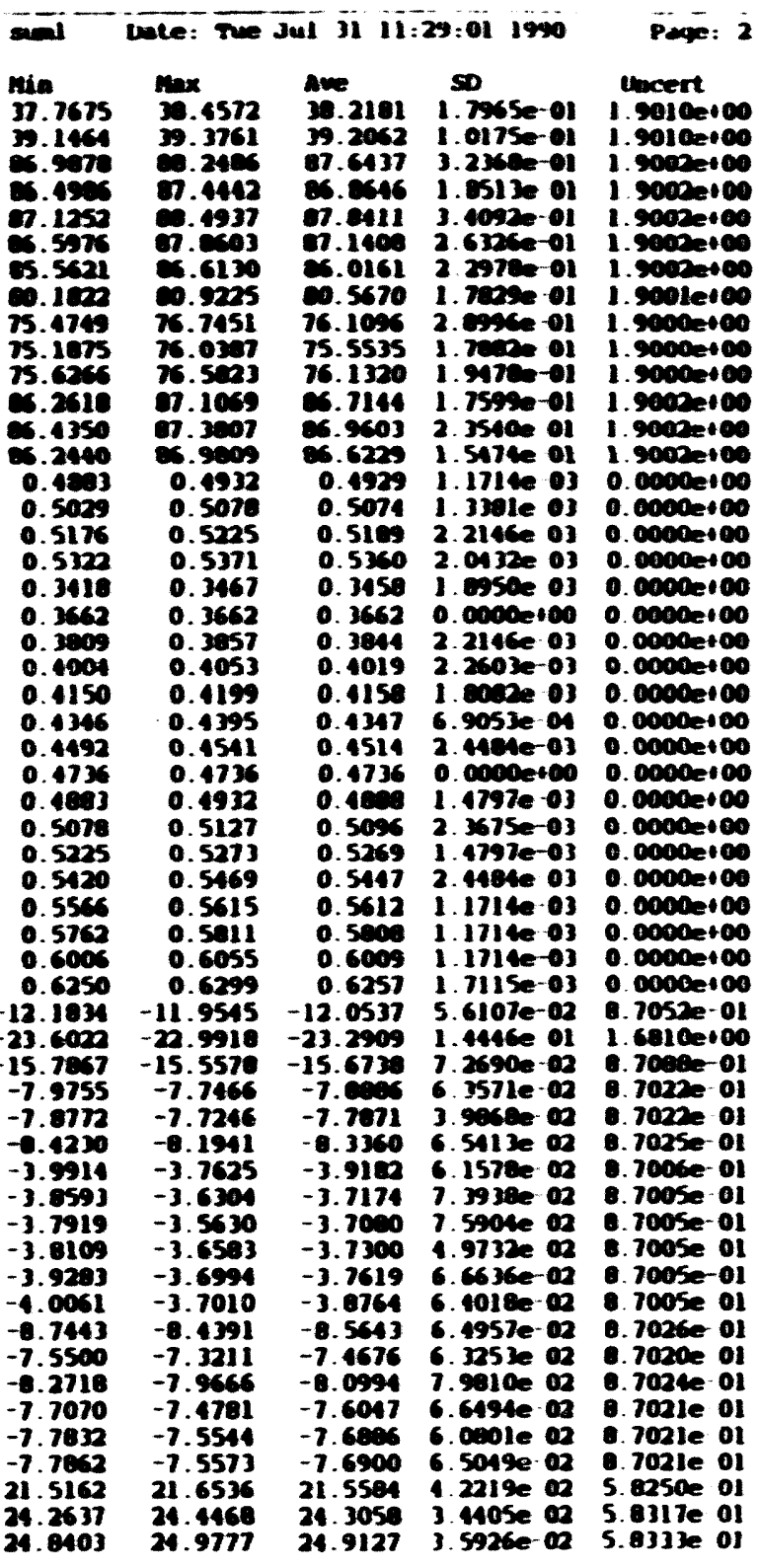




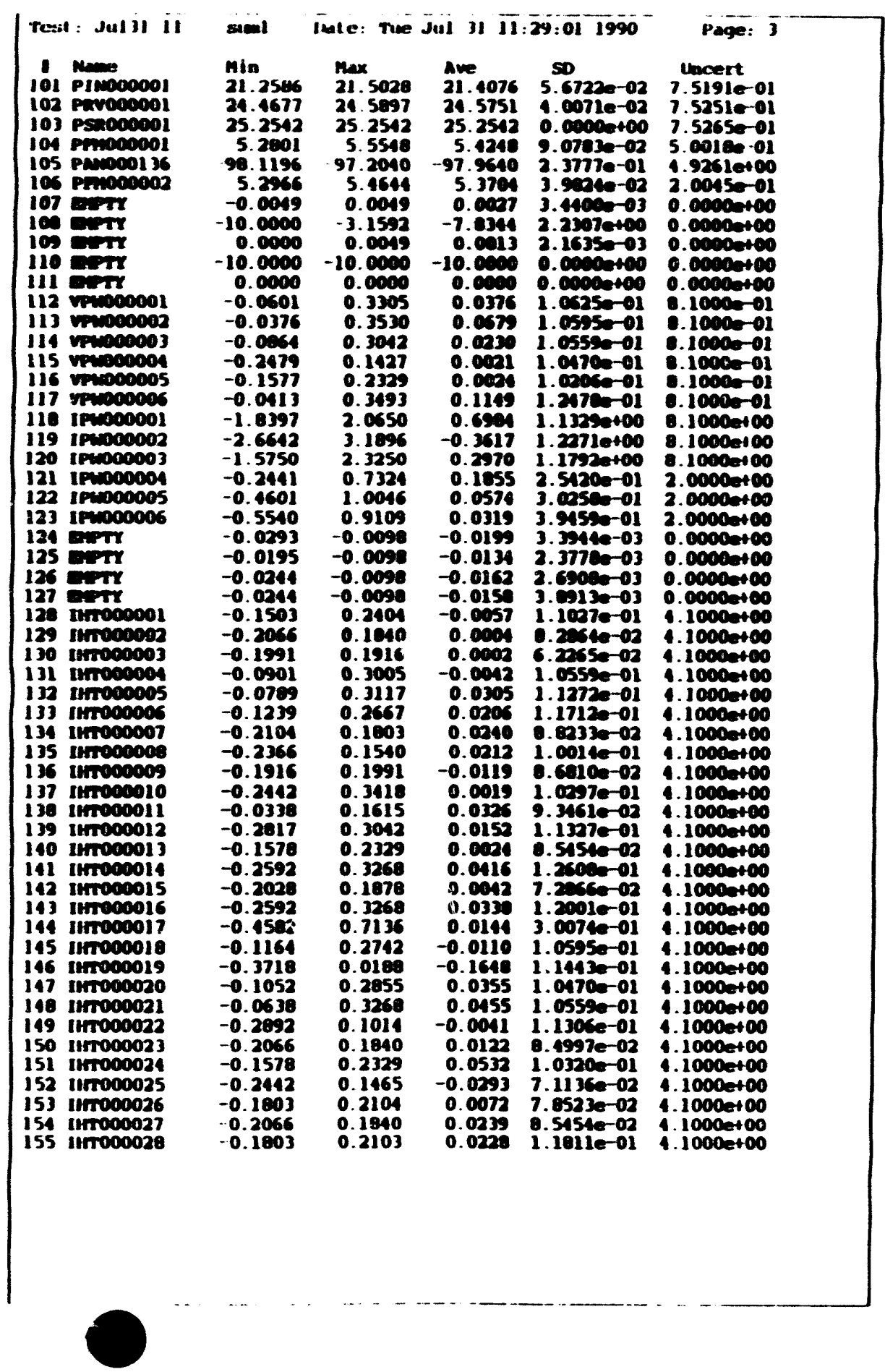

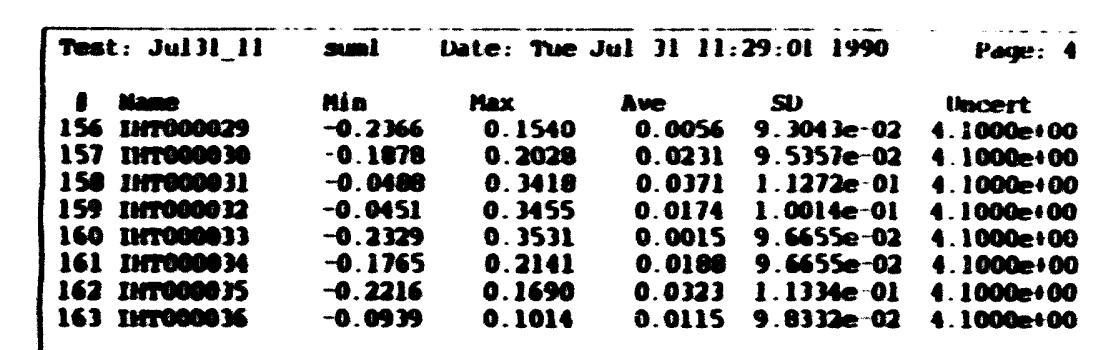




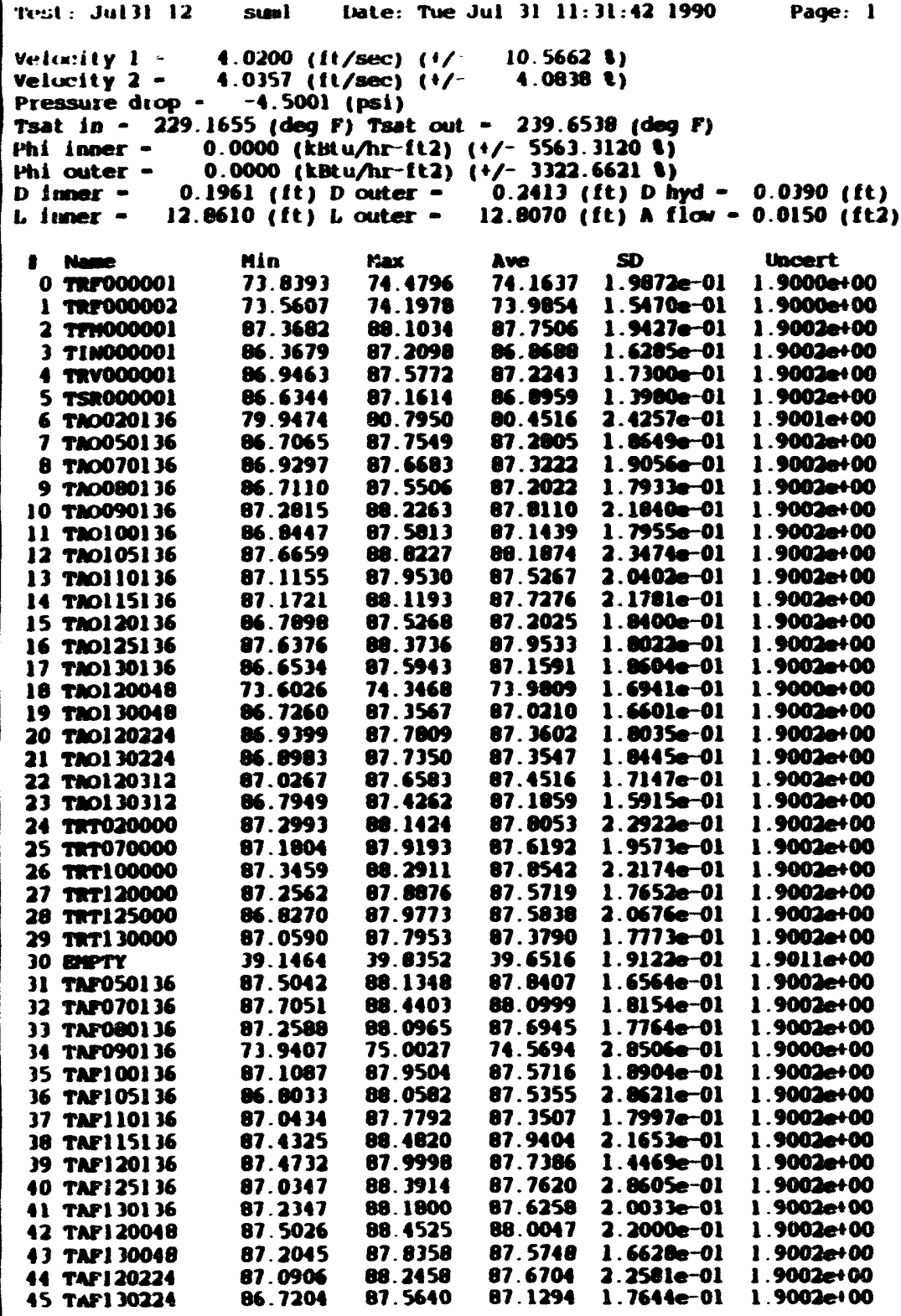

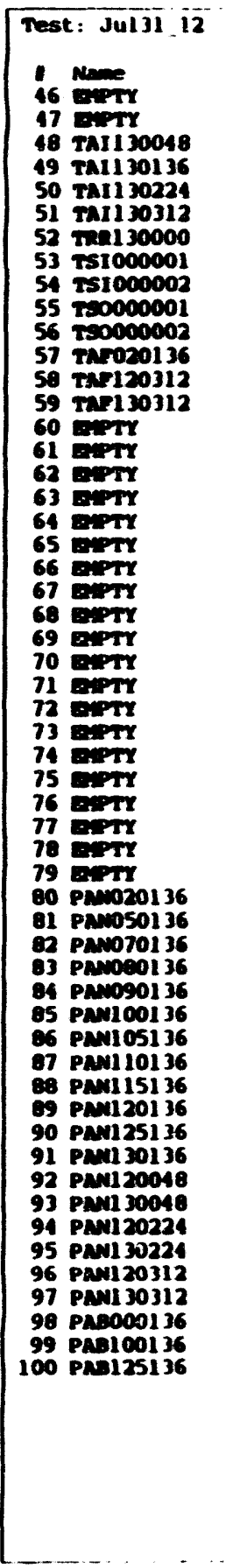

senl wate: The Jui il $11: 31: 42$ 1930 Payge: 2

Min Max Ave SD $\begin{array}{llllll}38.9160 & 39.3761 & 39.2475 & 1.2414 e-01 & 1.90102100\end{array}$

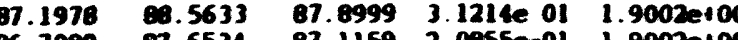
86.7357 $87.6534 \quad 87.1159$ 2. $97.0194 .9656 \quad 87.774$ 2.3942e-01 1.9002e+00 05.7717 7173 06.3012 2.5363e-01 1.90022000 $\$ 0.1822 \quad 00.9225 \quad 00.4913$ 1.7054e-01 1.9001et00 $\begin{array}{lllllll}75.5797 & 76.6392 & 76.1530 & 2.7379 e-01 & 1.9000 e+00\end{array}$ $\begin{array}{lllllll}75.1875 & 76.0397 & 75.5634 & 1.9625 e & 01 & 1.9000 e+100\end{array}$ $\begin{array}{llllll}75.6272 & 76.5823 & 76.1575 & 1.9606 e-01 & 1.90002+00\end{array}$ $8.5799 \quad 67.5307 \quad 67.0402 \quad 2.1004$ e $01 \quad 1.9002 e 100$ $\begin{array}{llllllll}0.7494 & 87.0004 & 87.1787 & 2.4093 e & 01 & 1.90022+100\end{array}$ 66.4530 $\quad 87.4002 \quad 86.9460 \quad 2.1395$ on $1.9002 e+00$ $0.4932 \quad 0.5029 \quad 0.4900 \quad 9.85860-04 \quad 0.00002+00$ $0.5078 \quad 0.5127 \quad 0.5125 \quad 9.6655$ of 0.00002100 $0.5371 \quad 0.5420 \quad 0.54131 .7415-030.0000200$ $0.3467 \quad 0.3467 \quad 0.3467 \quad 0.0000400 \quad 0.00004100$ $\begin{array}{llllllll}0.3662 & 0.3711 & 0.3680 & 2.3675 e-03 & 0.0000=100\end{array}$ $\begin{array}{llllllll}0.3857 & 0.3857 & 0.3657 & 0.0000 e+00 & 0.00000100\end{array}$

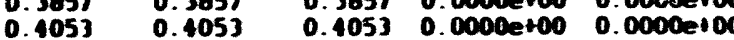
$\begin{array}{llllll}0.4150 & 0.4199 & 0.4197 & 9.6655 e^{-04} & 0.0000400\end{array}$ $\begin{array}{llllll}0.4346 & 0.4395 & 0.4384 & 2.0432 e^{-03} & 0.0000100\end{array}$ $\begin{array}{lllllllll}0.4492 & 0.4541 & 0.4540 & 6.90538-04 & 0.0000 e 100\end{array}$ $\begin{array}{llllll}0.4736 & 0.4785 & 0.4780 & 1.4797 e-03 & 0.00002100\end{array}$ $\begin{array}{llllll}0.4883 & 0.4932 & 0.4927 & 1.4797 e-03 & 0.00000100\end{array}$ $\begin{array}{lllll}0.5127 & 0.5127 & 0.5127 & 0.0002+100 & 0.00000+00\end{array}$

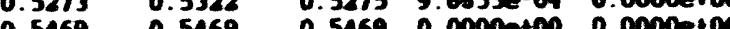
$0.5566 \quad 0.5615 \quad 0.56140 .90520-040.00000100$ $0.5762 \quad 0.5811 \quad 0.5140 .90530-040.00000400$ $0.6005 \quad 0.6055 \quad 0.6054 \quad 6.90535-040.00000+00$ $\begin{array}{llllll}0.6250 & 0.6299 & 0.6297 & 9.6655 \text { e o4 } & 0.0000=100\end{array}$ $\begin{array}{llllll}15.5403 & -15.2352 & -15.3710 & 7.7514 e^{-02} & 0.7005 e-01\end{array}$ $\begin{array}{llllll}-29.8583 & -29.4005 & -29.6569 & 1.1302 e-01 & 1.6816 e 100\end{array}$ -19.9065 -19.7540 -19.821 5.4800 e 02 o.714le ol $\begin{array}{llllllll}-10.1801 & -9.8066 & -9.9958 & 8.3348 e-02 & 8.7036 e & 01\end{array}$ $\begin{array}{llllll}-10.0134 & -9.7845 & -9.9432 & 5.5232 e^{-02} & 0.7036 e & 01\end{array}$

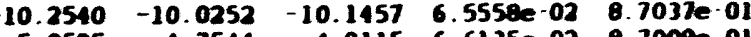

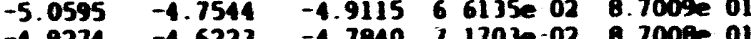

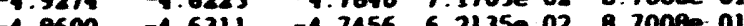

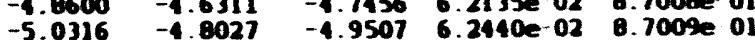
$-4.9964-4.8438-4.9399 \quad 4.977 e^{-02} 0.7009=01$ $-4.9900-4.7691-4.8005$ 5.6022e-02 B.7009e. ol $-10.0043-10.5754-10.64105 .9717 e^{-02} \quad$ g.7041e-01

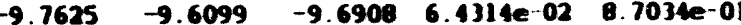

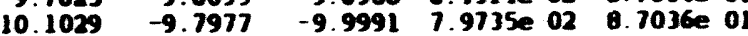
$\begin{array}{lllllll}-9.9195 & -9.6906 & -9.8279 & 7.7069 e^{2} 02 & 8.7035 e \cdot 01\end{array}$

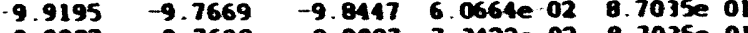
$\begin{array}{lllllll}-9.9987 & -9.7698 & -9.9087 & 7.3422 e & 02 & \text { B.7035e } 01\end{array}$ $20.4634 \quad 20.6465 \quad 20.5485 \quad 3.8143602 \quad 5.8227 e$ ol

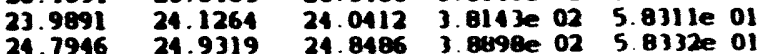




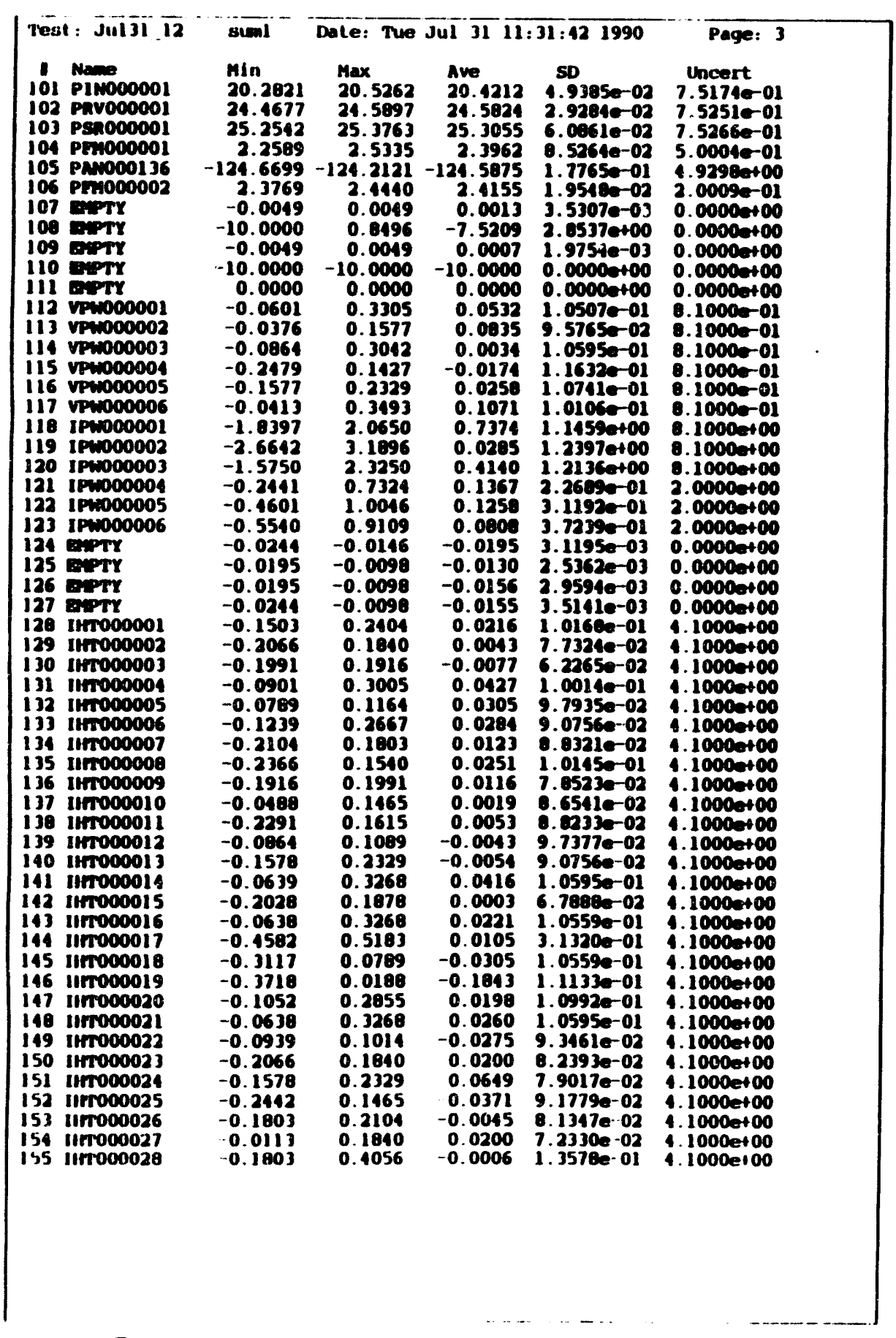

\begin{tabular}{|c|c|c|c|c|c|}
\hline Test: Jul31_12 & $\operatorname{sinl}$ & Date: Tue & ul 31 11: & $31: 421990$ & Page: 1 \\
\hline 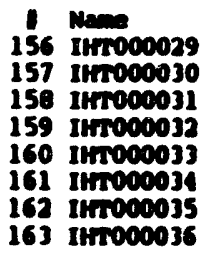 & $\begin{array}{l}\text { Min } \\
-0.0413 \\
-0.1878 \\
-0.0489 \\
-0.2404 \\
-0.0376 \\
-0.1765 \\
-0.2216 \\
-0.0939\end{array}$ & $\begin{array}{l}\operatorname{Max} \\
0.3493 \\
0.2028 \\
0.3418 \\
0.1502 \\
0.3531 \\
0.2141 \\
0.1690 \\
0.2967\end{array}$ & $\begin{array}{l}\text { Awe } \\
0.0134 \\
0.0192 \\
0.0644 \\
0.0057 \\
0.0093 \\
0.0227 \\
0.0401 \\
0.0115\end{array}$ & $\begin{array}{l}\text { SD } \\
9.6976 e-02 \\
9.9902 e-02 \\
1.2534 e-01 \\
9.5112 e-02 \\
9.3043 e-02 \\
8.3612 e-02 \\
1.0985 e-01 \\
1.0595 e-01\end{array}$ & $\begin{array}{c}\text { Uncert } \\
4.1000=+00 \\
1.1000=+00 \\
4.1000=+00 \\
1.1000=+00 \\
4.1000=+00 \\
1.1000=+00 \\
1.1000++00 \\
4.1000=+00\end{array}$ \\
\hline
\end{tabular}




\begin{tabular}{|c|c|c|c|c|c|c|}
\hline \multicolumn{6}{|c|}{ 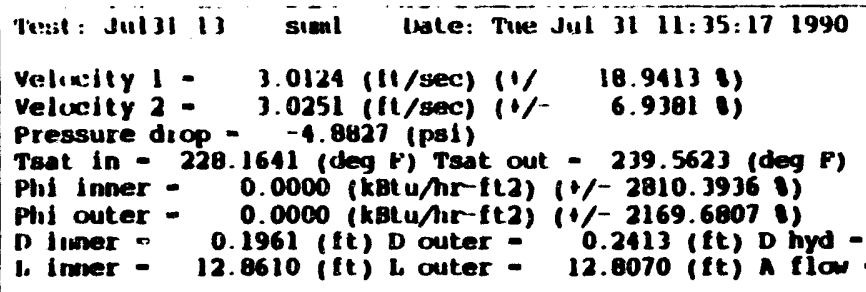 } & $\begin{array}{l}\text { Page: } 1 \\
\\
0.0390 \text { (ft) } \\
0.0150 \text { (ft2) }\end{array}$ \\
\hline $\begin{array}{l}1 \\
0 \\
1 \\
2 \\
3 \\
\vdots \\
5 \\
6 \\
7 \\
8 \\
9 \\
10 \\
11 \\
12 \\
13 \\
14 \\
15 \\
16 \\
17 \\
18 \\
19 \\
20 \\
21 \\
22 \\
23 \\
24 \\
25 \\
26 \\
27 \\
28 \\
29 \\
30 \\
31 \\
32 \\
33 \\
34 \\
35 \\
36 \\
37 \\
38 \\
39 \\
10 \\
11 \\
12 \\
13 \\
14 \\
15\end{array}$ & 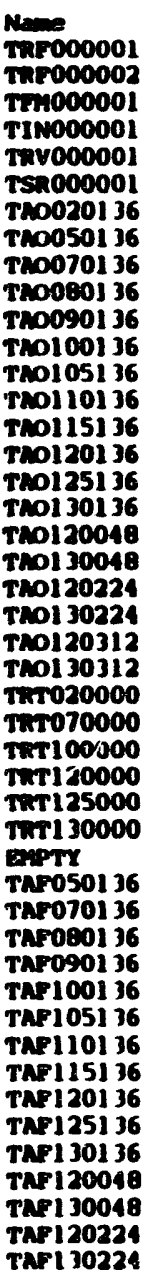 & $\begin{array}{l}\text { Min } \\
73.8393 \\
73.7731 \\
87.7806 \\
86.9986 \\
86.8421 \\
86.6344 \\
80.0531 \\
87.2281 \\
87.0356 \\
87.0255 \\
87.7007 \\
87.0545 \\
87.9807 \\
87.3237 \\
87.4974 \\
87.1050 \\
87.8972 \\
86.9660 \\
74.1328 \\
86.9354 \\
87.2542 \\
87.1069 \\
87.3422 \\
87.1009 \\
87.5100 \\
87.9968 \\
87.7646 \\
87.4659 \\
87.3476 \\
87.2686 \\
38.9168 \\
87.8183 \\
88.0189 \\
87.5716 \\
714.0469 \\
87.5293 \\
87.3251 \\
87.2524 \\
87.7462 \\
87.6822 \\
87.4536 \\
87.5491 \\
67.0189 \\
87.4142 \\
87.6148 \\
86.9313\end{array}$ & 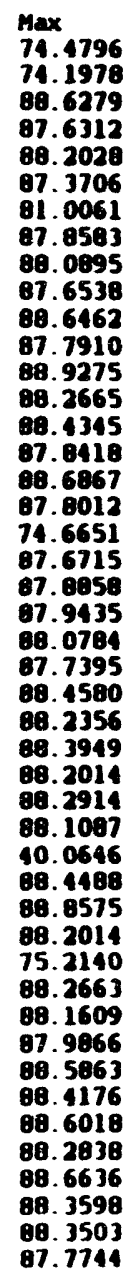 & $\begin{array}{l}\text { Ave } \\
74.1552 \\
71.0024 \\
88.1619 \\
87.2519 \\
87.3331 \\
87.0549 \\
80.4912 \\
87.5563 \\
87.6259 \\
87.4528 \\
88.1421 \\
87.3914 \\
88.1607 \\
87.8056 \\
87.9925 \\
87.4797 \\
88.2173 \\
87.4076 \\
74.4053 \\
87.2553 \\
87.6120 \\
87.6126 \\
87.6703 \\
87.4246 \\
88.0454 \\
87.8644 \\
88.1555 \\
87.8277 \\
87.8467 \\
87.6179 \\
39.6424 \\
89.1208 \\
88.4212 \\
87.9403 \\
74.6668 \\
87.8452 \\
87.7646 \\
87.6183 \\
88.2376 \\
87.9685 \\
88.1409 \\
87.9147 \\
88.2919 \\
87.8934 \\
87.9763 \\
87.4120\end{array}$ & 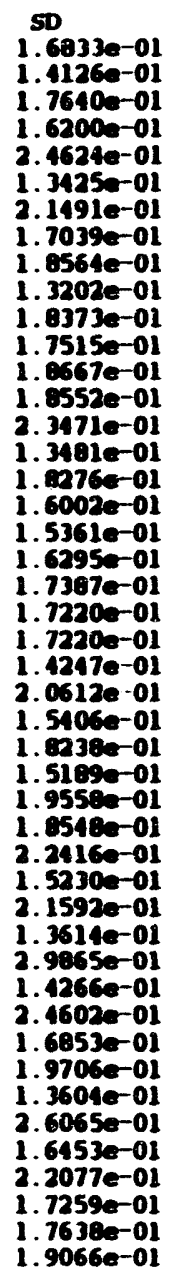 & $\begin{array}{l}\text { Uncert } \\
1.9000 e+00 \\
1.9000 e+00 \\
1.9002 e+00 \\
1.9002 e+00 \\
1.9002 e+00 \\
1.9002 e+00 \\
1.9001 e+00 \\
1.9002 e+00 \\
1.9002 e+00 \\
1.9002 e+00 \\
1.9002 e+00 \\
1.9002 e+00 \\
1.9002 e+00 \\
1.9002 e+00 \\
1.9002 e+00 \\
1.9002 e+00 \\
1.9002 e+00 \\
1.9002 e+00 \\
1.9000 e+00 \\
1.9002 e+00 \\
1.9002 e+00 \\
1.9002 e+00 \\
1.9002 e+00 \\
1.9002 e+00 \\
1.9002 e+00 \\
1.9002 e+00 \\
1.9002 e+00 \\
1.9002 e+00 \\
1.9002 e+00 \\
1.9002 e+00 \\
1.9011 e+00 \\
1.9002 e+00 \\
1.9002 e+00 \\
1.9002 e+00 \\
1.9000 e+00 \\
1.9002 e+00 \\
1.9002 e+00 \\
1.9002 e+00 \\
1.9002 e+00 \\
1.9002 e+00 \\
1.9002 e+00 \\
1.9002 e+00 \\
1.9002 e+00 \\
1.9002 e+00 \\
1.9002 e+00 \\
1.9002 e+00\end{array}$ \\
\hline
\end{tabular}

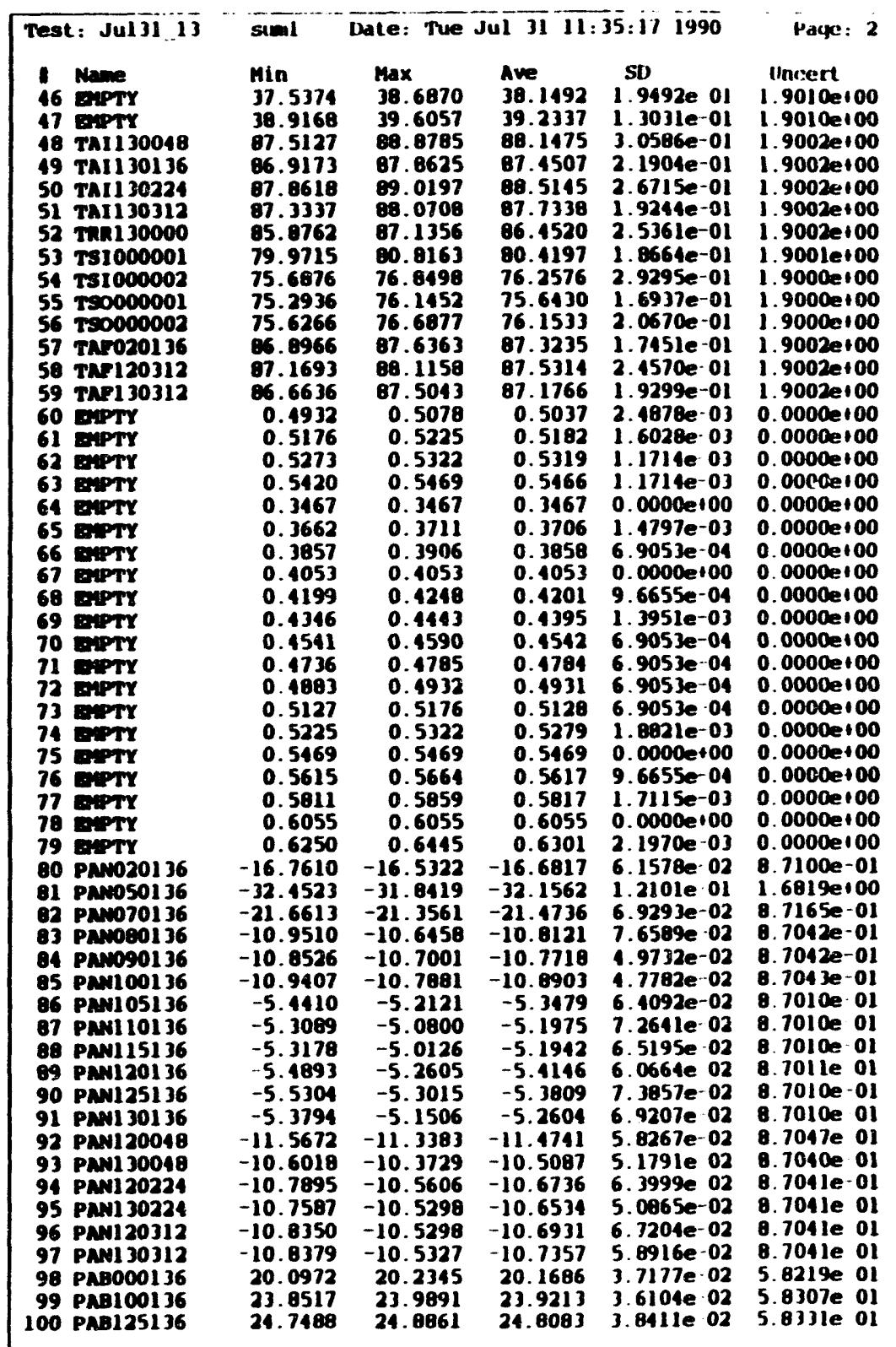




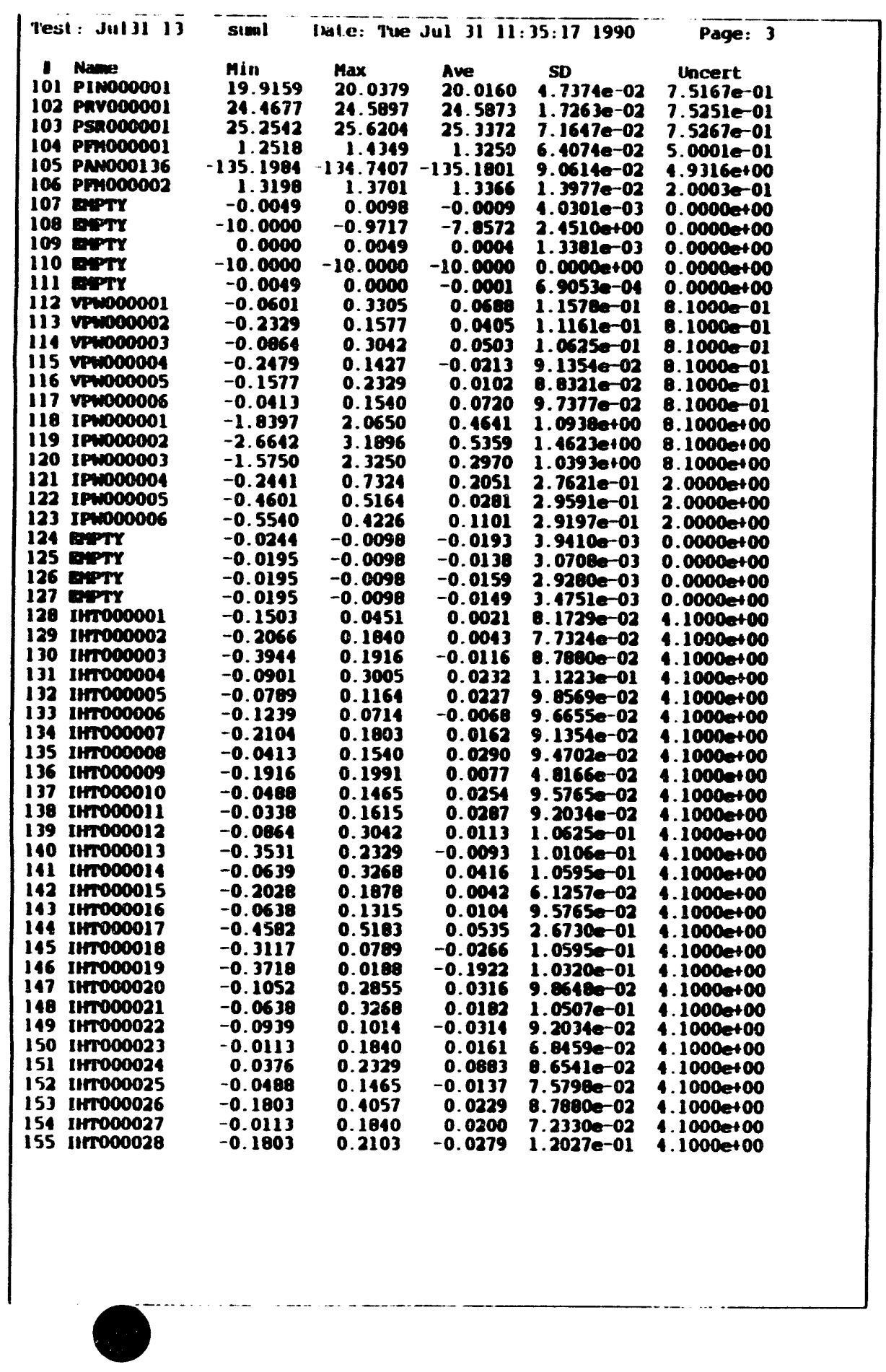

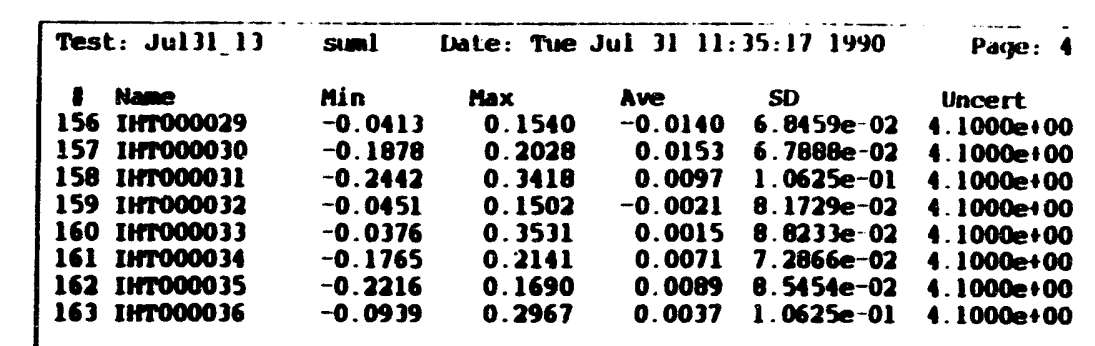




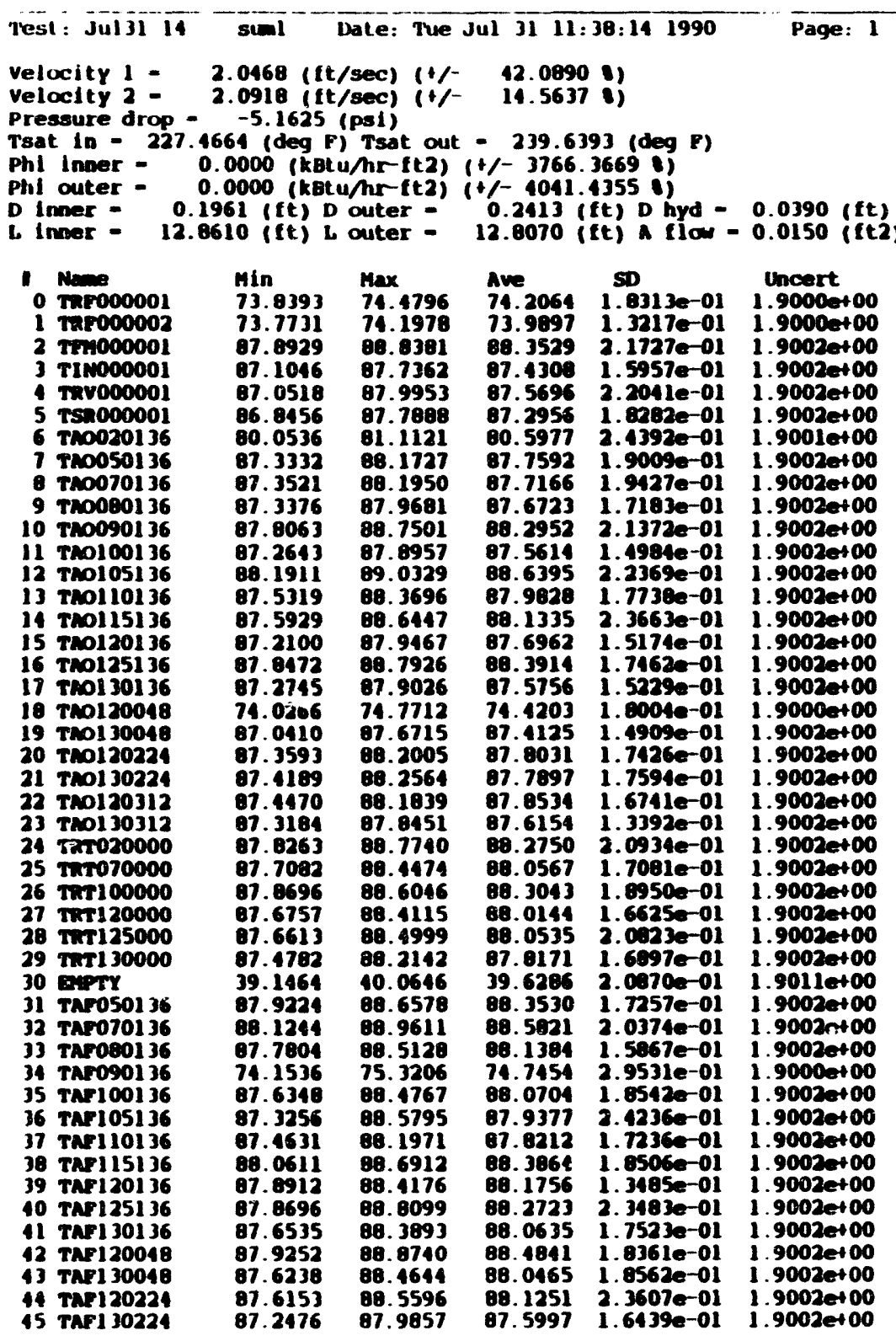

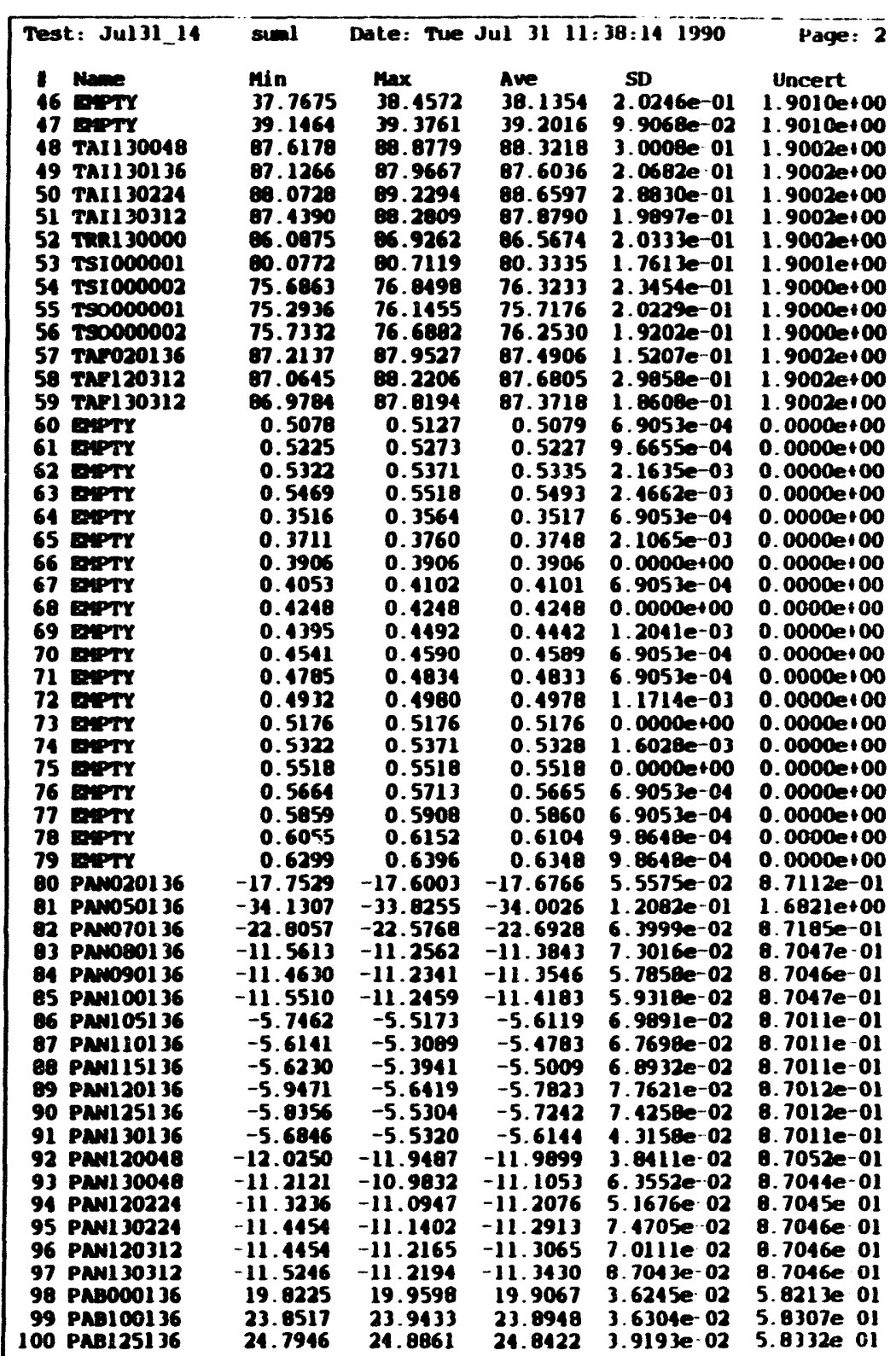




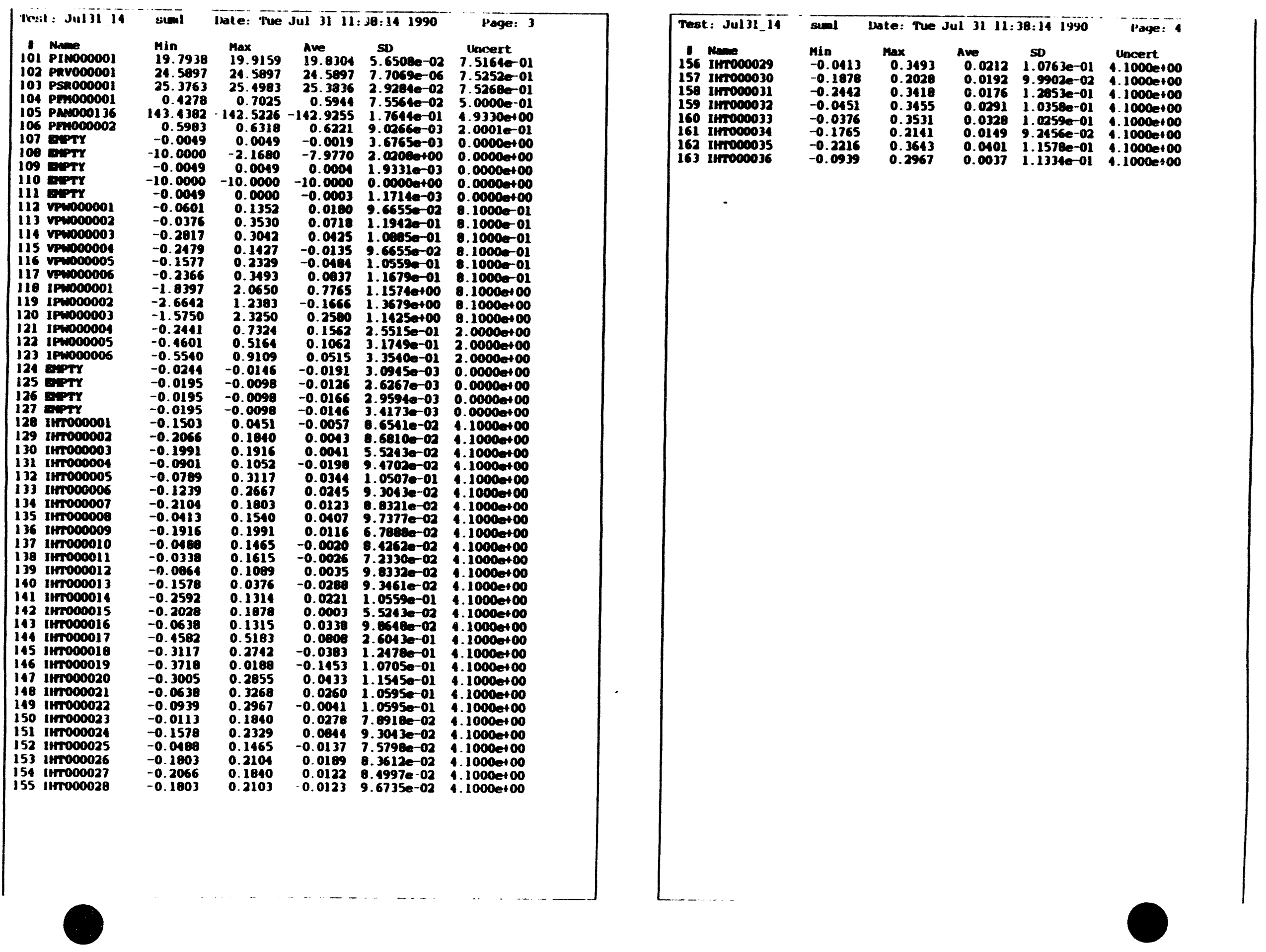




\begin{tabular}{|c|c|c|c|c|c|}
\hline \multicolumn{3}{|c|}{ 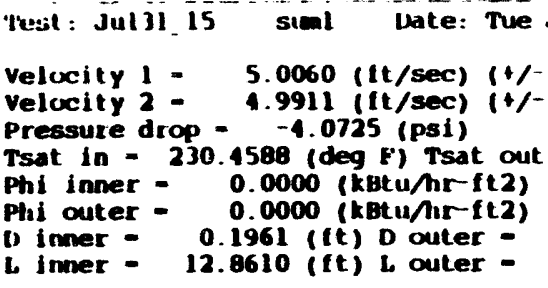 } & \multicolumn{2}{|c|}{ 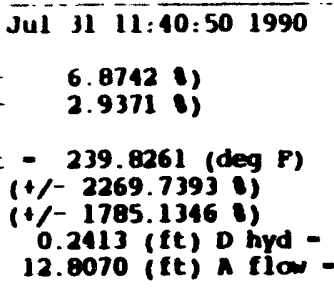 } & $\begin{array}{l}0390 \text { (ft) } \\
0150 \text { (fte }\end{array}$ \\
\hline 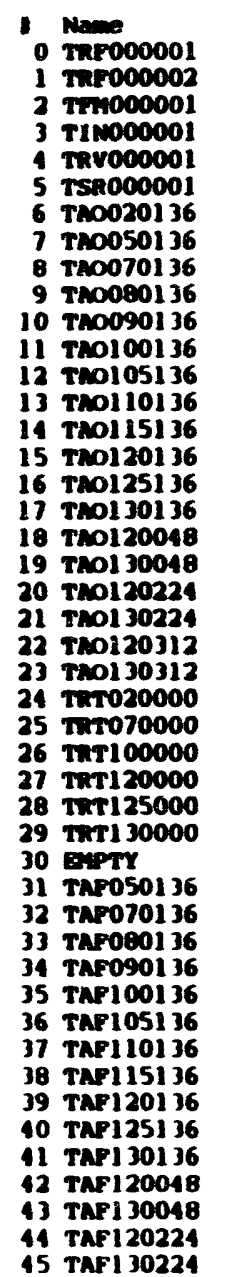 & $\begin{array}{l}\text { Min } \\
73.8393 \\
73.7731 \\
87.8929 \\
87.1046 \\
87.3661 \\
87.1579 \\
80.2640 \\
87.5422 \\
87.4572 \\
87.4432 \\
88.0153 \\
87.3693 \\
88.1019 \\
87.8456 \\
86.0130 \\
87.6301 \\
88.1622 \\
87.3801 \\
74.1328 \\
87.4590 \\
87.6747 \\
87.6269 \\
87.6576 \\
87.1234 \\
87.9319 \\
87.9196 \\
88.1839 \\
87.8853 \\
87.0699 \\
87.7927 \\
39.1464 \\
88.1329 \\
89.4361 \\
87.9882 \\
74.2598 \\
87.8448 \\
87.6384 \\
87.5666 \\
87.9567 \\
87.9967 \\
87.7646 \\
87.9678 \\
88.0308 \\
87.7293 \\
87.8248 \\
87.4588\end{array}$ & $\begin{array}{l}\text { Max } \\
74.4796 \\
74.1978 \\
88.8376 \\
87.9465 \\
88.5182 \\
87.6833 \\
81.1122 \\
88.3815 \\
88.3003 \\
88.0731 \\
88.8556 \\
88.0007 \\
89.2431 \\
88.5771 \\
86.7494 \\
88.1567 \\
88.8965 \\
88.2150 \\
74.6645 \\
87.8807 \\
88.2005 \\
88.2538 \\
88.2887 \\
89.0544 \\
88.8792 \\
88.5523 \\
88.9187 \\
88.5164 \\
89.7083 \\
88.3182 \\
40.0646 \\
88.8667 \\
89.2745 \\
88.6178 \\
75.3206 \\
88.4767 \\
88.7875 \\
88.3005 \\
89.1095 \\
88.6265 \\
89.0179 \\
88.5985 \\
89.0851 \\
88.5693 \\
88.6630 \\
88.0910\end{array}$ & $\begin{array}{l}\text { Ave } \\
74.1338 \\
74.0449 \\
88.4190 \\
87.4769 \\
87.7241 \\
87.1167 \\
80.6145 \\
87.9136 \\
87.9273 \\
87.7973 \\
88.4877 \\
87.7332 \\
88.8118 \\
88.1865 \\
88.3857 \\
87.8681 \\
88.5420 \\
87.7450 \\
74.3522 \\
87.6424 \\
87.9666 \\
87.9725 \\
89.0173 \\
87.7869 \\
88.4307 \\
88.2425 \\
88.5175 \\
88.1610 \\
88.2200 \\
87.9845 \\
39.5827 \\
88.4698 \\
88.7821 \\
88.2589 \\
74.7664 \\
88.2049 \\
88.1706 \\
87.9422 \\
88.5033 \\
88.3592 \\
88.3845 \\
88.2140 \\
88.6445 \\
88.1720 \\
88.2673 \\
87.7345\end{array}$ & $\begin{array}{l}50 \\
1.6070 e-01 \\
1.4255 e-01 \\
2.29800-01 \\
1.5363 e-01 \\
2.1267 e-01 \\
1.3452 e-01 \\
2.1306 e-01 \\
1.7237 e-01 \\
1.7935 e-01 \\
1.5258 e-01 \\
2.0582 e-01 \\
1.5050 e-01 \\
2.1162 e-01 \\
1.5222 e-01 \\
1.9496 e-01 \\
1.3743 e-01 \\
1.4373 e-01 \\
1.4802 e-01 \\
1.3920 e-01 \\
1.2355 e-01 \\
1.3914 e-01 \\
1.5405 e-01 \\
1.6061 e-01 \\
1.4439 e-01 \\
1.6995 e-01 \\
1.5565 e-01 \\
1.8304 e-01 \\
1.5594 e-01 \\
2.0118 e-01 \\
1.2514 e-01 \\
2.2362 e-01 \\
1.7505 e-01 \\
1.6915 e-01 \\
1.7319 e-01 \\
2.5013 e-01 \\
1.7154 e-01 \\
2.7429 e-01 \\
1.5448 e-01 \\
2.4355 e-01 \\
1.3116 e-01 \\
2.7264 e-01 \\
1.6504 e-01 \\
2.3960 e-01 \\
1.7524 e-01 \\
1.9696 e-01 \\
1.5916 e-01\end{array}$ & $\begin{array}{l}\text { Uncert } \\
1.90000+00 \\
1.90000+00 \\
1.90020+00 \\
1.9002 e+00 \\
1.9002 e+00 \\
1.9002 e+00 \\
1.9001+00 \\
1.9002 e+00 \\
1.9002 e+00 \\
1.9002 e+00 \\
1.9002 e+00\end{array}$ \\
\hline
\end{tabular}

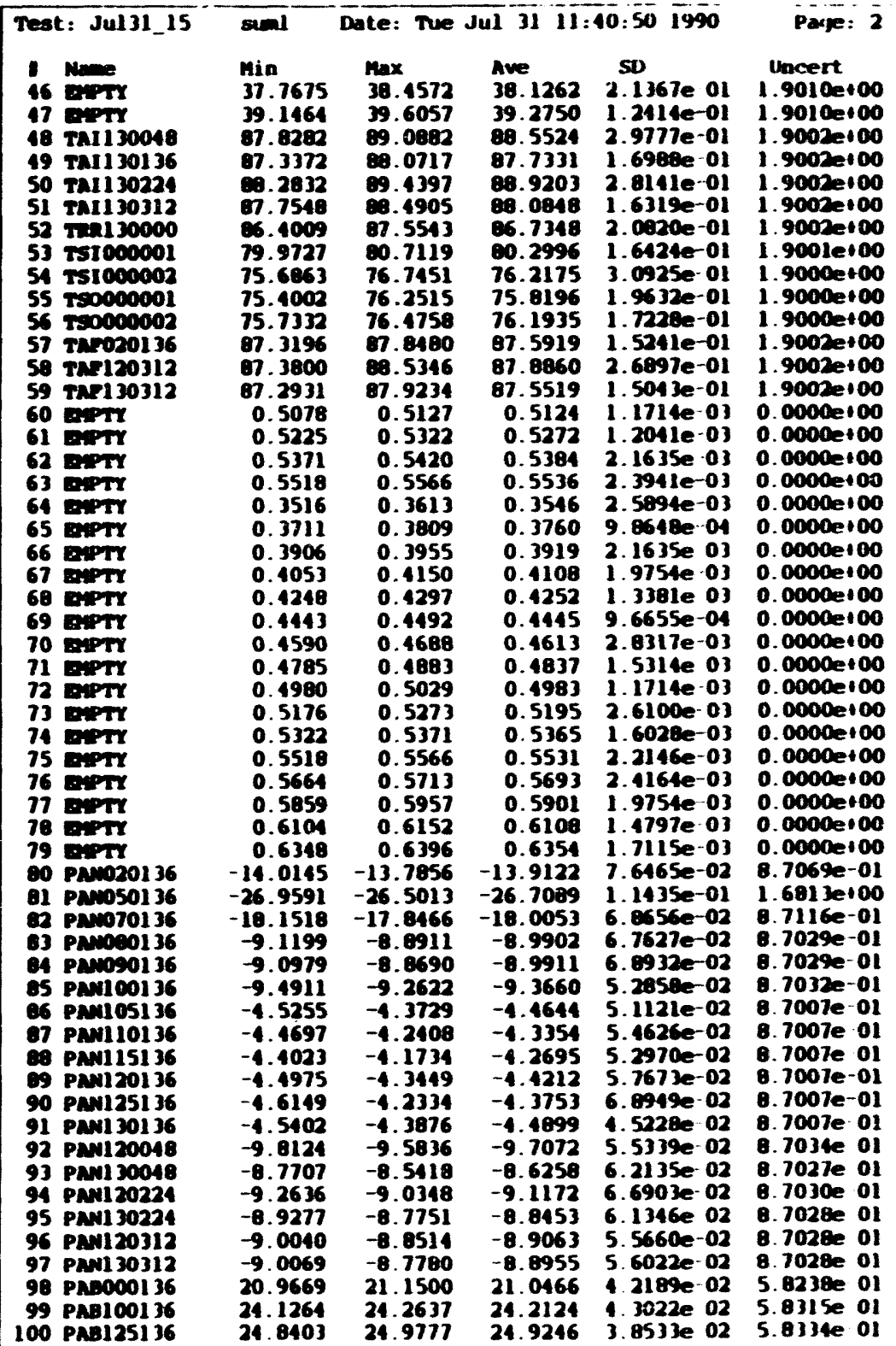




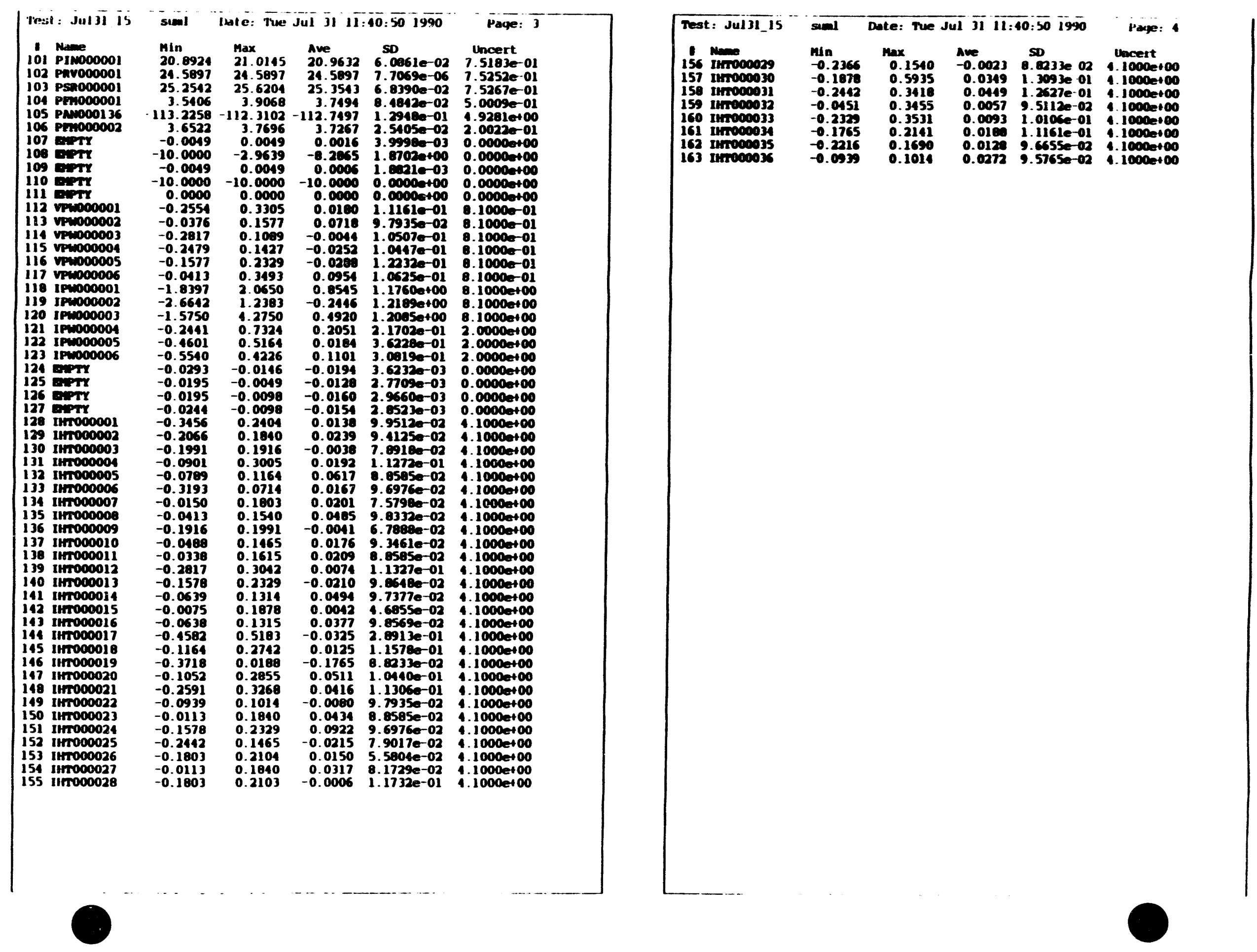




\begin{tabular}{|c|c|c|c|c|c|}
\hline \multicolumn{6}{|c|}{ 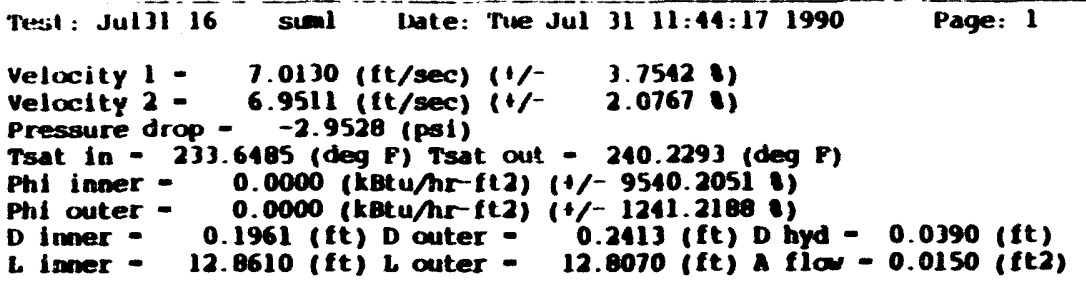 } \\
\hline 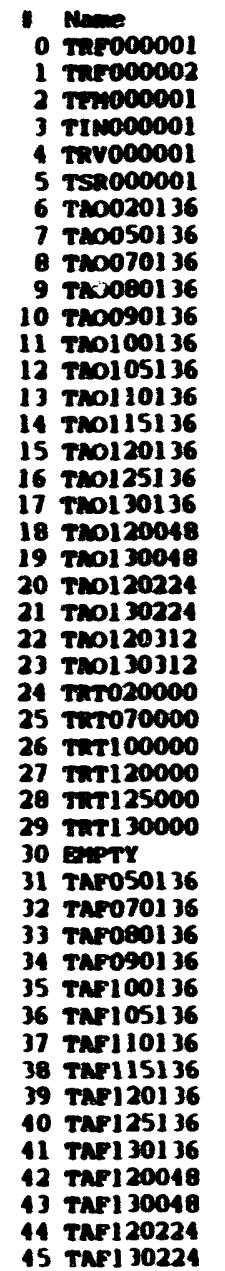 & $\begin{array}{l}\text { Min } \\
73.8393 \\
73.7731 \\
87.9979 \\
87.2094 \\
87.5737 \\
87.3676 \\
80.1592 \\
87.7516 \\
87.5631 \\
87.6515 \\
88.1198 \\
87.4741 \\
88.1019 \\
87.9491 \\
88.1183 \\
87.5251 \\
88.3712 \\
87.5870 \\
74.6633 \\
87.4596 \\
87.7790 \\
87.8348 \\
87.7629 \\
87.5328 \\
80.0371 \\
86.0251 \\
89.1832 \\
87.9900 \\
87.8699 \\
87.7927 \\
39.1464 \\
86.2364 \\
89.1361 \\
80.0914 \\
74.2594 \\
87.9502 \\
87.7422 \\
87.7750 \\
89.1649 \\
89.1001 \\
87.9722 \\
89.0733 \\
89.2420 \\
87.9383 \\
89.1382 \\
87.5638 \\
\end{array}$ & 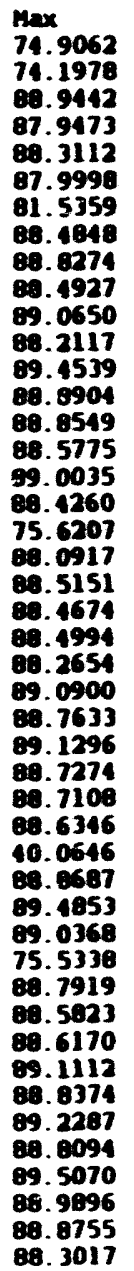 & 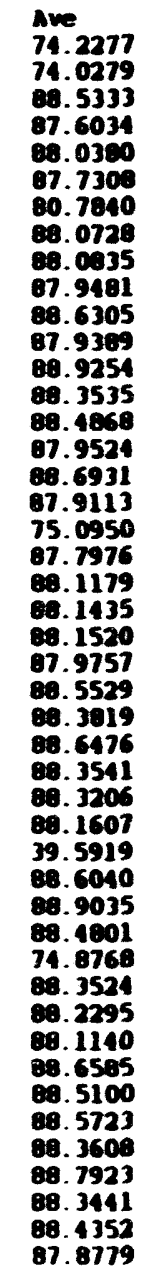 & 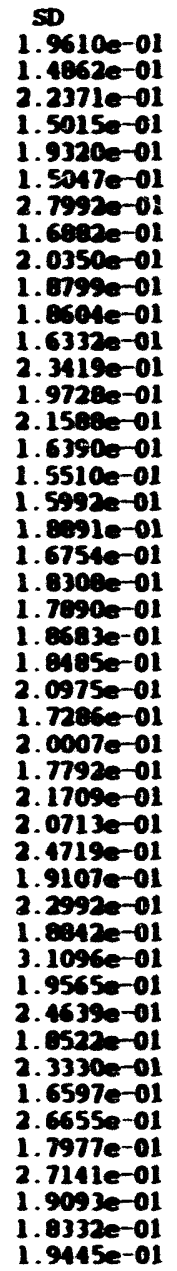 & $\begin{array}{l}\text { Uncert } \\
1.90000+00 \\
1.90000+00 \\
1.90020+00 \\
1.90020+00 \\
1.9002 e+00 \\
1.90020+00 \\
1.9001 e+00\end{array}$ \\
\hline
\end{tabular}

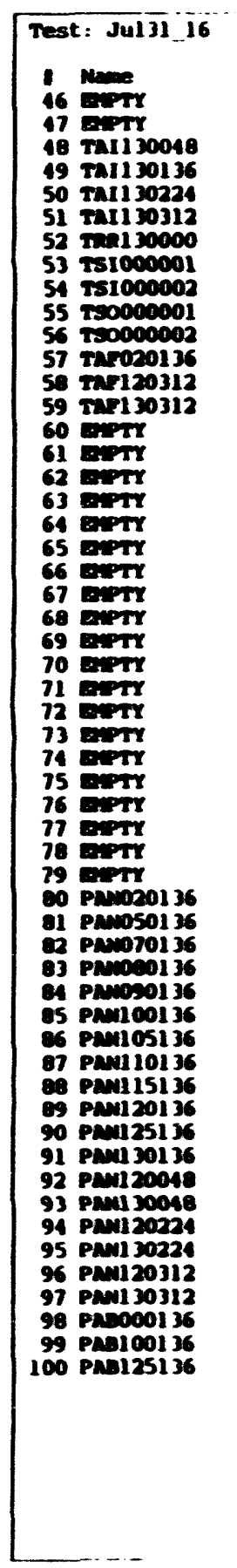

sal Date: Tue Jul 31 11:41:i7 $19 \% 0$ Paye:

Min 37.7675

39.1464

67.9332
87.1414

6.5905

a.

79.9715

75.7923

75.6132

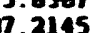

0.589

67.2927

0.5127

0.5371

0.5566

0. 3760

0. 3906

0.4102

0.1413

0.1590

0.4034

0.5176

0.5322
0.5518

0.5664

0.6104

0.6348

$-10.1235$

$-19.6349$

13.2690

$-6.8311$

$-7.3549$

-3.4574
-3.1727

-3.1727
-3.1816

$-3.18005$

$-3.2416$

38.4572

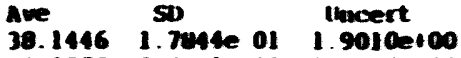
39.1970 9.6091e-02 1.90100100 207 1.9002e160 . 4402 con

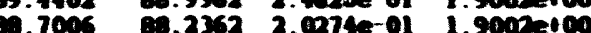

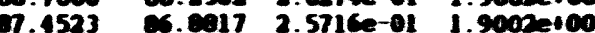
0.7137 c.3674 1.6237e-01 i.9001etce 76.050376 .30662 .7616 - 01 1 76.57117 .0369 1.7913e $01 \quad 1.9000=400$

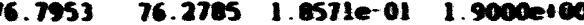
07.7568 1.0707e ol $1.90002+c 0$ c.5353 8.0163 2.cos3e ol 1.9002etco 07.7198 1.8526e ol $1.9002=100$ 0.51770 .5148 2.4404e 03 0.00000100

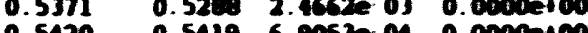

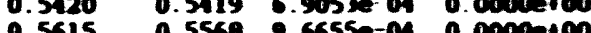
0.36130 .3553 i.cosse-04 0.00000000 0.3906 0.3764 2.1700-03 0.00000000 $0.39550 .39432 .1055 e-030.0000100$ $0.1150 \quad 0.41392 .1055 e 030.00000+00$ $0.42970 .42752 .44840 .03 \quad 0.00000+00$ $0.1492 \quad 0.4148$ 1.4797e $03 \quad 0.0000=100$ 0.16390 .4634 l.4797e $03 \quad 0.0000=100$ 0.48330 .1812 1.0002e $03 \quad 0.0000=100$ $0.52930 .49952 .2503 e-03 \quad 0.00000+00$ $0.5469 \quad 0.5372$ i.5566e-01 $0.0000=100$ $0.5615 \quad 0.55512 .50340-03 \quad 0.0000=+00$ $0.5713 \quad 0.5706$ 1.711se-03 $0.0000=100$ 0.62520 .651213750 .030 .00000100 $0.63 \%$ 0.6357 1.9730030 .00000000 $-9.0183-99467.25500 .7036-01$ -19.0245 - 19.4050 i. $211 \mathrm{e}-01$ 1.6807ec00

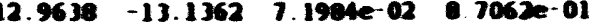
$-6.5259-6.6511 \quad 9.4716 e^{2} 0.7016 e 01$ $-6.2750-6.4306$ 6.7116e-02 a.7015e 01 $-7.0497-7.18407 .10167^{-02}$. 0.7015 - 01 $-3.1522-3.2926 \quad 6.9550002$ 8. 7004e ol -2.9430 - -3.0445 6.2497e.02 a 7003e ol -2.0754 - 3.0651 6.2362e.02 a.7003e ol $-2.9304-3.0355$ s. $-3.2432-3.3210$ s. $\begin{array}{llllll} & -7.1422 & -7.3054 & 7.0551 e-02 & 0.7019 & 01\end{array}$ $-6.2530-6.0241-6.11267 .7621$ e 02 e.7013e ol $-6.1100-6.1811-6.2742$ 7.607e 02 . 70140 o1 $\begin{array}{llll}-6.5625 & 6.2574-6.4115 & 7.3097 e 02 & 0.7015 \text { ol }\end{array}$ $-6.1892-6.2603-6.3946$. 9091e 02 0.7015 ol $22.248722 .3860 \quad 22.3118 \quad 30006025.0260$ ol

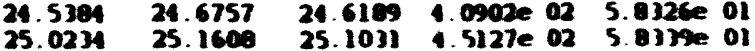




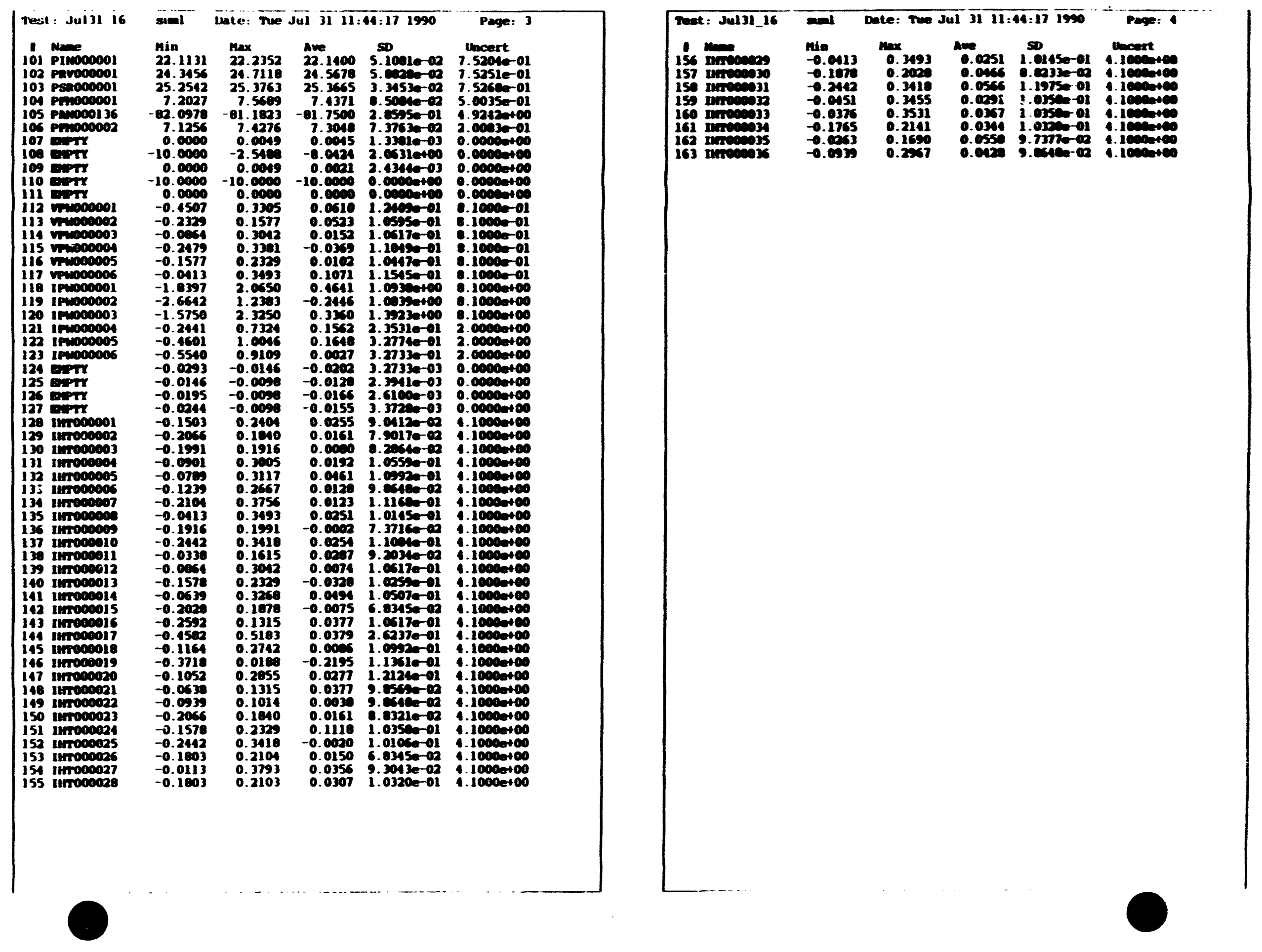




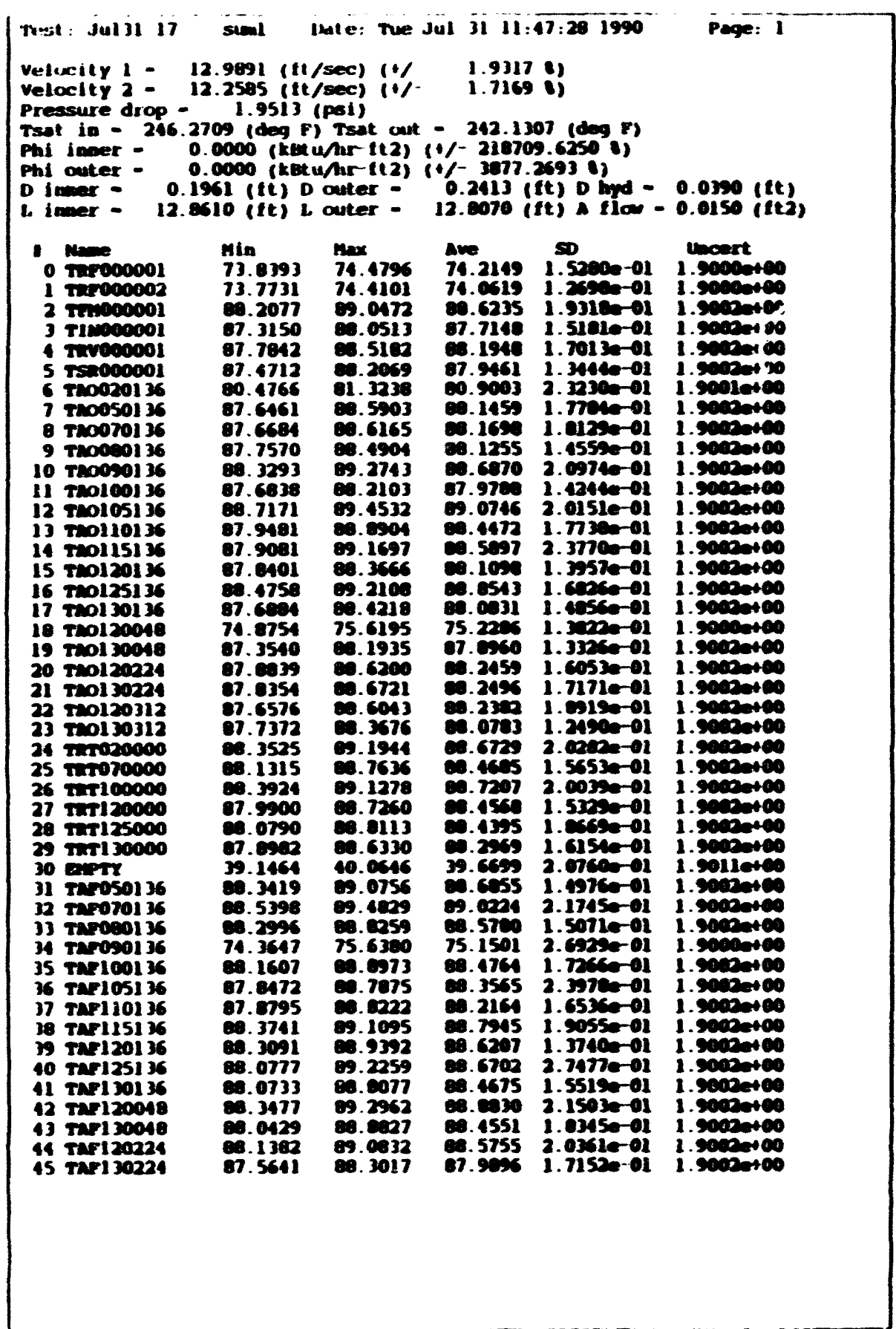

\begin{tabular}{|c|c|c|c|c|c|}
\hline 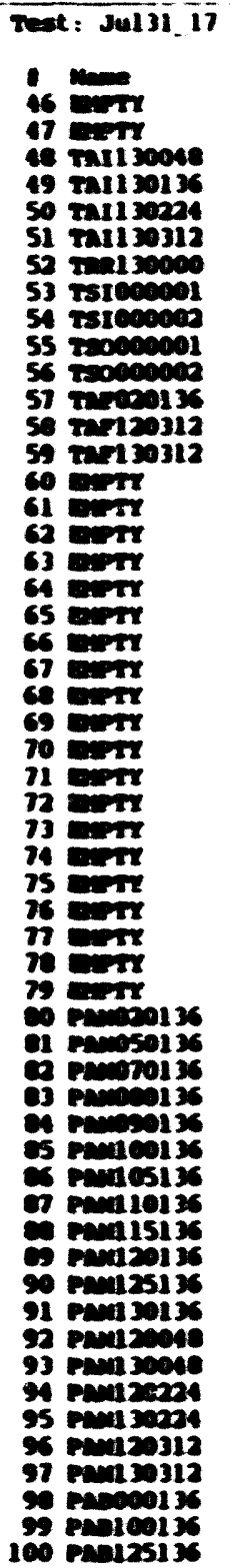 & 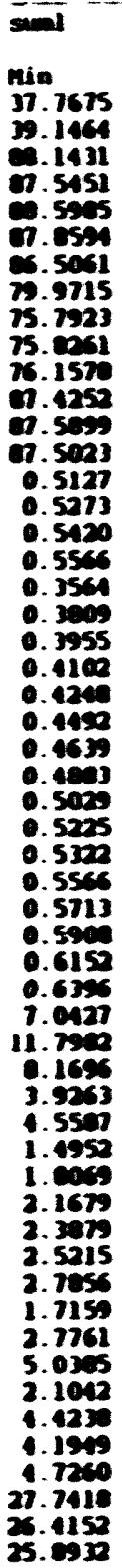 & 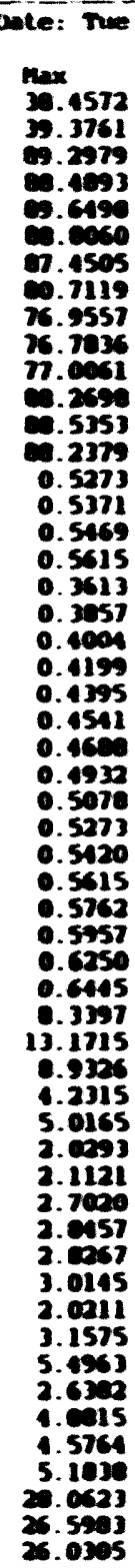 & 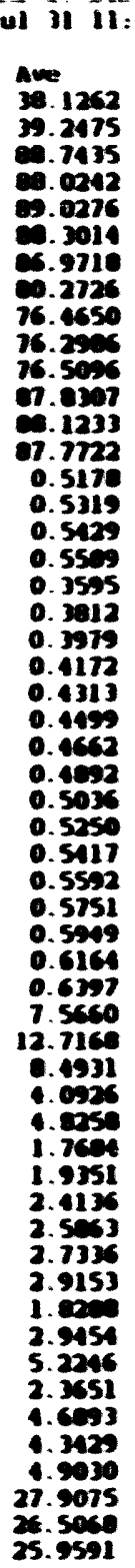 & 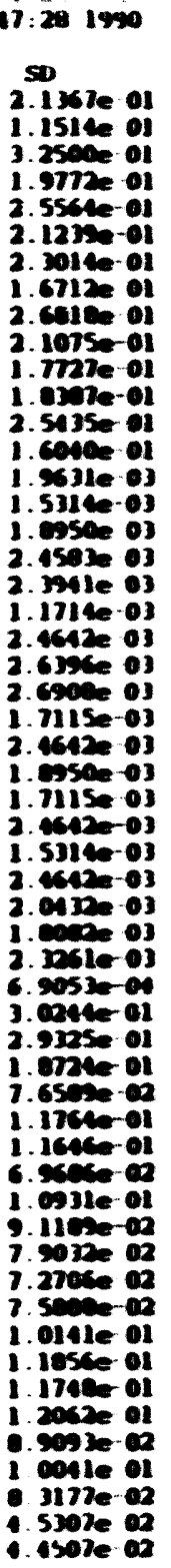 & 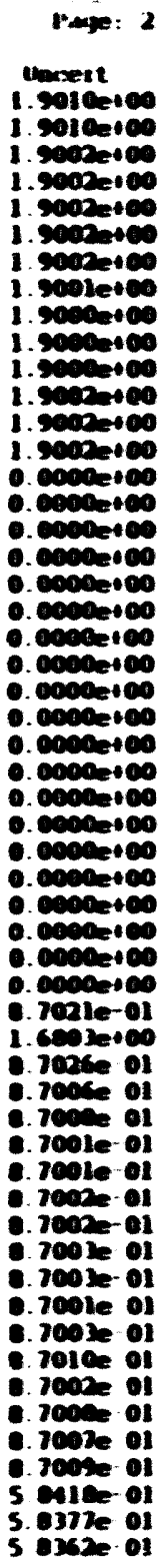 \\
\hline
\end{tabular}




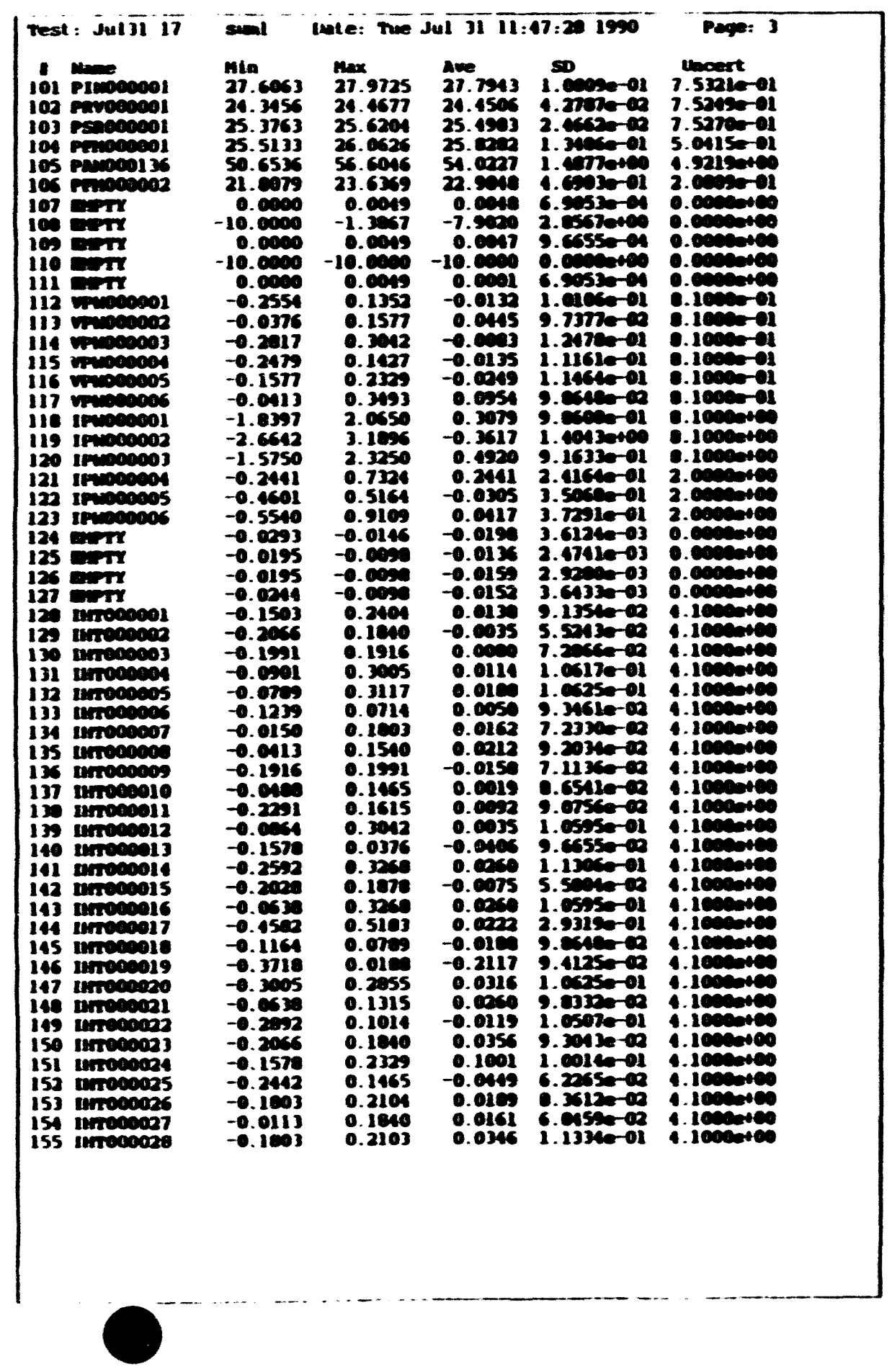

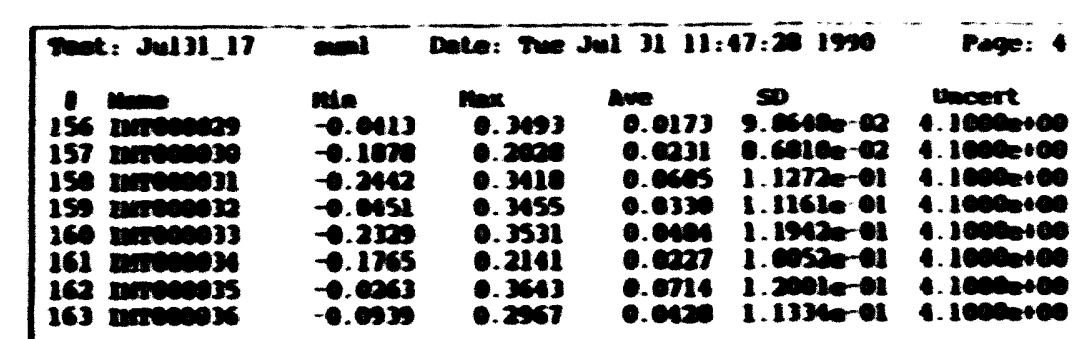




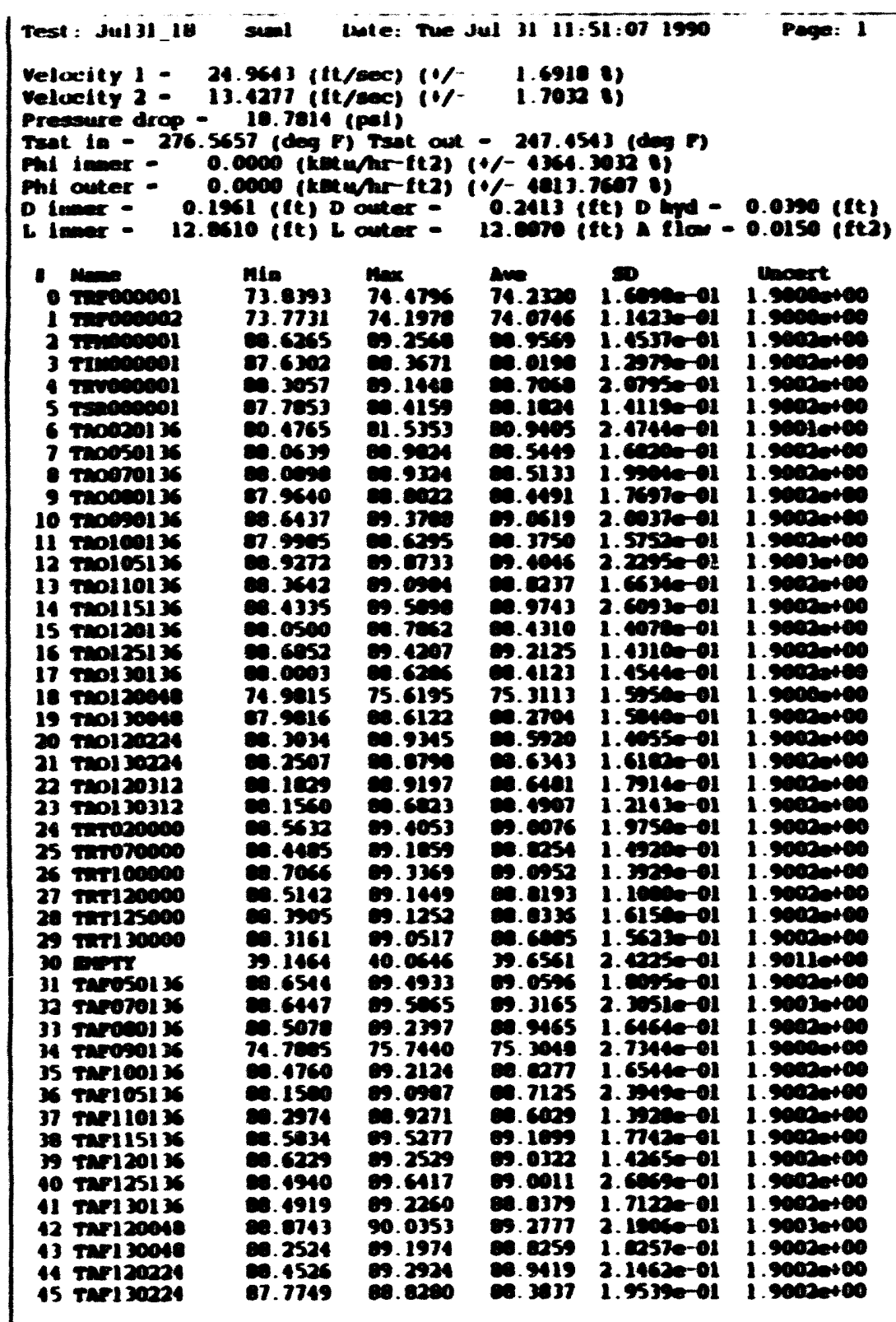

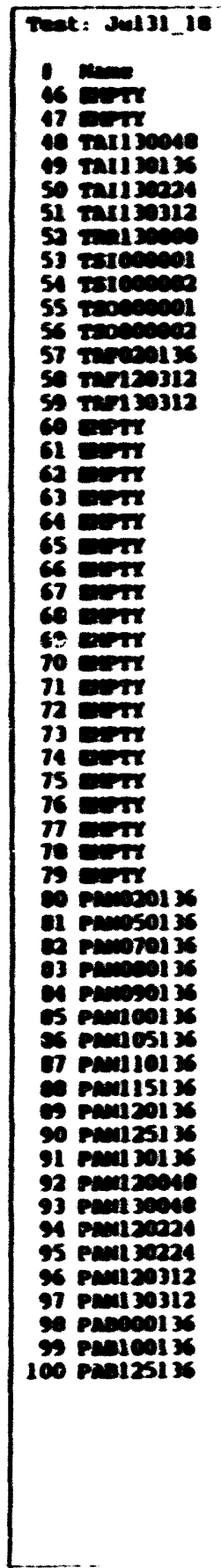

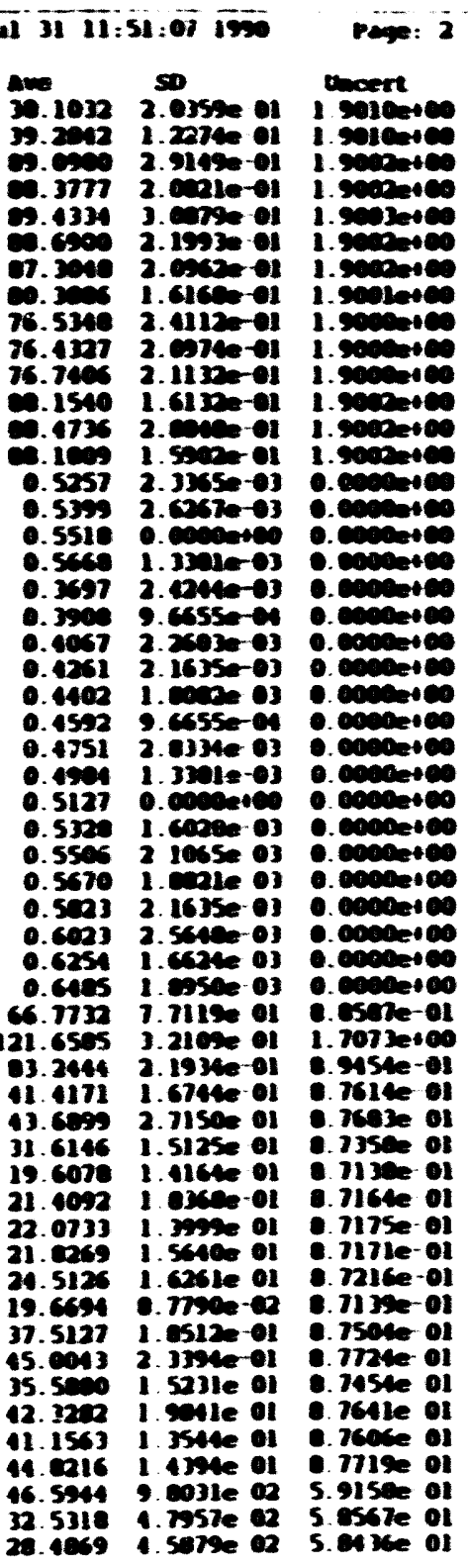




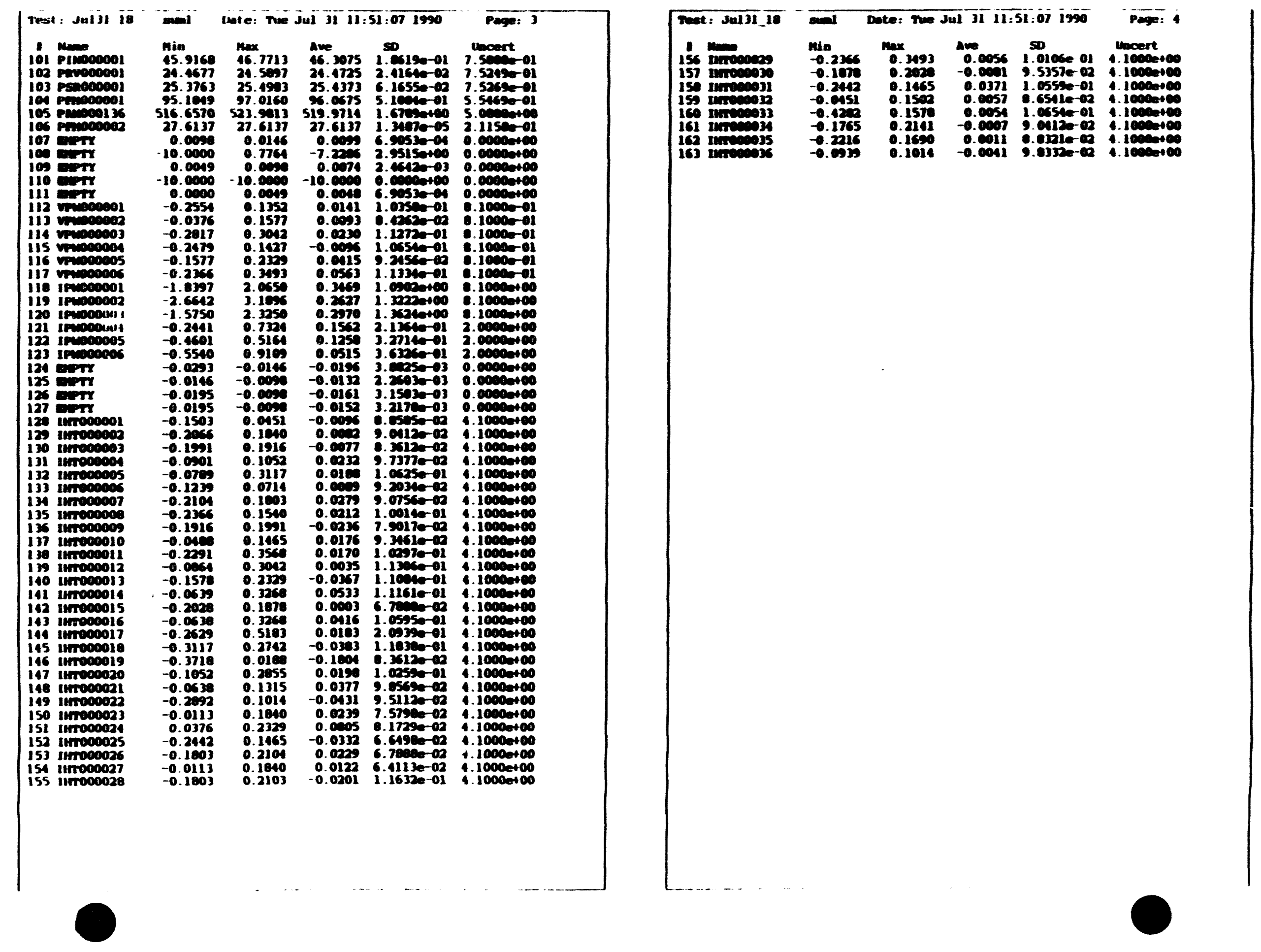




\begin{tabular}{|c|c|c|c|c|c|}
\hline \multicolumn{6}{|c|}{ 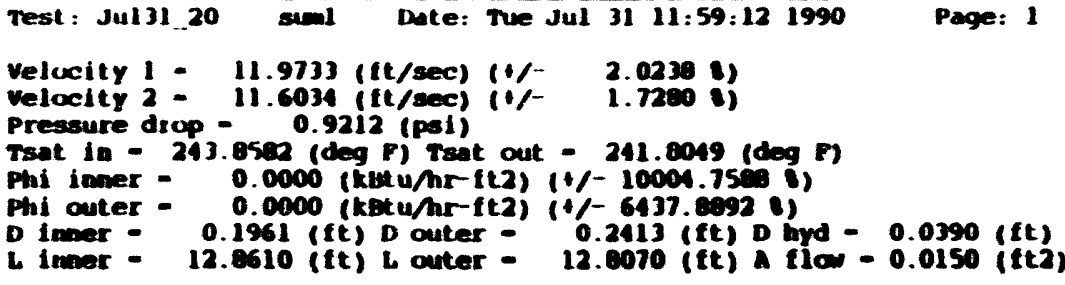 } \\
\hline 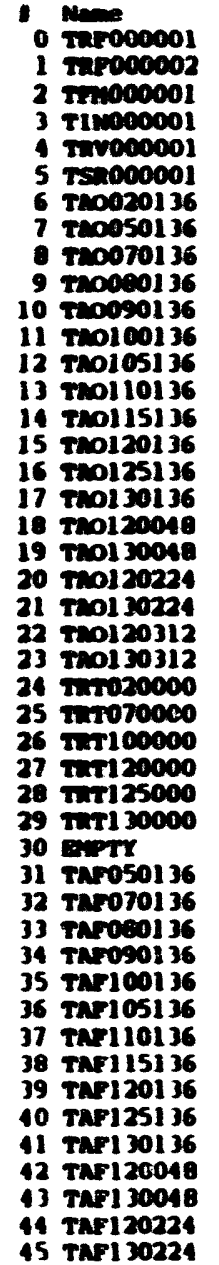 & 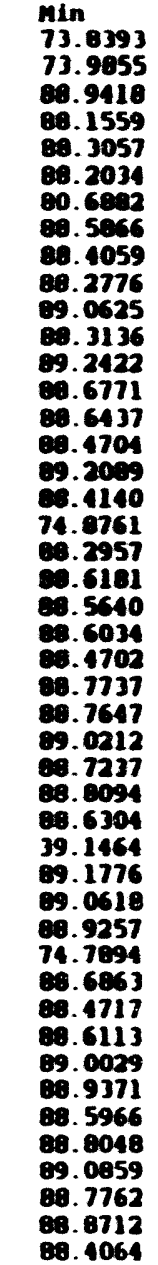 & $\begin{array}{l}\max \\
74.6929 \\
74.1970 \\
89.6772 \\
89.7875 \\
89.1448 \\
80.9377 \\
81.8529 \\
89.2165 \\
89.3539 \\
89.2216 \\
89.6929 \\
69.0405 \\
90.2933 \\
89.5141 \\
89.8005 \\
89.1015 \\
89.8392 \\
89.0421 \\
75.5141 \\
60.9261 \\
89.3544 \\
89.2953\end{array}$ & 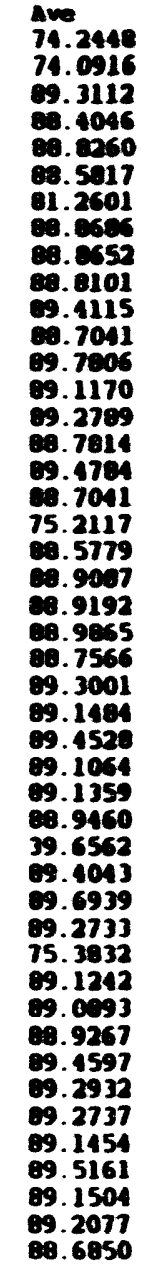 & 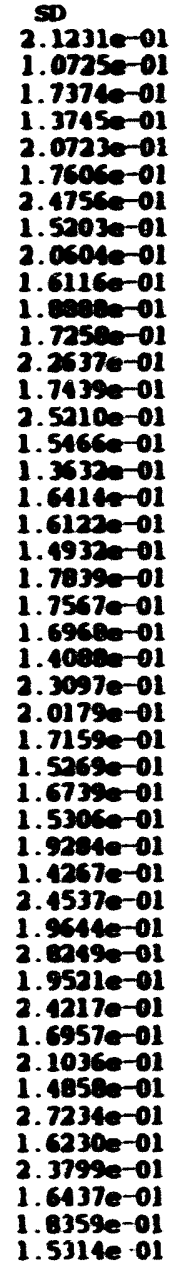 & \\
\hline
\end{tabular}

\begin{tabular}{|c|c|c|c|c|c|}
\hline $\begin{array}{l}x \\
x \\
x \\
30048 \\
31136 \\
30224 \\
30312 \\
30000 \\
00001 \\
00002 \\
00001 \\
00002 \\
20136 \\
20312\end{array}$ & 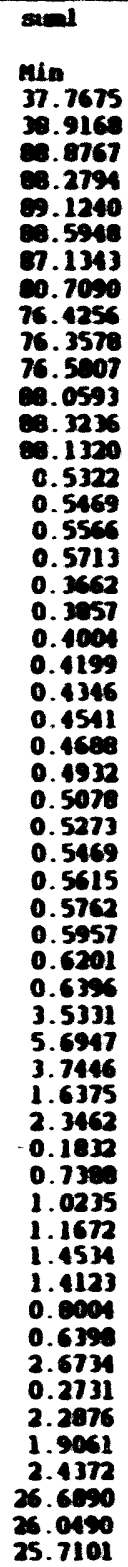 & 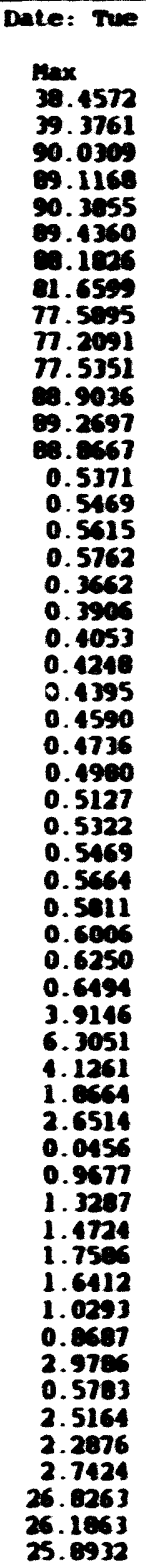 & 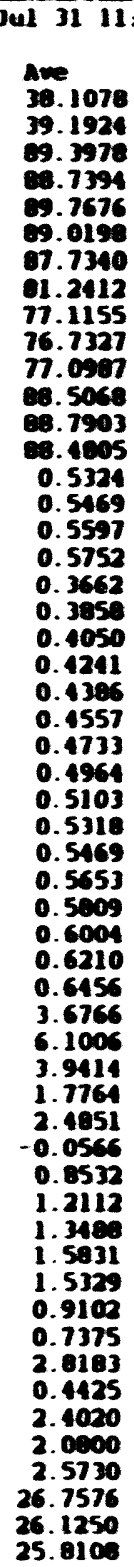 & 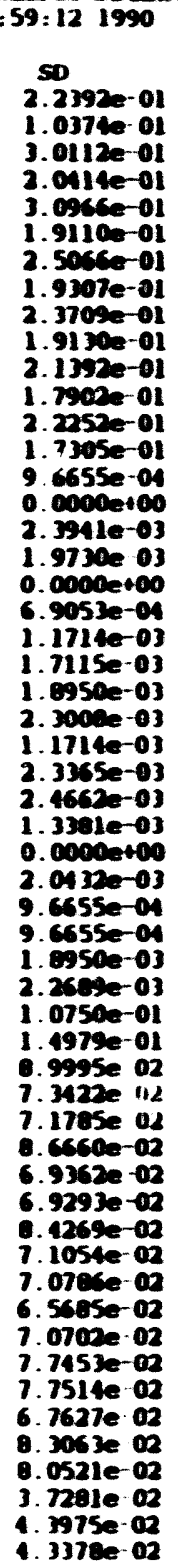 & 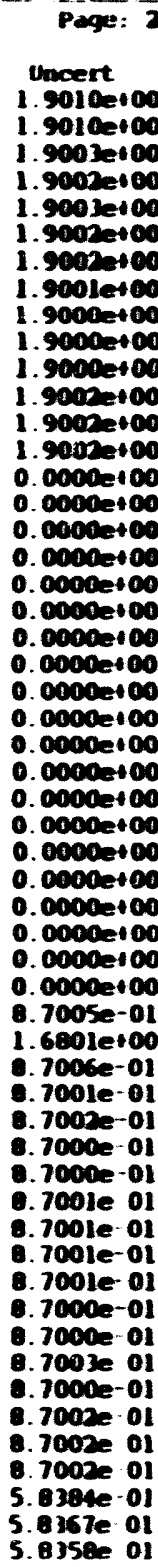 \\
\hline
\end{tabular}




\begin{tabular}{|c|c|c|c|c|c|c|}
\hline \multicolumn{2}{|c|}{ IYest : JuI31 20} & sienl & nat & ul & & $e: 3$ \\
\hline 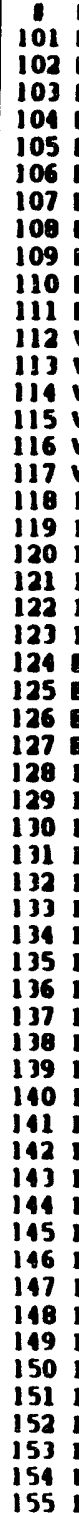 & 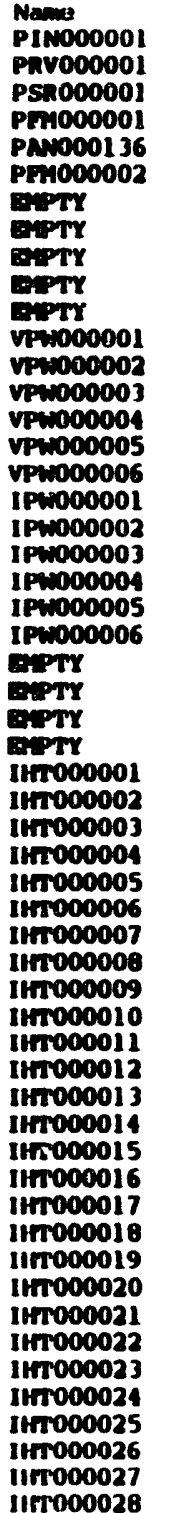 & $\begin{array}{r}\text { Min } \\
26.5077 \\
24.4677 \\
25.3763 \\
21.5765 \\
24.1033 \\
19.8279 \\
0.0049 \\
-10.0000 \\
0.0000 \\
-10.0000 \\
0.0000 \\
-0.0601 \\
-0.0376 \\
-0.2017 \\
-0.2479 \\
-0.1577 \\
-0.0413 \\
-1.8397 \\
-2.6642 \\
-1.5750 \\
-0.2441 \\
-0.4601 \\
-0.5540 \\
-0.0244 \\
-0.0195 \\
-0.0244 \\
-0.0244 \\
-0.1503 \\
-0.2066 \\
-0.1991 \\
-0.0901 \\
-0.0789 \\
-0.1239 \\
-0.2104 \\
-0.0413 \\
-0.1916 \\
-0.0488 \\
-0.0338 \\
-0.0864 \\
-0.1578 \\
-0.2592 \\
-0.2028 \\
-0.0638 \\
-0.4582 \\
-0.3117 \\
-0.3718 \\
-0.1052 \\
-0.0638 \\
-0.2892 \\
-0.0113 \\
-0.1578 \\
-0.2442 \\
-0.1803 \\
-0.0113 \\
-0.1803\end{array}$ & $\begin{array}{r}\text { Max } \\
26.6297 \\
24.5897 \\
25.4983 \\
22.3089 \\
26.8499 \\
21.1367 \\
0.0049 \\
-0.5957 \\
0.0049 \\
-10.0000 \\
0.0000 \\
0.1352 \\
0.1577 \\
0.1089 \\
0.1427 \\
0.2329 \\
0.3493 \\
4.0173 \\
3.1896 \\
2.3250 \\
0.7324 \\
0.5164 \\
0.9109 \\
-0.0146 \\
-0.0096 \\
-0.0098 \\
-0.0098 \\
0.2404 \\
0.1840 \\
0.5822 \\
0.3005 \\
0.3117 \\
0.2667 \\
0.1803 \\
0.3493 \\
0.1991 \\
0.1465 \\
0.1615 \\
0.3042 \\
0.2329 \\
0.1314 \\
0.1878 \\
0.3268 \\
0.5183 \\
0.2742 \\
0.0188 \\
0.2855 \\
0.3268 \\
0.1014 \\
0.1840 \\
0.2329 \\
0.1465 \\
0.0150 \\
0.1840 \\
0.2103\end{array}$ & $\begin{array}{r}\text { Ave } \\
26.5419 \\
24.4701 \\
25.4812 \\
21.9171 \\
25.5041 \\
20.5736 \\
0.0049 \\
-6.6372 \\
0.0016 \\
-10.0000 \\
0.0000 \\
0.0219 \\
0.0405 \\
0.0112 \\
-0.0369 \\
0.0337 \\
0.0759 \\
0.4250 \\
0.1066 \\
0.4530 \\
0.1758 \\
0.0965 \\
0.0027 \\
-0.0193 \\
-0.0133 \\
-0.0161 \\
-0.00161 \\
0.0060 \\
-0.0074 \\
0.0119 \\
0.0075 \\
0.0383 \\
-0.0146 \\
0.0006 \\
0.0173 \\
0.0077 \\
0.0176 \\
0.0287 \\
0.0230 \\
-0.0288 \\
0.0533 \\
0.0042 \\
0.0299 \\
0.0066 \\
-0.0227 \\
-0.2351 \\
0.0198 \\
0.0221 \\
-0.0392 \\
0.0395 \\
0.0922 \\
-0.0110 \\
0.0072 \\
0.0278 \\
-0.0123\end{array}$ & 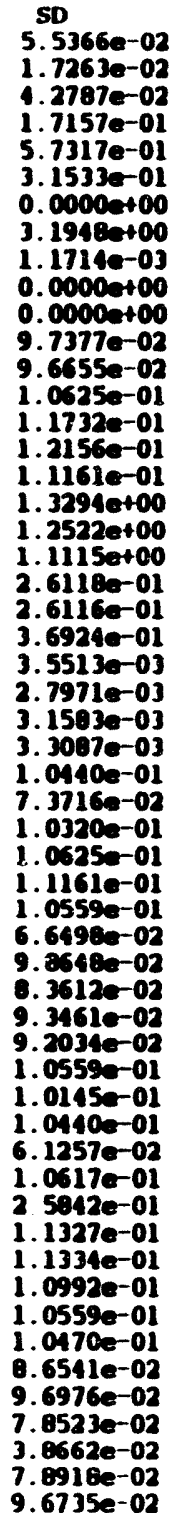 & 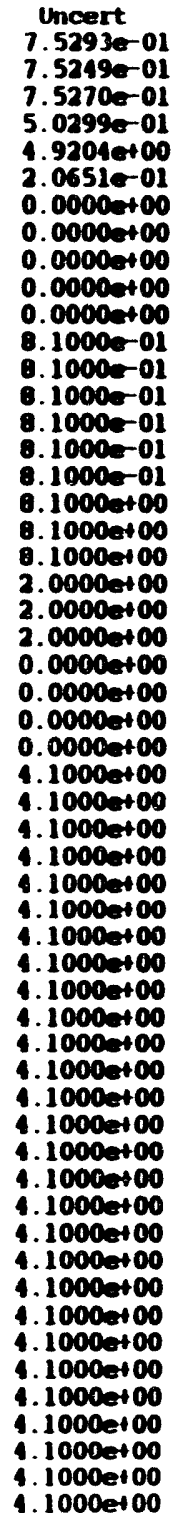 \\
\hline
\end{tabular}

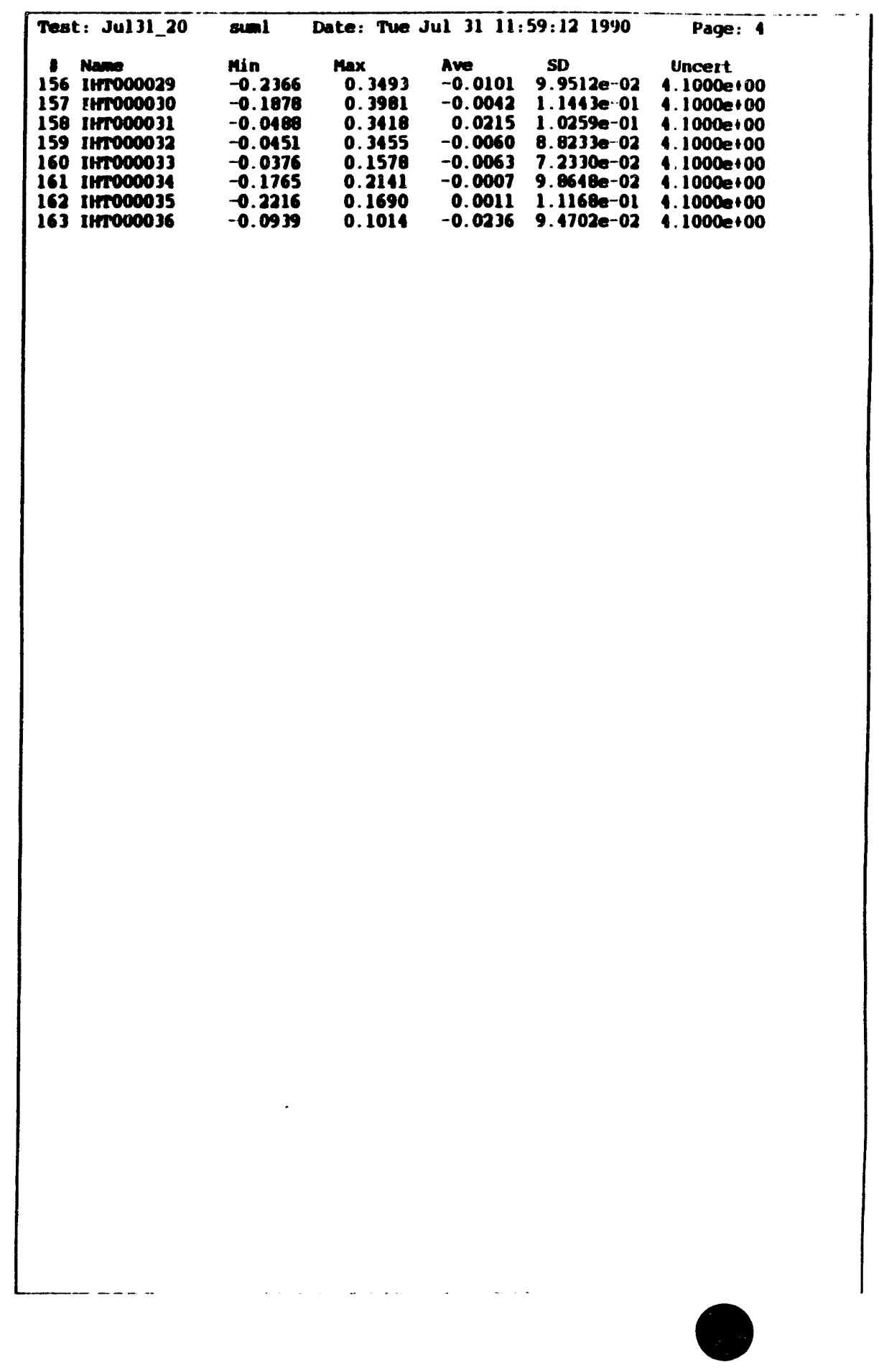




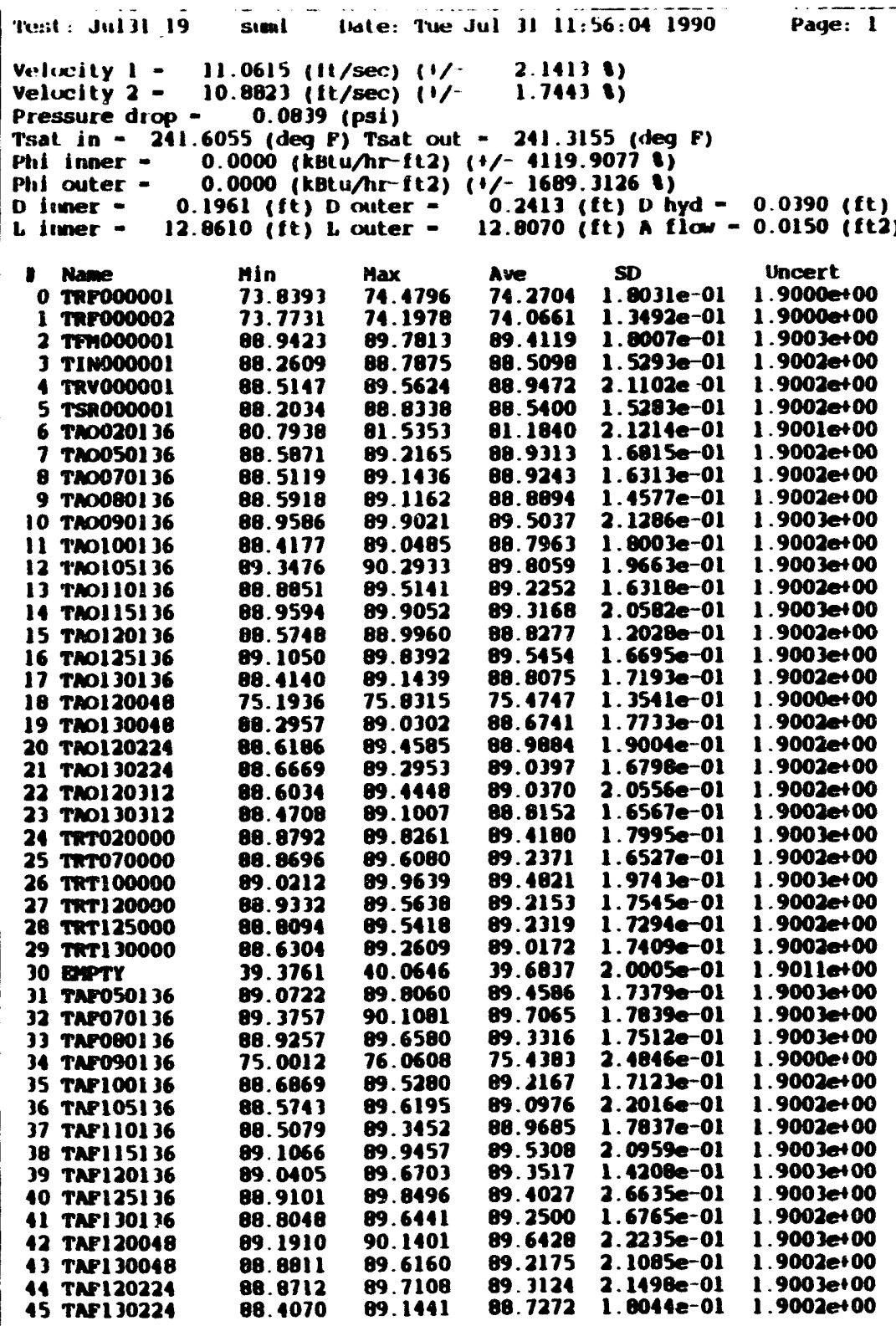

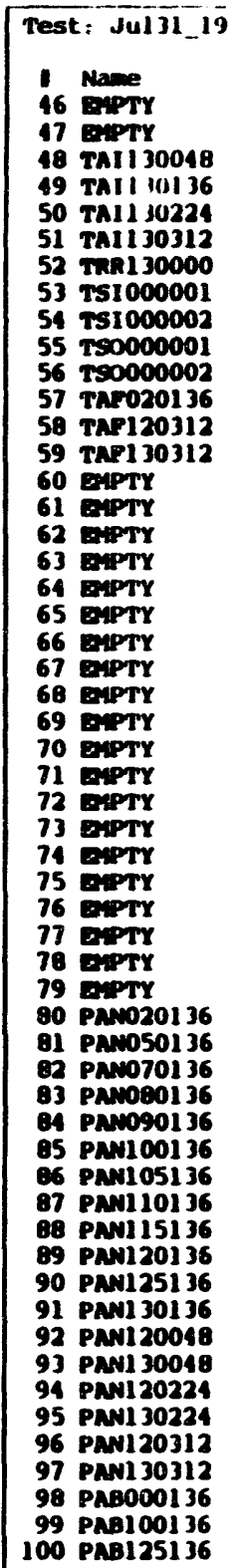

sual vate: Tre Jui 31 il:56:04 1990 packe: 2 Min Max Ave SD Ansert $\begin{array}{lll}\text { s.0672e } 01 & 1.9010 e 100\end{array}$ $39.1464 \quad 39.6057 \quad 39.2705$ 1.4079e ol $1.9010 e 100$

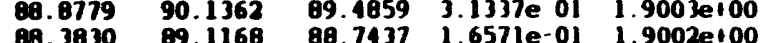
$\begin{array}{lllll}80.3830 & 89.1168 & 88.7437 & 1.6571 \mathrm{e}-01 & 1.9002 e 100\end{array}$

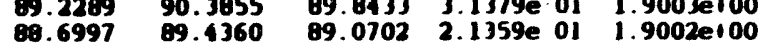
$07.3437 \quad 09.2876 \quad 87.7006$ 1.9420e-01 $1.9002 e+00$ $\begin{array}{llllll}80.7095 & 81.3434 & 81.0266 & 1.4940 e-01 & 1.9001 e 100\end{array}$ $\begin{array}{llllll} & \end{array}$ $\begin{array}{llllll}76.2513 & 76.9964 & 76.6434 & 2.0658 \mathrm{e} \cdot 01 & 1.9000 \mathrm{e} 00\end{array}$ $86.1652 \quad 89.0090 \quad 88.5914$ 1.7821e-01 1.9002 et00 $\begin{array}{lllll}80.1291 & 89.3738 & 88.9455 & 2.7778 e .01 & 1.9002 e 100\end{array}$ $\begin{array}{llllll}88.2365 & 80.8667 & 88.5266 & 1.3346 e-01 & 1.9002 e 100\end{array}$ $\begin{array}{llllll}0.5322 & 0.5371 & 0.5337 & 2.2603 \text { e } 03 & 0.0000100\end{array}$ $0.5469 \quad 0.5518 \quad 0.5473 \quad 1.3381 e^{-03} 0.0000100$ $\begin{array}{lllll}0.5566 & 0.5664 & 0.5605 & 2.2058 e-03 & 0.0000100\end{array}$ $\begin{array}{llllll}0.5713 & 0.5762 & 0.5758 & 1.3381 \text { e.03 } & 0.0000 \text { e } 00\end{array}$ $\begin{array}{llllll}0.3613 & 0.3662 & 0.3660 & 9.6655 \text { e } 04 & 0.0000 & 00\end{array}$ $\begin{array}{lllll}0.3809 & 0.3906 & 0.3860 & 1.5314 \text { e } 03 & 0.0000100\end{array}$ $\begin{array}{llllll}0.4004 & 0.4053 & 0.4045 & 1.8082 e & 03 & 0.0000 e 100\end{array}$ 0.41990 .42480 .42272 .4484 ( 0.430 .0000100 $0.45410 .4590 \quad 0.4544$ 1.1714e 030.0000100

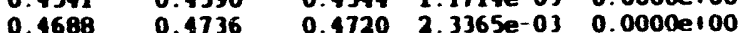
$\begin{array}{lllll}0.4688 & 0.4736 & 0.4720 & 2.3365 e-03 & 0.0000 \\ 0.4932 & 0.4980 & 0.4939 & 1.6082 \mathrm{e} .03 & 0.0000100\end{array}$ $\begin{array}{llllll}0.4932 & 0.4980 & 0.4939 & 1.6028 \mathrm{e} & 03 & 0.0000100 \\ 0.5078 & 0.5127 & 0.5084 & 1.6028 \mathrm{-03} & 0.0000100\end{array}$ $\begin{array}{llllll}0.5273 & 0.5322 & 0.5285 & 2.1065 e & 03 & 0.0000 \text { e } 100\end{array}$ $\begin{array}{llllll}0.5420 & 0.5469 & 0.5160 & 1.6950 \mathrm{e}-03 & 0.0000 \mathrm{e} & 00\end{array}$

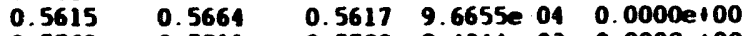
$\begin{array}{lllll}0.5762 & 0.5811 & 0.5782 & 2.4344 e-03 & 0.0000 \text { t00 }\end{array}$ $\begin{array}{llllll}0.5957 & 0.6006 & 0.5980 & 2.4642 e .03 & 0.0000 e 100\end{array}$ $\begin{array}{lllll}0.6201 & 0.6201 & 0.6201 & 0.00002100 & 0.0000 \mathrm{l} 100\end{array}$ $\begin{array}{lllll}0.6396 & 0.6494 & 0.6445 & 9.8648 e^{-04} 0.0000400\end{array}$ $0.4814 \quad 0.9392 \quad 0.6828$ 1.076 3e-01 8.7000 - 01

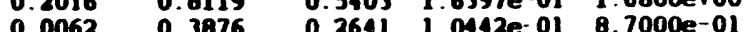
$\begin{array}{lllll}0.00635 & 0.1879 & -0.0318 & 9.9463 e^{-02} & 8.7000 e^{01}\end{array}$ $\begin{array}{llllllll}0.3626 & 0.6677 & 0.4984 & 7.2772 \mathrm{e} .02 & 8.7000 \mathrm{e} & 01\end{array}$ $-1.7854-1.1039-1.5733,7.9032 e-02$ B.7001e ol $\begin{array}{llllll}-0.2530 & 0.0522 & -0.0577 & 7.4178 e^{-02} & \text { B.7000e } 01\end{array}$ $\begin{array}{lllllll}0.1000 & 0.4131 & 0.2621 & 6.6279 e-02 & 8.7000 e & 01\end{array}$ $\begin{array}{llllll}0.0991 & 0.4806 & 0.2868 & 8.4719 e-02 & 8.7000 e & 01\end{array}$ $\begin{array}{llllll}0.6142 & 0.6430 & 0.7240 & 6.1962 \mathrm{e}-02 & 8.7000 \mathrm{e}-01\end{array}$ $\begin{array}{llllll}0.3442 & 0.6494 & 0.5334 & 5.3926 e^{-02} & 8.7000 & 01\end{array}$ $\begin{array}{llllll}-0.0389 & 0.1900 & 0.0847 & 5.3149 e-02 & 0.7000 e & 01\end{array}$ $\begin{array}{llllll}1.1149 & -0.6572 & 0.9440 & 0.7953 \text { e-02 } & \text { 8.7000e-01 }\end{array}$ $\begin{array}{llllll}0.6134 & 0.9186 & 0.7706 & 8.7750 e-02 & 8.7000 e & 01\end{array}$ $0.3802 \quad 0.6091 \quad 0.4824 \quad 6.0699 e-02 \quad 8.7000 e-01$ $\begin{array}{lllll}0.0750 & 0.3039 & 0.2383 & 6.5413 e^{-02} & 8.7000 e-01\end{array}$ $0.3773 \quad 0.7588 \quad 0.5711$ 8.4719e 02 8.7000e 01 $\begin{array}{llllll}25.6361 & 25.7734 & 25.7203 & 3.6245 e^{-02} & 5.8355 \text { e } 01\end{array}$

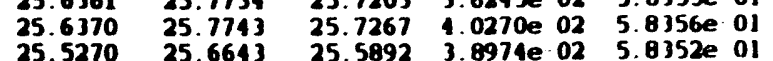




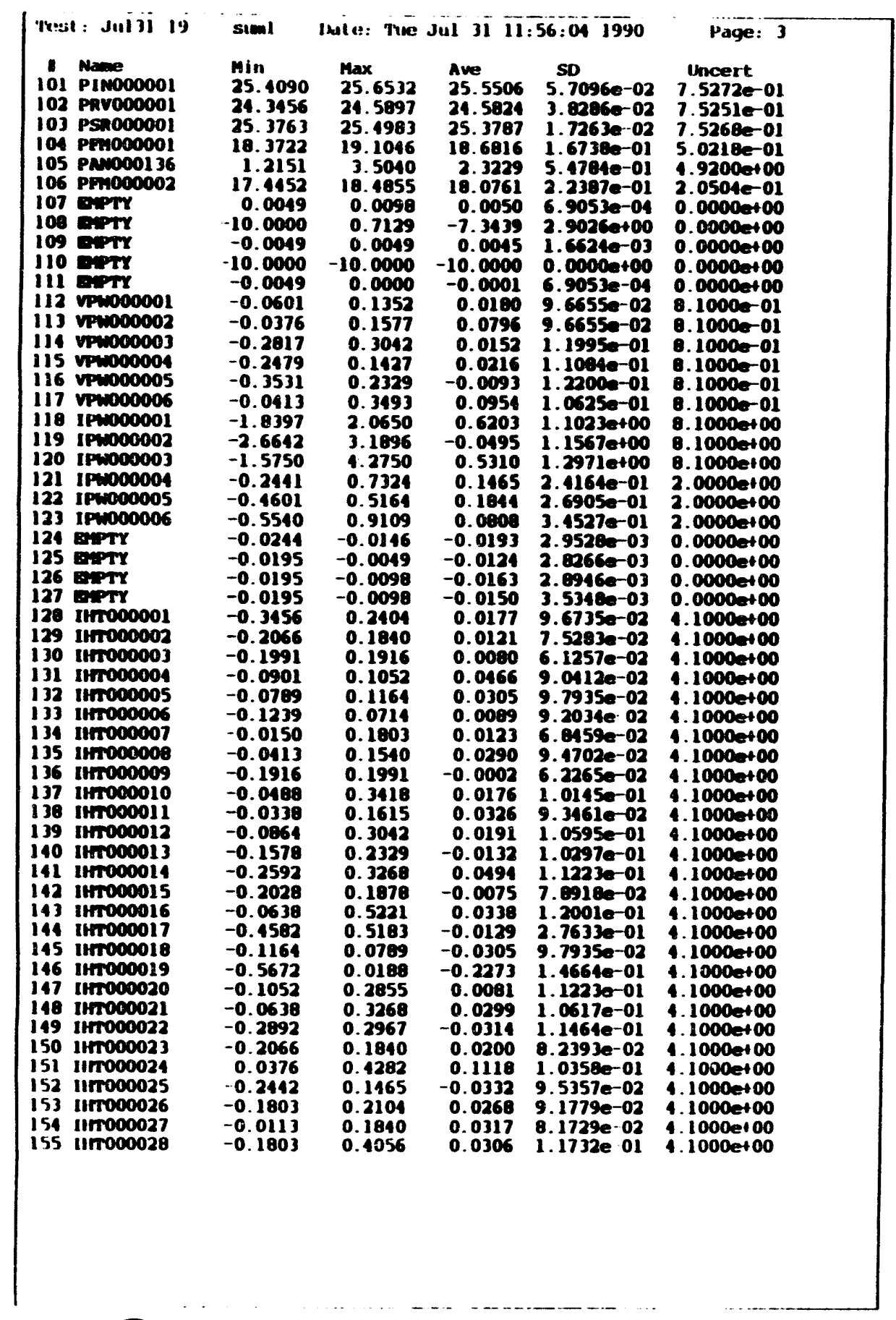

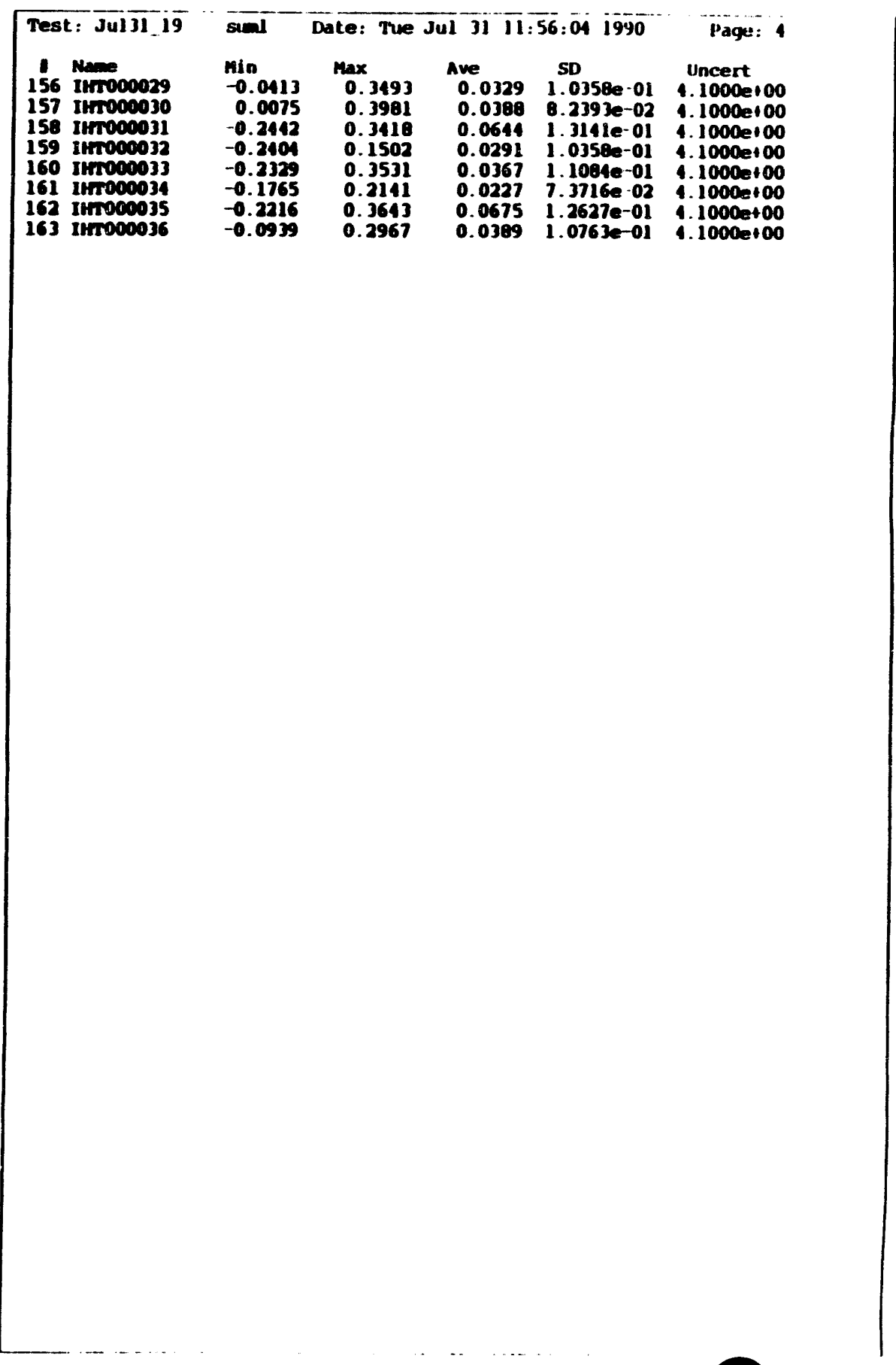




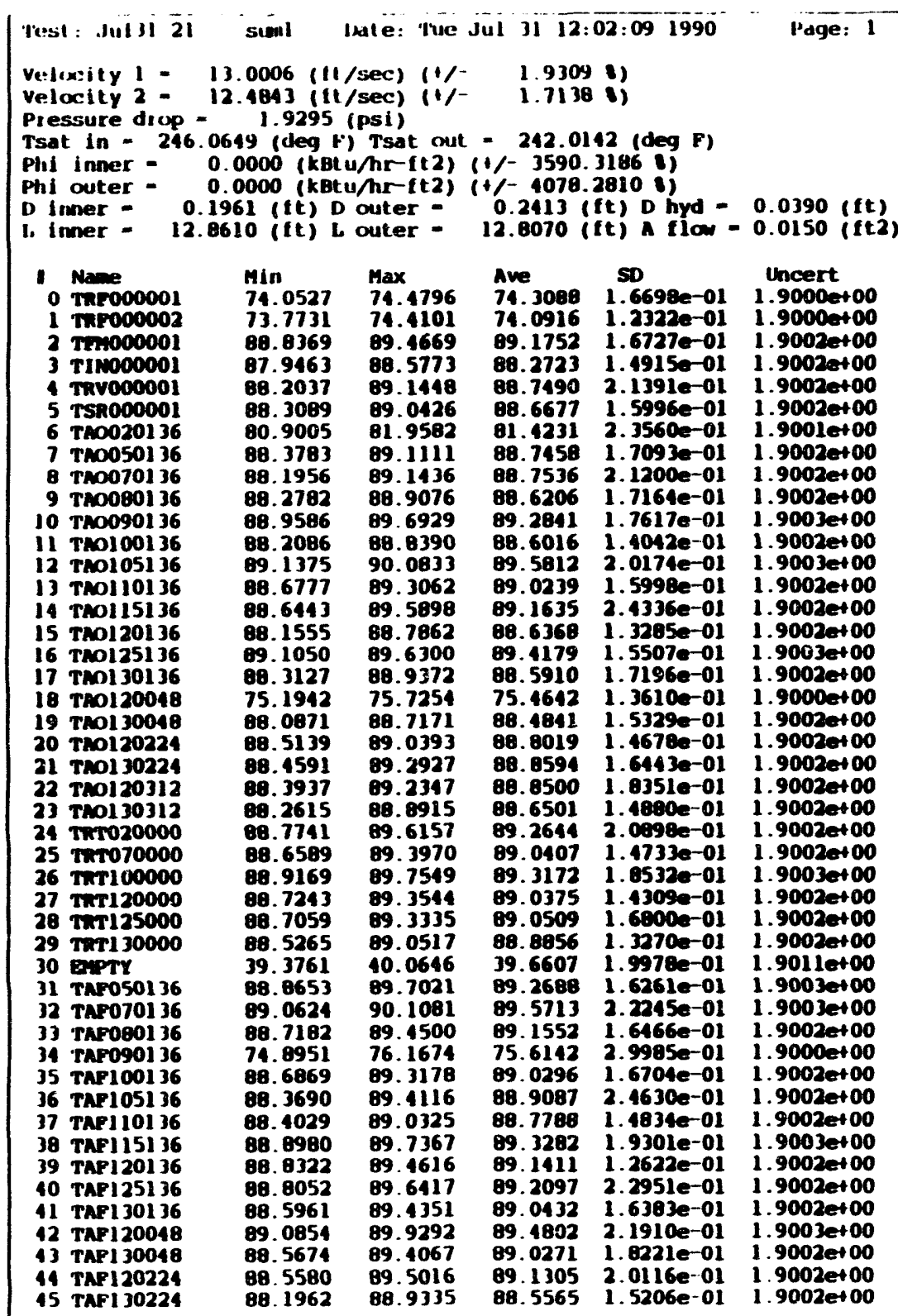

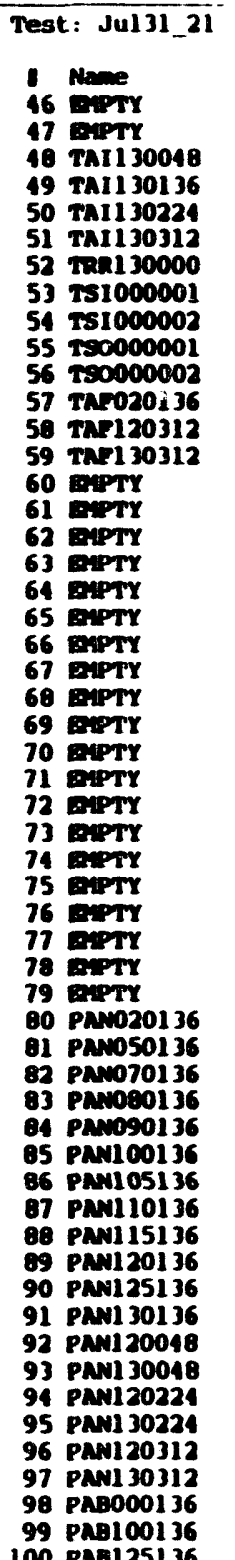

suml Date: Tue Jul 3i 12:02:09 1990 Pagk: 2

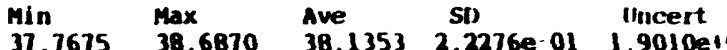
$38.9168 \quad 39.3761 \quad 39.2062 \quad 1.2107 e-01 \quad 1.9010 e+00$ $\begin{array}{llllll}08.7725 & 09.9267 & 09.3414 & 2.4437 e-01 & 1.9003 e 100\end{array}$ $\begin{array}{lllll}88.0689 & 89.0119 & 88.6080 & 2.1061 \text { e-01 } & 1.9002 e 100\end{array}$ $\begin{array}{lllll}09.1246 & 90.0701 \quad 89.5596 & 2.4672 \mathrm{e}-01 & 1.9003 \mathrm{e} 00\end{array}$ $\begin{array}{lllll}8.4902 & 89.2260 \quad 88.8750 & 1.9526 e-01 & 1.9002 e+00\end{array}$ $\begin{array}{lllll}87.1343 & 89.0784 & 87.6527 & 2.2752 e-01 & 1.9002 e+100\end{array}$ $\begin{array}{llllll}81.0256 & 81.6587 & 81.3825 & 1.59396-01 & 1.9001 e+00\end{array}$ 76.358777 .8005 77.2573 2.7436e-01 1.9000e+00 76.099177 .053277 .3975 1.9771e-01 1.9000et00 $09.0593 \quad 7978 \quad 09.4201 \quad 1.6930-01 \quad 1.90020100$ $\begin{array}{llllll}80.2193 & 89.1642 & 88.6668 & 2.8260 e-01 & 1.9002 e^{2}\end{array}$ $\begin{array}{llllll}87.9224 & 88.6573 & 80.3739 & 1.7135 e-01 & 1.9002 e+00\end{array}$ $\begin{array}{llllll}0.5273 & 0.5322 & 0.5287 & 2.2146 e-03 & 0.0000 \text { et00 }\end{array}$ $0.5420 \quad 0.5420 \quad 0.54200 .0000+00 \quad 0.0000$ et00 $\begin{array}{llllll}0.5518 & 0.5615 & 0.5559 & 2.0598 e-03 & 0.0000 \text { et } 00\end{array}$ $\begin{array}{lllll}0.5664 & 0.5713 & 0.5710 & 1.1714 e-03 & 0.0000 e+00\end{array}$ $\begin{array}{lllll}0.3564 & 0.3662 & 0.3657 & 1.7784 e^{-03} & 0.0000 \text { et00 }\end{array}$ $\begin{array}{llllll}0.3857 & 0.3906 & 0.3860 & 1.1714 e^{-03} & 0.0000400\end{array}$ $\begin{array}{lllll}0.4004 & 0.4053 & 0.4049 & 1.3381 e-03 & 0.0000 e^{00} \\ 0.4199 & 0.4248 & 0.4245 & 1.17140-03 & 0.00000100\end{array}$ $0.4960 .42400 .4251 .1714 e-030.0000$ $0.4346 \quad 0.443 \quad 0.4388$ 1.9754e 030.0000100 $0.46880 .47360 .47356 .90530-040.0000400$ $0.49320 .4980 \quad 0.49721 .8950 e-030.0000$ et 00 $\begin{array}{llllll}0.5078 & 0.5127 & 0.5124 & 1.1714 e-03 & 0.0000 e+00\end{array}$ $\begin{array}{lllll}0.5322 & 0.5322 & 0.5322 & 0.0000 e+00 & 0.0000 e+00\end{array}$ $\begin{array}{llllll}0.5469 & 0.5510 & 0.5472 & 1.1714 e-03 & 0.0000+00\end{array}$ $0.5615 \quad 0.57130 .56651 .2041 e^{-03} 0.0000 e^{00}$ $\begin{array}{llllll}0.5713 & 0.5859 & 0.5812 & 1.8429 e-03 & 0.0000 e+00\end{array}$ $\begin{array}{llllll}0.6006 & 0.6055 & 0.6009 & 1.1714 e-03 & 0.0000100\end{array}$ $\begin{array}{llllll}0.6201 & 0.6299 & 0.6250 & 9.8648 e-04 & 0.0000 \text { et } 00\end{array}$ $\begin{array}{llllll}0.6494 & 0.6494 & 0.6494 & 0.0000+00 & 0.0000 \text { et } 00\end{array}$ $\begin{array}{lllll}6.9664 & 0.1871 & 7.3967 & 2.5596 e^{-01} & 8.7020 e-01\end{array}$ $11.9508 \quad 13.1715 \quad 12.6008$ 2.6053e-01 $1.6803 e 100$ $\begin{array}{lllll}0.0933 & 8.7037 & 8.3604 & 1.5278 e^{-01} & 1.7025 e^{-01}\end{array}$ $\begin{array}{llll}.5587 & 5.1691 & 1.7815,2219 e^{-01} 8.70089-01\end{array}$ $1.4952 \quad 1.9530 \quad 1.7058 \quad 1.1175 e^{-01}$ B.7001e-01 $1.7306 \quad 2.0358 \quad 1.9107 \quad 7.6698 \mathrm{e}-02 \quad 8.7001 \mathrm{e}-01$ $\begin{array}{llllll}2.1679 & 2.5494 & 2.3663 & 0.0092 e-02 & 0.7002 e-01\end{array}$ $\begin{array}{llllll}2.3879 & 2.6931 & 2.5481 & 8.0462 e-02 & 8.7002 e & 01\end{array}$ $\begin{array}{lllllll}2.5215 & 2.9030 & 2.7184 & 0.0225 e & 02 & 8.7003 e-01\end{array}$ $\begin{array}{llllll}2.5567 & 2.8619 & 2.7185 & 7.6512 \mathrm{e}-02 & \mathbf{8} .7003 \mathrm{e} & 01\end{array}$

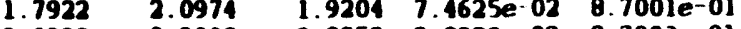
$\begin{array}{lllll}2.6998 & 3.2338 & 2.6859 & 9.8888 e-02 & 0.7003 e-01\end{array}$

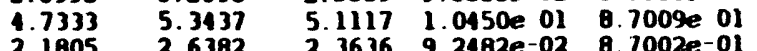
$2.1805 \quad 2.6382 \quad 2.3636 \quad 9.2482 e-02 \quad 8.7002 e-01$

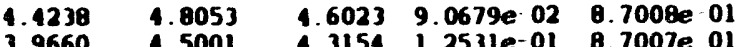

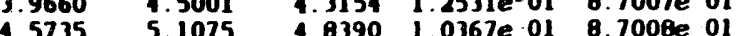
$27.604527 .970727 .8077 \quad 7.6911$ e 02 5415e-01 $26.3236 \quad 26.5525$ $\begin{array}{llllll}25.8016 & 25.9847 & 25.9060 & 4.3417 \mathrm{e} .02 & 5.8360 \mathrm{e} & 01\end{array}$ 


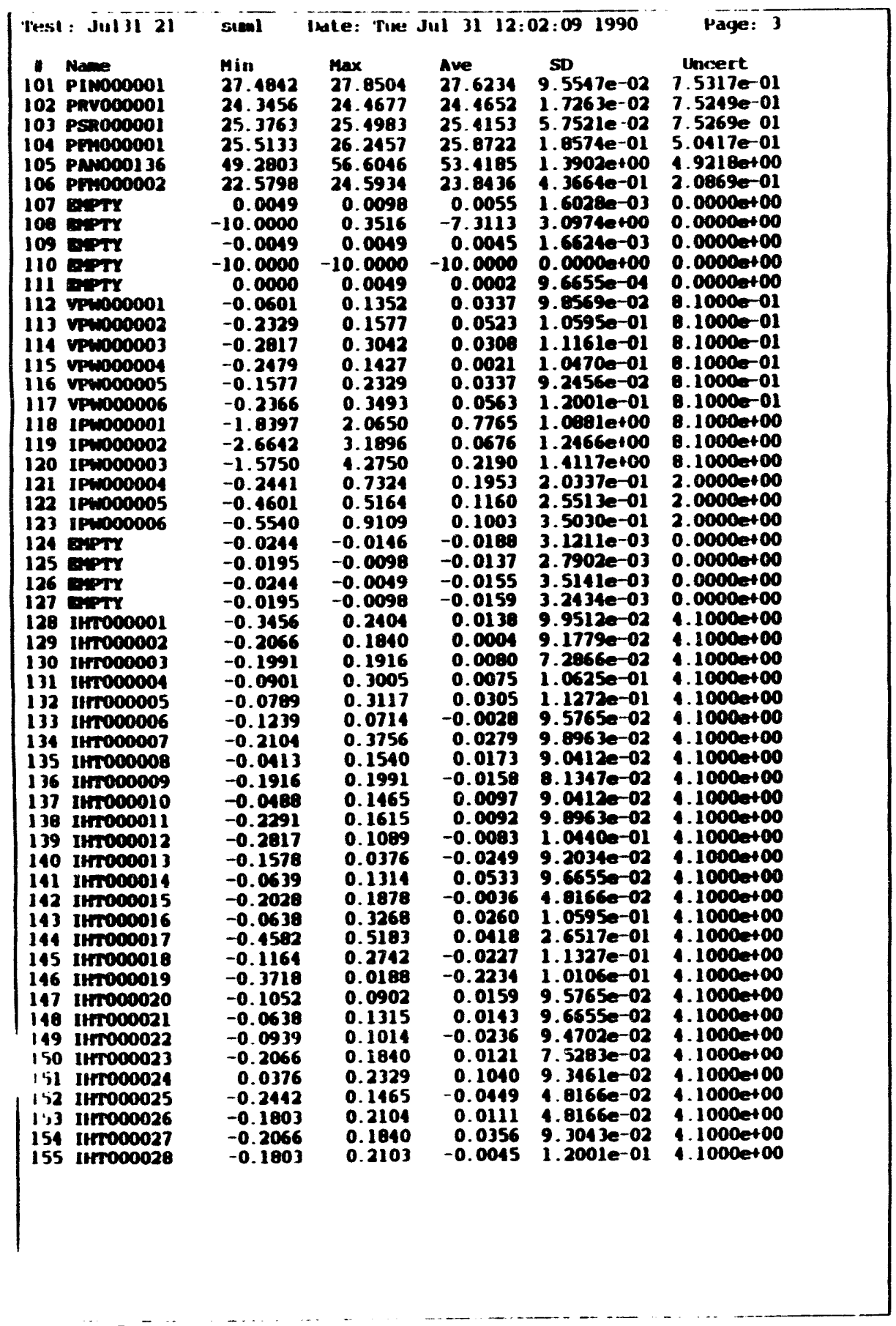

\begin{tabular}{|c|c|c|c|c|c|}
\hline Test: Julji_21 & sual & Date: Tue & $4131 \overline{12}$ & $02: 091990$ & Page: \\
\hline 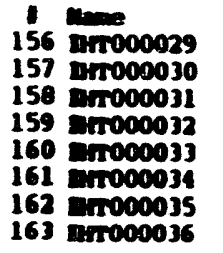 & $\begin{array}{l}\text { Min } \\
-0.2366 \\
-0.1878 \\
-0.2412 \\
-0.2404 \\
-0.2339 \\
-0.1765 \\
-0.2216 \\
-0.0939\end{array}$ & $\begin{array}{l}\operatorname{Max} \\
0.1540 \\
0.2028 \\
0.3418 \\
0.3455 \\
0.1578 \\
0.2141 \\
0.1690 \\
0.1014\end{array}$ & $\begin{array}{l}\text { Ave } \\
-0.0023 \\
-0.0120 \\
0.0254 \\
-0.0099 \\
-0.0024 \\
-0.0203 \\
0.0089 \\
0.0037\end{array}$ & $\begin{array}{l}\text { SD } \\
8.8233 \mathrm{e}-02 \\
1.1334 \mathrm{e}-01 \\
1.2409 \mathrm{e}-01 \\
9.4125 \mathrm{e}-02 \\
9.4125 \mathrm{e}-02 \\
9.6655 \mathrm{e}-02 \\
8.5454 \mathrm{e}-02 \\
9.8648 \mathrm{e}-02\end{array}$ & $\begin{array}{l}\text { Uncert } \\
1.1000 \text { et } 00 \\
1.1000 \text { et } 00 \\
1.1000 \text { et } 00 \\
1.1000 \text { et } 00 \\
1.1000 \text { et } 00 \\
1.1000 \text { e } 00 \\
1.1000=100 \\
1.1000 \text { et } 00\end{array}$ \\
\hline
\end{tabular}




\begin{tabular}{|c|c|c|c|c|c|c|}
\hline \multicolumn{6}{|c|}{ 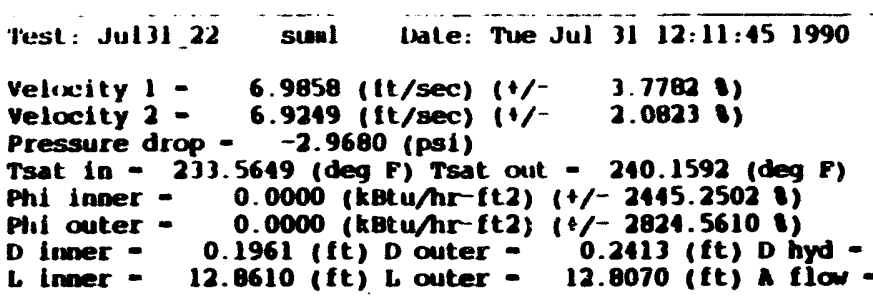 } & age: 1 \\
\hline 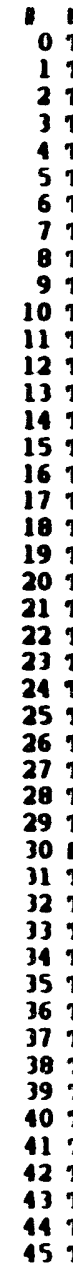 & 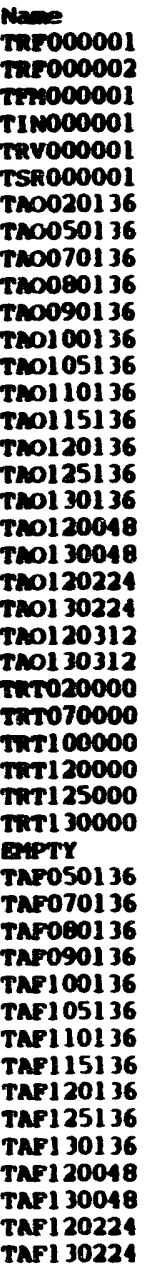 & $\begin{array}{l}\text { Min } \\
74.0527 \\
73.9855 \\
88.5224 \\
87.6304 \\
87.7842 \\
88.2054 \\
81.2176 \\
88.0656 \\
88.0895 \\
87.9655 \\
88.5407 \\
87.894 \\
88.7171 \\
88.2636 \\
88.3288 \\
88.0506 \\
88.6662 \\
87.8990 \\
75.5123 \\
87.7738 \\
88.0944 \\
88.0459 \\
87.9734 \\
87.9478 \\
88.4580 \\
88.1414 \\
88.4991 \\
88.4101 \\
88.2889 \\
88.1077 \\
39.1464 \\
88.4474 \\
88.7502 \\
88.4073 \\
75.6366 \\
88.2662 \\
87.9521 \\
88.0901 \\
88.3753 \\
88.4161 \\
88.1827 \\
88.2826 \\
88.6631 \\
88.2534 \\
88.2442 \\
87.8796\end{array}$ & $\begin{array}{l}\text { Max } \\
74.6929 \\
74.1101 \\
89.1526 \\
88.1568 \\
88.7265 \\
88.8338 \\
82.2757 \\
88.6952 \\
88.7220 \\
88.5959 \\
89.2743 \\
88.5254 \\
89.6633 \\
88.8904 \\
89.1704 \\
89.4720 \\
89.2113 \\
88.1210 \\
76.1499 \\
88.2990 \\
88.6200 \\
88.7770 \\
88.8145 \\
89.6823 \\
89.1949 \\
88.9747 \\
89.2332 \\
88.8314 \\
89.0222 \\
88.8424 \\
40.0646 \\
89.2845 \\
89.4829 \\
89.0340 \\
76.5906 \\
89.0027 \\
89.1010 \\
88.7204 \\
89.3186 \\
89.1495 \\
89.3313 \\
88.9132 \\
89.2962 \\
88.9881 \\
89.1886 \\
88.5121\end{array}$ & $\begin{array}{l}\text { Ave } \\
74.3472 \\
74.1468 \\
88.8357 \\
87.8559 \\
88.2353 \\
88.5426 \\
81.7319 \\
88.3619 \\
88.3742 \\
88.2621 \\
88.9200 \\
88.1700 \\
89.1693 \\
88.6336 \\
88.7559 \\
88.2675 \\
89.0370 \\
88.1988 \\
75.7951 \\
88.0912 \\
88.3826 \\
89.4276 \\
88.4717 \\
88.2778 \\
88.8476 \\
88.6603 \\
88.9285 \\
88.6145 \\
88.6386 \\
88.4796 \\
39.6102 \\
88.8973 \\
89.1339 \\
88.7436 \\
76.1690 \\
88.6175 \\
88.5555 \\
88.3697 \\
88.9186 \\
88.7490 \\
88.8191 \\
88.6502 \\
89.0072 \\
88.6379 \\
88.6786 \\
88.1265\end{array}$ & $\begin{array}{l}\text { s0 } \\
1.6636 e-01 \\
1.1795 e-01 \\
1.8794 e-01 \\
1.2034 e-01 \\
2.1792 e-01 \\
1.3622 e-01 \\
2.1791 e-01 \\
1.5913 e-01 \\
1.8587 e-01 \\
1.4937 e-01 \\
1.8574 e-01 \\
1.5730 e-01 \\
2.0176 e-01 \\
1.6532 e-01 \\
1.9967 e-01 \\
1.0930 e-01 \\
1.5527 e-01 \\
1.4909 e-01 \\
1.5414 e-01 \\
1.5365 e-01 \\
1.5139 e-01 \\
1.6051 e-01 \\
1.7368 e-01 \\
1.5292 e-01 \\
1.6909 e-01 \\
1.4519 e-01 \\
1.8734 e-01 \\
1.0502 e-01 \\
1.9779 e-01 \\
1.4977 e-01 \\
2.2477 e-01 \\
1.8486 e-01 \\
1.7367 e-01 \\
1.3593 e-01 \\
2.2063 e-01 \\
1.5852 e-01 \\
2.7275 e-01 \\
1.6365 e-01 \\
1.9632 e-01 \\
1.3033 e-01 \\
2.5016 e-01 \\
1.5901 e-01 \\
1.8916 e-01 \\
1.7435 e-01 \\
2.0107 e-01 \\
1.7757 e-01\end{array}$ & 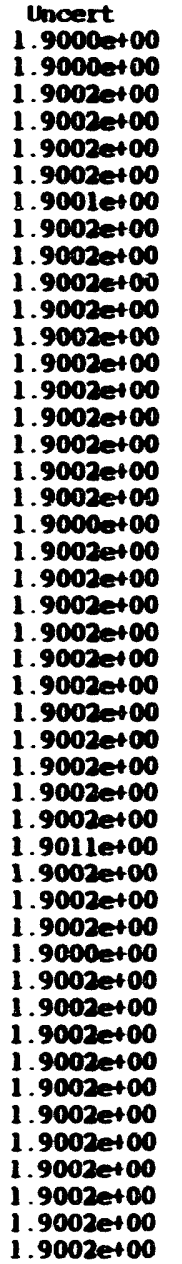 \\
\hline
\end{tabular}

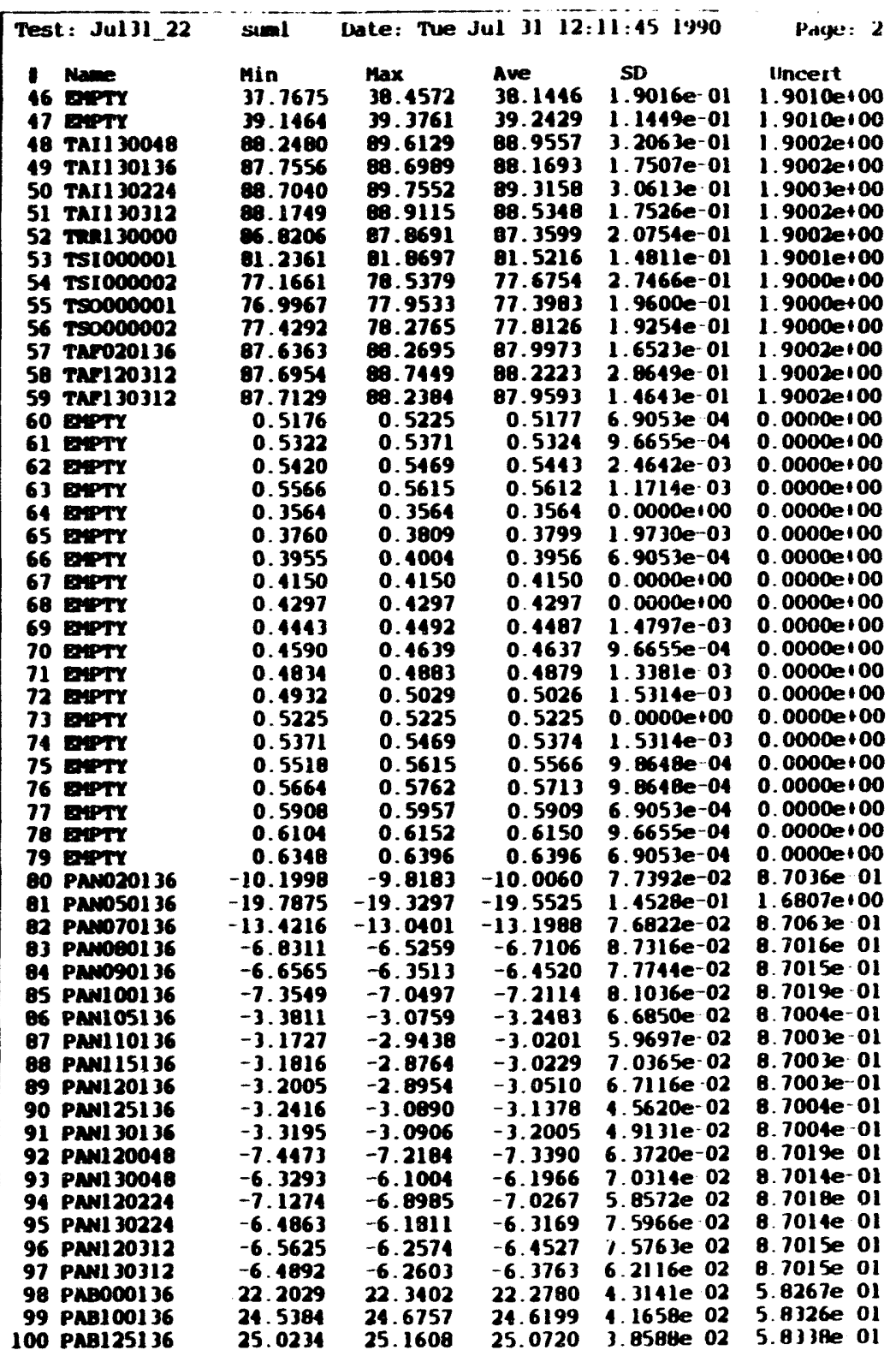




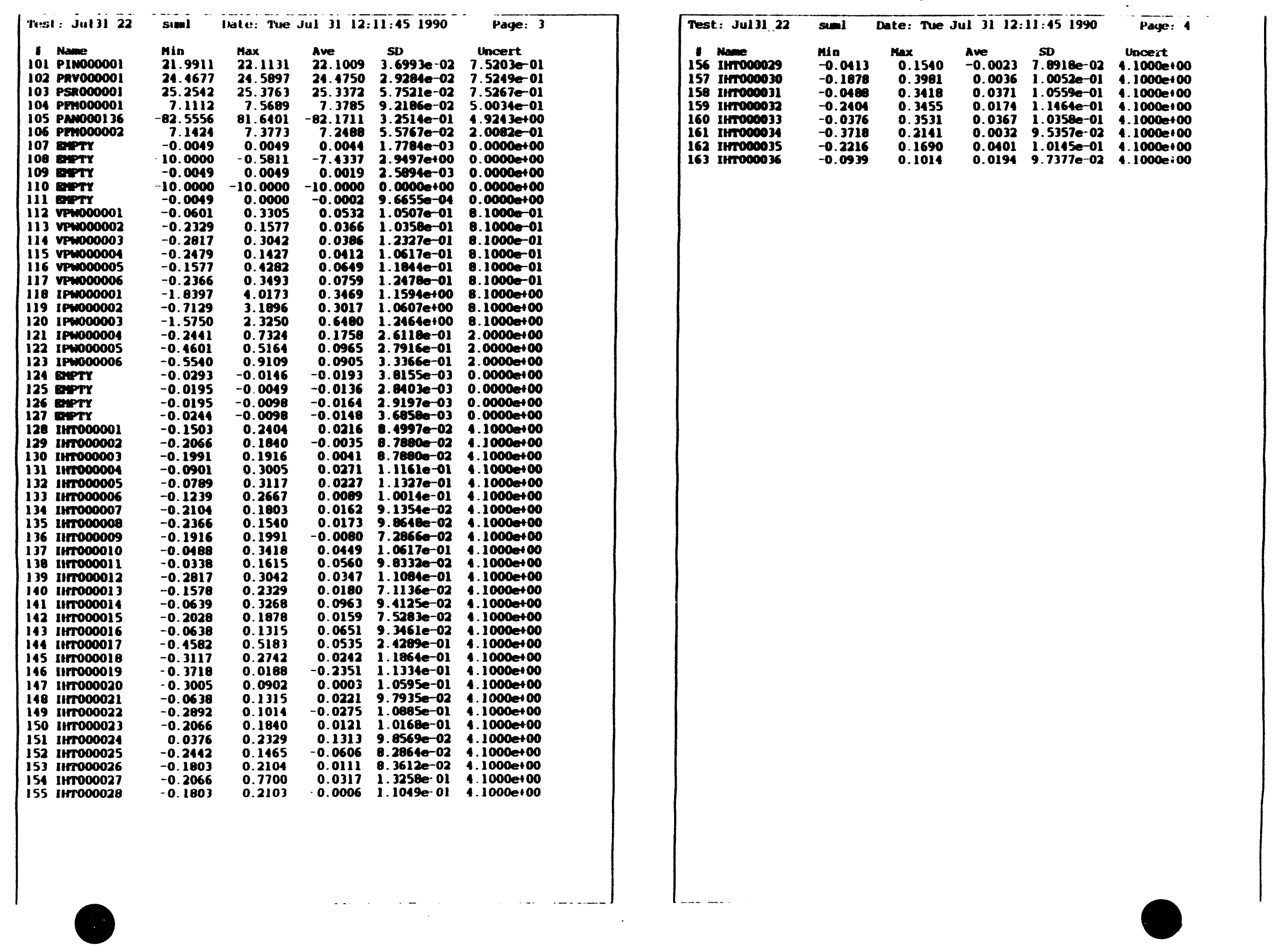




\begin{tabular}{|c|c|c|c|c|c|c|}
\hline \multicolumn{7}{|c|}{ 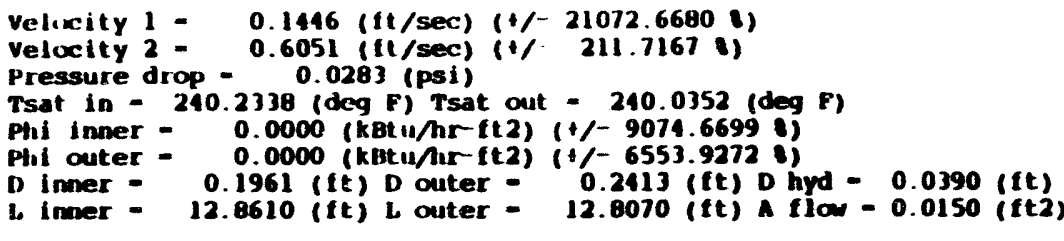 } \\
\hline 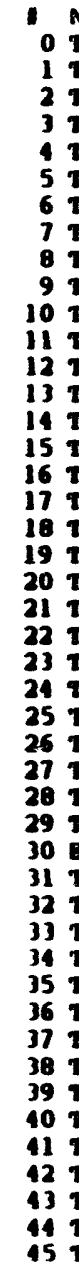 & 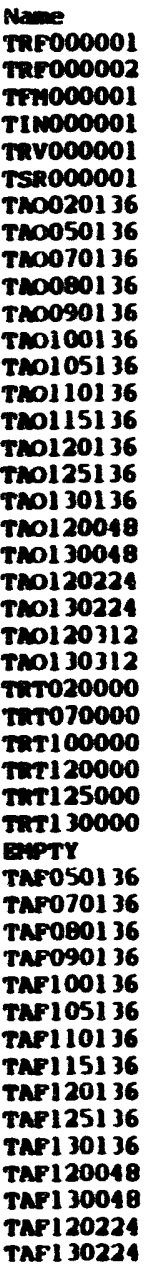 & $\begin{array}{l}\text { Min } \\
74.0527 \\
73.9855 \\
88.1034 \\
86.6838 \\
87.4716 \\
88.2054 \\
81.5347 \\
87.7527 \\
87.6678 \\
87.7588 \\
88.2258 \\
87.2660 \\
88.5070 \\
88.0555 \\
88.0138 \\
87.7357 \\
88.3722 \\
87.3837 \\
75.6189 \\
87.4604 \\
87.7797 \\
87.6300 \\
87.7626 \\
87.6340 \\
88.1424 \\
89.1293 \\
88.2894 \\
88.0958 \\
87.8718 \\
87.7937 \\
39.1464 \\
88.3433 \\
89.5434 \\
89.1986 \\
75.6375 \\
88.0553 \\
87.6412 \\
87.7771 \\
88.0623 \\
88.2066 \\
87.9745 \\
87.9696 \\
88.3462 \\
87.8353 \\
88.0348 \\
87.2476\end{array}$ & $\begin{array}{l}\operatorname{Max} \\
74.6929 \\
74.1101 \\
88.9430 \\
87.3154 \\
88.3107 \\
88.8352 \\
82.3812 \\
88.3831 \\
88.6162 \\
88.3872 \\
89.0659 \\
88.2103 \\
89.1526 \\
88.6849 \\
88.8549 \\
88.3671 \\
89.0029 \\
88.0117 \\
76.2559 \\
87.8920 \\
88.3060 \\
88.3619 \\
89.3943 \\
88.2638 \\
88.8794 \\
88.6578 \\
69.2332 \\
88.6219 \\
88.7103 \\
88.3192 \\
40.0646 \\
88.9716 \\
89.3799 \\
88.7232 \\
76.8030 \\
88.8972 \\
88.7875 \\
88.5115 \\
89.0058 \\
88.8369 \\
89.0202 \\
88.5997 \\
89.1906 \\
88.5703 \\
88.7700 \\
88.0907\end{array}$ & $\begin{array}{l}\text { Ave } \\
74.3856 \\
74.1935 \\
88.5380 \\
87.0269 \\
87.8970 \\
88.4593 \\
81.8694 \\
81.0782 \\
88.1043 \\
88.0329 \\
88.5977 \\
87.9018 \\
88.9087 \\
88.3512 \\
88.4743 \\
87.9822 \\
88.6226 \\
87.7444 \\
75.9605 \\
87.6480 \\
88.0849 \\
88.0582 \\
88.1606 \\
87.8551 \\
88.5318 \\
88.3559 \\
89.6610 \\
89.3128 \\
89.3471 \\
88.0777 \\
39.6194 \\
88.6009 \\
88.8632 \\
88.4485 \\
76.1607 \\
88.3608 \\
80.2562 \\
88.0942 \\
88.6536 \\
89.4861 \\
88.5657 \\
88.2529 \\
88.7790 \\
88.1982 \\
88.4066 \\
87.7597\end{array}$ & 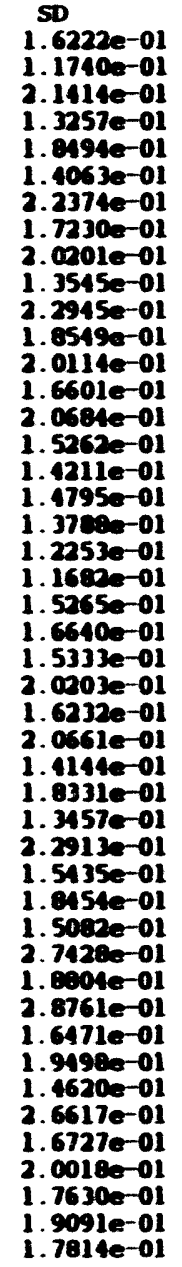 & $\begin{array}{r}\text { Uncert } \\
1.9000 e+00 \\
1.9000 e+00 \\
1.9002 e+00 \\
1.9002 e+00 \\
1.9002 e+00 \\
1.9002 e+00 \\
1.9001 e+00 \\
1.9002 e+00 \\
1.9002 e+10 \\
1.9002 e+00 \\
1.9002 e+00 \\
1.9002 e+00 \\
1.9002 e+00 \\
1.9002 e+00 \\
1.9002 e+00 \\
1.9002 e+00 \\
1.9002 e+00 \\
1.9002 e+00 \\
1.9000 e+00 \\
1.9002 e+00 \\
1.9002 e+00 \\
1.9002 e+00 \\
1.9002 e+00 \\
1.9002 e+00 \\
1.9002 e+00 \\
1.9002 e+00 \\
1.9002 e+00\end{array}$ \\
\hline
\end{tabular}

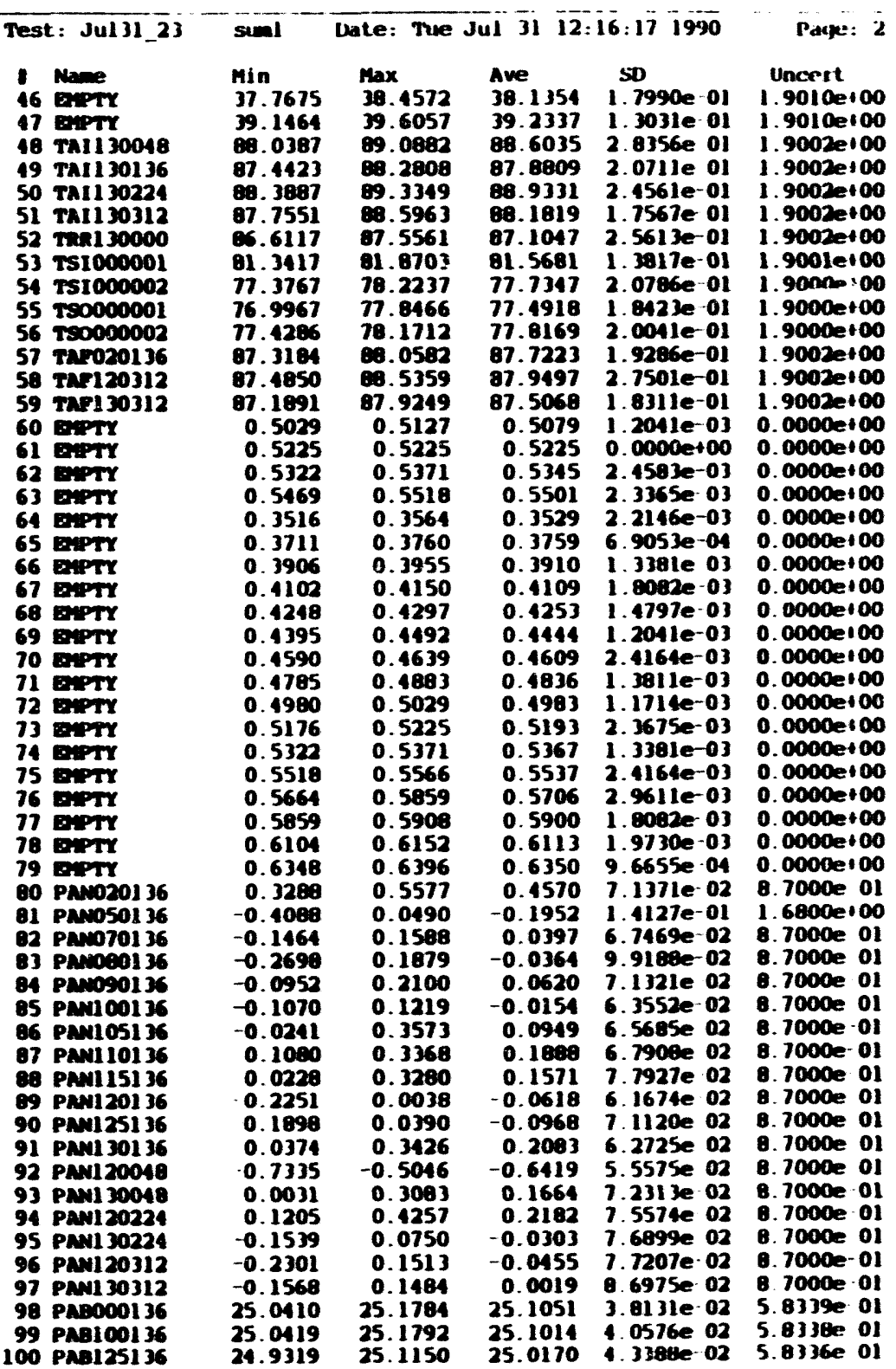




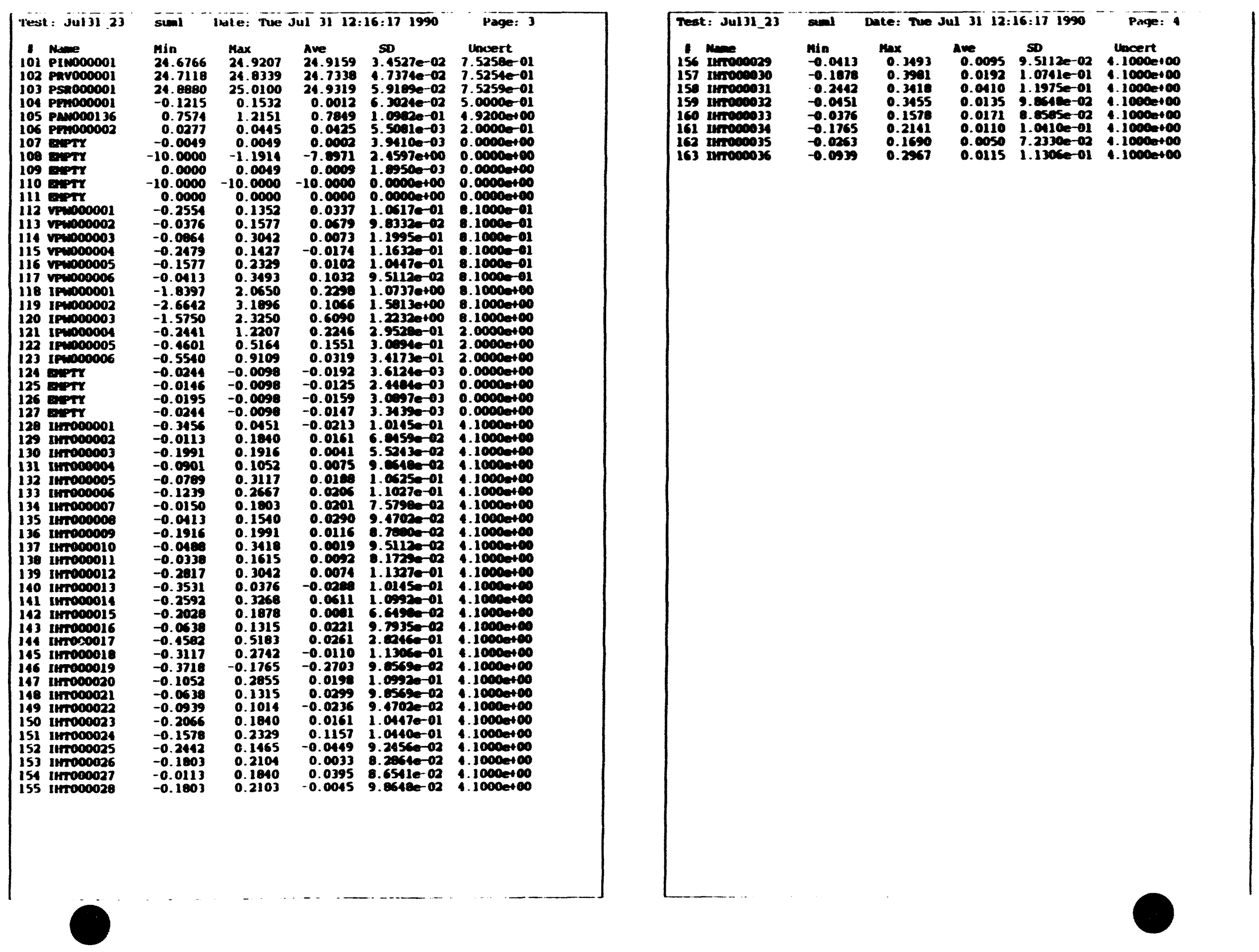




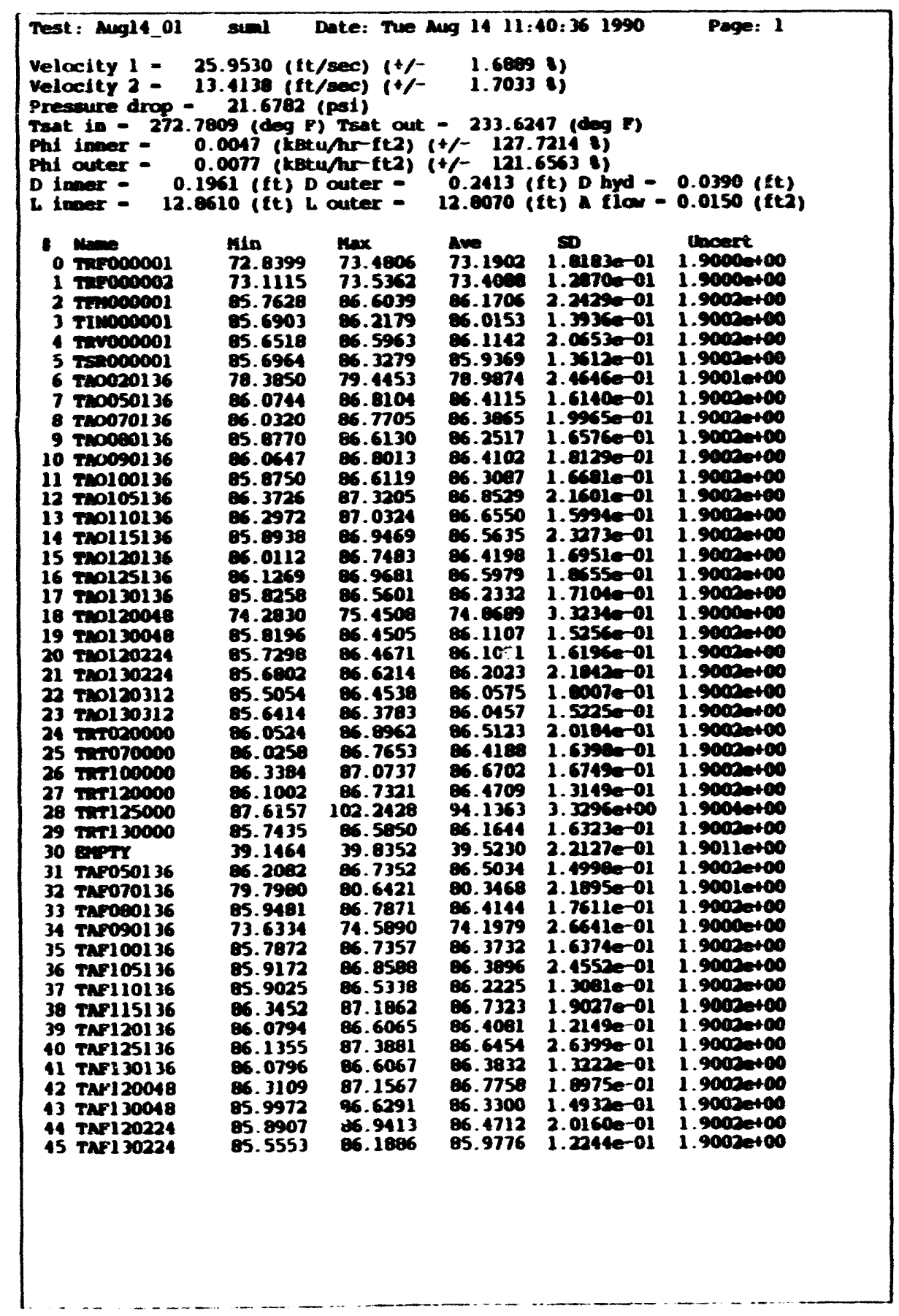

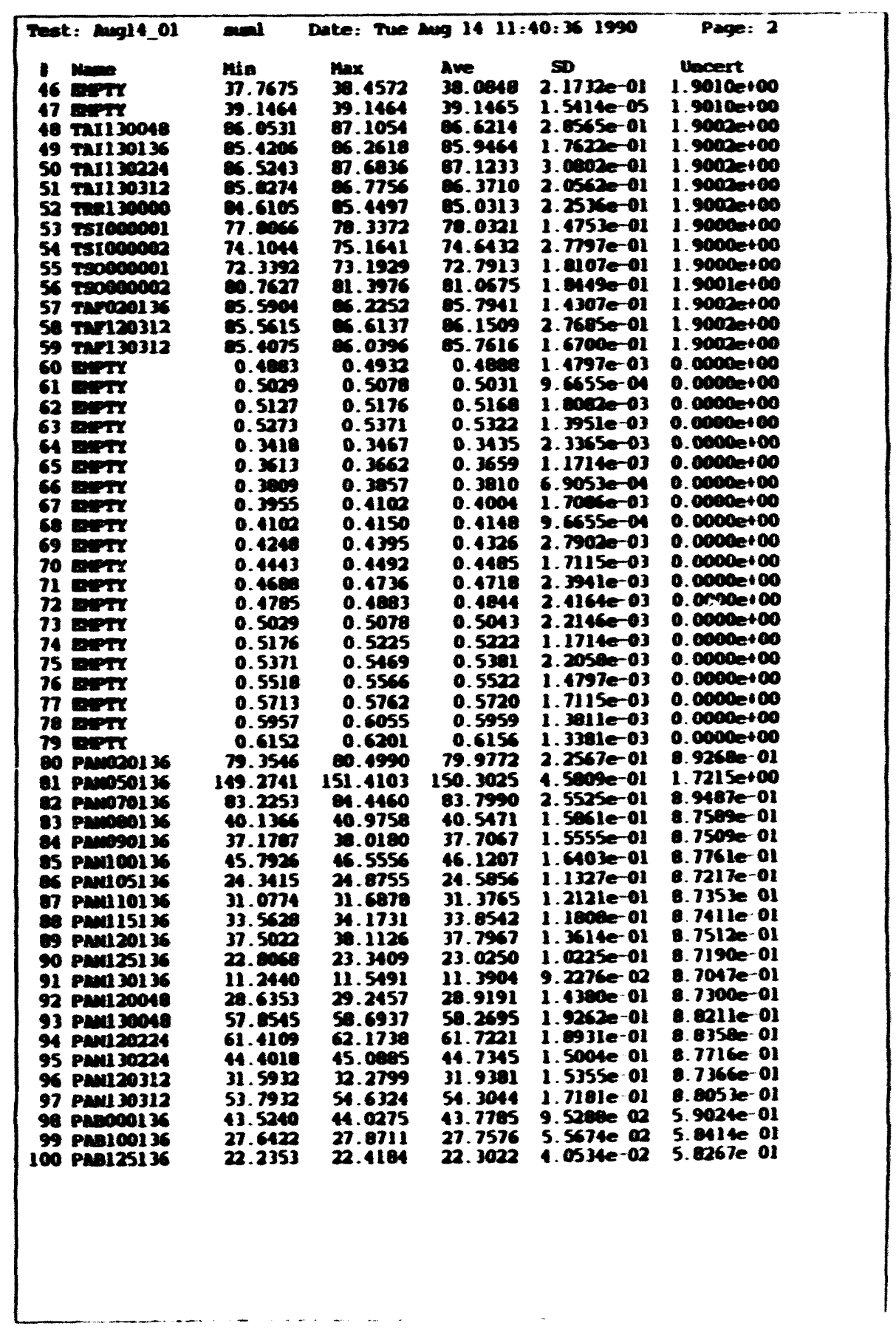




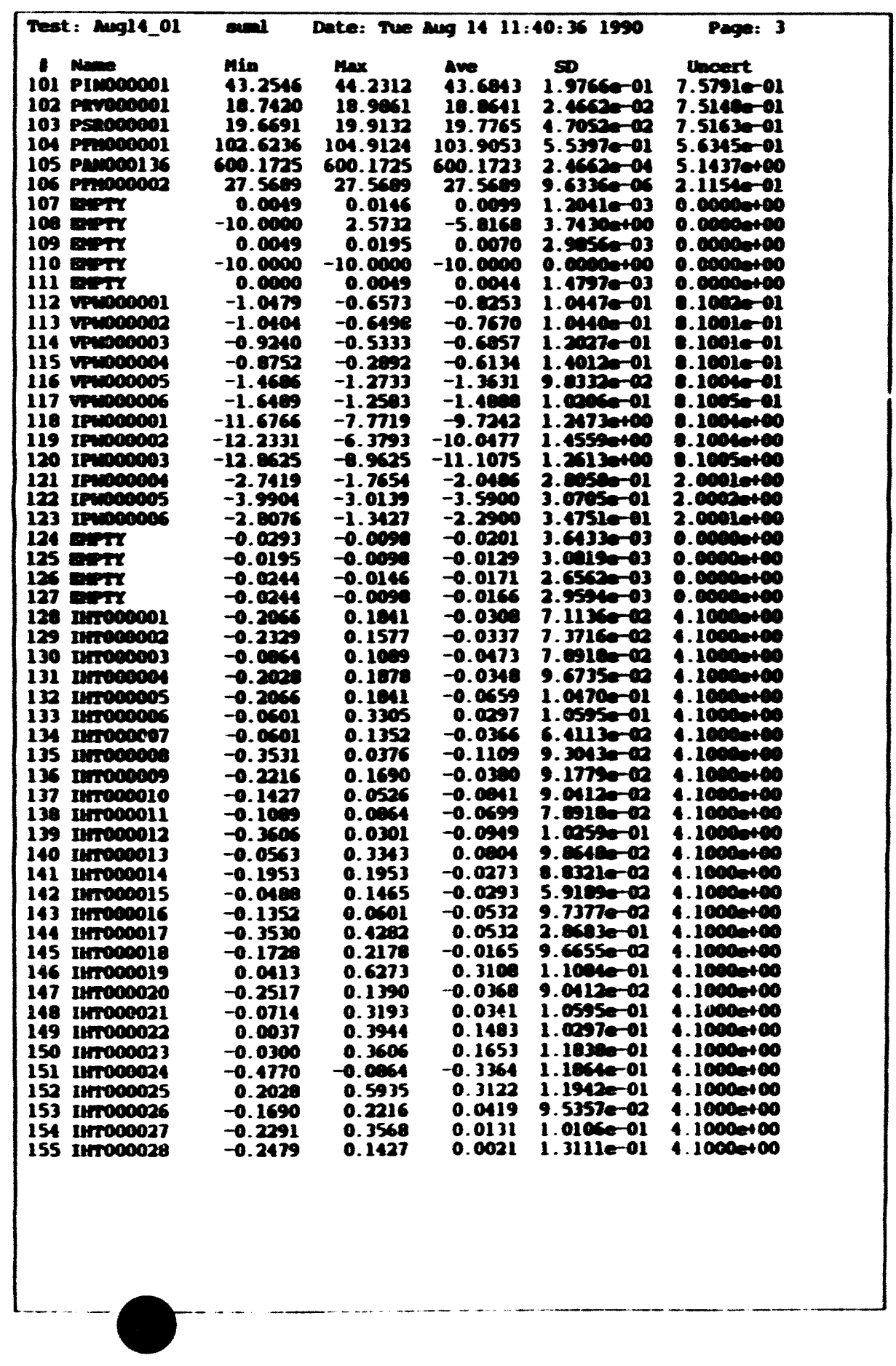

\begin{tabular}{|c|c|c|c|c|}
\hline 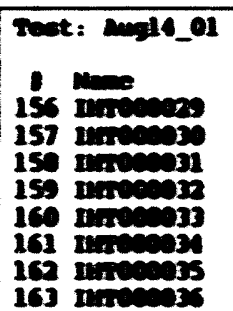 & 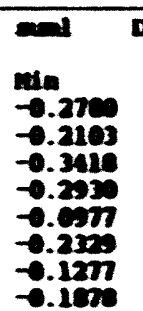 & $\begin{array}{l}\text { Dte: } 701 \\
\max \\
0.1127 \\
0.3756 \\
0.2411 \\
0.0977 \\
0.0976 \\
0.1577 \\
0.2600 \\
0.2000\end{array}$ & 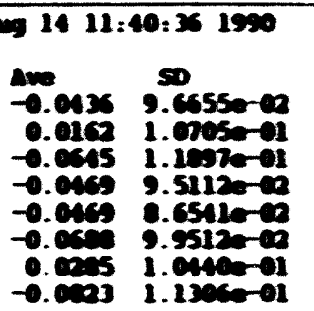 & \\
\hline
\end{tabular}



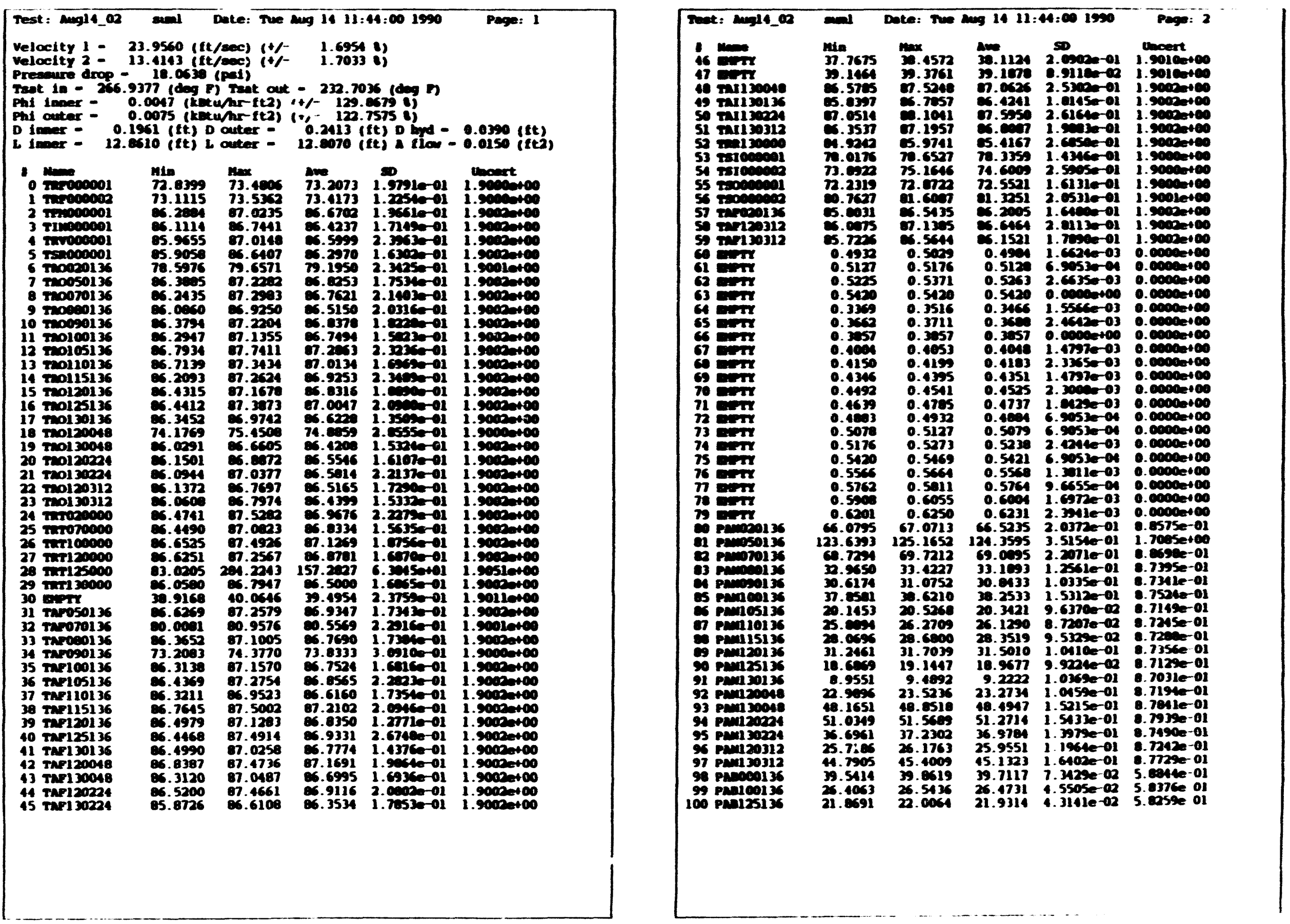

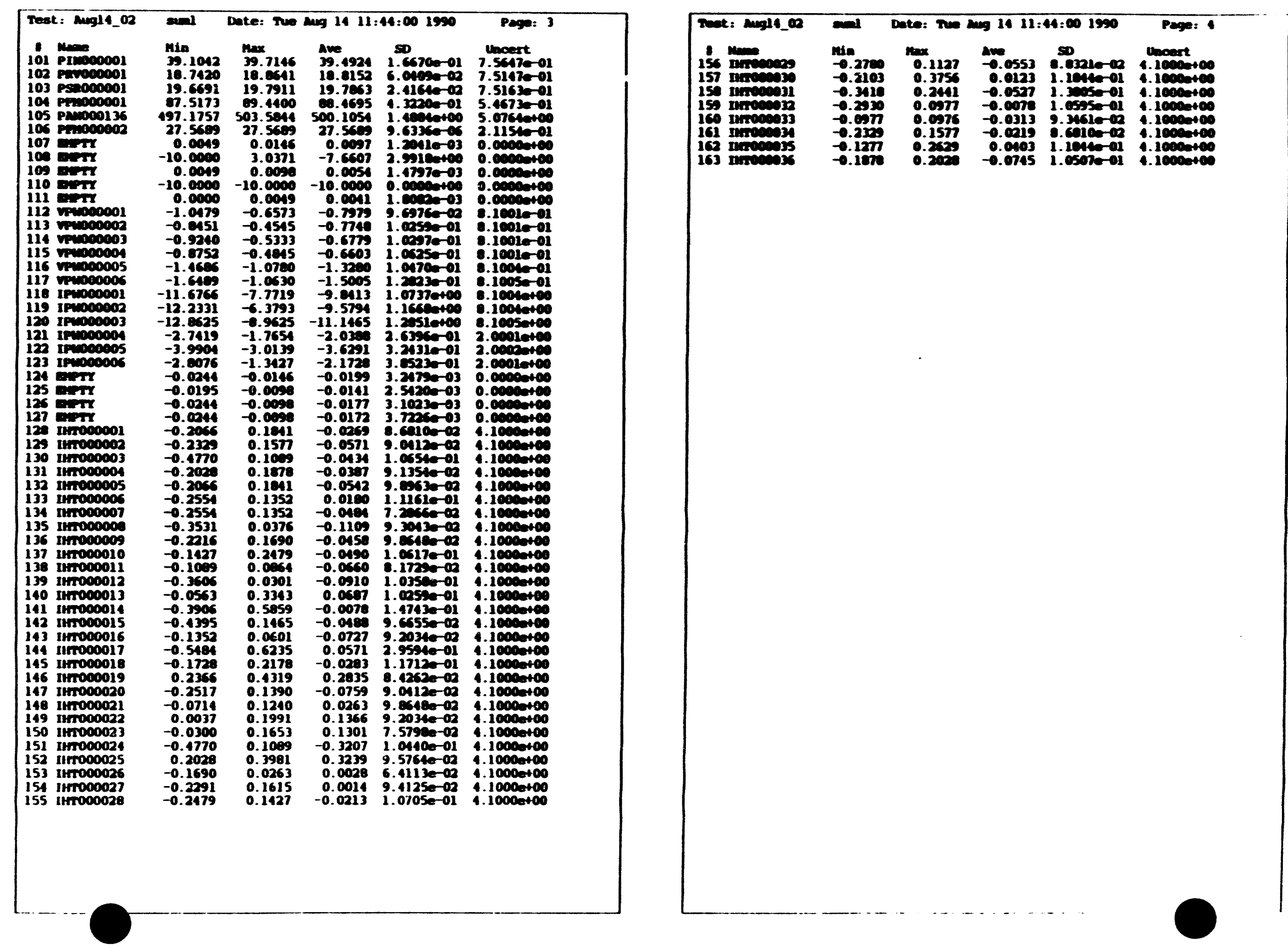


\begin{tabular}{|c|c|c|c|c|c|}
\hline \multicolumn{5}{|c|}{ 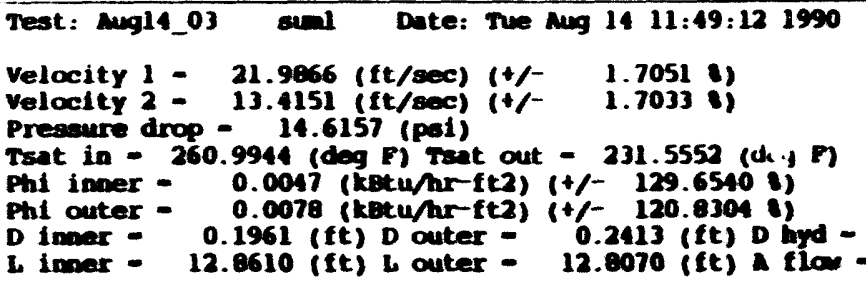 } & $\begin{array}{c}\text { Page: } 1 \\
0.0390 \text { (ft) } \\
0.0150 \text { (ft2) }\end{array}$ \\
\hline 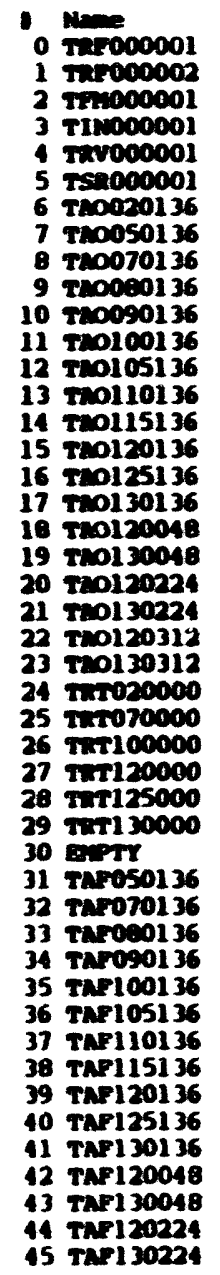 & $\begin{array}{l}\text { Min } \\
73.0535 \\
73.1115 \\
86.9171 \\
86.6380 \\
86.5949 \\
86.4300 \\
78.8095 \\
87.1209 \\
87.0872 \\
86.8181 \\
87.1137 \\
87.0296 \\
87.4251 \\
87.3411 \\
86.9462 \\
86.9572 \\
87.2607 \\
86.9670 \\
74.7074 \\
86.7628 \\
86.8659 \\
66.8046 \\
86.6639 \\
86.6900 \\
87.2121 \\
87.0940 \\
87.2910 \\
87.1504 \\
79.1375 \\
66.8977 \\
39.1464 \\
87.1510 \\
80.4290 \\
87.0962 \\
73.6334 \\
87.0514 \\
66.9593 \\
96.8452 \\
87.2691 \\
87.2309 \\
86.9692 \\
87.0238 \\
87.3681 \\
87.0471 \\
87.1500 \\
86.6116\end{array}$ & 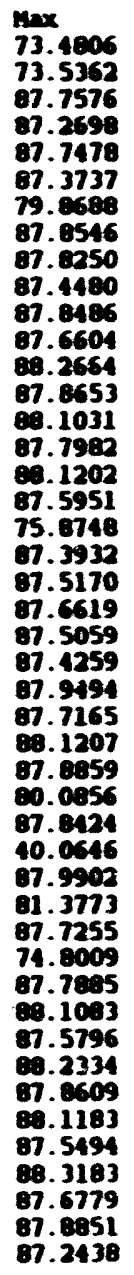 & $\begin{array}{l}\text { Av } \\
73.2628 \\
73.3579 \\
87.3772 \\
87.0100 \\
87.1629 \\
86.0600 \\
79.3503 \\
87.4417 \\
87.4563 \\
87.1436 \\
87.4560 \\
87.3220 \\
87.9235 \\
87.6070 \\
87.5667 \\
87.3547 \\
87.7278 \\
87.2296 \\
75.2360 \\
87.0342 \\
87.1448 \\
87.2663 \\
87.1333 \\
87.0122 \\
87.6357 \\
87.4319 \\
87.7197 \\
87.4806 \\
79.6204 \\
87.2862 \\
39.5138 \\
87.6021 \\
80.9706 \\
87.4047 \\
71.2130 \\
87.3653 \\
87.4545 \\
87.2123 \\
87.7277 \\
87.4644 \\
87.6477 \\
87.3202 \\
87.8435 \\
87.3395 \\
87.5469 \\
86.9424\end{array}$ & 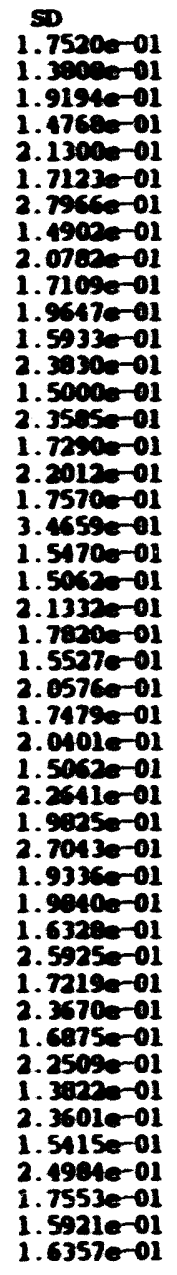 & 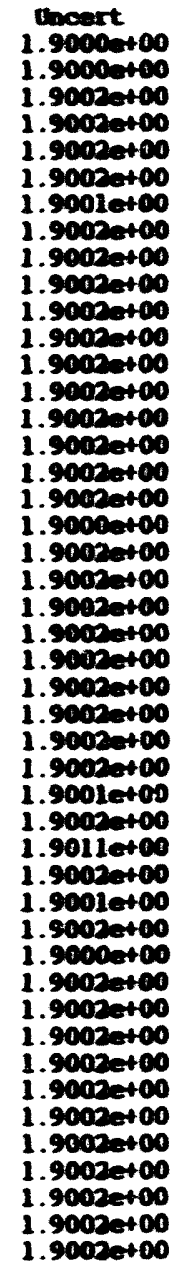 \\
\hline
\end{tabular}

\begin{tabular}{|c|c|c|c|c|c|}
\hline 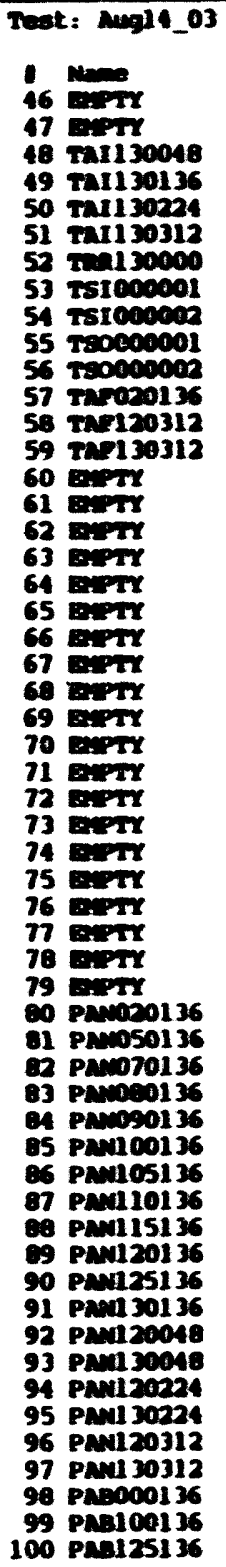 & 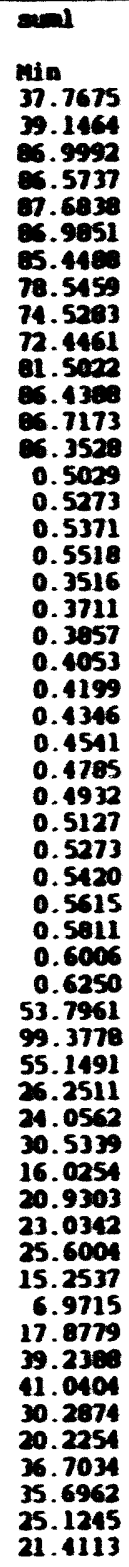 & 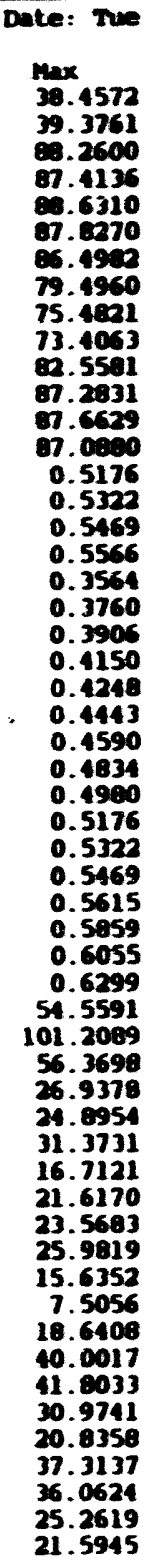 & 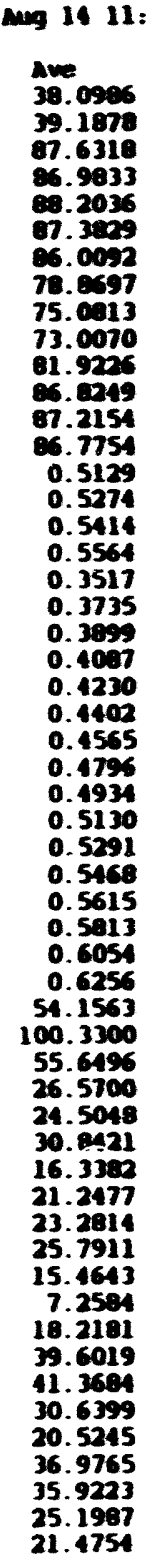 & 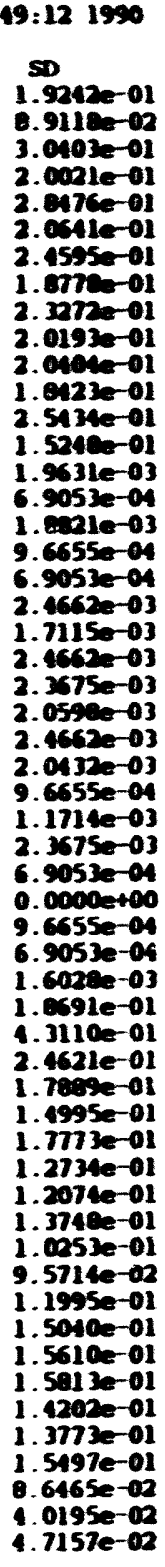 & 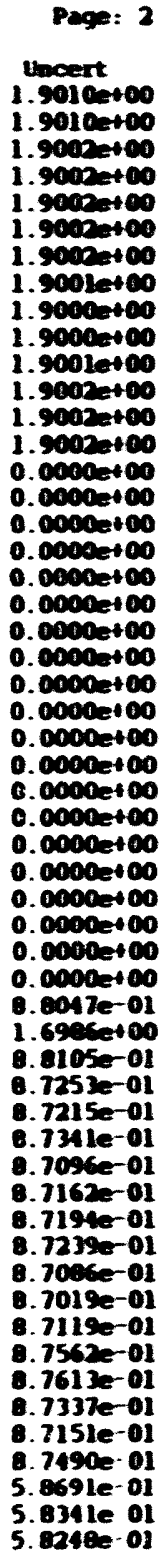 \\
\hline
\end{tabular}



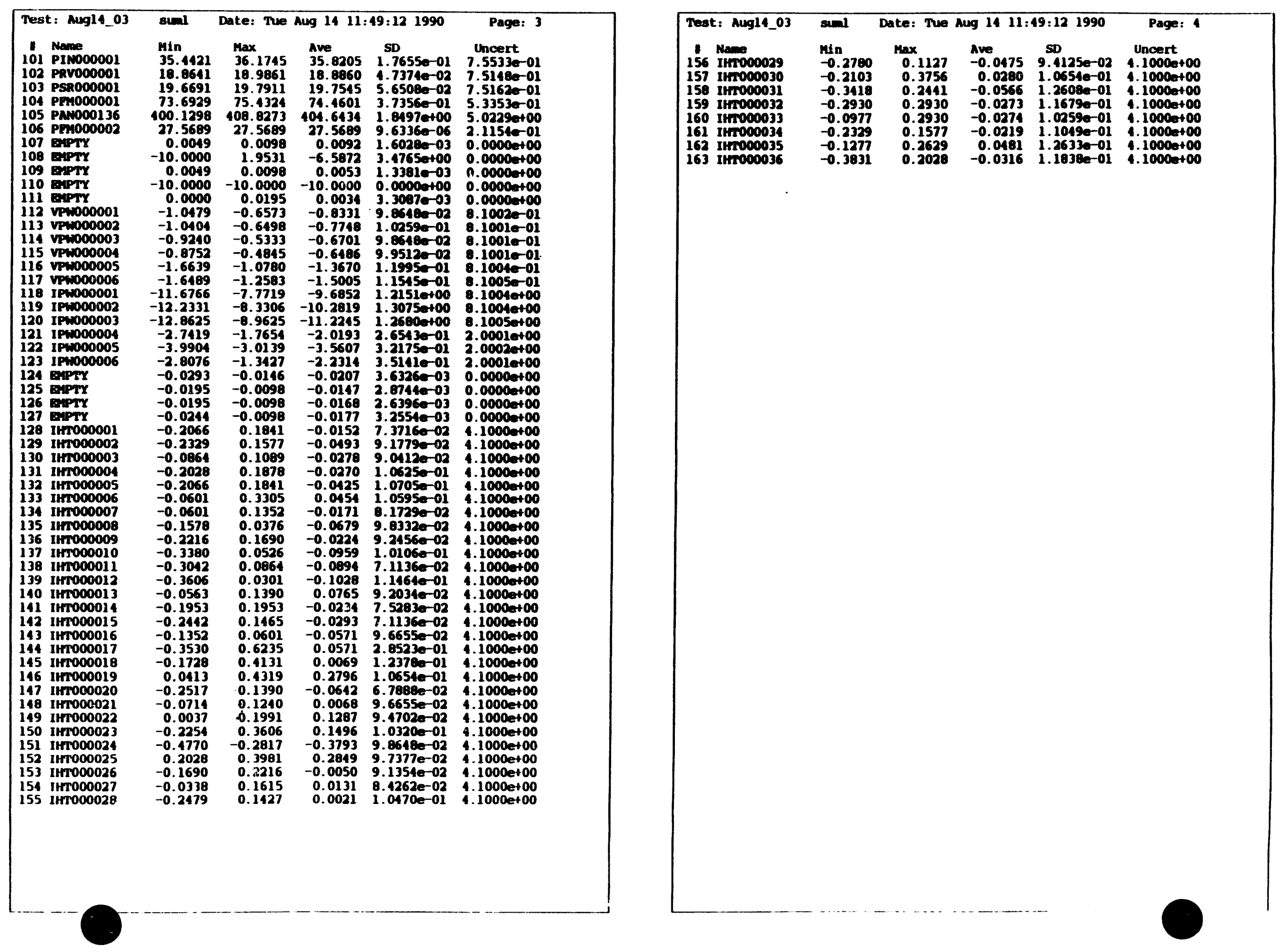


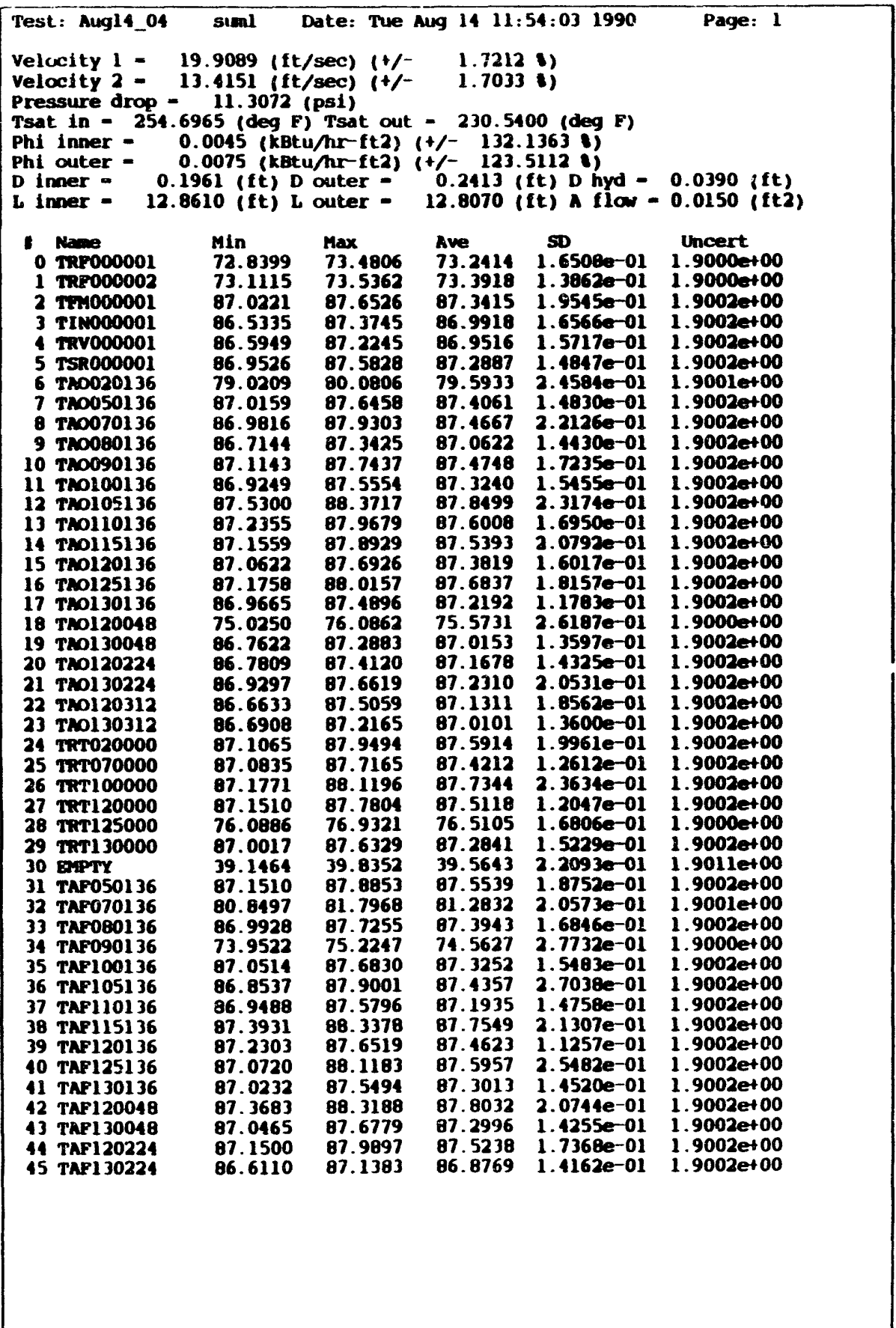

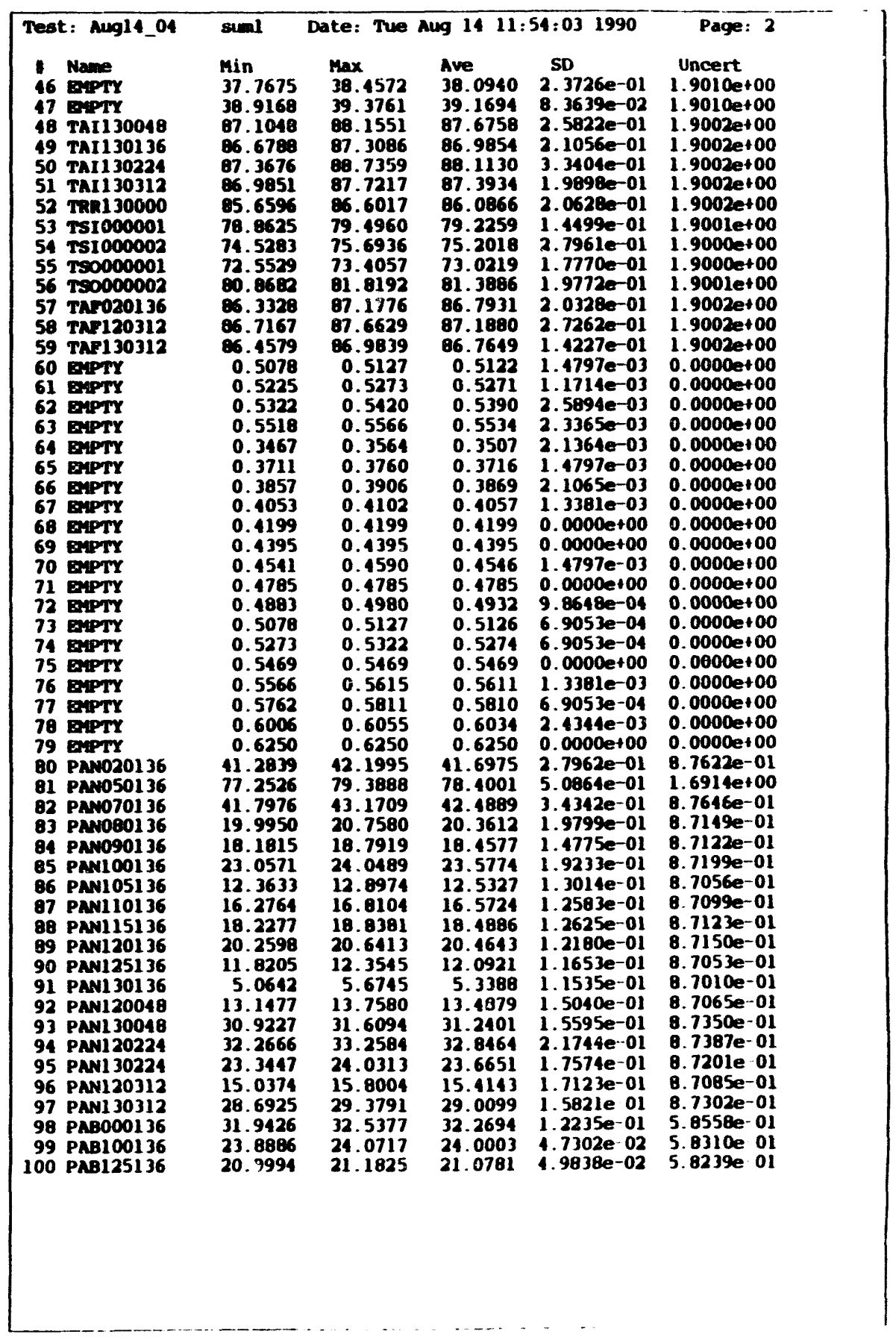



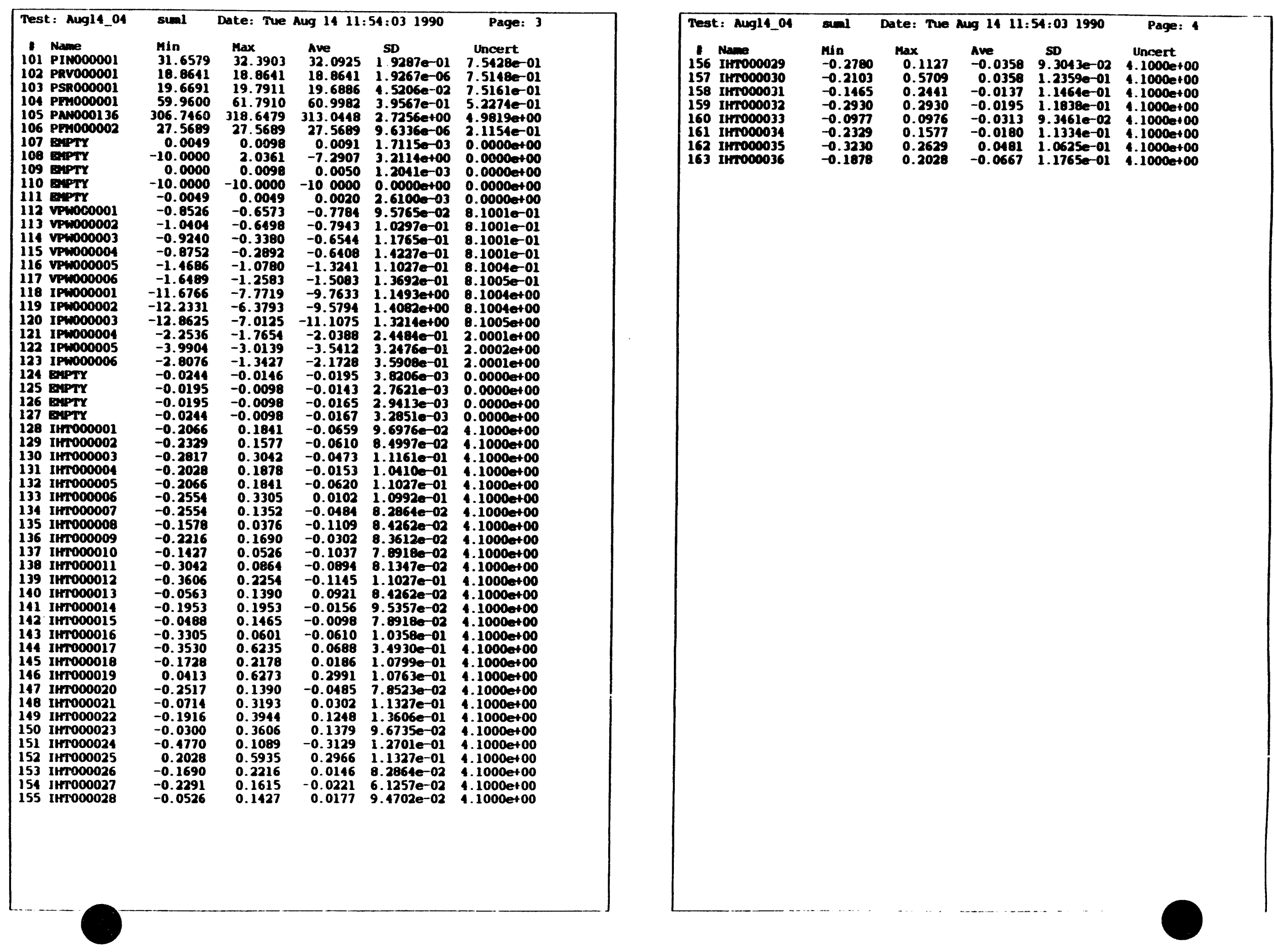


\begin{tabular}{|c|c|c|c|c|c|c|}
\hline \multicolumn{4}{|c|}{ 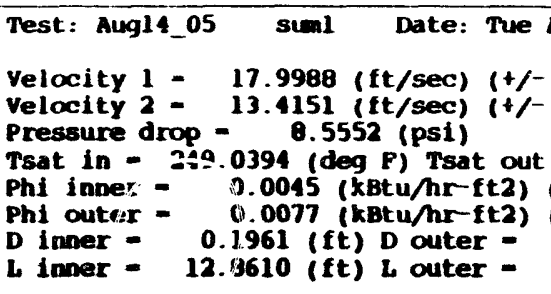 } & \multicolumn{2}{|c|}{ 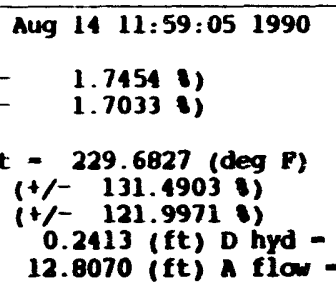 } & $\begin{array}{l}\text { Page: } 1 \\
0.0390 \text { (ft) } \\
0.0150 \text { (ft2) }\end{array}$ \\
\hline 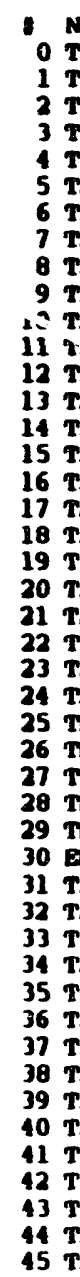 & 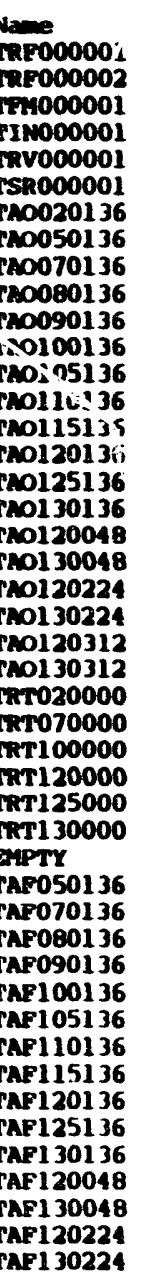 & $\begin{array}{l}\text { M1n } \\
72.8399 \\
73.1115 \\
86.9179 \\
86.6377 \\
86.8041 \\
86.8479 \\
79.3390 \\
87.0153 \\
86.9818 \\
86.6093 \\
87.0081 \\
86.9243 \\
87.2142 \\
87.2355 \\
87.1562 \\
86.8522 \\
87.1752 \\
86.7600 \\
75.1316 \\
86.7622 \\
86.7809 \\
86.8241 \\
86.6639 \\
86.5851 \\
87.0014 \\
87.0835 \\
87.1765 \\
87.1498 \\
75.0345 \\
86.7921 \\
39.1464 \\
87.1510 \\
81.0596 \\
86.8872 \\
74.0570 \\
86.9458 \\
86.8543 \\
86.7396 \\
87.2885 \\
87.0212 \\
86.8636 \\
87.0232 \\
87.2631 \\
86.8367 \\
87.0450 \\
86.3999\end{array}$ & $\begin{array}{l}\text { Max } \\
73.6941 \\
73.7485 \\
87.7576 \\
87.2698 \\
87.5387 \\
87.5828 \\
80.3984 \\
87.6458 \\
87.8250 \\
87.4480 \\
87.7437 \\
87.5563 \\
88.2664 \\
87.8653 \\
87.9979 \\
87.5883 \\
88.2251 \\
87.4937 \\
76.2986 \\
87.2896 \\
87.5170 \\
87.6619 \\
87.6114 \\
87.3220 \\
87.9494 \\
87.6107 \\
88.0152 \\
87.6762 \\
75.5645 \\
87.5290 \\
39.8352 \\
87.9902 \\
82.0065 \\
87.7250 \\
75.1195 \\
87.6835 \\
87.9001 \\
87.5796 \\
88.0240 \\
87.7574 \\
88.0128 \\
87.5504 \\
88.2125 \\
87.5738 \\
87.8851 \\
87.2438\end{array}$ & $\begin{array}{l}\text { Ave } \\
73.2970 \\
73.1216 \\
87.3124 \\
86.9751 \\
87.0858 \\
87.1970 \\
79.8390 \\
87.3313 \\
87.3992 \\
86.9916 \\
87.4248 \\
87.2320 \\
87.8541 \\
87.5555 \\
87.5436 \\
87.3106 \\
87.6588 \\
87.1289 \\
75.7937 \\
87.0030 \\
87.1134 \\
87.1691 \\
87.0765 \\
86.9349 \\
87.5829 \\
87.3450 \\
87.6676 \\
87.4533 \\
75.3144 \\
87.2047 \\
39.5000 \\
87.5333 \\
81.5018 \\
87.3114 \\
74.6350 \\
87.2874 \\
87.4071 \\
87.1854 \\
87.6421 \\
87.3790 \\
87.5378 \\
87.2932 \\
87.7524 \\
87.2956 \\
87.4569 \\
86.8769\end{array}$ & $\begin{array}{c}\text { sD } \\
1.8309 e-01 \\
1.3019 e-01 \\
1.9836 e-01 \\
1.4892 e-01 \\
1.6732 e-01 \\
1.5664 e-01 \\
2.5946 e-01 \\
1.6406 e-01 \\
1.9158 e-01 \\
2.0725 e-01 \\
1.7082 e-01 \\
1.6834 e-01 \\
2.1760 e-01 \\
1.6655 e-01 \\
2.2723 e-01 \\
1.6827 e-01 \\
2.2443 e-01 \\
1.6515 e-01 \\
2.9731 e-01 \\
1.3941 e-01 \\
1.4781 e-01 \\
2.0690 e-01 \\
1.8152 e-01 \\
1.7183 e-01 \\
1.8332 e-01 \\
1.2281 e-01 \\
2.0598 e-01 \\
1.6540 e-01 \\
1.5387 e-01 \\
1.7103 e-01 \\
2.1858 e-01 \\
1.8695 e-01 \\
2.0726 e-01 \\
1.9280 e-01 \\
2.9084 e-01 \\
1.6558 e-01 \\
2.1937 e-01 \\
1.6635 e-01 \\
2.1589 e-01 \\
1.6431 e-01 \\
2.6192 e-01 \\
1.2965 e-01 \\
2.3139 e-01 \\
1.5873 e-01 \\
1.8838 e-01 \\
1.5686 e-01\end{array}$ & 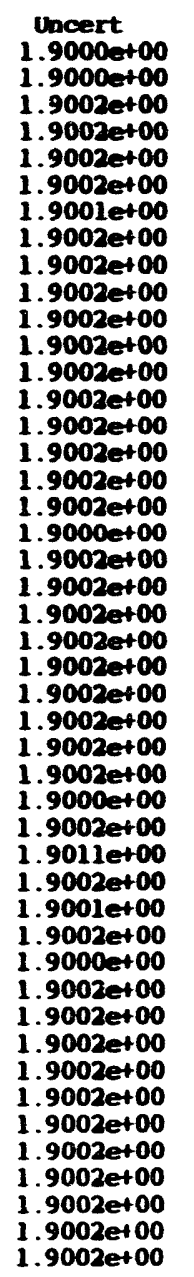 \\
\hline
\end{tabular}

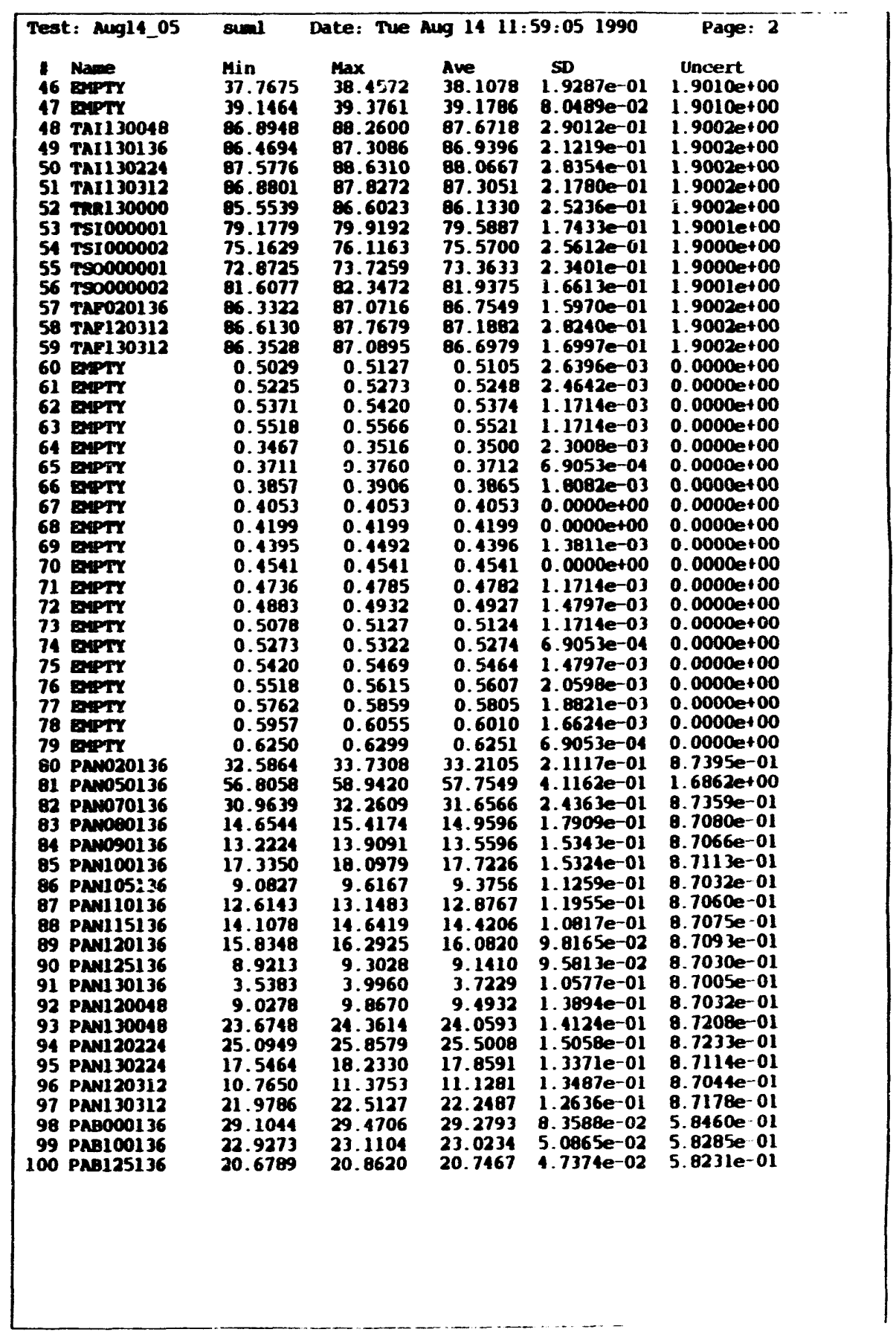



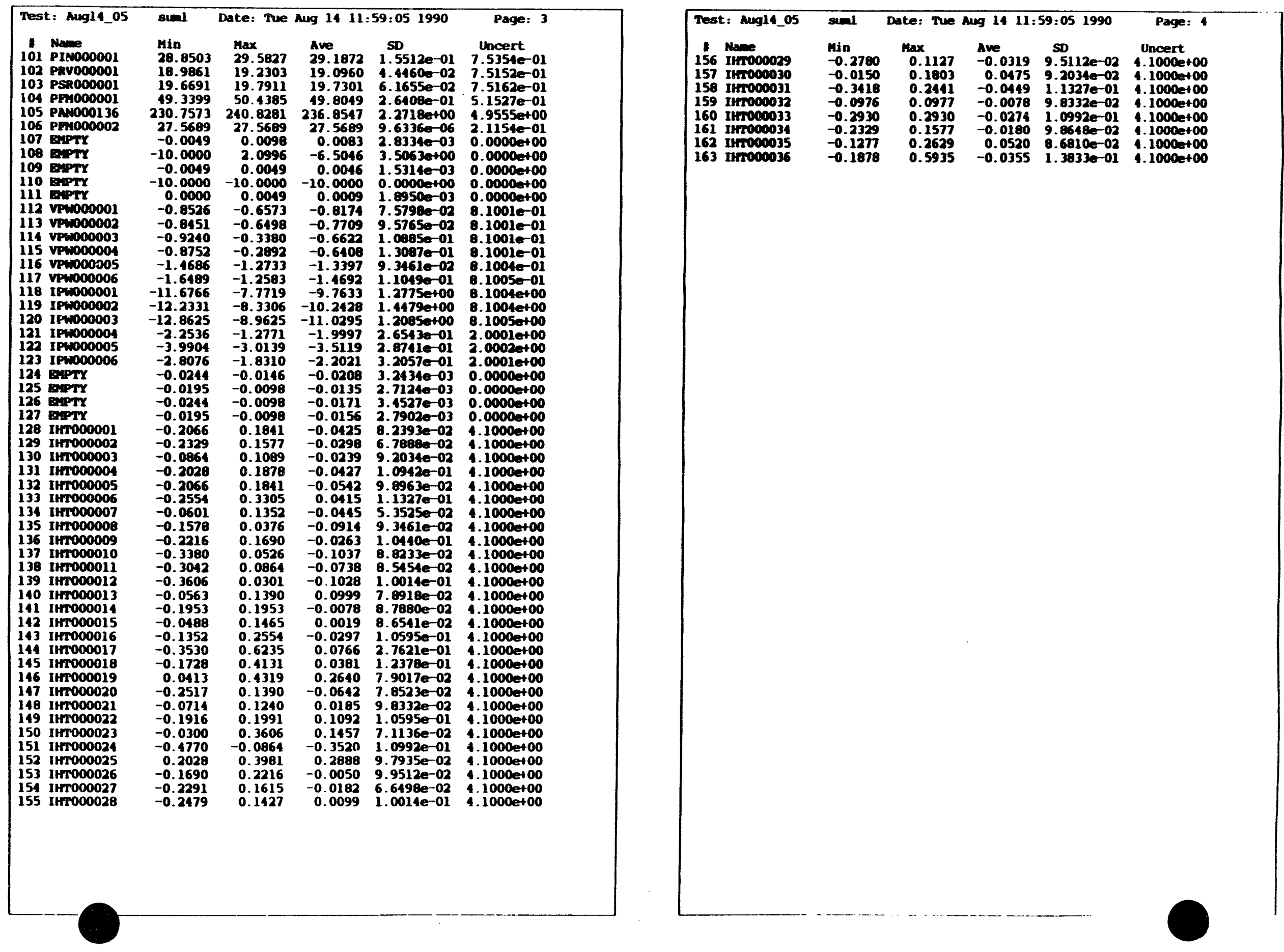


\begin{tabular}{|c|c|c|c|c|c|c|}
\hline \multicolumn{4}{|c|}{ 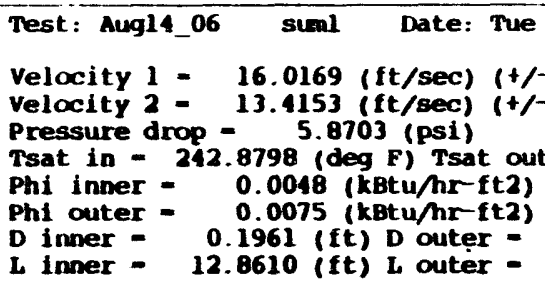 } & \multicolumn{2}{|c|}{ 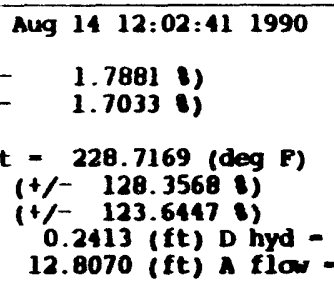 } & 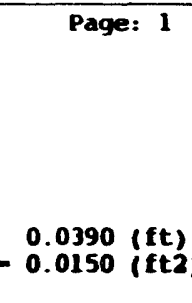 \\
\hline $\begin{array}{l}1 \\
0 \\
1 \\
2 \\
3 \\
4 \\
5 \\
6 \\
7 \\
8 \\
9 \\
10 \\
11 \\
12 \\
13 \\
14 \\
15 \\
16 \\
17 \\
18 \\
19 \\
20 \\
21 \\
22 \\
23 \\
24 \\
25 \\
26 \\
27 \\
28 \\
29 \\
30 \\
31 \\
32 \\
33 \\
34 \\
35 \\
36 \\
37 \\
38 \\
39 \\
10 \\
41 \\
12 \\
43 \\
14 \\
15\end{array}$ & 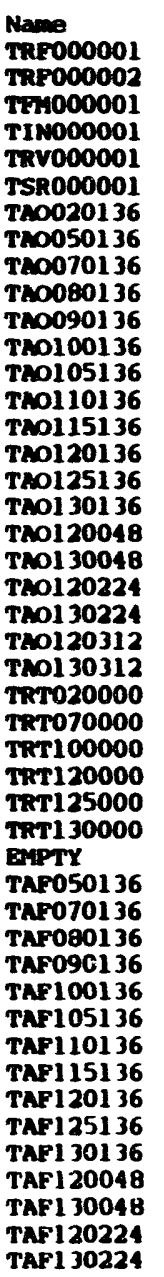 & $\begin{array}{l}\text { Min } \\
72.8399 \\
73.1115 \\
87.0221 \\
86.9536 \\
86.6985 \\
86.9520 \\
79.4446 \\
87.3298 \\
87.0872 \\
86.8176 \\
87.2187 \\
87.1346 \\
87.6350 \\
87.3411 \\
87.2615 \\
87.0622 \\
87.1752 \\
87.0720 \\
75.2371 \\
86.8692 \\
86.8854 \\
86.9297 \\
86.8738 \\
86.7947 \\
87.4228 \\
87.2940 \\
87.3860 \\
87.2546 \\
74.8237 \\
87.1073 \\
39.1464 \\
87.3596 \\
81.2694 \\
87.2011 \\
74.2696 \\
87.1564 \\
87.1676 \\
86.9488 \\
87.4986 \\
87.3358 \\
87.2803 \\
87.2327 \\
87.5789 \\
87.0465 \\
87.1500 \\
86.8217\end{array}$ & $\begin{array}{l}\text { Max } \\
73.6941 \\
73.7485 \\
87.9672 \\
87.4801 \\
87.8527 \\
87.6878 \\
80.5039 \\
87.9595 \\
88.0358 \\
87.6567 \\
88.0580 \\
87.7659 \\
88.5814 \\
88.1783 \\
88.1028 \\
87.9031 \\
88.3306 \\
87.7006 \\
76.4045 \\
87.4988 \\
87.7269 \\
87.7644 \\
87.7162 \\
87.5315 \\
88.2655 \\
88.0333 \\
88.2256 \\
87.9908 \\
75.5645 \\
87.7379 \\
39.8352 \\
88.0957 \\
82.1120 \\
88.0393 \\
75.3307 \\
87.8940 \\
88.1083 \\
87.6846 \\
88.3383 \\
87.9664 \\
88.3265 \\
87.7599 \\
88.3183 \\
87.7834 \\
88.0946 \\
87.3494\end{array}$ & $\begin{array}{l}\text { Ave } \\
73.3312 \\
73.4598 \\
87.5033 \\
87.2044 \\
87.1843 \\
87.2955 \\
79.9597 \\
87.6051 \\
87.5531 \\
87.2821 \\
87.6406 \\
87.4942 \\
88.0581 \\
87.7953 \\
87.6929 \\
87.5522 \\
87.8454 \\
87.3593 \\
75.8426 \\
87.2230 \\
87.3214 \\
87.3880 \\
87.2764 \\
87.1592 \\
87.8167 \\
87.6386 \\
87.8500 \\
87.6443 \\
75.1820 \\
87.4165 \\
39.4725 \\
87.7406 \\
81.6886 \\
87.5597 \\
74.8492 \\
87.5253 \\
87.6094 \\
87.3718 \\
87.9082 \\
87.6489 \\
87.7860 \\
87.5301 \\
87.9445 \\
87.4865 \\
87.6771 \\
87.1110\end{array}$ & $\begin{array}{c}\text { SD } \\
1.8929 e-01 \\
1.3402 e-01 \\
2.1529 e-01 \\
1.3093 e-01 \\
2.2404 e-01 \\
1.5849 e-01 \\
2.6809 e-01 \\
1.5847 e-01 \\
2.1830 e-01 \\
1.8605 e-01 \\
2.0054 e-01 \\
1.6322 e-01 \\
2.2825 e-01 \\
1.6479 e-01 \\
2.1170 e-01 \\
1.9536 e-01 \\
2.1181 e-01 \\
1.5196 e-01 \\
2.6866 e-01 \\
1.5582 e-01 \\
1.7649 e-01 \\
2.4595 e-01 \\
1.7881 e-01 \\
1.5638 e-01 \\
1.9009 e-01 \\
1.7289 e-01 \\
2.1721 e-01 \\
1.6887 e-01 \\
1.9099 e-01 \\
1.4305 e-01 \\
2.2766 e-01 \\
1.6407 e-01 \\
1.8947 e-01 \\
1.9045 e-01 \\
2.5733 e-01 \\
1.7192 e-01 \\
2.4425 e-01 \\
1.6361 e-01 \\
1.8138 e-01 \\
1.6078 e-01 \\
2.6564 e-01 \\
1.2162 e-01 \\
1.7871 e-01 \\
1.6546 e-01 \\
2.1992 e-01 \\
1.3437 e-01\end{array}$ & $\begin{array}{l}\text { Uncert } \\
1.9000 e+00 \\
1.9000 e+00 \\
1.9002 e+00 \\
1.9002 e+00 \\
1.9002 e+00 \\
1.9002 e+00 \\
1.9001 e+00 \\
1.9002 e+00 \\
1.9002 e+00 \\
1.9002 e+00 \\
1.9002 e+00 \\
1.9002 e+00 \\
1.9002 e+00 \\
1.9002 e+00 \\
1.9002 e+00 \\
1.9002 e+00 \\
1.9002 e+00 \\
1.9002 e+00 \\
1.9000 e+00 \\
1.9002 e+00 \\
1.9002 e+00 \\
1.9002 e+00 \\
1.9002 e+00 \\
1.9002 e+00 \\
1.9002 e+00 \\
1.9002 e+00 \\
1.9002 e+00 \\
1.9002 e+00 \\
1.9000 e+00 \\
1.9002 e+00 \\
1.9010 e+00 \\
1.9002 e+00 \\
1.9001 e+00 \\
1.9002 e+00 \\
1.9000 e+00 \\
1.9002 e+00 \\
1.9002 e+00 \\
1.9002 e+00 \\
1.9002 e+00 \\
1.9002 e+00 \\
1.9002 e+00 \\
1.9022 e+00 \\
1.9002 e+00 \\
1.9002 e+00 \\
1.9002 e+00 \\
1.9002 e+00\end{array}$ \\
\hline
\end{tabular}

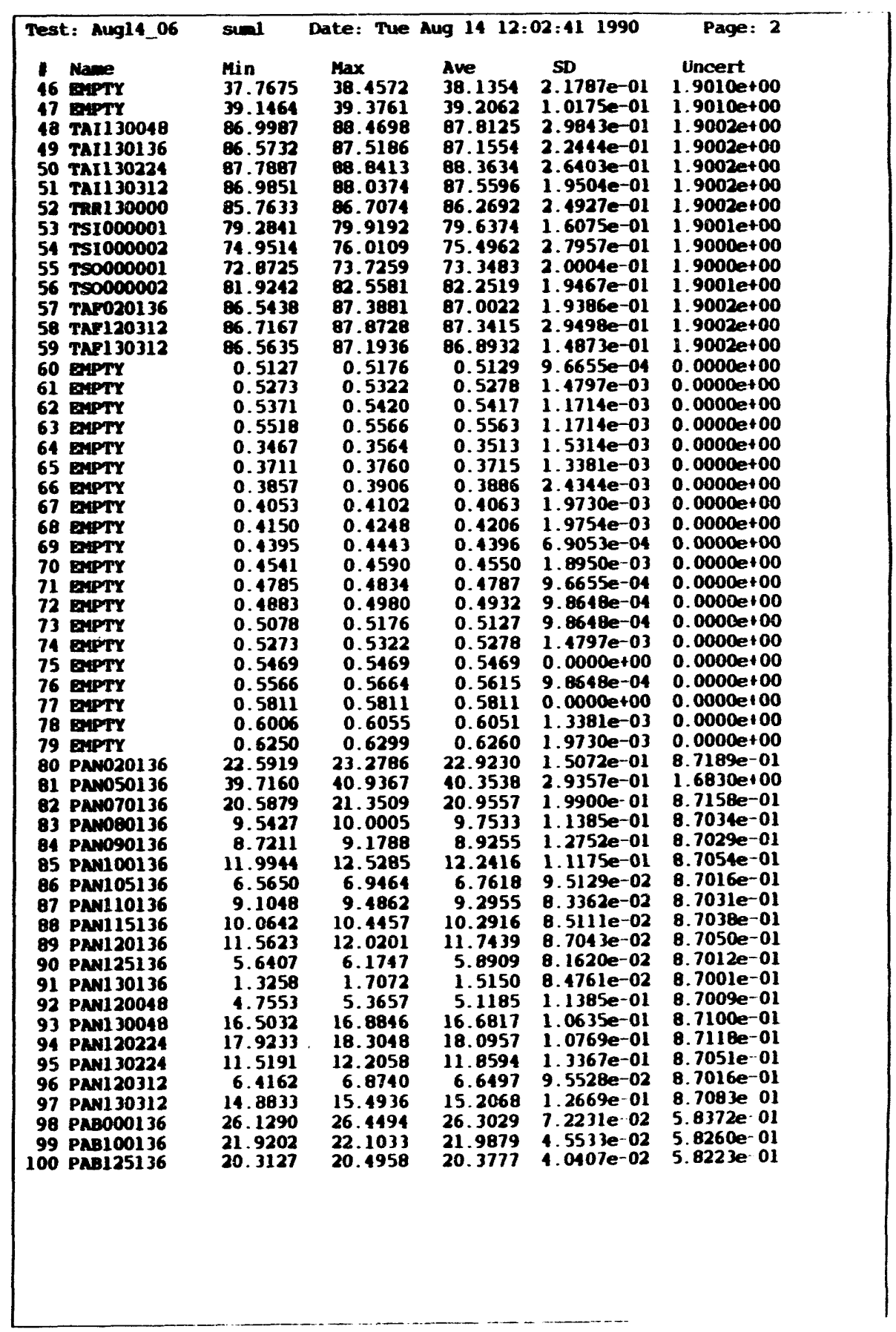



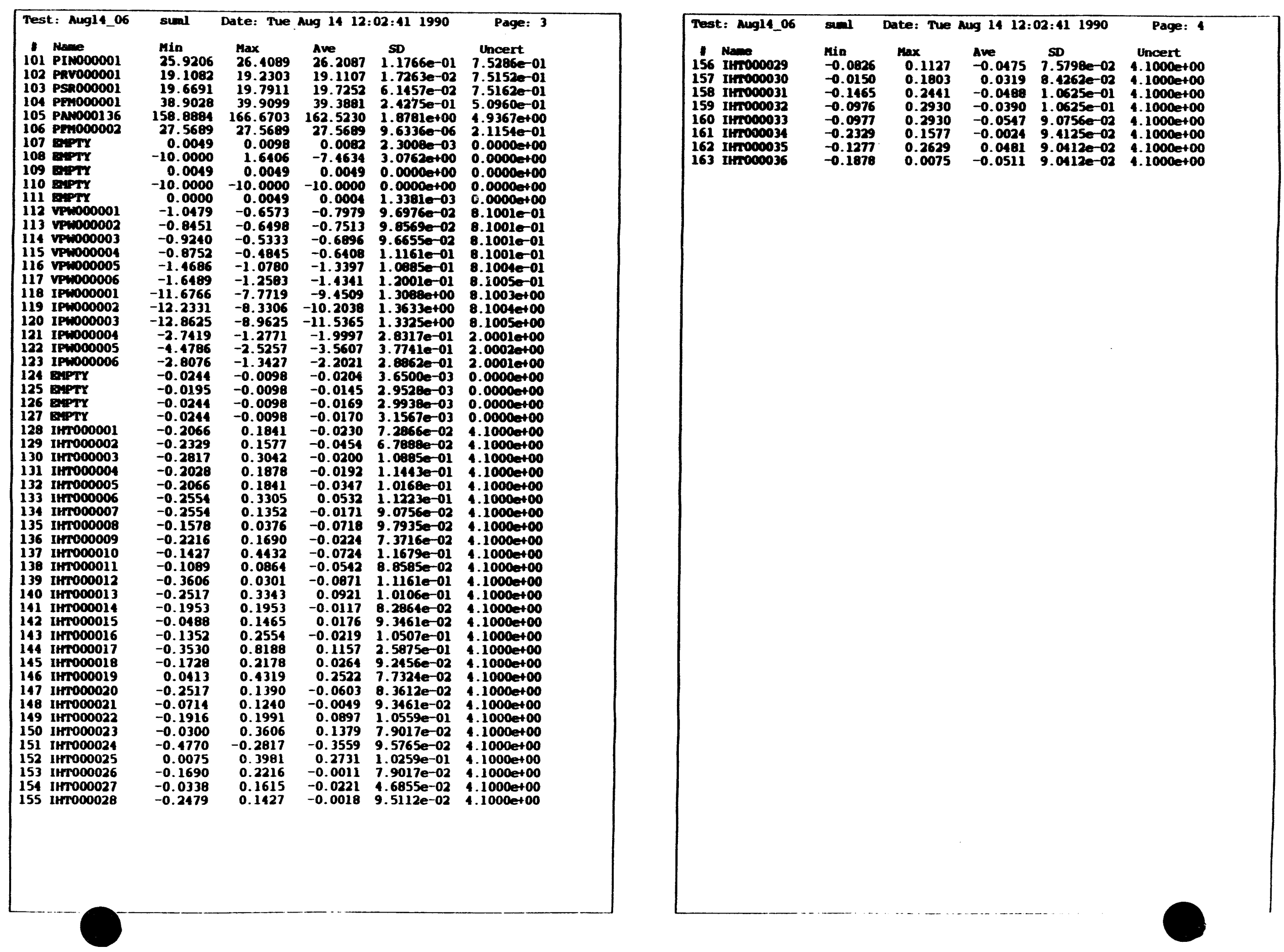


\begin{tabular}{|c|c|c|c|c|c|c|}
\hline \multicolumn{4}{|c|}{ 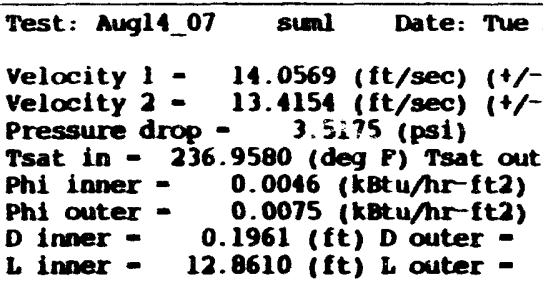 } & \multicolumn{2}{|c|}{$\begin{array}{l}\text { Aug } 1412: 06: 021990 \\
1.8644 \text { :) } \\
1.7033 \text { :) } \\
-227.9219 \text { (cleg F) } \\
(+/-130.7655 \text { s) } \\
(+/-122.9316 \text { : }) \\
0.2113 \text { (ft) D hyd - } \\
12.8070 \text { (ft) A flow - }\end{array}$} & $\begin{array}{l}\text { Page: } 1 \\
0.0390 \text { (ft) } \\
0.0150 \text { (ft2) }\end{array}$ \\
\hline $\begin{array}{l}1 \\
1 \\
2 \\
3 \\
1 \\
5 \\
6 \\
7 \\
8 \\
9 \\
10 \\
11 \\
12 \\
13 \\
14 \\
15 \\
16 \\
17 \\
18 \\
19 \\
20 \\
21 \\
22 \\
23 \\
24 \\
25 \\
26 \\
27 \\
28 \\
29 \\
30 \\
31 \\
32 \\
33 \\
34 \\
35 \\
36 \\
37 \\
38 \\
39 \\
40 \\
11 \\
42 \\
43 \\
44 \\
45\end{array}$ & 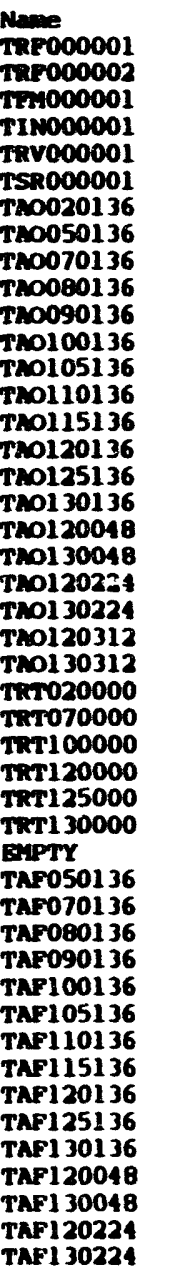 & $\begin{array}{l}\text { Min } \\
73.0535 \\
73.3239 \\
87.1277 \\
87.0586 \\
87.0134 \\
86.9541 \\
79.5508 \\
87.4347 \\
87.1921 \\
87.0282 \\
87.4282 \\
87.3443 \\
87.7403 \\
87.6542 \\
87.4717 \\
87.3772 \\
87.5952 \\
87.0720 \\
75.5557 \\
87.0771 \\
86.9909 \\
87.1378 \\
86.8745 \\
86.9003 \\
87.3177 \\
87.4004 \\
87.5954 \\
87.4651 \\
74.8237 \\
87.2123 \\
39.1464 \\
87.4651 \\
81.3755 \\
87.3061 \\
74.3763 \\
87.2619 \\
87.2726 \\
87.1600 \\
87.6036 \\
87.5449 \\
87.3859 \\
87.3383 \\
87.5794 \\
87.3619 \\
87.4645 \\
86.9277\end{array}$ & $\begin{array}{l}\max \\
73.6941 \\
73.7485 \\
88.0713 \\
87.5848 \\
87.8513 \\
87.5828 \\
80.5045 \\
88.0633 \\
88.1410 \\
87.6567 \\
88.3728 \\
87.9754 \\
88.5820 \\
88.3870 \\
88.1025 \\
88.0080 \\
88.3306 \\
87.8020 \\
76.6169 \\
87.6024 \\
87.7269 \\
87.8699 \\
87.7162 \\
87.5298 \\
88.2651 \\
88.0333 \\
88.3300 \\
87.9914 \\
75.8917 \\
87.9479 \\
39.8352 \\
88.1993 \\
82.2162 \\
88.1421 \\
75.1371 \\
87.9989 \\
88.3164 \\
87.7887 \\
88.4427 \\
87.9664 \\
88.4320 \\
87.9693 \\
88.5295 \\
87.9931 \\
88.2001 \\
87.4542\end{array}$ & $\begin{array}{l}\text { Ave } \\
73.2841 \\
73.4598 \\
87.6395 \\
87.2779 \\
87.3410 \\
87.2807 \\
80.0676 \\
87.7030 \\
87.6817 \\
87.3298 \\
87.7556 \\
87.5894 \\
88.1526 \\
87.9011 \\
87.7958 \\
87.6633 \\
87.9353 \\
87.4685 \\
76.0904 \\
87.2667 \\
87.4199 \\
87.4771 \\
87.3710 \\
87.2659 \\
87.8609 \\
87.7168 \\
87.9691 \\
87.7384 \\
75.4535 \\
87.5106 \\
39.5506 \\
87.8387 \\
81.7745 \\
87.6782 \\
74.9359 \\
87.6073 \\
87.7653 \\
87.4656 \\
88.0274 \\
87.7260 \\
87.8378 \\
87.5823 \\
88.0481 \\
87.5724 \\
87.7670 \\
87.1258\end{array}$ & 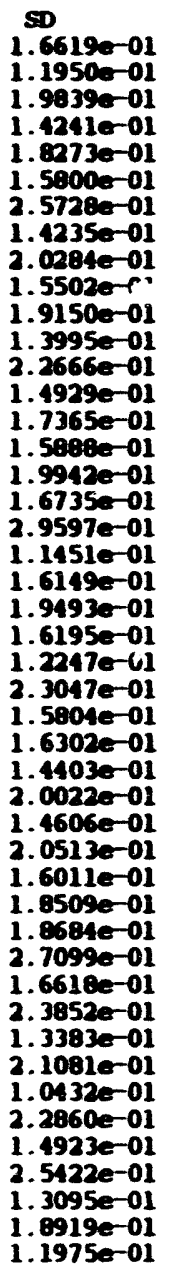 & $\begin{array}{c}\text { Uncert } \\
1.9000 e+00 \\
1.9000 e+00 \\
1.9002 e+00 \\
1.9002 e+00 \\
1.9002 e+00 \\
1.9002 e+00 \\
1.9001 e+00 \\
1.9002 e+00 \\
1.9002 e+00 \\
1.9002 e+00 \\
1.9002 e+00 \\
1.9002 e+00 \\
1.9002 e+00 \\
1.9002 e+00 \\
1.9002 e+00 \\
1.9002 e+00 \\
1.9002 e+00 \\
1.9002 e+00 \\
1.9000 e+00 \\
1.90020+00 \\
1.9002 e+00 \\
1.9002 e+00 \\
1.9002 e+00 \\
1.9002 e+00 \\
1.9002 e+00 \\
1.9002 e+00 \\
1.9002 e+00 \\
1.9002 e+00 \\
1.9000 e+00 \\
1.9002 e+00 \\
1.9011 e+00 \\
1.9002 e+00 \\
1.9001 e+00 \\
1.9002 e+00 \\
1.9000 e+00 \\
1.9002 e+00 \\
1.9002 e+00 \\
1.9002 e+00 \\
1.9002 e+00 \\
1.9002 e+00 \\
1.9002 e+00 \\
1.9002 e+00 \\
1.9002 e+00 \\
1.9002 e+00 \\
1.9002 e+00 \\
1.9002 e+00\end{array}$ \\
\hline
\end{tabular}

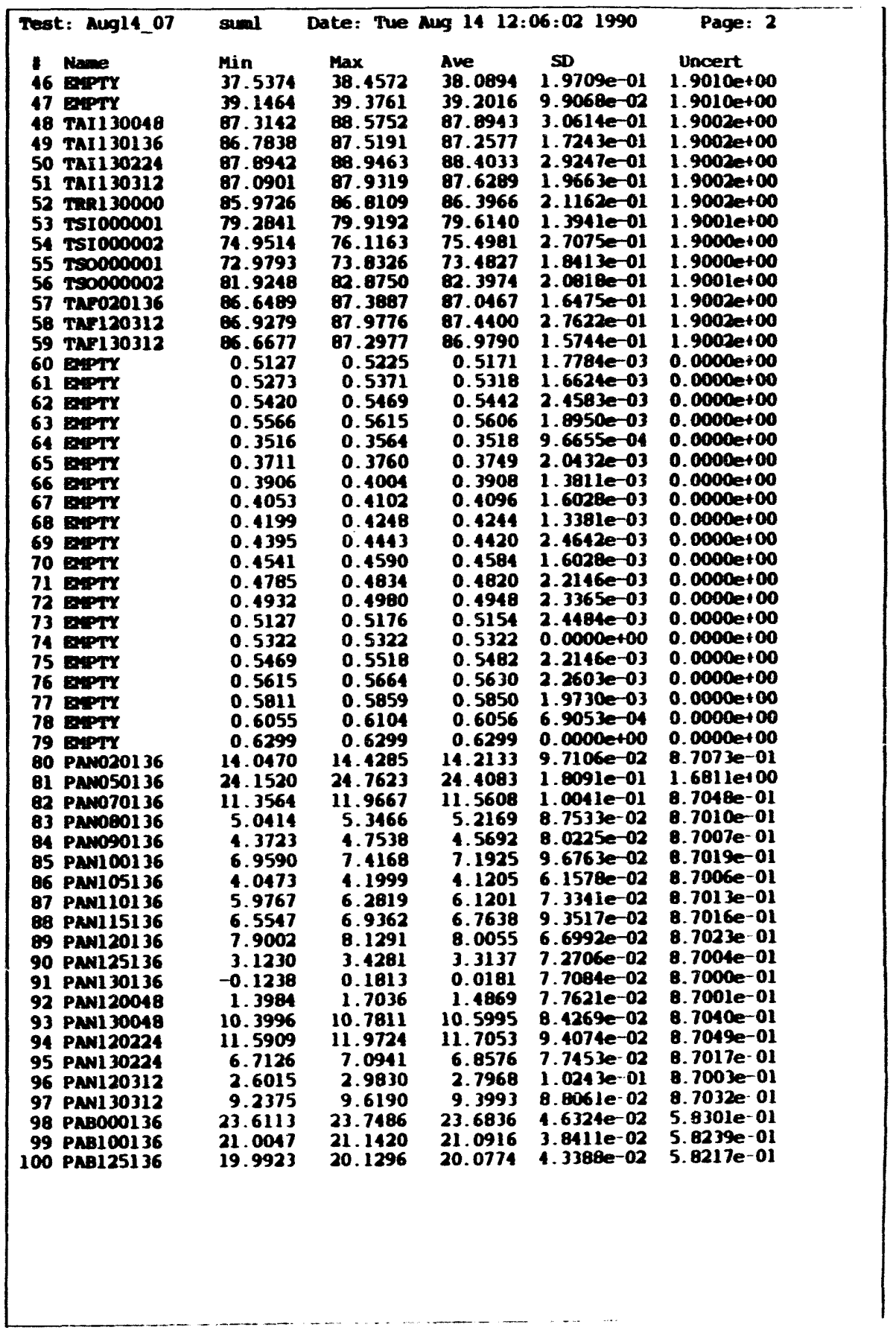



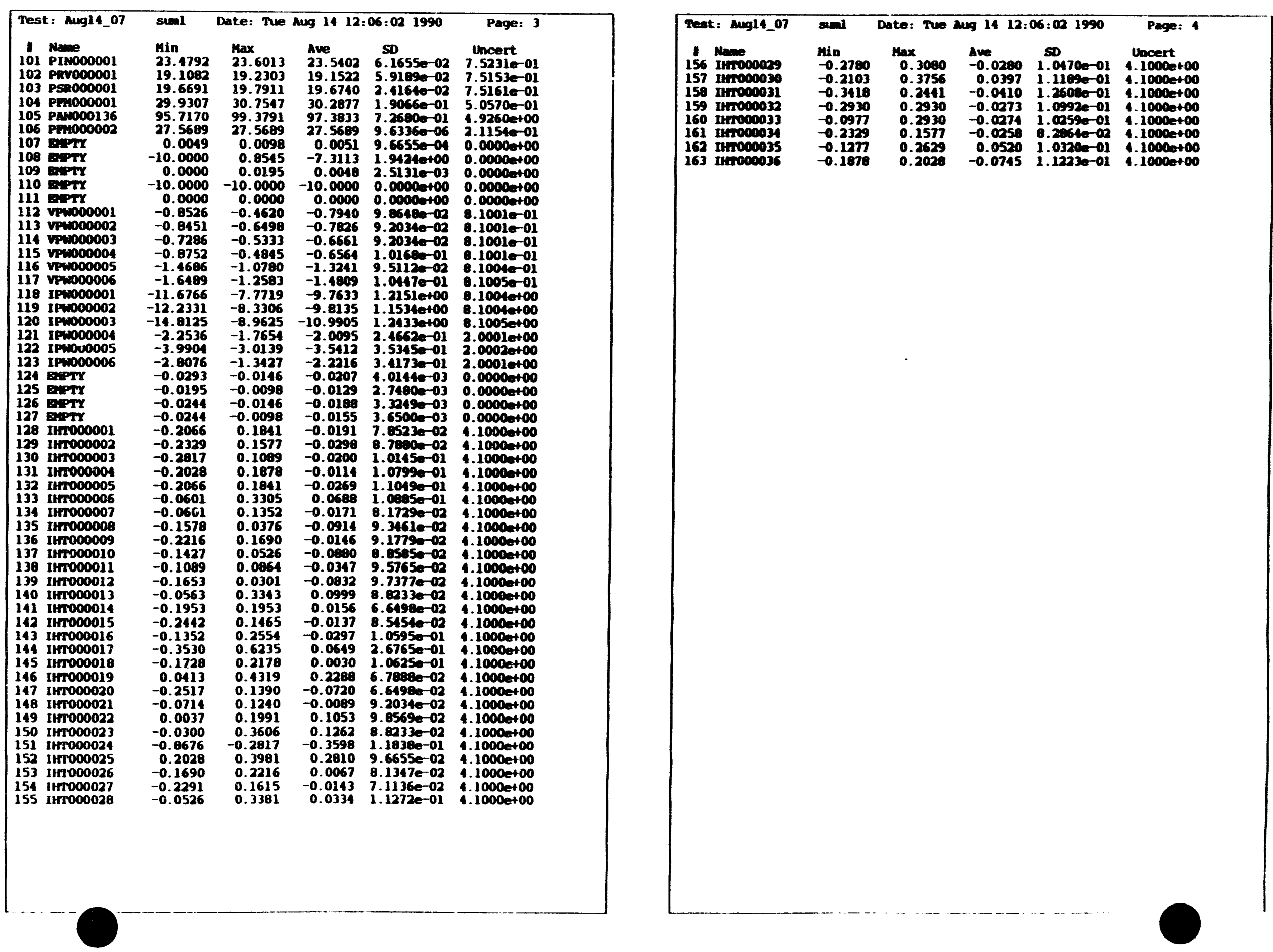


\begin{tabular}{|c|c|c|c|c|c|c|}
\hline \multicolumn{4}{|c|}{ 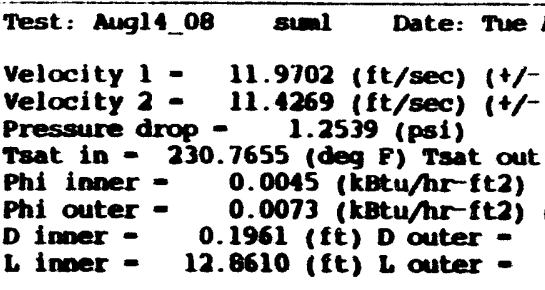 } & \multicolumn{2}{|c|}{$\begin{array}{l}\text { Aug } 1412: 12: 441990 \\
2.0240 \text { :) } \\
1.7315 \mathrm{~B}) \\
-227.0917 \text { (deg F) } \\
(+/-132.0604 \mathrm{~B}) \\
(+/-124.7036 \mathrm{~s}) \\
0.2413 \text { (ft) D hyd - } \\
12.8070 \text { (ft) A flow - }\end{array}$} & $\begin{array}{l}0.0390 \text { (ft) } \\
0.0150 \text { (ft2) }\end{array}$ \\
\hline $\begin{array}{l}1 \\
1 \\
1 \\
2 \\
3 \\
1 \\
5 \\
6 \\
7 \\
8 \\
9 \\
10 \\
11 \\
12 \\
13 \\
14 \\
15 \\
16 \\
17 \\
18 \\
19 \\
20 \\
21 \\
22 \\
23 \\
24 \\
25 \\
26 \\
27 \\
28 \\
29 \\
30 \\
31 \\
32 \\
33 \\
34 \\
35 \\
36 \\
37 \\
38 \\
39 \\
40 \\
41 \\
12 \\
13 \\
44 \\
15\end{array}$ & 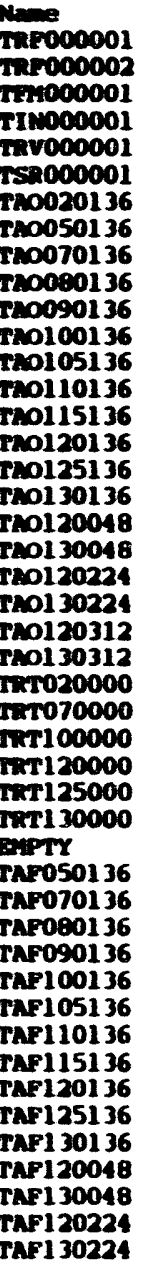 & $\begin{array}{l}\text { Min } \\
73.0535 \\
73.3239 \\
87.5465 \\
87.2689 \\
87.2226 \\
87.0576 \\
79.8682 \\
87.5386 \\
87.6145 \\
87.1319 \\
87.5326 \\
87.5539 \\
88.0554 \\
87.8624 \\
87.6819 \\
87.4822 \\
87.9092 \\
87.4860 \\
75.7682 \\
87.2863 \\
87.3059 \\
87.3458 \\
87.2948 \\
87.2154 \\
87.8441 \\
87.6118 \\
87.9097 \\
87.6749 \\
75.0345 \\
87.5264 \\
39.1464 \\
87.7792 \\
81.4793 \\
87.6178 \\
74.2696 \\
87.5780 \\
87.3759 \\
87.3685 \\
87.7081 \\
87.7539 \\
87.5942 \\
87.5478 \\
87.8966 \\
87.5716 \\
87.6747 \\
87.1387\end{array}$ & $\begin{array}{l}\text { Max } \\
73.6941 \\
73.7485 \\
89.3864 \\
88.0060 \\
88.0623 \\
88.0023 \\
80.8216 \\
88.2737 \\
88.4569 \\
88.2843 \\
88.3728 \\
88.2899 \\
88.8967 \\
88.4896 \\
88.8374 \\
88.3233 \\
86.7493 \\
88.4261 \\
77.0405 \\
87.8129 \\
88.1471 \\
89.2883 \\
88.1369 \\
88.0552 \\
88.5809 \\
88.4552 \\
88.7495 \\
86.5155 \\
75.5663 \\
88.1574 \\
40.0646 \\
88.5138 \\
82.3222 \\
88.3524 \\
75.5423 \\
88.4197 \\
88.1219 \\
87.9991 \\
88.7574 \\
88.2802 \\
88.6401 \\
88.2835 \\
88.8452 \\
88.3076 \\
88.4095 \\
87.7712\end{array}$ & $\begin{array}{l}\text { Ave } \\
73.3482 \\
73.5065 \\
87.9793 \\
87.6017 \\
87.6133 \\
87.5069 \\
80.3513 \\
87.9707 \\
88.0273 \\
87.6308 \\
88.0407 \\
87.8653 \\
88.4127 \\
88.1723 \\
89.1405 \\
87.8902 \\
88.2832 \\
87.7591 \\
76.3364 \\
87.5516 \\
87.7097 \\
87.7856 \\
87.6403 \\
87.5343 \\
88.2020 \\
87.9998 \\
88.3168 \\
88.0364 \\
75.2855 \\
87.7790 \\
39.5414 \\
88.1526 \\
81.9722 \\
87.9703 \\
75.0081 \\
87.9398 \\
88.0032 \\
87.7378 \\
88.2914 \\
87.9939 \\
88.1090 \\
87.8339 \\
88.3690 \\
87.8452 \\
88.0857 \\
87.1126\end{array}$ & 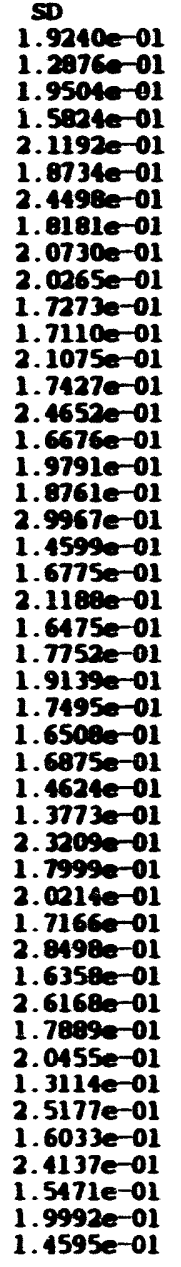 & 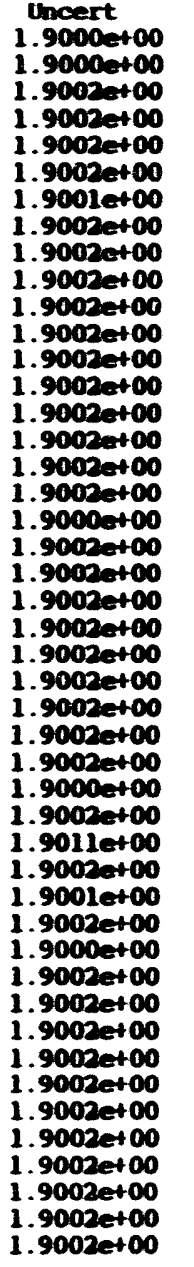 \\
\hline
\end{tabular}

\begin{tabular}{|c|c|c|c|c|c|}
\hline 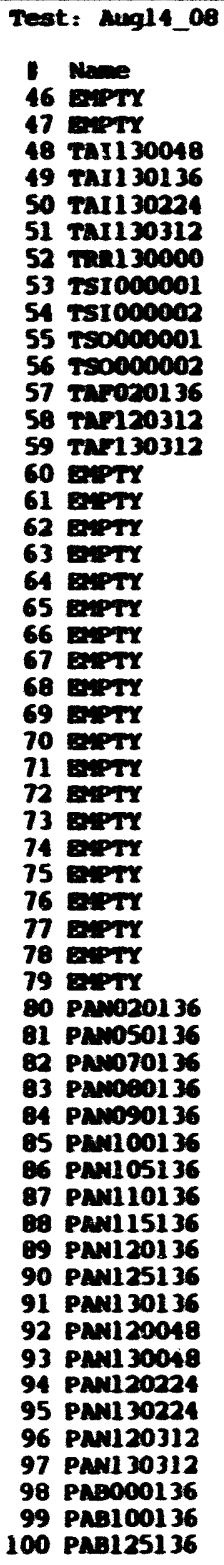 & 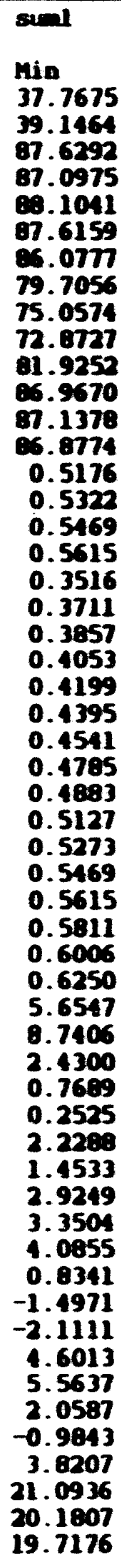 & $\begin{array}{l}\text { Date: Tue } \\
\text { Max } \\
38.4572 \\
39.3761 \\
69.6796 \\
87.9376 \\
89.0517 \\
89.2475 \\
87.2312 \\
80.3404 \\
76.3297 \\
73.9392 \\
83.0958 \\
87.8116 \\
69.1873 \\
87.6138 \\
0.5273 \\
0.5371 \\
0.5518 \\
0.5713 \\
0.3564 \\
0.3760 \\
0.3906 \\
0.4102 \\
0.4248 \\
0.4443 \\
0.4689 \\
0.4834 \\
0.4960 \\
0.5176 \\
0.5371 \\
0.5518 \\
0.5664 \\
0.5859 \\
0.6104 \\
0.6299 \\
5.9598 \\
9.3510 \\
2.7351 \\
0.9978 \\
0.5576 \\
2.5340 \\
1.7585 \\
3.1538 \\
3.6556 \\
1.6196 \\
1.1393 \\
-1.2682 \\
-1.8060 \\
1.9065 \\
5.7926 \\
2.3639 \\
-0.6791 \\
3.9733 \\
21.2309 \\
20.3160 \\
19.8092\end{array}$ & $\begin{array}{l}\text { ueg } 1412 \\
\text { Ave } \\
38.0986 \\
39.2245 \\
6.2512 \\
87.5218 \\
88.6853 \\
87.9317 \\
86.6648 \\
60.0180 \\
75.6126 \\
73.5092 \\
62.7054 \\
87.3891 \\
87.7297 \\
67.2770 \\
0.5223 \\
0.5370 \\
0.5486 \\
0.5646 \\
0.3521 \\
0.3749 \\
0.3905 \\
0.4098 \\
0.1245 \\
0.4115 \\
0.4599 \\
0.4814 \\
0.4939 \\
0.5144 \\
0.5316 \\
0.5471 \\
0.5618 \\
0.5823 \\
0.6056 \\
0.6297 \\
5.8317 \\
9.0702 \\
2.5963 \\
0.8971 \\
0.3694 \\
2.3914 \\
1.6104 \\
3.0577 \\
3.4969 \\
4.3846 \\
0.9944 \\
-1.4071 \\
-1.9326 \\
4.7951 \\
5.6614 \\
2.2158 \\
-0.8424 \\
3.9061 \\
21.1659 \\
20.3567 \\
19.7671\end{array}$ & 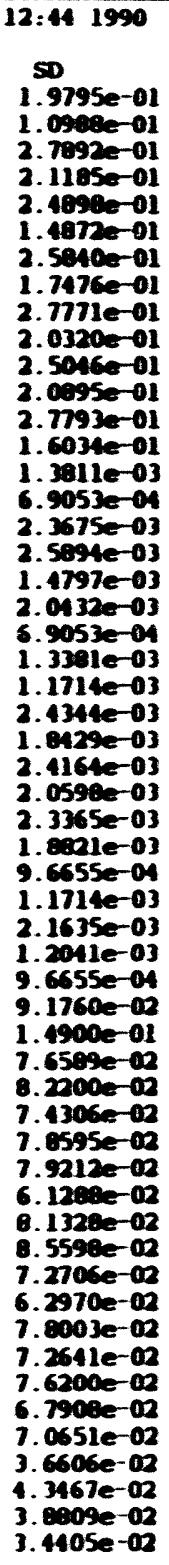 & 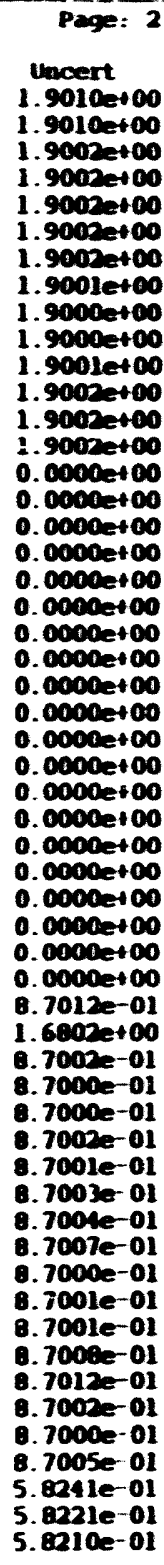 \\
\hline
\end{tabular}




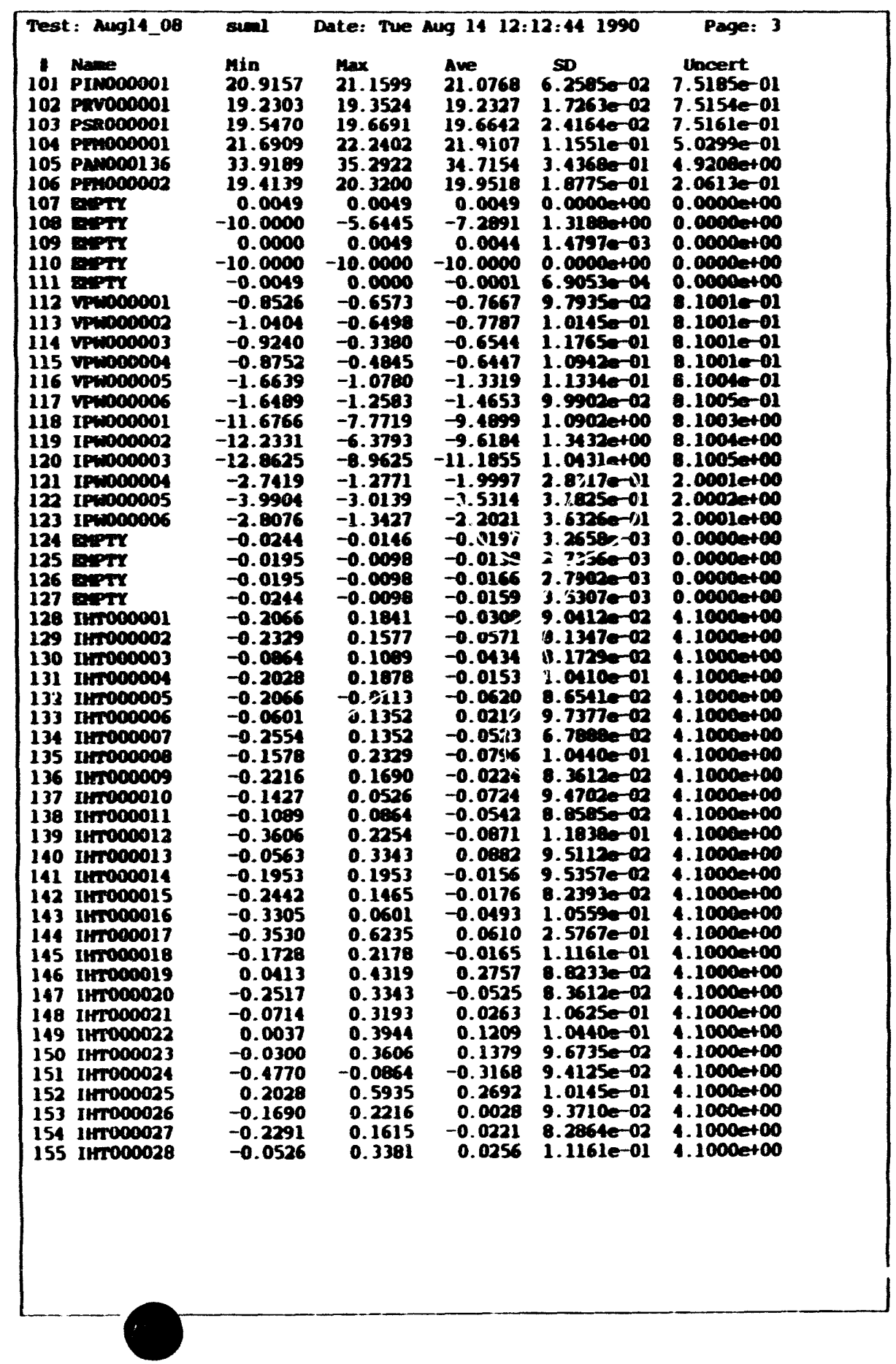

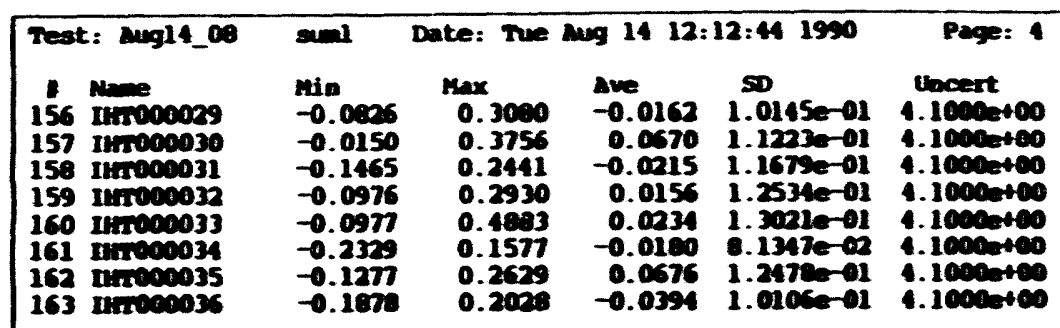




\begin{tabular}{|c|c|c|c|c|c|}
\hline \multicolumn{3}{|c|}{ 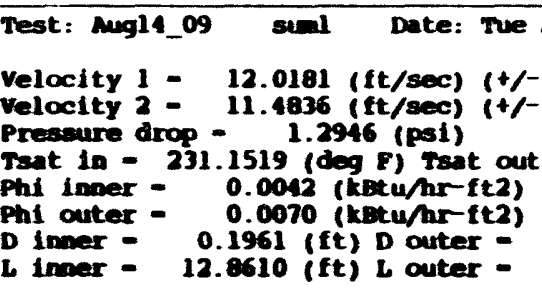 } & \multicolumn{2}{|c|}{ 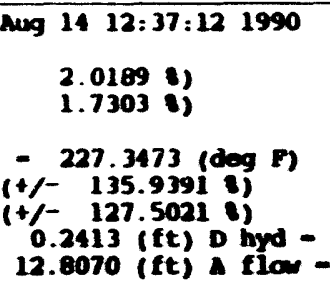 } & $\begin{array}{l}0.0390 \text { (ft) } \\
0.0150 \text { (ftz) }\end{array}$ \\
\hline 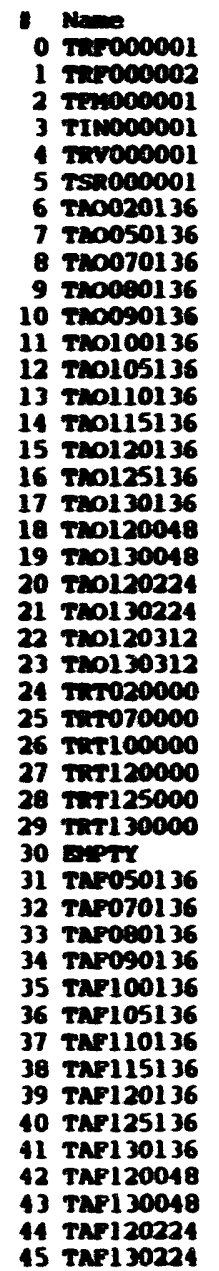 & 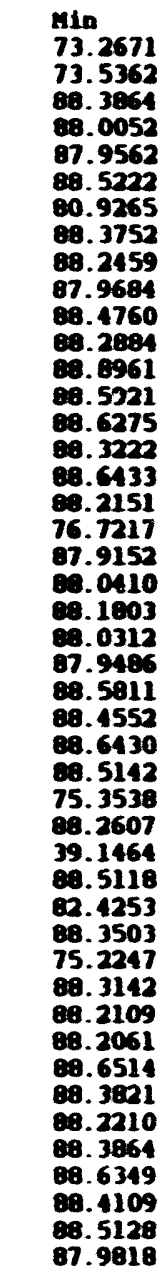 & 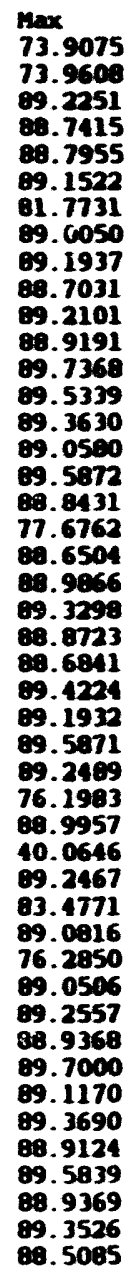 & 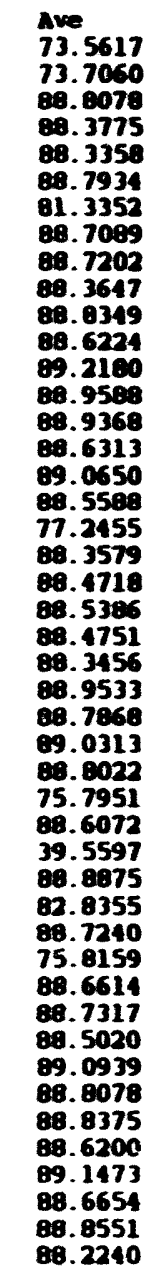 & 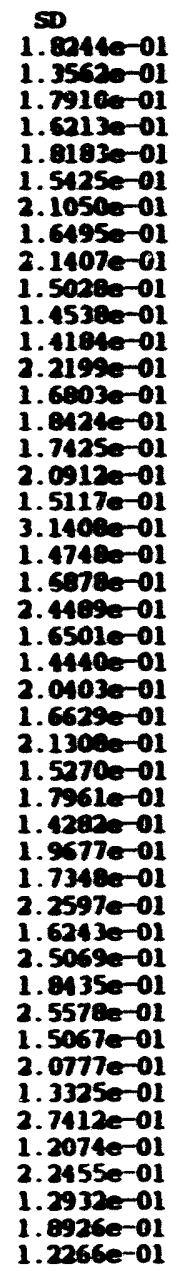 & 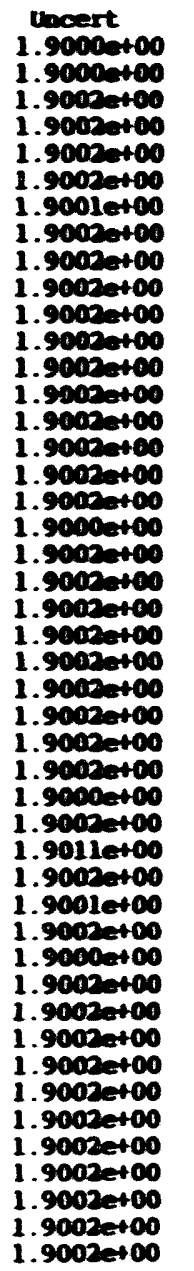 \\
\hline
\end{tabular}

\begin{tabular}{|c|c|c|c|c|c|}
\hline 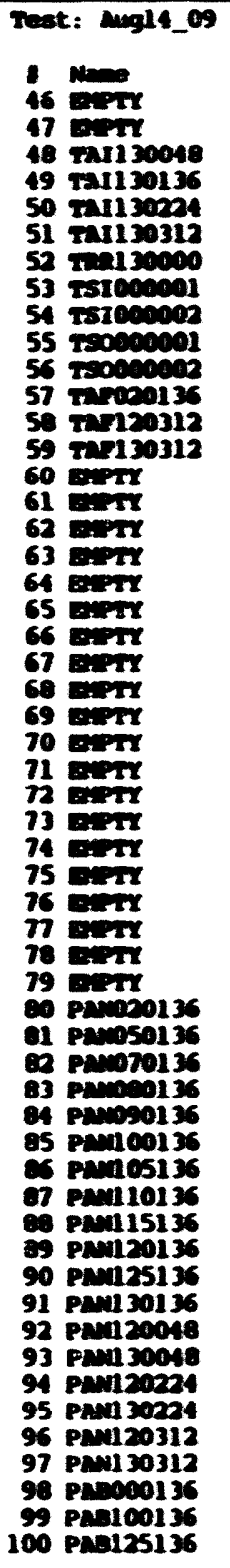 & 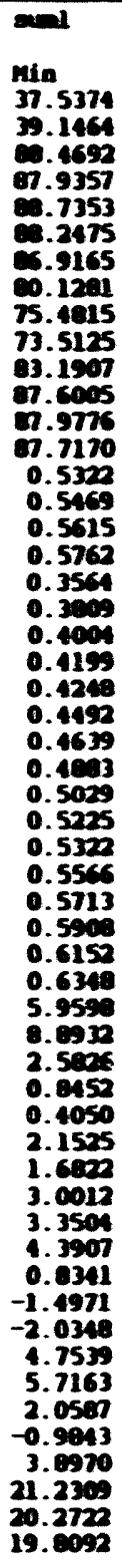 & 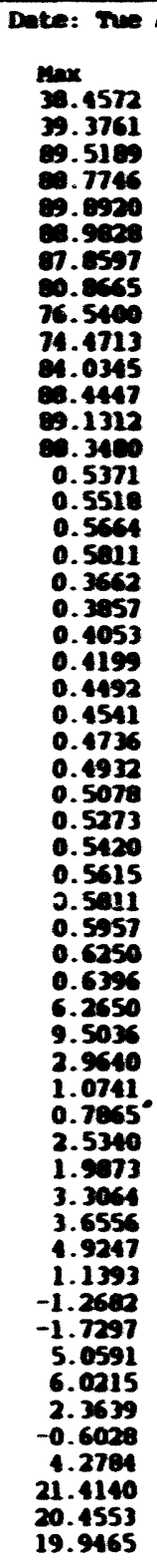 & 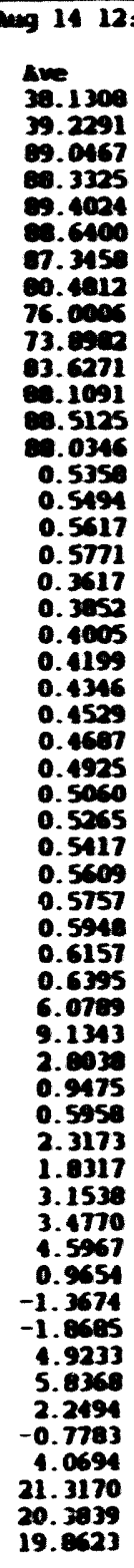 & 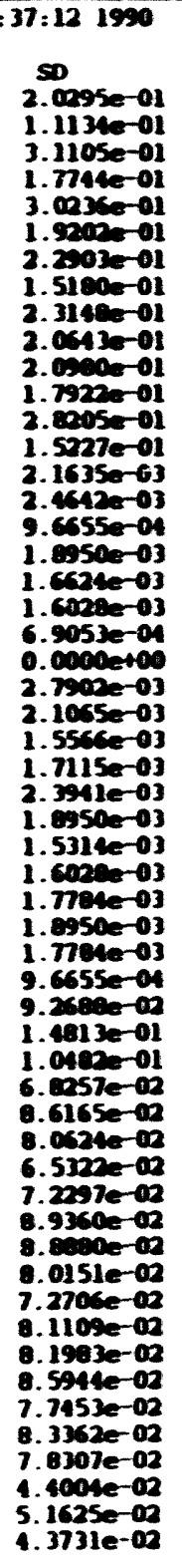 & 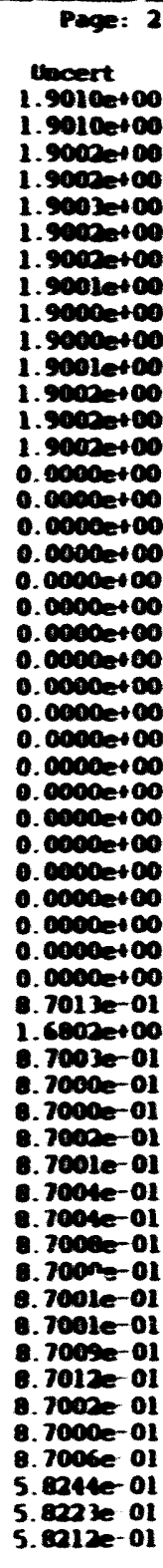 \\
\hline
\end{tabular}




\begin{tabular}{|c|c|c|c|c|c|c|}
\hline $\begin{array}{l}\text { Test } \\
11 \\
101 \\
102 \\
103 \\
104 \\
105 \\
105 \\
107 \\
109 \\
109 \\
110 \\
111 \\
112 \\
113 \\
114 \\
115 \\
116 \\
117 \\
118 \\
119 \\
120 \\
121 \\
122 \\
123 \\
124 \\
125 \\
126 \\
127 \\
120 \\
129 \\
130 \\
131 \\
132 \\
133 \\
134 \\
135 \\
136 \\
137 \\
139 \\
139 \\
140 \\
141 \\
142 \\
143 \\
144 \\
145 \\
146 \\
147 \\
148 \\
119 \\
150 \\
151 \\
152 \\
153 \\
154 \\
155\end{array}$ & 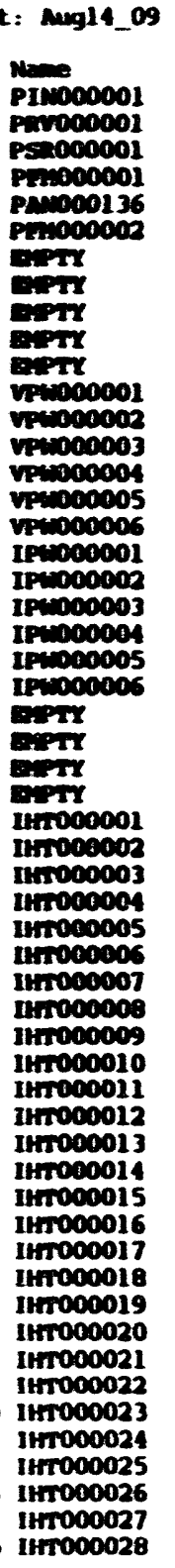 & 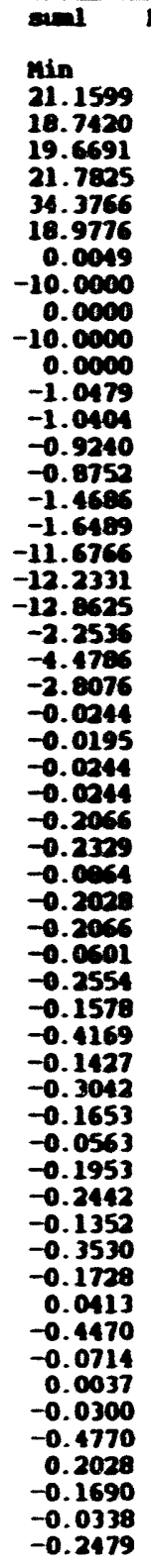 & $\begin{array}{l}\text { Date: Tue } \\
\text { Max } \\
21.5261 \\
19.1000 \\
19.7911 \\
22.4234 \\
37.1232 \\
20.8905 \\
0.0098 \\
-3.0615 \\
0.0049 \\
-10.0000 \\
0.0000 \\
-0.6573 \\
-0.4545 \\
-0.5333 \\
-0.4915 \\
-0.8927 \\
-1.2583 \\
-7.7719 \\
-6.3793 \\
-8.9625 \\
-1.2771 \\
-3.0139 \\
-1.3427 \\
0.0146 \\
-0.0698 \\
-0.0098 \\
-0.0098 \\
0.1841 \\
0.1577 \\
0.3042 \\
0.1878 \\
0.1841 \\
0.1352 \\
0.1352 \\
0.0376 \\
0.1690 \\
0.2479 \\
0.2017 \\
0.0301 \\
0.3343 \\
0.1953 \\
0.1465 \\
0.0601 \\
0.6235 \\
0.2178 \\
0.4319 \\
0.1390 \\
0.1240 \\
0.1991 \\
0.5559 \\
-0.0864 \\
0.3981 \\
0.2216 \\
0.1615 \\
0.3381\end{array}$ & 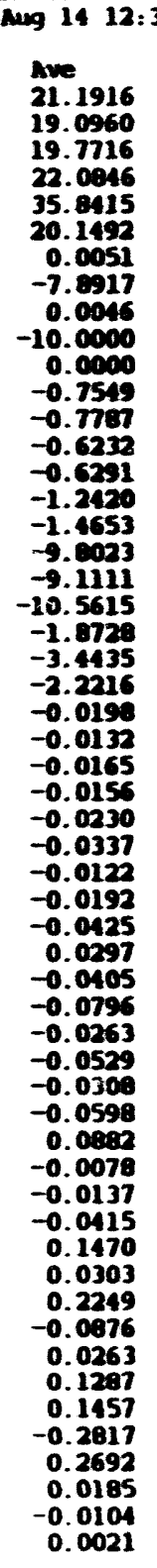 & 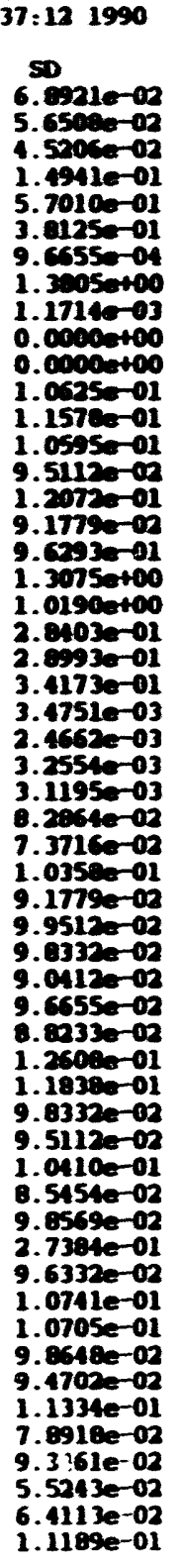 & 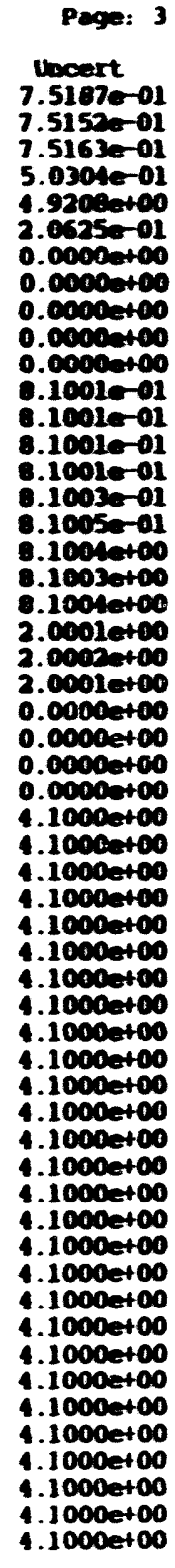 \\
\hline
\end{tabular}

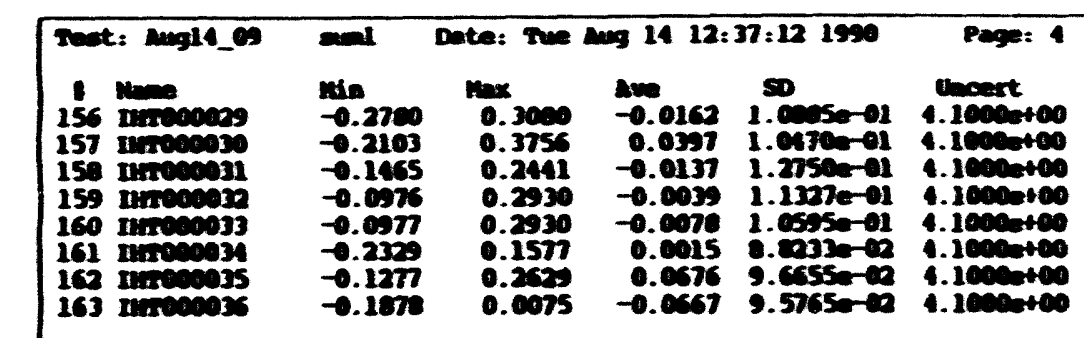




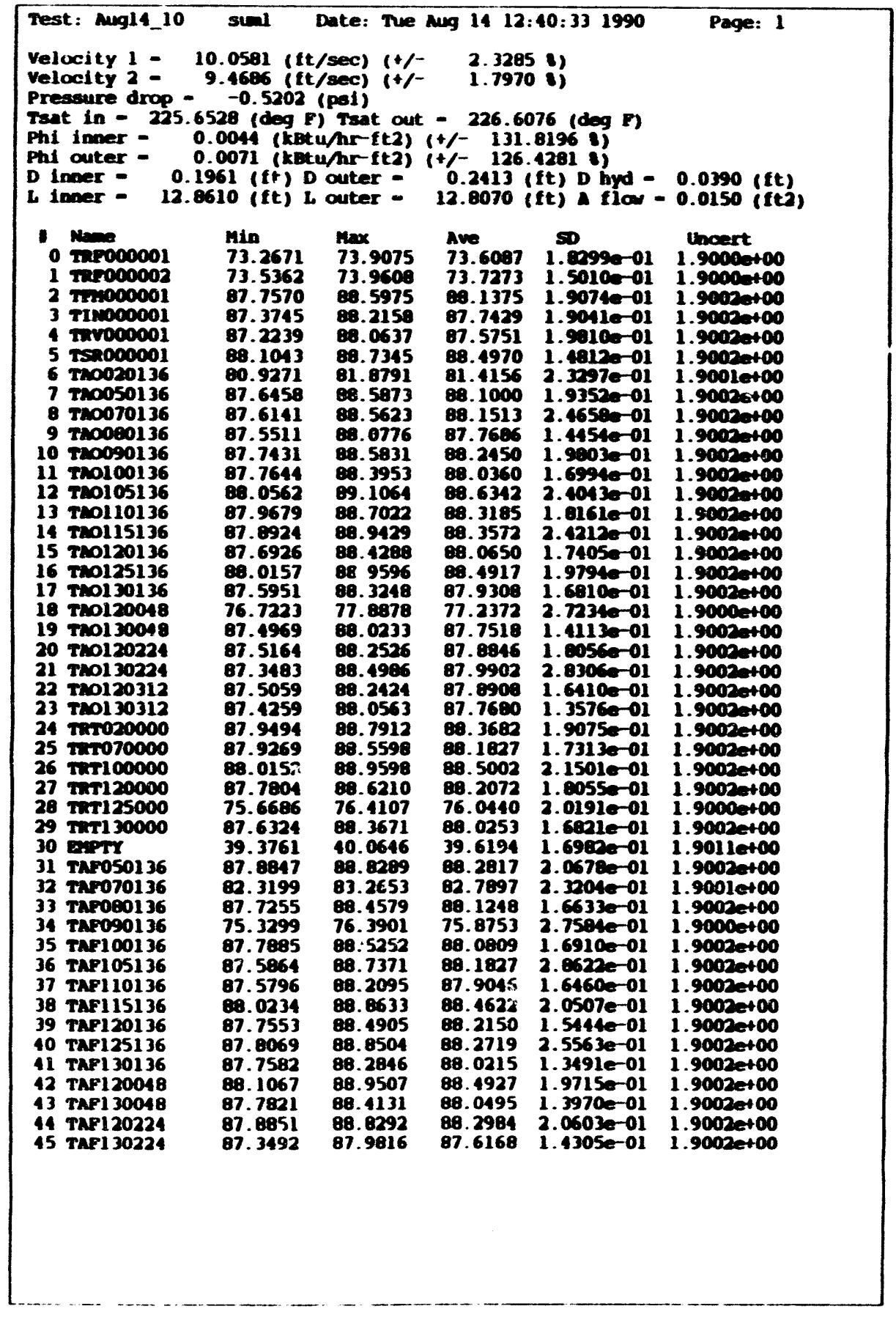

\begin{tabular}{|c|c|c|c|c|c|}
\hline 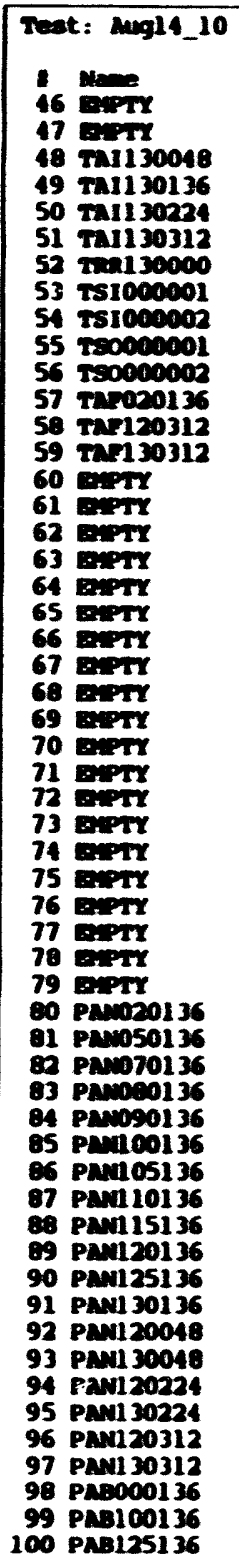 & 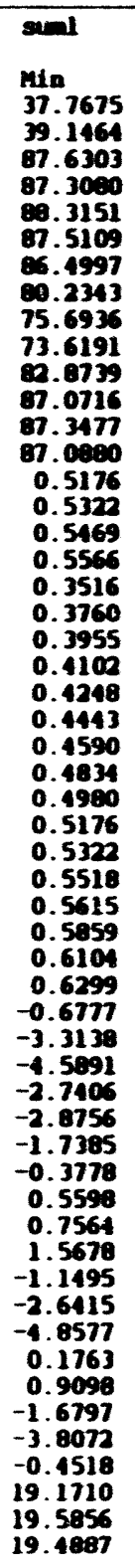 & 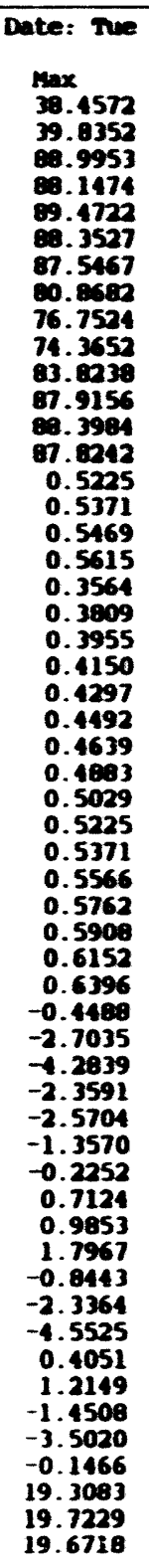 & 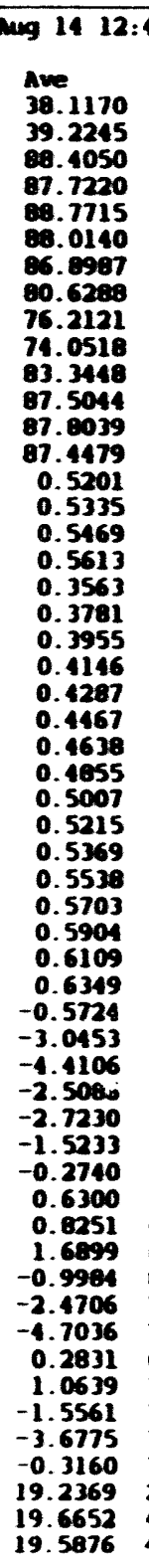 & 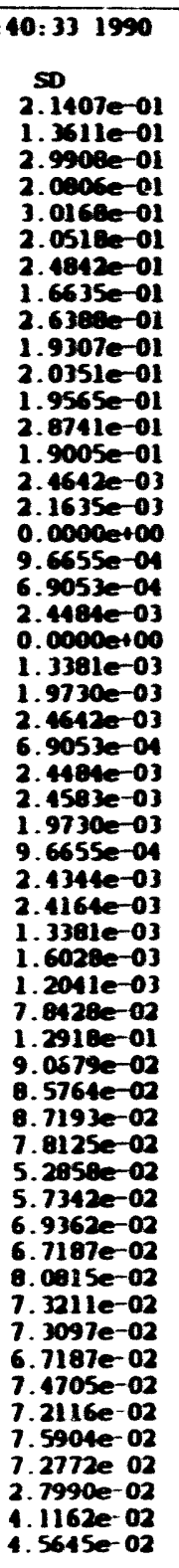 & 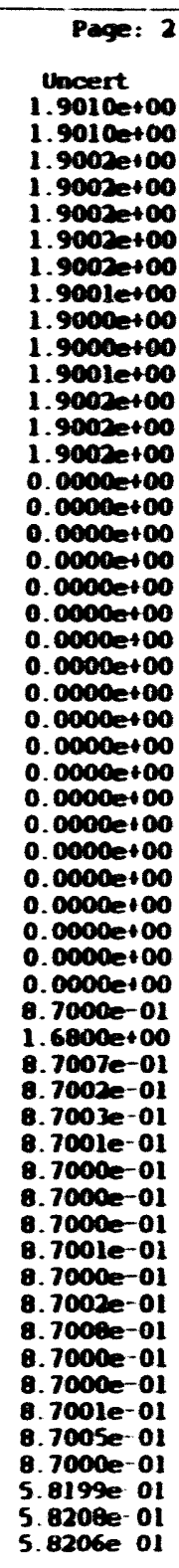 \\
\hline
\end{tabular}



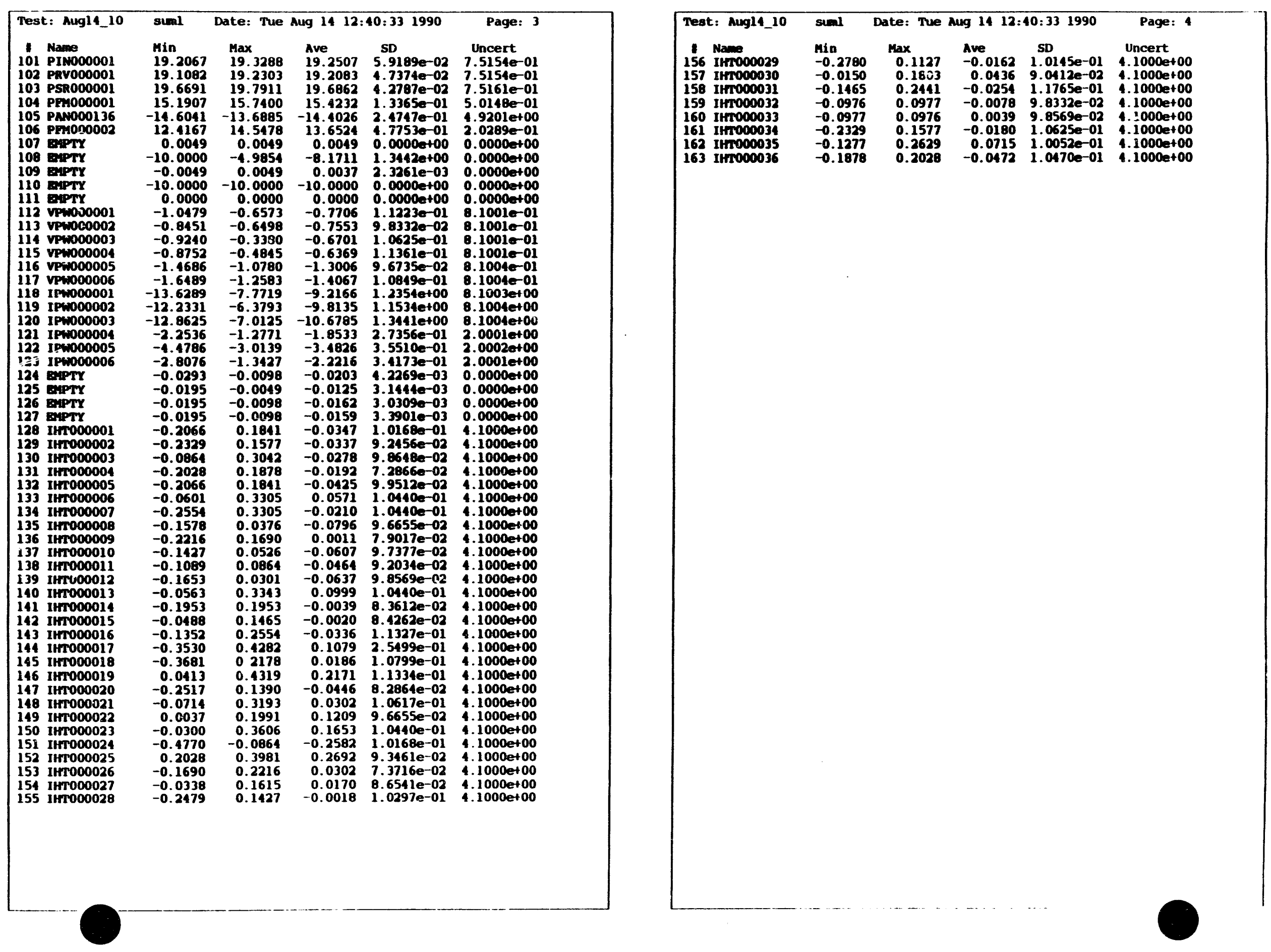


\begin{tabular}{|c|c|c|c|c|c|c|}
\hline \multicolumn{3}{|c|}{ 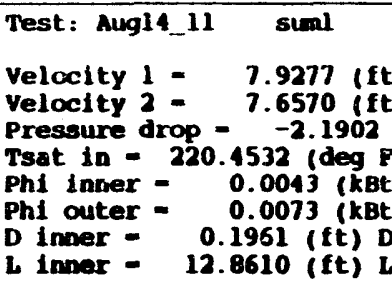 } & \multicolumn{3}{|c|}{ 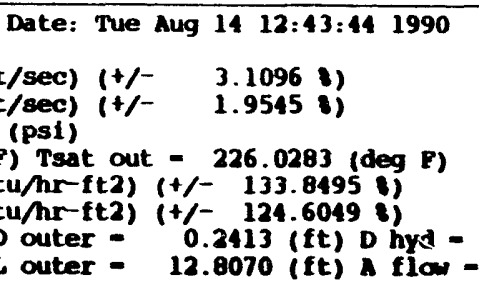 } & $\begin{array}{r}\text { Page: } 1 \\
\\
0.0390 \text { (ft) } \\
=0.0150 \text { (ft2) }\end{array}$ \\
\hline 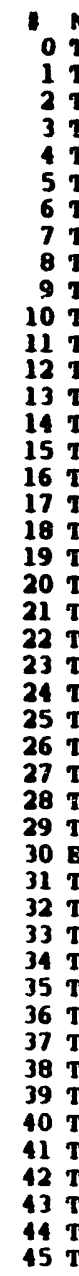 & 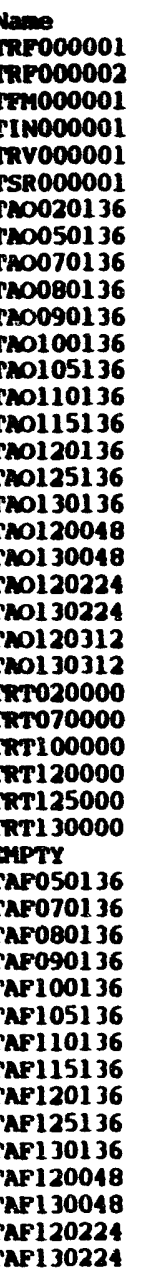 & $\begin{array}{l}\text { Min } \\
73.4806 \\
73.5362 \\
87.1432 \\
86.9541 \\
86.8069 \\
87.8968 \\
81.0326 \\
87.3332 \\
87.4029 \\
87.1356 \\
87.3259 \\
87.1364 \\
87.7407 \\
87.5545 \\
87.4723 \\
87.0640 \\
87.5978 \\
87.2862 \\
76.8282 \\
87.0798 \\
87.2021 \\
87.1427 \\
87.1901 \\
87.1120 \\
87.5280 \\
87.3993 \\
87.5976 \\
87.3617 \\
75.9871 \\
87.3189 \\
39.1464 \\
87.1679 \\
82.3216 \\
87.4139 \\
75.3313 \\
87.2619 \\
87.3804 \\
87.2664 \\
87.7102 \\
87.5478 \\
87.3904 \\
87.3403 \\
87.6835 \\
87.3635 \\
87.3615 \\
86.9269\end{array}$ & $\begin{array}{l}\text { Max } \\
74.1210 \\
73.9608 \\
88.0730 \\
87.5853 \\
87.4364 \\
88.4222 \\
81.9845 \\
88.0673 \\
88.1403 \\
87.6609 \\
88.1646 \\
87.7668 \\
88.5822 \\
88.1812 \\
88.2092 \\
87.9043 \\
88.3316 \\
87.8092 \\
78.2051 \\
87.6051 \\
87.7287 \\
87.7699 \\
87.7171 \\
87.6381 \\
88.3702 \\
88.0318 \\
88.3322 \\
88.0977 \\
76.6212 \\
87.8444 \\
39.8352 \\
88.0971 \\
83.0581 \\
88.0409 \\
76.4966 \\
87.9995 \\
88.3209 \\
87.7915 \\
88.4448 \\
87.9673 \\
88.3316 \\
87.8664 \\
88.6332 \\
87.8898 \\
88.2010 \\
87.4545\end{array}$ & $\begin{array}{l}\text { Ave } \\
73.6642 \\
73.7867 \\
87.6912 \\
87.2195 \\
87.1049 \\
88.1903 \\
81.4854 \\
87.6850 \\
87.7444 \\
87.3454 \\
87.7868 \\
87.5312 \\
88.1992 \\
87.9008 \\
87.8804 \\
87.6182 \\
87.9915 \\
87.4621 \\
77.4808 \\
87.2564 \\
87.4294 \\
87.4896 \\
87.4428 \\
87.2677 \\
87.9407 \\
87.7408 \\
88.0296 \\
87.7524 \\
76.2867 \\
87.5542 \\
39.6240 \\
87.8243 \\
82.7545 \\
87.6902 \\
75.9496 \\
87.5948 \\
87.7316 \\
87.4263 \\
88.0292 \\
87.6993 \\
87.8665 \\
87.6385 \\
88.0848 \\
87.6199 \\
87.8106 \\
87.2180\end{array}$ & $\begin{array}{l}5 D \\
1.4946 e-01 \\
1.2642 e-01 \\
1.7441 e-01 \\
1.3144 e-01 \\
1.8129 e-01 \\
1.3224 e-01 \\
2.0607 e-01 \\
1.5363 e-01 \\
1.7499 e-01 \\
1.4193 e-01 \\
2.0202 e-01 \\
1.4002 e-01 \\
2.1003 e-01 \\
1.5432 e-01 \\
2.2334 e-01 \\
1.8257 e-01 \\
1.6761 e-01 \\
1.5249 e-01 \\
3.1750 e-01 \\
1.50566 e-01 \\
1.4768 e-01 \\
1.7797 e-01 \\
1.4576 e-01 \\
1.2052 e-01 \\
1.8524 e-01 \\
1.2886 e-01 \\
1.6761 e-01 \\
1.6305 e-01 \\
1.6012 e-01 \\
1.2650 e-01 \\
2.0658 e-01 \\
1.4047 e-01 \\
1.9972 e-01 \\
1.3830 e-01 \\
2.7765 e-01 \\
1.7192 e-01 \\
2.3342 e-01 \\
1.2218 e-01 \\
1.8936 e-01 \\
1.2196 e-01 \\
2.6414 e-01 \\
1.1110 e-01 \\
2.2362 e-01 \\
1.3449 e-01 \\
1.7349 e-01 \\
1.0973 e-01\end{array}$ & $\begin{array}{c}\text { Uncert } \\
1.9000 e+00 \\
1.9000 e+00 \\
1.9002 e+00 \\
1.9002 e+00 \\
1.9002 e+00 \\
1.9002 e+00 \\
1.9001 e+00 \\
1.90022 e+00 \\
1.9002 e+00 \\
1.9002 e+00 \\
1.9002 e+00 \\
1.9002 e+00 \\
1.9002 e+00 \\
1.9002 e e+00 \\
1.9002 e+00 \\
1.9002 e+00 \\
1.9002 e+00 \\
1.9002 e+00 \\
1.9000 e+00 \\
1.9002 e+00 \\
1.9002 e+00 \\
1.9002 e+00 \\
1.9002 e+00 \\
1.9002 e+00 \\
1.9002 e+00 \\
1.9002 e+00 \\
1.9002 e+00 \\
1.9002 e+00 \\
1.9000 e+00 \\
1.9002 e+00 \\
1.9011 e+00 \\
1.9002 e+00 \\
1.9001 e+00 \\
1.9002 e+00 \\
1.9000 e+00 \\
1.9002 e+00 \\
1.9002 e+00 \\
1.9002 e+00 \\
1.9002 e+00 \\
1.9002 e+00 \\
1.9002 e+00 \\
1.9002 e+00 \\
1.9002 e+00 \\
1.9002 e+00 \\
1.9002 e+00 \\
1.9002 e+00\end{array}$ \\
\hline
\end{tabular}

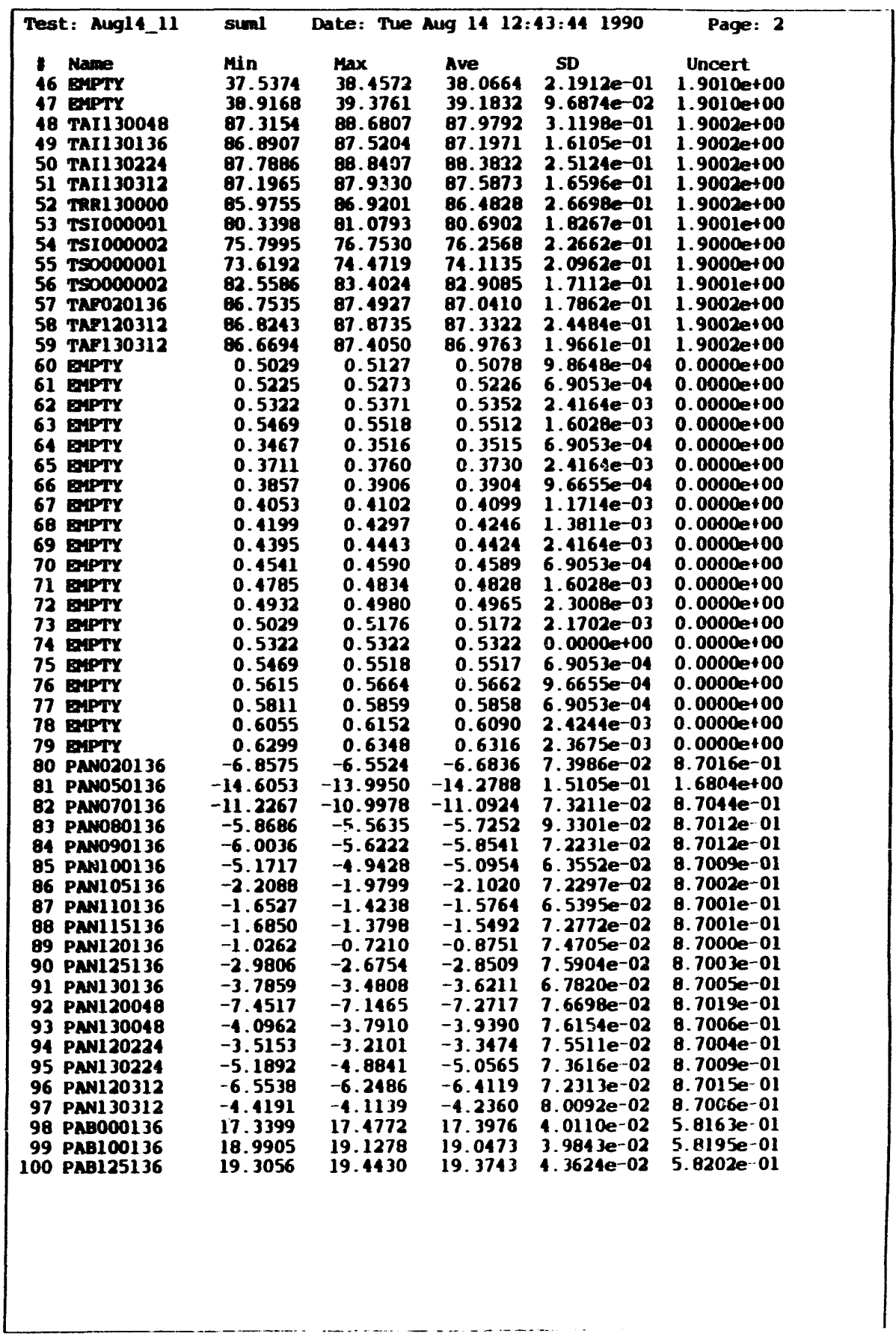




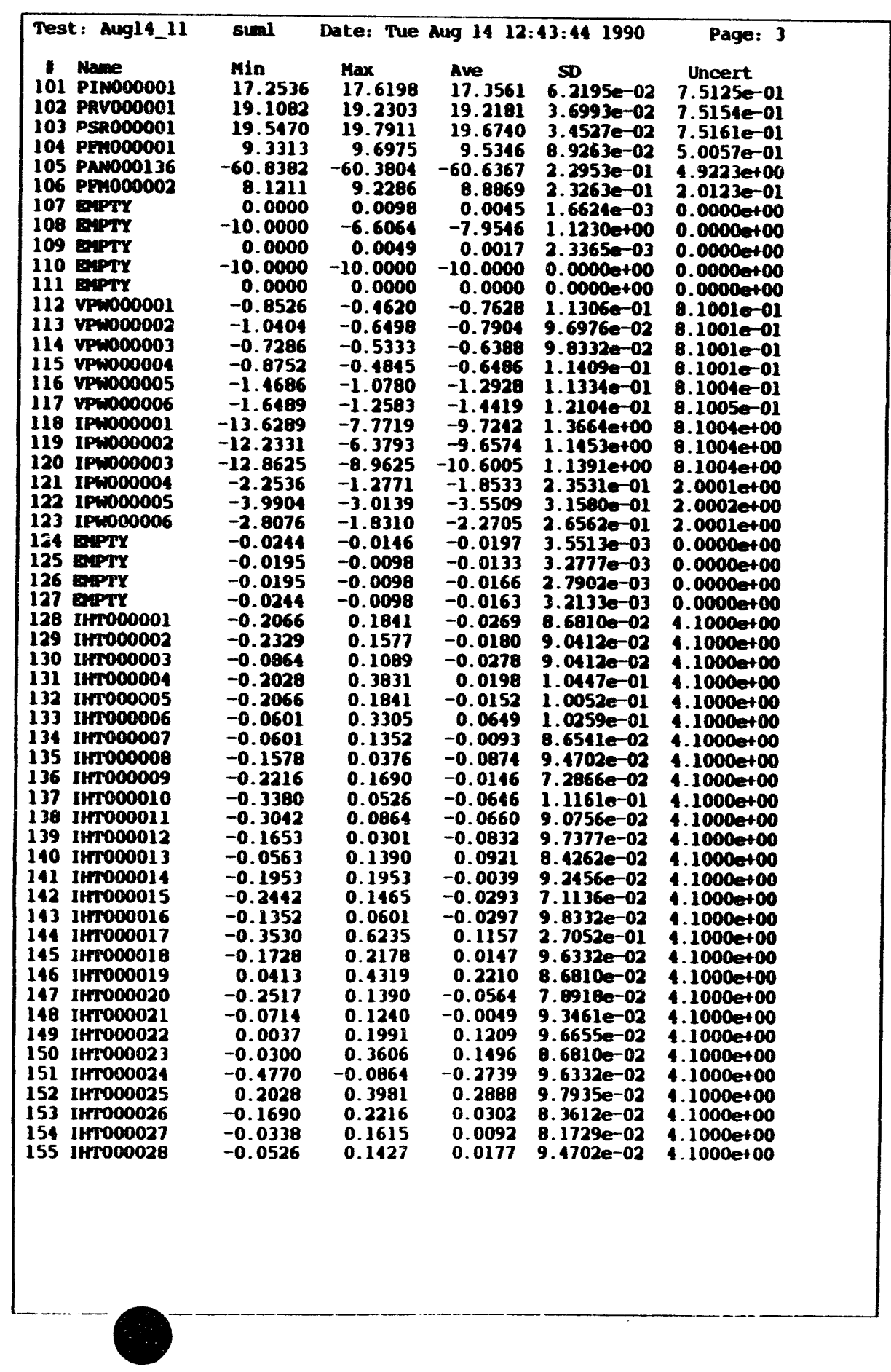

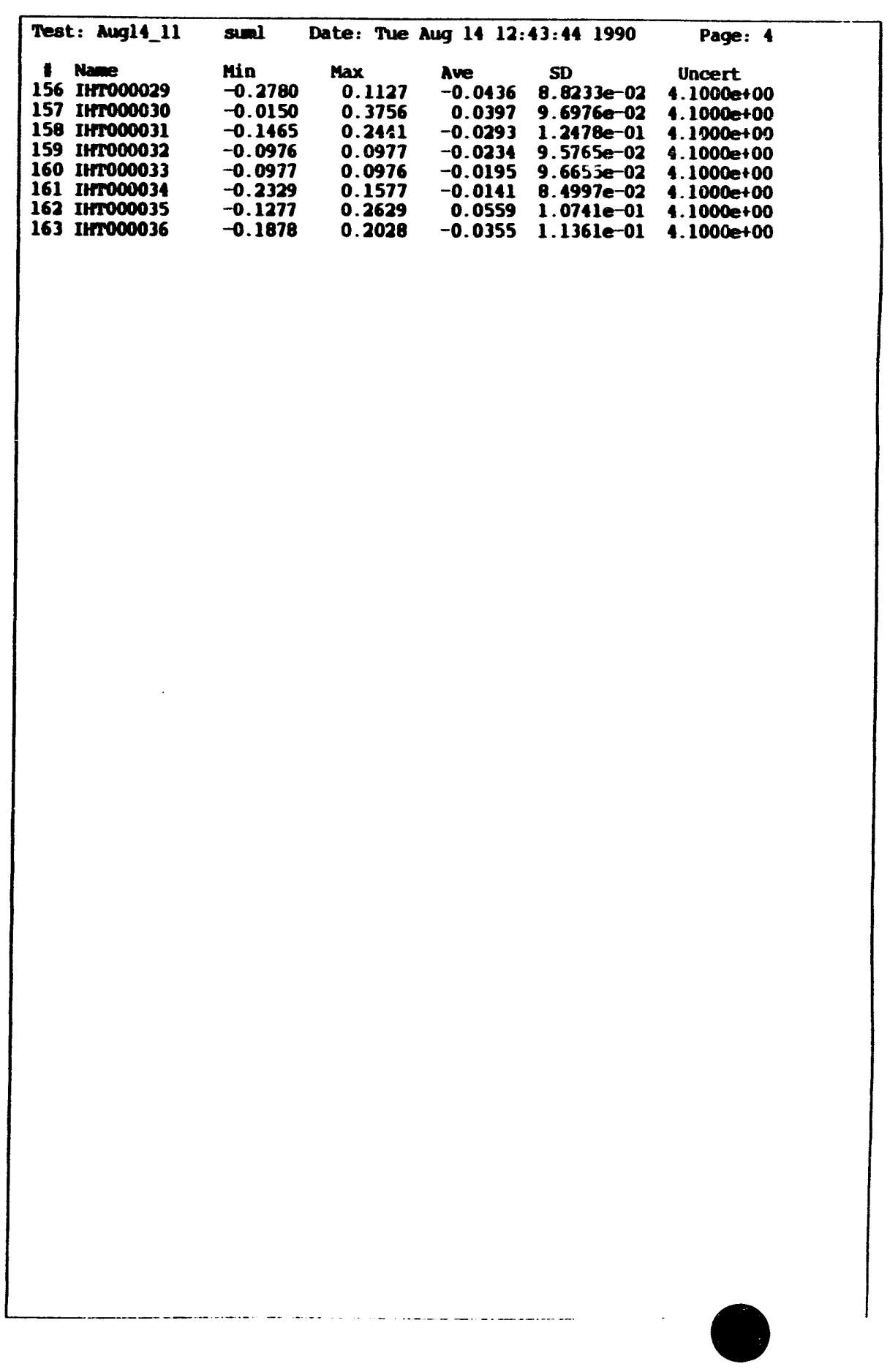




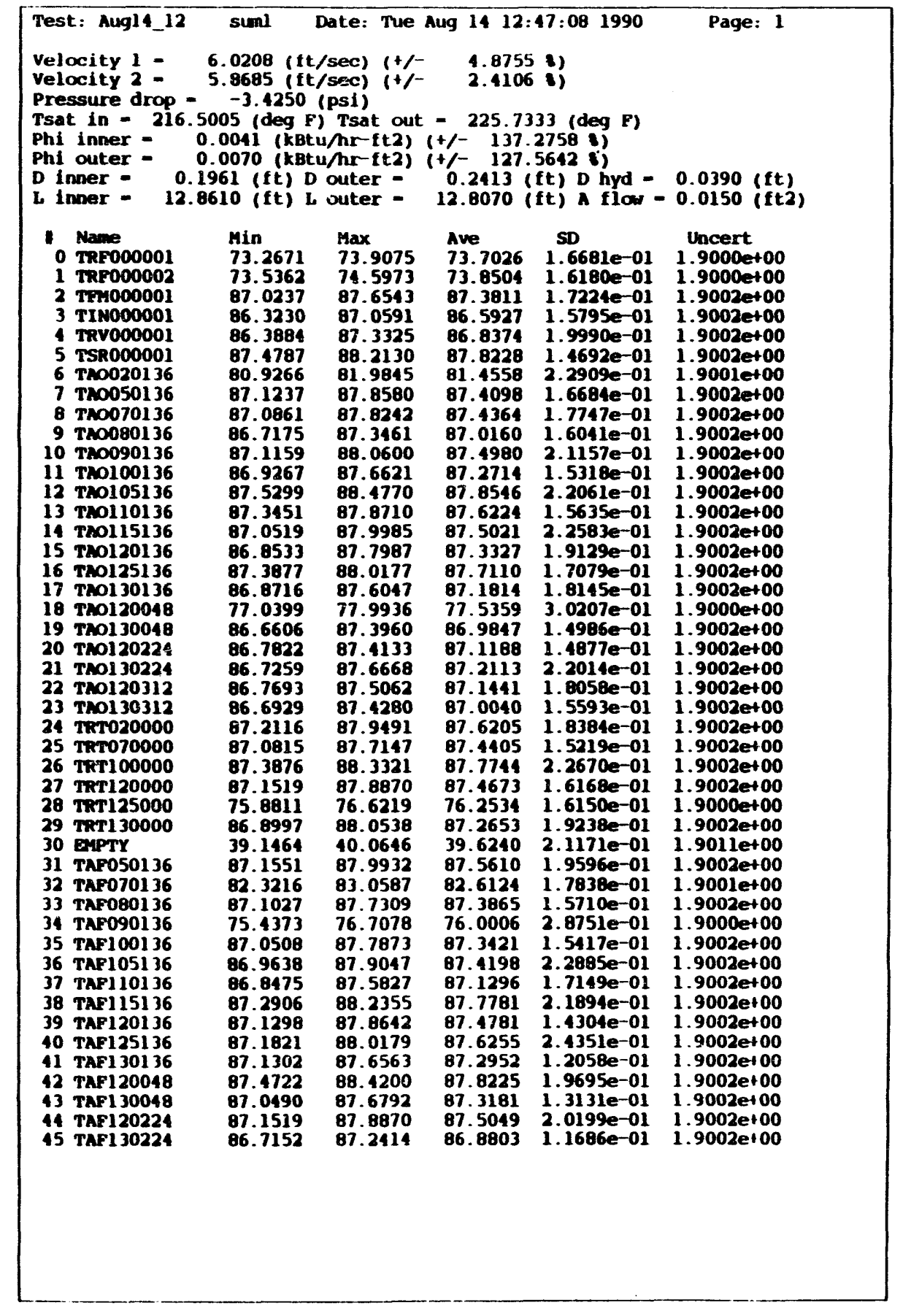

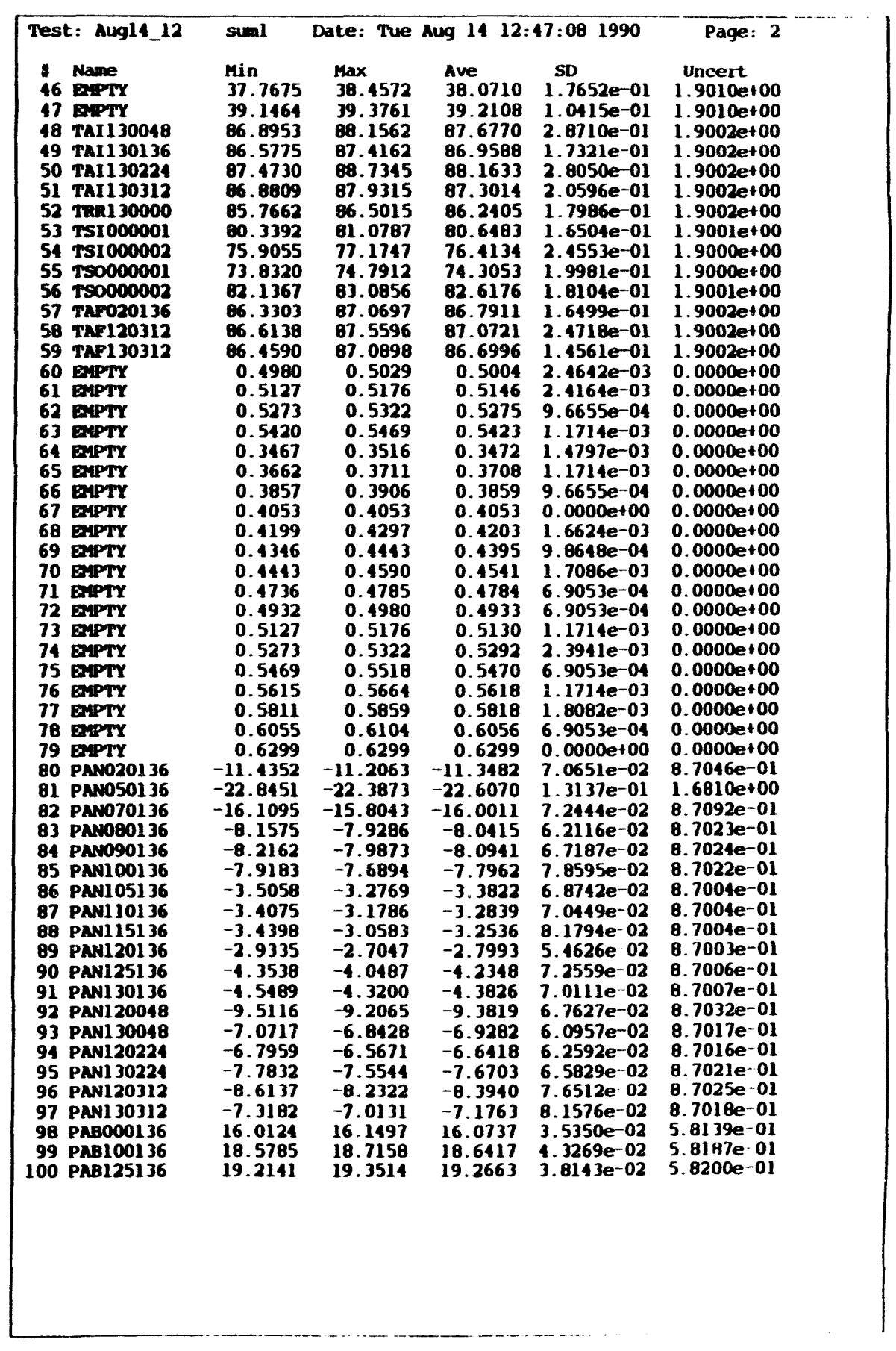




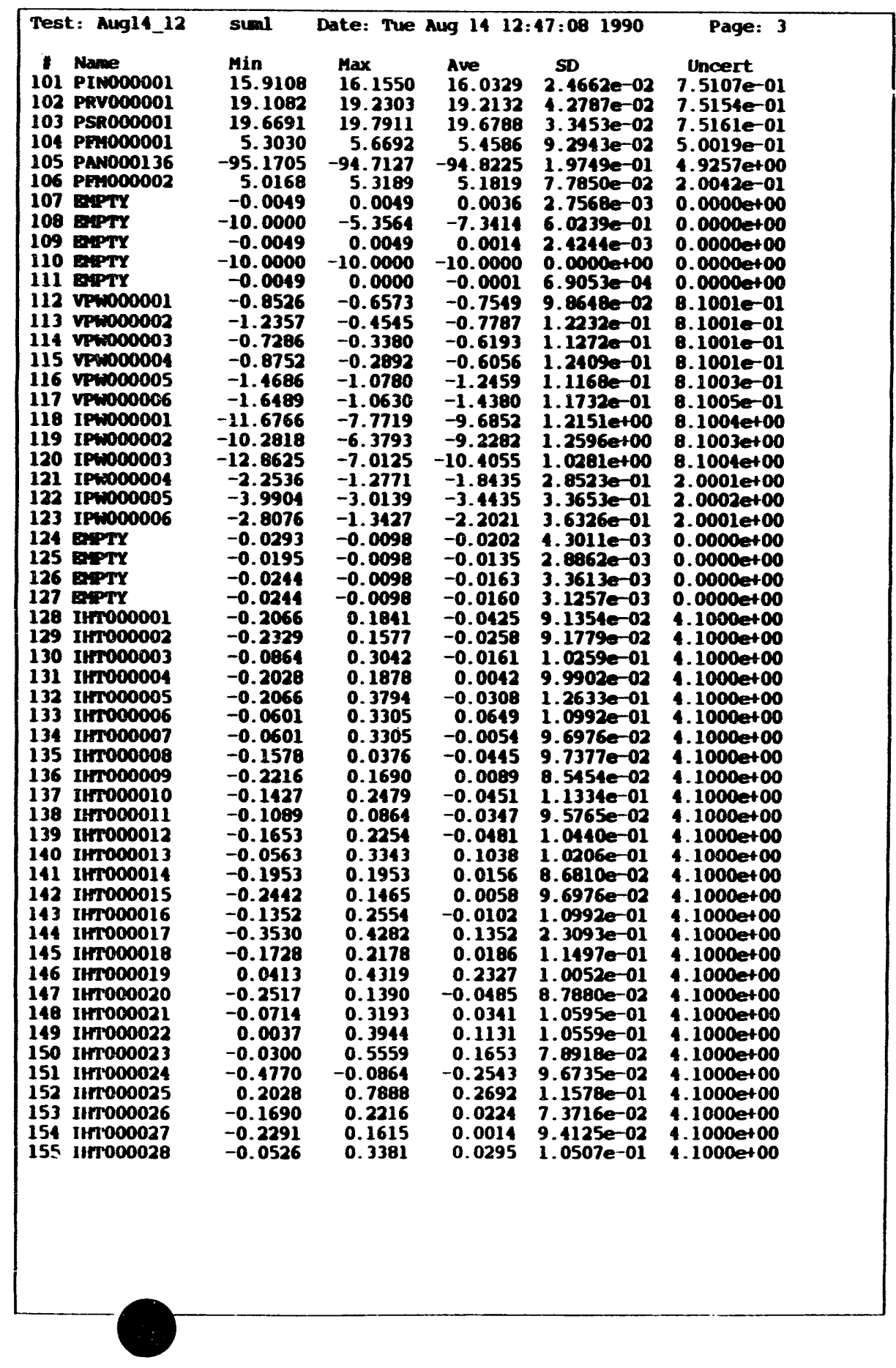

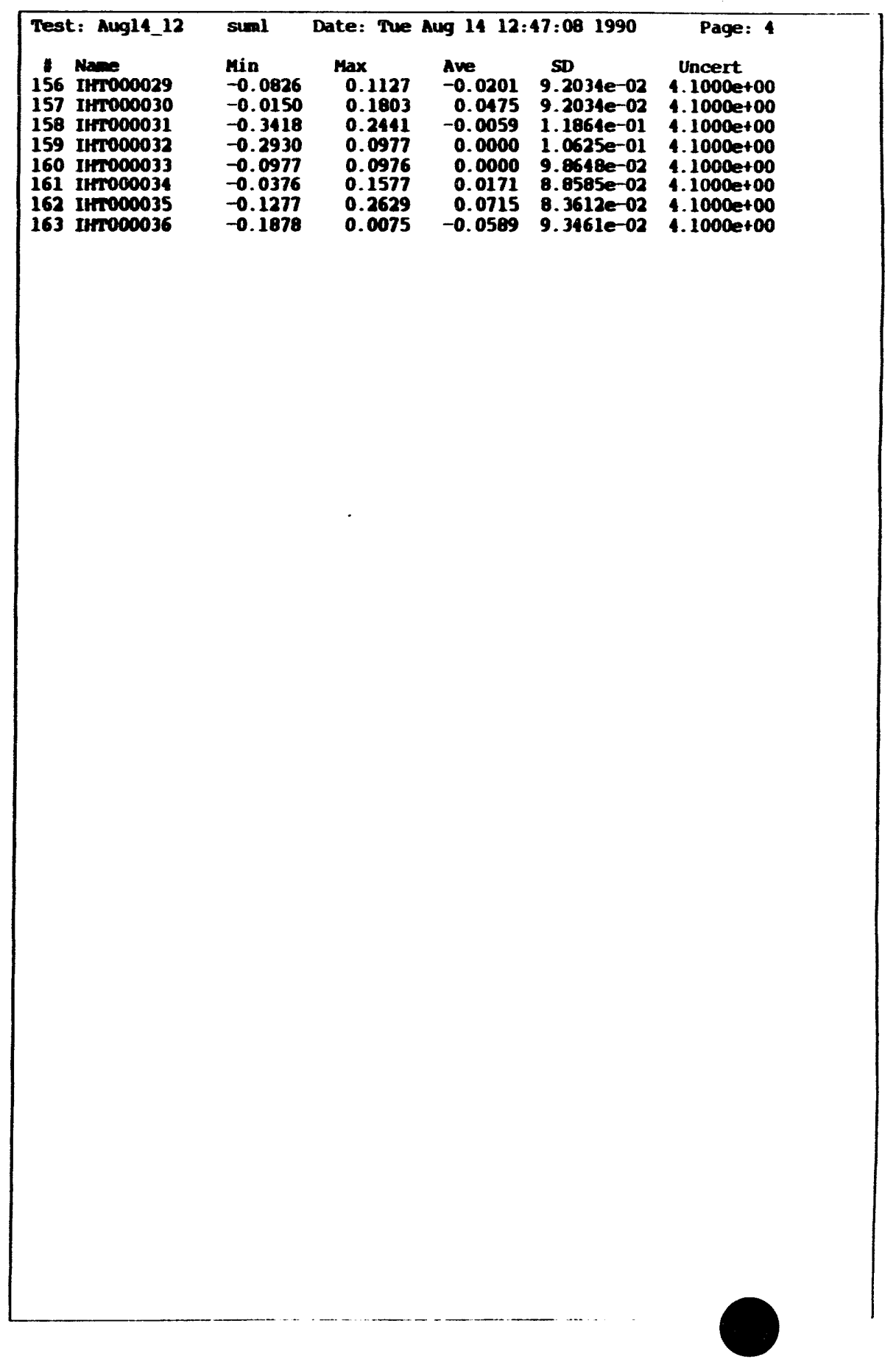




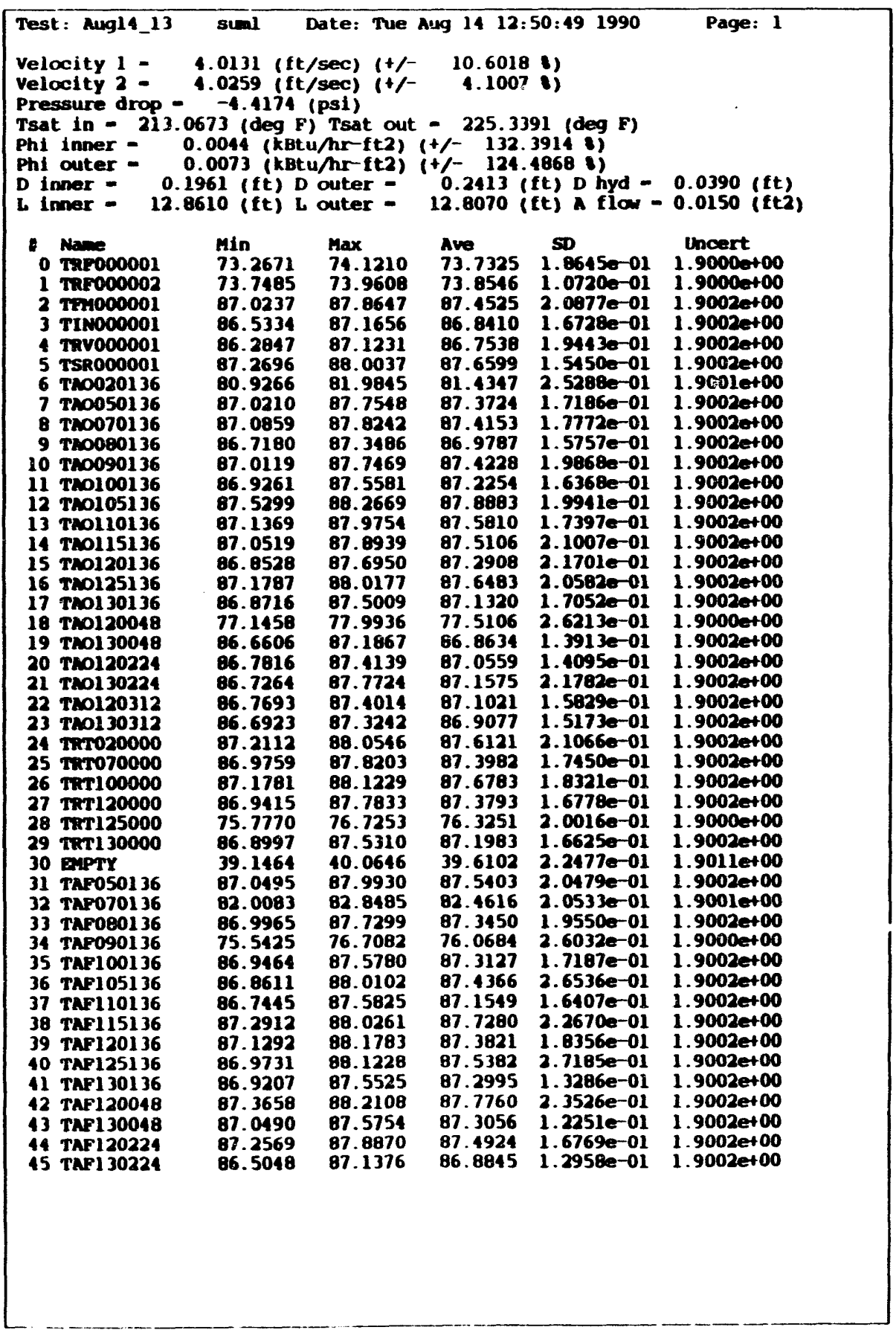

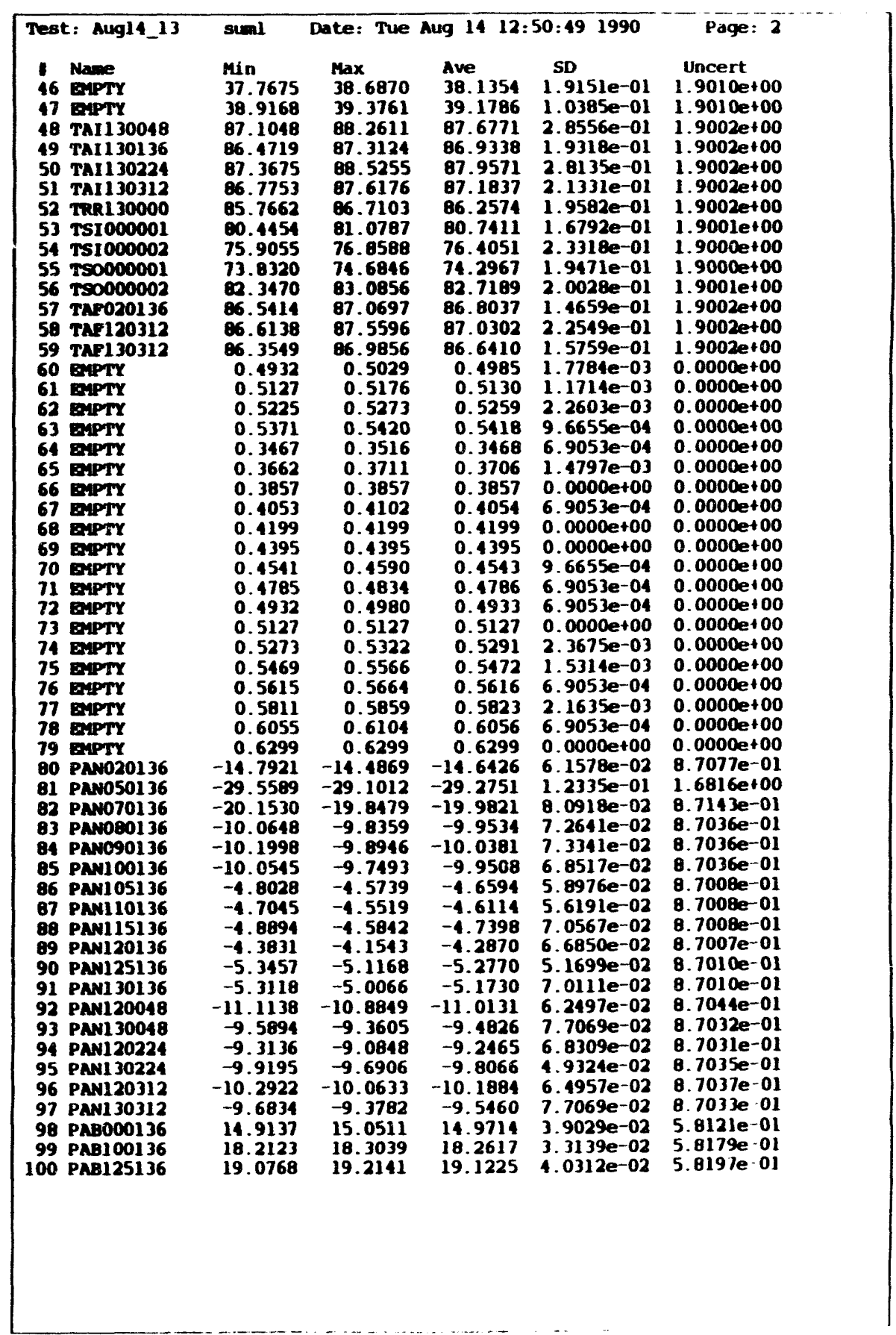




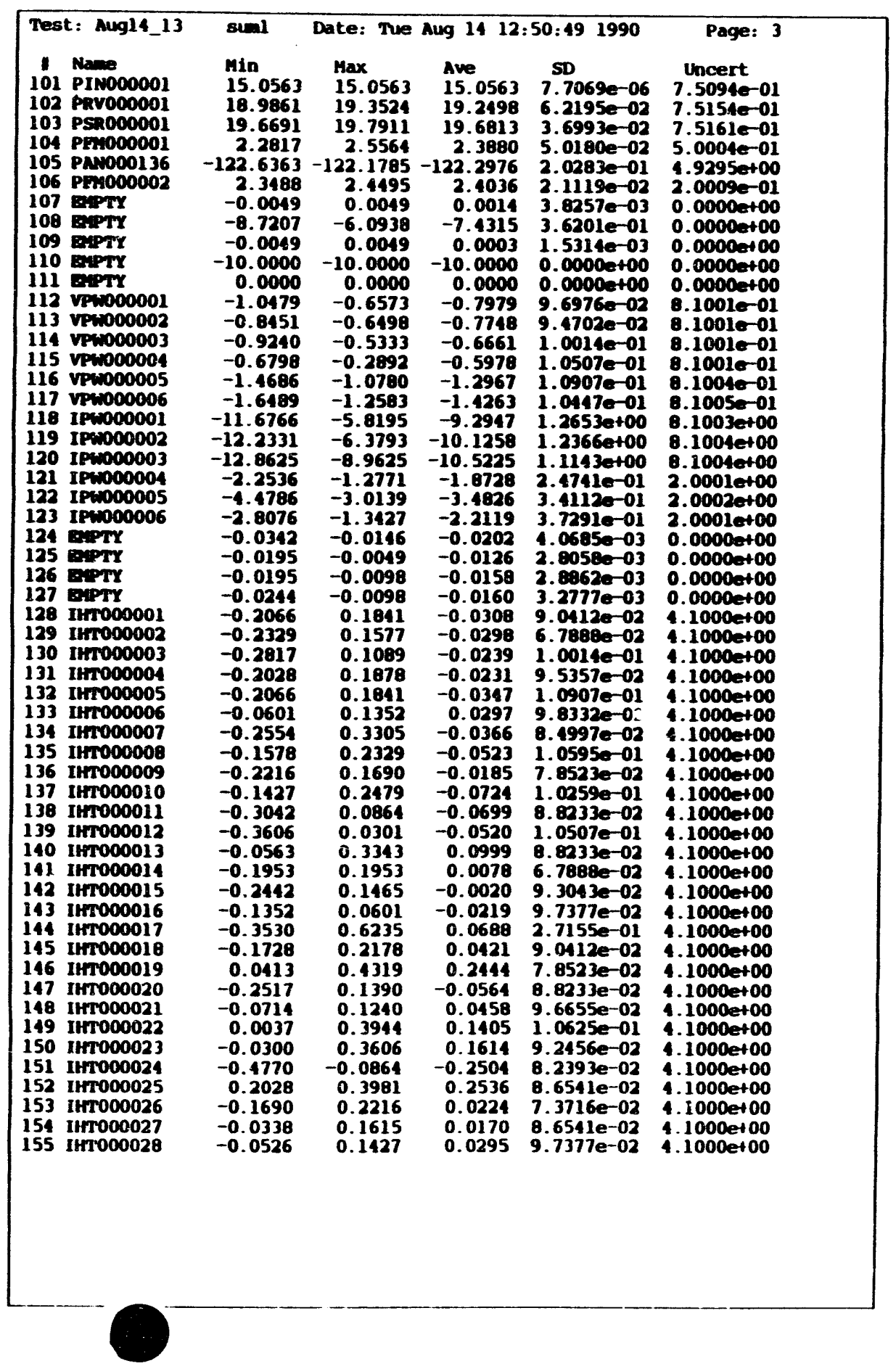

\begin{tabular}{|c|c|c|c|c|c|}
\hline Test: Au & al & Date: Tue & $\operatorname{lug} 14$ & 491990 & age: \\
\hline 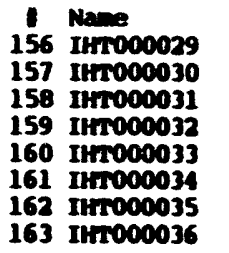 & $\begin{array}{l}\min \\
-0.2780 \\
-0.0150 \\
-0.1465 \\
-0.0976 \\
-0.0977 \\
-0.2329 \\
-0.1277 \\
-0.1878\end{array}$ & $\begin{array}{l}\operatorname{Max} \\
0.1127 \\
0.1803 \\
0.2441 \\
0.0977 \\
0.0976 \\
0.1577 \\
0.2629 \\
0.2028\end{array}$ & $\begin{array}{l}\text { Ave } \\
-0.0319 \\
0.0670 \\
-0.0098 \\
-0.0039 \\
-0.0117 \\
-0.0063 \\
0.0559 \\
-0.0355\end{array}$ & $\begin{array}{l}\text { SD } \\
9.5112 e-02 \\
9.7377 e-02 \\
1.1334 e-01 \\
9.8569 e-02 \\
9.7935 e-02 \\
9.1354 e-02 \\
7.2866 e-02 \\
9.8963 e-02\end{array}$ & $\begin{array}{l}\text { Uncert } \\
4.1000 \text { et00 } \\
4.1000 \text { et } 00 \\
4.1000 \text { et } 00 \\
4.1000 \text { et } 00 \\
4.1000 \text { et } 00 \\
4.1000 \text { et } 00 \\
4.1000 \text { et } 00 \\
4.1000 \text { et } 00\end{array}$ \\
\hline
\end{tabular}




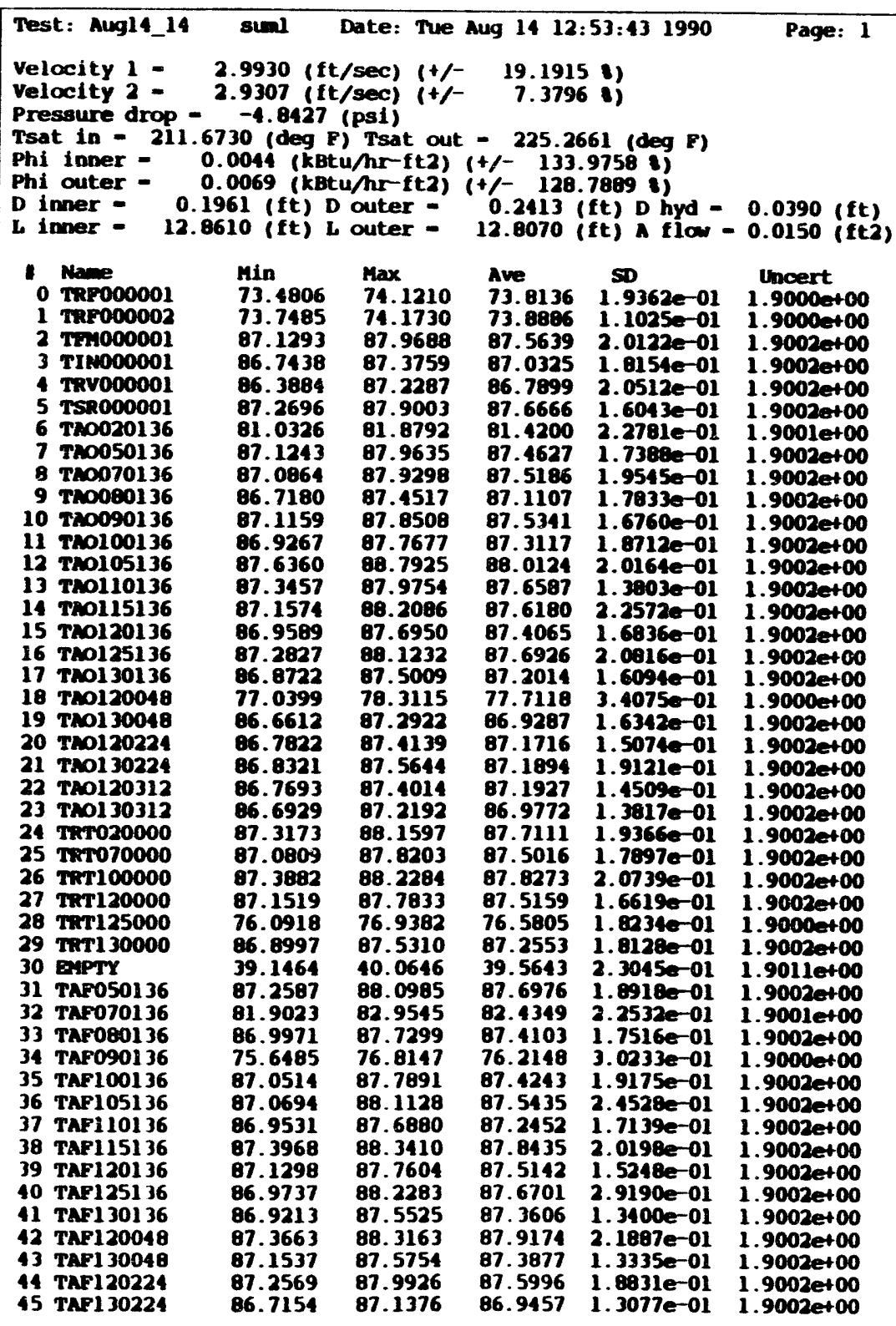

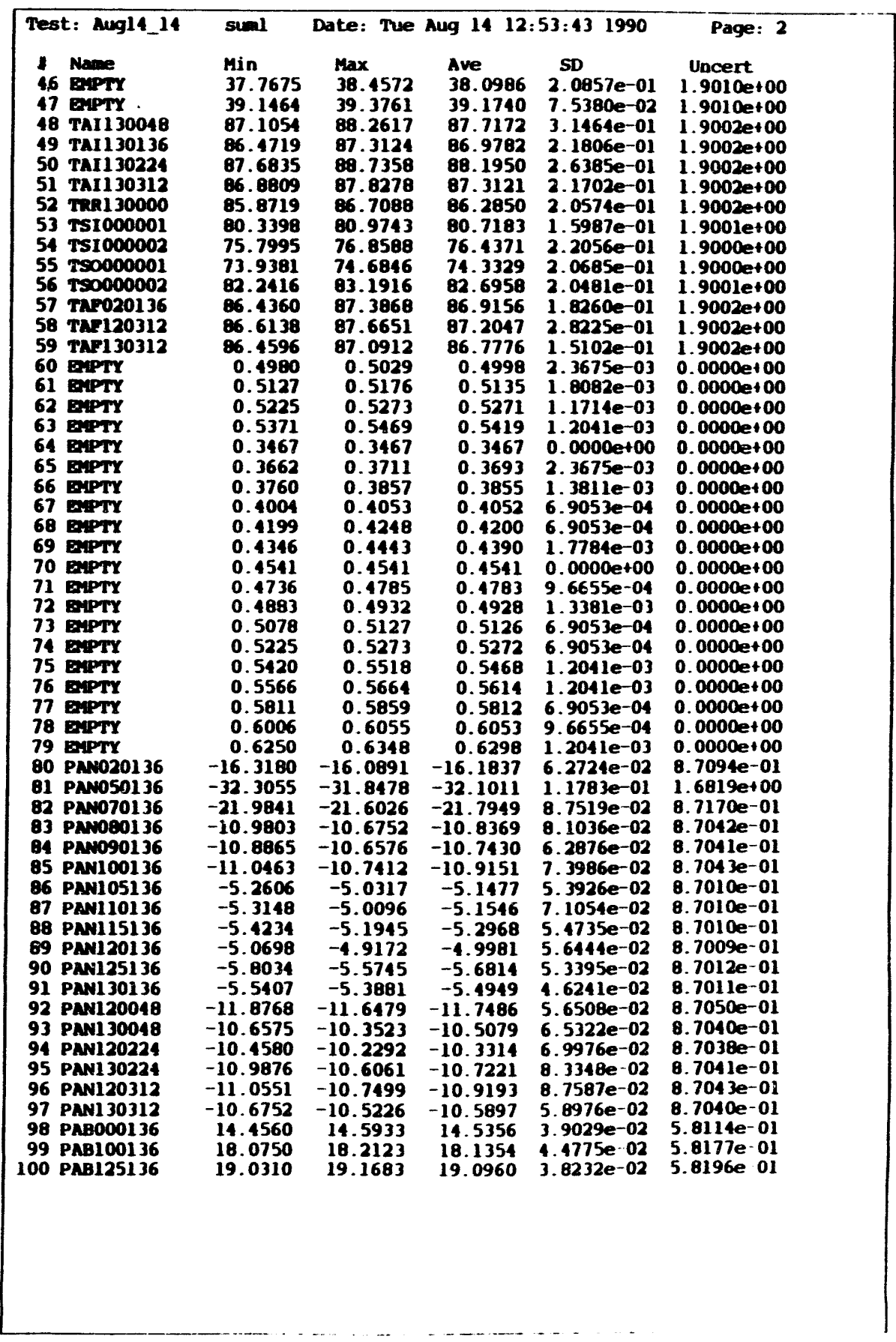



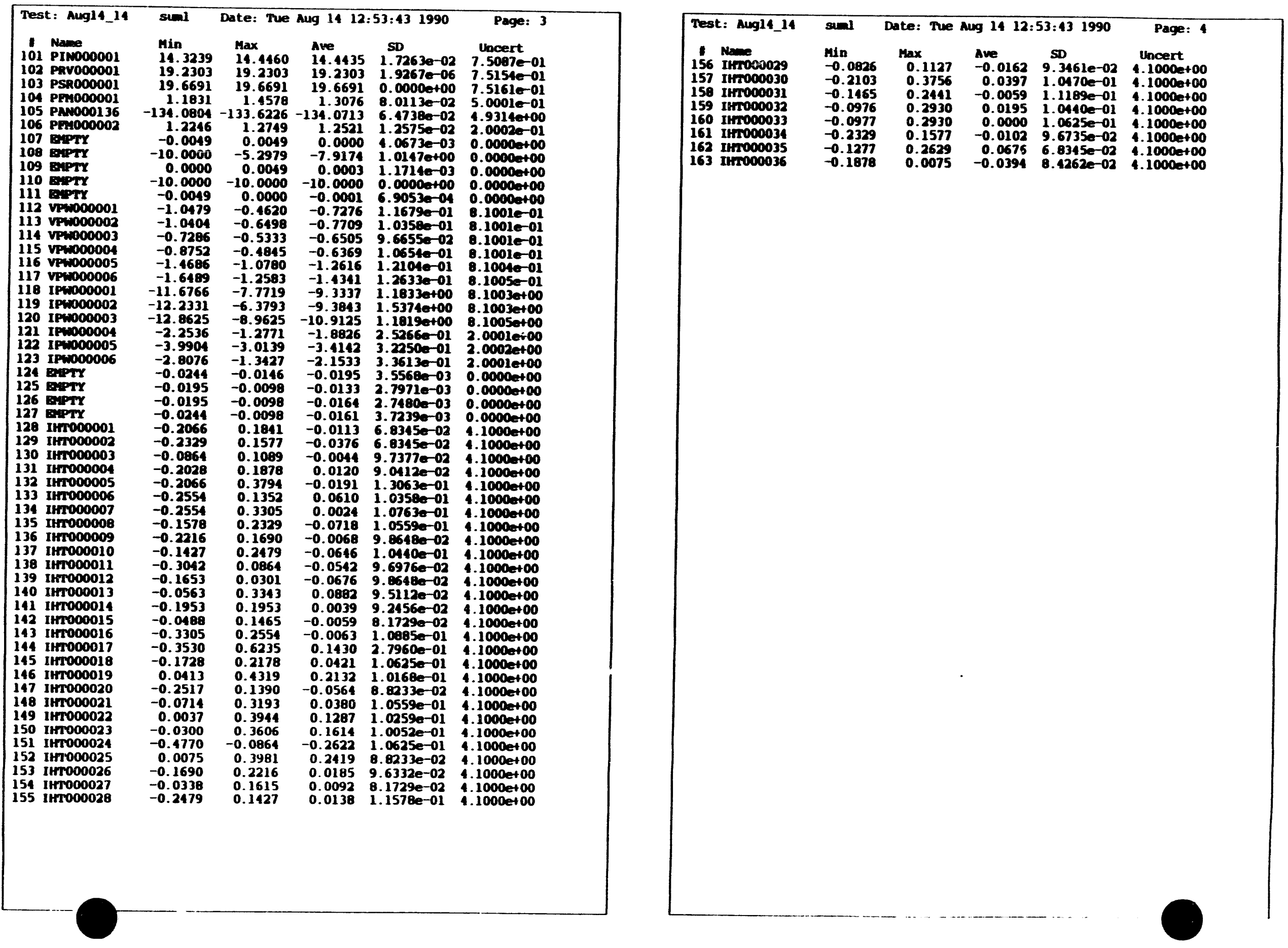


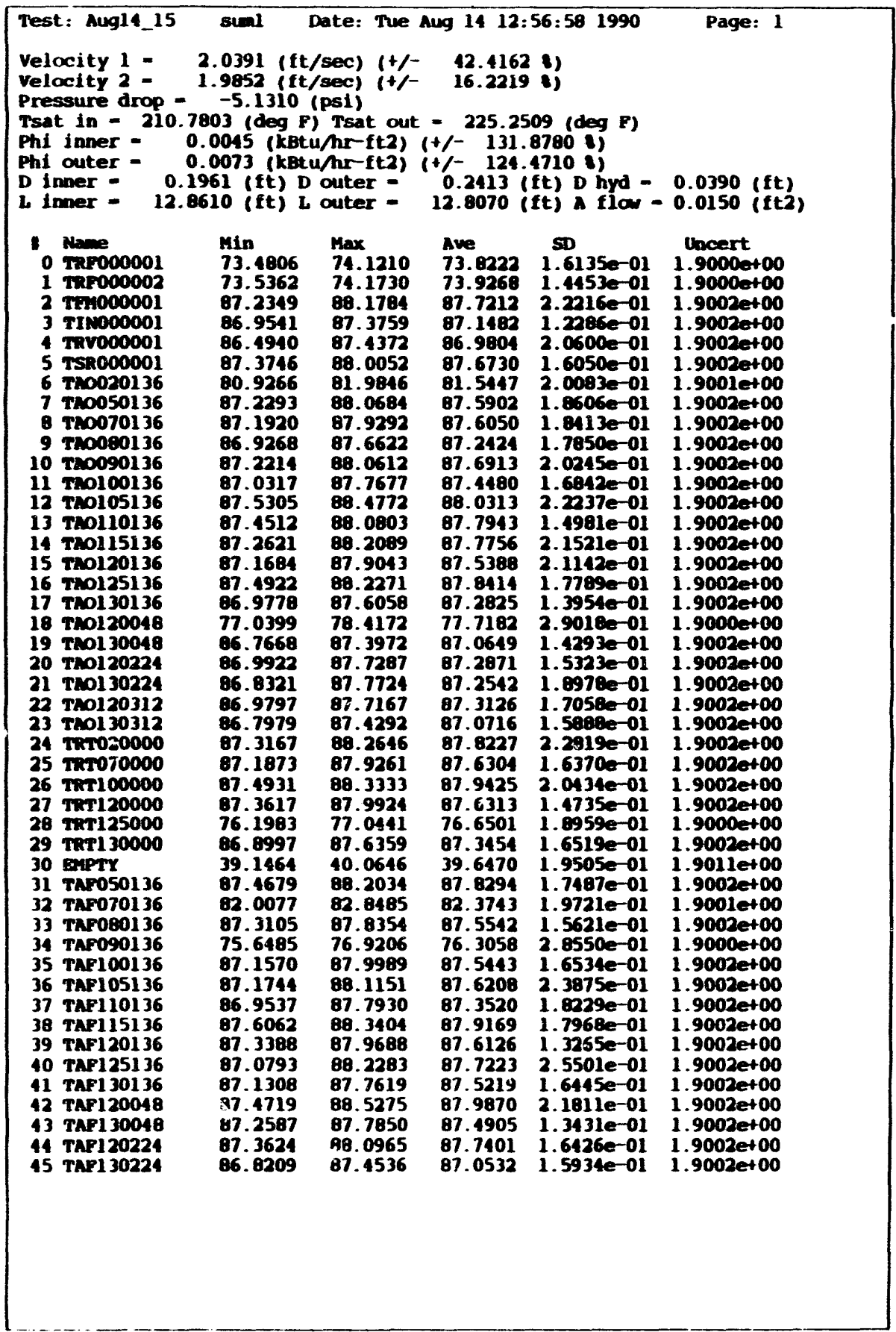

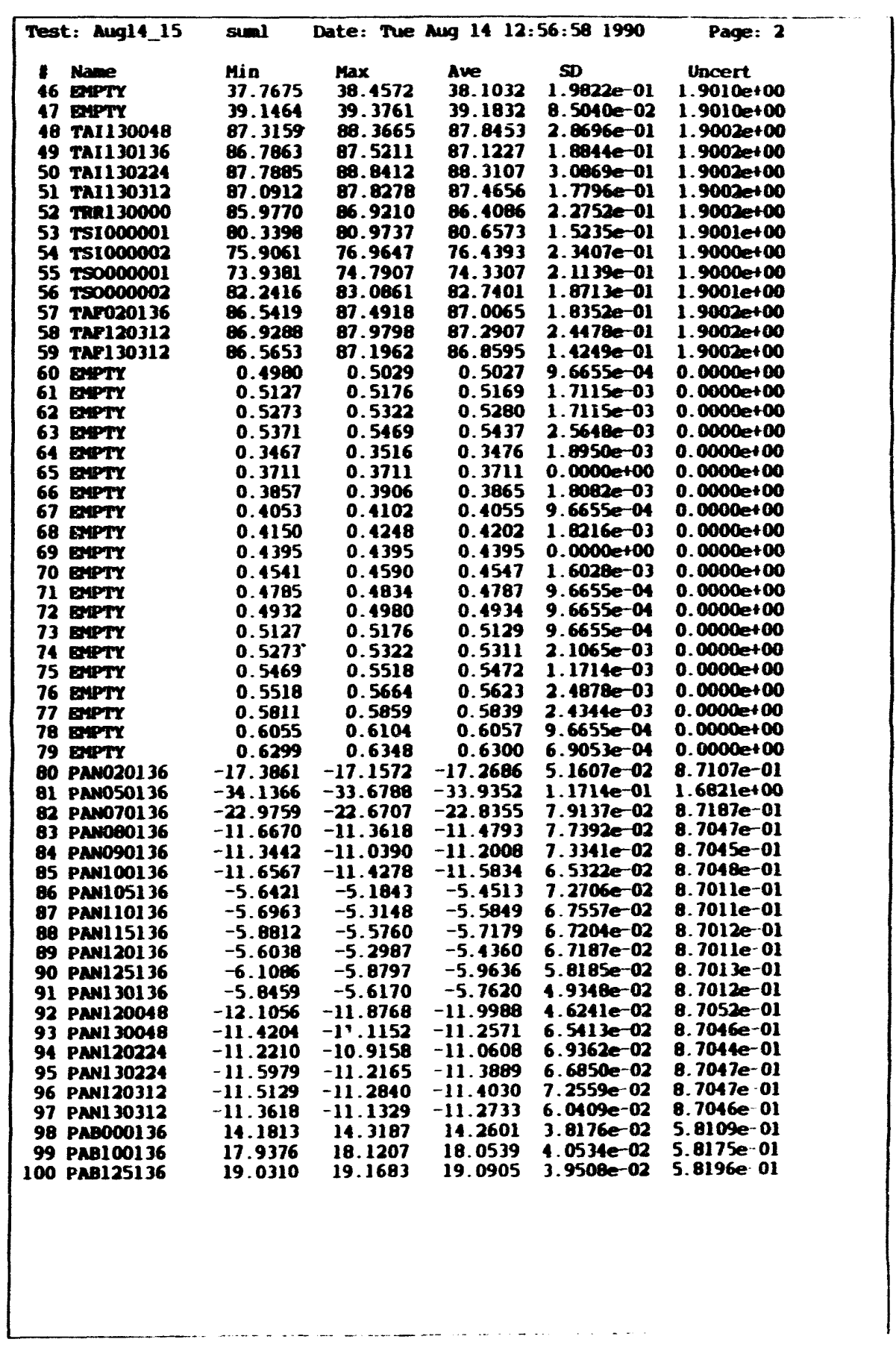




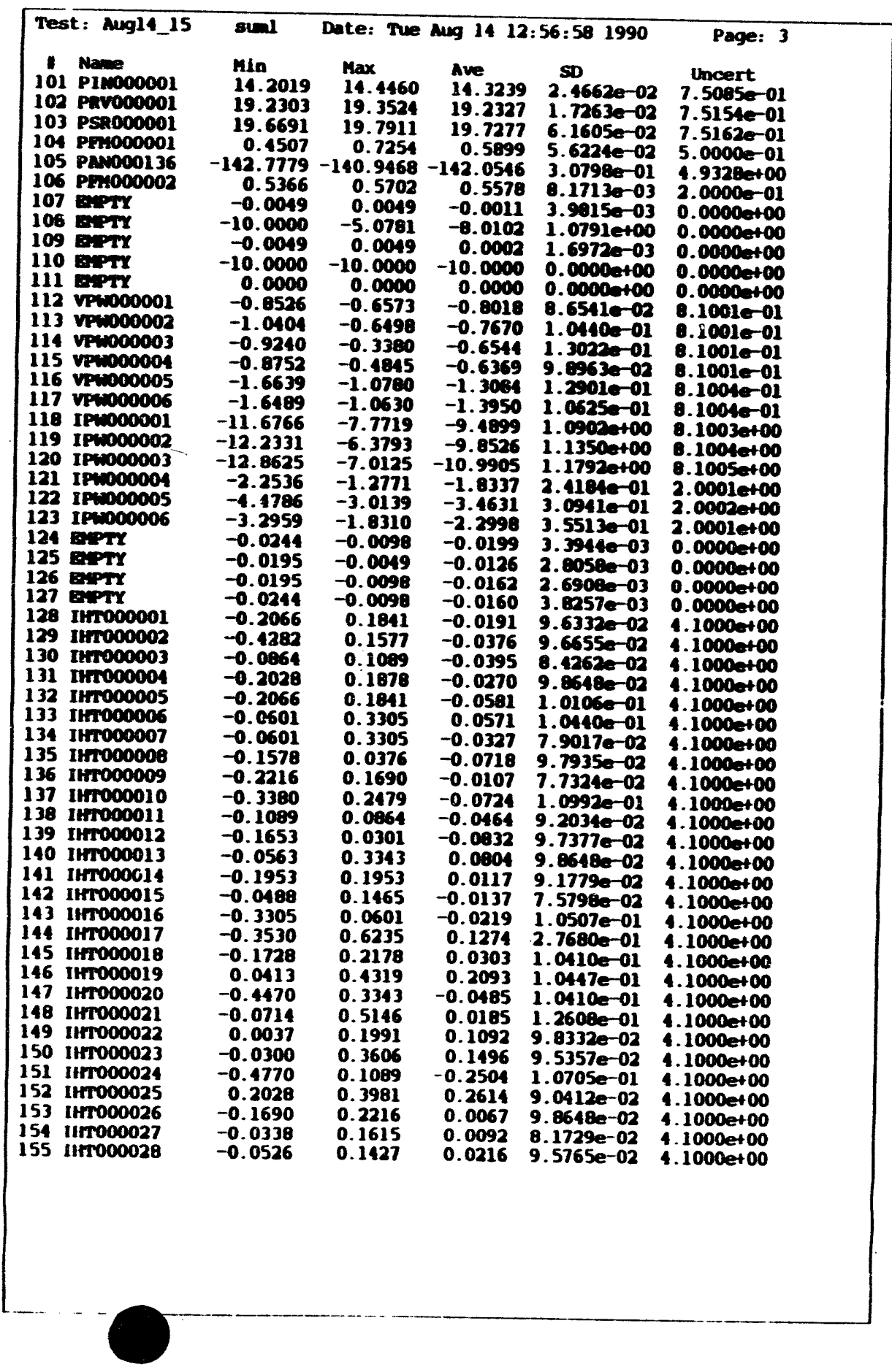

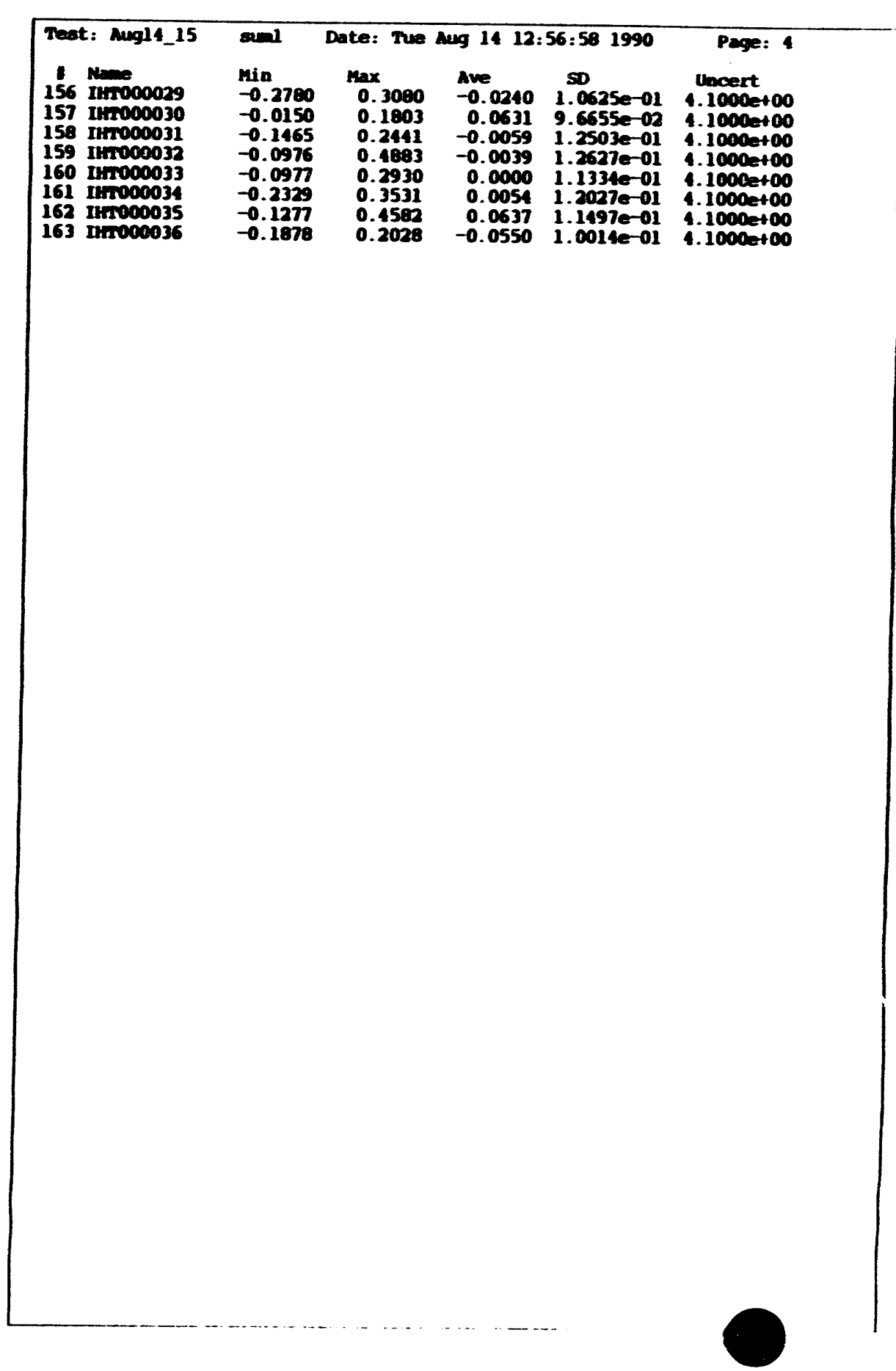




\begin{tabular}{|c|c|c|c|c|c|}
\hline 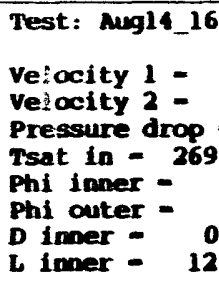 & $\begin{array}{c}\text { sumal } \\
25.0271 \text { (ft) } \\
13.4153 \text { (ft) } \\
20.0215 \\
9811 \text { (deg F } \\
0.0044 \text { (kBti } \\
0.0071 \text { (kBtt } \\
1961 \text { (ft) D } \\
8610 \text { (ft) L }\end{array}$ & $\begin{array}{l}\text { ate: Tue } \\
\text { sec) }(+/- \\
\text { sec) (t/- } \\
\text { psi) } \\
\text { Tsat out } \\
\text { Mr-f(2) } \\
\text { Mr-ft2) } \\
\text { outer - } \\
\text { outer - }\end{array}$ & $\begin{array}{r}1413: \\
1.6916 \\
1.7033 \\
=233.13 \\
1+/-133 . \\
1+/-126 \\
0.2413 \\
12.8070\end{array}$ & $\begin{array}{l}00: 201990 \\
\text { () } \\
\text { ह) } \\
71 \text { (deg F) } \\
1326 \text { ह) } \\
9062 \text { :) } \\
\text { ft) D hyd - } \\
\text { ft) A flow - }\end{array}$ & $\begin{array}{l}0.0390 \text { (ft) } \\
0.0150 \text { (ft2) }\end{array}$ \\
\hline 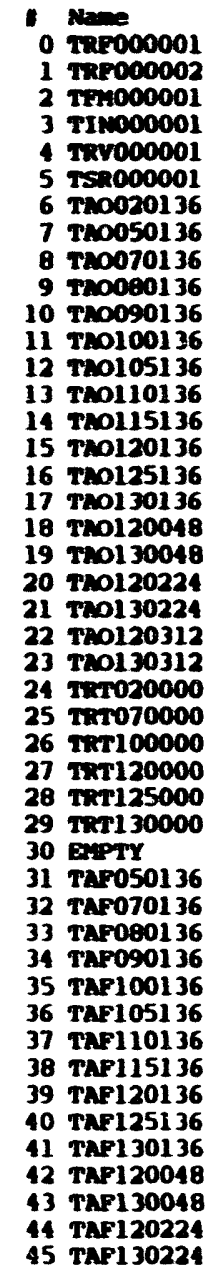 & $\begin{array}{l}\text { Hin } \\
73.4806 \\
73.7485 \\
87.2335 \\
86.8994 \\
87.1217 \\
87.3746 \\
81.1387 \\
87.3332 \\
87.0864 \\
87.0324 \\
87.2208 \\
87.3461 \\
87.6360 \\
87.3457 \\
87.3677 \\
87.0634 \\
87.3803 \\
87.0792 \\
77.1464 \\
86.8705 \\
86.9922 \\
86.9346 \\
86.9799 \\
86.9025 \\
87.4223 \\
87.1873 \\
87.5976 \\
87.3617 \\
76.5129 \\
87.3189 \\
39.3761 \\
87.2587 \\
82.0077 \\
87.3105 \\
75.8602 \\
87.0514 \\
87.1721 \\
86.9537 \\
87.5007 \\
87.3388 \\
87.1821 \\
87.1308 \\
87.5763 \\
87.1537 \\
87.3615 \\
86.8214\end{array}$ & $\begin{array}{l}\text { Max } \\
74.1210 \\
74.1730 \\
87.8647 \\
87.5855 \\
87.8555 \\
68.1087 \\
82.0906 \\
87.9635 \\
88.0347 \\
87.7653 \\
87.9563 \\
87.8726 \\
88.6873 \\
88.2884 \\
88.2089 \\
88.0098 \\
88.4375 \\
87.8127 \\
78.3115 \\
87.6064 \\
87.7287 \\
88.0853 \\
87.8215 \\
87.5336 \\
88.2646 \\
88.1365 \\
88.3333 \\
88.0979 \\
77.3591 \\
87.8454 \\
40.0646 \\
88.2034 \\
82.8503 \\
88.0431 \\
76.9198 \\
88.1044 \\
88.0102 \\
87.7930 \\
88.1458 \\
87.9694 \\
88.5413 \\
87.8669 \\
88.5269 \\
87.8900 \\
88.2014 \\
87.3486\end{array}$ & $\begin{array}{l}\text { Ave } \\
73.8392 \\
73.9990 \\
87.5724 \\
87.2218 \\
87.5056 \\
87.7548 \\
81.5679 \\
87.6636 \\
87.5437 \\
87.4243 \\
87.6474 \\
87.5676 \\
88.1343 \\
87.8841 \\
87.8281 \\
87.6585 \\
87.9065 \\
87.5 n 05 \\
77.8055 \\
87.2262 \\
87.3312 \\
87.4314 \\
87.3904 \\
87.2498 \\
87.8036 \\
87.6872 \\
87.9698 \\
87.6880 \\
76.9136 \\
87.5321 \\
39.6791 \\
87.7857 \\
82.4647 \\
87.6192 \\
76.3715 \\
87.6094 \\
87.7024 \\
87.4212 \\
87.9568 \\
87.6901 \\
87.8248 \\
87.5954 \\
88.0184 \\
87.5975 \\
87.7674 \\
87.1479\end{array}$ & $\begin{array}{l}\text { sD } \\
1.6945 e-01 \\
1.4660 e-01 \\
1.8640 e-01 \\
1.4913 e-01 \\
1.9105 e-01 \\
1.5695 e-01 \\
2.2039 e-01 \\
1.4985 e-01 \\
2.3866 e-01 \\
1.6775 e-01 \\
1.9238 e-01 \\
1.4432 e-01 \\
2.0900 e-01 \\
1.6932 e-01 \\
2.1747 e-01 \\
1.8705 e-01 \\
1.9014 e-01 \\
1.7977 e-01 \\
2.7982 e-01 \\
1.5143 e-01 \\
1.5802 e-01 \\
2.0547 e-01 \\
1.8607 e-01 \\
1.4444 e-01 \\
2.0059 e-01 \\
1.7151 e-01 \\
1.8383 e-01 \\
1.7019 e-01 \\
2.0402 e-01 \\
1.5952 e-01 \\
2.0966 e-01 \\
2.0814 e-01 \\
1.9435 e-01 \\
1.8967 e-01 \\
2.4175 e-01 \\
2.1506 e-01 \\
2.2960 e-01 \\
1.7374 e-01 \\
1.9619 e-01 \\
1.3691 e-01 \\
2.9970 e-01 \\
1.5900 e-01 \\
2.4837 e-01 \\
1.6069 e-01 \\
1.7996 e-01 \\
1.5965 e-01\end{array}$ & $\begin{array}{r}\text { Uncert } \\
1.9000 e+00 \\
1.9000 e+00 \\
1.9002 e+00 \\
1.9002 e+00 \\
1.9002 e+00 \\
1.9002 e+00 \\
1.9001 e+00 \\
1.9002 e+00 \\
1.9002 e+00 \\
1.9002 e+00 \\
1.9002 e+00 \\
1.9002 e+00 \\
1.9002 e+00 \\
1.9002 e+00 \\
1.9002 e+00 \\
1.9002 e+00 \\
1.9002 e+00 \\
1.9002 e+00 \\
1.9000 e+00 \\
1.9002 e+00 \\
1.9002 e+00 \\
1.9002 e+00 \\
1.9002 e+00 \\
1.9002 e+00 \\
1.91 .02 e+00 \\
1.9002 e+00 \\
1.9002 e+00 \\
1.9002 e+00 \\
1.9000 e+00 \\
1.9002 e+00 \\
1.9011 e+00 \\
1.9002 e+00 \\
1.9001 e+00 \\
1.9002 e+00 \\
1.9000 e+00 \\
1.9002 e+00 \\
1.9002 e+00 \\
1.9002 e+00 \\
1.9002 e+00 \\
1.9002 e+00 \\
1.9002 e+00 \\
1.9002 e+00 \\
1.9002 e+00 \\
1.9002 e+00 \\
1.9002 e+00 \\
1.9002 e+00\end{array}$ \\
\hline
\end{tabular}

\begin{tabular}{|c|c|c|c|c|c|}
\hline 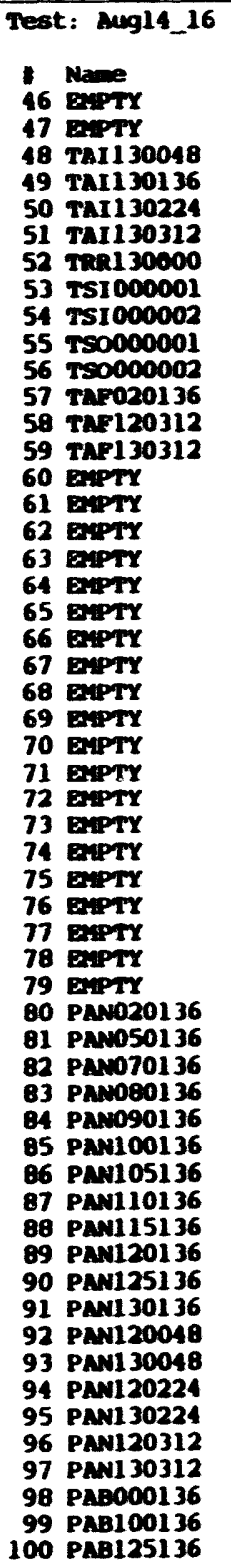 & $\begin{array}{l}\text { sual } \\
\text { Min } \\
37.5374 \\
39.1464 \\
87.1054 \\
86.6813 \\
87.6836 \\
87.0915 \\
85.9770 \\
80.3398 \\
75.7995 \\
73.8320 \\
82.4530 \\
86.5419 \\
86.6139 \\
86.5647 \\
0.5029 \\
0.5127 \\
0.5225 \\
0.5420 \\
0.3564 \\
0.3809 \\
0.3955 \\
0.4150 \\
0.4297 \\
0.4492 \\
0.4639 \\
0.4883 \\
0.5029 \\
0.5225 \\
0.5371 \\
0.5566 \\
0.5713 \\
0.5908 \\
0.6152 \\
0.6348 \\
73.9377 \\
136.1515 \\
76.2062 \\
37.0849 \\
33.8981 \\
41.9780 \\
22.1289 \\
28.1782 \\
31.4266 \\
34.2979 \\
20.9757 \\
10.0996 \\
25.8125 \\
53.2005 \\
56.1466 \\
40.7397 \\
28.7703 \\
49.5970 \\
41.5556 \\
27.0014 \\
22.0522\end{array}$ & $\begin{array}{l}\text { Date: Tue } \\
\text { Max } \\
38.4572 \\
39.3761 \\
69.3665 \\
87.5217 \\
88.8412 \\
87.9327 \\
87.0245 \\
80.9743 \\
76.8582 \\
74.7907 \\
83.2969 \\
87.3868 \\
87.8748 \\
87.4059 \\
0.5078 \\
0.5225 \\
0.5322 \\
0.5469 \\
0.3613 \\
0.3857 \\
0.4004 \\
0.4199 \\
0.4297 \\
0.4541 \\
0.4688 \\
0.4932 \\
0.5078 \\
0.5273 \\
0.5420 \\
0.5615 \\
0.5762 \\
0.5957 \\
0.6250 \\
0.6396 \\
75.1584 \\
137.9826 \\
77.5032 \\
37.7715 \\
34.4321 \\
42.5883 \\
22.5104 \\
28.7123 \\
31.9606 \\
34.8320 \\
21.3572 \\
10.4047 \\
26.4991 \\
54.0398 \\
56.9095 \\
11.2738 \\
29.1511 \\
50.3600 \\
12.1049 \\
27.1845 \\
22.1895\end{array}$ & $\begin{array}{l}\text { Aug } 1413: \\
\text { Ave } \\
38.0986 \\
39.2016 \\
87.9482 \\
87.1417 \\
88.3127 \\
87.4761 \\
86.4569 \\
80.6975 \\
76.3528 \\
74.3583 \\
82.8393 \\
86.9914 \\
87.3810 \\
86.9624 \\
0.5030 \\
0.5177 \\
0.5298 \\
0.5462 \\
0.3600 \\
0.3810 \\
0.3968 \\
0.4153 \\
0.4297 \\
0.4494 \\
0.4646 \\
0.4885 \\
0.5033 \\
0.5226 \\
0.5400 \\
0.5567 \\
0.5717 \\
0.5914 \\
0.6155 \\
0.6378 \\
74.5176 \\
136.8809 \\
76.7555 \\
37.3458 \\
34.2490 \\
12.3335 \\
22.3441 \\
28.4834 \\
31.6631 \\
34.5481 \\
21.1573 \\
10.2384 \\
26.0856 \\
53.5713 \\
56.5479 \\
11.0052 \\
28.9580 \\
19.9007 \\
11.7872 \\
27.0911 \\
22.1053\end{array}$ & $\begin{array}{c}00.201990 \\
50 \\
2.1368 e-01 \\
9.9068 e-02 \\
3.0194 e-01 \\
2.1275 e-01 \\
3.3510 e-01 \\
1.9696 e-01 \\
2.3772 e-01 \\
1.4922 e-01 \\
2.6295 e-01 \\
2.1130 e-01 \\
2.2311 e-01 \\
1.8201 e-01 \\
2.2477 e-01 \\
1.9829 e-01 \\
6.9053 e-04 \\
1.2041 e-03 \\
2.6562 e-03 \\
1.7115 e-03 \\
2.2146 e-03 \\
6.9053 e-04 \\
2.1635 e-03 \\
1.1714 e-03 \\
0.0000 e+00 \\
9.6655 e-04 \\
1.7115 e-03 \\
9.6655 e-04 \\
1.3381 e-03 \\
6.9053 e-04 \\
2.4164 e-03 \\
6.9053 e-04 \\
1.3381 e-03 \\
1.6028 e-03 \\
1.5314 e-03 \\
2.3941 e-03 \\
2.2653 e-01 \\
1.0110 e-01 \\
2.4517 e-01 \\
1.3965 e-01 \\
1.1008 e-01 \\
1.3579 e-01 \\
9.4628 e-02 \\
1.1839 e-01 \\
1.3894 e-01 \\
1.3083 e-01 \\
1.1315 e-01 \\
1.0065 e-01 \\
1.3890 e-01 \\
1.5795 e-01 \\
1.7081 e-01 \\
1.3807 e-01 \\
1.0478 e-01 \\
1.5901 e-01 \\
1.1152 e-01 \\
4.7121 e-02 \\
4.2742 e-02\end{array}$ & 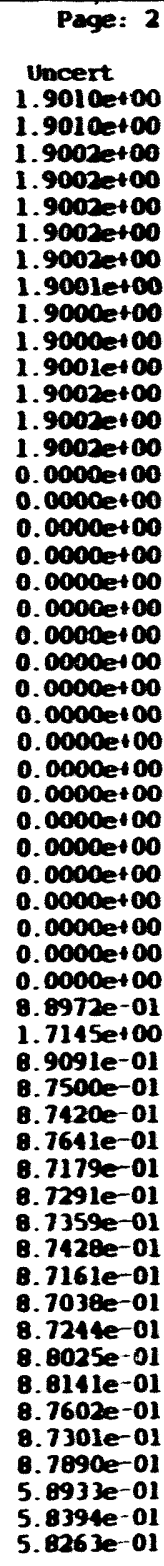 \\
\hline
\end{tabular}




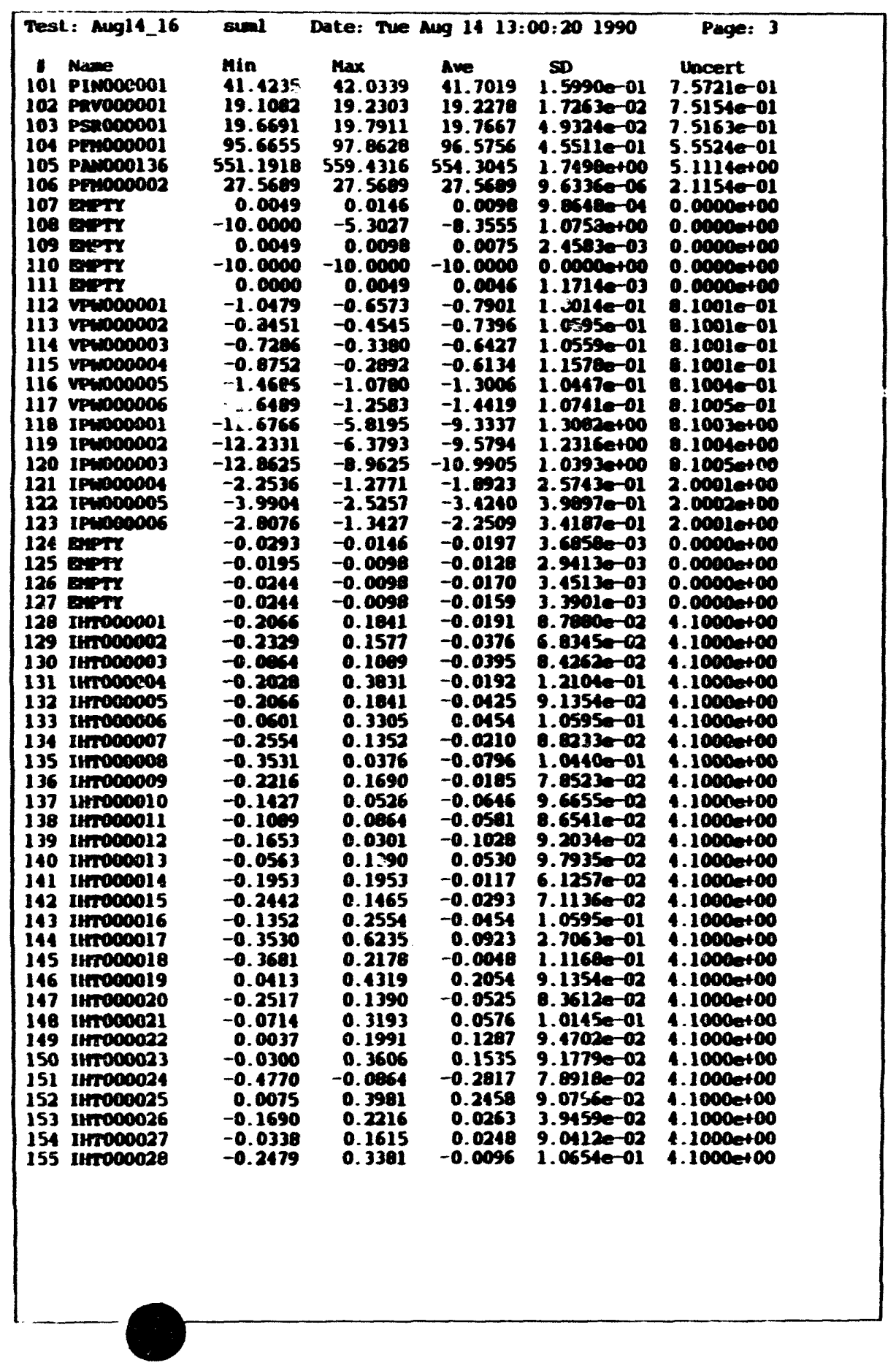

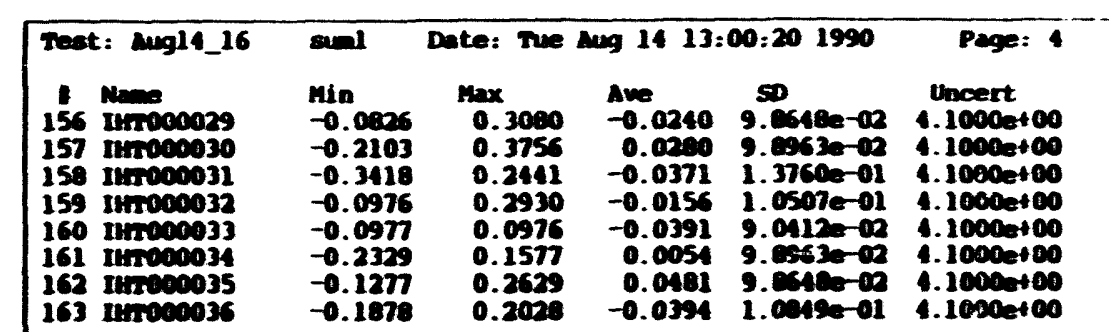



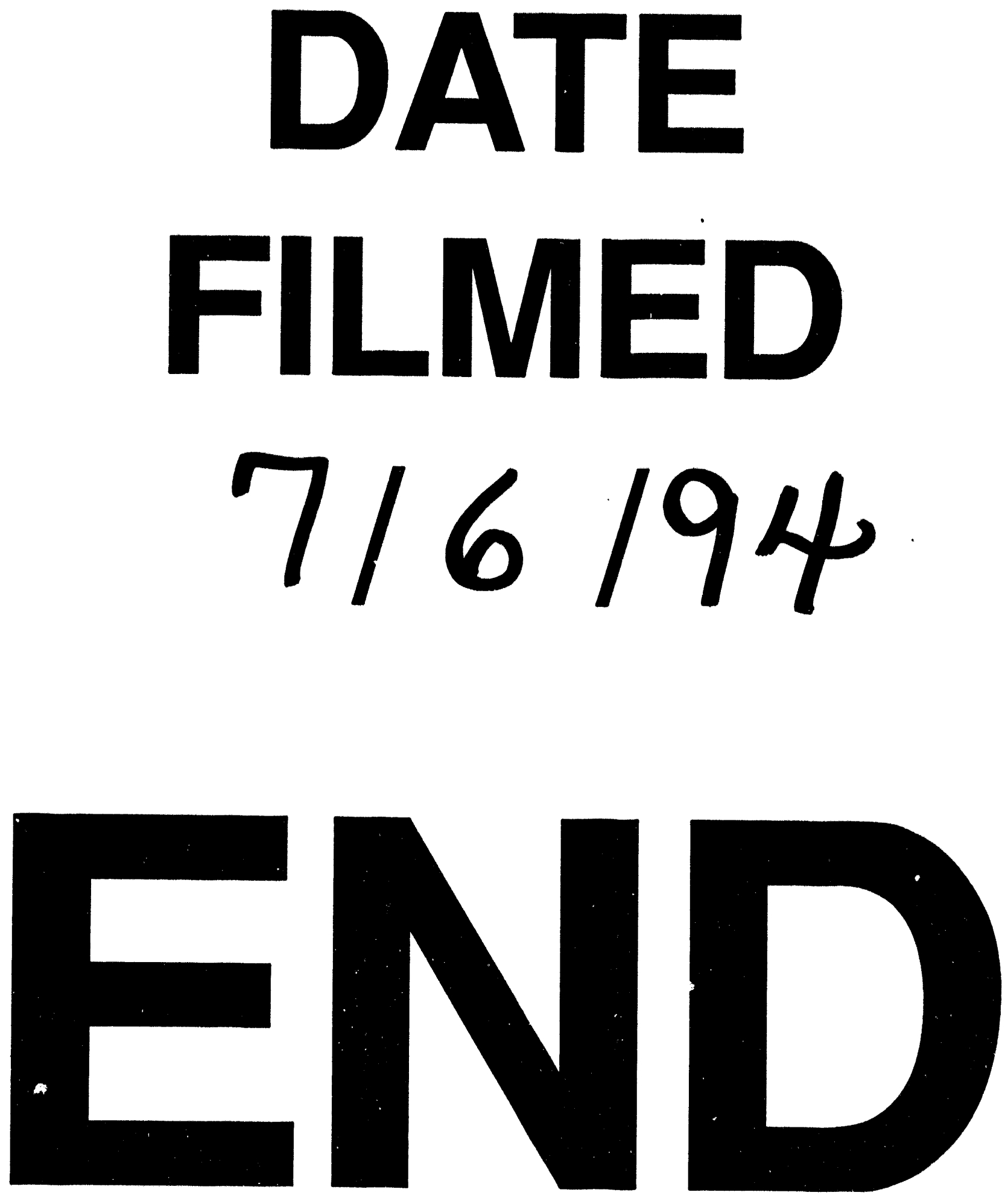